\title{
General Method for Enantioselective Three-Component Carboarylation of Alkenes Enabled by Visible-Light Dual Photoredox/Nickel Catalysis
}

Lei Guo, $\uparrow, \S$ Mingbin Yuan,,$\S \S$ Yanyan Zhang, $\dagger$ Fang Wang, $\uparrow$ Shengqing Zhu, $\uparrow$ Osvaldo Gutierrez,\$,* Lingling Chu†,**

$\dagger$ State Key Laboratory for Modification of Chemical Fibers and Polymer Materials, College of Chemistry, Chemical Engineering and Biotechnology, Center for Advanced Low-Dimension Materials, Donghua University, Shanghai 201620, China $\$$ Department of Chemistry and Biochemistry, University of Maryland, College Park, Maryland 20742, United States 


\section{Table of Contents}

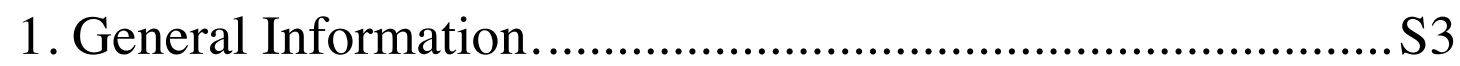

2. Optimization of the Reaction Conditions ...............................S4

3. General Procedures for photoredox/nickel-catalyzed

asymmetric, three-component 1,2-alkylarylation of alkenes ....S8

5. Crystallographic Data for Compound 28 ……..................S107

6. Synthesis of derivatives of piragliatin and esflurbiprofen S114

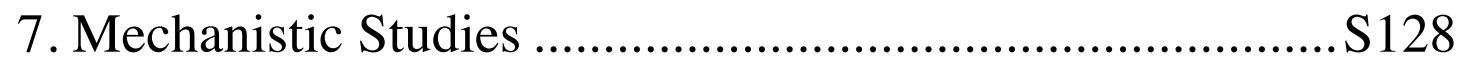

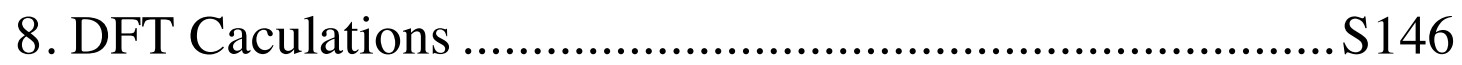

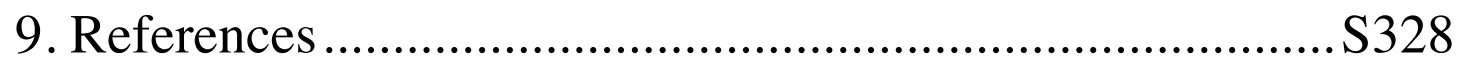

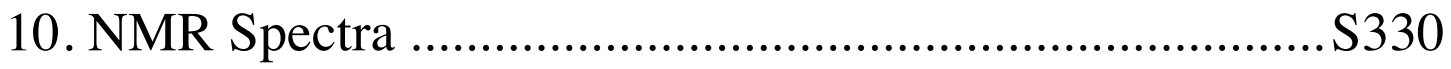




\section{General Information.}

Commercial reagents were purchased from Adamas, Aldrich, TCI, Energy Chemical, Bide, Leyan and J\&K chemical, and were used as received. All reactions were carried out in oven-dried glassware under an atmosphere of nitrogen unless otherwise noted. Chromatographic purification of products was accomplished by flash chromatography using silica gel. Thin-layer chromatography (TLC) was performed on Silicycle $250 \mathrm{~mm}$ silica gel F-254 plates. ${ }^{1} \mathrm{H},{ }^{13} \mathrm{C}$ and ${ }^{19} \mathrm{~F}$ NMR spectra were recorded on Bruker 400 (400, 100 and $375 \mathrm{MHz}$ ) and Bruker $600(600,150$ and $565 \mathrm{MHz})$, and are internally referenced to residual solvent signals (for $\mathrm{CDCl}_{3}, 7.26$ and $77.0 \mathrm{ppm}$, and for Acetoned6, 2.05, 29.84 and 206.26 ppm). Data for ${ }^{1} \mathrm{H}$ NMR and ${ }^{19} \mathrm{~F}$ NMR are reported as follows: chemical shift $(\delta \mathrm{ppm})$, multiplicity $(\mathrm{s}=$ singlet, $\mathrm{d}=$ doublet, $\mathrm{t}=$ triplet, $\mathrm{m}=$ multiplet, br $=$ broad), integration, coupling constant $(\mathrm{Hz}) \cdot{ }^{13} \mathrm{C}$ spectra were reported as chemical shifts in ppm and multiplicity where appropriate. High resolution mass spectra were obtained at Shanghai Institute of Organic Chemistry mass spectrometry facilities. IR spectra were collected with Bruker-TENSOR27 spectrometer and only major peaks were reported in $\mathrm{cm}^{-1}$. Optical rotations were measured on an automatic polarimeter with $[\alpha]_{\mathrm{D}}^{25}$ values reported in degress; concentration (c) is in $\mathrm{g} / 100 \mathrm{~mL}$. Photochemical experiments have been performed using $10 \mathrm{~W}$ 415-420 nm LED light (commercialized from WATTCAS ${ }^{\mathrm{TM}}$ ). High-pressure liquid chromatography (HPLC) analysis on chiral stationary phase was performed on an Agilent 1260-series instrument. Chiralpak IA-H, IB-H, IC-H, or IG-H columns with hexane: ${ }^{i} \mathrm{PrOH}$ as the eluents were used. Alkenes ${ }^{1}$ and alkyl trifluoroborates ${ }^{2}$ were prepared according to the previously reported procedure. 


\section{Optimization of the Reaction Conditions}

Table S1. Chiral ligand effect.

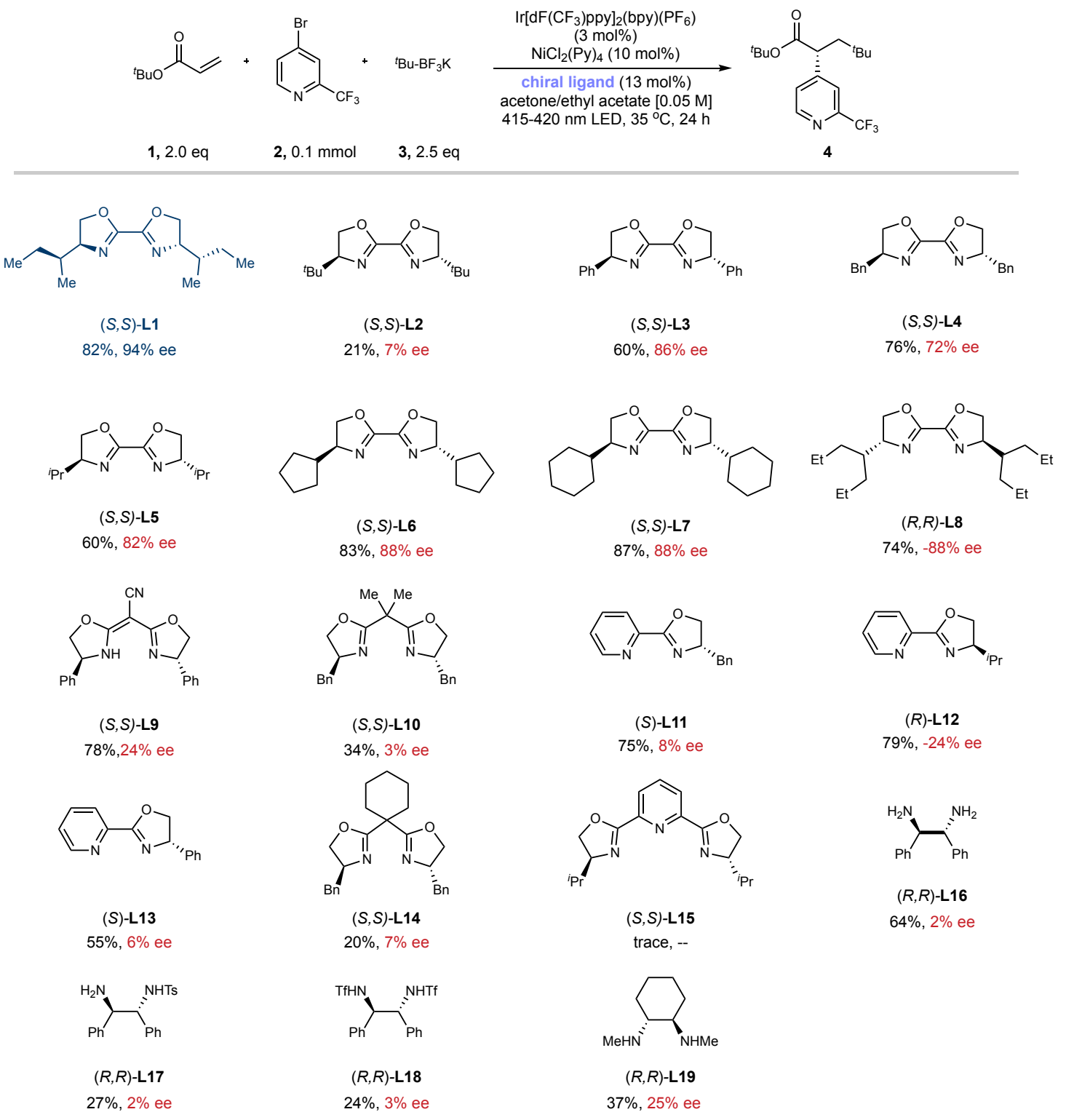


Table S2. Control experiment

\begin{tabular}{|c|c|c|c|}
\hline & & $\begin{array}{c}\operatorname{Ir}\left[\mathrm{dF}\left(\mathrm{CF}_{3}\right) \mathrm{ppyy}\right]_{2}(\mathrm{bpy})\left(\mathrm{PF}_{6}\right) \\
(3 \mathrm{~mol}) \\
\mathrm{NiCl}_{2}(\mathrm{Py})_{4}(10 \mathrm{~mol} \%) \\
(\mathrm{S}, \mathrm{S}) \cdot \mathrm{L} 1(13 \mathrm{~mol} \%) \\
\text { acetone/ethyl acetate }[0.05 \mathrm{M}] \\
415-420 \mathrm{~nm} \text { LED, } 35{ }^{\circ} \mathrm{C}, 24 \mathrm{~h}\end{array}$ & \\
\hline Entry & Control reaction & GC yield & ee \\
\hline 1 & w/o photocatalyst & $0 \%$ & -- \\
\hline 2 & w/o nickel catalyst & $0 \%$ & -- \\
\hline 3 & w/o ligand & $0 \%$ & -- \\
\hline 4 & w/o light & $0 \%$ & -- \\
\hline
\end{tabular}

Table S3. Photocatalyst effect.

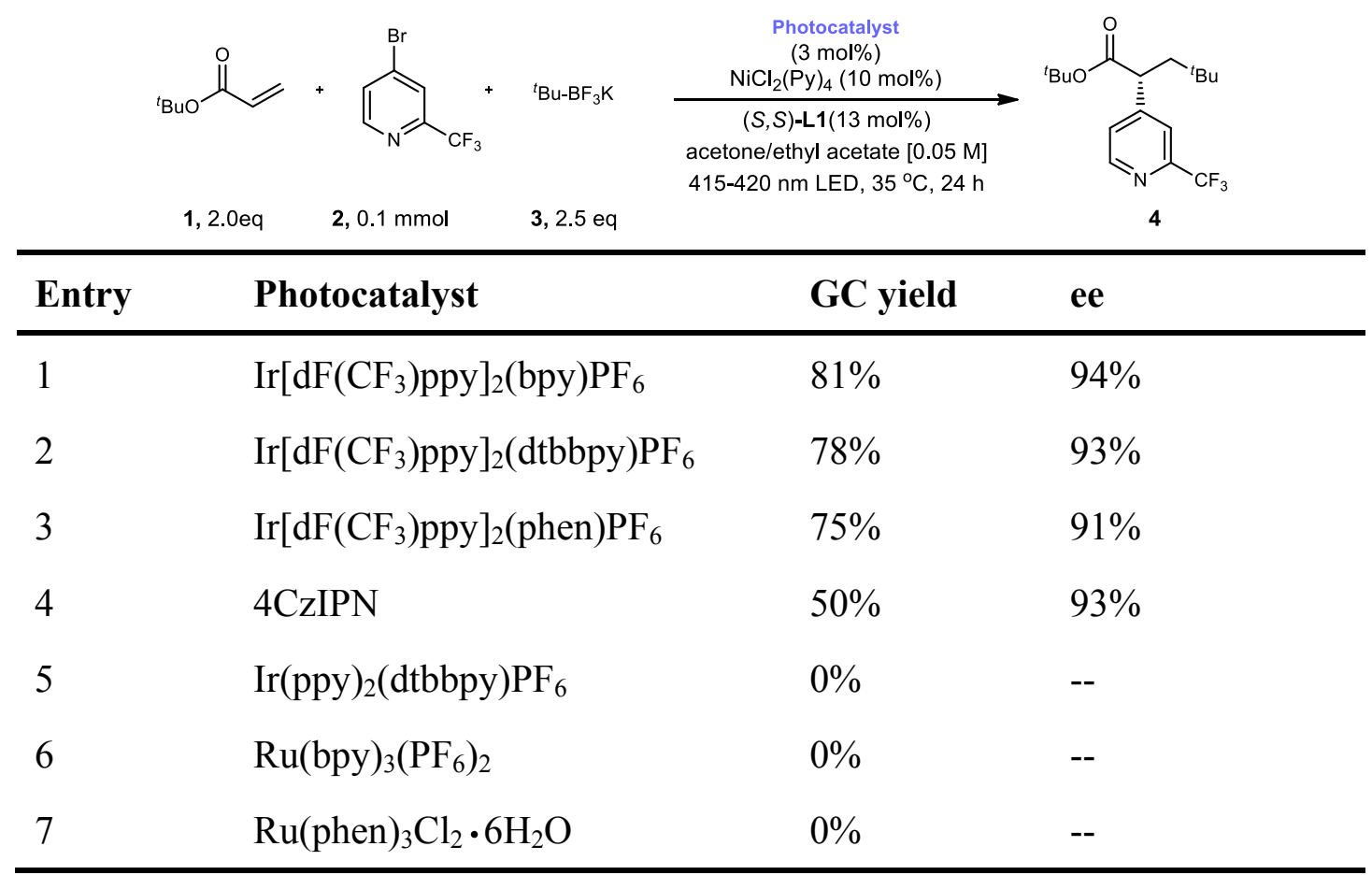


Table S4. Nickel catalyst effect.

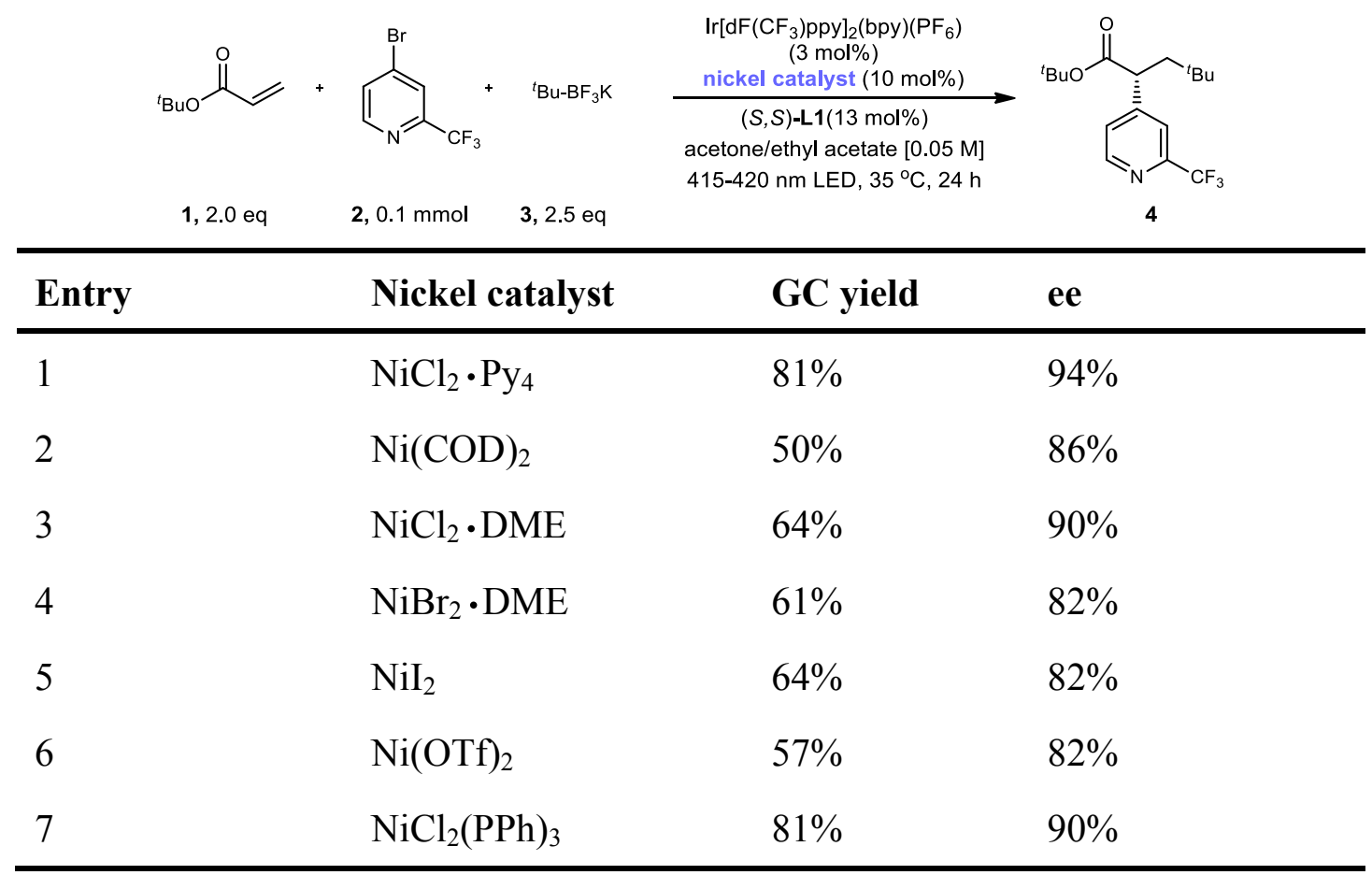

Table S5. Solvent effect.

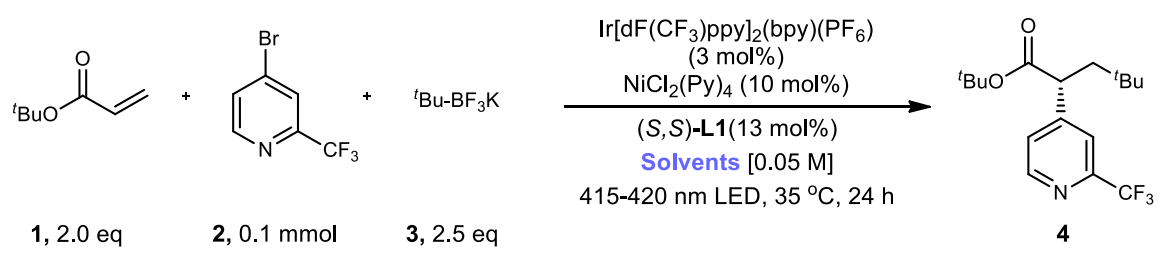

\begin{tabular}{|c|c|c|c|}
\hline Entry & Solvents & GC yield & ee \\
\hline 1 & DMSO & $6 \%$ & -- \\
\hline 2 & DMA & $58 \%$ & $66 \%$ \\
\hline 3 & Hexane & $0 \%$ & -- \\
\hline 4 & Toluene & $0 \%$ & -- \\
\hline 5 & acetone & $77 \%$ & $93 \%$ \\
\hline 6 & DME & $85 \%$ & $93 \%$ \\
\hline 7 & THF & $89 \%$ & $92 \%$ \\
\hline 8 & ethyl acetate & $84 \%$ & $90 \%$ \\
\hline 9 & acetone/ethyl acetate $2: 1$ & $83 \%$ & $94 \%$ \\
\hline
\end{tabular}


Table S6. Temperature effect.

\begin{tabular}{|c|c|c|c|c|c|}
\hline & ${ }^{\mathrm{B}} \mathrm{B}$ & & ${ }^{\mathrm{t}} \mathrm{Bu}^{-\mathrm{BF}_{3} \mathrm{~K}}$ & 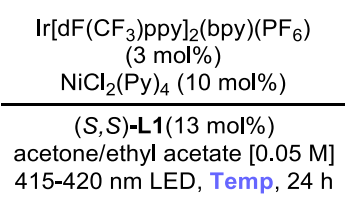 & \\
\hline & $1,2.0$ eq & 2, $0.1 \mathrm{mmol}$ & $3,2.5 \mathrm{eq}$ & & \\
\hline Entry & & Temp & & GC yield & ee \\
\hline 1 & & $30^{\circ} \mathrm{C}$ & & $76 \%$ & $94 \%$ \\
\hline 2 & & $20^{\circ} \mathrm{C}$ & & $81 \%$ & $95 \%$ \\
\hline 3 & & $10^{\circ} \mathrm{C}$ & & $82 \%$ & $95 \%$ \\
\hline 4 & & $0^{\circ} \mathrm{C}$ & & $73 \%$ & $95 \%$ \\
\hline 5 & & $-10^{\circ} \mathrm{C}$ & & $64 \%$ & $96 \%$ \\
\hline
\end{tabular}

Table S7. LED light effect (WATTCAS ${ }^{\text {TM }}$ WP-TEC-1020SL)<smiles>CCC(C)(C)C(=O)C=CC(=O)OC(C)(C)C(F)(F)c1ccnc(Br)c1</smiles>
$1,2.0 \mathrm{eq}$ 2, $0.1 \mathrm{mmol}$

$3,2.5$ eq

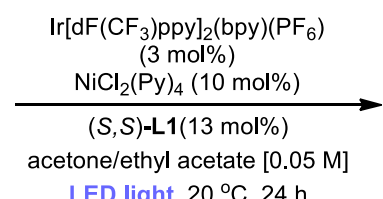

LED light, $20^{\circ} \mathrm{C}, 24 \mathrm{~h}$

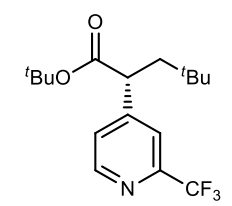

4

\begin{tabular}{|c|c|c|c|}
\hline Entry & LED light & GC yield & ee \\
\hline 1 & $90 \mathrm{~W}$ blue LED $33-35^{\circ} \mathrm{C}$ & $83 \%$ & $94 \%$ \\
\hline 2 & $10 \mathrm{~W} 395-400 \mathrm{~nm} 20^{\circ} \mathrm{C}$ & $74 \%$ & $89 \%$ \\
\hline 3 & $10 \mathrm{~W} 400-405 \mathrm{~nm} 20^{\circ} \mathrm{C}$ & $80 \%$ & $92 \%$ \\
\hline 4 & $10 \mathrm{~W} 415-420 \mathrm{~nm} 20^{\circ} \mathrm{C}$ & $81 \%$ & $95 \%$ \\
\hline 5 & $10 \mathrm{~W} 435-440 \mathrm{~nm} 20^{\circ} \mathrm{C}$ & $80 \%$ & $93 \%$ \\
\hline 6 & $10 \mathrm{~W} 460-465 \mathrm{~nm} 20^{\circ} \mathrm{C}$ & $82 \%$ & $93 \%$ \\
\hline
\end{tabular}




\section{General Procedures for photoredox/nickel-catalyzed asymmetric, three-component 1,2-alkylarylation of alkenes}

To a flame-dried $8 \mathrm{~mL}$ reaction vial was charged with (4S,4'S)-4,4'-di ((S)-sec-butyl)4,4',5,5'-tetrahydro-2,2'-bioxazole [(S,S)-L1] $(0.013 \mathrm{mmol}, 13 \mathrm{~mol} \%)$ [Note: 4,4'-ditert-butyl-2,2'-bipyridine (dtbbpy) for the corresponding racemic reactions], $\mathrm{NiCl}_{2} \bullet \mathrm{Py}_{4}$

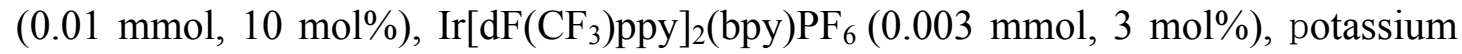
alkyl trifluoroborate $(0.25 \mathrm{mmol}, 2.5$ equiv. $)$, aryl bromide $(0.1 \mathrm{mmol}, 1.0$ equiv., if solid), the vial was capped. After evacuated and backfilled nitrogen three times, acetone/ethyl acetate 2:1 [0.05 M] was added via a syringe, aryl bromide $(0.1 \mathrm{mmol}$, 1.0 equiv., if liquid), followed by the addition of olefins ( $0.2 \mathrm{mmol}, 2.0$ equiv.). The reaction mixture was then irradiated with a $10 \mathrm{~W}$ 415-420nm LED lamp with cooling from circulating cold ethanol for $24 \mathrm{~h}$ at $20^{\circ} \mathrm{C}$. The reaction was quenched with $\mathrm{H}_{2} \mathrm{O}$, extracted with ethyl acetate. The combined organic layers were dried with $\mathrm{Mg}_{2} \mathrm{SO}_{4}$, filtered, and concentrated in vacuo. The crude material was purified by flash chromatography to afford the product.

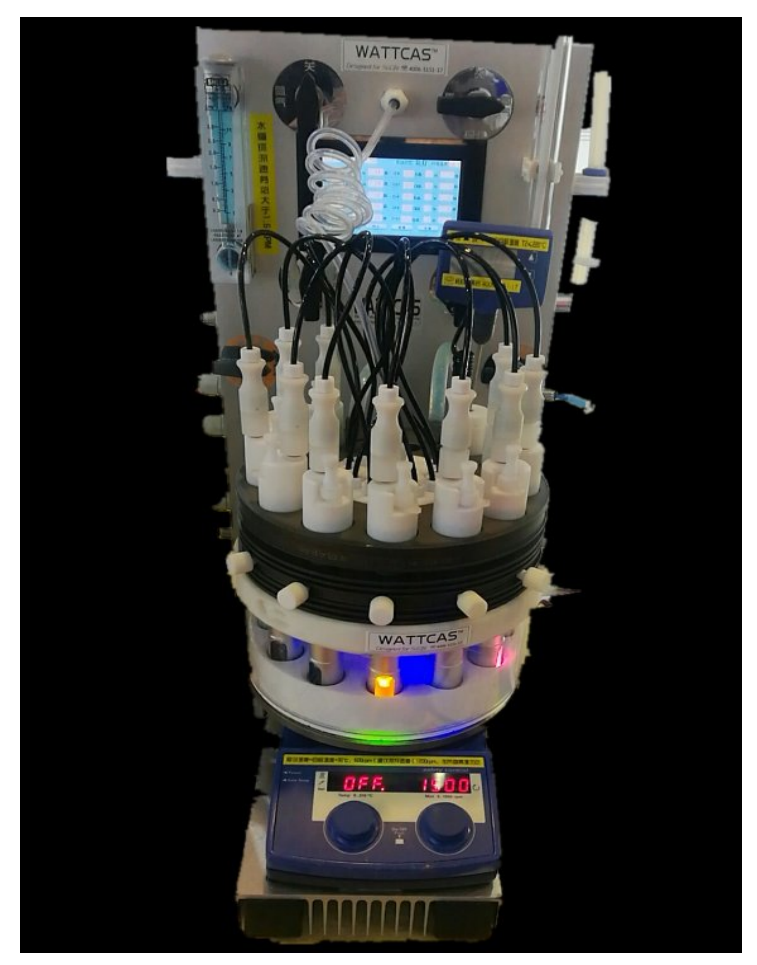

Figure S1 Experimental setup 


\section{Characterization Data for Products}<smiles>CC(C)(C)C[C@@H](C(=O)OC(C)(C)C)c1ccnc(C(F)(F)F)c1</smiles>

\section{(R)-tert-Butyl 4,4-dimethyl-2-(2-(trifluoromethyl)pyridin-4-yl)pentanoate (4)}

The product was prepared according to the general procedure with (4S,4'S)-4,4'-di((S)sec-butyl)-4,4',5,5'-tetrahydro-2,2'-bioxazole [(S,S)-L1] $(3.3 \mathrm{mg}, 0.013 \mathrm{mmol}, 13$ mol\%), $\mathrm{NiCl}_{2} \bullet \mathrm{Py}_{4}(4.5 \mathrm{mg}, 0.01 \mathrm{mmol}, 10 \mathrm{~mol} \%), \operatorname{Ir}\left[\mathrm{dF}_{\left(\mathrm{CF}_{3}\right) \text { ppy }}\right]_{2}(\mathrm{bpy}) \mathrm{PF}_{6}(3.2 \mathrm{mg}$, $0.003 \mathrm{mmol}, 3 \mathrm{~mol} \%$ ), 4-bromo-2-(trifluoromethyl)pyridine (13.7 uL, $0.1 \mathrm{mmol}, 1.0$ equiv.), ${ }^{t} \mathrm{BuBF}_{3} \mathrm{~K}$ (41.0 mg, $0.25 \mathrm{mmol}, 2.5$ equiv.) and tert-butyl acrylate (29.0 uL, 0.2 mmol, 2.0 equiv.) in acetone/ethyl acetate (1.3/0.7, v/v, $2 \mathrm{~mL})$ were used. After 24 hours, the product was isolated by flash chromatography (PE: ethyl acetate $=20: 1$ ) as a colorless oil ( $27.3 \mathrm{mg}, 82 \%$ yield, $95 \%$ ee).

${ }^{1} \mathbf{H}$ NMR $\left(400 \mathrm{MHz}, \mathrm{CDCl}_{3}\right) \delta 8.63(\mathrm{~d}, J=5.0 \mathrm{~Hz}, 1 \mathrm{H}), 7.62(\mathrm{br}, 1 \mathrm{H}), 7.43(\mathrm{dd}, J=5.0$, $1.3 \mathrm{~Hz}, 1 \mathrm{H}), 3.60(\mathrm{dd}, J=9.2,3.5 \mathrm{~Hz}, 1 \mathrm{H}), 2.28(\mathrm{dd}, J=14.0,9.2 \mathrm{~Hz}, 1 \mathrm{H}), 1.47(\mathrm{dd}, J$ $=14.0,3.5 \mathrm{~Hz}, 1 \mathrm{H}), 1.38(\mathrm{~s}, 9 \mathrm{H}), 0.92(\mathrm{~s}, 9 \mathrm{H})$.

${ }^{19}$ F NMR $\left(375 \mathrm{MHz}, \mathrm{CDCl}_{3}\right) \delta-67.99(\mathrm{~s})$

${ }^{13}$ C NMR (100 MHz, $\left.\mathrm{CDCl}_{3}\right) \delta 171.77,152.40,150.13,148.50(\mathrm{q}, J=34.4 \mathrm{~Hz}), 125.55$, $121.50(\mathrm{q}, J=274.4 \mathrm{~Hz}), 119.77(\mathrm{q}, J=2.7 \mathrm{~Hz}), 81.73,49.04,46.83,31.19,29.38$, 27.72 .

HRMS (ESI): $\mathrm{C}_{17} \mathrm{H}_{25} \mathrm{~F}_{3} \mathrm{NO}_{2}^{+}(\mathrm{M}+\mathrm{H}): 332.1837$, found: 332.1827 .

$[\alpha]_{\mathrm{D}}^{25}=-12.4613\left(\mathrm{c}=0.2600, \mathrm{CHCl}_{3}\right)$

Chiral HPLC: CHIRALPAK IC, $25{ }^{\circ} \mathrm{C},{ }^{i} \mathrm{PrOH}-$ hexanes 5/95, $0.5 \mathrm{~mL} / \mathrm{min}, 254 \mathrm{~nm}, \mathrm{t}_{\mathrm{R}}$ (major) $=9.3 \mathrm{~min}, \mathrm{t}_{\mathrm{R}}($ minor $)=8.2 \mathrm{~min}$. 


\section{4 racemic}

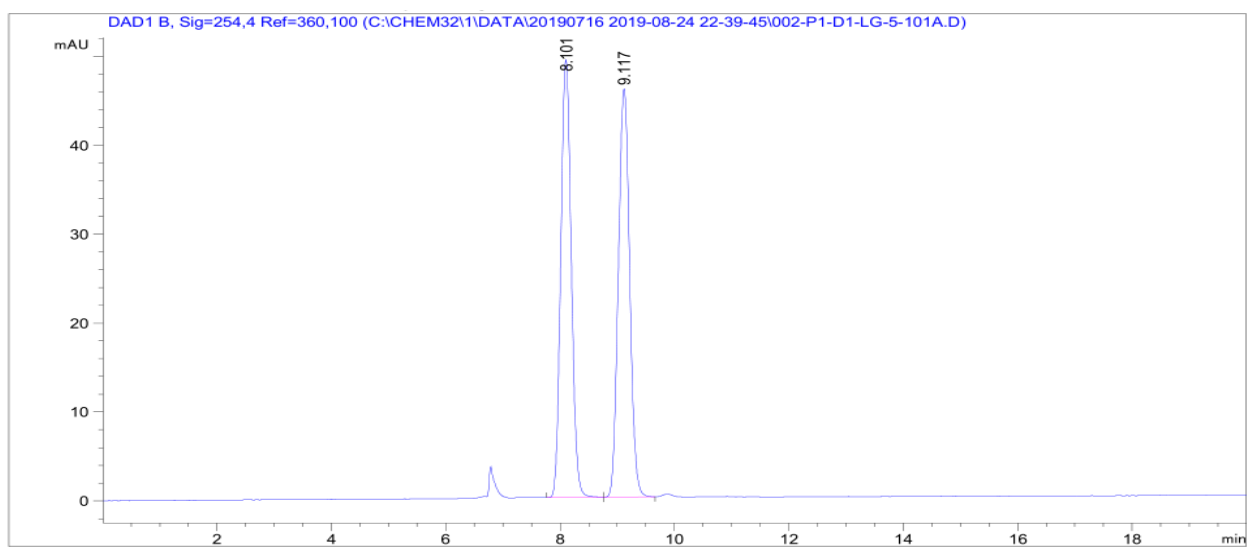

$\begin{array}{cccccc}\begin{array}{c}\text { Peak RetTime Type } \\ \text { \# } \\ {[\mathrm{min}]}\end{array} & \begin{array}{c}\text { Width } \\ {[\mathrm{min}]}\end{array} & \begin{array}{c}\text { Area } \\ {[\mathrm{mAU} \text { s }]}\end{array} & \begin{array}{c}\text { Height } \\ {[\mathrm{mAU}]}\end{array} & \begin{array}{c}\text { Area } \\ \%\end{array} \\ 1 & 8.101 \text { BB } & 0.2009 & 629.11060 & 49.30000 & 49.8690 \\ 2 & 9.117 \text { BB } & 0.2166 & 632.41559 & 45.92959 & 50.1310\end{array}$

\section{4 enantioenriched, $95 \%$ ee}

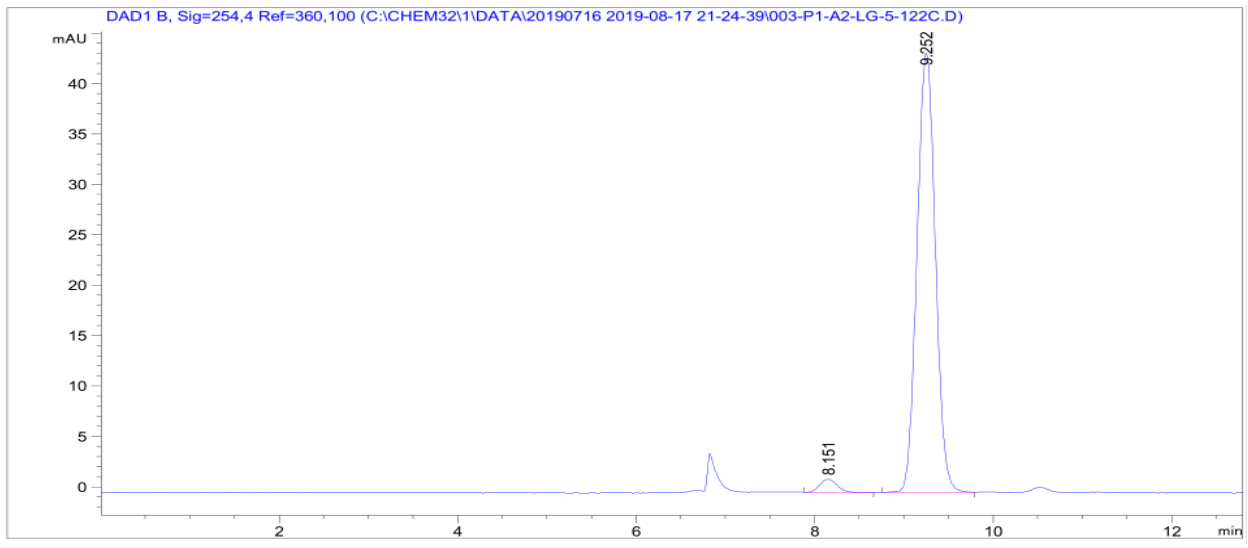

\begin{tabular}{cccccc}
$\begin{array}{c}\text { Peak RetTime Type } \\
\text { \# } \\
{[\mathrm{min}]}\end{array}$ & $\begin{array}{c}\text { Width } \\
{[\mathrm{min}]}\end{array}$ & $\begin{array}{c}\text { Area } \\
{[\mathrm{mAU} \text { s }]}\end{array}$ & $\begin{array}{c}\text { Height } \\
{[\mathrm{mAU}]}\end{array}$ & $\begin{array}{c}\text { Area } \\
\%\end{array}$ \\
\hline 1 & 8.151 BB & 0.2105 & 17.39006 & 1.29610 & 2.7192 \\
2 & 9.252 BB & 0.2247 & 622.15021 & 43.54199 & 97.2808
\end{tabular}<smiles>CO[SH](=O)(OC)c1ccc([C@H](CC(C)(C)C)C(=O)OCC(C)(C)C)cc1</smiles> 


\section{(R)-tert-Butyl 4,4-dimethyl-2-(4-(methylsulfonyl)phenyl)pentanoate (5)}

The product was prepared according to the general procedure with (4S,4'S)-4,4'-di((S)sec-butyl)-4,4',5,5'-tetrahydro-2,2'-bioxazole [(S,S)-L1] $(3.3 \mathrm{mg}, 0.013 \mathrm{mmol}, 13$

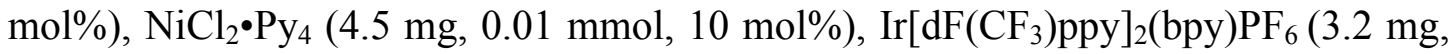
$0.003 \mathrm{mmol}, 3 \mathrm{~mol} \%$ ), 1-bromo-4-(methylsulfonyl)benzene (23.5 mg, $0.1 \mathrm{mmol}, 1.0$ equiv.), ${ }^{t} \mathrm{BuBF}_{3} \mathrm{~K}$ (41.0 mg, $0.25 \mathrm{mmol}, 2.5$ equiv.) and tert-butyl acrylate (29.0 uL, 0.2 mmol, 2.0 equiv.) in acetone/ethyl acetate (1.3/0.7, v/v, $2 \mathrm{~mL})$ were used. After 24 hours, the product was isolated by flash chromatography (PE: ethyl acetate $=20: 1)$ as a white solid (24.6 mg, 72\% yield, 97\% ee).

${ }^{1} \mathbf{H}$ NMR $\left(400 \mathrm{MHz}, \mathrm{CDCl}_{3}\right) \delta 7.87(\mathrm{~d}, J=8.3 \mathrm{~Hz}, 2 \mathrm{H}), 7.51(\mathrm{~d}, J=8.3 \mathrm{~Hz}, 2 \mathrm{H}), 3.62$ $(\mathrm{dd}, J=9.0,3.6 \mathrm{~Hz}, 1 \mathrm{H}), 3.05(\mathrm{~s}, 3 \mathrm{H}), 2.28(\mathrm{dd}, J=14.0,9.1 \mathrm{~Hz}, 1 \mathrm{H}), 1.48(\mathrm{dd}, J=$ 14.0, 3.6 Hz, 1H), 1.38 (s, 9H), $0.91(\mathrm{~s}, 9 \mathrm{H})$.

${ }^{13}$ C NMR $\left(100 \mathrm{MHz}, \mathrm{CDCl}_{3}\right) \delta 172.730,147.940,138.970,128.710,127.620,81.190$, $49.360,47.200,44.510,31.160,29.440,27.800$.

HRMS (ESI): $\mathrm{C}_{18} \mathrm{H}_{29} \mathrm{O}_{4} \mathrm{~S}^{+}(\mathrm{M}+\mathrm{H}): 341.1787$, found: 341.1785 .

$[\alpha]_{\mathrm{D}}^{25}=-20.2322\left(\mathrm{c}=0.4300, \mathrm{CHCl}_{3}\right)$

Chiral HPLC: CHIRALPAK IB, $25{ }^{\circ} \mathrm{C},{ }^{i} \mathrm{PrOH}-h$ exanes $1 / 100,0.5 \mathrm{~mL} / \mathrm{min}, 230 \mathrm{~nm}$, $t_{R}($ major $)=42.4 \mathrm{~min}, t_{R}($ minor $)=45.5 \mathrm{~min}$.

\section{5 racemic}

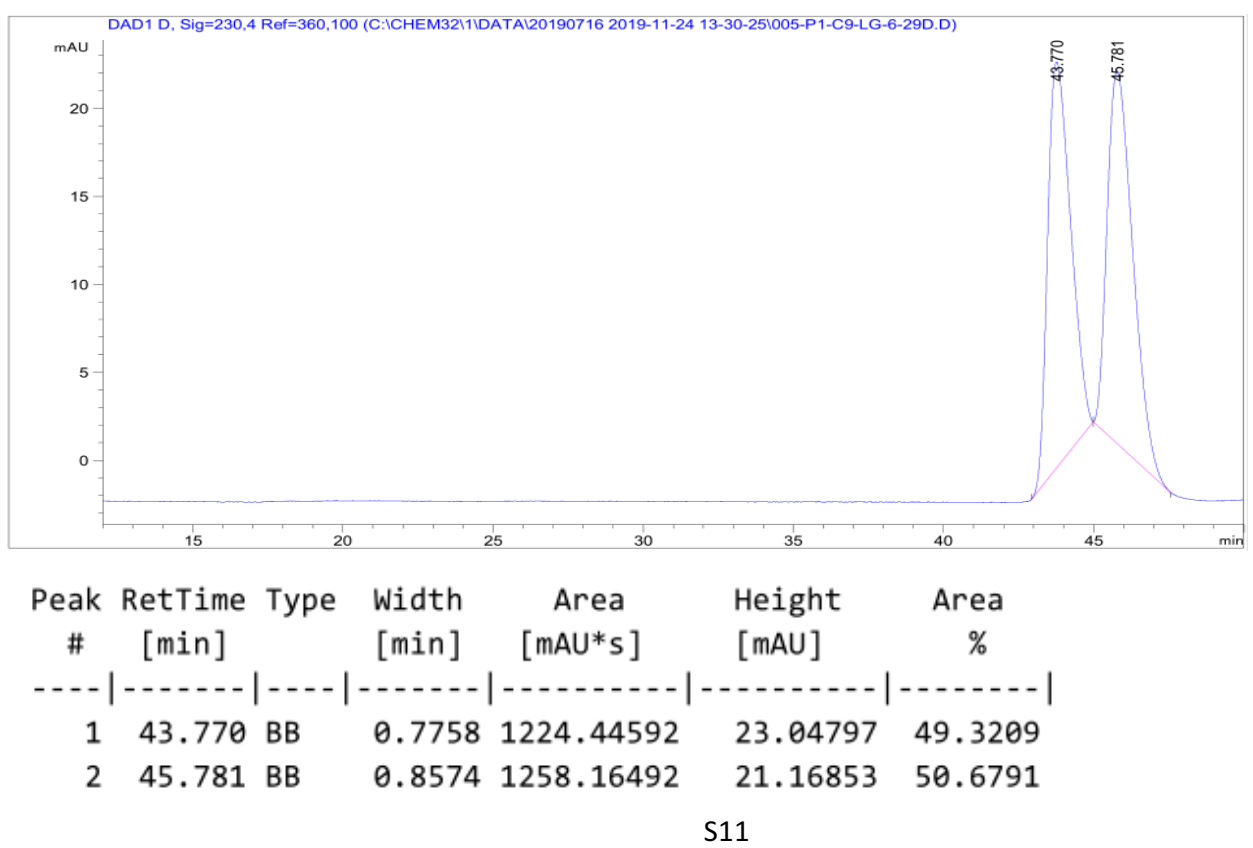



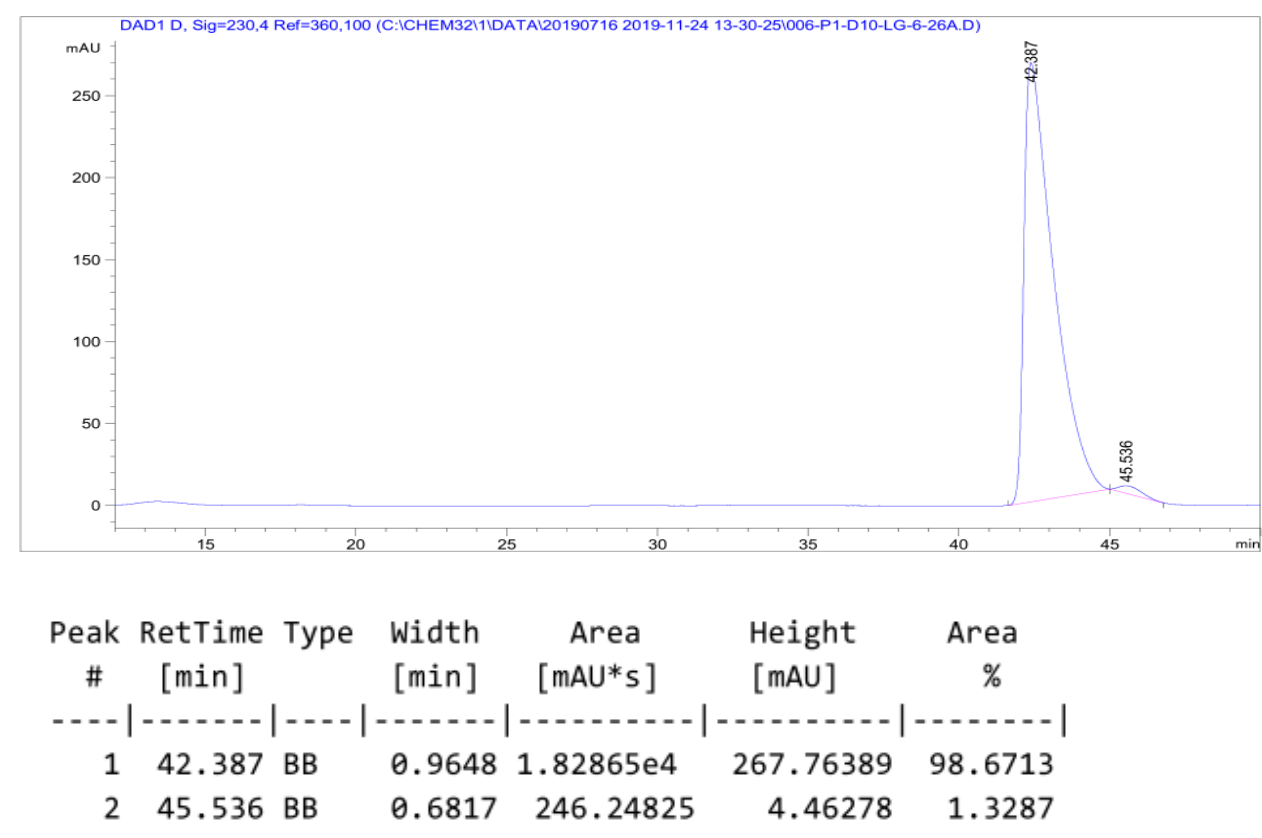<smiles>COC(=O)c1ccc(C(CC(C)(C)C)C(=O)OC(C)(C)C)cc1</smiles>

\section{(R)-Methyl 4-(1-(tert-butoxy)-4,4-dimethyl-1-oxopentan-2-yl)benzoate (6)}

The product was prepared according to the general procedure with (4S,4'S)-4,4'-di((S)sec-butyl)-4,4',5,5'-tetrahydro-2,2'-bioxazole [(S,S)-L1] (3.3 mg, $0.013 \mathrm{mmol}, 13$ mol\%), $\mathrm{NiCl}_{2} \bullet \mathrm{Py}_{4}(4.5 \mathrm{mg}, 0.01 \mathrm{mmol}, 10 \mathrm{~mol} \%), \operatorname{Ir}\left[\mathrm{dF}\left(\mathrm{CF}_{3}\right) \mathrm{ppy}\right]_{2}(\mathrm{bpy}) \mathrm{PF}_{6}(3.2 \mathrm{mg}$, $0.003 \mathrm{mmol}, 3 \mathrm{~mol} \%$ ), methyl 4-bromobenzoate (21.5 mg, $0.1 \mathrm{mmol}, 1.0$ equiv.), ${ }^{t} \mathrm{BuBF}_{3} \mathrm{~K}$ (41.0 mg, $0.25 \mathrm{mmol}, 2.5$ equiv.) and tert-butyl acrylate $(29.0 \mathrm{uL}, 0.2 \mathrm{mmol}$, 2.0 equiv.) in acetone/ethyl acetate $(1.3 / 0.7, \mathrm{v} / \mathrm{v}, 2 \mathrm{~mL})$ were used. After 24 hours, the product was isolated by flash chromatography (PE: ethyl acetate $=20: 1)$ as a white solid (22.5 mg, 70\% yield, 97\% ee).

${ }^{1} \mathbf{H}$ NMR $\left(400 \mathrm{MHz}, \mathrm{CDCl}_{3}\right) \delta 7.96(\mathrm{~d}, J=8.4 \mathrm{~Hz}, 2 \mathrm{H}), 7.37(\mathrm{~d}, J=8.3 \mathrm{~Hz}, 2 \mathrm{H}), 3.90$ (s, 3H), $3.58(\mathrm{dd}, J=9.0,3.7 \mathrm{~Hz}, 1 \mathrm{H}), 2.27(\mathrm{dd}, J=14.0,9.0 \mathrm{~Hz}, 1 \mathrm{H}), 1.50(\mathrm{dd}, J=$ 14.0, $3.8 \mathrm{~Hz}, 1 \mathrm{H}), 1.36(\mathrm{~s}, 9 \mathrm{H}), 0.90(\mathrm{~s}, 9 \mathrm{H})$. 
${ }^{13}$ C NMR (100 MHz, $\left.\mathrm{CDCl}_{3}\right) \delta 173.15,166.98,146.82,129.80,128.65,127.75,80.74$, 52.03, 49.41, 46.90, 31.09, 29.47, 27.78 .

HRMS (ESI): $\mathrm{C}_{19} \mathrm{H}_{29} \mathrm{O}_{4}{ }^{+}(\mathrm{M}+\mathrm{H}): 321.2066$, found: 321.2061 .

$[\alpha]_{\mathrm{D}}^{25}=-28.3204\left(\mathrm{c}=0.2567, \mathrm{CHCl}_{3}\right)$

Chiral HPLC: CHIRALPAK IC, $25{ }^{\circ} \mathrm{C},{ }^{i} \mathrm{PrOH}-$ hexanes 1/100, $0.3 \mathrm{~mL} / \mathrm{min}, 254 \mathrm{~nm}$, $t_{R}($ major $)=29.9 \min , t_{R}($ minor $)=27.9 \mathrm{~min}$.

\section{6 racemic}

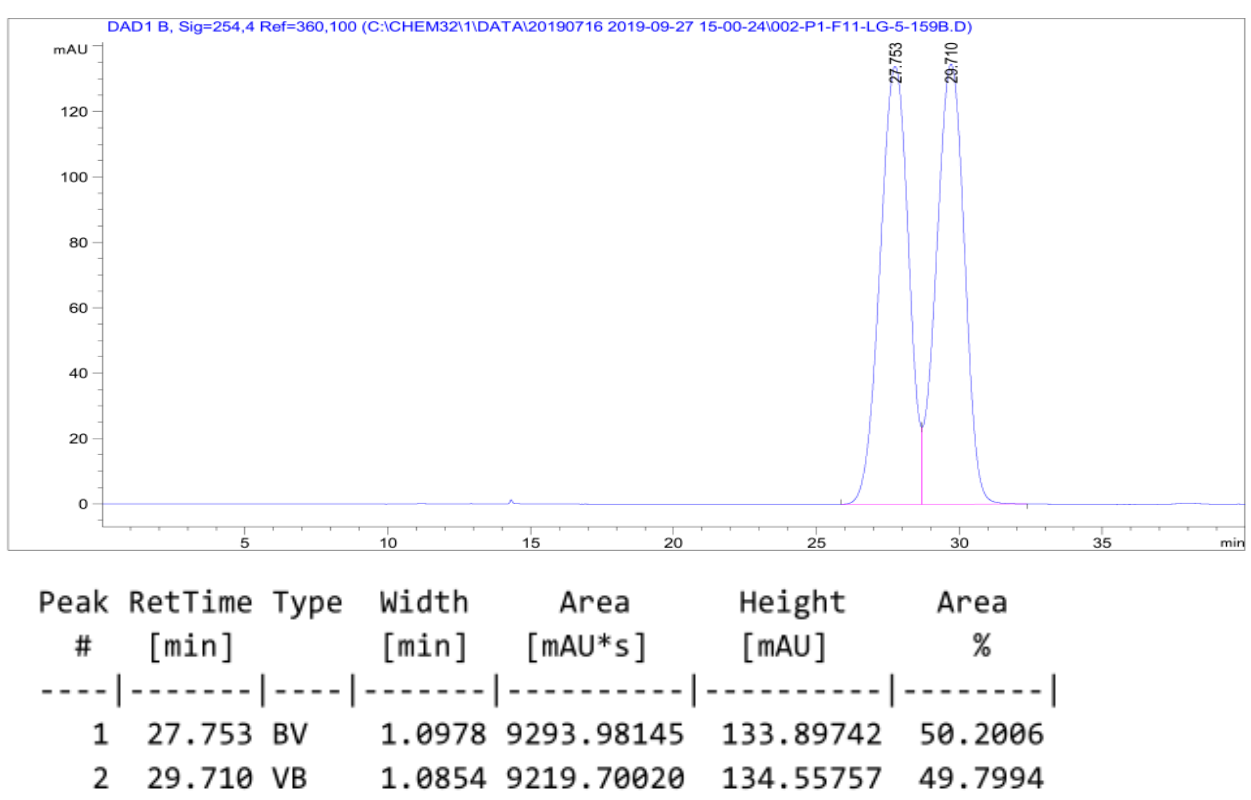

6 enantioenriched, $97 \%$ ee

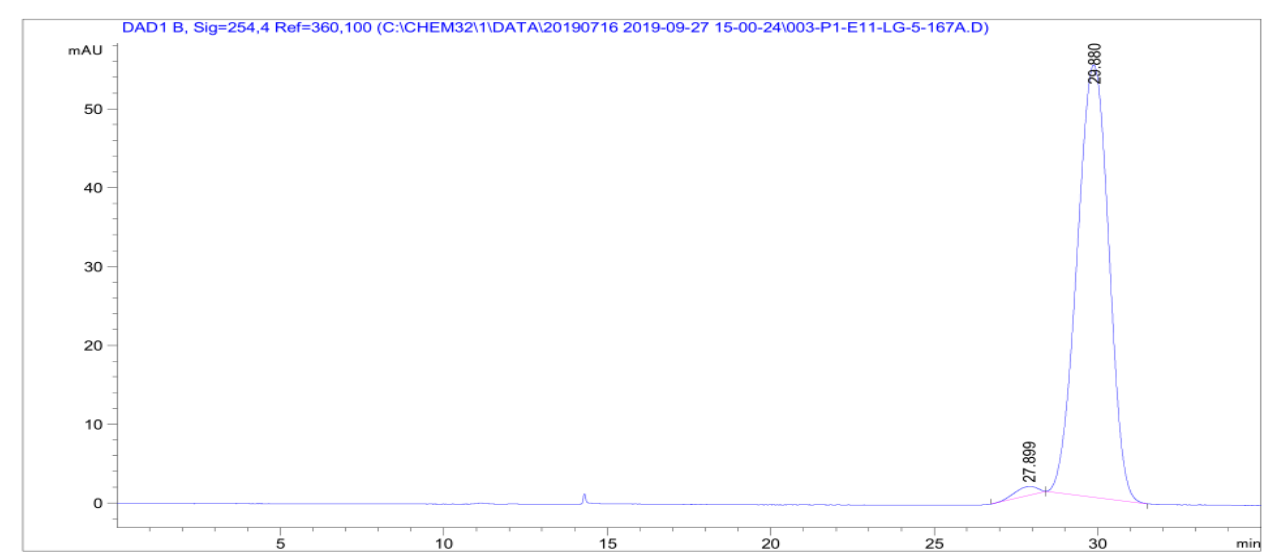




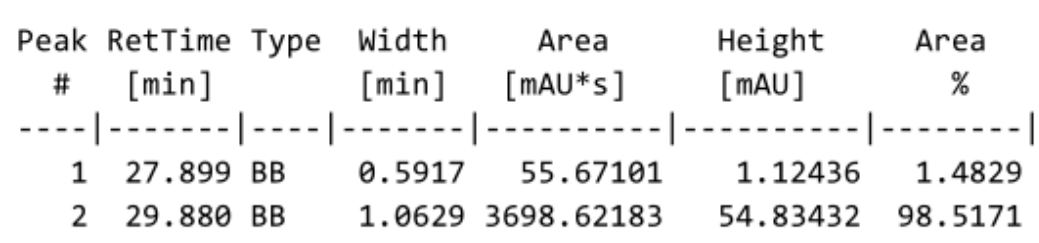<smiles>CC(C)(C)OC(=O)C(CBr)c1ccc(C(=O)c2ccccc2)cc1</smiles>

\section{(R)-tert-Butyl 2-(4-benzoylphenyl)-4,4-dimethylpentanoate (7)}

The product was prepared according to the general procedure with (4S,4'S)-4,4'-di((S)sec-butyl)-4,4',5,5'-tetrahydro-2,2'-bioxazole [(S,S)-L1] (3.3 mg, $0.013 \mathrm{mmol}, 13$ mol\%), $\mathrm{NiCl}_{2} \bullet \mathrm{Py}_{4}(4.5 \mathrm{mg}, 0.01 \mathrm{mmol}, 10 \mathrm{~mol} \%), \operatorname{Ir}\left[\mathrm{dF}\left(\mathrm{CF}_{3}\right) \mathrm{ppy}\right]_{2}(\mathrm{bpy}) \mathrm{PF}_{6}(3.2 \mathrm{mg}$, $0.003 \mathrm{mmol}, 3 \mathrm{~mol} \%$ ), (4-bromophenyl)(phenyl)methanone (26.1 mg, $0.1 \mathrm{mmol}, 1.0$ equiv.), ${ }^{t} \mathrm{BuBF}_{3} \mathrm{~K}$ (41.0 mg, $0.25 \mathrm{mmol}, 2.5$ equiv.) and tert-butyl acrylate (29.0 uL, 0.2 mmol, 2.0 equiv.) in acetone/ethyl acetate $(1.3 / 0.7, \mathrm{v} / \mathrm{v}, 2 \mathrm{~mL})$ were used. After 24 hours, the product was isolated by flash chromatography $(P E$ : ethyl acetate $=20: 1)$ as a white solid ( $25.6 \mathrm{mg}, 70 \%$ yield, $92 \%$ ee).

${ }^{1} \mathbf{H}$ NMR $\left(400 \mathrm{MHz}, \mathrm{CDCl}_{3}\right) \delta$ 7.80-7.74 (m, 4H), 7.60-7.56 (m, 1H), $7.47(\mathrm{t}, J=7.6$ $\mathrm{Hz}, 2 \mathrm{H}), 7.42$ (d, $J=8.3 \mathrm{~Hz}, 2 \mathrm{H}), 3.62(\mathrm{dd}, J=9.1,3.5 \mathrm{~Hz}, 1 \mathrm{H}), 2.30$ (dd, $J=14.0,9.2$ $\mathrm{Hz}, 1 \mathrm{H}), 1.53$ (dd, $J=14.0,3.6 \mathrm{~Hz}, 1 \mathrm{H}), 1.39$ (s, 9H), 0.93 (s, 9H).

${ }^{13}$ C NMR $\left(100 \mathrm{MHz}, \mathrm{CDCl}_{3}\right) \delta 196.35,173.16,146.43,137.69,136.01,132.29,130.41$, $129.95,128.23,127.63,80.81,49.40,47.10,31.12,29.47,27.81$.

HRMS (ESI): $\mathrm{C}_{24} \mathrm{H}_{31} \mathrm{O}_{3}{ }^{+}(\mathrm{M}+\mathrm{H})$ : 367.2273, found: 367.2261 .

$[\alpha]_{\mathrm{D}}^{25}=-16.7351\left(\mathrm{c}=0.3633, \mathrm{CHCl}_{3}\right)$

Chiral HPLC: CHIRALPAK IC, $25{ }^{\circ} \mathrm{C},{ }^{i} \mathrm{PrOH}-h$ exanes 5/95, $0.5 \mathrm{~mL} / \mathrm{min}, 254 \mathrm{~nm}, \mathrm{t}_{\mathrm{R}}$ (major) $=17.2 \mathrm{~min}, \mathrm{t}_{\mathrm{R}}($ minor $)=16.4 \mathrm{~min}$. 


\section{7 racemic}

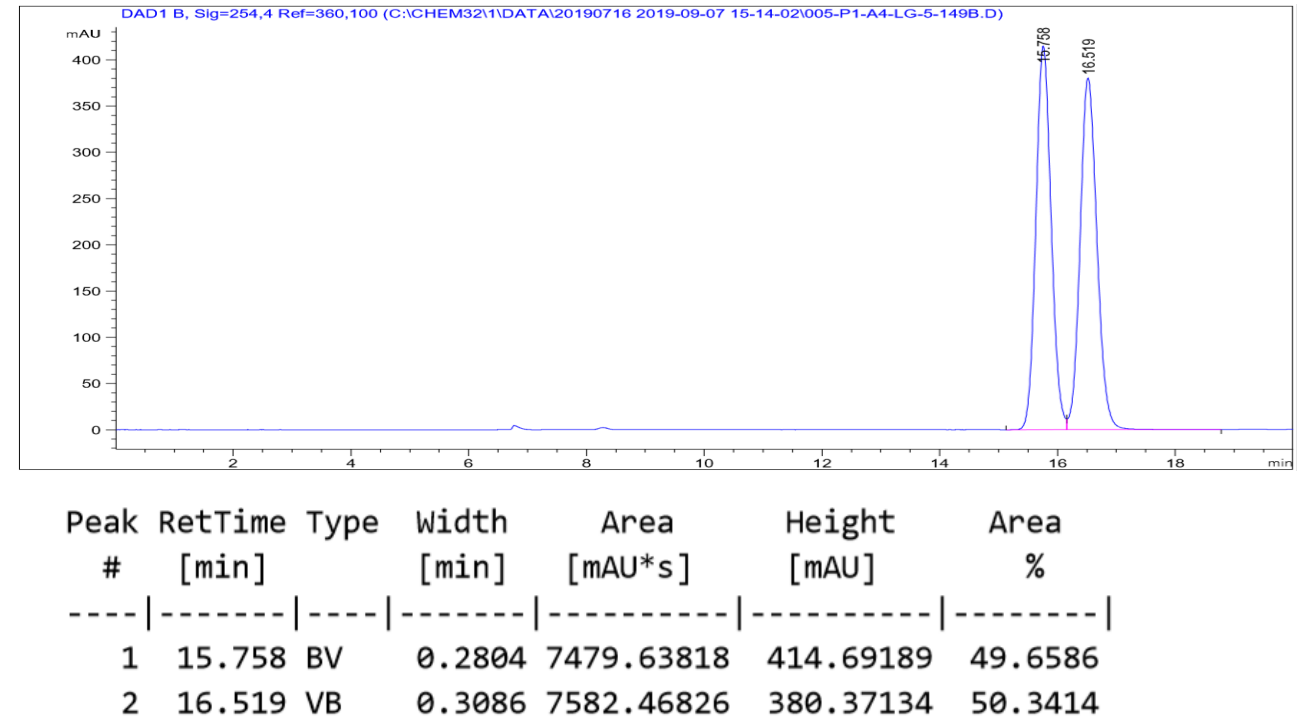

7 enantioenriched, $92 \%$ ee

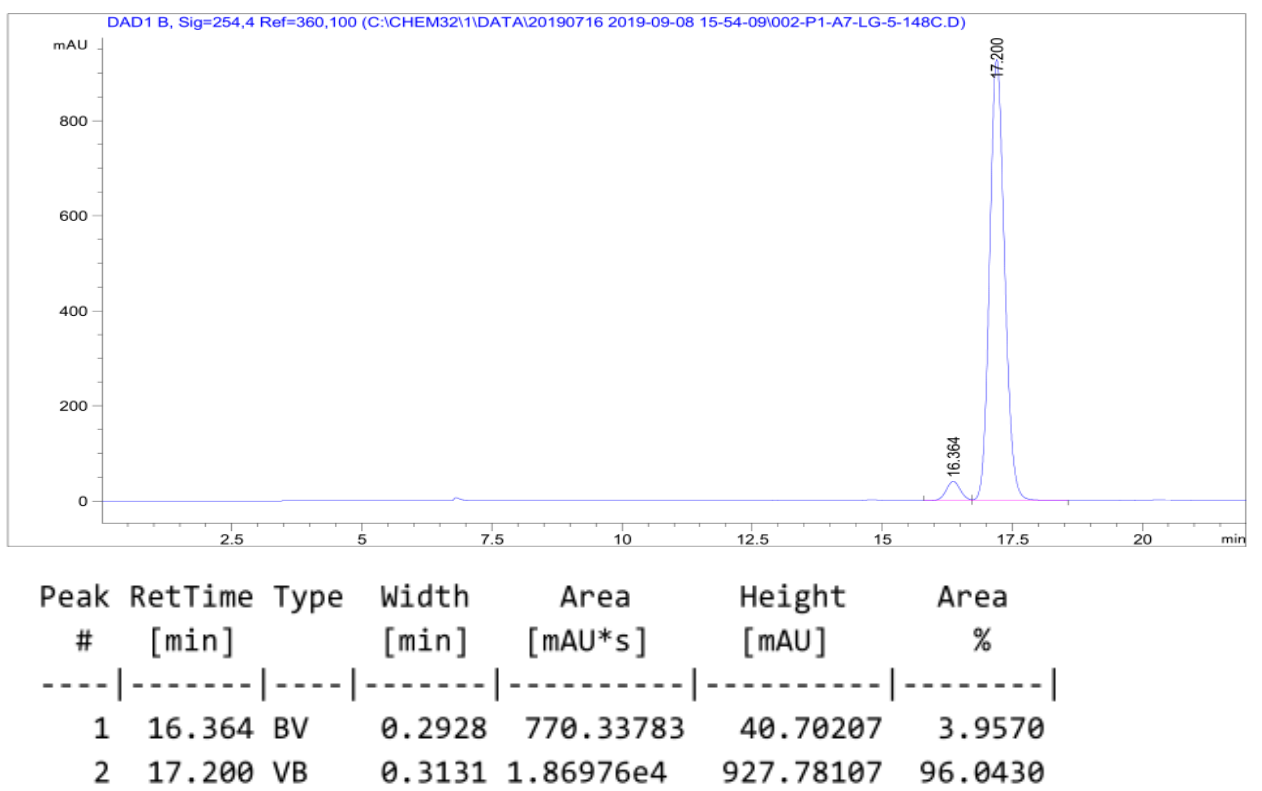<smiles>CC(C)(C)CC(=O)OC(C)(C)C</smiles>

(R)-tert-Butyl 2-(3-acetylphenyl)-4,4-dimethylpentanoate (8) 
The product was prepared according to the general procedure with (4S,4'S)-4,4'-di((S)sec-butyl)-4,4',5,5'-tetrahydro-2,2'-bioxazole [(S,S)-L1] $(3.3 \mathrm{mg}, 0.013 \mathrm{mmol}, 13$ mol\%), $\mathrm{NiCl}_{2} \cdot \mathrm{Py}_{4}(4.5 \mathrm{mg}, 0.01 \mathrm{mmol}, 10 \mathrm{~mol} \%), \operatorname{Ir}\left[\mathrm{dF}\left(\mathrm{CF}_{3}\right) \mathrm{ppy}\right]_{2}(\mathrm{bpy}) \mathrm{PF}_{6}(3.2 \mathrm{mg}$, $0.003 \mathrm{mmol}, 3 \mathrm{~mol} \%)$, 1-(3-bromophenyl)ethanone (13.2 uL, $0.1 \mathrm{mmol}, 1.0$ equiv.), ${ }^{t} \mathrm{BuBF}_{3} \mathrm{~K}$ (41.0 mg, $0.25 \mathrm{mmol}, 2.5$ equiv.) and tert-butyl acrylate (29.0 uL, $0.2 \mathrm{mmol}$, 2.0 equiv.) in acetone/ethyl acetate $(1.3 / 0.7, \mathrm{v} / \mathrm{v}, 2 \mathrm{~mL})$ were used. After 24 hours, the product was isolated by flash chromatography (PE: ethyl acetate $=20: 1)$ as a colorless oil (18.6 mg, 61\% yield, $92 \%$ ee).

${ }^{1} \mathbf{H}$ NMR $\left(400 \mathrm{MHz}, \mathrm{CDCl}_{3}\right) \delta 7.89(\mathrm{~s}, 1 \mathrm{H}), 7.82(\mathrm{~d}, J=7.7 \mathrm{~Hz}, 1 \mathrm{H}), 7.53(\mathrm{~d}, J=7.8$ $\mathrm{Hz}, 1 \mathrm{H}), 7.39$ (t, $J=7.7 \mathrm{~Hz}, 1 \mathrm{H}), 3.60$ (dd, $J=9.2,3.6 \mathrm{~Hz}, 1 \mathrm{H}), 2.60$ (s, 3H), 2.29 (dd, $J=14.0,9.2 \mathrm{~Hz}, 1 \mathrm{H}), 1.50(\mathrm{dd}, J=14.0,3.6 \mathrm{~Hz}, 1 \mathrm{H}), 1.37$ (s, 9H), 0.91 (s, 9H).

${ }^{13}$ C NMR $\left(100 \mathrm{MHz}, \mathrm{CDCl}_{3}\right) \delta 198.10,173.45,142.22,137.34,132.41,128.74,127.66$, $126.85,80.73,49.23,47.19,31.11,29.50,27.82,26.69$.

HRMS (ESI): $\mathrm{C}_{19} \mathrm{H}_{29} \mathrm{O}_{3}{ }^{+}(\mathrm{M}+\mathrm{H}): 305.2117$, found: 305.2108 .

$[\alpha]_{\mathrm{D}}^{25}=-19.8776\left(\mathrm{c}=0.1967, \mathrm{CHCl}_{3}\right)$

Chiral HPLC: CHIRALPAK IC, $25{ }^{\circ} \mathrm{C},{ }^{i} \mathrm{PrOH}-h$ exanes $2 / 98,0.5 \mathrm{~mL} / \mathrm{min}, 250 \mathrm{~nm}, \mathrm{t}_{\mathrm{R}}$ (major) $=16.0 \mathrm{~min}, \mathrm{t}_{\mathrm{R}}($ minor $)=15.2 \mathrm{~min}$.

\section{8 racemic}

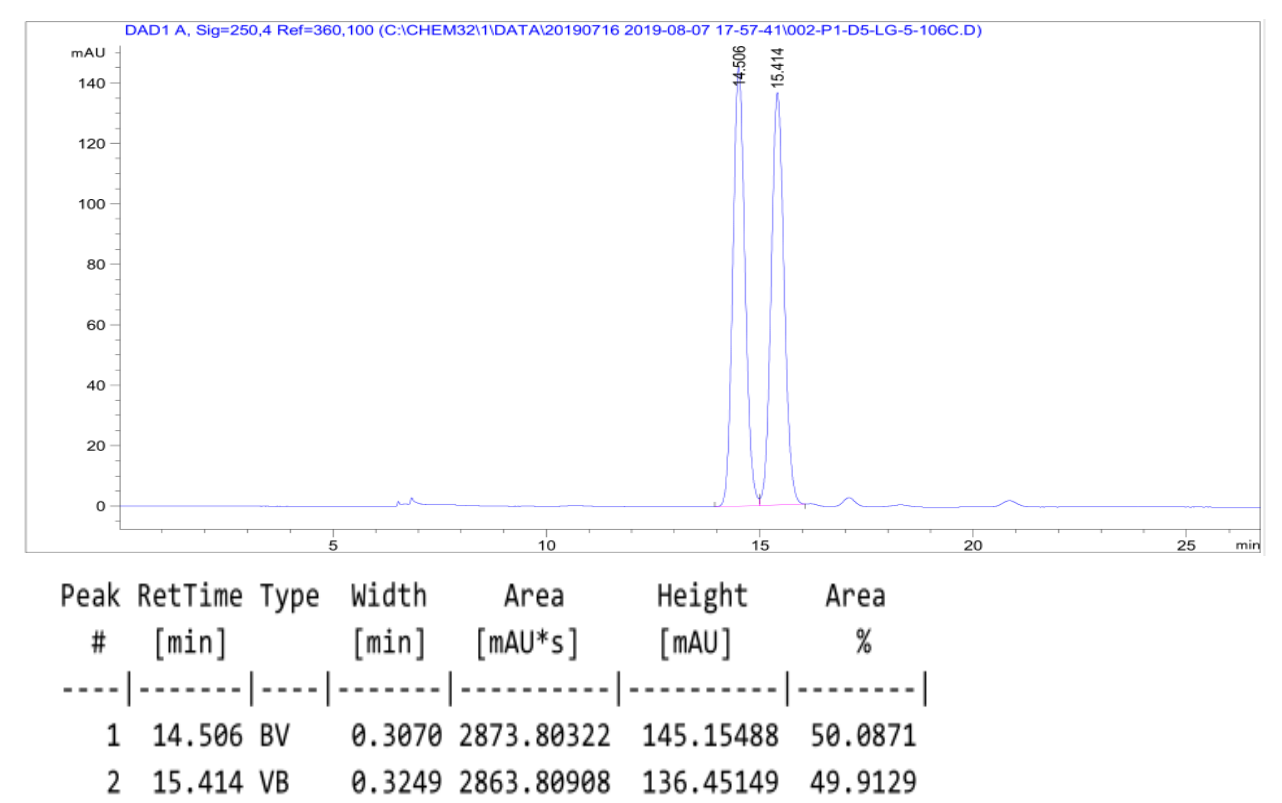




\section{8 enantioenriched, $92 \%$ ee}

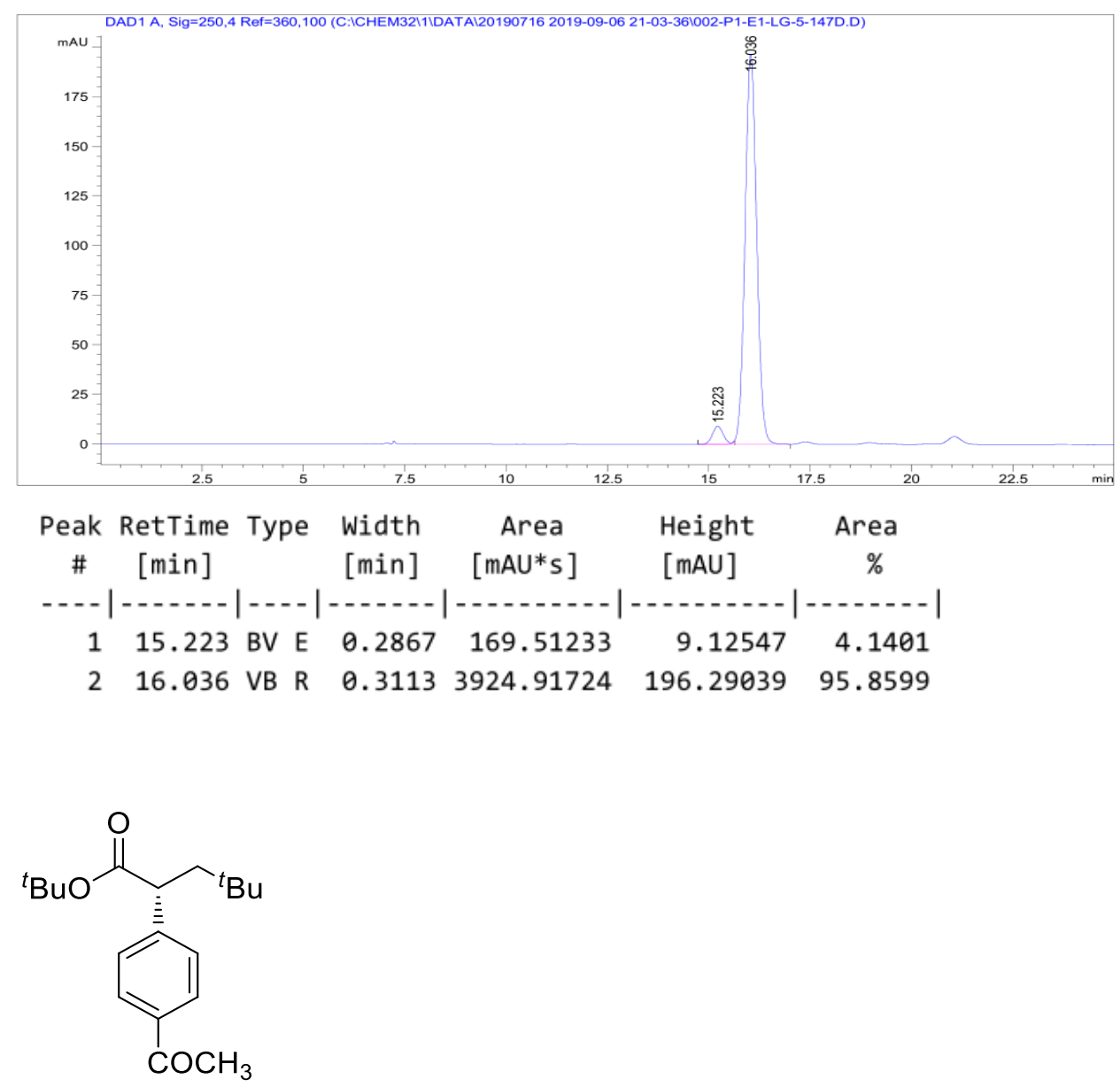

(R)-tert-Butyl 2-(4-acetylphenyl)-4,4-dimethylpentanoate (9)

The product was prepared according to the general procedure with (4S,4'S)-4,4'-di((S)sec-butyl)-4,4',5,5'-tetrahydro-2,2'-bioxazole [(S,S)-L1] $(3.3 \mathrm{mg}, 0.013 \mathrm{mmol}, 13$ mol\%), $\mathrm{NiCl}_{2} \bullet \mathrm{Py}_{4}(4.5 \mathrm{mg}, 0.01 \mathrm{mmol}, 10 \mathrm{~mol} \%), \operatorname{Ir}\left[\mathrm{dF}\left(\mathrm{CF}_{3}\right) \text { ppy }\right]_{2}(\mathrm{bpy}) \mathrm{PF}_{6}(3.2 \mathrm{mg}$, $0.003 \mathrm{mmol}, 3 \mathrm{~mol} \%$ ), 1-(4-bromophenyl)ethanone (19.9 mg, $0.1 \mathrm{mmol}, 1.0$ equiv.), ${ }^{t} \mathrm{BuBF}_{3} \mathrm{~K}(41.0 \mathrm{mg}, 0.25 \mathrm{mmol}, 2.5$ equiv.) and tert-butyl acrylate (29.0 uL, $0.2 \mathrm{mmol}$, 2.0 equiv.) in Acetone/ethyl acetate (1.3/0.7, v/v, $2 \mathrm{~mL})$ were used. After 24 hours, the product was isolated by flash chromatography (PE: ethyl acetate $=20: 1)$ as a colorless oil (22.3 mg, 73\% yield, 90\% ee).

${ }^{1}$ H NMR (400 MHz, $\left.\mathrm{CDCl}_{3}\right) \delta 7.89(\mathrm{~d}, J=8.4 \mathrm{~Hz}, 2 \mathrm{H}), 7.39(\mathrm{~d}, J=8.3 \mathrm{~Hz}, 2 \mathrm{H}), 3.59$ $(\mathrm{dd}, J=9.0,3.7 \mathrm{~Hz}, 1 \mathrm{H}), 2.58(\mathrm{~s}, 3 \mathrm{H}), 2.27(\mathrm{dd}, J=14.0,9.0 \mathrm{~Hz}, 1 \mathrm{H}), 1.50(\mathrm{dd}, J=$ 14.0, 3.7 Hz, 1H), $1.36(\mathrm{~s}, 9 \mathrm{H}), 0.91(\mathrm{~s}, 9 \mathrm{H})$.

${ }^{13} \mathrm{C}$ NMR $\left(100 \mathrm{MHz}, \mathrm{CDCl}_{3}\right) \delta 197.79,173.10,147.07,135.74,128.61,127.93,80.81$, 49.40, 46.98, 31.11, 29.47, 27.79, 26.57. 
HRMS (ESI): $\mathrm{C}_{19} \mathrm{H}_{29} \mathrm{O}_{3}{ }^{+}(\mathrm{M}+\mathrm{H})$ : 305.2117, found: 305.2109.

$[\alpha]_{\mathrm{D}}^{25}=-25.3562\left(\mathrm{c}=0.2733, \mathrm{CHCl}_{3}\right)$

Chiral HPLC: CHIRALPAK IC, $25{ }^{\circ} \mathrm{C},{ }^{i} \mathrm{PrOH}-h$ exanes 2/98, $0.5 \mathrm{~mL} / \mathrm{min}, 254 \mathrm{~nm}, \mathrm{t}_{\mathrm{R}}$ $($ major $)=8.5 \mathrm{~min}, \mathrm{t}_{\mathrm{R}}($ minor $)=6.2 \mathrm{~min}$.

\section{9 racemic}

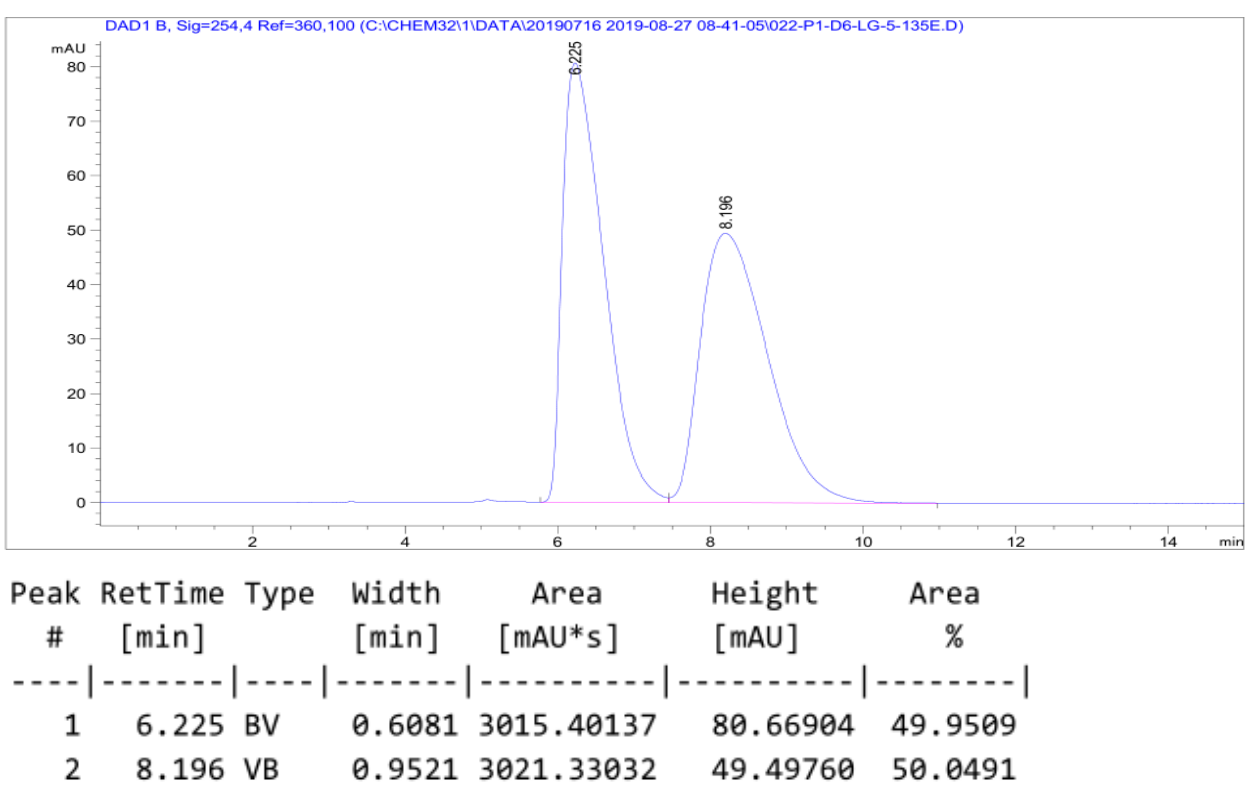

\section{9 enantioenriched, $90 \%$ ee}

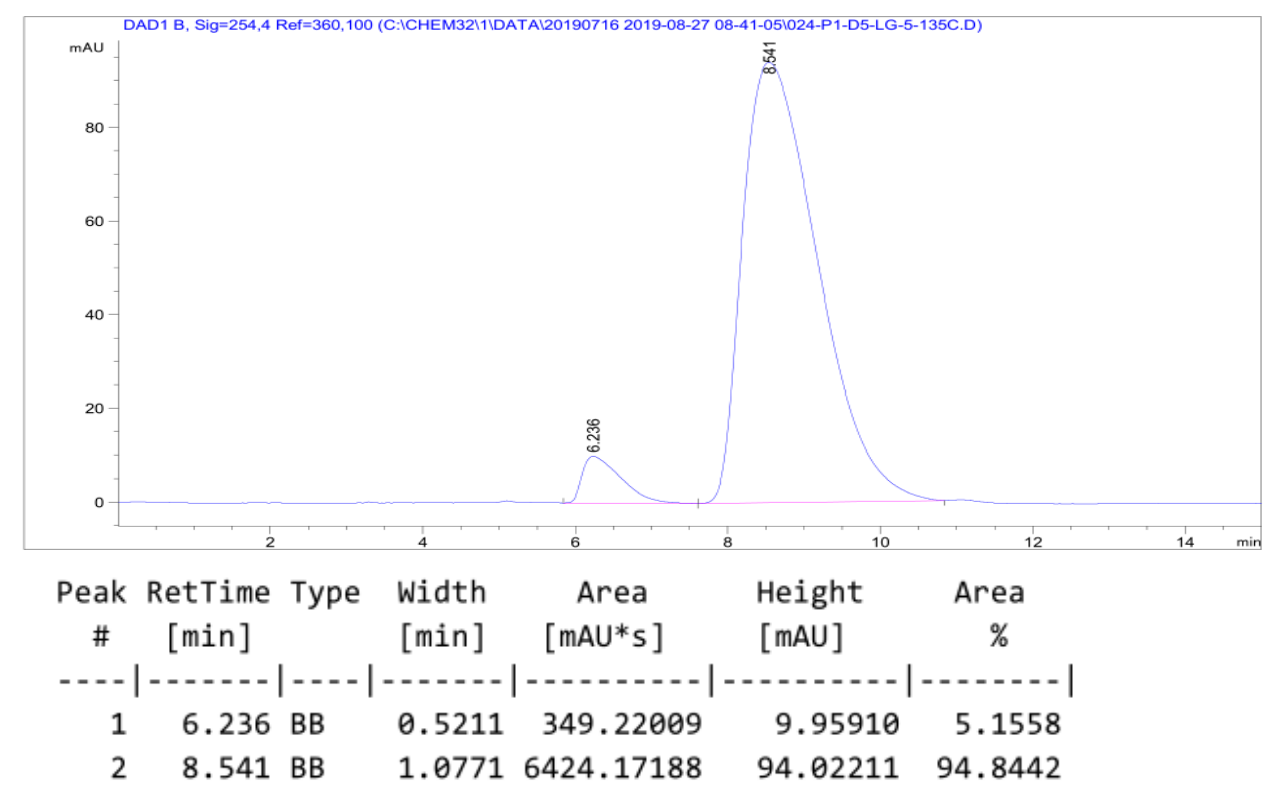


<smiles>CCOC(=O)C(CC(C)(C)C)c1ccc(C=O)cc1</smiles>

\section{(R)-tert-Butyl 2-(4-formylphenyl)-4,4-dimethylpentanoate (10)}

The product was prepared according to the general procedure with (4S,4'S)-4,4'-di((S)sec-butyl)-4,4',5,5'-tetrahydro-2,2'-bioxazole [(S,S)-L1] (3.3 mg, $0.013 \mathrm{mmol}, 13$

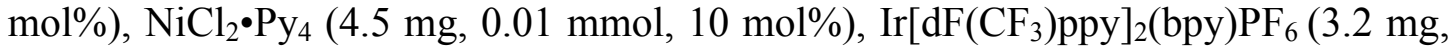
$0.003 \mathrm{mmol}, 3 \mathrm{~mol} \%)$, 4-bromobenzaldehyde (18.5 mg, $0.1 \mathrm{mmol}, 1.0$ equiv.), ${ }^{t} \mathrm{BuBF}_{3} \mathrm{~K}(41.0 \mathrm{mg}, 0.25 \mathrm{mmol}, 2.5$ equiv.) and tert-butyl acrylate $(29.0 \mathrm{uL}, 0.2 \mathrm{mmol}$, 2.0 equiv.) in acetone/ethyl acetate $(1.3 / 0.7, \mathrm{v} / \mathrm{v}, 2 \mathrm{~mL})$ were used. After 24 hours, the product was isolated by flash chromatography $(\mathrm{PE}$ : ethyl acetate $=20: 1)$ as a white solid (19.6 mg, 67\% yield, 93\% ee).

${ }^{1}$ H NMR $\left(400 \mathrm{MHz}, \mathrm{CDCl}_{3}\right) \delta 9.98(\mathrm{~s}, 1 \mathrm{H}), 7.82(\mathrm{~d}, J=8.2 \mathrm{~Hz}, 2 \mathrm{H}), 7.47(\mathrm{~d}, J=8.2$ $\mathrm{Hz}, 2 \mathrm{H}), 3.61$ (dd, $J=9.0,3.7 \mathrm{~Hz}, 1 \mathrm{H}), 2.29$ (dd, $J=14.0,9.0 \mathrm{~Hz}, 1 \mathrm{H}), 1.51$ (dd, $J=$ 14.0, 3.7 Hz, 1H), 1.37 (s, 9H), $0.91(\mathrm{~s}, 9 \mathrm{H})$.

${ }^{13} \mathbf{C}$ NMR $\left(100 \mathrm{MHz}, \mathrm{CDCl}_{3}\right) \delta 191.91,172.92,148.60,135.12,129.99,128.41,80.95$, 49.60, 46.97, 31.13, 29.46, 27.79.

HRMS (ESI): $\mathrm{C}_{18} \mathrm{H}_{27} \mathrm{O}_{3}{ }^{+}(\mathrm{M}+\mathrm{H}): 291.1960$, found: 291.1951 .

$[\alpha]_{\mathrm{D}}^{25}=-23.2669\left(\mathrm{c}=0.3033, \mathrm{CHCl}_{3}\right)$

Chiral HPLC: CHIRALPAK IA, $25{ }^{\circ} \mathrm{C},{ }^{i} \mathrm{PrOH}-h$ exanes 1/300, $1.0 \mathrm{~mL} / \mathrm{min}, 270 \mathrm{~nm}$, $t_{R}($ major $)=9.5 \mathrm{~min}, t_{R}($ minor $)=9.1 \mathrm{~min}$.

\section{0 racemic}

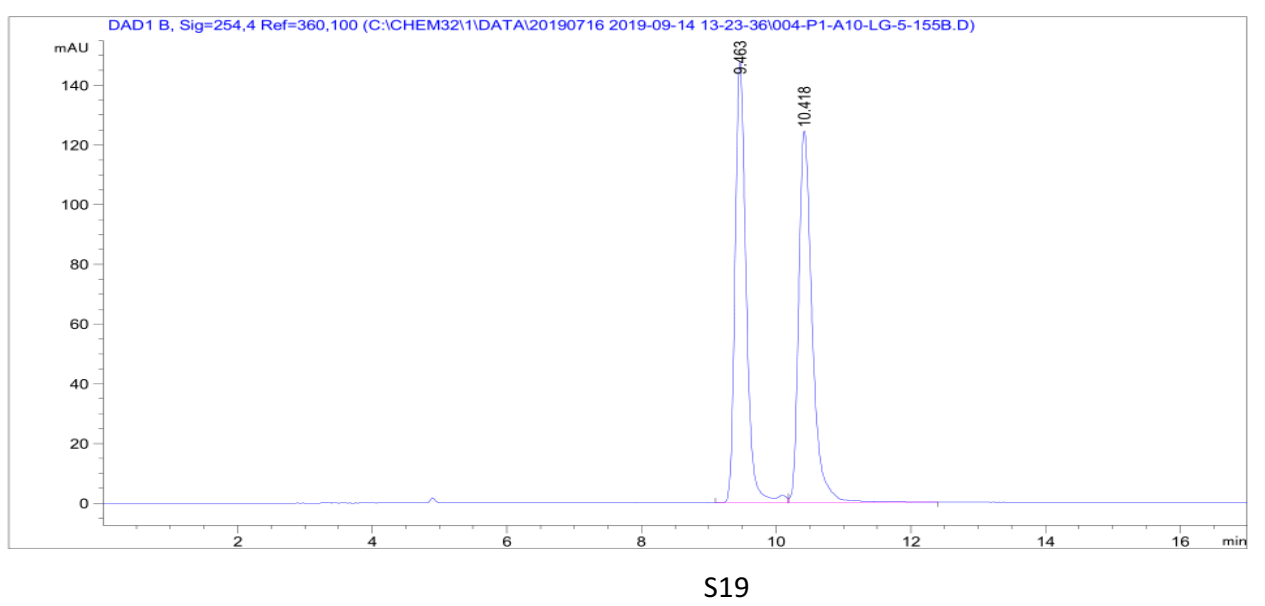




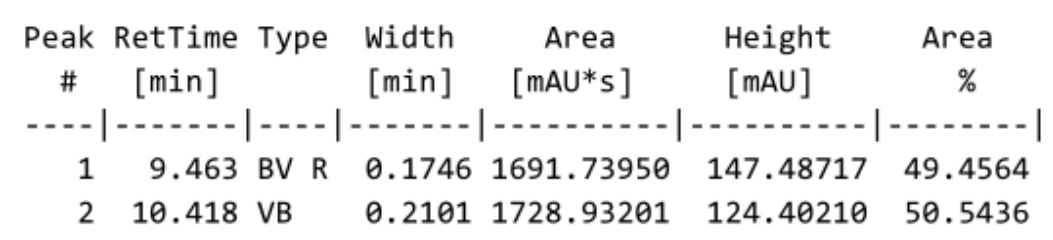

10 enantioenriched, $93 \%$ ee

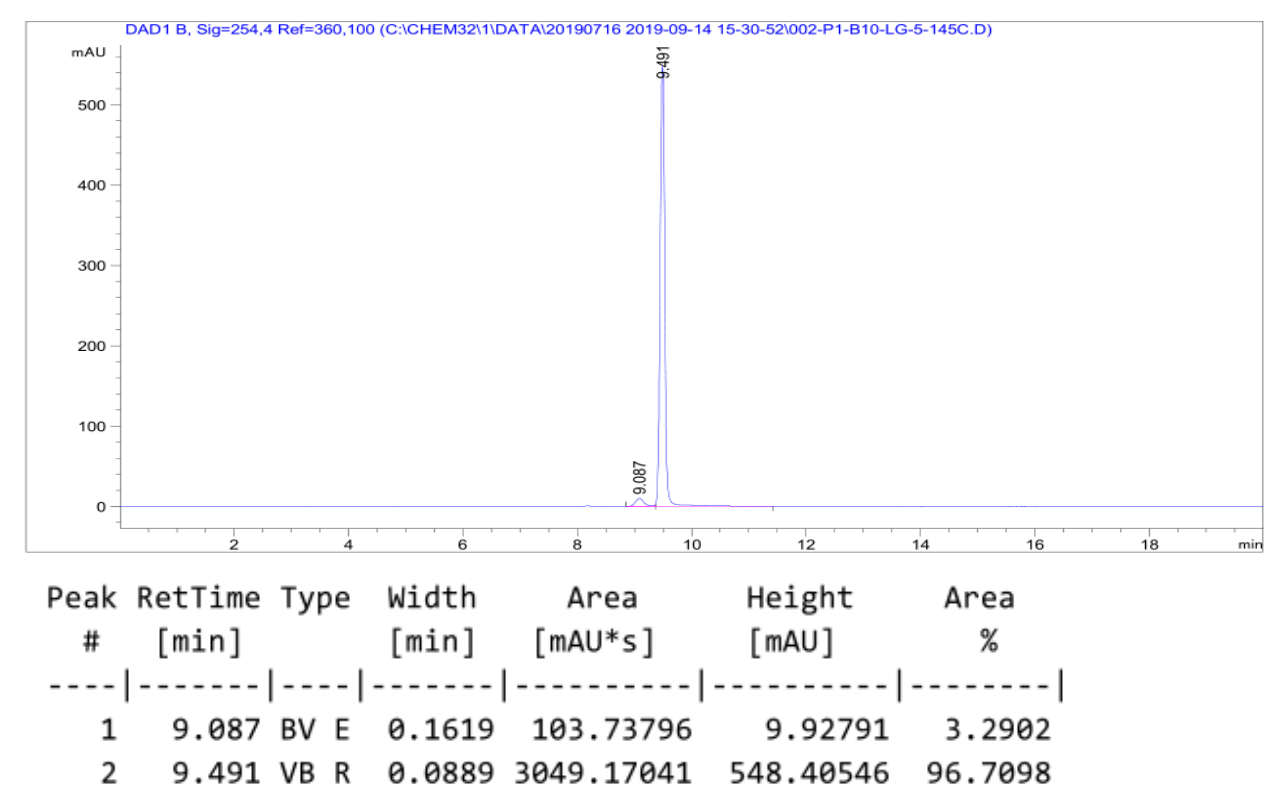

CN

\section{(R)-tert-Butyl 2-(4-cyanophenyl)-4,4-dimethylpentanoate (11)}

The product was prepared according to the general procedure with (4S,4'S)-4,4'-di((S)sec-butyl)-4,4',5,5'-tetrahydro-2,2'-bioxazole $[(S, S)$-L1 $](3.3 \mathrm{mg}, 0.013 \mathrm{mmol}, 13$ mol\%), $\mathrm{NiCl}_{2} \cdot \mathrm{Py}_{4}(4.5 \mathrm{mg}, 0.01 \mathrm{mmol}, 10 \mathrm{~mol} \%), \operatorname{Ir}\left[\mathrm{dF}\left(\mathrm{CF}_{3}\right) \mathrm{ppy}\right]_{2}(\mathrm{bpy}) \mathrm{PF}_{6}(3.2 \mathrm{mg}$, $0.003 \mathrm{mmol}, 3 \mathrm{~mol} \%$ ), 4-bromobenzonitrile (18.2 mg, $0.1 \mathrm{mmol}, 1.0$ equiv.), ${ }^{t} \mathrm{BuBF}_{3} \mathrm{~K}$ (41.0 mg, $0.25 \mathrm{mmol}, 2.5$ equiv.) and tert-butyl acrylate (29.0 uL, $0.2 \mathrm{mmol}, 2.0$ equiv.) in acetone/ethyl acetate $(1.3 / 0.7, \mathrm{v} / \mathrm{v}, 2 \mathrm{~mL})$ were used. After 24 hours, the product was isolated by flash chromatography (PE: ethyl acetate $=20: 1)$ as a white solid $(20.4 \mathrm{mg}$, $71 \%$ yield, $93 \%$ ee). 
${ }^{1} \mathbf{H}$ NMR $\left(400 \mathrm{MHz}, \mathrm{CDCl}_{3}\right) \delta 7.59(\mathrm{~d}, J=8.3 \mathrm{~Hz}, 2 \mathrm{H}), 7.41(\mathrm{~d}, J=8.3 \mathrm{~Hz}, 2 \mathrm{H}), 3.57$ (dd, $J=9.0,3.7 \mathrm{~Hz}, 1 \mathrm{H}), 2.25(\mathrm{dd}, J=14.0,9.0 \mathrm{~Hz}, 1 \mathrm{H}), 1.46(\mathrm{dd}, J=14.0,3.7 \mathrm{~Hz}$, 1H), $1.36(\mathrm{~s}, 9 \mathrm{H}), 0.90(\mathrm{~s}, 9 \mathrm{H})$.

${ }^{13}$ C NMR (100 MHz, $\left.\mathrm{CDCl}_{3}\right) \delta 172.68,146.95,132.31,128.53,118.80,110.71,81.13$, 49.49, 46.97, 31.11, 29.42, 27.77.

HRMS (ESI): $\mathrm{C}_{18} \mathrm{H}_{26} \mathrm{NO}_{2}^{+}(\mathrm{M}+\mathrm{H}): 288.1964$, found: 288.1954 .

$[\alpha]_{\mathrm{D}}^{25}=-22.3676\left(\mathrm{c}=0.3733, \mathrm{CHCl}_{3}\right)$

Chiral HPLC: CHIRALPAK IA, $25{ }^{\circ} \mathrm{C},{ }^{i} \mathrm{PrOH}-$ hexanes 5/95, $0.5 \mathrm{~mL} / \mathrm{min}, 254 \mathrm{~nm}, \mathrm{t}_{\mathrm{R}}$ (major) $=8.8 \mathrm{~min}, \mathrm{t}_{\mathrm{R}}($ minor $)=8.3 \mathrm{~min}$.

\section{1 racemic}

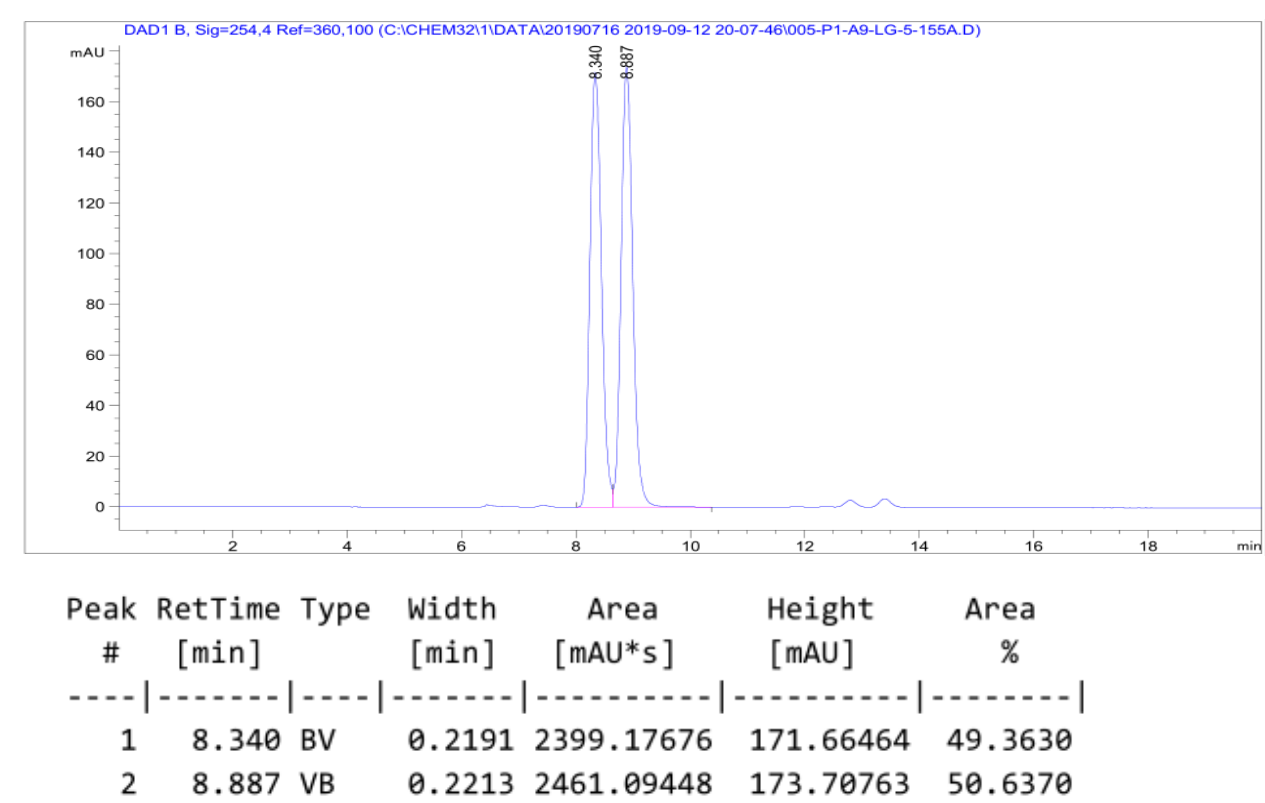

11 enantioenriched, $93 \%$ ee

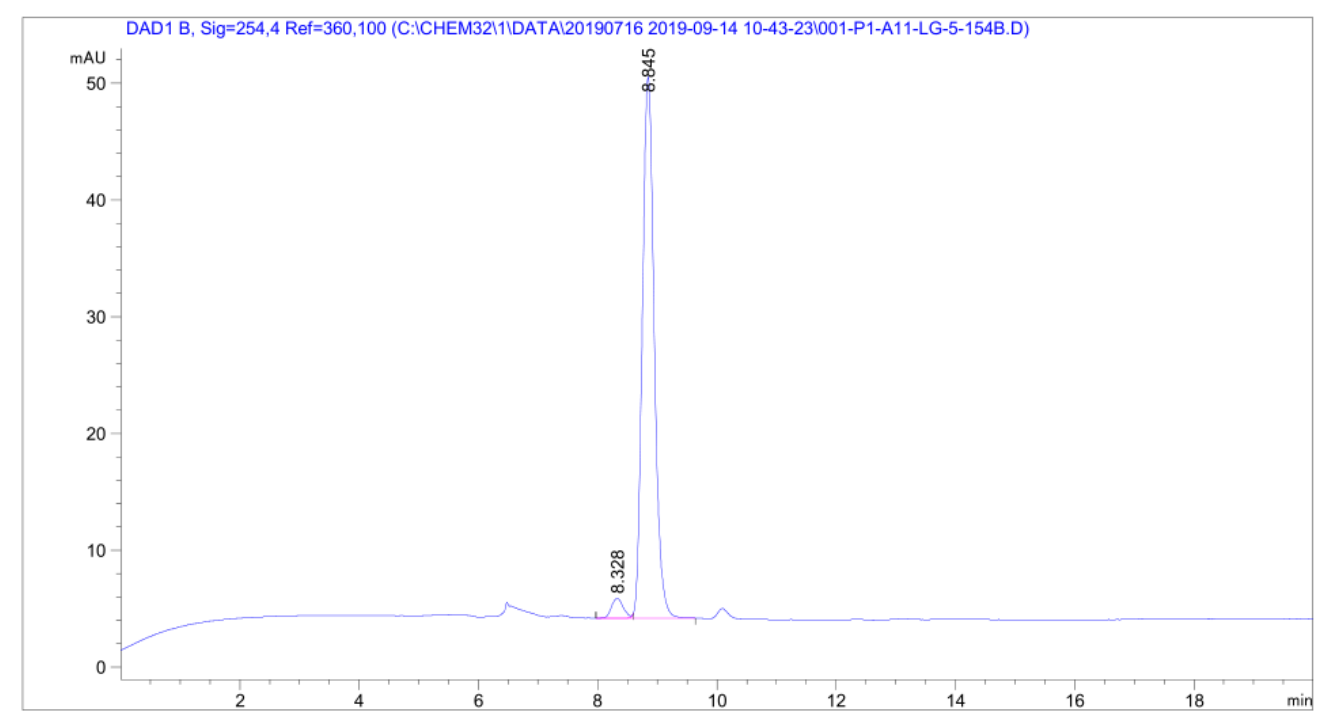




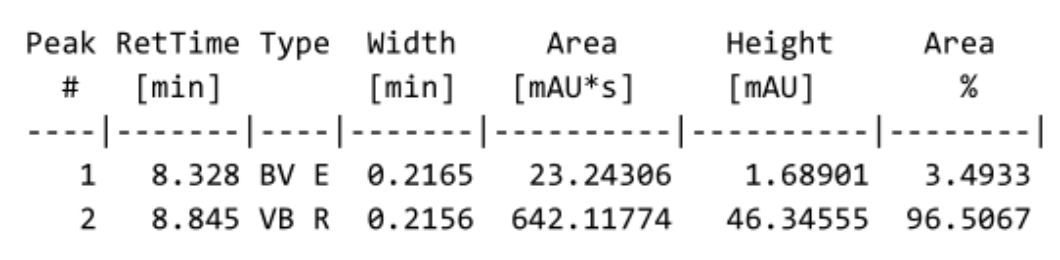<smiles>CC(C)(C)OC(=O)C(CBr)c1ccc(C(F)(F)F)cc1</smiles>

(R)-tert-Butyl 4,4-dimethyl-2-(4-(trifluoromethyl)phenyl)pentanoate (12)

The product was prepared according to the general procedure with (4S,4'S)-4,4'-di((S)sec-butyl)-4,4',5,5'-tetrahydro-2,2'-bioxazole $[(S, S)$-L1 $]$ (3.3 mg, $0.013 \mathrm{mmol}, 13$ mol\%), $\left.\mathrm{NiCl}_{2} \cdot \mathrm{Py}_{4}(4.5 \mathrm{mg}, 0.01 \mathrm{mmol}, 10 \mathrm{~mol} \%), \operatorname{Ir}\left[\mathrm{dF}_{(\mathrm{CF}}\right) \mathrm{ppy}\right]_{2}(\mathrm{bpy}) \mathrm{PF}_{6}(3.2 \mathrm{mg}$, $0.003 \mathrm{mmol}, 3 \mathrm{~mol} \%$ ), 1-bromo-4-(trifluoromethyl)benzene (14.0 uL, $0.1 \mathrm{mmol}, 1.0$ equiv.), ${ }^{t} \mathrm{BuBF}_{3} \mathrm{~K}(41.0 \mathrm{mg}, 0.25 \mathrm{mmol}, 2.5$ equiv.) and tert-butyl acrylate (29.0 uL, 0.2 mmol, 2.0 equiv.) in acetone/ethyl acetate $(1.3 / 0.7, \mathrm{v} / \mathrm{v}, 2 \mathrm{~mL})$ were used. After 24 hours, the product was isolated by flash chromatography (PE: ethyl acetate $=20: 1)$ as a white solid (20.4 mg, 62\% yield, 92\% ee).

${ }^{1}$ H NMR (400 MHz, CDCl3) $\delta 7.48(\mathrm{~d}, \mathrm{~J}=8.1 \mathrm{~Hz}, 2 \mathrm{H}), 7.35(\mathrm{~d}, \mathrm{~J}=8.2 \mathrm{~Hz}, 2 \mathrm{H}), 3.52$ (dd, J = 9.2, $3.5 \mathrm{~Hz}, 1 \mathrm{H}), 2.21$ (dd, J = 14.0, $9.2 \mathrm{~Hz}, 1 \mathrm{H}), 1.43-1.39$ (m, 1H), 1.30 (s, $9 \mathrm{H}), 0.84(\mathrm{~s}, 9 \mathrm{H})$.

${ }^{19}$ F NMR $\left(375 \mathrm{MHz}, \mathrm{CDCl}_{3}\right) \delta-62.45(\mathrm{~s})$

${ }^{13}$ C NMR $\left(100 \mathrm{MHz}, \mathrm{CDCl}_{3}\right) \delta 173.13,145.61,129.04(\mathrm{q}, J=34.6 \mathrm{~Hz}), 128.03,125.42$ (q, $J=3.7 \mathrm{~Hz}), 124.20$ (q, $J=271.8 \mathrm{~Hz}), 80.87,49.22,47.14,31.10,29.45,27.80$.

HRMS (ESI): $\mathrm{C}_{18} \mathrm{H}_{26} \mathrm{~F}_{3} \mathrm{O}_{2}^{+}(\mathrm{M}+\mathrm{H}): 331.1885$, found: 331.1876 .

$[\alpha]_{\mathrm{D}}^{25}=-19.2946\left(\mathrm{c}=0.5333, \mathrm{CHCl}_{3}\right)$

Chiral HPLC: CHIRALPAK IA, $25{ }^{\circ} \mathrm{C}$, Hexanes, $0.5 \mathrm{~mL} / \mathrm{min}, 230 \mathrm{~nm}, \mathrm{t}_{\mathrm{R}}$ (major) $=10.8 \mathrm{~min}, \mathrm{t}_{\mathrm{R}}($ minor $)=10.3 \mathrm{~min}$. 


\section{2 racemic}

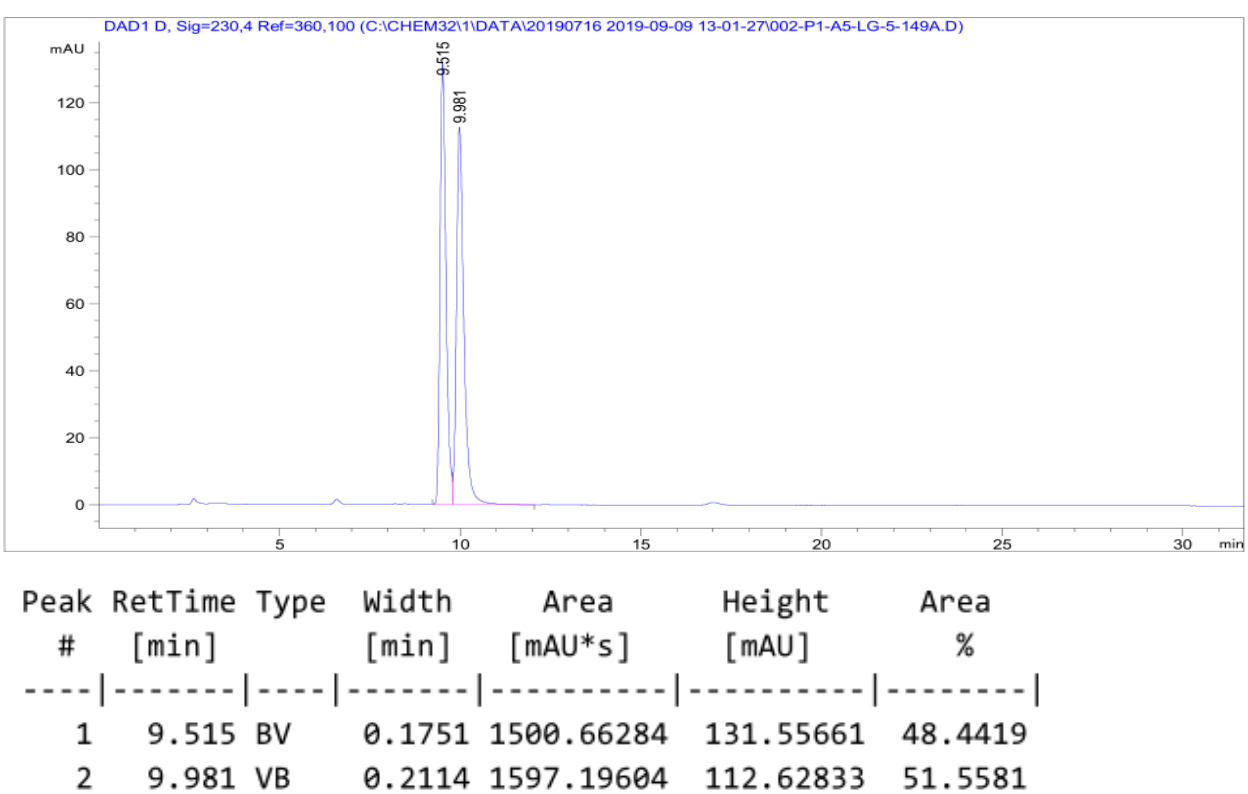

12 enantioenriched, $92 \%$ ee

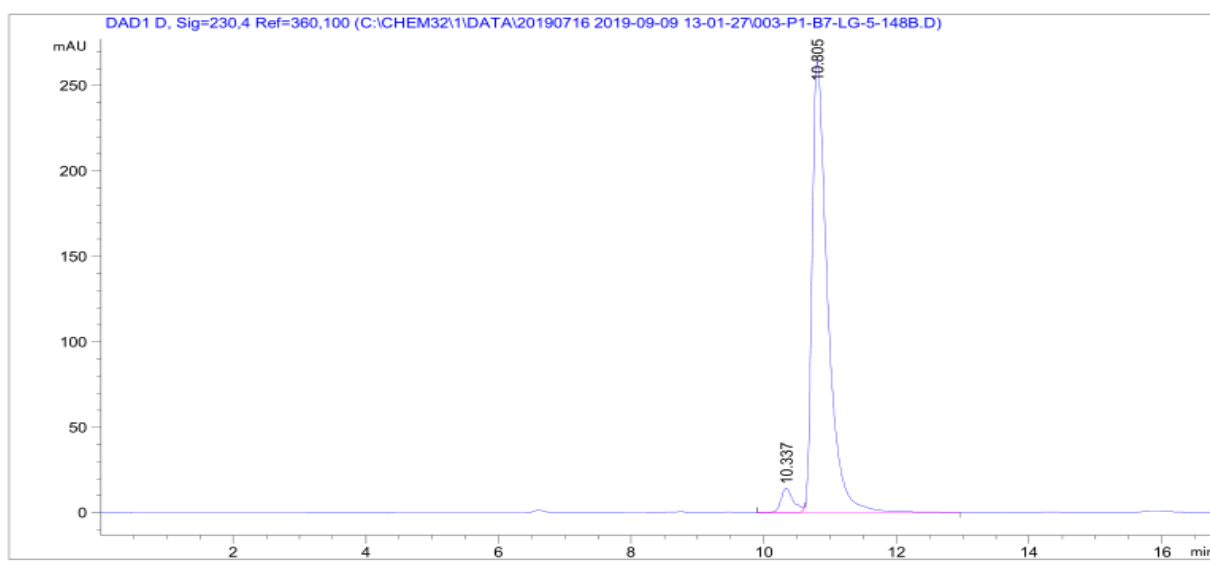

\begin{tabular}{cccccc}
$\begin{array}{c}\text { Peak RetTime Type Width } \\
\text { \# }[\text { min] }\end{array}$ [min] & $\begin{array}{c}\text { Area } \\
{[\text { mAU* }]}\end{array}$ & $\begin{array}{l}\text { Height } \\
\text { [mAU] }\end{array}$ & \multicolumn{1}{c}{$\begin{array}{c}\text { Area } \\
\%\end{array}$} \\
\hdashline & 10.337 BV E & 0.1936 & 185.68454 & 14.28755 & 4.1115 \\
2 & 10.805 VB R & 0.2431 & 4330.52100 & 264.35825 & 95.8885
\end{tabular}<smiles>COC(=O)c1ccc([C@H](CC(C)(C)C)C(=O)OC(C)(C)C)cc1F</smiles>

(R)-Methyl 4-(1-(tert-butoxy)-4,4-dimethyl-1-oxopentan-2-yl)-2-fluorobenzoate (13) 
The product was prepared according to the general procedure with $(4 \mathrm{~S}, 4$ 'S)-4,4'-di((S)sec-butyl)-4,4',5,5'-tetrahydro-2,2'-bioxazole [(S,S)-L1] $(3.3 \mathrm{mg}, 0.013 \mathrm{mmol}, 13$ mol\%), $\mathrm{NiCl}_{2} \cdot \mathrm{Py}_{4}(4.5 \mathrm{mg}, 0.01 \mathrm{mmol}, 10 \mathrm{~mol} \%), \operatorname{Ir}\left[\mathrm{dF}\left(\mathrm{CF}_{3}\right) \mathrm{ppy}\right]_{2}(\mathrm{bpy}) \mathrm{PF}_{6}(3.2 \mathrm{mg}$, $0.003 \mathrm{mmol}, 3 \mathrm{~mol} \%$ ), methyl 4-bromo-2-fluorobenzoate (23.3 mg, $0.1 \mathrm{mmol}, 1.0$ equiv.), ${ }^{t} \mathrm{BuBF}_{3} \mathrm{~K}(41.0 \mathrm{mg}, 0.25 \mathrm{mmol}, 2.5$ equiv.) and tert-butyl acrylate (29.0 uL, 0.2 mmol, 2.0 equiv.) in acetone/ethyl acetate (1.3/0.7, v/v, $2 \mathrm{~mL})$ were used. After 24 hours, the product was isolated by flash chromatography $(P E$ : ethyl acetate $=20: 1)$ as a white solid (24.6 mg, 73\% yield, 92\% ee).

${ }^{1}$ H NMR $\left(400 \mathrm{MHz}, \mathrm{CDCl}_{3}\right) \delta 7.86(\mathrm{t}, J=7.8 \mathrm{~Hz}, 1 \mathrm{H}), 7.13(\mathrm{dd}, J=8.1,1.6 \mathrm{~Hz}, 1 \mathrm{H})$, 7.11-7.08 (m, 1H), $3.91(\mathrm{~s}, 3 \mathrm{H}), 3.55(\mathrm{dd}, J=9.0,3.7 \mathrm{~Hz}, 1 \mathrm{H}), 2.23(\mathrm{dd}, J=14.0,9.0$ $\mathrm{Hz}, 1 \mathrm{H}), 1.47$ (dd, $J=14.0,3.8 \mathrm{~Hz}, 1 \mathrm{H}), 1.36$ (s, 9H), 0.89 (s, 9H).

${ }^{19}$ F NMR $\left(375 \mathrm{MHz}, \mathrm{CDCl}_{3}\right) \delta-109.19(\mathrm{dd}, J=11.8,7.6 \mathrm{~Hz})$.

${ }^{13}$ C NMR $\left(100 \mathrm{MHz}, \mathrm{CDCl}_{3}\right) \delta$ 172.55, $164.75(\mathrm{~d}, J=3.7 \mathrm{~Hz}), 161.86(\mathrm{~d}, J=260.1$ Hz), 149.09 (d, $J=8.6 \mathrm{~Hz}), 132.17,123.39$ (d, $J=3.3 \mathrm{~Hz}), 116.90$ (d, $J=10.1 \mathrm{~Hz})$, $116.15(\mathrm{~d}, J=23.2 \mathrm{~Hz}), 81.08,52.23,49.17,46.79,31.07,29.41,27.75$.

HRMS (ESI): $\mathrm{C}_{19} \mathrm{H}_{27} \mathrm{FO}_{4} \mathrm{Na}^{+}(\mathrm{M}+\mathrm{Na}): 361.1791$, found: 361.1784 .

$[\alpha]_{\mathrm{D}}^{25}=-19.1791\left(\mathrm{c}=0.3900, \mathrm{CHCl}_{3}\right)$

Chiral HPLC: CHIRALPAK IC, $25{ }^{\circ} \mathrm{C},{ }^{i} \mathrm{PrOH}-$ hexanes $1 / 100,0.5 \mathrm{~mL} / \mathrm{min}, 250 \mathrm{~nm}$, $\mathrm{t}_{\mathrm{R}}($ major $)=33.1 \mathrm{~min}, \mathrm{t}_{\mathrm{R}}($ minor $)=32.1 \mathrm{~min}$

\section{3 racemic}

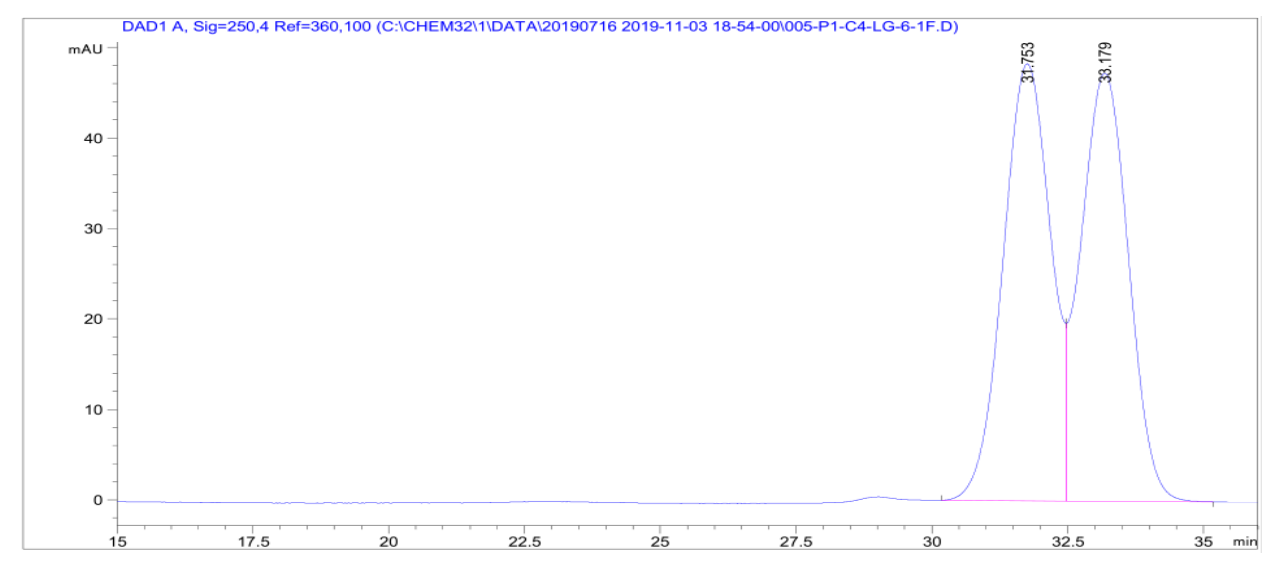




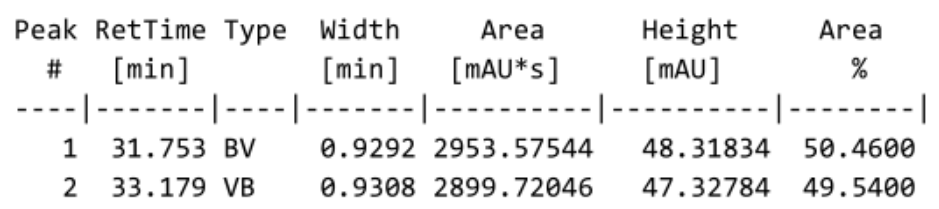

\section{3 enantioenriched, $92 \%$ ee}

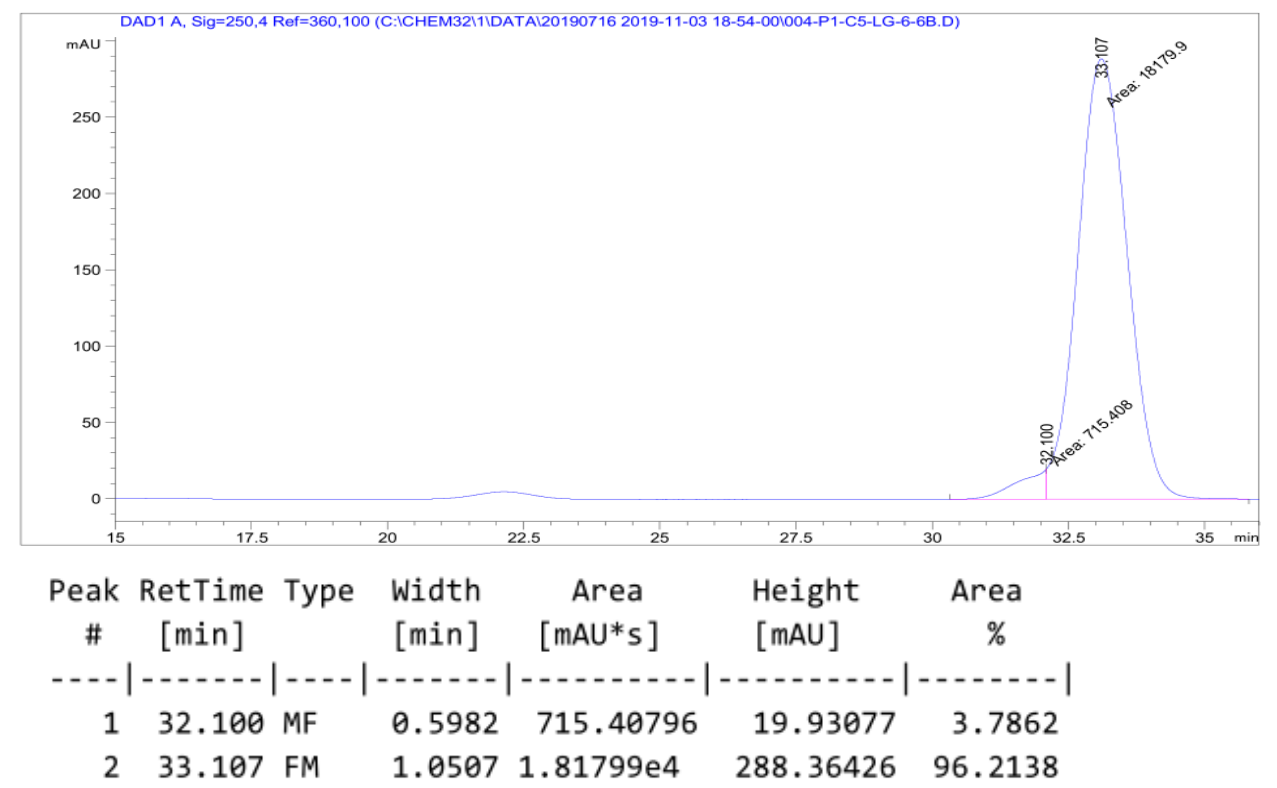<smiles>CC(C)(C)OC(=O)C(CBr)c1ccc2c(c1)COC2=O</smiles>

\section{(R)-tert-Butyl 4,4-dimethyl-2-(1-oxo-1,3-dihydroisobenzofuran-5-yl)pentanoate} (14)

The product was prepared according to the general procedure with (4S,4'S)-4,4'-di((S)sec-butyl)-4,4',5,5'-tetrahydro-2,2'-bioxazole [(S,S)-L1] $(3.3 \mathrm{mg}, 0.013 \mathrm{mmol}, 13$

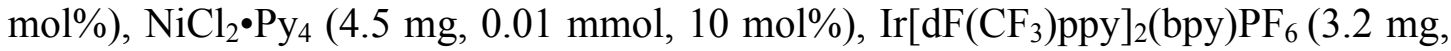
$0.003 \mathrm{mmol}, 3 \mathrm{~mol} \%)$, 5-bromoisobenzofuran-1(3H)-one (21.3 mg, $0.1 \mathrm{mmol}, 1.0$ equiv.), ${ }^{t} \mathrm{BuBF}_{3} \mathrm{~K}$ (41.0 mg, $0.25 \mathrm{mmol}, 2.5$ equiv.) and tert-butyl acrylate (29.0 uL, 0.2 mmol, 2.0 equiv.) in acetone/ethyl acetate (1.3/0.7, v/v, $2 \mathrm{~mL})$ were used. After 24 
hours, the product was isolated by flash chromatography (PE: ethyl acetate $=20: 1)$ as a white solid (24.9 mg, 78\% yield, 91\% ee).

${ }^{1} \mathbf{H}$ NMR $\left(400 \mathrm{MHz}, \mathrm{CDCl}_{3}\right) \delta 7.84(\mathrm{~d}, J=7.9 \mathrm{~Hz}, 1 \mathrm{H}), 7.46(\mathrm{~d}, J=8.0 \mathrm{~Hz}, 1 \mathrm{H}), 7.43$ (s, 1H), 5.29 (s, 2H), $3.65(\mathrm{dd}, J=9.1,3.5 \mathrm{~Hz}, 1 \mathrm{H}), 2.30(\mathrm{dd}, J=14.0,9.1 \mathrm{~Hz}, 1 \mathrm{H})$, 1.51-1.47 (m, 1H), $1.37(\mathrm{~s}, 9 \mathrm{H}), 0.91(\mathrm{~s}, 9 \mathrm{H})$.

${ }^{13}$ C NMR $\left(100 \mathrm{MHz}, \mathrm{CDCl}_{3}\right) \delta 172.87,170.84,148.54,147.08,129.04,125.80,124.42$, $121.10,81.17,69.48,49.63,47.37,31.16,29.42,27.78$.

HRMS (ESI): $\mathrm{C}_{19} \mathrm{H}_{27} \mathrm{O}_{4}^{+}(\mathrm{M}+\mathrm{H}): 319.1909$, found: 319.1899 .

$[\alpha]_{\mathrm{D}}^{25}=-13.1942\left(\mathrm{c}=0.3600, \mathrm{CHCl}_{3}\right)$

Chiral HPLC: CHIRALPAK IC, $25{ }^{\circ} \mathrm{C},{ }^{i} \mathrm{PrOH}-$ hexanes 10/90, $1.0 \mathrm{~mL} / \mathrm{min}, 210 \mathrm{~nm}$, $\mathrm{t}_{\mathrm{R}}($ major $)=25.2 \mathrm{~min}, \mathrm{t}_{\mathrm{R}}($ minor $)=24.1 \mathrm{~min}$.

\section{4 racemic}

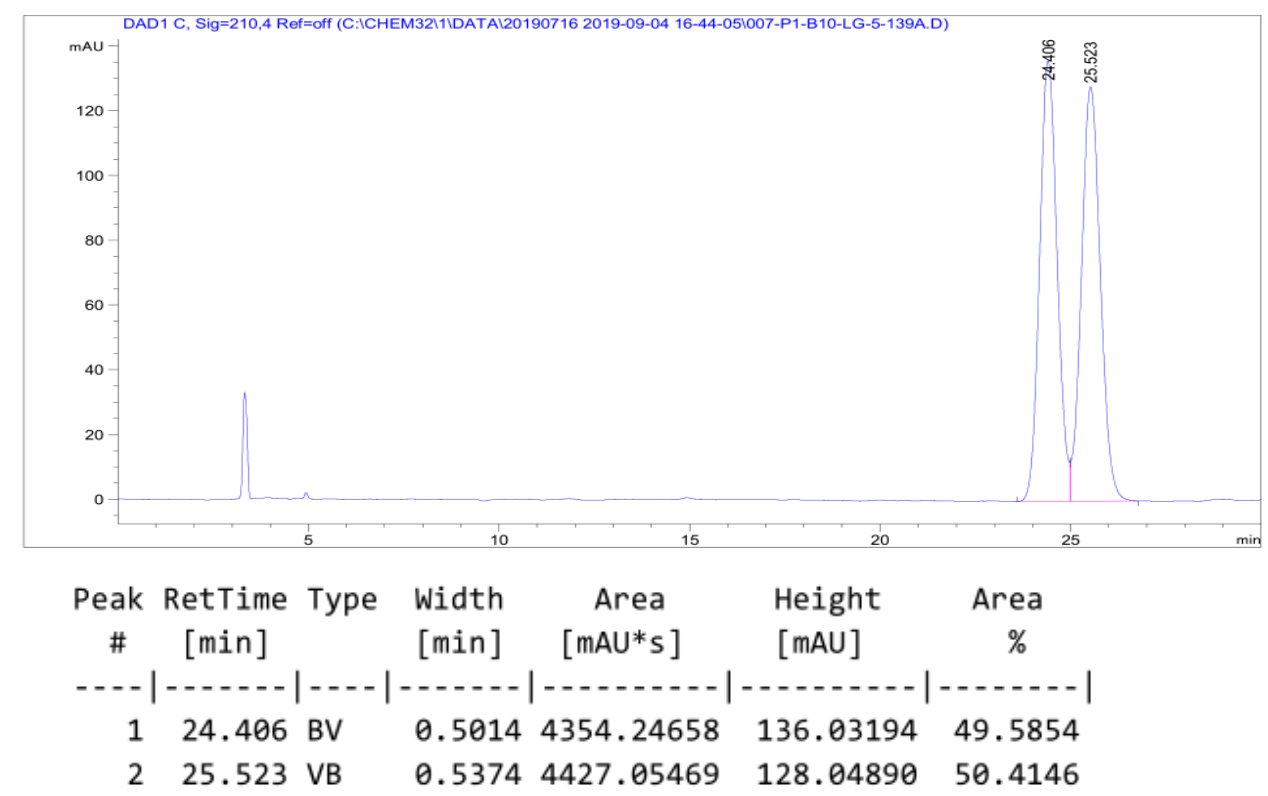




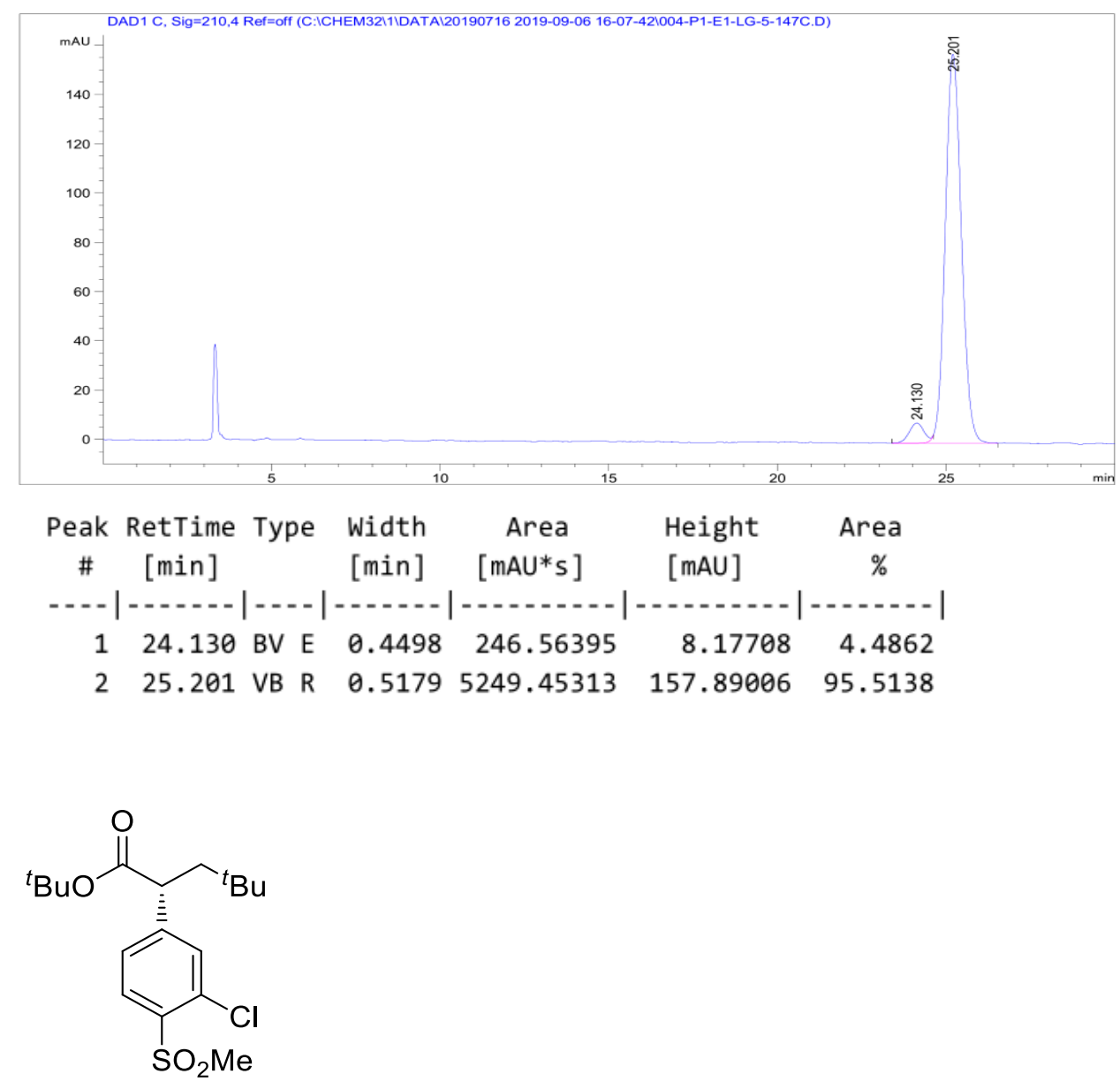

(R)-tert-Butyl 2-(3-chloro-4-(methylsulfonyl)phenyl)-4,4-dimethylpentanoate (15)

The product was prepared according to the general procedure with (4S,4'S)-4,4'-di((S)sec-butyl)-4,4',5,5'-tetrahydro-2,2'-bioxazole [(S,S)-L1] $(3.3 \mathrm{mg}, 0.013 \mathrm{mmol}, 13$ $\mathrm{mol} \%), \mathrm{NiCl}_{2} \bullet \mathrm{Py}_{4}(4.5 \mathrm{mg}, 0.01 \mathrm{mmol}, 10 \mathrm{~mol} \%), \operatorname{Ir}\left[\mathrm{dF}\left(\mathrm{CF}_{3}\right) \text { ppy }\right]_{2}(\mathrm{bpy}) \mathrm{PF}_{6}(3.2 \mathrm{mg}$, $0.003 \mathrm{mmol}, 3 \mathrm{~mol} \%$ ), 4-bromo-2-chloro-1-(methylsulfonyl)benzene (27.0 mg, 0.1 mmol, 1.0 equiv.), ${ }^{t} \mathrm{BuBF}_{3} \mathrm{~K}$ (41.0 mg, $0.25 \mathrm{mmol}, 2.5$ equiv.) and tert-butyl acrylate (29.0 uL, $0.2 \mathrm{mmol}, 2.0$ equiv.) in acetone/ethyl acetate $(1.3 / 0.7, \mathrm{v} / \mathrm{v}, 2 \mathrm{~mL})$ were used. After 24 hours, the product was isolated by flash chromatography (PE: ethyl acetate= 20:1) as a white solid (24.4 $\mathrm{mg}, 65 \%$ yield, $94 \%$ ee).

${ }^{1} \mathbf{H}$ NMR $\left(400 \mathrm{MHz}, \mathrm{CDCl}_{3}\right) \delta 8.06(\mathrm{~d}, J=8.2 \mathrm{~Hz}, 1 \mathrm{H}), 7.50(\mathrm{~s}, 1 \mathrm{H}), 7.39(\mathrm{~d}, J=8.2$, 1H), $3.57(\mathrm{dd}, J=9.1,3.5 \mathrm{~Hz}, 1 \mathrm{H}), 3.25(\mathrm{~s}, 3 \mathrm{H}), 2.25$ (dd, $J=14.0,9.1 \mathrm{~Hz}, 1 \mathrm{H}), 1.45$ $(\mathrm{dd}, J=14.0,3.5 \mathrm{~Hz}, 1 \mathrm{H}), 1.38(\mathrm{~s}, 9 \mathrm{H}), 0.91(\mathrm{~s}, 9 \mathrm{H})$.

${ }^{13}$ C NMR $\left(100 \mathrm{MHz}, \mathrm{CDCl}_{3}\right) \delta 172.17,149.36,136.32,132.52,131.04,130.93,126.79$, $81.52,49.00,47.16,42.78,31.17,29.39,27.78$. 
HRMS (ESI): $\mathrm{C}_{18} \mathrm{H}_{28} \mathrm{ClO}_{4} \mathrm{~S}^{+}(\mathrm{M}+\mathrm{H}): 375.1397$, found: 375.1394 .

$[\alpha]_{\mathrm{D}}^{25}=-14.6007\left(\mathrm{c}=0.1767, \mathrm{CHCl}_{3}\right)$

Chiral HPLC: CHIRALPAK IB, $25{ }^{\circ} \mathrm{C},{ }^{i} \mathrm{PrOH}-$ hexanes $1 / 100,0.5 \mathrm{~mL} / \mathrm{min}, 230 \mathrm{~nm}$, $t_{R}($ major $)=22.6 \mathrm{~min}, t_{R}($ minor $)=24.7 \mathrm{~min}$

\section{5 racemic}

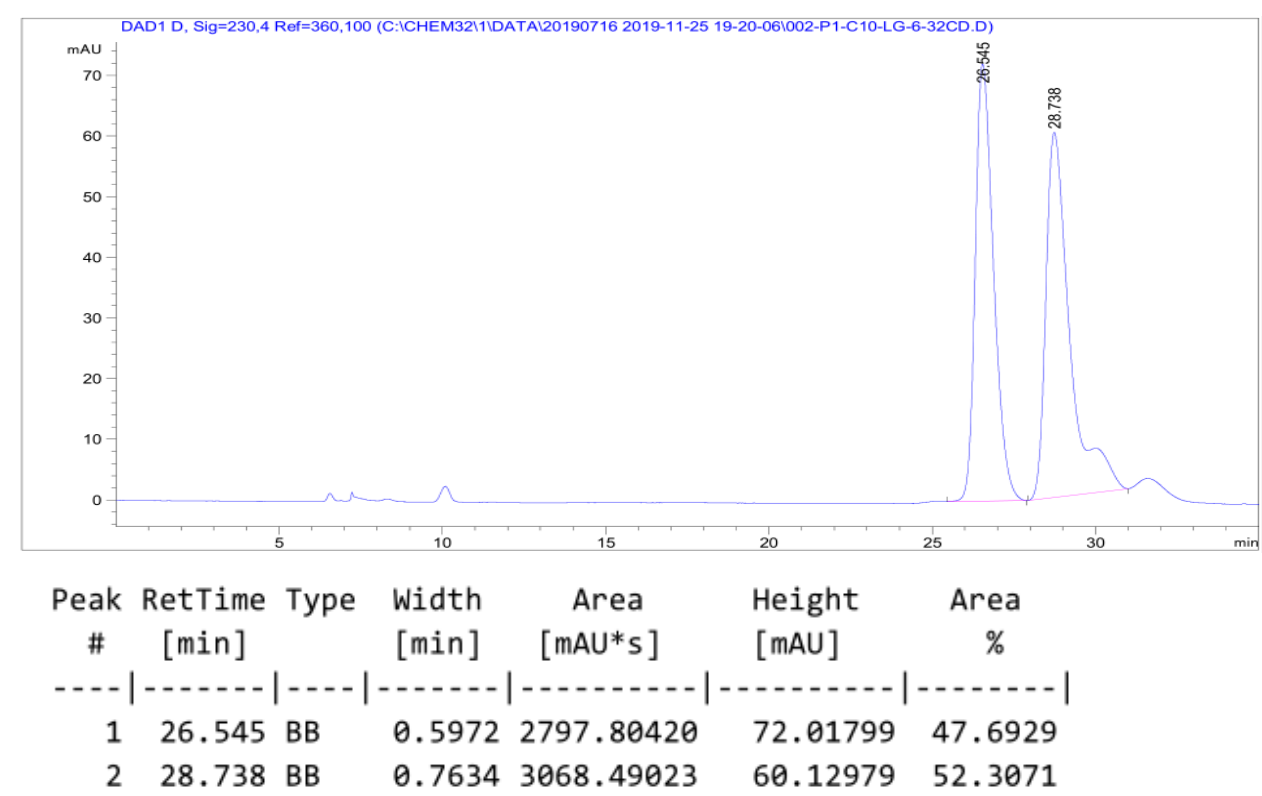

\section{5 enantioenriched, $94 \%$ ee}

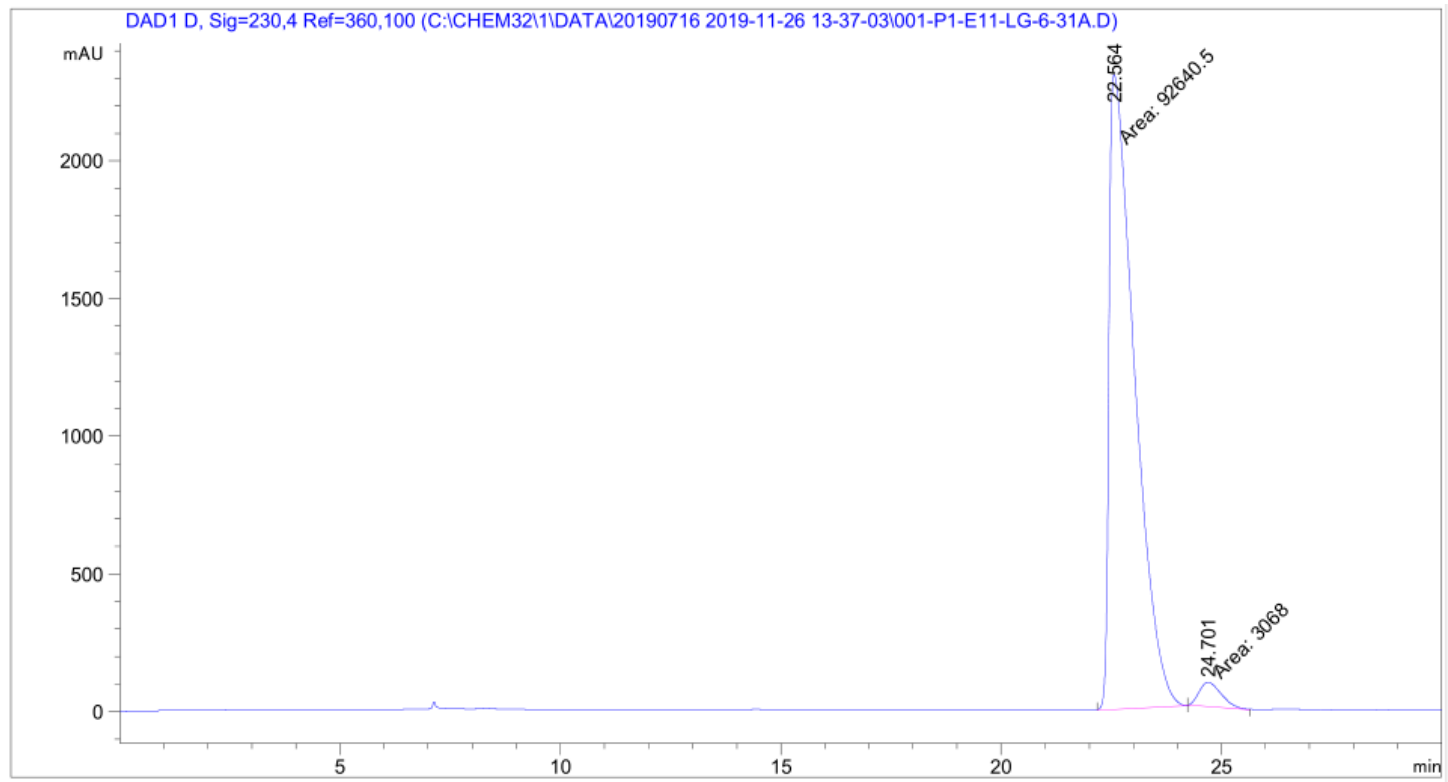




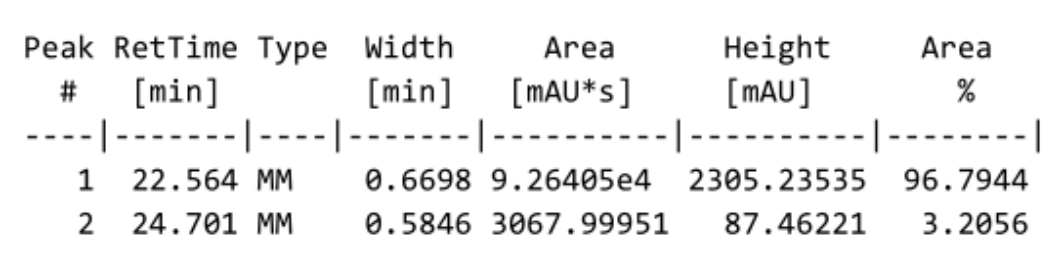<smiles>CCCCC(C(=O)OCC)c1cc(Cl)cc(C(=O)OCC)c1</smiles>

(R)-Methyl 3-(1-(tert-butoxy)-4,4-dimethyl-1-oxopentan-2-yl)-5-chlorobenzoate (16)

The product was prepared according to the general procedure with (4S,4'S)-4,4'-di((S)sec-butyl)-4,4',5,5'-tetrahydro-2,2'-bioxazole [(S,S)-L1] $(3.3 \mathrm{mg}, 0.013 \mathrm{mmol}, 13$ mol\%), $\mathrm{NiCl}_{2} \bullet \mathrm{Py}_{4}(4.5 \mathrm{mg}, 0.01 \mathrm{mmol}, 10 \mathrm{~mol} \%), \operatorname{Ir}\left[\mathrm{dF}\left(\mathrm{CF}_{3}\right) \mathrm{ppy}\right]_{2}(\mathrm{bpy}) \mathrm{PF}_{6}(3.2 \mathrm{mg}$, $0.003 \mathrm{mmol}, 3 \mathrm{~mol} \%$ ), methyl 3-bromo-5-chlorobenzoate $(25.0 \mathrm{mg}, 0.1 \mathrm{mmol}, 1.0$ equiv.), ${ }^{t} \mathrm{BuBF}_{3} \mathrm{~K}(41.0 \mathrm{mg}, 0.25 \mathrm{mmol}, 2.5$ equiv.) and tert-butyl acrylate (29.0 uL, 0.2 mmol, 2.0 equiv.) in acetone/ethyl acetate $(1.3 / 0.7, \mathrm{v} / \mathrm{v}, 2 \mathrm{~mL})$ were used. After 24 hours, the product was isolated by flash chromatography $(P E$ : ethyl acetate $=20: 1)$ as a white solid (19.9 mg, 56\% yield, 94\% ee).

${ }^{1} \mathbf{H}$ NMR $\left(400 \mathrm{MHz}, \mathrm{CDCl}_{3}\right) \delta 7.87(\mathrm{dt}, J=6.1,1.5 \mathrm{~Hz}, 2 \mathrm{H}), 7.50(\mathrm{t}, J=1.8 \mathrm{~Hz}, 1 \mathrm{H})$, $3.92(\mathrm{~s}, 3 \mathrm{H}), 3.56(\mathrm{dd}, J=9.1,3.7 \mathrm{~Hz}, 1 \mathrm{H}), 2.25(\mathrm{dd}, J=14.0,9.1 \mathrm{~Hz}, 1 \mathrm{H}), 1.48(\mathrm{dd}, J$ $=14.0,3.7 \mathrm{~Hz}, 1 \mathrm{H}), 1.38(\mathrm{~s}, 9 \mathrm{H}), 0.91(\mathrm{~s}, 9 \mathrm{H})$.

${ }^{13}$ C NMR $\left(101 \mathrm{MHz}, \mathrm{CDCl}_{3}\right) \delta 172.85,165.85,143.86,134.52,132.22,131.87,128.13$, 127.27, 81.11, 52.42, 49.00, 47.11, 31.14, 29.49, 27.83 .

HRMS (ESI): $\mathrm{C}_{19} \mathrm{H}_{28} \mathrm{ClO}_{4}^{+}(\mathrm{M}+\mathrm{H}): 355.1676$, found: 355.1670 .

$[\alpha]_{\mathrm{D}}^{25}=6.0008\left(\mathrm{c}=0.2133, \mathrm{CHCl}_{3}\right)$

Chiral HPLC: CHIRALPAK IA, $25{ }^{\circ} \mathrm{C}$, Hexanes, $1.0 \mathrm{~mL} / \mathrm{min}, 280 \mathrm{~nm}, \mathrm{t}_{\mathrm{R}}$ (major) $=24.3 \mathrm{~min}, \mathrm{t}_{\mathrm{R}}($ minor $)=22.9 \mathrm{~min}$. 


\section{6 racemic}

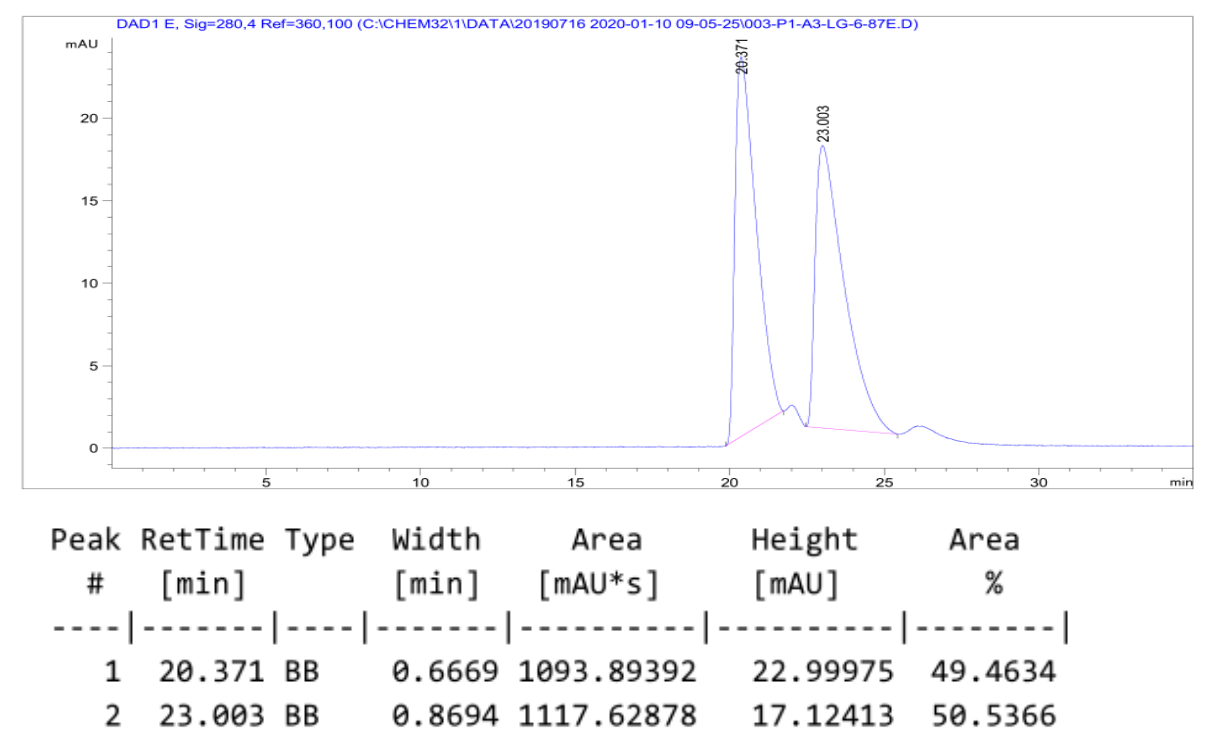

16 enantioenriched, $94 \%$ ee

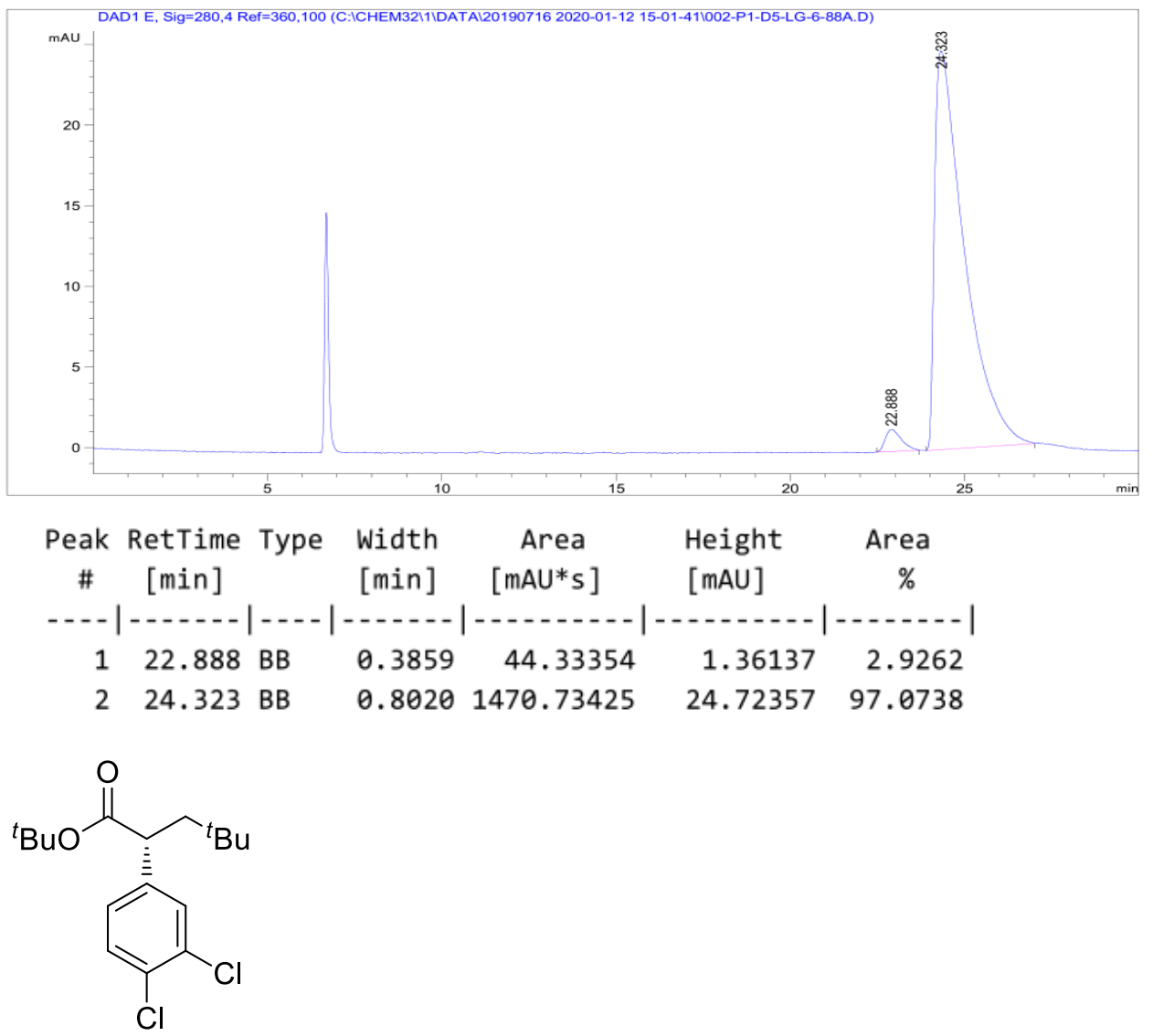

(R)-tert-Butyl 2-(3,4-dichlorophenyl)-4,4-dimethylpentanoate (17)

The product was prepared according to the general procedure with (4S,4'S)-4,4'-di((S)sec-butyl)-4,4',5,5'-tetrahydro-2,2'-bioxazole [(S,S)-L1] $(3.3 \mathrm{mg}, 0.013 \mathrm{mmol}, 13$ 


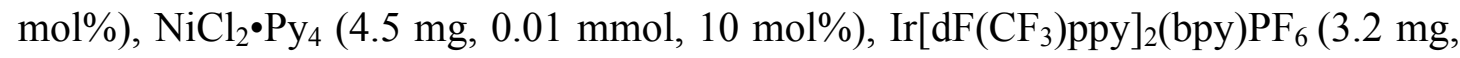
$0.003 \mathrm{mmol}, 3 \mathrm{~mol} \%$ ), 4-bromo-1,2-dichlorobenzene (12.8 uL, $0.1 \mathrm{mmol}, 1.0$ equiv.), ${ }^{t} \mathrm{BuBF}_{3} \mathrm{~K}$ (41.0 mg, $0.25 \mathrm{mmol}, 2.5$ equiv.) and tert-butyl acrylate (29.0 uL, $0.2 \mathrm{mmol}$, 2.0 equiv.) in acetone/ethyl acetate $(1.3 / 0.7, \mathrm{v} / \mathrm{v}, 2 \mathrm{~mL})$ were used. After 24 hours, the product was isolated by flash chromatography (PE: ethyl acetate $=20: 1)$ as a white solid (17.8 mg, 54\% yield, 92\% ee).

${ }^{1} \mathbf{H}$ NMR $\left(400 \mathrm{MHz}, \mathrm{CDCl}_{3}\right) \delta 7.39(\mathrm{~s}, 1 \mathrm{H}), 7.35(\mathrm{~d}, J=8.2 \mathrm{~Hz}, 1 \mathrm{H}), 7.15(\mathrm{~d}, J=8.2$ $\mathrm{Hz}, 1 \mathrm{H}), 3.47$ (d, $J=9.0 \mathrm{~Hz}, 1 \mathrm{H}), 2.24-2.18(\mathrm{~m}, 1 \mathrm{H}), 1.44$ (d, $J=13.9 \mathrm{~Hz}, 1 \mathrm{H}), 1.37$ $(\mathrm{s}, 9 \mathrm{H}), 0.89(\mathrm{~s}, 9 \mathrm{H})$.

${ }^{13}$ C NMR $\left(101 \mathrm{MHz}, \mathrm{CDCl}_{3}\right) \delta 172.98,141.78,132.38,130.80,130.38,129.74,127.11$, $80.99,48.55,47.09,31.09,29.47,27.83$.

HRMS (ESI): $\mathrm{C}_{17} \mathrm{H}_{25} \mathrm{Cl}_{2} \mathrm{O}_{2}^{+}(\mathrm{M}+\mathrm{H}): 331.1232$, found: 331.1229 .

$[\alpha]_{\mathrm{D}}^{25}=-16.8282\left(\mathrm{c}=0.700, \mathrm{CHCl}_{3}\right)$

Chiral HPLC: CHIRALPAK IA, $25{ }^{\circ} \mathrm{C}$, Hexanes, $0.5 \mathrm{~mL} / \mathrm{min}, 230 \mathrm{~nm}, \mathrm{t}_{\mathrm{R}}$ (major) $=11.3 \mathrm{~min}, \mathrm{t}_{\mathrm{R}}($ minor $)=10.4 \mathrm{~min}$.

\section{7 racemic}

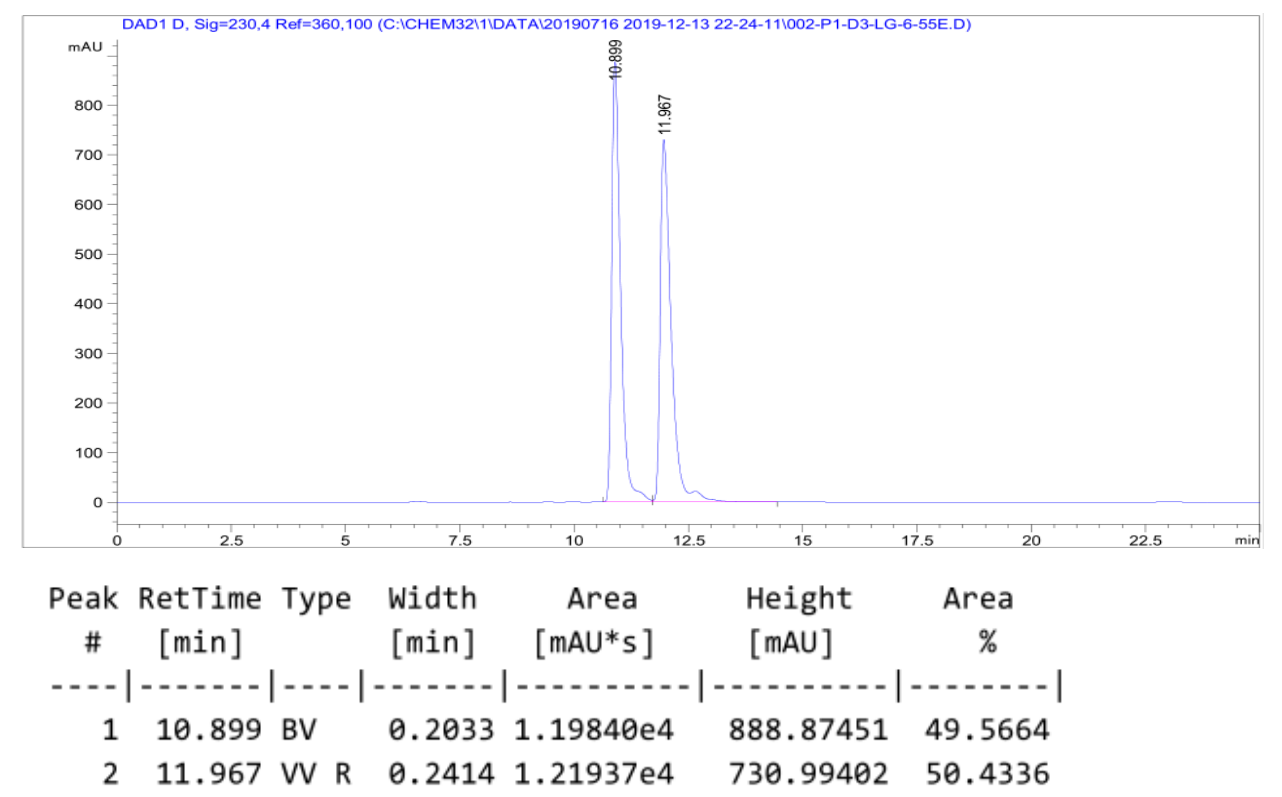




\section{7 enantioenriched, $92 \%$ ee}

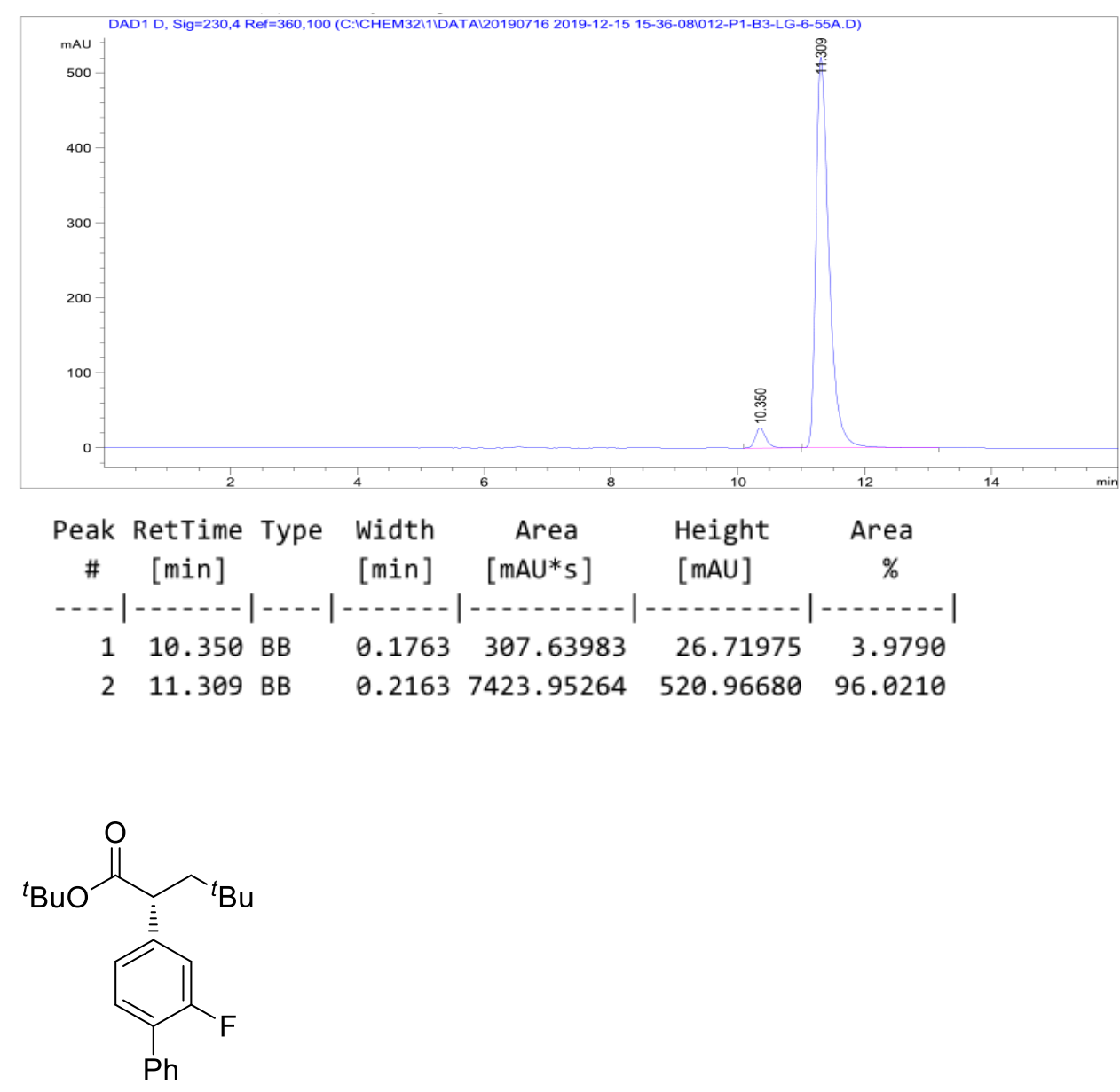

(R)-tert-Butyl 2-(2-fluoro-[1,1'-biphenyl]-4-yl)-4,4-dimethylpentanoate (18)

The product was prepared according to the general procedure with (4S,4'S)-4,4'-di((S)sec-butyl)-4,4',5,5'-tetrahydro-2,2'-bioxazole [(S,S)-L1] $(3.3 \mathrm{mg}, 0.013 \mathrm{mmol}, 13$ $\mathrm{mol} \%$ ), $\mathrm{NiCl}_{2} \bullet \mathrm{Py}_{4}(4.5 \mathrm{mg}, 0.01 \mathrm{mmol}, 10 \mathrm{~mol} \%), \operatorname{Ir}\left[\mathrm{dF}\left(\mathrm{CF}_{3}\right) \text { ppy }\right]_{2}(\mathrm{bpy}) \mathrm{PF}_{6}(3.2 \mathrm{mg}$, $0.003 \mathrm{mmol}, 3 \mathrm{~mol} \%$ ), 4-bromo-2-fluoro-1,1'-biphenyl (25.1 mg, $0.1 \mathrm{mmol}, 1.0$ equiv.), ${ }^{t} \mathrm{BuBF}_{3} \mathrm{~K}(41.0 \mathrm{mg}, 0.25 \mathrm{mmol}, 2.5$ equiv.) and tert-butyl acrylate (29.0 uL, $0.2 \mathrm{mmol}$, 2.0 equiv.) in acetone/ethyl acetate $(1.3 / 0.7, \mathrm{v} / \mathrm{v}, 2 \mathrm{~mL})$ were used. After 24 hours, the product was isolated by flash chromatography $(\mathrm{PE}$ : ethyl acetate $=20: 1)$ as a white solid (20.1 mg, 56\% yield, 90\% ee).

${ }^{1} \mathbf{H}$ NMR $\left(400 \mathrm{MHz} \mathrm{CDCl}_{3}\right) \delta$ 7.55-7.53 (m, 2H), 7.45-7.41 (m, 2H), 7.39-7.33 (m, 2H), 7.16-7.10 (m, 2H), 3.56 (dd, $J=9.3,3.4 \mathrm{~Hz}, 1 \mathrm{H}), 2.28(\mathrm{dd}, J=14.0,9.3 \mathrm{~Hz}, 1 \mathrm{H})$, $1.53(\mathrm{dd}, J=14.0,3.5 \mathrm{~Hz}, 1 \mathrm{H}), 1.41(\mathrm{~s}, 9 \mathrm{H}), 0.94(\mathrm{~s}, 9 \mathrm{H})$.

${ }^{19}$ F NMR $\left(375 \mathrm{MHz}, \mathrm{CDCl}_{3}\right) \delta-117.87--118.01(\mathrm{~m})$. 
${ }^{13}$ C NMR (100 MHz, $\left.\mathrm{CDCl}_{3}\right) \delta 173.34,159.6(\mathrm{~d}, J=247.8 \mathrm{~Hz}), 143.05(\mathrm{~d}, J=7.8 \mathrm{~Hz})$, $135.62(\mathrm{~d}, J=3.8 \mathrm{~Hz}), 130.59$ (d, $J=4.0 \mathrm{~Hz}), 128.93$ (d, $J=3.0 \mathrm{~Hz}), 128.39,127.54$, $127.3(\mathrm{~d}, J=13.7 \mathrm{~Hz}), 123.66(\mathrm{~d}, J=3.4 \mathrm{~Hz}), 115.29(\mathrm{~d}, J=23.8 \mathrm{~Hz}), 80.77,48.83$, $47.18,31.08,29.48,27.86$.

HRMS (ESI): $\mathrm{C}_{23} \mathrm{H}_{30} \mathrm{FO}_{2}^{+}(\mathrm{M}+\mathrm{H}): 357.2230$, found: 357.2227 .

$[\alpha]_{\mathrm{D}}^{25}=-10.4060\left(\mathrm{c}=0.3200, \mathrm{CHCl}_{3}\right)$

Chiral HPLC: CHIRALPAK IA, $25{ }^{\circ} \mathrm{C},{ }^{i} \mathrm{PrOH}-h$ exanes $1 / 100,0.5 \mathrm{~mL} / \mathrm{min}, 250 \mathrm{~nm}$, $t_{R}($ major $)=8.3 \mathrm{~min}, t_{R}($ minor $)=7.9 \mathrm{~min}$.

\section{8 racemic}

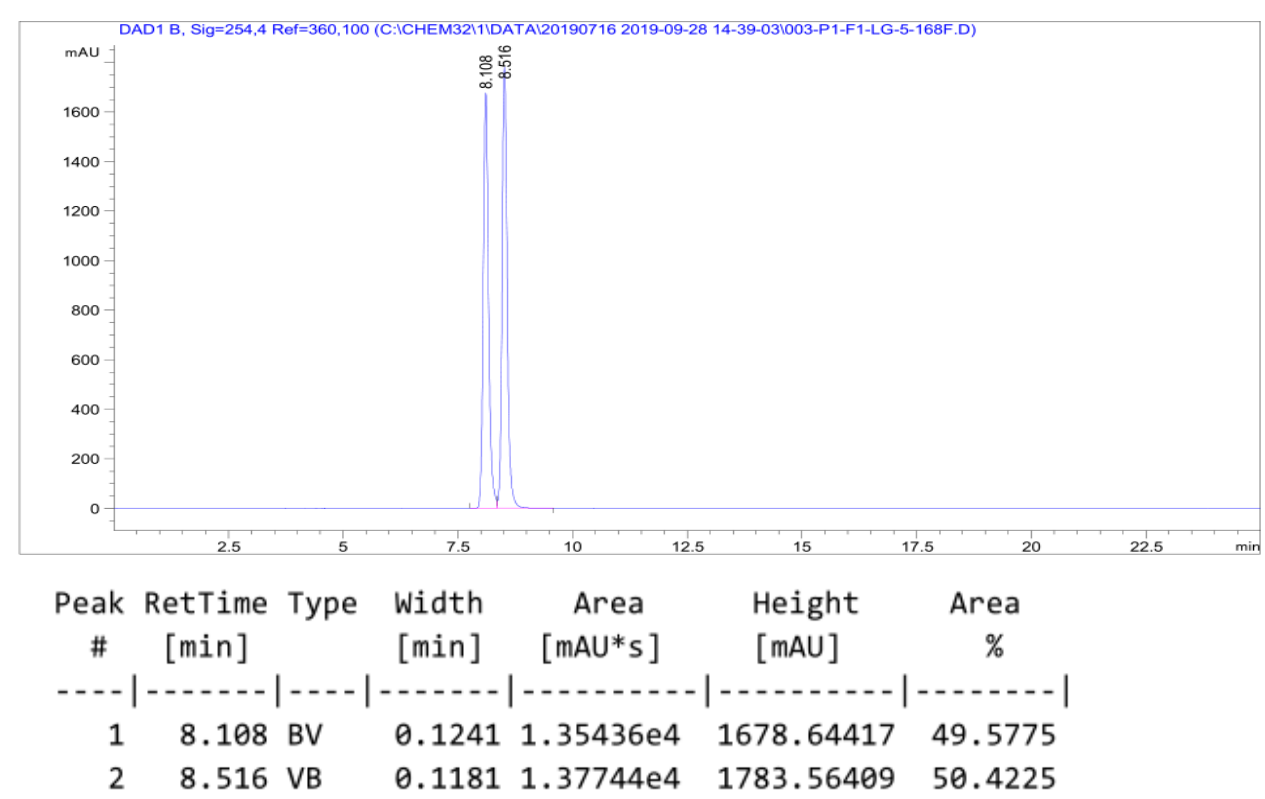

\section{8 enantioenriched, $90 \%$ ee}

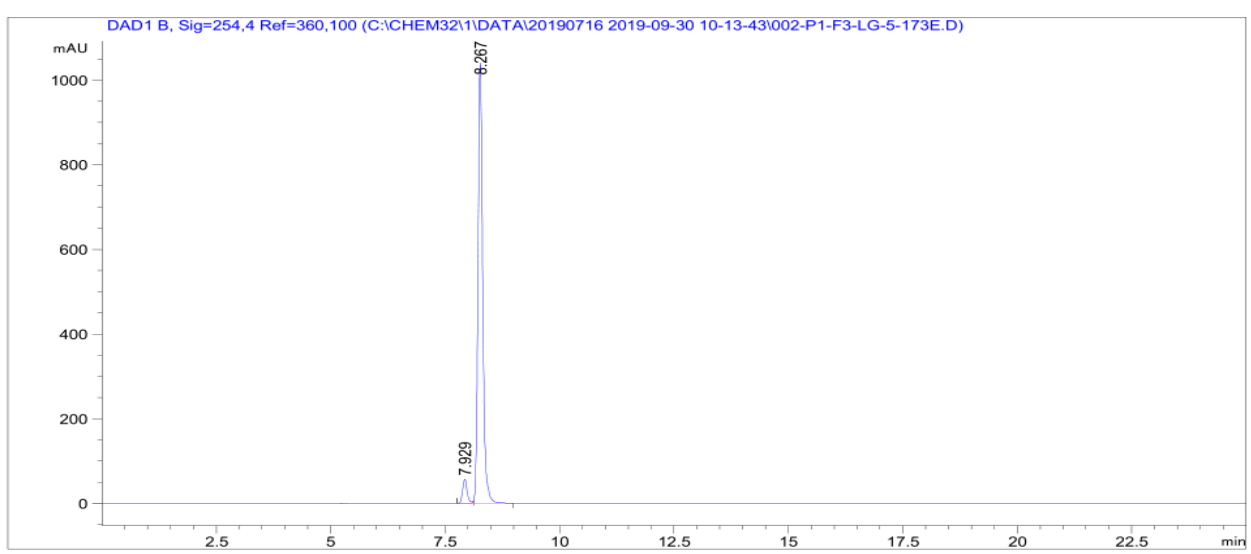




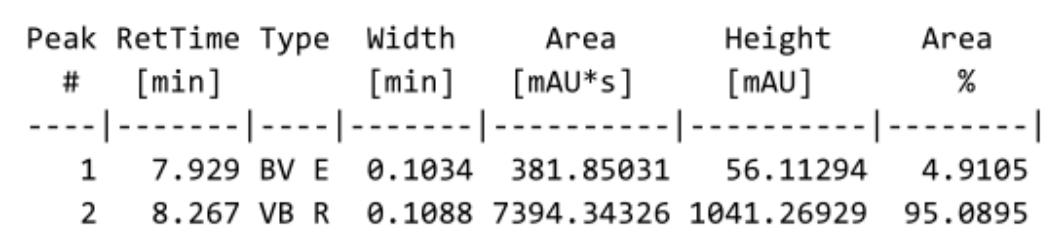

(BuO

(R)-tert-Butyl 2-([1,1'-biphenyl]-4-yl)-4,4-dimethylpentanoate (19)

The product was prepared according to the general procedure with (4S,4'S)-4,4'-di((S)sec-butyl)-4,4',5,5'-tetrahydro-2,2'-bioxazole $[(S, S)$-L1 $]$ (3.3 mg, $0.013 \mathrm{mmol}, 13$ $\mathrm{mol} \%$ ), $\left.\mathrm{NiCl}_{2} \cdot \mathrm{Py}_{4}(4.5 \mathrm{mg}, 0.01 \mathrm{mmol}, 10 \mathrm{~mol} \%), \operatorname{Ir}\left[\mathrm{dF}_{(\mathrm{CF}}\right) \mathrm{ppy}\right]_{2}(\mathrm{bpy}) \mathrm{PF}_{6}(3.2 \mathrm{mg}$, $0.003 \mathrm{mmol}, 3 \mathrm{~mol} \%$ ), 4-bromo-1,1'-biphenyl (23.3 mg, $0.1 \mathrm{mmol}, 1.0$ equiv.), ${ }^{t} \mathrm{BuBF}_{3} \mathrm{~K}$ (41.0 mg, $0.25 \mathrm{mmol}, 2.5$ equiv.) and tert-butyl acrylate $(29.0 \mathrm{uL}, 0.2 \mathrm{mmol}$, 2.0 equiv.) in acetone/ethyl acetate $(1.3 / 0.7, \mathrm{v} / \mathrm{v}, 2 \mathrm{~mL})$ were used. After 24 hours, the product was isolated by flash chromatography $(\mathrm{PE}$ : ethyl acetate $=20: 1)$ as a white solid (18.0 mg, 53\% yield, $85 \%$ ee).

${ }^{1} \mathbf{H}$ NMR $\left(400 \mathrm{MHz}, \mathrm{CDCl}_{3}\right) \delta 7.59(\mathrm{~d}, J=7.3 \mathrm{~Hz}, 2 \mathrm{H}), 7.53(\mathrm{~d}, J=8.2 \mathrm{~Hz}, 2 \mathrm{H}), 7.43$ (t, $J=7.6 \mathrm{~Hz}, 2 \mathrm{H}), 7.37(\mathrm{~d}, J=8.2 \mathrm{~Hz}, 2 \mathrm{H}), 7.33(\mathrm{t}, J=7.3 \mathrm{~Hz}, 1 \mathrm{H}), 3.58(\mathrm{dd}, J=9.4$, $3.2 \mathrm{~Hz}, 1 \mathrm{H}), 2.31(\mathrm{dd}, J=14.0,9.5 \mathrm{~Hz}, 1 \mathrm{H}), 1.53(\mathrm{dd}, J=14.0,3.3 \mathrm{~Hz}, 1 \mathrm{H}), 1.40$ (s, 9H), $0.94(\mathrm{~s}, 9 \mathrm{H})$.

${ }^{13}$ C NMR $\left(100 \mathrm{MHz}, \mathrm{CDCl}_{3}\right) \delta 173.86,140.80,140.72,139.50,128.70,128.01,127.15$, $127.13,127.00,80.43,48.88,47.25,31.08,29.49,27.85$.

HRMS (ESI): $\mathrm{C}_{23} \mathrm{H}_{31} \mathrm{O}_{2}^{+}(\mathrm{M}+\mathrm{H}): 339.2324$, found: 339.2318 .

$[\alpha]_{\mathrm{D}}^{25}=-13.9110\left(\mathrm{c}=0.6333, \mathrm{CHCl}_{3}\right)$

Chiral HPLC: CHIRALPAK IA, $25{ }^{\circ} \mathrm{C},{ }^{i} \mathrm{PrOH}-$ hexanes 1/300, $1.0 \mathrm{~mL} / \mathrm{min}, 270 \mathrm{~nm}$, $\mathrm{t}_{\mathrm{R}}($ major $)=5.7 \mathrm{~min}, \mathrm{t}_{\mathrm{R}}($ minor $)=5.2 \mathrm{~min}$ 


\section{9 racemic}

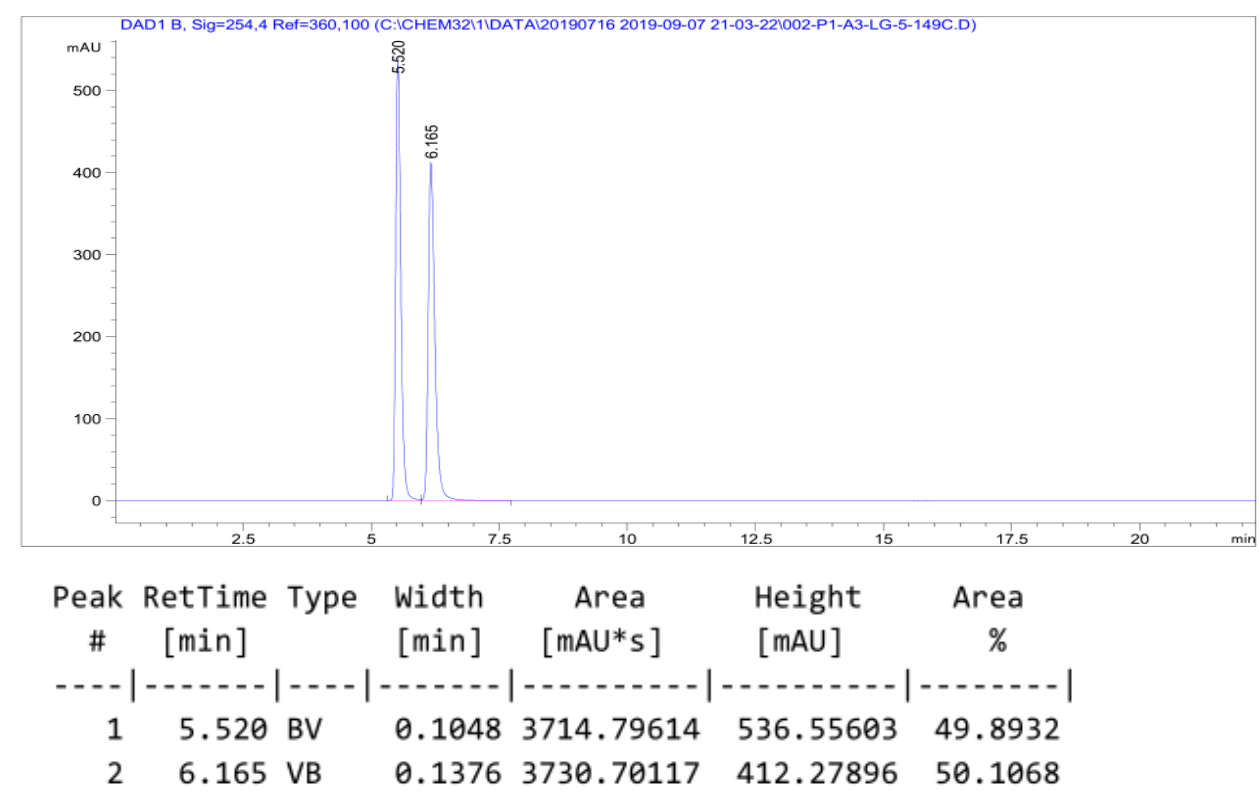

19 enantioenriched, $85 \%$ ee

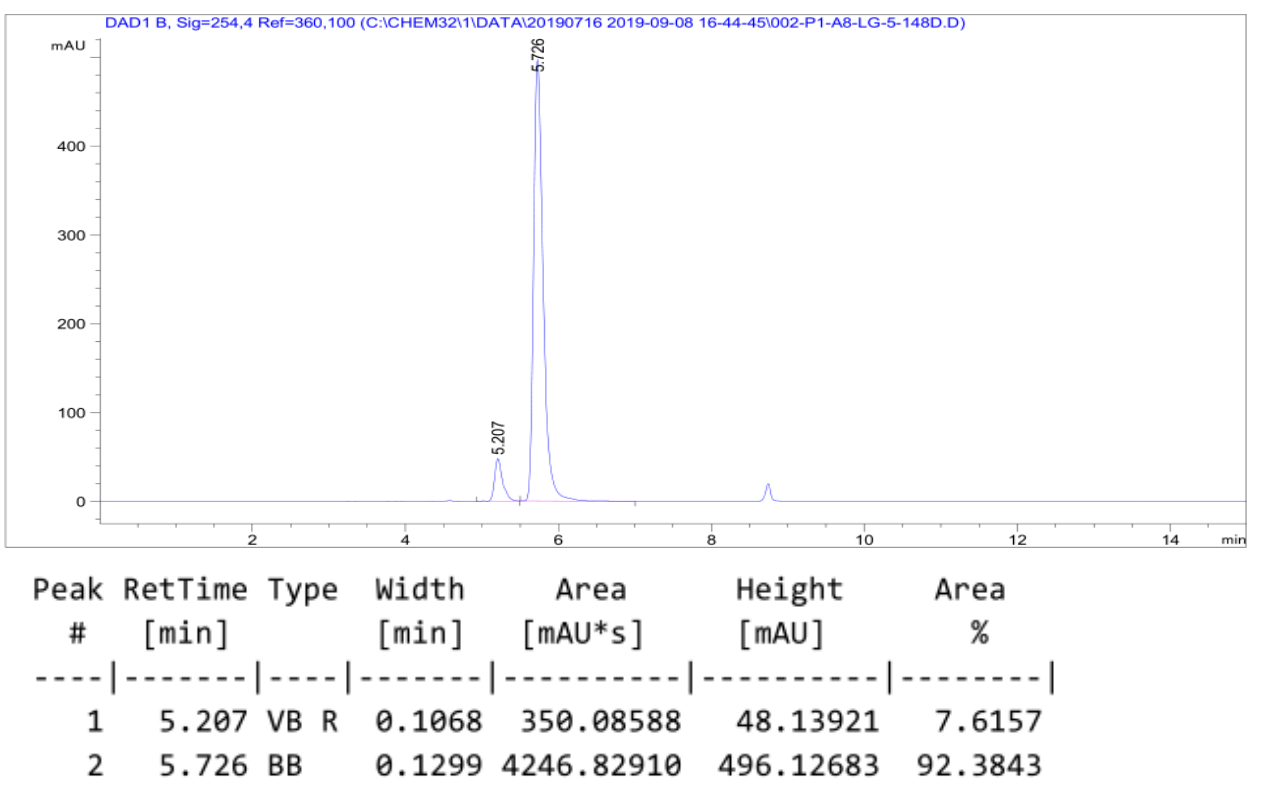<smiles>CCC(C)(C)CC(C(=O)OCC(C)(C)C)c1ccc(S(C)(C)C)cc1</smiles>

(R)-tert-Butyl 4,4-dimethyl-2-(4-(methylthio)phenyl)pentanoate (20) 
The product was prepared according to the general procedure with $(4 \mathrm{~S}, 4$ 'S)-4,4'-di((S)sec-butyl)-4,4',5,5'-tetrahydro-2,2'-bioxazole [(S,S)-L1] $(3.3 \mathrm{mg}, 0.013 \mathrm{mmol}, 13$ mol\%), $\mathrm{NiCl}_{2} \cdot \mathrm{Py}_{4}(4.5 \mathrm{mg}, 0.01 \mathrm{mmol}, 10 \mathrm{~mol} \%), \operatorname{Ir}\left[\mathrm{dF}\left(\mathrm{CF}_{3}\right) \mathrm{ppy}\right]_{2}(\mathrm{bpy}) \mathrm{PF}_{6}(3.2 \mathrm{mg}$, $0.003 \mathrm{mmol}, 3 \mathrm{~mol} \%$ ), (4-bromophenyl)(methyl)sulfane (20.3 mg, $0.1 \mathrm{mmol}, 1.0$ equiv.), ${ }^{t} \mathrm{BuBF}_{3} \mathrm{~K}(41.0 \mathrm{mg}, 0.25 \mathrm{mmol}, 2.5$ equiv.) and tert-butyl acrylate (29.0 uL, 0.2 mmol, 2.0 equiv.) in acetone/ethyl acetate (1.3/0.7, v/v, $2 \mathrm{~mL})$ were used. After 24 hours, the product was isolated by flash chromatography $(P E$ : ethyl acetate $=20: 1)$ as a white solid (18.9 mg, 61\% yield, 83\% ee).

${ }^{1} \mathbf{H}$ NMR $\left(400 \mathrm{MHz}, \mathrm{CDCl}_{3}\right) \delta 7.20(\mathrm{q}, J=8.5 \mathrm{~Hz}, 4 \mathrm{H}), 3.48(\mathrm{dd}, J=9.3,3.5 \mathrm{~Hz}, 1 \mathrm{H})$, $2.47(\mathrm{~s}, 3 \mathrm{H}), 2.23$ (dd, $J=14.0,9.3 \mathrm{~Hz}, 1 \mathrm{H}), 1.46$ (dd, $J=14.0,3.5 \mathrm{~Hz}, 1 \mathrm{H}), 1.37$ (s, 9H), $0.90(\mathrm{~s}, 9 \mathrm{H})$.

${ }^{13}$ C NMR (100 MHz, $\left.\mathrm{CDCl}_{3}\right) \delta 173.79,138.56,136.45,128.13,126.71,80.40,48.69$, $47.10,31.03,29.48,27.82,15.90$.

HRMS (ESI): $\mathrm{C}_{18} \mathrm{H}_{29} \mathrm{O}_{2} \mathrm{~S}^{+}(\mathrm{M}+\mathrm{H}): 309.1888$, found: 309.1884 .

$[\alpha]_{\mathrm{D}}^{25}=-20.5765\left(\mathrm{c}=0.2600, \mathrm{CHCl}_{3}\right)$

Chiral HPLC: CHIRALPAK IA, $25{ }^{\circ} \mathrm{C},{ }^{i} \mathrm{PrOH}-$ hexanes 1/500, $0.5 \mathrm{~mL} / \mathrm{min}, 254 \mathrm{~nm}$, $t_{R}($ major $)=12.0 \mathrm{~min}, t_{R}($ minor $)=11.0 \mathrm{~min}$

\section{0 racemic}

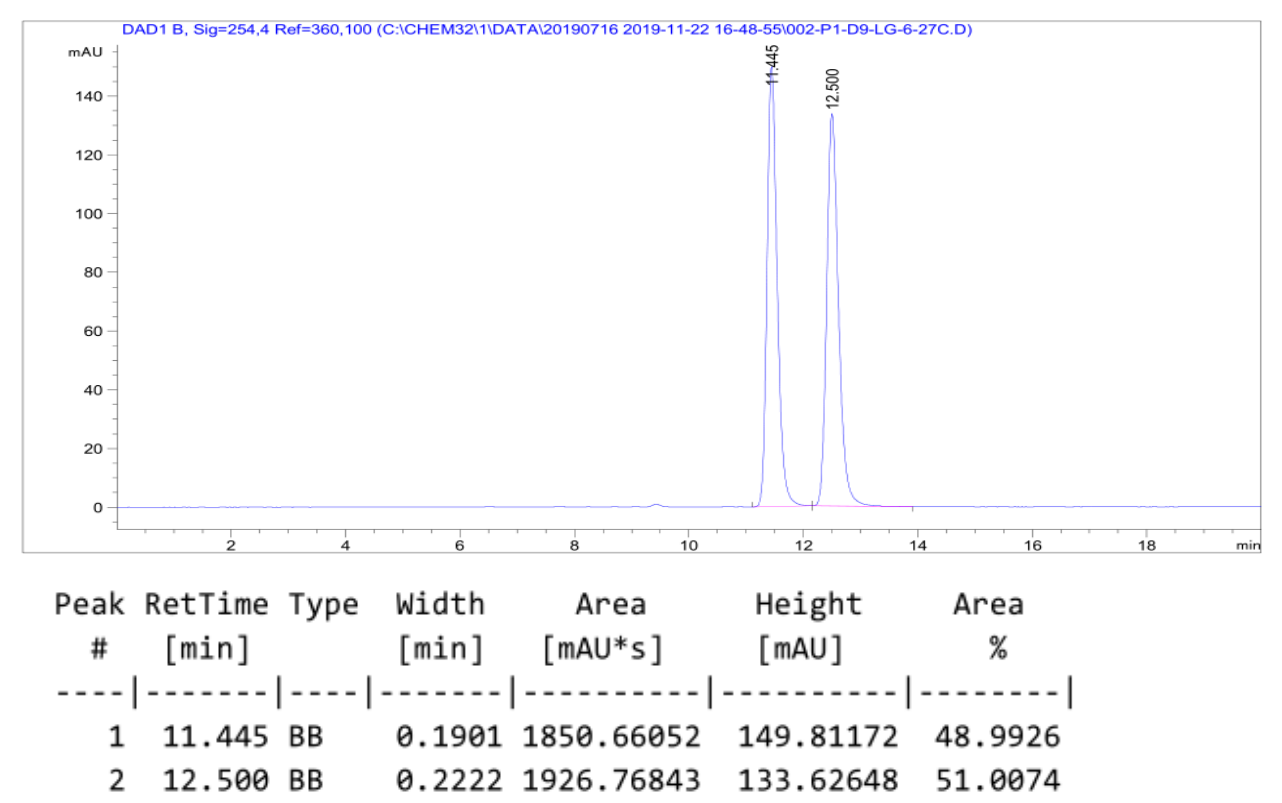




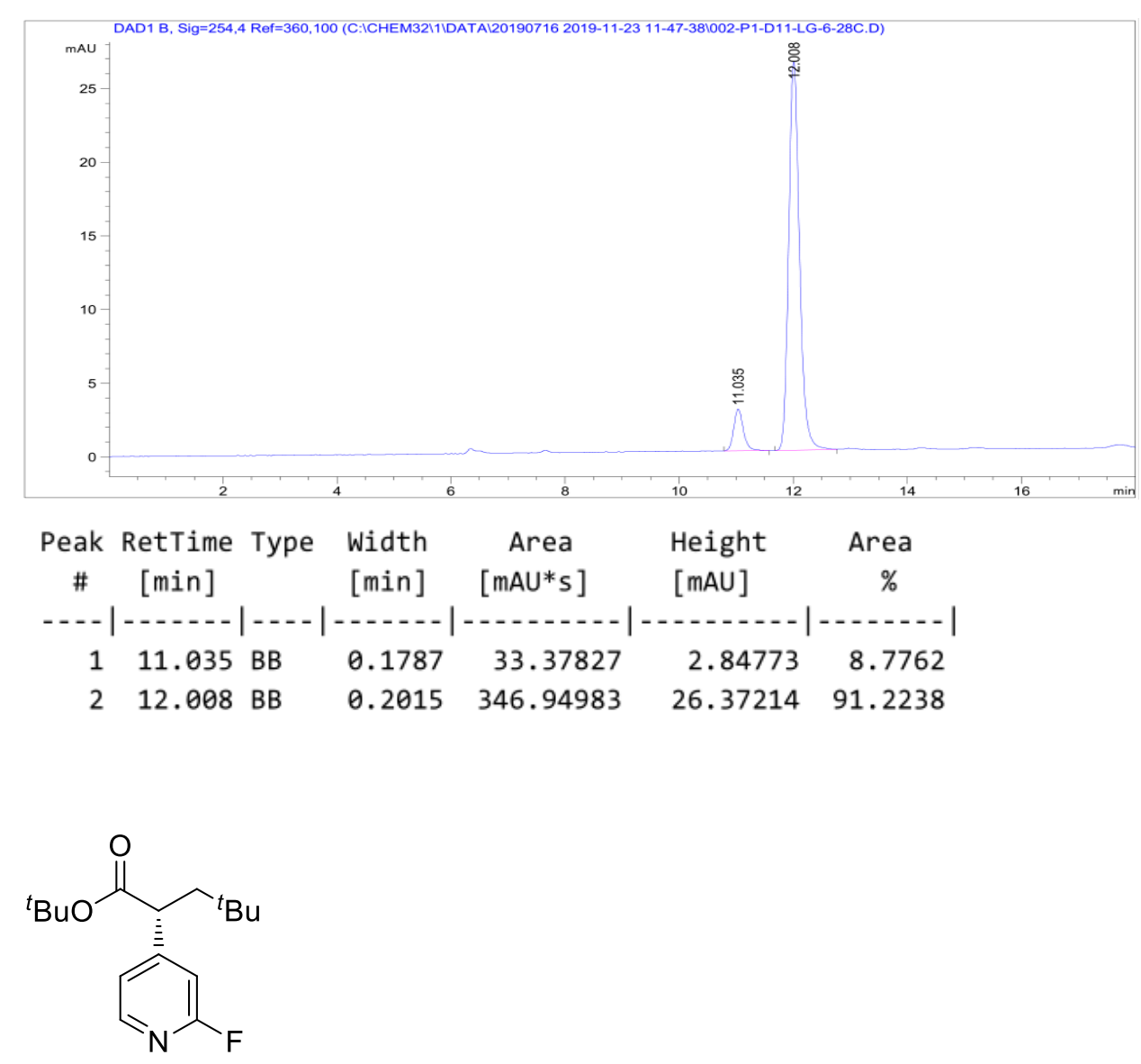

(R)-tert-Butyl 2-(2-fluoropyridin-4-yl)-4,4-dimethylpentanoate (21)

The product was prepared according to the general procedure with (4S,4'S)-4,4'-di((S)sec-butyl)-4,4',5,5'-tetrahydro-2,2'-bioxazole [(S,S)-L1] (3.3 mg, $0.013 \mathrm{mmol}, 13$ mol\%), $\mathrm{NiCl}_{2} \cdot \mathrm{Py}_{4}(4.5 \mathrm{mg}, 0.01 \mathrm{mmol}, 10 \mathrm{~mol} \%), \operatorname{Ir}\left[\mathrm{dF}\left(\mathrm{CF}_{3}\right) \mathrm{ppy}\right]_{2}(\mathrm{bpy}) \mathrm{PF}_{6}(3.2 \mathrm{mg}$, $0.003 \mathrm{mmol}, 3 \mathrm{~mol} \%$ ), 4-bromo-2-fluoropyridine (10.3 uL, $0.1 \mathrm{mmol}, 1.0$ equiv.), ${ }^{t} \mathrm{BuBF}_{3} \mathrm{~K}$ (41.0 mg, $0.25 \mathrm{mmol}, 2.5$ equiv.) and tert-butyl acrylate (29.0 uL, $0.2 \mathrm{mmol}$, 2.0 equiv.) in acetone/ethyl acetate $(1.3 / 0.7, \mathrm{v} / \mathrm{v}, 2 \mathrm{~mL})$ were used. After 24 hours, the product was isolated by flash chromatography (PE: ethyl acetate $=20: 1)$ as a colorless oil (19.7 mg, 70\% yield, 92\% ee).

${ }^{1} \mathbf{H}$ NMR $\left(400 \mathrm{MHz}, \mathrm{CDCl}_{3}\right) \delta 8.13(\mathrm{~d}, J=5.2 \mathrm{~Hz}, 1 \mathrm{H}), 7.12(\mathrm{dt}, J=5.2,1.6 \mathrm{~Hz}, 1 \mathrm{H})$, 6.87 (br, 1H), 3.55 (dd, $J=9.1,3.5 \mathrm{~Hz}, 1 \mathrm{H}), 2.25(\mathrm{dd}, J=14.0,9.1 \mathrm{~Hz}, 1 \mathrm{H}), 1.48-1.44$ (m, 1H), $1.38(\mathrm{~s}, 9 \mathrm{H}), 0.91(\mathrm{~s}, 9 \mathrm{H})$.

${ }^{19}$ F NMR $\left(375 \mathrm{MHz}, \mathrm{CDCl}_{3}\right) \delta-68.10(\mathrm{~s})$ 
${ }^{13}$ C NMR (100 MHz, $\left.\mathrm{CDCl}_{3}\right) \delta 171.93,164.01(\mathrm{~d}, J=238.6 \mathrm{~Hz}), 156.20(\mathrm{~d}, J=7.8$ Hz), $147.62(\mathrm{~d}, J=15.2 \mathrm{~Hz}), 120.71(\mathrm{~d}, J=3.8 \mathrm{~Hz}), 108.52$ (d, $J=37.7 \mathrm{~Hz}), 81.51$, $48.87,46.69,31.12,29.38,27.75$.

HRMS (ESI): $\mathrm{C}_{16} \mathrm{H}_{25} \mathrm{FNO}_{2}{ }^{+}(\mathrm{M}+\mathrm{H}): 282.1869$, found: 282.1858 .

$[\alpha]_{\mathrm{D}}^{25}=-18.0565\left(\mathrm{c}=0.5167, \mathrm{CHCl}_{3}\right)$

Chiral HPLC: CHIRALPAK IC, $25{ }^{\circ} \mathrm{C},{ }^{i} \mathrm{PrOH}-h$ exanes 5/95, $0.5 \mathrm{~mL} / \mathrm{min}, 254 \mathrm{~nm}, \mathrm{t}_{\mathrm{R}}$ $($ major $)=14.4 \mathrm{~min}, \mathrm{t}_{\mathrm{R}}($ minor $)=12.0 \mathrm{~min}$.

\section{1 racemic}

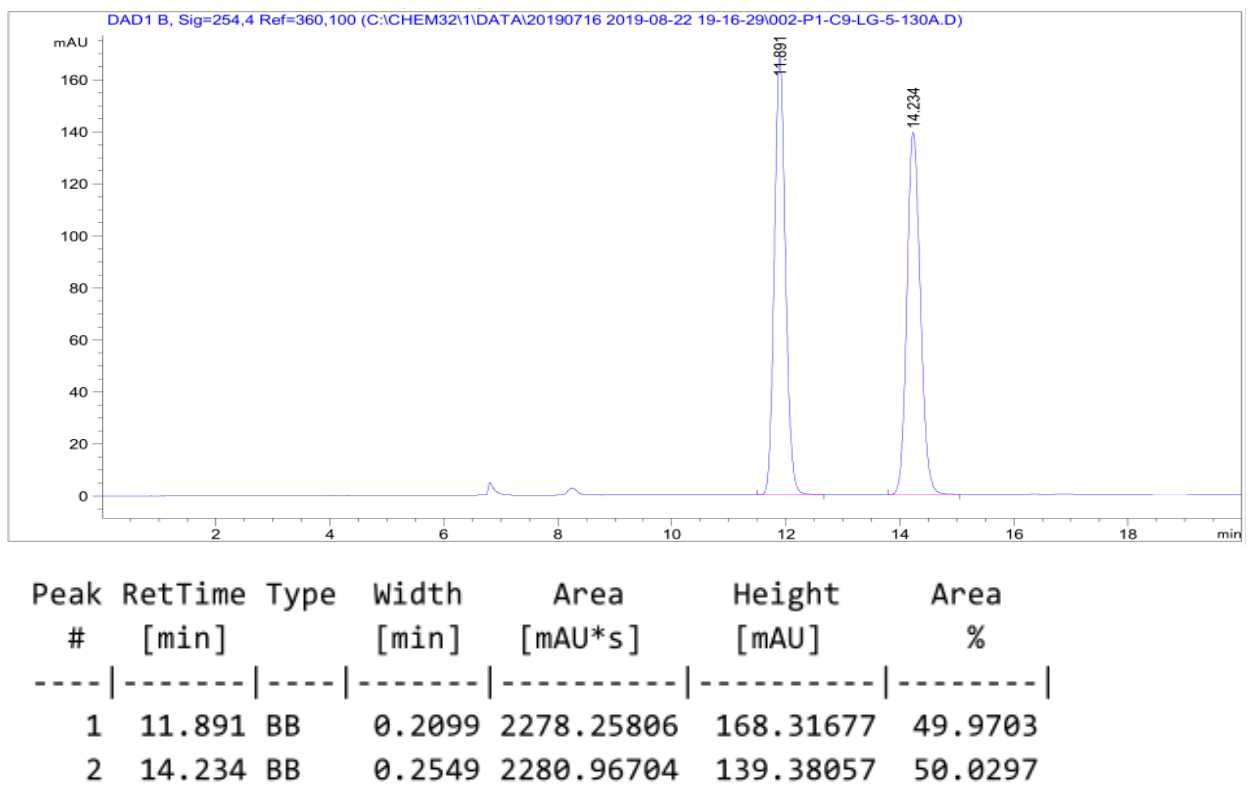

\section{1 enantioenriched, $92 \%$ ee}

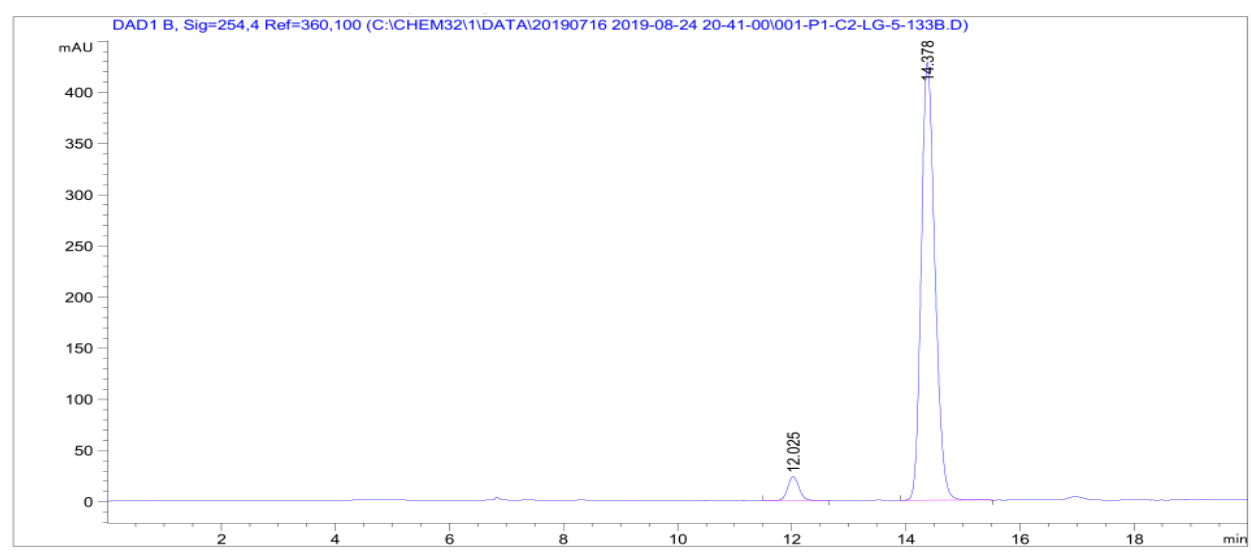




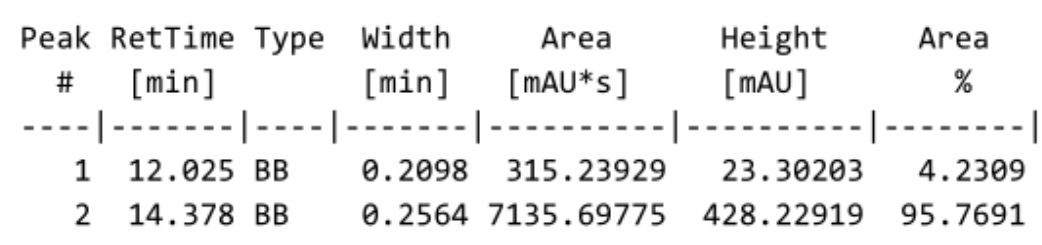<smiles>CCCCOC(=O)CC(C)(C)C</smiles>

\section{(R)-tert-Butyl 2-(2,6-dichloropyridin-4-yl)-4,4-dimethylpentanoate (22)}

The product was prepared according to the general procedure with (4S,4'S)-4,4'-di((S)sec-butyl)-4,4',5,5'-tetrahydro-2,2'-bioxazole [(S,S)-L1] $(3.3 \mathrm{mg}, 0.013 \mathrm{mmol}, 13$ mol\%), $\mathrm{NiCl}_{2} \cdot \mathrm{Py}_{4}(4.5 \mathrm{mg}, 0.01 \mathrm{mmol}, 10 \mathrm{~mol} \%), \operatorname{Ir}\left[\mathrm{dF}\left(\mathrm{CF}_{3}\right) \text { ppy }\right]_{2}(\mathrm{bpy}) \mathrm{PF}_{6}(3.2 \mathrm{mg}$, $0.003 \mathrm{mmol}, 3 \mathrm{~mol} \%$ ), 4-bromo-2,6-dichloropyridine (22.7 mg, $0.1 \mathrm{mmol}, 1.0$ equiv.), ${ }^{t} \mathrm{BuBF}_{3} \mathrm{~K}$ (41.0 mg, $0.25 \mathrm{mmol}, 2.5$ equiv.) and tert-butyl acrylate (29.0 uL, $0.2 \mathrm{mmol}$, 2.0 equiv.) in Acetone/ethyl acetate (1.3/0.7, v/v, $2 \mathrm{~mL})$ were used. After 24 hours, the product was isolated by flash chromatography $(\mathrm{PE}$ : ethyl acetate $=20: 1)$ as a white solid (19.4 mg, 58\% yield, 95\% ee).

${ }^{1} \mathbf{H}$ NMR $\left(400 \mathrm{MHz}, \mathrm{CDCl}_{3}\right) \delta 7.21(\mathrm{~s}, 2 \mathrm{H}), 3.47(\mathrm{dd}, J=9.2,3.4 \mathrm{~Hz}, 1 \mathrm{H}), 2.22(\mathrm{dd}, J$ $=14.0,9.2 \mathrm{~Hz}, 1 \mathrm{H}), 1.44-1.41(\mathrm{~m}, 1 \mathrm{H}), 1.40(\mathrm{~s}, 9 \mathrm{H}), 0.91(\mathrm{~s}, 9 \mathrm{H})$.

${ }^{13}$ C NMR $\left(100 \mathrm{MHz}, \mathrm{CDCl}_{3}\right) \delta 171.33,156.07,150.64,122.08,81.97,48.58,46.79$, $31.19,29.34,27.76$.

HRMS (ESI): $\mathrm{C}_{16} \mathrm{H}_{24} \mathrm{Cl}_{2} \mathrm{NO}_{2}{ }^{+}(\mathrm{M}+\mathrm{H}): 332.1184$, found: 332.1175 .

$[\alpha]_{\mathrm{D}}^{25}=-11.5673\left(\mathrm{c}=0.3700, \mathrm{CHCl}_{3}\right)$

Chiral HPLC: CHIRALPAK IC, $25{ }^{\circ} \mathrm{C},{ }^{i} \mathrm{PrOH}-$ hexanes $1 / 300,0.5 \mathrm{~mL} / \mathrm{min}, 254 \mathrm{~nm}$, $t_{R}($ major $)=18.7 \mathrm{~min}, t_{R}($ minor $)=14.3 \mathrm{~min}$. 


\section{2 racemic}

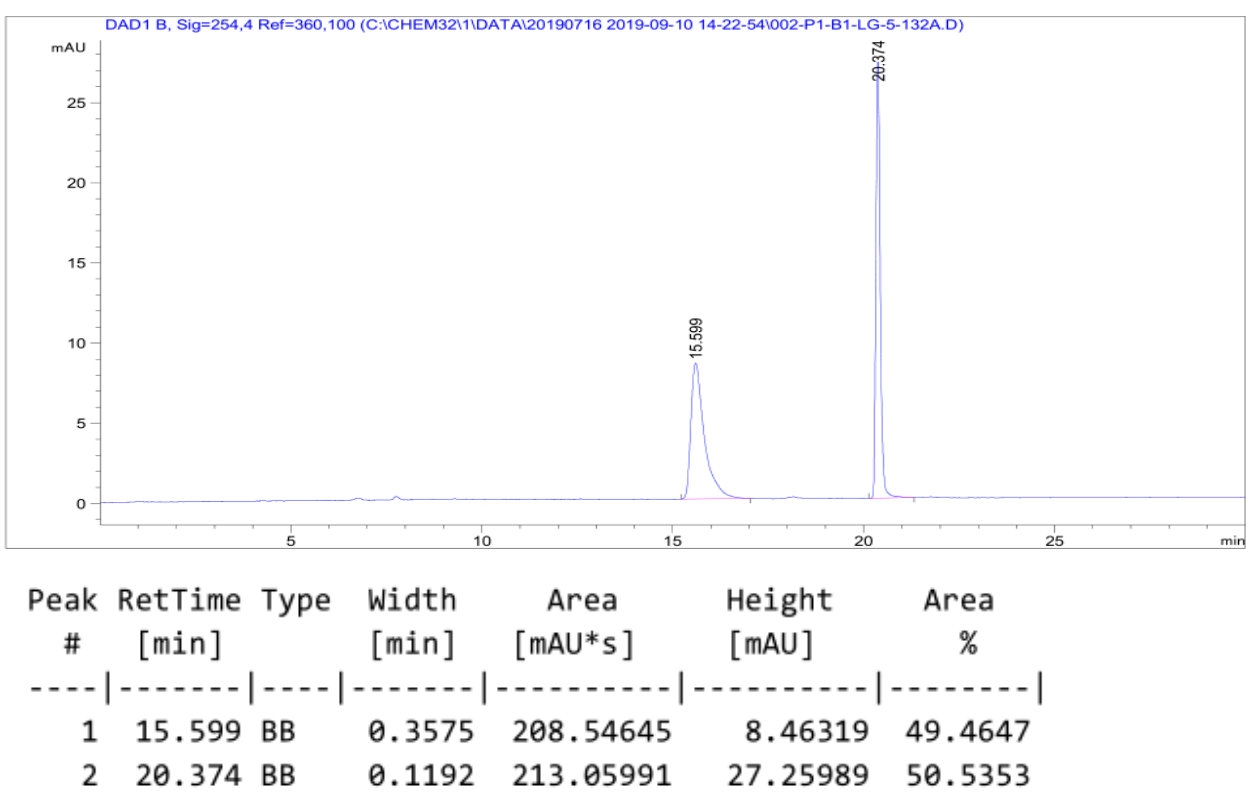

22 enantioenriched, $95 \%$ ee

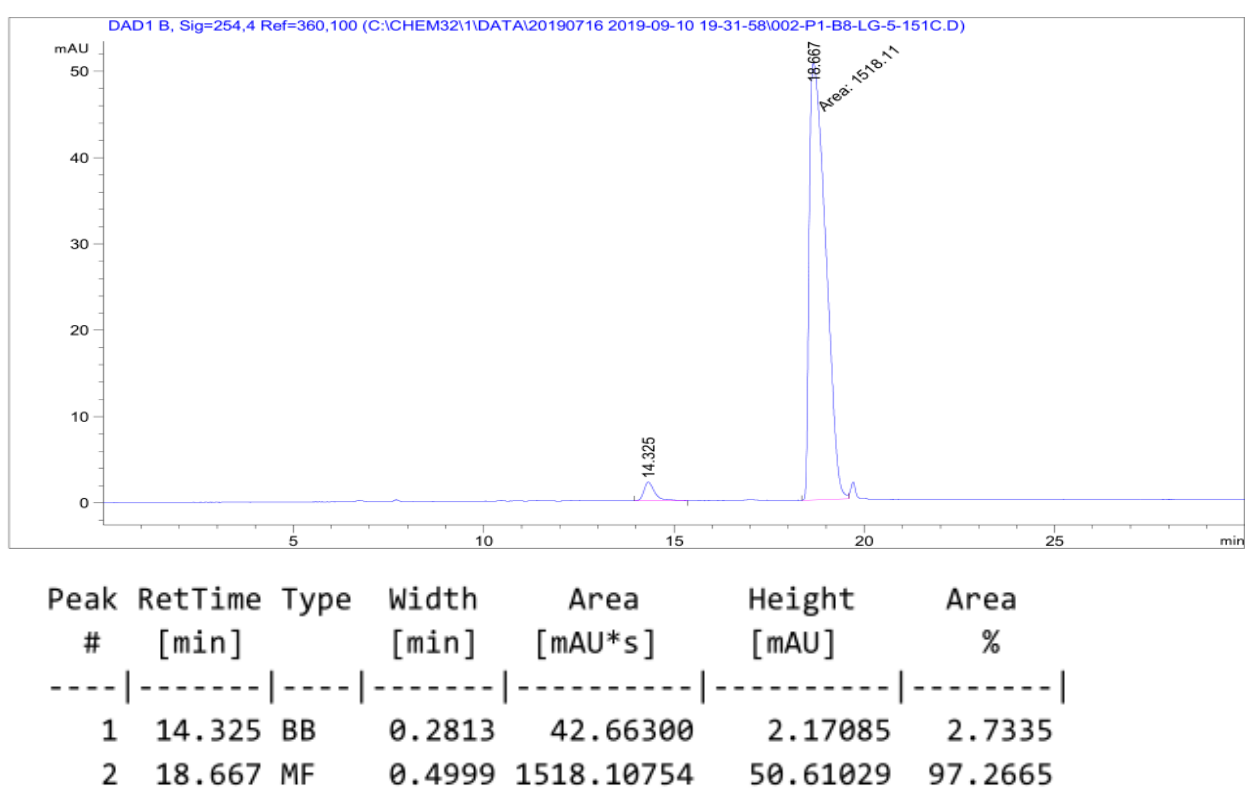<smiles>CC(C)(C)COC(=O)CC(C)(C)C</smiles>

(R)-tert-Butyl 2-(2-chloropyridin-4-yl)-4,4-dimethylpentanoate (23) 
The product was prepared according to the general procedure with (4S,4'S)-4,4'-di((S)sec-butyl)-4,4',5,5'-tetrahydro-2,2'-bioxazole [(S,S)-L1] (3.3 mg, $0.013 \mathrm{mmol}, 13$ mol\%), $\mathrm{NiCl}_{2} \cdot \mathrm{Py}_{4}(4.5 \mathrm{mg}, 0.01 \mathrm{mmol}, 10 \mathrm{~mol} \%), \operatorname{Ir}\left[\mathrm{dF}\left(\mathrm{CF}_{3}\right) \mathrm{ppy}\right]_{2}(\mathrm{bpy}) \mathrm{PF}_{6}(3.2 \mathrm{mg}$, $0.003 \mathrm{mmol}, 3 \mathrm{~mol} \%$ ), 4-bromo-2-chloropyridine (11.1 uL, $0.1 \mathrm{mmol}, 1.0$ equiv.), ${ }^{t} \mathrm{BuBF}_{3} \mathrm{~K}$ (41.0 mg, $0.25 \mathrm{mmol}, 2.5$ equiv.) and tert-butyl acrylate $(29.0 \mathrm{uL}, 0.2 \mathrm{mmol}$, 2.0 equiv.) in acetone/ethyl acetate $(1.3 / 0.7, \mathrm{v} / \mathrm{v}, 2 \mathrm{~mL})$ were used. After 24 hours, the product was isolated by flash chromatography (PE: ethyl acetate $=20: 1)$ as a white solid (21.2 mg, 71\% yield, 94\% ee).

${ }^{1} \mathbf{H}$ NMR $\left(400 \mathrm{MHz}, \mathrm{CDCl}_{3}\right) \delta 8.29(\mathrm{~d}, J=5.1 \mathrm{~Hz}, 1 \mathrm{H}), 7.27(\mathrm{~s}, 1 \mathrm{H}), 7.17(\mathrm{dd}, J=5.1$, $1.3 \mathrm{~Hz}, 1 \mathrm{H}), 3.49$ (dd, $J=9.1,3.5 \mathrm{~Hz}, 1 \mathrm{H}), 2.24$ (dd, $J=14.0,9.2 \mathrm{~Hz}, 1 \mathrm{H}), 1.44$ (dd, $J$ $=14.0,3.5 \mathrm{~Hz}, 1 \mathrm{H}), 1.39(\mathrm{~s}, 9 \mathrm{H}), 0.91(\mathrm{~s}, 9 \mathrm{H})$.

${ }^{13}$ C NMR $\left(101 \mathrm{MHz}, \mathrm{CDCl}_{3}\right) \delta 171.88,153.65,151.76,149.71,123.45,121.70,81.57$, 48.72, 46.71, 31.15, 29.38, 27.77.

HRMS (ESI): $\mathrm{C}_{16} \mathrm{H}_{25} \mathrm{ClNO}_{2}^{+}(\mathrm{M}+\mathrm{H}): 298.1574$, found: 298.1565 .

$[\alpha]_{\mathrm{D}}^{25}=-2.8437\left(\mathrm{c}=0.3200, \mathrm{CHCl}_{3}\right)$

Chiral HPLC: CHIRALPAK IC, $25{ }^{\circ} \mathrm{C},{ }^{i} \mathrm{PrOH}-h$ exanes 5/95, $0.5 \mathrm{~mL} / \mathrm{min}, 254 \mathrm{~nm}, \mathrm{t}_{\mathrm{R}}$ $($ major $)=17.2 \mathrm{~min}, \mathrm{t}_{\mathrm{R}}($ minor $)=12.1 \mathrm{~min}$.

\section{3 racemic}
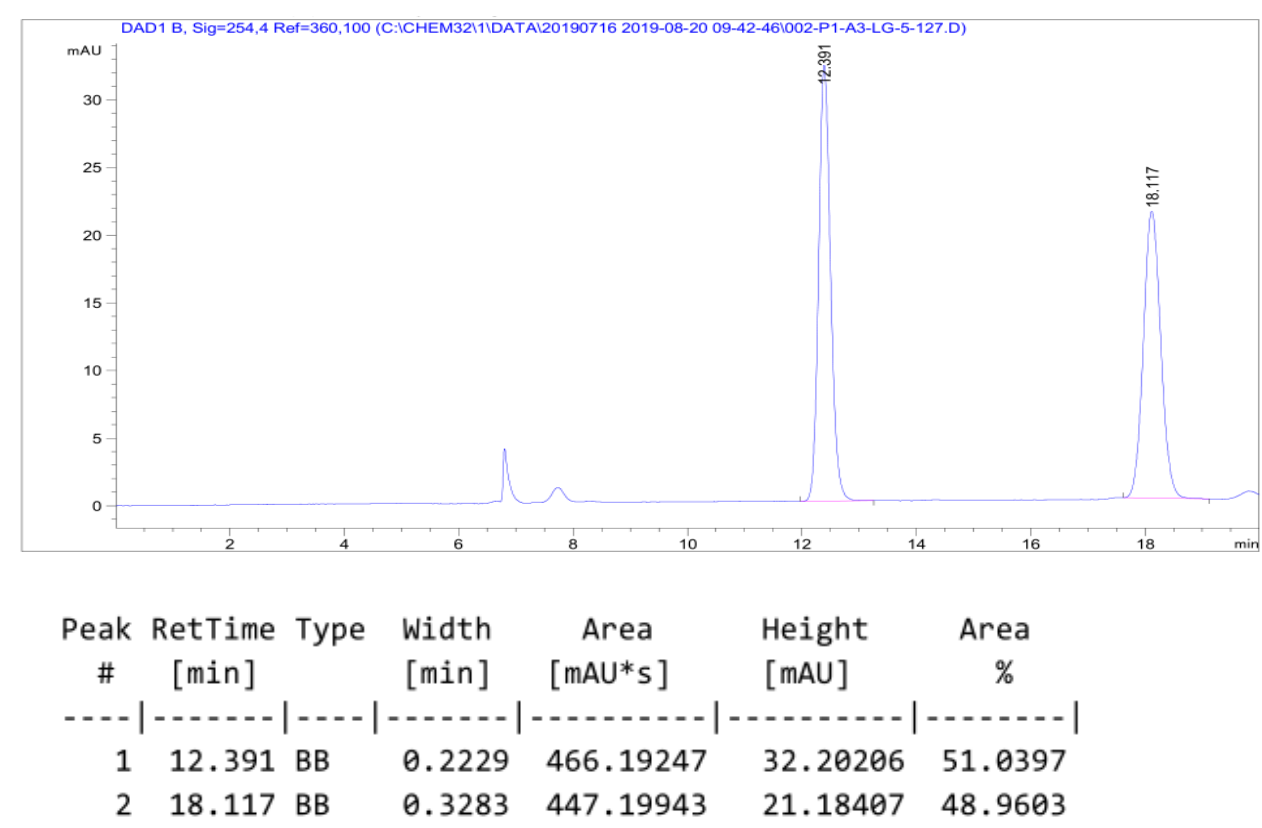


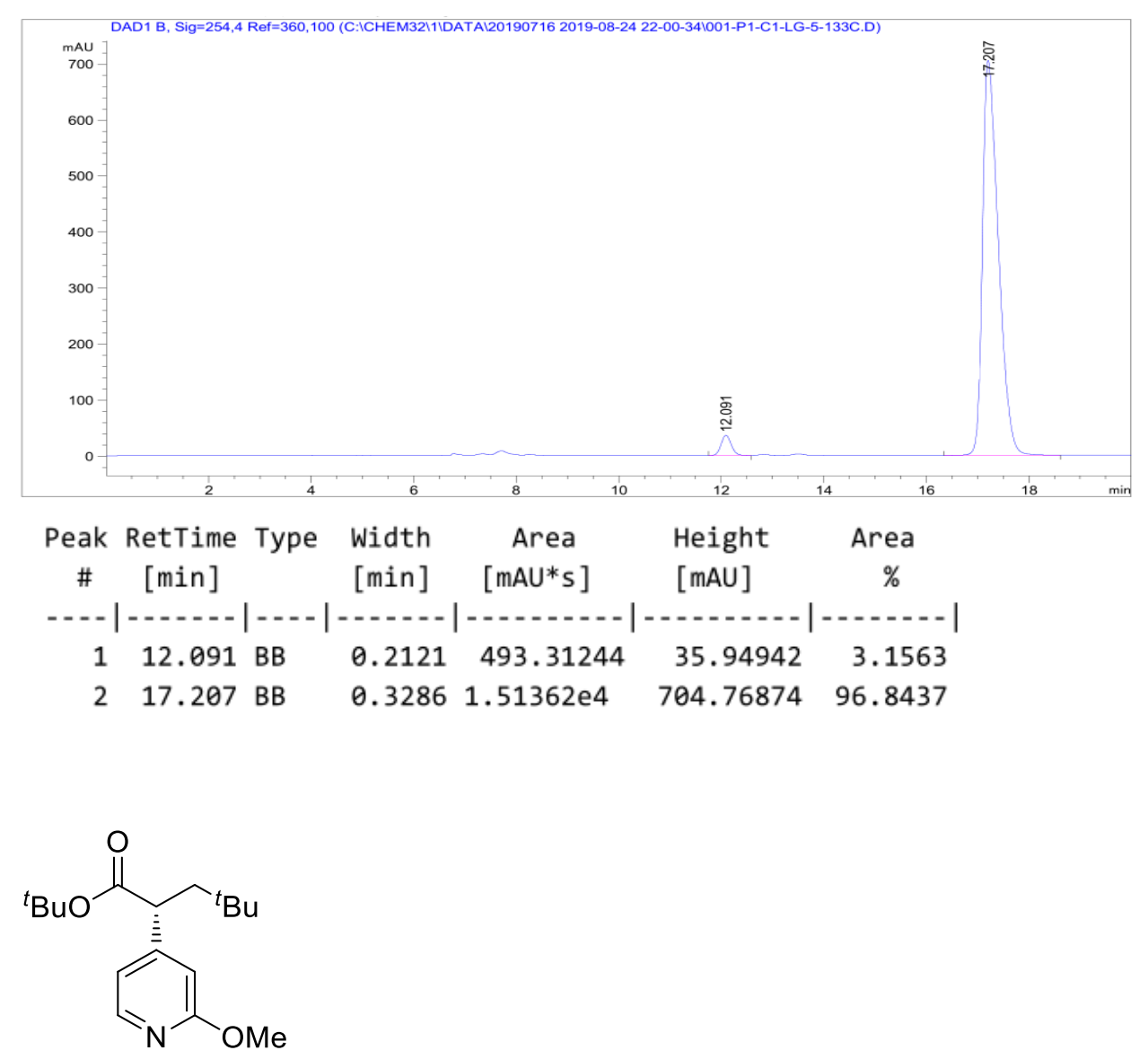

(R)-tert-Butyl 2-(2-methoxypyridin-4-yl)-4,4-dimethylpentanoate (24)

The product was prepared according to the general procedure with (4S,4'S)-4,4'-di((S)sec-butyl)-4,4',5,5'-tetrahydro-2,2'-bioxazole $[(S, S)$-L1 $]$ (3.3 mg, $0.013 \mathrm{mmol}, 13$ mol\%), $\mathrm{NiCl}_{2} \cdot \mathrm{Py}_{4}\left(4.5 \mathrm{mg}, 0.01 \mathrm{mmol}, 10 \mathrm{~mol} \%\right.$ ), $\operatorname{Ir}\left[\mathrm{dF}\left(\mathrm{CF}_{3}\right) \text { ppy }\right]_{2}(\mathrm{bpy}) \mathrm{PF}_{6}(3.2 \mathrm{mg}$, $0.003 \mathrm{mmol}, 3 \mathrm{~mol} \%$ ), 4-bromo-2-methoxypyridine (12.5 uL, $0.1 \mathrm{mmol}, 1.0$ equiv.), ${ }^{t} \mathrm{BuBF}_{3} \mathrm{~K}$ (41.0 mg, $0.25 \mathrm{mmol}, 2.5$ equiv.) and tert-butyl acrylate (29.0 uL, $0.2 \mathrm{mmol}$, 2.0 equiv.) in acetone/ethyl acetate $(1.3 / 0.7, \mathrm{v} / \mathrm{v}, 2 \mathrm{~mL})$ were used. After 24 hours, the product was isolated by flash chromatography (PE: ethyl acetate $=20: 1)$ as a colorless oil (17.5 mg, 60\% yield, $91 \%$ ee).

${ }^{1} \mathbf{H}$ NMR $\left(400 \mathrm{MHz}, \mathrm{CDCl}_{3}\right) \delta 8.06(\mathrm{~d}, J=5.4 \mathrm{~Hz}, 1 \mathrm{H}), 6.82(\mathrm{dd}, J=5.4,1.4 \mathrm{~Hz}, 1 \mathrm{H})$, $6.66(\mathrm{~s}, 1 \mathrm{H}), 3.91$ (s, 3H), 3.45 (dd, $J=9.2,3.4 \mathrm{~Hz}, 1 \mathrm{H}), 2.22(\mathrm{dd}, J=14.0,9.2 \mathrm{~Hz}$, 1H), 1.44 (dd, $J=14.0,3.5 \mathrm{~Hz}, 1 \mathrm{H}), 1.38$ (s, 9H), 0.90 (s, 9H).

${ }^{13}$ C NMR $\left(100 \mathrm{MHz}, \mathrm{CDCl}_{3}\right) \delta 172.49,164.48,153.16,146.81,116.33,109.70,80.99$, 53.39, 48.78, 46.58, 31.06, 29.39, 27.78. 
HRMS (ESI): $\mathrm{C}_{17} \mathrm{H}_{28} \mathrm{NO}_{3}{ }^{+}(\mathrm{M}+\mathrm{H}): 294.2069$, found: 294.2060 .

$[\alpha]_{\mathrm{D}}^{25}=-15.6784\left(\mathrm{c}=0.4133, \mathrm{CHCl}_{3}\right)$

Chiral HPLC: CHIRALPAK IC, $25{ }^{\circ} \mathrm{C},{ }^{i} \mathrm{PrOH}$-hexanes 5/95, $0.5 \mathrm{~mL} / \mathrm{min}, 254 \mathrm{~nm}, \mathrm{t}_{\mathrm{R}}$ (major) $=9.3 \mathrm{~min}, \mathrm{t}_{\mathrm{R}}($ minor $)=8.2 \mathrm{~min}$.

\section{4 racemic}

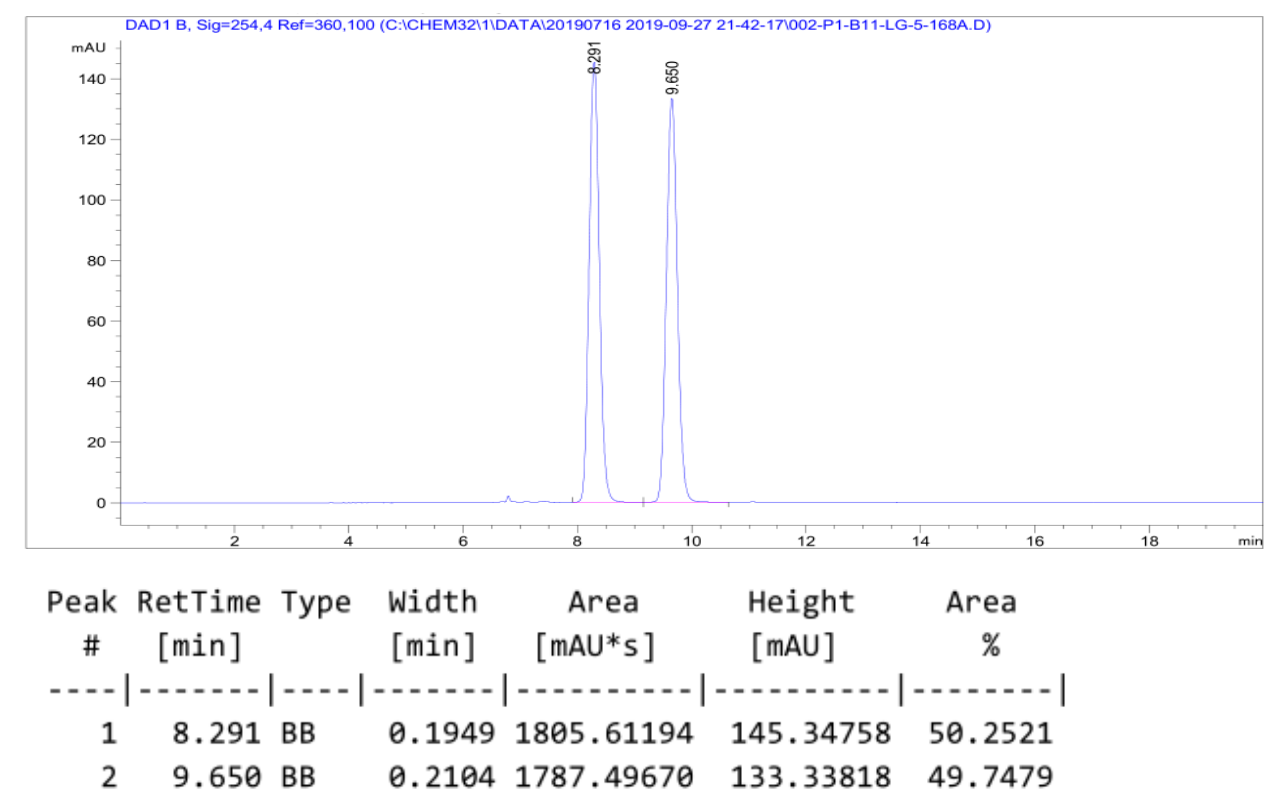

24 enantioenriched, $91 \%$ ee

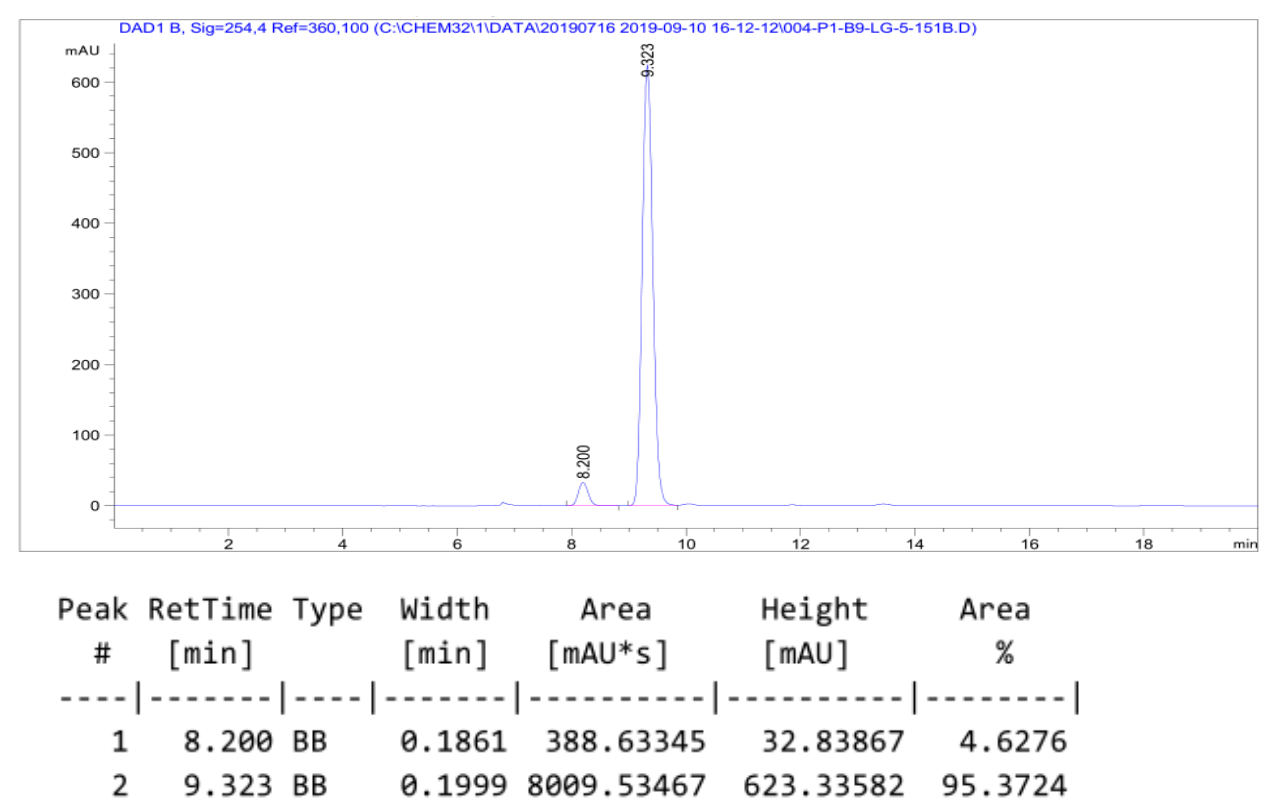


<smiles>CCCCC(C(=O)OCCC)c1ccc2sc3ccccc3c2c1</smiles>

\section{(R)-tert-Butyl 2-(dibenzo[b,d]thiophen-2-yl)-4,4-dimethylpentanoate (25)}

The product was prepared according to the general procedure with $(4 \mathrm{~S}, 4$ 'S)-4,4'-di((S)sec-butyl)-4,4',5,5'-tetrahydro-2,2'-bioxazole [(S,S)-L1] $(3.3 \mathrm{mg}, 0.013 \mathrm{mmol}, 13$ $\mathrm{mol} \%), \mathrm{NiCl}_{2} \bullet \mathrm{Py}_{4}(4.5 \mathrm{mg}, 0.01 \mathrm{mmol}, 10 \mathrm{~mol} \%), \operatorname{Ir}\left[\mathrm{dF}\left(\mathrm{CF}_{3}\right) \text { ppy }\right]_{2}(\mathrm{bpy}) \mathrm{PF}_{6}(3.2 \mathrm{mg}$, $0.003 \mathrm{mmol}, 3 \mathrm{~mol} \%$ ), 2-bromodibenzo[b,d]thiophene (26.3 mg, $0.1 \mathrm{mmol}, 1.0$ equiv.), ${ }^{t} \mathrm{BuBF}_{3} \mathrm{~K}$ (41.0 mg, $0.25 \mathrm{mmol}, 2.5$ equiv.) and tert-butyl acrylate (29.0 uL, $0.2 \mathrm{mmol}$, 2.0 equiv.) in acetone/ethyl acetate $(1.3 / 0.7, \mathrm{v} / \mathrm{v}, 2 \mathrm{~mL})$ were used. After 24 hours, the product was isolated by flash chromatography $(\mathrm{PE}$ : ethyl acetate $=20: 1)$ as a white solid (20.1 mg, 55\% yield, 91\% ee).

${ }^{1} \mathbf{H}$ NMR $\left(400 \mathrm{MHz}, \mathrm{CDCl}_{3}\right) \delta$ 8.19-8.17 (m, 1H), $8.09(\mathrm{~s}, 1 \mathrm{H}), 7.85-7.83(\mathrm{~m}, 1 \mathrm{H}), 7.78$ $(\mathrm{d}, J=8.3 \mathrm{~Hz}, 1 \mathrm{H}), 7.47-7.42(\mathrm{~m}, 3 \mathrm{H}), 3.72(\mathrm{dd}, J=9.2,3.5 \mathrm{~Hz}, 1 \mathrm{H}), 2.39$ (dd, $J=14.0$, 9.2 Hz, 1H), $1.61(\mathrm{dd}, J=14.0,3.5 \mathrm{~Hz}, 1 \mathrm{H}), 1.39$ (s, 9H), 0.96 (s, 9H).

${ }^{13}$ C NMR $\left(100 \mathrm{MHz}, \mathrm{CDCl}_{3}\right) \delta 173.92,139.81,138.18,137.82,135.77,135.47,126.70$, $126.67,124.30,122.82,122.73,121.63,120.57,80.51,49.29,47.53,31.15,29.57$, 27.86.

HRMS (ESI): $\mathrm{C}_{23} \mathrm{H}_{29} \mathrm{O}_{2} \mathrm{~S}^{+}(\mathrm{M}+\mathrm{H}): 369.1888$, found: 369.1883 .

$[\alpha]_{\mathrm{D}}^{25}=-14.7330\left(\mathrm{c}=0.3000, \mathrm{CHCl}_{3}\right)$

Chiral HPLC: CHIRALPAK IA, $25{ }^{\circ} \mathrm{C},{ }^{i} \mathrm{PrOH}$-hexanes $1 / 100,0.5 \mathrm{~mL} / \mathrm{min}, 254 \mathrm{~nm}$, $\mathrm{t}_{\mathrm{R}}($ major $)=10.2 \mathrm{~min}, \mathrm{t}_{\mathrm{R}}($ minor $)=10.0 \mathrm{~min}$ 


\section{5 racemic}

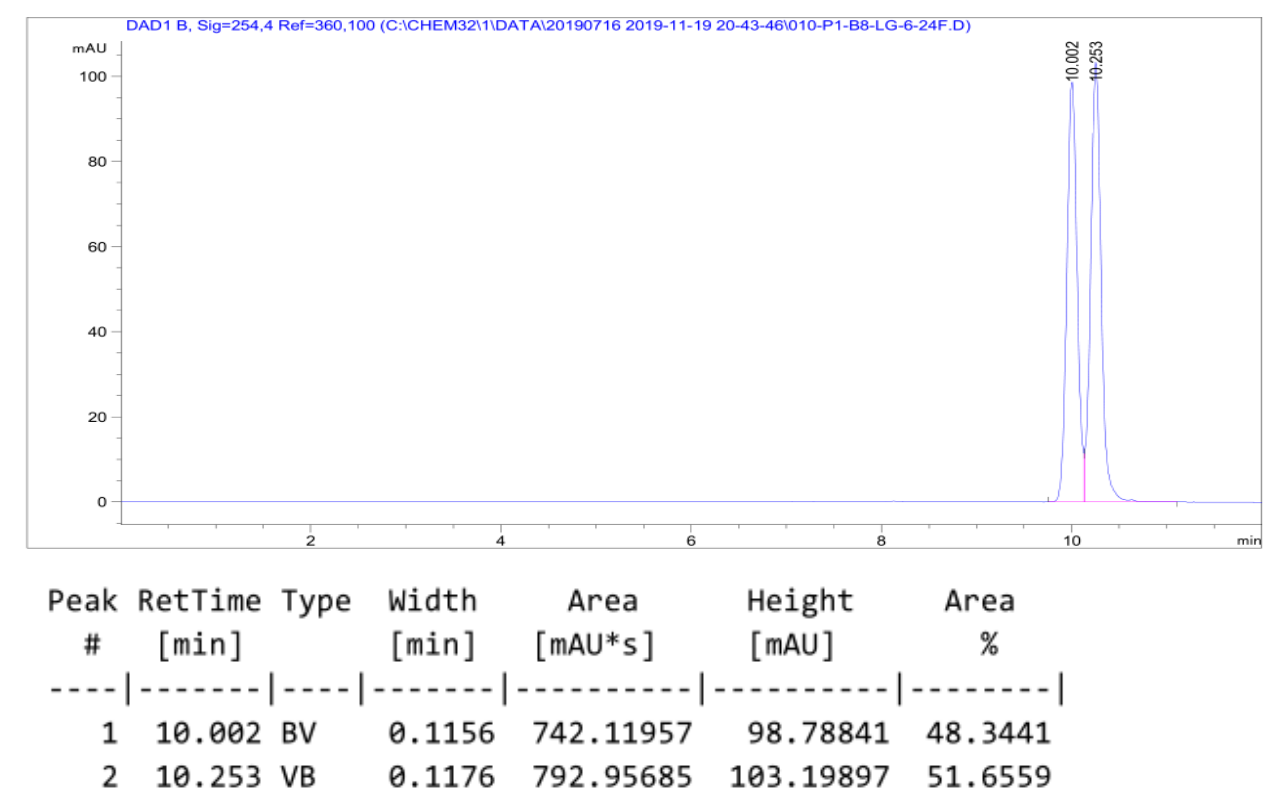

\section{5 enantioenriched, $91 \%$ ee}

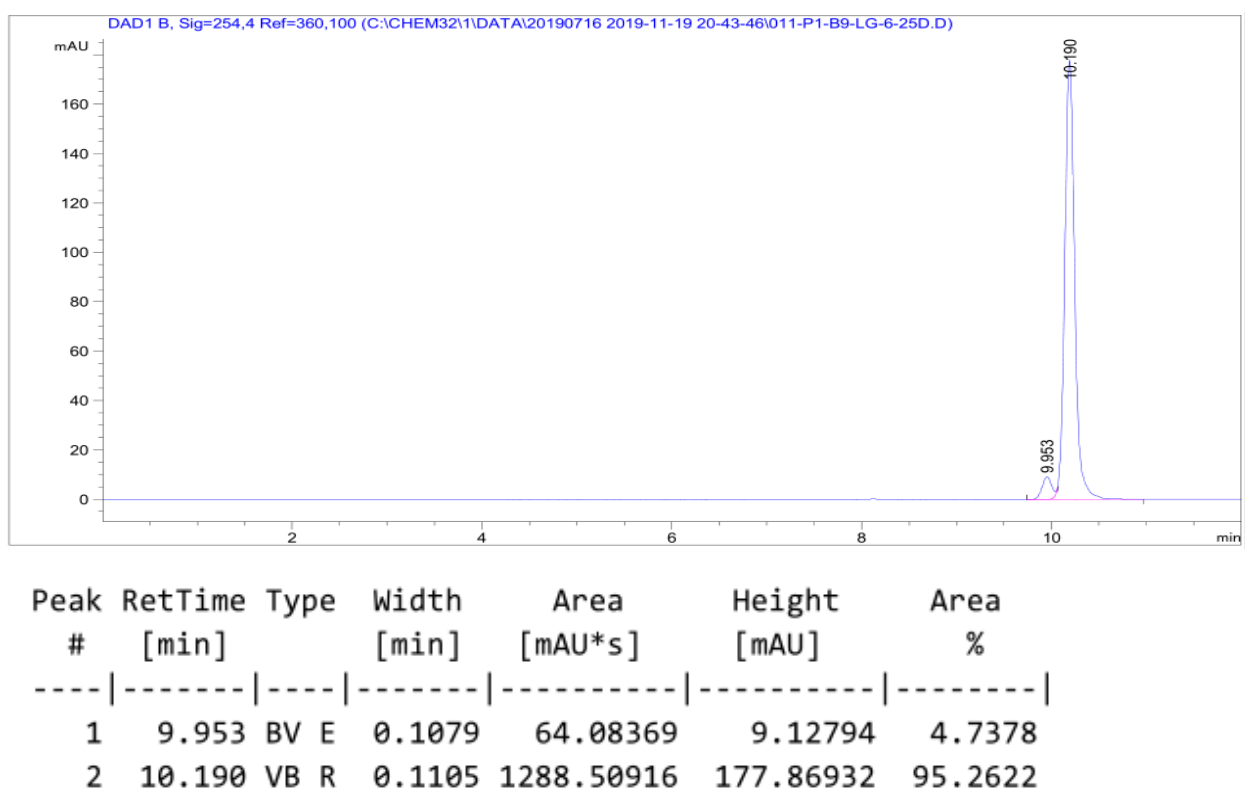<smiles>CCC(C)(C)CC(C(=O)OCC(C)(C)C)c1cnc(C)nc1</smiles>

(R)-tert-Butyl 4,4-dimethyl-2-(2-methylpyrimidin-5-yl)pentanoate (26)

The product was prepared according to the general procedure with $(4 \mathrm{~S}, 4$ 'S)-4,4'-di((S)sec-butyl)-4,4',5,5'-tetrahydro-2,2'-bioxazole [(S,S)-L1] $(3.3 \mathrm{mg}, 0.013 \mathrm{mmol}, 13$ 


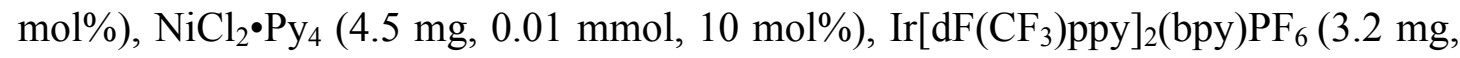
$0.003 \mathrm{mmol}, 3 \mathrm{~mol} \%$ ), 5-bromo-2-methylpyrimidine (17.3 mg, $0.1 \mathrm{mmol}, 1.0$ equiv.), ${ }^{t} \mathrm{BuBF}_{3} \mathrm{~K}$ (41.0 mg, $0.25 \mathrm{mmol}, 2.5$ equiv.) and tert-butyl acrylate (29.0 uL, $0.2 \mathrm{mmol}$, 2.0 equiv.) in acetone/ethyl acetate $(1.3 / 0.7, \mathrm{v} / \mathrm{v}, 2 \mathrm{~mL})$ were used. After 24 hours, the product was isolated by flash chromatography (PE: ethyl acetate $=20: 1)$ as a white solid (21.5 mg, 77\% yield, 88\% ee).

${ }^{1} \mathrm{H}$ NMR (400 MHz, $\left.\mathrm{CDCl}_{3}\right) \delta 8.57(\mathrm{~s}, 2 \mathrm{H}), 3.48(\mathrm{dd}, J=8.9,4.1 \mathrm{~Hz}, 1 \mathrm{H}), 2.71(\mathrm{~s}, 3 \mathrm{H})$, $2.26(\mathrm{dd}, J=14.0,8.9 \mathrm{~Hz}, 1 \mathrm{H}), 1.48(\mathrm{dd}, J=13.6,3.6 \mathrm{~Hz}, 1 \mathrm{H}), 1.38(\mathrm{~s}, 9 \mathrm{H}), 0.91$ (s, 9H).

${ }^{13} \mathrm{C}$ NMR $\left(101 \mathrm{MHz}, \mathrm{CDCl}_{3}\right) \delta 172.45,166.68,156.20,131.45,81.54,46.75,44.70$, $31.17,29.45,27.78,25.60$.

HRMS (ESI): $\mathrm{C}_{16} \mathrm{H}_{27} \mathrm{~N}_{2} \mathrm{O}_{2}^{+}(\mathrm{M}+\mathrm{H}): 279.2073$, found: 279.2060 .

$[\alpha]_{\mathrm{D}}^{25}=-4.5830\left(\mathrm{c}=0.5433, \mathrm{CHCl}_{3}\right)$

Chiral HPLC: CHIRALPAK IA, $25{ }^{\circ} \mathrm{C},{ }^{i} \mathrm{PrOH}-$ hexanes 10/90, $1.0 \mathrm{~mL} / \mathrm{min}, 210 \mathrm{~nm}$, $\mathrm{t}_{\mathrm{R}}($ major $)=7.7 \mathrm{~min}, \mathrm{t}_{\mathrm{R}}($ minor $)=6.0 \mathrm{~min}$

\section{6 racemic}
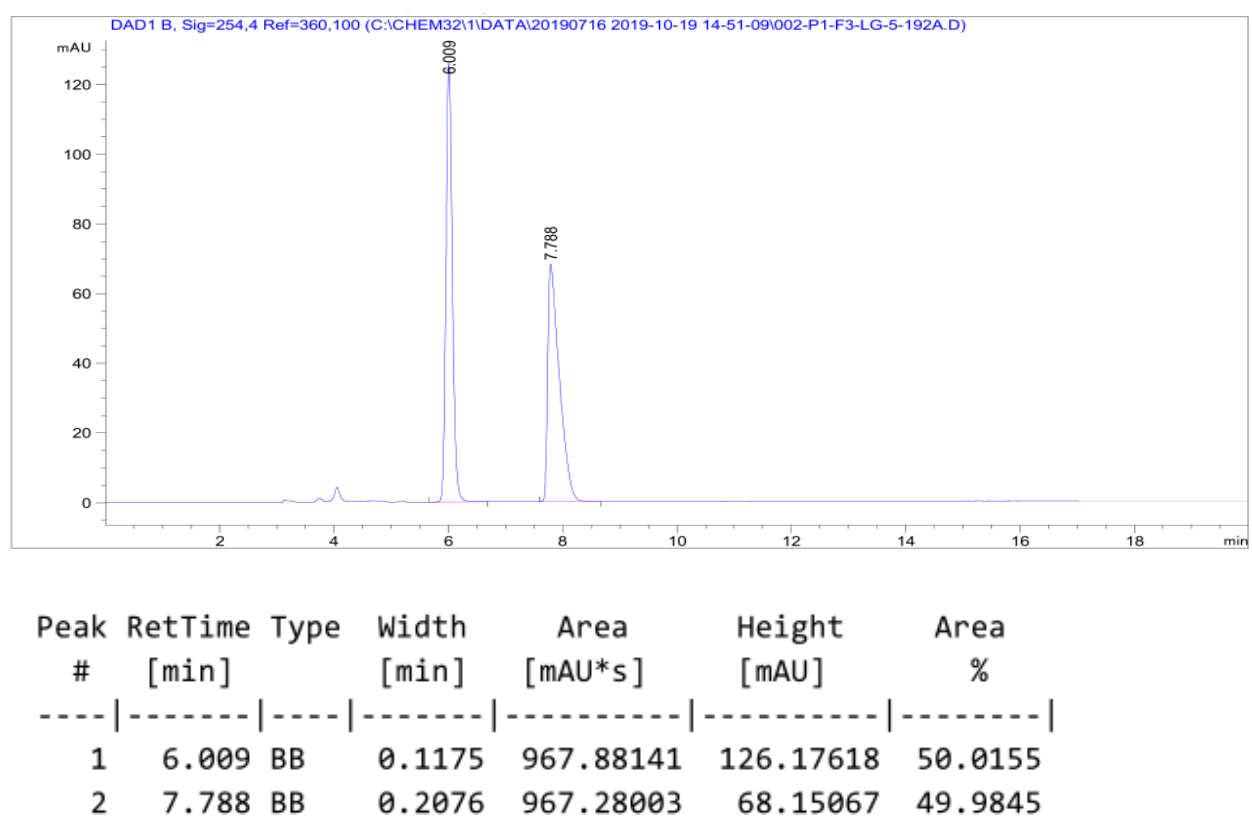


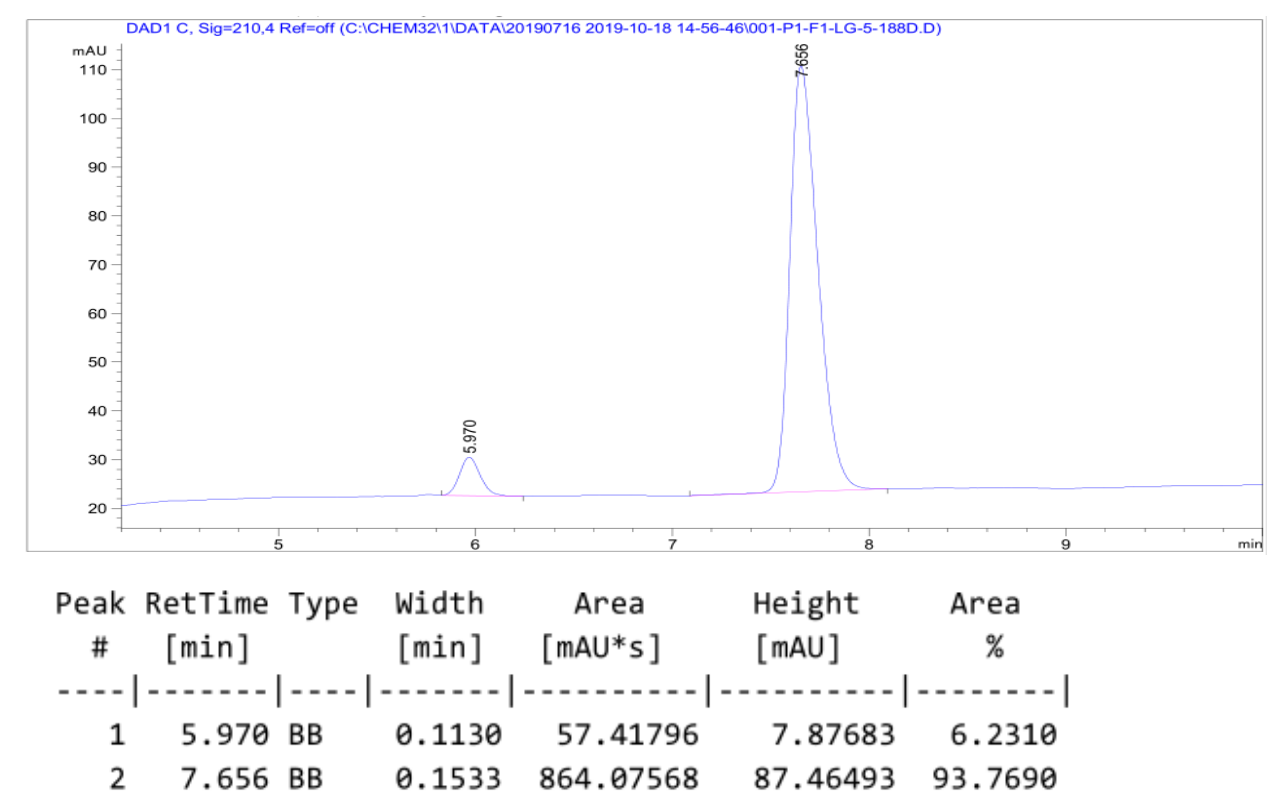<smiles>CCCCC(C(=O)OCC)c1cnc(S(C)(C)C)nc1</smiles>

(R)-tert-Butyl 4,4-dimethyl-2-(2-(methylthio)pyrimidin-5-yl)pentanoate (27)

The product was prepared according to the general procedure with (4S,4'S)-4,4'-di((S)sec-butyl)-4,4',5,5'-tetrahydro-2,2'-bioxazole [(S,S)-L1] $(3.3 \mathrm{mg}, 0.013 \mathrm{mmol}, 13$ mol\%), $\mathrm{NiCl}_{2} \cdot \mathrm{Py}_{4}(4.5 \mathrm{mg}, 0.01 \mathrm{mmol}, 10 \mathrm{~mol} \%), \operatorname{Ir}\left[\mathrm{dF}\left(\mathrm{CF}_{3}\right) \mathrm{ppy}\right]_{2}(\mathrm{bpy}) \mathrm{PF}_{6}(3.2 \mathrm{mg}$, $0.003 \mathrm{mmol}, 3 \mathrm{~mol} \%$ ), 5-bromo-2-(methylthio)pyrimidine (20.5 mg, $0.1 \mathrm{mmol}, 1.0$ equiv.), ${ }^{t} \mathrm{BuBF}_{3} \mathrm{~K}$ (41.0 mg, $0.25 \mathrm{mmol}, 2.5$ equiv.) and tert-butyl acrylate (29.0 uL, 0.2 mmol, 2.0 equiv.) in acetone/ethyl acetate (1.3/0.7, v/v, $2 \mathrm{~mL})$ were used. After 24 hours, the product was isolated by flash chromatography $(P E$ : ethyl acetate $=20: 1)$ as a white solid (23.9 mg, 77\% yield, $86 \%$ ee).

${ }^{1}$ H NMR (400 MHz, $\left.\mathrm{CDCl}_{3}\right) \delta 8.47(\mathrm{~s}, 2 \mathrm{H}), 3.43(\mathrm{dd}, J=8.8,4.1 \mathrm{~Hz}, 1 \mathrm{H}), 2.55(\mathrm{~s}, 3 \mathrm{H})$, $2.24(\mathrm{dd}, J=14.0,8.9 \mathrm{~Hz}, 1 \mathrm{H}), 1.46$ (dd, $J=14.0,4.1 \mathrm{~Hz}, 1 \mathrm{H}), 1.39$ (s, 9H), 0.90 (s, 9H).

${ }^{13}$ C NMR $\left(100 \mathrm{MHz}, \mathrm{CDCl}_{3}\right) \delta 172.46,171.08,156.46,129.59,81.55,46.75,44.46$, $31.16,29.45,27.78,14.11$. 
HRMS (ESI): $\mathrm{C}_{16} \mathrm{H}_{27} \mathrm{~N}_{2} \mathrm{O}_{2} \mathrm{~S}^{+}(\mathrm{M}+\mathrm{H}): 311.1793$, found: 311.1780 .

$[\alpha]_{\mathrm{D}}^{25}=-10.7445\left(\mathrm{c}=0.4700, \mathrm{CHCl}_{3}\right)$

Chiral HPLC: CHIRALPAK IA, $25{ }^{\circ} \mathrm{C},{ }^{i} \mathrm{PrOH}$-hexanes $1 / 100,0.5 \mathrm{~mL} / \mathrm{min}, 260 \mathrm{~nm}$, $t_{R}($ major $)=14.7 \mathrm{~min}, t_{R}($ minor $)=13.7 \mathrm{~min}$

\section{7 racemic}

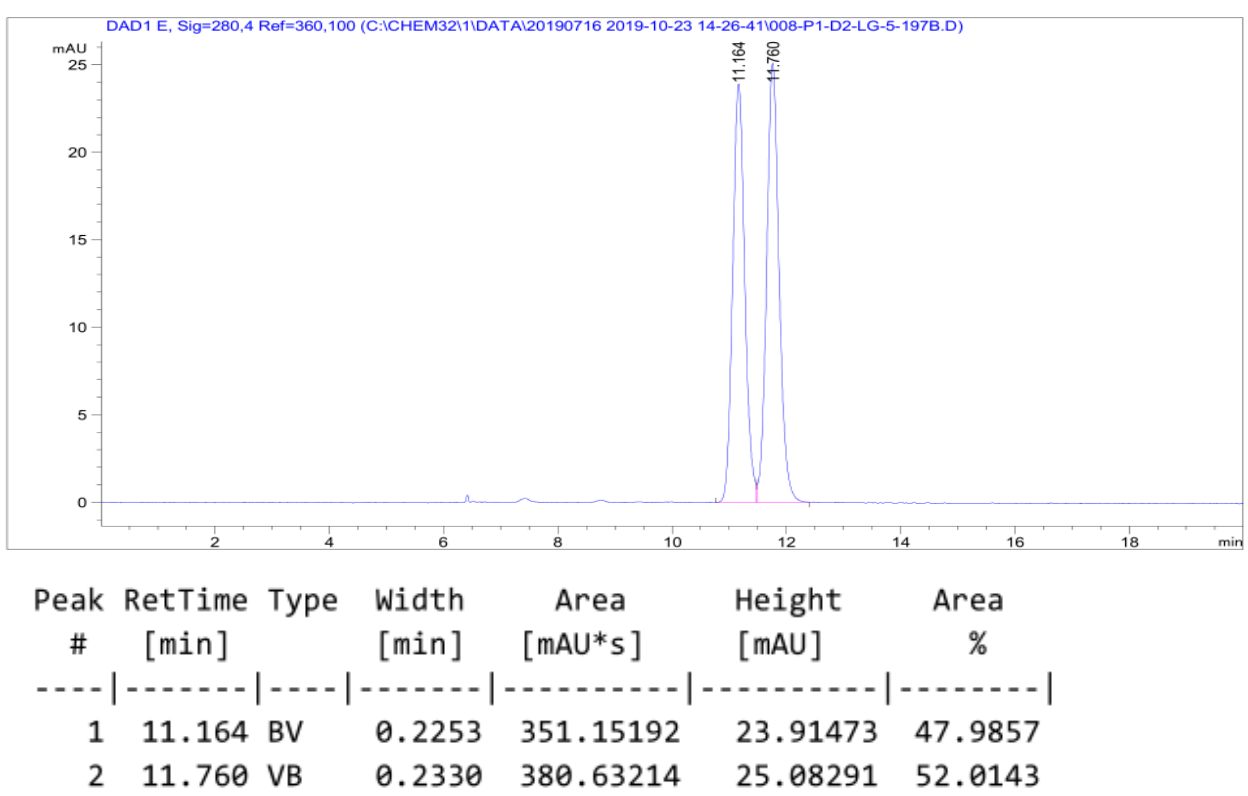

\section{7 enantioenriched, $86 \%$ ee}

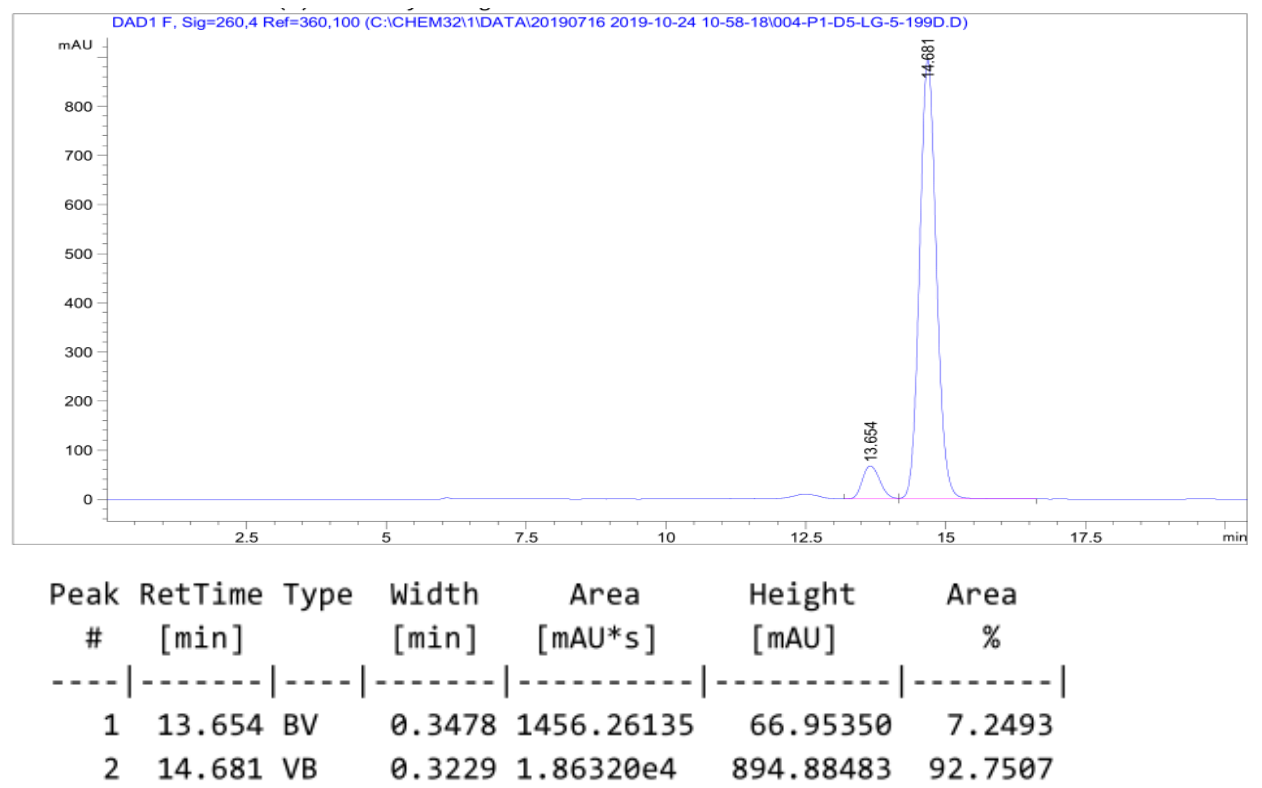


<smiles>COc1ncc(C(CC(C)(C)C)C(=O)OC(C)(C)C)cn1</smiles>

\section{(R)-tert-Butyl 2-(2-methoxypyrimidin-5-yl)-4,4-dimethylpentanoate (28)}

The product was prepared according to the general procedure with (4S,4'S)-4,4'-di((S)sec-butyl)-4,4',5,5'-tetrahydro-2,2'-bioxazole $[(S, S)$-L1 $](3.3 \mathrm{mg}, 0.013 \mathrm{mmol}, 13$ mol\%), $\mathrm{NiCl}_{2} \cdot \mathrm{Py}_{4}(4.5 \mathrm{mg}, 0.01 \mathrm{mmol}, 10 \mathrm{~mol} \%), \operatorname{Ir}\left[\mathrm{dF}\left(\mathrm{CF}_{3}\right) \mathrm{ppy}\right]_{2}(\mathrm{bpy}) \mathrm{PF}_{6}(3.2 \mathrm{mg}$, $0.003 \mathrm{mmol}, 3 \mathrm{~mol} \%$ ), 5-bromo-2-methoxypyrimidine (18.9 mg, $0.1 \mathrm{mmol}, 1.0$ equiv.), ${ }^{t} \mathrm{BuBF}_{3} \mathrm{~K}(41.0 \mathrm{mg}, 0.25 \mathrm{mmol}, 2.5$ equiv.) and tert-butyl acrylate $(29.0 \mathrm{uL}, 0.2 \mathrm{mmol}$, 2.0 equiv.) in acetone/ethyl acetate $(1.3 / 0.7, \mathrm{v} / \mathrm{v}, 2 \mathrm{~mL})$ were used. After 24 hours, the product was isolated by flash chromatography (PE: ethyl acetate $=20: 1)$ as a white solid (16.5 mg, 56\% yield, $82 \%$ ee).

${ }^{1}$ H NMR (400 MHz, $\mathrm{CDCl}_{3}$ ) $\delta 8.44$ (s, 2H), 3.99 (s, 3H), $3.45(\mathrm{dd}, J=8.8,4.2 \mathrm{~Hz}, 1 \mathrm{H})$, $2.24(\mathrm{dd}, J=14.0,8.9 \mathrm{~Hz}, 1 \mathrm{H}), 1.49(\mathrm{dd}, J=6.3,4.3 \mathrm{~Hz}, 1 \mathrm{H}), 1.38(\mathrm{~s}, 9 \mathrm{H}), 0.90$ (s, 9H).

${ }^{13}$ C NMR $\left(100 \mathrm{MHz}, \mathrm{CDCl}_{3}\right) \delta 172.74,164.79,158.39,128.03,81.40,54.87,46.81$, $44.09,31.12,29.46,27.79$.

HRMS (ESI): $\mathrm{C}_{16} \mathrm{H}_{27} \mathrm{~N}_{2} \mathrm{O}_{3}{ }^{+}(\mathrm{M}+\mathrm{H}): 295.2022$, found: 295.2009.

$[\alpha]_{\mathrm{D}}^{25}=-10.9064\left(\mathrm{c}=0.1467, \mathrm{CHCl}_{3}\right)$

Chiral HPLC: CHIRALPAK IA, $25^{\circ} \mathrm{C},{ }^{i} \mathrm{PrOH}-$ hexanes 5/95, $0.5 \mathrm{~mL} / \mathrm{min}, 280 \mathrm{~nm}, \mathrm{t}_{\mathrm{R}}$ (major) $=17.8 \mathrm{~min}, \mathrm{t}_{\mathrm{R}}($ minor $)=14.5 \mathrm{~min}$.

\section{8 racemic}




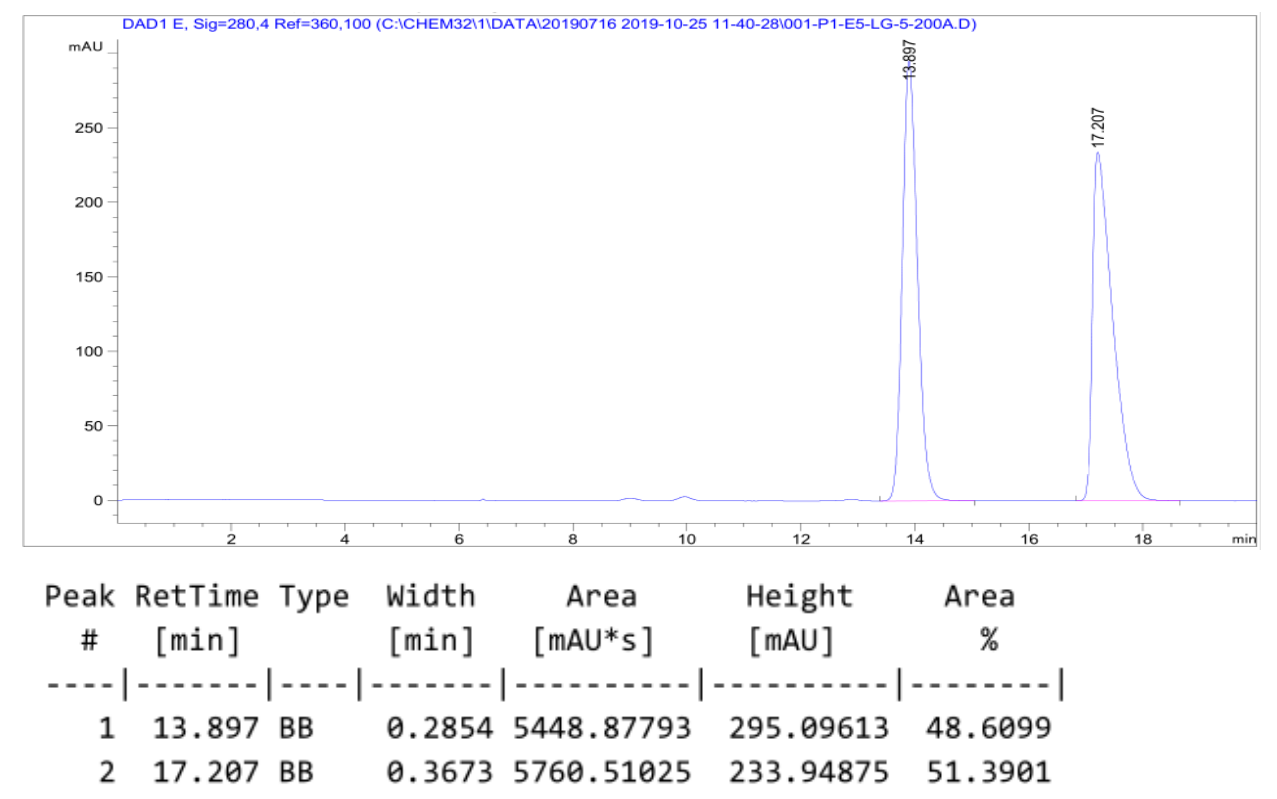

28 enantioenriched, $82 \%$ ee

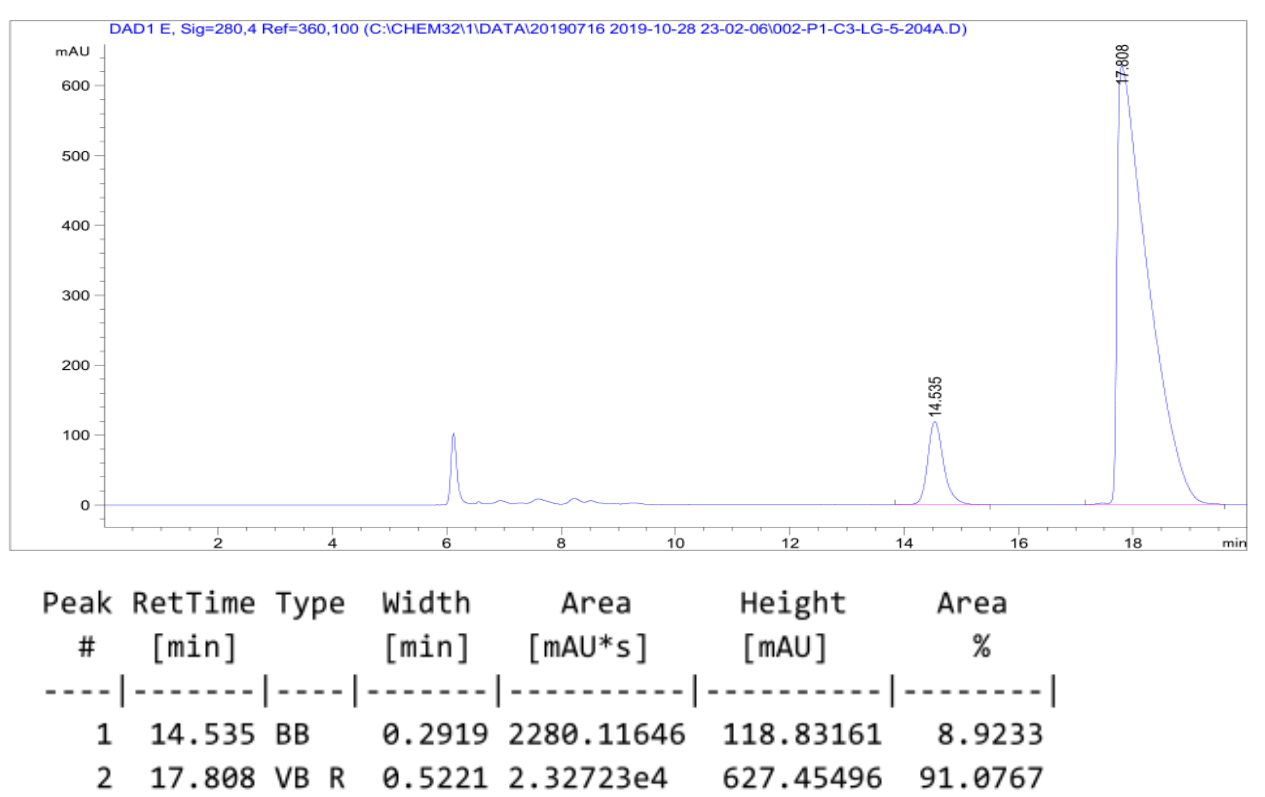<smiles>CCOC(=O)C(CC(C)(C)CC)c1csc(C(=O)OC)c1</smiles>

(R)-Methyl 4-(1-(tert-butoxy)-4,4-dimethyl-1-oxopentan-2-yl)thiophene-2-

Carboxylate (29) 
The product was prepared according to the general procedure with $(4 \mathrm{~S}, 4$ 'S)-4,4'-di((S)sec-butyl)-4,4',5,5'-tetrahydro-2,2'-bioxazole [(S,S)-L1] (3.3 mg, $0.013 \mathrm{mmol}, 13$ mol\%), $\mathrm{NiCl}_{2} \cdot \mathrm{Py}_{4}(4.5 \mathrm{mg}, 0.01 \mathrm{mmol}, 10 \mathrm{~mol} \%), \operatorname{Ir}\left[\mathrm{dF}\left(\mathrm{CF}_{3}\right) \mathrm{ppy}\right]_{2}(\mathrm{bpy}) \mathrm{PF}_{6}(3.2 \mathrm{mg}$, $0.003 \mathrm{mmol}, 3 \mathrm{~mol} \%$ ), methyl 4-bromothiophene-2-carboxylate (13.3 uL, $0.1 \mathrm{mmol}$, 1.0 equiv.), ${ }^{t} \mathrm{BuBF}_{3} \mathrm{~K}(41.0 \mathrm{mg}, 0.25 \mathrm{mmol}, 2.5$ equiv.) and tert-butyl acrylate (29.0 uL, $0.2 \mathrm{mmol}, 2.0$ equiv.) in Acetone/ethyl acetate (1.3/0.7, v/v, $2 \mathrm{~mL})$ were used. After 24 hours, the product was isolated by flash chromatography $(\mathrm{PE}$ : ethyl acetate $=20: 1)$ as a white solid (18.3 mg, 56\% yield, $80 \%$ ee).

${ }^{1} \mathbf{H}$ NMR $\left(400 \mathrm{MHz}, \mathrm{CDCl}_{3}\right) \delta 7.73(\mathrm{~d}, J=1.5 \mathrm{~Hz}, 1 \mathrm{H}), 7.31(\mathrm{~d}, J=1.2 \mathrm{~Hz}, 1 \mathrm{H}), 3.87$ (s, 3H), $3.63(\mathrm{dd}, J=9.1,3.9 \mathrm{~Hz}, 1 \mathrm{H}), 2.18(\mathrm{dd}, J=14.0,9.1 \mathrm{~Hz}, 1 \mathrm{H}), 1.51(\mathrm{dd}, J=$ 14.0, 3.9 Hz, 1H), 1.39 (s, 9H), 0.90 (s, 9H).

${ }^{13}$ C NMR $\left(100 \mathrm{MHz}, \mathrm{CDCl}_{3}\right) \delta 173.02,162.60,142.50,133.49,133.38,128.03,80.90$, $52.15,46.96,44.86,30.97,29.43,27.82$.

HRMS (ESI): $\mathrm{C}_{17} \mathrm{H}_{27} \mathrm{O}_{4} \mathrm{~S}^{+}(\mathrm{M}+\mathrm{H})$ : 327.1630 , found: 327.1623 .

$[\alpha]_{\mathrm{D}}^{25}=-2.9466\left(\mathrm{c}=0.3733, \mathrm{CHCl}_{3}\right)$

Chiral HPLC: CHIRALPAK IC, $25{ }^{\circ} \mathrm{C},{ }^{i} \mathrm{PrOH}$-hexanes 1/100, $0.5 \mathrm{~mL} / \mathrm{min}, 254 \mathrm{~nm}$, $\mathrm{t}_{\mathrm{R}}($ major $)=18.7 \mathrm{~min}, \mathrm{t}_{\mathrm{R}}($ minor $)=16.9 \mathrm{~min}$

\section{9 racemic}
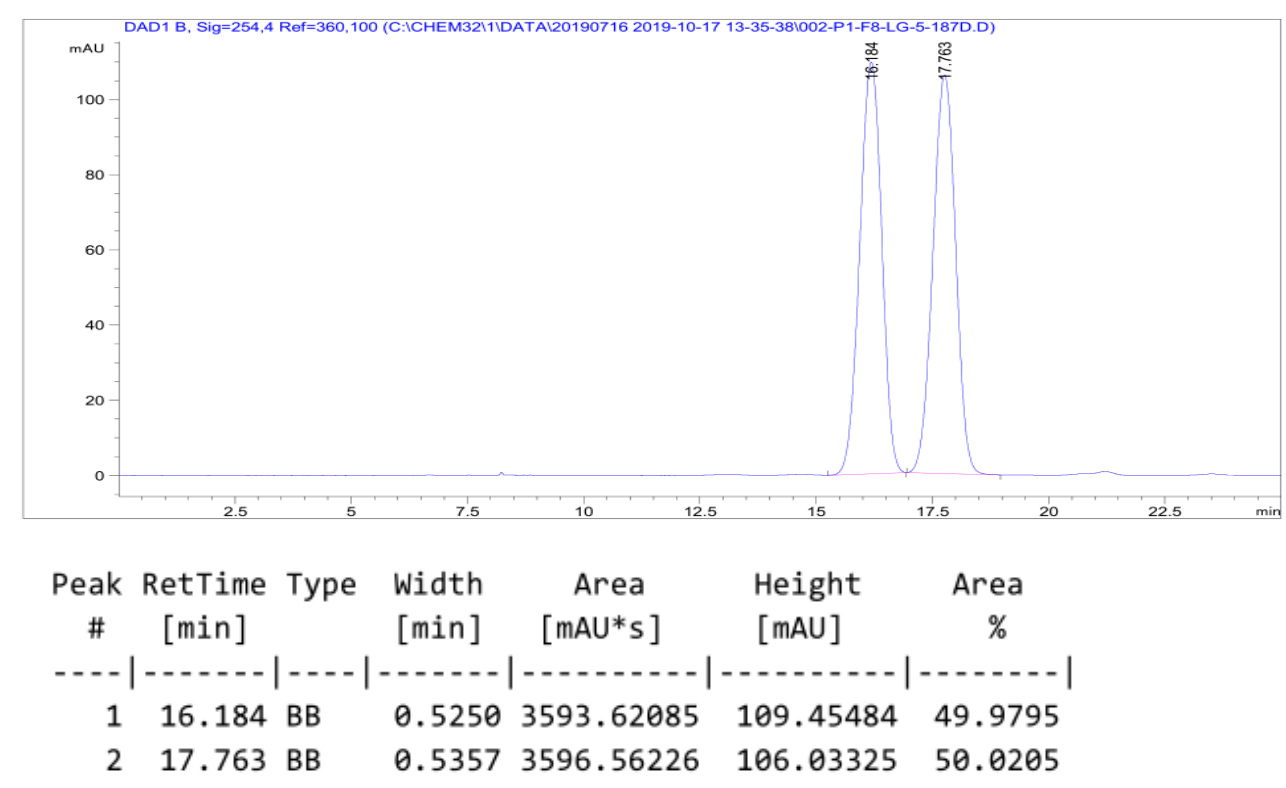

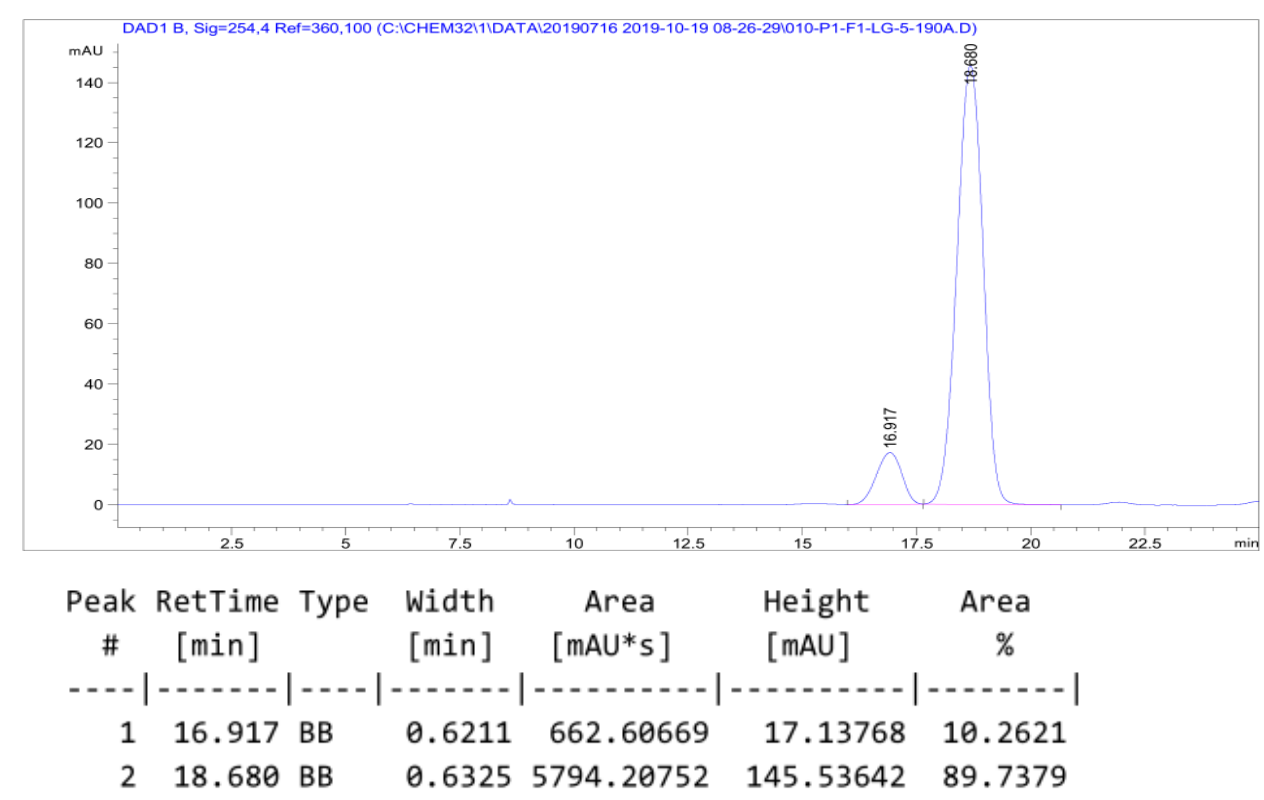

(1)

(R)-tert-Butyl 3-((3S,5S,7S)-adamantan-1-yl)-2-(2-(trifluoromethyl)pyridin-4-yl)

\section{Propanoate (30)}

The product was prepared according to the general procedure with (4S,4'S)-4,4'-di((S)sec-butyl)-4,4',5,5'-tetrahydro-2,2'-bioxazole [(S,S)-L1] (3.3 mg, $0.013 \mathrm{mmol}, 13$ mol\%), $\mathrm{NiCl}_{2} \bullet \mathrm{Py}_{4}(4.5 \mathrm{mg}, 0.01 \mathrm{mmol}, 10 \mathrm{~mol} \%), \operatorname{Ir}\left[\mathrm{dF}\left(\mathrm{CF}_{3}\right) \mathrm{ppy}\right]_{2}(\mathrm{bpy}) \mathrm{PF}_{6}(3.2 \mathrm{mg}$, $0.003 \mathrm{mmol}, 3 \mathrm{~mol} \%$ ), 4-bromo-2-(trifluoromethyl)pyridine (13.7 uL, $0.1 \mathrm{mmol}, 1.0$ equiv.), potassium ((3r,5r,7r)-adamantan-1-yl)trifluoroborate $(60.5 \mathrm{mg}, 0.25 \mathrm{mmol}, 2.5$ equiv.) and tert-butyl acrylate $(29.0 \mathrm{uL}, 0.2 \mathrm{mmol}, 2.0$ equiv.) in acetone/ethyl acetate $(1.3 / 0.7, \mathrm{v} / \mathrm{v}, 2 \mathrm{~mL})$ were used. After 24 hours, the product was isolated by flash chromatography $(\mathrm{PE}$ : ethyl acetate $=20: 1)$ as a white solid $(25.1 \mathrm{mg}, 61 \%$ yield, $96 \%$ ee).

${ }^{1} \mathbf{H}$ NMR $\left(400 \mathrm{MHz}, \mathrm{CDCl}_{3}\right) \delta 8.63(\mathrm{~d}, J=5.0 \mathrm{~Hz}, 1 \mathrm{H}), 7.62(\mathrm{~s}, 1 \mathrm{H}), 7.42(\mathrm{~d}, J=5.0$ Hz, 1H), $3.66(\mathrm{dd}, J=9.1,3.5 \mathrm{~Hz}, 1 \mathrm{H}), 2.14(\mathrm{dd}, J=14.1,9.2 \mathrm{~Hz}, 1 \mathrm{H}), 1.95$ (br, 3H), 1.71-1.59 (m, 7H), 1.53-1.41 (m, 5H), $1.38(\mathrm{~s}, 9 \mathrm{H}), 1.34-1.30(\mathrm{~m}, 1 \mathrm{H})$. 
${ }^{19}$ F NMR $\left(375 \mathrm{MHz}, \mathrm{CDCl}_{3}\right) \delta-67.95$ (s).

${ }^{13}$ C NMR $\left(100 \mathrm{MHz}, \mathrm{CDCl}_{3}\right) \delta 171.87,152.66,150.14,148.44(\mathrm{q}, J=34.3 \mathrm{~Hz}), 125.63$, $121.53(\mathrm{q}, J=274.5 \mathrm{~Hz}), 119.85(\mathrm{q}, J=2.7 \mathrm{~Hz}), 81.70,47.58,47.13,42.30,36.83$, $33.07,28.49,27.78$.

HRMS (ESI): $\mathrm{C}_{23} \mathrm{H}_{31} \mathrm{~F}_{3} \mathrm{NO}_{2}{ }^{+}(\mathrm{M}+\mathrm{H}): 410.2307$, found: 410.2302 .

$[\alpha]_{\mathrm{D}}^{25}=-10.8495\left(\mathrm{c}=0.7567, \mathrm{CHCl}_{3}\right)$

Chiral HPLC: CHIRALPAK IC, $25{ }^{\circ} \mathrm{C},{ }^{i} \mathrm{PrOH}$-hexanes 5/95, $1.0 \mathrm{~mL} / \mathrm{min}, 254 \mathrm{~nm}, \mathrm{t}_{\mathrm{R}}$ (major) $=10.0 \mathrm{~min}, \mathrm{t}_{\mathrm{R}}($ minor $)=8.7 \mathrm{~min}$

\section{0 racemic}

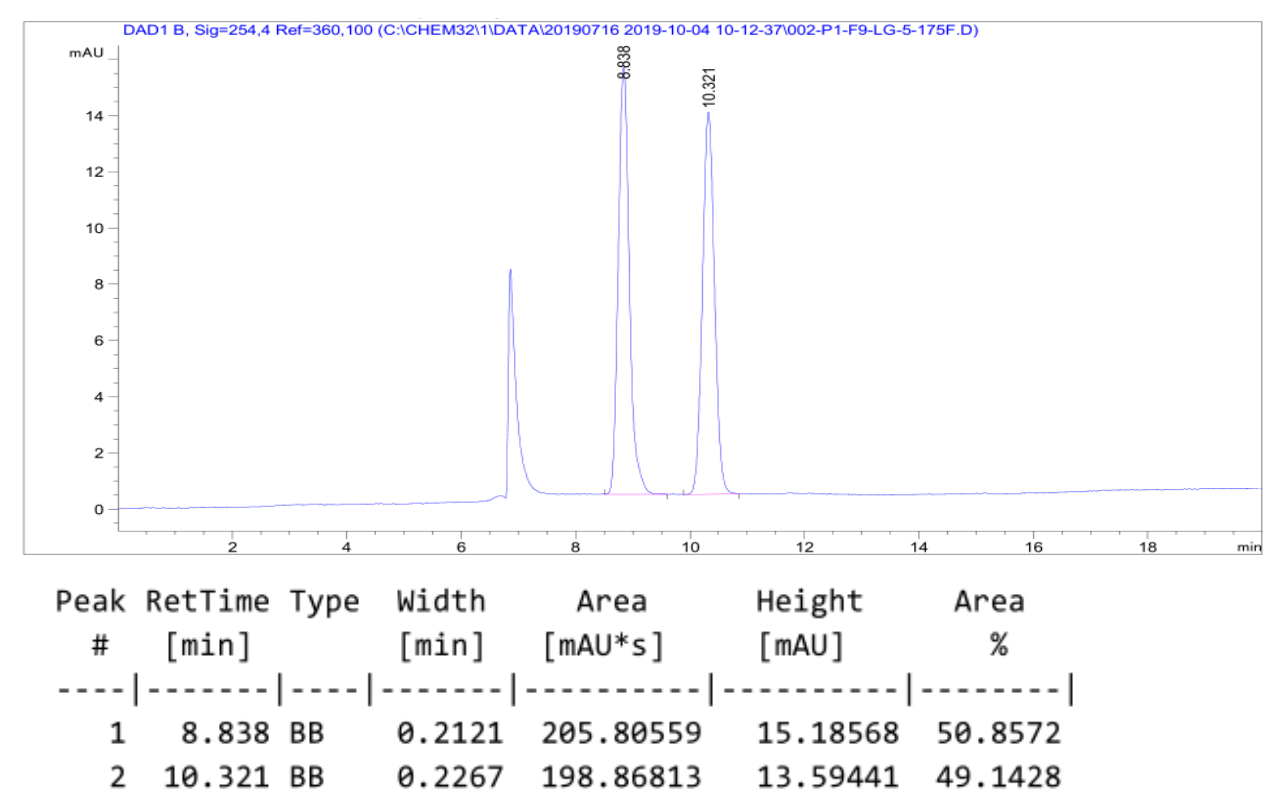

30 enantioenriched, $96 \%$ ee

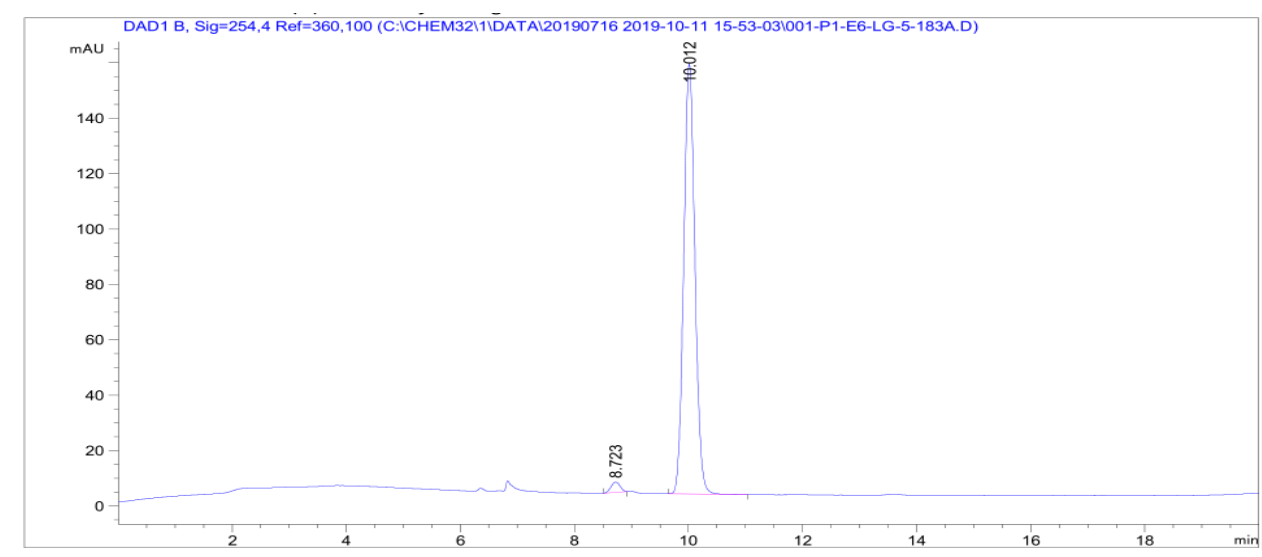




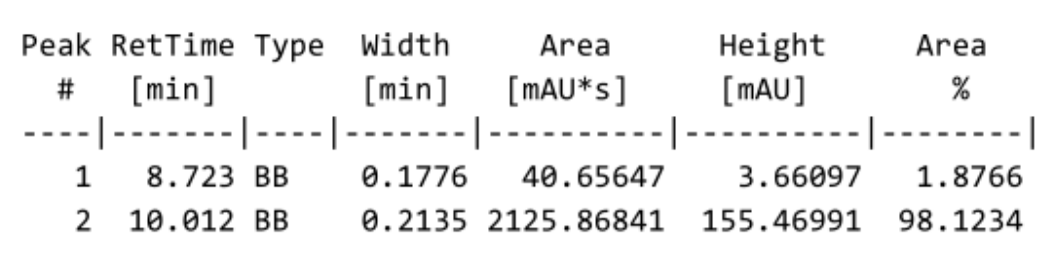



(R)-tert-Butyl 3-(1-methylcyclohexyl)-2-(2-(trifluoromethyl)pyridin-4-yl)

\section{Propanoate (31)}

The product was prepared according to the general procedure with (4S,4'S)-4,4'-di((S)sec-butyl)-4,4',5,5'-tetrahydro-2,2'-bioxazole [(S,S)-L1] $(3.3 \mathrm{mg}, 0.013 \mathrm{mmol}, 13$ mol\%), $\mathrm{NiCl}_{2} \cdot \mathrm{Py}_{4}(4.5 \mathrm{mg}, 0.01 \mathrm{mmol}, 10 \mathrm{~mol} \%), \operatorname{Ir}\left[\mathrm{dF}\left(\mathrm{CF}_{3}\right) \mathrm{ppy}\right]_{2}(\mathrm{bpy}) \mathrm{PF}_{6}(3.2 \mathrm{mg}$, $0.003 \mathrm{mmol}, 3 \mathrm{~mol} \%$ ), 4-bromo-2-(trifluoromethyl)pyridine (13.7 uL, $0.1 \mathrm{mmol}, 1.0$ equiv.), potassium (1-methylcyclohexyl)trifluoroborate $(51.0 \mathrm{mg}, 0.25 \mathrm{mmol}, 2.5$ equiv.) and tert-butyl acrylate $(29.0 \mathrm{uL}, 0.2 \mathrm{mmol}, 2.0$ equiv.) in acetone/ethyl acetate $(1.3 / 0.7, \mathrm{v} / \mathrm{v}, 2 \mathrm{~mL})$ were used. After 24 hours, the product was isolated by flash chromatography (PE: ethyl acetate $=20: 1)$ as a colorless oil $(31.6 \mathrm{mg}, 85 \%$ yield, 94\% ee).

${ }^{1} \mathbf{H}$ NMR $\left(400 \mathrm{MHz}, \mathrm{CDCl}_{3}\right) \delta 8.63(\mathrm{~d}, J=5.0 \mathrm{~Hz}, 1 \mathrm{H}), 7.63(\mathrm{~s}, 1 \mathrm{H}), 7.44(\mathrm{~d}, J=5.0$ $\mathrm{Hz}, 1 \mathrm{H}), 3.62(\mathrm{dd}, J=9.0,3.4 \mathrm{~Hz}, 1 \mathrm{H}), 2.29(\mathrm{dd}, J=14.1,9.0 \mathrm{~Hz}, 1 \mathrm{H}), 1.48(\mathrm{dd}, J=$ 14.1, 3.4 Hz, 1H), 1.45-1.41 (m, 4H), 1.38 (s, 9H), 1.30-1.16 (m, 6H), 0.88 (s, 3H).

${ }^{19}$ F NMR $\left(375 \mathrm{MHz}, \mathrm{CDCl}_{3}\right) \delta-67.95(\mathrm{~s})$.

${ }^{13}$ C NMR $\left(100 \mathrm{MHz}, \mathrm{CDCl}_{3}\right) \delta 171.92,152.64,150.11,148.42(\mathrm{q}, J=34.4 \mathrm{~Hz}), 125.59$, $121.48(\mathrm{q}, J=274.54 \mathrm{~Hz}), 119.78(\mathrm{q}, J=2.7 \mathrm{~Hz}), 81.68,48.00,37.82,37.59,33.58$, 27.70, 26.17, 21.84, 21.81 .

HRMS (ESI): $\mathrm{C}_{20} \mathrm{H}_{29} \mathrm{~F}_{3} \mathrm{NO}_{2}^{+}(\mathrm{M}+\mathrm{H}): 372.2150$, found: 372.2138 .

$[\alpha]_{\mathrm{D}}^{25}=-5.8758\left(\mathrm{c}=0.7267, \mathrm{CHCl}_{3}\right)$ 
Chiral HPLC: CHIRALPAK IC, $25{ }^{\circ} \mathrm{C},{ }^{i} \mathrm{PrOH}$-hexanes 5/95, $0.5 \mathrm{~mL} / \mathrm{min}, 254 \mathrm{~nm}, \mathrm{t}_{\mathrm{R}}$ (major) $=9.8 \mathrm{~min}, \mathrm{t}_{\mathrm{R}}($ minor $)=8.5 \mathrm{~min}$

\section{1 racemic}

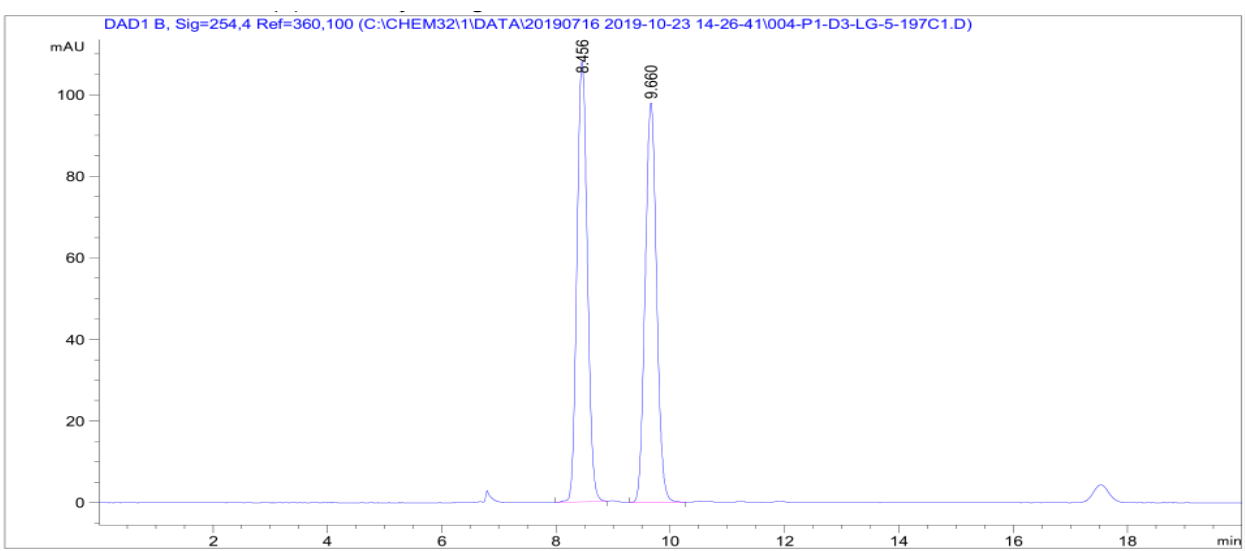

\begin{tabular}{|c|c|c|c|c|c|c|}
\hline $\begin{array}{l}\text { eak } \\
\#\end{array}$ & $\begin{array}{c}\text { RetTime } \\
\text { [min] }\end{array}$ & Type & $\begin{array}{l}\text { Width } \\
\text { [min] }\end{array}$ & $\begin{array}{c}\text { Area } \\
{\left[\mathrm{mAU}^{*} \mathrm{~s}\right]}\end{array}$ & $\begin{array}{l}\text { Height } \\
{[\mathrm{mAU}]}\end{array}$ & $\begin{array}{c}\text { Area } \\
\%\end{array}$ \\
\hline & & & & & & \\
\hline 1 & & & & 1361 & 10 & 50 \\
\hline 2 & $\theta$ & 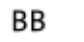 & 161 & 1360.50000 & 7.91948 & 49.989 \\
\hline
\end{tabular}

31 enantioenriched, $94 \%$ ee

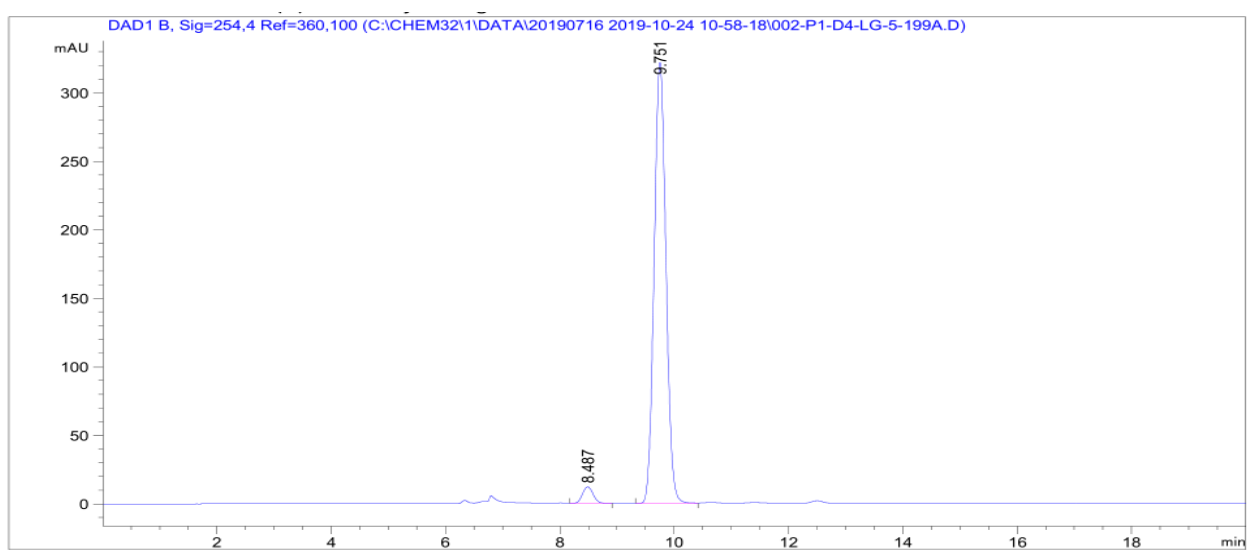

\begin{tabular}{|c|c|c|c|c|c|c|}
\hline $\begin{array}{c}\text { Peak } \\
\#\end{array}$ & $\begin{array}{c}\text { RetTime } \\
\text { [min] }\end{array}$ & Type & $\begin{array}{l}\text { Width } \\
\text { [min] }\end{array}$ & $\begin{array}{c}\text { Area } \\
{\left[\mathrm{mAU}^{*} \mathrm{~s}\right]}\end{array}$ & $\begin{array}{l}\text { Height } \\
{[\mathrm{mAU}]}\end{array}$ & $\begin{array}{c}\text { Area } \\
\%\end{array}$ \\
\hline & & & & & - & 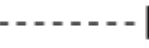 \\
\hline 1 & 8 & 3B & 0.2047 & 154.39224 & 11.94897 & 3.2373 \\
\hline 2 & 9.751 & BB & 234 & 4614.78467 & 321.58234 & 96.7627 \\
\hline
\end{tabular}


<smiles>CCCCOC(=O)[C@H](CC(C)(C)CCc1ccccc1)c1ccnc(C(F)(F)F)c1</smiles>

(R)-tert-Butyl 4,4-dimethyl-6-phenyl-2-(2-(trifluoromethyl)pyridin-4-yl)

\section{Hexanoate (32)}

The product was prepared according to the general procedure with (4S,4'S)-4,4'-di((S)sec-butyl)-4,4',5,5'-tetrahydro-2,2'-bioxazole [(S,S)-L1] $(3.3 \mathrm{mg}, 0.013 \mathrm{mmol}, 13$ mol\%), $\mathrm{NiCl}_{2} \bullet \mathrm{Py}_{4}(4.5 \mathrm{mg}, 0.01 \mathrm{mmol}, 10 \mathrm{~mol} \%), \operatorname{Ir}\left[\mathrm{dF}_{\left(\mathrm{CF}_{3}\right) \text { ppy }}\right]_{2}(\mathrm{bpy}) \mathrm{PF}_{6}(3.2 \mathrm{mg}$, $0.003 \mathrm{mmol}, 3 \mathrm{~mol} \%$ ), 4-bromo-2-(trifluoromethyl)pyridine (13.7 uL, $0.1 \mathrm{mmol}, 1.0$ equiv.), potassium (1,1-dimethyl-3-phenylbutanoate)trifluoroborate (63.5 mg, 0.25 mmol, 2.5 equiv.) and tert-butyl acrylate (29.0 uL, $0.2 \mathrm{mmol}, 2.0$ equiv.) in acetone/ethyl acetate $(1.3 / 0.7, \mathrm{v} / \mathrm{v}, 2 \mathrm{~mL})$ were used. After 24 hours, the product was isolated by flash chromatography (PE: ethyl acetate $=20: 1)$ as a white solid $(26.3 \mathrm{mg}$, $62 \%$ yield, $92 \%$ ee).

${ }^{1}$ H NMR $\left(400 \mathrm{MHz}, \mathrm{CDCl}_{3}\right) \delta 8.65(\mathrm{~d}, J=5.0 \mathrm{~Hz}, 1 \mathrm{H}), 7.64(\mathrm{~s}, 1 \mathrm{H}), 7.45(\mathrm{~d}, J=4.8$ $\mathrm{Hz}, 1 \mathrm{H}), 7.29-7.27$ (m, 2H), 7.19-7.14 (m, 3H), 3.63 (dd, $J=9.0,3.2 \mathrm{~Hz}, 1 \mathrm{H}), 2.62-$ $2.47(\mathrm{~m}, 2 \mathrm{H}), 2.36(\mathrm{dd}, J=14.1,9.1 \mathrm{~Hz}, 1 \mathrm{H}), 1.57-1.52(\mathrm{~m}, 3 \mathrm{H}), 1.36(\mathrm{~s}, 9 \mathrm{H}), 0.97$ (s, $6 \mathrm{H})$.

${ }^{19}$ F NMR $\left(375 \mathrm{MHz}, \mathrm{CDCl}_{3}\right) \delta-67.94(\mathrm{~s})$.

${ }^{13}$ C NMR (100 MHz, $\left.\mathrm{CDCl}_{3}\right) \delta 171.75,152.32,150.19,148.50$ (q, $\left.J=34.3 \mathrm{~Hz}\right), 142.70$, 128.37, 128.21, 125.72, 125.55, 121.46 (q, $J=274.5 \mathrm{~Hz}), 119.76$ (q, $J=2.7 \mathrm{~Hz}), 81.85$, $48.51,44.99,44.35,33.88,30.55,27.69,26.97,26.75$.

HRMS (ESI): $\mathrm{C}_{24} \mathrm{H}_{31} \mathrm{~F}_{3} \mathrm{NO}_{2}{ }^{+}(\mathrm{M}+\mathrm{H}): 422.2307$, found: 422.2301 .

$[\alpha]_{\mathrm{D}}^{25}=-16.5516\left(\mathrm{c}=0.5667, \mathrm{CHCl}_{3}\right)$

Chiral HPLC: CHIRALPAK IC, $25{ }^{\circ} \mathrm{C},{ }^{i} \mathrm{PrOH}-h$ exanes 5/95, $0.5 \mathrm{~mL} / \mathrm{min}, 254 \mathrm{~nm}, \mathrm{t}_{\mathrm{R}}$ (major) $=10.9 \mathrm{~min}, \mathrm{t}_{\mathrm{R}}($ minor $)=9.4 \mathrm{~min}$ 


\section{2 racemic}

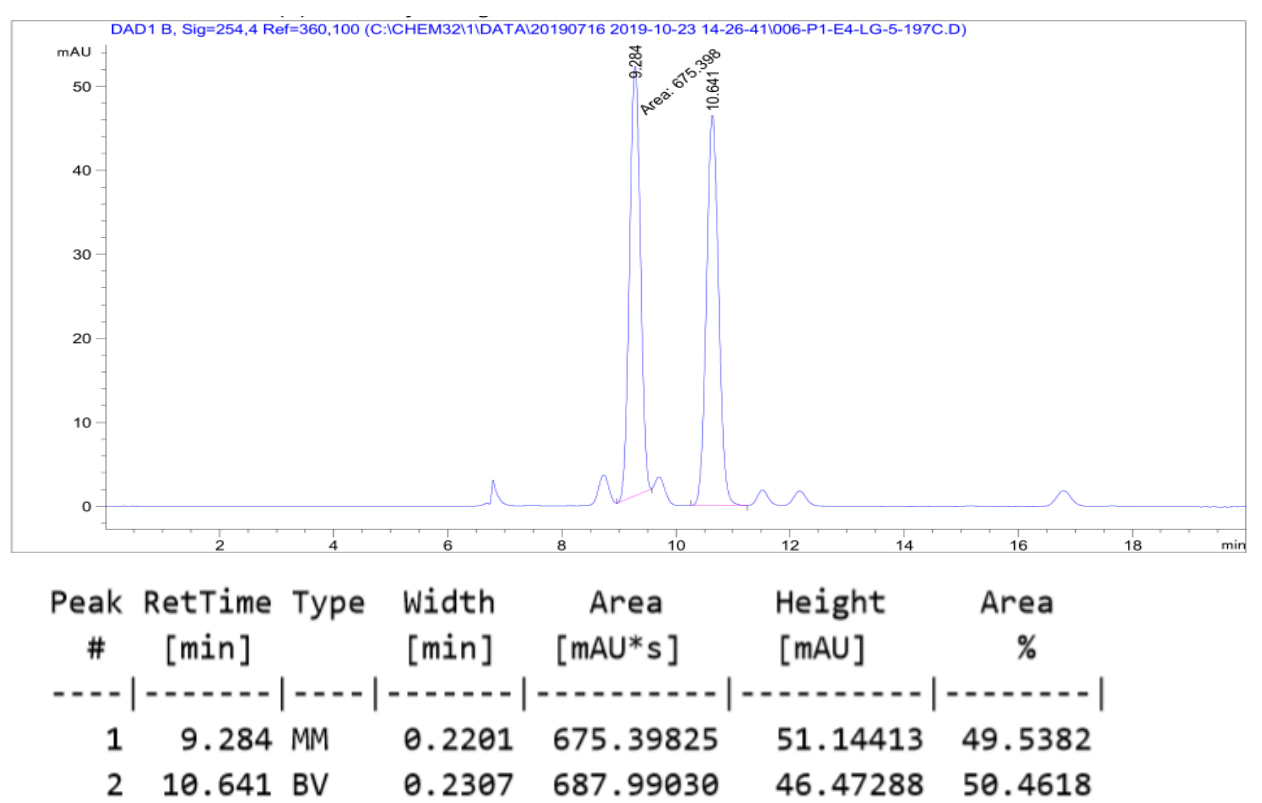

32 enantioenriched, $92 \%$ ee

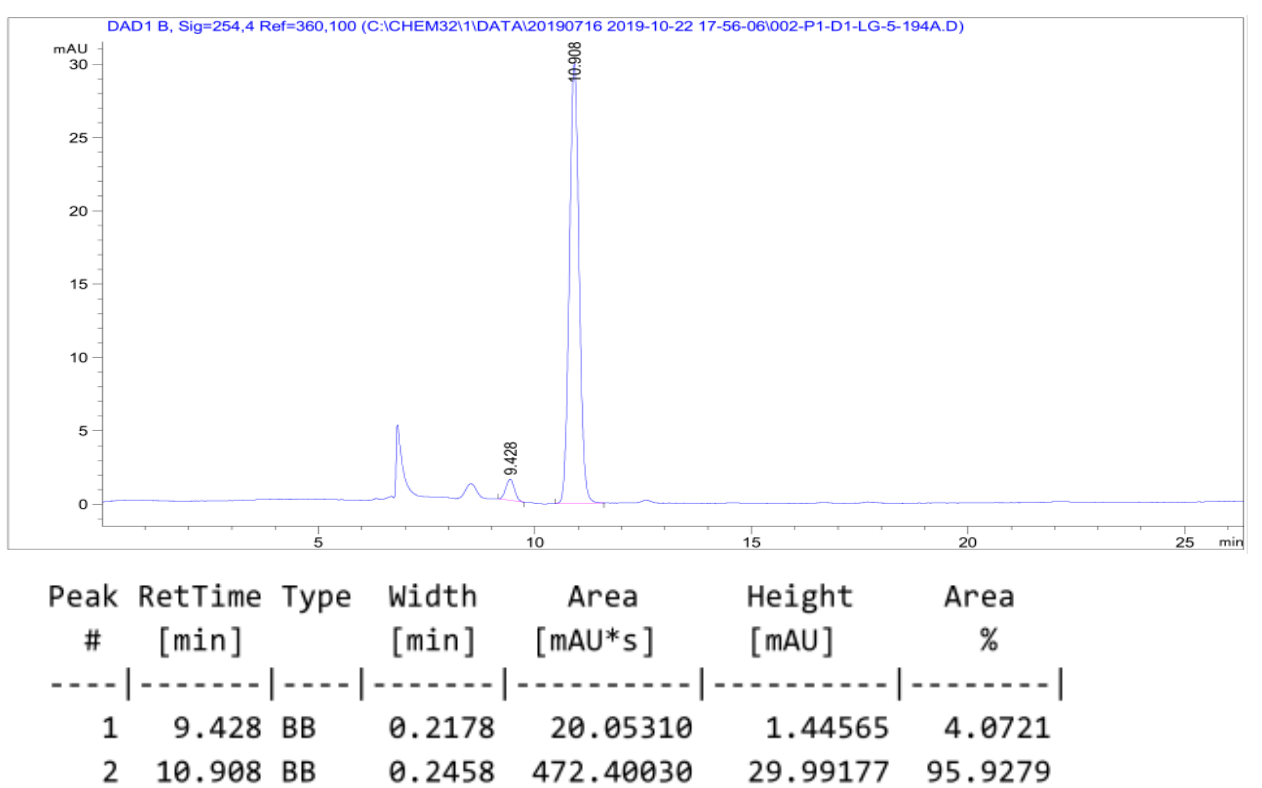<smiles>CC(C)(C)OC(=O)[C@H](CCl)c1ccnc(C(F)(F)F)c1</smiles>

(R)-tert-Butyl 3-cyclohexyl-2-(2-(trifluoromethyl)pyridin-4-yl)propanoate (33) 
The product was prepared according to the general procedure with (4S,4'S)-4,4'-di((S)sec-butyl)-4,4',5,5'-tetrahydro-2,2'-bioxazole [(S,S)-L1] $(3.3 \mathrm{mg}, 0.013 \mathrm{mmol}, 13$ mol\%), $\mathrm{NiCl}_{2} \cdot \mathrm{Py}_{4}(4.5 \mathrm{mg}, 0.01 \mathrm{mmol}, 10 \mathrm{~mol} \%), \operatorname{Ir}\left[\mathrm{dF}\left(\mathrm{CF}_{3}\right) \mathrm{ppy}\right]_{2}(\mathrm{bpy}) \mathrm{PF}_{6}(3.2 \mathrm{mg}$, $0.003 \mathrm{mmol}, 3 \mathrm{~mol} \%$ ), 4-bromo-2-(trifluoromethyl)pyridine (13.7 uL, $0.1 \mathrm{mmol}, 1.0$ equiv.), potassium cyclohexyltrifluoroborate (47.5 mg, $0.25 \mathrm{mmol}, 2.5$ equiv.) and tertbutyl acrylate $(29.0 \mathrm{uL}, 0.2 \mathrm{mmol}, 2.0$ equiv.) in acetone/ethyl acetate $(1.3 / 0.7, \mathrm{v} / \mathrm{v}, 2$ $\mathrm{mL}$ ) were used. After 24 hours, the product was isolated by flash chromatography (PE: ethyl acetate $=20: 1)$ as a colorless oil $(24.3 \mathrm{mg}, 68 \%$ yield, $90 \%$ ee $)$.

${ }^{1}$ H NMR $\left(400 \mathrm{MHz}, \mathrm{CDCl}_{3}\right) \delta 8.65(\mathrm{~d}, J=5.0 \mathrm{~Hz}, 1 \mathrm{H}), 7.63(\mathrm{~s}, 1 \mathrm{H}), 7.44(\mathrm{dd}, J=5.0$, $1.3 \mathrm{~Hz}, 1 \mathrm{H}), 3.66(\mathrm{dd}, J=8.6,7.0 \mathrm{~Hz}, 1 \mathrm{H}), 2.01-1.94(\mathrm{~m}, 1 \mathrm{H}), 1.75-1.67$ (m, 4H), 1.58$1.54(\mathrm{~m}, 2 \mathrm{H}), 1.41$ (s, 9H), 1.19-1.12 (m, 4H), 0.97-0.88 (m, 2H).

${ }^{19}$ F NMR $\left(375 \mathrm{MHz}, \mathrm{CDCl}_{3}\right) \delta-67.96$ (s)

${ }^{13}$ C NMR $\left(100 \mathrm{MHz}, \mathrm{CDCl}_{3}\right) \delta 171.44,151.07,150.10,148.47$ (q, $\left.J=34.3 \mathrm{~Hz}\right), 125.83$, 121.55 (q, $J=274.1 \mathrm{~Hz}), 120.03(\mathrm{q}, J=2.7 \mathrm{~Hz}), 81.81,49.60,40.90,35.50,33.07$, $33.02,27.89,26.35,26.05$.

HRMS (ESI): $\mathrm{C}_{19} \mathrm{H}_{27} \mathrm{~F}_{3} \mathrm{NO}_{2}^{+}(\mathrm{M}+\mathrm{H}): 358.1994$, found: 358.1983 .

$[\alpha]_{\mathrm{D}}^{25}=-4.5696\left(\mathrm{c}=0.3567, \mathrm{CHCl}_{3}\right)$

Chiral HPLC: CHIRALPAK IC, $25{ }^{\circ} \mathrm{C},{ }^{i} \mathrm{PrOH}-h$ exanes 5/95, $0.5 \mathrm{~mL} / \mathrm{min}, 254 \mathrm{~nm}, \mathrm{t}_{\mathrm{R}}$ $($ major $)=10.9 \mathrm{~min}, \mathrm{t}_{\mathrm{R}}($ minor $)=8.9 \mathrm{~min}$.

\section{3 racemic}

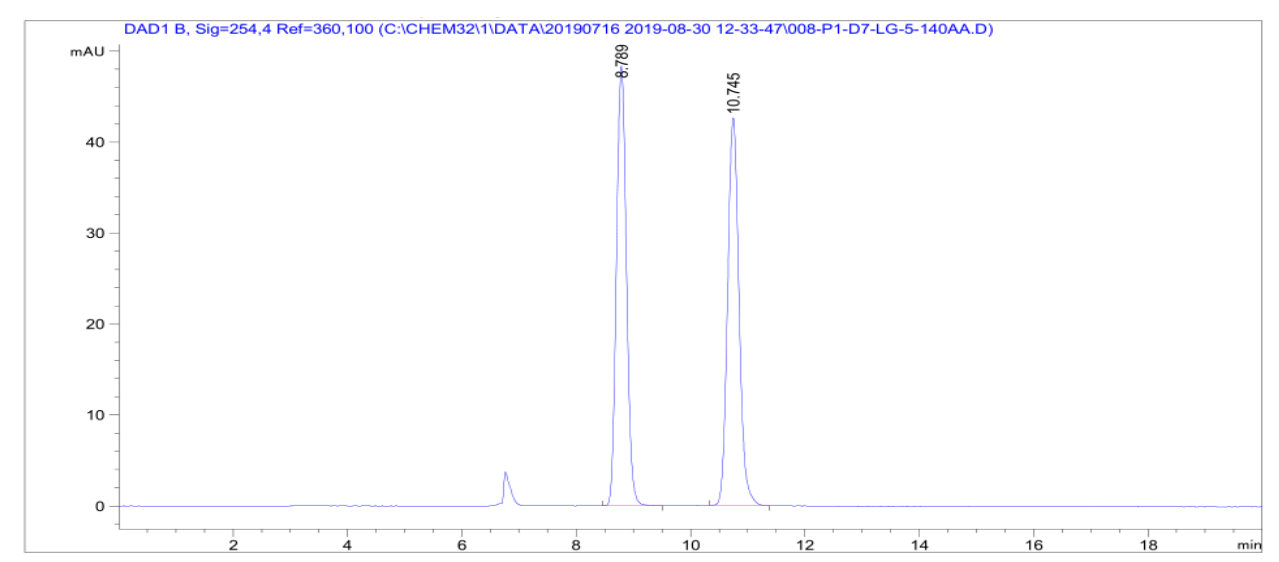




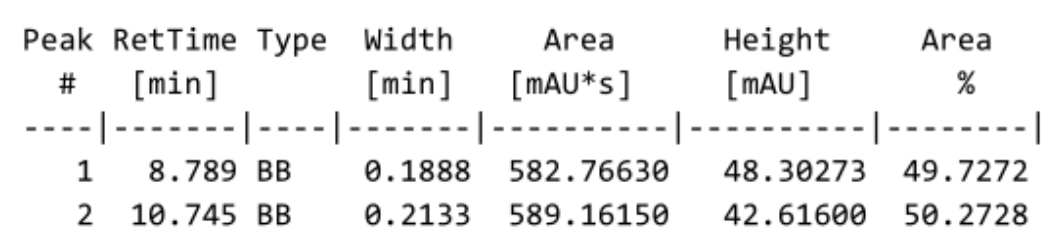

33 enantioenriched, $90 \%$ ee

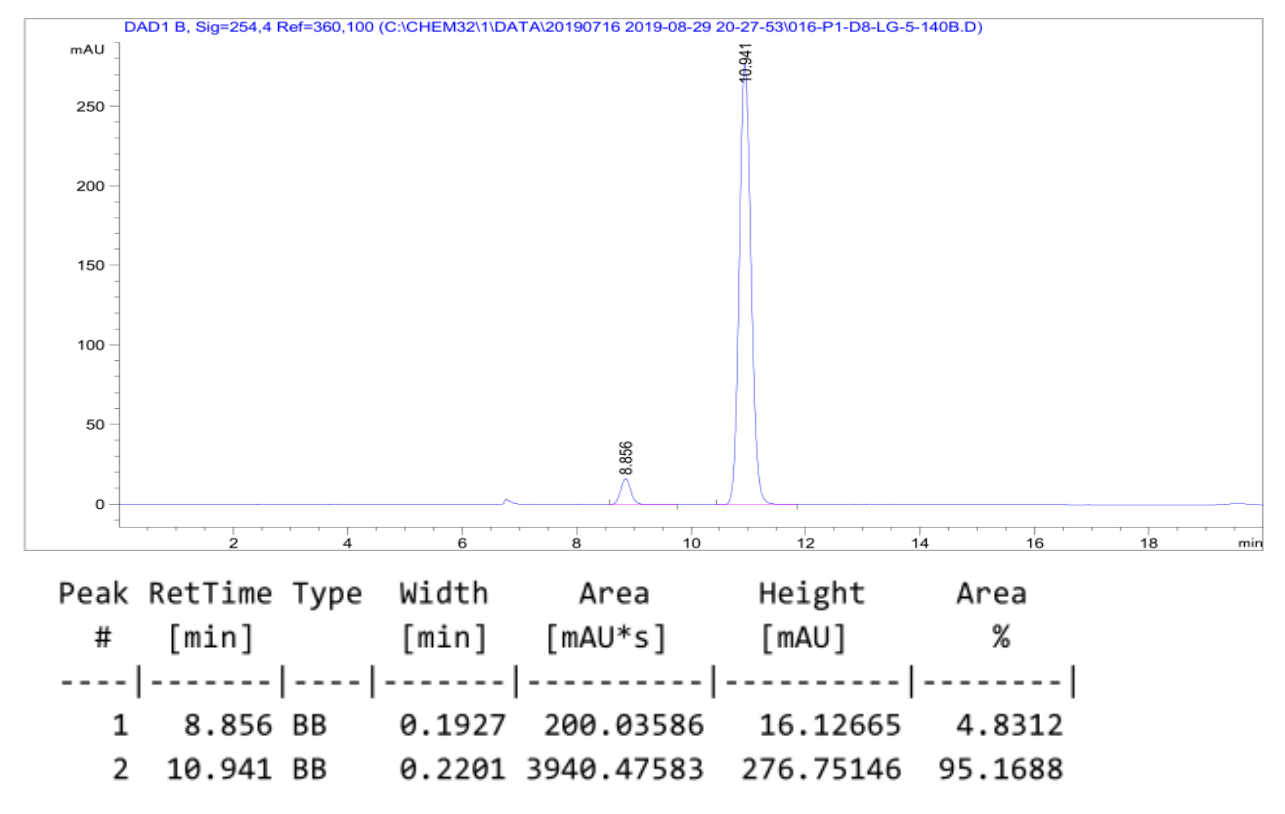<smiles>CCCCOC(=O)[C@H](CC1CCOCC1)c1ccnc(C(F)(F)F)c1</smiles>

(R)-tert-Butyl 3-(tetrahydro-2H-pyran-4-yl)-2-(2-(trifluoromethyl)pyridin-4-yl)

\section{Propanoate (34)}

The product was prepared according to the general procedure with (4S,4'S)-4,4'-di((S)sec-butyl)-4,4',5,5'-tetrahydro-2,2'-bioxazole [(S,S)-L1] $(3.3 \mathrm{mg}, 0.013 \mathrm{mmol}, 13$ mol\%), $\mathrm{NiCl}_{2} \cdot \mathrm{Py}_{4}(4.5 \mathrm{mg}, 0.01 \mathrm{mmol}, 10 \mathrm{~mol} \%), \operatorname{Ir}\left[\mathrm{dF}\left(\mathrm{CF}_{3}\right) \mathrm{ppy}\right]_{2}(\mathrm{bpy}) \mathrm{PF}_{6}(3.2 \mathrm{mg}$, $0.003 \mathrm{mmol}, 3 \mathrm{~mol} \%$ ), 4-bromo-2-(trifluoromethyl)pyridine (13.7 uL, $0.1 \mathrm{mmol}, 1.0$ equiv.), potassium tetrahydro-2H-pyran-4-trifluoroborate (48.0 mg, $0.25 \mathrm{mmol}, 2.5$ equiv.) and tert-butyl acrylate $(29.0 \mathrm{uL}, 0.2 \mathrm{mmol}, 2.0$ equiv.) in acetone/ethyl acetate $(1.3 / 0.7, \mathrm{v} / \mathrm{v}, 2 \mathrm{~mL})$ were used. After 24 hours, the product was isolated by flash 
chromatography (PE: ethyl acetate $=20: 1)$ as a colorless oil $(19.1 \mathrm{mg}, 53 \%$ yield, $88 \%$ ee).

${ }^{1}$ H NMR $\left(400 \mathrm{MHz}, \mathrm{CDCl}_{3}\right) \delta 8.67(\mathrm{~d}, J=5.0 \mathrm{~Hz}, 1 \mathrm{H}), 7.63(\mathrm{~s}, 1 \mathrm{H}), 7.43(\mathrm{~d}, J=4.9$ $\mathrm{Hz}, 1 \mathrm{H}), 3.96-3.93(\mathrm{~m}, 2 \mathrm{H}), 3.65(\mathrm{dd}, J=8.7,6.8 \mathrm{~Hz}, 1 \mathrm{H}), 3.31(\mathrm{t}, J=12.3 \mathrm{~Hz}, 2 \mathrm{H})$, 2.09-2.02 (m, 1H), 1.67-1.58 (m, 3H), 1.46-1.43 (m, 1H), $1.40(\mathrm{~s}, 9 \mathrm{H}), 1.36-1.27(\mathrm{~m}$, $2 \mathrm{H})$.

${ }^{19}$ F NMR $\left(375 \mathrm{MHz}, \mathrm{CDCl}_{3}\right) \delta-67.97$ (s).

${ }^{13}$ C NMR $\left(100 \mathrm{MHz}, \mathrm{CDCl}_{3}\right) \delta 171.06,150.57,150.24,148.62(\mathrm{q}, J=34.4 \mathrm{~Hz}), 125.73$, $121.49(\mathrm{q}, J=274.3 \mathrm{~Hz}), 119.92(\mathrm{q}, J=2.6 \mathrm{~Hz}), 82.11,67.73,67.72,49.13,40.23$, $33.00,32.82,32.73,27.87$.

HRMS (ESI): $\mathrm{C}_{18} \mathrm{H}_{25} \mathrm{~F}_{3} \mathrm{NO}_{3}{ }^{+}(\mathrm{M}+\mathrm{H}): 360.1787$, found: 360.1772 .

$[\alpha]_{\mathrm{D}}^{25}=-7.5262\left(\mathrm{c}=0.3800, \mathrm{CHCl}_{3}\right)$

Chiral HPLC: CHIRALPAK IC, $25{ }^{\circ} \mathrm{C},{ }^{i} \mathrm{PrOH}-h$ exanes $15 / 85,1.0 \mathrm{~mL} / \mathrm{min}, 254 \mathrm{~nm}$, $\mathrm{t}_{\mathrm{R}}($ major $)=9.0 \mathrm{~min}, \mathrm{t}_{\mathrm{R}}($ minor $)=8.0 \mathrm{~min}$

\section{4 racemic}

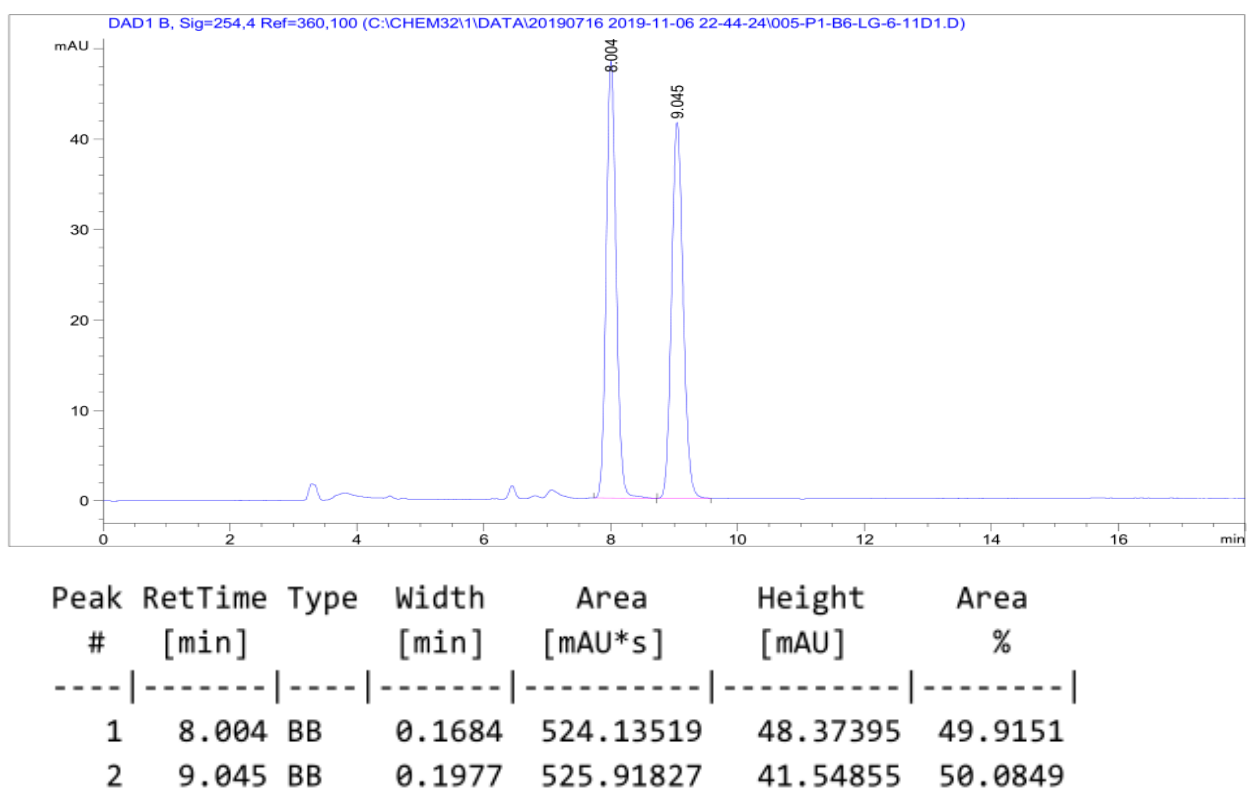




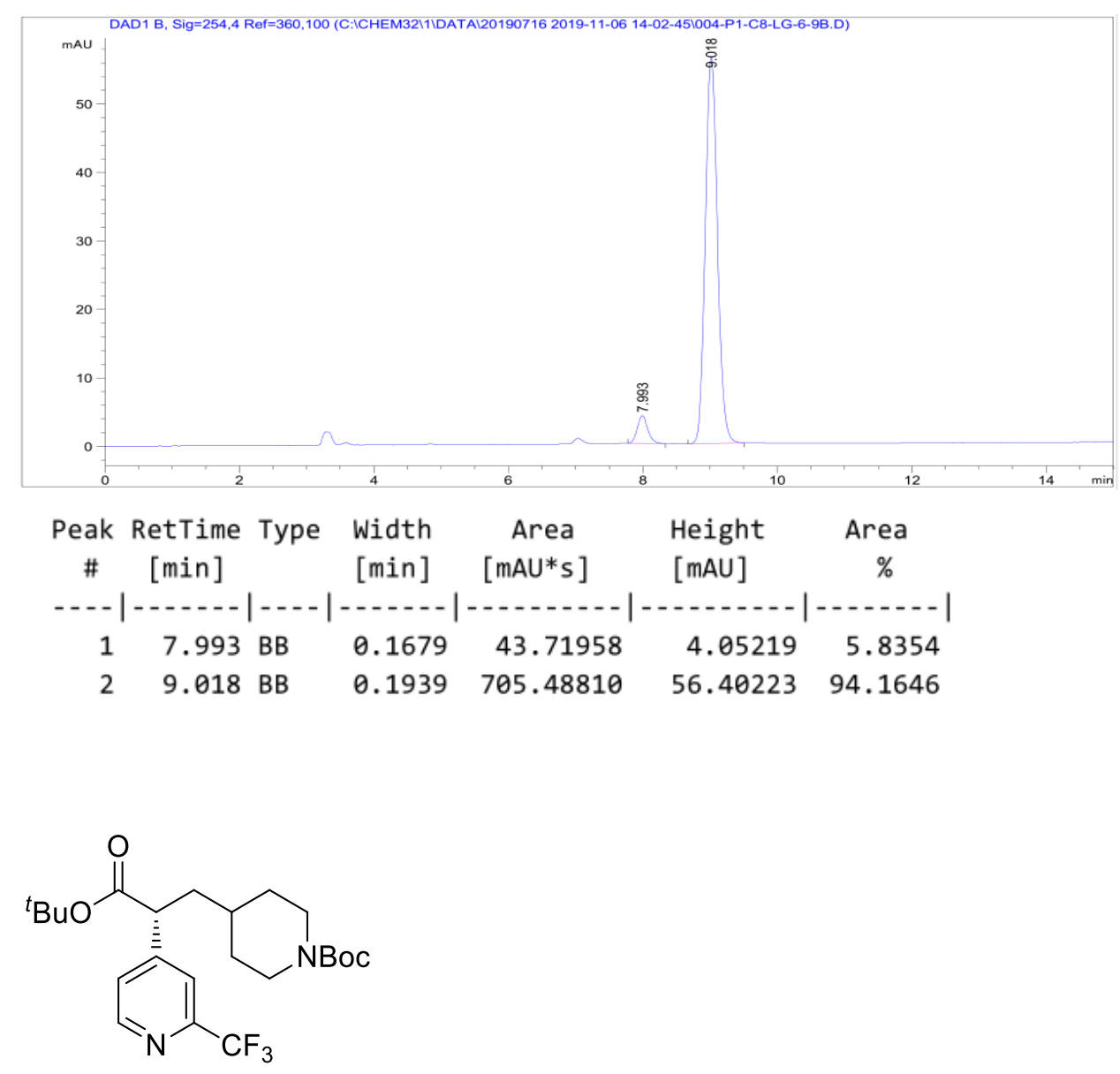

(R)-tert-Butyl4-(3-(tert-butoxy)-3-oxo-2-(2-(trifluoromethyl)pyridin-4-

\section{yl)propyl)piperidine-1-carboxylate (35)}

The product was prepared according to the general procedure with (4S,4'S)-4,4'-di((S)sec-butyl)-4,4',5,5'-tetrahydro-2,2'-bioxazole [(S,S)-L1] (3.3 mg, $0.013 \mathrm{mmol}, 13$ mol\%), $\mathrm{NiCl}_{2} \cdot \mathrm{Py}_{4}(4.5 \mathrm{mg}, 0.01 \mathrm{mmol}, 10 \mathrm{~mol} \%), \operatorname{Ir}\left[\mathrm{dF}\left(\mathrm{CF}_{3}\right) \mathrm{ppy}\right]_{2}(\mathrm{bpy}) \mathrm{PF}_{6}(3.2 \mathrm{mg}$, $0.003 \mathrm{mmol}, 3 \mathrm{~mol} \%$ ), 4-bromo-2-(trifluoromethyl)pyridine (13.7 uL, $0.1 \mathrm{mmol}, 1.0$ equiv.), potassium ( $N$-Boc-piperidin-4-yl)trifluoroborate $(72.5 \mathrm{mg}, 0.25 \mathrm{mmol}, 2.5$ equiv.) and tert-butyl acrylate $(29.0 \mathrm{uL}, 0.2 \mathrm{mmol}, 2.0$ equiv.) in acetone/ethyl acetate $(1.3 / 0.7, \mathrm{v} / \mathrm{v}, 2 \mathrm{~mL})$ were used. After 24 hours, the product was isolated by flash chromatography (PE: ethyl acetate $=4: 1)$ as a white solid $(36.2 \mathrm{mg}, 79 \%$ yield, $80 \%$ ee). ${ }^{1} \mathbf{H}$ NMR $\left(400 \mathrm{MHz}, \mathrm{CDCl}_{3}\right) \delta 8.67(\mathrm{~d}, J=5.0 \mathrm{~Hz}, 1 \mathrm{H}), 7.62(\mathrm{~s}, 1 \mathrm{H}), 7.43(\mathrm{dd}, J=5.0$, $1.1 \mathrm{~Hz}, 1 \mathrm{H}), 4.08$ (br, 2H), $3.65(\mathrm{dd}, J=8.8,6.8 \mathrm{~Hz}, 1 \mathrm{H}), 2.62$ (t, $J=11.5 \mathrm{~Hz}, 2 \mathrm{H})$, 2.08-2.01 (m, 1H), 1.70-1.66 (m, 2H), 1.61-1.56 (m, 1H), $1.44(\mathrm{~s}, 9 \mathrm{H}), 1.40(\mathrm{~s}, 9 \mathrm{H})$, $1.35-1.30(\mathrm{~m}, 1 \mathrm{H}), 1.18-1.07(\mathrm{~m}, 2 \mathrm{H})$. 
${ }^{19}$ F NMR $\left(375 \mathrm{MHz}, \mathrm{CDCl}_{3}\right) \delta-67.96(\mathrm{~s})$.

${ }^{13}$ C NMR (101 MHz, $\left.\mathrm{CDCl}_{3}\right) \delta 171.06,154.78,150.55,150.26,148.62$ (q, $J=34.4$ $\mathrm{Hz}), 125.72,121.48$ (q, $J=274.4 \mathrm{~Hz}), 119.91$ (q, $J=2.7 \mathrm{~Hz}), 82.16,79.47,49.35,43.80$, $39.89,34.02,31.92,28.45,27.88$.

HRMS (ESI): $\mathrm{C}_{23} \mathrm{H}_{34} \mathrm{~F}_{3} \mathrm{~N}_{2} \mathrm{O}_{4}{ }^{+}(\mathrm{M}+\mathrm{H}): 459.2471$, found: 459.2465 .

$[\alpha]_{\mathrm{D}}^{25}=-8.8259\left(\mathrm{c}=0.4600, \mathrm{CHCl}_{3}\right)$

Chiral HPLC: CHIRALPAK IC, $25^{\circ} \mathrm{C},{ }^{i}$ PrOH-hexanes 10/90, $1.0 \mathrm{~mL} / \mathrm{min}, 210 \mathrm{~nm}$, $t_{R}($ major $)=16.8 \mathrm{~min}, t_{R}($ minor $)=15.0 \mathrm{~min}$

\section{5 racemic}
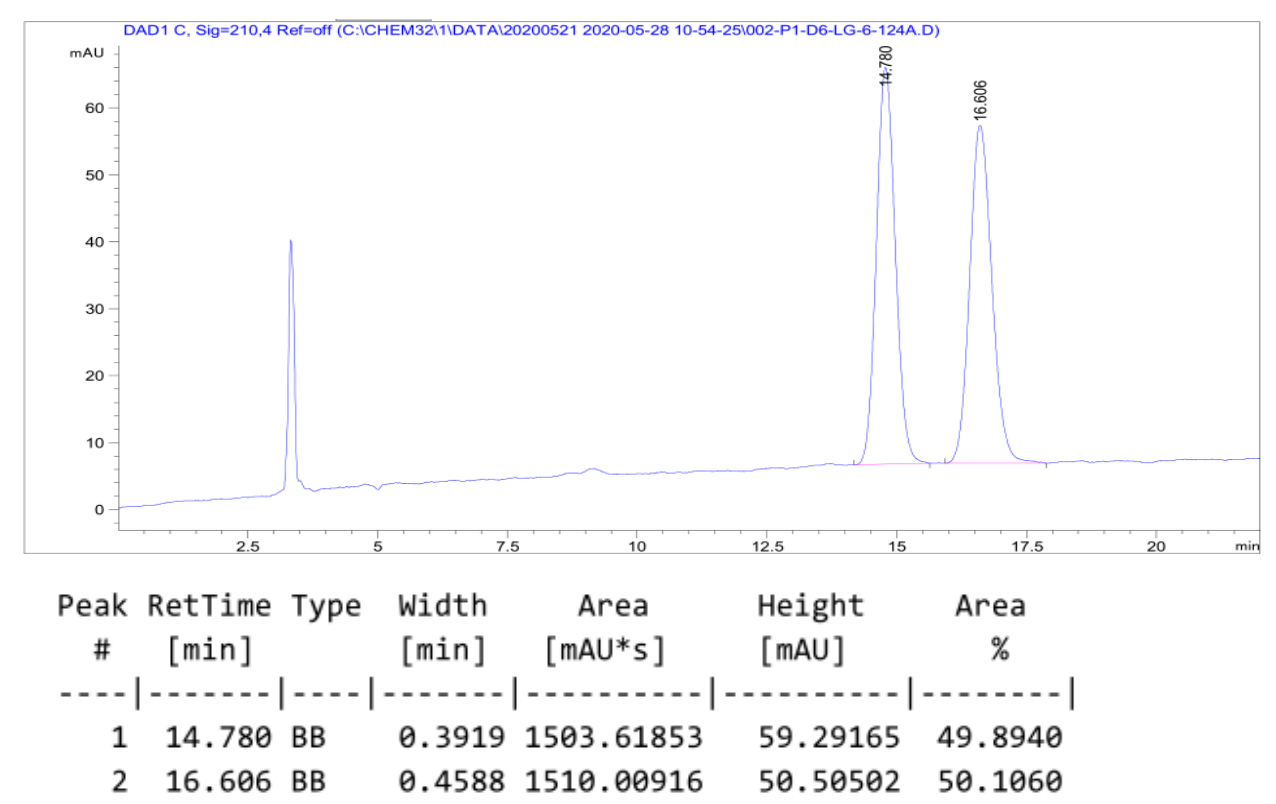

35 enantioenriched, $80 \%$ ee

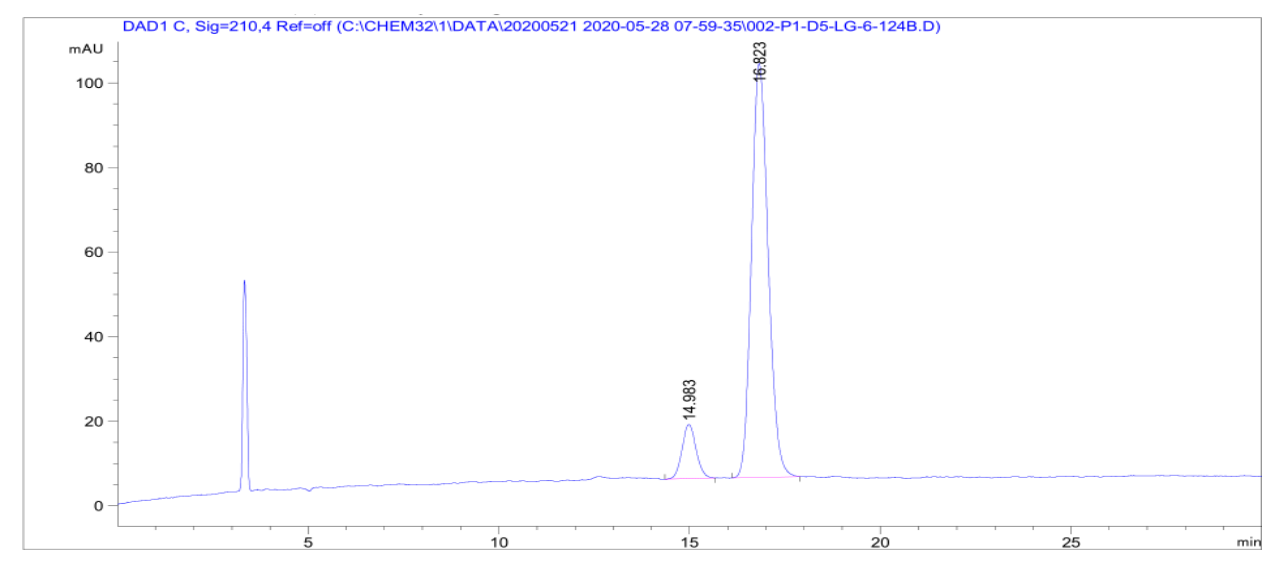




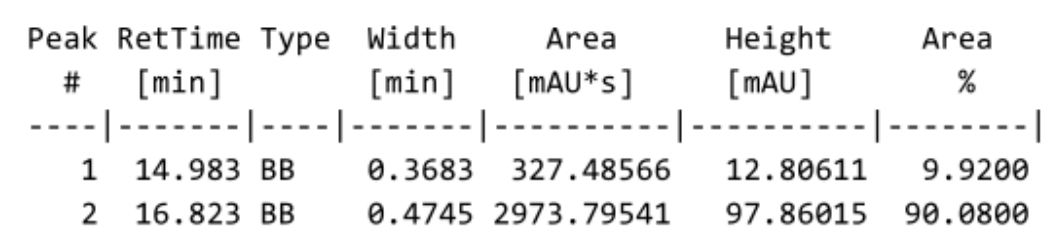<smiles>CCCCOC(=O)[C@H](CC1CCC1)c1ccnc(C(F)(F)F)c1</smiles>

(R)-tert-Butyl 3-cyclobutyl-2-(2-(trifluoromethyl)pyridin-4-yl)propanoate (36)

The product was prepared according to the general procedure with $(4 \mathrm{~S}, 4$ 'S)-4,4'-di((S)sec-butyl)-4,4',5,5'-tetrahydro-2,2'-bioxazole [(S,S)-L1] $(3.3 \mathrm{mg}, 0.013 \mathrm{mmol}, 13$ $\mathrm{mol} \%$ ), $\mathrm{NiCl}_{2} \bullet \mathrm{Py}_{4}(4.5 \mathrm{mg}, 0.01 \mathrm{mmol}, 10 \mathrm{~mol} \%), \operatorname{Ir}\left[\mathrm{dF}\left(\mathrm{CF}_{3}\right) \text { ppy }\right]_{2}(\mathrm{bpy}) \mathrm{PF}_{6}(3.2 \mathrm{mg}$, $0.003 \mathrm{mmol}, 3 \mathrm{~mol} \%$ ), 4-bromo-2-(trifluoromethyl)pyridine (13.7 uL, $0.1 \mathrm{mmol}, 1.0$ equiv.), potassium cyclobutyltrifluoroborate ( $40.5 \mathrm{mg}, 0.25 \mathrm{mmol}, 2.5$ equiv.) and tertbutyl acrylate (29.0 uL, $0.2 \mathrm{mmol}, 2.0$ equiv.) in acetone/ethyl acetate (1.3/0.7, v/v, 2 $\mathrm{mL}$ ) were used. After 24 hours, the product was isolated by flash chromatography (PE: ethyl acetate $=20: 1)$ as a colorless oil $(13.5 \mathrm{mg}, 41 \%$ yield, $90 \%$ ee $)$.

${ }^{1} \mathbf{H}$ NMR $\left(400 \mathrm{MHz}, \mathrm{CDCl}_{3}\right) \delta 8.65(\mathrm{~d}, J=5.0 \mathrm{~Hz}, 1 \mathrm{H}), 7.62(\mathrm{~s}, 1 \mathrm{H}), 7.42(\mathrm{~d}, J=4.9$ Hz, 1H), 3.45 (t, $J=7.3 \mathrm{~Hz}, 1 \mathrm{H}), 2.20-2.12(\mathrm{~m}, 2 \mathrm{H}), 2.06-1.92(\mathrm{~m}, 2 \mathrm{H}), 1.87-1.78(\mathrm{~m}$, 3H), 1.71-1.64 (m, 1H), 1.60-1.53 (m, 1H), $1.41(\mathrm{~s}, 9 \mathrm{H})$.

${ }^{19}$ F NMR $\left(375 \mathrm{MHz}, \mathrm{CDCl}_{3}\right) \delta-67.97$ (s).

${ }^{13}$ C NMR $\left(100 \mathrm{MHz}, \mathrm{CDCl}_{3}\right) \delta 171.19,150.70,150.03,148.40(\mathrm{q}, J=34.4 \mathrm{~Hz}), 125.84$, $121.52(\mathrm{q}, J=274.4 \mathrm{~Hz}), 120.04(\mathrm{q}, J=2.6 \mathrm{~Hz}), 81.79,50.46,40.30,33.89,28.21$, $28.03,27.85,18.32$.

HRMS (ESI): $\mathrm{C}_{17} \mathrm{H}_{23} \mathrm{~F}_{3} \mathrm{NO}_{2}^{+}(\mathrm{M}+\mathrm{H}): 330.1681$, found: 330.1670 .

$[\alpha]_{\mathrm{D}}^{25}=-4.8042\left(\mathrm{c}=0.4267, \mathrm{CHCl}_{3}\right)$

Chiral HPLC: CHIRALPAK IA, $25{ }^{\circ} \mathrm{C},{ }^{i} \mathrm{PrOH}-$ hexanes 5/95, $0.5 \mathrm{~mL} / \mathrm{min}, 254 \mathrm{~nm}, \mathrm{t}_{\mathrm{R}}$ (major) $=11.7 \mathrm{~min}, \mathrm{t}_{\mathrm{R}}($ minor $)=9.1 \mathrm{~min}$ 


\section{6 racemic}

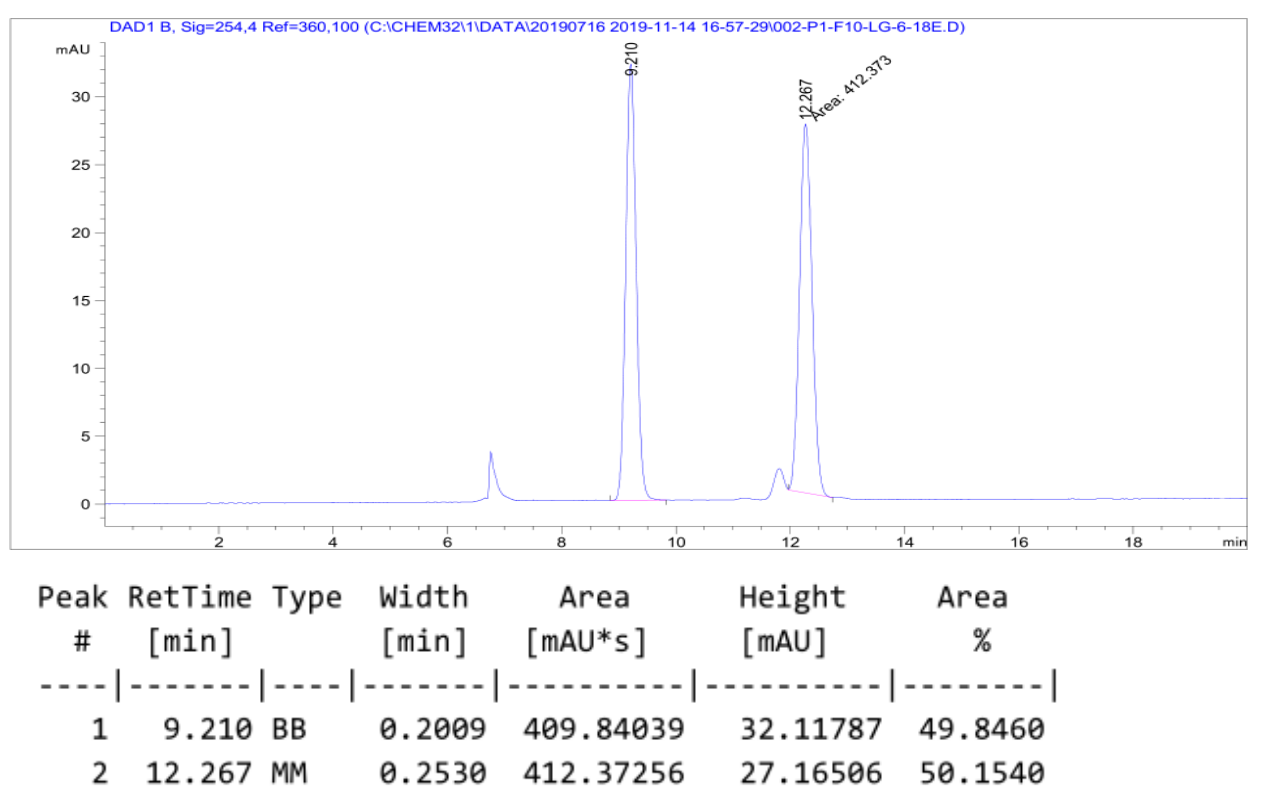

36 enantioenriched, $90 \%$ ee

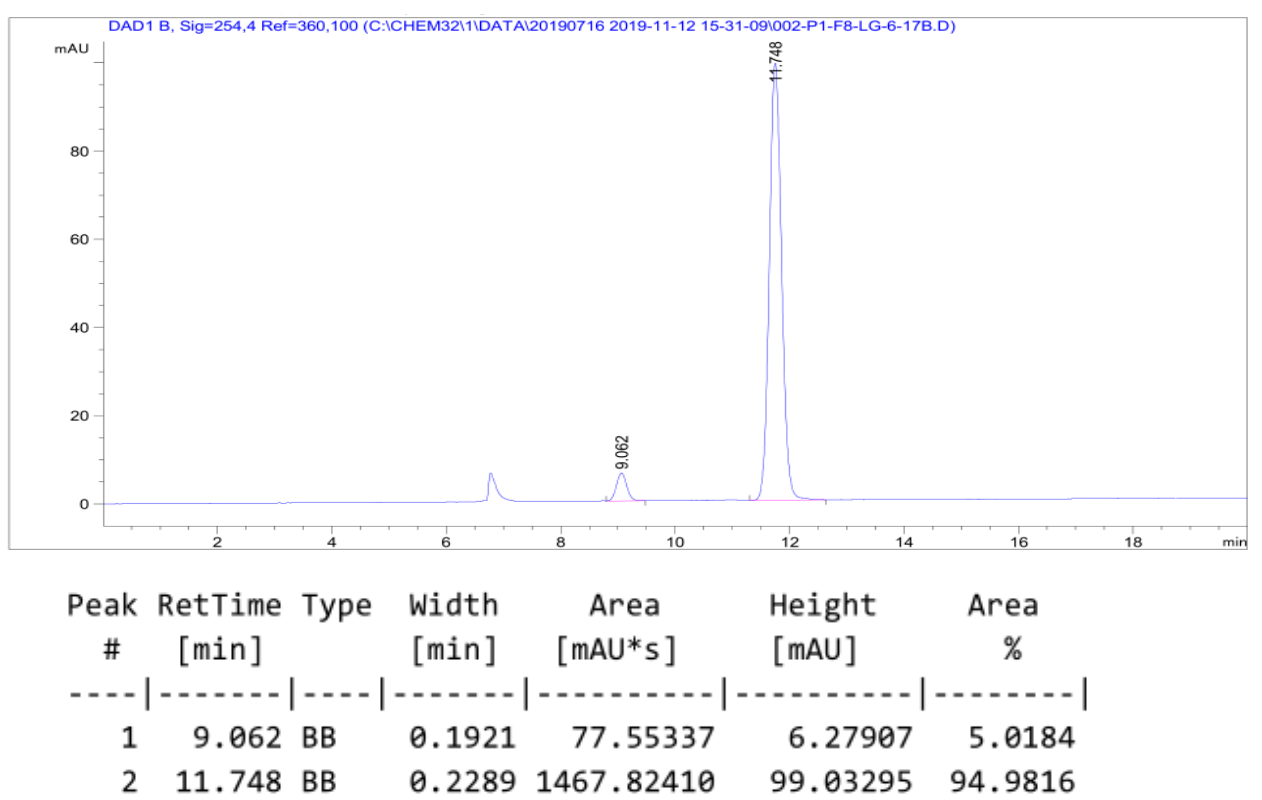<smiles>CCCCOC(=O)[C@H](CC1CCCC1)c1ccnc(C(F)(F)F)c1</smiles>

(R)-tert-Butyl 3-cyclopentyl-2-(2-(trifluoromethyl)pyridin-4-yl)propanoate (37) 
The product was prepared according to the general procedure with $(4 \mathrm{~S}, 4$ 'S)-4,4'-di((S)sec-butyl)-4,4',5,5'-tetrahydro-2,2'-bioxazole [(S,S)-L1] $(3.3 \mathrm{mg}, 0.013 \mathrm{mmol}, 13$ mol\%), $\mathrm{NiCl}_{2} \cdot \mathrm{Py}_{4}(4.5 \mathrm{mg}, 0.01 \mathrm{mmol}, 10 \mathrm{~mol} \%), \operatorname{Ir}\left[\mathrm{dF}\left(\mathrm{CF}_{3}\right) \mathrm{ppy}\right]_{2}(\mathrm{bpy}) \mathrm{PF}_{6}(3.2 \mathrm{mg}$, $0.003 \mathrm{mmol}, 3 \mathrm{~mol} \%$ ), 4-bromo-2-(trifluoromethyl)pyridine (13.7 uL, $0.1 \mathrm{mmol}, 1.0$ equiv.), potassium cyclopentyltrifluoroboranuide ( $44.0 \mathrm{mg}, 0.25 \mathrm{mmol}, 2.5$ equiv.) and tert-butyl acrylate $(29.0 \mathrm{uL}, 0.2 \mathrm{mmol}, 2.0$ equiv.) in acetone/ethyl acetate $(1.3 / 0.7, \mathrm{v} / \mathrm{v}$, $2 \mathrm{~mL}$ ) were used. After 24 hours, the product was isolated by flash chromatography (PE: ethyl acetate $=20: 1)$ as a colorless oil $(19.0 \mathrm{mg}, 55 \%$ yield, 91\% ee).

${ }^{1} \mathbf{H}$ NMR $\left(400 \mathrm{MHz}, \mathrm{CDCl}_{3}\right) \delta 8.65(\mathrm{~d}, J=5.0 \mathrm{~Hz}, 1 \mathrm{H}), 7.64(\mathrm{~s}, 1 \mathrm{H}), 7.44(\mathrm{~d}, J=5.0$ $\mathrm{Hz}, 1 \mathrm{H}), 3.57$ (t, $J=7.7 \mathrm{~Hz}, 1 \mathrm{H}), 2.09$ (dt, $J=13.4,7.7 \mathrm{~Hz}, 1 \mathrm{H}), 1.77-1.72(\mathrm{~m}, 3 \mathrm{H})$, 1.63-1.59 (m, 3H), 1.52-1.46 (m, 2H), $1.40(\mathrm{~s}, 9 \mathrm{H}), 1.16-1.07$ (m, 2H).

${ }^{19}$ F NMR $\left(377 \mathrm{MHz}, \mathrm{CDCl}_{3}\right) \delta-67.96(\mathrm{~s})$

${ }^{13}$ C NMR $\left(100 \mathrm{MHz}, \mathrm{CDCl}_{3}\right) \delta 171.37,150.91,150.08,148.45$ (q, $\left.J=34.5 \mathrm{~Hz}\right), 125.86$, $121.55(\mathrm{q}, J=274.4 \mathrm{~Hz}), 120.05(\mathrm{q}, J=2.4 \mathrm{~Hz}), 81.81,51.64,39.65,37.90,32.62$, $32.38,27.88,25.06,25.03$.

HRMS (ESI): $\mathrm{C}_{18} \mathrm{H}_{25} \mathrm{~F}_{3} \mathrm{NO}_{2}{ }^{+}(\mathrm{M}+\mathrm{H}): 344.1837$, found: 344.1823 .

$[\alpha]_{\mathrm{D}}^{25}=-14.1549\left(\mathrm{c}=0.4267, \mathrm{CHCl}_{3}\right)$

Chiral HPLC: CHIRALPAK IC, $25{ }^{\circ} \mathrm{C},{ }^{i} \mathrm{PrOH}-h$ exanes 5/95, $1.0 \mathrm{~mL} / \mathrm{min}, 254 \mathrm{~nm}, \mathrm{t}_{\mathrm{R}}$ $($ major $)=5.5 \mathrm{~min}, \mathrm{t}_{\mathrm{R}}($ minor $)=4.4 \mathrm{~min}$.

\section{7 racemic}

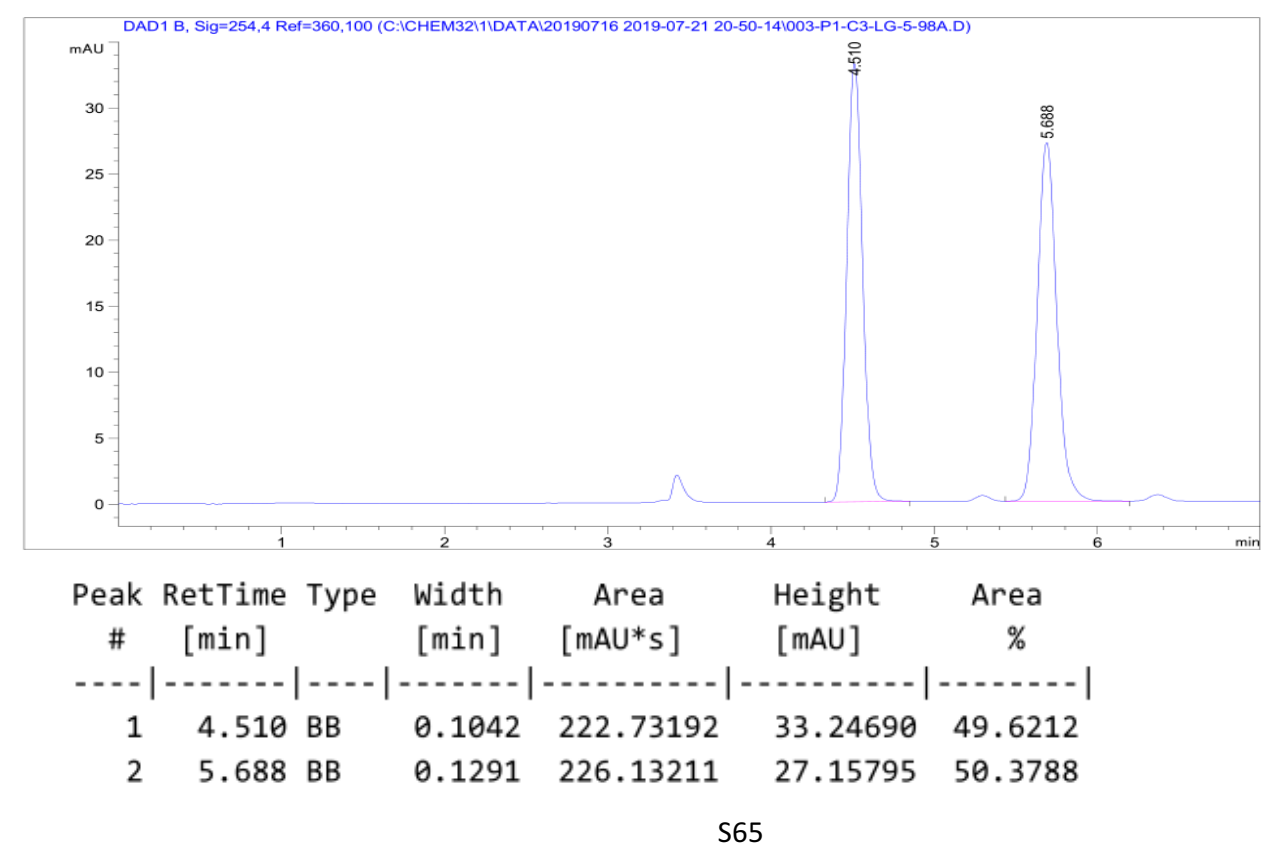




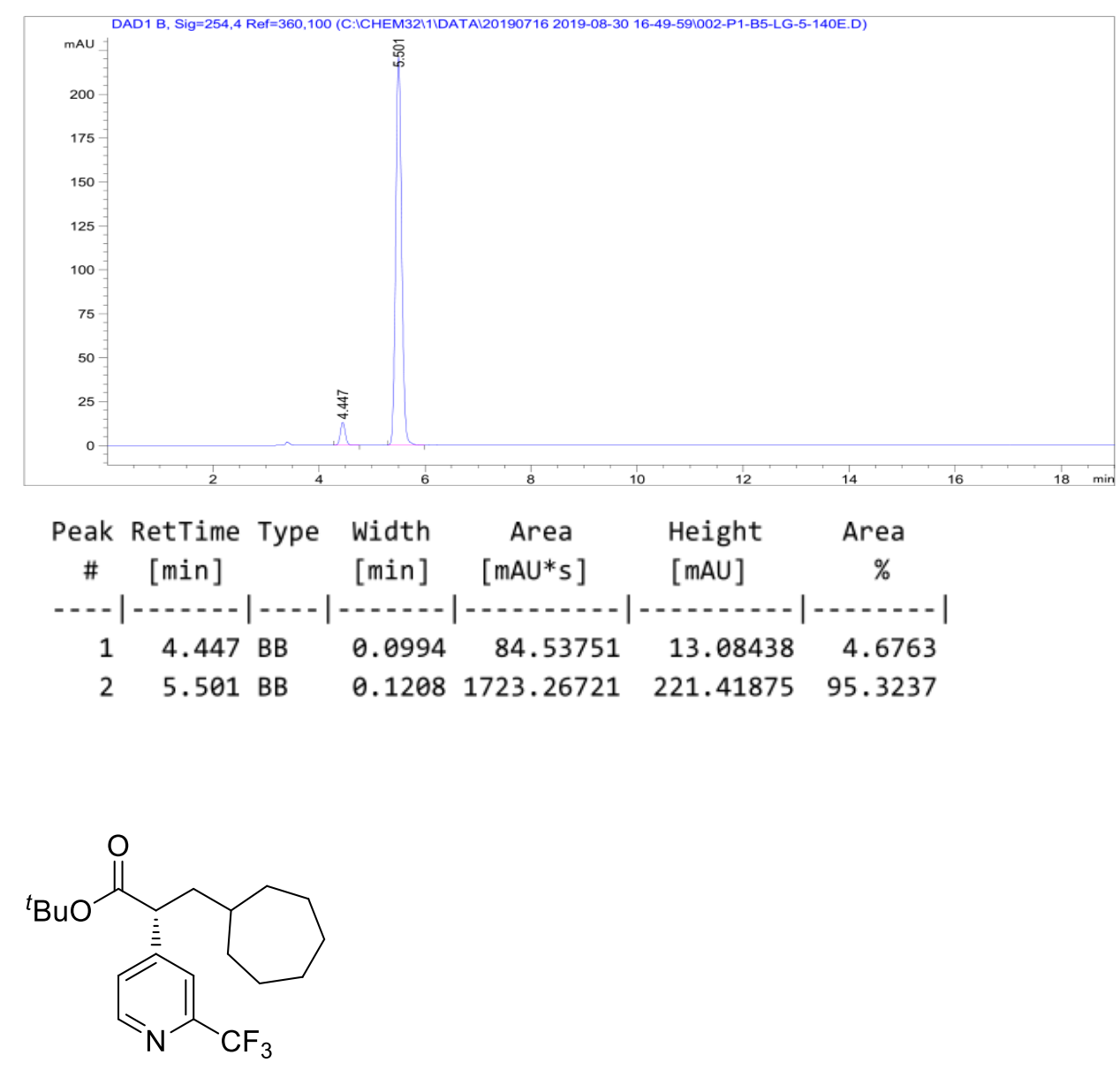

(R)-tert-Butyl 3-cycloheptyl-2-(2-(trifluoromethyl)pyridin-4-yl)propanoate (38)

The product was prepared according to the general procedure with (4S,4'S)-4,4'-di((S)sec-butyl)-4,4',5,5'-tetrahydro-2,2'-bioxazole [(S,S)-L1] $(3.3 \mathrm{mg}, 0.013 \mathrm{mmol}, 13$ mol\%), $\mathrm{NiCl}_{2} \cdot \mathrm{Py}_{4}(4.5 \mathrm{mg}, 0.01 \mathrm{mmol}, 10 \mathrm{~mol} \%), \operatorname{Ir}\left[\mathrm{dF}\left(\mathrm{CF}_{3}\right) \mathrm{ppy}\right]_{2}(\mathrm{bpy}) \mathrm{PF}_{6}(3.2 \mathrm{mg}$, $0.003 \mathrm{mmol}, 3 \mathrm{~mol} \%$ ), 4-bromo-2-(trifluoromethyl)pyridine (13.7 uL, $0.1 \mathrm{mmol}, 1.0$ equiv.), potassium cycloheptyltrifluoroborate (51.0 mg, $0.25 \mathrm{mmol}, 2.5$ equiv.) and tert-butyl acrylate $(29.0 \mathrm{uL}, 0.2 \mathrm{mmol}, 2.0$ equiv.) in acetone/ethyl acetate $(1.3 / 0.7, \mathrm{v} / \mathrm{v}$, $2 \mathrm{~mL}$ ) were used. After 24 hours, the product was isolated by flash chromatography (PE: ethyl acetate $=20: 1)$ as a colorless oil (23.8 mg, 64\% yield, 90\% ee).

${ }^{1} \mathbf{H}$ NMR $\left(400 \mathrm{MHz}, \mathrm{CDCl}_{3}\right) \delta 8.65(\mathrm{~d}, J=5.0 \mathrm{~Hz}, 1 \mathrm{H}), 7.63(\mathrm{~s}, 1 \mathrm{H}), 7.44(\mathrm{~d}, J=4.9$ $\mathrm{Hz}, 1 \mathrm{H}), 3.65-3.61$ (m, 1H), 2.03-1.95 (m, 1H), 1.74-1.66 (m, 3H), 1.60-1.57 (m, 2H), 1.53-1.46 (m, 5H), $1.41(\mathrm{~s}, 9 \mathrm{H}), 1.37-1.33(\mathrm{~m}, 2 \mathrm{H}), 1.23-1.15(\mathrm{~m}, 2 \mathrm{H})$.

${ }^{19}$ F NMR $\left(375 \mathrm{MHz}, \mathrm{CDCl}_{3}\right) \delta-67.94(\mathrm{~s})$. 
${ }^{13}$ C NMR $\left(100 \mathrm{MHz}, \mathrm{CDCl}_{3}\right) \delta 171.47,151.00,150.12,148.44(\mathrm{q}, J=34.5 \mathrm{~Hz}), 125.88$, $121.55(\mathrm{q}, J=274.5 \mathrm{~Hz}), 120.09$ (q, $J=2.7 \mathrm{~Hz}), 81.83,50.17,41.52,36.86,34.32$, 34.14, 28.46, 28.42, 27.90, 26.16, 26.13.

HRMS (ESI): $\mathrm{C}_{20} \mathrm{H}_{29} \mathrm{~F}_{3} \mathrm{NO}_{2}^{+}(\mathrm{M}+\mathrm{H}): 372.2150$, found: 372.2138 .

$[\alpha]_{\mathrm{D}}^{25}=-4.1116\left(\mathrm{c}=0.3867, \mathrm{CHCl}_{3}\right)$

Chiral HPLC: CHIRALPAK IC, $25{ }^{\circ} \mathrm{C},{ }^{i} \mathrm{PrOH}-h$ exanes 5/95, $0.5 \mathrm{~mL} / \mathrm{min}, 254 \mathrm{~nm}, \mathrm{t}_{\mathrm{R}}$ (major) $=11.9 \mathrm{~min}, \mathrm{t}_{\mathrm{R}}$ (minor) $=9.2 \mathrm{~min}$.

\section{8 racemic}

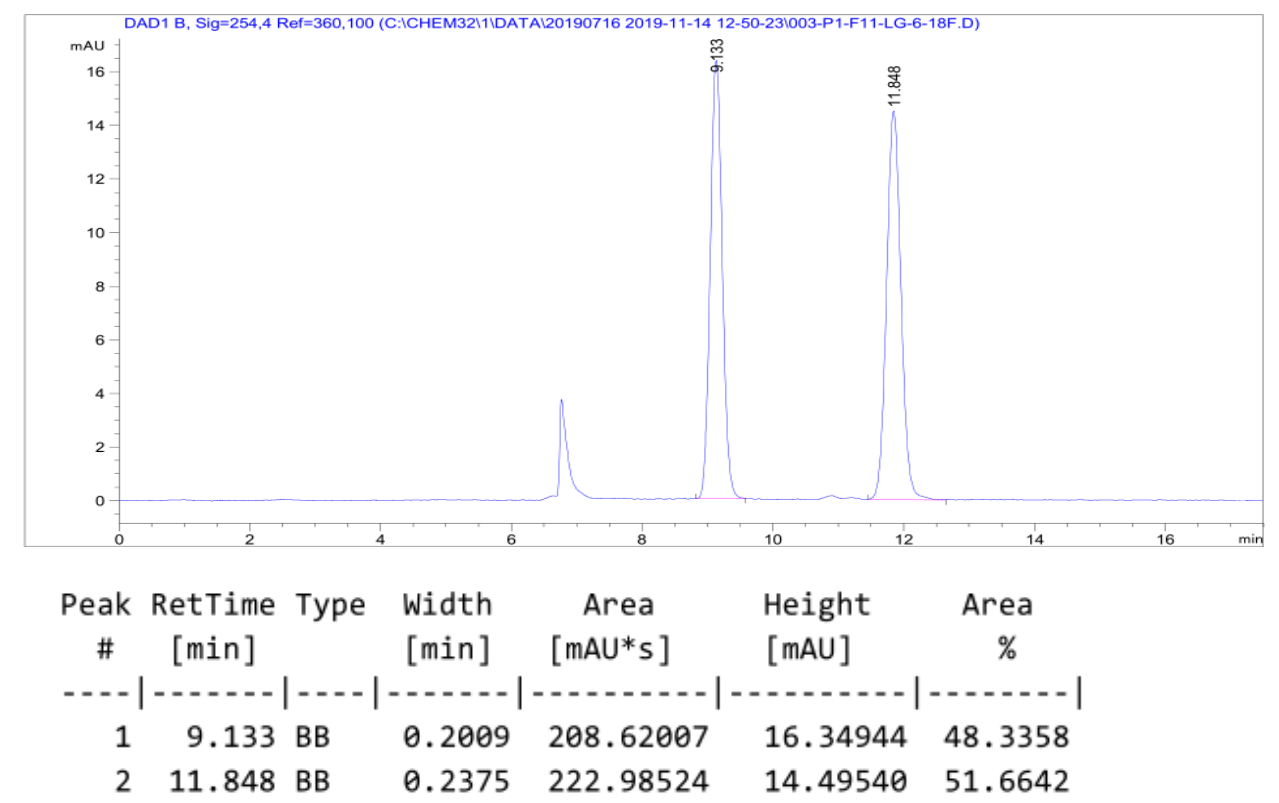

38 enantioenriched, $90 \%$ ee

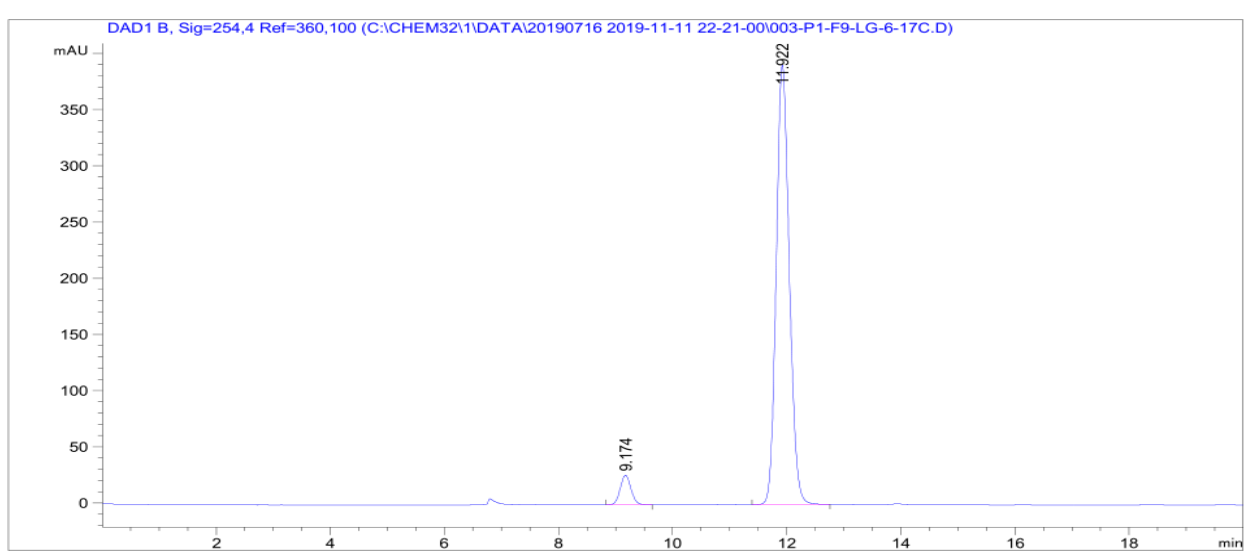




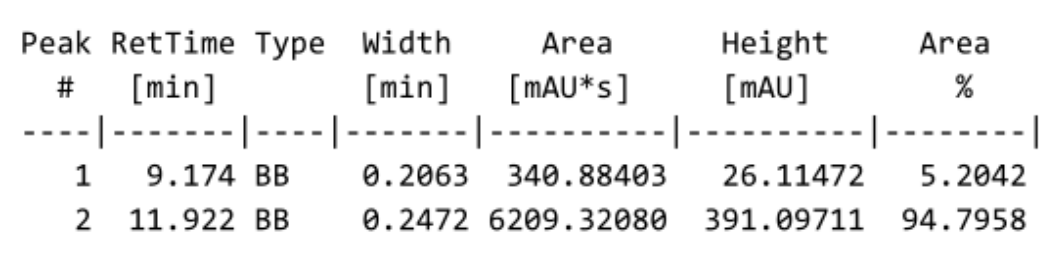<smiles>CCCCOC(=O)[C@H](CC1CCCCCCC1)c1ccnc(C(F)(F)F)c1</smiles>

\section{(R)-tert-Butyl 3-cyclooctyl-2-(2-(trifluoromethyl)pyridin-4-yl)propanoate (39)}

The product was prepared according to the general procedure with (4S,4'S)-4,4'-di((S)sec-butyl)-4,4',5,5'-tetrahydro-2,2'-bioxazole [(S,S)-L1] $(3.3 \mathrm{mg}, 0.013 \mathrm{mmol}, 13$ mol\%), $\mathrm{NiCl}_{2} \bullet \mathrm{Py}_{4}(4.5 \mathrm{mg}, 0.01 \mathrm{mmol}, 10 \mathrm{~mol} \%), \operatorname{Ir}\left[\mathrm{dF}\left(\mathrm{CF}_{3}\right) \text { ppy }\right]_{2}(\mathrm{bpy}) \mathrm{PF}_{6}(3.2 \mathrm{mg}$, $0.003 \mathrm{mmol}, 3 \mathrm{~mol} \%$ ), 4-bromo-2-(trifluoromethyl)pyridine (13.7 uL, $0.1 \mathrm{mmol}, 1.0$ equiv.), potassium cyclooctyltrifluoroborate $(54.5 \mathrm{mg}, 0.25 \mathrm{mmol}, 2.5$ equiv.) and tertbutyl acrylate (29.0 uL, $0.2 \mathrm{mmol}, 2.0$ equiv.) in acetone/ethyl acetate (1.3/0.7, v/v, 2 $\mathrm{mL}$ ) were used. After 24 hours, the product was isolated by flash chromatography (PE: ethyl acetate $=20: 1)$ as a white solid $(21.3 \mathrm{mg}, 55 \%$ yield, $90 \%$ ee $)$.

${ }^{1} \mathbf{H}$ NMR $\left(400 \mathrm{MHz}, \mathrm{CDCl}_{3}\right) \delta 8.66(\mathrm{~d}, J=5.0 \mathrm{~Hz}, 1 \mathrm{H}), 7.64(\mathrm{~s}, 1 \mathrm{H}), 7.45(\mathrm{~d}, J=5.0$ $\mathrm{Hz}, 1 \mathrm{H}), 3.64(\mathrm{dd}, J=8.8,6.7 \mathrm{~Hz}, 1 \mathrm{H}), 2.04-1.94(\mathrm{~m}, 1 \mathrm{H}), 1.62(\mathrm{t}, J=9.8 \mathrm{~Hz}, 4 \mathrm{H})$, $1.59-1.49(\mathrm{~m}, 4 \mathrm{H}), 1.45(\mathrm{~d}, J=18.0 \mathrm{~Hz}, 4 \mathrm{H}), 1.41(\mathrm{~s}, 9 \mathrm{H}), 1.30(\mathrm{dd}, J=26.2,15.1$ $\mathrm{Hz}, 4 \mathrm{H})$.

${ }^{19}$ F NMR $\left(375 \mathrm{MHz}, \mathrm{CDCl}_{3}\right) \delta-67.94$ (s)

${ }^{13}$ C NMR (100 MHz, $\left.\mathrm{CDCl}_{3}\right) \delta 171.43,151.00,150.09,148.40$ (q, $\left.J=34.3 \mathrm{~Hz}\right), 125.81$, $121.51(\mathrm{q}, J=274.2 \mathrm{~Hz}), 120.04(\mathrm{q}, J=2.7 \mathrm{~Hz}), 81.79,50.13,41.59,34.80,32.27$, $31.76,27.88,27.13,27.02,26.22,25.32,25.10$.

HRMS (ESI): $\mathrm{C}_{21} \mathrm{H}_{31} \mathrm{~F}_{3} \mathrm{NO}_{2}{ }^{+}(\mathrm{M}+\mathrm{H})$ : 386.2307 , found: 386.2293 .

$[\alpha]_{\mathrm{D}}^{25}=-2.8759\left(\mathrm{c}=0.4733, \mathrm{CHCl}_{3}\right)$

Chiral HPLC: CHIRALPAK IC, $25{ }^{\circ} \mathrm{C},{ }^{i} \mathrm{PrOH}-$ hexanes 5/95, $0.5 \mathrm{~mL} / \mathrm{min}, 254 \mathrm{~nm}, \mathrm{t}_{\mathrm{R}}$ (major) $=11.8 \mathrm{~min}, \mathrm{t}_{\mathrm{R}}($ minor $)=9.2 \mathrm{~min}$. 


\section{9 racemic}

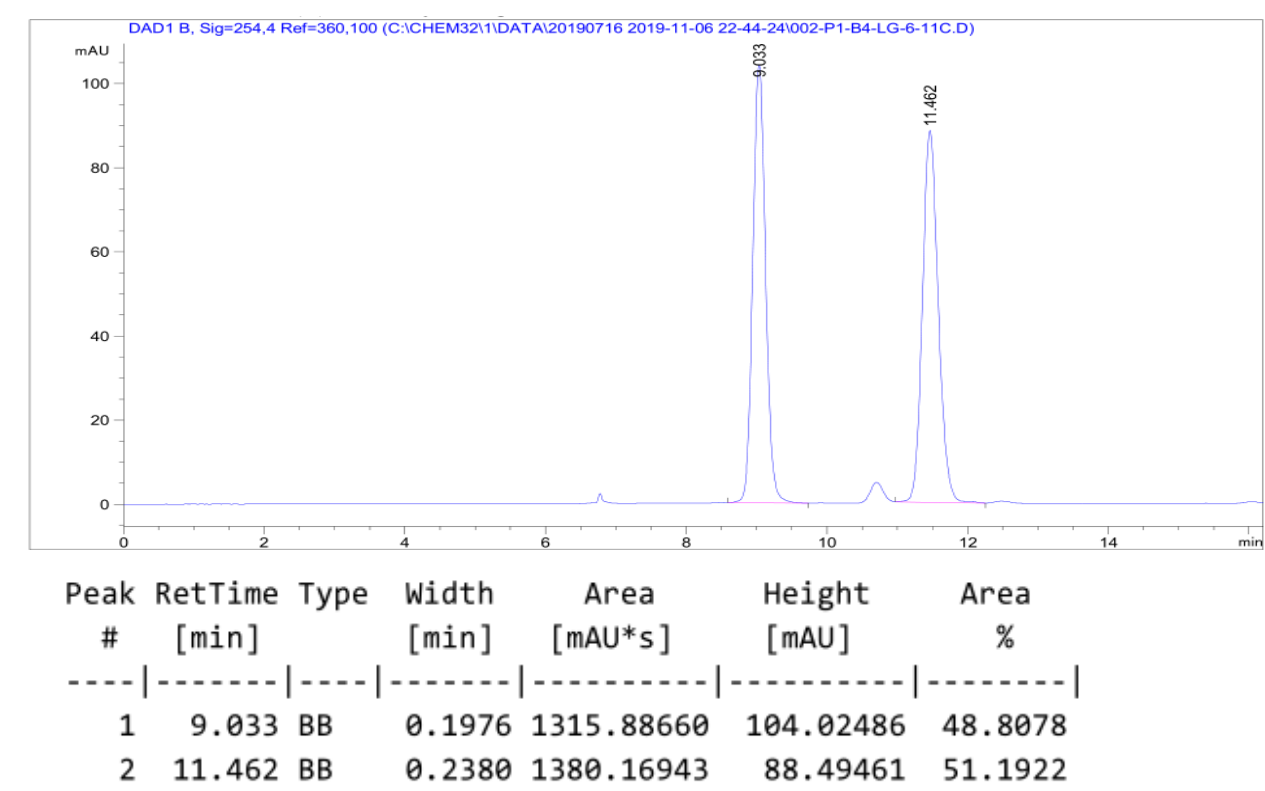

39 enantioenriched, $90 \%$ ee

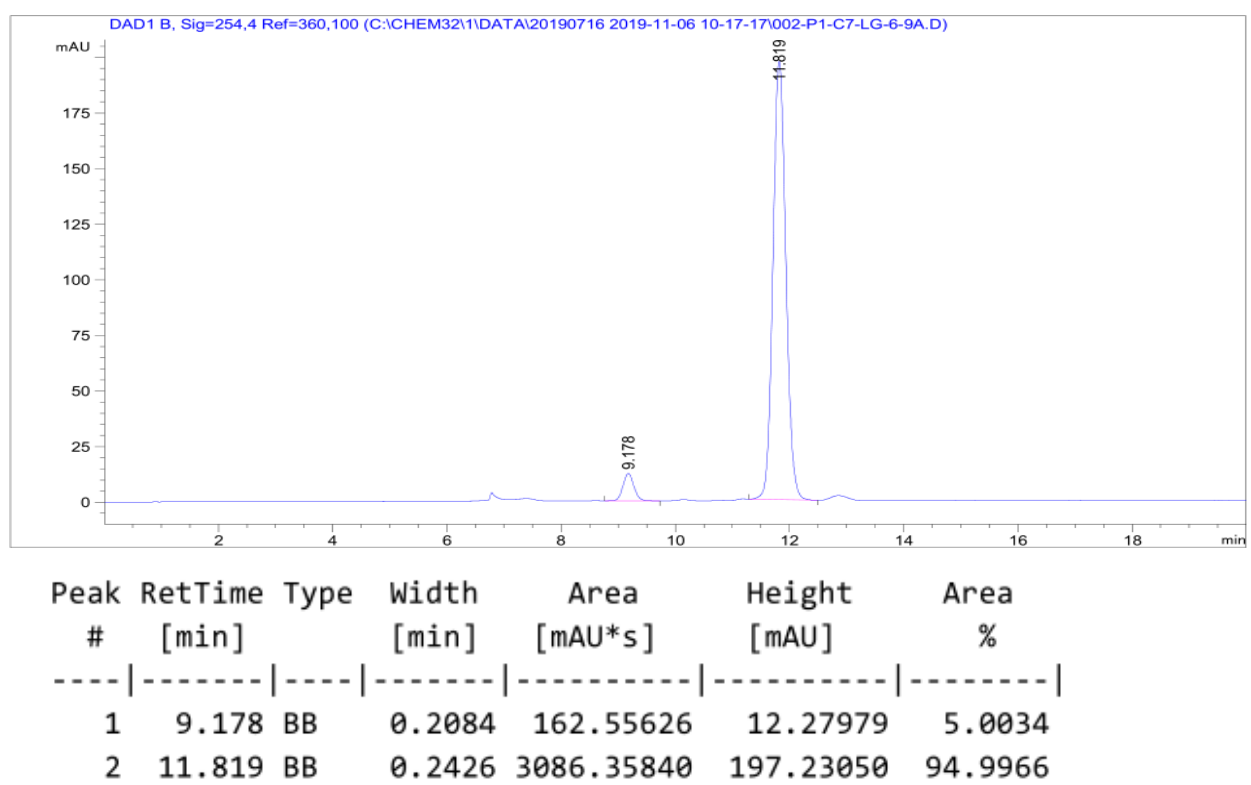<smiles>CCC(C)OC(=O)[C@H](CC1CC2CCC1C2)c1ccnc(C(F)(F)F)c1</smiles>

(R)-tert-Butyl-3-((1R,2S,4S)-bicyclo[2.2.1]heptan-2-yl)-2-(2-

(trifluoromethyl)pyridine-4-yl) propanoate (40) 
The product was prepared according to the general procedure with $(4 \mathrm{~S}, 4$ 'S)-4,4'-di((S)sec-butyl)-4,4',5,5'-tetrahydro-2,2'-bioxazole [(S,S)-L1] $(3.3 \mathrm{mg}, 0.013 \mathrm{mmol}, 13$ mol\%), $\mathrm{NiCl}_{2} \cdot \mathrm{Py}_{4}(4.5 \mathrm{mg}, 0.01 \mathrm{mmol}, 10 \mathrm{~mol} \%), \operatorname{Ir}\left[\mathrm{dF}\left(\mathrm{CF}_{3}\right) \mathrm{ppy}\right]_{2}(\mathrm{bpy}) \mathrm{PF}_{6}(3.2 \mathrm{mg}$, $0.003 \mathrm{mmol}, 3 \mathrm{~mol} \%$ ), 4-bromo-2-(trifluoromethyl)pyridine (13.7 uL, $0.1 \mathrm{mmol}, 1.0$ equiv.), potassium bicyclo[2.2.1] heptan-2-yltrifluoroborate (50.5 mg, $0.25 \mathrm{mmol}, 2.5$ equiv.) and tert-butyl acrylate $(29.0 \mathrm{uL}, 0.2 \mathrm{mmol}, 2.0$ equiv.) in acetone/ethyl acetate $(1.3 / 0.7, \mathrm{v} / \mathrm{v}, 2 \mathrm{~mL})$ were used. After 24 hours, the product was isolated by flash chromatography $(\mathrm{PE}$ : ethyl acetate $=20: 1)$ as a colorless oil $(24.8 \mathrm{mg}, 67 \%$ yield, $\mathrm{dr}=$ $1: 1,95 \%$ ee).

${ }^{1} \mathbf{H}$ NMR $\left(400 \mathrm{MHz}, \mathrm{CDCl}_{3}\right) \delta 8.65(\mathrm{dd}, J=4.4,2.8 \mathrm{~Hz}, 1 \mathrm{H}), 7.62(\mathrm{~s}, 1 \mathrm{H}), 7.43(\mathrm{~d}, J=$ $4.8 \mathrm{~Hz}, 1 \mathrm{H}), 3.54(\mathrm{t}, J=7.5 \mathrm{~Hz}, 1 \mathrm{H}), 2.20(\mathrm{br}, 1 \mathrm{H}), 2.10-1.66(\mathrm{~m}, 3 \mathrm{H}), 1.56-1.40(\mathrm{~m}$, $3 \mathrm{H}), 1.40$ (br, 9H), 1.29-1.24 (m, 2H), 1.11-1.00 (m, 4H).

${ }^{19}$ F NMR $\left(375 \mathrm{MHz}, \mathrm{CDCl}_{3}\right) \delta-67.95(\mathrm{~s})$.

${ }^{13}$ C NMR (100 MHz, $\left.\mathrm{CDCl}_{3}\right) \delta 171.41$ (171.35), 150.95 (150.72), 150.06, 148.37 (q, $J$ $=33.8 \mathrm{~Hz}), 126.00$ (125.82), 121.42 (q, $J=274.2 \mathrm{~Hz}), 120.14$ (q, $J=2.7 \mathrm{~Hz})(120.02$ q, $J=2.7 \mathrm{~Hz}$ ), 81.80 (81.79), 50.50 (50.41), 40.89 (40.85), 40.37 (40.08), 39.83 (39.5), 38.00 (37.62), 36.51, 35.33 (35.25), 29.87, 28.49, 27.85 .

HRMS (ESI): $\mathrm{C}_{20} \mathrm{H}_{27} \mathrm{~F}_{3} \mathrm{NO}_{2}^{+}(\mathrm{M}+\mathrm{H}): 370.1994$, found: 370.1988 .

$[\alpha]_{\mathrm{D}}^{25}=-4.6784\left(\mathrm{c}=0.6733, \mathrm{CHCl}_{3}\right)$

Chiral HPLC: CHIRALPAK IA, $25{ }^{\circ} \mathrm{C},{ }^{i} \mathrm{PrOH}-$ hexanes 1/500, $0.5 \mathrm{~mL} / \mathrm{min}, 250 \mathrm{~nm}$, $t_{R}($ major $)=14.9 \mathrm{~min}, t_{R}($ minor $)=13.1 \mathrm{~min}$

\section{0 racemic}

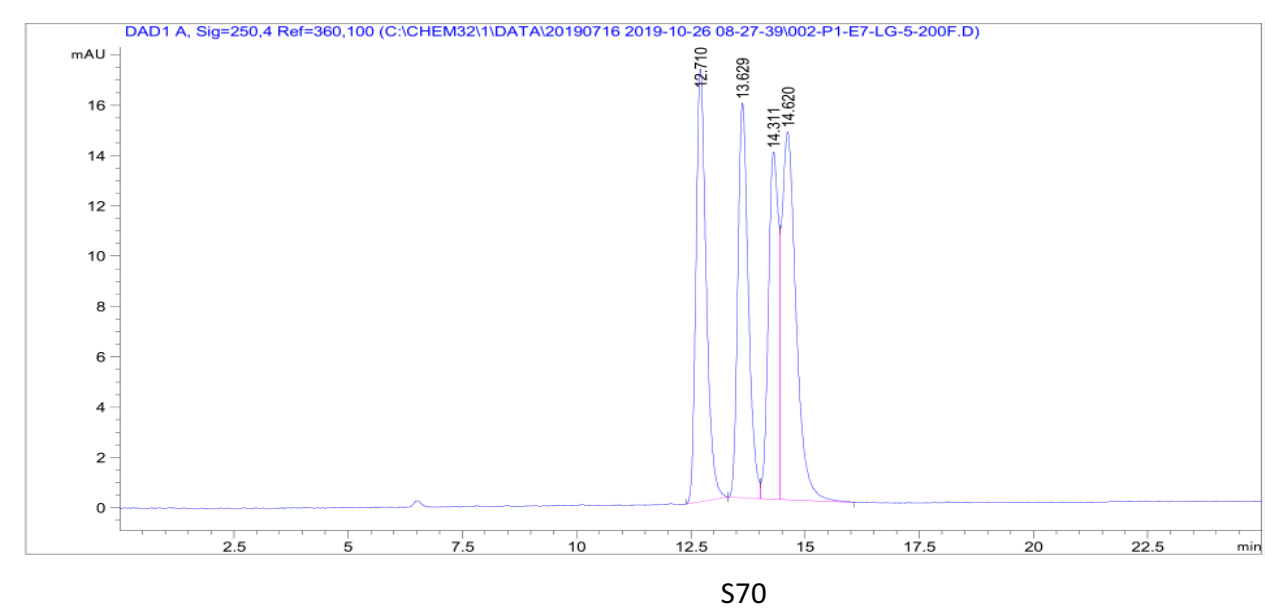




\begin{tabular}{|c|c|c|c|c|c|c|}
\hline $\begin{array}{c}\text { Peak } \\
\#\end{array}$ & $\begin{array}{c}\text { RetTime } \\
\text { [min] }\end{array}$ & Type & $\begin{array}{l}\text { Width } \\
\text { [min] }\end{array}$ & $\begin{array}{c}\text { Area } \\
{\left[\mathrm{mAU}^{*} \mathrm{~s}\right]}\end{array}$ & $\begin{array}{l}\text { Height } \\
{[\mathrm{mAU}]}\end{array}$ & $\begin{array}{c}\text { Area } \\
\%\end{array}$ \\
\hline & & & & $-1-1$ & 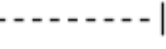 & 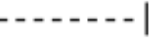 \\
\hline 1 & 12.710 & BB & 0.2363 & 265.94458 & 17.20543 & 25.3566 \\
\hline 2 & 13.629 & BV & 0.2448 & 251.46935 & 15.70508 & 23.9765 \\
\hline 3 & 14.311 & VV & 0.2350 & 214.04848 & 13.79881 & 20.4086 \\
\hline 4 & 14.620 & VB & 0.3171 & 317.35410 & 14.62838 & 30.2583 \\
\hline
\end{tabular}

40 enantioenriched, $95 \%$ ee
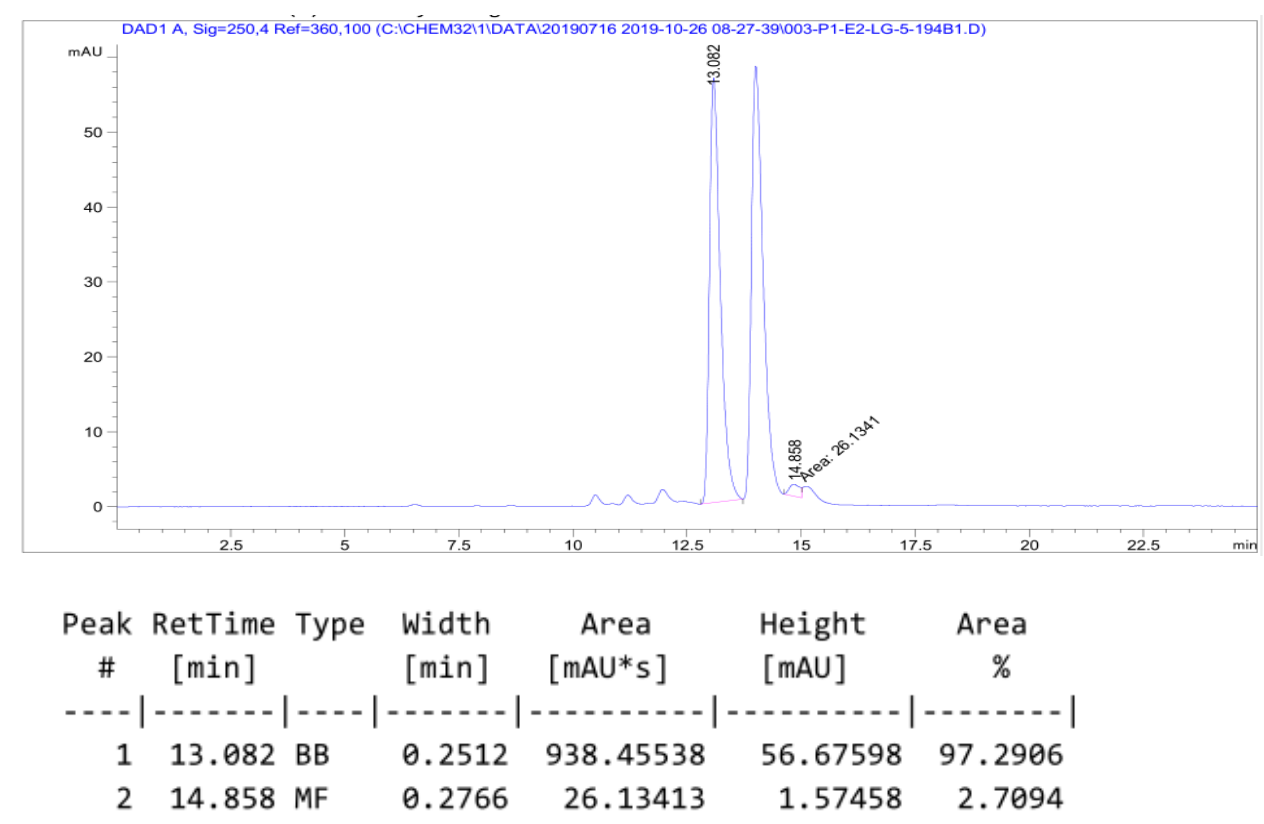<smiles>CCCCOC(=O)[C@H](CC(C)C)c1ccnc(C(F)(F)F)c1</smiles>

\section{(R)-tert-Butyl 4-methyl-2-(2-(trifluoromethyl)pyridin-4-yl)pentanoate (41)}

The product was prepared according to the general procedure with (4S,4'S)-4,4'-di((S)sec-butyl)-4,4',5,5'-tetrahydro-2,2'-bioxazole $[(S, S)$-L1 $]$ (3.3 mg, $0.013 \mathrm{mmol}, 13$ mol\%), $\mathrm{NiCl}_{2} \cdot \mathrm{Py}_{4}(4.5 \mathrm{mg}, 0.01 \mathrm{mmol}, 10 \mathrm{~mol} \%), \operatorname{Ir}\left[\mathrm{dF}\left(\mathrm{CF}_{3}\right) \mathrm{ppy}\right]_{2}(\mathrm{bpy}) \mathrm{PF}_{6}(3.2 \mathrm{mg}$, $0.003 \mathrm{mmol}, 3 \mathrm{~mol} \%$ ), 4-bromo-2-(trifluoromethyl)pyridine (13.7 uL, $0.1 \mathrm{mmol}, 1.0$ equiv.), potassium isopropyltrifluoroborate $(37.5 \mathrm{mg}, 0.25 \mathrm{mmol}, 2.5 \mathrm{equiv}$.$) and tert-$ butyl acrylate $(29.0 \mathrm{uL}, 0.2 \mathrm{mmol}, 2.0$ equiv.) in acetone/ethyl acetate $(1.3 / 0.7, \mathrm{v} / \mathrm{v}, 2$ $\mathrm{mL}$ ) were used. After 24 hours, the product was isolated by flash chromatography (PE: ethyl acetate $=20: 1)$ as a colorless oil $(17.8 \mathrm{mg}, 56 \%$ yield, $90 \%$ ee $)$. 
${ }^{1}$ H NMR $\left(400 \mathrm{MHz}, \mathrm{CDCl}_{3}\right) \delta 8.65(\mathrm{~d}, J=5.0 \mathrm{~Hz}, 1 \mathrm{H}), 7.64(\mathrm{~s}, 1 \mathrm{H}), 7.45-7.44(\mathrm{~m}$, 1H), $3.62(\mathrm{dd}, J=8.4,7.0 \mathrm{~Hz}, 1 \mathrm{H}), 2.00-1.94(\mathrm{~m}, 1 \mathrm{H}), 1.58-1.55(\mathrm{~m}, 1 \mathrm{H}), 1.52-1.45$ (mii, 1H), 1.41 (s, 9H), 0.94 (d, $J=3.2 \mathrm{~Hz}, 3 \mathrm{H}), 0.92$ (d, $J=3.1 \mathrm{~Hz}, 3 \mathrm{H})$.

${ }^{19}$ F NMR $\left(375 \mathrm{MHz}, \mathrm{CDCl}_{3}\right) \delta-67.97$ (s)

${ }^{13}$ C NMR (100 MHz, $\left.\mathrm{CDCl}_{3}\right) \delta 171.34,150.93,150.11,148.49$ (q, $\left.J=34.4 \mathrm{~Hz}\right), 125.81$, 121.54 (q, $J=274.4 \mathrm{~Hz}), 120.03(\mathrm{q}, J=2.7 \mathrm{~Hz}), 81.85,50.35,42.27,27.87,26.05$, 22.35, 22.32 .

HRMS (ESI): $\mathrm{C}_{16} \mathrm{H}_{23} \mathrm{~F}_{3} \mathrm{NO}_{2}^{+}(\mathrm{M}+\mathrm{H}): 318.1681$, found: 318.1671 .

$[\alpha]_{\mathrm{D}}^{25}=-3.5833\left(\mathrm{c}=0.2400, \mathrm{CHCl}_{3}\right)$

Chiral HPLC: CHIRALPAK IC, $25{ }^{\circ} \mathrm{C},{ }^{i} \mathrm{PrOH}-h$ exanes 5/95, $0.5 \mathrm{~mL} / \mathrm{min}, 254 \mathrm{~nm}, \mathrm{t}_{\mathrm{R}}$ $($ major $)=10.6 \mathrm{~min}, \mathrm{t}_{\mathrm{R}}(\operatorname{minor})=8.6 \mathrm{~min}$.

\section{1 racemic}

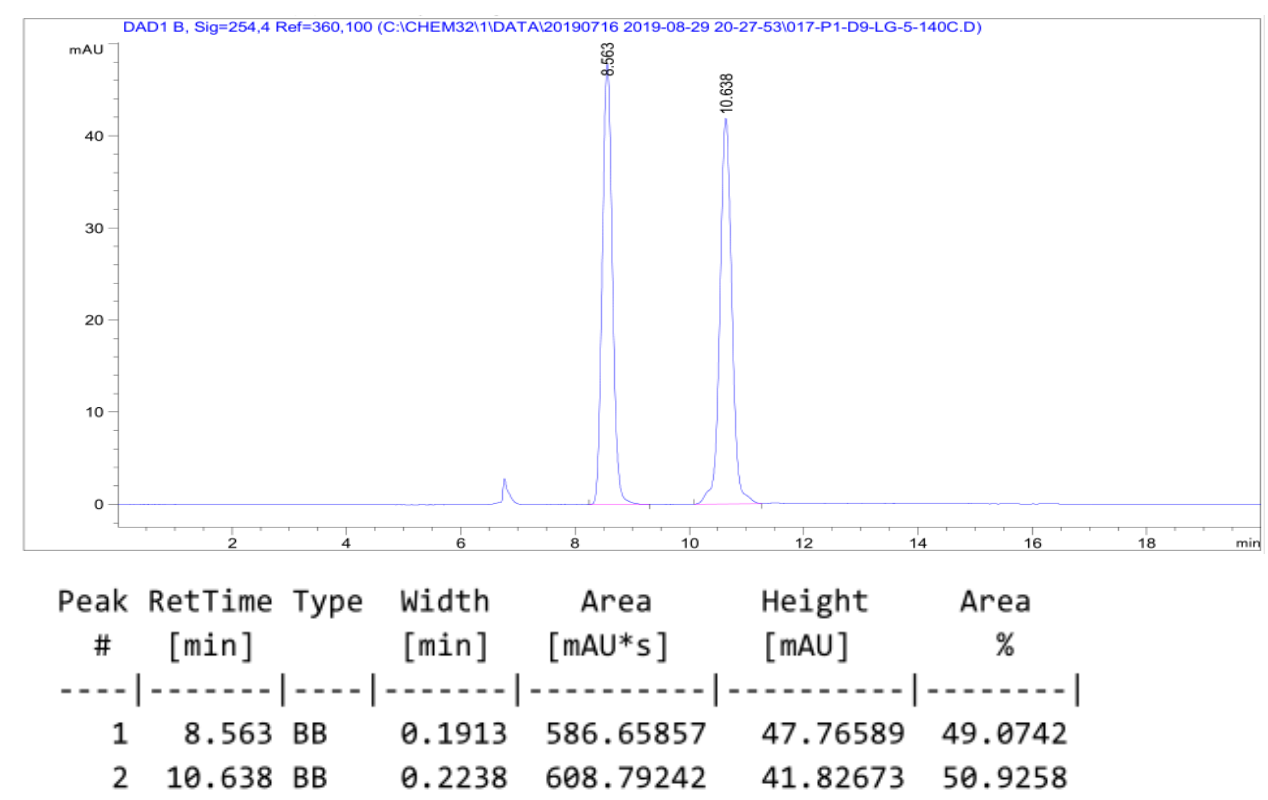




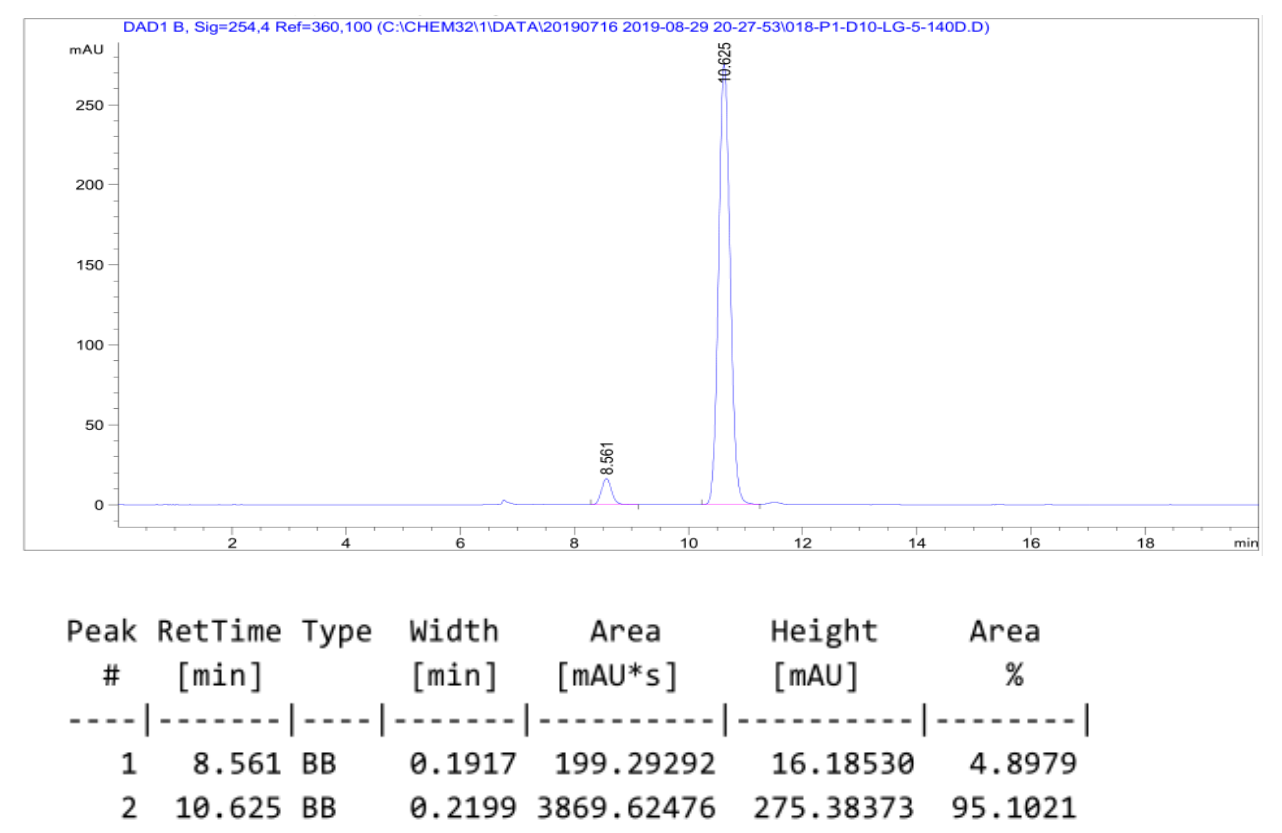<smiles>COC(=O)C(CBr)c1ccnc(C(F)(F)F)c1</smiles>

(R)-Methyl 4,4-dimethyl-2-(2-(trifluoromethyl)pyridin-4-yl)pentanoate (42)

The product was prepared according to the general procedure with (4S,4'S)-4,4'-di((S)sec-butyl)-4,4',5,5'-tetrahydro-2,2'-bioxazole $[(S, S)$-L1 $]$ (3.3 mg, $0.013 \mathrm{mmol}, 13$ $\mathrm{mol} \%$ ), $\mathrm{NiCl}_{2} \cdot \mathrm{Py}_{4}(4.5 \mathrm{mg}, 0.01 \mathrm{mmol}, 10 \mathrm{~mol} \%), \operatorname{Ir}\left[\mathrm{dF}\left(\mathrm{CF}_{3}\right) \mathrm{ppy}\right]_{2}(\mathrm{bpy}) \mathrm{PF}_{6}(3.2 \mathrm{mg}$, $0.003 \mathrm{mmol}, 3 \mathrm{~mol} \%$ ), 4-bromo-2-(trifluoromethyl)pyridine (13.7 uL, $0.1 \mathrm{mmol}, 1.0$ equiv.), ${ }^{t} \mathrm{BuBF}_{3} \mathrm{~K}$ (41.0 mg, $0.25 \mathrm{mmol}, 2.5$ equiv.) and methyl acrylate (18.0 uL, 0.2 mmol, 2.0 equiv.) in acetone/ethyl acetate (1.3/0.7, v/v, $2 \mathrm{~mL})$ were used. After 24 hours, the product was isolated by flash chromatography $(P E$ : ethyl acetate $=20: 1)$ as a colorless oil (25.7 mg, 89\% yield, $90 \%$ ee).

${ }^{1}$ H NMR $\left(400 \mathrm{MHz}, \mathrm{CDCl}_{3}\right) \delta 8.65(\mathrm{~d}, J=5.0 \mathrm{~Hz}, 1 \mathrm{H}), 7.65(\mathrm{br}, 1 \mathrm{H}), 7.47$ (dd, $J=5.0$, $1.5 \mathrm{~Hz}, 1 \mathrm{H}), 3.74(\mathrm{dd}, J=9.2,3.8 \mathrm{~Hz}, 1 \mathrm{H}), 3.69(\mathrm{~s}, 3 \mathrm{H}), 2.32(\mathrm{dd}, J=14.0,9.2 \mathrm{~Hz}, 1 \mathrm{H})$, $1.56(\mathrm{dd}, J=14.0,3.9 \mathrm{~Hz}, 1 \mathrm{H}), 0.92(\mathrm{~s}, 9 \mathrm{H})$.

${ }^{19}$ F NMR (375 MHz, $\left.\mathrm{CDCl}_{3}\right) \delta-67.96(\mathrm{~s})$. 
${ }^{13}$ C NMR $\left(100 \mathrm{MHz}, \mathrm{CDCl}_{3}\right) \delta 173.32,151.55,150.27,148.66(\mathrm{q}, J=34.60 \mathrm{~Hz})$, 125.73, $121.45(\mathrm{q}, J=274.6 \mathrm{~Hz}), 119.89$ (q, $J=2.7 \mathrm{~Hz}), 52.61,47.76,47.15,31.17$, 29.29 .

HRMS (ESI): $\mathrm{C}_{14} \mathrm{H}_{19} \mathrm{~F}_{3} \mathrm{NO}_{2}^{+}(\mathrm{M}+\mathrm{H}): 290.1368$, found: 290.1357.

$[\alpha]_{\mathrm{D}}^{25}=-24.2409\left(\mathrm{c}=0.2867, \mathrm{CHCl}_{3}\right)$

Chiral HPLC: CHIRALPAK IC, $25{ }^{\circ} \mathrm{C},{ }^{i} \mathrm{PrOH}-$ hexanes 5/95, $0.5 \mathrm{~mL} / \mathrm{min}, 254 \mathrm{~nm}, \mathrm{t}_{\mathrm{R}}$ $($ major $)=13.8 \mathrm{~min}, \mathrm{t}_{\mathrm{R}}($ minor $)=10.1 \mathrm{~min}$.

\section{2 racemic}

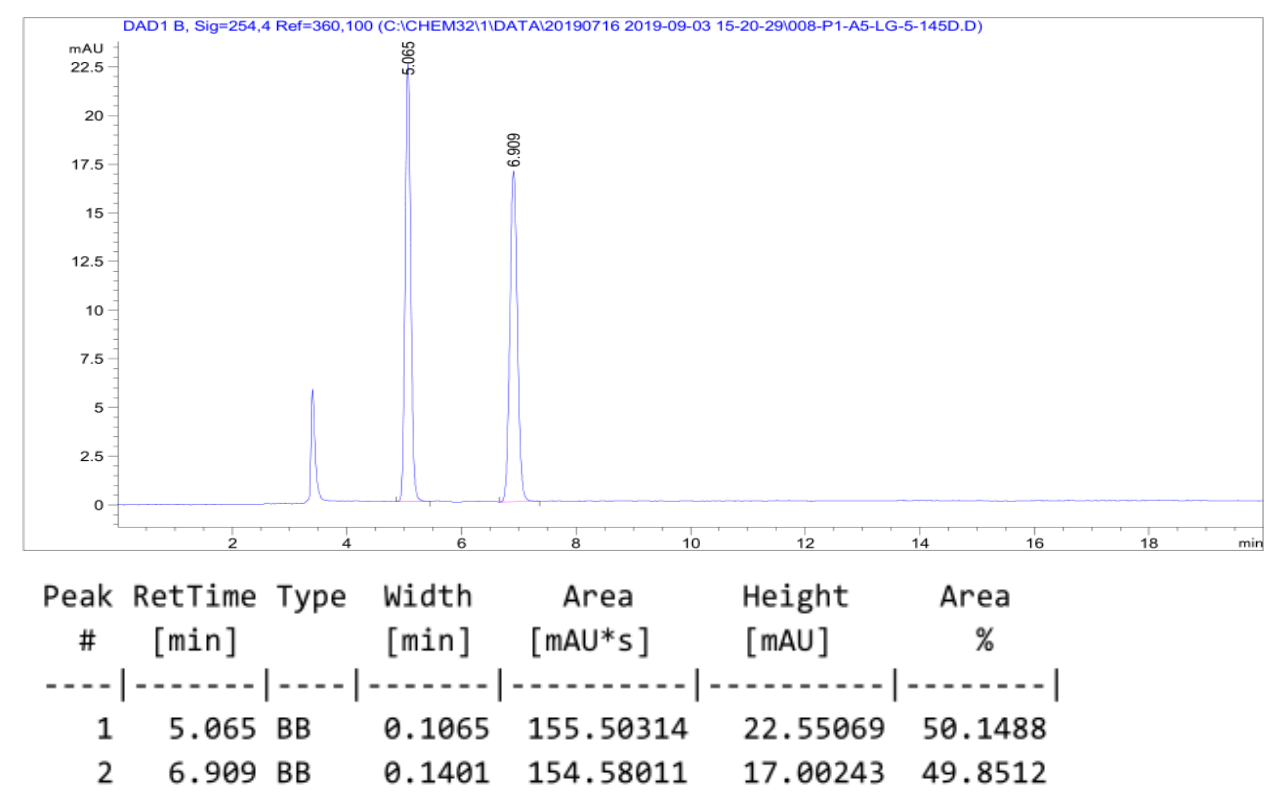

42 enantioenriched, $90 \%$ ee

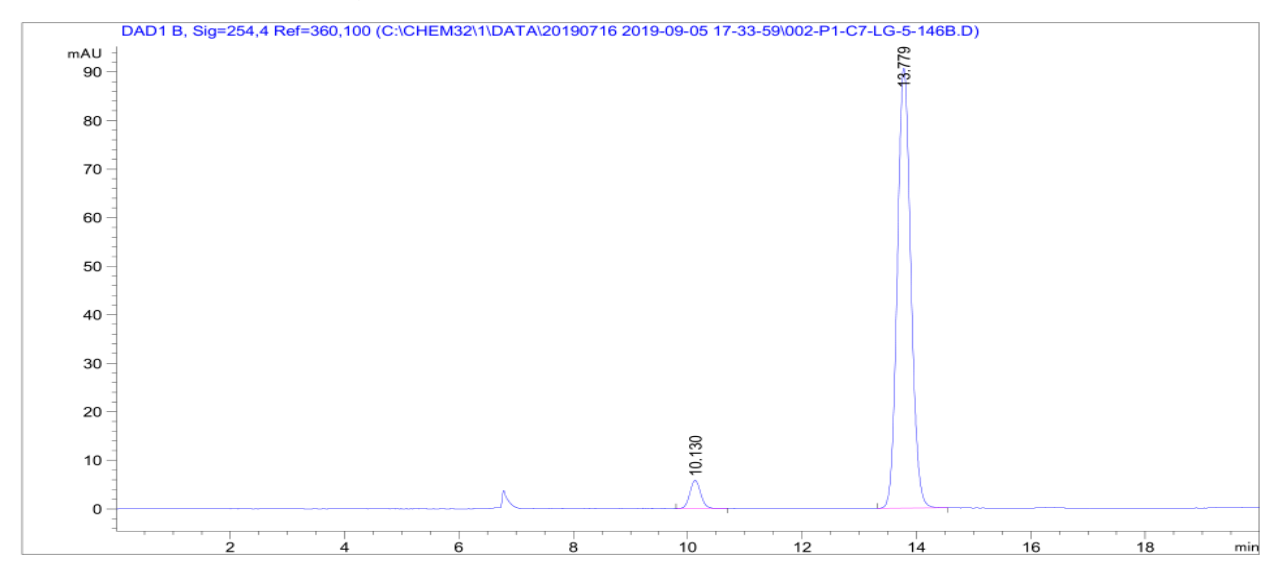




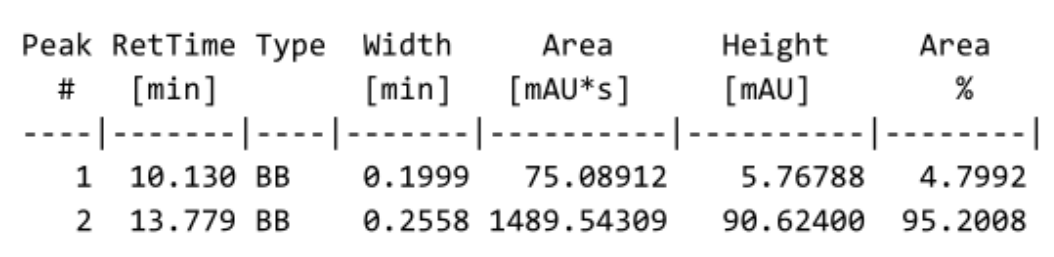<smiles>CC(C)(C)C[C@H](C(=O)Oc1ccccc1)c1ccnc(C(F)(F)F)c1</smiles>

\section{(R)-Phenyl 4,4-dimethyl-2-(2-(trifluoromethyl)pyridin-4-yl)pentanoate (43)}

The product was prepared according to the general procedure with (4S,4'S)-4,4'-di((S)sec-butyl)-4,4',5,5'-tetrahydro-2,2'-bioxazole [(S,S)-L1] $(3.3 \mathrm{mg}, 0.013 \mathrm{mmol}, 13$ mol\%), $\mathrm{NiCl}_{2} \cdot \mathrm{Py}_{4}(4.5 \mathrm{mg}, 0.01 \mathrm{mmol}, 10 \mathrm{~mol} \%), \operatorname{Ir}\left[\mathrm{dF}\left(\mathrm{CF}_{3}\right) \text { ppy }\right]_{2}(\mathrm{bpy}) \mathrm{PF}_{6}(3.2 \mathrm{mg}$, $0.003 \mathrm{mmol}, 3 \mathrm{~mol} \%$ ), 4-bromo-2-(trifluoromethyl)pyridine (13.7 uL, $0.1 \mathrm{mmol}, 1.0$ equiv.), ${ }^{t} \mathrm{BuBF}_{3} \mathrm{~K}$ (41.0 mg, $0.25 \mathrm{mmol}, 2.5$ equiv.) and phenyl acrylate (27.5 uL, 0.2 mmol, 2.0 equiv.) in acetone/ethyl acetate (1.3/0.7, v/v, $2 \mathrm{~mL})$ were used. After 24 hours, the product was isolated by flash chromatography (PE: ethyl acetate $=20: 1)$ as a colorless oil (31.9 mg, 91\% yield, 87\% ee).

${ }^{1} \mathbf{H}$ NMR $\left(400 \mathrm{MHz}, \mathrm{CDCl}_{3}\right) \delta 8.71(\mathrm{~d}, J=5.0 \mathrm{~Hz}, 1 \mathrm{H}), 7.75(\mathrm{~s}, 1 \mathrm{H}), 7.56(\mathrm{dd}, J=5.0$, $1.2 \mathrm{~Hz}, 1 \mathrm{H}), 7.36$ (t, $J=7.9 \mathrm{~Hz}, 2 \mathrm{H}), 7.23$ (t, $J=7.4 \mathrm{~Hz}, 1 \mathrm{H}), 6.98-6.96$ (m, 2H), 3.97 $(\mathrm{dd}, J=9.4,3.5 \mathrm{~Hz}, 1 \mathrm{H}), 2.46(\mathrm{dd}, J=14.1,9.4 \mathrm{~Hz}, 1 \mathrm{H}), 1.65(\mathrm{dd}, J=14.1,3.5 \mathrm{~Hz}$, 1H), $1.02(\mathrm{~s}, 9 \mathrm{H})$.

${ }^{19}$ F NMR $\left(375 \mathrm{MHz}, \mathrm{CDCl}_{3}\right) \delta-67.93$ (s)

${ }^{13}$ C NMR (100 MHz, $\left.\mathrm{CDCl}_{3}\right) \delta 171.40,151.15,150.46,150.37,148.88$ (q, $J=34.5$ $\mathrm{Hz}), 129.53,126.23,125.68,(\mathrm{~d}, J=17.9 \mathrm{~Hz}), 121.41$ (q, $J=274.3 \mathrm{~Hz}), 121.00,119.87$ (q, $J=2.6 \mathrm{~Hz}), 47.96,47.14,31.33,29.40$.

HRMS (ESI): $\mathrm{C}_{19} \mathrm{H}_{21} \mathrm{~F}_{3} \mathrm{NO}_{2}^{+}(\mathrm{M}+\mathrm{H}): 352.1524$, found: 352.1513 .

$[\alpha]_{\mathrm{D}}^{25}=-27.0735\left(\mathrm{c}=0.5400, \mathrm{CHCl}_{3}\right)$

Chiral HPLC: CHIRALPAK IC, $25{ }^{\circ} \mathrm{C},{ }^{i} \mathrm{PrOH}$-hexanes 5/95, $1.0 \mathrm{~mL} / \mathrm{min}, 254 \mathrm{~nm}, \mathrm{t}_{\mathrm{R}}$ $($ major $)=9.9 \mathrm{~min}, \mathrm{t}_{\mathrm{R}}(\operatorname{minor})=5.1 \mathrm{~min}$. 


\section{3 racemic}

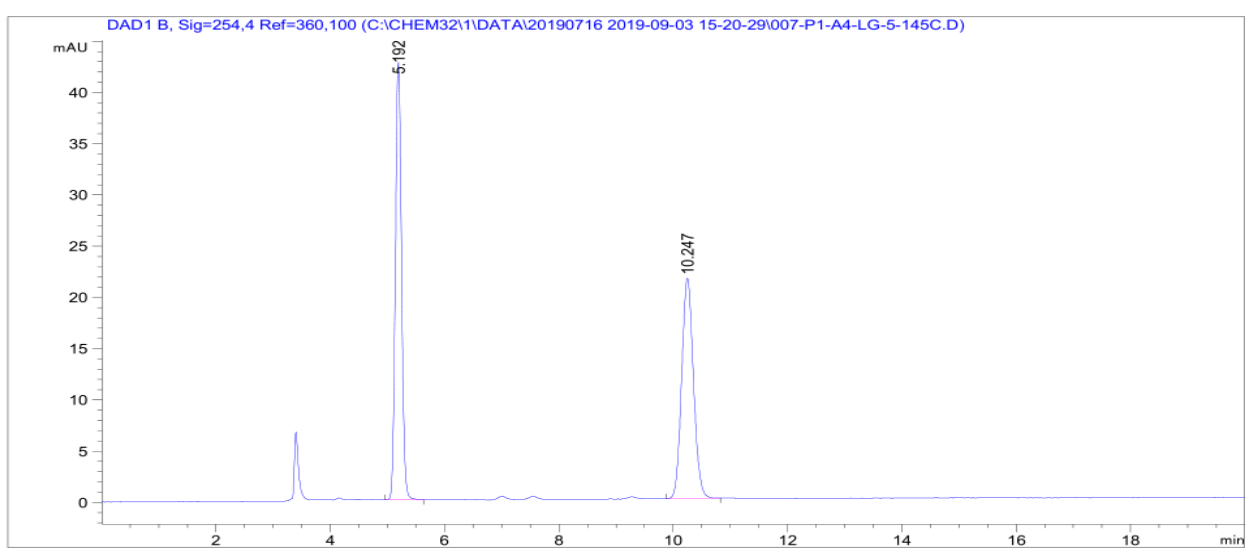

\begin{tabular}{|c|c|c|c|c|c|c|}
\hline $\begin{array}{l}\text { eak } \\
\text { e }\end{array}$ & $\begin{array}{c}\text { RetTime } \\
\text { [min] }\end{array}$ & Type & $\begin{array}{l}\text { Width } \\
\text { [min] }\end{array}$ & $\begin{array}{c}\text { Area } \\
{\left[\mathrm{mAU}^{*} \mathrm{~s}\right]}\end{array}$ & $\begin{array}{l}\text { Height } \\
\text { [mAU] }\end{array}$ & $\begin{array}{c}\text { Area } \\
\%\end{array}$ \\
\hline & & & & & & \\
\hline 1 & & & & 6877 & 795 & 50 \\
\hline 2 & 10 & BB & 236 & 309.07431 & 1.51475 & 49.734 \\
\hline
\end{tabular}

43 enantioenriched, $87 \%$ ee

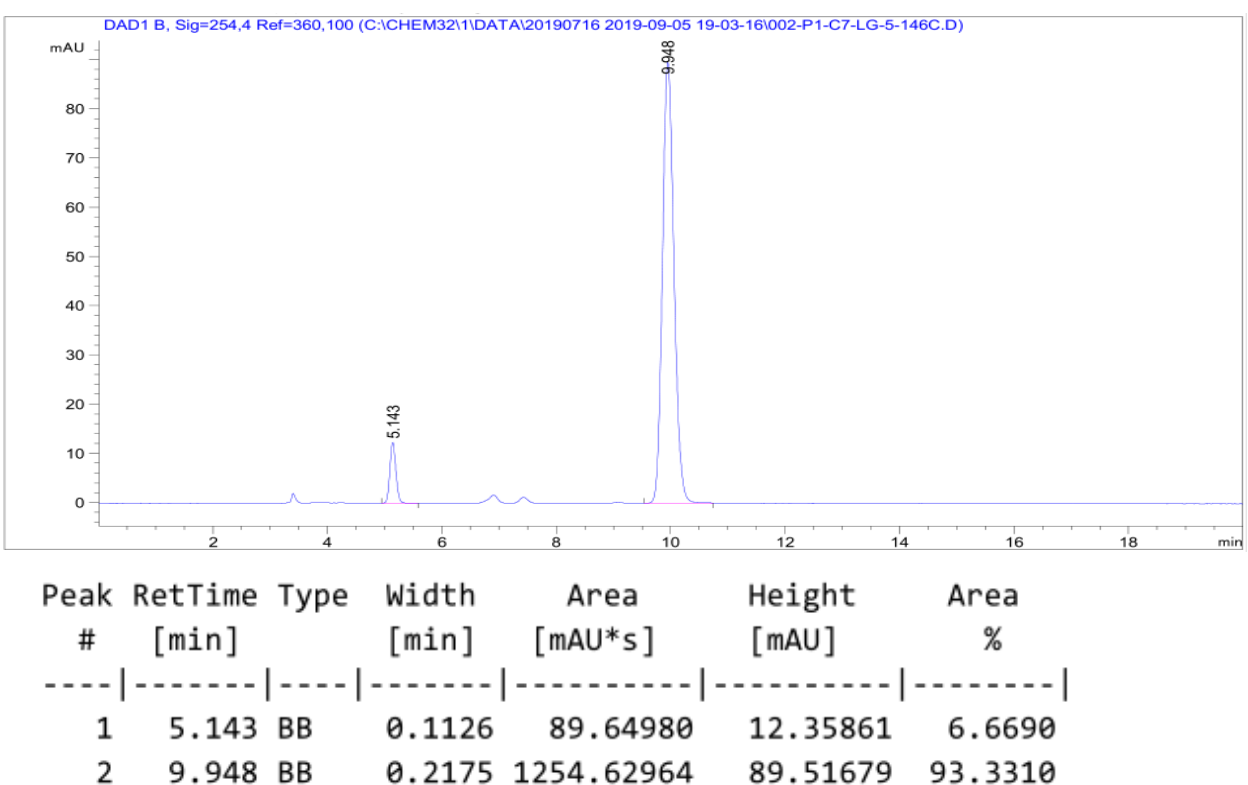<smiles>CC(C)(C)CC[C@@H](C(=O)OC1CCCCC1)c1ccnc(C(F)(F)F)c1</smiles>

(R)-Cyclohexyl 4,4-dimethyl-2-(2-(trifluoromethyl)pyridin-4-yl)pentanoate (44) 
The product was prepared according to the general procedure with $(4 \mathrm{~S}, 4$ 'S)-4,4'-di((S)sec-butyl)-4,4',5,5'-tetrahydro-2,2'-bioxazole [(S,S)-L1] $(3.3 \mathrm{mg}, 0.013 \mathrm{mmol}, 13$ mol\%), $\mathrm{NiCl}_{2} \cdot \mathrm{Py}_{4}(4.5 \mathrm{mg}, 0.01 \mathrm{mmol}, 10 \mathrm{~mol} \%), \operatorname{Ir}\left[\mathrm{dF}\left(\mathrm{CF}_{3}\right) \mathrm{ppy}\right]_{2}(\mathrm{bpy}) \mathrm{PF}_{6}(3.2 \mathrm{mg}$, $0.003 \mathrm{mmol}, 3 \mathrm{~mol} \%$ ), 4-bromo-2-(trifluoromethyl)pyridine (13.7 uL, $0.1 \mathrm{mmol}, 1.0$ equiv.), ${ }^{t} \mathrm{BuBF}_{3} \mathrm{~K}$ (41.0 mg, $0.25 \mathrm{mmol}, 2.5$ equiv.) and cyclohexyl acrylate (31.6 uL, $0.2 \mathrm{mmol}, 2.0$ equiv.) in acetone/ethyl acetate $(1.3 / 0.7, \mathrm{v} / \mathrm{v}, 2 \mathrm{~mL})$ were used. After 24 hours, the product was isolated by flash chromatography (PE: ethyl acetate $=20: 1)$ as a colorless oil (32.2 mg, 90\% yield, 90\% ee).

${ }^{1} \mathbf{H}$ NMR $\left(400 \mathrm{MHz}, \mathrm{CDCl}_{3}\right) \delta 8.64(\mathrm{~d}, J=5.0 \mathrm{~Hz}, 1 \mathrm{H}), 7.65(\mathrm{~s}, 1 \mathrm{H}), 7.45(\mathrm{dd}, J=5.0$, $1.4 \mathrm{~Hz}, 1 \mathrm{H}), 4.77-4.71(\mathrm{~m}, 1 \mathrm{H}), 3.69(\mathrm{dd}, J=9.3,3.5 \mathrm{~Hz}, 1 \mathrm{H}), 2.33(\mathrm{dd}, J=14.0,9.3$ $\mathrm{Hz}, 1 \mathrm{H}), 1.83-1.80(\mathrm{~m}, 1 \mathrm{H}), 1.70-1.59$ (m, 4H), 1.55-1.50 (m, 2H), 1.40-1.29 (m, 4H), $0.92(\mathrm{~s}, 9 \mathrm{H})$.

${ }^{19}$ F NMR $\left(375 \mathrm{MHz}, \mathrm{CDCl}_{3}\right) \delta-67.98(\mathrm{~s})$

${ }^{13}$ C NMR $\left(100 \mathrm{MHz}, \mathrm{CDCl}_{3}\right) \delta 172.16,152.08,150.18,148.57(\mathrm{q}, J=34.2 \mathrm{~Hz}), 125.67$, 121.50(q, $J=274.3 \mathrm{~Hz}), 119.86$ (q, $J=2.5 \mathrm{~Hz}$ ), 73.85, 48.35, 46.90, 31.26, 31.23, 29.38, $25.23,23.60,23.55$.

HRMS (ESI): $\mathrm{C}_{19} \mathrm{H}_{27} \mathrm{~F}_{3} \mathrm{NO}_{2}^{+}(\mathrm{M}+\mathrm{H}): 358.1994$, found: 358.1983 .

$[\alpha]_{\mathrm{D}}^{25}=-8.3352\left(\mathrm{c}=0.4067, \mathrm{CHCl}_{3}\right)$

Chiral HPLC: CHIRALPAK IC, $25{ }^{\circ} \mathrm{C},{ }^{i} \mathrm{PrOH}-h$ exanes 5/95, $0.5 \mathrm{~mL} / \mathrm{min}, 254 \mathrm{~nm}, \mathrm{t}_{\mathrm{R}}$ $($ major $)=12.6 \mathrm{~min}, \mathrm{t}_{\mathrm{R}}($ minor $)=8.8 \mathrm{~min}$.

\section{4 racemic}

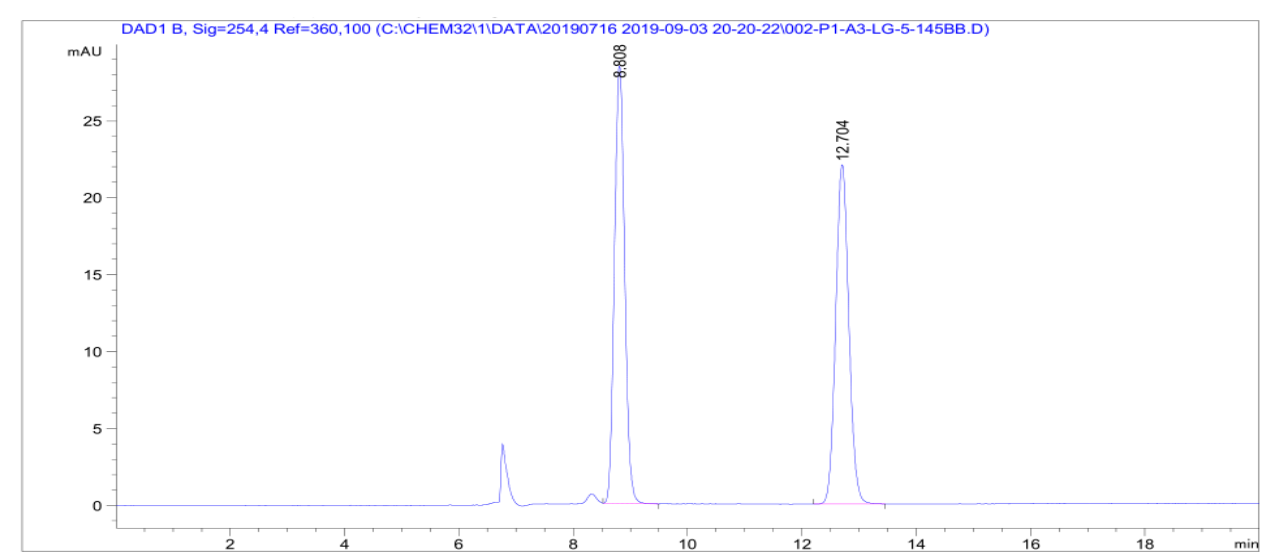




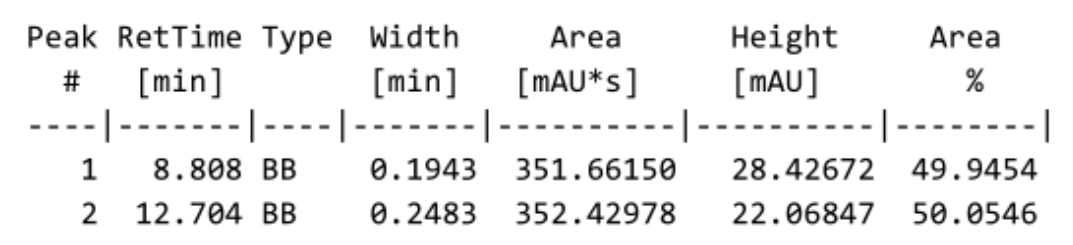

\section{4 enantioenriched, $90 \%$ ee}

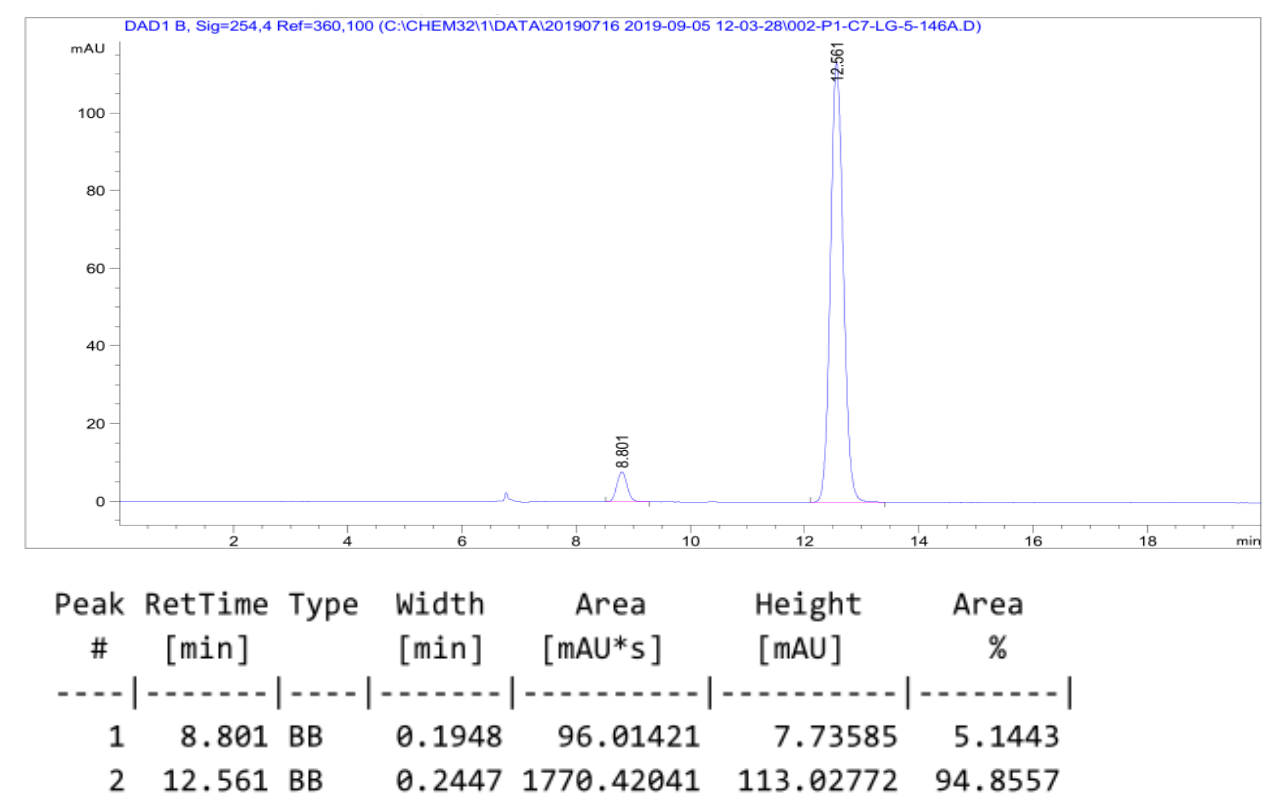<smiles>CCCCOC(=O)C(C)(Br)OC(=O)CCCC</smiles>

\section{(R)-Butyl 4,4-dimethyl-2-(2-(trifluoromethyl)pyridin-4-yl)pentanoate (45)}

The product was prepared according to the general procedure with (4S,4'S)-4,4'-di((S)sec-butyl)-4,4',5,5'-tetrahydro-2,2'-bioxazole [(S,S)-L1] $(3.3 \mathrm{mg}, 0.013 \mathrm{mmol}, 13$ mol\%), $\mathrm{NiCl}_{2} \bullet \mathrm{Py}_{4}(4.5 \mathrm{mg}, 0.01 \mathrm{mmol}, 10 \mathrm{~mol} \%), \operatorname{Ir}\left[\mathrm{dF}\left(\mathrm{CF}_{3}\right) \text { ppy }\right]_{2}(\mathrm{bpy}) \mathrm{PF}_{6}(3.2 \mathrm{mg}$, $0.003 \mathrm{mmol}, 3 \mathrm{~mol} \%$ ), 4-bromo-2-(trifluoromethyl)pyridine (13.7 uL, $0.1 \mathrm{mmol}, 1.0$ equiv.), ${ }^{t} \mathrm{BuBF}_{3} \mathrm{~K}$ (41.0 mg, $0.25 \mathrm{mmol}, 2.5$ equiv.) and butyl acrylate (28.7 uL, 0.2 mmol, 2.0 equiv.) in acetone/ethyl acetate (1.3/0.7, v/v, $2 \mathrm{~mL})$ were used. After 24 hours, the product was isolated by flash chromatography (PE: ethyl acetate $=20: 1)$ as a colorless oil (26.8 mg, $81 \%$ yield, $90 \%$ ee). 
${ }^{1} \mathbf{H}$ NMR $\left(400 \mathrm{MHz}, \mathrm{CDCl}_{3}\right) \delta 8.64(\mathrm{~d}, J=5.0 \mathrm{~Hz}, 1 \mathrm{H}), 7.65(\mathrm{~s}, 1 \mathrm{H}), 7.46(\mathrm{dd}, J=5.0$, $1.1 \mathrm{~Hz}, 1 \mathrm{H}), 4.12-4.01(\mathrm{~m}, 2 \mathrm{H}), 3.71(\mathrm{dd}, J=9.2,3.8 \mathrm{~Hz}, 1 \mathrm{H}), 2.32(\mathrm{dd}, J=14.0,9.2$ $\mathrm{Hz}, 1 \mathrm{H}), 1.59-1.55$ (m, 2H), 1.33-1.25 (m, 3H), 0.92 (s, 9H), 0.88 (t, J=7.4 Hz, 3H).

${ }^{19}$ F NMR $\left(375 \mathrm{MHz}, \mathrm{CDCl}_{3}\right) \delta-67.99$ (s)

${ }^{13} \mathrm{C} \mathrm{NMR}\left(100 \mathrm{MHz}, \mathrm{CDCl}_{3}\right) \delta 172.85,151.80,150.21,148.59(\mathrm{q}, J=34.5 \mathrm{~Hz}), 125.70$, $121.45(\mathrm{q}, J=274.2 \mathrm{~Hz}), 119.88(\mathrm{q}, J=2.8 \mathrm{~Hz}), 65.43,48.03,46.98,31.18,30.35$, $29.31,19.01,13.53$.

HRMS (ESI): $\mathrm{C}_{17} \mathrm{H}_{25} \mathrm{~F}_{3} \mathrm{NO}_{2}^{+}(\mathrm{M}+\mathrm{H}): 332.1837$, found: 332.1823 .

$[\alpha]_{\mathrm{D}}^{25}=-9.3476\left(\mathrm{c}=0.4600, \mathrm{CHCl}_{3}\right)$

Chiral HPLC: CHIRALPAK IA, $25{ }^{\circ} \mathrm{C},{ }^{i} \mathrm{PrOH}-$ hexanes 5/95, $0.5 \mathrm{~mL} / \mathrm{min}, 254 \mathrm{~nm}, \mathrm{t}_{\mathrm{R}}$ (major) $=12.0 \mathrm{~min}, \mathrm{t}_{\mathrm{R}}($ minor $)=9.0 \mathrm{~min}$.

\section{5 racemic}
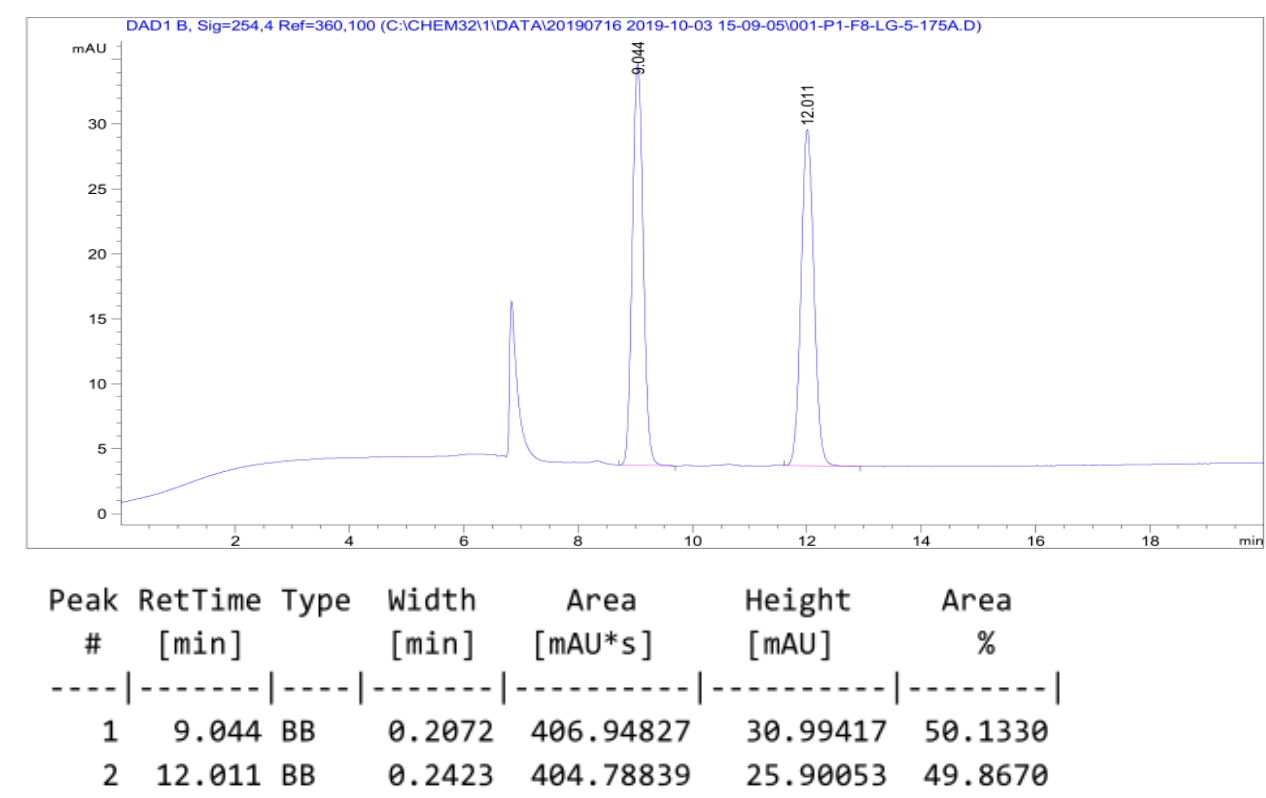

45 enantioenriched, $90 \%$ ee

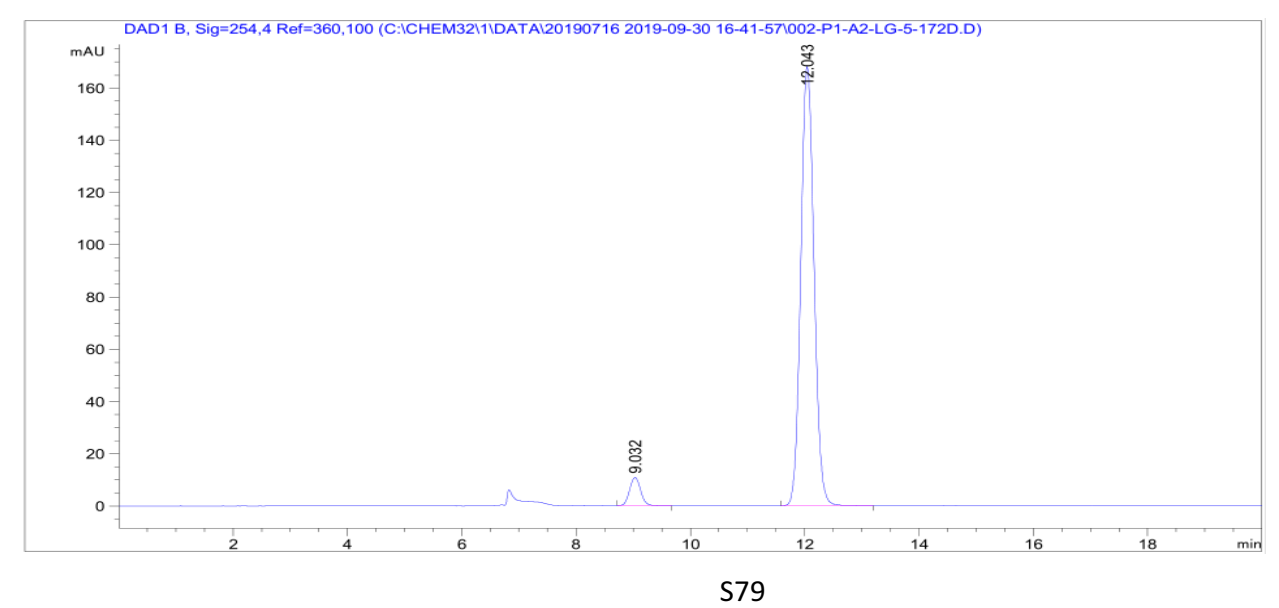




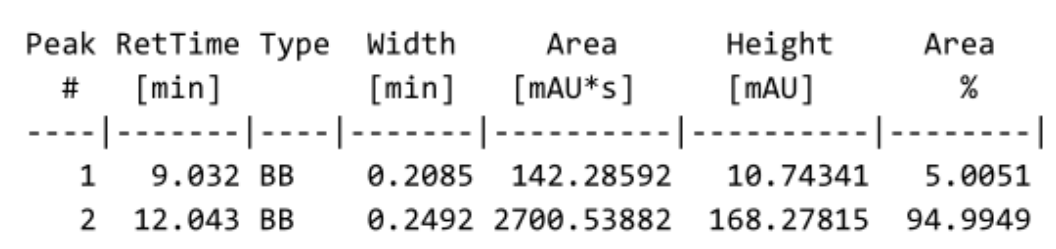<smiles>CC(C)(C)C[C@@H](C(=O)OCCCCO)c1ccnc(C(F)(F)F)c1</smiles>

(R)-4-Hydroxybutyl 4,4-dimethyl-2-(2-(trifluoromethyl)pyridin-4-yl)pentanoate (46)

The product was prepared according to the general procedure with (4S,4'S)-4,4'-di((S)sec-butyl)-4,4',5,5'-tetrahydro-2,2'-bioxazole [(S,S)-L1] $(3.3 \mathrm{mg}, 0.013 \mathrm{mmol}, 13$ mol\%), $\mathrm{NiCl}_{2} \bullet \mathrm{Py}_{4}(4.5 \mathrm{mg}, 0.01 \mathrm{mmol}, 10 \mathrm{~mol} \%), \operatorname{Ir}\left[\mathrm{dF}\left(\mathrm{CF}_{3}\right) \mathrm{ppy}\right]_{2}(\mathrm{bpy}) \mathrm{PF}_{6}(3.2 \mathrm{mg}$, $0.003 \mathrm{mmol}, 3 \mathrm{~mol} \%$ ), 4-bromo-2-(trifluoromethyl)pyridine (13.7 uL, $0.1 \mathrm{mmol}, 1.0$ equiv.), ${ }^{t} \mathrm{BuBF}_{3} \mathrm{~K}$ (41.0 mg, $0.25 \mathrm{mmol}, 2.5$ equiv.) and 4-hydroxybutyl acrylate (27.7 uL, $0.2 \mathrm{mmol}, 2.0$ equiv.) in acetone/ethyl acetate (1.3/0.7, v/v, $2 \mathrm{~mL})$ were used. After 24 hours, the product was isolated by flash chromatography (PE: ethyl acetate $=20: 1)$ as a colorless oil (28.5 mg, $82 \%$ yield, $90 \%$ ee).

${ }^{1}$ H NMR $\left(400 \mathrm{MHz} \mathrm{CDCl}_{3}\right) \delta 8.65(\mathrm{~d}, J=5.0 \mathrm{~Hz}, 1 \mathrm{H}), 7.64(\mathrm{~s}, 1 \mathrm{H}), 7.46(\mathrm{dd}, J=5.0$, $1.1 \mathrm{~Hz}, 1 \mathrm{H}), 4.16-4.04(\mathrm{~m}, 2 \mathrm{H}), 3.72(\mathrm{dd}, J=9.1,3.8 \mathrm{~Hz}, 1 \mathrm{H}), 3.63(\mathrm{t}, J=6.3 \mathrm{~Hz}, 2 \mathrm{H})$, $2.32(\mathrm{dd}, J=14.0,9.1 \mathrm{~Hz}, 1 \mathrm{H}), 1.73-1.66(\mathrm{~m}, 2 \mathrm{H}), 1.57-1.52(\mathrm{~m}, 4 \mathrm{H}), 0.91(\mathrm{~s}, 9 \mathrm{H})$.

${ }^{19}$ F NMR $\left(375 \mathrm{MHz}, \mathrm{CDCl}_{3}\right) \delta-67.95$ (s)

${ }^{13}$ C NMR $\left(100 \mathrm{MHz}, \mathrm{CDCl}_{3}\right) \delta 172.84,151.71,150.24,148.60(\mathrm{q}, J=34.6 \mathrm{~Hz}), 125.70$, $121.43(\mathrm{q} J=274.2 \mathrm{~Hz}), 119.86$ (q, $J=2.6 \mathrm{~Hz}), 65.39,62.13,47.98,46.98,31.18,29.32$, $28.88,24.89$.

HRMS (ESI): $\mathrm{C}_{17} \mathrm{H}_{25} \mathrm{~F}_{3} \mathrm{NO}_{3}{ }^{+}(\mathrm{M}+\mathrm{H}): 348.1787$, found: 348.1775 .

$[\alpha]_{\mathrm{D}}^{25}=-18.3624\left(\mathrm{c}=0.3567, \mathrm{CHCl}_{3}\right)$

Chiral HPLC: CHIRALPAK IC, $25{ }^{\circ} \mathrm{C},{ }^{i} \mathrm{PrOH}-$ hexanes 10/90, $1.0 \mathrm{~mL} / \mathrm{min}, 254 \mathrm{~nm}$, $\mathrm{t}_{\mathrm{R}}($ major $)=10.1 \mathrm{~min}, \mathrm{t}_{\mathrm{R}}($ minor $)=8.9 \mathrm{~min}$. 


\section{6 racemic}

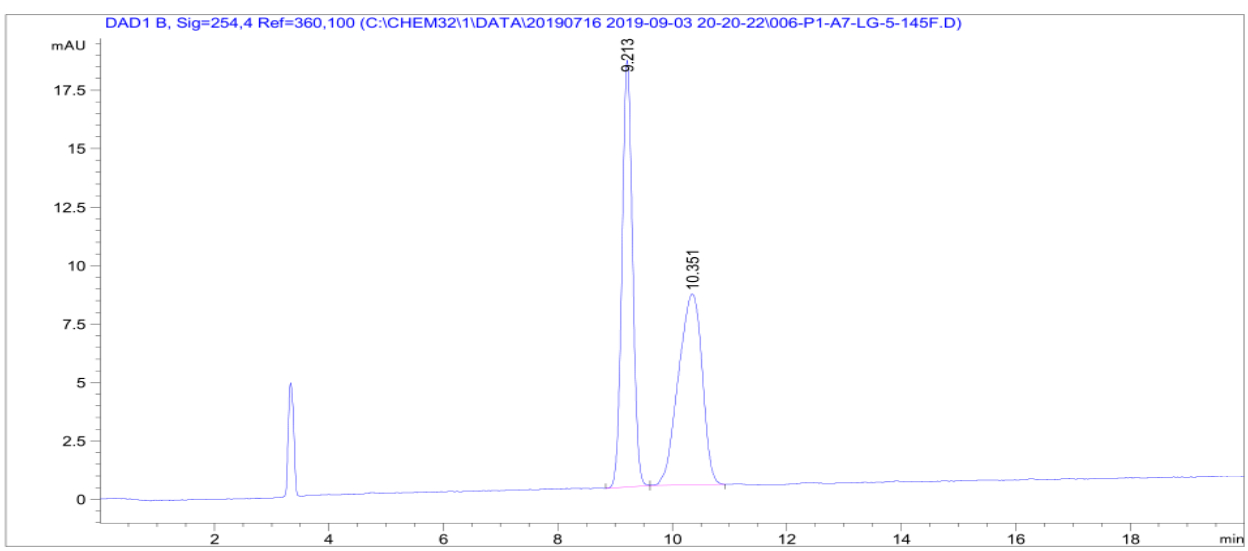

\begin{tabular}{cccccc}
$\begin{array}{c}\text { Peak RetTime Type } \\
\text { \# } \\
{[\text { [min] }}\end{array}$ & $\begin{array}{c}\text { Width } \\
{[\text { min] }}\end{array}$ & $\begin{array}{c}\text { Area } \\
{[\text { mAU*s] }}\end{array}$ & $\begin{array}{l}\text { Height } \\
{[\text { [mAU] }}\end{array}$ & $\begin{array}{c}\text { Area } \\
\%\end{array}$ \\
\hline 1 & 9.213 BB & 0.1973 & 233.67113 & 18.25550 & 49.8178 \\
2 & 10.351 BB & 0.4505 & 235.38002 & 8.16015 & 50.1822
\end{tabular}

\section{6 enantioenriched, $90 \%$ ee}

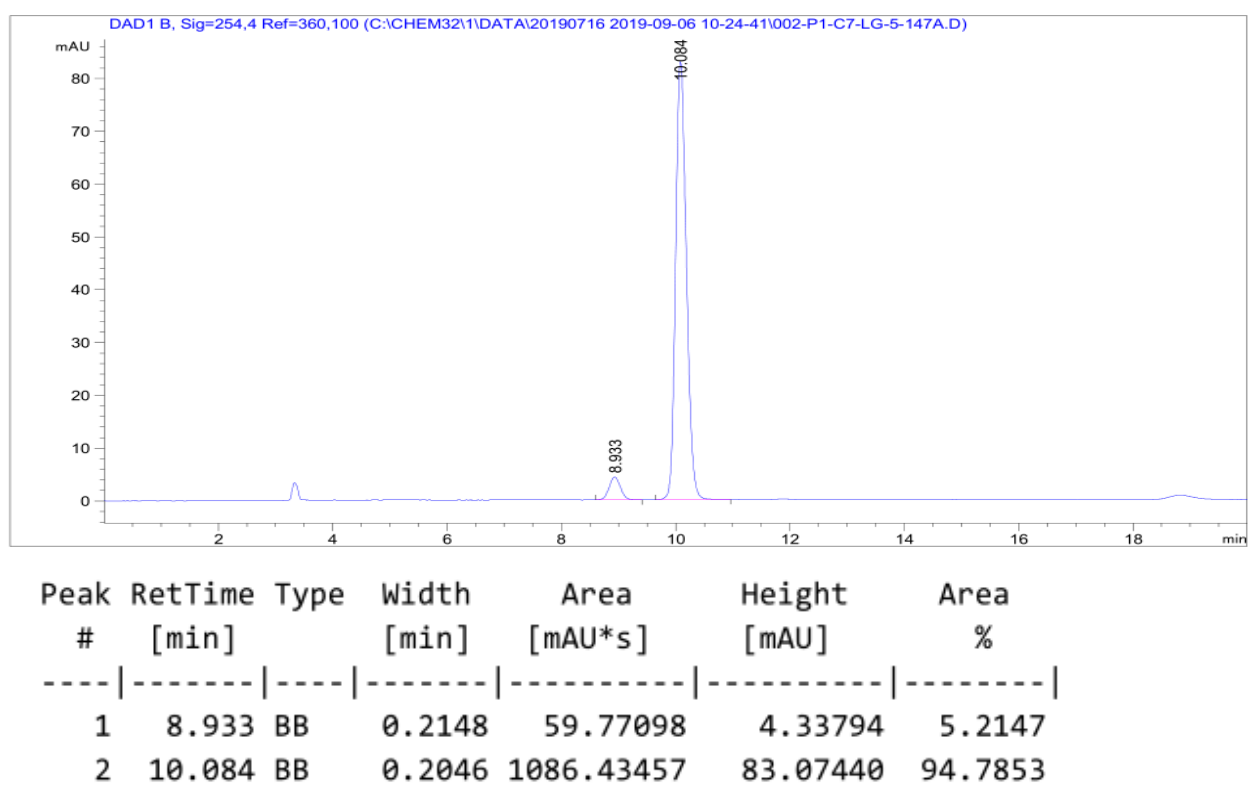


<smiles>COCCOC(=O)[C@H](CCC(C)(C)C)c1ccnc(C(F)(F)F)c1</smiles>

(R)-2-Methoxyethyl 4,4-dimethyl-2-(2-(trifluoromethyl)pyridin-4-yl)pentanoate (47)

The product was prepared according to the general procedure with (4S,4'S)-4,4'-di((S)sec-butyl)-4,4',5,5'-tetrahydro-2,2'-bioxazole [(S,S)-L1] $(3.3 \mathrm{mg}, 0.013 \mathrm{mmol}, 13$ mol\%), $\mathrm{NiCl}_{2} \bullet \mathrm{Py}_{4}(4.5 \mathrm{mg}, 0.01 \mathrm{mmol}, 10 \mathrm{~mol} \%), \operatorname{Ir}\left[\mathrm{dF}_{\left(\mathrm{CF}_{3}\right) \text { ppy }}\right]_{2}(\mathrm{bpy}) \mathrm{PF}_{6}(3.2 \mathrm{mg}$, $0.003 \mathrm{mmol}, 3 \mathrm{~mol} \%$ ), 4-bromo-2-(trifluoromethyl)pyridine (13.7 uL, $0.1 \mathrm{mmol}, 1.0$ equiv.), ${ }^{t} \mathrm{BuBF}_{3} \mathrm{~K}$ (41.0 mg, $0.25 \mathrm{mmol}, 2.5$ equiv.) and 2-methoxyethyl acrylate (25.7 uL, $0.2 \mathrm{mmol}, 2.0$ equiv.) in acetone/ethyl acetate $(1.3 / 0.7, \mathrm{v} / \mathrm{v}, 2 \mathrm{~mL})$ were used. After 24 hours, the product was isolated by flash chromatography $(\mathrm{PE}$ : ethyl acetate $=20: 1)$ as a colorless oil (23.4 mg, 70\% yield, $86 \%$ ee).

${ }^{1} \mathbf{H}$ NMR $\left(400 \mathrm{MHz}, \mathrm{CDCl}_{3}\right) \delta 8.64(\mathrm{~d}, J=5.0 \mathrm{~Hz}, 1 \mathrm{H}), 7.66(\mathrm{~s}, 1 \mathrm{H}), 7.46(\mathrm{dd}, J=5.0$, $1.5 \mathrm{~Hz}, 1 \mathrm{H}), 4.30-4.25(\mathrm{~m}, 1 \mathrm{H}), 4.21-4.16(\mathrm{~m}, 1 \mathrm{H}), 3.77$ (dd, $J=9.2,3.7 \mathrm{~Hz}, 1 \mathrm{H}), 3.53-$ $3.51(\mathrm{~m}, 2 \mathrm{H}), 3.29$ (s, 3H), $2.33(\mathrm{dd}, J=14.0,9.3 \mathrm{~Hz}, 1 \mathrm{H}), 1.55(\mathrm{dd}, J=14.0,3.7 \mathrm{~Hz}$, 1H), $0.91(\mathrm{~s}, 9 \mathrm{H})$.

${ }^{19}$ F NMR $\left(375 \mathrm{MHz}, \mathrm{CDCl}_{3}\right) \delta-67.97$ (s).

${ }^{13}$ C NMR $\left(100 \mathrm{MHz}, \mathrm{CDCl}_{3}\right) \delta 172.79,151.58,150.21,148.59$ (q, $\left.J=34.6 \mathrm{~Hz}\right), 125.73$, $121.44(\mathrm{q}, J=274.5 \mathrm{~Hz}), 119.87$ (q, $J=2.6 \mathrm{~Hz}), 70.10,64.32$, 58.80, 47.82, 47.07, $31.19,29.28$.

HRMS (ESI): $\mathrm{C}_{16} \mathrm{H}_{23} \mathrm{~F}_{3} \mathrm{NO}_{3}{ }^{+}(\mathrm{M}+\mathrm{H}): 334.1630$, found: 334.1619 .

$[\alpha]_{\mathrm{D}}^{25}=-12.8459\left(\mathrm{c}=0.3900, \mathrm{CHCl}_{3}\right)$

Chiral HPLC: CHIRALPAK IC, $25{ }^{\circ} \mathrm{C},{ }^{i} \mathrm{PrOH}-$ hexanes 5/95, $1.0 \mathrm{~mL} / \mathrm{min}, 254 \mathrm{~nm}, \mathrm{t}_{\mathrm{R}}$ (major) $=8.1 \mathrm{~min}, \mathrm{t}_{\mathrm{R}}($ minor $)=6.2 \mathrm{~min}$. 


\section{7 racemic}

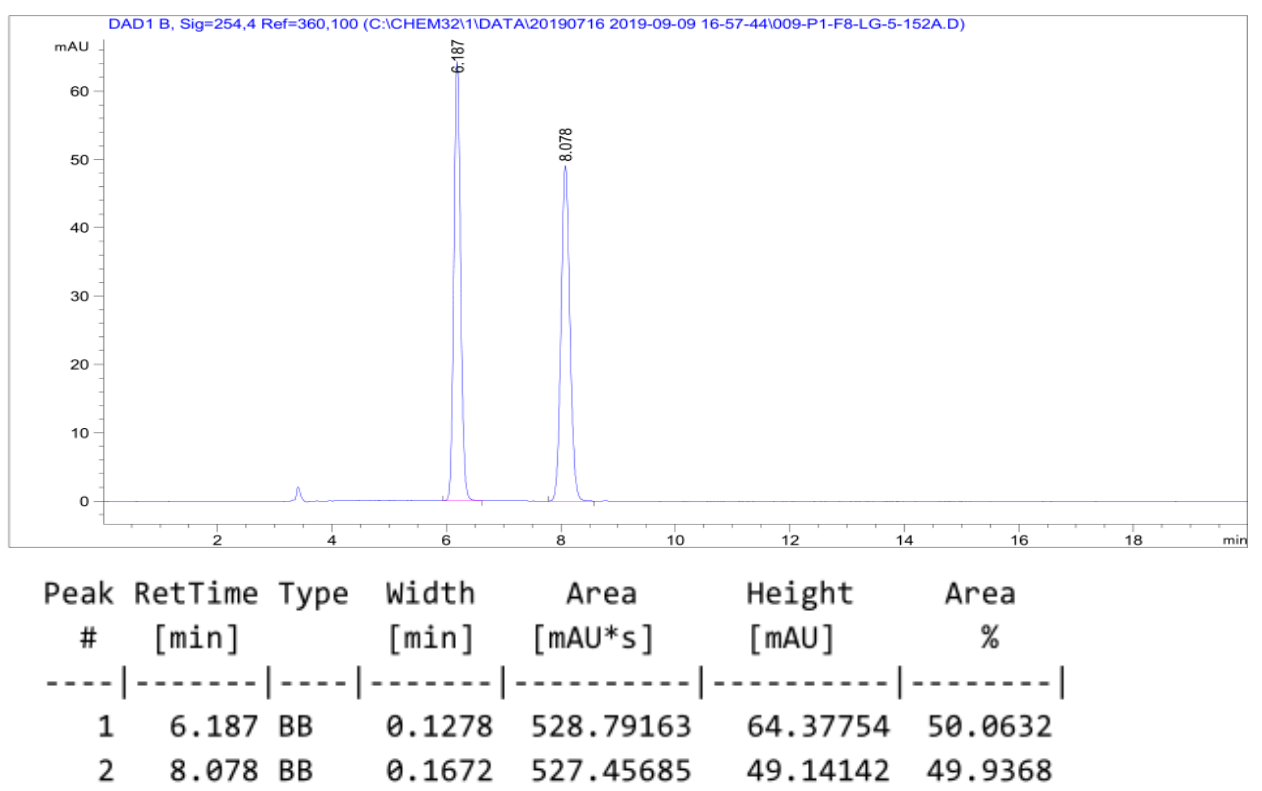

47 enantioenriched, $86 \%$ ee

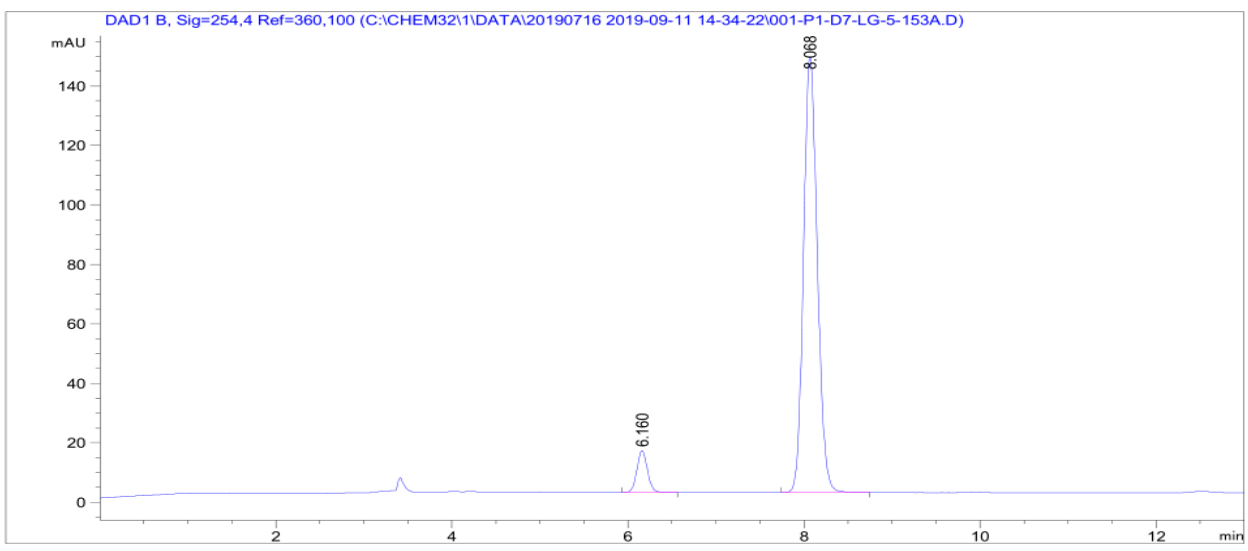

\begin{tabular}{|c|c|c|c|c|c|c|}
\hline $\begin{array}{c}\text { Peak } \\
\#\end{array}$ & $\begin{array}{c}\text { RetTime } \\
\text { [min] }\end{array}$ & Type & $\begin{array}{l}\text { Width } \\
\text { [min] }\end{array}$ & $\begin{array}{c}\text { Area } \\
{\left[\mathrm{mAU}^{*} \mathrm{~s}\right]}\end{array}$ & $\begin{array}{l}\text { Height } \\
{[\mathrm{mAU}]}\end{array}$ & $\begin{array}{c}\text { Area } \\
\%\end{array}$ \\
\hline & & & & - & & - \\
\hline 1 & & B & 0.1283 & 115.32841 & 13.97865 & 6.7533 \\
\hline 2 & 8.068 & BB & .1689 & 1592.40442 & 146.42616 & 93.2467 \\
\hline
\end{tabular}<smiles>CC(C)(C)C[C@@H](C(=O)OCC1CCCO1)c1ccnc(C(F)(F)F)c1</smiles>

(2R)-(Tetrahydrofuran-2-yl)methyl4,4-dimethyl-2-(2-(trifluoromethyl) Pyridine 4-yl)pentanoate (48) 
The product was prepared according to the general procedure with $(4 \mathrm{~S}, 4$ 'S)-4,4'-di((S)sec-butyl)-4,4',5,5'-tetrahydro-2,2'-bioxazole [(S,S)-L1] $(3.3 \mathrm{mg}, 0.013 \mathrm{mmol}, 13$ mol\%), $\mathrm{NiCl}_{2} \cdot \mathrm{Py}_{4}(4.5 \mathrm{mg}, 0.01 \mathrm{mmol}, 10 \mathrm{~mol} \%), \operatorname{Ir}\left[\mathrm{dF}\left(\mathrm{CF}_{3}\right) \mathrm{ppy}\right]_{2}(\mathrm{bpy}) \mathrm{PF}_{6}(3.2 \mathrm{mg}$, $0.003 \mathrm{mmol}, 3 \mathrm{~mol} \%)$, 4-bromo-2-(trifluoromethyl)pyridine (13.7 uL, $0.1 \mathrm{mmol}, 1.0$ equiv.), ${ }^{t} \mathrm{BuBF}_{3} \mathrm{~K}$ (41.0 mg, $0.25 \mathrm{mmol}, 2.5$ equiv.) and (tetrahydrofuran-2-yl)methyl acrylate $(29.4 \mathrm{uL}, 0.2 \mathrm{mmol}, 2.0$ equiv.) in acetone/ethyl acetate $(1.3 / 0.7, \mathrm{v} / \mathrm{v}, 2 \mathrm{~mL})$ were used. After 24 hours, the product was isolated by flash chromatography (PE: ethyl acetate $=20: 1)$ as a colorless oil $(29.0 \mathrm{mg}, 81 \%$ yield, $\mathrm{dr}=1: 1,88 \%$ ee $)$.

${ }^{1}$ H NMR $\left(400 \mathrm{MHz}, \mathrm{CDCl}_{3}\right) \delta 8.64(\mathrm{~d}, J=5.0 \mathrm{~Hz}, 1 \mathrm{H}), 7.66(\mathrm{~s}, 1 \mathrm{H}), 7.47(\mathrm{~d}, J=4.2$ $\mathrm{Hz}, 1 \mathrm{H}), 4.22-4.13(\mathrm{~m}, 1 \mathrm{H}), 4.07-3.95(\mathrm{~m}, 2 \mathrm{H}), 3.82-3.71(\mathrm{~m}, 3 \mathrm{H}), 2.33$ (dd, $J=14.1$, $9.0 \mathrm{~Hz}, 1 \mathrm{H}), 1.98-1.90(\mathrm{~m}, 1 \mathrm{H}), 1.89-1.80(\mathrm{~m}, 2 \mathrm{H}), 1.59-1.52(\mathrm{~m}, 1 \mathrm{H}), 1.49-1.43(\mathrm{~m}$, $1 \mathrm{H}), 0.91(\mathrm{~s}, 9 \mathrm{H})$.

${ }^{19}$ F NMR $\left(375 \mathrm{MHz}, \mathrm{CDCl}_{3}\right) \delta-67.97$ (s)

${ }^{13}$ C NMR (100 MHz, $\left.\mathrm{CDCl}_{3}\right) \delta 172.79$ (172.75), 151.62 (151.61), 150.22, 148.60 (q, $J$ $=34.7 \mathrm{~Hz}), 125.76,121.44(\mathrm{q}, J=274.1 \mathrm{~Hz}), 119.91(\mathrm{q}, J=1.6 \mathrm{~Hz}), 76.21$ (76.17), 68.39 (68.36), 67.31 (67.27), 47.85 (47.84), 47.12 (46.98), 31.19, 29.33, 27.90 (27.85), $25.61(25.56)$.

HRMS (ESI): $\mathrm{C}_{18} \mathrm{H}_{25} \mathrm{~F}_{3} \mathrm{NO}_{3}{ }^{+}(\mathrm{M}+\mathrm{H}): 360.1787$, found: 360.1772 .

$[\alpha]_{\mathrm{D}}^{25}=-9.1447\left(\mathrm{c}=0.6233, \mathrm{CHCl}_{3}\right)$

Chiral HPLC: CHIRALPAK IC, $25{ }^{\circ} \mathrm{C},{ }^{i} \mathrm{PrOH}-h$ exanes 5/95, $1.0 \mathrm{~mL} / \mathrm{min}, 254 \mathrm{~nm}, \mathrm{t}_{\mathrm{R}}$ $($ major $)=10.0 \min , t_{R}($ minor $)=8.1 \mathrm{~min} ; \mathrm{t}^{\prime}{ }_{\mathrm{R}}($ major $)=11.7 \mathrm{~min}, \mathrm{t}_{\mathrm{R}}($ minor $)=8.9 \mathrm{~min}$

\section{8 racemic}

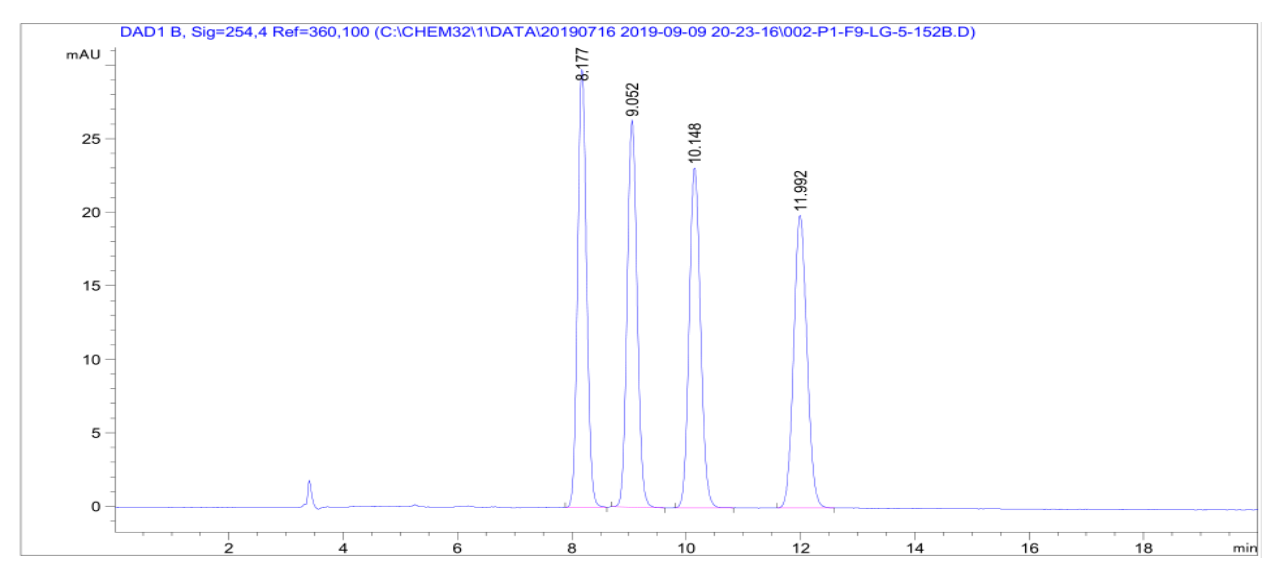




\begin{tabular}{|c|c|c|c|c|c|c|}
\hline $\begin{array}{l}\text { eak } \\
\text { \# }\end{array}$ & $\begin{array}{l}\text { RetTime } \\
{[\mathrm{min}]}\end{array}$ & Type & $\begin{array}{l}\text { Width } \\
\text { [min] }\end{array}$ & $\begin{array}{c}\text { Area } \\
{\left[\mathrm{mAU}{ }^{*} \mathrm{~s}\right]}\end{array}$ & $\begin{array}{l}\text { Height } \\
{[\mathrm{mAU}]}\end{array}$ & $\begin{array}{c}\text { Area } \\
\%\end{array}$ \\
\hline & & & & (1) & & 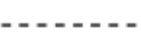 \\
\hline 1 & 8.177 & BB & 0.1683 & 322.76459 & 29.81340 & 25.1825 \\
\hline 2 & 9.05 & $B B$ & 0.1872 & 318.41507 & 26.30672 & 24.8431 \\
\hline 3 & 10.148 & BB & 0.2146 & 318.32904 & 23.12237 & 24.8364 \\
\hline 4 & 1.992 & BB & 0.2508 & 322.19363 & 9.90465 & 25.1379 \\
\hline
\end{tabular}

48 enantioenriched, $88 \%$ ee

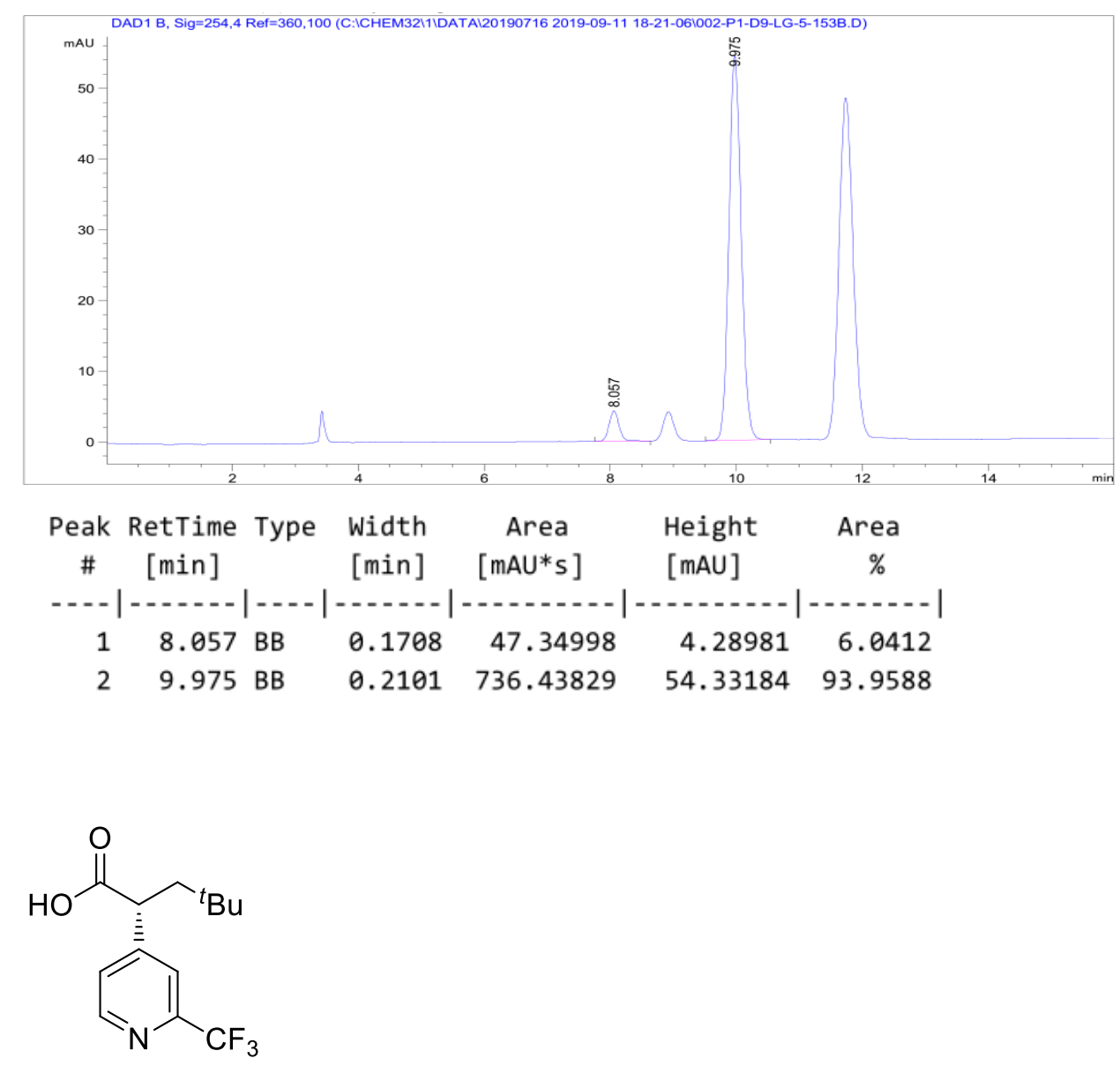

(R)-4,4-Dimethyl-2-(2-(trifluoromethyl)pyridin-4-yl)pentanoic acid (49)

The product was prepared according to the general procedure with (4S,4'S)-4,4'-di((S)sec-butyl)-4,4',5,5'-tetrahydro-2,2'-bioxazole $[(S, S)$-L1 $]$ (3.3 mg, $0.013 \mathrm{mmol}, 13$

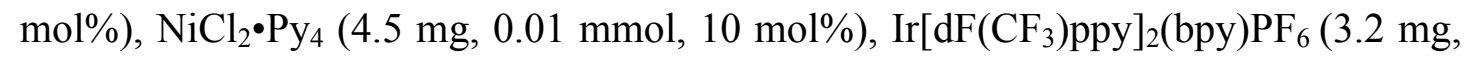
$0.003 \mathrm{mmol}, 3 \mathrm{~mol} \%$ ), 4-bromo-2-(trifluoromethyl)pyridine (13.7 uL, $0.1 \mathrm{mmol}, 1.0$ equiv.), ${ }^{t} \mathrm{BuBF}_{3} \mathrm{~K}(41.0 \mathrm{mg}, 0.25 \mathrm{mmol}, 2.5$ equiv.) and acrylic acid (13.7 uL, $0.2 \mathrm{mmol}$, 2.0 equiv.) in acetone/ethyl acetate $(1.3 / 0.7, \mathrm{v} / \mathrm{v}, 2 \mathrm{~mL})$ were used. After 24 hours, the product was isolated by flash chromatography $(\mathrm{PE}$ : ethyl acetate $=4: 1)$ as a colorless oil (14.3 mg, 52\% yield, $84 \%$ ee). 
${ }^{1} \mathbf{H}$ NMR $\left(400 \mathrm{MHz}, \mathrm{CDCl}_{3}\right) \delta 8.68(\mathrm{~d}, J=5.0 \mathrm{~Hz}, 1 \mathrm{H}), 7.67(\mathrm{~s}, 1 \mathrm{H}), 7.49(\mathrm{~d}, J=5.0$ $\mathrm{Hz}, 1 \mathrm{H}), 3.75(\mathrm{dd}, J=8.6,4.2 \mathrm{~Hz}, 1 \mathrm{H}), 2.31(\mathrm{dd}, J=14.1,8.7 \mathrm{~Hz}, 1 \mathrm{H}), 1.60(\mathrm{dd}, J=$ 14.1, 4.2 Hz, 1H), 0.93 (s, 9H).

${ }^{19}$ F NMR $\left(375 \mathrm{MHz}, \mathrm{CDCl}_{3}\right) \delta-67.93(\mathrm{~s})$.

${ }^{13}$ C NMR (100 MHz, $\left.\mathrm{CDCl}_{3}\right) \delta 176.82,151.23,150.27,148.64(\mathrm{q}, J=34.2 \mathrm{~Hz}), 125.94$, $121.44(\mathrm{q}, J=274.6 \mathrm{~Hz}), 120.04(\mathrm{q}, J=2.8 \mathrm{~Hz}), 47.56,46.82,31.27,29.33$.

HRMS (ESI): $\mathrm{C}_{13} \mathrm{H}_{16} \mathrm{~F}_{3} \mathrm{NO}_{2} \mathrm{Na}^{+}(\mathrm{M}+\mathrm{Na}): 298.1031$, found: 298.1025 .

$[\alpha]_{\mathrm{D}}^{25}=-8.1293\left(\mathrm{c}=0.1267, \mathrm{CHCl}_{3}\right)$

Chiral HPLC: CHIRALPAK IC, $25{ }^{\circ} \mathrm{C},{ }^{i} \mathrm{PrOH}-$ hexanes 5/95 (0.1\% TFA), $0.7 \mathrm{~mL} / \mathrm{min}$, $254 \mathrm{~nm}, \mathrm{t}_{\mathrm{R}}($ major $)=7.3 \mathrm{~min}, \mathrm{t}_{\mathrm{R}}($ minor $)=6.3 \mathrm{~min}$

\section{9 racemic}

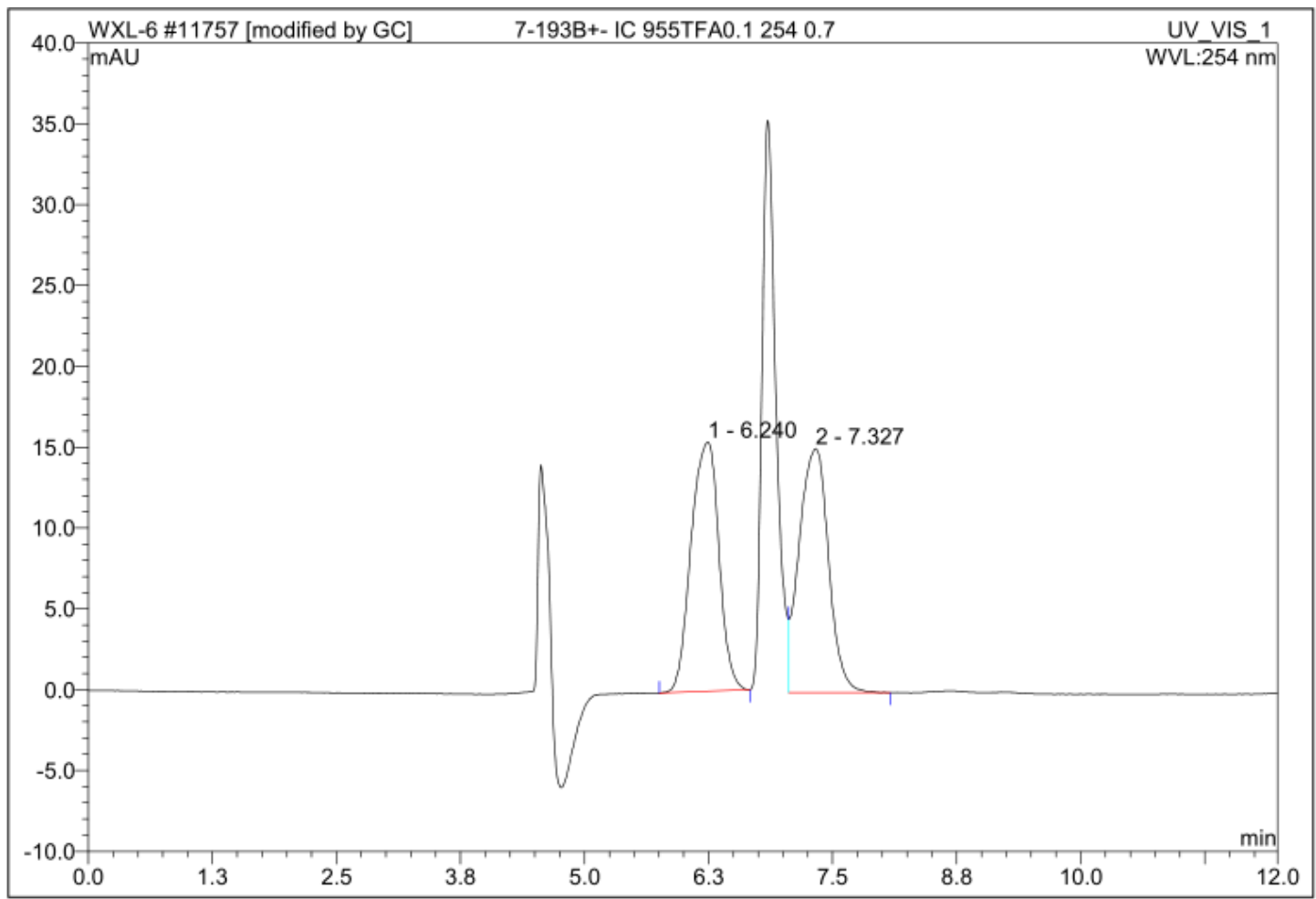

\begin{tabular}{|r|ccrrrrr|}
\hline No. & $\begin{array}{c}\text { Ret.Time } \\
\text { min }\end{array}$ & Peak Name & $\begin{array}{c}\text { Height } \\
\text { mAU }\end{array}$ & $\begin{array}{c}\text { Area } \\
\text { mAU*min }\end{array}$ & $\begin{array}{c}\text { Rel.Area } \\
\%\end{array}$ & Amount & Type \\
\hline 1 & 6.24 & n.a. & 15.410 & 5.021 & 49.17 & n.a. & BMB $^{*}$ \\
2 & 7.33 & n.a. & 15.086 & 5.191 & 50.83 & n.a. & MB $^{*}$ \\
\hline Total: & & & 30.496 & 10.212 & 100.00 & 0.000 & \\
\hline
\end{tabular}




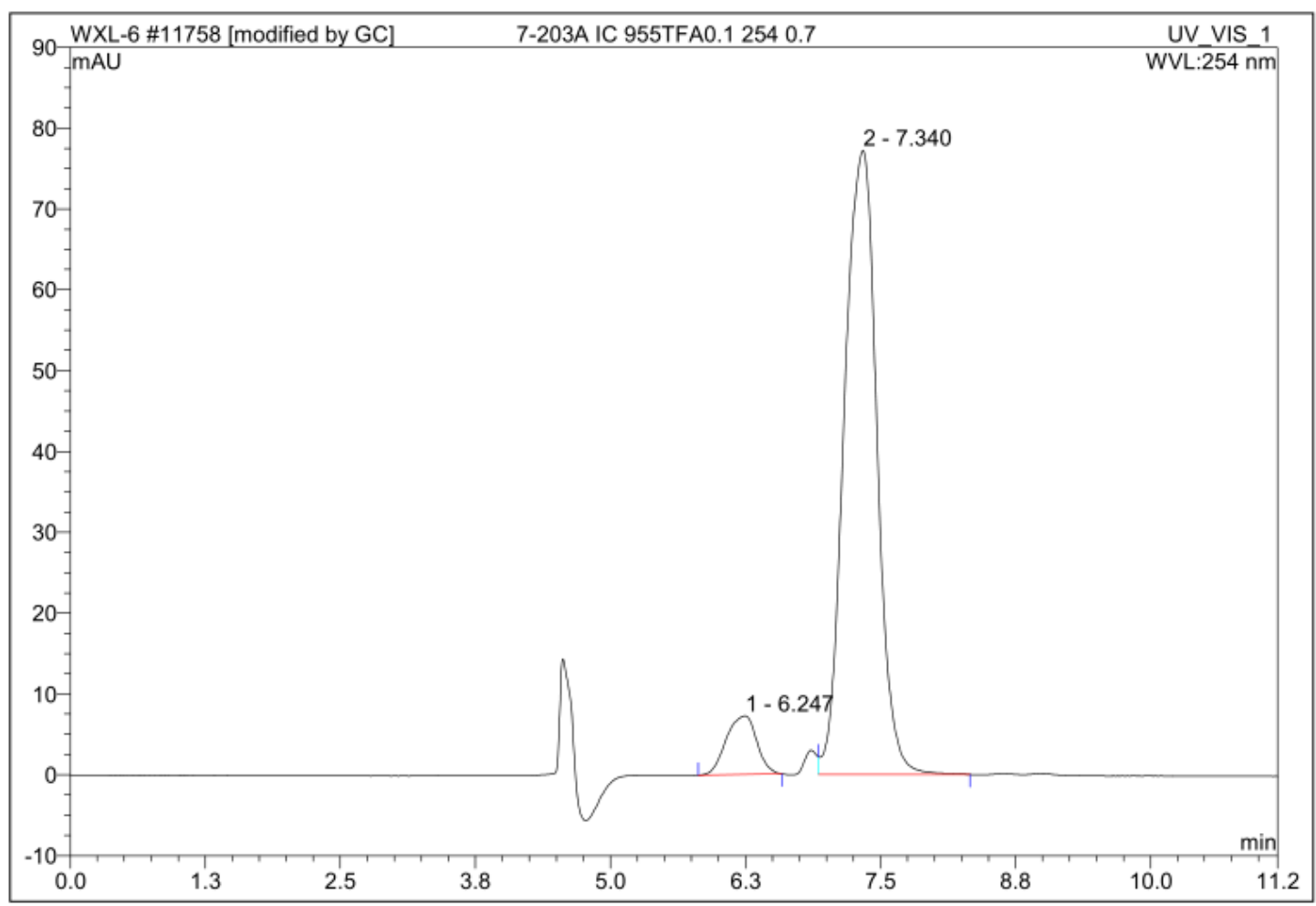

\begin{tabular}{|r|ccrrrrr|}
\hline No. & $\begin{array}{c}\text { Ret.Time } \\
\text { min }\end{array}$ & Peak Name & $\begin{array}{c}\text { Height } \\
\text { mAU }\end{array}$ & $\begin{array}{c}\text { Area } \\
\text { mAU*min }\end{array}$ & $\begin{array}{r}\text { Rel.Area } \\
\%\end{array}$ & Amount & Type \\
\hline 1 & 6.25 & n.a. & 7.224 & 2.362 & 8.03 & n.a. & BMB $^{*}$ \\
2 & 7.34 & n.a. & 77.236 & 27.035 & 91.97 & n.a. & MB $^{*}$ \\
\hline Total: & & & 84.461 & 29.397 & 100.00 & 0.000 & \\
\hline
\end{tabular}<smiles>CCC(C)[C@H](C(=O)OC)c1ccnc(C(F)(F)F)c1</smiles>

\section{(2R,3S)-Methyl3,4,4-trimethyl-2-(2-(trifluoromethyl)pyridin-4-yl)pentanoate (50)}

The product was prepared according to the general procedure with $(4 \mathrm{~S}, 4$ 'S)-4,4'-di((S)sec-butyl)-4,4',5,5'-tetrahydro-2,2'-bioxazole [(S,S)-L1] $(3.3 \mathrm{mg}, 0.013 \mathrm{mmol}, 13$ $\mathrm{mol} \%), \mathrm{NiCl}_{2} \bullet \mathrm{Py}_{4}(4.5 \mathrm{mg}, 0.01 \mathrm{mmol}, 10 \mathrm{~mol} \%), \operatorname{Ir}\left[\mathrm{dF}_{\left(\mathrm{CF}_{3}\right) \mathrm{ppy}}\right]_{2}(\mathrm{bpy}) \mathrm{PF}_{6}(3.2 \mathrm{mg}$, $0.003 \mathrm{mmol}, 3 \mathrm{~mol} \%$ ), 4-bromo-2-(trifluoromethyl)pyridine (13.7 uL, $0.1 \mathrm{mmol}, 1.0$ equiv.), ${ }^{t} \mathrm{BuBF}_{3} \mathrm{~K}$ (41.0 mg, $0.25 \mathrm{mmol}, 2.5$ equiv.) and $(E)$-methyl but-2-enoate (21.2 uL, $0.2 \mathrm{mmol}, 2.0$ equiv.) in acetone/ethyl acetate $(1.3 / 0.7, \mathrm{v} / \mathrm{v}, 2 \mathrm{~mL})$ were used. After 
24 hours, the product was isolated by flash chromatography (PE: ethyl acetate $=20: 1)$ as a colorless oil $(5.0 \mathrm{mg}, 16 \%$ yield, $87 \%$ ee, dr 6:1).

${ }^{1} \mathbf{H}$ NMR $\left(400 \mathrm{MHz}, \mathrm{CDCl}_{3}\right) \delta 8.65(\mathrm{~d}, J=5.0 \mathrm{~Hz}, 1 \mathrm{H}), 7.69(\mathrm{~s}, 1 \mathrm{H}), 7.53(\mathrm{~d}, J=4.9$ $\mathrm{Hz}, 1 \mathrm{H}), 3.93(\mathrm{~d}, J=5.4 \mathrm{~Hz}, 1 \mathrm{H}), 3.68(\mathrm{~s}, 3 \mathrm{H}), 1.87-1.80(\mathrm{~m}, 1 \mathrm{H}), 1.01(\mathrm{~d}, J=7.1 \mathrm{~Hz}$, $3 \mathrm{H}), 0.89(\mathrm{~s}, 9 \mathrm{H})$.

${ }^{19}$ F NMR $\left(375 \mathrm{MHz}, \mathrm{CDCl}_{3}\right) \delta-67.90(\mathrm{~s})$.

${ }^{13}$ C NMR (100 MHz, $\left.\mathrm{CDCl}_{3}\right) \delta 172.16,151.44,149.93,148.35$ (q, $\left.J=34.3 \mathrm{~Hz}\right), 126.42$, 121.50 (q, $J=274.3 \mathrm{~Hz}), 120.56(\mathrm{q}, J=2.7 \mathrm{~Hz}), 52.02,48.79,34.44,28.12, \quad 11.50$.

HRMS (ESI): $\mathrm{C}_{15} \mathrm{H}_{21} \mathrm{~F}_{3} \mathrm{NO}_{2}{ }^{+}$(M+H): 304.1524, found: 304.15117.

$[\alpha]_{\mathrm{D}}^{25}=-3.3008\left(\mathrm{c}=0.1333, \mathrm{CHCl}_{3}\right)$.

Chiral HPLC: CHIRALPAK IC, $25{ }^{\circ} \mathrm{C},{ }^{i} \mathrm{PrOH}-h$ exanes 5/95, $0.5 \mathrm{~mL} / \mathrm{min}, 254 \mathrm{~nm}, \mathrm{t}_{\mathrm{R}}$ (major) $=12.5 \mathrm{~min}, \mathrm{t}_{\mathrm{R}}($ minor $)=9.9 \mathrm{~min}$.

\section{0 racemic}

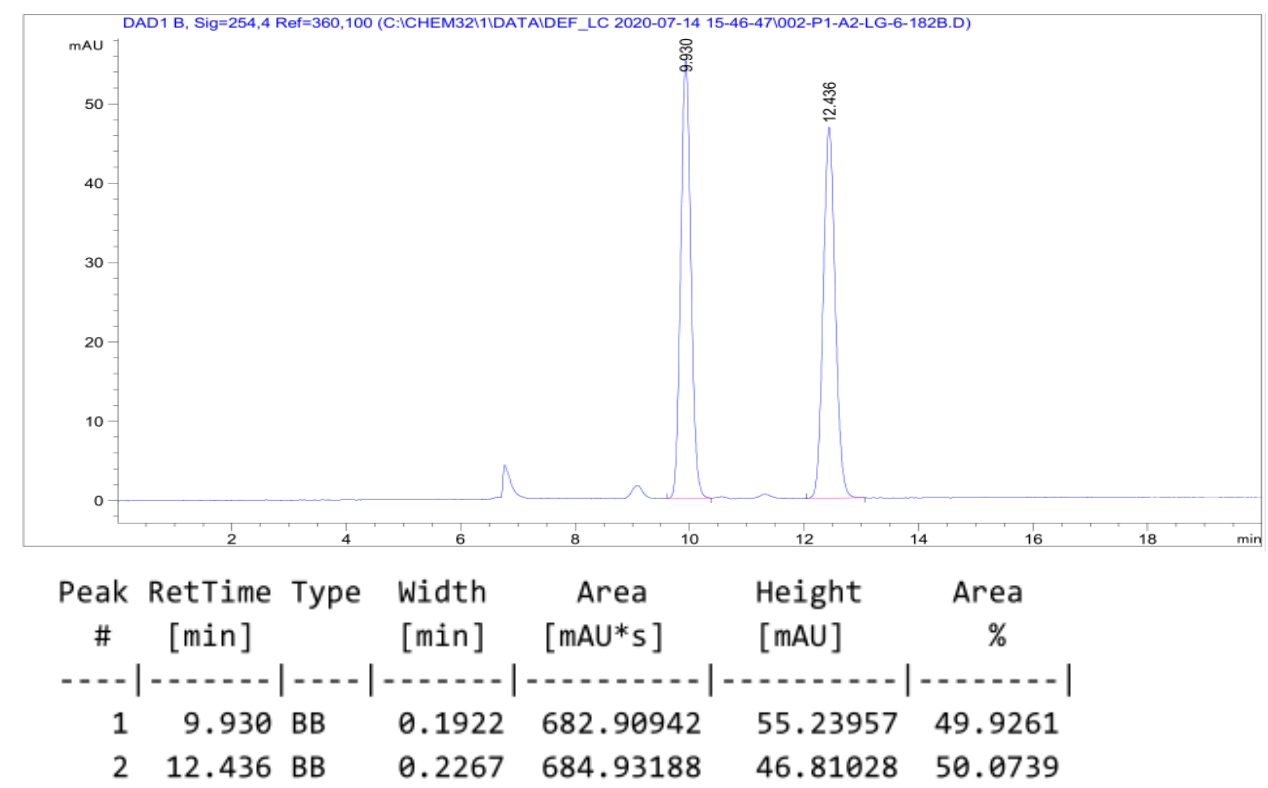




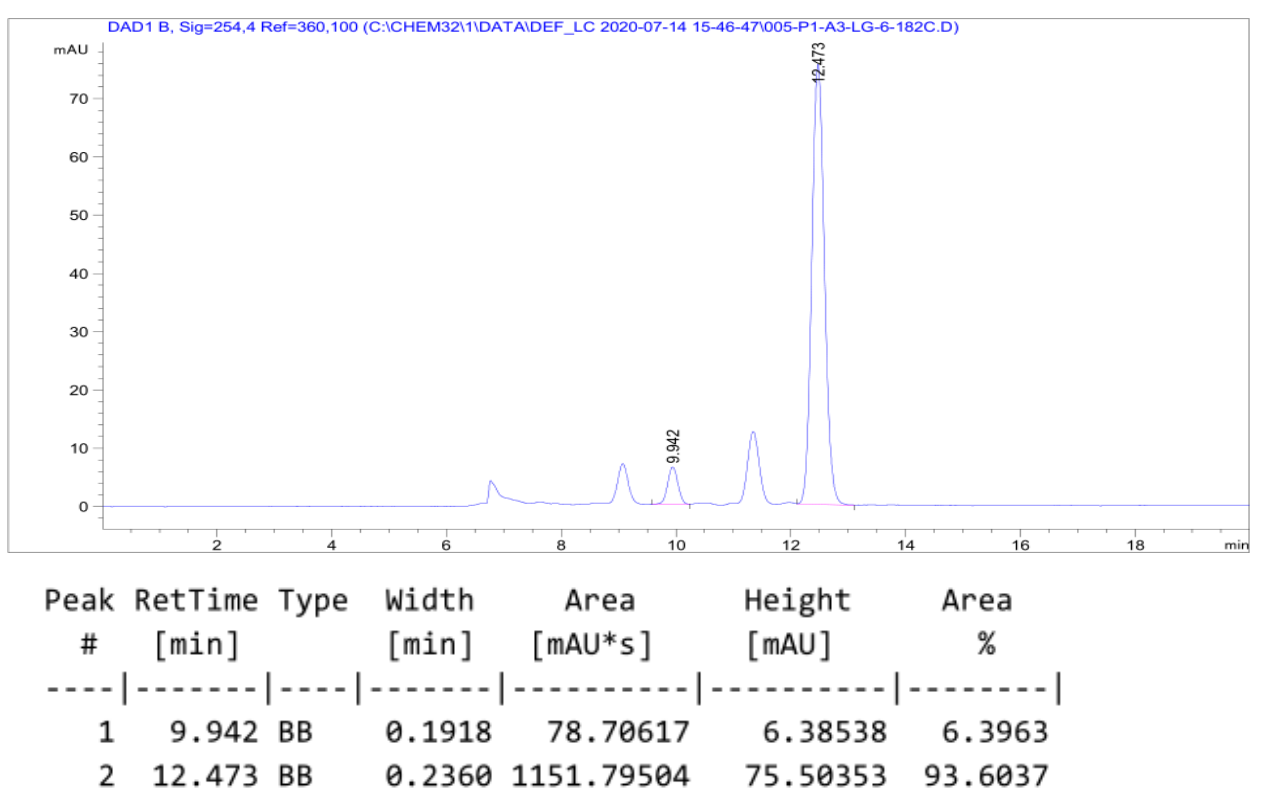<smiles>O=C1OC[C@@H](Br)[C@H]1c1ccnc(C(F)(F)F)c1</smiles>

(3R,4R)-4-(tert-Butyl)-3-(2-(trifluoromethyl)pyridin-4-yl)dihydrofuran-2(3H)-one (51)

The product was prepared according to the general procedure with (4S,4'S)-4,4'-di((S)sec-butyl)-4,4',5,5'-tetrahydro-2,2'-bioxazole [(S,S)-L1] $(3.3 \mathrm{mg}, 0.013 \mathrm{mmol}, 13$

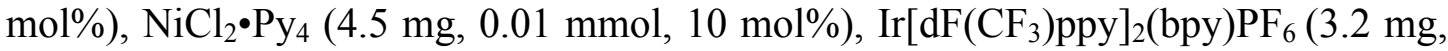
$0.003 \mathrm{mmol}, 3 \mathrm{~mol} \%$ ), 4-bromo-2-(trifluoromethyl)pyridine (13.7 uL, $0.1 \mathrm{mmol}, 1.0$ equiv.), ${ }^{t} \mathrm{BuBF}_{3} \mathrm{~K}$ (41.0 mg, $0.25 \mathrm{mmol}, 2.5$ equiv.) and furan-2(5H)-one (14.2 uL, 0.2 mmol, 2.0 equiv.) in acetone/ethyl acetate $(1.3 / 0.7, \mathrm{v} / \mathrm{v}, 2 \mathrm{~mL})$ were used. After 24 hours, the product was isolated by flash chromatography (PE: ethyl acetate $=10: 1)$ as a white solid (27.4 mg, 92\% yield, 15\% ee, dr 24:1).

${ }^{1}$ H NMR $\left(400 \mathrm{MHz}, \mathrm{CDCl}_{3}\right) \delta 8.73(\mathrm{~d}, J=5.0 \mathrm{~Hz}, 1 \mathrm{H}), 7.57(\mathrm{br}, 1 \mathrm{H}), 7.40(\mathrm{~d}, J=5.0$ $\mathrm{Hz}, 1 \mathrm{H}), 4.50(\mathrm{dd}, J=9.4,8.3 \mathrm{~Hz}, 1 \mathrm{H}), 4.25-4.21(\mathrm{~m}, 1 \mathrm{H}), 3.72(\mathrm{~d}, J=9.8 \mathrm{~Hz}, 1 \mathrm{H})$, $2.71(\mathrm{dd}, J=18.1,8.5 \mathrm{~Hz}, 1 \mathrm{H}), 0.91(\mathrm{~s}, 9 \mathrm{H})$.

${ }^{19}$ F NMR $\left(375 \mathrm{MHz}, \mathrm{CDCl}_{3}\right) \delta-68.00(\mathrm{~s})$. 
${ }^{13}$ C NMR (101 MHz, $\left.\mathrm{CDCl}_{3}\right) \delta 175.54,150.66,149.21,149.06(\mathrm{q}, J=34.7 \mathrm{~Hz}), 126.29$, $121.31(\mathrm{q}, J=274.6 \mathrm{~Hz}), 120.27$ (q, $J=2.7 \mathrm{~Hz}), 68.33,53.65,48.26,32.31,27.25$.

HRMS (ESI): $\mathrm{C}_{14} \mathrm{H}_{17} \mathrm{~F}_{3} \mathrm{NO}_{2}{ }^{+}(\mathrm{M}+\mathrm{H}): 288.1211$, found: 288.1208 .

$[\alpha]_{\mathrm{D}}^{25}=-7.1874\left(\mathrm{c}=0.3200, \mathrm{CHCl}_{3}\right)$

Chiral HPLC: CHIRALPAK IA, $25{ }^{\circ} \mathrm{C},{ }^{i} \mathrm{PrOH}-$ hexanes 5/95, $1.0 \mathrm{~mL} / \mathrm{min}, 254 \mathrm{~nm}, \mathrm{t}_{\mathrm{R}}$ (major) $=16.0 \mathrm{~min}, \mathrm{t}_{\mathrm{R}}($ minor $)=17.1 \mathrm{~min}$.

\section{1 racemic}

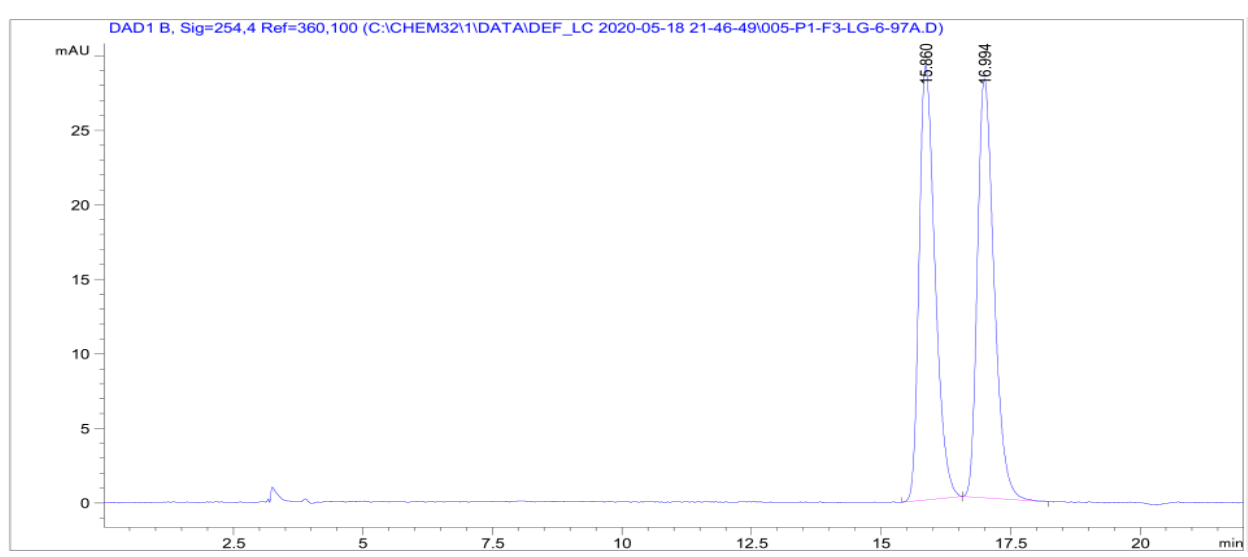

\begin{tabular}{|c|c|c|c|c|c|c|}
\hline $\begin{array}{c}\text { Peak } \\
\#\end{array}$ & $\begin{array}{c}\text { RetTime } \\
\text { [min] }\end{array}$ & Type & $\begin{array}{l}\text { Width } \\
\text { [min] }\end{array}$ & $\begin{array}{c}\text { Area } \\
{\left[\mathrm{mAU}^{*} \mathrm{~s}\right]}\end{array}$ & $\begin{array}{l}\text { Height } \\
\text { [mAU] }\end{array}$ & $\begin{array}{c}\text { Area } \\
\%\end{array}$ \\
\hline & & & & . & & \\
\hline 1 & 15.860 & BB & 0.3277 & 619.04626 & 29.15750 & 49.8136 \\
\hline 2 & 16.994 & BB & 0.3410 & 623.68030 & 28.08968 & 50.1864 \\
\hline
\end{tabular}

51 enantioenriched, $15 \%$ ee

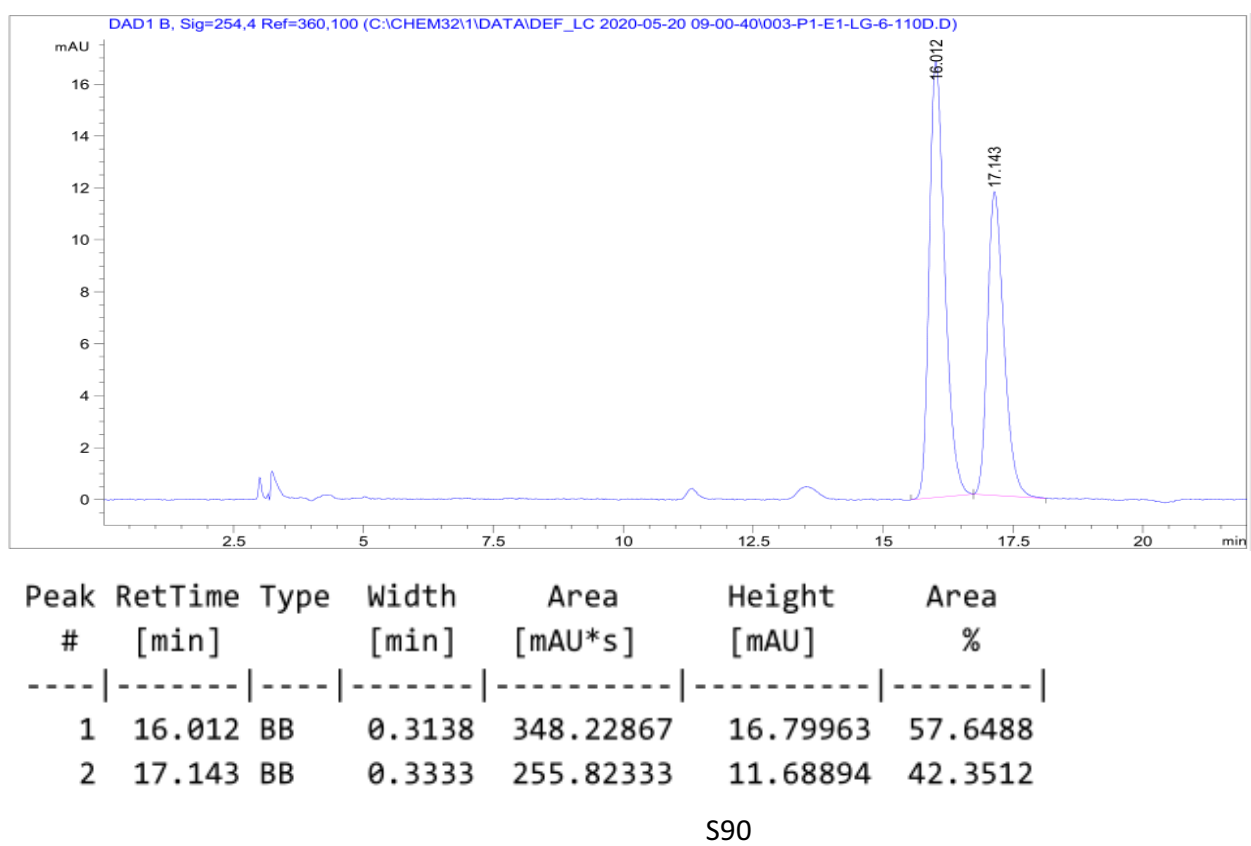


<smiles>NC(=O)[C@H](CC(F)(Br)Br)c1ccnc(C(F)(F)F)c1</smiles>

\section{(R)-4,4-Dimethyl-2-(2-(trifluoromethyl)pyridin-4-yl)pentanamide (52)}

The product was prepared according to the general procedure with (4S,4'S)-4,4'-di((S)sec-butyl)-4,4',5,5'-tetrahydro-2,2'-bioxazole [(S,S)-L1] $(3.3 \mathrm{mg}, 0.013 \mathrm{mmol}, 13$ $\mathrm{mol} \%), \mathrm{NiCl}_{2} \bullet \mathrm{Py}_{4}(4.5 \mathrm{mg}, 0.01 \mathrm{mmol}, 10 \mathrm{~mol} \%), \operatorname{Ir}\left[\mathrm{dF}\left(\mathrm{CF}_{3}\right) \text { ppy }\right]_{2}(\mathrm{bpy}) \mathrm{PF}_{6}(3.2 \mathrm{mg}$, $0.003 \mathrm{mmol}, 3 \mathrm{~mol} \%$ ), 4-bromo-2-(trifluoromethyl)pyridine (13.7 uL, $0.1 \mathrm{mmol}, 1.0$ equiv.), ${ }^{t} \mathrm{BuBF}_{3} \mathrm{~K}$ (41.0 mg, $0.25 \mathrm{mmol}, 2.5$ equiv.) and acrylamide (14.2 mg, $0.2 \mathrm{mmol}$, 2.0 equiv.) in acetone/ethyl acetate $(1.3 / 0.7, \mathrm{v} / \mathrm{v}, 2 \mathrm{~mL})$ were used. After 24 hours, the product was isolated by flash chromatography (PE: ethyl acetate $=10: 1)$ as a white solid (23.0 mg, 84\% yield, 83\% ee).

${ }^{1}$ H NMR $\left(400 \mathrm{MHz}, \mathrm{CDCl}_{3}\right) \delta 8.65(\mathrm{~d}, J=4.8 \mathrm{~Hz}, 1 \mathrm{H}), 7.66(\mathrm{~s}, 1 \mathrm{H}), 7.50(\mathrm{~d}, J=4.8$ $\mathrm{Hz}, 1 \mathrm{H}), 5.63$ (br, 2H), 3.52 (dd, $J=8.0,4.0 \mathrm{~Hz}, 1 \mathrm{H}), 2.38(\mathrm{dd}, J=14.1,8.3 \mathrm{~Hz}, 1 \mathrm{H})$, $1.54(\mathrm{dd}, J=14.1,4.1 \mathrm{~Hz}, 1 \mathrm{H}), 0.93(\mathrm{~s}, 9 \mathrm{H})$.

${ }^{19}$ F NMR $\left(375 \mathrm{MHz}, \mathrm{CDCl}_{3}\right) \delta-67.90(\mathrm{~s})$.

${ }^{13}$ C NMR $\left(100 \mathrm{MHz}, \mathrm{CDCl}_{3}\right) \delta 173.78,152.53,150.32,148.67$ (q, $\left.J=34.3 \mathrm{~Hz}\right), 125.54$, $121.46(\mathrm{q}, J=274.5 \mathrm{~Hz}), 119.73(\mathrm{q}, J=2.4 \mathrm{~Hz}), 48.88,47.04,31.23,29.46$.

HRMS (ESI): $\mathrm{C}_{13} \mathrm{H}_{18} \mathrm{~F}_{3} \mathrm{~N}_{2} \mathrm{O}^{+}(\mathrm{M}+\mathrm{H}): 275.1371$, found: 275.1368 .

$[\alpha]_{\mathrm{D}}^{25}=-36.3212\left(\mathrm{c}=0.2833, \mathrm{CHCl}_{3}\right)$

Chiral HPLC: CHIRALPAK IB, $25{ }^{\circ} \mathrm{C},{ }^{i} \mathrm{PrOH}-$ hexanes 10/95, $0.5 \mathrm{~mL} / \mathrm{min}, 254 \mathrm{~nm}$, $t_{R}($ major $)=13.5 \mathrm{~min}, t_{R}($ minor $)=14.7 \mathrm{~min}$. 


\section{2 racemic}

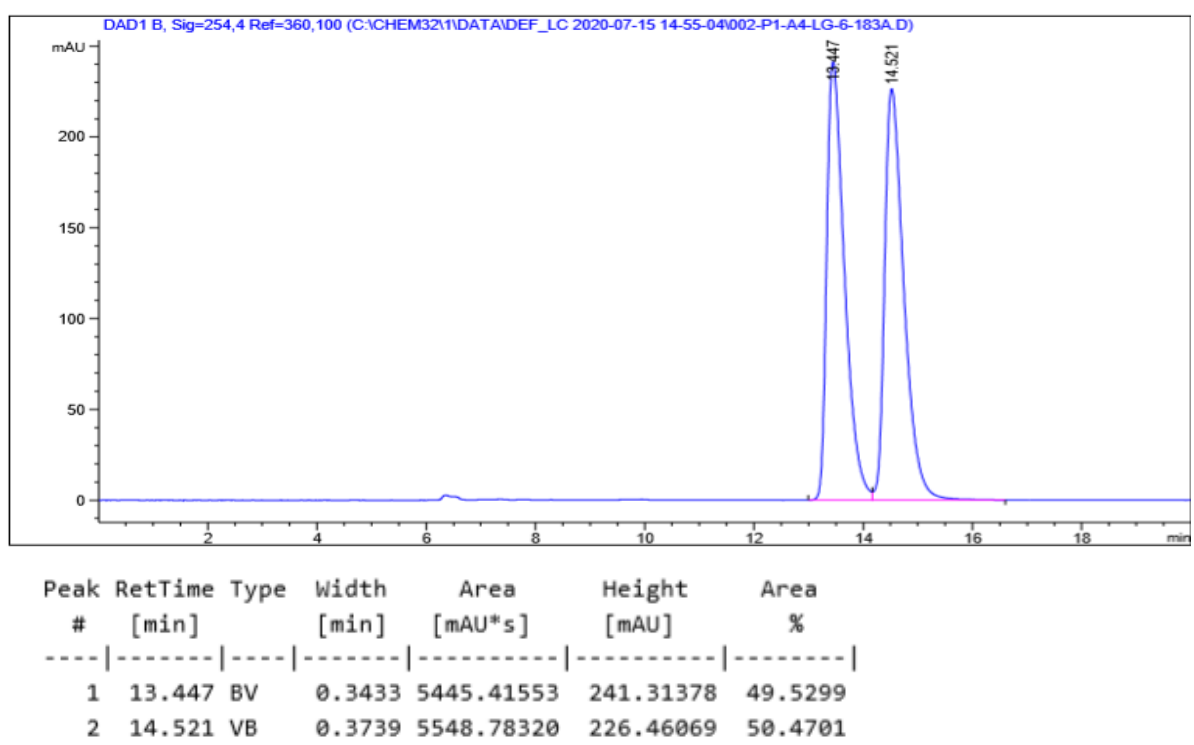

52 enantioenriched, $83 \%$ ee

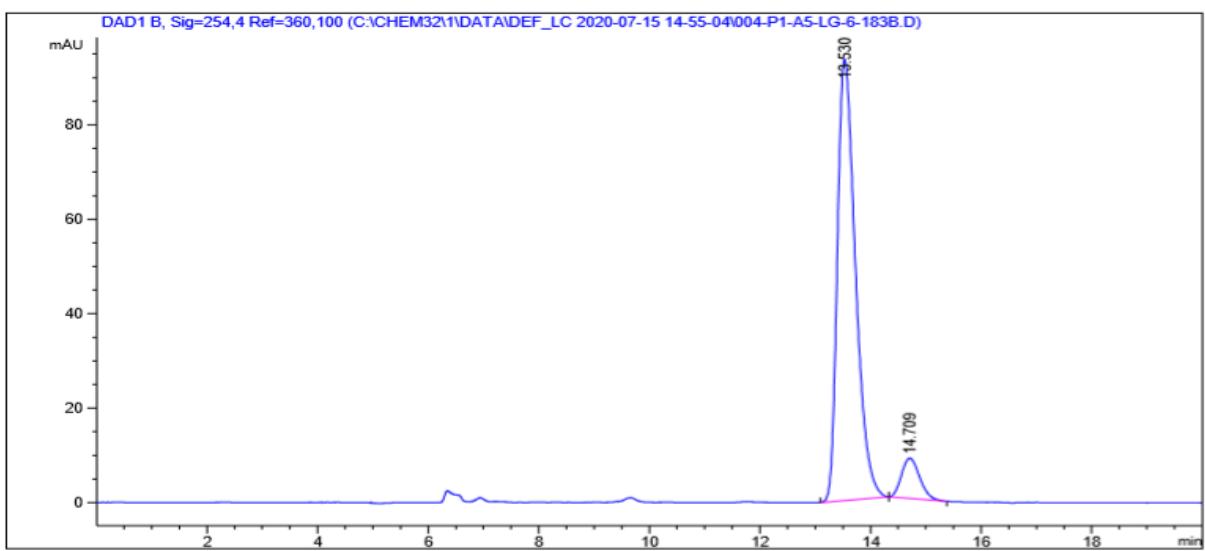

\begin{tabular}{cccccc}
$\begin{array}{c}\text { Peak RetTime Type Width } \\
\text { \# [min] }\end{array}$ & $\begin{array}{c}\text { Area } \\
\text { [min] }\end{array}$ [mAU*s] & $\begin{array}{c}\text { Height } \\
\text { [mAU] }\end{array}$ & $\begin{array}{c}\text { Area } \\
\%\end{array}$ \\
\hline 1 & 13.530 BB & 0.3505 & 2117.75366 & 93.40865 & 91.5614 \\
2 & 14.709 BB & 0.3529 & 195.17880 & 8.53024 & 8.4386
\end{tabular}<smiles>CC(C)(C)C[C@@H](C(=O)NC(C)(C)C)c1ccnc(C(F)(F)F)c1</smiles>

(R)-N-(tert-Butyl)-4,4-dimethyl-2-(2-(trifluoromethyl)pyridin-4-yl)pentanamide (53) 
The product was prepared according to the general procedure with (4S,4'S)-4,4'-di((S)sec-butyl)-4,4',5,5'-tetrahydro-2,2'-bioxazole [(S,S)-L1] $(3.3 \mathrm{mg}, 0.013 \mathrm{mmol}, 13$ mol\%), $\mathrm{NiCl}_{2} \cdot \mathrm{Py}_{4}(4.5 \mathrm{mg}, 0.01 \mathrm{mmol}, 10 \mathrm{~mol} \%), \operatorname{Ir}\left[\mathrm{dF}\left(\mathrm{CF}_{3}\right) \mathrm{ppy}\right]_{2}(\mathrm{bpy}) \mathrm{PF}_{6}(3.2 \mathrm{mg}$, $0.003 \mathrm{mmol}, 3 \mathrm{~mol} \%$ ), 4-bromo-2-(trifluoromethyl)pyridine (13.7 uL, $0.1 \mathrm{mmol}, 1.0$ equiv.), ${ }^{t} \mathrm{BuBF}_{3} \mathrm{~K}$ (41.0 mg, $0.25 \mathrm{mmol}, 2.5$ equiv.) and $N$-(tert-butyl)acrylamide (25.4 $\mathrm{mg}, 0.2 \mathrm{mmol}, 2.0$ equiv.) in acetone/ethyl acetate $(1.3 / 0.7, \mathrm{v} / \mathrm{v}, 2 \mathrm{~mL})$ were used. After 24 hours, the product was isolated by flash chromatography (PE: ethyl acetate $=10: 1)$ as a white solid (26.9 mg, $81 \%$ yield, $61 \%$ ee).

${ }^{1}$ H NMR $\left(400 \mathrm{MHz}, \mathrm{CDCl}_{3}\right) \delta 8.63(\mathrm{~d}, J=5.0 \mathrm{~Hz}, 1 \mathrm{H}), 7.62(\mathrm{~s}, 1 \mathrm{H}), 7.48(\mathrm{~d}, J=5.0$ $\mathrm{Hz}, 1 \mathrm{H}), 5.34$ (s, 1H), 3.29 (dd, $J=8.8,3.4 \mathrm{~Hz}, 1 \mathrm{H}), 2.37(\mathrm{dd}, J=14.0,8.9 \mathrm{~Hz}, 1 \mathrm{H})$, $1.41(\mathrm{dd}, J=14.0,3.5 \mathrm{~Hz}, 1 \mathrm{H}), 1.30(\mathrm{~s}, 9 \mathrm{H}), 0.93$ (s, 9H).

${ }^{19}$ F NMR $\left(375 \mathrm{MHz}, \mathrm{CDCl}_{3}\right) \delta-67.91(\mathrm{~s})$.

${ }^{13}$ C NMR (101 MHz, $\left.\mathrm{CDCl}_{3}\right) \delta 170.48,153.32,150.22,148.50(\mathrm{q}, \mathrm{J}=34.4 \mathrm{~Hz}), 125.26$, 121.52 (q, $J=274.2 \mathrm{~Hz}), 119.55(\mathrm{q}, J=2.7 \mathrm{~Hz}), 51.68,50.39,47.39,31.22,29.49$, 28.51 .

HRMS (ESI): $\mathrm{C}_{17} \mathrm{H}_{26} \mathrm{~F}_{3} \mathrm{~N}_{2} \mathrm{O}^{+}(\mathrm{M}+\mathrm{H}): 331.1997$, found: 331.2001 .

$[\alpha]_{\mathrm{D}}^{25}=-15.6591\left(\mathrm{c}=0.2267, \mathrm{CHCl}_{3}\right)$

Chiral HPLC: CHIRALPAK IA, $25{ }^{\circ} \mathrm{C},{ }^{i} \mathrm{PrOH}-$ hexanes 5/95, $1.0 \mathrm{~mL} / \mathrm{min}, 254 \mathrm{~nm}, \mathrm{t}_{\mathrm{R}}$ (major) $=8.8 \mathrm{~min}, \mathrm{t}_{\mathrm{R}}($ minor $)=6.7 \mathrm{~min}$.

\section{3 racemic}

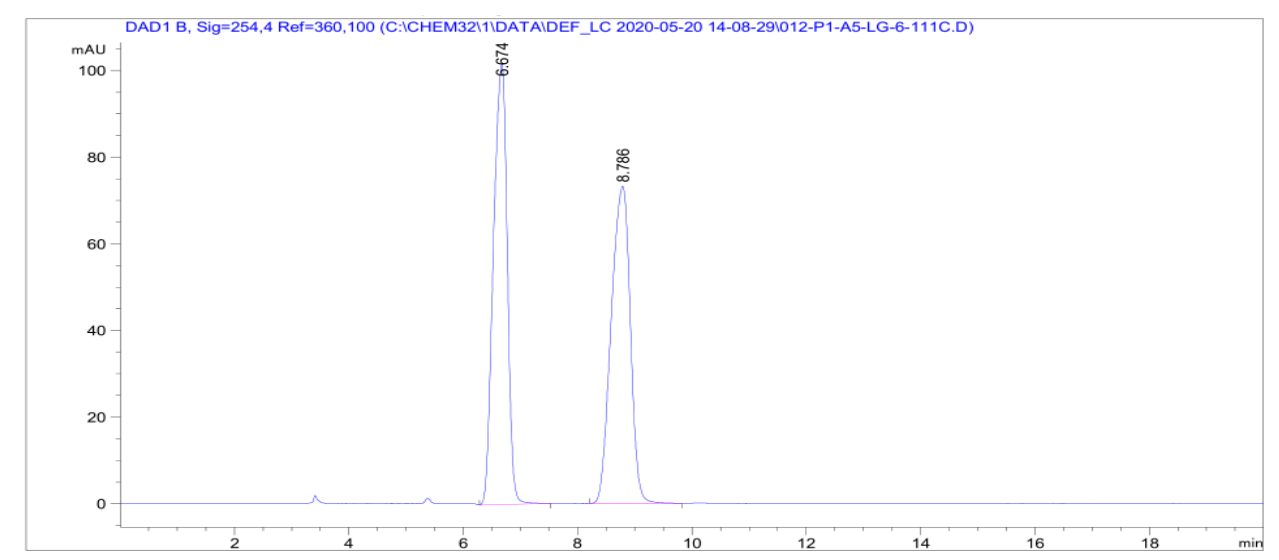




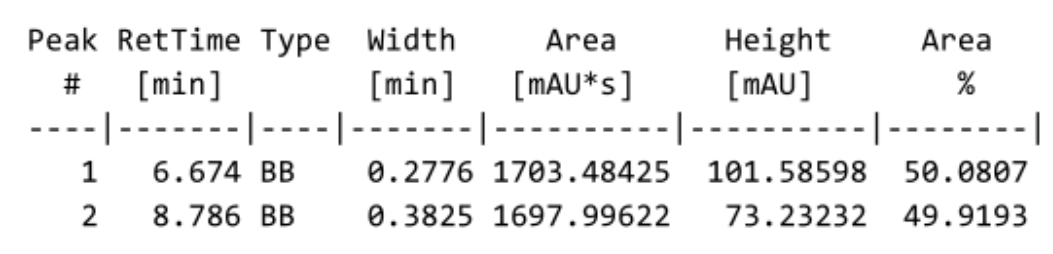

\section{3 enantioenriched, $61 \%$ ee}

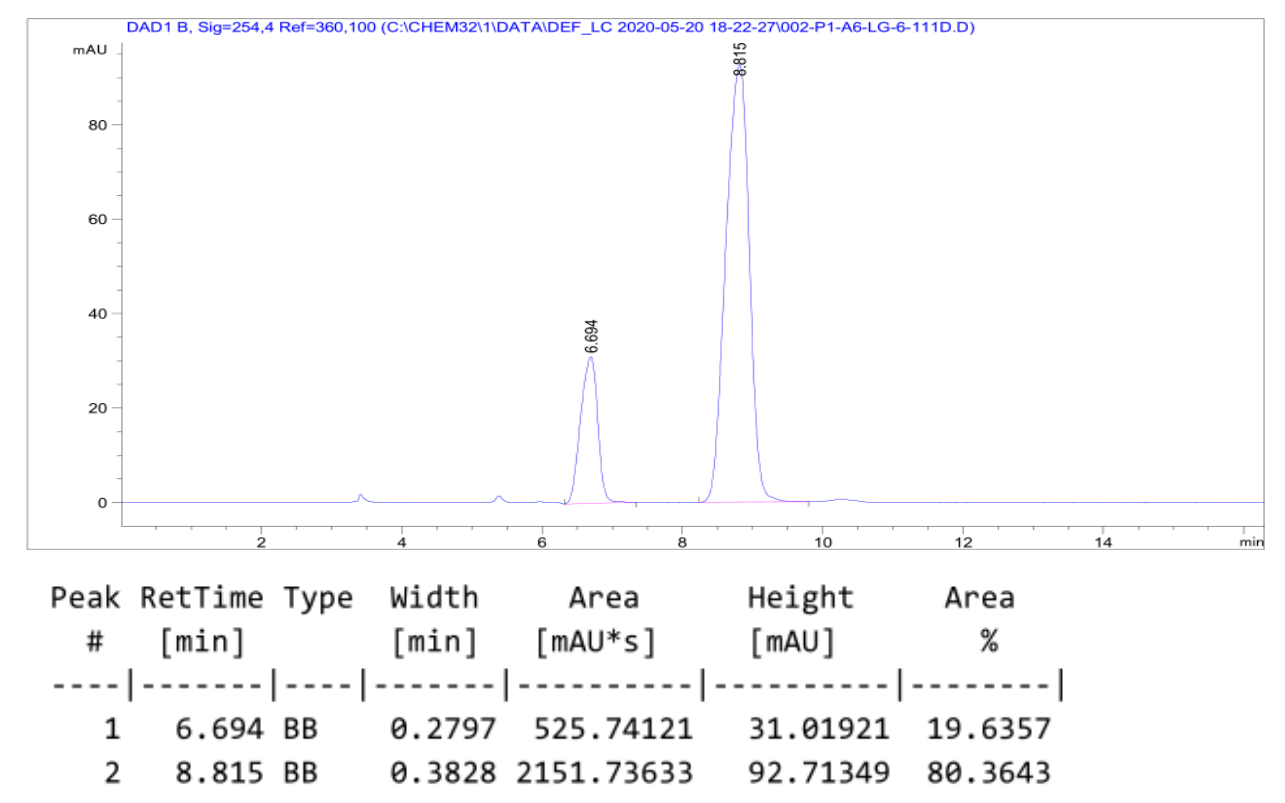<smiles>CCC(=O)[C@H](CC(C)(C)C)c1ccnc(C(F)(F)F)c1</smiles>

\section{(R)-6,6-Dimethyl-4-(2-(trifluoromethyl)pyridin-4-yl)heptan-3-one (54)}

The product was prepared according to the general procedure with (4S,4'S)-4,4'-di((S)sec-butyl)-4,4',5,5'-tetrahydro-2,2'-bioxazole [(S,S)-L1] $(3.3 \mathrm{mg}, 0.013 \mathrm{mmol}, 13$ mol\%), $\mathrm{NiCl}_{2} \cdot \mathrm{Py}_{4}(4.5 \mathrm{mg}, 0.01 \mathrm{mmol}, 10 \mathrm{~mol} \%), \operatorname{Ir}\left[\mathrm{dF}\left(\mathrm{CF}_{3}\right) \mathrm{ppy}\right]_{2}(\mathrm{bpy}) \mathrm{PF}_{6}(3.2 \mathrm{mg}$, $0.003 \mathrm{mmol}, 3 \mathrm{~mol} \%$ ), 4-bromo-2-(trifluoromethyl)pyridine (13.7 uL, $0.1 \mathrm{mmol}, 1.0$ equiv.), ${ }^{t} \mathrm{BuBF}_{3} \mathrm{~K}$ (41.0 mg, $0.25 \mathrm{mmol}, 2.5$ equiv.) and pent-1-en-3-one (19.8 uL, 0.2 mmol, 2.0 equiv.) in acetone/ethyl acetate (1.3/0.7, v/v, $2 \mathrm{~mL})$ were used. After 24 hours, the product was isolated by flash chromatography (PE: ethyl acetate $=20: 1$ ) as a colorless oil (12.1 mg, 42\% yield, $64 \%$ ee). 
${ }^{1} \mathbf{H}$ NMR $\left(400 \mathrm{MHz}, \mathrm{CDCl}_{3}\right) \delta 8.64(\mathrm{~d}, J=4.9 \mathrm{~Hz}, 1 \mathrm{H}), 7.57(\mathrm{~s}, 1 \mathrm{H}), 7.39(\mathrm{~d}, J=4.9$ $\mathrm{Hz}, 1 \mathrm{H}), 3.88$ (dd, $J=7.5,4.6 \mathrm{~Hz}, 1 \mathrm{H}), 2.61-2.40$ (m, 2H), 2.35 (dd, $J=14.1,7.6 \mathrm{~Hz}$, $1 \mathrm{H}), 1.50(\mathrm{dd}, J=14.1,4.6 \mathrm{~Hz}, 1 \mathrm{H}), 1.01(\mathrm{t}, J=7.2 \mathrm{~Hz}, 3 \mathrm{H}), 0.86(\mathrm{~s}, 9 \mathrm{H})$.

${ }^{19}$ F NMR $\left(375 \mathrm{MHz}, \mathrm{CDCl}_{3}\right) \delta-67.95$ (s).

${ }^{13}$ C NMR (100 MHz, $\left.\mathrm{CDCl}_{3}\right) \delta 208.70,151.74,150.31,148.73(\mathrm{q}, J=34.2 \mathrm{~Hz}), 125.96$, 121.55 (q, $J=274.4 \mathrm{~Hz}), 120.12$ (q, $J=2.9 \mathrm{~Hz}), 54.59$, 46.27, 36.03, 31.22, 29.62, 7.79. HRMS (ESI): $\mathrm{C}_{15} \mathrm{H}_{21} \mathrm{~F}_{3} \mathrm{NO}^{+}(\mathrm{M}+\mathrm{H}): 288.1575$, found: 288.1574 .

$[\alpha]_{\mathrm{D}}^{25}=-81.9949\left(\mathrm{c}=0.1733, \mathrm{CHCl}_{3}\right)$

Chiral HPLC: CHIRALPAK IC, $25{ }^{\circ} \mathrm{C},{ }^{i}$ PrOH-hexanes 5/95, $0.5 \mathrm{~mL} / \mathrm{min}, 254 \mathrm{~nm}, \mathrm{t}_{\mathrm{R}}$ (major) $=14.3 \mathrm{~min}, \mathrm{t}_{\mathrm{R}}($ minor $)=9.9 \mathrm{~min}$.

\section{4 racemic}
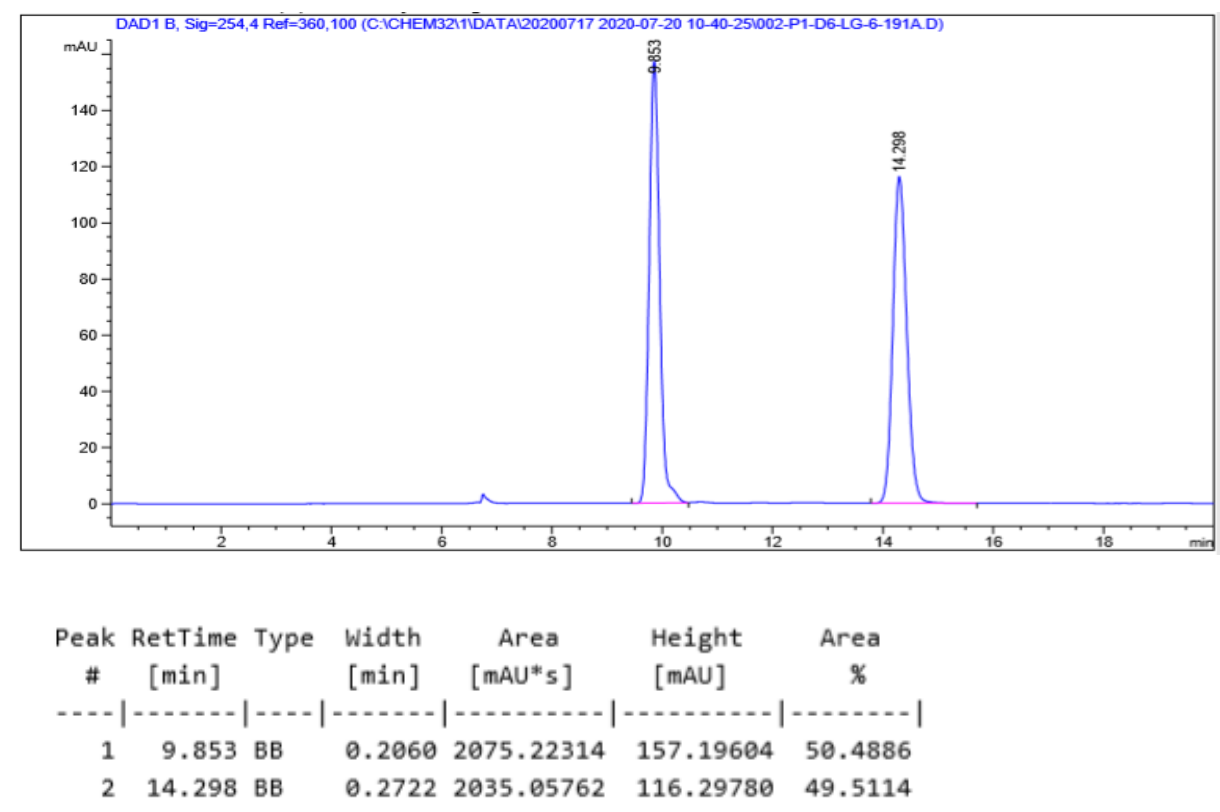


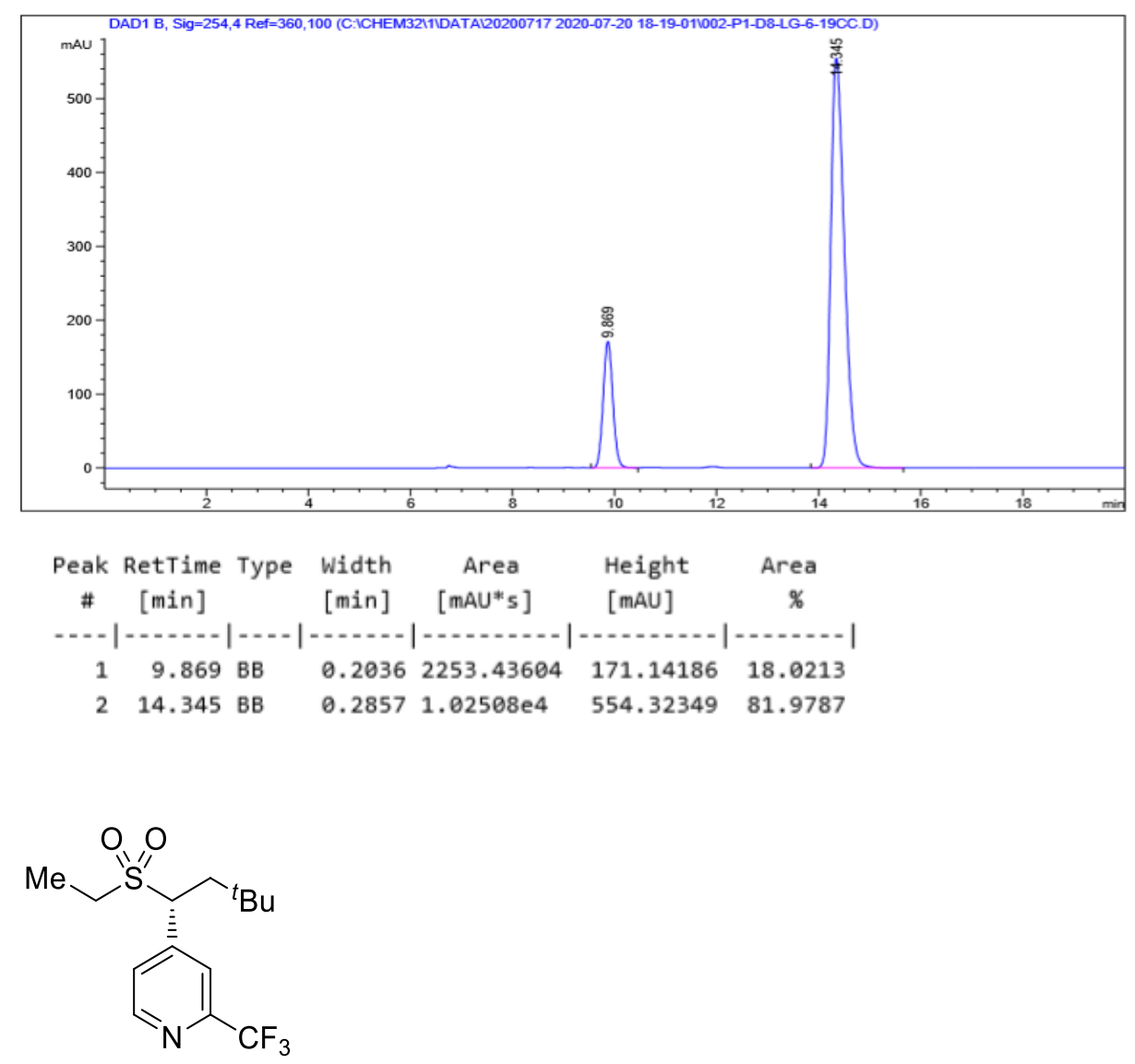

(R)-4-(1-(Ethylsulfonyl)-3,3-dimethylbutyl)-2-(trifluoromethyl)pyridine (55)

The product was prepared according to the general procedure with (4S,4'S)-4,4'-di((S)sec-butyl)-4,4',5,5'-tetrahydro-2,2'-bioxazole [(S,S)-L1] (3.3 mg, $0.013 \mathrm{mmol}, 13$ mol\%), $\mathrm{NiCl}_{2} \bullet \mathrm{Py}_{4}(4.5 \mathrm{mg}, 0.01 \mathrm{mmol}, 10 \mathrm{~mol} \%), \operatorname{Ir}\left[\mathrm{dF}\left(\mathrm{CF}_{3}\right) \mathrm{ppy}\right]_{2}(\mathrm{bpy}) \mathrm{PF}_{6}(3.2 \mathrm{mg}$, $0.003 \mathrm{mmol}, 3 \mathrm{~mol} \%$ ), 4-bromo-2-(trifluoromethyl)pyridine (13.7 uL, $0.1 \mathrm{mmol}, 1.0$ equiv.), ${ }^{t} \mathrm{BuBF}_{3} \mathrm{~K}$ (41.0 mg, $0.25 \mathrm{mmol}, 2.5$ equiv.) and (ethylsulfonyl)ethene (20.9 uL, $0.2 \mathrm{mmol}, 2.0$ equiv.) in acetone/ethyl acetate (1.3/0.7, v/v, $2 \mathrm{~mL})$ were used. After 24 hours, the product was isolated by flash chromatography (PE: ethyl acetate $=10: 1)$ as a colorless oil ( $26.6 \mathrm{mg}, 82 \%$ yield, $90 \%$ ee).

${ }^{1} \mathbf{H}$ NMR $\left(400 \mathrm{MHz}, \mathrm{CDCl}_{3}\right) \delta 8.78(\mathrm{~d}, J=5.0 \mathrm{~Hz}, 1 \mathrm{H}), 7.78(\mathrm{~s}, 1 \mathrm{H}), 7.64(\mathrm{~d}, J=4.8$ $\mathrm{Hz}, 1 \mathrm{H}), 4.15(\mathrm{~d}, J=9.5 \mathrm{~Hz}, 1 \mathrm{H}), 2.82-2.70(\mathrm{~m}, 2 \mathrm{H}), 2.42(\mathrm{dd}, J=14.4,1.3 \mathrm{~Hz}, 1 \mathrm{H})$, 2.07 (dd, $J=14.4,10.3 \mathrm{~Hz}, 1 \mathrm{H}), 1.34$ (t, $J=7.5 \mathrm{~Hz}, 3 \mathrm{H}), 0.84$ (s, 9H).

${ }^{19}$ F NMR (377 MHz, $\left.\mathrm{CDCl}_{3}\right) \delta-67.91(\mathrm{~s})$. 
${ }^{13}$ C NMR (100 MHz, $\left.\mathrm{CDCl}_{3}\right) \delta 150.71,149.13(\mathrm{q}, J=35.0 \mathrm{~Hz}), 146.45,127.33,121.34$ (q, $J=2.7 \mathrm{~Hz}), 121.20$ (q, $J=274.5 \mathrm{~Hz}), 64.60,44.94,40.82,31.41,29.72,6.05$.

HRMS (ESI): $\mathrm{C}_{14} \mathrm{H}_{21} \mathrm{~F}_{3} \mathrm{NO}_{2} \mathrm{~S}^{+}(\mathrm{M}+\mathrm{H}): 324.1245$, found: 324.1246 .

$[\alpha]_{\mathrm{D}}^{25}=+21.3942\left(\mathrm{c}=0.4833, \mathrm{CHCl}_{3}\right)$

Chiral HPLC: CHIRALPAK IA, $25{ }^{\circ} \mathrm{C},{ }^{i} \mathrm{PrOH}-$ hexanes 5/95, $1.0 \mathrm{~mL} / \mathrm{min}, 254 \mathrm{~nm}, \mathrm{t}_{\mathrm{R}}$ (major) $=9.5 \mathrm{~min}, \mathrm{t}_{\mathrm{R}}($ minor $)=12.1 \mathrm{~min}$.

\section{5 racemic}

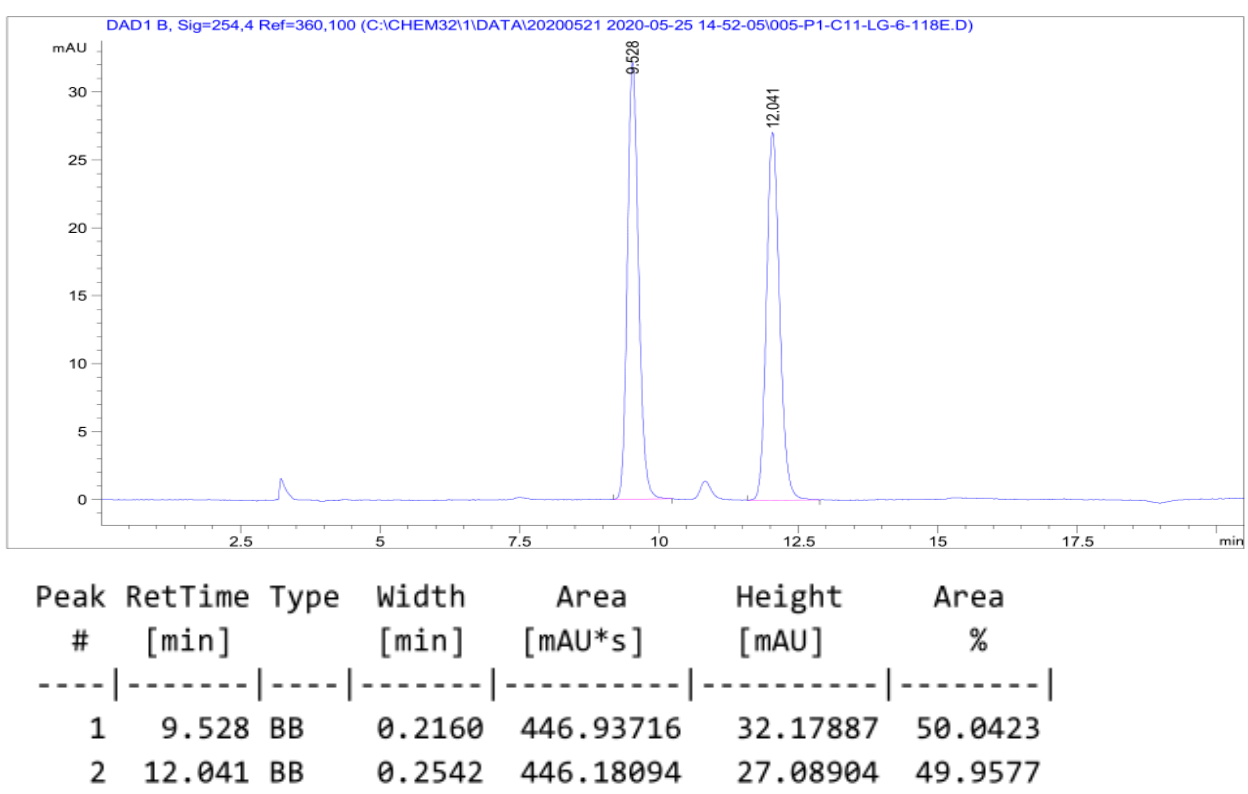

\section{5 enantioenriched, $90 \%$ ee}

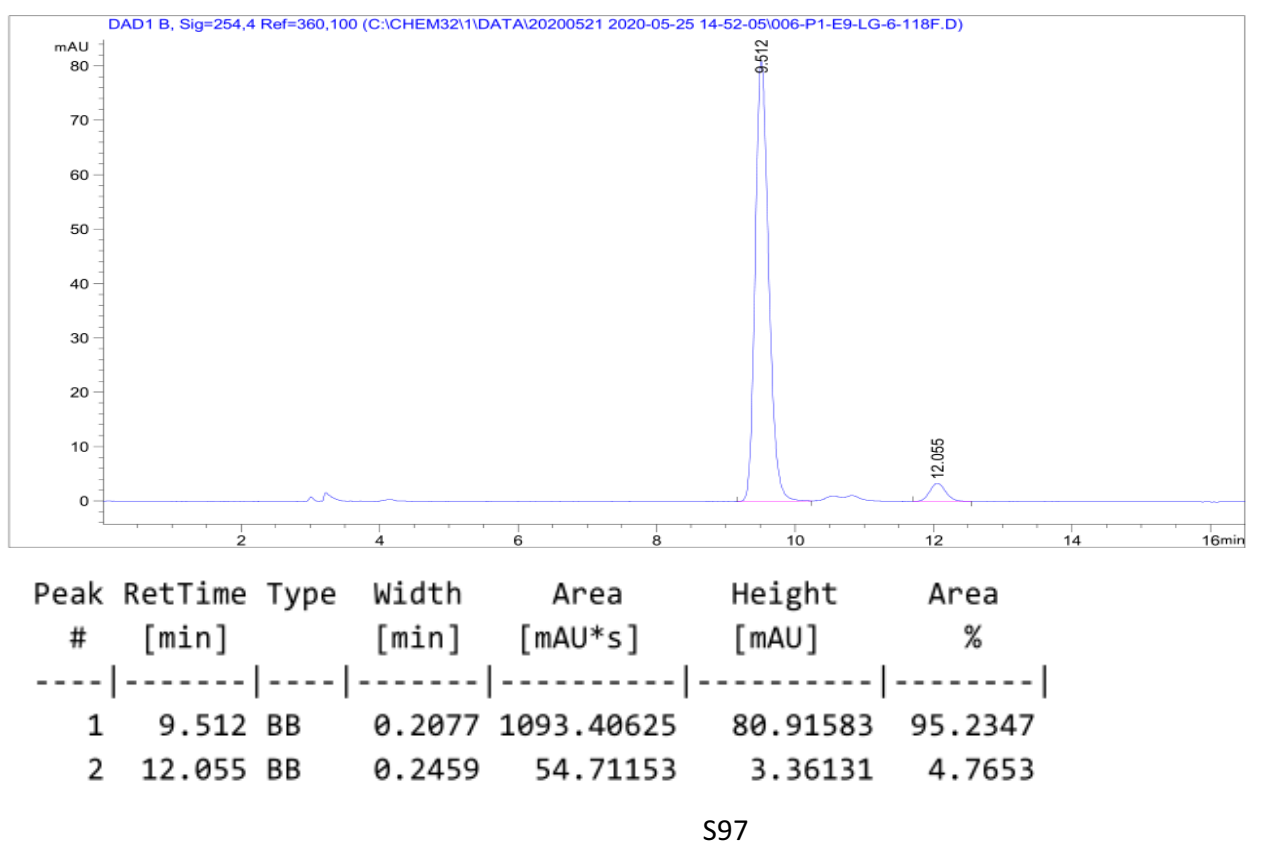


<smiles>O=S(=O)(c1ccccc1)[C@H](CCBr)c1ccnc(C(F)(F)F)c1</smiles>

(R)-4-(3,3-Dimethyl-1-(phenylsulfonyl)butyl)-2-(trifluoromethyl)pyridine (56)

The product was prepared according to the general procedure with $(4 \mathrm{~S}, 4$ 'S)-4,4'-di((S)sec-butyl)-4,4',5,5'-tetrahydro-2,2'-bioxazole [(S,S)-L1] $(3.3 \mathrm{mg}, 0.013 \mathrm{mmol}, 13$

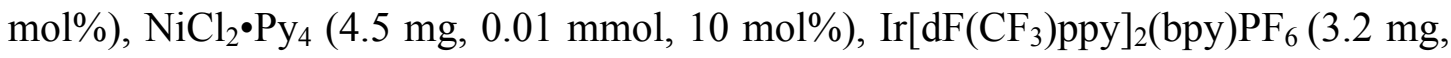
$0.003 \mathrm{mmol}, 3 \mathrm{~mol} \%$ ), 4-bromo-2-(trifluoromethyl)pyridine (13.7 uL, $0.1 \mathrm{mmol}, 1.0$ equiv.), ${ }^{t} \mathrm{BuBF}_{3} \mathrm{~K}$ (41.0 mg, $0.25 \mathrm{mmol}, 2.5$ equiv.) and (vinylsulfonyl)benzene (33.6 $\mathrm{mg}, 0.2 \mathrm{mmol}, 2.0$ equiv.) in acetone/ethyl acetate $(1.3 / 0.7, \mathrm{v} / \mathrm{v}, 2 \mathrm{~mL})$ were used. After 24 hours, the product was isolated by flash chromatography $(\mathrm{PE}$ : ethyl acetate $=10: 1)$ as a white solid (30.0mg, $81 \%$ yield, $81 \%$ ee).

${ }^{1}$ H NMR $\left(400 \mathrm{MHz}, \mathrm{CDCl}_{3}\right) \delta 8.63(\mathrm{~d}, J=5.0 \mathrm{~Hz}, 1 \mathrm{H})$, 7.64-7.60 (m, 1H), 7.54-7.52 (m, 2H), 7.44 (t, $J=7.8 \mathrm{~Hz}, 2 \mathrm{H}), 7.38$ (d, $J=4.3 \mathrm{~Hz}, 1 \mathrm{H}), 7.29$ (br, 1H), 4.14 (dd, $J=$ 10.3, $1.4 \mathrm{~Hz}, 1 \mathrm{H}), 2.48$ (dd, $J=14.4,1.6 \mathrm{~Hz}, 1 \mathrm{H}), 2.10$ (dd, $J=14.4,10.4 \mathrm{~Hz}, 1 \mathrm{H})$, $0.79(\mathrm{~s}, 9 \mathrm{H})$.

${ }^{19}$ F NMR $\left(375 \mathrm{MHz}, \mathrm{CDCl}_{3}\right) \delta-68.08(\mathrm{~s})$.

${ }^{13}$ C NMR (101 MHz, $\left.\mathrm{CDCl}_{3}\right) \delta 150.11,148.45$ (q, $\left.J=34.9 \mathrm{~Hz}\right), 146.01,136.19,134.26$, 129.09, 128.97, 127.42, 121.65 (q, $J=2.4 \mathrm{~Hz}), 121.11(\mathrm{q}, J=274.5 \mathrm{~Hz}), 68.49,40.39$, $31.36,29.68$.

HRMS (ESI): $\mathrm{C}_{18} \mathrm{H}_{21} \mathrm{~F}_{3} \mathrm{NO}_{2} \mathrm{~S}^{+}(\mathrm{M}+\mathrm{H}): 372.1245$, found: 372.1247 .

$[\alpha]_{\mathrm{D}}^{25}=+50.6996\left(\mathrm{c}=0.5067, \mathrm{CHCl}_{3}\right)$

Chiral HPLC: CHIRALPAK IB, $25{ }^{\circ} \mathrm{C},{ }^{i} \mathrm{PrOH}$-hexanes 5/95, $1.0 \mathrm{~mL} / \mathrm{min}, 254 \mathrm{~nm}, \mathrm{t}_{\mathrm{R}}$ (major $)=19.0 \mathrm{~min}, \mathrm{t}_{\mathrm{R}}($ minor $)=17.8 \mathrm{~min}$. 


\section{6 racemic}

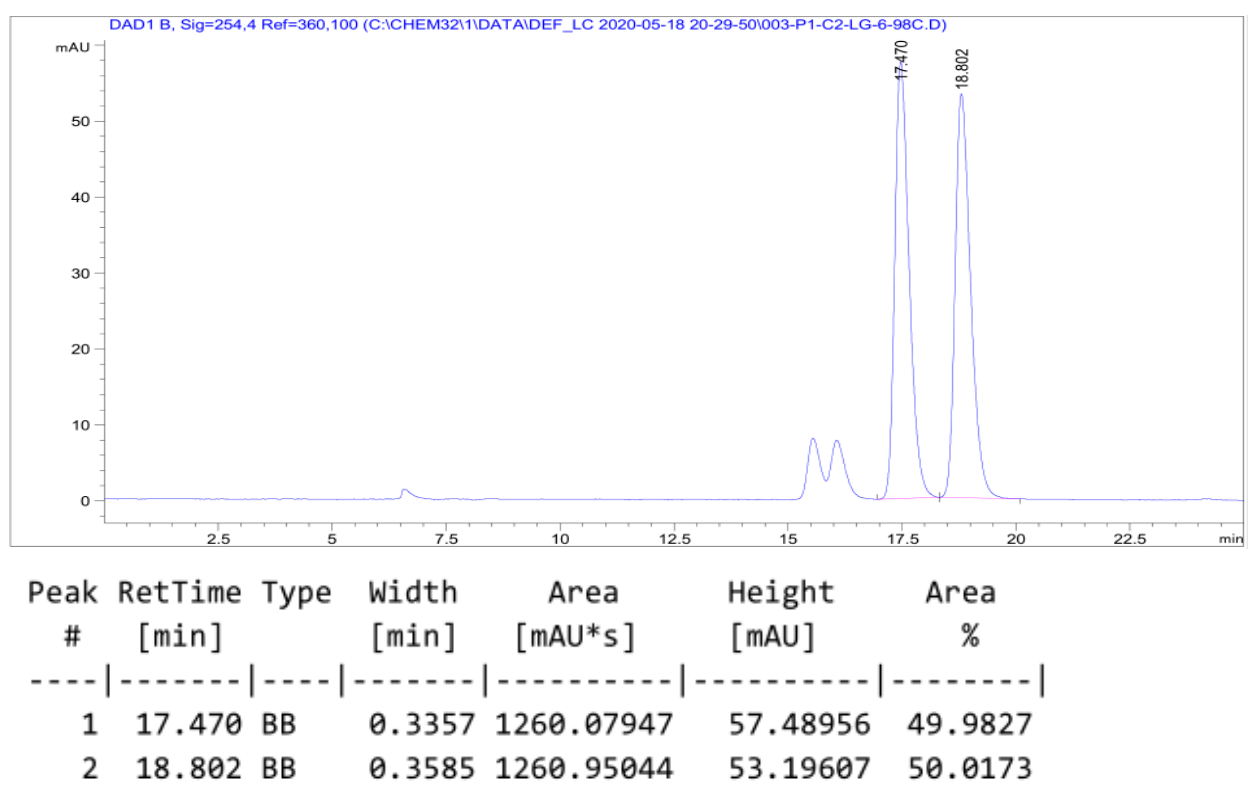

56 enantioenriched, $81 \%$ ee

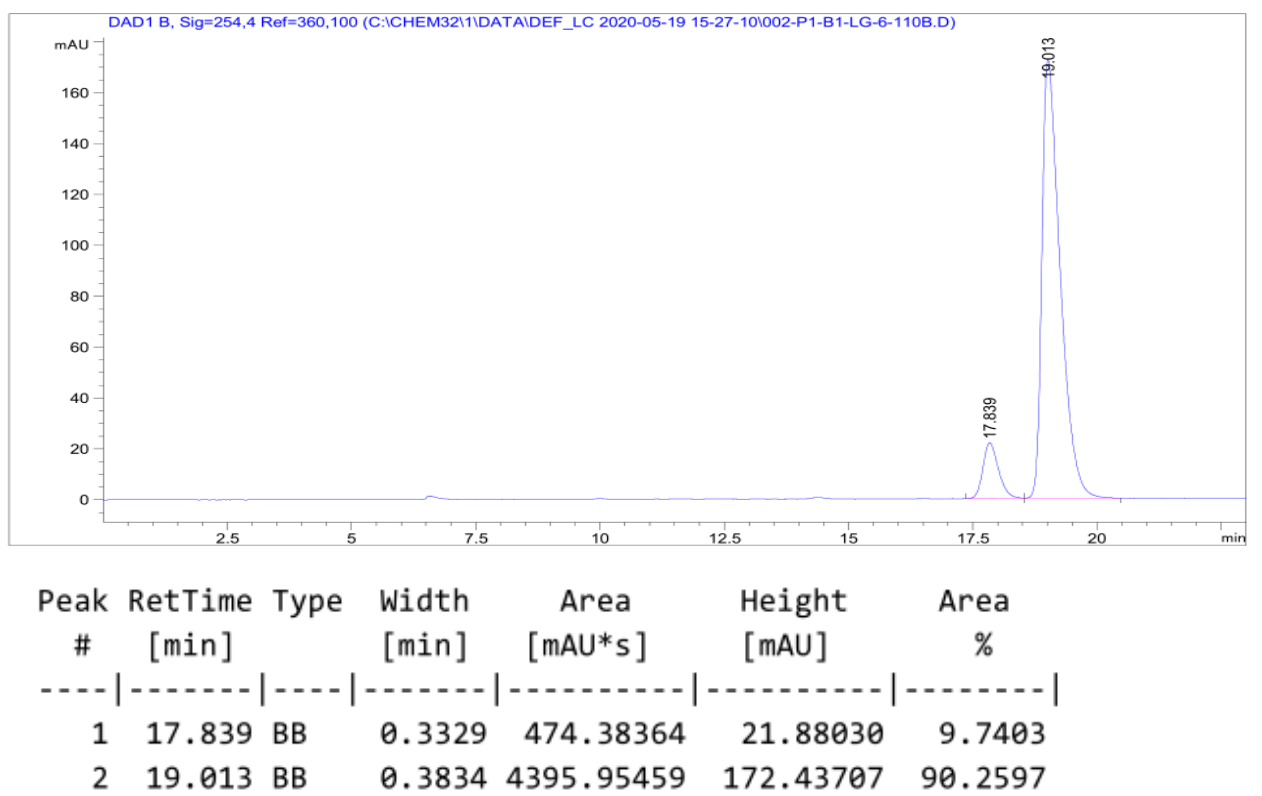<smiles>CCOC(=O)P(CCC(C)(C)C)[C@H](CC(C)(C)C)c1ccnc(C(F)(F)F)c1</smiles>

(R)-Diethyl (3,3-dimethyl-1-(2-(trifluoromethyl)pyridin-4-yl)butyl)phosphonate (57) 
The product was prepared according to the general procedure with (4S,4'S)-4,4'-di((S)sec-butyl)-4,4',5,5'-tetrahydro-2,2'-bioxazole [(S,S)-L1] $(3.3 \mathrm{mg}, 0.013 \mathrm{mmol}, 13$ mol\%), $\mathrm{NiCl}_{2} \cdot \mathrm{Py}_{4}(4.5 \mathrm{mg}, 0.01 \mathrm{mmol}, 10 \mathrm{~mol} \%), \operatorname{Ir}\left[\mathrm{dF}\left(\mathrm{CF}_{3}\right) \mathrm{ppy}\right]_{2}(\mathrm{bpy}) \mathrm{PF}_{6}(3.2 \mathrm{mg}$, $0.003 \mathrm{mmol}, 3 \mathrm{~mol} \%$ ), 4-bromo-2-(trifluoromethyl)pyridine (13.7 uL, $0.1 \mathrm{mmol}, 1.0$ equiv.), ${ }^{t} \mathrm{BuBF}_{3} \mathrm{~K}$ (41.0 mg, $0.25 \mathrm{mmol}, 2.5$ equiv.) and diethyl vinylphosphonate (30.7 $\mathrm{uL}, 0.2 \mathrm{mmol}, 2.0$ equiv.) in acetone/ethyl acetate $(1.3 / 0.7, \mathrm{v} / \mathrm{v}, 2 \mathrm{~mL})$ were used. After 24 hours, the product was isolated by flash chromatography (PE: ethyl acetate $=10: 1)$ as a white solid (32.7 $\mathrm{mg}, 89 \%$ yield, $91 \%$ ee).

${ }^{1} \mathbf{H}$ NMR $\left(400 \mathrm{MHz}, \mathrm{CDCl}_{3}\right) \delta 8.65(\mathrm{~d}, J=5.0 \mathrm{~Hz}, 1 \mathrm{H}), 7.66(\mathrm{br}, 1 \mathrm{H}), 7.51(\mathrm{~d}, J=4.7$ $\mathrm{Hz}, 1 \mathrm{H}), 4.14-4.03$ (m, 2H), 4.01-3.93 (m, 1H), 3.8892-3.83 (m, 1H), 3.21 (dd, $J=25.3$, $9.1 \mathrm{~Hz}, 1 \mathrm{H}), 2.12(\mathrm{t}, J=14.8 \mathrm{~Hz}, 1 \mathrm{H}), 2.01-1.94(\mathrm{~m}, 1 \mathrm{H}), 1.26(\mathrm{t}, J=7.0 \mathrm{~Hz}, 3 \mathrm{H}), 1.16$ ( $\mathrm{t}, J=7.0 \mathrm{~Hz}, 3 \mathrm{H}), 0.79(\mathrm{~s}, 9 \mathrm{H})$.

${ }^{19}$ F NMR (375 MHz, $\left.\mathrm{CDCl}_{3}\right) \delta-67.98(\mathrm{~s})$.

${ }^{13}$ C NMR (101 MHz, $\left.\mathrm{CDCl}_{3}\right) \delta 150.38(\mathrm{~d}, J=7.5 \mathrm{~Hz}), 149.90(\mathrm{~d}, J=2.6 \mathrm{~Hz}), 148.30$ (q, $J=34.4 \mathrm{~Hz}), 127.26(\mathrm{~d}, J=6.4 \mathrm{~Hz}), 121.53(\mathrm{q}, J=274.1 \mathrm{~Hz}), 121.41(\mathrm{q}, J=3.1$ Hz), $62.86(\mathrm{~d}, J=7.2 \mathrm{~Hz}), 62.53(\mathrm{~d}, J=7.3 \mathrm{~Hz}), 42.35$ (d, $J=4.3 \mathrm{~Hz}), 41.60(\mathrm{~d}, J=$ $135.1 \mathrm{~Hz}), 32.00$ (d, $J=13.9 \mathrm{~Hz}), 29.67,16.35$ (d, $J=6.1 \mathrm{~Hz}), 16.19$ (d, $J=5.8 \mathrm{~Hz})$.

HRMS (ESI): $\mathrm{C}_{16} \mathrm{H}_{26} \mathrm{~F}_{3} \mathrm{NO}_{3} \mathrm{P}^{+}(\mathrm{M}+\mathrm{H})$ : 368.1602, found: 368.1659 .

$[\alpha]_{\mathrm{D}}^{25}=-5.0891\left(\mathrm{c}=0.2633, \mathrm{CHCl}_{3}\right)$

Chiral HPLC: CHIRALPAK IA, $25{ }^{\circ} \mathrm{C},{ }^{i} \mathrm{PrOH}-h$ exanes 5/95, $1.0 \mathrm{~mL} / \mathrm{min}, 254 \mathrm{~nm}, \mathrm{t}_{\mathrm{R}}$ (major) $=8.9 \mathrm{~min}, \mathrm{t}_{\mathrm{R}}($ minor $)=10.1 \mathrm{~min}$.

\section{7 racemic}

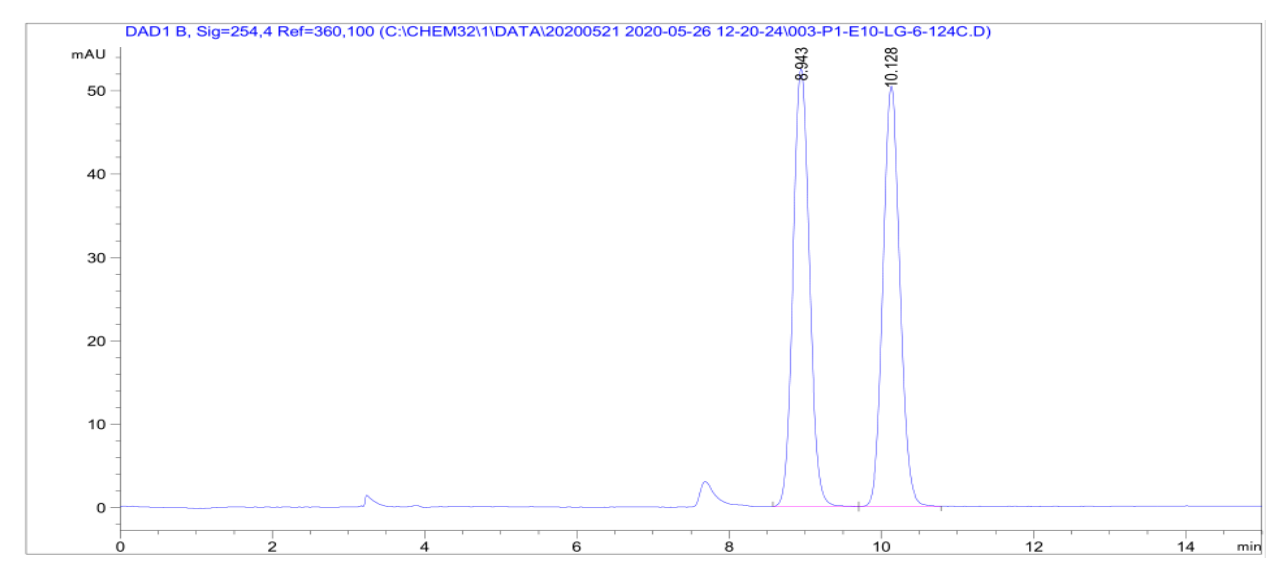




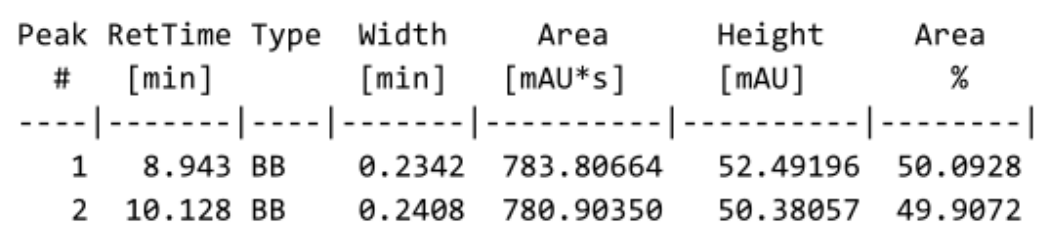

\section{7 enantioenriched, $91 \%$ ee}

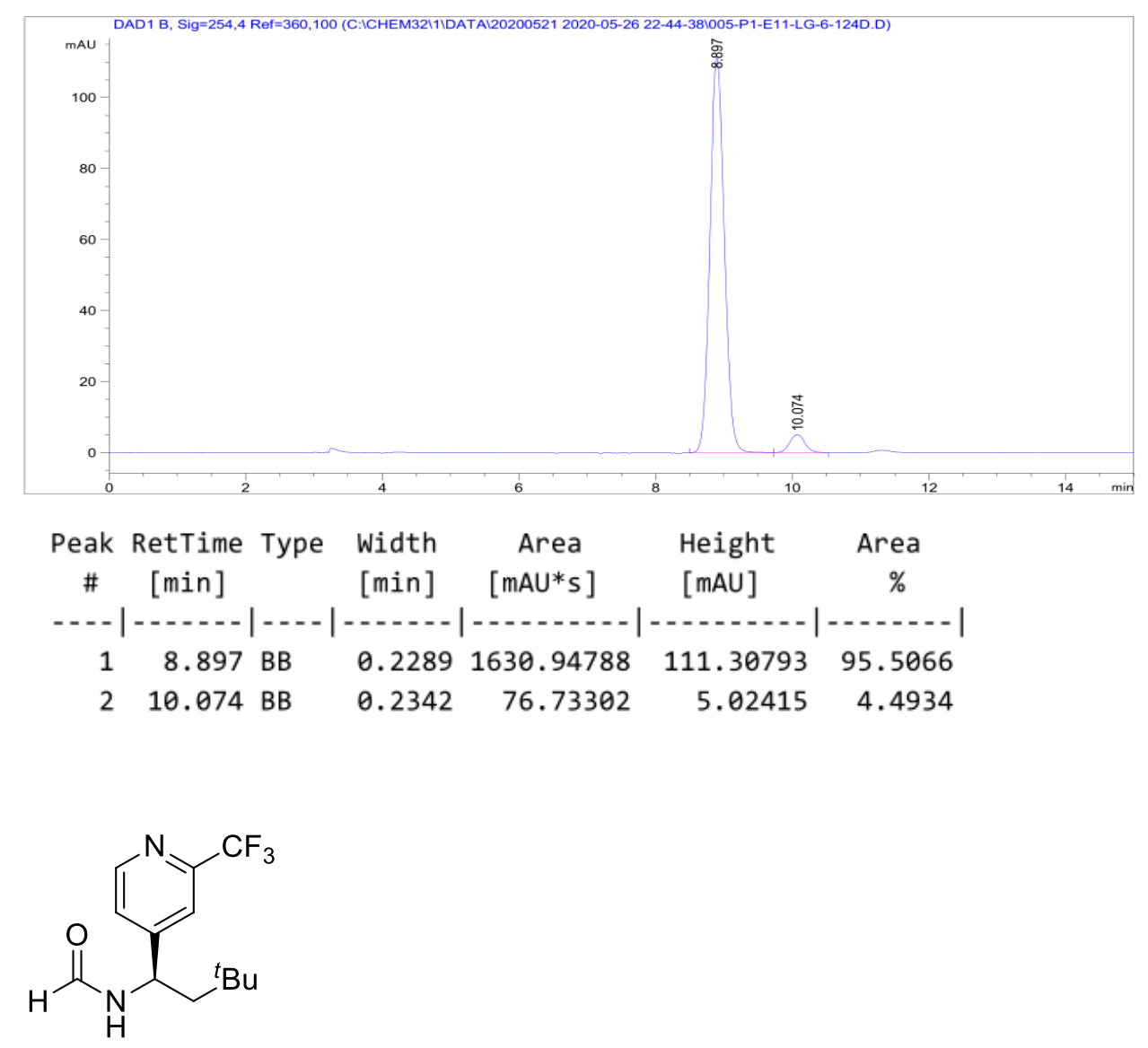

(R)-N-(3,3-Dimethyl-1-(2-(trifluoromethyl)pyridin-4-yl)butyl)formamide $\quad$ 58)

The product was prepared according to the general procedure with (4S,4'S)-4,4'-di((S)sec-butyl)-4,4',5,5'-tetrahydro-2,2'-bioxazole $[(S, S)$-L1 $]$ (3.3 mg, $0.013 \mathrm{mmol}, 13$ mol\%), $\mathrm{NiCl}_{2} \cdot \mathrm{Py}_{4}(4.5 \mathrm{mg}, 0.01 \mathrm{mmol}, 10 \mathrm{~mol} \%), \operatorname{Ir}\left[\mathrm{dF}\left(\mathrm{CF}_{3}\right) \mathrm{ppy}\right]_{2}(\mathrm{bpy}) \mathrm{PF}_{6}(3.2 \mathrm{mg}$, $0.003 \mathrm{mmol}, 3 \mathrm{~mol} \%$ ), 4-bromo-2-(trifluoromethyl)pyridine (13.7 uL, $0.1 \mathrm{mmol}, 1.0$ equiv.), ${ }^{t} \mathrm{BuBF}_{3} \mathrm{~K}$ (41.0 mg, $0.25 \mathrm{mmol}, 2.5$ equiv.) and $N$-vinylformamide (14.0 uL, $0.2 \mathrm{mmol}, 2.0$ equiv.) in acetone/ethyl acetate (1.3/0.7, v/v, $2 \mathrm{~mL})$ were used. After 24 hours, the product was isolated by flash chromatography $(\mathrm{PE}$ : ethyl acetate $=10: 1)$ as a colorless oil (20.8 mg, 76\% yield, $89 \%$ ee). 
${ }^{1}$ H NMR $\left(400 \mathrm{MHz}, \mathrm{CDCl}_{3}\right) \delta 8.67(\mathrm{~d}, J=5.0 \mathrm{~Hz}, 1 \mathrm{H}), 8.21(\mathrm{~s}, 1 \mathrm{H}), 7.58(\mathrm{br}, 1 \mathrm{H})$, $7.41(\mathrm{dd}, J=5.0,1.3 \mathrm{~Hz}, 1 \mathrm{H}), 5.95(\mathrm{~d}, J=7.2 \mathrm{~Hz}, 1 \mathrm{H}), 5.19(\mathrm{dd}, J=13.7,6.7 \mathrm{~Hz}, 1 \mathrm{H})$, $1.70(\mathrm{~d}, J=6.3 \mathrm{~Hz}, 2 \mathrm{H}), 1.02(\mathrm{~s}, 9 \mathrm{H})$.

${ }^{19}$ F NMR $\left(375 \mathrm{MHz}, \mathrm{CDCl}_{3}\right) \delta-67.94(\mathrm{~s})$.

${ }^{13}$ C NMR $\left(100 \mathrm{MHz}, \mathrm{CDCl}_{3}\right) \delta 160.30,154.73,150.41,148.78(\mathrm{q}, J=34.2 \mathrm{~Hz}), 124.14$, 121.46 (q, $J=274.6 \mathrm{~Hz}), 117.91(\mathrm{q}, J=2.8 \mathrm{~Hz}), 50.23,49.03,31.19,29.87$.

HRMS (ESI): $\mathrm{C}_{13} \mathrm{H}_{18} \mathrm{~F}_{3} \mathrm{~N}_{2} \mathrm{O}^{+}(\mathrm{M}+\mathrm{H}): 275.1371$, found: 275.1368 .

$[\alpha]_{\mathrm{D}}^{25}=56.9654\left(0.0567, \mathrm{CHCl}_{3}\right)$

Chiral HPLC: CHIRALPAK IA, $25{ }^{\circ} \mathrm{C},{ }^{i} \mathrm{PrOH}-$ hexanes 5/95, $0.5 \mathrm{~mL} / \mathrm{min}, 254 \mathrm{~nm}, \mathrm{t}_{\mathrm{R}}$ (major) $=9.8 \mathrm{~min}, \mathrm{t}_{\mathrm{R}}($ minor $)=8.7 \mathrm{~min}$.

\section{8 racemic}

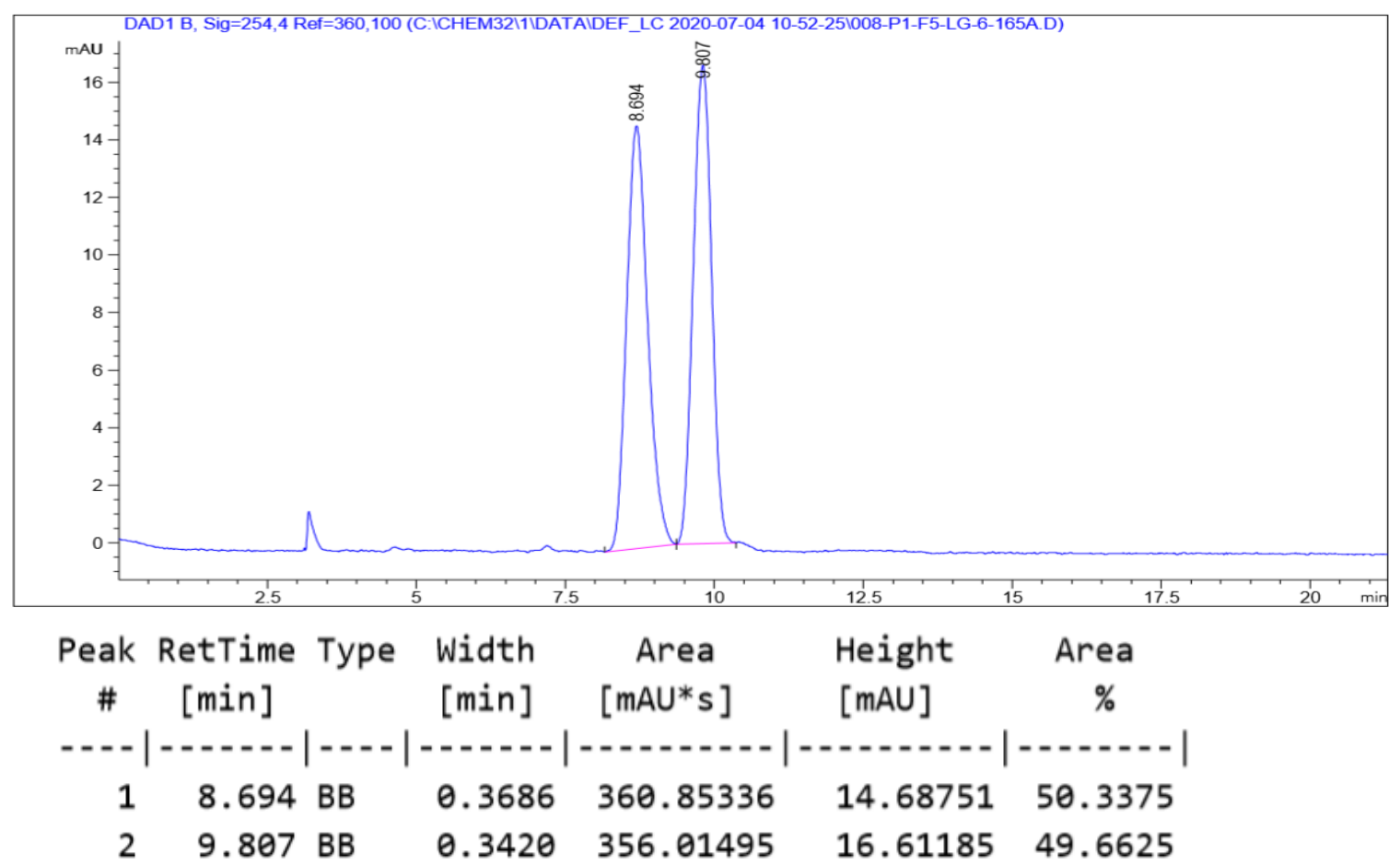



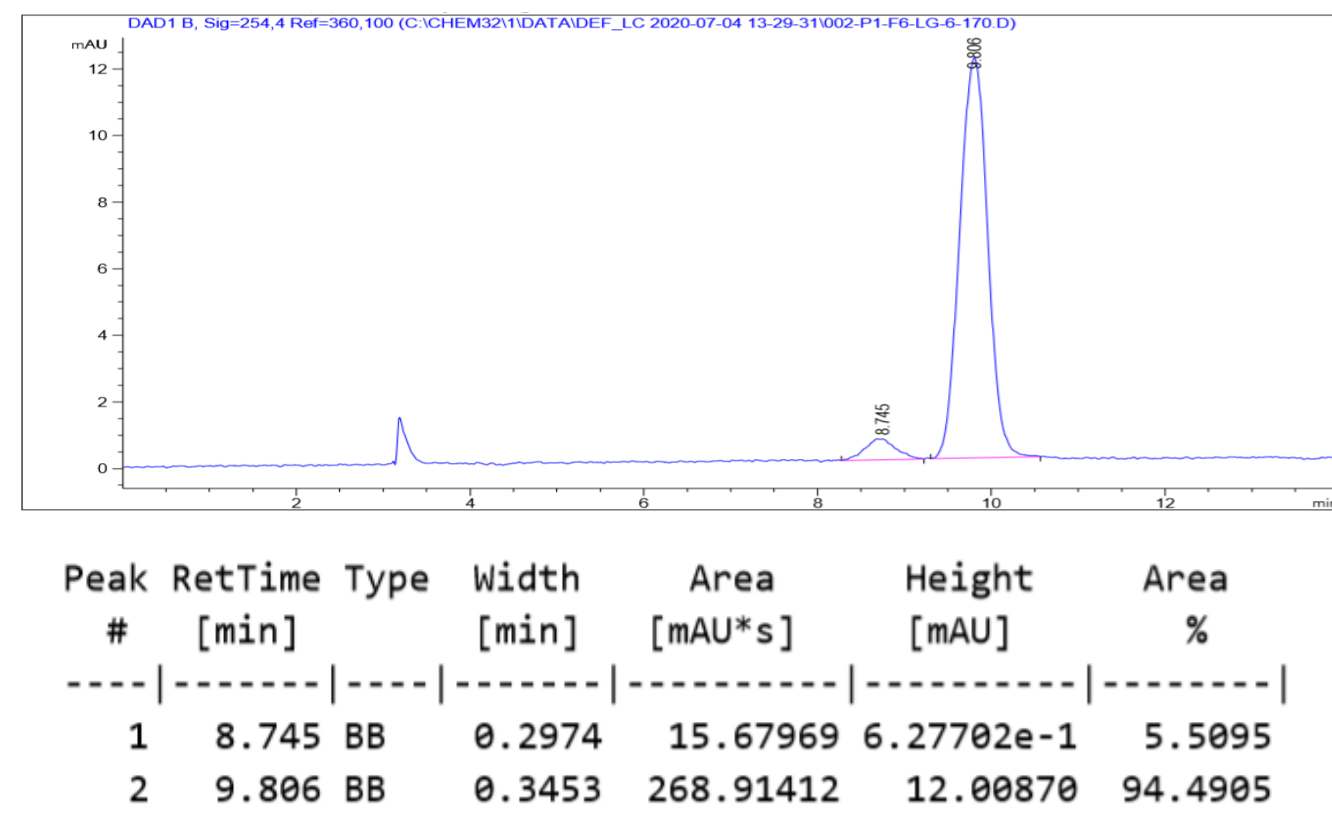<smiles>CC(=O)N[C@@H](CC(C)(C)C)c1ccnc(C(F)(F)F)c1</smiles>

(R)-N-(3,3-Dimethyl-1-(2-(trifluoromethyl)pyridin-4-yl)butyl)acetamide (59)

The product was prepared according to the general procedure with (4S,4'S)-4,4'-di((S)sec-butyl)-4,4',5,5'-tetrahydro-2,2'-bioxazole [(S,S)-L1] $(3.3 \mathrm{mg}, 0.013 \mathrm{mmol}, 13$ $\mathrm{mol} \%), \mathrm{NiCl}_{2} \bullet \mathrm{Py}_{4}(4.5 \mathrm{mg}, 0.01 \mathrm{mmol}, 10 \mathrm{~mol} \%), \operatorname{Ir}\left[\mathrm{dF}\left(\mathrm{CF}_{3}\right) \text { ppy }\right]_{2}(\mathrm{bpy}) \mathrm{PF}_{6}(3.2 \mathrm{mg}$, $0.003 \mathrm{mmol}, 3 \mathrm{~mol} \%$ ), 4-bromo-2-(trifluoromethyl)pyridine (13.7 uL, $0.1 \mathrm{mmol}, 1.0$ equiv.), ${ }^{t} \mathrm{BuBF}_{3} \mathrm{~K}$ (41.0 mg, $0.25 \mathrm{mmol}, 2.5$ equiv.) and $N$-vinylacetamide (17.0 mg, 0.2 mmol, 2.0 equiv.) in acetone/ethyl acetate (1.3/0.7, v/v, $2 \mathrm{~mL})$ were used. After 24 hours, the product was isolated by flash chromatography $(\mathrm{PE}$ : ethyl acetate $=4: 1)$ as a colorless oil (10.0 mg, 35\% yield, 90\% ee).

${ }^{1}$ H NMR $\left(400 \mathrm{MHz}, \mathrm{CDCl}_{3}\right) \delta 8.64(\mathrm{~d}, J=5.0 \mathrm{~Hz}, 1 \mathrm{H}), 7.55(\mathrm{~s}, 1 \mathrm{H}), 7.39(\mathrm{~d}, J=4.9$ Hz, 1H), 5.91 (br, 1H), 5.10 (dd, $J=13.2,7.1 \mathrm{~Hz}, 1 \mathrm{H}), 2.01$ (s, 3H), 1.65 (d, $J=6.1$ $\mathrm{Hz}, 2 \mathrm{H}), 0.99$ (s, 9H).

${ }^{19}$ F NMR (375 MHz, $\left.\mathrm{CDCl}_{3}\right) \delta-67.91$ (s). 
${ }^{13}$ C NMR $\left(100 \mathrm{MHz}, \mathrm{CDCl}_{3}\right) \delta 169.31,155.43,150.28,148.64(\mathrm{q}, J=34.4 \mathrm{~Hz}), 124.22$, $121.52(\mathrm{q}, J=274.5 \mathrm{~Hz}), 117.98(\mathrm{q}, J=2.5 \mathrm{~Hz}), 50.27,50.23,31.10,29.87,23.28$.

HRMS (ESI): $\mathrm{C}_{14} \mathrm{H}_{20} \mathrm{~F}_{3} \mathrm{~N}_{2} \mathrm{O}^{+}(\mathrm{M}+\mathrm{H})$ : 289.1528, found: 289.1525 .

$[\alpha]_{\mathrm{D}}^{25}=47.5365\left(\mathrm{c}=0.0467, \mathrm{CHCl}_{3}\right)$

Chiral HPLC: CHIRALPAK IC, $25{ }^{\circ} \mathrm{C},{ }^{i} \mathrm{PrOH}$-hexanes 10/90, $1.0 \mathrm{~mL} / \mathrm{min}, 254 \mathrm{~nm}$, $t_{R}($ major $)=17.5 \mathrm{~min}, t_{R}(\operatorname{minor})=12.2 \mathrm{~min}$.

\section{9 racemic}

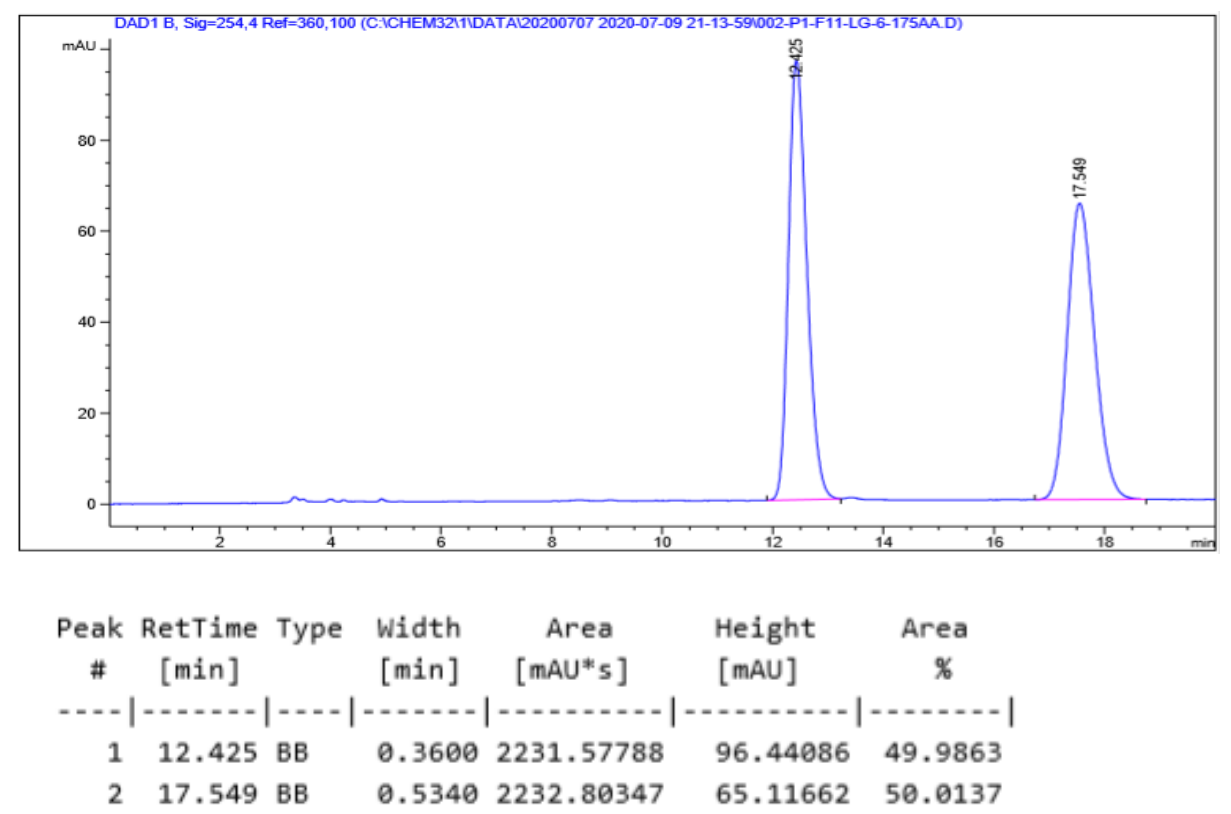

59 enantioenriched, $90 \%$ ee

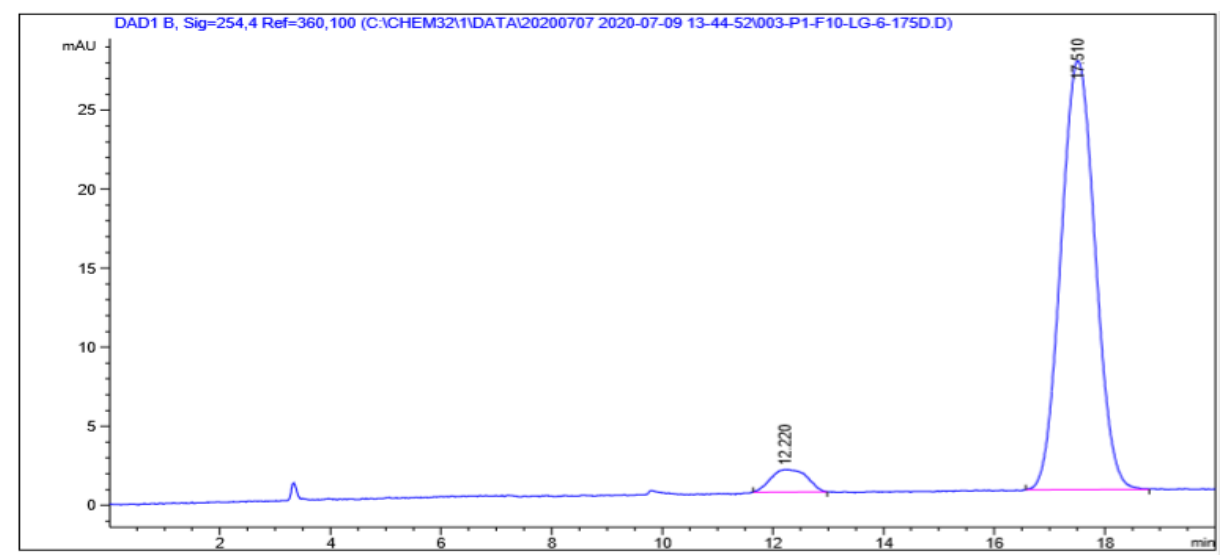




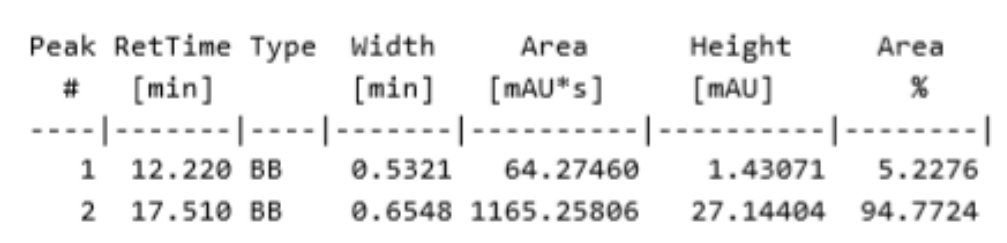<smiles>O=C(OC(CCBr)c1ccnc(C(F)(F)F)c1)c1ccccc1</smiles>

\section{(R)-3,3-Dimethyl-1-(2-(trifluoromethyl)pyridin-4-yl)butyl benzoate (60)}

The product was prepared according to the general procedure with (4S,4'S)-4,4'-di((S)sec-butyl)-4,4',5,5'-tetrahydro-2,2'-bioxazole [(S,S)-L1] $(3.3 \mathrm{mg}, 0.013 \mathrm{mmol}, 13$ mol\%), $\mathrm{NiCl}_{2} \bullet \mathrm{Py}_{4}(4.5 \mathrm{mg}, 0.01 \mathrm{mmol}, 10 \mathrm{~mol} \%), \operatorname{Ir}\left[\mathrm{dF}\left(\mathrm{CF}_{3}\right) \mathrm{ppy}\right]_{2}(\mathrm{bpy}) \mathrm{PF}_{6}(3.2 \mathrm{mg}$, $0.003 \mathrm{mmol}, 3 \mathrm{~mol} \%$ ), 4-bromo-2-(trifluoromethyl)pyridine (13.7 uL, $0.1 \mathrm{mmol}, 1.0$ equiv.), ${ }^{t} \mathrm{BuBF}_{3} \mathrm{~K}$ (41.0 mg, $0.25 \mathrm{mmol}, 2.5$ equiv.) and vinyl benzoate ( $27.6 \mathrm{uL}, 0.2$ mmol, 2.0 equiv.) in acetone/ethyl acetate (1.3/0.7, v/v, $2 \mathrm{~mL})$ were used. After 24 hours, the product was isolated by flash chromatography $(\mathrm{PE}$ : ethyl acetate $=10: 1)$ as a colorless oil (17.6 mg, 50\% yield, 78\% ee).

${ }^{1} \mathbf{H}$ NMR $\left(400 \mathrm{MHz}, \mathrm{CDCl}_{3}\right) \delta 8.68(\mathrm{~d}, J=4.9 \mathrm{~Hz}, 1 \mathrm{H}), 8.09(\mathrm{~d}, J=7.3 \mathrm{~Hz}, 2 \mathrm{H}), 7.65$ $-7.60(\mathrm{~m}, 2 \mathrm{H}), 7.51-7.47(\mathrm{~m}, 3 \mathrm{H}), 6.11(\mathrm{dd}, J=9.9,1.9 \mathrm{~Hz}, 1 \mathrm{H}), 2.12(\mathrm{dd}, J=15.0$, $10.0 \mathrm{~Hz}, 1 \mathrm{H}), 1.67$ (dd, $J=15.0,10.0 \mathrm{~Hz}, 1 \mathrm{H}), 1.05$ (s, 9H).

${ }^{19}$ F NMR $\left(375 \mathrm{MHz}, \mathrm{CDCl}_{3}\right) \delta-67.97$ (s).

${ }^{13}$ C NMR (101 MHz, $\left.\mathrm{CDCl}_{3}\right) \delta 165.58,153.42,150.40,148.69$ (q, J = 34.6 Hz), 133.58, 129.74, 129.42, 128.66, 123.43, 121.42 (q, J = 274.4 Hz), 117.59 (q, J = 2.6 $\mathrm{Hz}), 72.85,49.99,30.83,29.95$.

HRMS (ESI): $\mathrm{C}_{19} \mathrm{H}_{21} \mathrm{~F}_{3} \mathrm{NO}_{2}^{+}(\mathrm{M}+\mathrm{H}): 352.1524$, found: 352.1521 .

$[\alpha]_{\mathrm{D}}^{25}=-22.7532\left(\mathrm{c}=0.6900, \mathrm{CHCl}_{3}\right)$

Chiral HPLC: CHIRALPAK IC, $25{ }^{\circ} \mathrm{C},{ }^{i} \mathrm{PrOH}-$ hexanes 5/95, $1.0 \mathrm{~mL} / \mathrm{min}, 254 \mathrm{~nm}, \mathrm{t}_{\mathrm{R}}$ (major) $=6.5 \mathrm{~min}, \mathrm{t}_{\mathrm{R}}($ minor $)=5.6 \mathrm{~min}$. 


\section{0 racemic}

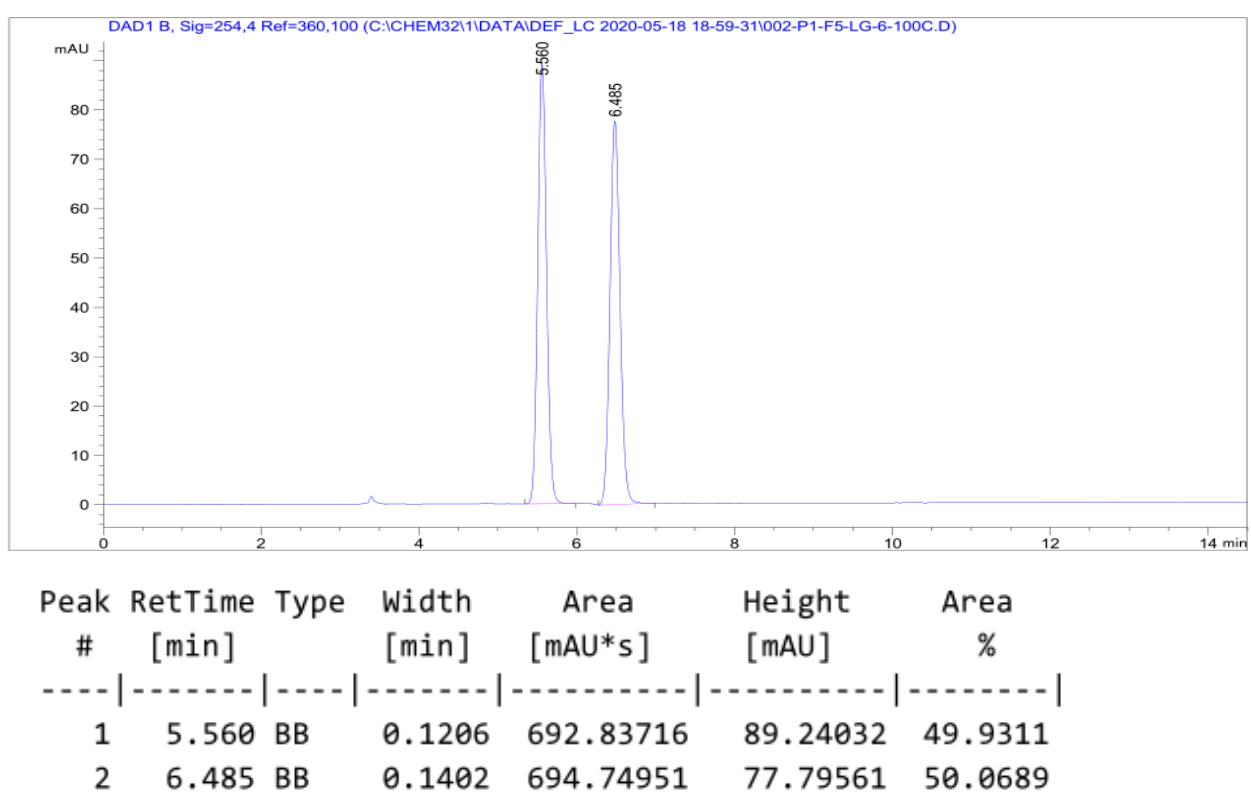

60 enantioenriched, $78 \%$ ee

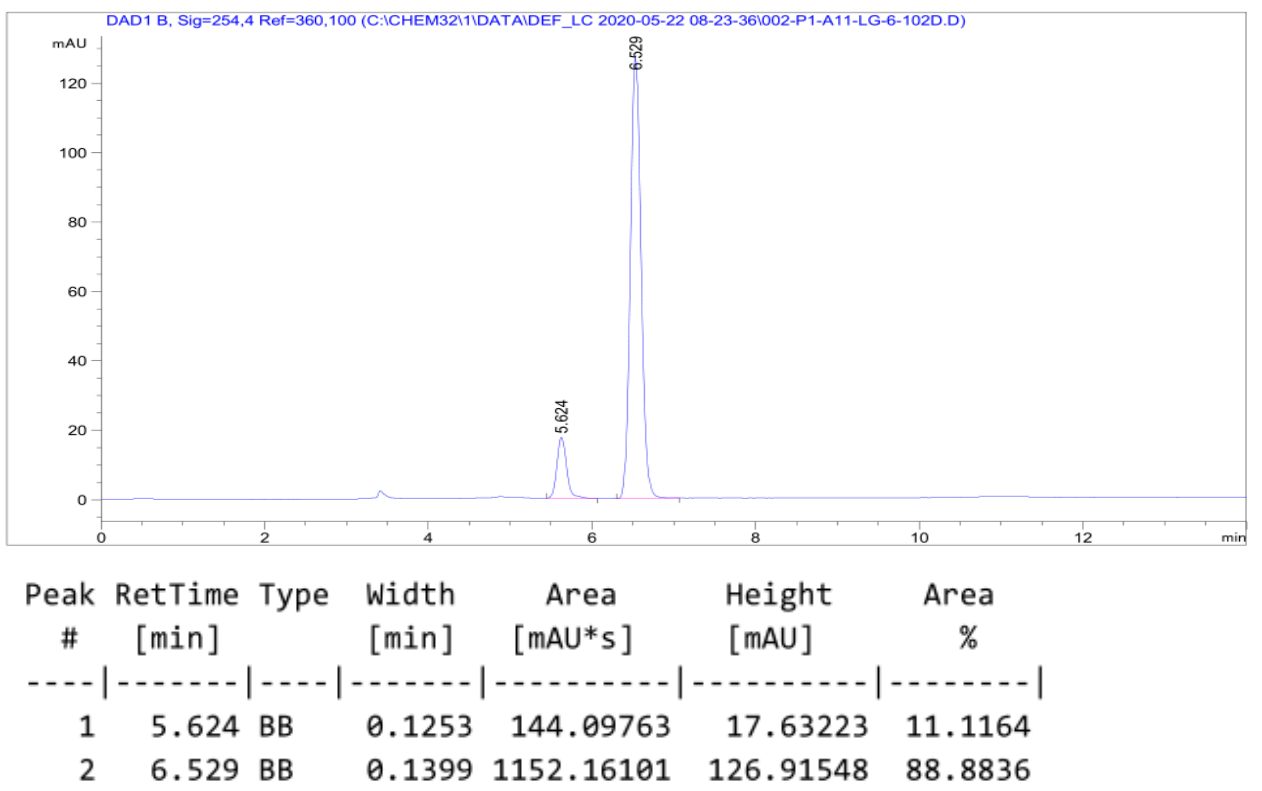




\section{Crystallographic Data for Compound 28}<smiles>COc1ncc([C@H](CC(C)(C)C)C(=O)OC(C)(C)C)cn1</smiles>

28

(CCDC 2021714)

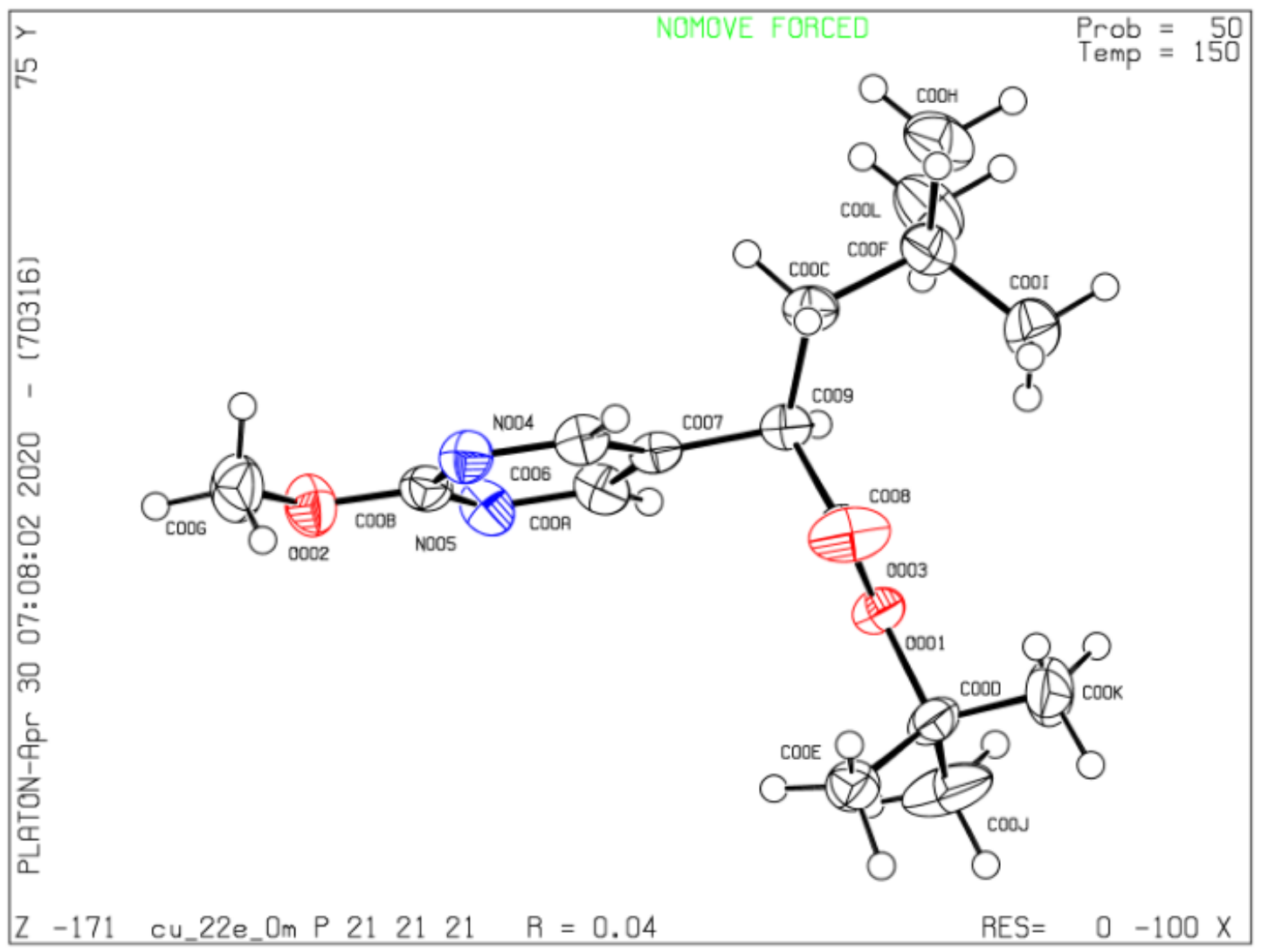

Table 1 Crystal data and structure refinement for compound 28

Identification code

Empirical formula

Formula weight

Temperature/K

Crystal system

Space group

$$
\begin{aligned}
& \mathrm{a} / \AA, \mathrm{b} / \AA, \mathrm{c} / \AA \\
& \alpha{ }^{\circ}, \beta /{ }^{\circ}, \gamma /{ }^{\circ} \\
& \text { Volume } / \AA^{3}
\end{aligned}
$$

cu_22E_0m

$\mathrm{C}_{16} \mathrm{H}_{26} \mathrm{~N}_{2} \mathrm{O}_{3}$

294.39

150.0

Triclinic

$\mathrm{P} 2{ }_{1} 2{ }_{1} 2_{1}$

10.5288(2),

11.5127(3),

14.2502(3)

$90,90,90$

1727.34(7) 
Z

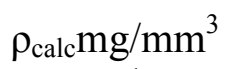

$\mathrm{m} / \mathrm{mm}^{-1}$

$\mathrm{F}(000)$

Crystal size $/ \mathrm{mm}^{3}$

Theta range for data collection

Index ranges

Reflections collected

Independent reflections

Data/restraints/parameters

Goodness-of-fit on $\mathrm{F}^{2}$

Final $R$ indexes $[I>2 \sigma(I)]$

Final $\mathrm{R}$ indexes [all data]

Largest diff. peak/hole / e $\AA^{-3}$

Flack parameter
4

1.132

0.628

640

$0.2 \times 0.15 \times 0.1$

5.223 to $79.510^{\circ}$

$-9 \leq \mathrm{h} \leq 12,-14 \leq \mathrm{k}$

$\leq 14,-18 \leq 1 \leq 18$

26335

$3691[\mathrm{R}$ (int) =

0.0560]

$3691 / 0 / 197$

1.069

$\mathrm{R}_{1}=0.0412, \mathrm{wR}_{2}=$

0.0939

$\mathrm{R}_{1}=0.0490, \mathrm{wR}_{2}=$

0.0992

$0.203 /-0.158$

$0.15(9)$

Table 2 Atomic Coordinates $\left(\AA \times 10^{4}\right)$ and Equivalent Isotropic Displacement Parameters $\left(\AA^{2} \times 10^{3}\right)$ for cu_ 22E_ $0 \mathrm{~m}$. $U_{\text {eq }}$ is defined as $1 / 3$ of of the trace of the orthogonalised $U_{I J}$ tensor.

$\begin{array}{lllll}\text { Atom } & \boldsymbol{x} & \boldsymbol{y} & \boldsymbol{z} & \mathbf{U}(\mathbf{e q}) \\ \mathrm{O} 001 & 4709.4(14) & 8314.3(14) & 3941.7(10) & 32.9(3) \\ \mathrm{O} 002 & 6101.6(17) & 2839.2(14) & 4864.8(13) & 44.6(4) \\ \mathrm{O} 003 & 6704.4(16) & 8517.3(19) & 4486.7(13) & 52.4(5) \\ \mathrm{N} 004 & 6917(17) & 4645.6(17) & 5189.5(14) & 36.5(4) \\ \text { N005 } & 4734.1(18) & 4314.7(17) & 4853.5(14) & 38.3(4) \\ \text { C006 } & 6657(2) & 5774(2) & 5307.2(16) & 33.8(5) \\ \text { C007 } & 5446.5(18) & 6231.6(19) & 5232.1(14) & 28.6(4) \\ \text { C008 } & 5623(2) & 8187(2) & 4575.9(15) & 32.2(5) \\ \text { C009 } & 5148.5(19) & 7501.9(19) & 5417.9(14) & 29.8(4) \\ \text { C00A } & 4513(19) & 5442(2) & 4984.8(15) & 34.9(5)\end{array}$




$\begin{array}{lllll}\mathrm{C} 00 \mathrm{~B} & 5933(2) & 3985.1(19) & 4980.7(15) & 33.8(5) \\ \mathrm{C} 00 \mathrm{C} & 5758(2) & 7927(2) & 6337.2(16) & 35.6(5) \\ \mathrm{C} 00 \mathrm{D} & 4947(2) & 8920(2) & 3036.8(15) & 40.1(6) \\ \mathrm{C} 00 \mathrm{E} & 6005(2) & 8335(2) & 2500.9(17) & 40.4(5) \\ \mathrm{C} 00 \mathrm{~F} & 5147(3) & 8991(2) & 6810.3(17) & 43.3(6) \\ \mathrm{C} 00 \mathrm{G} & 7372(3) & 2398(3) & 4977(2) & 53(7) \\ \mathrm{C} 00 \mathrm{H} & 5987(4) & 9324(3) & 7640(2) & 68.8(9) \\ \mathrm{C} 00 \mathrm{I} & 5054(4) & 10021(3) & 6144(2) & 63.1(9) \\ \mathrm{C} 00 \mathrm{~J} & 3689(3) & 8740(4) & 2529(2) & 71.4(12) \\ \mathrm{C} 00 \mathrm{~K} & 5212(5) & 10179(3) & 3233(2) & 75(11) \\ \mathrm{C} 00 \mathrm{~L} & 3826(3) & 8678(3) & 7171(3) & 66.3(9)\end{array}$

Table 3 Anisotropic Displacement Parameters $\left(\AA^{2} \times 10^{3}\right)$ for cu_22E_0m. The Anisotropic displacement factor exponent takes the form: $2 \pi^{2}\left[h^{2} a^{* 2} U_{11}+\ldots+2 h k a \times b \times U_{12}\right]$

$\begin{array}{lllllll}\text { Atom } & \mathbf{U}_{\mathbf{1 1}} & \mathbf{U}_{\mathbf{2 2}} & \mathbf{U}_{\mathbf{3 3}} & \mathbf{U}_{\mathbf{2 3}} & \mathbf{U}_{\mathbf{1 3}} & \mathbf{U}_{\mathbf{1 2}} \\ \mathrm{O} 001 & 29.4(7) & 40.7(9) & 28.5(7) & 5.8(6) & 0.5(6) & 3.4(7) \\ \mathrm{O} 002 & 42.8(9) & 38(9) & 52.9(10) & -4.1(8) & -2.5(8) & 6.3(7) \\ \mathrm{O} 003 & 34.7(9) & 82(14) & 40.3(9) & 7.9(10) & -3.9(7) & -23.8(9) \\ \mathrm{N} 004 & 29(9) & 42.4(11) & 38.3(10) & 0.6(9) & -2.4(8) & 4.2(8) \\ \mathrm{N} 005 & 30.6(9) & 41.3(10) & 43.1(10) & -9.1(8) & -1.6(8) & 1(8) \\ \text { C006 } & 26.7(10) & 40.4(13) & 34.4(11) & 1.3(9) & -1.4(8) & -3(9) \\ \text { C007 } & 24.7(9) & 37.9(11) & 23.3(9) & 2.5(8) & 0.1(7) & -0.4(8) \\ \text { C008 } & 29.1(10) & 36.5(12) & 30.9(10) & -0.1(9) & -1.2(8) & -1.8(8) \\ \text { C009 } & 25(9) & 36.6(11) & 27.9(10) & 0.4(8) & 0.3(8) & 0.1(8) \\ \text { C00A } & 25(11) & 44.7(12) & 35.1(11) & -6.1(10) & -1.2(9) & 2(8) \\ \text { C00B } & 33.9(11) & 39.7(12) & 27.7(10) & -0.8(9) & 2.1(9) & 3.8(8) \\ \text { C00C } & 34.3(11) & 43.4(13) & 29.1(10) & -1.1(9) & -2.7(9) & 1.4(10) \\ \text { C00D } & 46.5(14) & 44(13) & 29.9(11) & 11.8(10) & 7(10) & 14.4(11) \\ & & & 5109 & & & \end{array}$




$\begin{array}{lllllll}\text { C00E } & 41.7(13) & 44.6(14) & 34.8(11) & 2.4(10) & 5(10) & 7.5(11) \\ \text { C00F } & 51(14) & 41.7(14) & 37.2(12) & -6(10) & -3.9(11) & 3.8(12) \\ \text { C00G } & 49(15) & 46.5(14) & 63.5(17) & -0.5(13) & -4(13) & 16.2(11) \\ \text { C00H } & 90(2) & 67(2) & 49.9(16) & -21(15) & -18.5(16) & 6.7(18) \\ \text { C00I } & 96(3) & 39.7(15) & 53.7(16) & -3.4(13) & -12(16) & 2.6(15) \\ \text { C00J } & 45.5(16) & 132(4) & 36.9(13) & 25.8(18) & 1.8(12) & 25.2(18) \\ \text { C00K } & 130(3) & 35(14) & 59.8(17) & 12.1(13) & 37(2) & 20.7(19) \\ \text { C00L } & 61.7(19) & 72(2) & 64.9(19) & -19.9(17) & 19.9(16) & 11.7(17)\end{array}$

Table 4 Bond Lengths for cu_22E_0m.

$\begin{array}{llllllll}\text { Atom } & \text { Atom } & \text { Length } / \AA & & \text { Atom } & \text { Atom } & \text { Length/Å } \\ \mathrm{O} 001 & \mathrm{C} 008 & 1.328(3) & & \mathrm{C} 007 & \mathrm{C} 00 \mathrm{~A} & 1.385(3) \\ \mathrm{O} 001 & \mathrm{C} 00 \mathrm{D} & 1.487(3) & & \mathrm{C} 008 & \mathrm{C} 009 & 1.521(3) \\ \mathrm{O} 002 & \mathrm{C} 00 \mathrm{~B} & 1.341(3) & & \mathrm{C} 009 & \mathrm{C} 00 \mathrm{C} & 1.539(3) \\ \mathrm{O} 002 & \mathrm{C} 00 \mathrm{G} & 1.440(3) & & \mathrm{C} 00 \mathrm{C} & \mathrm{C} 00 \mathrm{~F} & 1.539(3) \\ \mathrm{O} 003 & \mathrm{C} 008 & 1.207(3) & & \mathrm{C} 00 \mathrm{D} & \mathrm{C} 00 \mathrm{E} & 1.509(3) \\ \mathrm{N} 004 & \mathrm{C} 006 & 1.338(3) & & \mathrm{C} 00 \mathrm{D} & \mathrm{C} 00 \mathrm{~J} & 1.523(4) \\ \mathrm{N} 004 & \mathrm{C} 00 \mathrm{~B} & 1.319(3) & & \mathrm{C} 00 \mathrm{D} & \mathrm{C} 00 \mathrm{~K} & 1.502(4) \\ \mathrm{N} 005 & \mathrm{C} 00 \mathrm{~A} & 1.332(3) & \mathrm{C} 00 \mathrm{~F} & \mathrm{C} 00 \mathrm{H} & 1.526(4) \\ \mathrm{N} 005 & \mathrm{C} 00 \mathrm{~B} & 1.330(3) & \mathrm{C} 00 \mathrm{~F} & \mathrm{C} 00 \mathrm{I} & 1.522(4) \\ \mathrm{C} 006 & \mathrm{C} 007 & 1.383(3) & \mathrm{C} 00 \mathrm{~F} & \mathrm{C} 00 \mathrm{~L} & 1.526(4) \\ \mathrm{C} 007 & \mathrm{C} 009 & 1.519(3) & & & \end{array}$

Table 5 Bond Angles for cu_22E_0m.

$\begin{array}{lllllllll}\text { Atom } & \text { Atom } & \text { Atom } & \text { Angle }^{\circ} & & \text { Atom } & \text { Atom } & \text { Atom } & \text { Angle } /^{\circ} \\ \text { O001 } & \text { C008 } & \text { C009 } & 110.87(17) & \text { C008 } & \text { C009 } & \text { C00C } & 111.70(18) \\ \text { O001 } & \text { C00D } & \text { C00E } & 110.70(18) & \text { C009 } & \text { C00C } & \text { C00F } & 116.86(19) \\ \text { O001 } & \text { C00D } & \text { C00J } & 101.6(2) & & \text { C00A } & \text { C007 } & \text { C009 } & 122.00(18) \\ \text { O001 } & \text { C00D } & \text { C00K } & 108.8(2) & & \text { C00B } & \text { O002 } & \text { C00G } & 117.2(2) \\ \text { O003 } & \text { C008 } & \text { O001 } & 125.2(2) & & \text { C00B } & \text { N004 } & \text { C006 } & 115.28(19)\end{array}$




$\begin{array}{lllllllll}\mathrm{O} 003 & \mathrm{C} 008 & \mathrm{C} 009 & 123.8(2) & \mathrm{C} 00 \mathrm{~B} & \mathrm{~N} 005 & \mathrm{C} 00 \mathrm{~A} & 115.13(19) \\ \mathrm{N} 004 & \mathrm{C} 006 & \mathrm{C} 007 & 123.3(2) & \mathrm{C} 00 \mathrm{E} & \mathrm{C} 00 \mathrm{D} & \mathrm{C} 00 \mathrm{~J} & 109.9(2) \\ \mathrm{N} 004 & \mathrm{C} 00 \mathrm{~B} & \mathrm{O} 002 & 119.4(2) & \mathrm{C} 00 \mathrm{H} & \mathrm{C} 00 \mathrm{~F} & \mathrm{C} 00 \mathrm{C} & 107.3(2) \\ \mathrm{N} 004 & \mathrm{C} 00 \mathrm{~B} & \mathrm{~N} 005 & 127.7(2) & \mathrm{C} 00 \mathrm{H} & \mathrm{C} 00 \mathrm{~F} & \mathrm{C} 00 \mathrm{~L} & 109.0(3) \\ \mathrm{N} 005 & \mathrm{C} 00 \mathrm{~A} & \mathrm{C} 007 & 123.48(19) & \mathrm{C} 00 \mathrm{I} & \mathrm{C} 00 \mathrm{~F} & \mathrm{C} 00 \mathrm{C} & 111.9(2) \\ \mathrm{N} 005 & \mathrm{C} 00 \mathrm{~B} & \mathrm{O} 002 & 112.9(2) & \mathrm{C} 00 \mathrm{I} & \mathrm{C} 00 \mathrm{~F} & \mathrm{C} 00 \mathrm{H} & 108.9(3) \\ \mathrm{C} 006 & \mathrm{C} 007 & \mathrm{C} 009 & 122.93(19) & \mathrm{C} 00 \mathrm{I} & \mathrm{C} 00 \mathrm{~F} & \mathrm{C} 00 \mathrm{~L} & 109.6(3) \\ \mathrm{C} 006 & \mathrm{C} 007 & \mathrm{C} 00 \mathrm{~A} & 115.1(2) & \mathrm{C} 00 \mathrm{~K} & \mathrm{C} 00 \mathrm{D} & \mathrm{C} 00 \mathrm{E} & 112.9(3) \\ \mathrm{C} 007 & \mathrm{C} 009 & \mathrm{C} 008 & 107.11(17) & \mathrm{C} 00 \mathrm{~K} & \mathrm{C} 00 \mathrm{D} & \mathrm{C} 00 \mathrm{~J} & 112.4(3) \\ \mathrm{C} 007 & \mathrm{C} 009 & \mathrm{C} 00 \mathrm{C} & 111.63(18) & \mathrm{C} 00 \mathrm{~L} & \mathrm{C} 00 \mathrm{~F} & \mathrm{C} 00 \mathrm{C} & 110.0(2) \\ \mathrm{C} 008 & \mathrm{O} 001 & \mathrm{C} 00 \mathrm{D} & 121.30(18) & & & & \end{array}$

Table 6 Torsion Angles for cu_22E_0m.
A $\quad$ B $\quad$ C $\quad$ D $\quad$ Angle $/^{\circ}$
O001 $\mathrm{C} 008 \quad \mathrm{C} 009 \quad \mathrm{C} 007$ 92.1(2)
O001 C008 C009 C00C -145.41(19)
O003 C008 C009 C007 $\quad$-84.7(3)
O003 C008 C009 C00C 37.9(3)
N004 C006 C007 C009 176.6(2)
N004 C006 C007 C00A -2.6(3)
C006 N004 C00B O002 -179.0(2)
C006 N004 C00B N005 1.8(3)
C006 $\quad 007 \quad C 009 \quad \mathrm{C} 008 \quad 75.3(2)$
C006 C007 C009 C00C -47.3(3)
$\begin{array}{lllll}\mathrm{C} 006 & \mathrm{C} 007 & \mathrm{C} 00 \mathrm{~A} & \mathrm{~N} 005 & 2.0(3)\end{array}$
C007 C009 C00C C00F -156.8(2)
C008 O001 C00D C00E 58.1(3)
C008 O001 C00D C00J 174.7(2)
C008 O001 C00D C00K -66.5(3) 


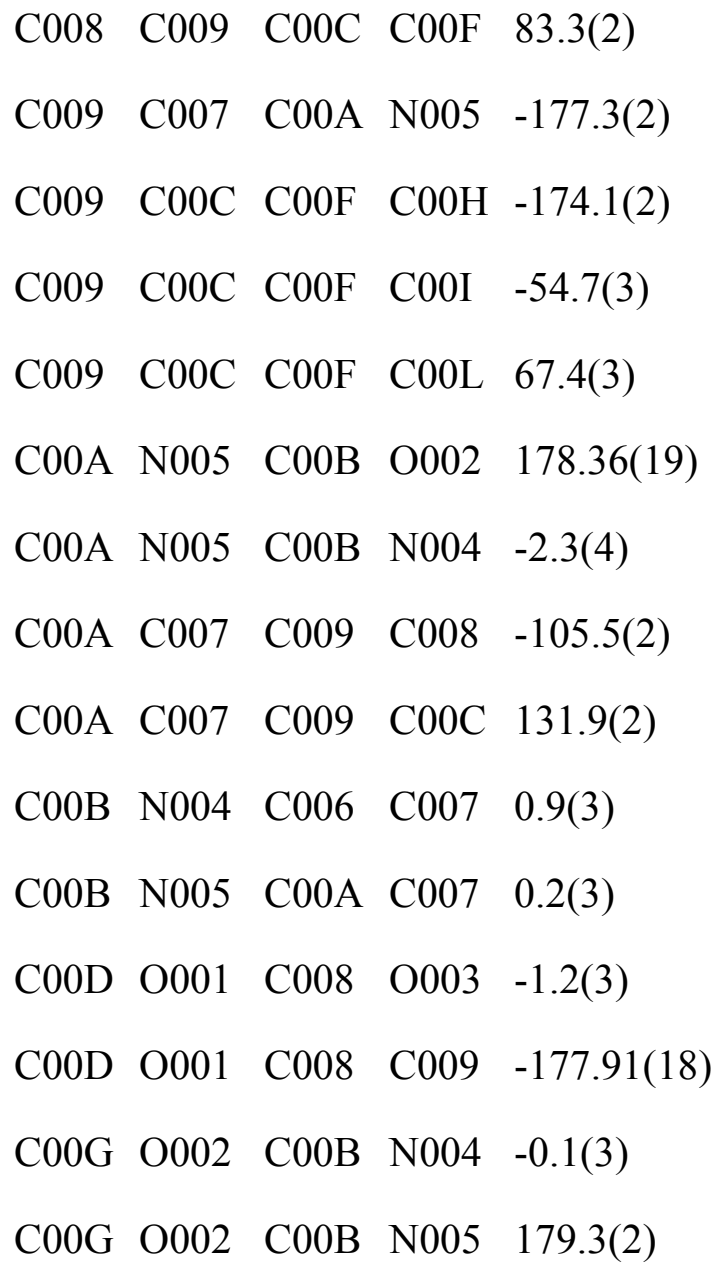

$\begin{array}{lllll}\text { Atom } & \boldsymbol{x} & \boldsymbol{y} & \boldsymbol{z} & \mathbf{U}(\mathbf{e q}) \\ \text { H006 } & 7337.57 & 6286.35 & 5449.47 & 41 \\ \text { H009 } & 4206.51 & 7596.85 & 5464.85 & 36 \\ \text { H00A } & 3670.03 & 5718.87 & 4904.53 & 42 \\ \text { H00B } & 6659.14 & 8113.08 & 6208.21 & 43 \\ \text { H00C } & 5745.85 & 7276.55 & 6791.34 & 43 \\ \text { H00D } & 6820.31 & 8510.26 & 2800.8 & 61 \\ \text { H00E } & 6013.67 & 8620.65 & 1853.16 & 61 \\ \text { H00F } & 5867.76 & 7492.74 & 2500.63 & 61 \\ \text { H00G } & 7940.9 & 2791.46 & 4535.19 & 79 \\ \text { H00H } & 7378.31 & 1560.67 & 4850.9 & 79\end{array}$




$\begin{array}{lllll}\text { H00I } & 7661.23 & 2539.79 & 5620.16 & 79 \\ \text { H00J } & 6036.78 & 8670.88 & 8079.52 & 103 \\ \text { H00K } & 5622.28 & 10000.03 & 7959.9 & 103 \\ \text { H00L } & 6840.31 & 9515.56 & 7413.92 & 103 \\ \text { H00M } & 5899.5 & 10206.71 & 5899.61 & 95 \\ \text { H00N } & 4714.16 & 10694.12 & 6481.94 & 95 \\ \text { H00O } & 4487.22 & 9824.78 & 5621.83 & 95 \\ \text { H00P } & 3542.86 & 7906.59 & 2438.45 & 107 \\ \text { H00Q } & 3718.1 & 9127.55 & 1917.47 & 107 \\ \text { H00R } & 2998.15 & 9068.52 & 2905.37 & 107 \\ \text { H00S } & 4524 & 10502.64 & 3615.68 & 113 \\ \text { H00T } & 5266.12 & 10605.55 & 2639.14 & 113 \\ \text { H00U } & 6017.63 & 10250.74 & 3572.26 & 113 \\ \text { H00V } & 3280.6 & 8464.22 & 6641.54 & 99 \\ \text { H00W } & 3459.59 & 9348.21 & 7498.08 & 99 \\ \text { H00X } & 3889.03 & 8021.56 & 7606.53 & 99\end{array}$




\section{Synthesis of derivatives of piragliatin and esflurbiprofen}

\subsection{Synthesis of piragliatin lead compound}<smiles>CCCCC(=O)C(CC1CCCC1)c1ccc(S(C)(=O)=O)c(Cl)c1</smiles>

(R)-tert-Butyl 2-(3-chloro-4-(methylsulfonyl)phenyl)-3-cyclopentylpropanoate (61)

The product was prepared according to the general procedure with (4S,4'S)-4,4'-di((S)sec-butyl)-4,4',5,5'-tetrahydro-2,2'-bioxazole [(S,S)-L1] (3.3 mg, $0.013 \mathrm{mmol}, 13$

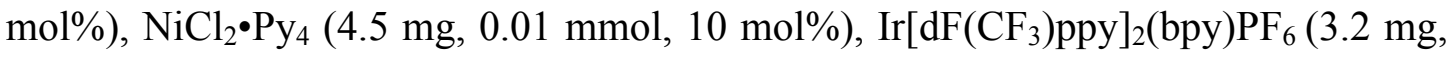
$0.003 \mathrm{mmol}, 3 \mathrm{~mol} \%$ ), 4-bromo-2-chloro-1-(methylsulfonyl)benzene (27.0 mg, 0.1 mmol, 1.0 equiv.), potassium cyclopentyltrifluoroboranuide $(52.8 \mathrm{mg}, 0.3 \mathrm{mmol}, 3.0$ equiv.) and tert-butyl acrylate $(29.0 \mathrm{uL}, 0.2 \mathrm{mmol}, 2.0$ equiv.) in acetone/ethyl acetate $(1.3 / 0.7, \mathrm{v} / \mathrm{v}, 2 \mathrm{~mL})$ were used. After 24 hours, the product was isolated by flash chromatography $(\mathrm{PE}$ : ethyl acetate $=10: 1)$ as a white solid $(29.0 \mathrm{mg}, 75 \%$ yield, $92 \%$ ee).

${ }^{1} \mathbf{H}$ NMR $\left(400 \mathrm{MHz}, \mathrm{CDCl}_{3}\right) \delta 8.08(\mathrm{~d}, J=8.2 \mathrm{~Hz}, 1 \mathrm{H}), 7.53(\mathrm{~s}, 1 \mathrm{H}), 7.41(\mathrm{dd}, J=8.2$, $1.4 \mathrm{~Hz}, 1 \mathrm{H}), 3.55(\mathrm{t}, J=7.7 \mathrm{~Hz}, 1 \mathrm{H}), 3.28(\mathrm{~s}, 3 \mathrm{H}), 2.08-2.01(\mathrm{~m}, 1 \mathrm{H}), 1.78-1.71(\mathrm{~m}, 3 \mathrm{H})$, 1.65-1.57 (m, 3H), 1.51-1.47 (m, 2H), $1.42(\mathrm{~s}, 9 \mathrm{H}), 1.17-1.05(\mathrm{~m}, 2 \mathrm{H})$.

${ }^{13}$ C NMR (101 MHz, $\left.\mathrm{CDCl}_{3}\right) \delta 171.88,147.84,136.41,132.51,131.35,130.88,127.15$, $81.66,51.60,42.79,39.93,37.84,32.69,32.30,27.95,25.09,25.05$.

HRMS (ESI): $\mathrm{C}_{19} \mathrm{H}_{28} \mathrm{ClO}_{4} \mathrm{~S}^{+}(\mathrm{M}+\mathrm{H}): 387.1397$, found: 387.1395 .

$[\alpha]_{\mathrm{D}}^{25}=-12.8934\left(\mathrm{c}=0.4033, \mathrm{CHCl}_{3}\right)$

Chiral HPLC: CHIRALPAK IB, $25{ }^{\circ} \mathrm{C},{ }^{i} \mathrm{PrOH}-h$ exanes $1 / 100,1.0 \mathrm{~mL} / \mathrm{min}, 230 \mathrm{~nm}$, $t_{R}($ major $)=19.3 \mathrm{~min}, t_{R}($ minor $)=21.3 \mathrm{~min}$.

\section{1 racemic}




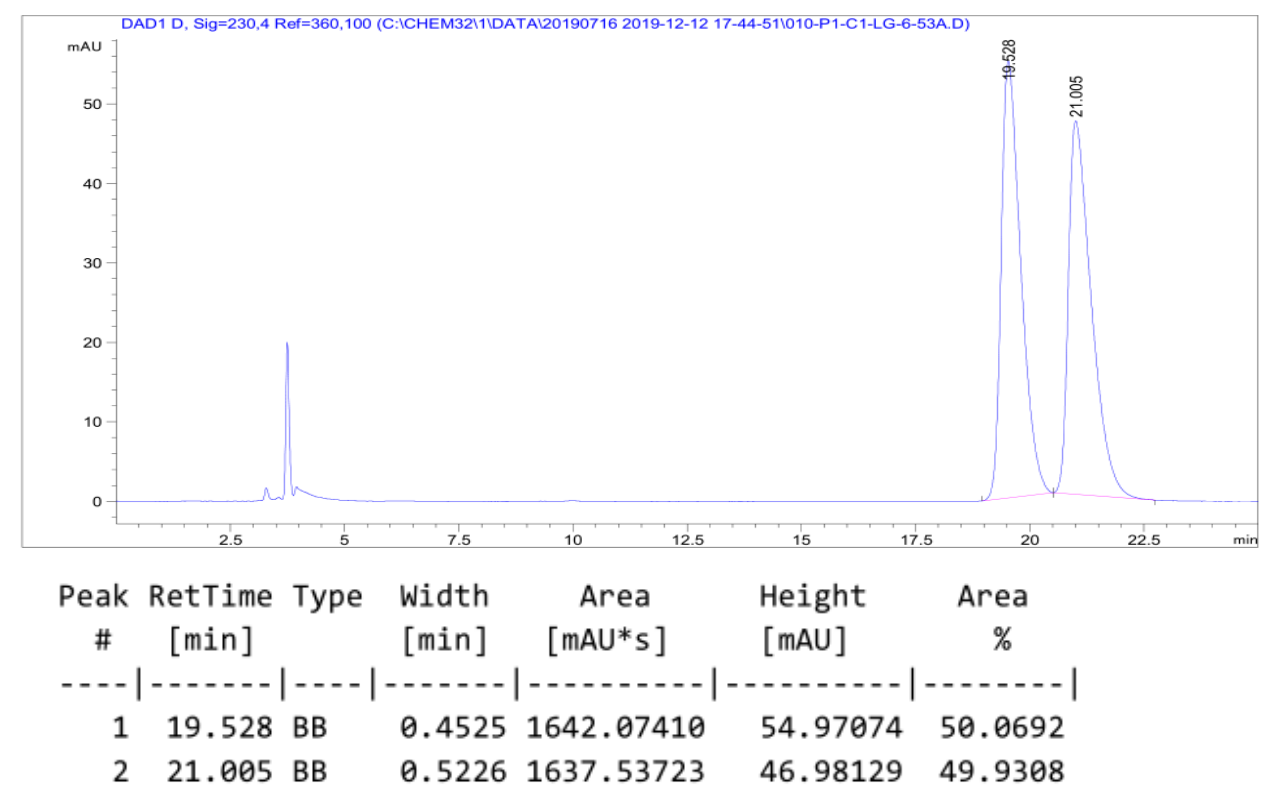

61 enantioenriched, $92 \%$ ee

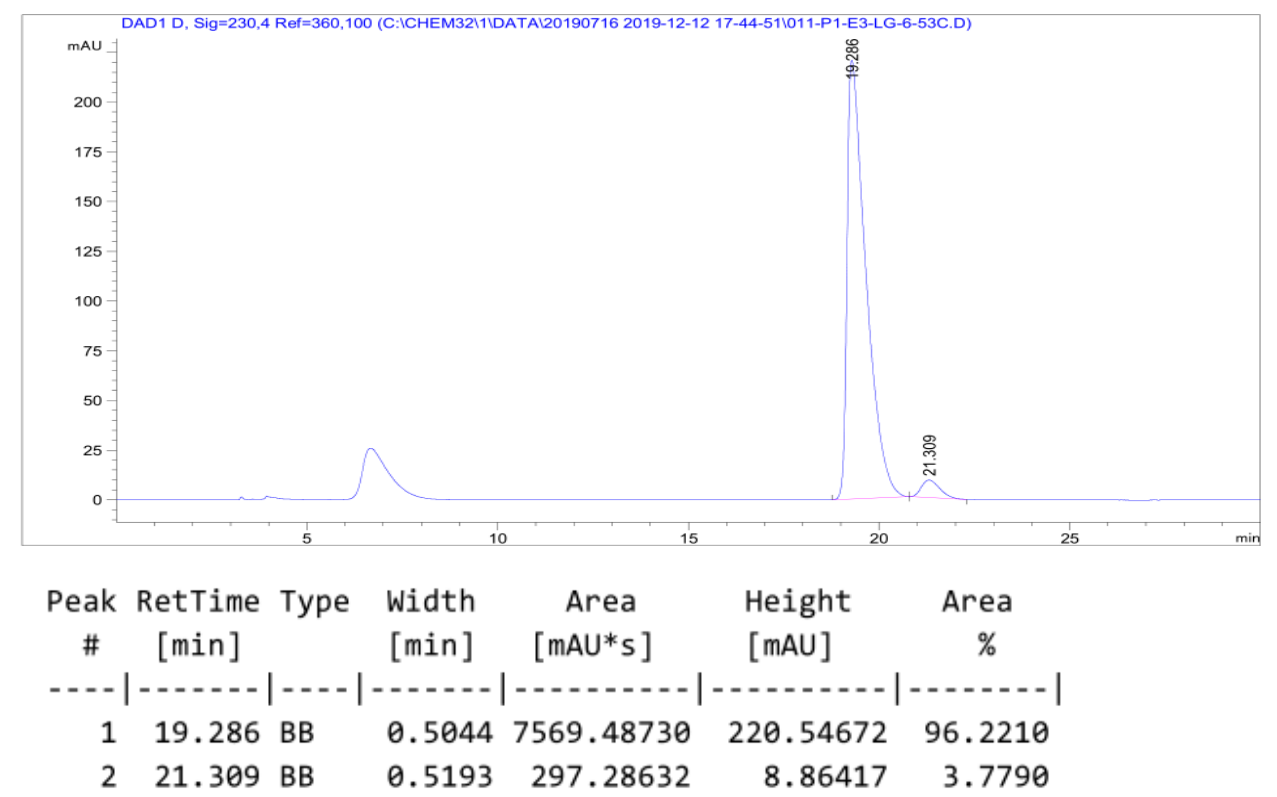

i

61<smiles>COc1ccc(C(CC2CCCC2)C(=O)Nc2cnccn2)cc1Cl</smiles>

62

(R)-2-(3-Chloro-4-(methylsulfonyl)phenyl)-3-cyclopentyl-N-(pyrazin-2-

yl)propanamide (62) 
The preparation and characterization data for the compound $\mathbf{6 2}$ is based on previously reported procedure ${ }^{3}$. A round-bottom flask was charged with $(R)$-tert-butyl 2-(3-chloro4-(methylsulfonyl)phenyl)-3-cyclopentylpropanoate 61 (38.7 mg, $0.1 \mathrm{mmol}, 1.0$ equiv.) and DCM [0.02M], to this solution, TFA (37.1 uL, $0.5 \mathrm{mmol}, 5.0$ equiv. ) was added dropwise at $0^{\circ} \mathrm{C}$. Then the mixture was stirred vigorously for 4 hours at room temperature, extracted with DCM three times, combined organic layer was washed with brine and dried over $\mathrm{MgSO}_{4}$ to obtain the crude acid. After drying under high vacuum, the acid was dissolved in anhydrous DCM [2.0 mL, 0.05M] in an oven dried vial, added oxalyl chloride $\left(9.3 \mu \mathrm{L}, 0.11 \mathrm{mmol}, 1.1\right.$ equiv.) and one drop of DMF at $0{ }^{\circ} \mathrm{C}$. The reaction was kept at $0{ }^{\circ} \mathrm{C}$ for 10 mins then room temperature for $2 \mathrm{~h}$ to get a soluble solution of acid chloride. A solution of 2-aminopyrazine (20.9 mg, $0.22 \mathrm{mmol}, 2.2$ equiv.), pyridine (17.7 $\mu \mathrm{L}, 0.22 \mathrm{mmol}, 2.2$ equiv.), in anhydrous THF [2.0 $\mathrm{mL}, 0.05 \mathrm{M}$ ] was added to the reaction and solid was crushed out, immediately. The reaction was left at room temperature for $24 \mathrm{~h}$, The crude material was purified by flash chromatography (PE:ethyl acetate $=2: 1)$ to afford the product as a white solid $(25.3 \mathrm{mg}, 62 \%$ yield over 2 steps.).

${ }^{1} \mathbf{H}$ NMR $\left(400 \mathrm{MHz}, \mathrm{CDCl}_{3}\right) \delta 9.53(\mathrm{~s}, 1 \mathrm{H}), 8.37(\mathrm{~d}, J=2.4 \mathrm{~Hz}, 1 \mathrm{H}), 8.23-8.22(\mathrm{~m}$, $1 \mathrm{H}), 8.16(\mathrm{br}, 1 \mathrm{H}), 8.13(\mathrm{~d}, J=8.2 \mathrm{~Hz}, 1 \mathrm{H}), 7.63(\mathrm{~d}, J=1.6 \mathrm{~Hz}, 1 \mathrm{H}), 7.50(\mathrm{dd}, J=8.2$, $1.6 \mathrm{~Hz}, 1 \mathrm{H}), 3.69$ (t, $J=7.5 \mathrm{~Hz}, 1 \mathrm{H}), 3.28(\mathrm{~s}, 3 \mathrm{H}), 2.23$ (dt, $J=13.7,7.5 \mathrm{~Hz}, 1 \mathrm{H}), 1.95-$ $1.88(\mathrm{~m}, 1 \mathrm{H}), 1.83-1.75(\mathrm{~m}, 2 \mathrm{H}), 1.69-1.61(\mathrm{~m}, 3 \mathrm{H}), 1.52-1.48(\mathrm{~m}, 2 \mathrm{H}), 1.20-1.11(\mathrm{~m}$, $2 \mathrm{H})$.

\subsection{Synthesis of esflurbiprofen derivatives}<smiles>CCCOC(=O)C(CC(C)(C)C)c1ccc(-c2ccccc2)c(F)c1</smiles>

\section{(R)-tert-Butyl 2-(2-fluoro-[1,1'-biphenyl]-4-yl)-4,4-dimethylpentanoate (63)}

The product was prepared according to the general procedure with (4S,4'S)-4,4'-di((S)sec-butyl)-4,4',5,5'-tetrahydro-2,2'-bioxazole [(S,S)-L1] $(3.3 \mathrm{mg}, 0.013 \mathrm{mmol}, 13$ mol\%), $\mathrm{NiCl}_{2} \bullet \mathrm{Py}_{4}(4.5 \mathrm{mg}, 0.01 \mathrm{mmol}, 10 \mathrm{~mol} \%), \operatorname{Ir}\left[\mathrm{dF}\left(\mathrm{CF}_{3}\right) \mathrm{ppy}\right]_{2}(\mathrm{bpy}) \mathrm{PF}_{6}(3.2 \mathrm{mg}$, 
$0.003 \mathrm{mmol}, 3 \mathrm{~mol} \%$ ), 4-bromo-2-fluoro-1,1'-biphenyl (25.1 mg, $0.1 \mathrm{mmol}, 1.0$ equiv.), ${ }^{t} \mathrm{BuBF}_{3} \mathrm{~K}$ (41.0 mg, $0.25 \mathrm{mmol}, 2.5$ equiv.) and tert-butyl acrylate $(29.0 \mathrm{uL}, 0.2 \mathrm{mmol}$, 2.0 equiv.) in Acetone/ethyl acetate (1.3/0.7, v/v, $2 \mathrm{~mL}$ ) were used. After 24 hours, the product was isolated by flash chromatography (PE: ethyl acetate $=20: 1)$ as a white solid (20.1 mg, 56\% yield, 90\% ee).

${ }^{1} \mathbf{H}$ NMR $\left(400 \mathrm{MHz}, \mathrm{CDCl}_{3}\right) \delta$ 7.55-7.53 (m, 2H), 7.45-7.41 (m, 2H), 7.39-7.33 (m, 2H), 7.16-7.10 (m, 2H), $3.56(\mathrm{dd}, J=9.3,3.4 \mathrm{~Hz}, 1 \mathrm{H}), 2.28(\mathrm{dd}, J=14.0,9.3 \mathrm{~Hz}, 1 \mathrm{H})$, $1.53(\mathrm{dd}, J=14.0,3.5 \mathrm{~Hz}, 1 \mathrm{H}), 1.41(\mathrm{~s}, 9 \mathrm{H}), 0.94(\mathrm{~s}, 9 \mathrm{H})$.

${ }^{19}$ F NMR $\left(375 \mathrm{MHz}, \mathrm{CDCl}_{3}\right) \delta-117.93(\mathrm{t}, J=11.0 \mathrm{~Hz})$.

${ }^{13}$ C NMR $\left(100 \mathrm{MHz}, \mathrm{CDCl}_{3}\right) \delta 173.34,159.6(\mathrm{~d}, J=247.8 \mathrm{~Hz}), 143.05(\mathrm{~d}, J=7.8 \mathrm{~Hz})$, 135.62 (135.61), 130.59 (d, $J=4.0 \mathrm{~Hz}), 128.93$ (d, $J=3.0 \mathrm{~Hz}), 128.39,127.54,127.3$ $(\mathrm{d}, J=13.7 \mathrm{~Hz}), 123.66(\mathrm{~d}, J=3.4 \mathrm{~Hz}), 115.29(\mathrm{~d}, J=23.8 \mathrm{~Hz}), 80.77,48.83(\mathrm{~s}), 47.18$, $31.08,29.48,27.86$.

HRMS (ESI): $\mathrm{C}_{23} \mathrm{H}_{30} \mathrm{FO}_{2}{ }^{+}(\mathrm{M}+\mathrm{H}): 357.2230$, found: 357.2227 .

$[\alpha]_{\mathrm{D}}^{25}=-10.4060\left(\mathrm{c}=0.3200, \mathrm{CHCl}_{3}\right)$

Chiral HPLC: CHIRALPAK IA, $25{ }^{\circ} \mathrm{C},{ }^{i} \mathrm{PrOH}-h$ hanes $1 / 100,0.5 \mathrm{~mL} / \mathrm{min}, 254 \mathrm{~nm}$, $\mathrm{t}_{\mathrm{R}}($ major $)=8.3 \mathrm{~min}, \mathrm{t}_{\mathrm{R}}($ minor $)=7.9 \mathrm{~min}$.

\section{3 racemic}

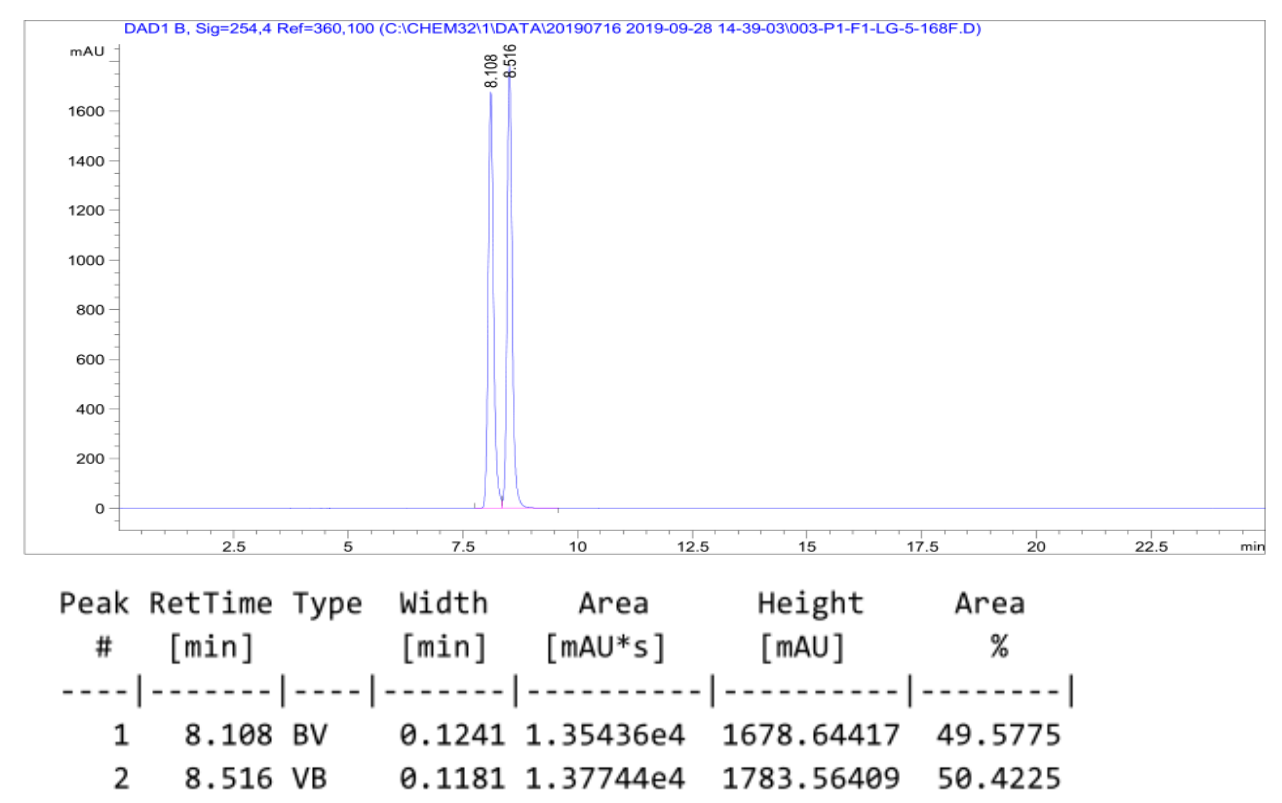




\section{3 enantioenriched, $90 \%$ ee}

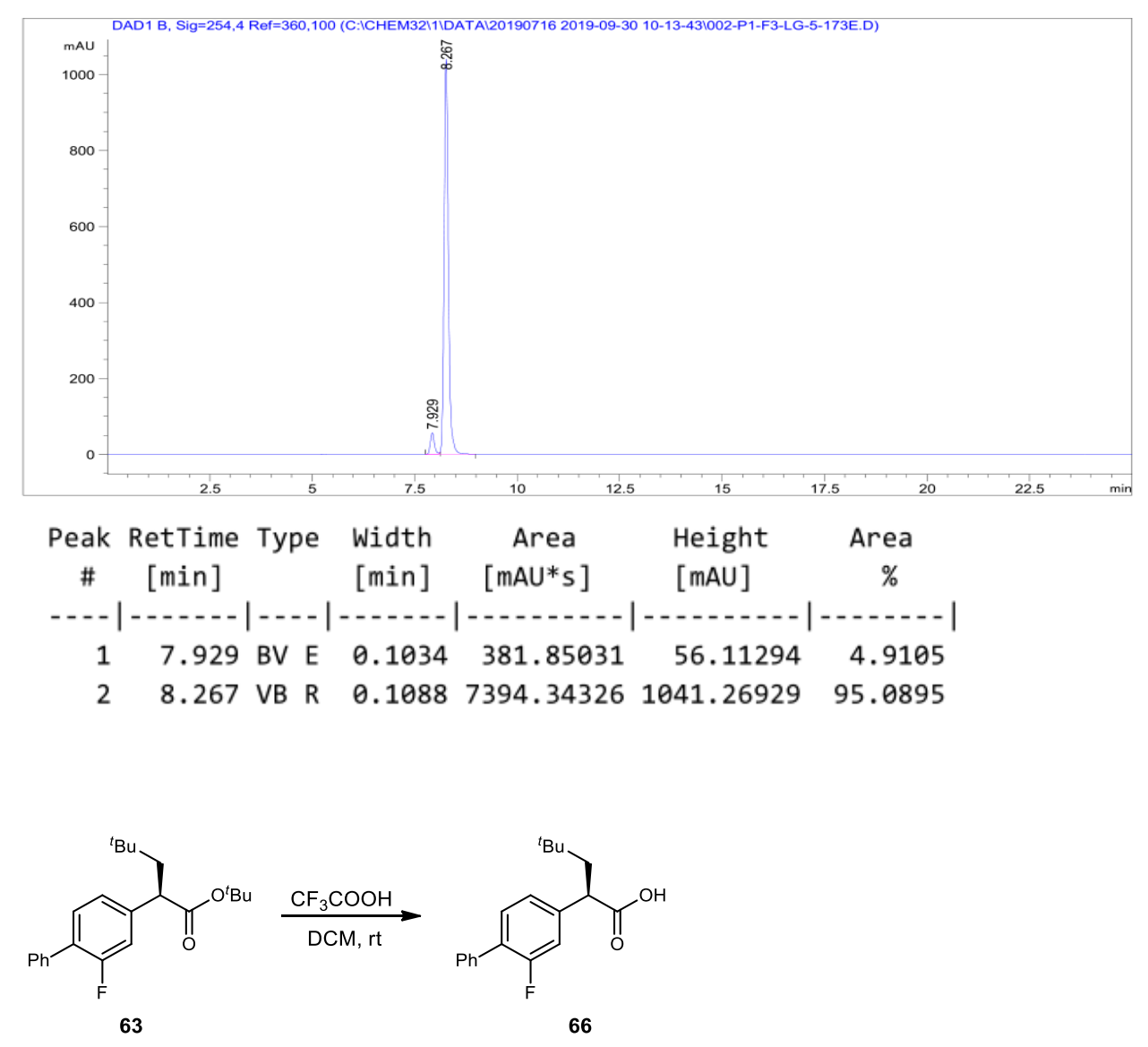

(R)-2-(2-Fluoro-[1,1'-biphenyl]-4-yl)-4,4-dimethylpentanoic acid (66)

The preparation and characterization data for the compound $\mathbf{6 6}$ is based on previously reported procedure ${ }^{4}$. A round-bottom flask was charged with $(R)$-tert-butyl 2-(2-fluoro[1,1'-biphenyl]-4-yl)-4-methylpentanoate 63 (57.8 mg, $0.16 \mathrm{mmol}, 1.0$ equiv.) and DCM $[0.02 \mathrm{M}]$, to this solution, TFA $(59.4 \mathrm{uL}, 0.8 \mathrm{mmol}, 5.0$ equiv. $)$ was added dropwise at $0{ }^{\circ} \mathrm{C}$. Then the mixture was stirred vigorously for 4 hours at room temperature, extracted with DCM three times, combined organic layer was washed with brine and dried over $\mathrm{MgSO}_{4}$. After drying under high vacuum without further purification to obtain the target product ( $48.7 \mathrm{mg},>99 \%$ yield, $89 \%$ ee)

${ }^{1} \mathbf{H}$ NMR $\left(400 \mathrm{MHz}, \mathrm{CDCl}_{3}\right) \delta 7.52(\mathrm{~d}, J=7.6 \mathrm{~Hz}, 2 \mathrm{H}), 7.42(\mathrm{t}, J=7.5 \mathrm{~Hz}, 2 \mathrm{H}), 7.39$ $7.33(\mathrm{~m}, 2 \mathrm{H}), 7.19-7.15(\mathrm{~m}, 2 \mathrm{H}), 3.69(\mathrm{dd}, J=8.5,4.2 \mathrm{~Hz}, 1 \mathrm{H}), 2.28(\mathrm{dd}, J=14.1,8.7$ $\mathrm{Hz}, 1 \mathrm{H}), 1.65$ (dd, $J=14.1,4.2 \mathrm{~Hz}, 1 \mathrm{H}), 0.93$ (s, 9H).

${ }^{19}$ F NMR $\left(375 \mathrm{MHz}, \mathrm{CDCl}_{3}\right) \delta-117.39(\mathrm{t}, J=9.7 \mathrm{~Hz})$. 
${ }^{13}$ C NMR (100 MHz, $\left.\mathrm{CDCl}_{3}\right) \delta 180.06,159.66(\mathrm{~d}, J=248.6 \mathrm{~Hz}), 141.47(\mathrm{~d}, J=7.6$ Hz), 135.40, 130.83 (d, $J=3.9 \mathrm{~Hz}), 128.94$ (d, $J=2.7 \mathrm{~Hz}), 128.44,128.02$ (d, $J=13.6$ Hz), 127.68, 124.00 (d, $J=3.2 \mathrm{~Hz}), 115.65$ (d, $J=23.7 \mathrm{~Hz}), 47.58,46.86,31.12,29.40$. HRMS (ESI): $\mathrm{C}_{19} \mathrm{H}_{21} \mathrm{FO}_{2} \mathrm{Na}(\mathrm{M}+\mathrm{Na}): 323.1423$, found: 323.1419.

$[\alpha]_{\mathrm{D}}^{25}=-31.8468\left(\mathrm{c}=0.2333, \mathrm{CHCl}_{3}\right)$

Chiral HPLC: CHIRALPAK IG, $25{ }^{\circ} \mathrm{C},{ }^{i} \mathrm{PrOH}-$ hexanes 5/95, $0.7 \mathrm{~mL} / \mathrm{min}, 214 \mathrm{~nm}, \mathrm{t}_{\mathrm{R}}$ (major) $=10.6 \mathrm{~min}, \mathrm{t}_{\mathrm{R}}($ minor $)=10.0 \mathrm{~min}$.

\section{6 racemic}

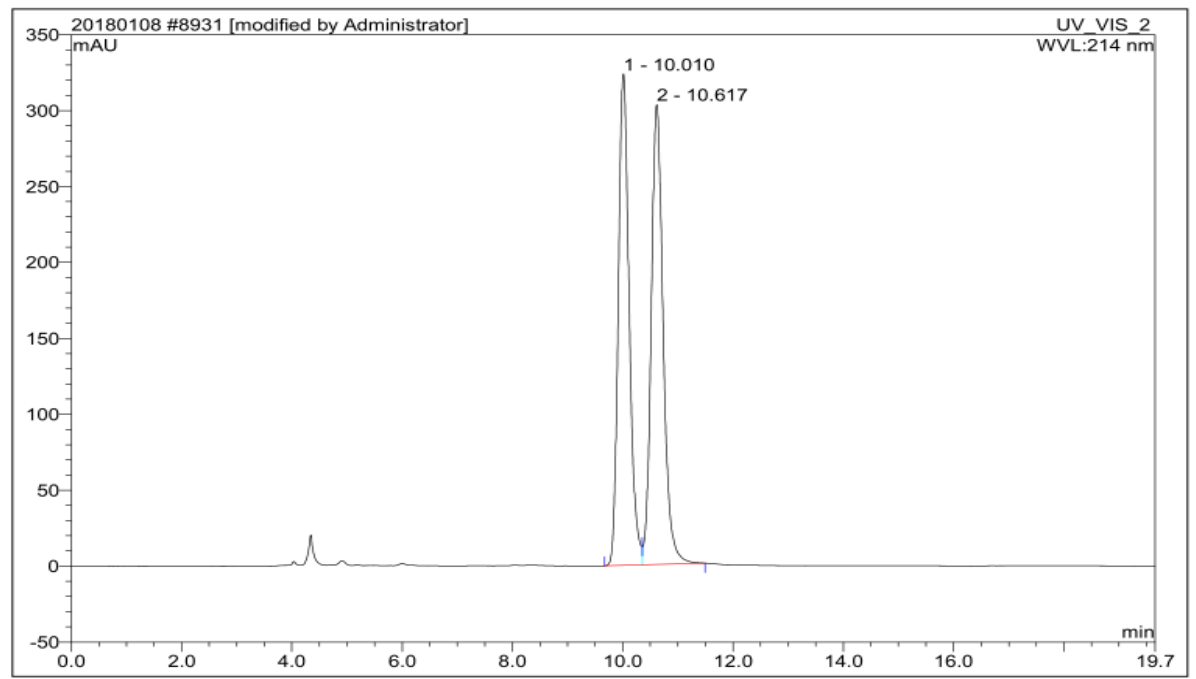

\begin{tabular}{|r|ccrrrrr|}
\hline No. & $\begin{array}{c}\text { Ret.Time } \\
\text { min }\end{array}$ & Peak Name & $\begin{array}{c}\text { Height } \\
\text { mAU }\end{array}$ & $\begin{array}{c}\text { Area } \\
\text { mAU*min }\end{array}$ & $\begin{array}{c}\text { Rel.Area } \\
\%\end{array}$ & Amount & Type \\
\hline 1 & 10.01 & n.a. & 323.734 & 74.196 & 49.36 & n.a. & BM \\
2 & 10.62 & n.a. & 303.056 & 76.125 & 50.64 & n.a. & MB \\
\hline Total: & & & 626.789 & 150.321 & 100.00 & 0.000 & \\
\hline
\end{tabular}




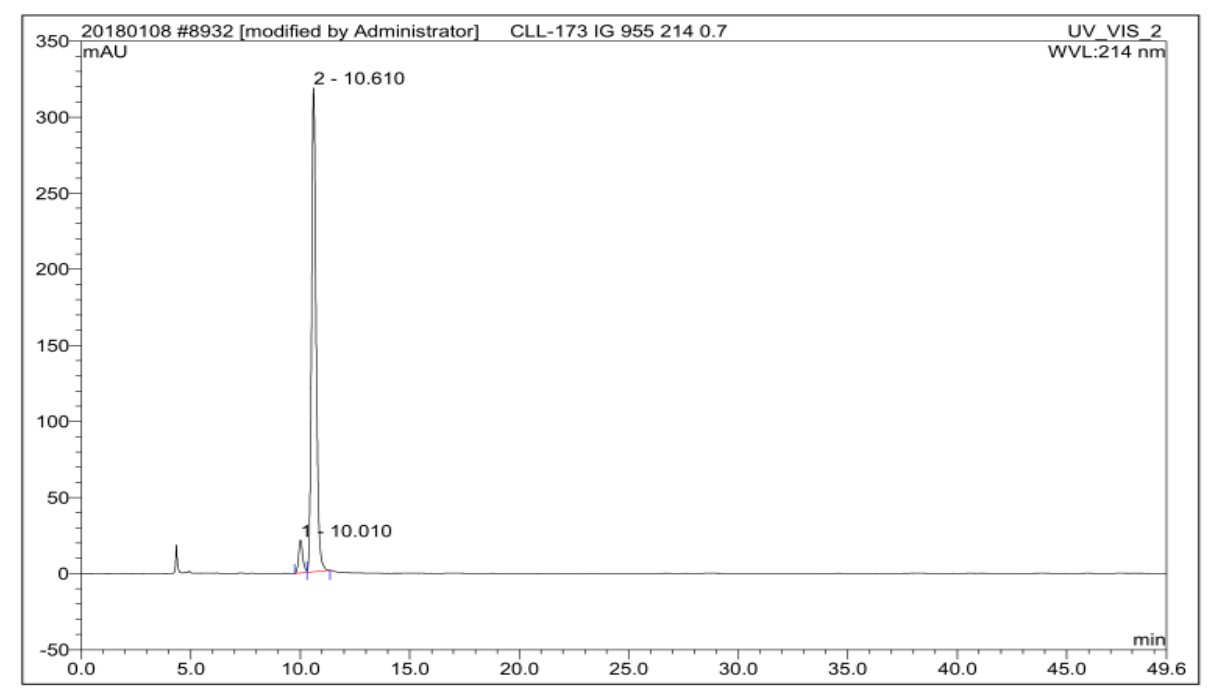

\begin{tabular}{|c|c|c|c|c|c|c|c|}
\hline No. & $\begin{array}{c}\text { Ret.Time } \\
\text { min }\end{array}$ & Peak Name & $\begin{array}{c}\text { Height } \\
\text { mAU }\end{array}$ & $\begin{array}{c}\text { Area } \\
\mathrm{mAU^{* } \operatorname { m i n }}\end{array}$ & $\begin{array}{c}\text { Rel.Area } \\
\%\end{array}$ & Amount & Type \\
\hline 1 & 10.01 & n.a. & 21.606 & 4.766 & 5.80 & n.a. & $\mathrm{BM}$ \\
\hline 2 & 10.61 & n.a. & 318.211 & 77.479 & 94.20 & n.a. & MB \\
\hline Total: & & & 339.817 & 82.245 & 100.00 & 0.000 & \\
\hline
\end{tabular}<smiles>CCCCC(C(=O)OCCC)c1ccc(-c2ccccc2)c(F)c1</smiles>

\section{(R)-tert-Butyl 2-(2-fluoro-[1,1'-biphenyl]-4-yl)-4-methylpentanoate (64)}

The product was prepared according to the general procedure with (4S,4'S)-4,4'-di((S)sec-butyl)-4,4',5,5'-tetrahydro-2,2'-bioxazole [(S,S)-L1] $(3.3 \mathrm{mg}, 0.013 \mathrm{mmol}, 13$ $\mathrm{mol} \%$ ), $\mathrm{NiCl}_{2} \bullet \mathrm{Py}_{4}(4.5 \mathrm{mg}, 0.01 \mathrm{mmol}, 10 \mathrm{~mol} \%), \operatorname{Ir}\left[\mathrm{dF}\left(\mathrm{CF}_{3}\right) \text { ppy }\right]_{2}(\mathrm{bpy}) \mathrm{PF}_{6}(3.2 \mathrm{mg}$, $0.003 \mathrm{mmol}, 3 \mathrm{~mol} \%$ ), 4-bromo-2-fluoro-1,1'-biphenyl (25.1 mg, $0.1 \mathrm{mmol}, 1.0$ equiv.), potassium isopropyltrifluoroborate $(45.0 \mathrm{mg}, 0.3 \mathrm{mmol}, 3.0$ equiv.) and tert-butyl acrylate $(29.0 \mathrm{uL}, 0.2 \mathrm{mmol}, 2.0$ equiv.) in acetone/ethyl acetate $(1.3 / 0.7, \mathrm{v} / \mathrm{v}, 2 \mathrm{~mL})$ were used. After 24 hours, the product was isolated by flash chromatography (PE: ethyl acetate $=20: 1)$ as a white solid $(17.5 \mathrm{mg}, 51 \%$ yield, $86 \%$ ee $)$.

${ }^{1} \mathbf{H}$ NMR $\left(400 \mathrm{MHz}, \mathrm{CDCl}_{3}\right) \delta 7.55(\mathrm{~d}, J=7.6 \mathrm{~Hz}, 2 \mathrm{H}), 7.44(\mathrm{t}, J=7.5 \mathrm{~Hz}, 2 \mathrm{H}), 7.40$ $7.34(\mathrm{~m}, 2 \mathrm{H}), 7.16-7.12(\mathrm{~m}, 2 \mathrm{H}), 3.57$ (t, $J=7.7 \mathrm{~Hz}, 1 \mathrm{H}), 1.98-1.90(\mathrm{~m}, 1 \mathrm{H}), 1.67-1.60$ 
$(\mathrm{m}, 1 \mathrm{H}), 1.56-1.49(\mathrm{~m}, 1 \mathrm{H}), 1.43(\mathrm{~s}, 9 \mathrm{H}), 0.95(\mathrm{~d}, J=2.6 \mathrm{~Hz}, 3 \mathrm{H}), 0.93(\mathrm{~d}, J=2.6 \mathrm{~Hz}$ $3 \mathrm{H})$.

${ }^{19}$ F NMR $\left(375 \mathrm{MHz}, \mathrm{CDCl}_{3}\right) \delta-118.06(\mathrm{t}, J=10.6 \mathrm{~Hz})$.

${ }^{13}$ C NMR (100 MHz, $\left.\mathrm{CDCl}_{3}\right) \delta 172.95,159.60(\mathrm{~d}, J=247.9 \mathrm{~Hz}), 141.38(\mathrm{~d}, J=7.6$ Hz), 135.60, 130.52 (d, $J=4.0 \mathrm{~Hz}), 128.93$ (d, $J=2.9 \mathrm{~Hz}), 128.39,127.54,127.42$ (d, $J=13.7 \mathrm{~Hz}), 123.91(\mathrm{~d}, J=3.2 \mathrm{~Hz}), 115.48(\mathrm{~d}, J=23.5 \mathrm{~Hz}), 80.86,50.15,42.54,27.95$, $25.92,22.54,22.37$.

HRMS (ESI): $\mathrm{C}_{22} \mathrm{H}_{28} \mathrm{FO}_{2}{ }^{+}$(M+H): 343.2073, found: 343.2069 .

$[\alpha]_{\mathrm{D}}^{25}=-6.2008\left(\mathrm{c}=0.4967, \mathrm{CHCl}_{3}\right)$

Chiral HPLC: CHIRALPAK IB, $25{ }^{\circ} \mathrm{C}$, Hexanes, $0.5 \mathrm{~mL} / \mathrm{min}$, $254 \mathrm{~nm}, \mathrm{t}_{\mathrm{R}}($ major $)=16.3 \mathrm{~min}, \mathrm{t}_{\mathrm{R}}($ minor $)=18.0 \mathrm{~min}$

\section{4 racemic}

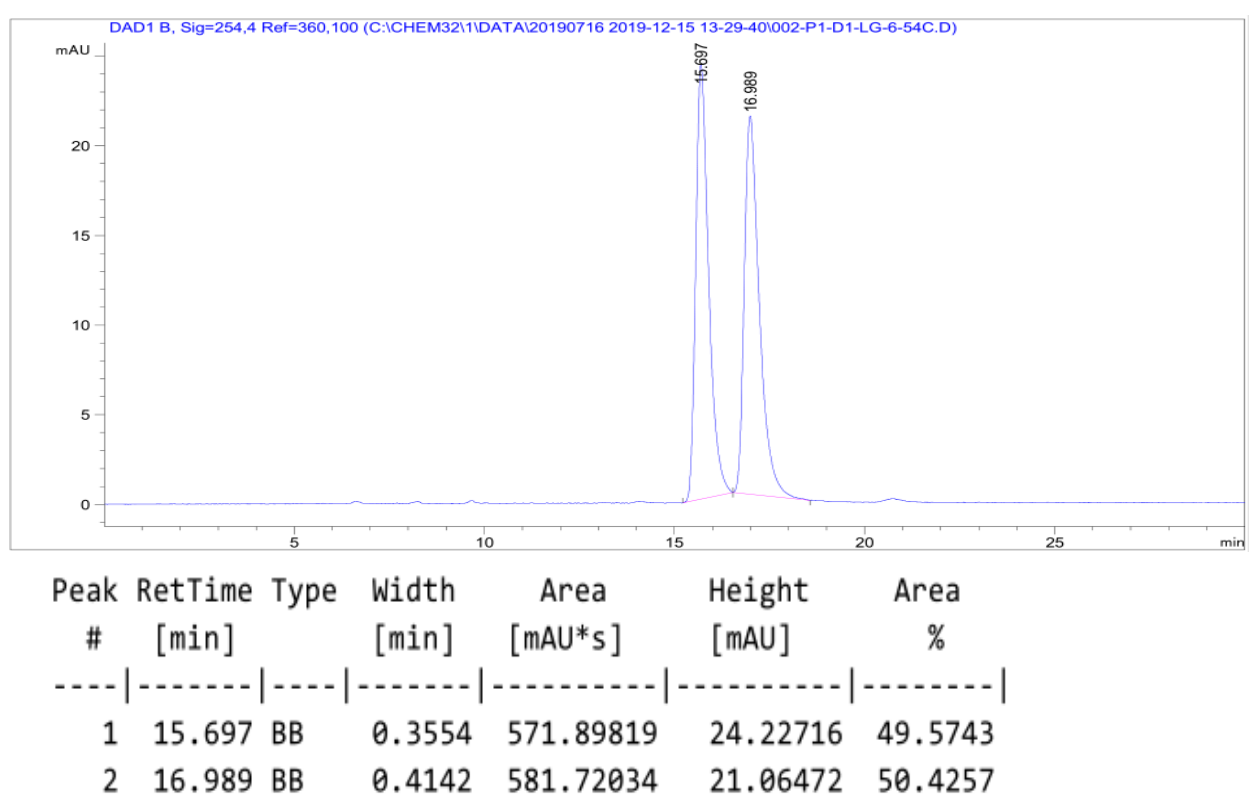

64 enantioenriched, $86 \%$ ee

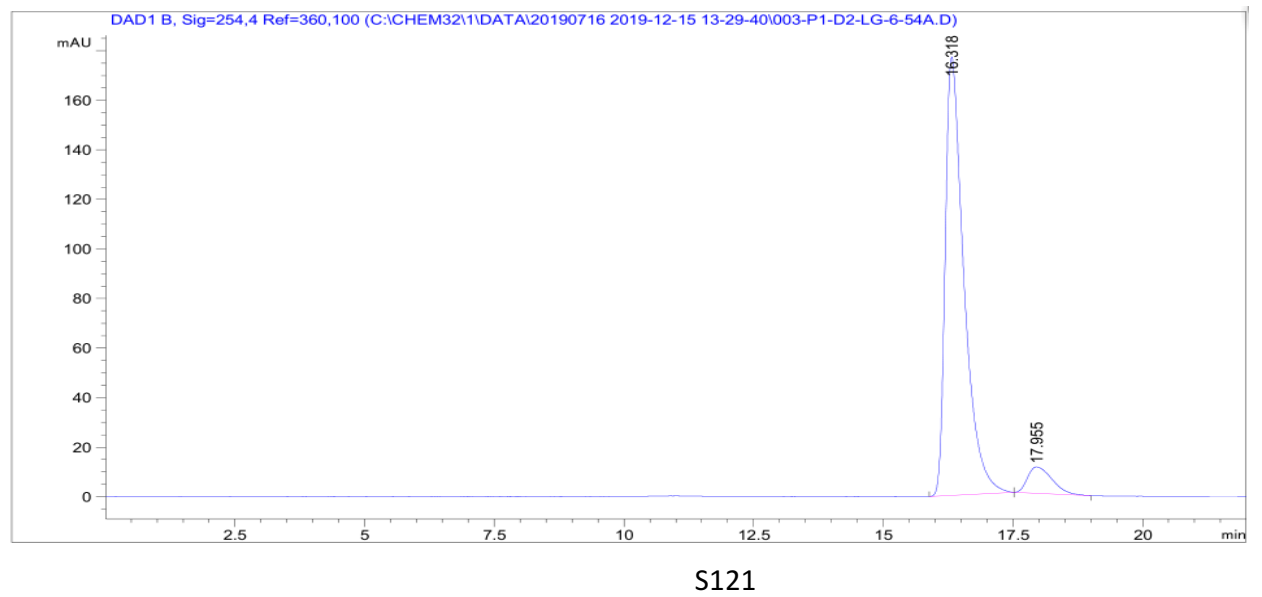




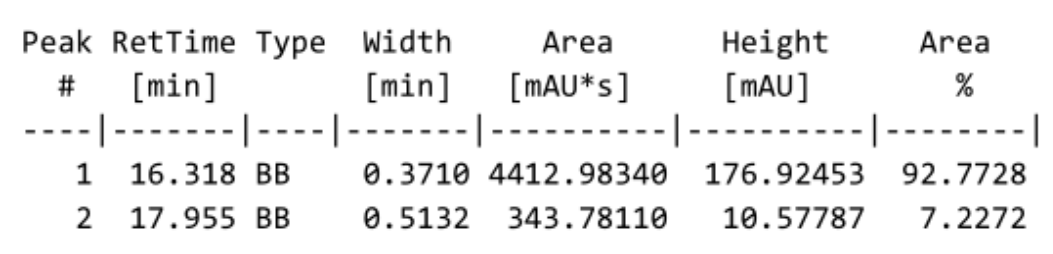

(c)

(R)-2-(2-Fluoro-[1,1'-biphenyl]-4-yl)-4-methylpentanoic acid (67)

The preparation and characterization data for the compound $\mathbf{6 7}$ is based on previously reported procedure ${ }^{4}$. A round-bottom flask was charged with $(R)$-tert-butyl 2-(2-fluoro[1,1'-biphenyl]-4-yl)-4-methylpentanoate 64 (34.2 mg, 0.1 mmol, 1.0 equiv.) and DCM $[0.02 \mathrm{M}]$, to this solution, TFA $(37.1 \mathrm{uL}, 0.5 \mathrm{mmol}, 5.0$ equiv. $)$ was added dropwise at $0^{\circ} \mathrm{C}$. Then the mixture was stirred vigorously for 4 hour at room temperatureerature, extracted with DCM three times, combined organic layer was washed with brine and dried over $\mathrm{MgSO}_{4}$. After drying under high vacuum without further purification to obtain the target product $(28.6 \mathrm{mg},>99 \%$ yield, $88 \%$ ee)

${ }^{1} \mathrm{H}$ NMR (400 MHz, $\left.\mathrm{CDCl}_{3}\right) \delta 7.53(\mathrm{~d}, J=6.6 \mathrm{~Hz}, 2 \mathrm{H}), 7.45-7.34(\mathrm{~m}, 4 \mathrm{H}), 7.18-7.14$ $(\mathrm{m}, 2 \mathrm{H}), 3.70(\mathrm{t}, J=7.4 \mathrm{~Hz}, 1 \mathrm{H}), 2.01-1.94(\mathrm{~m}, 1 \mathrm{H}), 1.76-1.69(\mathrm{~m}, 1 \mathrm{H}), 1.57-1.51(\mathrm{~m}$, $1 \mathrm{H}), 0.94(\mathrm{~d}, J=6.4 \mathrm{~Hz}, 6 \mathrm{H})$.

${ }^{19} \mathrm{~F}$ NMR $\left(375 \mathrm{MHz}, \mathrm{CDCl}_{3}\right) \delta-117.49(\mathrm{t}, J=10.6 \mathrm{~Hz})$.

${ }^{13} \mathrm{C} \mathrm{NMR}\left(100 \mathrm{MHz}, \mathrm{CDCl}_{3}\right) \delta 179.06,159.71(\mathrm{~d}, J=248.4 \mathrm{~Hz}), 139.89(\mathrm{~d}, J=7.7$ Hz), 135.44, 130.83 (d, $J=3.6 \mathrm{~Hz}), 128.96$ (d, $J=2.3 \mathrm{~Hz}), 128.46,128.15$ (d, $J=13.2$ $\mathrm{Hz}), 127.71,124.18(\mathrm{~d}, J=2.9 \mathrm{~Hz}), 115.77$ (d, $J=23.5 \mathrm{~Hz}), 48.88,42.00,25.77,22.60$, 22.17 .

HRMS (ESI): $\mathrm{C}_{18} \mathrm{H}_{19} \mathrm{FNaO}_{2}{ }^{+}(\mathrm{M}+\mathrm{H}): 309.1267$, found: 309.1267 .

$[\alpha]_{\mathrm{D}}^{25}=-18.4996\left(\mathrm{c}=0.0600, \mathrm{CHCl}_{3}\right)$

Chiral HPLC: CHIRALPAK IB, $25{ }^{\circ} \mathrm{C}, 99 / 1(1 / 1000 \mathrm{TFA}), 1.0 \mathrm{~mL} / \mathrm{min}, 254 \mathrm{~nm}$, $t_{R}($ major $)=14.4 \mathrm{~min}, t_{R}($ minor $)=16.3 \mathrm{~min}$ 


\section{7 racemic}

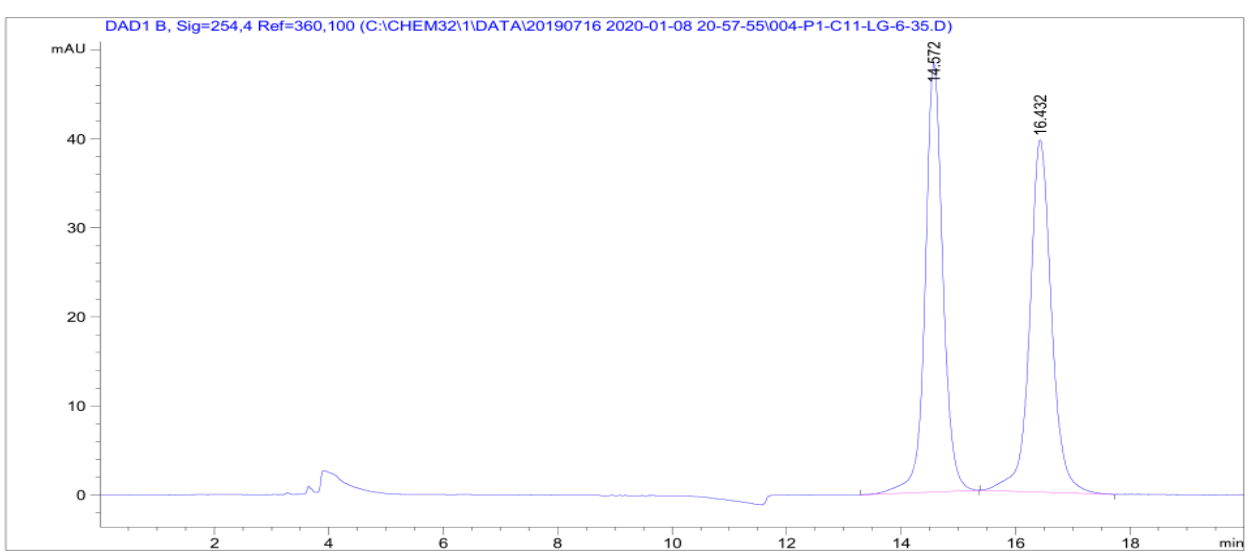

Peak RetTime Type Width Area Height Area

\# $[\mathrm{min}] \quad[\mathrm{min}] \quad\left[\mathrm{mAU}^{*} \mathrm{~s}\right] \quad[\mathrm{mAU}] \quad \%$

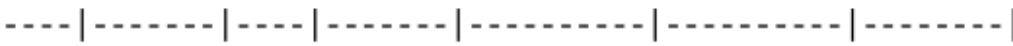

$\begin{array}{lllllll}1 & 14.572 & \text { BB } & 0.3250 & 1036.69629 & 48.18690 & 49.6630\end{array}$

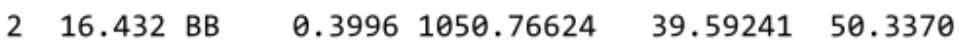

67 enantioenriched, $88 \%$ ee

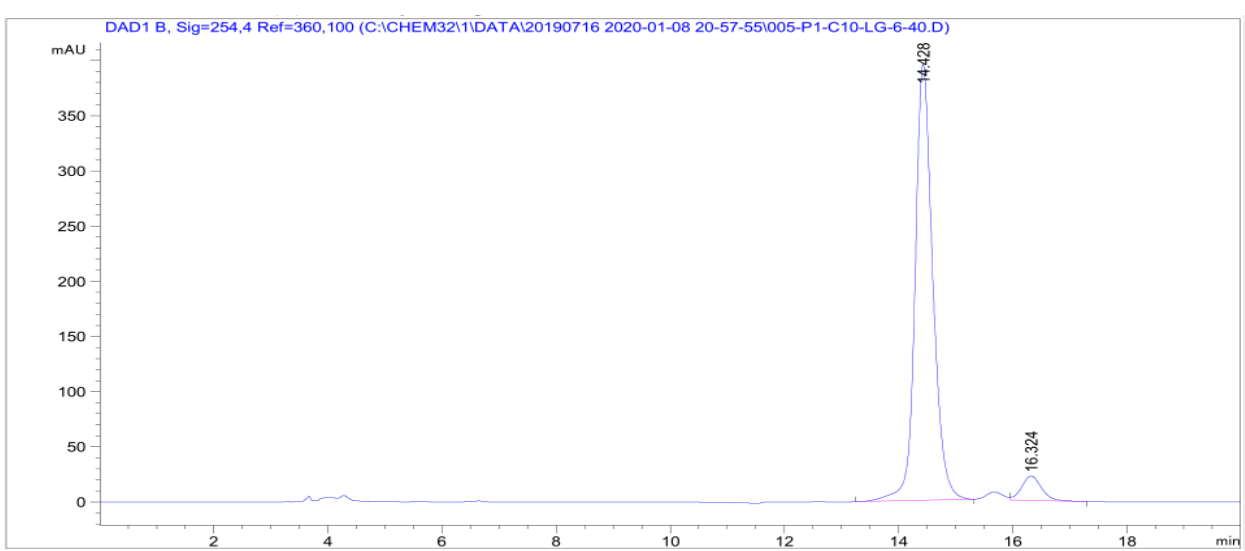

\begin{tabular}{cccccc}
$\begin{array}{c}\text { Peak RetTime Type } \\
\text { \# }\end{array}$ [min] & $\begin{array}{c}\text { Width } \\
{[\mathrm{min}]}\end{array}$ & $\begin{array}{c}\text { Area } \\
{[\mathrm{mAU} \text { s }]}\end{array}$ & $\begin{array}{c}\text { Height } \\
{[\mathrm{mAU}]}\end{array}$ & $\begin{array}{c}\text { Area } \\
\%\end{array}$ \\
\hline 1 & 14.428 BB & 0.3254 & 8511.65234 & 394.99243 & 94.0423 \\
2 & 16.324 VB & 0.3700 & 539.22729 & 22.15064 & 5.9577
\end{tabular}<smiles>CCCOC(=O)C(CC1CCCC1)c1ccc(-c2ccccc2)c(F)c1</smiles>

(R)-tert-Butyl 3-cyclopentyl-2-(2-fluoro-[1,1'-biphenyl]-4-yl)propanoate (65) 
The product was prepared according to the general procedure with $(4 \mathrm{~S}, 4$ 'S)-4,4'-di((S)sec-butyl)-4,4',5,5'-tetrahydro-2,2'-bioxazole [(S,S)-L1] $(3.3 \mathrm{mg}, 0.013 \mathrm{mmol}, 13$ mol\%), $\mathrm{NiCl}_{2} \cdot \mathrm{Py}_{4}(4.5 \mathrm{mg}, 0.01 \mathrm{mmol}, 10 \mathrm{~mol} \%), \operatorname{Ir}\left[\mathrm{dF}\left(\mathrm{CF}_{3}\right) \mathrm{ppy}\right]_{2}(\mathrm{bpy}) \mathrm{PF}_{6}(3.2 \mathrm{mg}$, $0.003 \mathrm{mmol}, 3 \mathrm{~mol} \%$ ), 4-bromo-2-fluoro-1,1'-biphenyl (25.1 mg, $0.1 \mathrm{mmol}, 1.0$ equiv.), potassium cyclopentyltrifluoroboranuide $(52.8 \mathrm{mg}, 0.3 \mathrm{mmol}, 3.0$ equiv.) and tert-butyl acrylate $(29.0 \mathrm{uL}, 0.2 \mathrm{mmol}, 2.0$ equiv.) in acetone/ethyl acetate $(1.3 / 0.7, \mathrm{v} / \mathrm{v}, 2 \mathrm{~mL})$ were used. After 24 hours, the product was isolated by flash chromatography (PE: ethyl acetate $=20: 1)$ as a white solid $(19.5 \mathrm{mg}, 53 \%$ yield, $87 \%$ ee).

${ }^{1} \mathbf{H}$ NMR $\left(400 \mathrm{MHz}, \mathrm{CDCl}_{3}\right) \delta$ 7.56-7.54 (m, 2H), $7.43(\mathrm{t}, J=7.4 \mathrm{~Hz}, 2 \mathrm{H}), 7.39-7.33$ (m, 2H), 7.15-7.11 (m, 2H), $3.52(\mathrm{t}, J=7.7 \mathrm{~Hz}, 1 \mathrm{H}), 2.05(\mathrm{dt}, J=13.2,7.6 \mathrm{~Hz}, 1 \mathrm{H})$, 1.81-1.67 (m, 4H), 1.65-1.58 (m, 2H), 1.52-1.47 (m, 2H), 1.42 (s, 9H), 1.19-1.08 (m, $2 \mathrm{H})$.

${ }^{19}$ F NMR (375 MHz, $\left.\mathrm{CDCl}_{3}\right) \delta-118.04--118.09(\mathrm{~m})$.

${ }^{13}$ C NMR $\left(100 \mathrm{MHz}, \mathrm{CDCl}_{3}\right) \delta 173.00,159.64(\mathrm{~d}, J=247.9 \mathrm{~Hz}), 141.46(\mathrm{~d}, J=7.7$ Hz), 135.67, 130.53 (d, $J=4.0 \mathrm{~Hz}), 128.96$ (d, $J=2.9 \mathrm{~Hz}), 128.42,127.56,127.45$ (d, $J=13.4 \mathrm{~Hz}), 123.97$ (d, $J=3.2 \mathrm{~Hz}), 115.53$ (d, $J=23.5 \mathrm{~Hz}), 80.86,51.49,39.94,37.94$, $32.72,32.43,28.01,25.13,25.10$.

HRMS (ESI): $\mathrm{C}_{24} \mathrm{H}_{30} \mathrm{FO}_{2}{ }^{+}$(M+H): 369.2230, found: 369.2227 .

$[\alpha]_{\mathrm{D}}^{25}=-11.1109\left(\mathrm{c}=0.0567, \mathrm{CHCl}_{3}\right)$

Chiral HPLC: CHIRALPAK IB, $25{ }^{\circ} \mathrm{C},{ }^{i} \mathrm{PrOH}-$ hexanes 1/100, $1.0 \mathrm{~mL} / \mathrm{min}, 254 \mathrm{~nm}$, $\mathrm{t}_{\mathrm{R}}$ (major) $=25.0 \mathrm{~min}, \mathrm{t}_{\mathrm{R}}($ minor $)=23.0 \mathrm{~min}$.

\section{5 racemic}

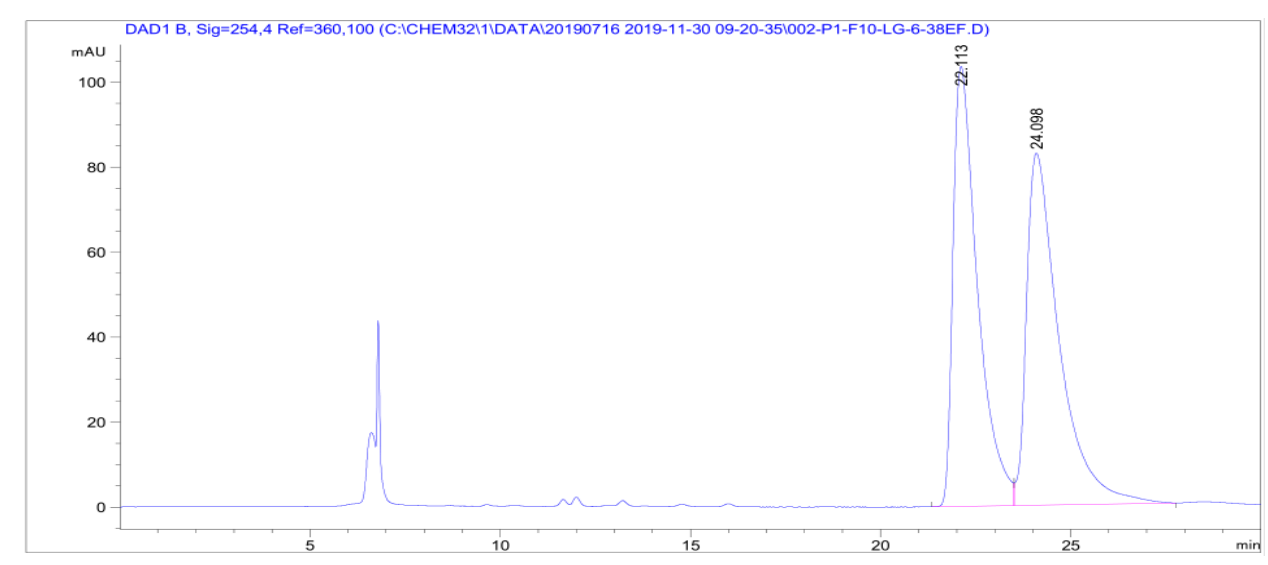




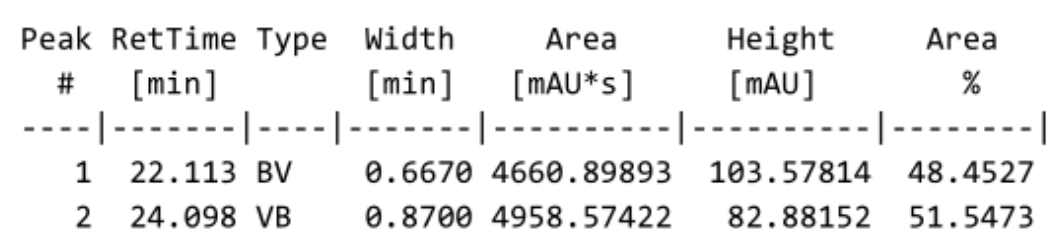

\section{5 enantioenriched, $87 \%$ ee}

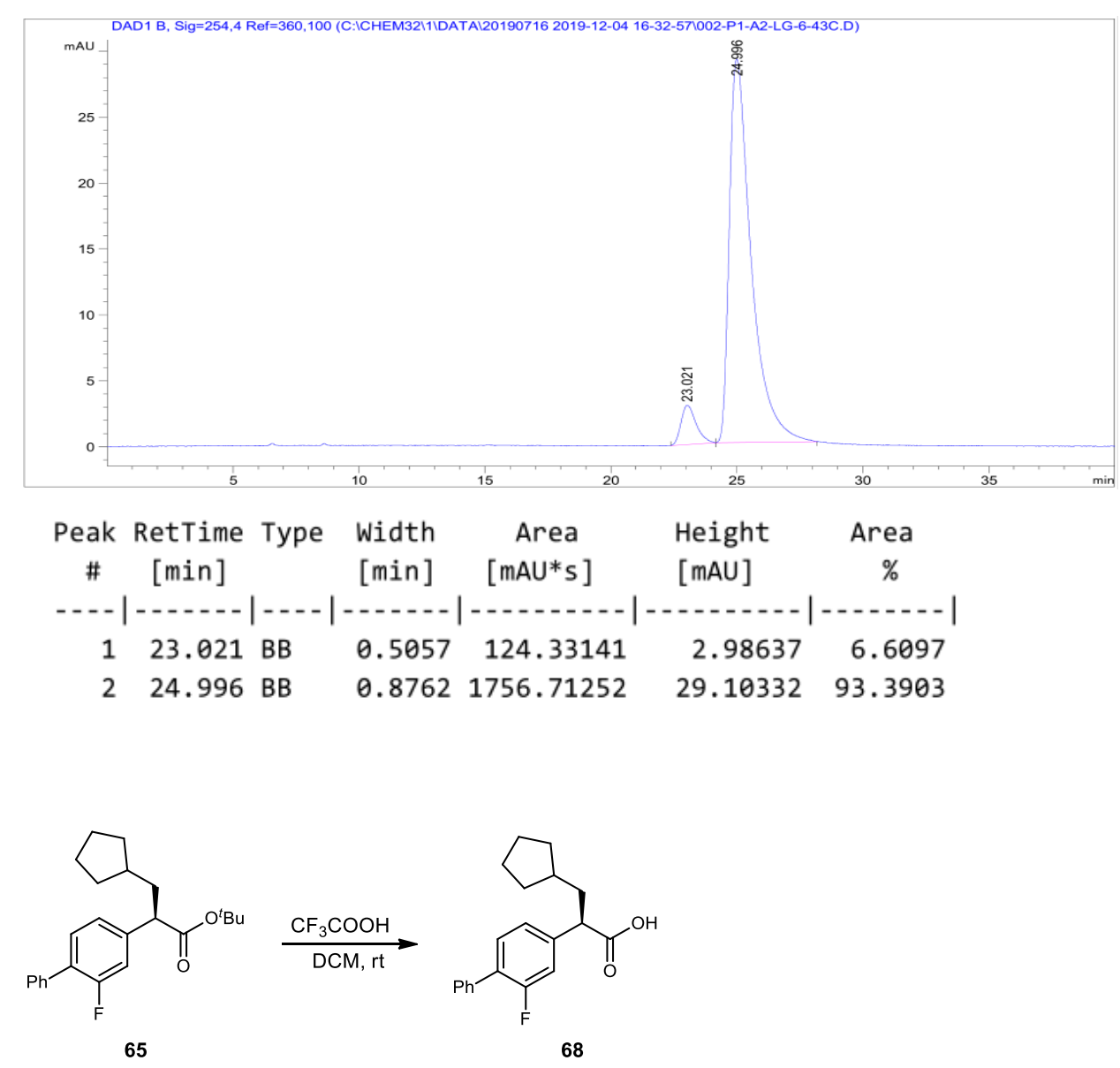

The preparation and characterization data for the compound $\mathbf{6 8}$ is based on previously reported procedure ${ }^{4}$. A round-bottom flask was charged with $(R)$-tert-butyl 3cyclopentyl-2-(2-fluoro-[1,1'-biphenyl]-4-yl)propanoate 65 (36.8 mg, $0.1 \mathrm{mmol}, 1.0$ equiv.) and DCM [0.02M], to this solution, TFA (37.1 uL, $0.5 \mathrm{mmol}, 5.0$ equiv. ) was added dropwise at $0^{\circ} \mathrm{C}$. Then the mixture was stirred vigorously for 4 hours at room temperature, extracted with DCM three times, combined organic layer were washed with brine and dried over $\mathrm{MgSO}_{4}$. After drying under high vacuum without further purification to obtain the target product ( $31.2 \mathrm{mg},>99 \%$ yield, $87 \%$ ee) 
${ }^{1}$ H NMR $\left(400 \mathrm{MHz}, \mathrm{CDCl}_{3}\right) \delta$ 7.54-7.52 (m, 2H), 7.45-7.34 (m, 4H), 7.18-7.15 (m, 2H), $3.65(\mathrm{t}, J=7.7 \mathrm{~Hz}, 1 \mathrm{H}), 2.14-2.04(\mathrm{~m}, 1 \mathrm{H}), 1.90-1.70(\mathrm{~m}, 4 \mathrm{H}), 1.63-1.51(\mathrm{~m}, 4 \mathrm{H})$, 1.19-1.09 (m, 2H).

${ }^{19}$ F NMR $\left(375 \mathrm{MHz}, \mathrm{CDCl}_{3}\right) \delta-117.49(\mathrm{t}, J=8.8 \mathrm{~Hz})$.

${ }^{13}$ C NMR (100 MHz, $\left.\mathrm{CDCl}_{3}\right) \delta 178.74,159.70(\mathrm{~d}, J=249.2 \mathrm{~Hz}), 139.97(\mathrm{~d}, J=7.5$ $\mathrm{Hz}), 135.46,130.80$ (d, $J=3.9 \mathrm{~Hz}), 128.96$ (d, $J=2.8 \mathrm{~Hz}), 128.45,128.13$ (d, $J=13.2$ $\mathrm{Hz}), 127.70,124.18$ (d, $J=3.0 \mathrm{~Hz}), 115.77$ (d, $J=23.6 \mathrm{~Hz}), 50.15,39.48,37.72,32.71$, $32.31,25.09,125.07$.

HRMS (ESI): $\mathrm{C}_{20} \mathrm{H}_{21} \mathrm{FNaO}_{2}^{+}(\mathrm{M}+\mathrm{Na})$ : 335.1423, found: 335.1425 .

$[\alpha]_{\mathrm{D}}^{25}=-46.4885\left(\mathrm{c}=0.1467, \mathrm{CHCl}_{3}\right)$

Chiral HPLC: CHIRALPAK IC, $25^{\circ} \mathrm{C},{ }^{i} \mathrm{PrOH}-h e x a n e s ~ 5 / 95(0.4 \%$ TFA $), 1.0 \mathrm{~mL} / \mathrm{min}$, $254 \mathrm{~nm}, \mathrm{t}_{\mathrm{R}}($ major $)=4.9 \mathrm{~min}, \mathrm{t}_{\mathrm{R}}($ minor $)=4.6 \mathrm{~min}$.

\section{8 racemic}

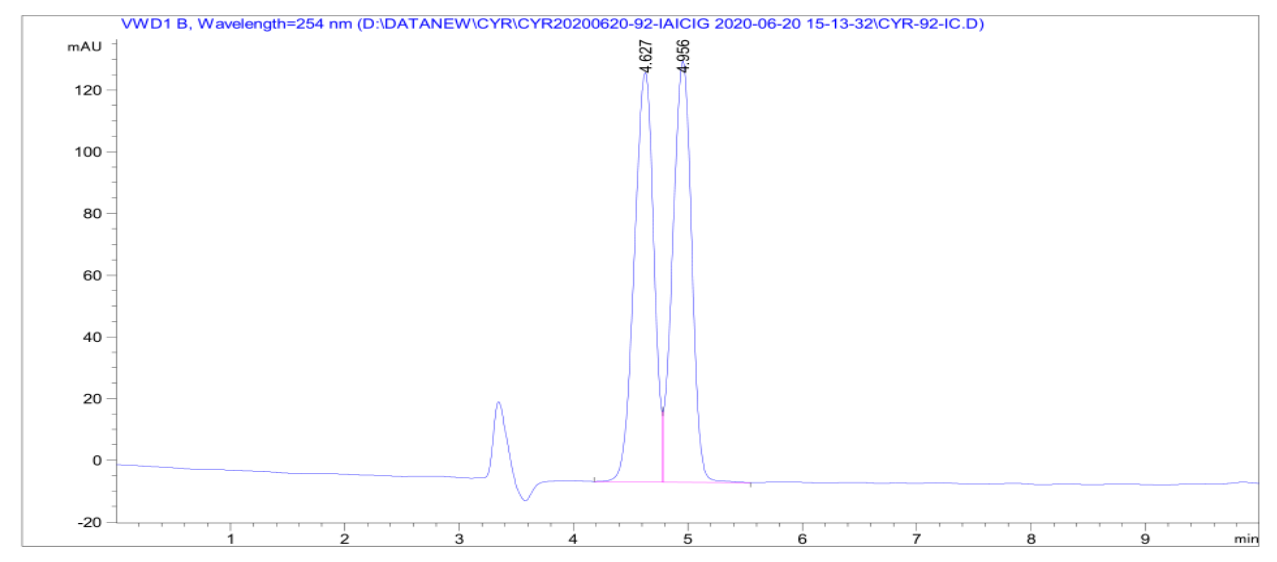

\begin{tabular}{|c|c|c|c|c|c|c|}
\hline $\begin{array}{c}\text { Peak } \\
\#\end{array}$ & $\begin{array}{c}\text { RetTime } \\
\text { [min] }\end{array}$ & Type & $\begin{array}{l}\text { Width } \\
\text { [min] }\end{array}$ & $\begin{array}{c}\text { Area } \\
{\left[\mathrm{mAU}^{*} \mathrm{~s}\right]}\end{array}$ & $\begin{array}{l}\text { Height } \\
{[\mathrm{mAU}]}\end{array}$ & $\begin{array}{c}\text { Area } \\
\frac{8}{\delta}\end{array}$ \\
\hline & - & & 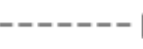 & 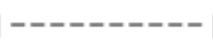 & ---ー-ー-ー- & $-------\mid$ \\
\hline 1 & 4.627 & BV & 60 & 1569.31250 & 2.67905 & 357 \\
\hline 2 & 4.956 & VB & 0.1824 & 1573.35437 & 136.52827 & 50.0643 \\
\hline
\end{tabular}


68 enantioenriched, $87 \%$ ee

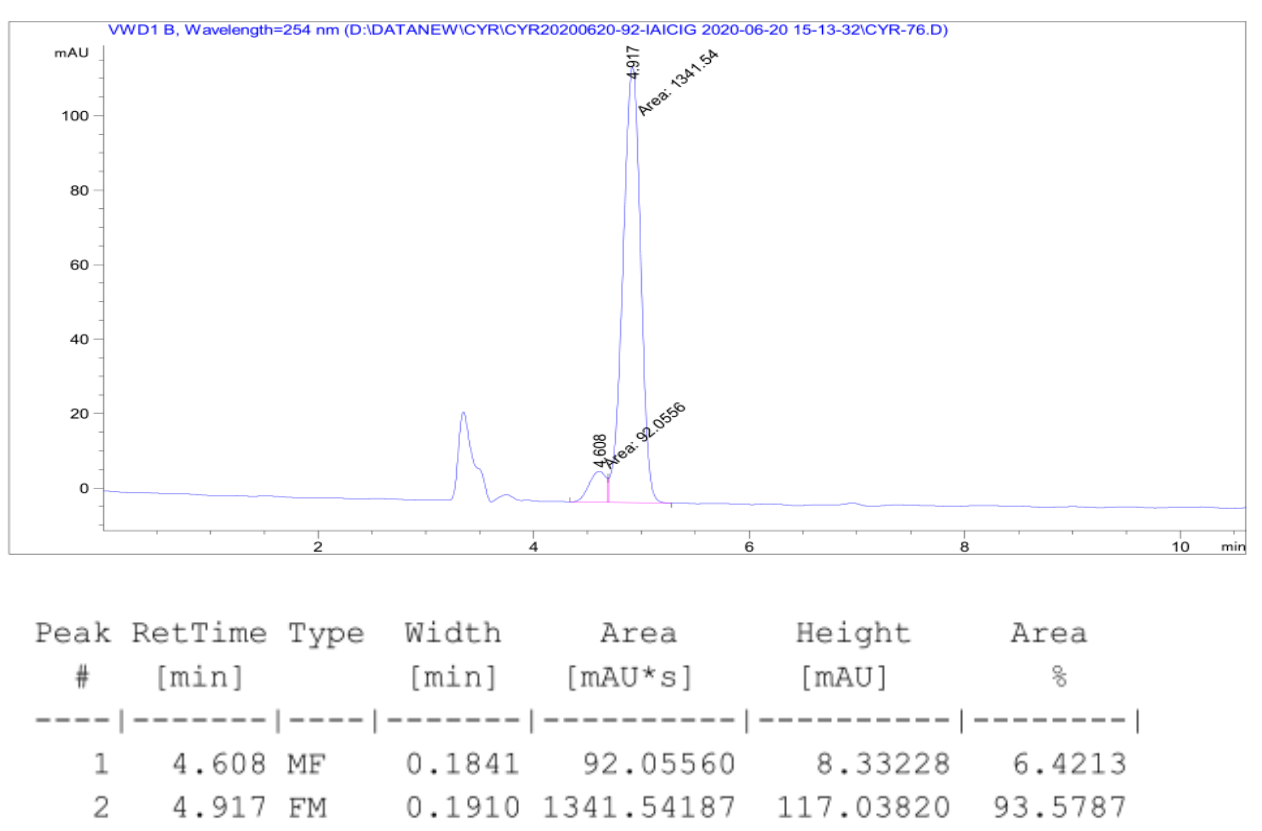




\section{Mechanistic Studies}

\subsection{Radical inhibition experiment}

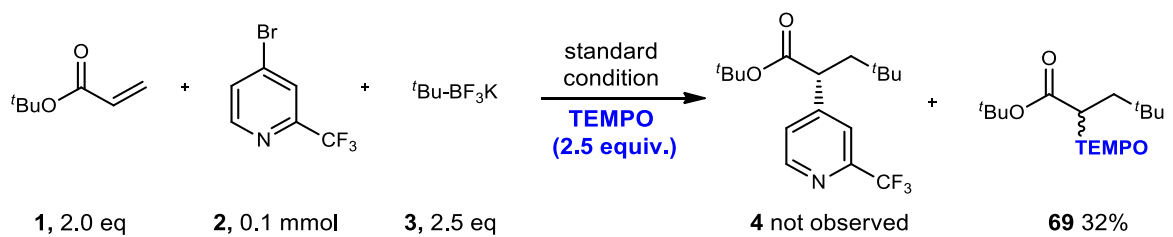

To a flame-dried $8 \mathrm{~mL}$ reaction vial was charged with (4S,4'S)-4,4'-di((S)-sec-butyl)4,4',5,5'-tetrahydro-2,2'-bioxazole $[(S, S)$-L1 $]$ (3.3 mg, $0.013 \mathrm{mmol}, 13 \mathrm{~mol} \%$ ), $\mathrm{NiCl}_{2} \bullet \mathrm{Py}_{4}(4.5 \mathrm{mg}, 0.01 \mathrm{mmol}, 10 \mathrm{~mol} \%), \operatorname{Ir}\left[\mathrm{dF}\left(\mathrm{CF}_{3}\right) \mathrm{ppy}\right]_{2}(\mathrm{bpy}) \mathrm{PF}_{6}(0.003 \mathrm{mmol}, 3$ mol\%), ${ }^{t} \mathrm{BuBF}_{3} \mathrm{~K}$ (41.0 mg, $0.25 \mathrm{mmol}, 2.5$ equiv.) and TEMPO (39.0 mg, $0.25 \mathrm{mmol}$, 2.5 equiv), the vial was capped. After evacuated and backfilled nitrogen three times, acetone/ethyl acetate 2:1 [0.05 M] was added via a syringe, 4-bromo-2(trifluoromethyl)pyridine $(13.7 \mathrm{uL}, 0.1 \mathrm{mmol}, 1.0$ equiv.), followed by the addition of tert-butyl acrylate $(29.0 \mathrm{uL}, 0.2 \mathrm{mmol}, 2.0$ equiv.). The reaction mixture was then irradiated with a $10 \mathrm{~W} 415-420 \mathrm{~nm}$ LED lamp with cooling from circulating cold ethanol for $24 \mathrm{~h}$ at $20{ }^{\circ} \mathrm{C}$. The reaction was quenched with $\mathrm{H}_{2} \mathrm{O}$, extracted with ethyl acetate. The combined organic layers were dried with $\mathrm{MgSO}_{4}$, filtered, and concentrated in vacuo. The crude material was purified by flash chromatography to afford the product 69.

${ }^{1}$ H NMR $\left(400 \mathrm{MHz}, \mathrm{CDCl}_{3}\right) \delta 4.13(\mathrm{dd}, J=10.3,2.9 \mathrm{~Hz}, 1 \mathrm{H}), 1.86(\mathrm{dd}, J=13.9,10.3$ $\mathrm{Hz}, 1 \mathrm{H}), 1.74(\mathrm{dd}, J=13.9,2.9 \mathrm{~Hz}, 1 \mathrm{H}), 1.53-1.50(\mathrm{~m}, 1 \mathrm{H}), 1.47$ (s, 9H), 1.45-1.43 (m, $3 \mathrm{H}), 1.31-1.25(\mathrm{~m}, 2 \mathrm{H}), 1.20(\mathrm{~s}, 3 \mathrm{H}), 1.11(\mathrm{~s}, 6 \mathrm{H}), 1.07(\mathrm{~s}, 3 \mathrm{H}), 0.93(\mathrm{~s}, 9 \mathrm{H})$.

${ }^{13} \mathrm{C}$ NMR $\left(100 \mathrm{MHz}, \mathrm{CDCl}_{3}\right) \delta 173.28,83.63,81.01,60.25,59.22,46.14,40.25,34.15$, $34.02,30.13,29.51,28.04,20.31,20.19,17.11$.

HRMS (ESI): $\mathrm{C}_{20} \mathrm{H}_{40} \mathrm{NO}_{3}{ }^{+}$(M+H): 342.3008 , found: 342.3002 . 


\subsection{Radical probe reaction}

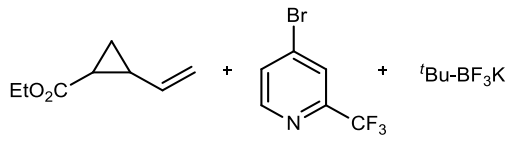

$70,2.0 \mathrm{eq}$

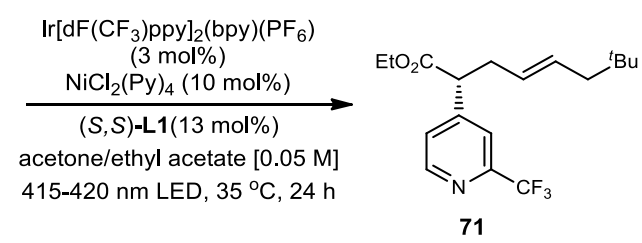

71

To a flame-dried $8 \mathrm{~mL}$ reaction vial was charged with (4S,4'S)-4,4'-di((S)-sec-butyl)4,4',5,5'-tetrahydro-2,2'-bioxazole $[(S, S)-\mathbf{L 1}]$ (3.3 mg, $0.013 \mathrm{mmol}, 13 \mathrm{~mol} \%)$, $\mathrm{NiCl}_{2} \bullet \mathrm{Py}_{4}(4.5 \mathrm{mg}, 0.01 \mathrm{mmol}, 10 \mathrm{~mol} \%), \operatorname{Ir}\left[\mathrm{dF}\left(\mathrm{CF}_{3}\right) \text { ppy }\right]_{2}(\mathrm{bpy}) \mathrm{PF}_{6}(0.003 \mathrm{mmol}, 3$ mol\%) and ${ }^{t} \mathrm{BuBF}_{3} \mathrm{~K}(41.0 \mathrm{mg}, 0.25 \mathrm{mmol}, 2.5$ equiv.), the vial was capped. After evacuated and backfilled nitrogen three times, acetone/ethyl acetate $(2: 1, \mathrm{v} / \mathrm{v})[0.05 \mathrm{M}]$ was added via a syringe, 4-bromo-2-(trifluoromethyl)pyridine (13.7 uL, $0.1 \mathrm{mmol}, 1.0$ equiv.), followed by the addition of ethyl 2-vinylcyclopropanecarboxylate ( $26.4 \mathrm{uL}, 0.2$ mmol, 2.0 equiv.). The reaction mixture was then irradiated with a $10 \mathrm{~W} 415-420 \mathrm{~nm}$ LED lamp with cooling from circulating cold ethanol for $24 \mathrm{~h}$ at $20{ }^{\circ} \mathrm{C}$. The reaction was quenched with $\mathrm{H}_{2} \mathrm{O}$, extracted with ethyl acetate. The combined organic layers were dried with $\mathrm{MgSO}_{4}$, filtered, and concentrated in vacuo. The crude material was purified by flash chromatography to afford the product 71, the product was isolated by flash chromatography $(\mathrm{PE}$ : ethyl acetate $=20: 1)$ as a colorless oil $(12.1 \mathrm{mg}, 35 \%$ yield, $71 \%$ ee, $E / Z$ 8:1).

${ }^{1}$ H NMR $\left(400 \mathrm{MHz}, \mathrm{CDCl}_{3}\right) \delta 8.67(\mathrm{~d}, J=5.0 \mathrm{~Hz}, 1 \mathrm{H}), 7.63(\mathrm{~s}, 1 \mathrm{H}), 7.45(\mathrm{~d}, J=3.8$ $\mathrm{Hz}, 1 \mathrm{H}), 5.55-5.45(\mathrm{~m}, 1 \mathrm{H}), 5.27-5.20(\mathrm{~m}, 1 \mathrm{H}), 4.22-4.09(\mathrm{~m}, 2 \mathrm{H}), 3.69(\mathrm{t}, J=7.7 \mathrm{~Hz}$, $1 \mathrm{H}), 2.84-2.77(\mathrm{~m}, 1 \mathrm{H}), 2.55-2.48(\mathrm{~m}, 1 \mathrm{H}), 1.81-1.79(\mathrm{~m}, 2 \mathrm{H}), 1.23(\mathrm{t}, J=7.2 \mathrm{~Hz}, 3 \mathrm{H})$, [0.85 (s, 1H), $0.77(\mathrm{~s}, 8 \mathrm{H}), E / Z$ 8:1].

${ }^{19}$ F NMR $\left(375 \mathrm{MHz}, \mathrm{CDCl}_{3}\right) \delta-67.99(\mathrm{~s})$.

${ }^{13}$ C NMR $\left(100 \mathrm{MHz}, \mathrm{CDCl}_{3}\right) \delta 171.65,150.16,149.57,148.22(\mathrm{q}, J=33.3 \mathrm{~Hz}), 131.94$, 127.01, 126.04, 124.23 (q, $J=277.8 \mathrm{~Hz}), 120.31$ (q, $J=3.0 \mathrm{~Hz}), 61.50,51.56,46.92$, $36.39,30.70,29.08,14.07$.

HRMS (ESI): $\mathrm{C}_{18} \mathrm{H}_{25} \mathrm{~F}_{3} \mathrm{NO}_{2}{ }^{+}(\mathrm{M}+\mathrm{H}): 344.1837$, found: 344.1835 .

$[\alpha]_{\mathrm{D}}^{25}=-9.6665\left(\mathrm{c}=0.1200, \mathrm{CHCl}_{3}\right)$ 
Chiral HPLC: CHIRALPAK IC, $25{ }^{\circ} \mathrm{C},{ }^{i} \mathrm{PrOH}-$ hexanes 5/95, $0.5 \mathrm{~mL} / \mathrm{min}, 254 \mathrm{~nm}, \mathrm{t}_{\mathrm{R}}$ $($ major $)=13.5 \mathrm{~min}, \mathrm{t}_{\mathrm{R}}($ minor $)=9.7 \mathrm{~min}$.

\section{1 racemic}

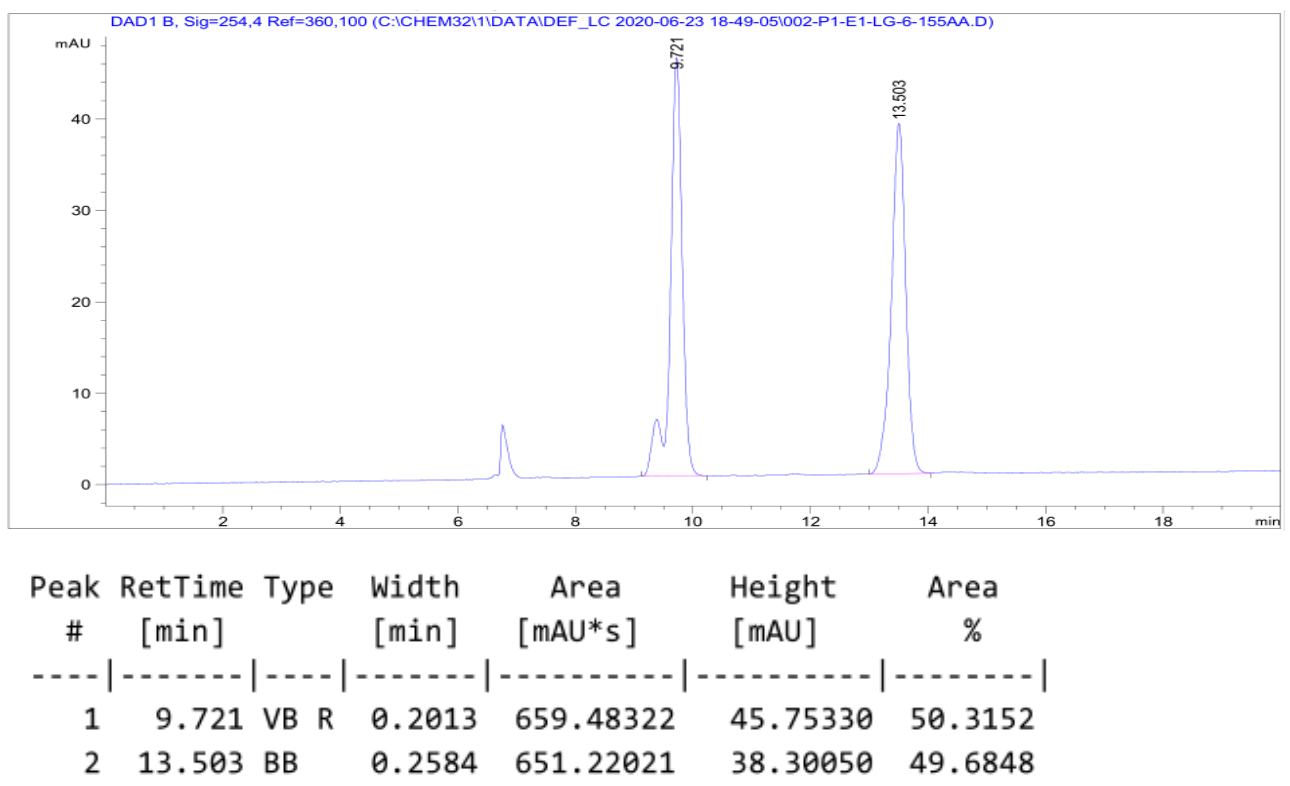

71 enantioenriched, $71 \%$ ee

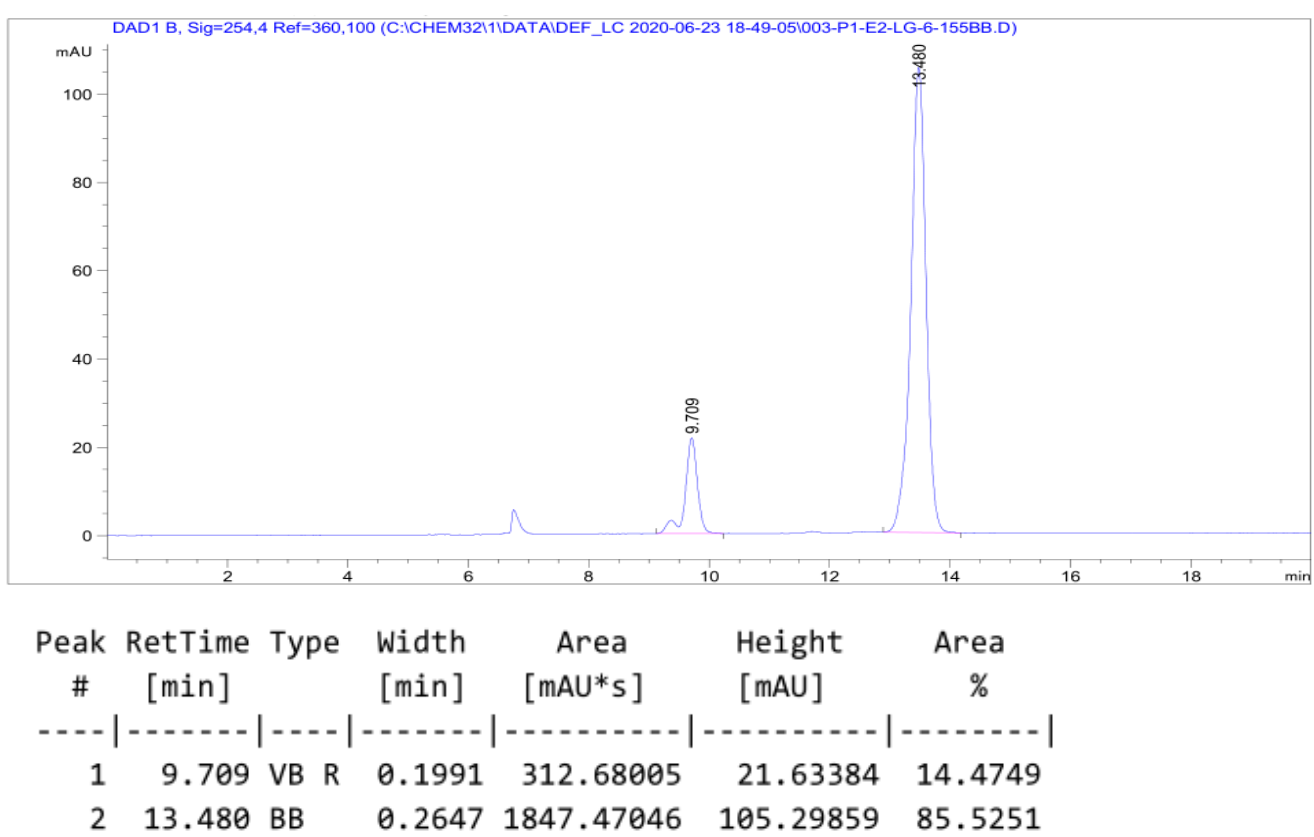




\subsection{Trapping of $\mathrm{Ni}(\mathrm{I})$-alkyl species with acetone}

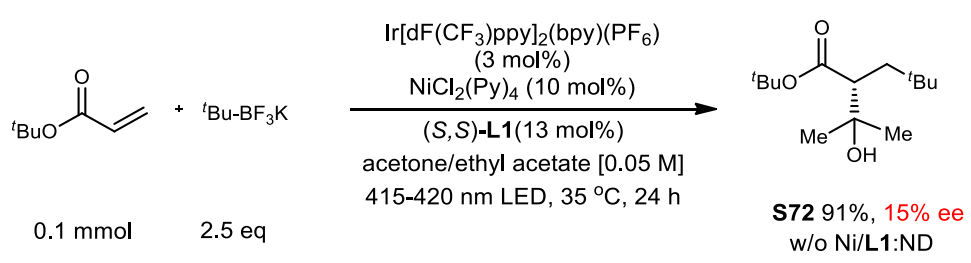

To a flame-dried $8 \mathrm{~mL}$ reaction vial was charged with (4S,4'S)-4,4'-di((S)-sec-butyl)4,4',5,5'-tetrahydro-2,2'-bioxazole $[(S, S)$-L1 $](3.3 \mathrm{mg}, 0.013 \mathrm{mmol}, 13 \mathrm{~mol} \%)$, $\mathrm{NiCl}_{2} \bullet \mathrm{Py}_{4}(4.5 \mathrm{mg}, 0.01 \mathrm{mmol}, 10 \mathrm{~mol} \%), \operatorname{Ir}\left[\mathrm{dF}\left(\mathrm{CF}_{3}\right) \text { ppy }\right]_{2}(\mathrm{bpy}) \mathrm{PF}_{6}(3.2 \mathrm{mg}, 0.003$ mmol, $3 \mathrm{~mol} \%$ ), and ${ }^{t} \mathrm{BuBF}_{3} \mathrm{~K}$ (41.0 $\mathrm{mg}, 0.25 \mathrm{mmol}, 2.5$ equiv.), the vial was capped. After evacuated and backfilled nitrogen three times, acetone/ethyl acetate 2:1 [0.05 M] was added via a syringe, followed by the addition of tert-butyl acrylate $(14.5 \mathrm{uL}, 0.1$ mmol, 1.0 equiv.). The reaction mixture was then irradiated with a $10 \mathrm{~W} 415-420 \mathrm{~nm}$ LED lamp with cooling from circulating cold ethanol for $24 \mathrm{~h}$ at $20{ }^{\circ} \mathrm{C}$. The reaction was quenched with $\mathrm{H}_{2} \mathrm{O}$, extracted with ethyl acetate. The combined organic layers were dried with $\mathrm{MgSO}_{4}$, filtered, and concentrated in vacuo. The crude material was purified by flash chromatography to afford the product S72, the product was isolated by flash chromatography (PE: ethyl acetate $=10: 1)$ as a white solid $(22.3 \mathrm{mg}, 91 \%$ yield, $15 \%$ ee).

(Note: there is no product is detected without $\mathbf{L} \mathbf{1}$ or nickel catalyst under this reaction condition)

${ }^{1}$ H NMR $\left(400 \mathrm{MHz}, \mathrm{CDCl}_{3}\right) \delta 2.50(\mathrm{~s}, 1 \mathrm{H}), 2.20(\mathrm{dd}, J=9.9,0.8 \mathrm{~Hz}, 1 \mathrm{H}), 1.66(\mathrm{dd}, J$ $=14.4,10.0 \mathrm{~Hz}, 1 \mathrm{H}), 1.40(\mathrm{~s}, 9 \mathrm{H}), 1.18(\mathrm{~s}, 3 \mathrm{H}), 1.13(\mathrm{~s}, 3 \mathrm{H}), 0.83(\mathrm{~s}, 9 \mathrm{H})$.

${ }^{13}$ C NMR $\left(100 \mathrm{MHz}, \mathrm{CDCl}_{3}\right) \delta 176.20,81.12,71.99,52.73,41.41,30.45,29.40,28.68$, $27.96,26.76$.

HRMS (ESI): $\mathrm{C}_{14} \mathrm{H}_{29} \mathrm{O}_{3}^{+}$(M+H): 245.2117, found: 245.2112 .

$[\alpha]_{\mathrm{D}}^{25}=-17.6237\left(\mathrm{c}=0.6333, \mathrm{CHCl}_{3}\right)$

Chiral HPLC: CHIRALPAK IA, $25{ }^{\circ} \mathrm{C},{ }^{i} \mathrm{PrOH}-$ hexanes 1/99, $1.0 \mathrm{~mL} / \mathrm{min}, 210 \mathrm{~nm}, \mathrm{t}_{\mathrm{R}}$ (major) $=8.0 \mathrm{~min}, \mathrm{t}_{\mathrm{R}}($ minor $)=7.5 \mathrm{~min}$. 


\section{2 racemic}

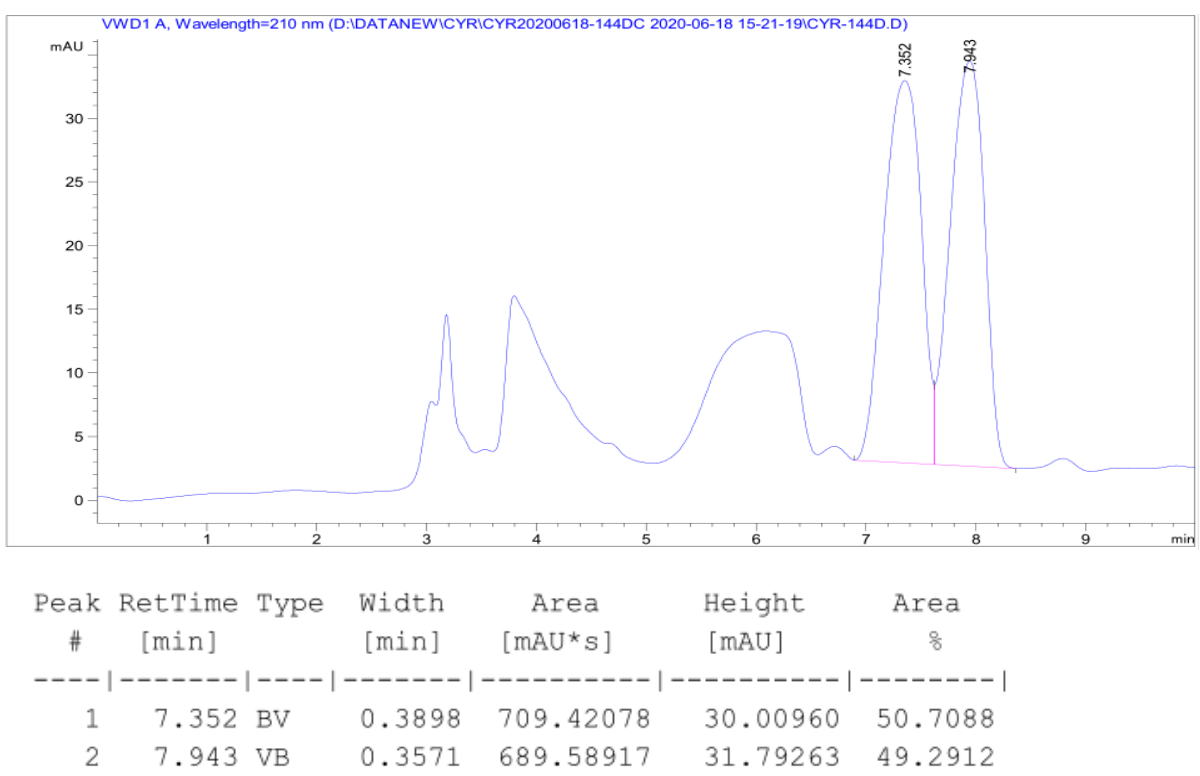

72 enantioenriched, $15 \%$ ee

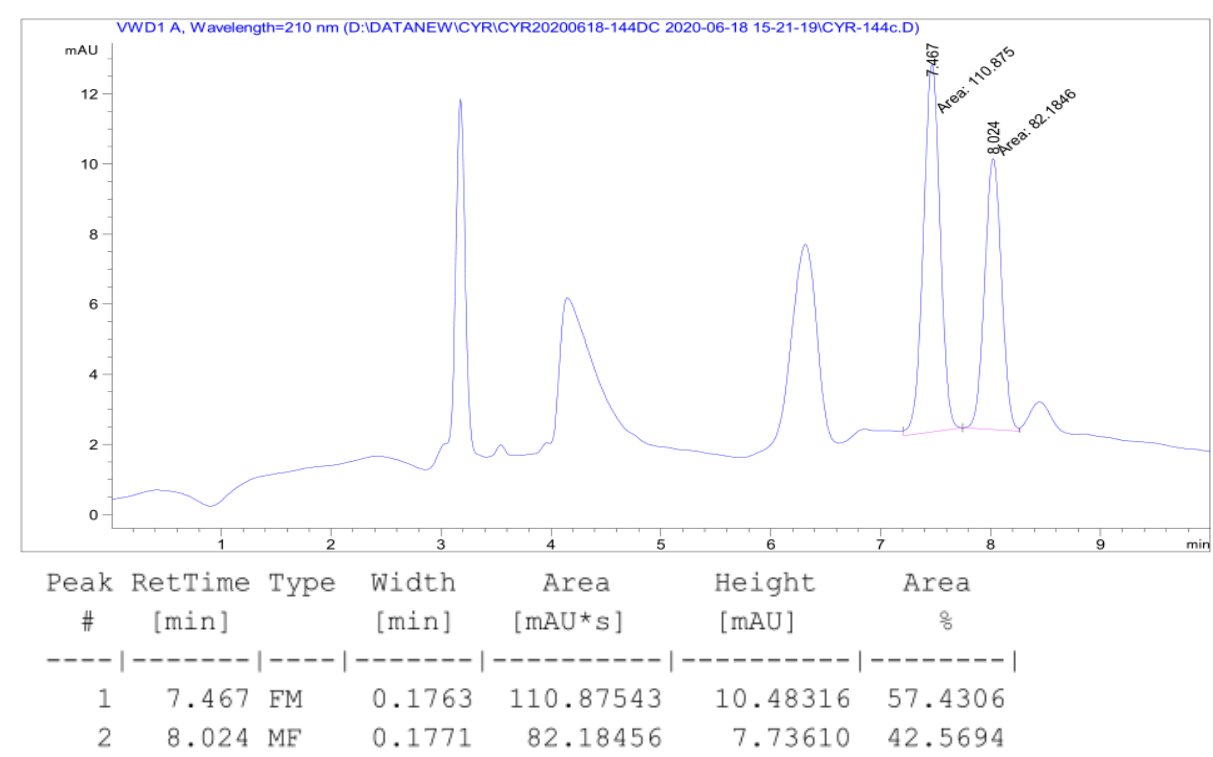




\subsection{Evaluation of styrenes}

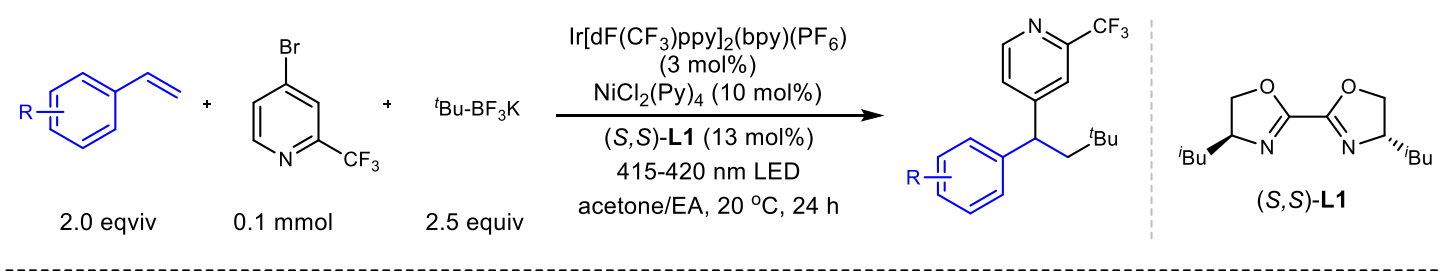

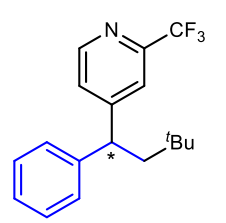

S73

$34 \%$ yield

ee not dermined*

*inseperable by chiral HPLCs teste

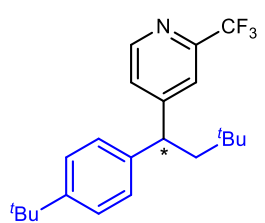

S74

$30 \%$ yield ee not dermined ${ }^{*}$

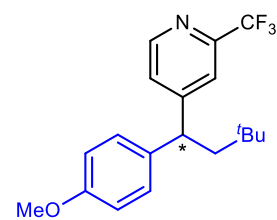

S75 $23 \%$ yield, $4 \%$ ee

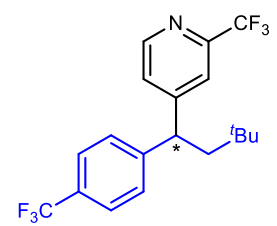

S76

$34 \%$ yield ee not dermined

Figure S2. Evaluation of styrenes under standard conditions<smiles>COc1ccc(C(CC(C)(C)C)c2ccnc(C(F)(F)F)c2)cc1</smiles>

\section{4-(1-(4-methoxyphenyl)-3,3-dimethylbutyl)-2-(trifluoromethyl)pyridine (S75)}

Following the general procedure, the product was isolated by flash chromatography $(\mathrm{PE}$ : ethyl acetate $=20: 1)$ as a colorless oil $(7.6 \mathrm{mg}, 23 \%$ yield, $4 \%$ ee).

${ }^{1} \mathbf{H}$ NMR $\left(400 \mathrm{MHz}, \mathrm{CDCl}_{3}\right) \delta 8.57(\mathrm{~d}, J=5.1 \mathrm{~Hz}, 1 \mathrm{H}), 7.56(\mathrm{br}, 1 \mathrm{H}), 7.37$ (dd, $J=5.1$, $1.4 \mathrm{~Hz}, 1 \mathrm{H}), 7.17(\mathrm{~d}, J=8.7 \mathrm{~Hz}, 2 \mathrm{H}), 6.84(\mathrm{~d}, J=8.7 \mathrm{~Hz}, 2 \mathrm{H}), 4.06(\mathrm{t}, J=6.8 \mathrm{~Hz}, 1 \mathrm{H})$, $3.78(\mathrm{~s}, 3 \mathrm{H}), 2.13(\mathrm{dd}, J=14.1,7.4 \mathrm{~Hz}, 1 \mathrm{H}), 2.01$ (dd, $J=14.1,5.9 \mathrm{~Hz}, 1 \mathrm{H}), 0.84$ (s, 9H).

${ }^{19}$ F NMR $\left(375 \mathrm{MHz}, \mathrm{CDCl}_{3}\right) \delta-67.90$ (s).

${ }^{13}$ C NMR $\left(100 \mathrm{MHz}, \mathrm{CDCl}_{3}\right) \delta 158.44,158.22,150.02,148.33$ (q, $\left.J=34.1 \mathrm{~Hz}\right), 135.75$, 128.70, 125.52, 121.61 (q, $J=274.1 \mathrm{~Hz}), 119.65$ (q, $J=2.8 \mathrm{~Hz}), 114.29,55.25,48.81$, $47.13,31.56,30.15$.

HRMS (ESI): $\mathrm{C}_{19} \mathrm{H}_{23} \mathrm{~F}_{3} \mathrm{NO}^{+}(\mathrm{M}+\mathrm{H}): 338.1732$, found:338.1728. 
$[\alpha]_{\mathrm{D}}^{25}=0.9231\left(\mathrm{c}=0.1300, \mathrm{CHCl}_{3}\right)$

Chiral HPLC: CHIRALPAK ODH, $25{ }^{\circ} \mathrm{C},{ }^{i} \mathrm{PrOH}$-hexanes 1/99, $0.7 \mathrm{~mL} / \mathrm{min}, 214$ $\mathrm{nm}, \mathrm{t}_{\mathrm{R}}($ major $)=8.8 \mathrm{~min}, \mathrm{t}_{\mathrm{R}}($ minor $)=9.5 \mathrm{~min}$.

\section{S75 racemic}

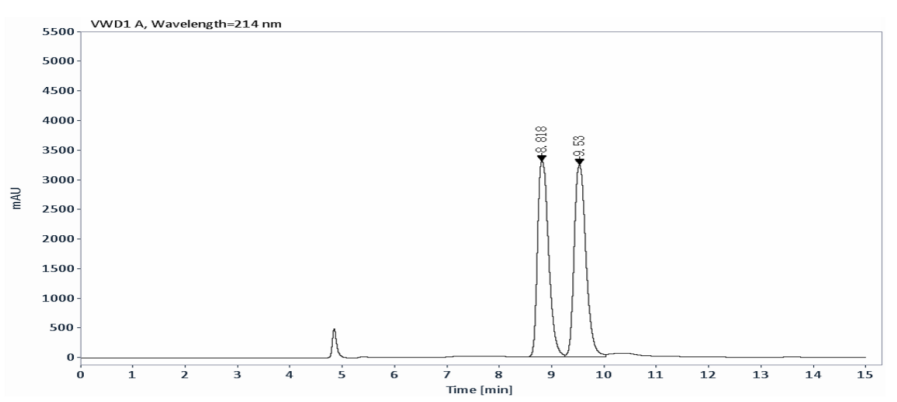

信号: VWD1 A, Wavelength=214 nm
\begin{tabular}{|l|l|l|l|l|l|}
\hline Ret.Time [min] & Name & Area [ $\boldsymbol{m A u}^{\star} \boldsymbol{m} \boldsymbol{m}$ ] & Height [mAu] & Rel. Area [\%] & Type \\
\hline 8.818 & n.a. & 48701.2617 & 3303.5938 & 49.0100 & BV \\
\hline 9.530 & n.a. & 50668.8359 & 3240.6204 & 50.9900 & VV \\
\hline
\end{tabular}

\section{S75 enantioenriched, $4 \%$ ee}

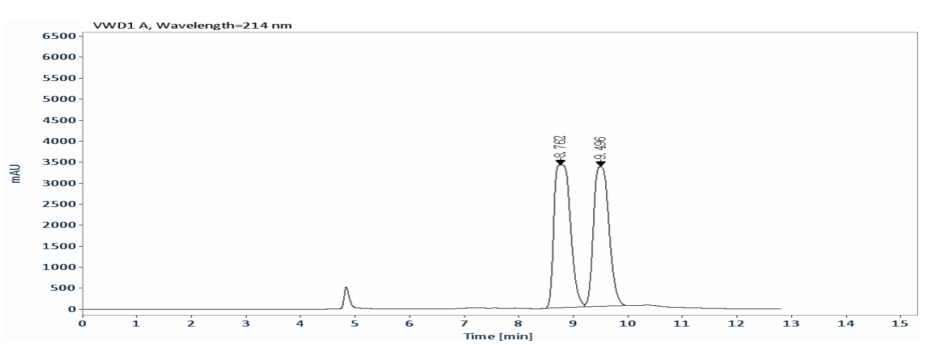

信号: VWD1 A, Wavelength=214 nm

\begin{tabular}{|l|l|l|l|l|l|}
\hline Ret.Time [min] & Name & Area [mA ${ }^{\star} \boldsymbol{m}$ in] & Height [mAu] & Rel. Area [\%] & Type \\
\hline 8.762 & n.a. & 71005.7891 & 3408.7500 & 52.0139 & BV \\
\hline 9.496 & n.a. & 65507.3633 & 3333.1318 & 47.9861 & VB \\
\hline
\end{tabular}<smiles>CC(C)(C)CC(c1ccccc1)c1ccnc(C(F)(F)F)c1</smiles>

\section{4-(3,3-dimethyl-1-phenylbutyl)-2-(trifluoromethyl)pyridine (S73)}

Following the general procedure, the product was isolated by flash chromatography (PE: ethyl acetate $=20: 1)$ as a colorless oil $(10.4 \mathrm{mg}, 34 \%$ yield $)$. 
${ }^{1} \mathbf{H}$ NMR $\left(400 \mathrm{MHz}, \mathrm{CDCl}_{3}\right) \delta 8.58(\mathrm{~d}, J=5.1 \mathrm{~Hz}, 1 \mathrm{H}), 7.59(\mathrm{~d}, J=1.3 \mathrm{~Hz}, 1 \mathrm{H}), 7.40$ $(\mathrm{dd}, J=5.0,1.4 \mathrm{~Hz}, 1 \mathrm{H}), 7.33-7.27$ (m, 3H), 7.25-7.19 (m, 2H), $4.11(\mathrm{t}, J=6.6 \mathrm{~Hz}$, 1H), $2.17(\mathrm{dd}, J=14.2,7.1 \mathrm{~Hz}, 1 \mathrm{H}), 2.05(\mathrm{dd}, J=14.2,6.1 \mathrm{~Hz}, 1 \mathrm{H}), 0.85(\mathrm{~s}, 9 \mathrm{H})$.

${ }^{19}$ F NMR $\left(375 \mathrm{MHz}, \mathrm{CDCl}_{3}\right) \delta-67.90(\mathrm{~s})$.

${ }^{13}$ C NMR $\left(100 \mathrm{MHz}, \mathrm{CDCl}_{3}\right) \delta 157.75,150.03,148.35$ (q, $\left.J=34.6 \mathrm{~Hz}\right), 143.74,128.94$, $127.73,126.91,125.62,121.58(\mathrm{q}, J=274.2 \mathrm{~Hz}), 119.73$ (q, $J=2.8 \mathrm{~Hz}), 48.66,47.98$, $31.59,30.11$.

HRMS (ESI): $\mathrm{C}_{18} \mathrm{H}_{21} \mathrm{~F}_{3} \mathrm{~N}^{+}(\mathrm{M}+\mathrm{H}): 308.1621$, found: 308.1623 .<smiles>CC(C)(C)c1ccc(C(CBr)c2ccnc(C(F)(F)F)c2)cc1</smiles>

\section{4-(1-(4-(tert-butyl)phenyl)-3,3-dimethylbutyl)-2-(trifluoromethyl)pyridine (S74)}

Following the general procedure, the product was isolated by flash chromatography (PE: ethyl acetate $=20: 1)$ as a colorless oil $(10.9 \mathrm{mg}, 30 \%$ yield $)$.

${ }^{1} \mathbf{H}$ NMR $\left(400 \mathrm{MHz}, \mathrm{CDCl}_{3}\right) \delta 8.57(\mathrm{~d}, J=5.1 \mathrm{~Hz}, 1 \mathrm{H}), 7.60(\mathrm{~d}, J=1.0 \mathrm{~Hz}, 1 \mathrm{H}), 7.41$ $(\mathrm{dd}, J=5.0,1.3 \mathrm{~Hz}, 1 \mathrm{H}), 7.30(\mathrm{~d}, J=8.4 \mathrm{~Hz}, 2 \mathrm{H}), 7.17(\mathrm{~d}, J=8.4 \mathrm{~Hz}, 2 \mathrm{H}), 4.07(\mathrm{t}, J$ $=6.6 \mathrm{~Hz}, 1 \mathrm{H}), 2.15-2.04(\mathrm{~m}, 2 \mathrm{H}), 1.28(\mathrm{~s}, 9 \mathrm{H}), 0.84(\mathrm{~s}, 9 \mathrm{H})$.

${ }^{19}$ F NMR $\left(375 \mathrm{MHz}, \mathrm{CDCl}_{3}\right) \delta-67.88(\mathrm{~s})$.

${ }^{13}$ C NMR $\left(100 \mathrm{MHz}, \mathrm{CDCl}_{3}\right) \delta 157.95,150.01,149.80,148.30(\mathrm{q}, J=34.2 \mathrm{~Hz}), 140.81$, 127.23, 125.80, , 125.74, 121.63 (q, $J=274.5 \mathrm{~Hz}), 119.85$ (q, $J=2.6 \mathrm{~Hz}), 48.81,47.57$, $34.41,31.60,31.32,30.14$.

HRMS (ESI): $\mathrm{C}_{22} \mathrm{H}_{29} \mathrm{~F}_{3} \mathrm{~N}^{+}(\mathrm{M}+\mathrm{H}): 364.2247$, found:364.2245.<smiles>CC(C)(C)CC(c1ccc(C(F)(F)F)cc1)c1ccnc(C(F)(F)F)c1</smiles> 


\section{(R)-4-(3,3-dimethyl-1-(4-(trifluoromethyl)phenyl)butyl)-2-(trifluoromethyl) pyridine (S76)}

Following the general procedure, the product was isolated by flash chromatography (PE: ethyl acetate $=20: 1)$ as a colorless oil $(17.1 \mathrm{mg}, 46 \%$ yield $)$.

${ }^{1} \mathbf{H}$ NMR $\left(400 \mathrm{MHz} \mathrm{CDCl}_{3}\right) \delta 8.61(\mathrm{~d}, J=5.1 \mathrm{~Hz}, 1 \mathrm{H}), 7.58-7.56(\mathrm{~m}, 3 \mathrm{H}), 7.40-7.38$ (m, 3H), $4.18(\mathrm{t}, J=6.6 \mathrm{~Hz}, 1 \mathrm{H}), 2.19-2.06(\mathrm{~m}, 2 \mathrm{H}), 0.86(\mathrm{~s}, 9 \mathrm{H})$.

${ }^{19}$ F NMR $\left(375 \mathrm{MHz}, \mathrm{CDCl}_{3}\right) \delta-62.53(\mathrm{~s}),-67.94(\mathrm{~s})$.

${ }^{13}$ C NMR $\left(100 \mathrm{MHz}, \mathrm{CDCl}_{3}\right) \delta 156.64,150.28,148.64(\mathrm{q}, J=34.3 \mathrm{~Hz}), 147.77,129.35$ (q, $J=32.6 \mathrm{~Hz}), 128.12,125.97(\mathrm{q}, J=3.7 \mathrm{~Hz}), 125.54,123.97(\mathrm{q}, J=271.9 \mathrm{~Hz})$, 121.49 (q, $J=274.4 \mathrm{~Hz}), 119.64$ (q, $J=2.7 \mathrm{~Hz}), 48.57,47.81,31.66,30.11$.

HRMS (ESI): $\mathrm{C}_{19} \mathrm{H}_{20} \mathrm{~F}_{6} \mathrm{~N}^{+}(\mathrm{M}+\mathrm{H}): 376.1494$, found:376.1491.

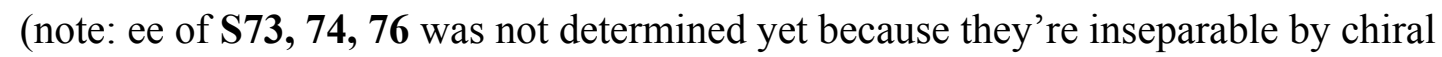
HPLC used in our lab as well as several labs in Shanghai.)

\subsection{Evaluation of chemoselectivity}

Following the general procedure, the reaction mixtures were analyzed by GC-MS to identify potential byproducts.

a) $3^{\circ}$-alkyl $\mathrm{BF}_{3} \mathrm{~K}$ in three-component reaction

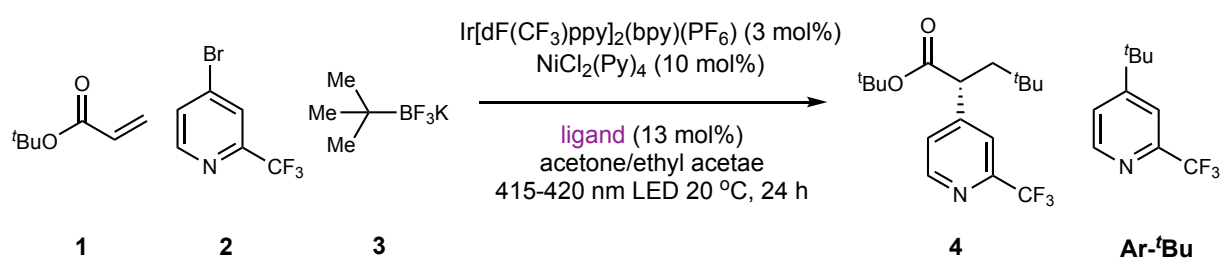

\begin{tabular}{llll}
\hline Entry & Ligand & Yield of $\mathbf{4}$ & Yield of $\mathbf{A r}^{-{ }^{t} \mathbf{B u}}$ \\
\hline 1 & dtbbpy & $83 \%$ & N.D. \\
2 & $(S, S)-\mathbf{L} \mathbf{1}$ & $82 \%$ & N.D. \\
\hline
\end{tabular}

b) $3^{\circ}$-alkyl $\mathrm{BF}_{3} \mathrm{~K}$ in two-component reaction. 


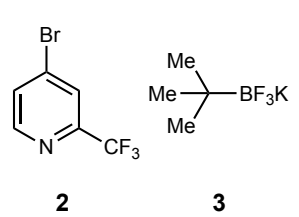

$\operatorname{Ir}\left[\mathrm{dF}\left(\mathrm{CF}_{3}\right) \text { ppy }\right]_{2}(\mathrm{bpy})\left(\mathrm{PF}_{6}\right)(3 \mathrm{~mol} \%)$ $\mathrm{NiCl}_{2}(\mathrm{Py})_{4}(10 \mathrm{~mol} \%)$

ligand (13 mol\%) acetone/ethyl acetae 415-420 nm LED $20^{\circ} \mathrm{C}, 24 \mathrm{~h}$

${ }_{{ }^{\mathrm{Bu}}} \stackrel{\mathrm{O}}{\mathrm{O}} \mathrm{L}_{\mathrm{B}_{\mathrm{Bu}}} \mathrm{L}$

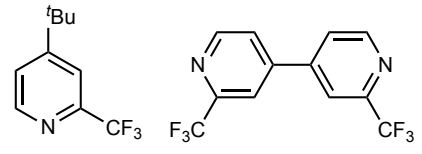

Ar- ${ }^{t} \mathrm{Bu}$

Ar-Ar

\begin{tabular}{llll}
\hline Entry & Ligand & Yield of Ar- ${ }^{+} \mathbf{B u}$ & Yield of Ar-Ar \\
\hline 1 & dtbbpy & N.D. & $45 \%$ \\
2 & $(S, S)-\mathbf{L 1}$ & trace & $15 \%$ \\
3 & L20 & $20 \%$ & $40 \%$ \\
\hline
\end{tabular}
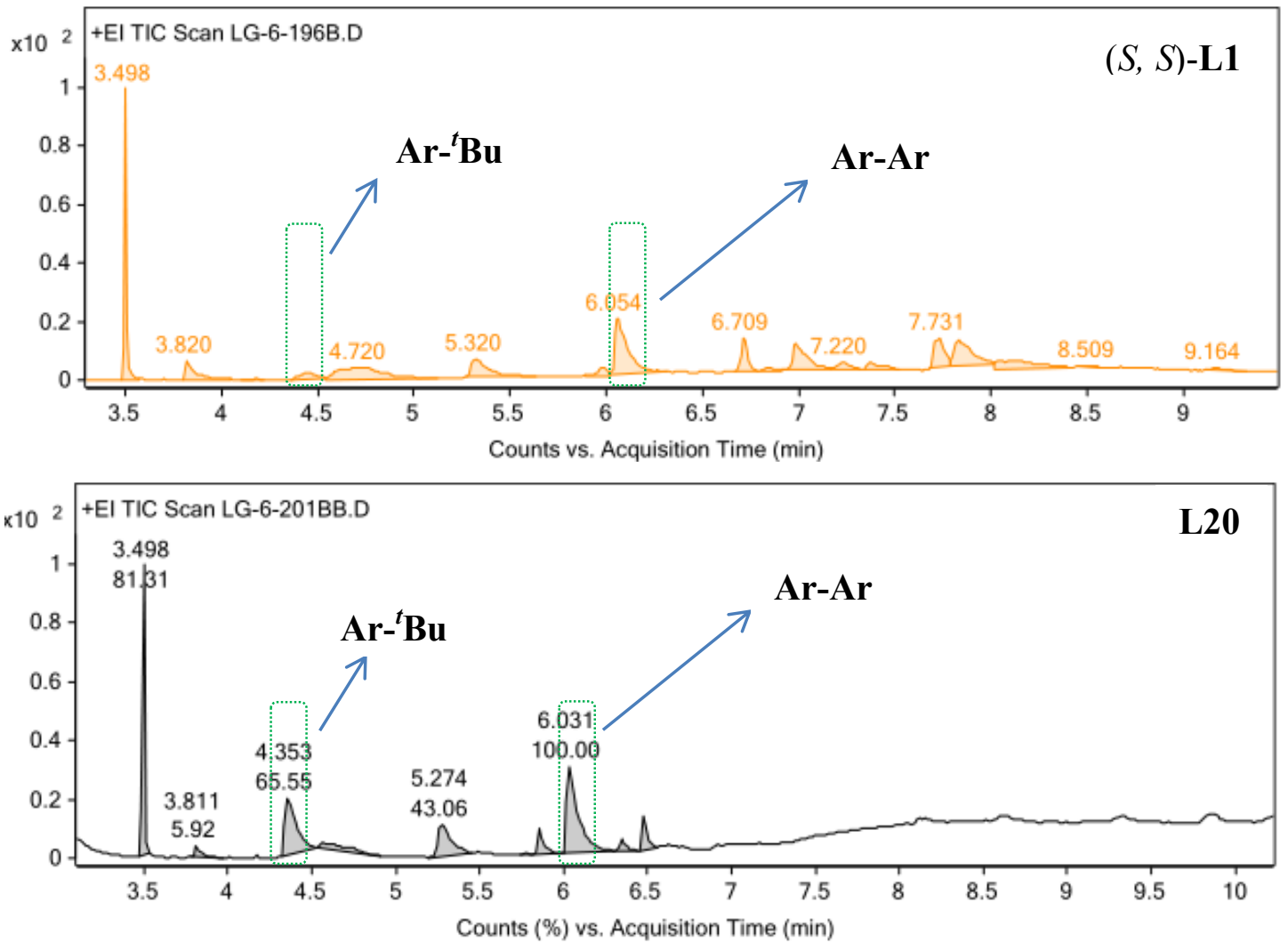

c) $\mathbf{2}^{\circ}$-alkyl $\mathrm{BF}_{3} \mathrm{~K}$ in three-component reaction.<smiles>C=CC(=O)OC(C)(C)C</smiles>

1<smiles>CCCCC(C(=O)OCCC)c1ccnc(C(F)(F)F)c1</smiles><smiles>CCCc1ccnc(C(F)(F)F)c1</smiles>

41 $\mathrm{Ar}^{-} \mathrm{Pr}$ 


\begin{tabular}{llll}
\hline Entry & Ligand & Yield of $\mathbf{4 1}$ & Yield of $\mathbf{A r}^{-}{ }^{\mathbf{P}} \mathbf{P r}$ \\
\hline 1 & dtbbpy & $62 \%$ & trace \\
2 & $(S, S)-\mathbf{L} \mathbf{1}$ & $56 \%$ & trace \\
\hline
\end{tabular}
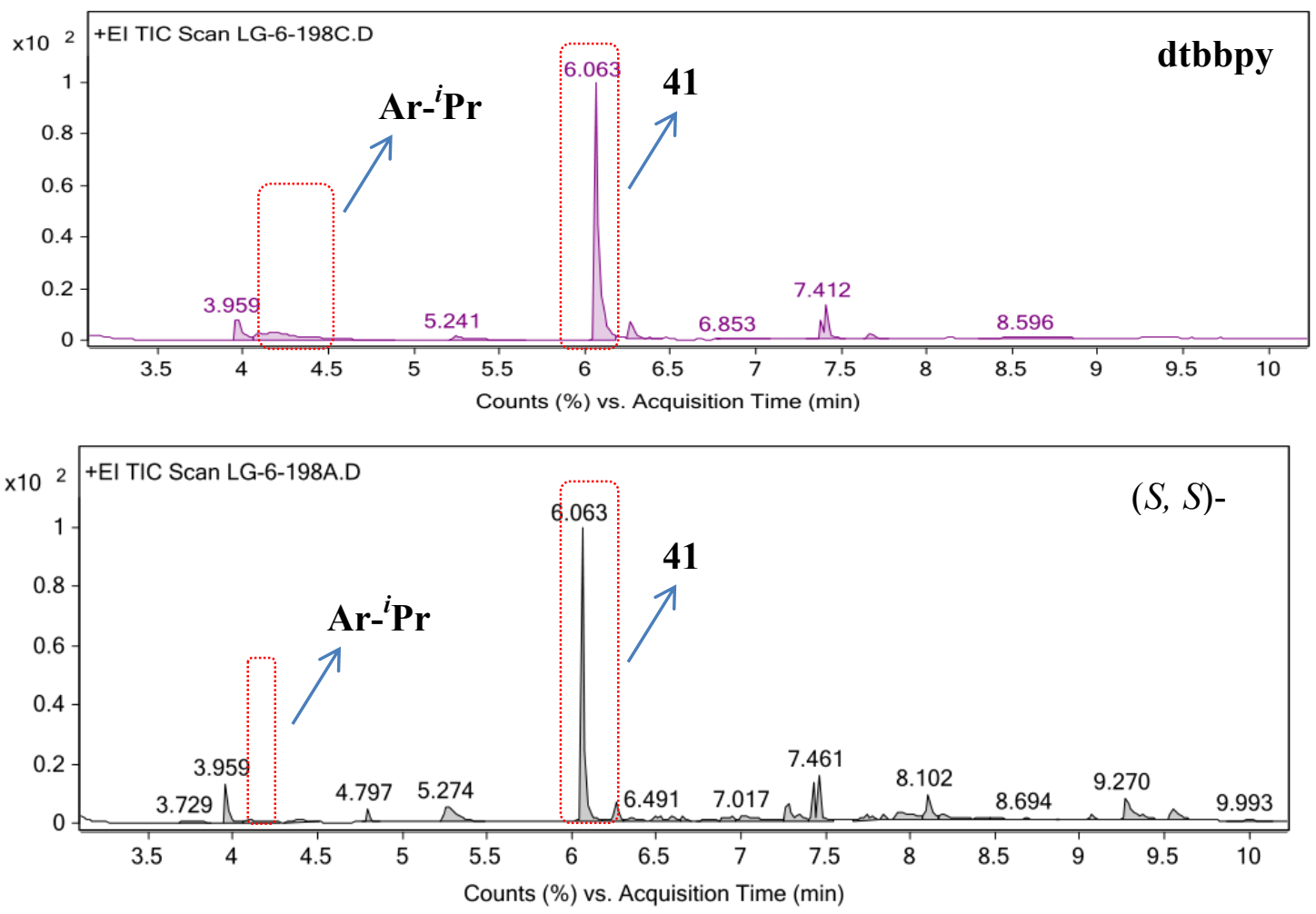

d) $2^{\circ}$-alkyl $\mathrm{BF}_{3} \mathrm{~K}$ in two-component reaction.<smiles>FC(F)c1cc(Br)ccn1</smiles>

2

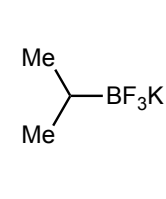

3'<smiles>CCCc1ccnc(C(F)(F)F)c1</smiles><smiles>FC(F)(F)c1cc(-c2ccnc(C(F)(F)F)c2)ccn1</smiles>
415-420 nm LED£" $20{ }^{\circ} \mathrm{C}$ $\operatorname{Ir}\left[\mathrm{dF}\left(\mathrm{CF}_{3}\right) \mathrm{ppy}\right]_{2}(\mathrm{bpy})\left(\mathrm{PF}_{6}\right)(3 \mathrm{~mol} \%)$

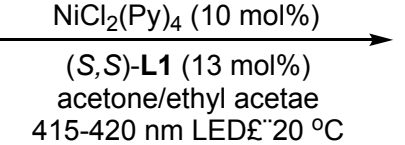

$\operatorname{Ar}^{-}{ }^{-} \operatorname{Pr} 56 \%$

Ar-Ar $8 \%$

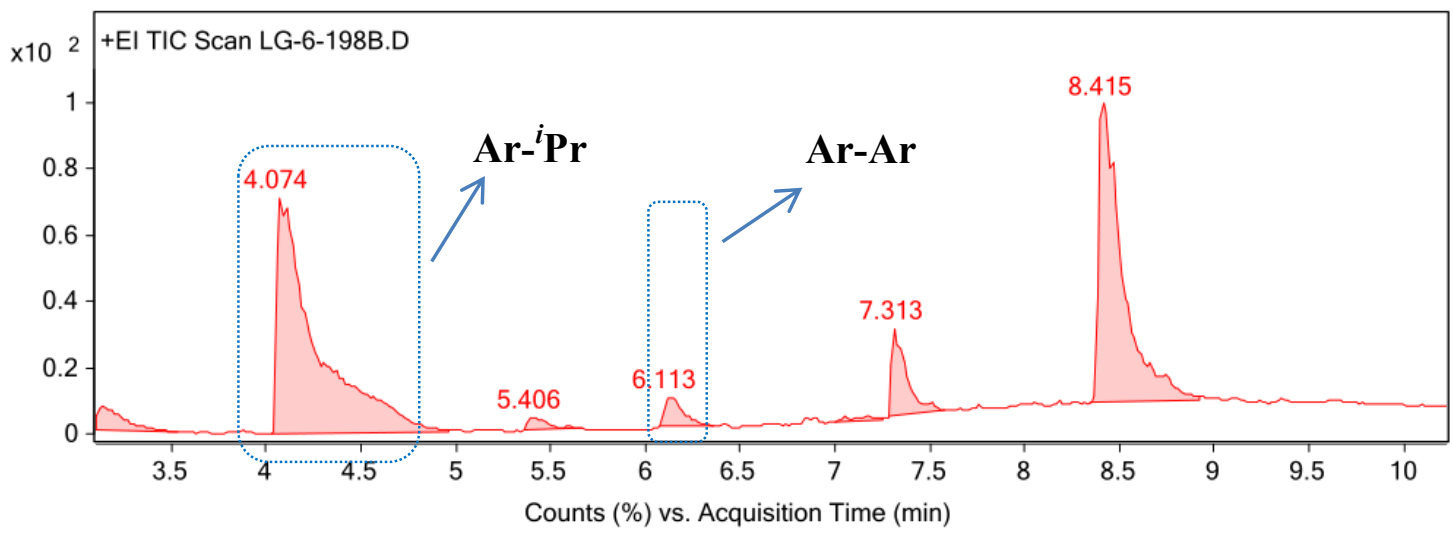


e) $\mathbf{1}^{\mathbf{0}}$-alkyl $\mathrm{BF}_{3} \mathrm{~K}$ in three-component reaction.

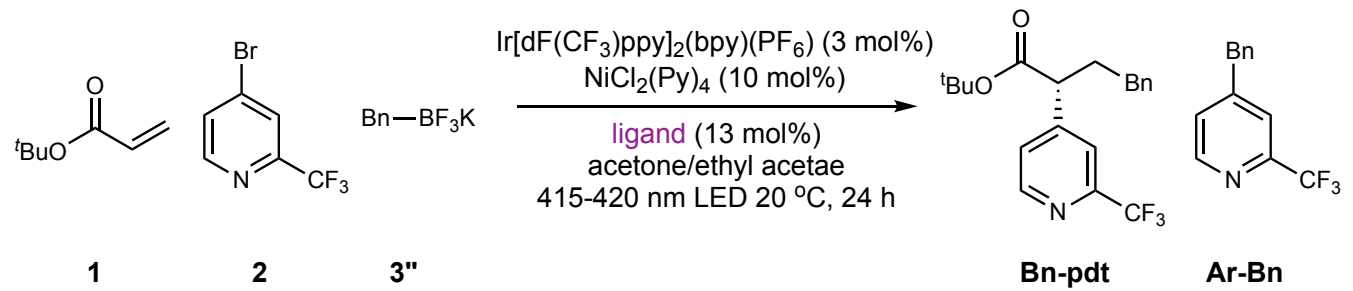

\begin{tabular}{llll}
\hline Entry & Ligand & Yield of 4' & Yield of Ar- ${ }^{i} \mathbf{P r}$ \\
\hline 1 & dtbbpy & N.D. & $95 \%$ \\
2 & $(S, S)-\mathbf{L 1}$ & N.D. & $87 \%$ \\
\hline
\end{tabular}
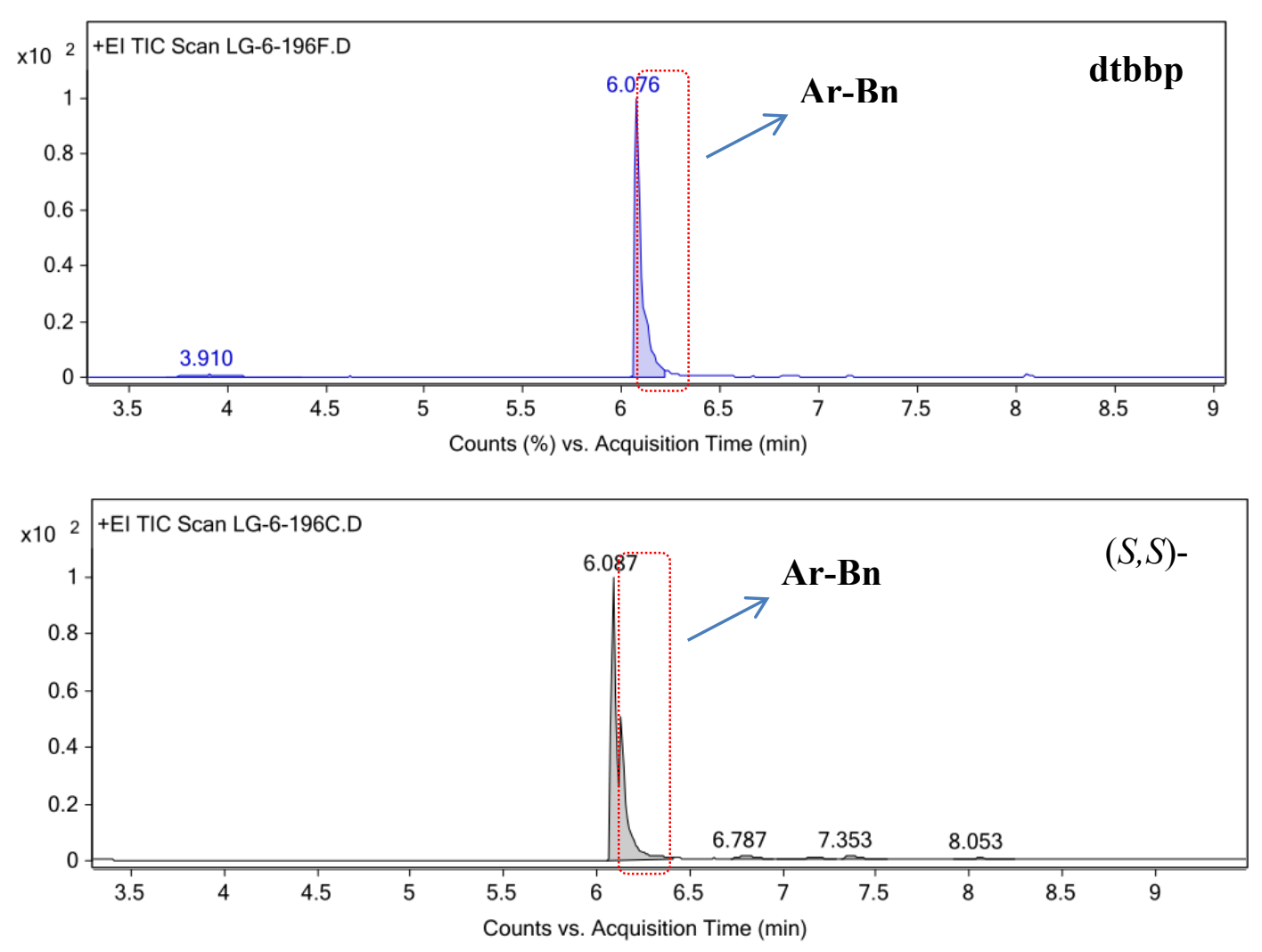

f) $\mathbf{1}^{\mathbf{0}}$-alkyl $\mathrm{BF}_{3} \mathrm{~K}$ in two-component reaction. 


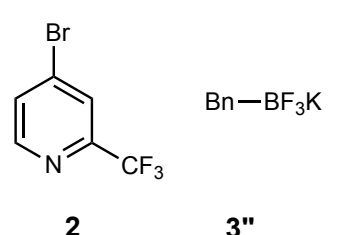

$\operatorname{Ir}\left[\mathrm{dF}\left(\mathrm{CF}_{3}\right) \mathrm{ppy}\right]_{2}(\mathrm{bpy})\left(\mathrm{PF}_{6}\right)(3 \mathrm{~mol} \%)$

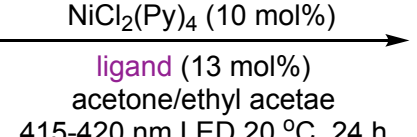<smiles>FC(F)(F)c1cc(Br)ccn1</smiles><smiles>FC(F)(F)c1cc(-c2ccnc(C(F)(F)F)c2)ccn1</smiles>
$415-420 \mathrm{~nm}$ LED $20^{\circ} \mathrm{C}, 24 \mathrm{~h}$

Ar-Bn $95 \% \quad$ Ar-Ar N.D.

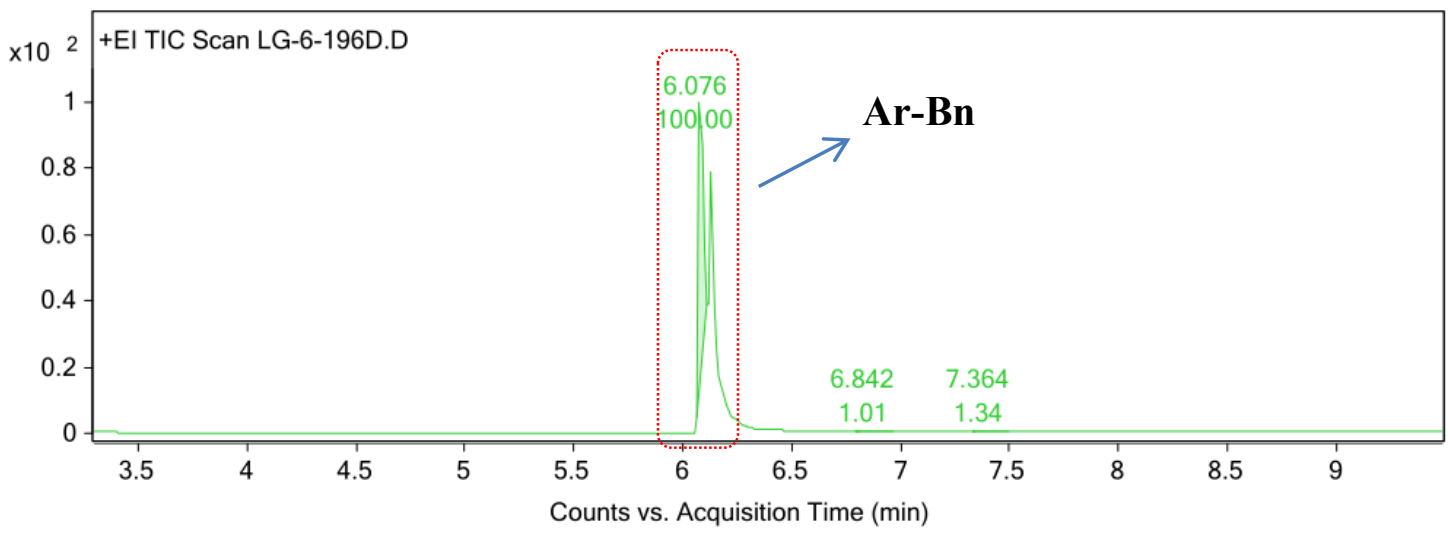

7.6. Effect of concentration of alkene on chemoselectivity<smiles>CCC(C)(C)C(C)(C)C(C)(C)C(F)(F)c1cc(Br)ccn1</smiles>

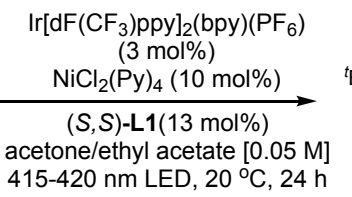<smiles>CC(C)(C)C[C@@H](C(=O)OC(C)(C)C)c1ccnc(C(F)(F)F)c1</smiles><smiles>CC(C)(C)c1ccnc(C(F)(F)F)c1</smiles>

$0.1 \mathrm{mmol}$ $x$ eq y eq

4 $\mathrm{Ar}-{ }^{t} \mathrm{Bu}$

\begin{tabular}{lllll}
\hline Entry & $\begin{array}{l}\text { Ar-Br } \\
\text { (equiv.) }\end{array}$ & $\begin{array}{l}{ }^{t} \mathbf{B u}_{-B F_{3} K} \\
\text { (equiv.) }\end{array}$ & Yield of 4 & $\begin{array}{l}\text { Yield of } \\
\mathbf{A r}-{ }^{\mathbf{t}} \mathbf{B u}\end{array}$ \\
\hline 1 & 1.0 & 2.0 & $65 \%$ & N.D. \\
2 & 1.0 & 3.0 & $79 \%$ & N.D. \\
3 & 1.5 & 2.0 & $75 \%$ & N.D. \\
4 & 1.5 & 3.0 & $78 \%$ & N.D. \\
5 & 2.0 & 2.0 & $75 \%$ & N.D. \\
6 & 3.0 & 3.0 & $70 \%$ & N.D. \\
\hline
\end{tabular}

7.7 Effect of concentration of nickel catalyst on chemoselectivity 


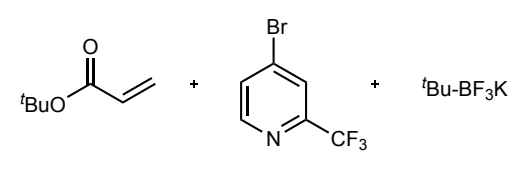

$1,2.0$ eq

$2,0.1 \mathrm{mmol}$

3, 2.5 eq
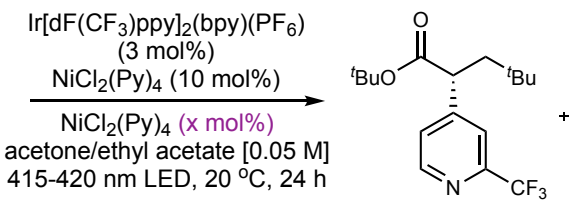<smiles>CC(C)(C)c1ccnc(C(F)(F)F)c1</smiles>

$\mathrm{Ar}^{-{ }^{\mathrm{B}} \mathrm{Bu}}$

\begin{tabular}{|c|c|c|c|}
\hline Entry & $\mathrm{NiCl}_{2}(\mathrm{Py})_{4}$ & Yield/EE of 4 & Yield of $\mathrm{Ar}-{ }^{t} \mathrm{Bu}$ \\
\hline 1 & $10 \mathrm{~mol} \%$ & $82 \%, 95 \%$ ee & N.D. \\
\hline 2 & $20 \mathrm{~mol} \%$ & $84 \%, 95 \%$ ee & trace \\
\hline
\end{tabular}

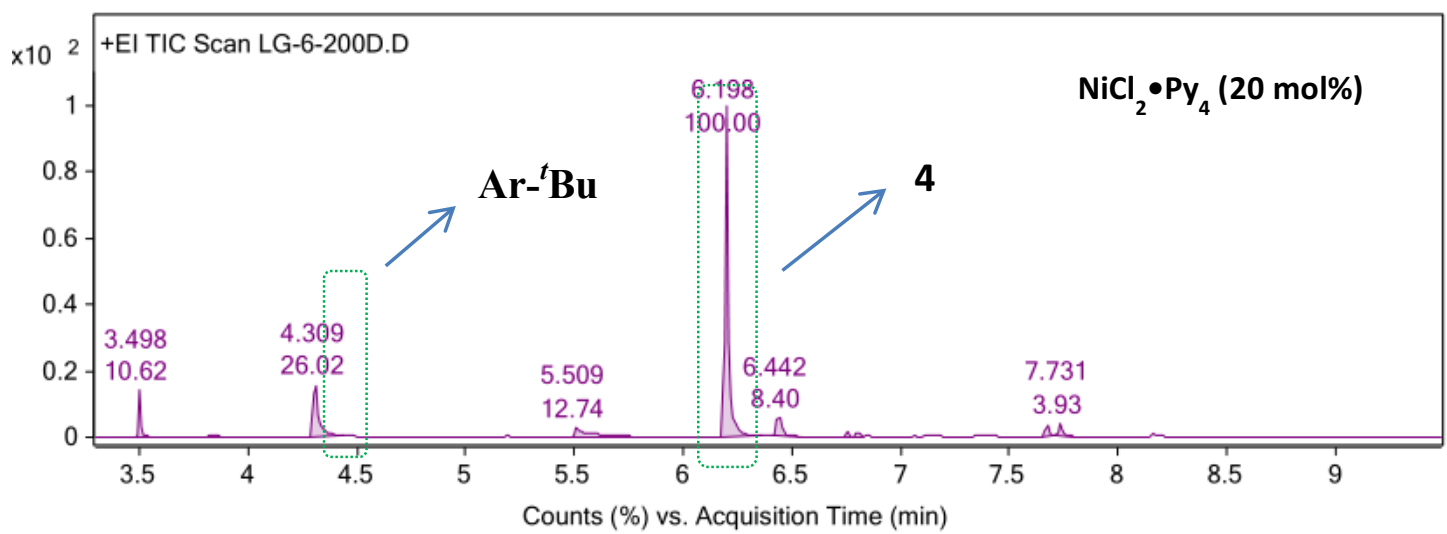

\subsection{Light/dark experiments}<smiles>C=CC(=O)OC(C)(C)C</smiles><smiles>CC(C)(c1cc(Br)ccn1)C(F)(F)Br</smiles>

1, 2.0 eq

2, $0.1 \mathrm{mmol}$

3, 2.5 equiv

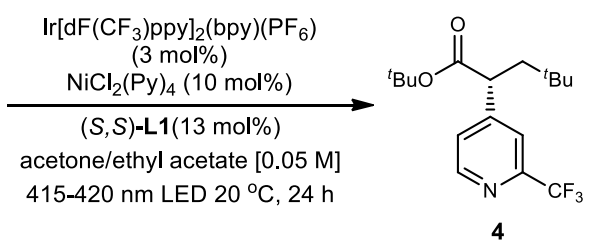

4

Nine standard reaction mixtures in $8 \mathrm{~mL}$ vials were charged with $(4 \mathrm{~S}, 4$ 'S)-4,4'-di((S)sec-butyl)-4,4',5,5'-tetrahydro-2,2'-bioxazole $[(S, S)-\mathbf{L 1}](0.013 \mathrm{mmol}, 13 \mathrm{~mol} \%)$, $\mathrm{NiCl}_{2} \bullet \mathrm{Py}_{4}(0.01 \mathrm{mmol}, 10 \mathrm{~mol} \%), \operatorname{Ir}\left[\mathrm{dF}\left(\mathrm{CF}_{3}\right) \mathrm{ppy}\right]_{2}(\mathrm{bpy}) \mathrm{PF}_{6}(0.003 \mathrm{mmol}, 3 \mathrm{~mol} \%)$, and ${ }^{t} \mathrm{BuBF}_{3} \mathrm{~K}$ (41.0 mg, $0.25 \mathrm{mmol}, 2.5$ equiv.), these vials were capped. After evacuated and backfilled nitrogen three times, acetone/ethyl acetate $2: 1[0.05 \mathrm{M}]$ was added via a syringe, 4-bromo-2-(trifluoromethyl) pyridine (13.7 uL, $0.1 \mathrm{mmol}, 1.0$ equiv.), followed by the addition of tert-butyl acrylate $(29.0 \mathrm{uL}, 0.2 \mathrm{mmol}, 2.0$ equiv.). The reaction mixture was then irradiated with a $10 \mathrm{~W} 415-420 \mathrm{~nm}$ LED lamp with cooling from circulating cold ethanol at $20{ }^{\circ} \mathrm{C}$. (Note: Only one vial was removed for analysis in every response time) 


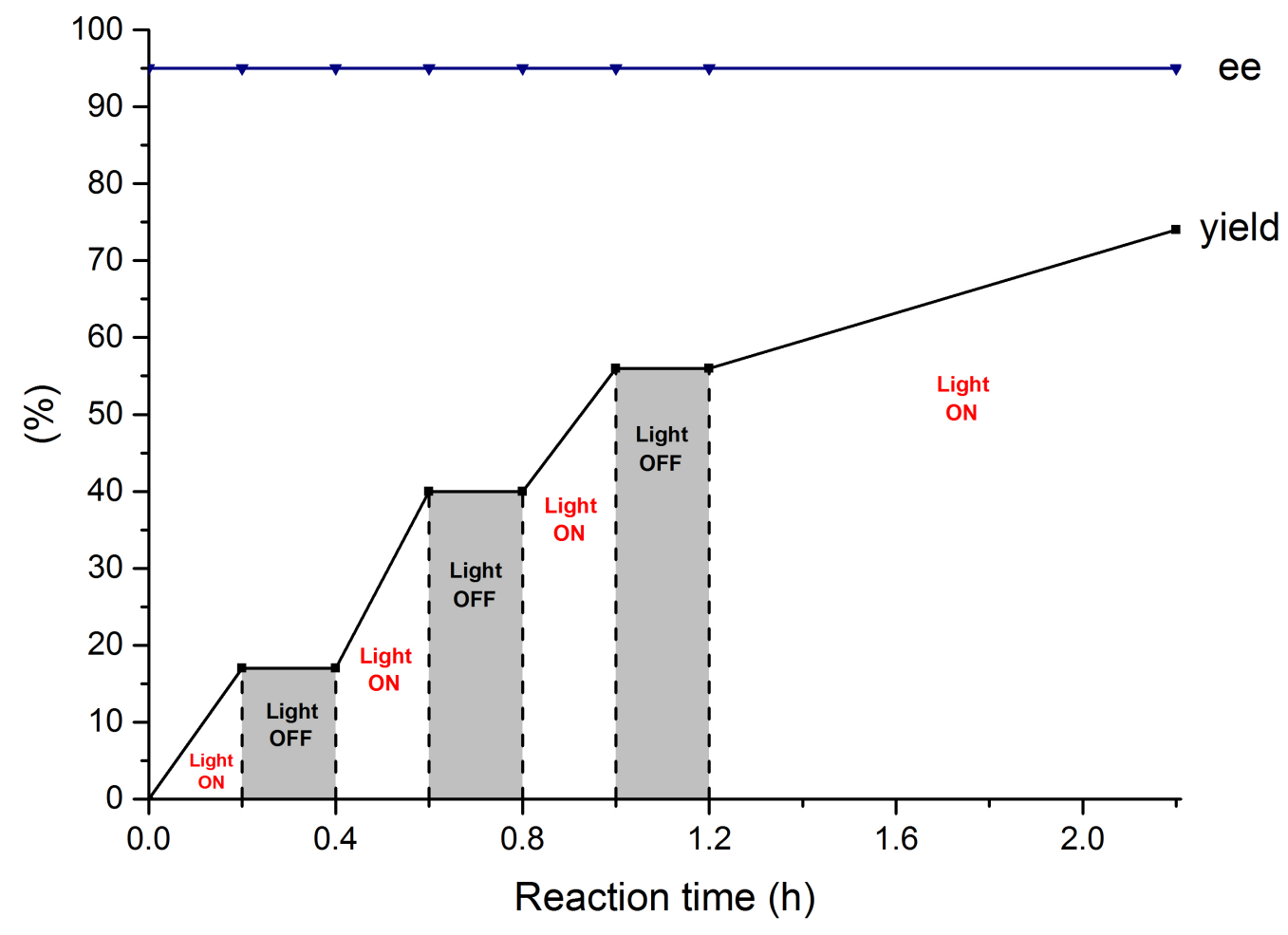

Figure S3 Light/dark experiments

\subsection{Stern-Volmer quenching experiments}

Stern-Volmer quenching experiments were carried by Edinburgh Fluorescence Spectrometer FS5, using a $0.01 \mathrm{mM}$ solution of photocatalyst $\operatorname{Ir}\left[\mathrm{dF}\left(\mathrm{CF}_{3}\right) \text { ppy }\right]_{2}(\mathrm{bpy}) \mathrm{PF}_{6}$ and variable concentrations $(0.05,0.10,0.15,0.20,0.25 \mathrm{mM})$ of tert-butyl acrylate, 4 bromo-2-(trifluoromethyl) pyridine and ${ }^{t} \mathrm{BuBF}_{3} \mathrm{~K}$ in mixed solvent acetone/ethyl acetate (2:1). The samples were prepared in $4 \mathrm{~mL}$ quartz cuvettes, equipped with PTFE stoppers, and sealed with parafilm inside nitrogen filled glove-box. The intensity of the emission peak at $496 \mathrm{~nm}\left(\lambda_{\text {ex }}=378 \mathrm{~nm}\right)$ expressed as the ratio $I_{0} / I$, where $I_{0}$ is the emission intensity of photocatalyst at $496 \mathrm{~nm}$ in the absence of a quencher and I is the observed intensity, as a function of the quencher concentration was measured. SternVolmer plots for each component are given in the Supplementary Figures below. 


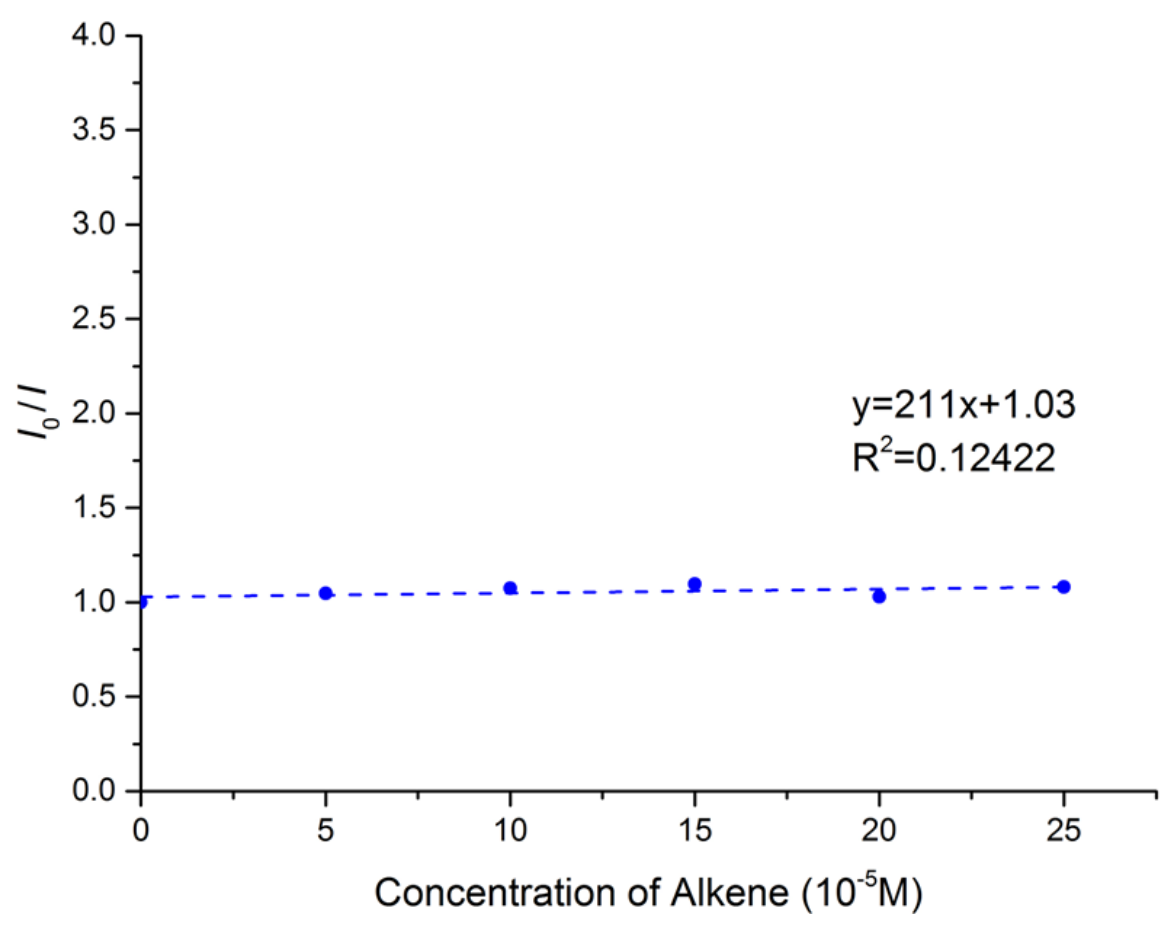

Figure S4 Stern-Volmer plot of photocatalyst $(0.01 \mathrm{mM})$ at different concentrations of tert-butyl acrylate

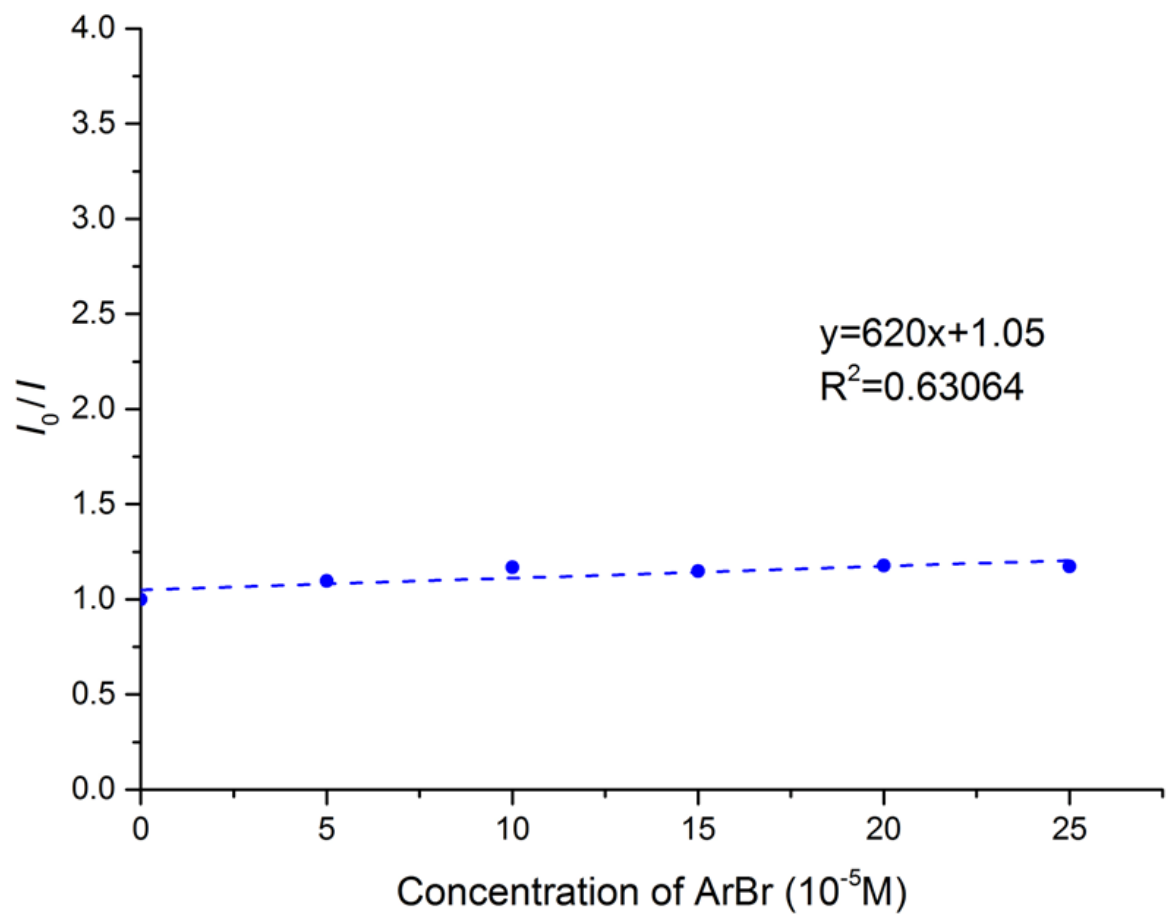

Figure S5 Stern-Volmer plot of photocatalyst $(0.01 \mathrm{mM})$ at different concentrations of 4-bromo-2-(trifluoromethyl) pyridine 


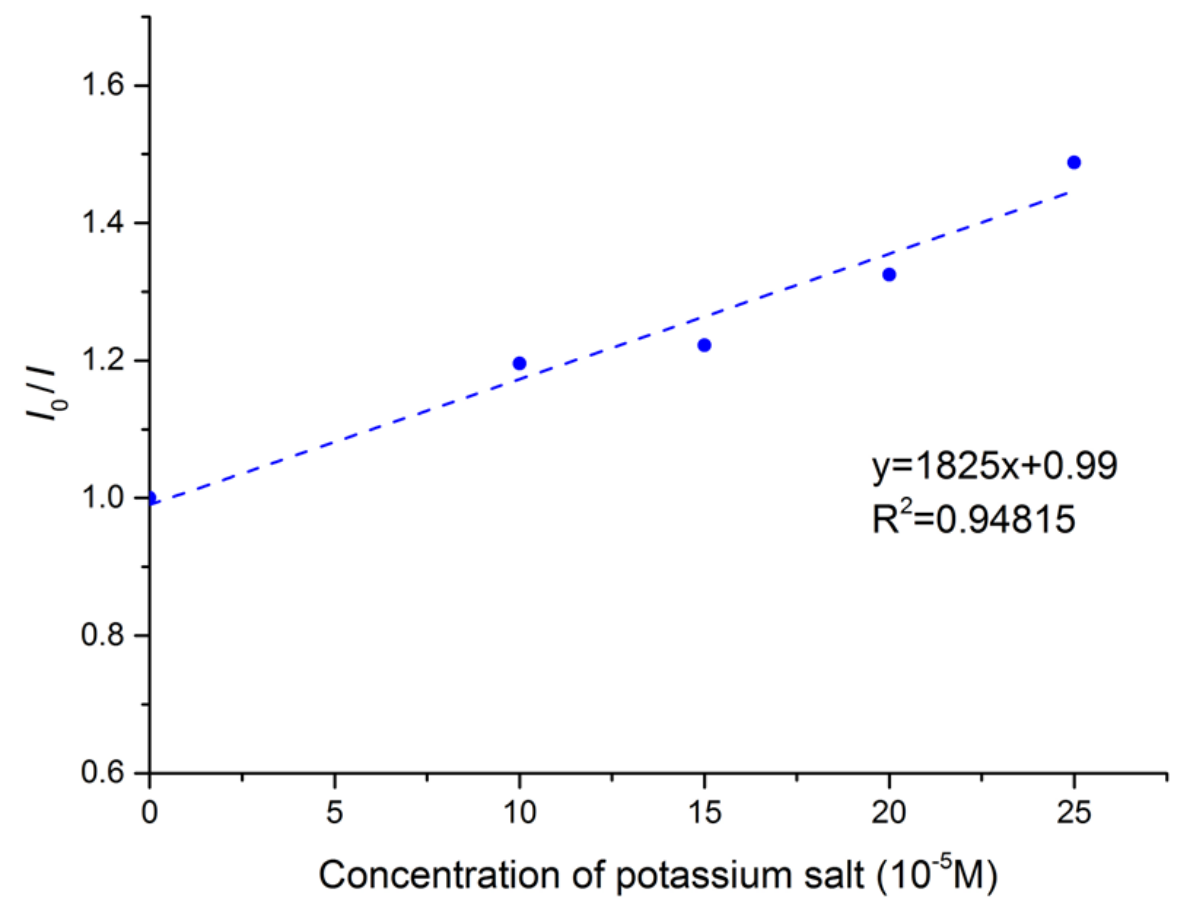

Figure S6 Stern-Volmer plot of photocatalyst $(0.01 \mathrm{mM})$ at different concentrations of ${ }^{t} \mathrm{BuBF}_{3} \mathrm{~K}$

\subsection{Identification and quantification of the likely resting state}

UV-Vis absorption spectra were collected on a PerkinElmer UV/VIS/NIR Spectrometer Lambda 950. All reagents were dispensed in stock solutions prepared volumetrically inside a nitrogen filled glove box.

A stock solution of ${ }^{i} \mathrm{Bu}-\mathrm{BiOx}-\mathrm{NiBr}_{2}$ was prepared for spectroscopic studies in the glove box without isolation. To a $8 \mathrm{~mL}$ reaction vial with a stir bar was added $\mathrm{NiBr}_{2} \cdot \mathrm{DME}$ (9.3 mg, $0.03 \mathrm{mmol}),{ }^{i} \mathrm{Bu}-\mathrm{BiOx}(7.6 \mathrm{mg}, 0.03 \mathrm{mmol})$ and DME $(3 \mathrm{~mL})$, the resulting red-orange solution was left to stir for $3 \mathrm{~h}$. For UV-vis analysis, the solution was diluted 8-fold with DME. 


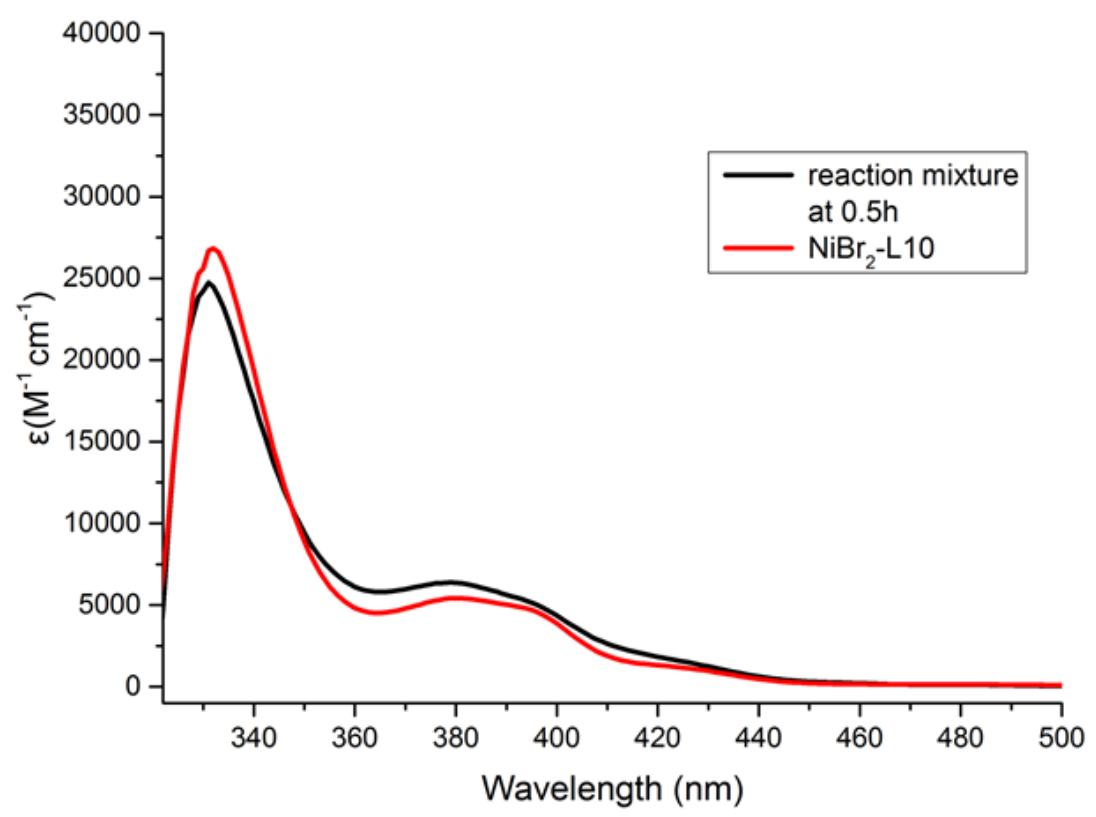

Figure S7 UV-vis spectraof the temperaturelate reaction in progress and of the stock solution of ${ }^{i} \mathrm{Bu}-\mathrm{BiOx}-\mathrm{NiBr}_{2}$ 


\section{DFT Caculations}

\subsection{Computational methods}

All optimizations were carried out without constraints at the (U)B3LYP $\left.{ }^{5}-\mathrm{D} 3(\mathrm{BJ})\right)^{6} / \mathrm{def} 2-$ $\mathrm{SVP}^{7}$ level of theory in THF solvent using the CPCM implicit solvent model ${ }^{8}$ with “opt=noeigen" keyword as implemented in Gaussian $16^{9}$. To compare energetics, we also carried out single point energy calculations using (U)B3LYP-D3(BJ)/def2TZVPP-CPCM(THF). All structural figures were generated using CLYview. ${ }^{10}$ Vibrational frequencies were computed at the same level to obtain thermal corrections (at $298 \mathrm{~K}$; enthalpic and free energy) and to characterize the stationary points as transition states (one and only one imaginary frequency) or minima (zero imaginary frequencies). Exhaustive conformational searches were performed for all intermediates to map out the lowest energy profile, and intrinsic reaction coordinate (IRCs) calculations were undertaken for selected transition state structures to ensure they connected the illustrated ground states.

Noncovalent interaction (NCI) analysis, also known as reduce density gradient (RDG) method, was performed on Multiwfn to study the possible effect of noncovalent interaction in the enantiodetermining radical addition step. ${ }^{11}$ Extension distance of 0 Bohr, medium quality grid (totally about 512000 points) were set by default. Further visualization of the color-filled RDG isosurface was realized by VMD, where RDG isosurface and color range were set as 0.5 , and -0.035 to 0.2 , respectively. ${ }^{12}$ 


\subsection{Computational results}

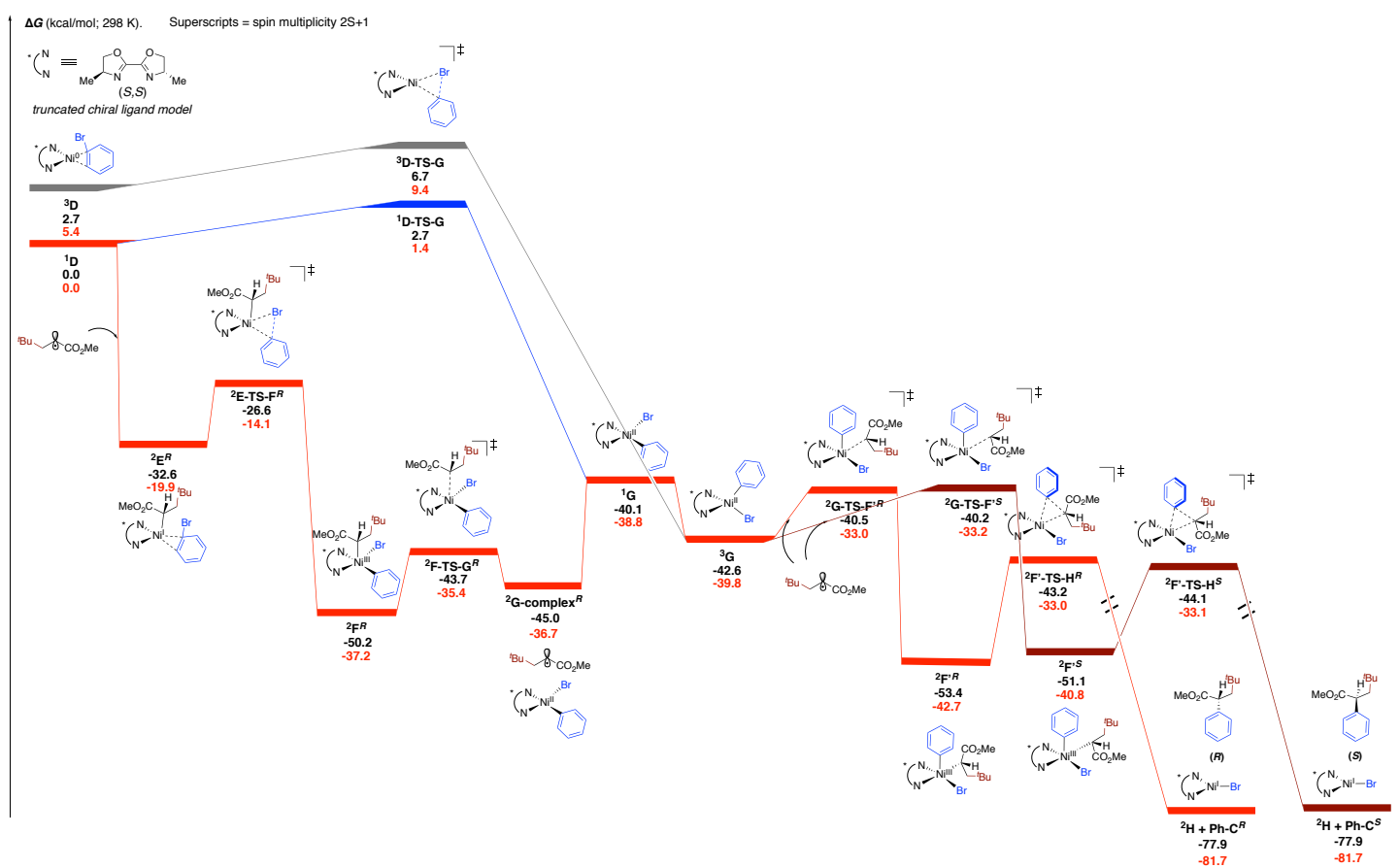

Scheme S1 Possible mechanistic pathways that lead to the formation of threecomponent alkylarylation product. Free energy values given were calculated at the UB3LYP-D3(BJ)/def2-SVP-CPCM(THF) (black) and UB3LYP-D3(BJ)/def2TZVPP-CPCM(THF)//UB3LYP-D3(BJ)/def2-SVP-CPCM(THF) (red) levels of method.

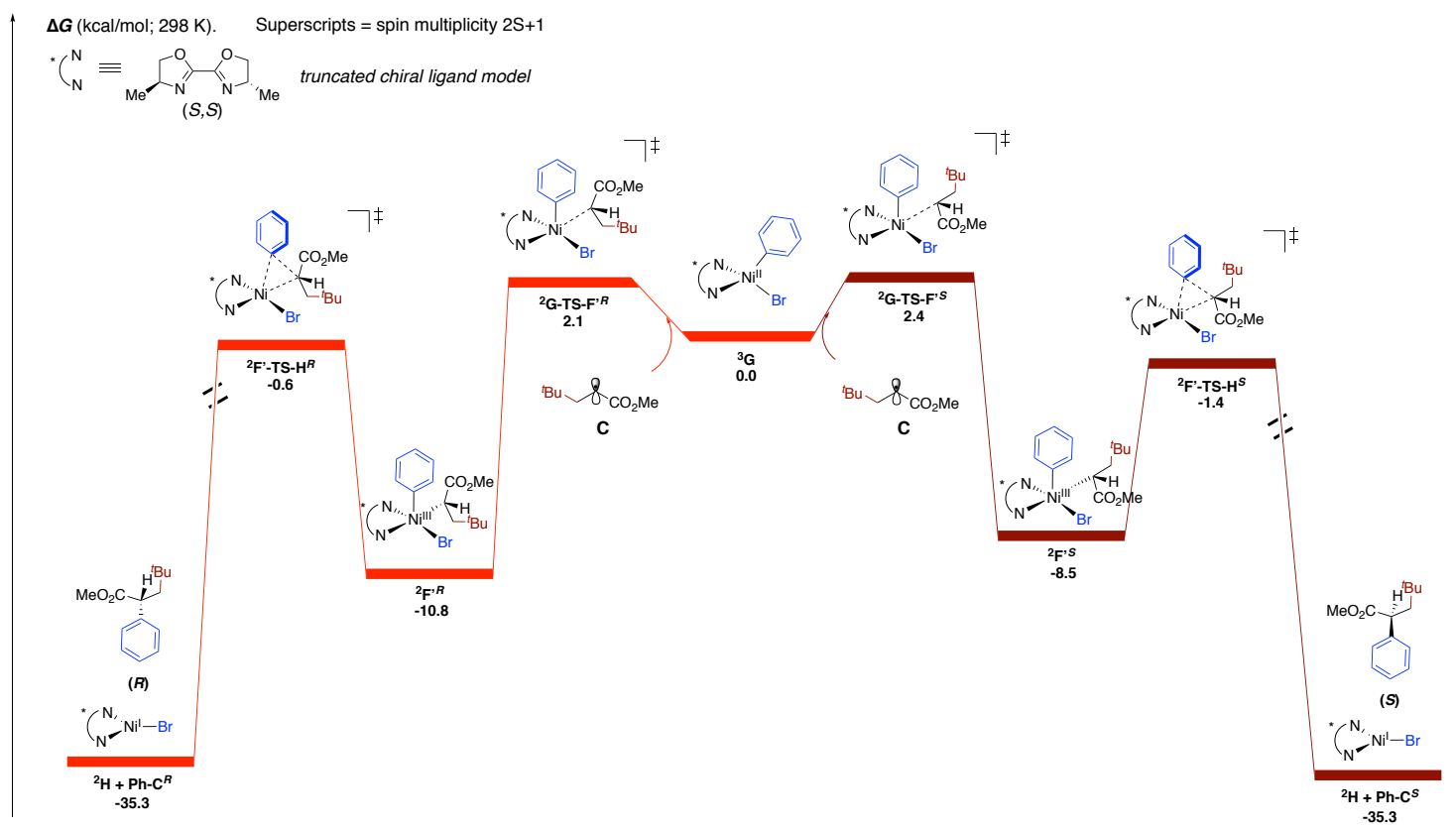

Scheme S2 Mechanistic pathways that lead to the formation of three-component alkylarylation products with different stereochemistry. Free energy values given were calculated at the UB3LYP-D3(BJ)/def2-SVP-CPCM(THF) level of method. 


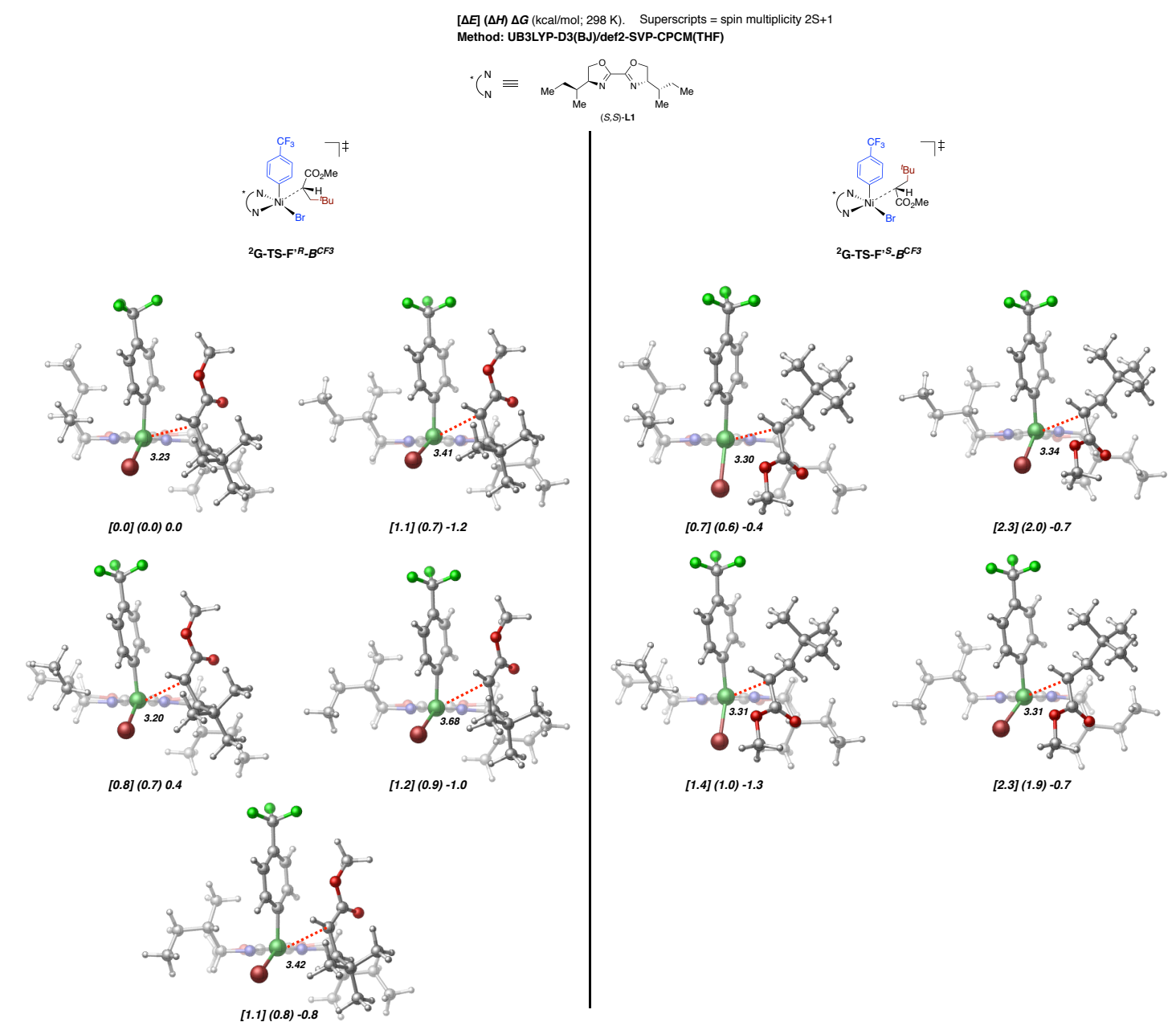

Scheme S3 Different located conformations of the enantiodetermining radical addition step with full ligand system. Energy values given were calculated at the UB3LYPD3(BJ)/def2-SVP-CPCM(THF) level of method.

Table S8. Enantioselectivity determined by electronic energies, enthalpies and free energies.

\begin{tabular}{cccccccc}
\hline \multirow{2}{*}{ Method } & \multicolumn{2}{c}{$E$} & \multicolumn{2}{c}{$H$} & \multicolumn{2}{c}{$\boldsymbol{G}$} \\
\cline { 2 - 7 } & configuration & ee (\%) & configuration & ee (\%) & configuration & ee (\%) \\
\hline UB3LYP-D3(BJ)/def2-SVP-CPCM(THF) & $R$ & 59.5 & $R$ & 54.6 & $R$ & 0.2 \\
\hline
\end{tabular}

The origin of enantioselectivity of this three-component alkylarylation of olefin was explored with focus on the enantiodetermining radical addition step with full ligand $(S, S)$-sec-Bu-BiOx $\mathbf{L 1}$ and $p-\mathrm{CF}_{3}-\mathrm{ArBr}$ as the model system. In particular, nine of the lowest-energy radical addition transition states were taken into consideration (Scheme S3). Boltzmann calculation was performed based on the electronic energy, enthalpy and 
free energy values of these conformations. As shown in Table S8, electronic energy values obtained with the optimization method ([UB3LYP-D3(BJ)/def2-SVP$\mathrm{CPCM}(\mathrm{THF})])$ predicted the correct enantiomer ( $R$-product) albeit with underestimated enantiomeric ratio ( $\sim 80: 20$ vs. $\sim 95: 5$ in experiment).
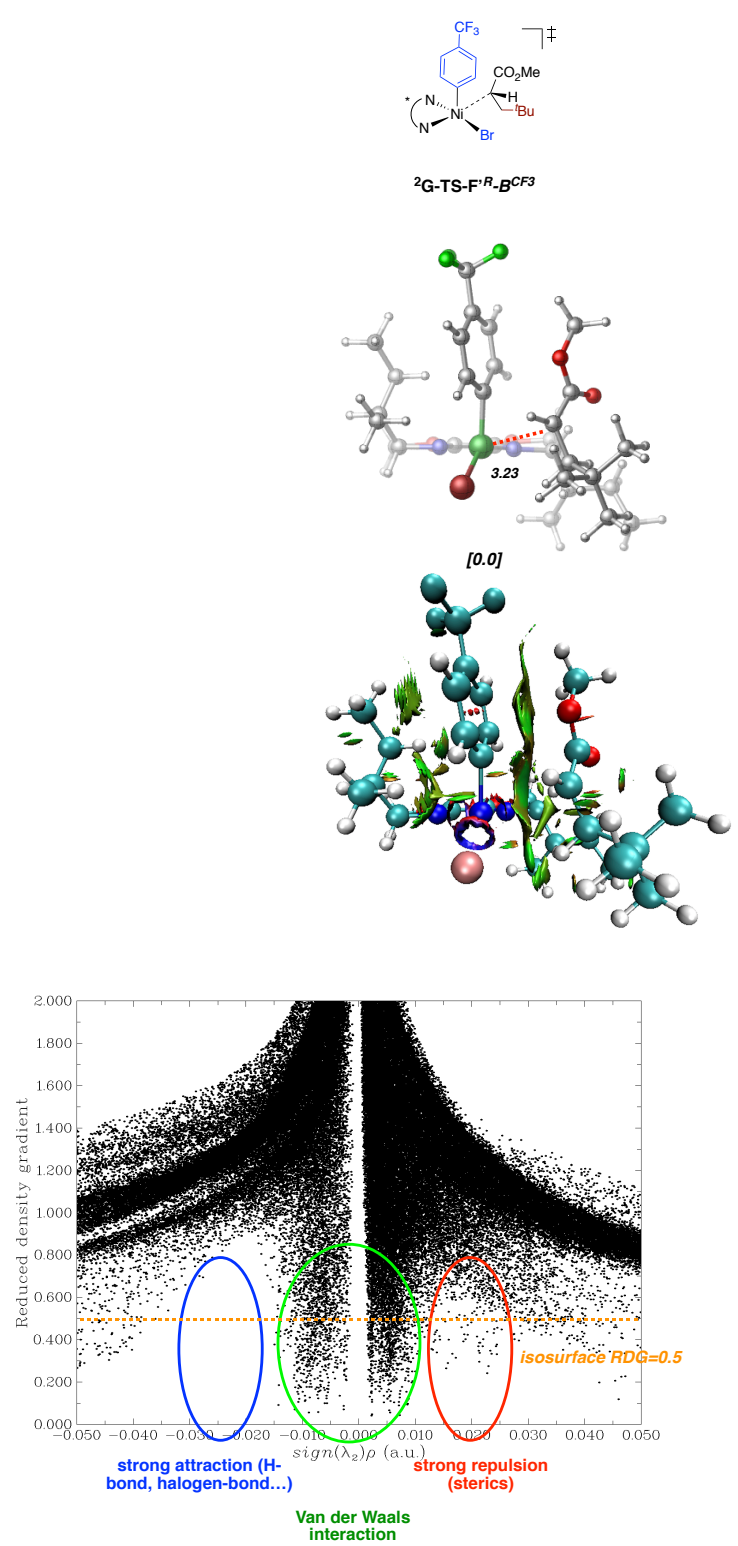
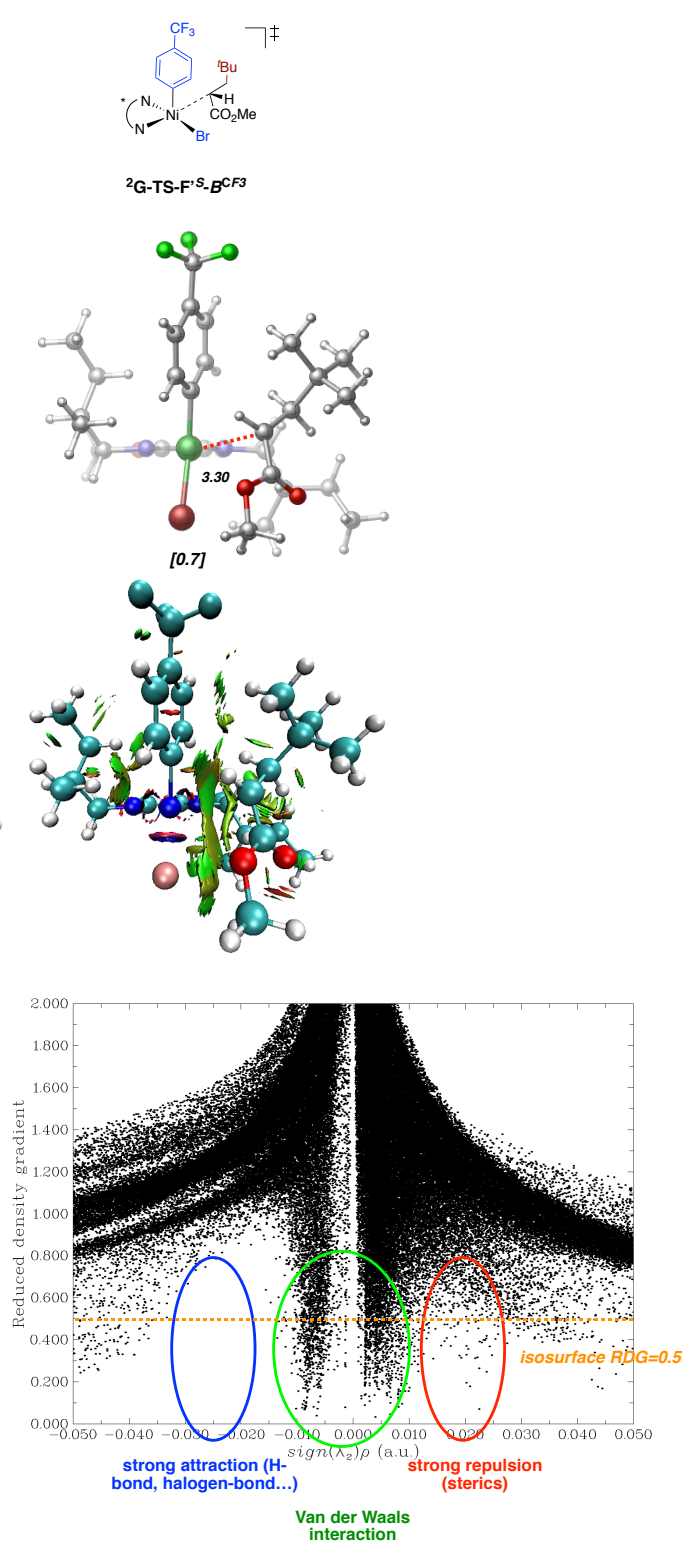

Scheme S4 Color-filled RDG isosurface and scatter map created from NCI analysis on the lowest-energy radical addition transition states. Electronic energy values given were calculated at the UB3LYP-D3(BJ)/def2-SVP-CPCM(THF) level of method. 
From the colored-filled RDG isosurface, it could be seen that both the $\mathrm{C}-\mathrm{H} \bullet \bullet \pi$ interaction (between alkyl chain and aryl ring) and the $\pi-\pi$ interactions (between aryl ring and ester moiety) are present in the system, thus emphasizing the effect of noncovalent interactions in the entiodetermining radical addition step.

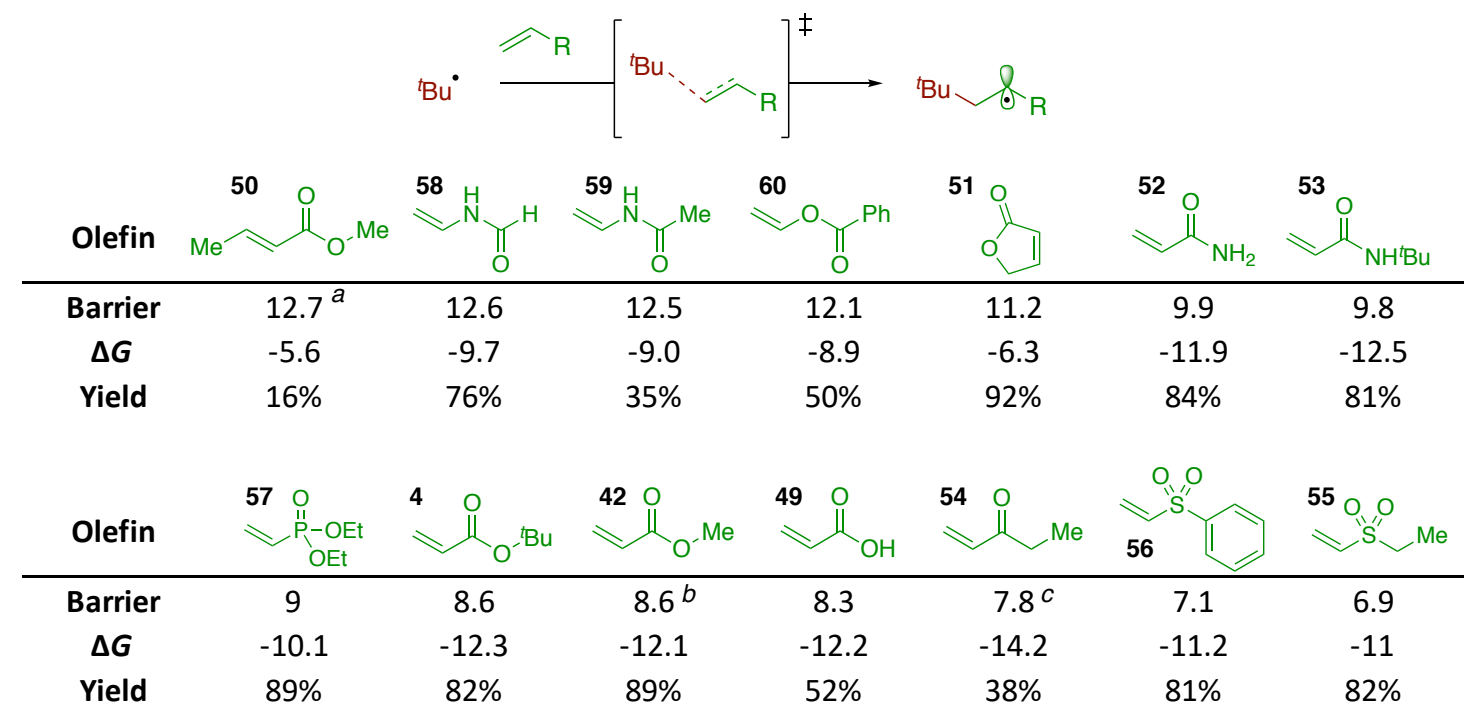

Scheme S5 Calculation of Giese addition of tert-Bu radical to different olefins. Free energy values given were calculated at the UB3LYP-D3(BJ)/def2-SVP-CPCM(THF) level of method.

The barrier and the thermodynamic drive of the Giese addition step were calculated between tert-butyl radical $\mathbf{A}$ and a series of different olefins that were tested in experiment as shown in Scheme S5. Based on the comparion between the computational results and the experimental yield (Scheme S5), a weak correlation can be found between the calculated barrier and the observed yield. Specifically, olefins found with higher barrier for radical addition (e.g., methyl (E)-but-2-enoate, vinyl amines and vinyl ether) generally have lower product yields. However, the correlation is weak since the selectivity of product (three-component vs two-component) depend on both the barrier for Giese addition and the relative concentration of olefins. Moreover, the calculation of Giese addition only focuses on the applicability of forming $\alpha$-carbonyl alkyl radical $\mathbf{C}$, while possible side reactions (e.g., hydrogen-atom-transfer 
with solvent THF, $\beta$-hydride elimination by $\mathrm{Ni}$ catalyst) could occur thus lower the product yield.

(a)
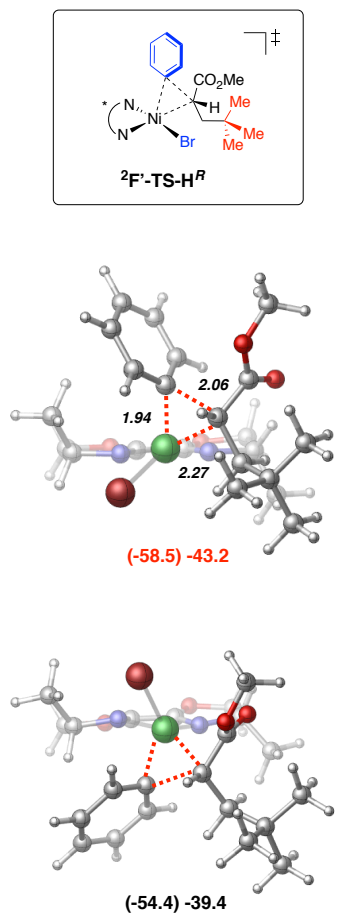

(b)
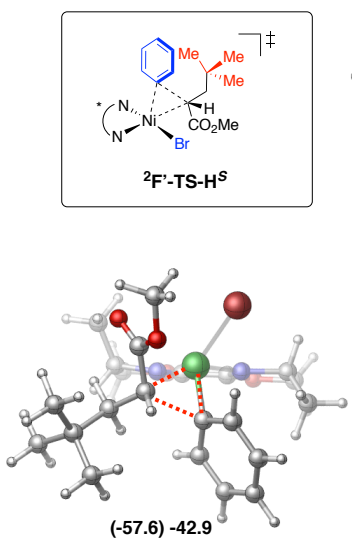

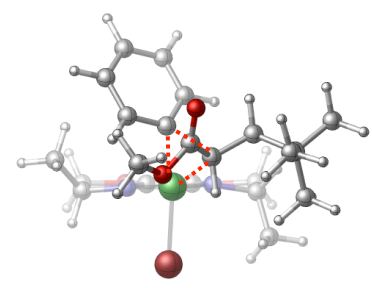

$(-57.7)-42.8$

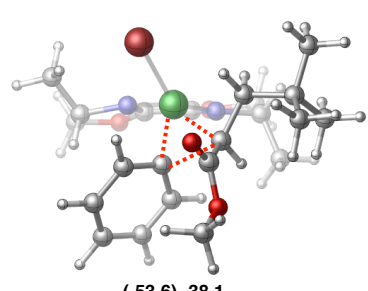

(-53.6) -38.1

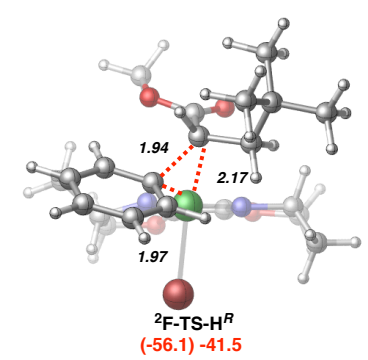

${ }^{2} \mathrm{~F}-\mathrm{TS}-\mathrm{H}^{R}$
$(-56.1)-41.5$

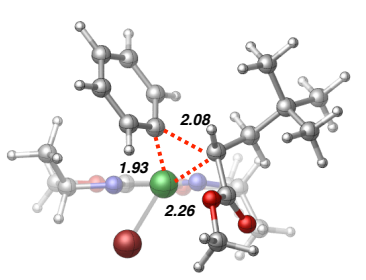

(-59.0) -44.1

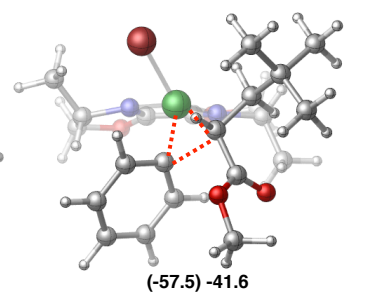

$(-57.5)-41.6$
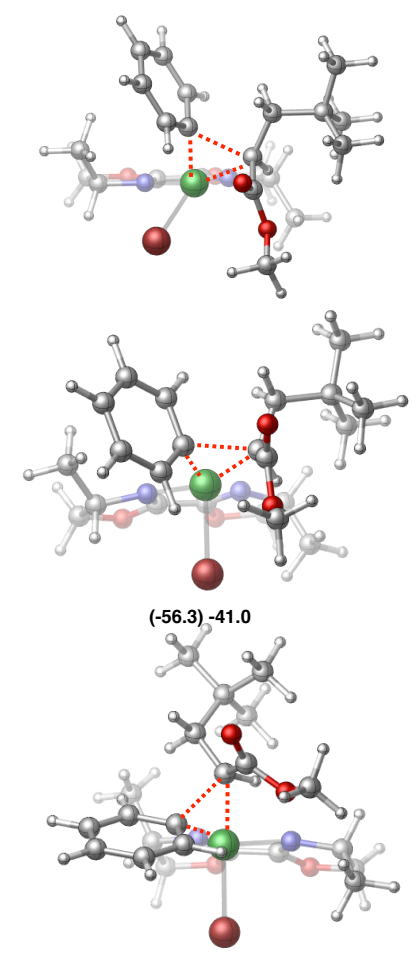

(-56.3) -42.1

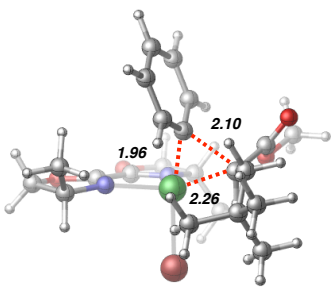

(-56.2) -41.3

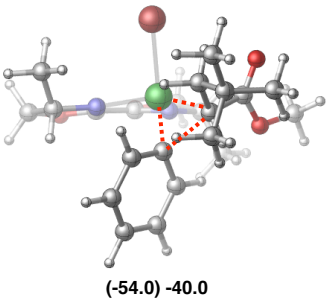

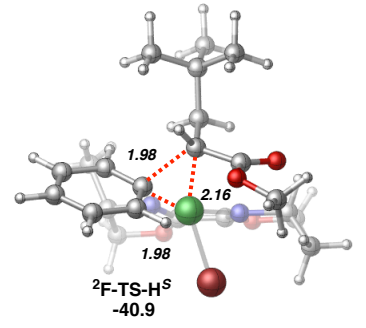




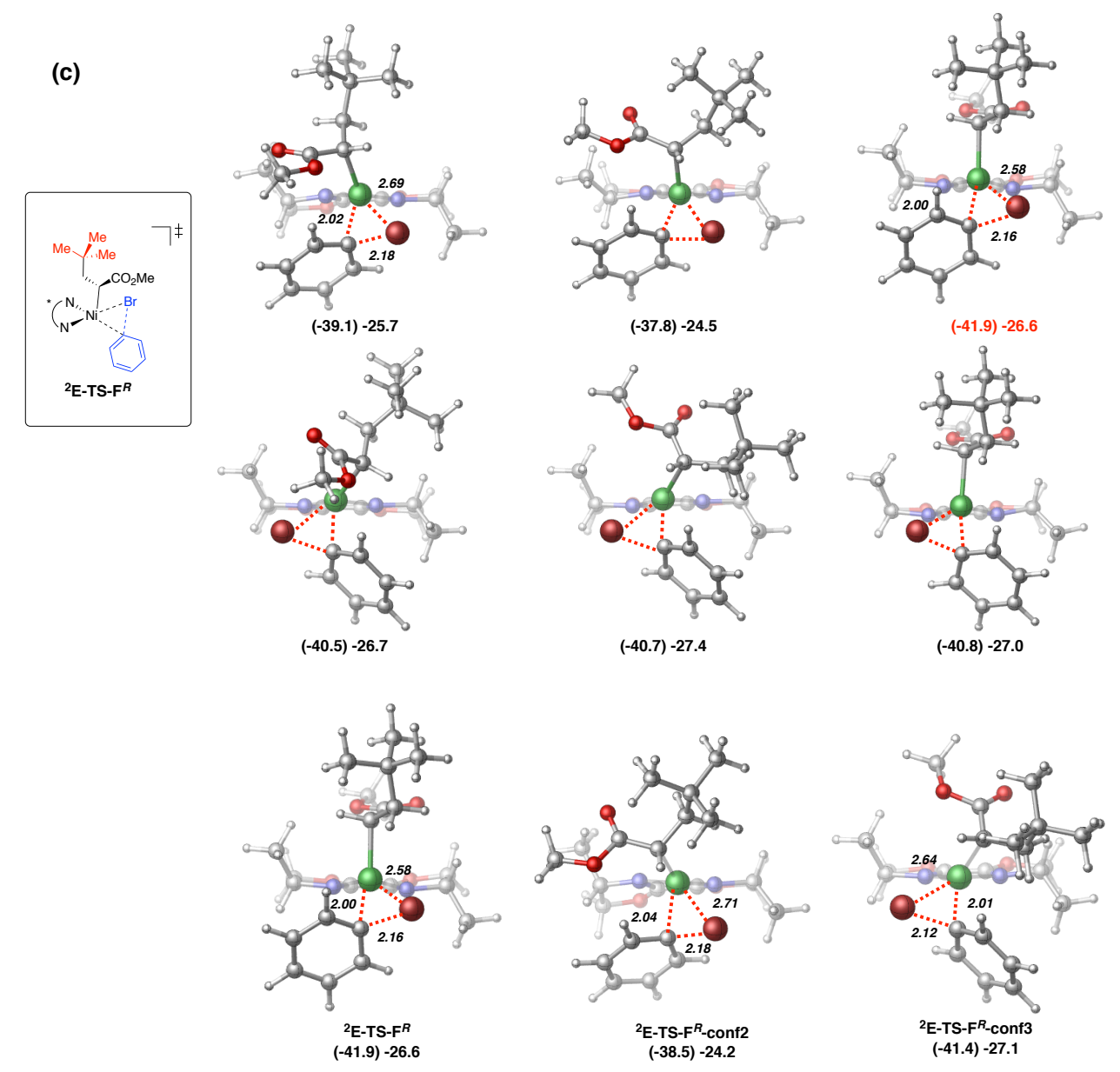

(d)
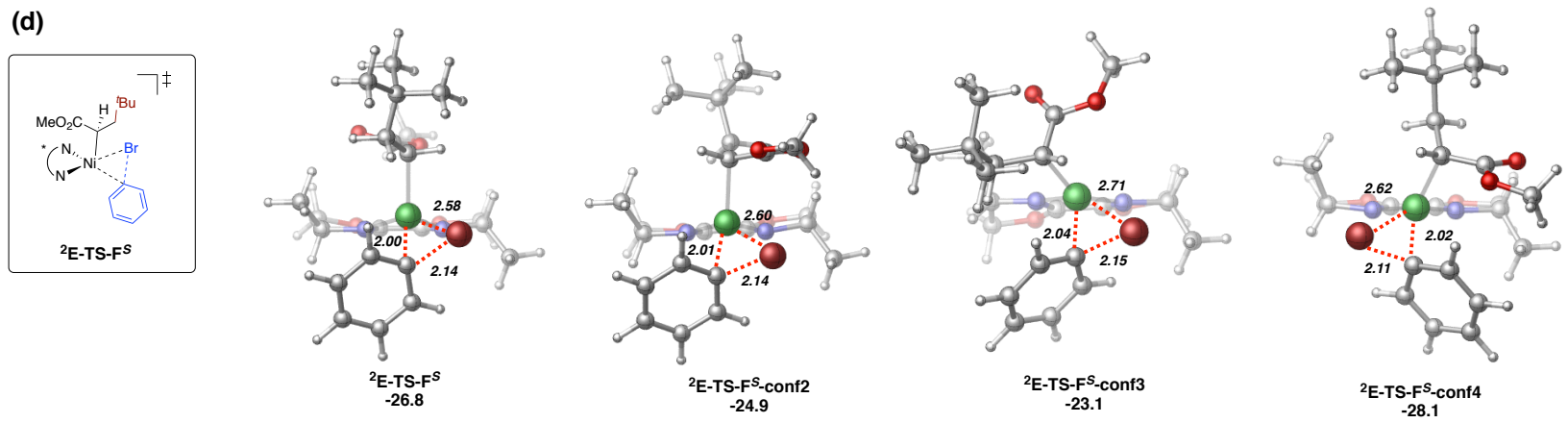


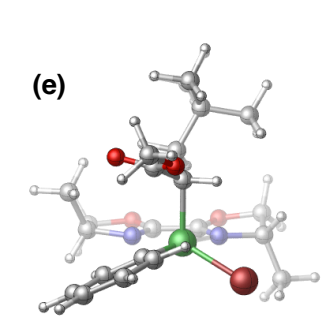

$(-57.3)-42.3$

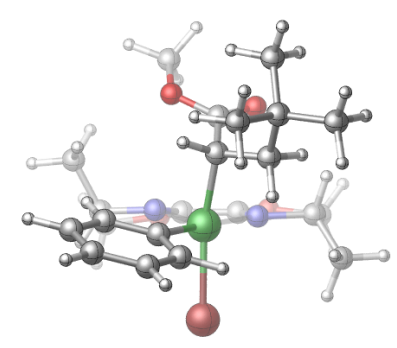

$(-68.8)-53.9$
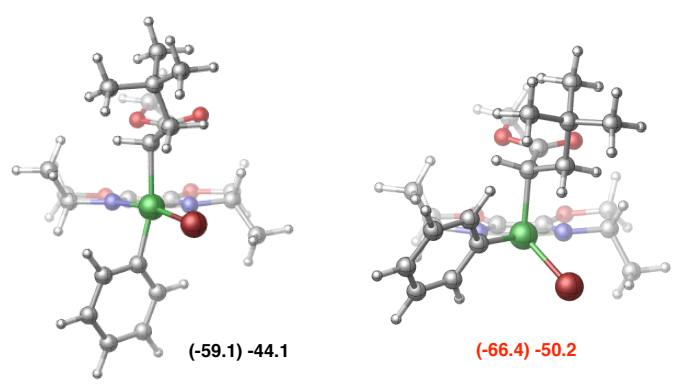

$(-66.4)-50.2$
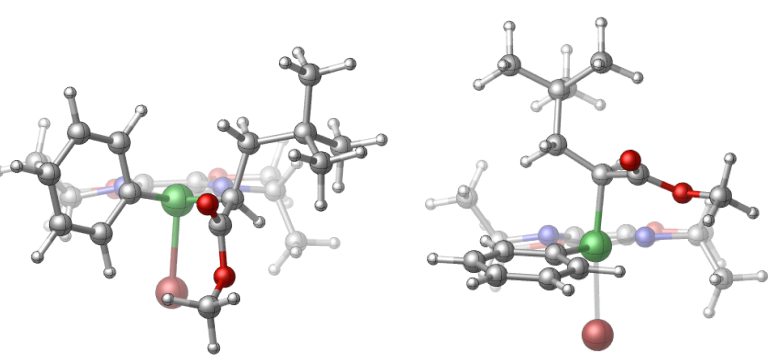

$(-65.9)-50.7$
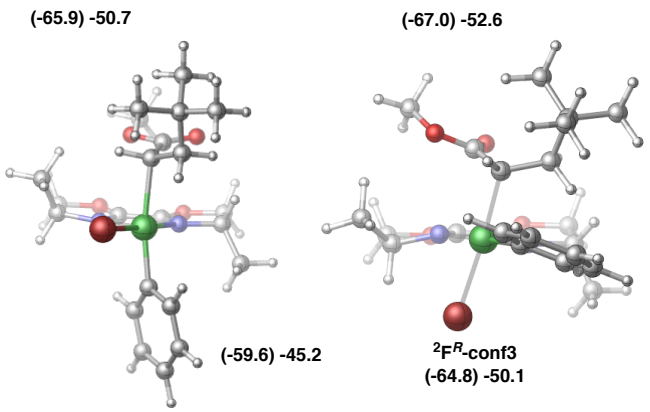

(f)
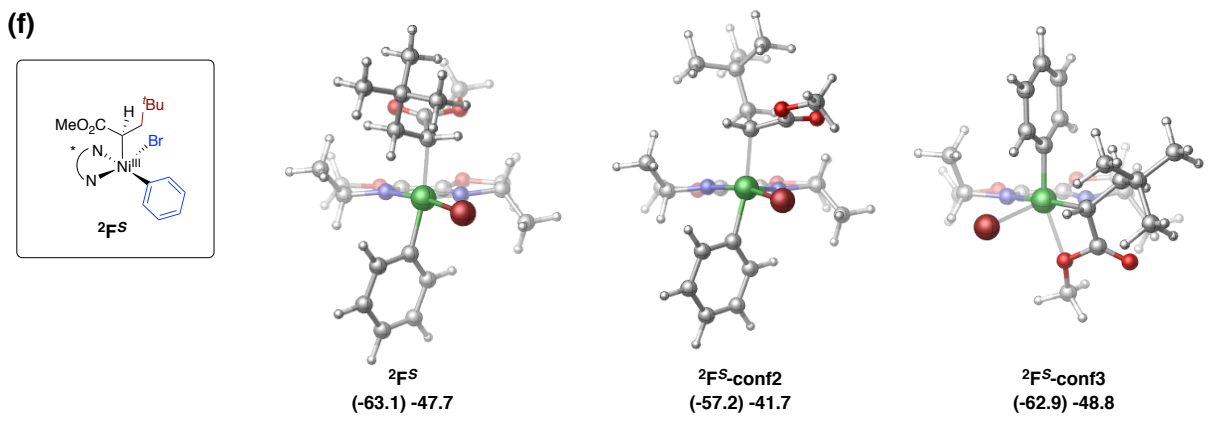

(g)
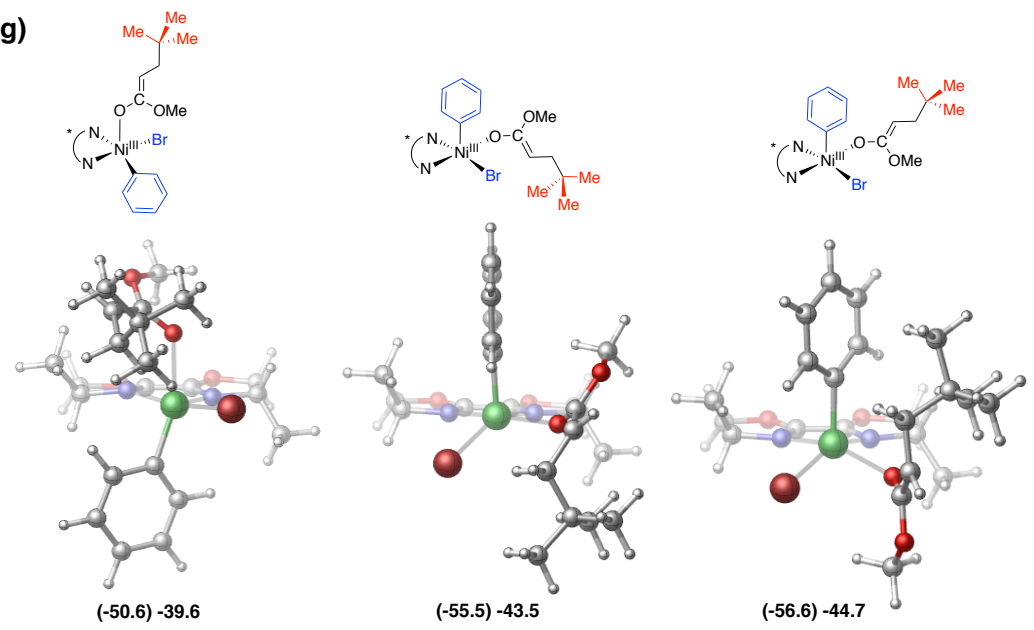

Scheme S6 Conformational search on key transition states (a) ${ }^{2} \mathbf{F}^{\prime}-\mathbf{T S}-\mathbf{H}^{R}$ (b) ${ }^{2} \mathbf{F}^{\prime}-\mathbf{T S}$ $\mathbf{H}^{S}$ (c) ${ }^{2} \mathbf{E}-$ TS-F $^{R}$ (d) ${ }^{2} \mathbf{E}-\mathbf{T S}-\mathbf{F}^{S}$ and intermediates (e) ${ }^{2} \mathbf{F}^{R}$ (f) ${ }^{2} \mathbf{F}^{S}$ (g) O-coordinated S153 
intermediates. Enthalpies (in parenthesis) and free energy values (out of parenthesis) given were calculated at the UB3LYP-D3(BJ)/def2-SVP-CPCM(THF) level of method. Conformations highlighted in red were the lowest-energy conformations of each species.

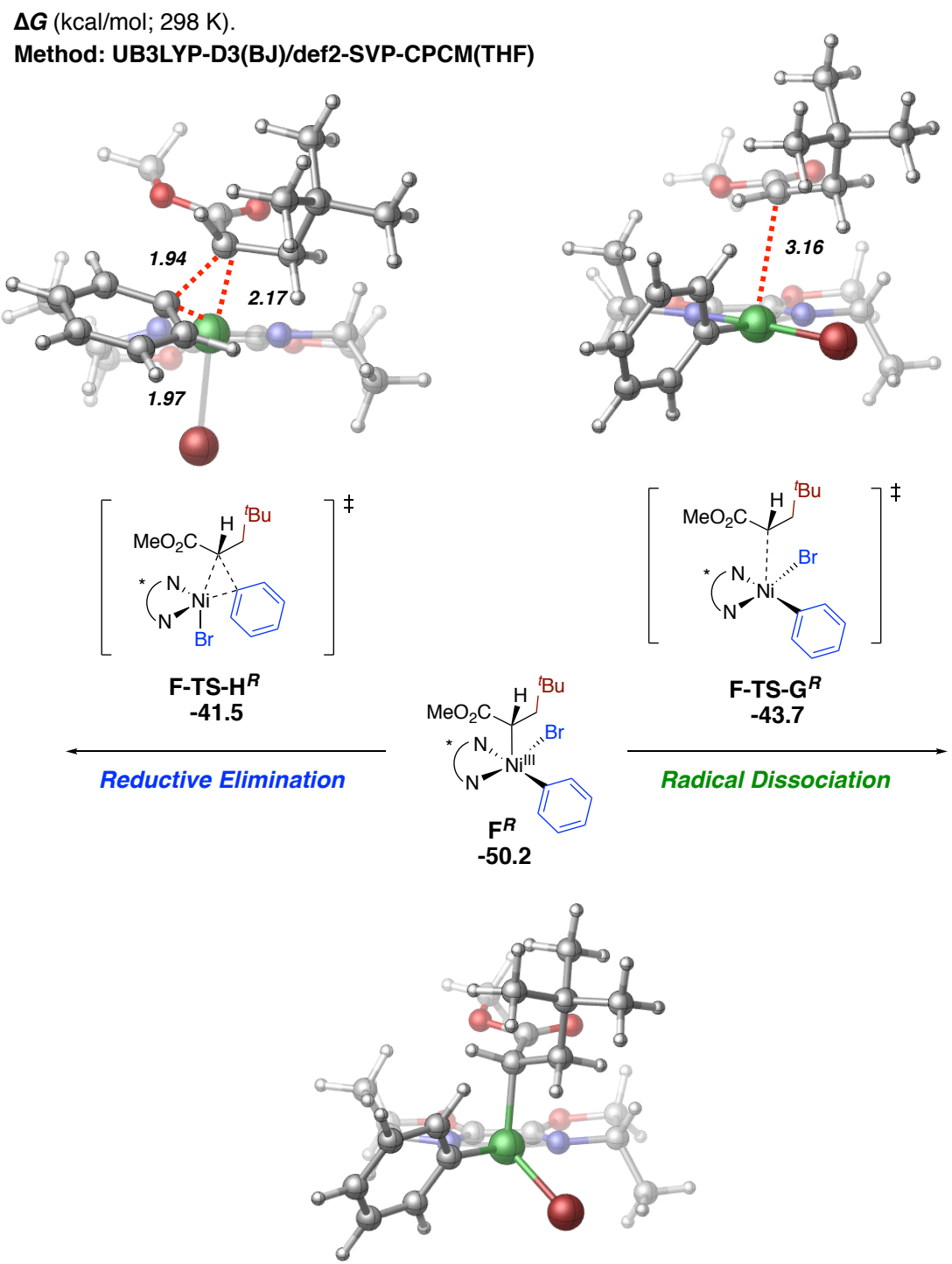

Scheme S7 Comparison between reductive elimination and radical dissociation pathways from intermediate $\mathbf{F}$. 

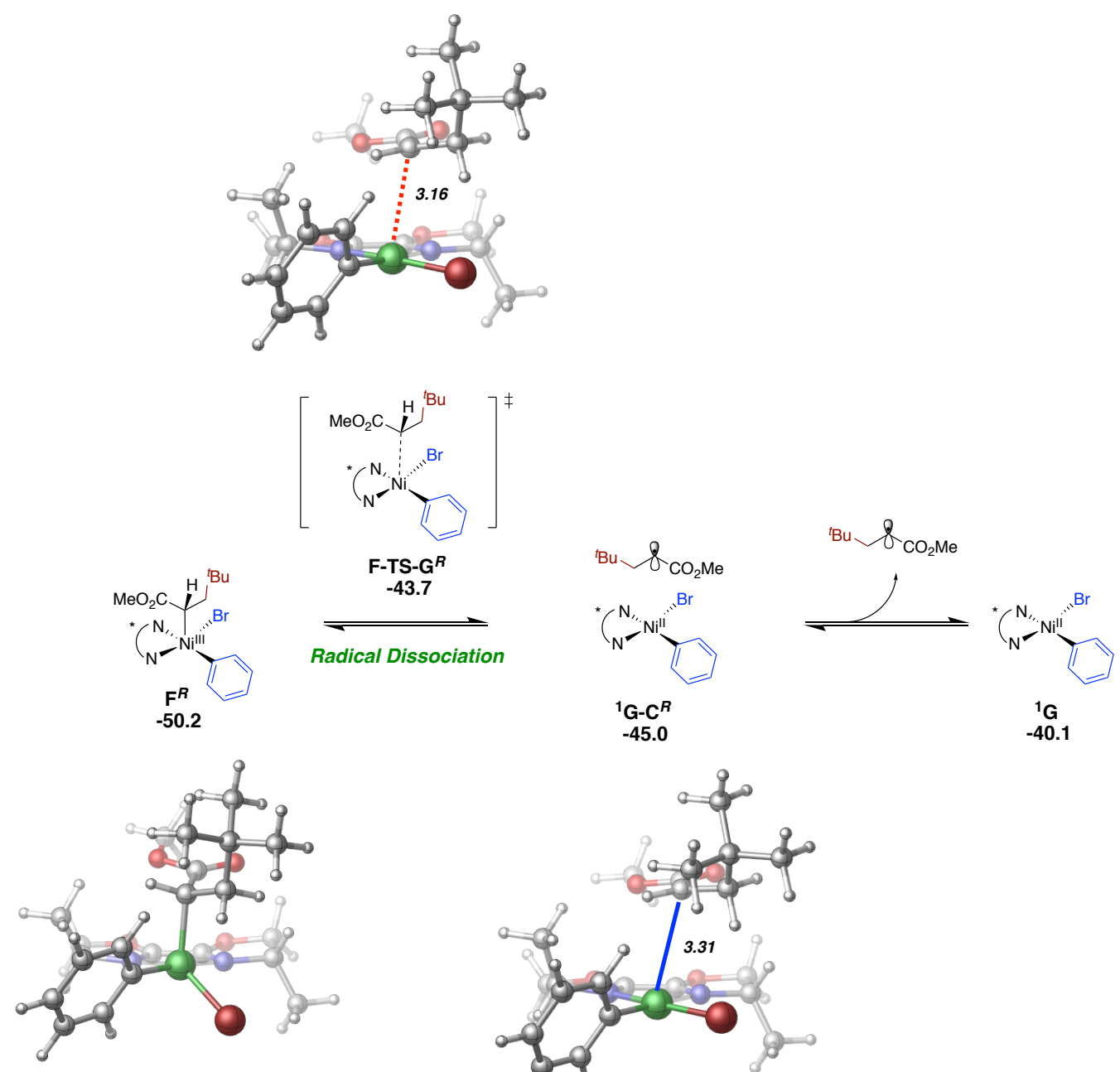

$\Delta G(\mathrm{kcal} / \mathrm{mol} ; 298 \mathrm{~K})$.

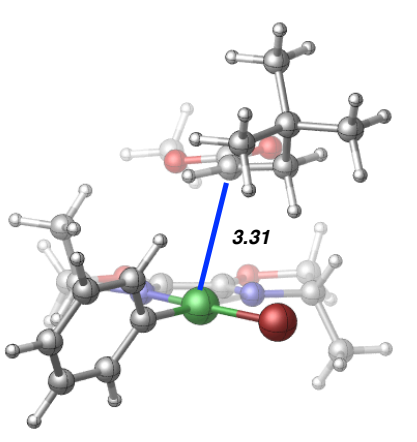

Method: UB3LYP-D3(BJ)/def2-SVP-CPCM(THF)

Scheme S8 Formation of alkyl radical Ni complex resulting from radical dissociation.

The radical addition transition state F-TS-G connects to two lower intermediate complexes which, as a complex but not as separated structures, are lower in energy than the transition state connecting them. However, decomplexation of the ${ }^{\mathbf{1}} \mathbf{G}-\mathbf{C}$ complex to separated $\mathbf{C}$ and ${ }^{\mathbf{1}} \mathbf{G}$ (as shown in Scheme 4) results in a higher energy. 


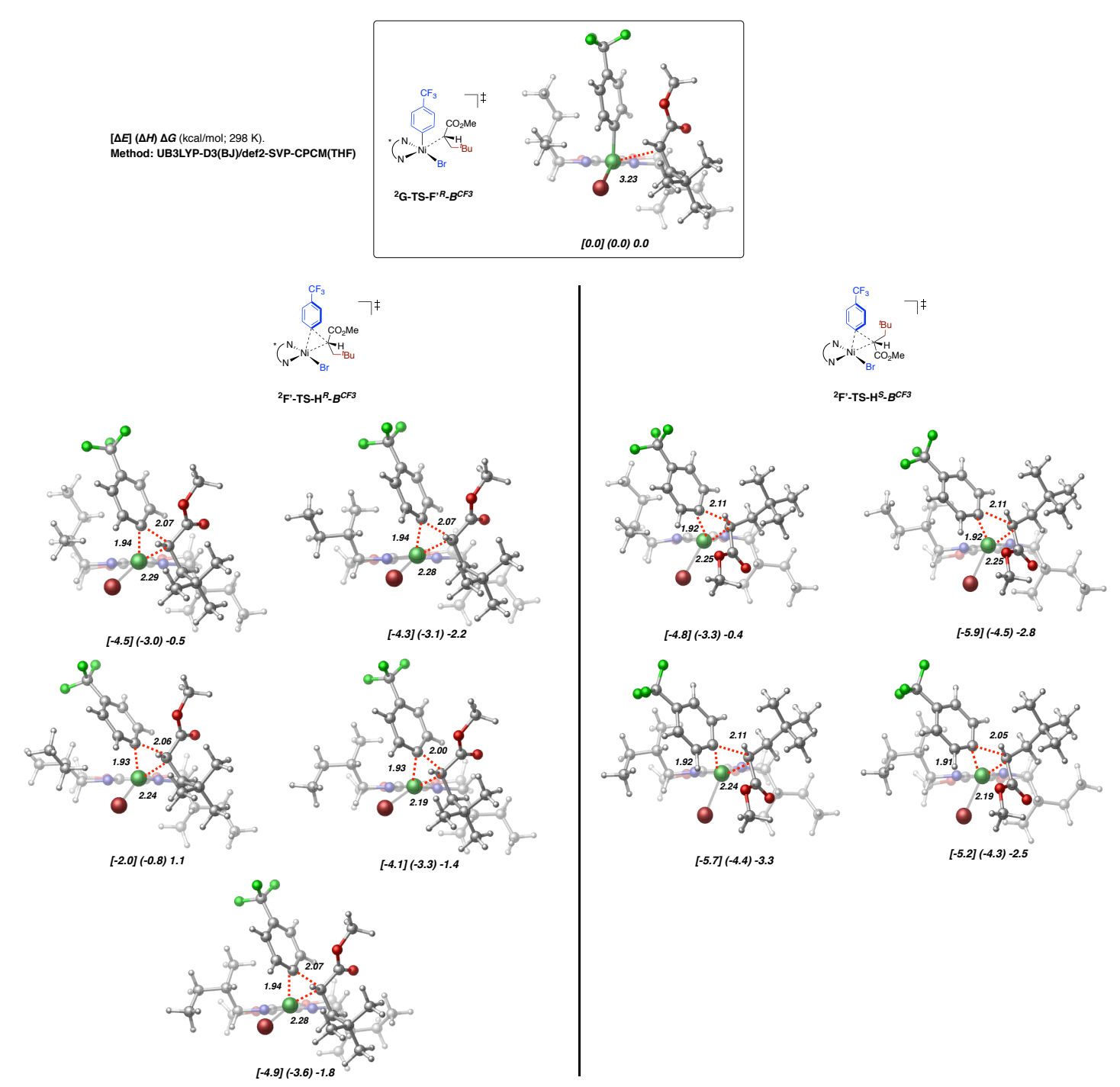

Scheme S9 Lowest energy diastereomeric reductive elimination transition states with full ligand. All relative energies are with respect to the lowest energy radical addition transition state (inset).

Based on the results obtained with the full ligand on the reductive elimination transition states, they were found all lower in energy than the radical addition (i.e. the lowestenergy conformer of G-TS-F' with full ligand). Specifically, the lowest energy reductive elimination step is found to be as much as $\sim 5 \mathrm{kcal} / \mathrm{mol}$ lower in energy than the lowest energy radical addition step. These results suggest that our calculations using the truncated ligand are adequate for exploring the mechanism and, in agreement with experiment, the substituents on the chiral ligand are crucial for inducing enantioselectivity. Moreover, we calculated the predicted enantioselectivity by Boltzmann distribution analysis based on the nine lowest energy conformers of 
reductive elimination transition states (as shown above) and, contrary to experiment, predicted the opposite stereochemistry. Specifically, the predicted product from the reductive elimination transition states is the $S$-product (calculated ee $\%=-68.7 \%$ ). The wrong stereochemistry of product predicted by the analysis on the reductive elimination step, in addition to the much higher energy for the radical addition versus reductive elimination, further supports our finding that radical addition is the stereodetermining step in this reaction system.

$\Delta G(\mathrm{kcal} / \mathrm{mol} ; 298 \mathrm{~K})$

Method: UB3LYP-D3(BJ)/def2-SVP-CPCM(THF)

(a)
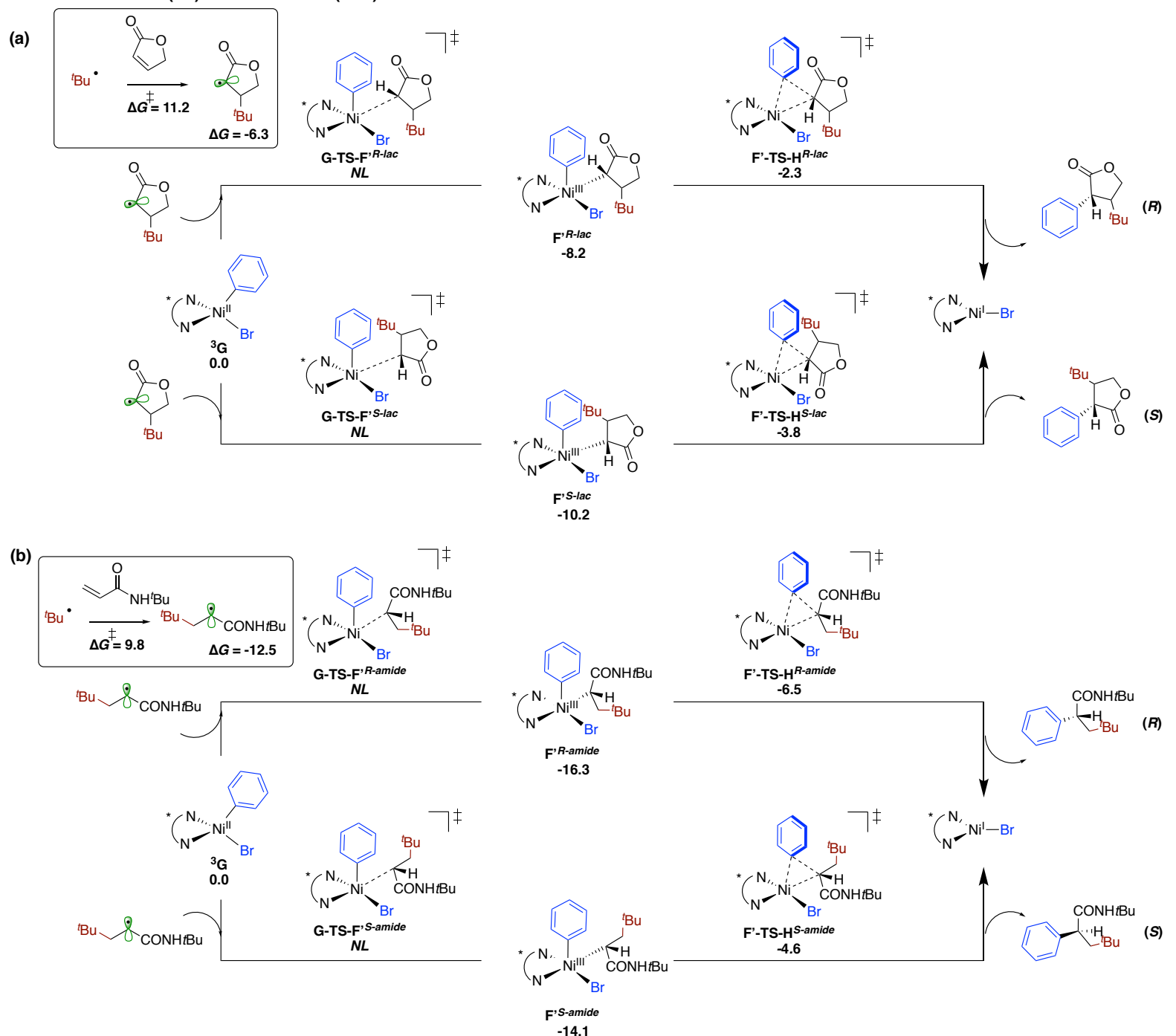

Scheme S10 Product-forming pathways via radical addition/reductive elimination with internal acrylate and acrylamide as olefin substrates.

We also calculated the radical addition/reductive elimination pathways leading to product formation with internal acrylate $\mathbf{5 1}$ and acrylamide $\mathbf{5 3}$ since they reveal 
diminished ee in experiment (15\% and 61\% ee, respectively, as shown in Scheme 2). The results suggest that, consistent with our model system (methyl acrylate), the formation of $\mathrm{Ni}(\mathrm{III})$ intermediate $\mathbf{F}$ ' for both systems is favorable (i.e., downhill in energy by $8-16 \mathrm{kcal} / \mathrm{mol}$ ), followed by fast reductive elimination (relative barrier of 6$10 \mathrm{kcal} / \mathrm{mol}$ ) to give the corresponding products. However, given that the energy for the reductive elimination transition states with respect to the tetrahedral $\mathrm{Ni}$ (II) species is lower by 2.3 to $6.5 \mathrm{kcal} / \mathrm{mol}$, these results suggest that the radical addition transition states are likely the stereodetermining step. Unfortunately, all attempts to locate the radical addition transition states failed and formdirectly the $\mathrm{Ni}(\mathrm{III})$ intermediates. Presumably, as observed previously for reactions in relatively flat energy surfaces, the low barriers make it very challenging to locate the stationary points corresponding to the radical addition transition states. Overall, the low barriers for the stereodetermining radical addition transition states will imply that achieving enantiocontrol in these substrates will be challenging as observed experimentally. Notably, we observed that in the internal acrylate case, its rigid structure prevents the carbonyl group from staying parallel to phenyl ligand as shown in the transition state of radical addition with methyl acrylate in the manuscript. As for the acrylamide case, the amide group also tends to stay away from the $\mathrm{Ph}$ ligand rather than staying parallel thus preventing $\pi-\pi$ interactions. Taken together, these results support that noncovalent interactions (in parallel orientation) between acrylate and $\mathrm{Ph}$ ligand are important for stereocontrol, and the modification of olefins (by their electronic and steric character) might lead to change in the resulted enantioselectivity. 


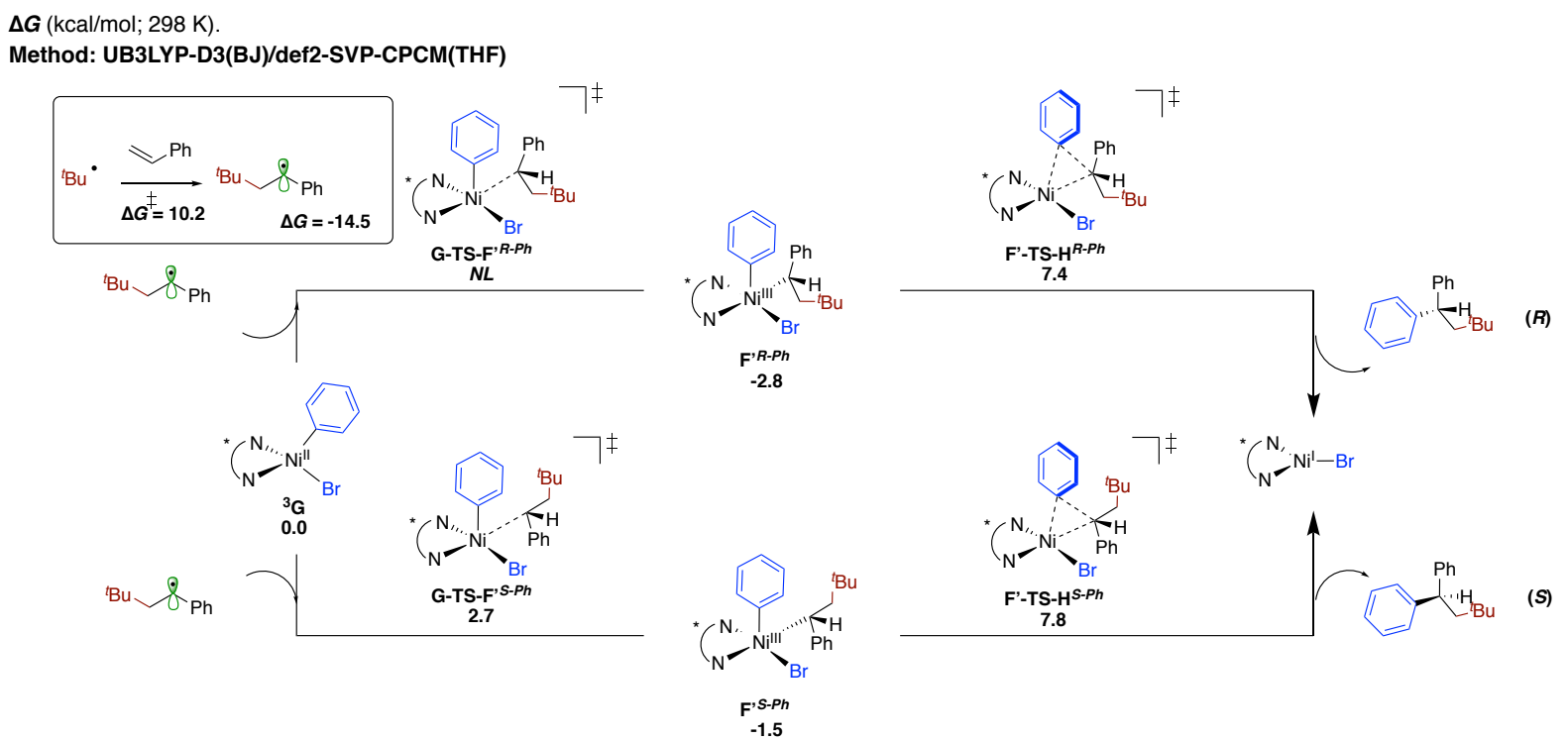

Scheme S11 Product-forming pathways via radical addition/reductive elimination with styrene as olefin substrate.

We also used computations to gain insights into the observed lower yields and enantioselectivity with styrenyl substrates (as shown before in the experimental part). In particular, we calculated the radical dissociation/reductive elimination pathway with vinylbenzene as a model substrate. Surprisingly, the radical addition transition state was found to be only $2.7 \mathrm{kcal} / \mathrm{mol}$ this time (for $S$-pathway, the $R$-pathway cannot be located). Also, it is noticeable that the generated $\mathrm{Ni}(\mathrm{III})$ intermediates $\mathbf{F}^{\mathbf{9}}$ in this case were found to be nearly isoenergetic (only $2 \sim 3 \mathrm{kcal} / \mathrm{mol}$ lower in energy) with the starting tetrahedral $\mathrm{Ni}$ (II) species (as comparison, the $\mathrm{Ni}(\mathrm{III})$ intermediate in methyl acrylate system is $\sim 10 \mathrm{kcal} / \mathrm{mol}$ lower in energy). Moreover, the reductive elimination transition states in this system $\mathbf{F}^{\prime}$-TS-H were found to be $\sim 8 \mathrm{kcal} / \mathrm{mol}$ higher than the starting $\mathrm{Ni}(\mathrm{II})$ species, while the energy of which was around $-1 \mathrm{kcal} / \mathrm{mol}$ in the methyl acrylate system. Overall, in contrast to the methyl acrylate system, reductive elimination was found to be higher in energy than radical addition in the styrene system, indicating a change in the identity of stereodetermining step. Altogether these suggest that the formation of $\mathrm{Ni}(\mathrm{III})$ intermediate in styrene system is reversible, which might be due to the stability of a secondary benzyl radical formed after Giese addition. Therefore, the formation of product is slower than the acrylate system and is consistent with the observed lower yields of styrenyl substrates in the experiment. 
$\Delta \boldsymbol{G}(\mathrm{kcal} / \mathrm{mol} ; 298 \mathrm{~K})$

Method: UB3LYP-D3(BJ)/def2-SVP-CPCM(THF)
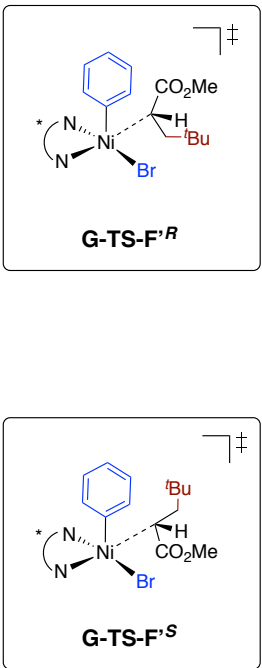
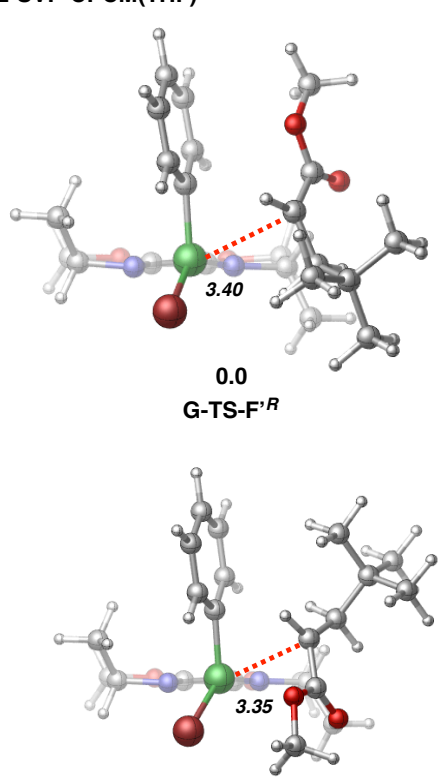

0.0

G-TS-F'S
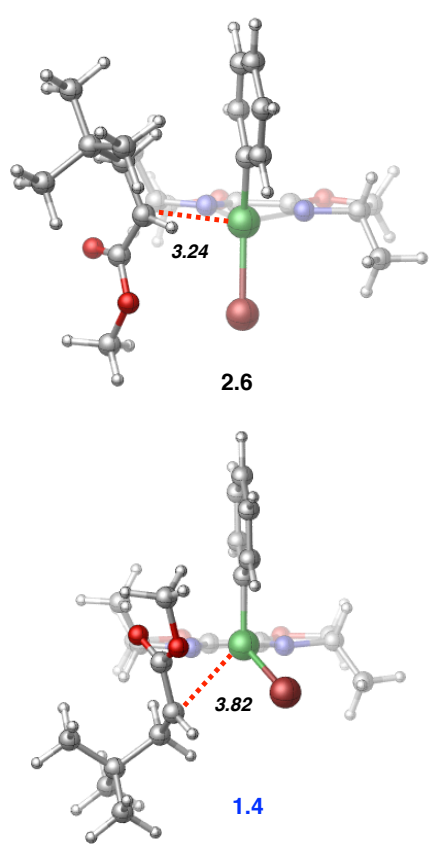

Scheme S12 Different conformations of radical addition to the tetrahedral Ni species

(G-TS-F'). Relative free energies were with respect to the lowest-energy radical addition transition states. 


\subsection{Coordinates and energies}

\section{Scheme 4}

A

C

$\mathrm{C}$

$\mathrm{H}$

$\mathrm{H}$

$\mathrm{H}$

$\mathrm{C}$

$\mathrm{H}$

$\mathrm{H}$

$\mathrm{H}$

C

$\mathrm{H}$

$\mathrm{H}$

$\mathrm{H}$
$-4.78826100$

$-6.18086900$

$-6.68885300$

$-6.19720600$

$-6.81119500$

$-3.70882600$

$-2.70404900$

$-3.80907100$

$-3.73501100$

$-4.59144200$

$-4.78837700$

$-5.27653500$

$-3.55683800$

$-5.14730900$

$-5.46619300$

$-6.15630800$

$-5.96654300$

$-4.56291000$

$-6.15188000$

$-5.70211900$

$-6.62898600$

$-6.97558700$

$-4.13276600$

$-4.56252600$

$-3.27539000$

$-3.75095300$

UB3LYP-D3(BJ)/def2-SVP-CPCM(THF)

Zero-point correction=

Thermal correction to Energy=

Thermal correction to Enthalpy=

Thermal correction to Gibbs Free Energy=

Sum of electronic and zero-point Energies=

Sum of electronic and thermal Energies=

Sum of electronic and thermal Enthalpies=

Sum of electronic and thermal Free Energies=

$\mathrm{HF}=-157.6986389$
$-2.55233700$

$-2.11142900$

$-2.82202500$

$-1.12853400$

$-2.05272000$

$-2.30512300$

$-2.37447600$

$-1.31566800$

$-3.05349300$

$-3.63281700$

$-4.64067500$

$-3.52233000$

$-3.65565900$

UB3LYP-D3(BJ)/def2-TZVPP-CPCM(THF)//UB3LYP-D3(BJ)/def2-SVPCPCM(THF)

$\mathrm{HF}=-157.877899$

\section{B}

$\begin{array}{lrrr}\text { C } & -4.30541400 & -3.77861800 & -0.90237400 \\ \mathrm{H} & -3.34032700 & -3.74703000 & -1.41607800 \\ \mathrm{C} & -4.41007900 & -4.35357000 & 0.30014300 \\ \mathrm{H} & -5.35744100 & -4.40341600 & 0.84228500 \\ \mathrm{H} & -5.16867100 & -3.33018600 & -1.39943300 \\ \mathrm{C} & -3.22744300 & -4.95957300 & 0.96455900 \\ \mathrm{O} & -2.10266100 & -4.99445900 & 0.51220900 \\ \mathrm{O} & -3.55935700 & -5.47871700 & 2.15823900 \\ \mathrm{C} & -2.50494100 & -6.09361700 & 2.90132800 \\ \mathrm{H} & -1.71034300 & -5.36587100 & 3.12371600 \\ \mathrm{H} & -2.95683000 & -6.45791800 & 3.83118900 \\ \mathrm{H} & -2.06803800 & -6.93059900 & 2.33658700\end{array}$

UB3LYP-D3(BJ)/def2-SVP-CPCM(THF)
0.115454 (Hartree/Particle)
0.121830
0.122774
0.086016
$-157.583185$
$-157.576809$
$-157.575865$
$-157.612623$


Zero-point correction=

Thermal correction to Energy=

Thermal correction to Enthalpy=

Thermal correction to Gibbs Free Energy=

Sum of electronic and zero-point Energies=

Sum of electronic and thermal Energies $=$

Sum of electronic and thermal Enthalpies=

Sum of electronic and thermal Free Energies=

$\mathrm{HF}=-306.2613926$

UB3LYP-D3(BJ)/de

CPCM(THF)

$\mathrm{HF}=-306.6188516$

\section{B-TS-C}

$\begin{array}{lrrr}\mathrm{C} & -4.63202900 & -3.79047800 & -1.09273600 \\ \mathrm{H} & -3.70695900 & -3.53738700 & -1.61309100 \\ \mathrm{C} & -4.57709000 & -4.42648200 & 0.10212500 \\ \mathrm{H} & -5.47327700 & -4.63252800 & 0.69119900 \\ \mathrm{H} & -5.55911300 & -3.34580000 & -1.45596100 \\ \mathrm{C} & -4.92896800 & -5.51713600 & -2.85803700 \\ \mathrm{C} & -6.40071800 & -5.74816600 & -2.71698100 \\ \mathrm{H} & -6.73369400 & -6.56139200 & -3.39582800 \\ \mathrm{H} & -6.66512500 & -6.05895000 & -1.69324300 \\ \mathrm{H} & -6.98805100 & -4.85314900 & -2.97687600 \\ \mathrm{C} & -4.01722800 & -6.57312100 & -2.32030300 \\ \mathrm{H} & -2.98094400 & -6.21511500 & -2.22830400 \\ \mathrm{H} & -4.34929100 & -6.92653600 & -1.33097100 \\ \mathrm{H} & -4.00697900 & -7.45612900 & -2.99303100 \\ \mathrm{C} & -4.45830500 & -4.71261500 & -4.02812200 \\ \mathrm{H} & -4.56445100 & -5.29350100 & -4.96881700 \\ \mathrm{H} & -5.04894800 & -3.79103600 & -4.15798200 \\ \mathrm{H} & -3.39455700 & -4.44055700 & -3.93976600 \\ \mathrm{C} & -3.30120700 & -4.93624700 & 0.62071100 \\ \mathrm{O} & -2.21178800 & -4.81305200 & 0.08945500 \\ \mathrm{O} & -3.46729400 & -5.58693900 & 1.79307300 \\ \mathrm{C} & -2.29112200 & -6.13205900 & 2.38710100 \\ \mathrm{H} & -1.55906200 & -5.34053500 & 2.60847200 \\ \mathrm{H} & -2.61187500 & -6.61931800 & 3.31571900 \\ \mathrm{H} & -1.81811700 & -6.86686700 & 1.71787400 \\ \mathrm{U} & - & \end{array}$

UB3LYP-D3(BJ)/def2-SVP-CPCM(THF)

Zero-point correction=

Thermal correction to Energy=

0.212760 (Hartree/Particle)

0.225980

0.226924

Thermal correction to Enthalpy=

0.172027
0.094952 (Hartree/Particle)

0.102527

$-306.166441$

$-306.159810$

$-306.158866$

$-306.196843$
0.101583

0.064550

Thermal correction to Gibbs Free Energy= 
Sum of electronic and zero-point Energies=

Sum of electronic and thermal Energies=

Sum of electronic and thermal Enthalpies=

Sum of electronic and thermal Free Energies= $\mathrm{HF}=-463.9677686$
$-463.755009$

$-463.741789$

$-463.740845$

$-463.795742$

UB3LYP-D3(BJ)/def2-TZVPP-CPCM(THF)//UB3LYP-D3(BJ)/def2-SVPCPCM(THF)

$\mathrm{HF}=-464.5019013$

C

$\begin{array}{lrrr}\mathrm{C} & -4.35990500 & -3.97061100 & -1.10299800 \\ \mathrm{H} & -3.33441400 & -3.60649700 & -1.26864000 \\ \mathrm{C} & -4.44155400 & -4.57066200 & 0.24863300 \\ \mathrm{H} & -5.40454100 & -4.73603500 & 0.73668800 \\ \mathrm{H} & -5.04462300 & -3.10584700 & -1.15778300 \\ \mathrm{C} & -4.73027400 & -4.93266800 & -2.28244300 \\ \mathrm{C} & -6.19100300 & -5.39005000 & -2.15531100 \\ \mathrm{H} & -6.46988000 & -6.03040700 & -3.00756200 \\ \mathrm{H} & -6.35503700 & -5.97132100 & -1.23445300 \\ \mathrm{H} & -6.87828000 & -4.52843300 & -2.14149300 \\ \mathrm{C} & -3.79873700 & -6.15402500 & -2.27369900 \\ \mathrm{H} & -2.74147400 & -5.84944400 & -2.31495900 \\ \mathrm{H} & -3.93773500 & -6.75858400 & -1.36350000 \\ \mathrm{H} & -4.00469000 & -6.80144800 & -3.14123500 \\ \mathrm{C} & -4.54730800 & -4.15435200 & -3.59312000 \\ \mathrm{H} & -4.79973200 & -4.78702400 & -4.45931900 \\ \mathrm{H} & -5.19742200 & -3.26496400 & -3.62229700 \\ \mathrm{H} & -3.50500400 & -3.81719200 & -3.71169400 \\ \mathrm{C} & -3.25138500 & -5.01070900 & 0.95197400 \\ \mathrm{O} & -2.10807800 & -4.91694900 & 0.52848600 \\ \mathrm{O} & -3.54188900 & -5.55432800 & 2.15601600 \\ \mathrm{C} & -2.43379000 & -6.02299900 & 2.92075200 \\ \mathrm{H} & -1.73272700 & -5.20318600 & 3.14038100 \\ \mathrm{H} & -2.84978600 & -6.42354500 & 3.85293900 \\ \mathrm{H} & -1.88880900 & -6.81176600 & 2.37971700 \\ \mathrm{H} & -\mathrm{B} & \end{array}$

UB3LYP-D3(BJ)/def2-SVP-CPCM(THF)

Zero-point correction=

0.217079 (Hartree/Particle)

Thermal correction to Energy=

0.229388

Thermal correction to Enthalpy=

0.230332

Thermal correction to Gibbs Free Energy=

0.178156

Sum of electronic and zero-point Energies=

$-463.789785$

Sum of electronic and thermal Energies=

$-463.777477$

Sum of electronic and thermal Enthalpies=

$-463.776533$

Sum of electronic and thermal Free Energies=

$-463.828708$ 


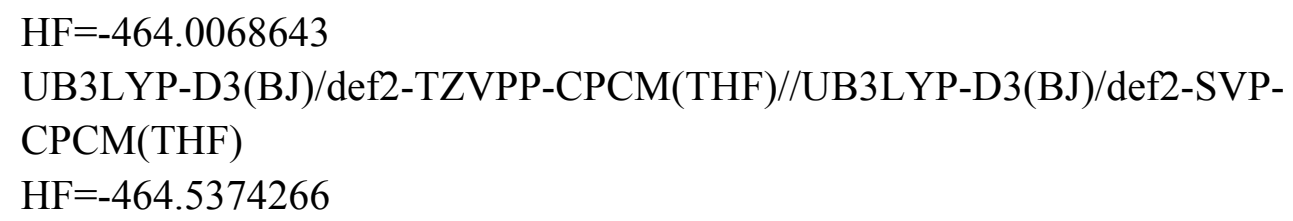

\section{Scheme 1}

${ }^{1} \mathbf{D}$

$\mathrm{Ni}$

$\mathrm{C}$

$-0.94258000$

1.43594000

$-0.77848100$

$-2.57987200$

2.01673500

$-1.41495100$

C

$-2.87097400$

0.15643900

$-3.56327400$

C

C

$-2.62500000$

2.46170800

$-2.78791900$

C

C

$-2.67279200$

0.57665500

$-1.15206900$

$-2.88348700$

$-0.30657700$

$-2.26948800$

$\mathrm{H}$

$-2.71669000$

1.55597000

$-3.81761300$

$-2.57180800$

3.53364800

$-2.99619900$

$\mathrm{H}$

$-3.04155300$

0.23099800

$-0.17810600$

$\mathrm{H}$

$\mathrm{H}$

$-3.08215900$

$-1.36483900$

$-2.07067100$

$-2.69456900$

1.91466900

$-4.85076900$

$\mathrm{H}$

$-3.01148800$

$-0.53147900$

$-4.40077800$

$\mathrm{Br}$

$-3.67354500$

3.27150700

$-0.17422200$

C

1.64688300

2.32929300

$-0.23793000$

C

2.16210900

4.49181200

$-0.19075600$

0.62733900

4.30435500

$-0.37050700$

$\mathrm{N}$

0.48390900

2.83948000

$-0.44611000$

$\mathrm{H}$

2.65349400

4.95190600

$-1.05845800$

0.28971400

4.72849300

$-1.32901500$

C

0.97481400

$-1.21602400$

$-0.43609500$

2.49442700

$-1.22045900$

$-0.09948100$

1.73489600

0.86589800

$-0.22252300$

0.63764000

0.21691900

$-0.39548000$

0.39909100

$-1.73009000$

0.34921400

3.11030500

$-1.71197800$

$-0.86461600$

2.42802300

5.04436000

0.72059300

2.72240700

$-1.64469700$

0.88754100

2.68736200

3.13435100

$-0.05520000$

2.86911300

0.19256300

$-0.06891400$

$-0.21515100$

4.87909100

0.76175300

$-0.11444200$

5.97500800

0.79237200

$-1.27298800$

4.62210200

0.60943000

0.11029200

4.46981600

1.73130900

0.62171700

$-1.80934700$

$-1.79522400$

$\mathrm{H}$

0.83470300

$-2.88912100$

$-1.81123500$

$\mathrm{H}$

$-0.44541300$

$-1.65188400$

$-2.00586200$

$\mathrm{H}$

1.20531900

$-1.32300100$

$-2.59267300$ 
UB3LYP-D3(BJ)/def2-SVP-CPCM(THF)

$\begin{array}{lc}\text { Zero-point correction }= & 0.291659 \text { (Hartree/Particle) } \\ \text { Thermal correction to Energy }= & 0.311891 \\ \text { Thermal correction to Enthalpy }= & 0.312835 \\ \text { Thermal correction to Gibbs Free Energy= } & 0.239949 \\ \text { Sum of electronic and zero-point Energies }= & -4884.933396 \\ \text { Sum of electronic and thermal Energies }= & -4884.913164 \\ \text { Sum of electronic and thermal Enthalpies }= & -4884.912219 \\ \text { Sum of electronic and thermal Free Energies }= & -4884.985105 \\ \text { HF }=-4885.2250546 & \end{array}$

UB3LYP-D3(BJ)/def2-TZVPP-CPCM(THF)//UB3LYP-D3(BJ)/def2-SVPCPCM(THF)

$\mathrm{HF}=-4886.6504419$

${ }^{3} \mathbf{D}$

$\begin{array}{lrrr}\mathrm{Ni} & -1.03209500 & 0.92430100 & -0.59379800 \\ \mathrm{C} & -2.57185900 & 0.47705500 & -1.82470700 \\ \mathrm{C} & -2.90496400 & 3.15067900 & -2.62571200 \\ \mathrm{C} & -3.07430000 & 1.44026800 & -0.89733100 \\ \mathrm{C} & -2.25722400 & 0.86490700 & -3.15774300 \\ \mathrm{C} & -2.40311800 & 2.18682300 & -3.53375500 \\ \mathrm{C} & -3.23712000 & 2.78129600 & -1.33594300 \\ \mathrm{H} & -3.53280000 & 1.11797900 & 0.04051500 \\ \mathrm{H} & -1.90373600 & 0.11432900 & -3.86613100 \\ \mathrm{H} & -2.13796000 & 2.48597000 & -4.55035600 \\ \mathrm{H} & -3.65449500 & 3.51326200 & -0.64067600 \\ \mathrm{H} & -3.03112000 & 4.18561000 & -2.94921400 \\ \mathrm{Br} & -2.93937600 & -1.41138900 & -1.50001500 \\ \mathrm{C} & 1.52700600 & 1.94093800 & -0.78901900 \\ \mathrm{C} & 1.98509600 & 3.69819300 & -2.07842100 \\ \mathrm{C} & 0.45759700 & 3.61360300 & -1.84568700 \\ \mathrm{~N} & 0.31572400 & 2.29761100 & -1.22238000 \\ \mathrm{H} & 2.26332800 & 3.36388100 & -3.09279600 \\ \mathrm{H} & -0.07641000 & 3.63879700 & -2.80858000 \\ \mathrm{C} & 0.95754900 & -1.16691100 & 0.89283300 \\ \mathrm{C} & 2.42206200 & -0.85964600 & 1.28675400 \\ \mathrm{C} & 1.64685600 & 0.77535500 & -0.01726900 \\ \mathrm{~N} & 0.53330500 & 0.10312500 & 0.30080600 \\ \mathrm{H} & 0.36074200 & -1.39116300 & 1.79242100 \\ \mathrm{H} & 3.11255900 & -1.69965000 & 1.12891300 \\ \mathrm{H} & 2.41029300 & 4.69481300 & -1.89596000 \\ \mathrm{H} & 2.50046100 & -0.51513800 & 2.33208600 \\ \mathrm{O} & 2.54786600 & 2.77938400 & -1.11876200 \\ \mathrm{O} & 2.81224900 & 0.22096200 & 0.41321100 \\ & & & \\ \mathrm{H} & & & \\ \mathrm{H} & & \end{array}$




$\begin{array}{lrrr}\mathrm{C} & -0.07543600 & 4.72824100 & -0.94358100 \\ \mathrm{H} & 0.01084700 & 5.71107000 & -1.43539800 \\ \mathrm{H} & -1.13305300 & 4.55032400 & -0.70412300 \\ \mathrm{H} & 0.49160700 & 4.75976500 & 0.00092700 \\ \mathrm{C} & 0.82464400 & -2.32332000 & -0.09988900 \\ \mathrm{H} & 1.12266200 & -3.27925200 & 0.36109500 \\ \mathrm{H} & -0.21646000 & -2.40840300 & -0.44453900 \\ \mathrm{H} & 1.46272000 & -2.14624600 & -0.98098500\end{array}$

UB3LYP-D3(BJ)/def2-SVP-CPCM(THF)

Zero-point correction=

0.290845 (Hartree/Particle)

Thermal correction to Energy=

0.311013

Thermal correction to Enthalpy=

0.311957

Thermal correction to Gibbs Free Energy=

Sum of electronic and zero-point Energies=

0.239012

$-4884.928978$

Sum of electronic and thermal Energies=

$-4884.908810$

$-4884.907866$

Sum of electronic and thermal Enthalpies=

$-4884.980811$

Sum of electronic and thermal Free Energies=

$\mathrm{HF}=-4885.2198229$

UB3LYP-D3(BJ)/def2-TZVPP-CPCM(THF)//UB3LYP-D3(BJ)/def2-SVP-

CPCM(THF)

$\mathrm{HF}=-4886.6409439$

\section{${ }^{1}$ D-TS-G}

$\begin{array}{lrrr}\mathrm{Ni} & -0.83110500 & 1.36977800 & -0.80861700 \\ \mathrm{C} & -2.31714400 & 1.88978200 & -1.66477700 \\ \mathrm{C} & -3.18775800 & 0.30912600 & -3.81514000 \\ \mathrm{C} & -2.52641600 & 2.48718600 & -2.92669500 \\ \mathrm{C} & -2.62024200 & 0.51017200 & -1.45985100 \\ \mathrm{C} & -3.05319700 & -0.27116700 & -2.56455000 \\ \mathrm{C} & -2.92457300 & 1.69026900 & -3.99046600 \\ \mathrm{H} & -2.32773200 & 3.55184600 & -3.06933900 \\ \mathrm{H} & -2.74668900 & 0.09942100 & -0.45083700 \\ \mathrm{H} & -3.33382300 & -1.31685200 & -2.40911800 \\ \mathrm{H} & -3.02383600 & 2.13355500 & -4.98541500 \\ \mathrm{H} & -3.52516000 & -0.28814500 & -4.66541700 \\ \mathrm{Br} & -3.65196600 & 3.38731200 & 0.05896100 \\ \mathrm{C} & 1.65377000 & 2.33211700 & -0.20371800 \\ \mathrm{C} & 2.08814500 & 4.50518200 & -0.33262300 \\ \mathrm{C} & 0.55266800 & 4.26353500 & -0.38253100 \\ \mathrm{~N} & 0.46745700 & 2.78929600 & -0.40782000 \\ \mathrm{H} & 2.50713400 & 4.86923800 & -1.28055600 \\ \mathrm{H} & 0.12276900 & 4.64660400 & -1.31988600 \\ \mathrm{C} & 1.06584600 & -1.23488200 & -0.27446200 \\ \mathrm{C} & 2.59302900 & -1.19450700 & 0.02867600\end{array}$




$\begin{array}{lrrr}\mathrm{C} & 1.78807200 & 0.87093500 & -0.13552300 \\ \mathrm{~N} & 0.70802200 & 0.19326700 & -0.29821400 \\ \mathrm{H} & 0.51735100 & -1.71257100 & 0.55236700 \\ \mathrm{H} & 3.20536900 & -1.66634300 & -0.75147000 \\ \mathrm{H} & 2.40113200 & 5.16037800 & 0.49008300 \\ \mathrm{H} & 2.85705800 & -1.60876200 & 1.01002800 \\ \mathrm{O} & 2.66201700 & 3.17917700 & -0.08560500 \\ \mathrm{O} & 2.93019400 & 0.23155900 & 0.04897300 \\ \mathrm{C} & -0.20972400 & 4.83624500 & 0.80606400 \\ \mathrm{H} & -0.13568500 & 5.93489800 & 0.80163100 \\ \mathrm{H} & -1.26966400 & 4.54753100 & 0.74630100 \\ \mathrm{H} & 0.21292200 & 4.46113100 & 1.75191900 \\ \mathrm{C} & 0.69145600 & -1.90971700 & -1.58862700 \\ \mathrm{H} & 0.93801000 & -2.98164200 & -1.55227800 \\ \mathrm{H} & -0.38710600 & -1.80127300 & -1.77349600 \\ \mathrm{H} & 1.23484700 & -1.45065500 & -2.42917900\end{array}$

UB3LYP-D3(BJ)/def2-SVP-CPCM(THF)

Zero-point correction=

Thermal correction to Energy=

0.291693 (Hartree/Particle)

Thermal correction to Enthalpy=

0.311482

0.312426

Thermal correction to Gibbs Free Energy=

Sum of electronic and zero-point Energies=

0.240116

$-4884.929204$

$-4884.909415$

$-4884.908471$

Sum of electronic and thermal Enthalpies=

$-4884.980781$

Sum of electronic and thermal Free Energies=

$\mathrm{HF}=-4885.220897$

UB3LYP-D3(BJ)/def2-TZVPP-CPCM(THF)//UB3LYP-D3(BJ)/def2-SVP-

CPCM(THF)

$\mathrm{HF}=-4886.6483641$

\section{${ }^{3}$ D-TS-G}

$\begin{array}{lrrr}\mathrm{Ni} & -1.01342900 & 1.23965900 & -0.00535300 \\ \mathrm{C} & -2.48634200 & 0.84282400 & -1.22759300 \\ \mathrm{C} & -2.62962200 & 2.32564400 & -3.58376200 \\ \mathrm{C} & -3.10521600 & 2.11345600 & -1.21085400 \\ \mathrm{C} & -2.04890700 & 0.25919400 & -2.43733800 \\ \mathrm{C} & -2.09612800 & 1.02326200 & -3.59728100 \\ \mathrm{C} & -3.13566100 & 2.85807400 & -2.39806200 \\ \mathrm{H} & -3.54014900 & 2.50879600 & -0.29151300 \\ \mathrm{H} & -1.63121000 & -0.74767700 & -2.44043000 \\ \mathrm{H} & -1.71206300 & 0.60136500 & -4.52932000 \\ \mathrm{H} & -3.57674700 & 3.85767700 & -2.38752800 \\ \mathrm{H} & -2.66506100 & 2.90923000 & -4.50576300 \\ \mathrm{Br} & -2.99577700 & -0.40911000 & 0.34928800\end{array}$




\begin{tabular}{|c|c|c|c|}
\hline $\mathrm{C}$ & 1.53125500 & 2.05298700 & -0.63596700 \\
\hline $\mathrm{C}$ & 1.91597100 & 4.13293300 & -1.34419700 \\
\hline $\mathrm{C}$ & 0.39054100 & 3.88459400 & -1.28815300 \\
\hline $\mathrm{N}$ & 0.31771400 & 2.61025600 & -0.57907300 \\
\hline $\mathrm{H}$ & 2.26675000 & 4.56153400 & -2.29259500 \\
\hline $\mathrm{H}$ & -0.00483400 & 3.75395600 & -2.31578900 \\
\hline C & 1.00594300 & -1.29641000 & 0.52241800 \\
\hline C & 2.55081500 & -1.19342500 & 0.53727400 \\
\hline $\mathrm{C}$ & 1.68521400 & 0.73588700 & -0.17448900 \\
\hline $\mathrm{N}$ & 0.61124600 & 0.09443700 & 0.29982700 \\
\hline $\mathrm{H}$ & 0.63938000 & -1.64850800 & 1.50106400 \\
\hline $\mathrm{H}$ & 3.05779900 & -2.02802500 & 0.03333200 \\
\hline $\mathrm{H}$ & 2.24504100 & 4.77827700 & -0.50968400 \\
\hline $\mathrm{H}$ & 2.94398900 & -1.09584500 & 1.56350000 \\
\hline $\mathrm{O}$ & 2.50905000 & 2.82792800 & -1.18139600 \\
\hline $\mathrm{O}$ & 2.84265100 & 0.02086100 & -0.18624400 \\
\hline $\mathrm{C}$ & -0.39630400 & 4.99316600 & -0.60515100 \\
\hline $\mathrm{H}$ & -0.30594000 & 5.94270000 & -1.15680700 \\
\hline $\mathrm{H}$ & -1.46214900 & 4.72521300 & -0.55756400 \\
\hline $\mathrm{H}$ & -0.03189400 & 5.14571400 & 0.42334900 \\
\hline $\mathrm{C}$ & 0.46633300 & -2.21934300 & -0.57218300 \\
\hline $\mathrm{H}$ & 0.77084500 & -3.26370300 & -0.39502600 \\
\hline $\mathrm{H}$ & -0.63242800 & -2.17727700 & -0.59308900 \\
\hline $\mathrm{H}$ & 0.84477000 & -1.90727700 & -1.55905500 \\
\hline \multicolumn{4}{|c|}{ UB3LYP-D3(BJ)/def2-SVP-CPCM(THF) } \\
\hline \multicolumn{3}{|c|}{ Zero-point correction $=$} & 0.290180 (Hartree/Particle) \\
\hline \multicolumn{3}{|c|}{ Thermal correction to Energy= } & 0.309933 \\
\hline \multicolumn{3}{|c|}{ Thermal correction to Enthalpy= } & 0.310878 \\
\hline \multicolumn{3}{|c|}{ Thermal correction to Gibbs Free Energy= } & 0.238909 \\
\hline \multicolumn{3}{|c|}{ Sum of electronic and zero-point Energies $=$} & -4884.923162 \\
\hline \multicolumn{3}{|c|}{ Sum of electronic and thermal Energies $=$} & -4884.903409 \\
\hline \multicolumn{3}{|c|}{ Sum of electronic and thermal Enthalpies $=$} & -4884.902464 \\
\hline \multicolumn{3}{|c|}{ Sum of electronic and thermal Free Energies $=$} & -4884.974432 \\
\hline \multicolumn{4}{|c|}{$H F=-4885.213342$} \\
\hline \multirow{2}{*}{\multicolumn{4}{|c|}{$\begin{array}{l}\text { UB3LYP-D3(BJ)/def2-TZVPP-CPCM(THF)//UB3LYP-D3(BJ)/def2-SVP- } \\
\text { CPCM(THF) } \\
\text { HF=-4886.6344331 }\end{array}$}} \\
\hline & & & \\
\hline \multicolumn{4}{|l|}{${ }^{1} \mathbf{G}$} \\
\hline $\mathrm{Ni}$ & -0.60698600 & 0.52697600 & -1.02379800 \\
\hline $\mathrm{C}$ & -2.04929000 & 1.27531300 & -1.96942100 \\
\hline $\mathrm{C}$ & -4.12342000 & 2.53188300 & -3.44255700 \\
\hline $\mathrm{C}$ & -1.83352900 & 1.74578900 & -3.27697500 \\
\hline $\mathrm{C}$ & -3.33474400 & 1.43752800 & -1.42323700 \\
\hline
\end{tabular}




\begin{tabular}{|c|c|c|c|}
\hline $\mathrm{C}$ & -4.35777100 & 2.06539400 & -2.14385400 \\
\hline $\mathrm{C}$ & -2.85652000 & 2.36327200 & -4.00959500 \\
\hline $\mathrm{H}$ & -0.84753500 & 1.63802100 & -3.74138100 \\
\hline $\mathrm{H}$ & -3.54684400 & 1.06436000 & -0.41752200 \\
\hline $\mathrm{H}$ & -5.34620500 & 2.18627800 & -1.69040200 \\
\hline $\mathrm{H}$ & -2.66040400 & 2.71653100 & -5.02644800 \\
\hline $\mathrm{H}$ & -4.92234800 & 3.01867100 & -4.00805400 \\
\hline $\mathrm{Br}$ & -1.64648100 & -1.56773600 & -0.88054800 \\
\hline $\mathrm{C}$ & 1.50965900 & 2.17738300 & -0.39391700 \\
\hline $\mathrm{C}$ & 1.44705100 & 4.30712400 & -1.00896300 \\
\hline $\mathrm{C}$ & 0.06975600 & 3.64041100 & -1.27033400 \\
\hline $\mathrm{N}$ & 0.34584500 & 2.22391100 & -0.94709800 \\
\hline $\mathrm{H}$ & 1.99715100 & 4.54807800 & -1.92882400 \\
\hline $\mathrm{H}$ & -0.20834200 & 3.70708200 & -2.33073400 \\
\hline $\mathrm{C}$ & 1.76040400 & -1.33335400 & 0.38566200 \\
\hline $\mathrm{C}$ & 3.03352700 & -0.78688700 & 1.09205900 \\
\hline $\mathrm{C}$ & 1.93892900 & 0.86587200 & 0.09987900 \\
\hline $\mathrm{N}$ & 1.11252100 & -0.09751300 & -0.08818300 \\
\hline $\mathrm{H}$ & 1.08469200 & -1.82525300 & 1.10015700 \\
\hline $\mathrm{H}$ & 3.96282700 & -1.26287900 & 0.75473100 \\
\hline $\mathrm{H}$ & 1.39292400 & 5.19183000 & -0.36268600 \\
\hline $\mathrm{H}$ & 2.97362700 & -0.81827500 & 2.18844600 \\
\hline $\mathrm{O}$ & 2.22054700 & 3.28531700 & -0.29788400 \\
\hline $\mathrm{O}$ & 3.09220500 & 0.62614800 & 0.70125700 \\
\hline $\mathrm{C}$ & -1.06011500 & 4.19226800 & -0.40835300 \\
\hline $\mathrm{H}$ & -1.23234300 & 5.25100100 & -0.65564900 \\
\hline $\mathrm{H}$ & -1.98608900 & 3.63191000 & -0.59083100 \\
\hline $\mathrm{H}$ & -0.80505600 & 4.11903500 & 0.66053300 \\
\hline $\mathrm{C}$ & 2.04796800 & -2.27708400 & -0.77785200 \\
\hline $\mathrm{H}$ & 2.53100600 & -3.19423100 & -0.40706200 \\
\hline $\mathrm{H}$ & 1.10681800 & -2.54509600 & -1.27644100 \\
\hline $\mathrm{H}$ & 2.71801100 & -1.79905600 & -1.50982800 \\
\hline \multicolumn{4}{|c|}{ UB3LYP-D3(BJ)/def2-SVP-CPCM(THF) } \\
\hline \multicolumn{3}{|c|}{ Zero-point correction $=$} & 0.294154 (Hartree/Particle) \\
\hline \multicolumn{3}{|c|}{ Thermal correction to Energy= } & 0.314093 \\
\hline \multicolumn{3}{|c|}{ Thermal correction to Enthalpy= } & 0.315037 \\
\hline \multicolumn{3}{|c|}{ Thermal correction to Gibbs Free Energy= } & 0.243493 \\
\hline \multicolumn{3}{|c|}{ Sum of electronic and zero-point Energies $=$} & -4884.998406 \\
\hline \multicolumn{3}{|c|}{ Sum of electronic and thermal Energies $=$} & -4884.978467 \\
\hline \multicolumn{3}{|c|}{ Sum of electronic and thermal Enthalpies $=$} & -4884.977523 \\
\hline \multicolumn{3}{|c|}{ Sum of electronic and thermal Free Energies $=$} & -4885.049067 \\
\hline \multicolumn{4}{|c|}{$H F=-4885.2925602$} \\
\hline \multicolumn{4}{|c|}{$\begin{array}{l}\text { UB3LYP-D3(BJ)/def2-TZVPP-CPCM(THF)//UB3LYP-D3(BJ)/def2-SVP- } \\
\text { CPCM(THF) }\end{array}$} \\
\hline
\end{tabular}




\begin{tabular}{|c|c|c|c|}
\hline \multicolumn{4}{|l|}{${ }^{3} \mathbf{G}$} \\
\hline $\mathrm{Ni}$ & -1.12100400 & 1.57322200 & 0.18362300 \\
\hline $\mathrm{C}$ & -1.85411200 & 1.37899100 & -1.64337000 \\
\hline $\mathrm{C}$ & -2.97596000 & 1.07046600 & -4.24117700 \\
\hline $\mathrm{C}$ & -3.24426200 & 1.26609300 & -1.83747800 \\
\hline $\mathrm{C}$ & -1.04692200 & 1.32817200 & -2.79462900 \\
\hline $\mathrm{C}$ & -1.59026900 & 1.17451300 & -4.07693100 \\
\hline $\mathrm{C}$ & -3.80325100 & 1.11784700 & -3.11421200 \\
\hline $\mathrm{H}$ & -3.91220600 & 1.28637600 & -0.96962000 \\
\hline $\mathrm{H}$ & 0.04355200 & 1.40555500 & -2.70847400 \\
\hline $\mathrm{H}$ & -0.93172500 & 1.13572100 & -4.95020700 \\
\hline $\mathrm{H}$ & -4.88841600 & 1.03456900 & -3.22969500 \\
\hline $\mathrm{H}$ & -3.40664400 & 0.95147700 & -5.23911600 \\
\hline $\mathrm{Br}$ & -2.56926900 & 0.84629100 & 1.96790900 \\
\hline $\mathrm{C}$ & 1.53734200 & 2.22908200 & -0.50719700 \\
\hline $\mathrm{C}$ & 2.14213500 & 4.27073500 & -1.14122400 \\
\hline $\mathrm{C}$ & 0.63779000 & 4.25109600 & -0.75141600 \\
\hline $\mathrm{N}$ & 0.45037200 & 2.87150200 & -0.27307100 \\
\hline $\mathrm{H}$ & 2.33548100 & 4.60695800 & -2.16660700 \\
\hline $\mathrm{H}$ & 0.00219300 & 4.36756000 & -1.64542700 \\
\hline $\mathrm{C}$ & 0.66632800 & -1.18657000 & 0.32716600 \\
\hline $\mathrm{C}$ & 2.14234200 & -1.35726200 & -0.13977200 \\
\hline $\mathrm{C}$ & 1.54762700 & 0.78393200 & -0.23563300 \\
\hline $\mathrm{N}$ & 0.45503100 & 0.26006900 & 0.18219000 \\
\hline $\mathrm{H}$ & 0.56060500 & -1.43490800 & 1.39544100 \\
\hline $\mathrm{H}$ & 2.23641800 & -1.94452600 & -1.06393600 \\
\hline $\mathrm{H}$ & 2.75669500 & 4.84892200 & -0.43565700 \\
\hline $\mathrm{H}$ & 2.80687500 & -1.76645600 & 0.63094300 \\
\hline $\mathrm{O}$ & 2.56359000 & 2.87234300 & -1.04342900 \\
\hline $\mathrm{O}$ & 2.59781300 & 0.00391300 & -0.43953000 \\
\hline $\mathrm{C}$ & 0.23693200 & 5.28100000 & 0.29395100 \\
\hline $\mathrm{H}$ & 0.38933600 & 6.29947400 & -0.09422900 \\
\hline $\mathrm{H}$ & -0.82595500 & 5.16552700 & 0.55223700 \\
\hline $\mathrm{H}$ & 0.83619900 & 5.15960300 & 1.20941000 \\
\hline $\mathrm{C}$ & -0.36131400 & -1.97249900 & -0.47670000 \\
\hline $\mathrm{H}$ & -0.19379800 & -3.05362200 & -0.35766200 \\
\hline $\mathrm{H}$ & -1.37261500 & -1.72882400 & -0.12100100 \\
\hline $\mathrm{H}$ & -0.29819200 & -1.71471700 & -1.54467700 \\
\hline
\end{tabular}

UB3LYP-D3(BJ)/def2-SVP-CPCM(THF)

Zero-point correction=

0.292282 (Hartree/Particle)

Thermal correction to Energy=

0.313210

Thermal correction to Enthalpy=

0.314155 


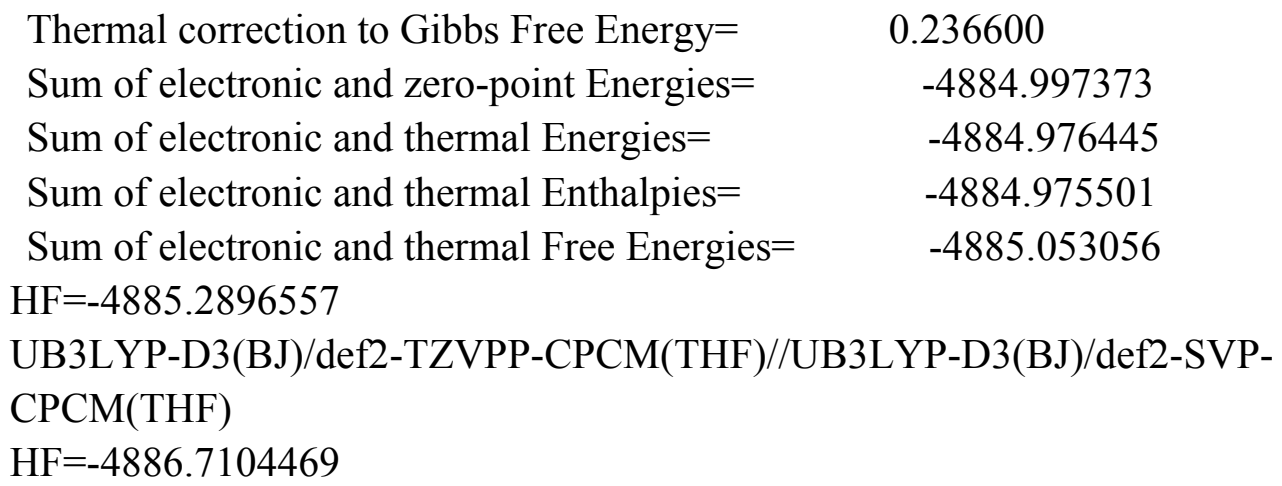

$\begin{array}{lrrr}{ }^{2} \mathbf{E}^{\boldsymbol{R}} & & & \\ \mathrm{Ni} & -0.71114700 & -0.02876000 & -0.12036400 \\ \mathrm{C} & -1.63492800 & 1.15436000 & -1.56325400 \\ \mathrm{C} & -0.22581300 & 3.48448800 & -0.90720300 \\ \mathrm{C} & -1.83453100 & 1.75529800 & -0.27967600 \\ \mathrm{C} & -0.75123300 & 1.73546500 & -2.51257700 \\ \mathrm{C} & -0.03468800 & 2.86500200 & -2.17044500 \\ \mathrm{C} & -1.11781000 & 2.95585300 & 0.00304800 \\ \mathrm{H} & -2.71771600 & 1.51481800 & 0.31535700 \\ \mathrm{H} & -0.64447700 & 1.27593500 & -3.49657700 \\ \mathrm{H} & 0.67184800 & 3.29277800 & -2.88524900 \\ \mathrm{H} & -1.30756300 & 3.46546500 & 0.95063700 \\ \mathrm{Br} & -3.03733200 & -0.02075900 & -2.23501600 \\ \mathrm{C} & -3.32849600 & 0.37064900 & 3.83990800 \\ \mathrm{H} & -2.31771300 & 0.79276500 & 3.95357900 \\ \mathrm{H} & -3.89523600 & 1.03027700 & 3.16141400 \\ \mathrm{H} & -3.81699800 & 0.40284100 & 4.82792900 \\ \mathrm{C} & -3.27863500 & -1.06610400 & 3.29575800 \\ \mathrm{C} & -2.62209200 & -1.07649900 & 1.88561300 \\ \mathrm{H} & -2.73799500 & -2.08559800 & 1.45738300 \\ \mathrm{H} & -3.23473500 & -0.41020700 & 1.25134800 \\ \mathrm{C} & -1.14782400 & -0.70395300 & 1.81899100 \\ \mathrm{C} & -4.71293900 & -1.60038100 & 3.15928200 \\ \mathrm{H} & -4.71283000 & -2.64104500 & 2.79524000 \\ \mathrm{H} & -5.24264800 & -1.58008100 & 4.12625900 \\ \mathrm{H} & -5.29397900 & -0.99452500 & 2.44403800 \\ \mathrm{H} & -0.87088200 & 0.17185100 & 2.41708300 \\ \mathrm{C} & -0.22566600 & -1.79916200 & 2.00981100 \\ \mathrm{O} & -0.41211000 & -2.99760700 & 1.79471500 \\ \mathrm{O} & 1.02148400 & -1.38151000 & 2.43343500 \\ \mathrm{C} & 1.99998600 & -2.38964300 & 2.64693100 \\ \mathrm{H} & 2.30973100 & -2.86431200 & 1.70342000 \\ \mathrm{H} & 2.86536000 & -1.88543700 & 3.09743200 \\ \mathrm{H} & -62768300 & -3.17232700 & 3.32517900\end{array}$




\begin{tabular}{|c|c|c|c|}
\hline $\mathrm{Ni}$ & -0.1251943 & 0.4790690 & -0.8701145 \\
\hline $\mathrm{C}$ & -0.9677233 & 1.6639030 & -2.3678395 \\
\hline $\mathrm{C}$ & 0.9903267 & 3.6498950 & -2.2907585 \\
\hline $\mathrm{C}$ & -0.8792743 & 2.5005410 & -1.2201245 \\
\hline $\mathrm{C}$ & -0.1047083 & 1.8336670 & -3.4771635 \\
\hline $\mathrm{C}$ & 0.8823197 & 2.8029390 & -3.4192155 \\
\hline $\mathrm{C}$ & 0.1157087 & 3.5148780 & -1.2268645 \\
\hline $\mathrm{H}$ & -1.6938783 & 2.5502870 & -0.4971625 \\
\hline $\mathrm{H}$ & -0.2162893 & 1.1905710 & -4.3510955 \\
\hline $\mathrm{H}$ & 1.5736687 & 2.9180440 & -4.2568725 \\
\hline $\mathrm{H}$ & 0.1641537 & 4.2062210 & -0.3828165 \\
\hline $\mathrm{Br}$ & -2.5957433 & 0.6264980 & -2.5905905 \\
\hline $\mathrm{H}$ & 1.7529787 & 4.4315690 & -2.2731465 \\
\hline $\mathrm{C}$ & 2.4925557 & -0.4455090 & -0.9999715 \\
\hline $\mathrm{C}$ & 4.1873067 & 0.6697220 & -0.0834745 \\
\hline $\mathrm{C}$ & 2.9442137 & 1.5930000 & -0.2165925 \\
\hline $\mathrm{N}$ & 1.9174757 & 0.6819920 & -0.7594655 \\
\hline $\mathrm{H}$ & 5.0760087 & 1.0422950 & -0.6080545 \\
\hline $\mathrm{H}$ & 3.1225207 & 2.3739410 & -0.9729275 \\
\hline $\mathrm{C}$ & -0.1561823 & -2.5795860 & -2.2064135 \\
\hline $\mathrm{C}$ & 0.9619507 & -3.6099220 & -1.8829145 \\
\hline $\mathrm{C}$ & 1.6802797 & -1.5402020 & -1.5123575 \\
\hline $\mathrm{N}$ & 0.4556377 & -1.2963570 & -1.8228385 \\
\hline $\mathrm{H}$ & -1.0340123 & -2.7449400 & -1.5629915 \\
\hline $\mathrm{H}$ & 1.2138007 & -4.2643320 & -2.7281845 \\
\hline $\mathrm{H}$ & 4.4411497 & 0.4528010 & 0.9650185 \\
\hline $\mathrm{H}$ & 0.7419437 & -4.2163830 & -0.9936635 \\
\hline $\mathrm{O}$ & 3.7871917 & -0.5844270 & -0.7083595 \\
\hline $\mathrm{O}$ & 2.1305617 & -2.7957070 & -1.5794175 \\
\hline $\mathrm{C}$ & 2.5125327 & 2.2363700 & 1.0927085 \\
\hline $\mathrm{H}$ & 3.3285437 & 2.8589430 & 1.4918975 \\
\hline $\mathrm{H}$ & 1.6370787 & 2.8790880 & 0.9304365 \\
\hline $\mathrm{H}$ & 2.2395087 & 1.4655900 & 1.8277875 \\
\hline $\mathrm{C}$ & -0.5880213 & -2.5678130 & -3.6695305 \\
\hline $\mathrm{H}$ & -1.0499343 & -3.5300490 & -3.9398785 \\
\hline $\mathrm{H}$ & -1.3192453 & -1.7664750 & -3.8445065 \\
\hline $\mathrm{H}$ & 0.2797967 & -2.4001880 & -4.3268765 \\
\hline $\mathrm{C}$ & -1.8513053 & 0.6287670 & 1.6527995 \\
\hline $\mathrm{H}$ & -2.4287003 & 1.2458140 & 0.9420385 \\
\hline $\mathrm{C}$ & -0.9107263 & -0.3025220 & 0.9100785 \\
\hline $\mathrm{H}$ & -1.4101993 & -1.1588300 & 0.4350265 \\
\hline $\mathrm{H}$ & -1.2413483 & 1.3315020 & 2.2444995 \\
\hline $\mathrm{C}$ & -2.8967683 & -0.0325210 & 2.5962515 \\
\hline $\mathrm{C}$ & -3.6452373 & 1.0875190 & 3.3358645 \\
\hline
\end{tabular}




$\begin{array}{lrrr}\mathrm{H} & -4.4319883 & 0.6796580 & 3.9921985 \\ \mathrm{H} & -2.9542513 & 1.6764320 & 3.9614195 \\ \mathrm{H} & -4.1257383 & 1.7791420 & 2.6237515 \\ \mathrm{C} & -2.2196493 & -0.9529710 & 3.6228485 \\ \mathrm{H} & -1.7226703 & -1.8022960 & 3.1292585 \\ \mathrm{H} & -1.4546543 & -0.4109980 & 4.1987215 \\ \mathrm{H} & -2.9628673 & -1.3626040 & 4.3273035 \\ \mathrm{C} & -3.9048163 & -0.8469420 & 1.7689625 \\ \mathrm{H} & -4.6759853 & -1.2966760 & 2.4161975 \\ \mathrm{H} & -4.4146493 & -0.2095100 & 1.0273535 \\ \mathrm{H} & -3.4087353 & -1.6651460 & 1.2236475 \\ \mathrm{C} & 0.2676307 & -0.7226280 & 1.6302125 \\ \mathrm{O} & 0.8273597 & -0.1562620 & 2.5681465 \\ \mathrm{O} & 0.8101887 & -1.8971860 & 1.1338385 \\ \mathrm{C} & 1.9821337 & -2.3714270 & 1.7793215 \\ \mathrm{H} & 1.7942397 & -2.5929390 & 2.8416115 \\ \mathrm{H} & 2.2764497 & -3.2869280 & 1.2503815 \\ \mathrm{H} & 2.8032787 & -1.6403840 & 1.7226035\end{array}$

UB3LYP-D3(BJ)/def2-SVP-CPCM(THF)

Zero-point correction=

Thermal correction to Energy=

Thermal correction to Enthalpy=

Thermal correction to Gibbs Free Energy=

Sum of electronic and zero-point Energies=

Sum of electronic and thermal Energies=

Sum of electronic and thermal Enthalpies=

Sum of electronic and thermal Free Energies= $\mathrm{HF}=-5349.3073465$

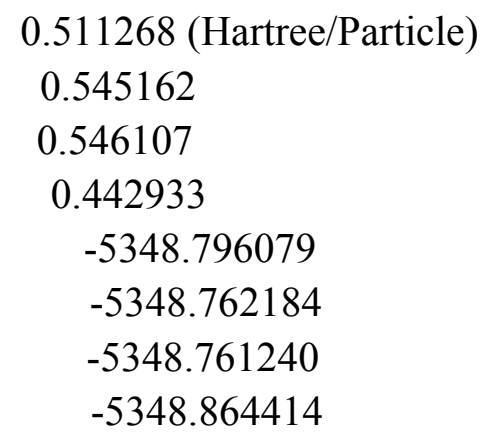

$\begin{array}{lrrr}{ }^{2} \mathbf{E}-\mathbf{T S}-\mathbf{F}^{\boldsymbol{R}} & & & \\ \mathrm{Ni} & -0.82109500 & -0.01116200 & -0.03852300 \\ \mathrm{C} & -1.31311000 & 1.30632800 & -1.45681600 \\ \mathrm{C} & 0.09510700 & 3.65992300 & -1.92928800 \\ \mathrm{C} & -1.48255800 & 2.39739600 & -0.58261500 \\ \mathrm{C} & -0.54776700 & 1.42024300 & -2.63185300 \\ \mathrm{C} & 0.17420900 & 2.58917500 & -2.84143400 \\ \mathrm{C} & -0.73559100 & 3.56246500 & -0.81304600 \\ \mathrm{H} & -2.16558700 & 2.32952500 & 0.26696100 \\ \mathrm{H} & -0.47187200 & 0.57721100 & -3.32013100 \\ \mathrm{H} & 0.81883700 & 2.67015300 & -3.72061000 \\ \mathrm{H} & -0.82731700 & 4.40236400 & -0.11968200 \\ \mathrm{Br} & -2.98488000 & -0.06199400 & -1.44777600 \\ \mathrm{C} & -3.25347900 & 0.11556900 & 4.18759100 \\ \mathrm{H} & -2.22937800 & 0.51118200 & 4.27577800\end{array}$


H

$\mathrm{H}$

C

C

$\mathrm{H}$

$\mathrm{H}$

C

C

$\mathrm{H}$

$\mathrm{H}$

$\mathrm{H}$

H

C

O

O

C

$\mathrm{H}$

$\mathrm{H}$

$\mathrm{H}$

C

$\mathrm{H}$

$\mathrm{H}$

$\mathrm{H}$

$\mathrm{H}$

C

C

C

N

$\mathrm{H}$

$\mathrm{H}$

C

C

C

N

$\mathrm{H}$

$\mathrm{H}$

$\mathrm{H}$

$\mathrm{H}$

O

O

C

$\mathrm{H}$

$\mathrm{H}$

$\mathrm{H}$

\begin{tabular}{rrr}
-3.85332900 & 0.85284100 & 3.62813900 \\
-3.67182100 & 0.04264400 & 5.20530300 \\
-3.26695600 & -1.25222700 & 3.48590400 \\
-2.71130600 & -1.11194300 & 2.03947100 \\
-2.88703300 & -2.06155500 & 1.50719800 \\
-3.34036100 & -0.36448200 & 1.52763900 \\
-1.23950600 & -0.75326900 & 1.90343400 \\
-4.71749200 & -1.75117600 & 3.39024700 \\
-4.76114300 & -2.74662300 & 2.91820100 \\
-5.18266600 & -1.82864200 & 4.38716900 \\
-5.33224200 & -1.06536600 & 2.78364800 \\
-0.92764700 & 0.09663100 & 2.52316400 \\
-0.31136300 & -1.85484100 & 2.01379800 \\
-0.49410700 & -3.03676100 & 1.72152800 \\
0.93766900 & -1.46046500 & 2.45940700 \\
1.91109400 & -2.48236300 & 2.62850200 \\
2.22770800 & -2.91076400 & 1.66536700 \\
2.77447300 & -2.00600200 & 3.11176900 \\
1.52926400 & -3.29663400 & 3.26270300 \\
-2.44111900 & -2.25211700 & 4.30964000 \\
-2.38416500 & -3.22998300 & 3.80831500 \\
-1.41044300 & -1.89343800 & 4.45467800 \\
-2.88840900 & -2.39702400 & 5.30731400 \\
0.66475600 & 4.57365600 & -2.11111000 \\
1.95651300 & -0.44672700 & -0.12751300 \\
3.40671700 & 0.90497300 & 0.88109400 \\
2.00240500 & 1.57312400 & 0.80682200 \\
1.18766400 & 0.54306500 & 0.14306700 \\
4.19199900 & 1.46670700 & 0.35953800 \\
2.02235600 & 2.45747900 & 0.14990100 \\
-0.29999200 & -2.94720500 & -1.44270900 \\
0.98958000 & -3.77979200 & -1.20397600 \\
1.35054900 & -1.64010800 & -0.71597700 \\
0.09471700 & -1.60473600 & -0.98601500 \\
-1.10129500 & -3.29450500 & -0.77524300 \\
1.33504900 & -4.32915400 & -2.08932500 \\
3.72504900 & 0.69340400 & 1.91205600 \\
0.89947200 & -4.45341900 & -0.34232000 \\
3.24505700 & -0.37557700 & 0.19961300 \\
2.00982800 & -2.78709200 & -0.86725700 \\
1.42250100 & 1.95807300 & 2.16164400 \\
2.06402700 & 2.70776900 & 2.64995300 \\
0.41889500 & 2.38859300 & 2.03565200 \\
1.34583600 & 1.06875400 & 2.80307500 \\
\hline
\end{tabular}




$\begin{array}{lrrr}\mathrm{C} & -0.77944200 & -2.92054900 & -2.88968800 \\ \mathrm{H} & -1.08090800 & -3.93022900 & -3.20850900 \\ \mathrm{H} & -1.64262400 & -2.24796400 & -2.98971900 \\ \mathrm{H} & 0.02096500 & -2.56727400 & -3.55902400\end{array}$

UB3LYP-D3(BJ)/def2-SVP-CPCM(THF)

Zero-point correction=

Thermal correction to Energy=

0.511327 (Hartree/Particle)

Thermal correction to Enthalpy=

Thermal correction to Gibbs Free Energy=

Sum of electronic and zero-point Energies=

Sum of electronic and thermal Energies=

Sum of electronic and thermal Enthalpies=

Sum of electronic and thermal Free Energies=

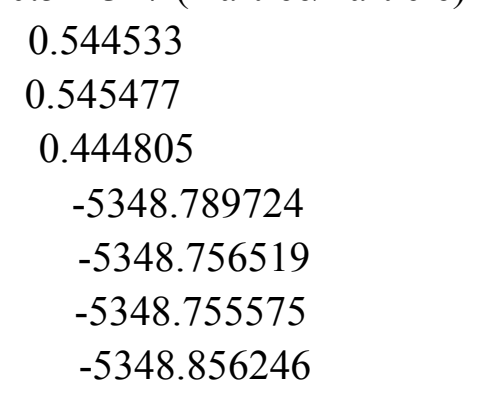

$\mathrm{HF}=-5349.3010513$

UB3LYP-D3(BJ)/def2-TZVPP-CPCM(THF)//UB3LYP-D3(BJ)/def2-SVPCPCM(THF)

$\mathrm{HF}=-5351.2370475$

$\begin{array}{lrrr}{ }^{2} \mathbf{E}-\mathbf{T S}-\mathbf{F}^{\boldsymbol{R}} \text {-conf2 } & & & \\ \mathrm{Ni} & -0.92244200 & 0.41923500 & -0.17564000 \\ \mathrm{C} & 0.12300900 & 2.04683500 & -0.82041900 \\ \mathrm{C} & 2.64991200 & 2.87904000 & 0.05620800 \\ \mathrm{C} & 0.22512300 & 2.94046000 & 0.26303800 \\ \mathrm{C} & 1.27473200 & 1.68143400 & -1.54789100 \\ \mathrm{C} & 2.53042900 & 2.06848500 & -1.08156600 \\ \mathrm{C} & 1.49194000 & 3.31955200 & 0.71125300 \\ \mathrm{H} & -0.66486800 & 3.26565200 & 0.79871400 \\ \mathrm{H} & 1.18416100 & 1.06183300 & -2.43941500 \\ \mathrm{H} & 3.42412200 & 1.73962600 & -1.61829100 \\ \mathrm{H} & 1.57108800 & 3.96564800 & 1.58960100 \\ \mathrm{Br} & -1.64914600 & 2.20765500 & -2.08447000 \\ \mathrm{H} & 3.63560200 & 3.18448600 & 0.41362200 \\ \mathrm{C} & 1.48649600 & -1.16383000 & 0.28513700 \\ \mathrm{C} & 3.03468300 & -0.51707500 & 1.73269900 \\ \mathrm{C} & 1.63334000 & -0.03697500 & 2.18621600 \\ \mathrm{~N} & 0.78795900 & -0.38814900 & 1.02458500 \\ \mathrm{H} & 3.66151300 & 0.29681200 & 1.34030200 \\ \mathrm{H} & 1.61051700 & 1.05447000 & 2.31005800 \\ \mathrm{C} & -0.53346300 & -1.93175100 & -2.63865200 \\ \mathrm{C} & 0.59992100 & -2.99323900 & -2.74124000 \\ \mathrm{C} & 0.89839300 & -1.70771200 & -0.94905000 \\ \mathrm{~N} & -0.16741900 & -1.17770200 & -1.42289000 \\ \mathrm{H} & -1.50480500 & -2.41557700 & -2.44681300 \\ \mathrm{H} & 1.22186000 & -2.87305800 & -3.63943900\end{array}$




$\begin{array}{lrrr}\mathrm{H} & 3.58129700 & -1.08419100 & 2.49592700 \\ \mathrm{H} & 0.24494800 & -4.02927700 & -2.67278500 \\ \mathrm{O} & 2.75008100 & -1.41833500 & 0.61669800 \\ \mathrm{O} & 1.44807800 & -2.73667400 & -1.58090400 \\ \mathrm{C} & 1.12583300 & -0.73024700 & 3.44773000 \\ \mathrm{H} & 1.72953900 & -0.41788000 & 4.31416800 \\ \mathrm{H} & 0.07266100 & -0.47447600 & 3.62653000 \\ \mathrm{H} & 1.21085400 & -1.82395700 & 3.34267500 \\ \mathrm{C} & -0.65447500 & -1.03456400 & -3.86271900 \\ \mathrm{H} & -1.41368100 & -0.25842900 & -3.69840300 \\ \mathrm{H} & 0.30485100 & -0.53960400 & -4.08030200 \\ \mathrm{H} & -0.94198700 & -1.63418500 & -4.73986000 \\ \mathrm{C} & -5.48566900 & 1.44159000 & 0.04109800 \\ \mathrm{H} & -4.87437000 & 2.34979800 & 0.15746500 \\ \mathrm{H} & -5.37739700 & 1.09661500 & -1.00066600 \\ \mathrm{H} & -6.53996600 & 1.72478200 & 0.19654700 \\ \mathrm{C} & -5.05835300 & 0.34627500 & 1.03127000 \\ \mathrm{C} & -3.58104000 & -0.06272900 & 0.76530300 \\ \mathrm{H} & -3.35119900 & -0.95067500 & 1.37782200 \\ \mathrm{H} & -3.53687800 & -0.39669800 & -0.28835600 \\ \mathrm{C} & -2.51714500 & 0.99438400 & 1.04778500 \\ \mathrm{C} & -5.94257900 & -0.89274600 & 0.82069200 \\ \mathrm{H} & -5.67961500 & -1.69093100 & 1.53437800 \\ \mathrm{H} & -7.00985600 & -0.65249200 & 0.95955500 \\ \mathrm{H} & -5.81845000 & -1.29807600 & -0.19746600 \\ \mathrm{H} & -2.77638000 & 1.99025100 & 0.67136200 \\ \mathrm{C} & -2.05505300 & 1.06145100 & 2.41516300 \\ \mathrm{H} & -2.00422700 & 0.15184100 & 3.24098200 \\ \mathrm{H} & -1.59835300 & 2.31698000 & 2.75275100 \\ \mathrm{C} & -0.99654200 & 2.46252800 & 4.02847500 \\ \mathrm{H} & -0.01731100 & 1.95851500 & 4.07360700 \\ \mathrm{H} & -0.85345500 & 3.54005000 & 4.18544300 \\ \mathrm{H} & -1.63362000 & 2.04794300 & 4.82396000 \\ \mathrm{H} & -5.23549200 & 0.85895800 & 2.46849500 \\ \mathrm{H} & -4.65721800 & 1.78024300 & 2.63727700 \\ \mathrm{H} & -6.29516600 & 1.08618100 & 2.67265300\end{array}$

UB3LYP-D3(BJ)/def2-SVP-CPCM(THF)

Zero-point correction=

Thermal correction to Energy=

0.512099 (Hartree/Particle)

0.545488

Thermal correction to Enthalpy=

0.546432

Thermal correction to Gibbs Free Energy=

Sum of electronic and zero-point Energies $=$

0.444184

$-5348.784424$

$-5348.751035$ 
Sum of electronic and thermal Enthalpies=

Sum of electronic and thermal Free Energies= $\mathrm{HF}=-5349.2965236$

\section{${ }^{2}$ E-TS-F ${ }^{R}$-conf3}

$\mathrm{Ni}$

$\mathrm{C}$

$\mathrm{C}$

C

$\mathrm{C}$

$\mathrm{C}$

$\mathrm{C}$

$\mathrm{H}$

$\mathrm{H}$

$\mathrm{H}$

$\mathrm{H}$

$\mathrm{Br}$

C

$\mathrm{H}$

$\mathrm{H}$

$\mathrm{H}$

C

$\mathrm{C}$

$\mathrm{H}$

$\mathrm{H}$

C

C

$\mathrm{H}$

$\mathrm{H}$

$\mathrm{H}$

$\mathrm{H}$

C

$\mathrm{O}$

$\mathrm{O}$

C

$\mathrm{H}$

$\mathrm{H}$

$\mathrm{H}$

C

$\mathrm{H}$

$\mathrm{H}$

$\mathrm{H}$

$\mathrm{H}$

C

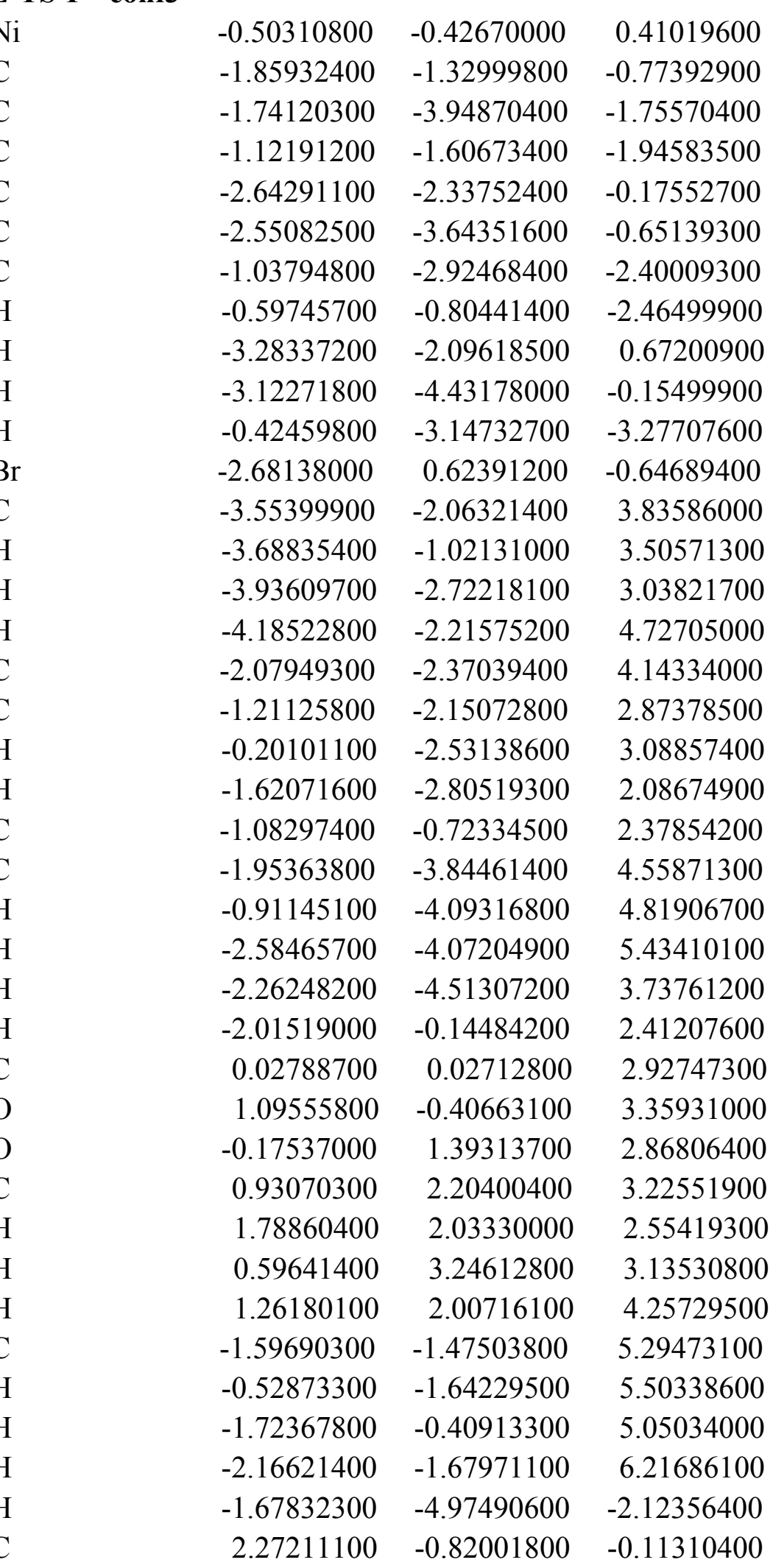

$-5348.750091$

$-5348.852339$ 


$\begin{array}{lrrr}\mathrm{C} & 3.39205500 & -2.58235400 & 0.64582100 \\ \mathrm{C} & 1.89650200 & -2.66945400 & 1.05846300 \\ \mathrm{~N} & 1.31005600 & -1.49257900 & 0.39885300 \\ \mathrm{H} & 4.09077100 & -2.59878700 & 1.49103300 \\ \mathrm{H} & 1.78041100 & -2.49474500 & 2.13990500 \\ \mathrm{C} & 0.69146700 & 2.18450800 & -1.37520100 \\ \mathrm{C} & 2.14427800 & 2.35456900 & -1.90518400 \\ \mathrm{C} & 1.95259700 & 0.45761800 & -0.75997300 \\ \mathrm{~N} & 0.73345900 & 0.85774700 & -0.73612300 \\ \mathrm{H} & -0.03077900 & 2.14460400 & -2.20485300 \\ \mathrm{H} & 2.63644900 & 3.26840600 & -1.54678000 \\ \mathrm{H} & 3.67772600 & -3.35139400 & -0.08767900 \\ \mathrm{H} & 2.22119400 & 2.29714200 & -2.99935700 \\ \mathrm{O} & 3.51538900 & -1.28212300 & -0.01128900 \\ \mathrm{O} & 2.87050900 & 1.21291500 & -1.35292900 \\ \mathrm{C} & 1.20095600 & -3.95929100 & 0.65140000 \\ \mathrm{H} & 1.65351200 & -4.81786500 & 1.17092200 \\ \mathrm{H} & 0.13510700 & -3.91468900 & 0.91240700 \\ \mathrm{H} & 1.28097300 & -4.11934300 & -0.43453400 \\ \mathrm{C} & 0.25964100 & 3.25528200 & -0.37886700 \\ \mathrm{H} & 0.21596700 & 4.23748400 & -0.87399600 \\ \mathrm{H} & -0.73451700 & 3.01981300 & 0.02530700 \\ \mathrm{H} & 0.97131100 & 3.31471300 & 0.45820800 \\ \mathrm{HB} 3 \mathrm{P}-\mathrm{B} 3) / \mathrm{H} & \mathrm{C} & \end{array}$

UB3LYP-D3(BJ)/def2-SVP-CPCM(THF)

Zero-point correction=

Thermal correction to Energy=

Thermal correction to Enthalpy=

Thermal correction to Gibbs Free Energy=

Sum of electronic and zero-point Energies=

Sum of electronic and thermal Energies=

Sum of electronic and thermal Enthalpies=

Sum of electronic and thermal Free Energies= $\mathrm{HF}=-5349.3003202$

${ }^{2} \mathbf{E}-\mathbf{T S}-\mathbf{F}^{S}$
$\mathrm{Ni}$
$\mathrm{C}$
$\mathrm{C}$
$\mathrm{C}$
$\mathrm{C}$
$\mathrm{C}$
$\mathrm{C}$
$\mathrm{H}$
$\mathrm{H}$

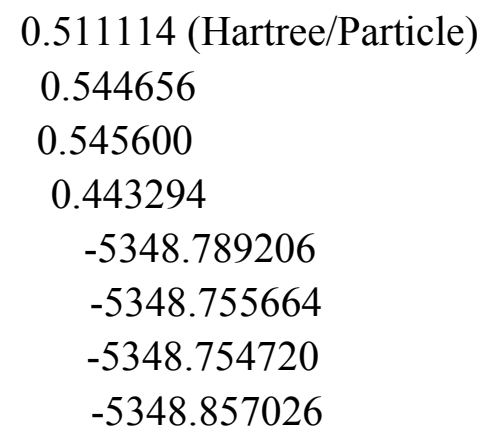


$\mathrm{H}$

$\mathrm{H}$

$\mathrm{Br}$

$\mathrm{H}$

C

C

C

$\mathrm{N}$

$\mathrm{H}$

$\mathrm{H}$

C

C

C

$\mathrm{N}$

$\mathrm{H}$

$\mathrm{H}$

$\mathrm{H}$

$\mathrm{H}$

$\mathrm{O}$

$\mathrm{O}$

C

$\mathrm{H}$

$\mathrm{H}$

$\mathrm{H}$

C

$\mathrm{H}$

$\mathrm{H}$

$\mathrm{H}$

C

$\mathrm{H}$

C

$\mathrm{H}$

$\mathrm{H}$

C

C

$\mathrm{H}$

$\mathrm{H}$

$\mathrm{H}$

C

$\mathrm{H}$

$\mathrm{H}$

$\mathrm{H}$

C

$\mathrm{H}$
1.04613900

$-0.37987200$

$-3.00526600$

1.12112000

1.87846600

3.24967300

2.07587800

1.19526200

4.24487800

2.41189300

$-0.46950400$

0.78137500

1.22234600

0.00587300

$-1.27691600$

1.16918900

3.13940600

0.62562700

3.11567000

1.79755100

1.38159700

2.09517300

0.55818100

0.97983800

$-0.99575800$

$-1.33562800$

$-1.84270000$

$-0.20604200$

$-2.22658400$

$-2.79843700$

$-1.46926500$

$-2.10418300$

$-1.49274900$

$-3.24181800$

$-3.77677900$

$-4.53713200$

$-2.96320200$

$-4.24092500$

$-2.57386800$

$-2.23190300$

$-1.69635300$

$-3.28296500$

$-4.41477600$

$-5.16225000$
2.42547900

4.43702000

0.19646400

4.37662900

$-0.65674600$

0.45104100

1.36258400

0.41269500

0.87910900

2.09956700

$-2.89001900$

$-3.80504900$

$-1.76402000$

$-1.60005100$

$-3.23429700$

$-4.15298900$

0.11494000

$-4.66355400$

$-0.73234900$

$-2.95321300$

2.08222500

2.74514700

2.69879700

1.34757600

$-2.77032700$

$-3.75201700$

$-2.07132300$

$-2.40186000$

0.30628700

0.95482000

$-0.75534200$

$-1.55396000$

0.96160100

$-0.19520600$

1.02951100

0.74044700

1.54997000

1.75156700

$-1.15665700$

$-2.07437700$

$-0.69215300$

$-1.45218900$

$-0.90938400$

$-1.24642500$
$-3.80857600$

$-0.26007200$

$-1.43914900$

$-2.25036800$

0.04564300

1.39060500

0.95882300

0.25021300

1.22543400

0.20801300

$-1.55098500$

$-1.41025500$

$-0.64336400$

$-1.02784800$

$-0.88512000$

$-2.37836400$

2.43280100

$-0.74450200$

0.54114000

$-0.80528100$

2.10373000

2.61764900

1.71808200

2.81528200

$-2.97577100$

$-3.34036300$

$-3.01150600$

$-3.64925600$

2.55302600

1.86556800

1.76988700

1.36439700

3.04972100

3.62090300

4.37954100

5.12409500

4.91144800

3.68706100

4.61496700

4.11225700

5.08910200

5.40628000

2.93035000

3.66760800 


$\begin{array}{lrrr}\mathrm{H} & -4.92127100 & -0.23834500 & 2.21665800 \\ \mathrm{H} & -4.07283600 & -1.79526700 & 2.37255000 \\ \mathrm{C} & -0.28301700 & -1.29541200 & 2.38802500 \\ \mathrm{O} & 0.44416400 & -0.77967100 & 3.23742400 \\ \mathrm{O} & 0.06678100 & -2.53138500 & 1.87337500 \\ \mathrm{C} & 1.21534400 & -3.15148700 & 2.43558300 \\ \mathrm{H} & 1.14834700 & -3.19683400 & 3.53292100 \\ \mathrm{H} & 1.25157100 & -4.16734700 & 2.02062700 \\ \mathrm{H} & 2.14062900 & -2.61912900 & 2.16524200\end{array}$

UB3LYP-D3(BJ)/def2-SVP-CPCM(THF)

Zero-point correction=

Thermal correction to Energy=

Thermal correction to Enthalpy=

Thermal correction to Gibbs Free Energy=

Sum of electronic and zero-point Energies=

Sum of electronic and thermal Energies=

Sum of electronic and thermal Enthalpies=

Sum of electronic and thermal Free Energies=

$\mathrm{HF}=-5349.3001745$

UB3LYP-D3(BJ)/def2-TZVPP-CPCM(THF)//UB3LYP-D3(BJ)/def2-SVPCPCM(THF)

$\mathrm{HF}=-5351.2363084$

\section{${ }^{2}$ E-TS-F ${ }^{S}$-conf2}

$\mathrm{Ni}$

$\mathrm{C}$

$\mathrm{C}$

$\mathrm{C}$

C

$\mathrm{C}$

C

$\mathrm{H}$

$\mathrm{H}$

$\mathrm{H}$

$\mathrm{H}$

$\mathrm{Br}$

$\mathrm{H}$

C

C

C

$\mathrm{N}$

$\mathrm{H}$

$\mathrm{H}$

C
$-0.81694700$

$-1.30099300$

0.24914600

$-1.31971800$

$-0.60828000$

0.18552800

$-0.50632700$

$-1.94785900$

$-0.64889400$

0.77206900

$-0.48670100$

$-3.07341200$

0.87071400

1.88485700

3.55292300

2.20495700

1.23100800

4.30967800

2.18710700

$-0.72142000$
0.17077800

0.07939500

1.58599700

$-1.26754800$

$3.84040500-1.79889600$

$2.66731800-0.36362000$

$1.67059400 \quad-2.49089400$

$2.78524900-2.73081900$

$3.77908100-0.62797700$

$2.63301700 \quad 0.52930000$

$0.84237400 \quad-3.20016100$

$2.83700000-3.65184700$

$4.60840500 \quad 0.08365600$

$0.38451600-1.18907400$

$4.71396700 \quad-2.00608300$

$-0.51124000-0.30615700$

$\begin{array}{ll}0.77229700 & 0.41595300\end{array}$

$\begin{array}{ll}1.54627000 & 0.48678200\end{array}$

$0.53951900 \quad 0.03722800$

$1.25564600-0.21422400$

$2.37124800 \quad-0.24327700$

$-2.84634400 \quad-1.22027700$ 


\begin{tabular}{|c|c|c|c|}
\hline $\mathrm{C}$ & 0.53062700 & -3.77103500 & -1.20207100 \\
\hline $\mathrm{C}$ & 1.11612500 & -1.66867900 & -0.75954000 \\
\hline $\mathrm{N}$ & -0.15451400 & -1.52595400 & -0.89631500 \\
\hline $\mathrm{H}$ & -1.42034100 & -3.09481500 & -0.40544500 \\
\hline $\mathrm{H}$ & 0.72138800 & -4.26086000 & -2.16720300 \\
\hline $\mathrm{H}$ & 3.97664100 & 0.56390900 & 1.40967100 \\
\hline $\mathrm{H}$ & 0.50769400 & -4.52505800 & -0.40391500 \\
\hline $\mathrm{O}$ & 3.21225000 & -0.50963200 & -0.19418000 \\
\hline $\mathrm{O}$ & 1.65212800 & -2.87583600 & -0.92859600 \\
\hline $\mathrm{C}$ & 1.85678900 & 2.08840900 & 1.86590300 \\
\hline $\mathrm{H}$ & 2.60571900 & 2.82873900 & 2.18647600 \\
\hline $\mathrm{H}$ & 0.87271800 & 2.57687300 & 1.84131300 \\
\hline $\mathrm{H}$ & 1.82323100 & 1.27638900 & 2.60692500 \\
\hline $\mathrm{C}$ & -1.46155800 & -2.83410500 & -2.55246800 \\
\hline $\mathrm{H}$ & -1.86589900 & -3.83515400 & -2.76855900 \\
\hline $\mathrm{H}$ & -2.29464000 & -2.11917500 & -2.51805600 \\
\hline $\mathrm{H}$ & -0.78496900 & -2.54625800 & -3.37293700 \\
\hline $\mathrm{C}$ & 0.05786900 & -1.66351500 & 2.32329200 \\
\hline $\mathrm{H}$ & 1.03287000 & -1.30706000 & 1.94911900 \\
\hline $\mathrm{C}$ & -1.05354300 & -0.68287300 & 2.00015000 \\
\hline $\mathrm{H}$ & -1.07757600 & 0.20532900 & 2.64616300 \\
\hline $\mathrm{H}$ & -0.14838200 & -2.60076800 & 1.78243400 \\
\hline $\mathrm{C}$ & 0.27298700 & -2.02032600 & 3.82127700 \\
\hline $\mathrm{C}$ & 1.32109000 & -3.14183900 & 3.89509900 \\
\hline $\mathrm{H}$ & 1.55254900 & -3.40819900 & 4.93979800 \\
\hline $\mathrm{H}$ & 0.96145900 & -4.05103500 & 3.38558300 \\
\hline $\mathrm{H}$ & 2.26230200 & -2.83516000 & 3.40867800 \\
\hline $\mathrm{C}$ & -1.03557100 & -2.50486900 & 4.46411400 \\
\hline $\mathrm{H}$ & -1.79087100 & -1.70434400 & 4.48245500 \\
\hline $\mathrm{H}$ & -1.46253500 & -3.35013500 & 3.90331700 \\
\hline $\mathrm{H}$ & -0.86278900 & -2.82906300 & 5.50388200 \\
\hline $\mathrm{C}$ & 0.79687600 & -0.79571300 & 4.58767000 \\
\hline $\mathrm{H}$ & 0.94771100 & -1.03060900 & 5.65430200 \\
\hline $\mathrm{H}$ & 1.76454600 & -0.45966000 & 4.17923100 \\
\hline $\mathrm{H}$ & 0.09235500 & 0.04836500 & 4.52698200 \\
\hline $\mathrm{C}$ & -2.36708200 & -1.27390200 & 1.85945500 \\
\hline $\mathrm{O}$ & -2.63813300 & -2.41003000 & 1.46778700 \\
\hline $\mathrm{O}$ & -3.38090100 & -0.40769900 & 2.18954100 \\
\hline $\mathrm{C}$ & -4.70223100 & -0.87570900 & 1.96990200 \\
\hline $\mathrm{H}$ & -4.92938600 & -1.75142600 & 2.59916300 \\
\hline $\mathrm{H}$ & -5.37388400 & -0.04768300 & 2.23344300 \\
\hline $\mathrm{H}$ & -4.85798700 & -1.15762200 & 0.91739100 \\
\hline
\end{tabular}

UB3LYP-D3(BJ)/def2-SVP-CPCM(THF)

Zero-point correction=

0.511041 (Hartree/Particle) 
Thermal correction to Energy=

Thermal correction to Enthalpy=

Thermal correction to Gibbs Free Energy=

Sum of electronic and zero-point Energies=

Sum of electronic and thermal Energies=

Sum of electronic and thermal Enthalpies=

Sum of electronic and thermal Free Energies=

$\mathrm{HF}=-5349.297156$

\section{${ }^{2}$ E-TS-F ${ }^{S}$-conf3}

$\mathrm{Ni}$

$\mathrm{C}$

C

$\mathrm{C}$

$\mathrm{C}$

C

$\mathrm{C}$

$\mathrm{H}$

$\mathrm{H}$

$\mathrm{H}$

$\mathrm{H}$

$\mathrm{Br}$

$\mathrm{H}$

$\mathrm{C}$

$\mathrm{C}$

C

$\mathrm{N}$

$\mathrm{H}$

$\mathrm{H}$

C

C

C

$\mathrm{N}$

$\mathrm{H}$

$\mathrm{H}$

$\mathrm{H}$

$\mathrm{H}$

$\mathrm{O}$

$\mathrm{O}$

C

$\mathrm{H}$

$\mathrm{H}$

$\mathrm{H}$

C

$\begin{array}{lrrr}\mathrm{Ni} & -0.88921500 & 0.32365200 & -0.17636200 \\ \mathrm{C} & -0.21389300 & 2.09469200 & -0.93191200 \\ \mathrm{C} & 2.05143300 & 3.50493700 & -0.06878200 \\ \mathrm{C} & -0.36091600 & 3.20496900 & -0.07458600 \\ \mathrm{C} & 1.05444200 & 1.79192700 & -1.47514600 \\ \mathrm{C} & 2.17976200 & 2.46980000 & -1.00284400 \\ \mathrm{H} & 0.77338600 & 3.87674700 & 0.37533300 \\ \mathrm{H} & -1.35209400 & 3.50344900 & 0.26228100 \\ \mathrm{H} & 1.15607600 & 1.00761800 & -2.22331400 \\ \mathrm{H} & 3.16606700 & 2.19313000 & -1.38456100 \\ \mathrm{Br} & 0.65561200 & 4.70388700 & 1.08035900 \\ \mathrm{H} & -1.87664600 & 1.76554300 & -2.25393800 \\ \mathrm{C} & 2.93503600 & 4.03837000 & 0.28781700 \\ \mathrm{C} & 1.45742200 & -1.32267300 & 0.33562400 \\ \mathrm{C} & 2.81692700 & -0.82838100 & 2.01126400 \\ \mathrm{~N} & 1.39631200 & -0.28933700 & 2.29085300 \\ \mathrm{H} & 0.73794700 & -0.48179100 & 0.98039500 \\ \mathrm{H} & 3.52580600 & -0.04436100 & 1.70586800 \\ \mathrm{C} & 1.40507000 & 0.78155500 & 2.53522900 \\ \mathrm{C} & -0.23773000 & -1.98838900 & -2.80670900 \\ \mathrm{C} & 0.76857100 & -3.17057100 & -2.72093300 \\ \mathrm{~N} & 0.98361800 & -1.83341700 & -0.95923600 \\ \mathrm{H} & -0.42731300 & -0.91205100 & 3.30402200 \\ \mathrm{H} & -1.27483200 & -2.35570900 & -2.83341200 \\ \mathrm{H} & 1.43938700 & -3.23998500 & -3.58705900 \\ \mathrm{H} & 3.24053100 & -1.42693300 & 2.82619700 \\ \mathrm{O} & 0.28801300 & -4.14280200 & -2.54455500 \\ \mathrm{O} & 2.61977600 & -1.70812200 & 0.85734400 \\ \mathrm{C} & 1.58189600 & -2.86424100 & -1.54838500 \\ \mathrm{H} & 0.65445400 & -1.09111300 & 3.36118800 \\ \mathrm{H} & 1.02605200 & -0.82493100 & 4.36245100 \\ \mathrm{C} & -2.16941300 & 3.20460200 \\ & -1.06435800 & -3.99585800\end{array}$

0.544433

0.545378

0.443586

$-5348.786115$

$-5348.752723$

$-5348.751778$

$-5348.853570$ 


$\begin{array}{lrrr}\mathrm{H} & -0.66427600 & -0.19563600 & -3.95261800 \\ \mathrm{H} & 1.04700400 & -0.70491200 & -4.00128000 \\ \mathrm{H} & -0.17654700 & -1.60791700 & -4.93555600 \\ \mathrm{C} & -1.80017500 & 1.58179600 & 2.47245800 \\ \mathrm{H} & -0.96434100 & 2.25199400 & 2.21045200 \\ \mathrm{C} & -2.32173100 & 0.86442400 & 1.24088700 \\ \mathrm{H} & -2.99938500 & 1.46474900 & 0.62055100 \\ \mathrm{H} & -1.37899100 & 0.83110700 & 3.15591000 \\ \mathrm{C} & -2.82783700 & 2.42594000 & 3.27715500 \\ \mathrm{C} & -2.13972900 & 2.91758000 & 4.56041800 \\ \mathrm{H} & -2.80809000 & 3.56340700 & 5.15407100 \\ \mathrm{H} & -1.83773900 & 2.06850200 & 5.19565000 \\ \mathrm{H} & -1.23267300 & 3.49858900 & 4.32342800 \\ \mathrm{C} & -4.05436400 & 1.57970000 & 3.65172600 \\ \mathrm{H} & -4.60824500 & 1.26248300 & 2.75472600 \\ \mathrm{H} & -3.75565000 & 0.67021800 & 4.19513300 \\ \mathrm{H} & -4.74700700 & 2.15453300 & 4.28901300 \\ \mathrm{C} & -3.27897400 & 3.64468100 & 2.45613700 \\ \mathrm{H} & -4.02232000 & 4.24176300 & 3.00998700 \\ \mathrm{H} & -2.42588900 & 4.30444700 & 2.22645400 \\ \mathrm{H} & -3.73914400 & 3.34078900 & 1.50275600 \\ \mathrm{C} & -2.86628100 & -0.45923300 & 1.47284600 \\ \mathrm{O} & -2.60029500 & -1.23871400 & 2.38574000 \\ \mathrm{O} & -3.73792000 & -0.85009500 & 0.47716700 \\ \mathrm{C} & -4.19167400 & -2.19228200 & 0.53322000 \\ \mathrm{H} & -4.69468500 & -2.41108000 & 1.48814400 \\ \mathrm{H} & -4.89968900 & -2.31781500 & -0.29698300 \\ \mathrm{H} & -3.35754400 & -2.90436500 & 0.41564400 \\ \mathrm{H} & -1300\end{array}$

UB3LYP-D3(BJ)/def2-SVP-CPCM(THF)

Zero-point correction=
0.512125 (Hartree/Particle) 0.545526
0.546471
0.443511
$-5348.782016$
$-5348.748615$
$-5348.747670$
$-5348.850630$

Thermal correction to Enthalpy=

Sum of electronic and thermal Free Energies=

$\mathrm{HF}=-5349.294141$

\section{${ }^{2}$ E-TS-F ${ }^{S}$-conf4}

$\mathrm{Ni}$

$\mathrm{C}$

$\mathrm{C}$

$\mathrm{C}$

$\begin{array}{ll}-0.39384000 & -0.63620900 \\ -1.81117900 & -1.32068100 \\ -1.83468200 & -3.80669700 \\ -1.26428400 & -1.44481000\end{array}$

0.45910400

$-0.80604600$

$-2.07917400$

$-2.09920800$ 


\begin{tabular}{|c|c|c|c|}
\hline $\mathrm{C}$ & -2.45823300 & -2.40899500 & -0.19070400 \\
\hline $\mathrm{C}$ & -2.43748300 & -3.65268300 & -0.82433800 \\
\hline $\mathrm{C}$ & -1.25246600 & -2.69918700 & -2.70982500 \\
\hline $\mathrm{H}$ & -0.82705600 & -0.57751600 & -2.59409700 \\
\hline $\mathrm{H}$ & -2.92084200 & -2.28111400 & 0.78681000 \\
\hline $\mathrm{H}$ & -2.90197100 & -4.50927500 & -0.32927600 \\
\hline $\mathrm{H}$ & -0.79042200 & -2.80860000 & -3.69436800 \\
\hline $\mathrm{Br}$ & -2.57632200 & 0.58192700 & -0.31380400 \\
\hline $\mathrm{H}$ & -1.82906900 & -4.78233900 & -2.56968400 \\
\hline $\mathrm{C}$ & 2.29220300 & -1.17040800 & -0.29679800 \\
\hline $\mathrm{C}$ & 3.35172700 & -3.04462100 & 0.26612100 \\
\hline $\mathrm{C}$ & 1.85838900 & -3.11523700 & 0.69550700 \\
\hline $\mathrm{N}$ & 1.32731500 & -1.81759200 & 0.24511300 \\
\hline $\mathrm{H}$ & 4.05649900 & -3.09943200 & 1.10596500 \\
\hline $\mathrm{H}$ & 1.73676700 & -3.14377100 & 1.78835700 \\
\hline $\mathrm{C}$ & 0.83906600 & 2.02914600 & -1.16306400 \\
\hline $\mathrm{C}$ & 2.26536100 & 2.16702900 & -1.76601000 \\
\hline $\mathrm{C}$ & 2.01708700 & 0.17525000 & -0.80931500 \\
\hline $\mathrm{N}$ & 0.82170700 & 0.63158000 & -0.69645800 \\
\hline $\mathrm{H}$ & 0.06801300 & 2.14948700 & -1.93895700 \\
\hline $\mathrm{H}$ & 2.83040800 & 3.02037500 & -1.36882100 \\
\hline $\mathrm{H}$ & 3.62022600 & -3.79554000 & -0.49088600 \\
\hline $\mathrm{H}$ & 2.27279000 & 2.19603700 & -2.86435300 \\
\hline $\mathrm{O}$ & 3.49956200 & -1.72645500 & -0.35023700 \\
\hline $\mathrm{O}$ & 2.95362000 & 0.94586500 & -1.35370400 \\
\hline $\mathrm{C}$ & 1.08077200 & -4.27706500 & 0.09437000 \\
\hline $\mathrm{H}$ & 1.50682800 & -5.23285300 & 0.43682300 \\
\hline $\mathrm{H}$ & 0.03176500 & -4.22809100 & 0.41365400 \\
\hline $\mathrm{H}$ & 1.11037000 & -4.24899400 & -1.00565900 \\
\hline $\mathrm{C}$ & 0.54983900 & 2.98204400 & -0.00691900 \\
\hline $\mathrm{H}$ & 0.55745600 & 4.02415100 & -0.36127500 \\
\hline $\mathrm{H}$ & -0.43703100 & 2.76479600 & 0.42527200 \\
\hline $\mathrm{H}$ & 1.30849200 & 2.87401300 & 0.78398800 \\
\hline $\mathrm{C}$ & 0.61682800 & -0.09347800 & 3.03116700 \\
\hline $\mathrm{H}$ & 0.96755300 & 0.70471100 & 2.35085500 \\
\hline $\mathrm{C}$ & -0.67366900 & -0.72567300 & 2.51966000 \\
\hline $\mathrm{H}$ & -1.57350000 & -0.11971900 & 2.68217000 \\
\hline $\mathrm{H}$ & 1.40331600 & -0.86519500 & 3.01546100 \\
\hline $\mathrm{C}$ & 0.57569500 & 0.53154500 & 4.45433700 \\
\hline $\mathrm{C}$ & 1.99986400 & 0.97799600 & 4.82023700 \\
\hline $\mathrm{H}$ & 2.02657600 & 1.46754800 & 5.80803200 \\
\hline $\mathrm{H}$ & 2.68746900 & 0.11661700 & 4.85081300 \\
\hline $\mathrm{H}$ & 2.39197700 & 1.69416800 & 4.07867600 \\
\hline $\mathrm{C}$ & 0.08222300 & -0.49266900 & 5.48727800 \\
\hline
\end{tabular}




\begin{tabular}{|c|c|c|c|}
\hline $\mathrm{H}$ & -0.95788600 & -0.79136000 & 5.28603900 \\
\hline $\mathrm{H}$ & 0.69885300 & -1.40430100 & 5.46647300 \\
\hline $\mathrm{H}$ & 0.12013300 & -0.07010200 & 6.50525100 \\
\hline $\mathrm{C}$ & -0.35172400 & 1.75709700 & 4.46235200 \\
\hline $\mathrm{H}$ & -0.37371200 & 2.23080300 & 5.45789100 \\
\hline $\mathrm{H}$ & -0.01164100 & 2.51371100 & 3.73533800 \\
\hline $\mathrm{H}$ & -1.38483100 & 1.48003900 & 4.20089700 \\
\hline $\mathrm{C}$ & -0.88734900 & -2.09898600 & 2.90117000 \\
\hline $\mathrm{O}$ & -0.04253800 & -2.96243200 & 3.14216400 \\
\hline $\mathrm{O}$ & -2.22583700 & -2.43568600 & 2.96310300 \\
\hline $\mathrm{C}$ & -2.51539300 & -3.80559300 & 3.19248900 \\
\hline $\mathrm{H}$ & -2.06572300 & -4.16147400 & 4.13219700 \\
\hline $\mathrm{H}$ & -3.60922300 & -3.88769300 & 3.24839600 \\
\hline $\mathrm{H}$ & -2.14043500 & -4.43996700 & 2.37257000 \\
\hline \multicolumn{4}{|c|}{ UB3LYP-D3(BJ)/def2-SVP-CPCM(THF) } \\
\hline \multicolumn{3}{|c|}{ Zero-point correction $=$} & 0.510891 (Hartree/Particle) \\
\hline \multicolumn{3}{|c|}{ Thermal correction to Energy= } & 0.544513 \\
\hline \multicolumn{3}{|c|}{ Thermal correction to Enthalpy= } & 0.545457 \\
\hline \multicolumn{3}{|c|}{ Thermal correction to Gibbs Free Energy= } & 0.442158 \\
\hline \multicolumn{3}{|c|}{ Sum of electronic and zero-point Energies $=$} & -5348.789902 \\
\hline \multicolumn{3}{|c|}{ Sum of electronic and thermal Energies $=$} & -5348.756280 \\
\hline \multicolumn{3}{|c|}{ Sum of electronic and thermal Enthalpies= } & -5348.755336 \\
\hline \multicolumn{3}{|c|}{ Sum of electronic and thermal Free Energies= } & -5348.858635 \\
\hline \multicolumn{4}{|c|}{$H F=-5349.3007929$} \\
\hline \multicolumn{4}{|l|}{${ }^{2} \mathbf{F}^{R}$} \\
\hline $\mathrm{Ni}$ & -0.71668400 & 0.00420100 & -0.18905700 \\
\hline $\mathrm{C}$ & -1.43494300 & 1.68778200 & -0.76461000 \\
\hline $\mathrm{C}$ & -2.17472000 & 4.13709100 & -1.93997800 \\
\hline $\mathrm{C}$ & -2.41252500 & 2.48619600 & -0.17292200 \\
\hline C & -0.84479700 & 2.10809500 & -1.96533800 \\
\hline $\mathrm{C}$ & -1.20873600 & 3.32756300 & -2.54713700 \\
\hline $\mathrm{C}$ & -2.77855200 & 3.70783100 & -0.75601800 \\
\hline $\mathrm{H}$ & -2.90497900 & 2.18102200 & 0.74688600 \\
\hline $\mathrm{H}$ & -0.09504200 & 1.48902700 & -2.46503500 \\
\hline $\mathrm{H}$ & -0.73400900 & 3.64184100 & -3.48068400 \\
\hline $\mathrm{H}$ & -3.54231700 & 4.32380000 & -0.27329900 \\
\hline $\mathrm{Br}$ & -2.40524000 & -1.10939800 & -1.56719500 \\
\hline $\mathrm{C}$ & -3.76753000 & 1.69956000 & 3.17604600 \\
\hline $\mathrm{H}$ & -2.89631200 & 2.36776500 & 3.09253200 \\
\hline $\mathrm{H}$ & -4.41698900 & 1.87185500 & 2.30283100 \\
\hline $\mathrm{H}$ & -4.33103300 & 2.00254100 & 4.07329500 \\
\hline $\mathrm{C}$ & -3.34734600 & 0.22467400 & 3.27700800 \\
\hline $\mathrm{C}$ & -2.55793600 & -0.21627900 & 2.00513300 \\
\hline
\end{tabular}


H

$\mathrm{H}$

C

C

$\mathrm{H}$

$\mathrm{H}$

$\mathrm{H}$

$\mathrm{H}$

C

O

O

C

$\mathrm{H}$

$\mathrm{H}$

$\mathrm{H}$

C

$\mathrm{H}$

$\mathrm{H}$

$\mathrm{H}$

$\mathrm{H}$

C

C

C

N

$\mathrm{H}$

$\mathrm{H}$

C

C

C

$\mathrm{N}$

$\mathrm{H}$

$\mathrm{H}$

$\mathrm{H}$

$\mathrm{H}$

O

O

C

$\mathrm{H}$

$\mathrm{H}$

$\mathrm{H}$

C

$\mathrm{H}$

$\mathrm{H}$

$\mathrm{H}$

$\begin{array}{rrr}-2.44813900 & -1.31152500 & 2.03415700 \\ -3.19649100 & -0.00504300 & 1.13475300 \\ -1.18057800 & 0.38904900 & 1.78868300 \\ -4.61174500 & -0.64749800 & 3.34782000 \\ -4.35074500 & -1.71198900 & 3.46339000 \\ -5.24497600 & -0.35871100 & 4.20260700 \\ -5.21418200 & -0.54586700 & 2.43034900 \\ -1.13729200 & 1.47805200 & 1.86972300 \\ -0.12550500 & -0.21794200 & 2.62574800 \\ -0.11490500 & -1.34821400 & 3.08582100 \\ 0.92203900 & 0.62249100 & 2.83701600 \\ 2.00220500 & 0.11845000 & 3.62399400 \\ 2.48645700 & -0.73684000 & 3.13027100 \\ 2.71702300 & 0.94422800 & 3.72179300 \\ 1.64888200 & -0.19977400 & 4.61534400 \\ -2.53010200 & 0.02821300 & 4.56331400 \\ -2.17140600 & -1.00654500 & 4.65703100 \\ -1.65114300 & 0.69163700 & 4.58939600 \\ -3.14696600 & 0.26646300 & 5.44526000 \\ -2.45757300 & 5.09137900 & -2.39081000 \\ 2.10403700 & -0.16838400 & 0.18342900 \\ 3.46967000 & 1.58194800 & 0.10201700 \\ 2.02388500 & 2.00612000 & -0.29264500 \\ 1.28724600 & 0.72673900 & -0.22930600 \\ 4.19761500 & 1.68191900 & -0.71322300 \\ 1.99403300 & 2.36346700 & -1.33282100 \\ 0.04887700 & -3.12470300 & 0.62311600 \\ 1.37460300 & -3.54894500 & 1.31003900 \\ 1.57757200 & -1.51466700 & 0.44522900 \\ 0.32915300 & -1.72434400 & 0.24153100 \\ -0.77193300 & -3.11096700 & 1.35214100 \\ 1.79160100 & -4.48788300 & 0.92566800 \\ 3.84150700 & 2.09434400 & 0.99929800 \\ 1.30089400 & -3.57483800 & 2.40406900 \\ 3.36329600 & 0.15830800 & 0.42441400 \\ 2.31688700 & -2.47551300 & 0.97294000 \\ 1.42180200 & 3.06622000 & 0.62293400 \\ 2.03258700 & 3.98085200 & 0.57471500 \\ 0.40006000 & 3.31126200 & 0.30611700 \\ 1.39101100 & 2.70334700 & 1.65913800 \\ -0.31831100 & -3.97679900 & -0.58650600 \\ -0.51227900 & -5.01130000 & -0.26344700 \\ -1.21606900 & -3.57403900 & -1.07295600 \\ 0.50492300 & -3.98794300 & -1.31833800\end{array}$


UB3LYP-D3(BJ)/def2-SVP-CPCM(THF)

$\begin{array}{lc}\text { Zero-point correction }= & 0.515294 \text { (Hartree/Particle) } \\ \text { Thermal correction to Energy }= & 0.548283 \\ \text { Thermal correction to Enthalpy }= & 0.549227 \\ \text { Thermal correction to Gibbs Free Energy= } & 0.450026 \\ \text { Sum of electronic and zero-point Energies }= & -5348.828533 \\ \text { Sum of electronic and thermal Energies }= & -5348.795544 \\ \text { Sum of electronic and thermal Enthalpies }= & -5348.794600 \\ \text { Sum of electronic and thermal Free Energies }= & -5348.893801 \\ \text { HF=-5349.3438272 }\end{array}$

UB3LYP-D3(BJ)/def2-TZVPP-CPCM(THF)//UB3LYP-D3(BJ)/def2-SVPCPCM(THF)

$\mathrm{HF}=-5351.279108$

\section{${ }^{2} \mathrm{~F}^{R}-\operatorname{conf3}$}

$\mathrm{Ni}$

C

$-0.49816600$

C

$-1.91566800$

$-0.63607300$

$-0.29100300$

C

$-3.99759100$

$-1.94067700$

$-0.40476400$

C

$-1.62658900$

$-3.81313300$

$-0.63762600$

$\mathrm{C}$

$-3.24922900$

$-3.29630700$

$-0.58656800$

$-4.28684200$

$-1.52219400$

$-0.35287600$

C

$-2.66411600$

$-2.45549300$

$-0.46888800$

$\mathrm{H}$

$-0.59114500$

$-4.23026400$

$-0.69955700$

$\mathrm{H}$

$-3.49505300$

$-3.63803700$

$-0.64013900$

$\mathrm{H}$

$-5.32555600$

$-0.46532500$

$-0.21365400$

$\mathrm{H}$

$-2.42506300$

$-2.11738500$

$-0.41924300$

$\mathrm{Br}$

$-5.28805800$

$-0.83963400$

C

$-0.50347700$

$-0.42141700$

$-2.73792900$

$\mathrm{H}$

$-2.99514000$

$-2.52934500$

3.22922300

$-3.39225600$

$-1.58024200$

2.83853900

$\mathrm{H}$

$-3.11944800$

$-3.28680200$

2.43925200

$\mathrm{H}$

$-3.61843900$

$-2.82194100$

4.09009200

C

C

$-1.52110300$

$-2.40119100$

3.64633900

$\mathrm{H}$

$-0.63948000$

$-2.07836100$

2.41007800

0.41619600

$-2.12025400$

2.71611300

$\mathrm{H}$

$-0.79320300$

$-2.88831900$

1.68752400

C

$-0.88589100$

$-0.71884100$

1.76919700

C

$-1.04220800$

$-3.74770100$

4.21209600

$\mathrm{H}$

0.01158100

$-3.69051700$

4.53072800

$\mathrm{H}$

$-1.64459100$

$-4.04898600$

5.08465200

$\mathrm{H}$

$-1.12284200$

$-4.54429800$

3.45433700

$\mathrm{H}$

$-1.93554100$

$-0.40828200$

1.79178700

C

$-0.04621600$

0.33768800

2.38551300

$\mathrm{O}$

1.09659600

0.20162000

2.79136200

$\mathrm{O}$

$-0.69249600$

1.52171100

2.49068000 


$\begin{array}{rrrr}\mathrm{C} & -0.00088100 & 2.55830200 & 3.18572400 \\ \mathrm{H} & 0.97001200 & 2.77618300 & 2.71822600 \\ \mathrm{H} & -0.64875700 & 3.44190000 & 3.14469500 \\ \mathrm{H} & 0.17626700 & 2.26873500 & 4.23262000 \\ \mathrm{C} & -1.37881300 & -1.31796400 & 4.72773800 \\ \mathrm{H} & -0.32589200 & -1.18831700 & 5.02390700 \\ \mathrm{H} & -1.75215800 & -0.34300100 & 4.37735200 \\ \mathrm{H} & -1.95616000 & -1.58805900 & 5.62702000 \\ \mathrm{H} & -4.80706400 & -4.54229000 & -0.72213300 \\ \mathrm{C} & 2.44500800 & -0.82987800 & 0.40187400 \\ \mathrm{C} & 3.51789300 & -2.59788200 & 1.21137200 \\ \mathrm{C} & 2.20228900 & -3.03167500 & 0.50601500 \\ \mathrm{~N} & 1.58687600 & -1.74243700 & 0.14215400 \\ \mathrm{H} & 3.48819000 & -2.72599400 & 2.30203200 \\ \mathrm{H} & 1.53612600 & -3.55280900 & 1.20837700 \\ \mathrm{C} & 1.02385800 & 2.26111500 & -0.85150400 \\ \mathrm{C} & 2.44109000 & 2.71589700 & -0.43700400 \\ \mathrm{C} & 2.15269100 & 0.56963100 & 0.04837600 \\ \mathrm{~N} & 0.95805200 & 0.91302600 & -0.25477700 \\ \mathrm{H} & 0.96971900 & 2.10186000 & -1.94212500 \\ \mathrm{H} & 2.43398400 & 3.35709200 & 0.45836900 \\ \mathrm{H} & 4.41686300 & -3.07798600 & 0.80347000 \\ \mathrm{H} & 3.01627300 & 3.19553300 & -1.23675300 \\ \mathrm{O} & 3.61172200 & -1.16647800 & 0.94177500 \\ \mathrm{O} & 3.12022500 & 1.47009200 & -0.07785500 \\ \mathrm{C} & 2.41660900 & -3.89306800 & -0.73555400 \\ \mathrm{H} & 2.85288100 & -4.86360300 & -0.45433700 \\ \mathrm{H} & 1.46230000 & -4.07635400 & -1.24978900 \\ \mathrm{H} & 3.09629400 & -3.39315200 & -1.44289200 \\ \mathrm{C} & -0.11444800 & 3.17333300 & -0.43480800 \\ \mathrm{H} & -0.03593300 & 4.13810200 & -0.95798200 \\ \mathrm{H} & -1.07487100 & 2.71201100 & -0.70733000 \\ \mathrm{H} & -0.10868300 & 3.35766200 & 0.64663400 \\ \mathrm{H} 3 \mathrm{~B} & & \end{array}$

UB3LYP-D3(BJ)/def2-SVP-CPCM(THF)

Zero-point correction=

Thermal correction to Energy=

Thermal correction to Enthalpy=

Thermal correction to Gibbs Free Energy=

Sum of electronic and zero-point Energies=

Sum of electronic and thermal Energies=

Sum of electronic and thermal Enthalpies=

Sum of electronic and thermal Free Energies= $\mathrm{HF}=-5349.3416708$

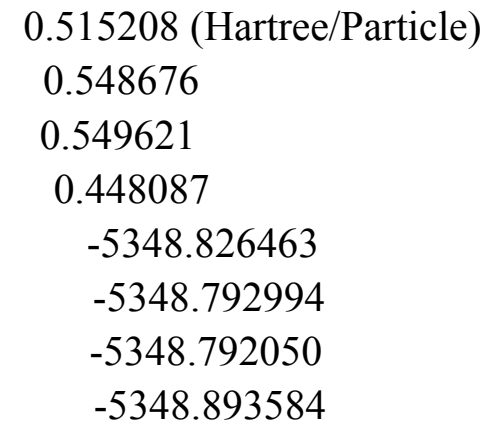




\begin{tabular}{|c|c|c|c|}
\hline$s$ & & & \\
\hline $\mathrm{Ni}$ & -0.3860868 & 0.4177150 & -0.8250254 \\
\hline $\mathrm{C}$ & 0.0815182 & 1.7363110 & -2.2108364 \\
\hline $\mathrm{C}$ & 0.8324282 & 3.8102380 & -3.9611274 \\
\hline $\mathrm{C}$ & -0.1749638 & 3.0736850 & -1.8805404 \\
\hline $\mathrm{C}$ & 0.7186892 & 1.4445190 & -3.4198314 \\
\hline $\mathrm{C}$ & 1.0889792 & 2.4766910 & -4.2937104 \\
\hline $\mathrm{C}$ & 0.1990592 & 4.1070650 & -2.7495114 \\
\hline $\mathrm{H}$ & -0.6774988 & 3.3260540 & -0.9411414 \\
\hline $\mathrm{H}$ & 0.9354092 & 0.4110190 & -3.6971244 \\
\hline $\mathrm{H}$ & 1.5823272 & 2.2333770 & -5.2392234 \\
\hline $\mathrm{H}$ & -0.0077188 & 5.1461340 & -2.4776964 \\
\hline $\mathrm{Br}$ & -2.6162768 & 0.3840390 & -1.6847114 \\
\hline $\mathrm{H}$ & 1.1232602 & 4.6140640 & -4.6420354 \\
\hline $\mathrm{C}$ & 2.3001742 & -0.1540690 & -0.4887834 \\
\hline $\mathrm{C}$ & 3.6107812 & 1.2228770 & 0.6511946 \\
\hline $\mathrm{C}$ & 2.2034732 & 1.8541020 & 0.4851816 \\
\hline $\mathrm{N}$ & 1.4555222 & 0.7539470 & -0.1502154 \\
\hline $\mathrm{H}$ & 4.4311912 & 1.8502010 & 0.2846346 \\
\hline $\mathrm{H}$ & 2.2251432 & 2.6815740 & -0.2440184 \\
\hline $\mathrm{C}$ & 0.1965022 & -2.5095570 & -2.2361444 \\
\hline $\mathrm{C}$ & 1.5676582 & -3.2420850 & -2.2891604 \\
\hline $\mathrm{C}$ & 1.7829782 & -1.2884840 & -1.2536494 \\
\hline $\mathrm{N}$ & 0.5557542 & -1.2236020 & -1.6198154 \\
\hline $\mathrm{H}$ & -0.4808428 & -3.0113420 & -1.5270544 \\
\hline $\mathrm{H}$ & 1.9698392 & -3.3173580 & -3.3098304 \\
\hline $\mathrm{H}$ & 3.8056732 & 0.9100540 & 1.6874796 \\
\hline $\mathrm{H}$ & 1.5653602 & -4.2301530 & -1.8145374 \\
\hline $\mathrm{O}$ & 3.5727422 & 0.0101470 & -0.1670414 \\
\hline $\mathrm{O}$ & 2.4777582 & -2.3850290 & -1.5240744 \\
\hline $\mathrm{C}$ & 1.5821892 & 2.3323450 & 1.7860906 \\
\hline $\mathrm{H}$ & 2.2122692 & 3.1155640 & 2.2340846 \\
\hline $\mathrm{H}$ & 0.5855562 & 2.7570430 & 1.6015266 \\
\hline $\mathrm{H}$ & 1.4857762 & 1.4911740 & 2.4859976 \\
\hline $\mathrm{C}$ & -0.4905918 & -2.3460730 & -3.5832574 \\
\hline $\mathrm{H}$ & -0.7605008 & -3.3325370 & -3.9897814 \\
\hline $\mathrm{H}$ & -1.4061238 & -1.7495240 & -3.4656284 \\
\hline $\mathrm{H}$ & 0.1705932 & -1.8414300 & -4.3043364 \\
\hline $\mathrm{C}$ & -1.5305928 & 0.4391730 & 1.7986466 \\
\hline $\mathrm{H}$ & -1.9738328 & 1.2638720 & 1.2139816 \\
\hline $\mathrm{C}$ & -0.9949318 & -0.6407160 & 0.8725766 \\
\hline $\mathrm{H}$ & -1.7682178 & -1.2929940 & 0.4586466 \\
\hline $\mathrm{H}$ & -0.6879778 & 0.8679760 & 2.3578296 \\
\hline $\mathrm{C}$ & -2.6111608 & -0.0151850 & 2.8231656 \\
\hline
\end{tabular}




$\begin{array}{lrrr}\mathrm{C} & -2.9124478 & 1.1796510 & 3.7418106 \\ \mathrm{H} & -3.6947538 & 0.9286780 & 4.4768496 \\ \mathrm{H} & -2.0117898 & 1.4882820 & 4.2975976 \\ \mathrm{H} & -3.2628948 & 2.0473600 & 3.1590026 \\ \mathrm{C} & -2.0973398 & -1.1896780 & 3.6706546 \\ \mathrm{H} & -1.9250938 & -2.0858470 & 3.0544866 \\ \mathrm{H} & -1.1483388 & -0.9367970 & 4.1684476 \\ \mathrm{H} & -2.8323968 & -1.4577130 & 4.4472366 \\ \mathrm{C} & -3.9003748 & -0.4293480 & 2.0977276 \\ \mathrm{H} & -4.6778718 & -0.7212120 & 2.8227656 \\ \mathrm{H} & -4.2940288 & 0.3982080 & 1.4865206 \\ \mathrm{H} & -3.7307638 & -1.2839900 & 1.4253076 \\ \mathrm{C} & 0.1121752 & -1.4300720 & 1.4131026 \\ \mathrm{O} & 1.0001732 & -1.0502830 & 2.1638236 \\ \mathrm{O} & 0.1146202 & -2.7139450 & 0.9433806 \\ \mathrm{C} & 1.1771452 & -3.5533320 & 1.3899126 \\ \mathrm{H} & 1.2093702 & -3.5987930 & 2.4882816 \\ \mathrm{H} & 0.9732342 & -4.5495800 & 0.9779556 \\ \mathrm{H} & 2.1500902 & -3.1924100 & 1.0239436\end{array}$

UB3LYP-D3(BJ)/def2-SVP-CPCM(THF)

Zero-point correction=

0.514544 (Hartree/Particle)
0.547766
0.548710
0.448208
-5348.823432
-5348.790210
-5348.789266
-5348.889768

Thermal correction to Energy=

Thermal correction to Enthalpy=

Thermal correction to Gibbs Free Energy=

Sum of electronic and zero-point Energies=

Sum of electronic and thermal Energies=

Sum of electronic and thermal Enthalpies=

Sum of electronic and thermal Free Energies= $\mathrm{HF}=-5349.3379762$

\section{${ }^{2} \mathbf{F}^{S}$-conf2}

$\mathrm{Ni}$

$\mathrm{C}$

$$
\begin{array}{rrr}
-0.79578000 & 0.37783400 & 0.21147100 \\
-0.45334900 & 1.67917900 & -1.23935900 \\
0.18379700 & 3.68020800 & -3.11408200 \\
-0.44678600 & 3.02828300 & -0.86405100 \\
-0.13860200 & 1.33687100 & -2.55681900 \\
0.17556900 & 2.33418900 & -3.49125000 \\
-0.12862100 & 4.02552100 & -1.79468600 \\
-0.70095400 & 3.32057000 & 0.15990200 \\
-0.13516300 & 0.29282400 & -2.87388000 \\
0.41518900 & 2.05233200 & -4.52064600 \\
-0.12846600 & 5.07484500 & -1.48622400 \\
-3.10580200 & 0.77300700 & -0.23680600 \\
0.43004300 & 4.45601300 & -3.84328300
\end{array}
$$

$\mathrm{C}$

$\mathrm{C}$

$\mathrm{C}$

$\mathrm{C}$

$\mathrm{C}$

$\mathrm{H}$

$\mathrm{H}$

$\mathrm{H}$

$\mathrm{H}$

$\mathrm{Br}$

$\mathrm{H}$ 


\begin{tabular}{|c|c|c|c|}
\hline $\mathrm{C}$ & 1.86265300 & -0.35648900 & -0.01660800 \\
\hline $\mathrm{C}$ & 3.45544500 & 0.93547600 & 0.83555600 \\
\hline $\mathrm{C}$ & 2.07678200 & 1.63296200 & 0.98370100 \\
\hline $\mathrm{N}$ & 1.15991700 & 0.58642900 & 0.50521700 \\
\hline $\mathrm{H}$ & 4.20438800 & 1.53181000 & 0.30239300 \\
\hline $\mathrm{H}$ & 1.98906000 & 2.47977800 & 0.28298100 \\
\hline $\mathrm{C}$ & -0.71840400 & -2.54118100 & -1.28303300 \\
\hline $\mathrm{C}$ & 0.55481300 & -3.37077000 & -1.61434600 \\
\hline $\mathrm{C}$ & 1.12478600 & -1.44864100 & -0.65349300 \\
\hline $\mathrm{N}$ & -0.14524200 & -1.29519400 & -0.75179900 \\
\hline $\mathrm{H}$ & -1.29480700 & -2.98499000 & -0.45509600 \\
\hline $\mathrm{H}$ & 0.72863300 & -3.47248700 & -2.69552700 \\
\hline $\mathrm{H}$ & 3.86106700 & 0.59591600 & 1.80022300 \\
\hline $\mathrm{H}$ & 0.58057700 & -4.35775200 & -1.13735800 \\
\hline $\mathrm{O}$ & 3.18174600 & -0.26036800 & 0.03404400 \\
\hline $\mathrm{O}$ & 1.66672100 & -2.58578000 & -1.06551900 \\
\hline $\mathrm{C}$ & 1.73930700 & 2.10218200 & 2.38923600 \\
\hline $\mathrm{H}$ & 2.45199700 & 2.87538000 & 2.71294500 \\
\hline $\mathrm{H}$ & 0.72857900 & 2.53502800 & 2.41112700 \\
\hline $\mathrm{H}$ & 1.77477800 & 1.26702100 & 3.10277200 \\
\hline $\mathrm{C}$ & -1.64944500 & -2.29294100 & -2.46053900 \\
\hline $\mathrm{H}$ & -2.09386400 & -3.24203300 & -2.79640800 \\
\hline $\mathrm{H}$ & -2.45827600 & -1.61375100 & -2.15518100 \\
\hline $\mathrm{H}$ & -1.10985600 & -1.84234400 & -3.30788100 \\
\hline $\mathrm{C}$ & 0.05993600 & -1.72801400 & 2.33070900 \\
\hline $\mathrm{H}$ & 1.04521200 & -1.33139500 & 2.05030100 \\
\hline $\mathrm{C}$ & -1.05076900 & -0.74233800 & 1.99917800 \\
\hline $\mathrm{H}$ & -1.09600200 & 0.10721200 & 2.69558800 \\
\hline $\mathrm{H}$ & -0.09181500 & -2.63623500 & 1.72666600 \\
\hline $\mathrm{C}$ & 0.19767900 & -2.17015000 & 3.81840800 \\
\hline $\mathrm{C}$ & 1.29300100 & -3.24762500 & 3.87600400 \\
\hline $\mathrm{H}$ & 1.47372400 & -3.57589900 & 4.91264600 \\
\hline $\mathrm{H}$ & 1.00671900 & -4.13314600 & 3.28529400 \\
\hline $\mathrm{H}$ & 2.24552400 & -2.86690900 & 3.47142300 \\
\hline $\mathrm{C}$ & -1.11389500 & -2.75866000 & 4.36196200 \\
\hline $\mathrm{H}$ & -1.90647900 & -1.99809500 & 4.41579900 \\
\hline $\mathrm{H}$ & -1.47762500 & -3.57706000 & 3.72234100 \\
\hline $\mathrm{H}$ & -0.96379300 & -3.15692000 & 5.37881300 \\
\hline $\mathrm{C}$ & 0.62398700 & -0.98010000 & 4.69170100 \\
\hline $\mathrm{H}$ & 0.72758000 & -1.28512200 & 5.74569700 \\
\hline $\mathrm{H}$ & 1.59723200 & -0.57937800 & 4.36381400 \\
\hline $\mathrm{H}$ & -0.11171000 & -0.16197300 & 4.65212300 \\
\hline $\mathrm{C}$ & -2.36173900 & -1.40907600 & 1.91713500 \\
\hline $\mathrm{O}$ & -2.59236400 & -2.47015700 & 1.35311800 \\
\hline
\end{tabular}




$\begin{array}{llll}\mathrm{O} & -3.33730600 & -0.74390200 & 2.57953900 \\ \mathrm{C} & -4.65618000 & -1.26372500 & 2.44859400 \\ \mathrm{H} & -4.72204000 & -2.28251800 & 2.86025600 \\ \mathrm{H} & -5.31174900 & -0.58830100 & 3.01255800 \\ \mathrm{H} & -4.95898800 & -1.28533900 & 1.39153000\end{array}$

UB3LYP-D3(BJ)/def2-SVP-CPCM(THF)

\begin{tabular}{|c|c|c|c|}
\hline \multicolumn{3}{|c|}{ Zero-point correction $=$} & 0.514312 (Hartree/Particle) \\
\hline \multicolumn{3}{|c|}{ Thermal correction to Energy= } & 0.547538 \\
\hline \multicolumn{3}{|c|}{ Thermal correction to Enthalpy= } & 0.548482 \\
\hline \multicolumn{3}{|c|}{ Thermal correction to Gibbs Free Energy= } & 0.448059 \\
\hline \multicolumn{3}{|c|}{ Sum of electronic and zero-point Energies $=$} & -5348.814053 \\
\hline \multicolumn{3}{|c|}{ Sum of electronic and thermal Energies $=$} & -5348.780827 \\
\hline \multicolumn{3}{|c|}{ Sum of electronic and thermal Enthalpies $=$} & -5348.779882 \\
\hline \multicolumn{3}{|c|}{$\begin{array}{l}\text { Sum of electronic and thermal Free Energies= } \\
\mathrm{HF}=-5349.3283643\end{array}$} & -5348.880306 \\
\hline \multicolumn{4}{|c|}{${ }^{2} F^{S}-\operatorname{conf} 3$} \\
\hline $\mathrm{Ni}$ & -1.24440900 & -0.01888700 & -0.11289100 \\
\hline $\mathrm{C}$ & -0.25123600 & 1.60382000 & -0.33557000 \\
\hline $\mathrm{C}$ & 1.24118700 & 3.96077700 & -0.74785400 \\
\hline $\mathrm{C}$ & -0.85514000 & 2.74064900 & -0.88651600 \\
\hline $\mathrm{C}$ & 1.10464500 & 1.65825300 & 0.00945700 \\
\hline $\mathrm{C}$ & 1.84801400 & 2.82862600 & -0.19837500 \\
\hline $\mathrm{C}$ & -0.11410600 & 3.91057000 & -1.09059700 \\
\hline $\mathrm{H}$ & -1.90933800 & 2.71085600 & -1.16482000 \\
\hline $\mathrm{H}$ & 1.60818600 & 0.79816500 & 0.45106800 \\
\hline $\mathrm{H}$ & 2.90695600 & 2.84918700 & 0.07414800 \\
\hline $\mathrm{H}$ & -0.60355900 & 4.78940500 & -1.51992300 \\
\hline $\mathrm{Br}$ & -3.22986400 & 0.34251400 & -1.77897800 \\
\hline $\mathrm{H}$ & 1.81851000 & 4.87504300 & -0.90610700 \\
\hline $\mathrm{C}$ & 1.12935800 & -1.82838900 & 0.30338900 \\
\hline $\mathrm{C}$ & 2.44987100 & -2.04872200 & 2.07965800 \\
\hline $\mathrm{C}$ & 1.10790600 & -1.36301800 & 2.47089000 \\
\hline $\mathrm{N}$ & 0.38139500 & -1.28004000 & 1.18341900 \\
\hline $\mathrm{H}$ & 3.33181500 & -1.40800000 & 2.20967400 \\
\hline $\mathrm{H}$ & 1.27909100 & -0.32992500 & 2.81463800 \\
\hline $\mathrm{C}$ & -0.29638700 & -1.15013400 & -2.97562300 \\
\hline $\mathrm{C}$ & 0.72309800 & -2.27612500 & -3.29149100 \\
\hline $\mathrm{C}$ & 0.73960300 & -1.81629600 & -1.11666200 \\
\hline $\mathrm{N}$ & -0.24167300 & -1.08784200 & -1.50115900 \\
\hline $\mathrm{H}$ & -1.31827300 & -1.42875000 & -3.26676200 \\
\hline $\mathrm{H}$ & 1.47654700 & -1.99364200 & -4.03734400 \\
\hline $\mathrm{H}$ & 2.60906900 & -3.00698600 & 2.59270400 \\
\hline $\mathrm{H}$ & 0.25159100 & -3.22643900 & -3.57701300 \\
\hline
\end{tabular}




$\begin{array}{lrrr}\mathrm{O} & 2.31137200 & -2.32548300 & 0.65423300 \\ \mathrm{O} & 1.41851900 & -2.50166400 & -2.02239000 \\ \mathrm{C} & 0.33350800 & -2.12682400 & 3.53828200 \\ \mathrm{H} & 0.92524800 & -2.16363900 & 4.46591600 \\ \mathrm{H} & -0.62867100 & -1.64561800 & 3.75139700 \\ \mathrm{H} & 0.14357200 & -3.16120200 & 3.21028100 \\ \mathrm{C} & 0.05449000 & 0.20328300 & -3.58337200 \\ \mathrm{H} & -0.67480300 & 0.95471200 & -3.25566100 \\ \mathrm{H} & 1.06008100 & 0.52350400 & -3.26999500 \\ \mathrm{H} & 0.02973100 & 0.13386000 & -4.68184900 \\ \mathrm{C} & -1.56072100 & 1.74640200 & 2.48149000 \\ \mathrm{H} & -0.72249200 & 2.29523500 & 2.03085200 \\ \mathrm{C} & -2.16431500 & 0.81011400 & 1.45213900 \\ \mathrm{H} & -2.98695000 & 1.24257300 & 0.86778600 \\ \mathrm{H} & -1.13393800 & 1.14708500 & 3.30219800 \\ \mathrm{C} & -2.52887200 & 2.79940600 & 3.08444500 \\ \mathrm{C} & -1.75879300 & 3.57208200 & 4.16722700 \\ \mathrm{H} & -2.38701500 & 4.36002700 & 4.61360300 \\ \mathrm{H} & -1.43343500 & 2.89914100 & 4.97740500 \\ \mathrm{H} & -0.86061500 & 4.05317200 & 3.74642800 \\ \mathrm{C} & -3.75744200 & 2.12865700 & 3.71737000 \\ \mathrm{H} & -4.36006100 & 1.59673700 & 2.96474100 \\ \mathrm{H} & -3.45917800 & 1.39495600 & 4.48150500 \\ \mathrm{H} & -4.40708000 & 2.88187500 & 4.19253000 \\ \mathrm{C} & -2.97978700 & 3.78310700 & 1.99223400 \\ \mathrm{H} & -3.63873400 & 4.55827900 & 2.41601900 \\ \mathrm{H} & -2.11469600 & 4.28265000 & 1.52817100 \\ \mathrm{H} & -3.53901800 & 3.27642000 & 1.19011700 \\ \mathrm{C} & -2.55133700 & -0.52877500 & 1.96558900 \\ \mathrm{O} & -2.68082000 & -0.92923200 & 3.09678800 \\ \mathrm{O} & -2.62370300 & -1.37045600 & 0.88104600 \\ \mathrm{C} & -2.77114000 & -2.77726600 & 1.09385400 \\ \mathrm{H} & -3.64724400 & -2.97077800 & 1.72640900 \\ \mathrm{H} & -2.90756200 & -3.21895700 & 0.10044700 \\ \mathrm{H} & -1.86956600 & -3.18265600 & 1.57385700\end{array}$

UB3LYP-D3(BJ)/def2-SVP-CPCM(THF)

Zero-point correction $=$

Thermal correction to Energy=

0.513722 (Hartree/Particle)

0.547438

0.548382

Thermal correction to Enthalpy=

0.445882

Thermal correction to Gibbs Free Energy=

Sum of electronic and zero-point Energies=

Sum of electronic and thermal Energies=

$-5348.823721$

$-5348.790005$

$-5348.789061$

Sum of electronic and thermal Enthalpies=

$-5348.891561$ 


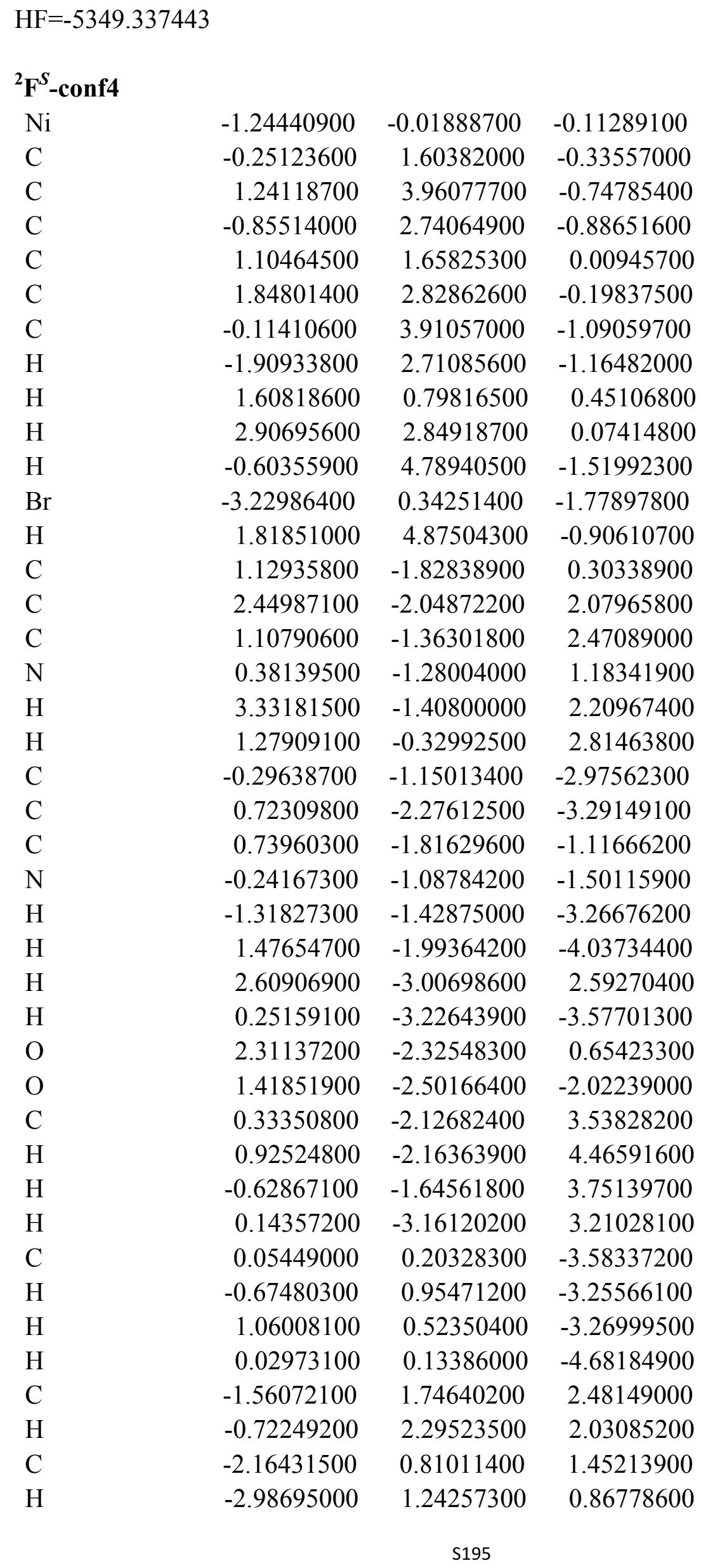




$\begin{array}{lrrr}\mathrm{H} & -1.13393800 & 1.14708500 & 3.30219800 \\ \mathrm{C} & -2.52887200 & 2.79940600 & 3.08444500 \\ \mathrm{C} & -1.75879300 & 3.57208200 & 4.16722700 \\ \mathrm{H} & -2.38701500 & 4.36002700 & 4.61360300 \\ \mathrm{H} & -1.43343500 & 2.89914100 & 4.97740500 \\ \mathrm{H} & -0.86061500 & 4.05317200 & 3.74642800 \\ \mathrm{C} & -3.75744200 & 2.12865700 & 3.71737000 \\ \mathrm{H} & -4.36006100 & 1.59673700 & 2.96474100 \\ \mathrm{H} & -3.45917800 & 1.39495600 & 4.48150500 \\ \mathrm{H} & -4.40708000 & 2.88187500 & 4.19253000 \\ \mathrm{C} & -2.97978700 & 3.78310700 & 1.99223400 \\ \mathrm{H} & -3.63873400 & 4.55827900 & 2.41601900 \\ \mathrm{H} & -2.11469600 & 4.28265000 & 1.52817100 \\ \mathrm{H} & -3.53901800 & 3.27642000 & 1.19011700 \\ \mathrm{C} & -2.55133700 & -0.52877500 & 1.96558900 \\ \mathrm{O} & -2.68082000 & -0.92923200 & 3.09678800 \\ \mathrm{O} & -2.62370300 & -1.37045600 & 0.88104600 \\ \mathrm{C} & -2.77114000 & -2.77726600 & 1.09385400 \\ \mathrm{H} & -3.64724400 & -2.97077800 & 1.72640900 \\ \mathrm{H} & -2.90756200 & -3.21895700 & 0.10044700 \\ \mathrm{H} & -1.86956600 & -3.18265600 & 1.57385700 \\ \mathrm{H} & -\mathrm{B} & \end{array}$

UB3LYP-D3(BJ)/def2-SVP-CPCM(THF)

Zero-point correction=

0.513722 (Hartree/Particle)

Thermal correction to Energy=

0.547438

Thermal correction to Enthalpy=

0.548382

Thermal correction to Gibbs Free Energy=

0.445882

$-5348.823721$

$-5348.790005$

$-5348.789061$

$-5348.891561$

Sum of electronic and thermal Free Energies= $\mathrm{HF}=-5349.337443$

$\begin{array}{lrrr}{ }^{2} \mathbf{F}-\mathbf{T S}-\mathbf{G}^{\boldsymbol{R}} & & & \\ \mathrm{Ni} & -0.65029000 & 0.08414400 & -0.61920200 \\ \mathrm{C} & -1.23302200 & 1.74718000 & -1.27518400 \\ \mathrm{C} & -1.95838100 & 4.31100600 & -2.24060100 \\ \mathrm{C} & -2.17032100 & 2.54087000 & -0.59256100 \\ \mathrm{C} & -0.68008200 & 2.25938500 & -2.46100100 \\ \mathrm{C} & -1.03893700 & 3.52556600 & -2.94288100 \\ \mathrm{C} & -2.52375200 & 3.81166200 & -1.06182400 \\ \mathrm{H} & -2.63631900 & 2.16809600 & 0.32156600 \\ \mathrm{H} & 0.04956600 & 1.66894000 & -3.02484700 \\ \mathrm{H} & -0.59458600 & 3.90013300 & -3.86981700 \\ \mathrm{H} & -3.24895600 & 4.41273700 & -0.50529000 \\ & & & \end{array}$




\begin{tabular}{|c|c|c|c|}
\hline $\mathrm{Br}$ & -2.71524900 & -0.90879600 & -1.13955500 \\
\hline $\mathrm{C}$ & -3.82833100 & 1.41204000 & 2.81772400 \\
\hline $\mathrm{H}$ & -2.98969800 & 2.12506300 & 2.79475700 \\
\hline $\mathrm{H}$ & -4.18383600 & 1.26939100 & 1.78494200 \\
\hline $\mathrm{H}$ & -4.64292100 & 1.87754400 & 3.39545200 \\
\hline $\mathrm{C}$ & -3.41977100 & 0.07140000 & 3.44584900 \\
\hline $\mathrm{C}$ & -2.30511300 & -0.60971200 & 2.59294200 \\
\hline $\mathrm{H}$ & -2.11235600 & -1.61306800 & 3.00098900 \\
\hline $\mathrm{H}$ & -2.69732100 & -0.73584500 & 1.56880100 \\
\hline $\mathrm{C}$ & -1.02184200 & 0.12438000 & 2.52113100 \\
\hline $\mathrm{C}$ & -4.63095700 & -0.87239600 & 3.45915100 \\
\hline $\mathrm{H}$ & -4.37185200 & -1.84260200 & 3.91292200 \\
\hline $\mathrm{H}$ & -5.46038200 & -0.43672500 & 4.03940700 \\
\hline $\mathrm{H}$ & -4.99348100 & -1.06283000 & 2.43615700 \\
\hline $\mathrm{H}$ & -0.96080800 & 1.12097000 & 2.08197700 \\
\hline $\mathrm{C}$ & 0.17313300 & -0.42142500 & 3.12565400 \\
\hline $\mathrm{O}$ & 0.25467100 & -1.51504100 & 3.67117700 \\
\hline $\mathrm{O}$ & 1.24534300 & 0.40484200 & 3.01686000 \\
\hline $\mathrm{C}$ & 2.45720500 & -0.03913000 & 3.63172800 \\
\hline $\mathrm{H}$ & 2.81188100 & -0.96904700 & 3.16542800 \\
\hline $\mathrm{H}$ & 3.18761100 & 0.76254800 & 3.47500200 \\
\hline $\mathrm{H}$ & 2.30433300 & -0.21296700 & 4.70688200 \\
\hline $\mathrm{C}$ & -2.91774700 & 0.29354000 & 4.88019200 \\
\hline $\mathrm{H}$ & -2.59067400 & -0.65457300 & 5.33669100 \\
\hline $\mathrm{H}$ & -2.06299900 & 0.98898000 & 4.90059600 \\
\hline $\mathrm{H}$ & -3.71394600 & 0.71947000 & 5.51193500 \\
\hline $\mathrm{H}$ & -2.23539200 & 5.30160500 & -2.61046400 \\
\hline $\mathrm{C}$ & 1.97115800 & -0.09741700 & 0.21540400 \\
\hline $\mathrm{C}$ & 3.29809300 & 1.67043400 & 0.03353500 \\
\hline $\mathrm{C}$ & 1.83166800 & 2.07538500 & -0.27521500 \\
\hline $\mathrm{N}$ & 1.13930500 & 0.76779500 & -0.25111100 \\
\hline $\mathrm{H}$ & 3.95192700 & 1.68667000 & -0.84881700 \\
\hline $\mathrm{H}$ & 1.74262900 & 2.50040100 & -1.28382300 \\
\hline $\mathrm{C}$ & -0.11563600 & -3.03007500 & 0.48351700 \\
\hline $\mathrm{C}$ & 1.13837800 & -3.48169300 & 1.28438000 \\
\hline $\mathrm{C}$ & 1.44334000 & -1.44117100 & 0.46505100 \\
\hline $\mathrm{N}$ & 0.21883000 & -1.63666900 & 0.13536800 \\
\hline $\mathrm{H}$ & -1.00745400 & -3.01386400 & 1.12636200 \\
\hline $\mathrm{H}$ & 1.58349700 & -4.41519200 & 0.91871900 \\
\hline $\mathrm{H}$ & 3.75208700 & 2.24927300 & 0.84717100 \\
\hline $\mathrm{H}$ & 0.96315100 & -3.52676300 & 2.36583800 \\
\hline $\mathrm{O}$ & 3.20842300 & 0.27704500 & 0.47909100 \\
\hline $\mathrm{O}$ & 2.11859400 & -2.40846500 & 1.06206000 \\
\hline $\mathrm{C}$ & 1.22957800 & 3.03431300 & 0.74661200 \\
\hline
\end{tabular}




$\begin{array}{rrrr}\mathrm{H} & 1.78818200 & 3.98275000 & 0.73625100 \\ \mathrm{H} & 0.18057000 & 3.24273000 & 0.50002700 \\ \mathrm{H} & 1.28008000 & 2.59862800 & 1.75507400 \\ \mathrm{C} & -0.39608600 & -3.85831900 & -0.76565000 \\ \mathrm{H} & -0.64244400 & -4.89296700 & -0.48159700 \\ \mathrm{H} & -1.24407500 & -3.42591800 & -1.31355600 \\ \mathrm{H} & 0.48522100 & -3.87646500 & -1.42610400\end{array}$

UB3LYP-D3(BJ)/def2-SVP-CPCM(THF)

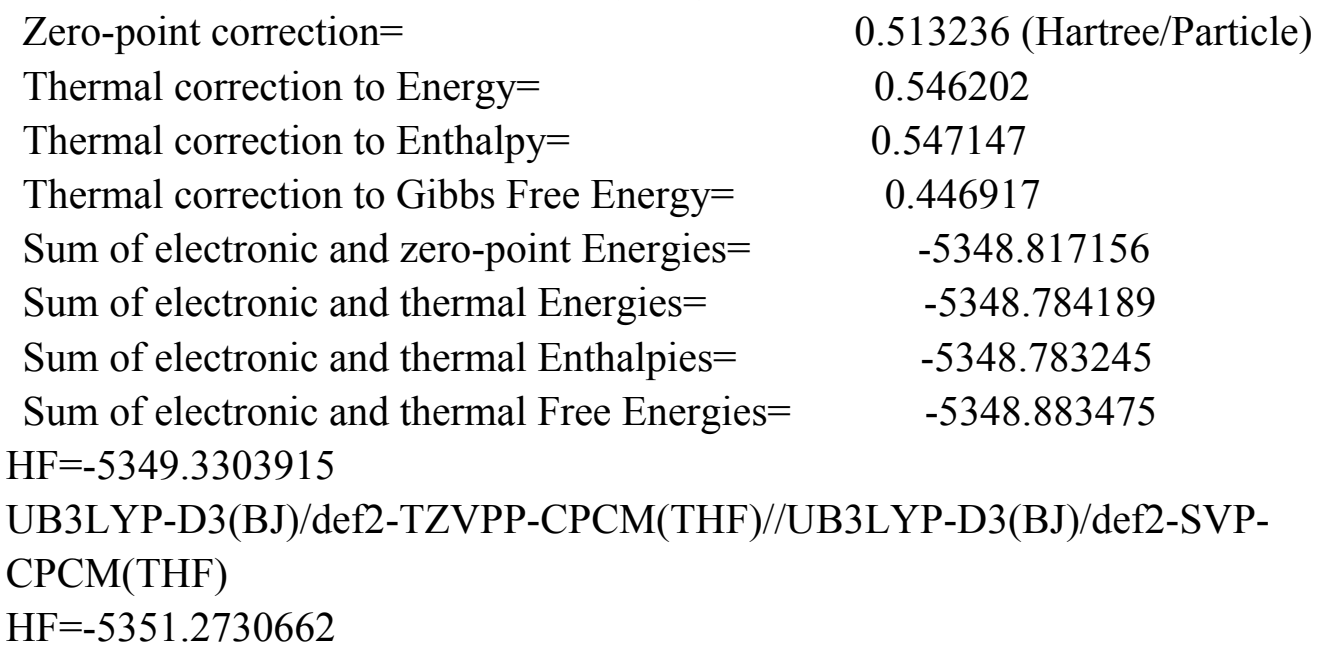

$\begin{array}{lrrr}{ }^{2} \text { G-complex }{ }^{R} & & & \\ \mathrm{Ni} & -0.70885300 & 0.14705700 & -0.57870400 \\ \mathrm{C} & -1.24483000 & 1.78858300 & -1.32173600 \\ \mathrm{C} & -1.89610100 & 4.29971600 & -2.46436300 \\ \mathrm{C} & -2.16732100 & 2.64953700 & -0.70363400 \\ \mathrm{C} & -0.66454600 & 2.20939000 & -2.53086100 \\ \mathrm{C} & -0.98791700 & 3.44813800 & -3.10117200 \\ \mathrm{C} & -2.48431900 & 3.89422800 & -1.26102700 \\ \mathrm{H} & -2.64997600 & 2.34801100 & 0.22788500 \\ \mathrm{H} & 0.05941900 & 1.56706900 & -3.04317600 \\ \mathrm{H} & -0.52434400 & 3.74950600 & -4.04526600 \\ \mathrm{H} & -3.19959700 & 4.54885800 & -0.75420200 \\ \mathrm{Br} & -2.82026900 & -0.80394300 & -0.96925700 \\ \mathrm{C} & -3.75426900 & 1.23963800 & 2.61779100 \\ \mathrm{H} & -2.94278800 & 1.98338900 & 2.64672800 \\ \mathrm{H} & -3.95454800 & 0.99398000 & 1.56344300 \\ \mathrm{H} & -4.65387600 & 1.71782400 & 3.03745500 \\ \mathrm{C} & -3.39782500 & -0.02491200 & 3.41373900 \\ \mathrm{C} & -2.17804400 & -0.74684800 & 2.77092400 \\ \mathrm{H} & -1.99446100 & -1.69522200 & 3.29785200 \\ \mathrm{H} & -2.45966600 & -0.99484800 & 1.72926500 \\ \mathrm{C} & -0.91900900 & 0.02684000 & 2.72736400 \\ \mathrm{C} & -4.58451500 & -0.99900500 & 3.37142500\end{array}$




\begin{tabular}{|c|c|c|c|}
\hline $\mathrm{H}$ & -4.36058600 & -1.92088900 & 3.93231600 \\
\hline $\mathrm{H}$ & -5.48321800 & -0.54124300 & 3.81530600 \\
\hline $\mathrm{H}$ & -4.82275800 & -1.28249200 & 2.33371700 \\
\hline $\mathrm{H}$ & -0.88358500 & 1.02282400 & 2.28385000 \\
\hline $\mathrm{C}$ & 0.30943500 & -0.51564500 & 3.26174800 \\
\hline $\mathrm{O}$ & 0.43211200 & -1.61723100 & 3.78269100 \\
\hline $\mathrm{O}$ & 1.36426000 & 0.32562700 & 3.10523400 \\
\hline $\mathrm{C}$ & 2.61634700 & -0.11832900 & 3.63300800 \\
\hline $\mathrm{H}$ & 2.94917300 & -1.03315100 & 3.12270700 \\
\hline $\mathrm{H}$ & 3.32800000 & 0.69439600 & 3.44974100 \\
\hline $\mathrm{H}$ & 2.53264100 & -0.31888800 & 4.71113000 \\
\hline $\mathrm{C}$ & -3.07585300 & 0.34142300 & 4.87009200 \\
\hline $\mathrm{H}$ & -2.80788200 & -0.55417600 & 5.45404700 \\
\hline $\mathrm{H}$ & -2.22925100 & 1.04502000 & 4.92543900 \\
\hline $\mathrm{H}$ & -3.94314700 & 0.81719500 & 5.35588200 \\
\hline $\mathrm{H}$ & -2.14577500 & 5.26933500 & -2.90300400 \\
\hline $\mathrm{C}$ & 1.91805400 & -0.05487300 & 0.22480200 \\
\hline $\mathrm{C}$ & 3.23670700 & 1.71961500 & 0.05094300 \\
\hline $\mathrm{C}$ & 1.76402000 & 2.12619400 & -0.21846900 \\
\hline $\mathrm{N}$ & 1.07964600 & 0.81491500 & -0.22173900 \\
\hline $\mathrm{H}$ & 3.86903600 & 1.74652500 & -0.84684300 \\
\hline $\mathrm{H}$ & 1.65283600 & 2.58424300 & -1.21014400 \\
\hline $\mathrm{C}$ & -0.17012500 & -2.98623800 & 0.48790000 \\
\hline $\mathrm{C}$ & 1.10969000 & -3.46479000 & 1.23019700 \\
\hline $\mathrm{C}$ & 1.39119700 & -1.40003200 & 0.46494500 \\
\hline $\mathrm{N}$ & 0.15424500 & -1.58177900 & 0.17420700 \\
\hline $\mathrm{H}$ & -1.03875700 & -2.99360000 & 1.16171000 \\
\hline $\mathrm{H}$ & 1.54008500 & -4.38632800 & 0.81930200 \\
\hline $\mathrm{H}$ & 3.70988500 & 2.28831900 & 0.86064600 \\
\hline $\mathrm{H}$ & 0.97232800 & -3.54497000 & 2.31511900 \\
\hline $\mathrm{O}$ & 3.15693500 & 0.32031400 & 0.48095700 \\
\hline $\mathrm{O}$ & 2.08356900 & -2.38589600 & 1.00914200 \\
\hline $\mathrm{C}$ & 1.16967300 & 3.04017600 & 0.84834400 \\
\hline $\mathrm{H}$ & 1.70793900 & 4.00030900 & 0.85657900 \\
\hline $\mathrm{H}$ & 0.11077000 & 3.23374000 & 0.63235500 \\
\hline $\mathrm{H}$ & 1.25358600 & 2.57358300 & 1.84101800 \\
\hline $\mathrm{C}$ & -0.49536400 & -3.77215400 & -0.77759400 \\
\hline $\mathrm{H}$ & -0.72883600 & -4.81669000 & -0.52017200 \\
\hline $\mathrm{H}$ & -1.36432200 & -3.32438100 & -1.27830300 \\
\hline $\mathrm{H}$ & 0.36048200 & -3.76478200 & -1.4709070 \\
\hline
\end{tabular}

UB3LYP-D3(BJ)/def2-SVP-CPCM(THF)

Zero-point correction=

0.513178 (Hartree/Particle)

Thermal correction to Energy=

0.547040

Thermal correction to Enthalpy=

0.547984 


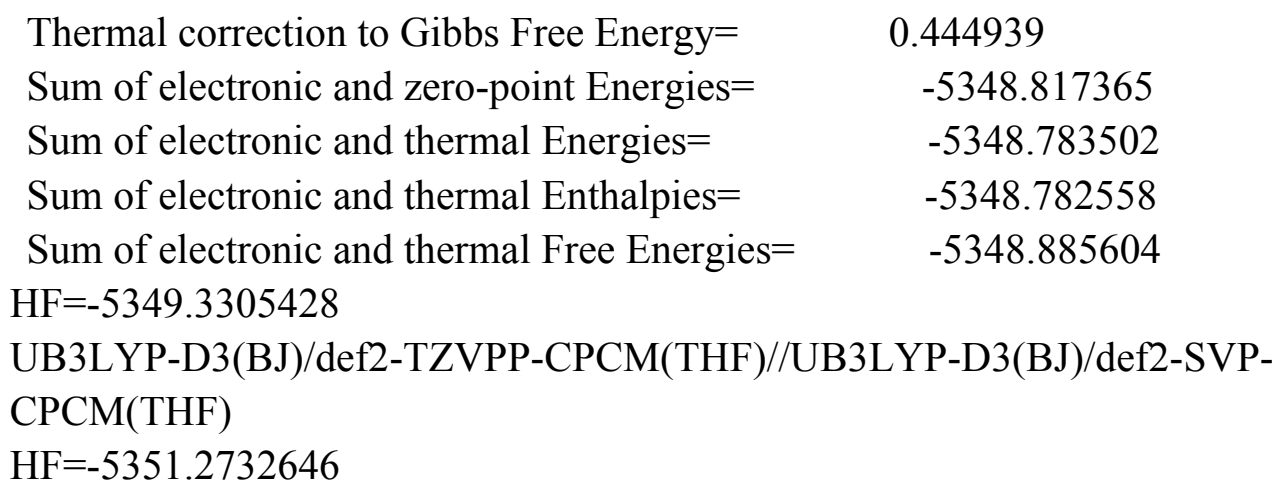

$\begin{array}{rrr}-0.95344700 & 0.61851600 & 0.25802000 \\ -0.45937400 & 1.74112700 & -1.31151600 \\ 0.28140900 & 3.42718600 & -3.47486900 \\ -1.08273600 & 2.98466300 & -1.51593300 \\ 0.54239000 & 1.36770400 & -2.22167900 \\ 0.90991600 & 2.19076400 & -3.29471900 \\ -0.71604800 & 3.82286600 & -2.57781100 \\ -1.88148300 & 3.30725900 & -0.84136200 \\ 1.06413600 & 0.41006800 & -2.11438100 \\ 1.68948900 & 1.86550800 & -3.99038100 \\ -1.21688700 & 4.78715100 & -2.70818600 \\ -3.33341600 & 0.89371900 & 0.36860800 \\ 0.56539000 & 4.07539600 & -4.30819900 \\ 1.62318800 & -0.43655700 & 0.40531000 \\ 3.31947400 & 0.75485200 & 1.20783600 \\ 1.98928900 & 1.54306000 & 1.36600100 \\ 0.99042100 & 0.57342900 & 0.89061700 \\ 4.08464400 & 1.28113400 & 0.62533800 \\ 1.95974500 & 2.40046700 & 0.67298600 \\ -1.06048000 & -2.41539100 & -0.99797500 \\ 0.16531700 & -3.33120800 & -1.28386500 \\ 0.82072500 & -1.48088100 & -0.23595000 \\ -0.43647600 & -1.24981200 & -0.35543200 \\ -1.72869400 & -2.86934300 & -0.24937800 \\ 0.37882400 & -3.44326700 & -2.35649500 \\ 3.73869800 & 0.43273300 & 2.17249400 \\ 0.09947300 & -4.31893500 & -0.81164300 \\ 2.94767600 & -0.45228600 & 0.46832400 \\ 1.30566900 & -2.63062900 & -0.68258000 \\ 1.69312100 & 2.01834800 & 2.78053700 \\ 2.45696200 & 2.73833500 & 3.11095100 \\ 0.71243000 & 2.51519500 & 2.81541800 \\ 1.68106100 & 1.17137900 & 3.48319600\end{array}$




$\begin{array}{lrrr}\mathrm{C} & -1.86104000 & -2.00765700 & -2.22696700 \\ \mathrm{H} & -2.31953500 & -2.89245600 & -2.69409900 \\ \mathrm{H} & -2.65885600 & -1.31011700 & -1.93346400 \\ \mathrm{H} & -1.21528600 & -1.50908700 & -2.96621600 \\ \mathrm{C} & 0.05416500 & -2.04873200 & 2.79869200 \\ \mathrm{H} & 0.96220600 & -1.52661400 & 2.46821400 \\ \mathrm{C} & -1.11546100 & -1.13872600 & 2.76491200 \\ \mathrm{H} & -1.07472900 & -0.15946500 & 3.24523600 \\ \mathrm{H} & -0.12712500 & -2.88848500 & 2.11159800 \\ \mathrm{C} & 0.38377100 & -2.64012500 & 4.21132000 \\ \mathrm{C} & 1.53714700 & -3.63720400 & 4.02881100 \\ \mathrm{H} & 1.83864700 & -4.06728600 & 4.99742600 \\ \mathrm{H} & 1.24240200 & -4.46631300 & 3.36565100 \\ \mathrm{H} & 2.41920300 & -3.14660300 & 3.58588400 \\ \mathrm{C} & -0.84018400 & -3.36461900 & 4.79098300 \\ \mathrm{H} & -1.66570700 & -2.66361600 & 4.99132200 \\ \mathrm{H} & -1.21296100 & -4.13427300 & 4.09739600 \\ \mathrm{H} & -0.58127700 & -3.85620400 & 5.74237500 \\ \mathrm{C} & 0.82414400 & -1.51505900 & 5.15977800 \\ \mathrm{H} & 1.09117700 & -1.92383400 & 6.14739000 \\ \mathrm{H} & 1.70799000 & -0.98779700 & 4.76460200 \\ \mathrm{H} & 0.02445300 & -0.77353500 & 5.31081400 \\ \mathrm{C} & -2.43667000 & -1.69531200 & 2.49827400 \\ \mathrm{O} & -2.64780600 & -2.71375900 & 1.85325200 \\ \mathrm{O} & -3.41643700 & -0.99511300 & 3.09436000 \\ \mathrm{C} & -4.75152900 & -1.42722900 & 2.83840200 \\ \mathrm{H} & -4.89650000 & -2.46626700 & 3.16998400 \\ \mathrm{H} & -5.40520600 & -0.75310800 & 3.40453400 \\ \mathrm{H} & -4.97366200 & -1.35285600 & 1.76431700 \\ \mathrm{~B} 3 \mathrm{~s} & & \end{array}$

UB3LYP-D3(BJ)/def2-SVP-CPCM(THF)

Zero-point correction=

Thermal correction to Energy=

0.511367 (Hartree/Particle)

0.545241

Thermal correction to Enthalpy=

0.546185

Thermal correction to Gibbs Free Energy=

0.441048

$-5348.803600$

$-5348.769726$

$-5348.768782$

$-5348.873919$

$\mathrm{HF}=-5349.3149668$

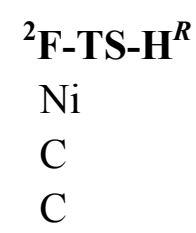

$-1.36141200$

$-3.11461200$

1.24562200

2.00729900

4.06136700
$-0.37638400$

0.08091300

$-0.38938700$ 


\begin{tabular}{|c|c|c|c|}
\hline $\mathrm{C}$ & -3.18896800 & 3.12798600 & 0.93047400 \\
\hline $\mathrm{C}$ & -4.04774400 & 1.90396500 & -0.96922700 \\
\hline $\mathrm{C}$ & -4.96709500 & 2.92740900 & -1.20857600 \\
\hline $\mathrm{C}$ & -4.10561600 & 4.15055300 & 0.68545700 \\
\hline $\mathrm{H}$ & -2.53735500 & 3.17337100 & 1.80109900 \\
\hline $\mathrm{H}$ & -4.03787300 & 1.03807900 & -1.63369000 \\
\hline $\mathrm{H}$ & -5.66218800 & 2.83663100 & -2.04741500 \\
\hline $\mathrm{H}$ & -4.13007900 & 5.01931400 & 1.34896000 \\
\hline $\mathrm{H}$ & -5.72167000 & 4.85884700 & -0.57665800 \\
\hline $\mathrm{C}$ & -3.09124700 & -0.93581600 & 0.22285500 \\
\hline $\mathrm{H}$ & -2.19009100 & -1.56112800 & 0.20962900 \\
\hline $\mathrm{C}$ & -2.77547700 & 0.33702800 & 1.00186300 \\
\hline $\mathrm{H}$ & -3.64644600 & 0.65482500 & 1.57889000 \\
\hline $\mathrm{H}$ & -3.32768600 & -0.70579100 & -0.82759600 \\
\hline $\mathrm{C}$ & -4.26514500 & -1.77757600 & 0.78735900 \\
\hline $\mathrm{C}$ & -5.58911200 & -1.00704700 & 0.66207900 \\
\hline $\mathrm{H}$ & -6.42551900 & -1.60731500 & 1.05457700 \\
\hline $\mathrm{H}$ & -5.57065000 & -0.06052100 & 1.22543800 \\
\hline $\mathrm{H}$ & -5.80843400 & -0.76396800 & -0.38954400 \\
\hline $\mathrm{C}$ & -4.01024800 & -2.14135100 & 2.25921200 \\
\hline $\mathrm{H}$ & -3.04806000 & -2.66249000 & 2.37789400 \\
\hline $\mathrm{H}$ & -3.99007100 & -1.24876000 & 2.90457600 \\
\hline $\mathrm{H}$ & -4.80908800 & -2.79942100 & 2.63713500 \\
\hline $\mathrm{C}$ & -4.35042400 & -3.06677500 & -0.04447100 \\
\hline $\mathrm{H}$ & -5.18250500 & -3.70298400 & 0.29752800 \\
\hline $\mathrm{H}$ & -4.51381500 & -2.83978100 & -1.11060300 \\
\hline $\mathrm{H}$ & -3.41982800 & -3.65149300 & 0.03758000 \\
\hline $\mathrm{C}$ & -1.67749300 & 0.18359500 & 2.01861200 \\
\hline $\mathrm{O}$ & -0.90517200 & -0.74887300 & 2.11694700 \\
\hline $\mathrm{O}$ & -1.68481600 & 1.20383800 & 2.90174600 \\
\hline $\mathrm{C}$ & -0.67945900 & 1.17284100 & 3.91414700 \\
\hline $\mathrm{H}$ & 0.32386900 & 1.19998300 & 3.46473900 \\
\hline $\mathrm{H}$ & -0.84100500 & 2.06358600 & 4.53220600 \\
\hline $\mathrm{H}$ & -0.76938200 & 0.26350400 & 4.52578700 \\
\hline $\mathrm{Br}$ & -0.80119300 & 2.30851600 & -2.56719200 \\
\hline $\mathrm{C}$ & 1.35400700 & 1.51713100 & 0.37372700 \\
\hline $\mathrm{C}$ & 2.26462900 & 3.46989500 & 0.90428600 \\
\hline $\mathrm{C}$ & 0.72502000 & 3.60586100 & 0.78049900 \\
\hline $\mathrm{N}$ & 0.28971000 & 2.20674000 & 0.56943000 \\
\hline $\mathrm{H}$ & 2.82970500 & 4.14569800 & 0.25125500 \\
\hline $\mathrm{H}$ & 0.45231300 & 4.14146300 & -0.14481400 \\
\hline $\mathrm{C}$ & 0.29117800 & -1.67083100 & -0.97424600 \\
\hline $\mathrm{C}$ & 1.82768100 & -1.87208600 & -0.86202600 \\
\hline $\mathrm{C}$ & 1.24685800 & 0.13810800 & -0.11864000 \\
\hline
\end{tabular}




$\begin{array}{lrrr}\mathrm{N} & 0.11324700 & -0.27861300 & -0.53199100 \\ \mathrm{H} & -0.23221800 & -2.31013000 & -0.24449200 \\ \mathrm{H} & 2.32277900 & -1.91724100 & -1.84367600 \\ \mathrm{H} & 2.61381300 & 3.57625200 & 1.94264600 \\ \mathrm{H} & 2.12131600 & -2.73901100 & -0.25810700 \\ \mathrm{O} & 2.54047600 & 2.10283100 & 0.48444100 \\ \mathrm{O} & 2.30256500 & -0.66932200 & -0.18465100 \\ \mathrm{C} & 0.09288700 & 4.29931600 & 1.97576600 \\ \mathrm{H} & 0.53307900 & 5.30172200 & 2.09177900 \\ \mathrm{H} & -0.98720600 & 4.42019600 & 1.83570400 \\ \mathrm{H} & 0.27381500 & 3.73370500 & 2.90109500 \\ \mathrm{C} & -0.28053000 & -1.90098000 & -2.36537000 \\ \mathrm{H} & -0.10613200 & -2.94011600 & -2.68357000 \\ \mathrm{H} & -1.36419300 & -1.71535000 & -2.36816300 \\ \mathrm{H} & 0.18728400 & -1.22036900 & -3.09234400\end{array}$

UB3LYP-D3(BJ)/def2-SVP-CPCM(THF)

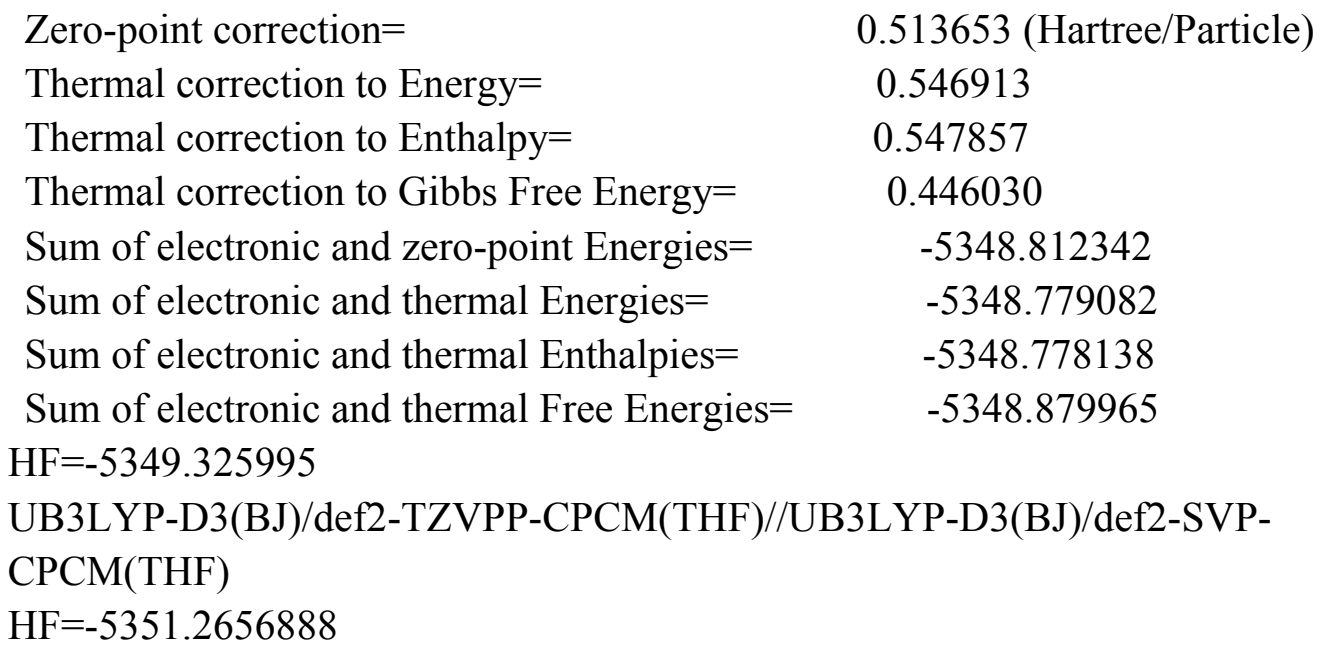

$\begin{array}{lrrr}{ }^{2} \mathbf{F}-\mathbf{T S}-\mathbf{H}^{S} & & & \\ \mathrm{Ni} & -1.59518000 & 1.00258500 & -0.43897600 \\ \mathrm{C} & -3.07256300 & 2.05375900 & 0.35638700 \\ \mathrm{C} & -4.55523600 & 4.45728300 & 0.47162600 \\ \mathrm{C} & -2.69199900 & 3.09862100 & 1.21500400 \\ \mathrm{C} & -4.25145100 & 2.20871900 & -0.40029900 \\ \mathrm{C} & -4.97108200 & 3.40265100 & -0.35268000 \\ \mathrm{C} & -3.41283500 & 4.29575800 & 1.25985500 \\ \mathrm{H} & -1.82251400 & 2.97834000 & 1.85752800 \\ \mathrm{H} & -4.58638200 & 1.39333400 & -1.03870800 \\ \mathrm{H} & -5.86919000 & 3.51067400 & -0.96678800 \\ \mathrm{H} & -3.07851800 & 5.09957200 & 1.92116100 \\ \mathrm{H} & -5.12468600 & 5.38899500 & 0.50667600 \\ \mathrm{Br} & -2.32484900 & 0.68384800 & -2.78016300 \\ \mathrm{C} & 1.31092500 & 1.73111300 & -0.21017300\end{array}$




\begin{tabular}{|c|c|c|c|}
\hline $\mathrm{C}$ & 2.08664400 & 3.80959000 & -0.21828600 \\
\hline $\mathrm{C}$ & 0.69666000 & 3.74617000 & 0.46687300 \\
\hline $\mathrm{N}$ & 0.27602500 & 2.34784900 & 0.22104200 \\
\hline $\mathrm{H}$ & 2.06282400 & 4.28505500 & -1.20937000 \\
\hline $\mathrm{H}$ & -0.02257300 & 4.42200200 & -0.01821700 \\
\hline $\mathrm{C}$ & 0.40935900 & -1.73404500 & -0.86125900 \\
\hline $\mathrm{C}$ & 1.96257500 & -1.77976200 & -0.91179400 \\
\hline $\mathrm{C}$ & 1.25596700 & 0.28926800 & -0.50632100 \\
\hline $\mathrm{N}$ & 0.12976100 & -0.30344300 & -0.61526900 \\
\hline $\mathrm{H}$ & 0.01815200 & -2.28658400 & 0.00526500 \\
\hline $\mathrm{H}$ & 2.35181800 & -2.05193500 & -1.90348500 \\
\hline $\mathrm{H}$ & 2.86635100 & 4.27297000 & 0.39898900 \\
\hline $\mathrm{H}$ & 2.41311500 & -2.42657400 & -0.14812000 \\
\hline $\mathrm{O}$ & 2.43937400 & 2.40788000 & -0.41446700 \\
\hline $\mathrm{O}$ & 2.38014500 & -0.40909400 & -0.63601500 \\
\hline $\mathrm{C}$ & 0.76627900 & 4.04665100 & 1.96358600 \\
\hline $\mathrm{H}$ & 1.10269000 & 5.08285400 & 2.12022200 \\
\hline $\mathrm{H}$ & -0.21380400 & 3.93109800 & 2.44379700 \\
\hline $\mathrm{H}$ & 1.47862700 & 3.37006300 & 2.46115400 \\
\hline $\mathrm{C}$ & -0.26356300 & -2.26217800 & -2.11896000 \\
\hline $\mathrm{H}$ & 0.00974900 & -3.31942000 & -2.26364900 \\
\hline $\mathrm{H}$ & -1.35233400 & -2.18430200 & -2.02276300 \\
\hline $\mathrm{H}$ & 0.04964500 & -1.68778800 & -3.00319800 \\
\hline $\mathrm{C}$ & -1.67132200 & 0.15551100 & 2.22174400 \\
\hline $\mathrm{H}$ & -1.07029100 & 1.07652100 & 2.24927700 \\
\hline $\mathrm{C}$ & -2.79638900 & 0.28424600 & 1.20131000 \\
\hline $\mathrm{H}$ & -3.73433200 & 0.56752100 & 1.68339800 \\
\hline $\mathrm{H}$ & -0.99130900 & -0.63857000 & 1.88220700 \\
\hline $\mathrm{C}$ & -2.12704600 & -0.15838200 & 3.66988100 \\
\hline $\mathrm{C}$ & -2.99105200 & -1.42928500 & 3.70141400 \\
\hline $\mathrm{H}$ & -3.25079900 & -1.69479400 & 4.73882600 \\
\hline $\mathrm{H}$ & -3.93630300 & -1.29507700 & 3.15164400 \\
\hline $\mathrm{H}$ & -2.45918600 & -2.28068700 & 3.24979800 \\
\hline $\mathrm{C}$ & -2.91603200 & 1.02521900 & 4.25199800 \\
\hline $\mathrm{H}$ & -2.29982000 & 1.93823800 & 4.27441300 \\
\hline $\mathrm{H}$ & -3.82050900 & 1.24758700 & 3.66386300 \\
\hline $\mathrm{H}$ & -3.23842600 & 0.80810900 & 5.28299200 \\
\hline $\mathrm{C}$ & -0.86252600 & -0.38317800 & 4.51351000 \\
\hline $\mathrm{H}$ & -1.12004100 & -0.58515800 & 5.56573000 \\
\hline $\mathrm{H}$ & -0.28265400 & -1.24060700 & 4.13525700 \\
\hline $\mathrm{H}$ & -0.20865300 & 0.50397100 & 4.48903200 \\
\hline $\mathrm{C}$ & -3.09927800 & -0.96617600 & 0.42365600 \\
\hline $\mathrm{O}$ & -2.39923100 & -1.95763600 & 0.35678300 \\
\hline $\mathrm{O}$ & -4.30428700 & -0.88514700 & -0.16467800 \\
\hline
\end{tabular}




$\begin{array}{llll}\mathrm{C} & -4.65092600 & -1.92813800 & -1.07759900 \\ \mathrm{H} & -4.50604400 & -2.91601200 & -0.61915300 \\ \mathrm{H} & -5.70641600 & -1.77378300 & -1.33118300 \\ \mathrm{H} & -4.03082300 & -1.84165900 & -1.98268900\end{array}$

UB3LYP-D3(BJ)/def2-SVP-CPCM(THF)

Zero-point correction=

Thermal correction to Energy=

0.514313 (Hartree/Particle)

Thermal correction to Enthalpy=

Thermal correction to Gibbs Free Energy=

Sum of electronic and zero-point Energies=

Sum of electronic and thermal Energies=

Sum of electronic and thermal Enthalpies=

Sum of electronic and thermal Free Energies= $\mathrm{HF}=-5349.3255474$

$\begin{array}{lrrr}{ }^{2} \mathbf{G}-\mathbf{T S}-F^{,} \boldsymbol{R} & & & \\ \mathrm{Ni} & -1.44907600 & 1.16914700 & -0.36769400 \\ \mathrm{C} & -1.93655600 & 1.24982600 & 1.54737300 \\ \mathrm{C} & -2.52591200 & 1.66939600 & 4.29540900 \\ \mathrm{C} & -1.13689500 & 0.69809000 & 2.56195300 \\ \mathrm{C} & -3.04134700 & 2.02106700 & 1.95214600 \\ \mathrm{C} & -3.33942900 & 2.22830000 & 3.30407500 \\ \mathrm{C} & -1.41859400 & 0.90307900 & 3.91856700 \\ \mathrm{H} & -0.26465300 & 0.08647300 & 2.30688600 \\ \mathrm{H} & -3.68714300 & 2.47407100 & 1.19265300 \\ \mathrm{H} & -4.20847800 & 2.83073900 & 3.58615600 \\ \mathrm{H} & -0.77500600 & 0.45898100 & 4.68408500 \\ \mathrm{H} & -2.75300400 & 1.82928000 & 5.35293300 \\ \mathrm{C} & -4.02419000 & -1.35321300 & -1.06236600 \\ \mathrm{H} & -3.40727300 & -2.26128000 & -1.12525900 \\ \mathrm{C} & -4.05582900 & -0.88749500 & 0.34322100 \\ \mathrm{H} & -4.58292800 & 0.02601200 & 0.61666900 \\ \mathrm{H} & -3.55464700 & -0.57823100 & -1.69312400 \\ \mathrm{C} & -5.42353400 & -1.64719100 & -1.68817000 \\ \mathrm{C} & -6.28570300 & -0.37502300 & -1.68829100 \\ \mathrm{H} & -7.23336300 & -0.55210000 & -2.22208000 \\ \mathrm{H} & -6.53732500 & -0.05469900 & -0.66536800 \\ \mathrm{H} & -5.76116300 & 0.45845300 & -2.18194500 \\ \mathrm{C} & -6.12565200 & -2.75839200 & -0.89407400 \\ \mathrm{H} & -5.51771000 & -3.67756000 & -0.87500300 \\ \mathrm{H} & -6.30410600 & -2.45065200 & 0.14897600 \\ \mathrm{H} & -7.10087600 & -3.00473600 & -1.34445000 \\ \mathrm{C} & -5.19382100 & -2.10776000 & -3.13490200 \\ \mathrm{H} & -6.15082300 & -2.34300200 & -3.62805200\end{array}$




$\begin{array}{lrrr}\mathrm{H} & -4.69204700 & -1.32288200 & -3.72371200 \\ \mathrm{H} & -4.56354100 & -3.01132700 & -3.16801800 \\ \mathrm{C} & -3.50821500 & -1.68210200 & 1.42402700 \\ \mathrm{O} & -2.79477700 & -2.67132900 & 1.28362000 \\ \mathrm{O} & -3.87835300 & -1.22803100 & 2.63633500 \\ \mathrm{C} & -3.35729400 & -1.92483100 & 3.76276900 \\ \mathrm{H} & -2.25825300 & -1.91731000 & 3.74864700 \\ \mathrm{H} & -3.71838600 & -1.38852900 & 4.64762800 \\ \mathrm{H} & -3.70867900 & -2.96832000 & 3.77549300 \\ \mathrm{Br} & -2.93119000 & 2.34219200 & -1.88181500 \\ \mathrm{C} & 1.33365200 & 1.33579200 & -0.00228100 \\ \mathrm{C} & 2.33670500 & 3.27342900 & 0.43003400 \\ \mathrm{C} & 0.84162400 & 3.51008800 & 0.06256700 \\ \mathrm{~N} & 0.36187000 & 2.14583600 & -0.20947900 \\ \mathrm{H} & 3.04244000 & 3.72846600 & -0.27620700 \\ \mathrm{H} & 0.75569000 & 4.09057000 & -0.86956600 \\ \mathrm{C} & -0.15783500 & -1.92898500 & -0.52342200 \\ \mathrm{C} & 1.26577700 & -2.31286100 & -0.03351400 \\ \mathrm{C} & 1.05700800 & -0.10278200 & -0.12867600 \\ \mathrm{~N} & -0.14505500 & -0.45850200 & -0.40427400 \\ \mathrm{H} & -0.93449400 & -2.32350300 & 0.14780000 \\ \mathrm{H} & 1.81734700 & -2.95448400 & -0.73245800 \\ \mathrm{H} & 2.58412500 & 3.58175800 & 1.45507400 \\ \mathrm{H} & 1.27547400 & -2.75887600 & 0.97032300 \\ \mathrm{O} & 2.51215100 & 1.82104800 & 0.35556500 \\ \mathrm{O} & 1.97892600 & -1.03498300 & 0.05403900 \\ \mathrm{C} & 0.01458700 & 4.17924800 & 1.15341600 \\ \mathrm{H} & 0.38770000 & 5.19812700 & 1.33792400 \\ \mathrm{H} & -1.03834700 & 4.23699500 & 0.84454200 \\ \mathrm{H} & 0.06334600 & 3.60129600 & 2.08807800 \\ \mathrm{C} & -0.45632900 & -2.34413500 & -1.96099500 \\ \mathrm{H} & -0.50001200 & -3.44136300 & -2.03573000 \\ \mathrm{H} & -1.42440200 & -1.93699200 & -2.28313200 \\ \mathrm{H} & -3281900 & -1.97525100 & -2.64632000\end{array}$

UB3LYP-D3(BJ)/def2-SVP-CPCM(THF)

Zero-point correction=

Thermal correction to Energy=

Thermal correction to Enthalpy=

Thermal correction to Gibbs Free Energy=

Sum of electronic and zero-point Energies=

Sum of electronic and thermal Energies=

Sum of electronic and thermal Enthalpies=

Sum of electronic and thermal Free Energies=

0.511376 (Hartree/Particle)

0.545355

0.546300

0.441225

$-5348.808231$

$-5348.774252$

$-5348.773307$

$-5348.878382$ 
UB3LYP-D3(BJ)/def2-TZVPP-CPCM(THF)//UB3LYP-D3(BJ)/def2-SVPCPCM(THF)

$\mathrm{HF}=-5351.2635068$

${ }^{2}$ G-TS-F ${ }^{S}$

$\mathrm{Ni}$

C

$-1.16710600$

1.21767400

$-0.93713300$

$-2.23585400$

2.11209700

0.46574000

$\mathrm{C}$

$-3.71213300$

3.56739700

2.41356500

C

$-1.76581600$

2.26542100

1.78172700

$\mathrm{C}$

$\mathrm{C}$

$-3.46643200$

2.71961000

0.15358600

$-4.20034700$

3.43453100

1.10895700

C

$-2.48720600$

2.98024900

2.74763400

$\mathrm{H}$

$\mathrm{H}$

$-0.81199900$

1.81569800

2.07884100

$\mathrm{H}$

$-3.86749500$

2.62639600

$-0.86108700$

$\mathrm{H}$

$-5.15747800$

3.88892800

0.83518000

$-2.09295100$

3.07824200

3.76375600

$\mathrm{H}$

$\mathrm{Br}$

$-4.28170900$

4.12272600

3.16349900

C

$-2.03350800$

1.38336900

$-3.19548100$

1.40820200

2.18266200

$-0.29276700$

C

2.04126200

4.23752400

$-0.85958200$

C

$\mathrm{N}$

0.65008200

3.91088100

$-1.47980800$

0.40595100

2.54222600

$-1.00280200$

$\mathrm{H}$

2.82295300

4.43632500

$-1.60309800$

$\mathrm{H}$

0.70730300

3.87473300

$-2.57932900$

C

0.49638000

$-1.13879700$

0.84992800

C

1.92544200

$-0.98516900$

1.44658100

C

1.35603200

0.84775800

0.32344200

0.28995300

0.15074200

0.16425900

$-0.25090700$

$-1.21461700$

1.65347900

$\mathrm{H}$

2.64499000

$-1.70669700$

1.03576000

$\mathrm{H}$

2.00472000

5.05709300

$-0.12852600$

$\mathrm{H}$

1.95375400

$-1.01494500$

2.54299100

2.42046900

3.02144400

$-0.13414200$

2.35697600

0.34992300

1.03036100

C

$-0.47391100$

4.85119700

$-1.06460200$

$\mathrm{H}$

$-0.26997700$

5.86923300

$-1.42973300$

$-1.42564900$

4.50425100

$-1.48980600$

$\mathrm{H}$

$-0.57632900$

4.87513600

0.03061000

0.33108300

$-2.30985100$

$-0.10965100$

$\mathrm{H}$

0.48318700

$-3.25893400$

0.42747400

$-0.67876800$

$-2.31326000$

$-0.54510400$

$\mathrm{H}$

1.07026400

$-2.24725900$

$-0.92373500$

C

$-3.07004700$

$-1.13329400$

1.35338000

$\mathrm{H}$

$-2.39395900$

$-0.33801900$

1.71043500 


\begin{tabular}{|c|c|c|c|}
\hline $\mathrm{C}$ & -3.73556600 & -0.66173200 & 0.11815600 \\
\hline $\mathrm{H}$ & -4.40265300 & 0.19844200 & 0.14596500 \\
\hline $\mathrm{H}$ & -2.45744300 & -2.01548600 & 1.11725900 \\
\hline $\mathrm{C}$ & -4.03025700 & -1.47958900 & 2.53007900 \\
\hline $\mathrm{C}$ & -4.98666400 & -2.60404200 & 2.10652500 \\
\hline $\mathrm{H}$ & -5.65003800 & -2.88916300 & 2.93909200 \\
\hline $\mathrm{H}$ & -5.62106100 & -2.28919500 & 1.26218700 \\
\hline $\mathrm{H}$ & -4.42937700 & -3.50168600 & 1.79245700 \\
\hline $\mathrm{C}$ & -4.83059400 & -0.23690900 & 2.95019500 \\
\hline $\mathrm{H}$ & -4.16169400 & 0.60275200 & 3.19591800 \\
\hline $\mathrm{H}$ & -5.50903100 & 0.10038900 & 2.15162000 \\
\hline $\mathrm{H}$ & -5.44712900 & -0.45799600 & 3.83644800 \\
\hline $\mathrm{C}$ & -3.16499400 & -1.94952500 & 3.70858900 \\
\hline $\mathrm{H}$ & -3.79203500 & -2.21851300 & 4.57393500 \\
\hline $\mathrm{H}$ & -2.56767100 & -2.83434500 & 3.43422500 \\
\hline $\mathrm{H}$ & -2.46901100 & -1.15644900 & 4.02734400 \\
\hline $\mathrm{C}$ & -3.63911800 & -1.38082900 & -1.13654100 \\
\hline $\mathrm{O}$ & -2.87391600 & -2.31091900 & -1.36533900 \\
\hline $\mathrm{O}$ & -4.51100200 & -0.91388400 & -2.05323900 \\
\hline $\mathrm{C}$ & -4.43773600 & -1.49279600 & -3.35331600 \\
\hline $\mathrm{H}$ & -4.57556900 & -2.58350900 & -3.30706600 \\
\hline $\mathrm{H}$ & -5.24272700 & -1.03296000 & -3.93926100 \\
\hline $\mathrm{H}$ & -3.46483700 & -1.26644900 & -3.81387900 \\
\hline \multicolumn{4}{|c|}{ UB3LYP-D3(BJ)/def2-SVP-CPCM(THF) } \\
\hline \multicolumn{3}{|c|}{ Zero-point correction $=$} & 0.511018 (Hartree/Particle) \\
\hline \multicolumn{3}{|c|}{ Thermal correction to Energy $=$} & 0.545176 \\
\hline \multicolumn{3}{|c|}{ Thermal correction to Enthalpy= } & 0.546120 \\
\hline \multicolumn{3}{|c|}{ Thermal correction to Gibbs Free Energy= } & 0.440427 \\
\hline \multicolumn{3}{|c|}{ Sum of electronic and zero-point Energies $=$} & -5348.807324 \\
\hline \multicolumn{3}{|c|}{ Sum of electronic and thermal Energies $=$} & -5348.773166 \\
\hline \multicolumn{3}{|c|}{ Sum of electronic and thermal Enthalpies= } & -5348.772222 \\
\hline \multicolumn{3}{|c|}{ Sum of electronic and thermal Free Energies= } & -5348.877915 \\
\hline \multicolumn{4}{|c|}{$H F=-5349.3183414$} \\
\hline \multirow{2}{*}{\multicolumn{4}{|c|}{$\begin{array}{l}\text { UB3LYP-D3(BJ)/def2-TZVPP-CPCM(THF)//UB3LYP-D3(BJ)/def2-SVP- } \\
\text { CPCM(THF) } \\
\text { HF=-5351.2631476 }\end{array}$}} \\
\hline & & & \\
\hline \multicolumn{4}{|c|}{${ }^{2} \mathrm{~F}^{, R}$} \\
\hline $\mathrm{Ni}$ & -1.48511000 & 1.00665900 & -0.05685400 \\
\hline $\mathrm{C}$ & -1.63243700 & 1.13354000 & 1.85697900 \\
\hline $\mathrm{C}$ & -1.67467200 & 1.62047400 & 4.61557800 \\
\hline $\mathrm{C}$ & -0.72152900 & 0.48489800 & 2.69366400 \\
\hline $\mathrm{C}$ & -2.58251100 & 2.00523200 & 2.39717100 \\
\hline $\mathrm{C}$ & -2.59861400 & 2.25109700 & 3.77480600 \\
\hline
\end{tabular}




\begin{tabular}{|c|c|c|c|}
\hline $\mathrm{C}$ & -0.73830600 & 0.73576700 & 4.07280400 \\
\hline $\mathrm{H}$ & -0.00567900 & -0.23229400 & 2.28840700 \\
\hline $\mathrm{H}$ & -3.30541500 & 2.49859700 & 1.74349200 \\
\hline $\mathrm{H}$ & -3.33975000 & 2.93824000 & 4.19225200 \\
\hline $\mathrm{H}$ & -0.01825100 & 0.23039700 & 4.72220500 \\
\hline $\mathrm{H}$ & -1.69023200 & 1.81193300 & 5.69129700 \\
\hline $\mathrm{C}$ & -2.82836000 & -0.98422100 & -1.35054900 \\
\hline $\mathrm{H}$ & -2.07249300 & -1.77970900 & -1.30937700 \\
\hline $\mathrm{C}$ & -2.93690700 & -0.36636300 & 0.03602900 \\
\hline $\mathrm{H}$ & -3.83769100 & 0.23429800 & 0.19021800 \\
\hline $\mathrm{H}$ & -2.47354800 & -0.23924600 & -2.08435900 \\
\hline $\mathrm{C}$ & -4.14938100 & -1.58280100 & -1.91631300 \\
\hline $\mathrm{C}$ & -5.19006800 & -0.47720100 & -2.14929400 \\
\hline $\mathrm{H}$ & -6.10160200 & -0.89705800 & -2.60487600 \\
\hline $\mathrm{H}$ & -5.48313700 & 0.01219900 & -1.20811800 \\
\hline $\mathrm{H}$ & -4.79600600 & 0.30398400 & -2.81749600 \\
\hline $\mathrm{C}$ & -4.71935300 & -2.63845500 & -0.95529300 \\
\hline $\mathrm{H}$ & -3.97649400 & -3.42187100 & -0.73774300 \\
\hline $\mathrm{H}$ & -5.02787200 & -2.19058500 & 0.00178200 \\
\hline $\mathrm{H}$ & -5.60663900 & -3.12121600 & -1.39584600 \\
\hline $\mathrm{C}$ & -3.80947300 & -2.24877300 & -3.25913400 \\
\hline $\mathrm{H}$ & -4.71019200 & -2.68510100 & -3.72060500 \\
\hline $\mathrm{H}$ & -3.38627900 & -1.51798800 & -3.96731800 \\
\hline $\mathrm{H}$ & -3.07191600 & -3.05686800 & -3.12518000 \\
\hline $\mathrm{C}$ & -2.75865600 & -1.34398100 & 1.14249200 \\
\hline $\mathrm{O}$ & -1.95654900 & -2.26090600 & 1.16162000 \\
\hline $\mathrm{O}$ & -3.61009600 & -1.12012800 & 2.16111500 \\
\hline $\mathrm{C}$ & -3.43666000 & -1.92446800 & 3.32587400 \\
\hline $\mathrm{H}$ & -2.43479500 & -1.76865800 & 3.75258600 \\
\hline $\mathrm{H}$ & -4.20268800 & -1.59852400 & 4.03952500 \\
\hline $\mathrm{H}$ & -3.56470400 & -2.99149900 & 3.09075500 \\
\hline $\mathrm{Br}$ & -2.87324100 & 2.73162000 & -1.31184100 \\
\hline $\mathrm{C}$ & 1.39060000 & 1.71692600 & -0.11416500 \\
\hline $\mathrm{C}$ & 2.00455000 & 3.85614100 & -0.04598100 \\
\hline $\mathrm{C}$ & 0.45323000 & 3.73999700 & -0.06937700 \\
\hline $\mathrm{N}$ & 0.24438200 & 2.28348700 & -0.11998300 \\
\hline $\mathrm{H}$ & 2.42379500 & 4.37130800 & -0.91951400 \\
\hline $\mathrm{H}$ & 0.03277700 & 4.16276100 & -0.99471900 \\
\hline $\mathrm{C}$ & 0.88005800 & -1.87738700 & -0.34422000 \\
\hline $\mathrm{C}$ & 2.35629100 & -1.78003500 & 0.12716600 \\
\hline $\mathrm{C}$ & 1.48608900 & 0.24567500 & -0.14477200 \\
\hline $\mathrm{N}$ & 0.44073300 & -0.46664200 & -0.32924300 \\
\hline $\mathrm{H}$ & 0.25138200 & -2.42926500 & 0.36721500 \\
\hline $\mathrm{H}$ & 3.06544500 & -2.30903600 & -0.52214900 \\
\hline
\end{tabular}




$\begin{array}{lrrr}\mathrm{H} & 2.38925200 & 4.31437200 & 0.87570400 \\ \mathrm{H} & 2.50381600 & -2.09105800 & 1.17108500 \\ \mathrm{O} & 2.47921200 & 2.47289900 & -0.07910200 \\ \mathrm{O} & 2.65867100 & -0.35192000 & 0.05163100 \\ \mathrm{C} & -0.24463900 & 4.37055200 & 1.12784100 \\ \mathrm{H} & -0.03509800 & 5.45093600 & 1.15565700 \\ \mathrm{H} & -1.32886400 & 4.22177100 & 1.04618800 \\ \mathrm{H} & 0.10230600 & 3.91554300 & 2.06791500 \\ \mathrm{C} & 0.73113200 & -2.47764900 & -1.74032900 \\ \mathrm{H} & 1.05315100 & -3.53022800 & -1.73224200 \\ \mathrm{H} & -0.31453700 & -2.43832300 & -2.07289700 \\ \mathrm{H} & 1.34818500 & -1.92904400 & -2.46941500\end{array}$

UB3LYP-D3(BJ)/def2-SVP-CPCM(THF)

Zero-point correction $=$

Thermal correction to Energy=

0.514885 (Hartree/Particle)

Thermal correction to Enthalpy=

0.548566

0.549510

Thermal correction to Gibbs Free Energy=

Sum of electronic and zero-point Energies=

0.447077

$-5348.831174$

Sum of electronic and thermal Energies=

$-5348.797494$

$-5348.796549$

Sum of electronic and thermal Enthalpies=

$-5348.898982$

Sum of electronic and thermal Free Energies=

$\mathrm{HF}=-5349.3460592$

UB3LYP-D3(BJ)/def2-TZVPP-CPCM(THF)//UB3LYP-D3(BJ)/def2-SVPCPCM(THF)

$\mathrm{HF}=-5351.2849533$

$\begin{array}{lrrr}{ }^{2} \mathbf{F}^{, S} & & & \\ \mathrm{Ni} & -1.15832800 & 1.02824200 & -0.04287800 \\ \mathrm{C} & -1.69719700 & 1.54618500 & 1.72867800 \\ \mathrm{C} & -2.26808000 & 2.54651800 & 4.28319300 \\ \mathrm{C} & -0.97276800 & 1.09321800 & 2.83330100 \\ \mathrm{C} & -2.71372900 & 2.49053800 & 1.89759000 \\ \mathrm{C} & -2.99618600 & 2.99114100 & 3.17436800 \\ \mathrm{C} & -1.25620200 & 1.59703800 & 4.11028400 \\ \mathrm{H} & -0.18121100 & 0.34926500 & 2.71346900 \\ \mathrm{H} & -3.28347900 & 2.84120100 & 1.03370900 \\ \mathrm{H} & -3.79229000 & 3.72988700 & 3.30086600 \\ \mathrm{H} & -0.68076400 & 1.24377500 & 4.97031200 \\ \mathrm{H} & -2.49178600 & 2.93543700 & 5.27951700 \\ \mathrm{Br} & -1.94475600 & 2.57466400 & -1.86583900 \\ \mathrm{C} & 1.75572700 & 1.50826100 & 0.28859400 \\ \mathrm{C} & 2.52108200 & 3.59800700 & 0.29276800 \\ \mathrm{C} & 0.98976500 & 3.58502600 & 0.02413500 \\ \mathrm{~N} & 0.66694000 & 2.15027100 & 0.09876700\end{array}$




\begin{tabular}{|c|c|c|c|}
\hline $\mathrm{H}$ & 3.11228000 & 4.04434500 & -0.51623700 \\
\hline $\mathrm{H}$ & 0.75890800 & 3.91060800 & -1.00219200 \\
\hline $\mathrm{C}$ & 0.96165300 & -2.03802800 & 0.28180400 \\
\hline $\mathrm{C}$ & 2.46587000 & -2.04135700 & 0.67365100 \\
\hline $\mathrm{C}$ & 1.73613500 & 0.03662700 & 0.37416800 \\
\hline $\mathrm{N}$ & 0.63404100 & -0.59903400 & 0.25121500 \\
\hline $\mathrm{H}$ & 0.35346300 & -2.51796800 & 1.06209200 \\
\hline $\mathrm{H}$ & 3.09802800 & -2.62330200 & -0.00945600 \\
\hline $\mathrm{H}$ & 2.78352800 & 4.07098100 & 1.24995800 \\
\hline $\mathrm{H}$ & 2.64792500 & -2.35754600 & 1.71007700 \\
\hline $\mathrm{O}$ & 2.89141300 & 2.18609800 & 0.38738600 \\
\hline $\mathrm{O}$ & 2.86442100 & -0.63983200 & 0.56730400 \\
\hline $\mathrm{C}$ & 0.16859000 & 4.41089100 & 1.00343200 \\
\hline $\mathrm{H}$ & 0.47167800 & 5.46771200 & 0.94723100 \\
\hline $\mathrm{H}$ & -0.89688000 & 4.33503100 & 0.75232800 \\
\hline $\mathrm{H}$ & 0.31030300 & 4.05248500 & 2.03426200 \\
\hline $\mathrm{C}$ & 0.66374500 & -2.67841200 & -1.07104700 \\
\hline $\mathrm{H}$ & 0.83850600 & -3.76433700 & -1.02353900 \\
\hline $\mathrm{H}$ & -0.37870700 & -2.48708800 & -1.36175100 \\
\hline $\mathrm{H}$ & 1.31935100 & -2.25300500 & -1.84765600 \\
\hline $\mathrm{C}$ & -2.41984000 & -1.43655300 & 0.97220900 \\
\hline $\mathrm{H}$ & -1.74900200 & -1.16555000 & 1.79833700 \\
\hline $\mathrm{C}$ & -2.75548100 & -0.21126000 & 0.13147700 \\
\hline $\mathrm{H}$ & -3.58767300 & 0.37127100 & 0.53119700 \\
\hline $\mathrm{H}$ & -1.86901000 & -2.14826800 & 0.34147500 \\
\hline $\mathrm{C}$ & -3.63775300 & -2.16993800 & 1.59499900 \\
\hline $\mathrm{C}$ & -3.10249000 & -3.42098900 & 2.30996900 \\
\hline $\mathrm{H}$ & -3.91902300 & -3.97950100 & 2.79589800 \\
\hline $\mathrm{H}$ & -2.60393700 & -4.09971800 & 1.59869400 \\
\hline $\mathrm{H}$ & -2.36941800 & -3.14888900 & 3.08707900 \\
\hline $\mathrm{C}$ & -4.63827900 & -2.59593900 & 0.50884300 \\
\hline $\mathrm{H}$ & -5.08805800 & -1.72667700 & 0.00619000 \\
\hline $\mathrm{H}$ & -4.14692100 & -3.21293900 & -0.26080000 \\
\hline $\mathrm{H}$ & -5.45805400 & -3.18845700 & 0.94659800 \\
\hline $\mathrm{C}$ & -4.33498000 & -1.26380600 & 2.62273400 \\
\hline $\mathrm{H}$ & -5.16791900 & -1.79648000 & 3.10969100 \\
\hline $\mathrm{H}$ & -3.63012900 & -0.94120400 & 3.40601100 \\
\hline $\mathrm{H}$ & -4.74907600 & -0.35773600 & 2.15458500 \\
\hline $\mathrm{C}$ & -3.02192300 & -0.53623400 & -1.30044400 \\
\hline $\mathrm{O}$ & -2.33697200 & -1.26314200 & -2.00007100 \\
\hline $\mathrm{O}$ & -4.13934200 & 0.05480600 & -1.75692700 \\
\hline $\mathrm{C}$ & -4.40284200 & -0.06888700 & -3.15319900 \\
\hline $\mathrm{H}$ & -4.44827300 & -1.12557100 & -3.45430400 \\
\hline $\mathrm{H}$ & -5.36947800 & 0.41865500 & -3.32754400 \\
\hline
\end{tabular}




\begin{tabular}{|c|c|}
\hline-3.61518400 & -3.72908100 \\
\hline \multicolumn{2}{|l|}{ UB3LYP-D3(BJ)/def2-SVP-CPCM(THF) } \\
\hline Zero-point correction $=$ & 0.514932 (Hartree/Particle) \\
\hline Thermal correction to Energy= & 0.548672 \\
\hline Thermal correction to Enthalpy= & 0.549616 \\
\hline Thermal correction to Gibbs Free Energy= & 0.446374 \\
\hline Sum of electronic and zero-point Energies= & -5348.826695 \\
\hline Sum of electronic and thermal Energies $=$ & -5348.792955 \\
\hline Sum of electronic and thermal Enthalpies= & -5348.792011 \\
\hline Sum of electronic and thermal Free Energies= & -5348.895254 \\
\hline \multicolumn{2}{|l|}{$H F=-5349.3416273$} \\
\hline \multicolumn{2}{|c|}{$\begin{array}{l}\text { UB3LYP-D3(BJ)/def2-TZVPP-CPCM(THF)//UB3LYP-D3(BJ)/def2-SVP- } \\
\text { CPCM(THF) }\end{array}$} \\
\hline $\begin{array}{l}\mathrm{CPCM}(\mathrm{THF}) \\
\mathrm{HF}=-53512810774\end{array}$ & \\
\hline
\end{tabular}

$\begin{array}{lrrr}{ }^{2} \mathbf{F}^{\prime}-\mathbf{T S}-\mathbf{H}^{\boldsymbol{R}} & & & \\ \mathrm{Ni} & -1.35061000 & 1.10235700 & -0.28688000 \\ \mathrm{C} & -1.90565700 & 0.92023100 & 1.56509100 \\ \mathrm{C} & -1.63770400 & 1.91075400 & 4.18887100 \\ \mathrm{C} & -0.92834100 & 0.39468600 & 2.43067700 \\ \mathrm{C} & -2.79021800 & 1.89920600 & 2.05240400 \\ \mathrm{C} & -2.64490400 & 2.39875600 & 3.34785600 \\ \mathrm{C} & -0.78404000 & 0.90368800 & 3.72406200 \\ \mathrm{H} & -0.28393600 & -0.41842200 & 2.09696600 \\ \mathrm{H} & -3.56858600 & 2.29331000 & 1.39670800 \\ \mathrm{H} & -3.32533500 & 3.17755200 & 3.70225900 \\ \mathrm{H} & -0.00254100 & 0.50211000 & 4.37456200 \\ \mathrm{H} & -1.52886600 & 2.30073100 & 5.20350100 \\ \mathrm{C} & -2.75675300 & -0.96251600 & -1.06634100 \\ \mathrm{H} & -1.99510000 & -1.74941400 & -1.02438000 \\ \mathrm{C} & -2.93097200 & -0.39521600 & 0.35085800 \\ \mathrm{H} & -3.86815200 & 0.15135400 & 0.46510200 \\ \mathrm{H} & -2.40072700 & -0.20634500 & -1.78800500 \\ \mathrm{C} & -4.05553500 & -1.55843900 & -1.68511500 \\ \mathrm{C} & -5.10984200 & -0.46212100 & -1.90176900 \\ \mathrm{H} & -5.99111200 & -0.87607300 & -2.41783700 \\ \mathrm{H} & -5.45705200 & -0.03379300 & -0.94883200 \\ \mathrm{H} & -4.70482400 & 0.36488900 & -2.50411600 \\ \mathrm{C} & -4.63687300 & -2.67001100 & -0.79670800 \\ \mathrm{H} & -3.88881000 & -3.45184700 & -0.59332700 \\ \mathrm{H} & -4.98998300 & -2.27856800 & 0.16946000 \\ \mathrm{H} & -5.49966000 & -3.14270100 & -1.29300500 \\ \mathrm{C} & -3.66237500 & -2.15502300 & -3.04685600 \\ \mathrm{H} & -4.54132600 & -2.57973200 & -3.55784900\end{array}$




$\begin{array}{lrrr}\mathrm{H} & -3.22691700 & -1.38562300 & -3.70478300 \\ \mathrm{H} & -2.91870500 & -2.95973200 & -2.92765300 \\ \mathrm{C} & -2.80650100 & -1.46659800 & 1.38088800 \\ \mathrm{O} & -1.98884300 & -2.36752700 & 1.35917900 \\ \mathrm{O} & -3.70163000 & -1.32776200 & 2.37230800 \\ \mathrm{C} & -3.57949600 & -2.23033500 & 3.47167200 \\ \mathrm{H} & -2.60013300 & -2.10892700 & 3.95847900 \\ \mathrm{H} & -4.38325300 & -1.97135300 & 4.17071200 \\ \mathrm{H} & -3.68565400 & -3.27284200 & 3.13778900 \\ \mathrm{Br} & -2.81648600 & 2.72935200 & -1.51829100 \\ \mathrm{C} & 1.47453000 & 1.72501800 & -0.06622400 \\ \mathrm{C} & 2.12259700 & 3.82627900 & 0.27860900 \\ \mathrm{C} & 0.58978200 & 3.76692200 & 0.01544100 \\ \mathrm{~N} & 0.35369500 & 2.32909700 & -0.18977800 \\ \mathrm{H} & 2.67898000 & 4.40569600 & -0.46904900 \\ \mathrm{H} & 0.32782300 & 4.28398600 & -0.92110600 \\ \mathrm{C} & 0.79903100 & -1.81411200 & -0.53277400 \\ \mathrm{C} & 2.26212600 & -1.82683100 & -0.01247100 \\ \mathrm{C} & 1.51209200 & 0.25763900 & -0.18017100 \\ \mathrm{~N} & 0.43521300 & -0.38535800 & -0.43611500 \\ \mathrm{H} & 0.12680100 & -2.37937700 & 0.12874600 \\ \mathrm{H} & 2.96316700 & -2.35294400 & -0.67283100 \\ \mathrm{H} & 2.37257000 & 4.18477700 & 1.28710500 \\ \mathrm{H} & 2.35728900 & -2.21059500 & 1.01310500 \\ \mathrm{O} & 2.56764900 & 2.43634000 & 0.18434800 \\ \mathrm{O} & 2.64260800 & -0.41585600 & 0.01291100 \\ \mathrm{C} & -0.27355800 & 4.31182600 & 1.14543000 \\ \mathrm{H} & -0.05563300 & 5.37876700 & 1.30712900 \\ \mathrm{H} & -1.33432100 & 4.19976900 & 0.88514800 \\ \mathrm{H} & -0.08577800 & 3.76257200 & 2.08060300 \\ \mathrm{C} & 0.66192900 & -2.31229000 & -1.97014100 \\ \mathrm{H} & 0.91903500 & -3.38123800 & -2.02401000 \\ \mathrm{H} & -23515700 & -2.18525700 & -2.33207000 \\ \mathrm{H} & -1.75608400 & -2.64074500\end{array}$

UB3LYP-D3(BJ)/def2-SVP-CPCM(THF)

Zero-point correction=

Thermal correction to Energy=

0.514921 (Hartree/Particle)

0.547925

Thermal correction to Enthalpy=

0.548869

0.448088

$-5348.815868$

$-5348.782863$

$-5348.781919$

$-5348.882700$

Sum of electronic and thermal Free Energies= $\mathrm{HF}=-5349.3307884$ 
UB3LYP-D3(BJ)/def2-TZVPP-CPCM(THF)//UB3LYP-D3(BJ)/def2-SVPCPCM(THF)

$\mathrm{HF}=-5351.2703752$

\section{${ }^{2} \mathbf{F}^{\prime}-\mathrm{TS}-\mathrm{H}^{S}$}

$\mathrm{Ni}$

C

$\mathrm{C}$

C

C

C

C

$\mathrm{H}$

$\mathrm{H}$

$\mathrm{H}$

$\mathrm{H}$

$\mathrm{H}$

$\mathrm{Br}$

C

C

C

$\mathrm{N}$

$\mathrm{H}$

$\mathrm{H}$

C

C

C

$\mathrm{N}$

$\mathrm{H}$

$\mathrm{H}$

$\mathrm{H}$

$\mathrm{H}$

$\mathrm{O}$

$\mathrm{O}$

C

$\mathrm{H}$

$\mathrm{H}$

$\mathrm{H}$

C

$\mathrm{H}$

$\mathrm{H}$

$\mathrm{H}$

C

$\mathrm{H}$

$-1.00974200$

$-2.05186800$

$-2.48245700$

$-1.42638300$

$-2.93020200$

$-3.13294900$

$-1.63000400$

$-0.75254400$

$-3.44224000$

$-3.80743800$

$-1.11455800$

$-2.64693900$

$-1.49108600$

1.80937100

2.60362800

1.12703600

0.76176900

3.31002000

1.05662000

0.87924900

2.38018100

1.73132700

0.60425800

0.25769500

2.99522500

2.72481000

2.54791900

2.94454800

2.82619700

0.18961600

0.51330200

$-0.83030300$

0.17179300

0.55454500

0.71604800

$-0.49171200$

1.20273000

$-2.32884900$

$-1.58795400$
0.95255300

1.41737600

3.12470100

1.16233300

2.50991500

3.36120400

2.02357900

0.31000500

2.68703800

4.21516100

1.82860400

3.79041800

2.37337200

1.40412500

3.46802700

3.50329100

2.07832900

3.96452000

3.85511700

$-2.08729200$

$-2.19122600$

$-0.06559900$

$-0.63969300$

$-2.62777300$

$-2.69830500$

3.86244500

$-2.64897300$

2.04584000

$-0.80330800$

4.32870600

5.38078500

4.27710600

3.94762000

$-2.56728600$

$-3.65351800$

$-2.33825500$

$-2.06321600$

$-1.54345800$

$-1.33308100$
$-0.16249500$

1.39617200

3.58755600

2.62403200

1.28190800

2.36870500

3.70922800

2.73427200

0.33433800

2.26437400

4.65340700

4.43792700

$-2.16167900$

0.49132600

0.71137100

0.22647100

0.21328100

0.03499200

$-0.81552100$

0.14732100

0.53447100

0.47373500

0.26986700

0.87428400

$-0.22054500$

1.73110800

1.51884200

0.74906600

0.61346600

1.09401500

1.10641200

0.69245900

2.12598900

$-1.26397000$

$-1.34216800$

$-1.51244000$

$-1.99835000$

1.08644000

1.86911200 


$\begin{array}{rrrr}\mathrm{C} & -2.81630900 & -0.26070100 & 0.44228500 \\ \mathrm{H} & -3.71702700 & 0.12053400 & 0.91958000 \\ \mathrm{H} & -1.81922900 & -2.13842600 & 0.31916200 \\ \mathrm{C} & -3.44562800 & -2.41278400 & 1.72120600 \\ \mathrm{C} & -2.79361700 & -3.71916200 & 2.20143700 \\ \mathrm{H} & -3.53359400 & -4.37652700 & 2.68587400 \\ \mathrm{H} & -2.34982000 & -4.27230300 & 1.35766800 \\ \mathrm{H} & -1.99250000 & -3.51635900 & 2.93110400 \\ \mathrm{C} & -4.53515300 & -2.74098100 & 0.68722500 \\ \mathrm{H} & -5.06201300 & -1.83574100 & 0.34699400 \\ \mathrm{H} & -4.10396900 & -3.23142400 & -0.19948500 \\ \mathrm{H} & -5.28925900 & -3.41761300 & 1.12050500 \\ \mathrm{C} & -4.06838100 & -1.68866500 & 2.92558600 \\ \mathrm{H} & -4.83781700 & -2.31727300 & 3.40201800 \\ \mathrm{H} & -3.30369800 & -1.45267300 & 3.68297400 \\ \mathrm{H} & -4.55115300 & -0.74313600 & 2.63263400 \\ \mathrm{C} & -3.12846200 & -0.39266900 & -1.01378700 \\ \mathrm{O} & -2.59858800 & -1.16483200 & -1.79455000 \\ \mathrm{O} & -4.15517900 & 0.40347400 & -1.36213400 \\ \mathrm{C} & -4.51991800 & 0.41151000 & -2.74312700 \\ \mathrm{H} & -4.78335100 & -0.60131600 & -3.08131100 \\ \mathrm{H} & -5.38800700 & 1.07668800 & -2.82397800 \\ \mathrm{H} & -3.68705500 & 0.79984800 & -3.34597800\end{array}$

UB3LYP-D3(BJ)/def2-SVP-CPCM(THF)

Zero-point correction=

0.514798 (Hartree/Particle)

Thermal correction to Energy=

0.547859

Thermal correction to Enthalpy=

0.548803

Thermal correction to Gibbs Free Energy=

Sum of electronic and zero-point Energies=

Sum of electronic and thermal Energies=

Sum of electronic and thermal Enthalpies=

Sum of electronic and thermal Free Energies=

0.447538

$-5348.816789$

$-5348.783728$

$-5348.782784$

$-5348.884048$

$\mathrm{HF}=-5349.3315868$

UB3LYP-D3(BJ)/def2-TZVPP-CPCM(THF)//UB3LYP-D3(BJ)/def2-SVPCPCM(THF)

$\mathrm{HF}=-5351.2701106$

$\begin{array}{lrrr}{ }^{2} \mathbf{H} & & & \\ \mathrm{Ni} & -0.49278500 & 0.48606900 & -0.92633700 \\ \mathrm{Br} & -2.70170700 & 0.22916600 & -1.64157700 \\ \mathrm{C} & 1.58555800 & 2.23323300 & -0.33883000 \\ \mathrm{C} & 1.42044200 & 4.42446900 & -0.70405400 \\ \mathrm{C} & 0.17113100 & 3.68833700 & -1.27100500 \\ \mathrm{~N} & 0.43723900 & 2.28506000 & -0.93050900\end{array}$




$\begin{array}{lrrr}\mathrm{H} & 1.99623000 & 4.96791500 & -1.46413600 \\ \mathrm{H} & 0.13666500 & 3.77347400 & -2.37022700 \\ \mathrm{C} & 1.80699400 & -1.28082200 & 0.42871900 \\ \mathrm{C} & 3.22778800 & -0.79995200 & 0.83706800 \\ \mathrm{C} & 2.01981600 & 0.92136100 & 0.10482000 \\ \mathrm{~N} & 1.18074700 & -0.05165800 & -0.08345700 \\ \mathrm{H} & 1.23658900 & -1.60388600 & 1.31594300 \\ \mathrm{H} & 4.01249700 & -1.19152200 & 0.17197200 \\ \mathrm{H} & 1.17587700 & 5.10269500 & 0.12666400 \\ \mathrm{H} & 3.48740400 & -1.02064900 & 1.88003700 \\ \mathrm{O} & 2.27134500 & 3.36366700 & -0.17215700 \\ \mathrm{O} & 3.19446800 & 0.64849700 & 0.67750700 \\ \mathrm{C} & -1.16308600 & 4.14641200 & -0.69499600 \\ \mathrm{H} & -1.38215300 & 5.18316800 & -0.99319400 \\ \mathrm{H} & -1.96647300 & 3.49160000 & -1.06332500 \\ \mathrm{H} & -1.14864300 & 4.09050000 & 0.40491100 \\ \mathrm{C} & 1.78026900 & -2.38957000 & -0.61364300 \\ \mathrm{H} & 2.21978900 & -3.31510600 & -0.21140700 \\ \mathrm{H} & 0.74081700 & -2.59529800 & -0.91033000 \\ \mathrm{H} & 2.34463400 & -2.09098100 & -1.51083300\end{array}$

UB3LYP-D3(BJ)/def2-SVP-CPCM(THF)

Zero-point correction $=$

Thermal correction to Energy=

0.201468 (Hartree/Particle)

Thermal correction to Enthalpy=

0.216791

0.217736

Thermal correction to Gibbs Free Energy=

Sum of electronic and zero-point Energies=

0.154564

$-4653.582635$

Sum of electronic and thermal Energies=

$-4653.567311$

$-4653.566367$

Sum of electronic and thermal Enthalpies=

$-4653.629539$

Sum of electronic and thermal Free Energies= $\mathrm{HF}=-4653.7841028$

UB3LYP-D3(BJ)/def2-TZVPP-CPCM(THF)//UB3LYP-D3(BJ)/def2-SVPCPCM(THF)

$\mathrm{HF}=-4654.9693954$

\section{$\mathrm{Ph}-\mathrm{C}^{R}$}

$\begin{array}{lrrr}\mathrm{C} & -2.06322000 & 0.75924400 & 1.40145300 \\ \mathrm{C} & -1.20219200 & 2.28742700 & 3.60407900 \\ \mathrm{C} & -0.87209400 & 0.43773300 & 2.07124800 \\ \mathrm{C} & -2.81642200 & 1.85302100 & 1.85059000 \\ \mathrm{C} & -2.39013000 & 2.61363900 & 2.94341800 \\ \mathrm{C} & -0.44554900 & 1.19617200 & 3.16438100 \\ \mathrm{H} & -0.28314700 & -0.41892100 & 1.73799500 \\ \mathrm{H} & -3.74583200 & 2.11199900 & 1.33666900 \\ \mathrm{H} & -2.98985700 & 3.46291800 & 3.27989700\end{array}$




$\begin{array}{lrrr}\mathrm{H} & 0.48290000 & 0.93218700 & 3.67678400 \\ \mathrm{H} & -0.86795600 & 2.87971500 & 4.45921300 \\ \mathrm{C} & -1.60285600 & 0.23179800 & -1.03371500 \\ \mathrm{H} & -0.67866100 & -0.35095500 & -0.89136000 \\ \mathrm{C} & -2.51854700 & -0.02834300 & 0.17859900 \\ \mathrm{H} & -3.54509800 & 0.28572700 & -0.04857200 \\ \mathrm{H} & -1.31145400 & 1.29385300 & -1.01530500 \\ \mathrm{C} & -2.19717300 & -0.07872800 & -2.42900000 \\ \mathrm{C} & -3.35495800 & 0.88441700 & -2.74212600 \\ \mathrm{H} & -3.74869700 & 0.70068700 & -3.75464000 \\ \mathrm{H} & -4.19307900 & 0.76390600 & -2.03788600 \\ \mathrm{H} & -3.02103000 & 1.93364800 & -2.69400800 \\ \mathrm{C} & -2.69990100 & -1.52919400 & -2.52464000 \\ \mathrm{H} & -1.90717600 & -2.24645900 & -2.26405800 \\ \mathrm{H} & -3.55585000 & -1.71073200 & -1.85594900 \\ \mathrm{H} & -3.03803600 & -1.74912400 & -3.54995200 \\ \mathrm{C} & -1.07884100 & 0.13306700 & -3.46354200 \\ \mathrm{H} & -1.45081000 & -0.04026600 & -4.48617800 \\ \mathrm{H} & -0.68469000 & 1.16127400 & -3.41659800 \\ \mathrm{H} & -0.23982800 & -0.55890800 & -3.28560400 \\ \mathrm{C} & -2.56802200 & -1.50467500 & 0.53818500 \\ \mathrm{O} & -1.61606500 & -2.25326800 & 0.54268100 \\ \mathrm{O} & -3.80230500 & -1.88577000 & 0.89516200 \\ \mathrm{C} & -3.95884400 & -3.24914300 & 1.30279600 \\ \mathrm{H} & -3.32061300 & -3.46916500 & 2.17085100 \\ \mathrm{H} & -5.01588200 & -3.36943000 & 1.56621800 \\ \mathrm{H} & -3.69041300 & -3.92966600 & 0.48155500 \\ \mathrm{HB} 3 \mathrm{P} & -\mathrm{d} & \end{array}$

UB3LYP-D3(BJ)/def2-SVP-CPCM(THF)

Zero-point correction=

Thermal correction to Energy=

0.312154 (Hartree/Particle)

Thermal correction to Enthalpy=

0.328961

0.329906

Thermal correction to Gibbs Free Energy=

Sum of electronic and zero-point Energies=

0.266965

$-695.263265$

$-695.246457$

Sum of electronic and thermal Energies=

$-695.245513$

Sum of electronic and thermal Enthalpies=

$-695.308454$

Sum of electronic and thermal Free Energies=

$\mathrm{HF}=-695.5754188$

UB3LYP-D3(BJ)/def2-TZVPP-CPCM(THF)//UB3LYP-D3(BJ)/def2-SVPCPCM(THF)

$\mathrm{HF}=-696.3521062$ 


\begin{tabular}{|c|c|c|c|}
\hline \multicolumn{4}{|c|}{$\mathbf{P h}-\mathbf{C}^{S}$} \\
\hline $\mathrm{C}$ & -1.62431900 & 0.30806800 & 1.47854800 \\
\hline $\mathrm{C}$ & -0.97130800 & 1.62015400 & 3.88275400 \\
\hline $\mathrm{C}$ & -0.57706300 & 1.23900000 & 1.52293900 \\
\hline $\mathrm{C}$ & -2.34077900 & 0.03958800 & 2.65557800 \\
\hline $\mathrm{C}$ & -2.01626100 & 0.69085200 & 3.84829100 \\
\hline $\mathrm{C}$ & -0.25194400 & 1.89228800 & 2.71546100 \\
\hline $\mathrm{H}$ & -0.01216700 & 1.45524500 & 0.61239200 \\
\hline $\mathrm{H}$ & -3.15043500 & -0.69241500 & 2.63609500 \\
\hline $\mathrm{H}$ & -2.58207200 & 0.46977900 & 4.75671900 \\
\hline $\mathrm{H}$ & 0.56743700 & 2.61514600 & 2.73178300 \\
\hline $\mathrm{H}$ & -0.71794600 & 2.12862900 & 4.81604900 \\
\hline $\mathrm{C}$ & -3.34687600 & 0.15666300 & -0.37390900 \\
\hline $\mathrm{H}$ & -3.41083100 & 1.23283700 & -0.14821900 \\
\hline $\mathrm{C}$ & -1.99587700 & -0.35884100 & 0.15939000 \\
\hline $\mathrm{H}$ & -1.19578000 & -0.13400900 & -0.55706100 \\
\hline $\mathrm{H}$ & -4.14795200 & -0.33410200 & 0.20176300 \\
\hline $\mathrm{C}$ & -3.61070500 & -0.03283900 & -1.88750600 \\
\hline $\mathrm{C}$ & -5.04911000 & 0.43504900 & -2.16660100 \\
\hline $\mathrm{H}$ & -5.28731100 & 0.35631100 & -3.23956600 \\
\hline $\mathrm{H}$ & -5.77832200 & -0.17669300 & -1.61120300 \\
\hline $\mathrm{H}$ & -5.19001600 & 1.48573100 & -1.86493100 \\
\hline $\mathrm{C}$ & -3.47183800 & -1.50511300 & -2.31028000 \\
\hline $\mathrm{H}$ & -2.43710600 & -1.86616800 & -2.20182700 \\
\hline $\mathrm{H}$ & -4.12396300 & -2.15782000 & -1.71068500 \\
\hline $\mathrm{H}$ & -3.74714300 & -1.62552400 & -3.37032700 \\
\hline $\mathrm{C}$ & -2.63766600 & 0.82863500 & -2.71044400 \\
\hline $\mathrm{H}$ & -2.85385600 & 0.73636200 & -3.78687900 \\
\hline $\mathrm{H}$ & -2.72396100 & 1.89304200 & -2.43787300 \\
\hline $\mathrm{H}$ & -1.58982300 & 0.52498300 & -2.55954300 \\
\hline $\mathrm{C}$ & -2.00977200 & -1.86560300 & 0.36138600 \\
\hline $\mathrm{O}$ & -2.93279200 & -2.49831600 & 0.82438600 \\
\hline $\mathrm{O}$ & -0.84640500 & -2.42377900 & -0.00083100 \\
\hline $\mathrm{C}$ & -0.72561200 & -3.83686400 & 0.19401700 \\
\hline $\mathrm{H}$ & -1.48292400 & -4.37315100 & -0.39627100 \\
\hline $\mathrm{H}$ & 0.28136000 & -4.10656700 & -0.14433600 \\
\hline $\mathrm{H}$ & -0.85397900 & -4.09334900 & 1.25564900 \\
\hline
\end{tabular}

UB3LYP-D3(BJ)/def2-SVP-CPCM(THF)

Zero-point correction=

Thermal correction to Energy=

0.312144 (Hartree/Particle)

0.328948

Thermal correction to Enthalpy=

0.329892

Thermal correction to Gibbs Free Energy=

Sum of electronic and zero-point Energies $=$

0.266992

$-695.263283$

$-695.246479$

Sum of electronic and thermal Energies= 
Sum of electronic and thermal Enthalpies=

Sum of electronic and thermal Free Energies=

$\mathrm{HF}=-695.575427$

UB3LYP-D3(BJ)/def2-TZVPP-CPCM(THF)//UB3LYP-D3(BJ)/def2-SVPCPCM(THF)

$\mathrm{HF}=-696.35212$
$-695.245535$

$-695.308435$ 


\section{Scheme 6}

G-TS-F ${ }^{\mathbf{A}}$

$\mathrm{Ni}$

C

C

C

C

C

C

$\mathrm{H}$

$\mathrm{H}$

$\mathrm{H}$

$\mathrm{H}$

$\mathrm{H}$

$\mathrm{C}$

$\mathrm{Br}$

C

C

C

$\mathrm{N}$

$\mathrm{H}$

$\mathrm{H}$

C

C

C

$\mathrm{N}$

$\mathrm{H}$

$\mathrm{H}$

$\mathrm{H}$

$\mathrm{H}$

$\mathrm{O}$

$\mathrm{O}$

C

$\mathrm{H}$

$\mathrm{H}$

$\mathrm{H}$

C

$\mathrm{H}$

$\mathrm{H}$

$\mathrm{H}$

C

$\mathrm{H}$

$\mathrm{H}$

$\mathrm{H}$
$-1.15616200$

$-1.51097400$

$-2.07618600$

$-0.59172800$

$-2.72082400$

$-3.00658700$

$-0.86239100$

0.37014400

$-3.46478000$

$-3.95906100$

$-0.12402200$

$-2.29439800$

$-3.43727700$

$-2.91022500$

1.50013700

1.94530000

0.41493300

0.32163400

2.41124400

0.06493500

0.96442300

2.50197500

1.63425600

0.56812000

0.46242000

3.08440100

2.20121600

2.82809800

2.52254900

2.79801500

$-0.45172800$

$-0.37202100$

$-1.50173300$

$-0.13966500$

0.55690000

0.84616500

$-0.53038600$

1.04605300

$-2.47611500$

$-1.85655800$

$-1.79213600$

$-2.99970300$
1.05293400

0.96460900

0.93597800

0.45465200

1.46922200

1.45633000

0.43507000

0.05296500

1.88238800

1.85446700

0.02709600

0.92264700

$-1.83511700$

2.07480700

2.00032300

4.16658600

3.94705000

2.48830100

4.75201600

4.40962600

$-1.58168800$

$-1.50914500$

0.53693300

$-0.16267300$

$-2.06466700$

$-1.93739000$

4.60201900

$-1.94867500$

2.82019000

$-0.07752700$

4.42998200

5.52289900

4.16517600

3.95670800

$-2.27673100$

$-3.33814700$

$-2.21730500$

$-1.80374700$

$-2.50339200$

$-3.25483400$

$-1.77438500$

$-3.01094000$
$-0.19875700$

1.74897900

4.54135300

2.68470500

2.26352400

3.63478500

4.06000500

2.34826300

1.57554700

3.99841700

4.75733000

5.61270700

$-0.04045300$

$-1.53231700$

$-0.11338500$

0.12170900

$-0.06938200$

$-0.22574600$

$-0.68080200$

$-1.00561900$

$-0.30487500$

$-0.07407800$

$-0.16314300$

$-0.31172800$

0.54724000

$-0.90111100$

1.09734400

0.87704200

0.07776300

$-0.02168400$

1.08686100

1.18964900

0.90046500

2.02996100

$-1.59711200$

$-1.56554000$

$-1.73444200$

$-2.46269900$

0.88983000

0.37455100

1.36842200

1.72246900 


$\begin{array}{lrrr}\mathrm{C} & -3.28788900 & -1.93493500 & -1.52496200 \\ \mathrm{H} & -2.74424100 & -1.06435500 & -1.94712400 \\ \mathrm{H} & -2.74409200 & -2.84439100 & -1.82886200 \\ \mathrm{H} & -4.27115500 & -1.94191900 & -2.02992600 \\ \mathrm{C} & -4.54307700 & -0.98876600 & 0.50386100 \\ \mathrm{H} & -5.53876700 & -1.41825900 & 0.26535700 \\ \mathrm{H} & -4.47778000 & -0.87411000 & 1.59583800 \\ \mathrm{H} & -4.52796700 & 0.02385700 & 0.06050700\end{array}$

UB3LYP-D3(BJ)/def2-SVP-CPCM(THF)

Zero-point correction=

Thermal correction to Energy=

0.408906 (Hartree/Particle)

0.437403

0.438347

Thermal correction to Enthalpy=

0.344341

Thermal correction to Gibbs Free Energy=

Sum of electronic and zero-point Energies=

$-5042.591832$

Sum of electronic and thermal Energies=

$-5042.563335$

Sum of electronic and thermal Enthalpies=

$-5042.562391$

Sum of electronic and thermal Free Energies=

$-5042.656397$

$\mathrm{HF}=-5043.0007384$

UB3LYP-D3(BJ)/def2-TZVPP-CPCM(THF)//UB3LYP-D3(BJ)/def2-SVP-

CPCM(THF)

$\mathrm{HF}=-5044.5965129$

$\begin{array}{crrr}\mathbf{F}^{\mathbf{A}} & & & \\ \mathrm{Ni} & -1.42218100 & 0.94115600 & 0.25189900 \\ \mathrm{C} & -1.51284000 & 1.13629900 & 2.15457700 \\ \mathrm{C} & -1.43360600 & 1.77136000 & 4.89878800 \\ \mathrm{C} & -0.48622900 & 0.64297700 & 2.96985100 \\ \mathrm{C} & -2.50142700 & 1.94710800 & 2.72589400 \\ \mathrm{C} & -2.46326100 & 2.26352300 & 4.08956700 \\ \mathrm{C} & -0.44443000 & 0.95932500 & 4.33428500 \\ \mathrm{H} & 0.29177800 & 0.00096700 & 2.54890800 \\ \mathrm{H} & -3.30378200 & 2.34566300 & 2.10044400 \\ \mathrm{H} & -3.24135100 & 2.90042400 & 4.52010400 \\ \mathrm{H} & 0.36515200 & 0.56794100 & 4.95710500 \\ \mathrm{H} & -1.40365400 & 2.01690600 & 5.96339600 \\ \mathrm{C} & -2.68024200 & -0.63234200 & 0.42132900 \\ \mathrm{Br} & -2.94264800 & 2.53403300 & -1.04161900 \\ \mathrm{C} & 1.35274400 & 1.94919300 & -0.11867300 \\ \mathrm{C} & 1.69928400 & 4.14771900 & -0.17228500 \\ \mathrm{C} & 0.18445900 & 3.84058200 & -0.00447800 \\ \mathrm{~N} & 0.15233500 & 2.36677600 & 0.00688300 \\ \mathrm{H} & 1.94381600 & 4.68455500 & -1.09784700 \\ \mathrm{H} & -0.39483700 & 4.16945400 & -0.88022800 \\ \mathrm{C} & 1.35530800 & -1.68840900 & -0.07009300\end{array}$




\begin{tabular}{|c|c|c|c|}
\hline $\mathrm{Ni}$ & -1.33009000 & 1.13461500 & 0.19667200 \\
\hline $\mathrm{C}$ & -1.69468100 & 0.97095200 & 2.06970700 \\
\hline $\mathrm{C}$ & -1.18499200 & 2.07612500 & 4.62744100 \\
\hline $\mathrm{C}$ & -0.60988000 & 0.52531800 & 2.85054600 \\
\hline $\mathrm{C}$ & -2.53882100 & 1.95715600 & 2.61468300 \\
\hline $\mathrm{C}$ & -2.28242900 & 2.50682800 & 3.87345900 \\
\hline $\mathrm{C}$ & -0.34937600 & 1.08212100 & 4.10600800 \\
\hline $\mathrm{H}$ & 0.05486000 & -0.25063600 & 2.47068000 \\
\hline $\mathrm{H}$ & -3.38051600 & 2.32655400 & 2.02886700 \\
\hline $\mathrm{H}$ & -2.94400700 & 3.28510200 & 4.26392000 \\
\hline $\mathrm{H}$ & 0.51253200 & 0.73182700 & 4.68074100 \\
\hline $\mathrm{H}$ & -0.98632600 & 2.50569800 & 5.61230000 \\
\hline $\mathrm{C}$ & -2.69347600 & -0.51910400 & 0.94696700 \\
\hline $\mathrm{Br}$ & -3.03559400 & 2.65797900 & -0.94020800 \\
\hline $\mathrm{C}$ & 1.39311900 & 2.02523600 & -0.20364700 \\
\hline $\mathrm{C}$ & 1.76198100 & 4.21933200 & -0.29181200 \\
\hline $\mathrm{C}$ & 0.24491300 & 3.93168200 & -0.10886200 \\
\hline $\mathrm{N}$ & 0.19258800 & 2.45903400 & -0.11523600 \\
\hline $\mathrm{H}$ & 2.00682300 & 4.71081600 & -1.24262500 \\
\hline $\mathrm{H}$ & -0.34157400 & 4.28725900 & -0.96902700 \\
\hline $\mathrm{C}$ & 1.27916700 & -1.60087700 & -0.02278100 \\
\hline $\mathrm{C}$ & 2.79992000 & -1.32455000 & -0.19778500 \\
\hline $\mathrm{C}$ & 1.64790400 & 0.57793400 & -0.17432400 \\
\hline $\mathrm{N}$ & 0.67881400 & -0.25035100 & -0.06486100 \\
\hline $\mathrm{H}$ & 1.07901400 & -2.02228900 & 0.97653800 \\
\hline $\mathrm{H}$ & 3.19655000 & -1.70768700 & -1.14939800 \\
\hline $\mathrm{H}$ & 2.19864500 & 4.78697800 & 0.54138000 \\
\hline $\mathrm{H}$ & 3.41733500 & -1.69020500 & 0.63235900 \\
\hline $\mathrm{O}$ & 2.38911600 & 2.89938000 & -0.30271000 \\
\hline $\mathrm{O}$ & 2.90031800 & 0.12681400 & -0.22827400 \\
\hline $\mathrm{C}$ & -0.35856700 & 4.49341900 & 1.17195800 \\
\hline $\mathrm{H}$ & -0.28251000 & 5.59176900 & 1.17423600 \\
\hline $\mathrm{H}$ & -1.41642700 & 4.20878600 & 1.23582000 \\
\hline $\mathrm{H}$ & 0.16409000 & 4.09911500 & 2.05713300 \\
\hline $\mathrm{C}$ & 0.70094800 & -2.53409200 & -1.07922400 \\
\hline $\mathrm{H}$ & 1.22649300 & -3.50103200 & -1.05297100 \\
\hline $\mathrm{H}$ & -0.36598300 & -2.72104100 & -0.89860200 \\
\hline $\mathrm{H}$ & 0.81634100 & -2.10022900 & -2.08443300 \\
\hline $\mathrm{C}$ & -2.15180000 & -1.71798700 & 1.71228300 \\
\hline $\mathrm{H}$ & -2.74573000 & -2.60949100 & 1.44086900 \\
\hline $\mathrm{H}$ & -1.10647000 & -1.93382400 & 1.45521500 \\
\hline $\mathrm{H}$ & -2.21786300 & -1.58534600 & 2.80026400 \\
\hline $\mathrm{C}$ & -2.67547600 & -0.84185600 & -0.56327200 \\
\hline $\mathrm{H}$ & -1.66485400 & -0.92344300 & -0.99090700 \\
\hline
\end{tabular}




$\begin{array}{lrrc}\text { H } & -3.14976200 & -1.83288700 & -0.69971300 \\ \text { H } & -3.24554600 & -0.11134100 & -1.15003400 \\ \mathrm{C} & -4.12241500 & -0.19650600 & 1.35144600 \\ \mathrm{H} & -4.77643200 & -1.02962100 & 1.03443500 \\ \mathrm{H} & -4.23173200 & -0.07706000 & 2.43804100 \\ \mathrm{H} & -4.47905500 & 0.71543100 & 0.85229700 \\ \text { UB3LYP-D3(BJ)/def2-SVP-CPCM(THF) } & \\ \text { Zero-point correction= } & 0.414825 \text { (Hartree/Particle) } \\ \text { Thermal correction to Energy= } & 0.440631 \\ \text { Thermal correction to Enthalpy= } & 0.441575 \\ \text { Thermal correction to Gibbs Free Energy= } & -357673 \\ \text { Sum of electronic and zero-point Energies= } & -5042.599705 \\ \text { Sum of electronic and thermal Energies }= & -5042.572954 \\ \text { Sum of electronic and thermal Enthalpies= } & -5042.656856 \\ \text { Sum of electronic and thermal Free Energies= } & \\ \text { HF=-5043.0145295 } & \\ \text { UB3LYP-D3(BJ)/def2-TZVPP-CPCM(THF)//UB3LYP-D3(BJ)/def2-SVP- } \\ \text { CPCM(THF) } \\ \text { HF=-5044.6073643 }\end{array}$

\section{Ph-tBu}

$\begin{array}{lrrr}\mathrm{C} & -2.65049300 & 0.95100600 & -1.34603200 \\ \mathrm{C} & -2.42329700 & 0.45341500 & -4.12915400 \\ \mathrm{C} & -2.99956600 & 1.92953600 & -2.29606700 \\ \mathrm{C} & -2.18509900 & -0.28185000 & -1.82899800 \\ \mathrm{C} & -2.07214700 & -0.52924300 & -3.20259600 \\ \mathrm{C} & -2.88940700 & 1.68882700 & -3.66636900 \\ \mathrm{H} & -3.36609900 & 2.90271100 & -1.96228600 \\ \mathrm{H} & -1.90225100 & -1.07217300 & -1.13408500 \\ \mathrm{H} & -1.70605400 & -1.50038300 & -3.54562700 \\ \mathrm{H} & -3.16918200 & 2.47065000 & -4.37713500 \\ \mathrm{H} & -2.33561800 & 0.26117800 & -5.20118800 \\ \mathrm{C} & -2.78736800 & 1.26039300 & 0.15323200 \\ \mathrm{C} & -2.36648000 & 0.07288900 & 1.03254300 \\ \mathrm{H} & -2.48216300 & 0.33938600 & 2.09439900 \\ \mathrm{H} & -2.98691400 & -0.81641500 & 0.84246700 \\ \mathrm{H} & -1.31290200 & -0.20240500 & 0.87099300 \\ \mathrm{C} & -4.25879100 & 1.60136900 & 0.47098200 \\ \mathrm{H} & -4.60694600 & 2.47716100 & -0.09668900 \\ \mathrm{H} & -4.91861700 & 0.75428400 & 0.22548200 \\ \mathrm{H} & -4.37615200 & 1.82770400 & 1.54307500 \\ \mathrm{C} & -1.89256400 & 2.46723000 & 0.50708100 \\ \mathrm{H} & -1.97889700 & 2.70651300 & 1.57932600 \\ \mathrm{H} & -0.83546700 & 2.24775400 & 0.28904000\end{array}$




\begin{tabular}{|c|c|}
\hline-2.17580800 & -0.06121100 \\
\hline \multicolumn{2}{|l|}{ UB3LYP-D3(BJ)/def2-SVP-CPCM(THF) } \\
\hline Zero-point correction $=$ & 0.212333 (Hartree/Particle) \\
\hline Thermal correction to Energy= & 0.222060 \\
\hline Thermal correction to Enthalpy= & 0.223005 \\
\hline Thermal correction to Gibbs Free Energy= & 0.177939 \\
\hline Sum of electronic and zero-point Energies $=$ & -389.058242 \\
\hline Sum of electronic and thermal Energies $=$ & -389.048515 \\
\hline Sum of electronic and thermal Enthalpies= & -389.047571 \\
\hline Sum of electronic and thermal Free Energies= & -389.092637 \\
\hline \multicolumn{2}{|l|}{$H F=-389.2705756$} \\
\hline \multicolumn{2}{|c|}{$\begin{array}{l}\text { UB3LYP-D3(BJ)/def2-TZVPP-CPCM(THF)//UB3LYP-D3(BJ)/def2-SVP- } \\
\text { CPCM(THF) }\end{array}$} \\
\hline $\begin{array}{l}\mathrm{CPCM}(\mathrm{THF}) \\
\mathrm{HF}=-3896985297\end{array}$ & \\
\hline
\end{tabular}

\section{Scheme 3}

$\begin{array}{lrrr}{ }^{2} \mathbf{G}-\mathbf{T S}-\mathbf{F}^{, \boldsymbol{R}} \mathbf{- B}^{\mathbf{C F 3}} & & & \\ \mathrm{Ni} & -1.47212800 & 1.12405500 & -0.23789000 \\ \mathrm{C} & -2.00755400 & 1.04105800 & 1.66180700 \\ \mathrm{C} & -2.52250000 & 1.35329100 & 4.42749500 \\ \mathrm{C} & -1.26667700 & 0.33930100 & 2.62921500 \\ \mathrm{C} & -3.03441800 & 1.87965400 & 2.12629600 \\ \mathrm{C} & -3.29556500 & 2.04284300 & 3.48880800 \\ \mathrm{C} & -1.50765000 & 0.49060100 & 3.99420300 \\ \mathrm{H} & -0.46404100 & -0.33781000 & 2.32603400 \\ \mathrm{H} & -3.64587100 & 2.43324400 & 1.40760900 \\ \mathrm{H} & -4.09064600 & 2.71117900 & 3.82432300 \\ \mathrm{H} & -0.90539500 & -0.05564000 & 4.72447500 \\ \mathrm{C} & -3.95110400 & -1.33196300 & -1.18780800 \\ \mathrm{H} & -3.32299100 & -2.23208700 & -1.23006600 \\ \mathrm{C} & -3.99915400 & -0.84420700 & 0.21022500 \\ \mathrm{H} & -4.50204100 & 0.09063200 & 0.45460900 \\ \mathrm{H} & -3.49930300 & -0.56005000 & -1.83268100 \\ \mathrm{C} & -5.34712000 & -1.67858300 & -1.80073200 \\ \mathrm{C} & -6.22371500 & -0.41921500 & -1.87438400 \\ \mathrm{H} & -7.17717200 & -0.64234300 & -2.37983300 \\ \mathrm{H} & -6.46247000 & -0.03199100 & -0.87170300 \\ \mathrm{H} & -5.71676300 & 0.38395700 & -2.43214400 \\ \mathrm{C} & -6.03978100 & -2.75276500 & -0.94906600 \\ \mathrm{H} & -5.41155300 & -3.65349800 & -0.85780300 \\ \mathrm{H} & -6.24922600 & -2.38369300 & 0.06788100 \\ \mathrm{H} & -6.99918200 & -3.05043300 & -1.40218200 \\ \mathrm{C} & -5.10511900 & -2.21883200 & -3.21740200 \\ \mathrm{H} & -6.05870600 & -2.47285600 & -3.70772000\end{array}$




\begin{tabular}{|c|c|c|c|}
\hline $\mathrm{H}$ & -4.58900300 & -1.47303900 & -3.84331600 \\
\hline $\mathrm{H}$ & -4.48430900 & -3.12888000 & -3.19275800 \\
\hline $\mathrm{C}$ & -3.61276100 & -1.70129500 & 1.31678400 \\
\hline $\mathrm{O}$ & -2.94629900 & -2.72624100 & 1.21324800 \\
\hline $\mathrm{O}$ & -4.07964000 & -1.25982200 & 2.50021100 \\
\hline $\mathrm{C}$ & -3.72720800 & -2.01793000 & 3.65427200 \\
\hline $\mathrm{H}$ & -2.64573900 & -2.20916100 & 3.67782300 \\
\hline $\mathrm{H}$ & -4.01384500 & -1.41235800 & 4.52111700 \\
\hline $\mathrm{H}$ & -4.26050800 & -2.98166000 & 3.66339000 \\
\hline $\mathrm{Br}$ & -2.92927600 & 2.33676000 & -1.73291500 \\
\hline $\mathrm{C}$ & 1.33532100 & 1.34374200 & 0.07952700 \\
\hline $\mathrm{C}$ & 2.42661700 & 3.27186900 & 0.24381200 \\
\hline $\mathrm{C}$ & 0.89908900 & 3.52937500 & 0.10629000 \\
\hline $\mathrm{N}$ & 0.35264300 & 2.16171600 & -0.02680300 \\
\hline $\mathrm{H}$ & 3.00833000 & 3.64274300 & -0.60961900 \\
\hline $\mathrm{H}$ & 0.68503500 & 4.06163900 & -0.83303900 \\
\hline $\mathrm{C}$ & -0.15228600 & -1.92230800 & -0.43388100 \\
\hline $\mathrm{C}$ & 1.26556600 & -2.30556400 & 0.06820400 \\
\hline $\mathrm{C}$ & 1.05792800 & -0.09654500 & -0.03088200 \\
\hline $\mathrm{N}$ & -0.14346800 & -0.45747200 & -0.29660500 \\
\hline $\mathrm{H}$ & -0.93376500 & -2.32942500 & 0.22355600 \\
\hline $\mathrm{H}$ & 1.82490700 & -2.96668800 & -0.60370900 \\
\hline $\mathrm{H}$ & 2.85622300 & 3.65629500 & 1.17768800 \\
\hline $\mathrm{H}$ & 1.26401500 & -2.72589700 & 1.08284600 \\
\hline $\mathrm{O}$ & 2.55758400 & 1.81539300 & 0.26182400 \\
\hline $\mathrm{O}$ & 1.98390700 & -1.02718000 & 0.13229100 \\
\hline $\mathrm{C}$ & 0.27356400 & 4.33385500 & 1.26476500 \\
\hline $\mathrm{H}$ & 0.89320100 & 5.24748400 & 1.34272100 \\
\hline $\mathrm{C}$ & -0.48056700 & -2.32279900 & -1.88735300 \\
\hline $\mathrm{H}$ & -1.41790600 & -1.79488400 & -2.12370700 \\
\hline $\mathrm{C}$ & -1.15089200 & 4.76001600 & 0.90255900 \\
\hline $\mathrm{H}$ & -1.58347300 & 5.40888200 & 1.67716300 \\
\hline $\mathrm{H}$ & -1.16493400 & 5.31606000 & -0.04772600 \\
\hline $\mathrm{H}$ & -1.80401800 & 3.88580000 & 0.78463000 \\
\hline $\mathrm{C}$ & 0.35959900 & 3.57658700 & 2.59977100 \\
\hline $\mathrm{H}$ & 1.40411800 & 3.26434300 & 2.77497200 \\
\hline $\mathrm{H}$ & -0.22461400 & 2.65147000 & 2.51632200 \\
\hline $\mathrm{C}$ & -0.12598700 & 4.36341200 & 3.81606200 \\
\hline $\mathrm{H}$ & -1.19281800 & 4.61967800 & 3.73207300 \\
\hline $\mathrm{H}$ & -0.01345700 & 3.76508600 & 4.73352100 \\
\hline $\mathrm{H}$ & 0.43839700 & 5.30200200 & 3.94817700 \\
\hline $\mathrm{C}$ & 0.57716900 & -1.85126700 & -2.88752200 \\
\hline $\mathrm{H}$ & 0.76387100 & -0.77058800 & -2.79079300 \\
\hline $\mathrm{H}$ & 1.53543500 & -2.38038300 & -2.75800600 \\
\hline
\end{tabular}




$\begin{array}{lrrr}\mathrm{H} & 0.24382700 & -2.02835000 & -3.91988700 \\ \mathrm{C} & -0.76842800 & -3.83082900 & -1.95486900 \\ \mathrm{H} & -1.51086800 & -4.07043100 & -1.17477600 \\ \mathrm{H} & 0.14569500 & -4.39282300 & -1.68941500 \\ \mathrm{C} & -1.28688800 & -4.31061300 & -3.30960800 \\ \mathrm{H} & -0.53466400 & -4.19457300 & -4.10490100 \\ \mathrm{H} & -1.56324000 & -5.37564400 & -3.26956900 \\ \mathrm{H} & -2.18221100 & -3.74312000 & -3.61225400 \\ \mathrm{C} & -2.77021000 & 1.49999800 & 5.89718000 \\ \mathrm{~F} & -3.22415900 & 0.34885100 & 6.45275000 \\ \mathrm{~F} & -3.67604900 & 2.45049600 & 6.18505600 \\ \mathrm{~F} & -1.64025000 & 1.81449500 & 6.57125700\end{array}$

UB3LYP-D3(BJ)/def2-SVP-CPCM(THF)

\begin{tabular}{|c|c|c|c|}
\hline \multicolumn{3}{|c|}{ Zero-point correction $=$} & 0.686644 (Hartree/Particle) \\
\hline \multicolumn{3}{|c|}{ Thermal correction to Energy= } & 0.732063 \\
\hline \multicolumn{3}{|c|}{ Thermal correction to Enthalpy= } & 0.733007 \\
\hline \multicolumn{3}{|c|}{ Thermal correction to Gibbs Free Energy= } & 0.602778 \\
\hline \multicolumn{3}{|c|}{ Sum of electronic and zero-point Energies $=$} & -5921.185894 \\
\hline \multicolumn{3}{|c|}{ Sum of electronic and thermal Energies $=$} & -5921.140475 \\
\hline \multicolumn{3}{|c|}{ Sum of electronic and thermal Enthalpies $=$} & -5921.139531 \\
\hline \multicolumn{3}{|c|}{ Sum of electronic and thermal Free Energies $=$} & -5921.269760 \\
\hline \multicolumn{4}{|c|}{$\mathrm{HF}=-5921.8725379$} \\
\hline \multicolumn{4}{|c|}{$\begin{array}{l}\text { UB3LYP-D3(B } \\
\text { CPCM(THF) }\end{array}$} \\
\hline \multicolumn{4}{|c|}{$H F=-5924.4763259$} \\
\hline \multicolumn{4}{|c|}{$\begin{array}{l}\text { PBE0-D3(BJ)/def2-TZVPP-CPCM(THF)//UB3LYP-D3(BJ)/def2-SVP-CPCM(THF) } \\
\mathrm{HF}=-5921.6820834\end{array}$} \\
\hline \multicolumn{4}{|c|}{$\begin{array}{l}\text { M06-D3/def2-TZVPP-CPCM(THF)//UB3LYP-D3(BJ)/def2-SVP-CF } \\
\mathrm{HF}=-5922.9199479\end{array}$} \\
\hline \multicolumn{4}{|c|}{ M06/def2-TZVPP-CPCM(THF)//UB3LYP-D3(BJ)/def2-SVP-CPCM(THF) } \\
\hline \multicolumn{4}{|c|}{$H F=-5922.8922488$} \\
\hline \multicolumn{4}{|c|}{${ }^{2} \mathrm{G}-\mathrm{TS}-\mathrm{F}{ }^{, R}-\mathrm{B}^{\mathrm{CF} 3}-\operatorname{conf} 2$} \\
\hline $\mathrm{Ni}$ & -1.37140200 & 1.13521400 & -0.26359900 \\
\hline $\mathrm{C}$ & -1.91200700 & 1.10204200 & 1.64075600 \\
\hline $\mathrm{C}$ & -2.59755500 & 1.27747100 & 4.38727500 \\
\hline $\mathrm{C}$ & -1.21929100 & 0.36988100 & 2.62159400 \\
\hline $\mathrm{C}$ & -2.95656600 & 1.93268400 & 2.08694700 \\
\hline $\mathrm{C}$ & -3.30628200 & 2.02146600 & 3.43625900 \\
\hline $\mathrm{C}$ & -1.54402300 & 0.45294600 & 3.97659600 \\
\hline $\mathrm{H}$ & -0.39487500 & -0.28957300 & 2.33666800 \\
\hline $\mathrm{H}$ & -3.51946800 & 2.52518000 & 1.35938100 \\
\hline $\mathrm{H}$ & -4.12524900 & 2.67056400 & 3.75391000 \\
\hline $\mathrm{H}$ & -0.98410500 & -0.12439000 & 4.71569600 \\
\hline
\end{tabular}




\begin{tabular}{|c|c|c|c|}
\hline $\mathrm{C}$ & -4.00280000 & -1.17973500 & -1.12999300 \\
\hline $\mathrm{H}$ & -3.35643900 & -2.05883600 & -1.25740900 \\
\hline $\mathrm{C}$ & -4.08622000 & -0.85287500 & 0.31123200 \\
\hline $\mathrm{H}$ & -4.61145200 & 0.03879600 & 0.65309100 \\
\hline $\mathrm{H}$ & -3.54004600 & -0.33078900 & -1.66396500 \\
\hline $\mathrm{C}$ & -5.37059600 & -1.46186200 & -1.82665600 \\
\hline $\mathrm{C}$ & -6.27738200 & -0.22459800 & -1.74187200 \\
\hline $\mathrm{H}$ & -7.20534000 & -0.38625500 & -2.31376300 \\
\hline $\mathrm{H}$ & -6.56354200 & -0.00202500 & -0.70214100 \\
\hline $\mathrm{H}$ & -5.77123700 & 0.66468400 & -2.15008600 \\
\hline $\mathrm{C}$ & -6.05439700 & -2.66420300 & -1.15947800 \\
\hline $\mathrm{H}$ & -5.40997600 & -3.55750800 & -1.19669200 \\
\hline $\mathrm{H}$ & -6.28204000 & -2.45695500 & -0.10135000 \\
\hline $\mathrm{H}$ & -7.00217400 & -2.90652600 & -1.66691800 \\
\hline $\mathrm{C}$ & -5.07767200 & -1.78263100 & -3.29944100 \\
\hline $\mathrm{H}$ & -6.00897800 & -2.00185500 & -3.84606800 \\
\hline $\mathrm{H}$ & -4.58180400 & -0.93390400 & -3.79747200 \\
\hline $\mathrm{H}$ & -4.41739600 & -2.66053800 & -3.38949300 \\
\hline $\mathrm{C}$ & -3.58785000 & -1.75629400 & 1.32879000 \\
\hline $\mathrm{O}$ & -2.89640900 & -2.74825400 & 1.11851000 \\
\hline $\mathrm{O}$ & -3.97780300 & -1.39588800 & 2.56722200 \\
\hline $\mathrm{C}$ & -3.53165700 & -2.21390600 & 3.64471200 \\
\hline $\mathrm{H}$ & -2.43882200 & -2.32749600 & 3.62068200 \\
\hline $\mathrm{H}$ & -3.83139000 & -1.69751100 & 4.56276000 \\
\hline $\mathrm{H}$ & -3.99393100 & -3.21224200 & 3.59283400 \\
\hline $\mathrm{Br}$ & -2.80664300 & 2.47638000 & -1.68218000 \\
\hline $\mathrm{C}$ & 1.42462800 & 1.39778900 & -0.01835600 \\
\hline $\mathrm{C}$ & 2.39569900 & 3.39454700 & 0.12273700 \\
\hline $\mathrm{C}$ & 0.85403700 & 3.55465000 & -0.02862100 \\
\hline $\mathrm{N}$ & 0.40608200 & 2.16336000 & -0.15869400 \\
\hline $\mathrm{H}$ & 2.95896600 & 3.77720100 & -0.73749500 \\
\hline $\mathrm{H}$ & 0.60836500 & 4.07591700 & -0.96654700 \\
\hline $\mathrm{C}$ & -0.02089700 & -1.91112700 & -0.32899400 \\
\hline $\mathrm{C}$ & 1.43218400 & -2.25576200 & 0.10031300 \\
\hline $\mathrm{C}$ & 1.18293600 & -0.05352600 & -0.06952100 \\
\hline $\mathrm{N}$ & -0.02222200 & -0.44298000 & -0.27616500 \\
\hline $\mathrm{H}$ & -0.75500300 & -2.28303200 & 0.39984500 \\
\hline $\mathrm{H}$ & 1.96681200 & -2.91795300 & -0.59085700 \\
\hline $\mathrm{H}$ & 2.80261500 & 3.81963000 & 1.04832400 \\
\hline $\mathrm{H}$ & 1.49689600 & -2.65744800 & 1.12011400 \\
\hline $\mathrm{O}$ & 2.61337900 & 1.94359600 & 0.17504300 \\
\hline $\mathrm{O}$ & 2.12831800 & -0.96272700 & 0.10126500 \\
\hline $\mathrm{C}$ & 0.11803200 & 4.27227100 & 1.11979000 \\
\hline $\mathrm{H}$ & -0.95312400 & 4.10632300 & 0.91082500 \\
\hline
\end{tabular}




$\begin{array}{lrrr}\text { C } & -0.45155700 & -2.39903000 & -1.72685200 \\ \mathrm{H} & -1.40661800 & -1.88481500 & -1.92053300 \\ \mathrm{C} & 0.43081300 & 3.66758900 & 2.48988500 \\ \mathrm{H} & -0.26659500 & 4.04718200 & 3.24985900 \\ \mathrm{H} & 0.32151100 & 2.57487400 & 2.47965500 \\ \mathrm{H} & 1.45092300 & 3.91823300 & 2.82474800 \\ \mathrm{C} & 0.38626300 & 5.78377100 & 1.05074900 \\ \mathrm{H} & 0.16770400 & 6.13123900 & 0.02614500 \\ \mathrm{H} & 1.46367200 & 5.97208700 & 1.21353600 \\ \mathrm{C} & -0.43023300 & 6.60858700 & 2.04453200 \\ \mathrm{H} & -0.16512500 & 6.37647900 & 3.08739400 \\ \mathrm{H} & -0.26261200 & 7.68622000 & 1.89298000 \\ \mathrm{H} & -1.50913300 & 6.41565500 & 1.92387300 \\ \mathrm{C} & 0.52684000 & -1.98503200 & -2.82786900 \\ \mathrm{H} & 0.72087900 & -0.90149200 & -2.80193200 \\ \mathrm{H} & 1.49225700 & -2.51030900 & -2.74265500 \\ \mathrm{H} & 0.11716700 & -2.21535900 & -3.82159900 \\ \mathrm{C} & -0.73976700 & -3.90752300 & -1.68350000 \\ \mathrm{H} & -1.42437600 & -4.09332400 & -0.83949100 \\ \mathrm{H} & 0.19344800 & -4.45454500 & -1.45476500 \\ \mathrm{C} & -1.35744700 & -4.46469400 & -2.96514100 \\ \mathrm{H} & -0.67191200 & -4.38951400 & -3.82326800 \\ \mathrm{H} & -1.62049500 & -5.52706400 & -2.84490300 \\ \mathrm{H} & -2.27977000 & -3.91983300 & -3.22690000 \\ \mathrm{C} & -3.00652000 & 1.30109800 & 5.82838100 \\ \mathrm{~F} & -3.63542400 & 2.44108800 & 6.17108900 \\ \mathrm{~F} & -1.95779200 & 1.15796300 & 6.66339300 \\ \mathrm{~F} & -3.86505800 & 0.29133800 & 6.13042400 \\ \mathrm{H} & -5600\end{array}$

UB3LYP-D3(BJ)/def2-SVP-CPCM(THF)

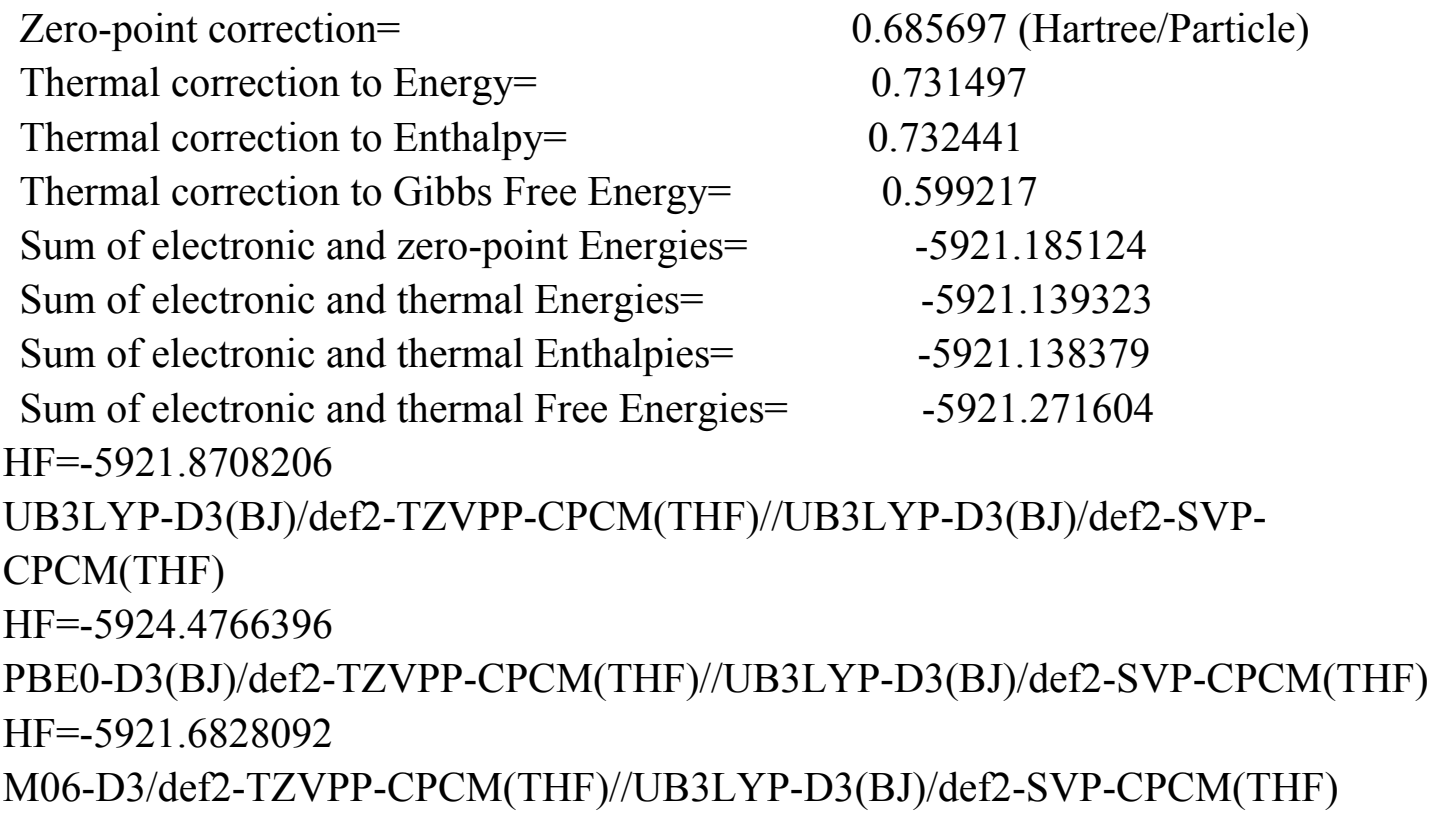




\begin{tabular}{|c|c|c|c|}
\hline \multicolumn{4}{|c|}{$H F=-5922.9181946$} \\
\hline & $-\mathrm{CPCM}(\mathrm{THF}) /$ & JB3LYP-D3(B & J)/def2-SVP-CPCM(THF) \\
\hline \multicolumn{4}{|c|}{$\mathrm{HF}=-5922.8916641$} \\
\hline \multicolumn{4}{|c|}{${ }^{2} \mathrm{G}-\mathrm{TS}-\mathrm{F}^{, R}-\mathrm{B}^{\mathrm{CF} 3}-\operatorname{conf3}$} \\
\hline $\mathrm{Ni}$ & -1.48322600 & 1.09496900 & -0.24003000 \\
\hline $\mathrm{C}$ & -1.99438200 & 1.19064300 & 1.66749100 \\
\hline $\mathrm{C}$ & -2.67173600 & 1.52498900 & 4.39660400 \\
\hline $\mathrm{C}$ & -1.19080300 & 0.66294000 & 2.69136100 \\
\hline $\mathrm{C}$ & -3.14182100 & 1.90352800 & 2.05591200 \\
\hline $\mathrm{C}$ & -3.48756800 & 2.06973200 & 3.39767600 \\
\hline $\mathrm{C}$ & -1.51411900 & 0.82332400 & 4.04074100 \\
\hline $\mathrm{H}$ & -0.28364400 & 0.10118300 & 2.44849700 \\
\hline $\mathrm{H}$ & -3.78872900 & 2.34415800 & 1.29137500 \\
\hline $\mathrm{H}$ & -4.38676700 & 2.62592500 & 3.67158500 \\
\hline $\mathrm{H}$ & -0.87237800 & 0.40151200 & 4.81727700 \\
\hline $\mathrm{C}$ & -3.92504900 & -1.44404800 & -1.09849900 \\
\hline $\mathrm{H}$ & -3.28313200 & -2.33431100 & -1.13744200 \\
\hline $\mathrm{C}$ & -3.92236600 & -0.90485900 & 0.28042300 \\
\hline $\mathrm{H}$ & -4.43553700 & 0.02780400 & 0.51044200 \\
\hline $\mathrm{H}$ & -3.51581100 & -0.68534000 & -1.78593000 \\
\hline $\mathrm{C}$ & -5.33885500 & -1.83753300 & -1.63797200 \\
\hline $\mathrm{C}$ & -6.23866900 & -0.59597300 & -1.72890900 \\
\hline $\mathrm{H}$ & -7.21014100 & -0.85629300 & -2.17923300 \\
\hline $\mathrm{H}$ & -6.43826600 & -0.16590100 & -0.73515200 \\
\hline $\mathrm{H}$ & -5.77107700 & 0.18741800 & -2.34590700 \\
\hline $\mathrm{C}$ & -5.97813200 & -2.88447200 & -0.71369600 \\
\hline $\mathrm{H}$ & -5.32906400 & -3.76844100 & -0.60595900 \\
\hline $\mathrm{H}$ & -6.15755900 & -2.47357900 & 0.29291300 \\
\hline $\mathrm{H}$ & -6.94736500 & -3.21991700 & -1.11669400 \\
\hline $\mathrm{C}$ & -5.14422700 & -2.43497400 & -3.03892200 \\
\hline $\mathrm{H}$ & -6.11264800 & -2.72005100 & -3.48071100 \\
\hline $\mathrm{H}$ & -4.66185300 & -1.71083500 & -3.71515300 \\
\hline $\mathrm{H}$ & -4.51115200 & -3.33585400 & -2.99986800 \\
\hline $\mathrm{C}$ & -3.47960800 & -1.71403200 & 1.40284400 \\
\hline $\mathrm{O}$ & -2.80466600 & -2.73448600 & 1.31095700 \\
\hline $\mathrm{O}$ & -3.90507600 & -1.23561200 & 2.58559100 \\
\hline $\mathrm{C}$ & -3.48935400 & -1.94295500 & 3.75105300 \\
\hline $\mathrm{H}$ & -2.39399300 & -2.03080600 & 3.77916200 \\
\hline $\mathrm{H}$ & -3.83596100 & -1.35232700 & 4.60538900 \\
\hline $\mathrm{H}$ & -3.92894900 & -2.95223600 & 3.77216100 \\
\hline $\mathrm{Br}$ & -2.93623300 & 2.14763600 & -1.86197500 \\
\hline $\mathrm{C}$ & 1.31677400 & 1.22773800 & 0.14230700 \\
\hline $\mathrm{C}$ & 2.38952700 & 3.08726200 & 0.69266500 \\
\hline
\end{tabular}




\begin{tabular}{|c|c|c|c|}
\hline $\mathrm{C}$ & 0.94155600 & 3.42149900 & 0.23614300 \\
\hline $\mathrm{N}$ & 0.36783000 & 2.07936200 & -0.00091300 \\
\hline $\mathrm{H}$ & 3.17178500 & 3.60236000 & 0.12371500 \\
\hline $\mathrm{H}$ & 0.94996800 & 3.94873300 & -0.73369600 \\
\hline $\mathrm{C}$ & -0.22031700 & -1.97765400 & -0.55127200 \\
\hline $\mathrm{C}$ & 1.18932700 & -2.40855300 & -0.07244400 \\
\hline $\mathrm{C}$ & 1.01152700 & -0.19792400 & -0.03741100 \\
\hline $\mathrm{N}$ & -0.20206300 & -0.52824000 & -0.28905200 \\
\hline $\mathrm{H}$ & -1.00589500 & -2.43694400 & 0.06415300 \\
\hline $\mathrm{H}$ & 1.73499500 & -3.05239400 & -0.77159100 \\
\hline $\mathrm{H}$ & 2.54369300 & 3.24931200 & 1.76877500 \\
\hline $\mathrm{H}$ & 1.18015500 & -2.86824700 & 0.92530300 \\
\hline $\mathrm{O}$ & 2.52934800 & 1.65278500 & 0.45716800 \\
\hline $\mathrm{O}$ & 1.92979900 & -1.14697500 & 0.04151600 \\
\hline $\mathrm{C}$ & 0.14387000 & 4.27095200 & 1.23951900 \\
\hline $\mathrm{H}$ & 0.02589600 & 3.66035300 & 2.15324900 \\
\hline $\mathrm{C}$ & -0.53029500 & -2.24298700 & -2.04048100 \\
\hline $\mathrm{H}$ & -1.46326300 & -1.69352000 & -2.24235200 \\
\hline $\mathrm{C}$ & 0.92612600 & 5.54380200 & 1.58543300 \\
\hline $\mathrm{H}$ & 0.37444700 & 6.15385200 & 2.31411700 \\
\hline $\mathrm{H}$ & 1.91074500 & 5.32910800 & 2.02754600 \\
\hline $\mathrm{H}$ & 1.08525900 & 6.15942300 & 0.68411300 \\
\hline $\mathrm{C}$ & -1.25169000 & 4.59472600 & 0.68920100 \\
\hline $\mathrm{H}$ & -1.71804500 & 3.66582000 & 0.33837200 \\
\hline $\mathrm{H}$ & -1.13907100 & 5.23164100 & -0.20706400 \\
\hline $\mathrm{C}$ & -2.18700600 & 5.26571900 & 1.69205500 \\
\hline $\mathrm{H}$ & -1.83657100 & 6.26634400 & 1.98981600 \\
\hline $\mathrm{H}$ & -3.19391000 & 5.38346200 & 1.26187400 \\
\hline $\mathrm{H}$ & -2.28816200 & 4.65499500 & 2.60307800 \\
\hline $\mathrm{C}$ & 0.54129700 & -1.68628500 & -2.98073300 \\
\hline $\mathrm{H}$ & 0.71563400 & -0.61484500 & -2.79835600 \\
\hline $\mathrm{H}$ & 1.50203900 & -2.21603900 & -2.87465000 \\
\hline $\mathrm{H}$ & 0.22796100 & -1.78476400 & -4.02965100 \\
\hline $\mathrm{C}$ & -0.81688000 & -3.73807100 & -2.25157100 \\
\hline $\mathrm{H}$ & -1.55973000 & -4.05543400 & -1.49971100 \\
\hline $\mathrm{H}$ & 0.09688500 & -4.32262200 & -2.03979000 \\
\hline $\mathrm{C}$ & -1.33437200 & -4.08569500 & -3.64655000 \\
\hline $\mathrm{H}$ & -0.58078100 & -3.89559000 & -4.42608200 \\
\hline $\mathrm{H}$ & -1.61206700 & -5.14933200 & -3.70853700 \\
\hline $\mathrm{H}$ & -2.22845700 & -3.49049800 & -3.89371000 \\
\hline $\mathrm{C}$ & -3.07651100 & 1.62670500 & 5.83551400 \\
\hline $\mathrm{F}$ & -3.77601700 & 2.74757600 & 6.09765600 \\
\hline $\mathrm{F}$ & -2.02027600 & 1.61137800 & 6.67187300 \\
\hline $\mathrm{F}$ & -3.87118200 & 0.59110400 & 6.21506300 \\
\hline
\end{tabular}


UB3LYP-D3(BJ)/def2-SVP-CPCM(THF)

$\begin{array}{lc}\text { Zero-point correction }= & 0.686586 \text { (Hartree/Particle) } \\ \text { Thermal correction to Energy= } & 0.731971 \\ \text { Thermal correction to Enthalpy= } & 0.732915 \\ \text { Thermal correction to Gibbs Free Energy= } & 0.602164 \\ \text { Sum of electronic and zero-point Energies }= & -5921.184703 \\ \text { Sum of electronic and thermal Energies }= & -5921.139319 \\ \text { Sum of electronic and thermal Enthalpies }= & -5921.138375 \\ \text { Sum of electronic and thermal Free Energies }= & -5921.269125 \\ \text { HF=-5921.8712896 } & \end{array}$

UB3LYP-D3(BJ)/def2-TZVPP-CPCM(THF)//UB3LYP-D3(BJ)/def2-SVP-

CPCM(THF)

$\mathrm{HF}=-5924.4753011$

PBE0-D3(BJ)/def2-TZVPP-CPCM(THF)//UB3LYP-D3(BJ)/def2-SVP-CPCM(THF)

$\mathrm{HF}=-5921.68136$

M06-D3/def2-TZVPP-CPCM(THF)//UB3LYP-D3(BJ)/def2-SVP-CPCM(THF)

$\mathrm{HF}=-5922.9172952$

M06/def2-TZVPP-CPCM(THF)//UB3LYP-D3(BJ)/def2-SVP-CPCM(THF)

$\mathrm{HF}=-5922.8903189$

${ }^{2}$ G-TS-F ${ }^{, R}-B^{\mathrm{CF} 3}$-conf4

$\begin{array}{lrrr}\mathrm{Ni} & -1.42605100 & 1.07321800 & -0.32155700 \\ \mathrm{C} & -2.02634100 & 1.11140900 & 1.56965200 \\ \mathrm{C} & -2.77010500 & 1.40904900 & 4.29265900 \\ \mathrm{C} & -1.36005100 & 0.41907800 & 2.59733300 \\ \mathrm{C} & -3.08188900 & 1.95831400 & 1.95766900 \\ \mathrm{C} & -3.45879400 & 2.10856700 & 3.29468400 \\ \mathrm{C} & -1.71302400 & 0.56241400 & 3.94012100 \\ \mathrm{H} & -0.53164000 & -0.25422300 & 2.35968900 \\ \mathrm{H} & -3.62818700 & 2.51872300 & 1.19315300 \\ \mathrm{H} & -4.28214800 & 2.77319500 & 3.56562400 \\ \mathrm{H} & -1.16926400 & 0.01880100 & 4.71574200 \\ \mathrm{C} & -4.15968700 & -1.18006400 & -0.76948400 \\ \mathrm{H} & -3.43677500 & -1.99641700 & -0.89599900 \\ \mathrm{C} & -4.34734800 & -0.92546300 & 0.67498200 \\ \mathrm{H} & -4.98378800 & -0.11086600 & 1.02126800 \\ \mathrm{H} & -3.73253200 & -0.27598000 & -1.24058500 \\ \mathrm{C} & -5.45376800 & -1.51605600 & -1.57099100 \\ \mathrm{C} & -6.44767900 & -0.34737600 & -1.48791600 \\ \mathrm{H} & -7.32499600 & -0.53992900 & -2.12624500 \\ \mathrm{H} & -6.81292800 & -0.19603100 & -0.46029300 \\ \mathrm{H} & -5.97944600 & 0.59210200 & -1.82333000 \\ \mathrm{C} & -6.09442500 & -2.79572600 & -1.01432900 \\ \mathrm{H} & -5.39072300 & -3.64263500 & -1.05744700\end{array}$


H

$\mathrm{H}$

C

$\mathrm{H}$

$\mathrm{H}$

$\mathrm{H}$

$\mathrm{C}$

$\mathrm{O}$

$\mathrm{O}$

C

$\mathrm{H}$

$\mathrm{H}$

$\mathrm{H}$

$\mathrm{Br}$

C

C

C

$\mathrm{N}$

$\mathrm{H}$

$\mathrm{H}$

C

C

C

$\mathrm{N}$

$\mathrm{H}$

$\mathrm{H}$

$\mathrm{H}$

$\mathrm{H}$

$\mathrm{O}$

$\mathrm{O}$

C

$\mathrm{H}$

C

$\mathrm{H}$

C

$\mathrm{H}$

$\mathrm{H}$

$\mathrm{H}$

C

$\mathrm{H}$

$\mathrm{H}$

C

$\mathrm{H}$

$\mathrm{H}$

\begin{tabular}{rrr}
-6.39739100 & -2.66364500 & 0.03691900 \\
-6.99146000 & -3.06890400 & -1.59333200 \\
-5.04326000 & -1.73146100 & -3.03540400 \\
-5.91993900 & -1.97344100 & -3.65754500 \\
-4.57039100 & -0.82590900 & -3.44848700 \\
-4.32312700 & -2.56038400 & -3.12860800 \\
-3.74111100 & -1.75933400 & 1.69054200 \\
-2.94787400 & -2.67290000 & 1.48429500 \\
-4.15398800 & -1.42928800 & 2.93115100 \\
-3.62023700 & -2.19239600 & 4.00896100 \\
-2.52345900 & -2.22911000 & 3.95474900 \\
-3.93320600 & -1.68285800 & 4.92636700 \\
-4.01068400 & -3.22219300 & 3.98632100 \\
-2.80594200 & 2.39664700 & -1.80087900 \\
1.36388200 & 1.13550600 & 0.04099200 \\
2.47655600 & 3.05748200 & 0.19295500 \\
0.96220900 & 3.32864100 & -0.04720000 \\
0.41367800 & 1.97200000 & -0.15924400 \\
3.11350200 & 3.38080000 & -0.63976200 \\
0.80875700 & 3.82152900 & -1.01759400 \\
-0.27026000 & -2.06969600 & -0.40274600 \\
1.12599300 & -2.50878200 & 0.11610300 \\
1.02260500 & -0.29510900 & -0.01982200 \\
-0.19489800 & -0.60431700 & -0.28378600 \\
-1.07185600 & -2.42692000 & 0.25808800 \\
1.66059400 & -3.20389300 & -0.54183600 \\
2.86312200 & 3.46917700 & 1.13323000 \\
1.09708500 & -2.91484100 & 1.13613700 \\
2.58078600 & 1.59573100 & 0.27924000 \\
1.90244800 & -1.26471200 & 0.16855600 \\
0.22561600 & 4.14311700 & 1.03435300 \\
-0.87891518500 & 6.33436400 & -0.34119600 \\
-0.58973300 & -2.47355500 & -1.86095200 \\
-1.55061900 & -1.98571100 & -2.09767300 \\
0.43888200 & 3.58569500 & 2.44237100 \\
-0.24606500 & 4.07719700 & 3.14971400 \\
0.23368900 & 2.50801700 & 2.48862500 \\
\hline .46470100 & 3.76533700 & 2.80333700 \\
\hline .66463500 & 5.76505700 & 1.14648900 \\
\hline
\end{tabular}




$\begin{array}{lrrr}\mathrm{H} & 0.76783500 & 5.96877300 & -1.20444300 \\ \mathrm{C} & 0.45301900 & -1.94959400 & -2.85186700 \\ \mathrm{H} & 0.56200000 & -0.85656300 & -2.78591200 \\ \mathrm{H} & 1.44362600 & -2.40462700 & -2.68749800 \\ \mathrm{H} & 0.15165200 & -2.19004100 & -3.88248900 \\ \mathrm{C} & -0.78118500 & -3.99535800 & -1.98841200 \\ \mathrm{H} & 0.10451800 & -4.50953400 & -1.57318000 \\ \mathrm{H} & -0.78604900 & -4.24686200 & -3.06211400 \\ \mathrm{C} & -2.05403500 & -4.54332000 & -1.34227100 \\ \mathrm{H} & -2.94883000 & -4.15084000 & -1.85118800 \\ \mathrm{H} & -2.08834200 & -5.64150300 & -1.41601000 \\ \mathrm{H} & -2.14253900 & -4.26996500 & -0.28093400 \\ \mathrm{C} & -3.20112300 & 1.51030100 & 5.72418800 \\ \mathrm{~F} & -4.09486000 & 0.54291200 & 6.05910500 \\ \mathrm{~F} & -3.80061700 & 2.68357500 & 6.00249700 \\ \mathrm{~F} & -2.17008100 & 1.37635200 & 6.58230000\end{array}$

UB3LYP-D3(BJ)/def2-SVP-CPCM(THF)

Zero-point correction=

0.685713 (Hartree/Particle)

Thermal correction to Energy=

0.731517

0.732461

Thermal correction to Enthalpy=

0.599268

$-5921.184889$

$-5921.139085$

$-5921.138141$

$-5921.271334$

Sum of electronic and thermal Free Energies=

$\mathrm{HF}=-5921.8706021$

UB3LYP-D3(BJ)/def2-TZVPP-CPCM(THF)//UB3LYP-D3(BJ)/def2-SVP-

CPCM(THF)

$\mathrm{HF}=-5924.4759634$

PBE0-D3(BJ)/def2-TZVPP-CPCM(THF)//UB3LYP-D3(BJ)/def2-SVP-CPCM(THF)

$\mathrm{HF}=-5921.6825104$

M06-D3/def2-TZVPP-CPCM(THF)//UB3LYP-D3(BJ)/def2-SVP-CPCM(THF)

$\mathrm{HF}=-5922.9185719$

M06/def2-TZVPP-CPCM(THF)//UB3LYP-D3(BJ)/def2-SVP-CPCM(THF)

$\mathrm{HF}=-5922.8913846$

${ }^{2} \mathrm{G}-\mathrm{TS}-\mathrm{F}^{, R}-B^{\mathrm{CF} 3}$-conf5

$\mathrm{Ni}$

$\mathrm{C}$

$\mathrm{C}$

$\mathrm{C}$

$\mathrm{C}$

C

C
$-1.37364500$

$-1.91525500$

$-2.60811500$

$-1.21778700$

$-2.96772200$

$-3.32102600$

$-1.54621100$
1.13174500

1.09805300

1.26306600

0.37097500

1.91915000

2.00258700

0.44890100
$-0.26327400$

1.64096100

4.38654500

2.62235100

2.08651000

3.43518900

3.97687000 


\begin{tabular}{|c|c|c|c|}
\hline $\mathrm{H}$ & -0.38677800 & -0.28048500 & 2.33826500 \\
\hline $\mathrm{H}$ & -3.53385300 & 2.50829800 & 1.35879200 \\
\hline $\mathrm{H}$ & -4.14636700 & 2.64394000 & 3.75211300 \\
\hline $\mathrm{H}$ & -0.98274500 & -0.12468000 & 4.71619900 \\
\hline $\mathrm{C}$ & -4.00721500 & -1.17484600 & -1.13838400 \\
\hline $\mathrm{H}$ & -3.35951400 & -2.05241200 & -1.26958400 \\
\hline $\mathrm{C}$ & -4.09420000 & -0.85717400 & 0.30475300 \\
\hline $\mathrm{H}$ & -4.62300800 & 0.03055900 & 0.65139100 \\
\hline $\mathrm{H}$ & -3.54334700 & -0.32232600 & -1.66604000 \\
\hline $\mathrm{C}$ & -5.37259800 & -1.45343500 & -1.84077200 \\
\hline $\mathrm{C}$ & -6.27909900 & -0.21613900 & -1.75369100 \\
\hline $\mathrm{H}$ & -7.20562100 & -0.37513900 & -2.32863700 \\
\hline $\mathrm{H}$ & -6.56790600 & 0.00268600 & -0.71389400 \\
\hline $\mathrm{H}$ & -5.77148200 & 0.67443800 & -2.15728400 \\
\hline $\mathrm{C}$ & -6.05895000 & -2.65841800 & -1.18101200 \\
\hline $\mathrm{H}$ & -5.41456100 & -3.55168400 & -1.21982000 \\
\hline $\mathrm{H}$ & -6.29010300 & -2.45559400 & -0.12278900 \\
\hline $\mathrm{H}$ & -7.00502300 & -2.89839500 & -1.69271500 \\
\hline $\mathrm{C}$ & -5.07471500 & -1.76807900 & -3.31390300 \\
\hline $\mathrm{H}$ & -6.00420600 & -1.98462200 & -3.86467000 \\
\hline $\mathrm{H}$ & -4.57683000 & -0.91743700 & -3.80663500 \\
\hline $\mathrm{H}$ & -4.41437400 & -2.64583100 & -3.40532700 \\
\hline $\mathrm{C}$ & -3.59000100 & -1.76270200 & 1.31755900 \\
\hline $\mathrm{O}$ & -2.89637400 & -2.75184900 & 1.10157200 \\
\hline $\mathrm{O}$ & -3.97696300 & -1.40763500 & 2.55845600 \\
\hline $\mathrm{C}$ & -3.52447100 & -2.22778700 & 3.63167400 \\
\hline $\mathrm{H}$ & -2.43092900 & -2.33424600 & 3.60593300 \\
\hline $\mathrm{H}$ & -3.82631100 & -1.71737500 & 4.55235700 \\
\hline $\mathrm{H}$ & -3.98046500 & -3.22877300 & 3.57580700 \\
\hline $\mathrm{Br}$ & -2.80231100 & 2.49791300 & -1.67032500 \\
\hline $\mathrm{C}$ & 1.42168700 & 1.40698200 & -0.03231800 \\
\hline $\mathrm{C}$ & 2.37831700 & 3.41376800 & 0.07649100 \\
\hline $\mathrm{C}$ & 0.83475800 & 3.55907300 & -0.06646000 \\
\hline $\mathrm{N}$ & 0.39645200 & 2.16342700 & -0.16980800 \\
\hline $\mathrm{H}$ & 2.93230300 & 3.79181800 & -0.79184200 \\
\hline $\mathrm{H}$ & 0.57963500 & 4.05317400 & -1.01431700 \\
\hline $\mathrm{C}$ & -0.00850200 & -1.91143800 & -0.31188000 \\
\hline $\mathrm{C}$ & 1.44867500 & -2.24612200 & 0.11173800 \\
\hline $\mathrm{C}$ & 1.18751500 & -0.04610600 & -0.07111400 \\
\hline $\mathrm{N}$ & -0.01646000 & -0.44300800 & -0.27148200 \\
\hline $\mathrm{H}$ & -0.73664300 & -2.28042400 & 0.42432100 \\
\hline $\mathrm{H}$ & 1.98337700 & -2.90858100 & -0.57917200 \\
\hline $\mathrm{H}$ & 2.78841700 & 3.85265500 & 0.99434300 \\
\hline $\mathrm{H}$ & 1.52077900 & -2.64281400 & 1.13296900 \\
\hline
\end{tabular}




$\begin{array}{lrrr}\text { O } & 2.60820500 & 1.96480000 & 0.14275700 \\ \text { O } & 2.13788700 & -0.94941600 & 0.10317500 \\ \mathrm{C} & 0.09873900 & 4.28644500 & 1.07602700 \\ \mathrm{H} & -0.97151500 & 4.10572100 & 0.87561300 \\ \mathrm{C} & -0.44626300 & -2.41306700 & -1.70253200 \\ \mathrm{H} & -1.40393800 & -1.90305800 & -1.89427500 \\ \mathrm{C} & 0.42371500 & 3.70527300 & 2.45278300 \\ \mathrm{H} & -0.24185800 & 4.14246700 & 3.21236700 \\ \mathrm{H} & 0.27534800 & 2.61728500 & 2.47940200 \\ \mathrm{H} & 1.45905900 & 3.92796900 & 2.75863300 \\ \mathrm{C} & 0.33945700 & 5.80396900 & 1.03189500 \\ \mathrm{H} & 1.41541300 & 6.01039600 & 1.18117100 \\ \mathrm{H} & -0.16897900 & 6.24277700 & 1.90667000 \\ \mathrm{C} & -0.15460200 & 6.50055400 & -0.23636600 \\ \mathrm{H} & -1.21151900 & 6.25786800 & -0.43499600 \\ \mathrm{H} & -0.07172600 & 7.59431800 & -0.14157100 \\ \mathrm{H} & 0.42410300 & 6.20926300 & -1.12739100 \\ \mathrm{C} & 0.52398100 & -2.00586800 & -2.81323600 \\ \mathrm{H} & 0.71641900 & -0.92184100 & -2.79694600 \\ \mathrm{H} & 1.49083800 & -2.52886700 & -2.73031600 \\ \mathrm{H} & 0.10819100 & -2.24465800 & -3.80242600 \\ \mathrm{C} & -0.73057000 & -3.92175300 & -1.64482300 \\ \mathrm{H} & -1.40848700 & -4.10176400 & -0.79433700 \\ \mathrm{H} & 0.20567400 & -4.46498900 & -1.41937200 \\ \mathrm{C} & -1.35743200 & -4.48992600 & -2.91720300 \\ \mathrm{H} & -0.67922600 & -4.41976900 & -3.78157800 \\ \mathrm{H} & -1.61726800 & -5.55190700 & -2.78689500 \\ \mathrm{H} & -2.28312400 & -3.94897400 & -3.17539900 \\ \mathrm{C} & -3.02110000 & 1.28038900 & 5.82661700 \\ \mathrm{~F} & -3.65769200 & 2.41573300 & 6.17060700 \\ \mathrm{~F} & -1.97391600 & 1.14116500 & 6.66416700 \\ \mathrm{~F} & -3.87460900 & 0.26484900 & 6.12357400 \\ \mathrm{HB} \mathrm{y} & \mathrm{r} & \end{array}$

UB3LYP-D3(BJ)/def2-SVP-CPCM(THF)

Zero-point correction=

Thermal correction to Energy=

0.685742 (Hartree/Particle)

0.731525

Thermal correction to Enthalpy=

0.732469

0.599763

$-5921.185051$

$-5921.139268$

$-5921.138324$

$-5921.271030$

Sum of electronic and thermal Free Energies=

$\mathrm{HF}=-5921.8707934$

UB3LYP-D3(BJ)/def2-TZVPP-CPCM(THF)//UB3LYP-D3(BJ)/def2-SVPCPCM(THF) 


\begin{tabular}{|c|c|c|c|}
\hline \multicolumn{4}{|c|}{$H F=-5924.4764462$} \\
\hline \multirow{2}{*}{\multicolumn{4}{|c|}{ PBE0-D3(BJ)/def2-TZVPP-CPCM(THF)//UB3LYP-D3(BJ)/def2-SVP-CPCM(THF) }} \\
\hline & & & \\
\hline \multicolumn{4}{|c|}{ M06-D3/def2-TZVPP-CPCM(THF)//UB3LYP-D3(BJ)/def2-SVP-CPCM(THF) } \\
\hline \multicolumn{4}{|c|}{$H F=-5922.918112$} \\
\hline \multicolumn{4}{|c|}{ M06/def2-TZVPP-CPCM(THF)//UB3LYP-D3(BJ)/def2-SVP-CPCM(THF) } \\
\hline \multicolumn{4}{|c|}{$\mathrm{HF}=-5922.891514$} \\
\hline \multicolumn{4}{|c|}{${ }^{2} \mathrm{G}-\mathrm{TS}-\mathrm{F}^{, S}-B^{\mathrm{CF} 3}$} \\
\hline $\mathrm{Ni}$ & -1.23489900 & 1.32346600 & -0.74674200 \\
\hline $\mathrm{C}$ & -2.25460700 & 2.08095700 & 0.77538100 \\
\hline $\mathrm{C}$ & -3.71012200 & 3.25270900 & 2.91616200 \\
\hline $\mathrm{C}$ & -1.74028200 & 2.12263300 & 2.08499000 \\
\hline $\mathrm{C}$ & -3.52435100 & 2.65811900 & 0.58054100 \\
\hline $\mathrm{C}$ & -4.24999200 & 3.23641100 & 1.62531200 \\
\hline $\mathrm{C}$ & -2.44688200 & 2.69433500 & 3.14534300 \\
\hline $\mathrm{H}$ & -0.75379200 & 1.70095000 & 2.29740900 \\
\hline $\mathrm{H}$ & -3.97674400 & 2.65776100 & -0.41675900 \\
\hline $\mathrm{H}$ & -5.23461900 & 3.67119500 & 1.44100300 \\
\hline $\mathrm{H}$ & -2.01961900 & 2.70902200 & 4.15050500 \\
\hline $\mathrm{Br}$ & -1.20830300 & 0.50719300 & -3.03117100 \\
\hline $\mathrm{C}$ & 1.39036900 & 2.31171900 & -0.33883500 \\
\hline $\mathrm{C}$ & 2.09984600 & 4.25090900 & -1.16298400 \\
\hline $\mathrm{C}$ & 0.61377600 & 4.01030200 & -1.55239200 \\
\hline $\mathrm{N}$ & 0.31876000 & 2.71661700 & -0.91269800 \\
\hline $\mathrm{H}$ & 2.77157500 & 4.31247300 & -2.02774100 \\
\hline $\mathrm{H}$ & 0.52480900 & 3.85907500 & -2.63979200 \\
\hline $\mathrm{C}$ & 0.51020400 & -0.96917300 & 0.92244500 \\
\hline $\mathrm{C}$ & 1.96027100 & -0.81465200 & 1.46378000 \\
\hline $\mathrm{C}$ & 1.36460700 & 0.99864300 & 0.32270900 \\
\hline $\mathrm{N}$ & 0.27068500 & 0.33233100 & 0.28535800 \\
\hline $\mathrm{H}$ & -0.19968700 & -1.08134200 & 1.75460200 \\
\hline $\mathrm{H}$ & 2.65598100 & -1.58698700 & 1.11421000 \\
\hline $\mathrm{H}$ & 2.24956900 & 5.12590400 & -0.51680500 \\
\hline $\mathrm{H}$ & 2.00869200 & -0.74243400 & 2.55778600 \\
\hline $\mathrm{O}$ & 2.47847000 & 3.06420100 & -0.39481100 \\
\hline $\mathrm{O}$ & 2.41818500 & 0.46859200 & 0.92217600 \\
\hline $\mathrm{C}$ & -0.37160700 & 5.12009200 & -1.13916500 \\
\hline $\mathrm{H}$ & 0.04870300 & 6.05634500 & -1.55213200 \\
\hline $\mathrm{C}$ & 0.26773900 & -2.11577200 & -0.07694900 \\
\hline $\mathrm{H}$ & -0.75395000 & -1.95943800 & -0.45728500 \\
\hline $\mathrm{C}$ & -3.09444500 & -1.23741700 & 1.39809600 \\
\hline $\mathrm{H}$ & -2.46027200 & -0.44080600 & 1.81954600 \\
\hline $\mathrm{C}$ & -3.63656000 & -0.77520000 & 0.10113400 \\
\hline
\end{tabular}


$\mathrm{H}$

$\mathrm{H}$

C

C

$\mathrm{H}$

$\mathrm{H}$

$\mathrm{H}$

C

$\mathrm{H}$

$\mathrm{H}$

$\mathrm{H}$

C

$\mathrm{H}$

$\mathrm{H}$

$\mathrm{H}$

C

$\mathrm{O}$

$\mathrm{O}$

$\mathrm{C}$

$\mathrm{H}$

$\mathrm{H}$

$\mathrm{H}$

C

$\mathrm{H}$

$\mathrm{H}$

$\mathrm{H}$

C

$\mathrm{H}$

$\mathrm{H}$

C

$\mathrm{H}$

$\mathrm{H}$

$\mathrm{H}$

C

$\mathrm{H}$

$\mathrm{H}$

$\mathrm{H}$

C

$\mathrm{H}$

$\mathrm{H}$

C

$\mathrm{H}$

$\mathrm{H}$

$\mathrm{H}$
$-4.20724900$

$-2.46722200$

$-4.16729700$

$-5.07925700$

$-5.80739400$

$-5.64491500$

$-4.49430900$

$-5.00278500$

$-4.36850900$

$-5.57582900$

$-5.72370400$

$-3.41782600$

$-4.12492400$

$-2.80099000$

$-2.75222300$

$-3.58512300$

$-3.00247800$

$-4.27559700$

$-4.22510000$

$-4.60721700$

$-4.85528800$

$-3.19098900$

1.21799600

0.94230500

1.15909400

2.26457500

0.28636300

$-0.35181800$

1.30664100

$-0.20880300$

0.45783900

$-0.26226200$

$-1.21269200$

$-1.73144000$

$-2.42409600$

$-1.62685600$

$-2.19466500$

$-0.46403400$

0.54995400

$-0.84149400$

$-1.34671800$

$-2.40220700$

$-1.30198400$

$-1.02800900$
0.15036900

$-2.12426500$

$-1.58586500$

$-2.70751400$

$-3.00570400$

$-2.38333200$

$-3.59815400$

$-0.34411800$

0.48515400

0.01522100

$-0.57634700$

$-2.06118800$

$-2.32852100$

$-2.94808300$

$-1.27112100$

$-1.60381900$

$-2.67688100$

$-1.06209300$

$-1.76465100$

$-2.79024300$

$-1.20044300$

$-1.80119200$

$-2.05615000$

$-2.81260900$

$-1.07841000$

$-2.24733600$

$-3.46645200$

$-3.38371100$

$-3.67529400$

$-4.63520400$

$-4.83136200$

$-5.56077700$

$-4.41816100$

4.87833400

5.71056000

4.76813200

3.96205800

5.26165200

5.39803100

4.31711000

6.41374100

6.25669200

6.50890500

7.37621900
0.03912300

1.22886300

2.47779400

1.95976300

2.73129600

1.07137200

1.67925600

2.82311800

3.16969500

1.95470300

3.62358800

3.73074600

4.53254300

3.51195200

4.11480800

$-1.08951000$

$-1.17845700$

$-2.11285300$

$-3.35258100$

$-3.23842500$

$-4.05046900$

$-3.72505600$

$-1.27439100$

$-2.02284200$

$-1.77451900$

$-0.98423700$

0.65591600

1.55347800

1.02755200

$-0.19570900$

$-1.04993800$

0.39878600

$-0.59174400$

$-1.80033300$

$-1.61163400$

$-2.89073100$

$-1.40863700$

0.38883200

0.80279800

0.80775300

0.86641000

0.59652500

1.96251300

0.43192400 


$\begin{array}{llll}\text { C } & -4.51411700 & 3.78047100 & 4.06574500 \\ \text { F } & -5.23257500 & 2.80223900 & 4.66979100 \\ F & -5.40025100 & 4.72155000 & 3.68708700 \\ F & -3.74049800 & 4.32559800 & 5.02587800\end{array}$

UB3LYP-D3(BJ)/def2-SVP-CPCM(THF)

$\begin{array}{lc}\text { Zero-point correction= } & 0.686304 \text { (Hartree/Particle) } \\ \text { Thermal correction to Energy= } & 0.731871 \\ \text { Thermal correction to Enthalpy= } & 0.732815 \\ \text { Thermal correction to Gibbs Free Energy= } & 0.601058 \\ \text { Sum of electronic and zero-point Energies }= & -5921.185076 \\ \text { Sum of electronic and thermal Energies }= & -5921.139509 \\ \text { Sum of electronic and thermal Enthalpies }= & -5921.138565 \\ \text { Sum of electronic and thermal Free Energies }= & -5921.270322 \\ \text { HF=-5921.8713796 } & \\ \text { UB3LYP-D3(BJ)/def2-TZVPP-CPCM(THF)//UB3LYP-D3(BJ)/def2-SVP- } \\ \text { CPCM(THF) }\end{array}$

PBE0-D3(BJ)/def2-TZVPP-CPCM(THF)//UB3LYP-D3(BJ)/def2-SVP-CPCM(THF) $\mathrm{HF}=-5921.6840862$

M06-D3/def2-TZVPP-CPCM(THF)//UB3LYP-D3(BJ)/def2-SVP-CPCM(THF)

$\mathrm{HF}=-5922.9212352$

M06/def2-TZVPP-CPCM(THF)//UB3LYP-D3(BJ)/def2-SVP-CPCM(THF)

$\mathrm{HF}=-5922.8937697$

$\begin{array}{lrrr}{ }^{2} \mathbf{G}-\mathbf{T S}-\mathbf{F}^{, \mathbf{S}_{\boldsymbol{B}} \boldsymbol{B}^{\mathbf{C F} 3} \text {-conf2 }} & & \\ \mathrm{Ni} & -1.10822300 & 1.25417000 & -0.79901600 \\ \mathrm{C} & -2.22918700 & 2.11502800 & 0.57950300 \\ \mathrm{C} & -3.84554200 & 3.41900800 & 2.51146200 \\ \mathrm{C} & -1.86131900 & 2.16275000 & 1.93670400 \\ \mathrm{C} & -3.42418900 & 2.76535200 & 0.22095300 \\ \mathrm{C} & -4.23065000 & 3.40712000 & 1.16568900 \\ \mathrm{C} & -2.64974000 & 2.80023900 & 2.89666600 \\ \mathrm{H} & -0.93288100 & 1.68704200 & 2.26679800 \\ \mathrm{H} & -3.74871900 & 2.76071700 & -0.82475500 \\ \mathrm{H} & -5.15986800 & 3.89361700 & 0.86157700 \\ \mathrm{H} & -2.34191200 & 2.81509600 & 3.94474100 \\ \mathrm{Br} & -1.79372500 & 1.05652000 & -3.10566200 \\ \mathrm{C} & 1.49389100 & 2.24489600 & -0.28788400 \\ \mathrm{C} & 2.16680600 & 4.20526000 & -1.09585900 \\ \mathrm{C} & 0.69179100 & 3.92699800 & -1.51197300 \\ \mathrm{~N} & 0.44574300 & 2.60328400 & -0.93044800 \\ \mathrm{H} & 2.86021700 & 4.24060600 & -1.94512000 \\ \mathrm{H} & 0.60732300 & 3.82954600 & -2.60531800 \\ \mathrm{C} & 0.57168000 & -1.02936600 & 0.95535900\end{array}$




\begin{tabular}{|c|c|c|c|}
\hline $\mathrm{C}$ & 2.03376500 & -0.91363600 & 1.47445400 \\
\hline $\mathrm{C}$ & 1.45211900 & 0.93508100 & 0.38300700 \\
\hline $\mathrm{N}$ & 0.34602300 & 0.28640700 & 0.34429200 \\
\hline $\mathrm{H}$ & -0.12581400 & -1.14650400 & 1.79759600 \\
\hline $\mathrm{H}$ & 2.71304100 & -1.68059600 & 1.08223600 \\
\hline $\mathrm{H}$ & 2.29551900 & 5.10211300 & -0.47746800 \\
\hline $\mathrm{H}$ & 2.10484600 & -0.88321100 & 2.56890900 \\
\hline $\mathrm{O}$ & 2.54338700 & 3.04962700 & -0.27273100 \\
\hline $\mathrm{O}$ & 2.49939900 & 0.38303500 & 0.97047400 \\
\hline $\mathrm{C}$ & -0.35986600 & 4.95077800 & -1.04322100 \\
\hline $\mathrm{H}$ & -1.33145200 & 4.49198800 & -1.29677900 \\
\hline $\mathrm{C}$ & 0.28620900 & -2.14721700 & -0.06491800 \\
\hline $\mathrm{H}$ & -0.75592600 & -1.99122200 & -0.38601700 \\
\hline $\mathrm{C}$ & -3.06255700 & -1.17717500 & 1.39825200 \\
\hline $\mathrm{H}$ & -2.41803600 & -0.37690700 & 1.79631400 \\
\hline $\mathrm{C}$ & -3.64187600 & -0.72581700 & 0.11352300 \\
\hline $\mathrm{H}$ & -4.23553600 & 0.18603500 & 0.06540800 \\
\hline $\mathrm{H}$ & -2.43654500 & -2.06333800 & 1.22022700 \\
\hline $\mathrm{C}$ & -4.10739600 & -1.51913700 & 2.50670800 \\
\hline $\mathrm{C}$ & -5.00656600 & -2.66979000 & 2.03151700 \\
\hline $\mathrm{H}$ & -5.72054700 & -2.95873400 & 2.81962200 \\
\hline $\mathrm{H}$ & -5.58774300 & -2.37971700 & 1.14132200 \\
\hline $\mathrm{H}$ & -4.40978900 & -3.55838200 & 1.76926900 \\
\hline $\mathrm{C}$ & -4.96215800 & -0.28673000 & 2.83980200 \\
\hline $\mathrm{H}$ & -4.33806500 & 0.56887300 & 3.13752200 \\
\hline $\mathrm{H}$ & -5.57289700 & 0.02985400 & 1.98040800 \\
\hline $\mathrm{H}$ & -5.65099700 & -0.50999900 & 3.67040200 \\
\hline $\mathrm{C}$ & -3.32522700 & -1.95129700 & 3.75530500 \\
\hline $\mathrm{H}$ & -4.01132600 & -2.22079900 & 4.57441000 \\
\hline $\mathrm{H}$ & -2.68994400 & -2.82659900 & 3.54229200 \\
\hline $\mathrm{H}$ & -2.67311100 & -1.13775300 & 4.11260400 \\
\hline $\mathrm{C}$ & -3.58921600 & -1.54421500 & -1.08320100 \\
\hline $\mathrm{O}$ & -2.91938000 & -2.56166200 & -1.21598300 \\
\hline $\mathrm{O}$ & -4.38165000 & -1.06262900 & -2.06032300 \\
\hline $\mathrm{C}$ & -4.32431500 & -1.73734400 & -3.31429400 \\
\hline $\mathrm{H}$ & -4.58797800 & -2.79970800 & -3.20224700 \\
\hline $\mathrm{H}$ & -5.04675700 & -1.23316400 & -3.96718700 \\
\hline $\mathrm{H}$ & -3.31401400 & -1.65717200 & -3.74207400 \\
\hline $\mathrm{C}$ & 1.16824600 & -2.03982700 & -1.31075400 \\
\hline $\mathrm{H}$ & 0.88347900 & -2.79911800 & -2.05265100 \\
\hline $\mathrm{H}$ & 1.05120200 & -1.05807600 & -1.79455200 \\
\hline $\mathrm{H}$ & 2.23643800 & -2.18913100 & -1.08075100 \\
\hline $\mathrm{C}$ & 0.35012200 & -3.52066900 & 0.62106700 \\
\hline $\mathrm{H}$ & -0.23662800 & -3.46798400 & 1.55534400 \\
\hline
\end{tabular}




$\begin{array}{lrrr}\mathrm{H} & 1.38984100 & -3.73933700 & 0.92701400 \\ \mathrm{C} & -0.19155500 & -4.66267000 & -0.23897700 \\ \mathrm{H} & 0.43160700 & -4.83597800 & -1.13018900 \\ \mathrm{H} & -0.21990000 & -5.60506100 & 0.33009600 \\ \mathrm{H} & -1.21217800 & -4.43184200 & -0.58108700 \\ \mathrm{C} & -0.32802000 & 5.17629300 & 0.46958600 \\ \mathrm{H} & -1.21259400 & 5.74084400 & 0.79675100 \\ \mathrm{H} & -0.34064700 & 4.22297500 & 1.01506400 \\ \mathrm{H} & 0.56131700 & 5.74918500 & 0.78013500 \\ \mathrm{C} & -0.22279500 & 6.24980400 & -1.85281700 \\ \mathrm{H} & -0.21659000 & 5.99370800 & -2.92646900 \\ \mathrm{H} & 0.76068500 & 6.70971000 & -1.64326400 \\ \mathrm{C} & -1.32804800 & 7.27081000 & -1.58698100 \\ \mathrm{H} & -1.30421300 & 7.64219500 & -0.55098800 \\ \mathrm{H} & -1.22726000 & 8.14207500 & -2.25260200 \\ \mathrm{H} & -2.32316800 & 6.82853000 & -1.75985900 \\ \mathrm{C} & -4.74609300 & 4.00817100 & 3.55462900 \\ \mathrm{~F} & -5.56928200 & 4.95048300 & 3.05673900 \\ \mathrm{~F} & -4.05755100 & 4.57562900 & 4.56601100 \\ \mathrm{~F} & -5.54157500 & 3.06811800 & 4.12226700\end{array}$

UB3LYP-D3(BJ)/def2-SVP-CPCM(THF)

Zero-point correction=

Thermal correction to Energy=

Thermal correction to Enthalpy=

Thermal correction to Gibbs Free Energy=

Sum of electronic and zero-point Energies=

Sum of electronic and thermal Energies=

Sum of electronic and thermal Enthalpies=

Sum of electronic and thermal Free Energies $=$
0.685422 (Hartree/Particle)

0.731471

0.732415

0.597969

$-5921.183404$

$-5921.137356$

$-5921.136412$

$-5921.270857$

$\mathrm{HF}=-5921.8688265$

UB3LYP-D3(BJ)/def2-TZVPP-CPCM(THF)//UB3LYP-D3(BJ)/def2-SVP-

CPCM(THF)

$\mathrm{HF}=-5924.4759019$

PBE0-D3(BJ)/def2-TZVPP-CPCM(THF)//UB3LYP-D3(BJ)/def2-SVP-CPCM(THF)

$\mathrm{HF}=-5921.682429$

M06-D3/def2-TZVPP-CPCM(THF)//UB3LYP-D3(BJ)/def2-SVP-CPCM(THF)

$\mathrm{HF}=-5922.9192238$

M06/def2-TZVPP-CPCM(THF)//UB3LYP-D3(BJ)/def2-SVP-CPCM(THF)

$\mathrm{HF}=-5922.892584$

${ }^{2}$ G-TS-F ${ }^{, S}-B^{\mathrm{CF} 3}$-conf3

$\begin{array}{lrrr}\mathrm{Ni} & -1.18601500 & 1.31387500 & -0.73781600 \\ \mathrm{C} & -2.19160100 & 2.13060400 & 0.76227900 \\ \mathrm{C} & -3.68810700 & 3.29703200 & 2.87579100\end{array}$




\begin{tabular}{|c|c|c|c|}
\hline $\mathrm{C}$ & -1.67564200 & 2.22531300 & 2.06770100 \\
\hline $\mathrm{C}$ & -3.47831700 & 2.66339200 & 0.55268900 \\
\hline $\mathrm{C}$ & -4.22531000 & 3.23603200 & 1.58495100 \\
\hline $\mathrm{C}$ & -2.40341200 & 2.79506900 & 3.11574700 \\
\hline $\mathrm{H}$ & -0.67746500 & 1.83661200 & 2.29051900 \\
\hline $\mathrm{H}$ & -3.92682100 & 2.62922400 & -0.44550900 \\
\hline $\mathrm{H}$ & -5.22458500 & 3.63138500 & 1.39074100 \\
\hline $\mathrm{H}$ & -1.97776200 & 2.84744700 & 4.12030500 \\
\hline $\mathrm{Br}$ & -1.33032500 & 0.58948300 & -3.04125000 \\
\hline $\mathrm{C}$ & 1.42849500 & 2.27528500 & -0.23556100 \\
\hline $\mathrm{C}$ & 2.07198000 & 4.32776400 & -0.76663300 \\
\hline $\mathrm{C}$ & 0.69768100 & 4.01442500 & -1.41644100 \\
\hline $\mathrm{N}$ & 0.38294300 & 2.69039400 & -0.84984300 \\
\hline $\mathrm{H}$ & 2.84444300 & 4.64941100 & -1.47364300 \\
\hline $\mathrm{H}$ & 0.80337800 & 3.88044400 & -2.50773600 \\
\hline $\mathrm{C}$ & 0.50825800 & -1.04441500 & 0.88568600 \\
\hline $\mathrm{C}$ & 1.95880200 & -0.92889300 & 1.43607000 \\
\hline $\mathrm{C}$ & 1.38760800 & 0.93517700 & 0.36641600 \\
\hline $\mathrm{N}$ & 0.28891000 & 0.27979500 & 0.28901200 \\
\hline $\mathrm{H}$ & -0.20505000 & -1.17129600 & 1.71244000 \\
\hline $\mathrm{H}$ & 2.64859900 & -1.68796000 & 1.04744700 \\
\hline $\mathrm{H}$ & 1.99462000 & 5.05101600 & 0.05850500 \\
\hline $\mathrm{H}$ & 2.00692200 & -0.91377300 & 2.53221200 \\
\hline $\mathrm{O}$ & 2.49645300 & 3.05638700 & -0.18079600 \\
\hline $\mathrm{O}$ & 2.42827400 & 0.37624000 & 0.96110500 \\
\hline $\mathrm{C}$ & -0.39560200 & 5.06139500 & -1.16120200 \\
\hline $\mathrm{H}$ & -0.58070600 & 5.07230400 & -0.07146000 \\
\hline $\mathrm{C}$ & 0.25227800 & -2.15617000 & -0.14859700 \\
\hline $\mathrm{H}$ & -0.77452500 & -1.98806800 & -0.50941100 \\
\hline $\mathrm{C}$ & -3.06473500 & -1.18763600 & 1.41428200 \\
\hline $\mathrm{H}$ & -2.42616500 & -0.38468600 & 1.81713700 \\
\hline $\mathrm{C}$ & -3.63304300 & -0.73922700 & 0.12382100 \\
\hline $\mathrm{H}$ & -4.19852800 & 0.18951300 & 0.06204500 \\
\hline $\mathrm{H}$ & -2.43799200 & -2.07397300 & 1.24035400 \\
\hline $\mathrm{C}$ & -4.11588400 & -1.53320700 & 2.51641600 \\
\hline $\mathrm{C}$ & -5.03512800 & -2.65961800 & 2.02220800 \\
\hline $\mathrm{H}$ & -5.74605100 & -2.95630600 & 2.81017700 \\
\hline $\mathrm{H}$ & -5.62036100 & -2.34090100 & 1.14460700 \\
\hline $\mathrm{H}$ & -4.45379000 & -3.54992200 & 1.73332000 \\
\hline $\mathrm{C}$ & -4.94659900 & -0.29124900 & 2.87120800 \\
\hline $\mathrm{H}$ & -4.30697200 & 0.53989400 & 3.20257300 \\
\hline $\mathrm{H}$ & -5.53517900 & 0.06410800 & 2.01155600 \\
\hline $\mathrm{H}$ & -5.65283100 & -0.52027000 & 3.68554500 \\
\hline $\mathrm{C}$ & -3.34089900 & -2.00025200 & 3.75689200 \\
\hline
\end{tabular}




$\begin{array}{lrrr}\mathrm{H} & -4.03158500 & -2.26383200 & 4.57408700 \\ \mathrm{H} & -2.72723000 & -2.88757700 & 3.53098400 \\ \mathrm{H} & -2.66902000 & -1.20702800 & 4.12310900 \\ \mathrm{C} & -3.61098600 & -1.58162500 & -1.05777600 \\ \mathrm{O} & -3.02807100 & -2.65447000 & -1.14914000 \\ \mathrm{O} & -4.32667000 & -1.05214700 & -2.06978700 \\ \mathrm{C} & -4.29586400 & -1.76226100 & -3.30575200 \\ \mathrm{H} & -4.66846200 & -2.78970600 & -3.17795800 \\ \mathrm{H} & -4.94308800 & -1.20665200 & -3.99482900 \\ \mathrm{H} & -3.26889100 & -1.79445200 & -3.69815800 \\ \mathrm{C} & 1.18683700 & -2.05520900 & -1.35559200 \\ \mathrm{H} & 0.90942900 & -2.79371300 & -2.12107300 \\ \mathrm{H} & 1.11339300 & -1.06420800 & -1.82710600 \\ \mathrm{H} & 2.23905400 & -2.24302900 & -1.08399000 \\ \mathrm{C} & 0.27952100 & -3.53020400 & 0.53904800 \\ \mathrm{H} & -0.34990500 & -3.47745100 & 1.44507600 \\ \mathrm{H} & 1.30361600 & -3.74994800 & 0.89346900 \\ \mathrm{C} & -0.22225300 & -4.67176500 & -0.34493700 \\ \mathrm{H} & 0.43639900 & -4.83933300 & -1.21134000 \\ \mathrm{H} & -0.26836400 & -5.61595900 & 0.22015900 \\ \mathrm{H} & -1.23043200 & -4.44450600 & -0.72377600 \\ \mathrm{C} & 0.08978500 & 6.44786600 & -1.59915700 \\ \mathrm{H} & -0.68038600 & 7.20954600 & -1.41370400 \\ \mathrm{H} & 0.99385000 & 6.76561900 & -1.05759600 \\ \mathrm{H} & -.31883600 & 6.45645900 & -2.67794200 \\ \mathrm{C} & -1.69869600 & 4.66027600 & -1.86918200 \\ \mathrm{H} & -1.91090500 & 3.60607100 & -1.64187400 \\ \mathrm{H} & -1.53845000 & 4.70691100 & -2.96118100 \\ \mathrm{C} & -2.91282800 & 5.49843000 & -1.47796000 \\ \mathrm{H} & -2.79709400 & 6.55658100 & -1.75973700 \\ \mathrm{~F} & -3.82125800 & 5.12272900 & -1.97393000 \\ \mathrm{H} & -3.08721200 & 5.45279500 & -0.39117500 \\ \mathrm{C} & -4.51717700 & 3.81241700 & 4.01319800 \\ \mathrm{~F} & -5.22366000 & 2.82193600 & 4.61184900 \\ \mathrm{~F} & -5.41848700 & 4.73287600 & 3.61893400 \\ \mathrm{H} & -3.76850000 & 4.37761500 & 4.98112900\end{array}$

UB3LYP-D3(BJ)/def2-SVP-CPCM(THF)

Zero-point correction=

0.685601 (Hartree/Particle)

Thermal correction to Energy=

0.731508

Thermal correction to Enthalpy=

0.732452

Thermal correction to Gibbs Free Energy=

Sum of electronic and zero-point Energies=

0.598530

$-5921.184769$

Sum of electronic and thermal Energies=

$-5921.138863$

Sum of electronic and thermal Enthalpies=

$-5921.137919$ 
Sum of electronic and thermal Free Energies=

$-5921.271841$

$\mathrm{HF}=-5921.8703707$

UB3LYP-D3(BJ)/def2-TZVPP-CPCM(THF)//UB3LYP-D3(BJ)/def2-SVP-

CPCM(THF)

$\mathrm{HF}=-5924.4759366$

PBE0-D3(BJ)/def2-TZVPP-CPCM(THF)//UB3LYP-D3(BJ)/def2-SVP-CPCM(THF)

$\mathrm{HF}=-5921.6829721$

M06-D3/def2-TZVPP-CPCM(THF)//UB3LYP-D3(BJ)/def2-SVP-CPCM(THF)

$\mathrm{HF}=-5922.9198895$

M06/def2-TZVPP-CPCM(THF)//UB3LYP-D3(BJ)/def2-SVP-CPCM(THF)

$\mathrm{HF}=-5922.8931311$

${ }^{2}$ G-TS-F ${ }^{S}-B^{\mathrm{CF} 3}$-conf4

$\mathrm{Ni}$

C

C

C

C

C

C

$\mathrm{H}$

$\mathrm{H}$

$\mathrm{H}$

$\mathrm{H}$

$\mathrm{Br}$

C

C

C

$\mathrm{N}$

$\mathrm{H}$

$\mathrm{H}$

$\mathrm{C}$

C

C

$\mathrm{N}$

$\mathrm{H}$

$\mathrm{H}$

$\mathrm{H}$

$\mathrm{H}$

$\mathrm{O}$

$\mathrm{O}$

$\mathrm{C}$

$\mathrm{H}$

C
$-1.15097800$

$-2.24427300$

$-3.78385300$

$-1.84608300$

$-3.42805900$

$-4.19686800$

$-2.59692600$

$-0.92397500$

$-3.77125700$

$-5.11867700$

$-2.26562600$

$-1.94299500$

1.44392400

2.14947200

0.68919300

0.41712800

2.87071800

0.64472500

0.52997000

1.92745200

1.38178000

0.29386200

$-0.23579800$

2.66268200

2.25949100

1.88513100

2.49673100

2.40549600

$-0.37589800$

$-1.33758600$

0.42431400
1.23714000

2.10895100

3.47091100

2.15847500

2.78912000

3.45886100

2.82372200

1.66155900

2.78627300

3.96821000

2.83925900

1.37048500

2.20070700

4.14985400

3.86886400

2.55818500

4.17536100

3.73757000

$-1.10866900$

$-0.93495400$

0.88866400

0.21556200

$-1.28572700$

$-1.69424400$

5.05330100

$-0.86646600$

3.00168300

0.35701800

4.90432000

4.43280100

$-2.20508600$
$-0.81694600$

0.58030300

2.53525800

1.92884400

0.24127400

1.19766600

2.90018300

2.24489800

$-0.79802600$

0.90869800

3.94105600

$-3.09803800$

$-0.23882600$

$-1.04928400$

$-1.51126500$

$-0.91372600$

$-1.87554800$

$-2.60156400$

0.91400500

1.57119000

0.42533800

0.31806300

1.67980800

1.27828200

$-0.43675900$

2.66609000

$-0.20187600$

1.07149200

$-1.10295800$

$-1.36864800$

$-0.16757100$ 
$\mathrm{H}$

C

$\mathrm{H}$

C

$\mathrm{H}$

$\mathrm{H}$

C

C

$\mathrm{H}$

$\mathrm{H}$

$\mathrm{H}$

C

$\mathrm{H}$

$\mathrm{H}$

$\mathrm{H}$

C

$\mathrm{H}$

$\mathrm{H}$

$\mathrm{H}$

C

$\mathrm{O}$

$\mathrm{O}$

$\mathrm{C}$

$\mathrm{H}$

$\mathrm{H}$

$\mathrm{H}$

C

$\mathrm{H}$

$\mathrm{H}$

$\mathrm{H}$

C

$\mathrm{H}$

$\mathrm{H}$

C

$\mathrm{H}$

$\mathrm{H}$

$\mathrm{H}$

C

$\mathrm{H}$

$\mathrm{H}$

$\mathrm{H}$

C

$\mathrm{H}$

$\mathrm{H}$
$-0.60404900$

$-3.12808000$

$-2.51180900$

$-3.65438300$

$-4.27283800$

$-2.48353200$

$-4.21658600$

$-5.08294800$

$-5.82983000$

$-5.62494500$

$-4.46644300$

$-5.09676700$

$-4.49494500$

$-5.67104800$

$-5.82050300$

$-3.48579100$

$-4.20475100$

$-2.83590400$

$-2.85560900$

$-3.52951800$

$-2.83830200$

$-4.27271100$

$-4.12938300$

$-4.36202200$

$-4.83325600$

$-3.10251300$

1.38875000

1.25872000

1.20233800

2.44423100

0.59813000

1.59706200

0.60191800

$-0.48568100$

$-1.48186600$

$-0.33980000$

$-0.48609600$

$-0.38719500$

$-1.26443600$

$-0.44955600$

0.50719400

$-0.24466000$

0.72729400

$-1.01001100$
$-2.13748500$

$-1.08764400$

$-0.27298800$

$-0.66713400$

0.22452400

$-1.96620400$

$-1.42355600$

$-2.59123000$

$-2.87310300$

$-2.32347700$

$-3.47848400$

$-0.19505600$

0.67091400

0.10337500

$-0.41359500$

$-1.82873700$

$-2.09002400$

$-2.70238100$

$-1.00428800$

$-1.50227000$

$-2.51165400$

$-1.04294400$

$-1.72166200$

$-2.79183300$

$-1.24466500$

$-1.60805600$

$-1.96108600$

$-2.73853100$

$-0.98889900$

$-1.99092200$

$-3.61173600$

$-3.70114000$

$-4.32632200$

$-4.02689800$

$-3.94961700$

$-5.06793100$

$-3.40001700$

5.18021400

5.78991400

4.25077800

5.73814800

6.20355500

6.68061900

6.90338300
$-0.55759600$

1.54548200

1.95797300

0.22852900

0.13907800

1.41122000

2.61243000

2.11795800

2.87766400

1.19655700

1.90058700

2.88882500

3.20280900

1.99827000

3.69058600

3.90072100

4.69390100

3.73022200

4.27200900

$-0.95039900$

$-1.03580900$

$-1.97623900$

$-3.22070600$

$-3.11538700$

$-3.91298500$

$-3.59885000$

$-1.33115400$

$-2.09907500$

$-1.81211000$

$-1.01261100$

0.42954300

0.89475500

$-0.41041800$

1.42507500

0.96305700

1.75267800

2.33111800

0.40115400

0.66563000

0.98311800

0.72339600

$-1.91398300$

$-1.68948100$

$-1.53866400$ 


$\begin{array}{lrrr}\text { C } & -0.40927400 & 6.04291800 & -3.42560800 \\ \mathrm{H} & -1.34172900 & 5.50702400 & -3.66800600 \\ \mathrm{H} & -0.44492400 & 7.02435400 & -3.92329100 \\ \mathrm{H} & 0.42201000 & 5.48065500 & -3.87999900 \\ \mathrm{C} & -4.64508600 & 4.09444800 & 3.59145900 \\ \mathrm{~F} & -5.44955100 & 5.05746200 & 3.10273800 \\ \mathrm{~F} & -3.91961800 & 4.64810200 & 4.58448700 \\ \mathrm{~F} & -5.45732400 & 3.18328800 & 4.18271200\end{array}$

UB3LYP-D3(BJ)/def2-SVP-CPCM(THF)

Zero-point correction=

0.685518 (Hartree/Particle)

Thermal correction to Energy=

0.731517

Thermal correction to Enthalpy=

0.732461

Thermal correction to Gibbs Free Energy=

Sum of electronic and zero-point Energies=

0.597970

$-5921.183405$

$-5921.137406$

$-5921.136461$

Sum of electronic and thermal Enthalpies=

$-5921.270953$

Sum of electronic and thermal Free Energies=

$\mathrm{HF}=-5921.8689228$

UB3LYP-D3(BJ)/def2-TZVPP-CPCM(THF)//UB3LYP-D3(BJ)/def2-SVP-

$\mathrm{CPCM}(\mathrm{THF})$

$\mathrm{HF}=-5924.475699$

PBE0-D3(BJ)/def2-TZVPP-CPCM(THF)//UB3LYP-D3(BJ)/def2-SVP-CPCM(THF)

$\mathrm{HF}=-5921.682036$

M06-D3/def2-TZVPP-CPCM(THF)//UB3LYP-D3(BJ)/def2-SVP-CPCM(THF)

$\mathrm{HF}=-5922.9186702$

M06/def2-TZVPP-CPCM(THF)//UB3LYP-D3(BJ)/def2-SVP-CPCM(THF)

$\mathrm{HF}=-5922.8915719$

$\begin{array}{lrrr}\begin{array}{l}\text { Scheme 5 } \\ \mathbf{B}^{\mathbf{5 0}}\end{array} & & & \\ \mathrm{C} & -4.34827600 & -3.68665100 & -0.81733300 \\ \mathrm{H} & -3.33791200 & -3.45612600 & -1.17650200 \\ \mathrm{C} & -4.44825500 & -4.42846400 & 0.29658200 \\ \mathrm{H} & -5.41657000 & -4.70410300 & 0.72255200 \\ \mathrm{C} & -3.24236300 & -4.91076300 & 1.00381100 \\ \mathrm{O} & -2.09126400 & -4.70242500 & 0.67704800 \\ \mathrm{O} & -3.57514600 & -5.63311400 & 2.09032000 \\ \mathrm{C} & -2.49364600 & -6.15718100 & 2.86121000 \\ \mathrm{H} & -1.85682400 & -5.34527900 & 3.24334000 \\ \mathrm{H} & -2.94776600 & -6.70607300 & 3.69458500 \\ \mathrm{H} & -1.87313900 & -6.83360600 & 2.25438200 \\ \mathrm{C} & -5.49370100 & -3.15002500 & -1.60704000 \\ \mathrm{H} & -5.43736900 & -2.04942100 & -1.66950400 \\ \mathrm{H} & -5.45077700 & -3.51694300 & -2.64721200\end{array}$




$\mathrm{H}-6.46486700-3.42980200$
UB3LYP-D3(BJ)/def2-SVP-CPCM(THF)
Zero-point correction=
Thermal correction to Energy=
Thermal correction to Enthalpy=
Thermal correction to Gibbs Free Energy=
Sum of electronic and zero-point Energies $=$
Sum of electronic and thermal Energies $=$
Sum of electronic and thermal Enthalpies $=$
Sum of electronic and thermal Free Energies=
HF=-345.5594917

\section{B-TS-C ${ }^{50}$}

\begin{tabular}{|c|c|c|c|}
\hline $\mathrm{C}$ & -4.92439700 & -3.85569000 & -1.07695700 \\
\hline $\mathrm{H}$ & -4.06866700 & -3.42791400 & -1.60709600 \\
\hline $\mathrm{C}$ & -4.64324200 & -4.54726400 & 0.07562600 \\
\hline $\mathrm{H}$ & -5.44124000 & -4.91169700 & 0.72709300 \\
\hline $\mathrm{C}$ & -4.93703300 & -5.50119400 & -2.75232900 \\
\hline $\mathrm{C}$ & -4.66605500 & -4.64972700 & -3.95653300 \\
\hline $\mathrm{H}$ & -4.66531300 & -5.27876900 & -4.87042400 \\
\hline $\mathrm{H}$ & -5.43588000 & -3.87703500 & -4.10667900 \\
\hline $\mathrm{H}$ & -3.68018600 & -4.16210400 & -3.90157100 \\
\hline $\mathrm{C}$ & -6.31707800 & -6.07228500 & -2.61899000 \\
\hline $\mathrm{H}$ & -6.50775100 & -6.43158200 & -1.59514200 \\
\hline $\mathrm{H}$ & -7.10128800 & -5.35234900 & -2.89401400 \\
\hline $\mathrm{H}$ & -6.42758300 & -6.94554900 & -3.29381900 \\
\hline $\mathrm{C}$ & -3.81970400 & -6.40244600 & -2.32087200 \\
\hline $\mathrm{H}$ & -3.68477600 & -7.20979500 & -3.06870600 \\
\hline $\mathrm{H}$ & -2.86580100 & -5.86421400 & -2.23237700 \\
\hline $\mathrm{H}$ & -4.03635800 & -6.88572200 & -1.35594000 \\
\hline $\mathrm{C}$ & -3.27293000 & -4.87051100 & 0.45333200 \\
\hline $\mathrm{O}$ & -2.25895700 & -4.52050300 & -0.13063200 \\
\hline $\mathrm{O}$ & -3.23023500 & -5.63164100 & 1.57463100 \\
\hline $\mathrm{C}$ & -1.93454500 & -5.99684900 & 2.04018200 \\
\hline $\mathrm{H}$ & -1.33319400 & -5.10540600 & 2.27605400 \\
\hline $\mathrm{H}$ & -2.08886400 & -6.59659500 & 2.94554400 \\
\hline $\mathrm{H}$ & -1.39598600 & -6.58790500 & 1.28346900 \\
\hline $\mathrm{C}$ & -6.26331700 & -3.21948600 & -1.31399900 \\
\hline $\mathrm{H}$ & -6.49090900 & -3.09426400 & -2.38154500 \\
\hline $\mathrm{H}$ & -7.07585600 & -3.79288300 & -0.84390000 \\
\hline $\mathrm{H}$ & -6.26842300 & -2.20911600 & -0.86787000 \\
\hline \multicolumn{4}{|c|}{ UB3LYP-D3(BJ)/def2-SVP-CPCM(THF) } \\
\hline \multicolumn{3}{|c|}{ Zero-point correction $=$} & 0.241505 (Hartree/Particle) \\
\hline \multicolumn{3}{|c|}{ Thermal correction to Energy= } & 0.255741 \\
\hline
\end{tabular}

0.122756 (Hartree/Particle)

$$
0.130927
$$

0.131871

0.090117

$-345.436736$

$-345.428565$

$-345.427621$

$-345.469375$ 
Thermal correction to Enthalpy=

Thermal correction to Gibbs Free Energy=

Sum of electronic and zero-point Energies $=$

Sum of electronic and thermal Energies=

Sum of electronic and thermal Enthalpies=

Sum of electronic and thermal Free Energies=

$\mathrm{HF}=-503.2621976$

$\mathbf{C}^{50}$

$\mathrm{C}$

$\mathrm{H}$

$\mathrm{C}$

$\mathrm{H}$

$\mathrm{C}$

$\mathrm{C}$

$\mathrm{H}$

$\mathrm{H}$

$\mathrm{H}$

$\mathrm{C}$

$\mathrm{H}$

$\mathrm{H}$

$\mathrm{H}$

$\mathrm{C}$

$\mathrm{H}$

$\mathrm{H}$

$\mathrm{H}$

C

$\mathrm{O}$

$\mathrm{O}$

$\mathrm{C}$

$\mathrm{H}$

$\mathrm{H}$

$\mathrm{H}$

C

$\mathrm{H}$

$\mathrm{H}$

$\mathrm{H}$
$-3.39259700$

$-4.46469400$

$-5.41705100$

$-4.76175000$

$-6.22758800$

$-6.41609300$

$-6.47567200$

$-6.92452000$

$-3.84389600$

$-2.78493700$

$-4.07274100$

$-3.97314000$

$-4.47727400$

$-4.63845600$

$-5.13572100$

$-3.43340400$

$-3.25161100$

$-2.11340600$

$-3.51283900$

$-2.38058700$

$-1.75233100$

$-2.77501100$

$-1.76515700$

$-5.33080900$

$-6.37558600$

$-4.98001300$

$-5.31586000$
$-4.43293900$
$-3.93831800$

$-3.61571300$

$-4.61815800$

$-4.86440400$

$-4.92767200$

$-5.38658800$

$-6.11526800$

$-5.87586700$

$-4.54744200$

$-6.15768900$

$-5.86172100$

$-6.75378800$

$-6.81207800$

$-4.22502600$

$-4.92318100$

$-3.35897600$

$-3.87511000$

$-4.99319100$

$-4.79071800$

$-5.61535500$

$-6.03015600$

$-5.16808900$

$-6.50956900$

$-6.74362300$

$-2.68897300$

$-2.94648100$

$-1.99355000$

$-2.15162800$

$-1.10111700$

$-1.26766700$

0.22256100

0.69891200

$-2.28700700$

$-2.24931700$

$-3.05397600$

$-1.29423900$

$-2.39439200$

$-2.17727500$

$-2.12123900$

$-1.27970100$

$-3.05397600$

$-3.62388000$

$-4.46099000$

$-3.78686800$

$-3.67254600$

0.92193100

0.52280200

2.09492800

2.85526400

3.12732800

3.75919200

2.28599900

$-1.06068800$

$-0.82888100$

$-0.28322200$

$-2.01972400$

UB3LYP-D3(BJ)/def2-SVP-CPCM(THF)

Zero-point correction=

Thermal correction to Energy=

0.244993 (Hartree/Particle)

0.258787

0.259731

0.204260

$-503.050147$

$-503.036353$

Sum of electronic and thermal Energies= 
Sum of electronic and thermal Enthalpies=

Sum of electronic and thermal Free Energies= $\mathrm{HF}=-503.29514$

$\begin{array}{lrr}-4.56510400 & -3.66964800 & -1.10279300 \\ -3.62457200 & -3.45078800 & -1.61688200 \\ -4.62587700 & -4.49517300 & -0.05017700 \\ -5.56158400 & -4.71771200 & 0.46751400 \\ -5.47304900 & -3.19162900 & -1.47306200 \\ -3.62401800 & -5.99537000 & 1.58386300 \\ -4.65236500 & -6.25741500 & 2.17841000 \\ -3.53267600 & -5.15895300 & 0.50609200 \\ -2.63433300 & -6.42049300 & 1.86527400 \\ -2.61401000 & -5.01927600 & 0.09489000\end{array}$

UB3LYP-D3(BJ)/def2-SVP-CPCM(THF)

Zero-point correction=
Thermal correction to Energy $=$
Thermal correction to Enthalpy $=$
Thermal correction to Gibbs Free Energy=
Sum of electronic and zero-point Energies $=$
Sum of electronic and thermal Energies $=$
Sum of electronic and thermal Enthalpies $=$
Sum of electronic and thermal Free Energies $=$
$\mathrm{HF}=-247.1294622$

$$
\begin{aligned}
& 0.079410 \text { (Hartree/Particle) } \\
& 0.084676 \\
& 0.085620 \\
& 0.051284 \\
& -247.050052 \\
& -247.044786 \\
& -247.043842 \\
& -247.078178
\end{aligned}
$$

\section{B-TS-C ${ }^{58}$}

$\begin{array}{llrr}\mathrm{C} & -4.32743700 & -3.65733800 & -0.81107300 \\ \mathrm{H} & -3.31350100 & -3.56434100 & -1.20793800 \\ \mathrm{C} & -4.56358900 & -4.30864900 & 0.36039700 \\ \mathrm{H} & -5.53749300 & -4.31009800 & 0.85114800 \\ \mathrm{H} & -5.06883200 & -2.94717200 & -1.17720700 \\ \mathrm{C} & -4.80728100 & -5.08751500 & -2.62239100 \\ \mathrm{C} & -6.21999700 & -5.47972000 & -2.31280700 \\ \mathrm{H} & -6.60637000 & -6.20221600 & -3.06203100 \\ \mathrm{H} & -6.29534600 & -5.96621600 & -1.32642400 \\ \mathrm{H} & -6.89829900 & -4.61096900 & -2.32178100 \\ \mathrm{C} & -3.76431400 & -6.14548400 & -2.41931800 \\ \mathrm{H} & -2.74307500 & -5.73828900 & -2.50245300 \\ \mathrm{H} & -3.86612400 & -6.63024700 & -1.43503700 \\ \mathrm{H} & -3.85491500 & -6.94587300 & -3.18385700 \\ \mathrm{C} & -4.59644100 & -4.13792400 & -3.76292400 \\ \mathrm{H} & -4.75659400 & -4.64428400 & -4.73851800 \\ \mathrm{H} & -5.29909500 & -3.28940000 & -3.72517700\end{array}$




$\begin{array}{llrr}\mathrm{H} & -3.56918200 & -3.73726300 & -3.77843500 \\ \mathrm{C} & -3.88515900 & -5.86673800 & 2.10797100 \\ \mathrm{O} & -4.95967500 & -5.90615700 & 2.68379500 \\ \mathrm{~N} & -3.62559500 & -5.12623700 & 0.99133900 \\ \mathrm{H} & -2.99349500 & -6.44223400 & 2.44422200 \\ \mathrm{H} & -2.68865500 & -5.17904900 & 0.60194800\end{array}$

UB3LYP-D3(BJ)/def2-SVP-CPCM(THF)

Zero-point correction=

0.197108 (Hartree/Particle)

Thermal correction to Energy=

0.208899

Thermal correction to Enthalpy=

0.209843

Thermal correction to Gibbs Free Energy=

Sum of electronic and zero-point Energies=

Sum of electronic and thermal Energies=

Sum of electronic and thermal Enthalpies $=$

Sum of electronic and thermal Free Energies=

0.158297

$-404.631839$

$-404.620048$

$-404.619104$

$-404.670649$

$\mathrm{HF}=-404.8289466$

\begin{tabular}{|c|c|c|c|}
\hline \multicolumn{4}{|c|}{$C^{58}$} \\
\hline $\mathrm{C}$ & -4.46605500 & -3.94501000 & -1.13898700 \\
\hline $\mathrm{H}$ & -3.42591300 & -3.58328400 & -1.24044900 \\
\hline $\mathrm{C}$ & -4.66823000 & -4.50891700 & 0.22108600 \\
\hline $\mathrm{H}$ & -5.63929900 & -4.57210000 & 0.70950300 \\
\hline $\mathrm{H}$ & -5.11592000 & -3.06049700 & -1.25351400 \\
\hline $\mathrm{C}$ & -4.75883500 & -4.90143500 & -2.34220700 \\
\hline $\mathrm{C}$ & -6.21971700 & -5.37192700 & -2.29083700 \\
\hline $\mathrm{H}$ & -6.45084600 & -6.01923000 & -3.15215200 \\
\hline $\mathrm{H}$ & -6.42239600 & -5.94635600 & -1.37327300 \\
\hline $\mathrm{H}$ & -6.91363900 & -4.51564200 & -2.31542100 \\
\hline $\mathrm{C}$ & -3.82135600 & -6.11712400 & -2.29260900 \\
\hline $\mathrm{H}$ & -2.76356300 & -5.80605000 & -2.31982300 \\
\hline $\mathrm{H}$ & -3.97847900 & -6.71103000 & -1.37910700 \\
\hline $\mathrm{H}$ & -3.99741800 & -6.77825200 & -3.15647700 \\
\hline $\mathrm{C}$ & -4.51446200 & -4.11756500 & -3.63988000 \\
\hline $\mathrm{H}$ & -4.70817300 & -4.74873700 & -4.52220200 \\
\hline $\mathrm{H}$ & -5.17463600 & -3.23717000 & -3.70238900 \\
\hline $\mathrm{H}$ & -3.47220600 & -3.76356900 & -3.70011200 \\
\hline $\mathrm{C}$ & -3.76369000 & -5.80125400 & 2.07822700 \\
\hline $\mathrm{O}$ & -4.81031500 & -5.90087200 & 2.70213100 \\
\hline $\mathrm{N}$ & -3.64138500 & -5.15101700 & 0.87940400 \\
\hline $\mathrm{H}$ & -2.79795800 & -6.23041100 & 2.42440800 \\
\hline $\mathrm{H}$ & -2.71597300 & -5.13596400 & 0.45813100 \\
\hline \multicolumn{4}{|c|}{ UB3LYP-D3(BJ)/def2-SVP-CPCM(THF) } \\
\hline \multicolumn{3}{|c|}{ Zero-point correction $=$} & 0.201266 (Hartree/Particle) \\
\hline \multicolumn{3}{|c|}{ Thermal correction to Energy= } & 0.212301 \\
\hline
\end{tabular}


Thermal correction to Enthalpy=

Thermal correction to Gibbs Free Energy=

Sum of electronic and zero-point Energies $=$

Sum of electronic and thermal Energies=

Sum of electronic and thermal Enthalpies=

Sum of electronic and thermal Free Energies=

$\mathrm{HF}=-404.8703729$

$\mathbf{B}^{\mathbf{5 9}}$
$\mathrm{C}$
$\mathrm{H}$
$\mathrm{C}$
$\mathrm{H}$
$\mathrm{H}$
$\mathrm{C}$
$\mathrm{O}$
$\mathrm{N}$
$\mathrm{H}$
$\mathrm{C}$
$\mathrm{H}$
$\mathrm{H}$
$\mathrm{H}$

$-3.63234200$

$-4.52789300$

$-3.33616500$

$-4.62783900-4.51556800$

H

$-5.58874300 \quad-4.81527400$

$-5.42803300 \quad-3.18382400$

$-3.69874300 \quad-6.05977900$

$-4.79262100-6.36331100$

$-2.61627900-4.93719000$

C

$-2.40441500 \quad-6.67286300$
0.213245

0.164072

$-404.669107$

$-404.658072$

$-404.657128$

$-404.706301$

UB3LYP-D3(BJ)/def2-SVP-CPCM(THF)

Zero-point correction=

Thermal correction to Energy=

$-1.06233200$

$-1.48308800$

$-0.05974000$

0.36441800

$-1.48492000$

1.58049200

2.03095400

0.55655900

0.22821000

2.07176900

1.41813800

2.06327500

3.08811400

Thermal correction to Enthalpy=

Thermal correction to Gibbs Free Energy=

Sum of electronic and zero-point Energies=

Sum of electronic and thermal Energies=

0.106762 (Hartree/Particle)

0.113702

0.114646

0.075705

$-286.322374$

$-286.315435$

$-286.314491$

$-286.353432$

Sum of electronic and thermal Free Energies= $\mathrm{HF}=-286.4291365$

\section{B-TS-C ${ }^{59}$}

$\mathrm{C}$

$\mathrm{H}$

$\mathrm{C}$

$\mathrm{H}$

$\mathrm{H}$

$\mathrm{C}$

$\mathrm{C}$

$\mathrm{H}$

$\mathrm{H}$

$\mathrm{H}$

$\begin{array}{ll}-4.34367600 & -3.64402200 \\ -3.34484200 & -3.49997000 \\ -4.52378300 & -4.34489500 \\ -5.48179300 & -4.39974100 \\ -5.11796900 & -2.94601700 \\ -4.81682400 & -5.03201200 \\ -6.20862300 & -5.48143700 \\ -6.58695300 & -6.19597200 \\ -6.24611400 & -5.99691100 \\ -6.91592400 & -4.63653000\end{array}$

$-0.81366500$

$-1.23334700$

0.33981400

0.85779200

$-1.13234300$

$-2.65307600$

$-2.32634900$

$-3.08733700$

$-1.35249600$

$-2.29635900$ 


$\begin{array}{lrrr}\mathrm{C} & -3.73609500 & -6.06119000 & -2.50577600 \\ \mathrm{H} & -2.73053800 & -5.61826000 & -2.59819300 \\ \mathrm{H} & -3.79957900 & -6.57783400 & -1.53454000 \\ \mathrm{H} & -3.81765900 & -6.84117100 & -3.29222500 \\ \mathrm{C} & -4.66356300 & -4.04448600 & -3.77053200 \\ \mathrm{H} & -4.83059600 & -4.52717700 & -4.75702500 \\ \mathrm{H} & -5.39155000 & -3.22042000 & -3.69166200 \\ \mathrm{H} & -3.65026200 & -3.61011900 & -3.79754100 \\ \mathrm{C} & -3.75932200 & -5.95886100 & 2.00120300 \\ \mathrm{O} & -4.84501900 & -6.01736700 & 2.56509300 \\ \mathrm{~N} & -3.54356100 & -5.15398100 & 0.91013700 \\ \mathrm{H} & -2.62153900 & -5.15516100 & 0.48706300 \\ \mathrm{C} & -2.56672100 & -6.77754600 & 2.44888800 \\ \mathrm{H} & -2.39786300 & -6.60235500 & 3.52187700 \\ \mathrm{H} & -2.80556700 & -7.84579200 & 2.32747500 \\ \mathrm{H} & -1.64450100 & -6.55324700 & 1.89529400\end{array}$

UB3LYP-D3(BJ)/def2-SVP-CPCM(THF)

Zero-point correction=

Thermal correction to Energy=

0.224357 (Hartree/Particle)

0.237918

0.238862

Thermal correction to Enthalpy=

0.182202

Thermal correction to Gibbs Free Energy=

Sum of electronic and zero-point Energies=

$-443.904042$

$-443.890481$

$-443.889537$

Sum of electronic and thermal Enthalpies=

$-443.946197$

$\mathrm{HF}=-444.1283986$

$C^{59}$
C
H
C
H
H
C
C
H
H
H
C
H
H
H
C
H

$\begin{array}{llr}-4.46771400 & -3.95188000 & -1.12904500 \\ -3.43147300 & -3.57726800 & -1.22723500 \\ -4.64780300 & -4.58149000 & 0.20537700 \\ -5.61032700 & -4.67423900 & 0.70539100 \\ -5.12525200 & -3.06798400 & -1.19320900 \\ -4.76845800 & -4.85145300 & -2.37369400 \\ -6.22403800 & -5.33823200 & -2.32528500 \\ -6.46159100 & -5.94612100 & -3.21322600 \\ -6.40816700 & -5.95716900 & -1.43310900 \\ -6.92604200 & -4.48856400 & -2.29935600 \\ -3.81969000 & -6.05923300 & -2.39684600 \\ -2.76517100 & -5.73697300 & -2.42405100 \\ -3.95781800 & -6.69785800 & -1.51101800 \\ -4.00242300 & -6.67935400 & -3.28933600 \\ -4.54957300 & -4.00350200 & -3.63515800 \\ -4.74962100 & -4.59289500 & -4.54462300\end{array}$




$\begin{array}{lrrr}\mathrm{H} & -5.21859700 & -3.12759700 & -3.64595800 \\ \mathrm{H} & -3.51157400 & -3.63657100 & -3.69210200 \\ \mathrm{C} & -3.72998400 & -5.99992200 & 1.96426700 \\ \mathrm{O} & -4.79891900 & -6.09903400 & 2.55882800 \\ \mathrm{~N} & -3.61368300 & -5.27166300 & 0.80220400 \\ \mathrm{H} & -2.70008400 & -5.23021300 & 0.36133600 \\ \mathrm{C} & -2.45785900 & -6.65922000 & 2.45123200 \\ \mathrm{H} & -2.21974900 & -6.28076600 & 3.45777600 \\ \mathrm{H} & -2.63073300 & -7.74248900 & 2.54602600 \\ \mathrm{H} & -1.59409500 & -6.49078400 & 1.79298300\end{array}$

UB3LYP-D3(BJ)/def2-SVP-CPCM(THF)

Zero-point correction=

Thermal correction to Energy=

0.228809 (Hartree/Particle)

0.241492

Thermal correction to Enthalpy=

0.242436

Thermal correction to Gibbs Free Energy=

0.189114

Sum of electronic and zero-point Energies $=$

$-443.940733$

$-443.928050$

$-443.927106$

Sum of electronic and thermal Enthalpies=

$-443.980428$

$\mathrm{HF}=-444.1695418$

$\begin{array}{cccc}\mathbf{B}^{\mathbf{6 0}} & & & \\ \mathrm{C} & -4.36664600 & -3.66755900 & -0.97464600 \\ \mathrm{H} & -3.35698800 & -3.44825700 & -1.32996100 \\ \mathrm{C} & -4.58701300 & -4.54986900 & -0.00200400 \\ \mathrm{H} & -5.56793300 & -4.81331900 & 0.39712600 \\ \mathrm{H} & -5.21043300 & -3.14841500 & -1.43106300 \\ \mathrm{C} & -3.78686300 & -6.11110100 & 1.57736200 \\ \mathrm{O} & -4.90209600 & -6.35832500 & 1.97433700 \\ \mathrm{O} & -3.53423200 & -5.21406100 & 0.58526800 \\ \mathrm{C} & -2.54034500 & -6.73138600 & 2.09525700 \\ \mathrm{C} & -1.27217000 & -6.40073000 & 1.58986800 \\ \mathrm{C} & -2.65704300 & -7.67868600 & 3.12566400 \\ \mathrm{C} & -0.13406800 & -7.01502500 & 2.11379100 \\ \mathrm{H} & -1.18559700 & -5.66517700 & 0.79034700 \\ \mathrm{C} & -1.51720300 & -8.28964800 & 3.64585900 \\ \mathrm{H} & -3.65006900 & -7.92308500 & 3.50587200 \\ \mathrm{C} & -0.25468400 & -7.95820400 & 3.14023500 \\ \mathrm{H} & 0.85169100 & -6.75753700 & 1.72035700 \\ \mathrm{H} & -1.61061500 & -9.02597000 & 4.44701700 \\ \mathrm{H} & 0.63871400 & -8.43709600 & 3.54787500 \\ \text { UB3LYP-D3(BJ)/def2-SVP-CPCM(THF) } & \\ \text { Zero-point correction= } & & 0.147935 \text { (Hartree/Particle) } \\ \text { Thermal correction to Energy= } & & 0.157453 \\ & & & \end{array}$


Thermal correction to Enthalpy=

Thermal correction to Gibbs Free Energy=

Sum of electronic and zero-point Energies=

Sum of electronic and thermal Energies=

Sum of electronic and thermal Enthalpies=

Sum of electronic and thermal Free Energies= $\mathrm{HF}=-497.889814$

\section{B-TS-C ${ }^{60}$}

$\mathrm{C}$

$\mathrm{H}$

$\mathrm{C}$

$\mathrm{H}$

$\mathrm{H}$

$\mathrm{C}$

$\mathrm{O}$

$\mathrm{O}$

$\mathrm{C}$

$\mathrm{C}$

$\mathrm{C}$

$\mathrm{C}$

$\mathrm{H}$

$\mathrm{C}$

$\mathrm{H}$

C

$\mathrm{H}$

$\mathrm{H}$

$\mathrm{H}$

C

C

$\mathrm{H}$

$\mathrm{H}$

$\mathrm{H}$

C

$\mathrm{H}$

$\mathrm{H}$

$\mathrm{H}$

C

$\mathrm{H}$

$\mathrm{H}$

$\mathrm{H}$

UB

$$
\begin{array}{rrr}
-4.45743200 & -3.66406000 & -0.88835300 \\
-3.43620300 & -3.41024200 & -1.17702200 \\
-4.68797500 & -4.44286600 & 0.19598200 \\
-5.66306100 & -4.65157900 & 0.63494800 \\
-5.26382800 & -3.02700300 & -1.25190700 \\
-3.87553800 & -6.03514300 & 1.74119900 \\
-4.97720800 & -6.22249000 & 2.21125900 \\
-3.63863700 & -5.15107700 & 0.74261800 \\
-2.63638900 & -6.73426300 & 2.16892400 \\
-1.39264100 & -6.49544500 & 1.56031100 \\
-2.73307400 & -7.66743200 & 3.21420400 \\
-0.26092800 & -7.18549800 & 1.99628600 \\
-1.32128600 & -5.77148900 & 0.74866400 \\
-1.59937200 & -8.35374800 & 3.64724300 \\
-3.70689300 & -7.84116200 & 3.67462500 \\
-0.36187600 & -8.11364100 & 3.03871700 \\
0.70487400 & -6.99922200 & 1.52136500 \\
-1.67831100 & -9.07830200 & 4.46073800 \\
0.52648600 & -8.65179400 & 3.37769700 \\
-4.56766900 & -4.98674200 & -2.81961900 \\
-5.92357800 & -5.60744600 & -2.67097700 \\
-6.12932800 & -6.31027100 & -3.50493700 \\
-6.00185600 & -6.18464600 & -1.73523400 \\
-6.72508300 & -4.85108800 & -2.67973400 \\
-3.39334000 & -5.89200000 & -2.59421400 \\
-2.44424200 & -5.33307200 & -2.55552200 \\
-3.49510900 & -6.46355900 & -1.65846200 \\
-3.30188800 & -6.62922100 & -3.41925100 \\
-4.41420500 & -3.92497700 & -3.86667900 \\
-4.41053200 & -4.37085000 & -4.88366800 \\
-5.24239500 & -3.19832200 & -3.83953300 \\
-3.46471500 & -3.37541200 & -3.75736900
\end{array}
$$$$
\text { Zero-point correction= }
$$

Thermal correction to Energy=
0.265540 (Hartree/Particle) 0.281653 
Thermal correction to Enthalpy=

Thermal correction to Gibbs Free Energy=

Sum of electronic and zero-point Energies=

Sum of electronic and thermal Energies=

Sum of electronic and thermal Enthalpies=

Sum of electronic and thermal Free Energies=

$\mathrm{HF}=-655.5899832$

$\mathrm{C}^{60}$

$\mathrm{C}$

$\mathrm{H}$

$\mathrm{C}$

$\mathrm{H}$

$\mathrm{H}$

$\mathrm{C}$

$\mathrm{O}$

$\mathrm{O}$

$\mathrm{C}$

$\mathrm{C}$

$\mathrm{C}$

$\mathrm{C}$

$\mathrm{H}$

$\mathrm{C}$

$\mathrm{H}$

C

$\mathrm{H}$

$\mathrm{H}$

$\mathrm{H}$

$\mathrm{C}$

C

$\mathrm{H}$

$\mathrm{H}$

$\mathrm{H}$

C

$\mathrm{H}$

$\mathrm{H}$

$\mathrm{H}$

C

$\mathrm{H}$

$\mathrm{H}$

$\mathrm{H}$

\section{UB3I}

$$
\begin{array}{rrr}
-4.50443900 & -3.93111100 & -1.22426500 \\
-3.51528500 & -3.44155600 & -1.17569800 \\
-4.76746400 & -4.61858700 & 0.05908300 \\
-5.74795600 & -4.83061200 & 0.48411400 \\
-5.25612400 & -3.13262900 & -1.34146100 \\
-3.90671500 & -6.09696800 & 1.68930300 \\
-4.99167400 & -6.24210900 & 2.20993600 \\
-3.71509700 & -5.29313300 & 0.60880500 \\
-2.64849000 & -6.75197900 & 2.12264500 \\
-1.42839300 & -6.55131900 & 1.45421000 \\
-2.69941800 & -7.60361900 & 3.23889400 \\
-0.27568200 & -7.19728600 & 1.90183100 \\
-1.39161300 & -5.89152200 & 0.58741800 \\
-1.54459500 & -8.24649000 & 3.68247600 \\
-3.65476400 & -7.74917600 & 3.74553100 \\
-0.33117200 & -8.04420700 & 3.01464800 \\
0.67127000 & -7.04034900 & 1.38062900 \\
-1.58827000 & -8.90757600 & 4.55083200 \\
0.57374100 & -8.54821400 & 3.36216600 \\
-4.54212600 & -4.81725900 & -2.51484700 \\
-5.91776800 & -5.48685400 & -2.64788600 \\
-5.97236900 & -6.08164100 & -3.57387500 \\
-6.11764100 & -6.16307500 & -1.80161200 \\
-6.72452800 & -4.73625800 & -2.68017800 \\
-3.44521600 & -5.89143300 & -2.45840200 \\
-2.44819400 & -5.43598900 & -2.34223700 \\
-3.59774600 & -6.58357700 & -1.61722200 \\
-3.44010700 & -6.48374000 & -3.38781900 \\
-4.29656700 & -3.89803500 & -3.72038700 \\
-4.31045400 & -4.47352100 & -4.66007400 \\
-5.07141400 & -3.11730800 & -3.79022500 \\
-3.31746000 & -3.39743300 & -3.64503300
\end{array}
$$

Zero-point correction=

Thermal correction to Energy=
0.269610 (Hartree/Particle)

0.285013 
Thermal correction to Enthalpy=

Thermal correction to Gibbs Free Energy=

Sum of electronic and zero-point Energies $=$

Sum of electronic and thermal Energies=

Sum of electronic and thermal Enthalpies=

Sum of electronic and thermal Free Energies=

$\mathrm{HF}=-655.6295692$

$\mathbf{B}^{\mathbf{5 1}}$
$\mathrm{C}$
$\mathrm{C}$
$\mathrm{C}$
$\mathrm{C}$
$\mathrm{O}$
$\mathrm{H}$
$\mathrm{H}$
$\mathrm{H}$
$\mathrm{O}$
$\mathrm{H}$

$-4.51608300$

C $\quad-4.65991600 \quad-3.78978400$

C $\quad-5.90782500-3.31224300$

C $\quad-6.64509400 \quad-3.69523500$

O $\quad-5.78574500-4.40415300$

$\begin{array}{lll}-4.26206600 & -5.58240300\end{array}$

$\begin{array}{lll}-3.85177500 & -3.69175000\end{array}$

$\begin{array}{lll}-3.85176393500 & -2.73737000\end{array}$

$-7.78330200-3.47210200$
0.285957

0.224987

$-655.359960$

$-655.344557$

$-655.343612$

$-655.404582$

UB3LYP-D3(BJ)/def2-SVP-CPCM(THF)

Zero-point correction=

Thermal correction to Energy=

0.21109900

$-1.08934600$

$-1.17938200$

0.04844300

0.84262600

0.07424900

$-1.81528500$

$-1.98323100$

Thermal correction to Enthalpy=

0.37510000

0.86016800

Thermal correction to Gibbs Free Energy=

Sum of electronic and zero-point Energies=

Sum of electronic and thermal Energies=

0.074597 (Hartree/Particle)

0.079227

0.080171

0.046664

$-304.987496$

$-304.982865$

$-304.981921$

$-305.015428$

Sum of electronic and thermal Free Energies= $\mathrm{HF}=-305.0620923$

\section{B-TS-C ${ }^{51}$}

$\mathrm{C}$

C

$\mathrm{H}$

$\mathrm{H}$

$\mathrm{H}$

$\mathrm{C}$

$\mathrm{H}$

$\mathrm{H}$

$\mathrm{H}$

C

$\mathrm{H}$

$\mathrm{H}$

$\mathrm{H}$

-4.81645600
-6.19565000
-6.46800500
-6.27713600
-6.94910500
-3.66905100
-2.70149100
-3.77419500
-3.61864400
-4.66148800
-4.73427200
-5.45213500
-3.68095400

$-5.25637100$

$-5.77927000$

$-6.52614000$

$-6.28594000$

$-4.97796900$

$-6.17733800$

$-5.65073900$

$-6.69643000$

$-6.96589100$

$-4.33884600$

$-4.91772300$

$-3.57383600$

$-3.83669600$
$-2.50313800$

$-2.24311200$

$-3.01595000$

$-1.26924200$

$-2.28981600$

$-2.21964000$

$-2.23650400$

$-1.25423700$

$-2.99789200$

$-3.67813700$

$-4.62081700$

$-3.70602400$

$-3.68463900$ 


$\begin{array}{lrrr}\mathrm{C} & -4.84757800 & -4.50578300 & 0.46282800 \\ \mathrm{C} & -4.56489200 & -3.68917200 & -0.77099600 \\ \mathrm{C} & -5.60685800 & -2.81570400 & -0.92292300 \\ \mathrm{C} & -6.66588800 & -3.17369200 & 0.02038500 \\ \mathrm{O} & -6.19888500 & -4.20169300 & 0.80776800 \\ \mathrm{H} & -4.74891300 & -5.59274200 & 0.32949600 \\ \mathrm{H} & -3.55662600 & -3.56268100 & -1.16349000 \\ \mathrm{H} & -5.71380600 & -2.01203500 & -1.64808600 \\ \mathrm{O} & -7.77716000 & -2.71987500 & 0.17369100 \\ \mathrm{H} & -4.18256100 & -4.20791000 & 1.29325100\end{array}$

UB3LYP-D3(BJ)/def2-SVP-CPCM(THF)

\begin{tabular}{|c|c|c|c|}
\hline \multicolumn{3}{|c|}{ Zero-point correction $=$} & $0.192859(\mathrm{H}$ \\
\hline \multicolumn{3}{|c|}{ Thermal correction to Energy= } & 0.203874 \\
\hline \multicolumn{3}{|c|}{ Thermal correction to Enthalpy= } & 0.204819 \\
\hline \multicolumn{3}{|c|}{ Thermal correction to Gibbs Free Energy= } & 0.155672 \\
\hline \multicolumn{3}{|c|}{ Sum of electronic and zero-point Energies= } & -462.573 \\
\hline \multicolumn{3}{|c|}{ Sum of electronic and thermal Energies= } & -462.562 \\
\hline \multicolumn{3}{|c|}{ Sum of electronic and thermal Enthalpies= } & -462.5611 \\
\hline \multicolumn{3}{|c|}{ Sum of electronic and thermal Free Energies $=$} & \\
\hline \multicolumn{4}{|c|}{$\mathrm{HF}=-462.7659456$} \\
\hline \multicolumn{4}{|c|}{$C^{51}$} \\
\hline $\mathrm{C}$ & -4.80134400 & -5.04512400 & -2.25515300 \\
\hline $\mathrm{C}$ & -6.17280700 & -5.73635200 & -2.21509600 \\
\hline $\mathrm{H}$ & -6.25317800 & -6.46662800 & -3.03544500 \\
\hline $\mathrm{H}$ & -6.33473600 & -6.28092100 & -1.27206800 \\
\hline $\mathrm{H}$ & -6.99164300 & -5.00909400 & -2.33226900 \\
\hline $\mathrm{C}$ & -3.67978200 & -6.08840000 & -2.13923100 \\
\hline $\mathrm{H}$ & -2.68794100 & -5.60932300 & -2.16638200 \\
\hline $\mathrm{H}$ & -3.75029700 & -6.67263400 & -1.20901400 \\
\hline $\mathrm{H}$ & -3.73553000 & -6.79922700 & -2.97865300 \\
\hline $\mathrm{C}$ & -4.64336300 & -4.29055500 & -3.58426800 \\
\hline $\mathrm{H}$ & -4.66342900 & -4.99837900 & -4.42777200 \\
\hline $\mathrm{H}$ & -5.45503300 & -3.56406000 & -3.74093400 \\
\hline $\mathrm{H}$ & -3.68526600 & -3.74756800 & -3.62262500 \\
\hline $\mathrm{C}$ & -4.85985800 & -4.62181800 & 0.34211700 \\
\hline $\mathrm{C}$ & -4.66272400 & -4.01685200 & -1.07027200 \\
\hline $\mathrm{C}$ & -5.69363900 & -2.95081000 & -1.06503200 \\
\hline $\mathrm{C}$ & -6.60020600 & -3.13183700 & 0.05427100 \\
\hline $\mathrm{O}$ & -6.13909000 & -4.16867300 & 0.81523700 \\
\hline $\mathrm{H}$ & -4.86608400 & -5.71864100 & 0.35764400 \\
\hline $\mathrm{H}$ & -3.65236600 & -3.58078900 & -1.16332700 \\
\hline $\mathrm{H}$ & -5.82913700 & -2.16486200 & -1.80553400 \\
\hline $\mathrm{O}$ & -7.61778400 & -2.53413300 & 0.33559000 \\
\hline
\end{tabular}




\begin{tabular}{|c|c|c|c|}
\hline $\mathrm{H}$ & -4.08651300 & -4.26779600 & 1.04099100 \\
\hline \multicolumn{4}{|c|}{ UB3LYP-D3(BJ)/def2-SVP-CPCM(THF) } \\
\hline \multicolumn{3}{|c|}{ Zero-point correction $=$} & 0.196634 (Hartree/Particle) \\
\hline \multicolumn{3}{|c|}{ Thermal correction to Energy= } & 0.207049 \\
\hline \multicolumn{3}{|c|}{ Thermal correction to Enthalpy= } & 0.207994 \\
\hline \multicolumn{3}{|c|}{ Thermal correction to Gibbs Free Energy= } & 0.160647 \\
\hline \multicolumn{3}{|c|}{ Sum of electronic and zero-point Energies $=$} & -462.602061 \\
\hline \multicolumn{3}{|c|}{ Sum of electronic and thermal Energies $=$} & -462.591646 \\
\hline \multicolumn{3}{|c|}{ Sum of electronic and thermal Enthalpies= } & -462.590702 \\
\hline \multicolumn{3}{|c|}{ Sum of electronic and thermal Free Energies $=$} & -462.638049 \\
\hline \multicolumn{4}{|c|}{$H F=-462.7986958$} \\
\hline \multicolumn{4}{|l|}{$B^{52}$} \\
\hline $\mathrm{C}$ & -4.32661400 & -3.80931400 & -0.91340900 \\
\hline $\mathrm{H}$ & -3.35746700 & -3.52047700 & -1.33028500 \\
\hline $\mathrm{C}$ & -4.37845700 & -4.47497600 & 0.24372700 \\
\hline $\mathrm{H}$ & -5.33730900 & -4.77357100 & 0.67939400 \\
\hline $\mathrm{H}$ & -5.23044300 & -3.53986600 & -1.46471500 \\
\hline $\mathrm{C}$ & -3.12388400 & -4.83285400 & 0.98130800 \\
\hline $\mathrm{O}$ & -2.00538000 & -4.54064300 & 0.57027400 \\
\hline $\mathrm{N}$ & -3.32532000 & -5.50719900 & 2.14216800 \\
\hline $\mathrm{H}$ & -4.24966800 & -5.74567700 & 2.47745900 \\
\hline $\mathrm{H}$ & -2.52309400 & -5.78274200 & 2.69697000 \\
\hline
\end{tabular}

UB3LYP-D3(BJ)/def2-SVP-CPCM(THF)

Zero-point correction=

Thermal correction to Energy=

0.079033 (Hartree/Particle)

0.084510

0.085454

Thermal correction to Enthalpy=

0.050766

Sum of electronic and zero-point Energies=

$-247.057752$

$-247.052274$

$-247.051330$

Sum of electronic and thermal Enthalpies=

Sum of electronic and thermal Free Energies= $\mathrm{HF}=-247.1367843$

$-247.086019$

\section{B-TS-C ${ }^{52}$}

$\begin{array}{lrrr}\mathrm{C} & -3.94966300 & -3.88942200 & -1.28334500 \\ \mathrm{H} & -2.95594900 & -4.17430800 & -1.63197800 \\ \mathrm{C} & -4.35036500 & -4.25870200 & -0.03803200 \\ \mathrm{H} & -5.29630500 & -3.89248100 & 0.37170600 \\ \mathrm{H} & -4.47491200 & -3.10344100 & -1.82692200 \\ \mathrm{C} & -4.80959300 & -5.48684900 & -2.84993100 \\ \mathrm{C} & -6.04517100 & -5.93991800 & -2.13642400 \\ \mathrm{H} & -6.57195500 & -6.71508800 & -2.73043200 \\ \mathrm{H} & -5.80629000 & -6.38523100 & -1.15793300\end{array}$




$\begin{array}{lrrr}\mathrm{H} & -6.75456900 & -5.11260000 & -1.97681300 \\ \mathrm{C} & -3.65988100 & -6.44830700 & -2.87458300 \\ \mathrm{H} & -2.76436400 & -6.00415600 & -3.33743400 \\ \mathrm{H} & -3.39290000 & -6.78301300 & -1.86086200 \\ \mathrm{H} & -3.92234100 & -7.34823300 & -3.46949200 \\ \mathrm{C} & -4.98948200 & -4.60112200 & -4.04459000 \\ \mathrm{H} & -5.31791400 & -5.19592600 & -4.92288300 \\ \mathrm{H} & -5.75690100 & -3.82907700 & -3.87522900 \\ \mathrm{H} & -4.04802000 & -4.10513900 & -4.33192800 \\ \mathrm{C} & -3.57249800 & -5.23223700 & 0.75916700 \\ \mathrm{O} & -2.52825300 & -5.75357500 & 0.35944900 \\ \mathrm{~N} & -4.09954300 & -5.53280200 & 1.97986600 \\ \mathrm{H} & -3.59994500 & -6.17412100 & 2.58426600 \\ \mathrm{H} & -4.93206700 & -5.08149100 & 2.33535800\end{array}$

UB3LYP-D3(BJ)/def2-SVP-CPCM(THF)

Zero-point correction=

0.196947 (Hartree/Particle)

Thermal correction to Energy=

0.208958

Thermal correction to Enthalpy=

0.209902

Thermal correction to Gibbs Free Energy=

Sum of electronic and zero-point Energies=

Sum of electronic and thermal Energies=

Sum of electronic and thermal Enthalpies=

Sum of electronic and thermal Free Energies=

0.158381

$-404.644342$

$-404.632331$

$-404.631387$

$-404.682908$

$\mathrm{HF}=-404.8412892$

$\begin{array}{lrrr}\mathbf{C}^{\mathbf{5 2}} & & & \\ \mathrm{C} & -4.32284300 & -3.95931800 & -1.10458200 \\ \mathrm{H} & -3.29235200 & -3.62056200 & -1.29117200 \\ \mathrm{C} & -4.38261800 & -4.53911300 & 0.25759200 \\ \mathrm{H} & -5.34873900 & -4.68041400 & 0.75095400 \\ \mathrm{H} & -4.99041000 & -3.08066900 & -1.16020500 \\ \mathrm{C} & -4.73259300 & -4.92788700 & -2.26382400 \\ \mathrm{C} & -6.20458300 & -5.34094500 & -2.11539300 \\ \mathrm{H} & -6.51022000 & -5.99156000 & -2.95075700 \\ \mathrm{H} & -6.37618900 & -5.89703100 & -1.18034300 \\ \mathrm{H} & -6.86713100 & -4.45990400 & -2.11416900 \\ \mathrm{C} & -3.83801400 & -6.17646100 & -2.24381100 \\ \mathrm{H} & -2.77274900 & -5.90360800 & -2.29331500 \\ \mathrm{H} & -3.98908800 & -6.76157100 & -1.32284200 \\ \mathrm{H} & -4.06866500 & -6.83063300 & -3.10024300 \\ \mathrm{C} & -4.54157300 & -4.17855700 & -3.59021700 \\ \mathrm{H} & -4.82472300 & -4.81624500 & -4.44332600 \\ \mathrm{H} & -5.16285500 & -3.26888900 & -3.62666200 \\ \mathrm{H} & -3.49051300 & -3.87693000 & -3.72641300\end{array}$




$\begin{array}{llll}\mathrm{C} & -3.16636000 & -4.98399000 & 0.94114400 \\ \mathrm{O} & -2.04296100 & -4.86955200 & 0.43797900 \\ \mathrm{~N} & -3.34956900 & -5.54175000 & 2.17364400 \\ \mathrm{H} & -2.54078100 & -5.86725200 & 2.68912600 \\ \mathrm{H} & -4.26449400 & -5.64183600 & 2.59287000\end{array}$

UB3LYP-D3(BJ)/def2-SVP-CPCM(THF)

Zero-point correction=

0.200702 (Hartree/Particle)

Thermal correction to Energy=

0.212116

Thermal correction to Enthalpy=

0.213061

Thermal correction to Gibbs Free Energy=

0.163209

Sum of electronic and zero-point Energies=

$-404.680086$

Sum of electronic and thermal Energies=

$-404.668671$

Sum of electronic and thermal Enthalpies=

$-404.667727$

Sum of electronic and thermal Free Energies=

$-404.717578$

$\mathrm{HF}=-404.8807879$

$\begin{array}{lrrr}\mathbf{B}^{\mathbf{5 3}} & & & \\ \mathrm{C} & -3.34273300 & -3.61119700 & -1.46426900 \\ \mathrm{H} & -2.76204300 & -4.05002500 & -2.27991300 \\ \mathrm{C} & -3.18618900 & -4.05918600 & -0.21405800 \\ \mathrm{H} & -3.76480000 & -3.62146000 & 0.59774400 \\ \mathrm{H} & -4.04439000 & -2.80720800 & -1.69825400 \\ \mathrm{C} & -2.21718700 & -5.16312800 & 0.07765200 \\ \mathrm{O} & -1.55665700 & -5.67845000 & -0.82528100 \\ \mathrm{~N} & -2.07152000 & -5.59613800 & 1.36220600 \\ \mathrm{H} & -1.38715400 & -6.34516400 & 1.41988400 \\ \mathrm{C} & -2.69170500 & -5.20364700 & 2.64781200 \\ \mathrm{C} & -2.37031500 & -3.74084500 & 3.00100300 \\ \mathrm{C} & -4.20906000 & -5.45935700 & 2.63761100 \\ \mathrm{C} & -2.04792300 & -6.11532100 & 3.70465200 \\ \mathrm{H} & -1.28154600 & -3.58073200 & 3.00674900 \\ \mathrm{H} & -2.81459500 & -3.02968100 & 2.29165300 \\ \mathrm{H} & -2.76136700 & -3.50181500 & 4.00170400 \\ \mathrm{H} & -4.41631200 & -6.51102200 & 2.38830300 \\ \mathrm{H} & -4.63238500 & -5.24848700 & 3.63148900 \\ \mathrm{H} & -4.73664800 & -4.82752200 & 1.91038600 \\ \mathrm{H} & -2.45584600 & -5.88938100 & 4.70000800 \\ \mathrm{H} & -2.25028300 & -7.17512600 & 3.48339300 \\ \mathrm{H} & -0.95724000 & -5.96579400 & 3.73881700\end{array}$

UB3LYP-D3(BJ)/def2-SVP-CPCM(THF)

Zero-point correction=

0.191313 (Hartree/Particle)

Thermal correction to Energy=

0.201941

Thermal correction to Enthalpy=

0.202885

Thermal correction to Gibbs Free Energy=

0.155604 
Sum of electronic and zero-point Energies=

Sum of electronic and thermal Energies=

Sum of electronic and thermal Enthalpies=

Sum of electronic and thermal Free Energies= $\mathrm{HF}=-404.2913611$

\section{B-TS-C $C^{53}$}

$\mathrm{C}$

$\mathrm{H}$

$\mathrm{C}$

$\mathrm{H}$

$\mathrm{H}$

$\mathrm{C}$

$\mathrm{C}$

$\mathrm{H}$

$\mathrm{H}$

$\mathrm{H}$

C

$\mathrm{H}$

$\mathrm{H}$

$\mathrm{H}$

$\mathrm{C}$

$\mathrm{H}$

$\mathrm{H}$

$\mathrm{H}$

C

$\mathrm{O}$

$\mathrm{N}$

$\mathrm{H}$

C

C

C

C

$\mathrm{H}$

$\mathrm{H}$

$\mathrm{H}$

$\mathrm{H}$

$\mathrm{H}$

$\mathrm{H}$

$\mathrm{H}$

$\mathrm{H}$

$\mathrm{H}$

$$
\begin{array}{rrr}
-3.46892500 & -3.72955100 & -1.51792400 \\
-2.81625100 & -4.08518700 & -2.31559600 \\
-3.24369800 & -4.12511400 & -0.23653800 \\
-3.82845700 & -3.68849000 & 0.57026300 \\
-4.11406200 & -2.87557600 & -1.72669500 \\
-5.15998000 & -5.18078200 & -2.43715500 \\
-5.86479900 & -4.21213200 & -3.33630900 \\
-6.65484100 & -4.72918700 & -3.92097000 \\
-6.36038200 & -3.40765500 & -2.76978600 \\
-5.17699600 & -3.75642300 & -4.06713300 \\
-5.87023600 & -5.60410200 & -1.18946700 \\
-5.19518300 & -6.13226900 & -0.49857500 \\
-6.31041300 & -4.74729600 & -0.65543100 \\
-6.69910700 & -6.30013100 & -1.43412000 \\
-4.27392100 & -6.20216300 & -3.08355500 \\
-4.87921200 & -7.04783400 & -3.47270400 \\
-3.71928800 & -5.78156900 & -3.93735200 \\
-3.54558700 & -6.61215800 & -2.36777100 \\
-2.28300000 & -5.20929600 & 0.05691100 \\
-1.64527100 & -5.76748300 & -0.84646700 \\
-2.09407400 & -5.61294200 & 1.35089200 \\
-1.41779100 & -6.36893100 & 1.40313500 \\
-2.69567000 & -5.20513000 & 2.63726000 \\
-2.39023500 & -3.73105600 & 2.95706000 \\
-4.21037800 & -5.47963000 & 2.65912400 \\
-2.02294000 & -6.08627200 & 3.70183900 \\
-1.30341100 & -3.55754200 & 2.94843300 \\
-2.84936600 & -3.04311300 & 2.23462300 \\
-2.77461400 & -3.47456600 & 3.95618900 \\
-4.40780400 & -6.53937900 & 2.43666100 \\
-4.62149400 & -5.25180500 & 3.65452700 \\
-4.75578300 & -4.87263200 & 1.92438000 \\
-2.41605100 & -5.84535300 & 4.69978800 \\
-2.21558700 & -7.15295300 & 3.50568200 \\
-0.93368200 & -5.92329200 & 3.71302300
\end{array}
$$

UB3LYP-D3(BJ)/def2-SVP-CPCM(THF)

Zero-point correction=

0.309387 (Hartree/Particle) 
Thermal correction to Energy=

Thermal correction to Enthalpy=

Thermal correction to Gibbs Free Energy=

Sum of electronic and zero-point Energies=

Sum of electronic and thermal Energies=

Sum of electronic and thermal Enthalpies=

Sum of electronic and thermal Free Energies=

$\mathrm{HF}=-561.9971912$

$\mathbf{C}^{53}$

$\mathrm{C}$

$\mathrm{H}$

$\mathrm{C}$

$\mathrm{H}$

$\mathrm{H}$

$\mathrm{C}$

$\mathrm{C}$

$\mathrm{H}$

$\mathrm{H}$

$\mathrm{H}$

$\mathrm{C}$

$\mathrm{H}$

$\mathrm{H}$

$\mathrm{H}$

$\mathrm{C}$

$\mathrm{H}$

$\mathrm{H}$

$\mathrm{H}$

C

$\mathrm{O}$

$\mathrm{N}$

$\mathrm{H}$

C

C

C

C

$\mathrm{H}$

$\mathrm{H}$

$\mathrm{H}$

$\mathrm{H}$

$\mathrm{H}$

$\mathrm{H}$

$\mathrm{H}$

$\mathrm{H}$

$\begin{array}{lrrr}C & & & \\ H & -3.65348000 & -3.71505200 & -1.50982100 \\ \mathrm{C} & -2.77549300 & -3.78703000 & -2.16835000 \\ \mathrm{H} & -3.24192200 & -4.07583700 & -0.13180000 \\ \mathrm{H} & -3.87701400 & -3.78670600 & 0.70324500 \\ \mathrm{C} & -4.01457300 & -2.67132500 & -1.52375000 \\ \mathrm{H} & -4.78992200 & -4.60790200 & -2.11136600 \\ \mathrm{H} & -5.03824300 & -4.14189000 & -3.55306200 \\ \mathrm{H} & -5.83907900 & -4.73535500 & -4.02323800 \\ \mathrm{C} & -5.34035700 & -3.08232700 & -3.58223600 \\ \mathrm{H} & -4.12937200 & -4.25215700 & -4.16623500 \\ \mathrm{H} & -6.07983200 & -4.44999100 & -1.29217500 \\ \mathrm{H} & -5.94636800 & -4.79666000 & -0.25536300 \\ \mathrm{C} & -6.40464200 & -3.39717600 & -1.25932200 \\ \mathrm{H} & -6.89619400 & -5.04161800 & -1.73709900 \\ \mathrm{H} & -4.35414400 & -6.08099800 & -2.11320600 \\ \mathrm{H} & -5.11680500 & -6.70863400 & -2.60202000 \\ \mathrm{C} & -3.40164100 & -6.21365400 & -2.64868900 \\ \mathrm{O} & -4.21510700 & -6.45833200 & -1.08771600 \\ \mathrm{~N} & -2.02732600 & -4.85548000 & 0.10059800 \\ \mathrm{H} & -1.25264400 & -5.11520000 & -0.83398200 \\ \mathrm{C} & -1.72931400 & -5.29880400 & 1.36362600 \\ \mathrm{C} & -0.86340200 & -5.82938000 & 1.37103700 \\ \mathrm{C} & -2.49411800 & -5.30736100 & 2.62744100 \\ \mathrm{C} & -2.67448900 & -3.88324000 & 3.18280100 \\ \mathrm{H} & -3.85043300 & -6.01502400 & 2.45517800 \\ \mathrm{H} & -1.64100900 & -6.11412500 & 3.61891300 \\ \mathrm{H} & -1.69553200 & -3.39448100 & 3.30052200 \\ \mathrm{H} & -3.29164200 & -3.25250100 & 2.52854700 \\ & -3.16479900 & -3.92134100 & 4.16755200 \\ \mathrm{H} & -3.70206500 & -7.03372800 & 2.06571900 \\ & -4.36636600 & -6.08545200 & 3.42487000 \\ & -4.51385400 & -5.48061000 & 1.76173300 \\ & -2.14107400 & -6.16680700 & 4.59643800 \\ & & & 3.25603100\end{array}$

0.326461

0.327405

0.264459

$-561.687804$

$-561.670730$

$-561.669786$

$-561.732732$ 


\begin{tabular}{|c|c|c|c|}
\hline $\mathrm{H}$ & -0.65621900 & -5.64202300 & 3.76189300 \\
\hline \multicolumn{4}{|c|}{ UB3LYP-D3(BJ)/def2-SVP-CPCM(THF) } \\
\hline \multicolumn{3}{|c|}{ Zero-point correction $=$} & 0.313095 (Hartree/Particle) \\
\hline \multicolumn{3}{|c|}{ Thermal correction to Energy= } & 0.329563 \\
\hline \multicolumn{3}{|c|}{ Thermal correction to Enthalpy= } & 0.330507 \\
\hline \multicolumn{3}{|c|}{ Thermal correction to Gibbs Free Energy= } & 0.268944 \\
\hline \multicolumn{3}{|c|}{ Sum of electronic and zero-point Energies $=$} & -561.724075 \\
\hline \multicolumn{3}{|c|}{ Sum of electronic and thermal Energies $=$} & -561.707606 \\
\hline \multicolumn{3}{|c|}{ Sum of electronic and thermal Enthalpies $=$} & -561.706662 \\
\hline \multicolumn{3}{|c|}{ Sum of electronic and thermal Free Energies $=$} & -561.768225 \\
\hline \multicolumn{4}{|c|}{$H F=-562.0371696$} \\
\hline \multicolumn{4}{|c|}{$B^{49}$} \\
\hline $\mathrm{C}$ & -4.29833200 & -3.78258200 & -0.89752700 \\
\hline $\mathrm{H}$ & -3.32638000 & -3.75874600 & -1.39857100 \\
\hline $\mathrm{C}$ & -4.42262100 & -4.35320000 & 0.30518200 \\
\hline $\mathrm{H}$ & -5.37775200 & -4.39497900 & 0.83417600 \\
\hline $\mathrm{H}$ & -5.15221600 & -3.33021300 & -1.40701400 \\
\hline $\mathrm{C}$ & -3.25143500 & -4.96411200 & 0.98475100 \\
\hline $\mathrm{O}$ & -2.12262700 & -5.00648000 & 0.54487400 \\
\hline $\mathrm{O}$ & -3.58789300 & -5.48230000 & 2.17940400 \\
\hline $\mathrm{H}$ & -2.78314700 & -5.86475900 & 2.57171600 \\
\hline \multicolumn{4}{|c|}{ UB3LYP-D3(BJ)/def2-SVP-CPCM(THF) } \\
\hline \multicolumn{3}{|c|}{ Zero-point correction $=$} & 0.067257 (Hartree/Particle) \\
\hline \multicolumn{3}{|c|}{ Thermal correction to Energy= } & 0.072257 \\
\hline \multicolumn{3}{|c|}{ Thermal correction to Enthalpy= } & 0.073201 \\
\hline \multicolumn{3}{|c|}{ Thermal correction to Gibbs Free Energy= } & 0.039266 \\
\hline \multicolumn{3}{|c|}{ Sum of electronic and zero-point Energies= } & -266.917836 \\
\hline \multicolumn{3}{|c|}{ Sum of electronic and thermal Energies $=$} & -266.912836 \\
\hline \multicolumn{3}{|c|}{ Sum of electronic and thermal Enthalpies $=$} & -266.911892 \\
\hline \multicolumn{3}{|c|}{ Sum of electronic and thermal Free Energies $=$} & -266.945827 \\
\hline \multicolumn{4}{|c|}{$H F=-266.9850931$} \\
\hline \multicolumn{4}{|c|}{ B-TS-C ${ }^{49}$} \\
\hline $\mathrm{C}$ & -4.63734400 & -3.79275400 & -1.07318800 \\
\hline $\mathrm{H}$ & -3.71081900 & -3.54542200 & -1.59369200 \\
\hline $\mathrm{C}$ & -4.58831700 & -4.43211800 & 0.11888400 \\
\hline $\mathrm{H}$ & -5.48742700 & -4.63420200 & 0.70488600 \\
\hline $\mathrm{H}$ & -5.56346500 & -3.35079000 & -1.44200500 \\
\hline $\mathrm{C}$ & -4.93229500 & -5.52507900 & -2.86675800 \\
\hline $\mathrm{C}$ & -6.40598700 & -5.74679200 & -2.73479100 \\
\hline $\mathrm{H}$ & -6.74076300 & -6.55652500 & -3.41708700 \\
\hline $\mathrm{H}$ & -6.67863400 & -6.05815000 & -1.71333900 \\
\hline $\mathrm{H}$ & -6.98603000 & -4.84752900 & -2.99646500 \\
\hline
\end{tabular}




$\begin{array}{lrrr}\mathrm{C} & -4.02808500 & -6.58261300 & -2.32049900 \\ \mathrm{H} & -2.99232700 & -6.22607700 & -2.21695100 \\ \mathrm{H} & -4.37142000 & -6.93695500 & -1.33534500 \\ \mathrm{H} & -4.01114300 & -7.46520000 & -2.99383600 \\ \mathrm{C} & -4.44788600 & -4.71371600 & -4.02604500 \\ \mathrm{H} & -4.54756500 & -5.28655000 & -4.97247100 \\ \mathrm{H} & -5.03382400 & -3.78888000 & -4.15435300 \\ \mathrm{H} & -3.38389100 & -4.44660300 & -3.92608900 \\ \mathrm{C} & -3.31465400 & -4.94176000 & 0.64277000 \\ \mathrm{O} & -2.22494200 & -4.82934100 & 0.11004900 \\ \mathrm{O} & -3.46812000 & -5.57938900 & 1.82449200 \\ \mathrm{H} & -2.58654800 & -5.88379900 & 2.10133400\end{array}$

UB3LYP-D3(BJ)/def2-SVP-CPCM(THF)

Zero-point correction $=$

Thermal correction to Energy=

0.185049 (Hartree/Particle)

Thermal correction to Enthalpy=

0.196666

0.197610

Thermal correction to Gibbs Free Energy=

Sum of electronic and zero-point Energies=

0.146509

Sum of electronic and thermal Energies=

$-424.506644$

$-424.495027$

Sum of electronic and thermal Enthalpies=

Sum of electronic and thermal Free Energies=

$-424.494083$

$-424.545184$

$\mathrm{HF}=-424.6916931$

$\begin{array}{crrr}\text { C }^{49} & & & \\ \mathrm{C} & -4.36394100 & -3.97578600 & -1.09652700 \\ \mathrm{H} & -3.33631400 & -3.61492200 & -1.25523200 \\ \mathrm{C} & -4.45664300 & -4.57712900 & 0.25330400 \\ \mathrm{H} & -5.42455000 & -4.74234700 & 0.73178200 \\ \mathrm{H} & -5.04572500 & -3.10885000 & -1.15370400 \\ \mathrm{C} & -4.73117600 & -4.93457000 & -2.27990400 \\ \mathrm{C} & -6.19415100 & -5.38715500 & -2.16213700 \\ \mathrm{H} & -6.47024800 & -6.02399300 & -3.01789200 \\ \mathrm{H} & -6.36553600 & -5.97078200 & -1.24411300 \\ \mathrm{H} & -6.87853600 & -4.52324100 & -2.14975200 \\ \mathrm{C} & -3.80353700 & -6.15881200 & -2.26803300 \\ \mathrm{H} & -2.74506600 & -5.85748000 & -2.30190300 \\ \mathrm{H} & -3.95038700 & -6.76533600 & -1.36036400 \\ \mathrm{H} & -4.00590700 & -6.80340300 & -3.13848500 \\ \mathrm{C} & -4.53792000 & -4.15392300 & -3.58769400 \\ \mathrm{H} & -4.78725900 & -4.78409600 & -4.45657300 \\ \mathrm{H} & -5.18506100 & -3.26246600 & -3.61884300 \\ \mathrm{H} & -3.49387300 & -3.81990500 & -3.69943400 \\ \mathrm{C} & -3.27223700 & -5.01549300 & 0.96766900 \\ \mathrm{O} & -2.12682000 & -4.92205900 & 0.54994300\end{array}$




$\begin{array}{llll}\mathrm{O} & -3.55467000 & -5.55760700 & 2.17442100 \\ \mathrm{H} & -2.70910600 & -5.82212500 & 2.57574900\end{array}$

UB3LYP-D3(BJ)/def2-SVP-CPCM(THF)

\begin{tabular}{|c|c|c|c|}
\hline \multicolumn{3}{|c|}{ Zero-point correction $=$} & $0.189390(\mathrm{Ha}$ \\
\hline \multicolumn{3}{|c|}{ Thermal correction to Energy= } & 0.200058 \\
\hline \multicolumn{3}{|c|}{ Thermal correction to Enthalpy= } & 0.201002 \\
\hline \multicolumn{3}{|c|}{ Thermal correction to Gibbs Free Energy= } & 0.152764 \\
\hline \multicolumn{3}{|c|}{ Sum of electronic and zero-point Energies= } & -424.541 \\
\hline \multicolumn{3}{|c|}{ Sum of electronic and thermal Energies $=$} & -424.53 \\
\hline \multicolumn{3}{|c|}{ Sum of electronic and thermal Enthalpies $=$} & \\
\hline \multicolumn{3}{|c|}{ Sum of electronic and thermal Free Energies $=$} & 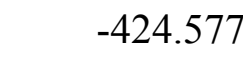 \\
\hline \multicolumn{4}{|c|}{$\mathrm{HF}=-424.7306232$} \\
\hline \multicolumn{4}{|l|}{$\mathbf{B}^{54}$} \\
\hline $\mathrm{C}$ & -4.29476500 & -3.72816200 & -1.00633700 \\
\hline $\mathrm{H}$ & -3.30791000 & -3.71932700 & -1.47891600 \\
\hline $\mathrm{C}$ & -4.45121300 & -4.29402900 & 0.19646900 \\
\hline $\mathrm{H}$ & -5.42856700 & -4.31437400 & 0.68855500 \\
\hline $\mathrm{H}$ & -5.12782900 & -3.26739700 & -1.54264500 \\
\hline $\mathrm{C}$ & -3.30344100 & -4.92143700 & 0.92119900 \\
\hline $\mathrm{O}$ & -2.17937200 & -4.94719300 & 0.44943800 \\
\hline $\mathrm{C}$ & -2.42599700 & -6.14882400 & 2.97560300 \\
\hline $\mathrm{H}$ & -1.63432400 & -5.40334700 & 3.14489400 \\
\hline $\mathrm{H}$ & -2.71764800 & -6.57025000 & 3.94905400 \\
\hline $\mathrm{H}$ & -1.99148500 & -6.95520100 & 2.36562500 \\
\hline $\mathrm{C}$ & -3.62318400 & -5.52190200 & 2.27534600 \\
\hline $\mathrm{H}$ & -4.43641900 & -6.25585900 & 2.12385800 \\
\hline $\mathrm{H}$ & -4.08060100 & -4.72271900 & 2.88779500 \\
\hline
\end{tabular}

UB3LYP-D3(BJ)/def2-SVP-CPCM(THF)

Zero-point correction=

0.117470 (Hartree/Particle)

Thermal correction to Energy=

0.124535

Thermal correction to Enthalpy=

0.125480

Thermal correction to Gibbs Free Energy=

Sum of electronic and zero-point Energies=

0.086478

Sum of electronic and thermal Energies=

$-270.258029$

$-270.250964$

$-270.250020$

Sum of electronic and thermal Enthalpies=

Sum of electronic and thermal Free Energies= $\mathrm{HF}=-270.3754997$

$-270.289022$

\section{B-TS-C ${ }^{54}$}

$\begin{array}{lrrr}\mathrm{C} & -4.68436300 & -3.73160000 & -1.08349500 \\ \mathrm{H} & -3.75427000 & -3.50917800 & -1.60973300 \\ \mathrm{C} & -4.64145400 & -4.38227300 & 0.09915400 \\ \mathrm{H} & -5.55525400 & -4.57076300 & 0.67056700\end{array}$




$\begin{array}{llll}\mathrm{H} & -5.60865200 & -3.30305200 & -1.47336300 \\ \mathrm{C} & -4.95922000 & -5.54104300 & -2.95125600 \\ \mathrm{C} & -6.43923300 & -5.72850200 & -2.85180900 \\ \mathrm{H} & -6.78142400 & -6.53848500 & -3.53113900 \\ \mathrm{H} & -6.74264500 & -6.02052200 & -1.83300100 \\ \mathrm{H} & -6.99227800 & -4.81903400 & -3.13646000 \\ \mathrm{C} & -4.08472600 & -6.59128400 & -2.34852300 \\ \mathrm{H} & -3.04828700 & -6.24268300 & -2.22607400 \\ \mathrm{H} & -4.45864500 & -6.90884100 & -1.36155800 \\ \mathrm{H} & -4.05789400 & -7.49811100 & -2.98959500 \\ \mathrm{C} & -4.42297200 & -4.73299700 & -4.08862400 \\ \mathrm{H} & -4.48816500 & -5.29957700 & -5.04252500 \\ \mathrm{H} & -4.99501400 & -3.80223200 & -4.23714900 \\ \mathrm{H} & -3.36154400 & -4.47479900 & -3.94536100 \\ \mathrm{C} & -3.37097600 & -4.90757800 & 0.64355100 \\ \mathrm{O} & -2.30145700 & -4.77337000 & 0.06027700 \\ \mathrm{C} & -2.12871600 & -6.15658400 & 2.48880900 \\ \mathrm{H} & -1.41262300 & -5.33310200 & 2.63155000 \\ \mathrm{H} & -2.25316200 & -6.67660300 & 3.45055300 \\ \mathrm{H} & -1.67811300 & -6.85930100 & 1.77156800 \\ \mathrm{C} & -3.46254200 & -5.63544800 & 1.97428400 \\ \mathrm{H} & -4.19448700 & -6.45483000 & 1.85021200 \\ \mathrm{H} & -3.93440000 & -4.94619500 & 2.69859200\end{array}$

UB3LYP-D3(BJ)/def2-SVP-CPCM(THF)

Zero-point correction=

Thermal correction to Energy=

Thermal correction to Enthalpy=

Thermal correction to Gibbs Free Energy=

Sum of electronic and zero-point Energies=

Sum of electronic and thermal Energies=

Sum of electronic and thermal Enthalpies=

Sum of electronic and thermal Free Energies= $\mathrm{HF}=-428.0823713$
0.235017 (Hartree/Particle)
0.248877
0.249821
0.193154
$-427.847354$
$-427.833495$
$-427.832550$
$-427.889217$




$\begin{array}{lrrr}\mathrm{H} & -6.88521200 & -4.49138000 & -2.21451800 \\ \mathrm{C} & -3.82637400 & -6.15857600 & -2.29609200 \\ \mathrm{H} & -2.76478300 & -5.86797800 & -2.32543700 \\ \mathrm{H} & -3.98405900 & -6.75435300 & -1.38317300 \\ \mathrm{H} & -4.02985400 & -6.80890200 & -3.16208700 \\ \mathrm{C} & -4.52583400 & -4.15430600 & -3.63454300 \\ \mathrm{H} & -4.76890600 & -4.78779500 & -4.50288000 \\ \mathrm{H} & -5.16570400 & -3.25814500 & -3.67911800 \\ \mathrm{H} & -3.47802800 & -3.82855100 & -3.73490100 \\ \mathrm{C} & -3.34022800 & -4.99736200 & 0.96546000 \\ \mathrm{O} & -2.19425400 & -4.88267400 & 0.51379800 \\ \mathrm{C} & -2.32606100 & -6.05512600 & 3.05254700 \\ \mathrm{H} & -1.64584800 & -5.20411700 & 3.20927900 \\ \mathrm{H} & -2.56303200 & -6.49312700 & 4.03380600 \\ \mathrm{H} & -1.77815100 & -6.80557700 & 2.46263000 \\ \mathrm{C} & -3.59399400 & -5.61585400 & 2.33376900 \\ \mathrm{H} & -4.28725100 & -6.46524500 & 2.19582400 \\ \mathrm{H} & -4.15962800 & -4.88180900 & 2.93576300\end{array}$

UB3LYP-D3(BJ)/def2-SVP-CPCM(THF)

Zero-point correction=

Thermal correction to Energy=

0.239853 (Hartree/Particle)

0.252557

0.253502

0.200159

$-427.884509$

$-427.871804$

$-427.870860$

$-427.924202$

Sum of electronic and thermal Free Energies= $\mathrm{HF}=-428.1243616$

$\begin{array}{cccc}\mathbf{B}^{57} & & & \\ \mathrm{C} & -4.21251500 & -4.28670700 & -1.36050400 \\ \mathrm{H} & -3.54196500 & -4.91968400 & -1.94951000 \\ \mathrm{C} & -4.16423900 & -4.29737100 & -0.02480900 \\ \mathrm{H} & -4.82260700 & -3.67119400 & 0.58614900 \\ \mathrm{H} & -4.91794900 & -3.65160000 & -1.90422100 \\ \mathrm{O} & -2.13794500 & -6.20377000 & -0.03064500 \\ \mathrm{O} & -3.88470400 & -6.03299800 & 1.95021500 \\ \mathrm{O} & -2.09586800 & -4.29652500 & 1.75663900 \\ \mathrm{C} & -3.32671100 & -7.05340400 & 2.80934200 \\ \mathrm{C} & -2.90743300 & -6.47528400 & 4.14696600 \\ \mathrm{H} & -2.4811900 & -7.53506600 & 2.29296100 \\ \mathrm{H} & -4.11975900 & -7.80579700 & 2.92999600 \\ \mathrm{H} & -2.54767000 & -7.28130100 & 4.80579200 \\ \mathrm{H} & -2.10161800 & -5.73869200 & 4.01808800\end{array}$




$\begin{array}{lrrr}\mathrm{H} & -3.75826600 & -5.97926200 & 4.63896000 \\ \mathrm{C} & -0.92989100 & -3.64906000 & 1.21337800 \\ \mathrm{C} & -0.05807700 & -3.18352900 & 2.35935300 \\ \mathrm{H} & -1.25472600 & -2.79795500 & 0.59008600 \\ \mathrm{H} & -0.39499300 & -4.36012900 & 0.56451000 \\ \mathrm{H} & 0.82864600 & -2.66046300 & 1.96909800 \\ \mathrm{H} & -0.60979100 & -2.49109200 & 3.01357300 \\ \mathrm{H} & 0.27978000 & -4.03969600 & 2.96331900 \\ \mathrm{P} & -2.96959900 & -5.31481800 & 0.83604600\end{array}$

UB3LYP-D3(BJ)/def2-SVP-CPCM(THF)

Zero-point correction=

Thermal correction to Energy=

Thermal correction to Enthalpy=

Thermal correction to Gibbs Free Energy=

Sum of electronic and zero-point Energies=

Sum of electronic and thermal Energies=

Sum of electronic and thermal Enthalpies=

Sum of electronic and thermal Free Energies= $\mathrm{HF}=-803.1385909$
0.189850 (Hartree/Particle)
0.202904
0.203848
0.148051
-802.948741
-802.935687
-802.934743
-802.990540

\section{B-TS-C $\mathbf{C}^{57}$}

$\begin{array}{lrrr}\mathrm{C} & -4.10219800 & -4.22923600 & -1.33902500 \\ \mathrm{H} & -3.47284400 & -4.92976300 & -1.89083900 \\ \mathrm{C} & -3.92449900 & -4.05971700 & 0.00316800 \\ \mathrm{H} & -4.45912000 & -3.28249200 & 0.55714200 \\ \mathrm{H} & -4.63105500 & -3.47220200 & -1.92139600 \\ \mathrm{O} & -2.26060200 & -6.27275500 & 0.17056500 \\ \mathrm{O} & -3.96200900 & -5.69200400 & 2.10269900 \\ \mathrm{O} & -1.91388000 & -4.29604300 & 1.84687400 \\ \mathrm{C} & -3.56504300 & -6.74115500 & 3.01261800 \\ \mathrm{C} & -3.06755300 & -6.16955900 & 4.32626800 \\ \mathrm{H} & -2.79873900 & -7.36636200 & 2.52728900 \\ \mathrm{H} & -4.46082500 & -7.36211800 & 3.16107600 \\ \mathrm{H} & -2.82981600 & -6.98730400 & 5.02495700 \\ \mathrm{H} & -2.16301000 & -5.56506600 & 4.16848000 \\ \mathrm{H} & -3.83714400 & -5.53187100 & 4.78816200 \\ \mathrm{C} & -0.71166500 & -3.78955400 & 1.24306900 \\ \mathrm{C} & 0.15192600 & -3.18168700 & 2.32695700 \\ \mathrm{H} & -0.97956500 & -3.03582900 & 0.48151900 \\ \mathrm{H} & -0.19142800 & -4.61446200 & 0.72994600 \\ \mathrm{H} & 1.07386300 & -2.76959800 & 1.88840800 \\ \mathrm{H} & -0.38292800 & -2.36805000 & 2.84064400 \\ \mathrm{H} & 0.43080900 & -3.94102300 & 3.07380200 \\ \mathrm{P} & -2.94286900 & -5.18879300 & 0.94836800 \\ & & & \\ & & 5268 & \\ & & & \\ & & & \\ & & & \\ & & & \end{array}$




$\begin{array}{lrrr}\mathrm{C} & -5.87714500 & -5.72790600 & -1.67615400 \\ \mathrm{C} & -6.67740300 & -5.51503000 & -0.42769100 \\ \mathrm{H} & -7.56614000 & -6.17840900 & -0.42875000 \\ \mathrm{H} & -6.09002400 & -5.74924500 & 0.47278800 \\ \mathrm{H} & -7.04202100 & -4.47910400 & -0.34579400 \\ \mathrm{C} & -5.11654000 & -7.01768800 & -1.76583600 \\ \mathrm{H} & -4.50108000 & -7.06844800 & -2.67789800 \\ \mathrm{H} & -4.45579100 & -7.16523500 & -0.89824900 \\ \mathrm{H} & -5.82190500 & -7.87341000 & -1.79875700 \\ \mathrm{C} & -6.43866600 & -5.18029200 & -2.95334400 \\ \mathrm{H} & -7.26397200 & -5.82693500 & -3.31744400 \\ \mathrm{H} & -6.85606800 & -4.16948600 & -2.82196900 \\ \mathrm{H} & -5.68216400 & -5.14966800 & -3.75415800\end{array}$

UB3LYP-D3(BJ)/def2-SVP-CPCM(THF)

\begin{tabular}{|c|c|c|c|}
\hline \multicolumn{3}{|c|}{ Zero-point correction $=$} & 0.308178 (Hartree/Particle) \\
\hline \multicolumn{3}{|c|}{ Thermal correction to Energy= } & 0.327564 \\
\hline \multicolumn{3}{|c|}{ Thermal correction to Enthalpy= } & 0.328508 \\
\hline \multicolumn{3}{|c|}{ Thermal correction to Gibbs Free Energy= } & 0.257760 \\
\hline \multicolumn{3}{|c|}{ Sum of electronic and zero-point Energies $=$} & -960.538331 \\
\hline \multicolumn{3}{|c|}{ Sum of electronic and thermal Energies $=$} & -960.518945 \\
\hline \multicolumn{3}{|c|}{ Sum of electronic and thermal Enthalpies $=$} & -960.518001 \\
\hline \multicolumn{3}{|c|}{ Sum of electronic and thermal Free Energies $=$} & -960.588749 \\
\hline \multicolumn{4}{|c|}{$H F=-960.8465089$} \\
\hline \multicolumn{4}{|c|}{$C^{57}$} \\
\hline $\mathrm{C}$ & -4.11628200 & -4.09356200 & -0.95005000 \\
\hline $\mathrm{H}$ & -3.25888400 & -4.49449100 & -1.51370000 \\
\hline $\mathrm{C}$ & -3.82739100 & -4.17556000 & 0.50607300 \\
\hline $\mathrm{H}$ & -4.36128800 & -3.54121300 & 1.22007700 \\
\hline $\mathrm{H}$ & -4.24650400 & -3.03588300 & -1.23769700 \\
\hline $\mathrm{O}$ & -1.93238200 & -6.14898100 & 0.14049400 \\
\hline $\mathrm{O}$ & -3.63006100 & -6.22689300 & 2.16964700 \\
\hline $\mathrm{O}$ & -1.73979800 & -4.59124200 & 2.22861700 \\
\hline $\mathrm{C}$ & -3.08955900 & -7.37969100 & 2.85514000 \\
\hline $\mathrm{C}$ & -2.70942800 & -7.03535900 & 4.28174500 \\
\hline $\mathrm{H}$ & -2.22855600 & -7.76675100 & 2.28712700 \\
\hline $\mathrm{H}$ & -3.88066400 & -8.14333500 & 2.82469600 \\
\hline $\mathrm{H}$ & -2.35528300 & -7.93834800 & 4.80372300 \\
\hline $\mathrm{H}$ & -1.91000600 & -6.28084800 & 4.29874300 \\
\hline $\mathrm{H}$ & -3.57775000 & -6.63696300 & 4.82891400 \\
\hline $\mathrm{C}$ & -0.46044300 & -4.07415000 & 1.81650300 \\
\hline $\mathrm{C}$ & -0.59046800 & -2.72596600 & 1.13179100 \\
\hline $\mathrm{H}$ & 0.02569200 & -4.80923200 & 1.15641300 \\
\hline $\mathrm{H}$ & 0.13210300 & -3.99370800 & 2.73941300 \\
\hline
\end{tabular}




\begin{tabular}{|c|c|c|c|}
\hline $\mathrm{H}$ & 0.40804800 & -2.31788800 & 0.90965200 \\
\hline $\mathrm{H}$ & -1.14051400 & -2.81439800 & 0.18144700 \\
\hline $\mathrm{H}$ & -1.12442700 & -2.01073500 & 1.77611000 \\
\hline $\mathrm{P}$ & -2.69842600 & -5.36950200 & 1.16340600 \\
\hline $\mathrm{C}$ & -5.39589400 & -4.87008000 & -1.41590200 \\
\hline $\mathrm{C}$ & -6.64193600 & -4.28590300 & -0.73498200 \\
\hline $\mathrm{H}$ & -7.55107000 & -4.79776200 & -1.08952000 \\
\hline $\mathrm{H}$ & -6.59631100 & -4.40553500 & 0.35881100 \\
\hline $\mathrm{H}$ & -6.75096300 & -3.21164400 & -0.95679500 \\
\hline $\mathrm{C}$ & -5.26308900 & -6.36086000 & -1.06825500 \\
\hline $\mathrm{H}$ & -4.33862000 & -6.78677500 & -1.48752700 \\
\hline $\mathrm{H}$ & -5.23634200 & -6.51770500 & 0.02083900 \\
\hline $\mathrm{H}$ & -6.11901000 & -6.92626600 & -1.47044900 \\
\hline $\mathrm{C}$ & -5.51059400 & -4.70242600 & -2.93749100 \\
\hline $\mathrm{H}$ & -6.40260900 & -5.22458000 & -3.31952400 \\
\hline $\mathrm{H}$ & -5.59450900 & -3.63965100 & -3.21687400 \\
\hline $\mathrm{H}$ & -4.62849400 & -5.11975800 & -3.44933000 \\
\hline \multicolumn{4}{|c|}{ UB3LYP-D3(BJ)/def2-SVP-CPCM(THF) } \\
\hline \multicolumn{3}{|c|}{ Zero-point correction $=$} & 0.312029 (Hartree/Particle) \\
\hline \multicolumn{3}{|c|}{ Thermal correction to Energy= } & 0.330780 \\
\hline \multicolumn{3}{|c|}{ Thermal correction to Enthalpy= } & 0.331724 \\
\hline \multicolumn{3}{|c|}{ Thermal correction to Gibbs Free Energy= } & 0.262579 \\
\hline \multicolumn{3}{|c|}{ Sum of electronic and zero-point Energies $=$} & -960.569876 \\
\hline \multicolumn{3}{|c|}{ Sum of electronic and thermal Energies $=$} & -960.551125 \\
\hline \multicolumn{3}{|c|}{ Sum of electronic and thermal Enthalpies $=$} & -960.550181 \\
\hline \multicolumn{3}{|c|}{ Sum of electronic and thermal Free Energies $=$} & -960.619326 \\
\hline \multicolumn{4}{|c|}{$H F=-960.8819047$} \\
\hline \multicolumn{4}{|c|}{$B^{56}$} \\
\hline $\mathrm{C}$ & -4.77335900 & -4.32150700 & -1.16309700 \\
\hline $\mathrm{H}$ & -4.19941800 & -5.09344900 & -1.68284100 \\
\hline $\mathrm{C}$ & -4.46428800 & -3.97443800 & 0.08358900 \\
\hline $\mathrm{H}$ & -4.97514300 & -3.21458500 & 0.68036900 \\
\hline $\mathrm{H}$ & -5.60062500 & -3.84039400 & -1.69147700 \\
\hline $\mathrm{S}$ & -3.06668500 & -4.72549700 & 0.90306700 \\
\hline $\mathrm{O}$ & -2.45153500 & -5.69464400 & -0.02271400 \\
\hline $\mathrm{O}$ & -2.25932200 & -3.63192500 & 1.47360700 \\
\hline $\mathrm{C}$ & -3.79556500 & -5.63854200 & 2.26120000 \\
\hline $\mathrm{C}$ & -4.24559200 & -6.94279000 & 2.03704800 \\
\hline $\mathrm{C}$ & -3.88898100 & -5.02778500 & 3.51386000 \\
\hline $\mathrm{C}$ & -4.81490200 & -7.64679100 & 3.09930600 \\
\hline $\mathrm{H}$ & -4.14081100 & -7.39399500 & 1.04931300 \\
\hline $\mathrm{C}$ & -4.46064000 & -5.74414200 & 4.56822000 \\
\hline $\mathrm{H}$ & -3.51003200 & -4.01444800 & 3.65286000 \\
\hline
\end{tabular}




$\begin{array}{llll}\mathrm{C} & -4.92334000 & -7.04775400 & 4.36010900 \\ \mathrm{H} & -5.17056300 & -8.66765000 & 2.94483400 \\ \mathrm{H} & -4.54061200 & -5.28436900 & 5.55549300 \\ \mathrm{H} & -5.36892300 & -7.60385300 & 5.18791600\end{array}$

UB3LYP-D3(BJ)/def2-SVP-CPCM(THF)

Zero-point correction=

0.143221 (Hartree/Particle)

Thermal correction to Energy=

0.153200

Thermal correction to Enthalpy=

0.154145

Thermal correction to Gibbs Free Energy=

Sum of electronic and zero-point Energies=

Sum of electronic and thermal Energies=

Sum of electronic and thermal Enthalpies=

Sum of electronic and thermal Free Energies= $\mathrm{HF}=-857.8040116$

0.106958

$-857.660791$

$-857.650811$

$-857.649867$

$-857.697054$

\section{B-TS-C $\mathbf{C}^{56}$}

$\begin{array}{llll}\mathrm{C} & -5.71322600 & -4.85997900 & -0.88427700 \\ \mathrm{H} & -5.18967200 & -3.90389300 & -0.92196900 \\ \mathrm{C} & -5.25344900 & -5.85522000 & -0.08502600 \\ \mathrm{H} & -5.75649100 & -6.81041000 & 0.07858500 \\ \mathrm{H} & -6.74209600 & -4.89494000 & -1.24552200 \\ \mathrm{C} & -4.94695100 & -5.27340100 & -3.09219100 \\ \mathrm{C} & -6.10694900 & -4.72368700 & -3.86428300 \\ \mathrm{H} & -5.89659600 & -4.76954200 & -4.95304300 \\ \mathrm{H} & -7.03008900 & -5.29900700 & -3.69119200 \\ \mathrm{H} & -6.29702800 & -3.66551800 & -3.62228700 \\ \mathrm{C} & -4.78458800 & -6.76123500 & -3.04857700 \\ \mathrm{H} & -4.06802200 & -7.06427800 & -2.27015600 \\ \mathrm{H} & -5.74143600 & -7.27417600 & -2.86407200 \\ \mathrm{H} & -4.39565300 & -7.12829100 & -4.02005500 \\ \mathrm{C} & -3.68443700 & -4.46558500 & -3.08724200 \\ \mathrm{H} & -3.12926300 & -4.62674400 & -4.03423200 \\ \mathrm{H} & -3.88630300 & -3.38568600 & -3.00875600 \\ \mathrm{H} & -3.01870000 & -4.75362800 & -2.26061500 \\ \mathrm{~S} & -3.61682800 & -5.79313600 & 0.56128900 \\ \mathrm{O} & -2.88592200 & -6.99493300 & 0.09958500 \\ \mathrm{O} & -3.04678900 & -4.45587400 & 0.28768100 \\ \mathrm{C} & -3.81629900 & -5.94706700 & 2.33529200 \\ \mathrm{C} & -3.75181300 & -7.21391700 & 2.92061500 \\ \mathrm{C} & -4.03199200 & -4.79327200 & 3.09471800 \\ \mathrm{C} & -3.92089500 & -7.32538400 & 4.30295500 \\ \mathrm{H} & -3.56267200 & -8.08939300 & 2.29815600 \\ \mathrm{C} & -4.19771400 & -4.91861700 & 4.47494100 \\ \mathrm{H} & -4.05974300 & -3.81807500 & 2.60649700\end{array}$




$\begin{array}{llll}\mathrm{C} & -4.14474300 & -6.18167200 & 5.07675200 \\ \mathrm{H} & -3.87224300 & -8.30790800 & 4.77730400 \\ \mathrm{H} & -4.36422600 & -4.02764600 & 5.08416700 \\ \mathrm{H} & -4.27477100 & -6.27385200 & 6.15735700\end{array}$

UB3LYP-D3(BJ)/def2-SVP-CPCM(THF)

Zero-point correction=

0.261634 (Hartree/Particle)

Thermal correction to Energy=

0.277997

Thermal correction to Enthalpy=

0.278941

Thermal correction to Gibbs Free Energy=

Sum of electronic and zero-point Energies=

Sum of electronic and thermal Energies=

Sum of electronic and thermal Enthalpies=

Sum of electronic and thermal Free Energies=

$\mathrm{HF}=-1015.5143562$

0.216065

$-1015.252722$

$-1015.236360$

$-1015.235415$

$-1015.298291$

$\begin{array}{crrr}\text { C }^{56} & & & \\ \mathrm{C} & -4.93940100 & -4.28736100 & -0.83907500 \\ \mathrm{H} & -4.09622000 & -3.63183400 & -0.57106100 \\ \mathrm{C} & -4.98856700 & -5.42369000 & 0.10734700 \\ \mathrm{H} & -5.88008300 & -6.03052200 & 0.28452700 \\ \mathrm{H} & -5.87181600 & -3.70753200 & -0.72796600 \\ \mathrm{C} & -4.79611900 & -4.67764500 & -2.35160300 \\ \mathrm{C} & -5.96850000 & -5.57408400 & -2.77504100 \\ \mathrm{H} & -5.91018700 & -5.79897400 & -3.85191500 \\ \mathrm{H} & -5.95842400 & -6.53287800 & -2.23332200 \\ \mathrm{H} & -6.93680100 & -5.08315600 & -2.58425100 \\ \mathrm{C} & -3.46783600 & -5.41298400 & -2.58260600 \\ \mathrm{H} & -2.61155500 & -4.79558900 & -2.27086300 \\ \mathrm{H} & -3.42393600 & -6.35399500 & -2.01400300 \\ \mathrm{H} & -3.34745900 & -5.65588100 & -3.65042100 \\ \mathrm{C} & -4.81673900 & -3.37581900 & -3.16463600 \\ \mathrm{H} & -4.70859500 & -3.59026900 & -4.23988900 \\ \mathrm{H} & -5.76318700 & -2.82976500 & -3.02146300 \\ \mathrm{H} & -3.99093300 & -2.70975100 & -2.86727900 \\ \mathrm{~S} & -3.52953000 & -6.07025000 & 0.83975800 \\ \mathrm{O} & -3.34617200 & -7.45836200 & 0.35939100 \\ \mathrm{O} & -2.44112000 & -5.08958400 & 0.65542500 \\ \mathrm{C} & -3.91216100 & -6.15748200 & 2.58499000 \\ \mathrm{C} & -3.65881200 & -5.04096700 & 3.38789400 \\ \mathrm{C} & -4.45632300 & -7.33500500 & 3.10535100 \\ \mathrm{C} & -3.96962700 & -5.10814200 & 4.74695800 \\ \mathrm{H} & -3.21757700 & -4.14464200 & 2.94979400 \\ \mathrm{C} & -4.76371400 & -7.38733600 & 4.46663400 \\ \mathrm{H} & -4.62191700 & -8.19188600 & 2.45107600\end{array}$




$\begin{array}{llll}\mathrm{C} & -4.52268600 & -6.27697100 & 5.28338200 \\ \mathrm{H} & -3.77570700 & -4.24764900 & 5.39075200 \\ \mathrm{H} & -5.18743400 & -8.29956800 & 4.89189000 \\ \mathrm{H} & -4.76374800 & -6.32387700 & 6.34767700\end{array}$

UB3LYP-D3(BJ)/def2-SVP-CPCM(THF)

Zero-point correction=

0.265352 (Hartree/Particle)

Thermal correction to Energy=

0.281141

Thermal correction to Enthalpy=

0.282085

Thermal correction to Gibbs Free Energy=

Sum of electronic and zero-point Energies=

Sum of electronic and thermal Energies=

Sum of electronic and thermal Enthalpies=

Sum of electronic and thermal Free Energies= $\mathrm{HF}=-1015.5485956$

0.220995

$-1015.283243$

$-1015.267455$

$-1015.266511$

$-1015.327601$

$\begin{array}{crrr}\mathbf{B}^{\mathbf{5 5}} & & & \\ \mathrm{C} & -4.74436700 & -4.17487400 & -1.11187600 \\ \mathrm{H} & -4.12203200 & -4.86581000 & -1.68723800 \\ \mathrm{C} & -4.48163000 & -3.94053000 & 0.17126700 \\ \mathrm{H} & -5.03909300 & -3.26263100 & 0.82281900 \\ \mathrm{H} & -5.57775600 & -3.67865600 & -1.61628100 \\ \mathrm{~S} & -3.07125900 & -4.71551300 & 0.94600600 \\ \mathrm{O} & -2.34181400 & -5.49949700 & -0.07097100 \\ \mathrm{O} & -2.35174300 & -3.67047800 & 1.70083300 \\ \mathrm{C} & -3.78643300 & -5.87518300 & 2.13884300 \\ \mathrm{H} & -4.44945300 & -5.27972700 & 2.78375000 \\ \mathrm{H} & -4.38934300 & -6.57893900 & 1.54697600 \\ \mathrm{C} & -2.67057300 & -6.55790400 & 2.91774100 \\ \mathrm{H} & -2.07035900 & -5.81730400 & 3.46530900 \\ \mathrm{H} & -3.10235400 & -7.26340900 & 3.64247300 \\ \mathrm{H} & -2.00874000 & -7.11521400 & 2.23926400\end{array}$

UB3LYP-D3(BJ)/def2-SVP-CPCM(THF)

Zero-point correction=

0.118171 (Hartree/Particle)

Thermal correction to Energy=

0.126491

Thermal correction to Enthalpy=

0.127436

Thermal correction to Gibbs Free Energy=

Sum of electronic and zero-point Energies=

0.085281

Sum of electronic and thermal Energies=

Sum of electronic and thermal Enthalpies=

Sum of electronic and thermal Free Energies= $\mathrm{HF}=-705.4770802$

$-705.358909$

$-705.350589$

$-705.349645$

$-705.391799$

\section{B-TS-C ${ }^{55}$}

$\mathrm{C}$ 


$\begin{array}{lrrr}\mathrm{H} & -4.97529000 & -3.81490000 & -0.88876900 \\ \mathrm{C} & -5.04270700 & -5.63293900 & 0.20739200 \\ \mathrm{H} & -5.56629500 & -6.53531500 & 0.53022600 \\ \mathrm{H} & -6.59347900 & -4.75111100 & -0.93582800 \\ \mathrm{C} & -5.01708900 & -5.47366500 & -2.86322900 \\ \mathrm{C} & -6.20817200 & -4.93785000 & -3.59709900 \\ \mathrm{H} & -6.10877600 & -5.13918500 & -4.68393000 \\ \mathrm{H} & -7.14607900 & -5.41188600 & -3.26722300 \\ \mathrm{H} & -6.30315900 & -3.84578100 & -3.48403200 \\ \mathrm{C} & -4.95011600 & -6.95225200 & -2.63541800 \\ \mathrm{H} & -4.17627000 & -7.20915900 & -1.89625800 \\ \mathrm{H} & -5.91403800 & -7.35973700 & -2.29239900 \\ \mathrm{H} & -4.69192300 & -7.46944100 & -3.58176100 \\ \mathrm{C} & -3.70890900 & -4.77490100 & -3.08032500 \\ \mathrm{H} & -3.26276800 & -5.10023600 & -4.04265900 \\ \mathrm{H} & -3.82893900 & -3.68125500 & -3.13069500 \\ \mathrm{H} & -2.98603300 & -5.00660200 & -2.28481000 \\ \mathrm{~S} & -3.34438400 & -5.57943200 & 0.67193900 \\ \mathrm{O} & -2.70641400 & -6.86176500 & 0.28615900 \\ \mathrm{O} & -2.74187100 & -4.32172700 & 0.17185100 \\ \mathrm{C} & -3.34351800 & -5.50642200 & 2.48079200 \\ \mathrm{H} & -3.87685400 & -4.58050200 & 2.74015200 \\ \mathrm{H} & -3.93349800 & -6.37073200 & 2.82001400 \\ \mathrm{C} & -1.91363800 & -5.53335300 & 3.00165300 \\ \mathrm{H} & -1.34410600 & -4.67672800 & 2.61306800 \\ \mathrm{H} & -1.91604400 & -5.48189000 & 4.10031200 \\ \mathrm{H} & -1.40728900 & -6.45958700 & 2.69490800 \\ \mathrm{HB} & -\mathrm{P} & \end{array}$

UB3LYP-D3(BJ)/def2-SVP-CPCM(THF)

Zero-point correction=

Thermal correction to Energy=

0.236530 (Hartree/Particle)

0.251222

0.252166

Thermal correction to Enthalpy=

0.193975

$-862.950880$

$-862.936188$

$-862.935244$

$-862.993435$

Sum of electronic and thermal Free Energies= $\mathrm{HF}=-863.1874102$

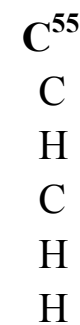

$\mathrm{C}$
$\mathrm{H}$
$\mathrm{C}$
$\mathrm{H}$
$\mathrm{H}$

$$
\begin{array}{ll}
-4.56687300 & -4.03659400 \\
-3.54133900 & -3.63591100 \\
-4.77873800 & -4.86255300 \\
-5.76623800 & -5.11129800 \\
-5.26722900 & -3.18464100
\end{array}
$$

$$
\begin{array}{r}
-0.89429200 \\
-0.87906200 \\
0.31540100 \\
0.71329200 \\
-0.85211700
\end{array}
$$




$\begin{array}{llll}\mathrm{C} & -4.78790700 & -4.76785000 & -2.26432800 \\ \mathrm{C} & -6.21103500 & -5.34024600 & -2.33003100 \\ \mathrm{H} & -6.39619900 & -5.79557900 & -3.31593600 \\ \mathrm{H} & -6.36690800 & -6.12012500 & -1.56820000 \\ \mathrm{H} & -6.96688000 & -4.55314500 & -2.17424200 \\ \mathrm{C} & -3.76316500 & -5.89991000 & -2.42925900 \\ \mathrm{H} & -2.73314300 & -5.51611500 & -2.36999900 \\ \mathrm{H} & -3.87798200 & -6.66511900 & -1.64721900 \\ \mathrm{H} & -3.89244100 & -6.39066400 & -3.40714800 \\ \mathrm{C} & -4.59364200 & -3.72659300 & -3.37520100 \\ \mathrm{H} & -4.72396800 & -4.19030900 & -4.36605000 \\ \mathrm{H} & -5.32346100 & -2.90567100 & -3.28638500 \\ \mathrm{H} & -3.58296100 & -3.28952200 & -3.33599600 \\ \mathrm{~S} & -3.45793300 & -5.76956500 & 1.03885200 \\ \mathrm{O} & -3.73472000 & -7.21628800 & 0.86278900 \\ \mathrm{O} & -2.17730800 & -5.23070100 & 0.53279300 \\ \mathrm{C} & -3.55647300 & -5.41922900 & 2.81144600 \\ \mathrm{H} & -3.42344800 & -4.33218200 & 2.90959800 \\ \mathrm{H} & -4.57706200 & -5.69040100 & 3.11886400 \\ \mathrm{C} & -2.49047600 & -6.21497700 & 3.55237900 \\ \mathrm{H} & -1.48685400 & -5.94821800 & 3.19107900 \\ \mathrm{H} & -2.54494400 & -5.99361900 & 4.62822600 \\ \mathrm{H} & -2.64124600 & -7.29412000 & 3.40797100 \\ \mathrm{UB} & -\mathrm{PY}-\mathrm{B})\end{array}$

UB3LYP-D3(BJ)/def2-SVP-CPCM(THF)

Zero-point correction=

Thermal correction to Energy=

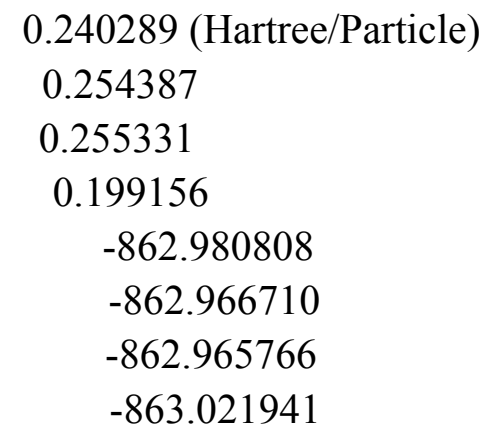

$\mathrm{HF}=-863.2210973$

\section{Scheme $\mathbf{S 7}$}

\section{F-TS-H ${ }^{R}$}

$\mathrm{Ni}$

$\mathrm{C}$

$\mathrm{C}$

C

C

$\mathrm{C}$

$\mathrm{C}$

$\mathrm{H}$

-1.36141200
-3.11461200
-4.99910900
-3.18896800
-4.04774400
-4.96709500
-4.10561600
-2.53735500

1.24562200

2.00729900

4.06136700

3.12798600

1.90396500

2.92740900

4.15055300

3.17337100
$-0.37638400$

0.08091300

$-0.38938700$

0.93047400

$-0.96922700$

$-1.20857600$

0.68545700

1.80109900 


\begin{tabular}{|c|c|c|c|}
\hline $\mathrm{H}$ & -4.03787300 & 1.03807900 & -1.63369000 \\
\hline $\mathrm{H}$ & -5.66218800 & 2.83663100 & -2.04741500 \\
\hline $\mathrm{H}$ & -4.13007900 & 5.01931400 & 1.34896000 \\
\hline $\mathrm{H}$ & -5.72167000 & 4.85884700 & -0.57665800 \\
\hline $\mathrm{C}$ & -3.09124700 & -0.93581600 & 0.22285500 \\
\hline $\mathrm{H}$ & -2.19009100 & -1.56112800 & 0.20962900 \\
\hline $\mathrm{C}$ & -2.77547700 & 0.33702800 & 1.00186300 \\
\hline $\mathrm{H}$ & -3.64644600 & 0.65482500 & 1.57889000 \\
\hline $\mathrm{H}$ & -3.32768600 & -0.70579100 & -0.82759600 \\
\hline $\mathrm{C}$ & -4.26514500 & -1.77757600 & 0.78735900 \\
\hline $\mathrm{C}$ & -5.58911200 & -1.00704700 & 0.66207900 \\
\hline $\mathrm{H}$ & -6.42551900 & -1.60731500 & 1.05457700 \\
\hline $\mathrm{H}$ & -5.57065000 & -0.06052100 & 1.22543800 \\
\hline $\mathrm{H}$ & -5.80843400 & -0.76396800 & -0.38954400 \\
\hline $\mathrm{C}$ & -4.01024800 & -2.14135100 & 2.25921200 \\
\hline $\mathrm{H}$ & -3.04806000 & -2.66249000 & 2.37789400 \\
\hline $\mathrm{H}$ & -3.99007100 & -1.24876000 & 2.90457600 \\
\hline $\mathrm{H}$ & -4.80908800 & -2.79942100 & 2.63713500 \\
\hline $\mathrm{C}$ & -4.35042400 & -3.06677500 & -0.04447100 \\
\hline $\mathrm{H}$ & -5.18250500 & -3.70298400 & 0.29752800 \\
\hline $\mathrm{H}$ & -4.51381500 & -2.83978100 & -1.11060300 \\
\hline $\mathrm{H}$ & -3.41982800 & -3.65149300 & 0.03758000 \\
\hline $\mathrm{C}$ & -1.67749300 & 0.18359500 & 2.01861200 \\
\hline $\mathrm{O}$ & -0.90517200 & -0.74887300 & 2.11694700 \\
\hline $\mathrm{O}$ & -1.68481600 & 1.20383800 & 2.90174600 \\
\hline $\mathrm{C}$ & -0.67945900 & 1.17284100 & 3.91414700 \\
\hline $\mathrm{H}$ & 0.32386900 & 1.19998300 & 3.46473900 \\
\hline $\mathrm{H}$ & -0.84100500 & 2.06358600 & 4.53220600 \\
\hline $\mathrm{H}$ & -0.76938200 & 0.26350400 & 4.52578700 \\
\hline $\mathrm{Br}$ & -0.80119300 & 2.30851600 & -2.56719200 \\
\hline $\mathrm{C}$ & 1.35400700 & 1.51713100 & 0.37372700 \\
\hline $\mathrm{C}$ & 2.26462900 & 3.46989500 & 0.90428600 \\
\hline $\mathrm{C}$ & 0.72502000 & 3.60586100 & 0.78049900 \\
\hline $\mathrm{N}$ & 0.28971000 & 2.20674000 & 0.56943000 \\
\hline $\mathrm{H}$ & 2.82970500 & 4.14569800 & 0.25125500 \\
\hline $\mathrm{H}$ & 0.45231300 & 4.14146300 & -0.14481400 \\
\hline $\mathrm{C}$ & 0.29117800 & -1.67083100 & -0.97424600 \\
\hline $\mathrm{C}$ & 1.82768100 & -1.87208600 & -0.86202600 \\
\hline $\mathrm{C}$ & 1.24685800 & 0.13810800 & -0.11864000 \\
\hline $\mathrm{N}$ & 0.11324700 & -0.27861300 & -0.53199100 \\
\hline $\mathrm{H}$ & -0.23221800 & -2.31013000 & -0.24449200 \\
\hline $\mathrm{H}$ & 2.32277900 & -1.91724100 & -1.84367600 \\
\hline $\mathrm{H}$ & 2.61381300 & 3.57625200 & 1.94264600 \\
\hline $\mathrm{H}$ & 2.12131600 & -2.73901100 & -0.25810700 \\
\hline
\end{tabular}




$\begin{array}{lrrr}\mathrm{O} & 2.54047600 & 2.10283100 & 0.48444100 \\ \mathrm{O} & 2.30256500 & -0.66932200 & -0.18465100 \\ \mathrm{C} & 0.09288700 & 4.29931600 & 1.97576600 \\ \mathrm{H} & 0.53307900 & 5.30172200 & 2.09177900 \\ \mathrm{H} & -0.98720600 & 4.42019600 & 1.83570400 \\ \mathrm{H} & 0.27381500 & 3.73370500 & 2.90109500 \\ \mathrm{C} & -0.28053000 & -1.90098000 & -2.36537000 \\ \mathrm{H} & -0.10613200 & -2.94011600 & -2.68357000 \\ \mathrm{H} & -1.36419300 & -1.71535000 & -2.36816300 \\ \mathrm{H} & 0.18728400 & -1.22036900 & -3.09234400\end{array}$

UB3LYP-D3(BJ)/def2-SVP-CPCM(THF)

Zero-point correction=
Thermal correction to Energy=
Thermal correction to Enthalpy=
Thermal correction to Gibbs Free Energy=
Sum of electronic and zero-point Energies $=$
Sum of electronic and thermal Energies $=$
Sum of electronic and thermal Enthalpies $=$
Sum of electronic and thermal Free Energies=
$\mathrm{HF}=-5349.325995$

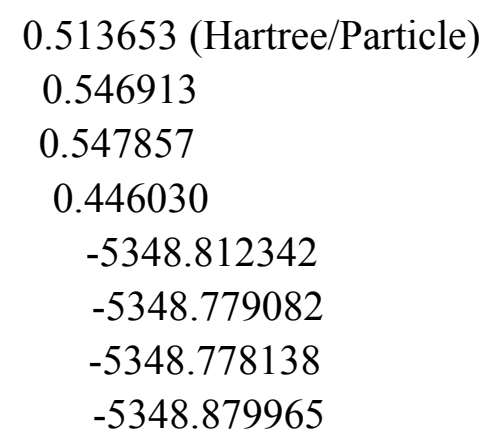

\section{Scheme S8}

${ }^{1} \mathrm{G}-\mathbf{C}^{\boldsymbol{R}}$

$\begin{array}{lrrr}\mathrm{Ni} & -0.70885300 & 0.14705700 & -0.57870400 \\ \mathrm{C} & -1.24483000 & 1.78858300 & -1.32173600 \\ \mathrm{C} & -1.89610100 & 4.29971600 & -2.46436300 \\ \mathrm{C} & -2.16732100 & 2.64953700 & -0.70363400 \\ \mathrm{C} & -0.66454600 & 2.20939000 & -2.53086100 \\ \mathrm{C} & -0.98791700 & 3.44813800 & -3.10117200 \\ \mathrm{C} & -2.48431900 & 3.89422800 & -1.26102700 \\ \mathrm{H} & -2.64997600 & 2.34801100 & 0.22788500 \\ \mathrm{H} & 0.05941900 & 1.56706900 & -3.04317600 \\ \mathrm{H} & -0.52434400 & 3.74950600 & -4.04526600 \\ \mathrm{H} & -3.19959700 & 4.54885800 & -0.75420200 \\ \mathrm{Br} & -2.82026900 & -0.80394300 & -0.96925700 \\ \mathrm{C} & -3.75426900 & 1.23963800 & 2.61779100 \\ \mathrm{H} & -2.94278800 & 1.98338900 & 2.64672800 \\ \mathrm{H} & -3.95454800 & 0.99398000 & 1.56344300 \\ \mathrm{H} & -4.65387600 & 1.71782400 & 3.03745500 \\ \mathrm{C} & -3.39782500 & -0.02491200 & 3.41373900 \\ \mathrm{C} & -2.17804400 & -0.74684800 & 2.77092400 \\ \mathrm{H} & -1.99446100 & -1.69522200 & 3.29785200 \\ \mathrm{H} & -2.45966600 & -0.99484800 & 1.72926500 \\ \mathrm{C} & -0.91900900 & 0.02684000 & 2.72736400\end{array}$




$\begin{array}{lrrr}\mathrm{C} & -4.58451500 & -0.99900500 & 3.37142500 \\ \mathrm{H} & -4.36058600 & -1.92088900 & 3.93231600 \\ \mathrm{H} & -5.48321800 & -0.54124300 & 3.81530600 \\ \mathrm{H} & -4.82275800 & -1.28249200 & 2.33371700 \\ \mathrm{H} & -0.88358500 & 1.02282400 & 2.28385000 \\ \mathrm{C} & 0.30943500 & -0.51564500 & 3.26174800 \\ \mathrm{O} & 0.43211200 & -1.61723100 & 3.78269100 \\ \mathrm{O} & 1.36426000 & 0.32562700 & 3.10523400 \\ \mathrm{C} & 2.61634700 & -0.11832900 & 3.63300800 \\ \mathrm{H} & 2.94917300 & -1.03315100 & 3.12270700 \\ \mathrm{H} & 3.32800000 & 0.69439600 & 3.44974100 \\ \mathrm{H} & 2.53264100 & -0.31888800 & 4.71113000 \\ \mathrm{C} & -3.07585300 & 0.34142300 & 4.87009200 \\ \mathrm{H} & -2.80788200 & -0.55417600 & 5.45404700 \\ \mathrm{H} & -2.22925100 & 1.04502000 & 4.92543900 \\ \mathrm{H} & -3.94314700 & 0.81719500 & 5.35588200 \\ \mathrm{H} & -2.14577500 & 5.26933500 & -2.90300400 \\ \mathrm{C} & 1.91805400 & -0.05487300 & 0.22480200 \\ \mathrm{C} & 3.23670700 & 1.71961500 & 0.05094300 \\ \mathrm{C} & 1.76402000 & 2.12619400 & -0.21846900 \\ \mathrm{~N} & 1.07964600 & 0.81491500 & -0.22173900 \\ \mathrm{H} & 3.86903600 & 1.74652500 & -0.84684300 \\ \mathrm{H} & 1.65283600 & 2.58424300 & -1.21014400 \\ \mathrm{C} & -0.17012500 & -2.98623800 & 0.48790000 \\ \mathrm{C} & 1.10969000 & -3.46479000 & 1.23019700 \\ \mathrm{C} & 1.39119700 & -1.40003200 & 0.46494500 \\ \mathrm{~N} & 0.15424500 & -1.58177900 & 0.17420700 \\ \mathrm{H} & -1.03875700 & -2.99360000 & 1.16171000 \\ \mathrm{H} & 1.54008500 & -4.38632800 & 0.81930200 \\ \mathrm{H} & 3.70988500 & 2.28831900 & 0.86064600 \\ \mathrm{H} & 0.97232800 & -3.54497000 & 2.31511900 \\ \mathrm{O} & 3.15693500 & 0.32031400 & 0.48095700 \\ \mathrm{O} & 2.08356900 & -2.38589600 & 1.00914200 \\ \mathrm{C} & 1.16967300 & 3.04017600 & 0.84834400 \\ \mathrm{H} & 1.70793900 & 4.00030900 & 0.85657900 \\ \mathrm{H} & 0.11077000 & 3.23374000 & 0.63235500 \\ \mathrm{H} & 1.25358600 & 2.57358300 & 1.84101800 \\ \mathrm{C} & -0.49536400 & -3.77215400 & -0.77759400 \\ \mathrm{H} & -0.72883600 & -4.81669000 & -0.52017200 \\ \mathrm{H} & -1.36432200 & -3.32438100 & -1.27830300 \\ \mathrm{H} & 0.36048200 & -3.76478200 & -1.47090700 \\ \mathrm{H} & & \end{array}$

UB3LYP-D3(BJ)/def2-SVP-CPCM(THF)

Zero-point correction=

0.513178 (Hartree/Particle)

Thermal correction to Energy= 
Thermal correction to Enthalpy=

Thermal correction to Gibbs Free Energy=

Sum of electronic and zero-point Energies=

Sum of electronic and thermal Energies=

Sum of electronic and thermal Enthalpies=

Sum of electronic and thermal Free Energies=

$\mathrm{HF}=-5349.3305428$
0.547984

0.444939

$-5348.817365$

$-5348.783502$

$-5348.782558$

$-5348.885604$

\section{Scheme S9}

\section{${ }^{2}$ F'-TS-H ${ }^{R}-B^{C F 3}$-Conf1}

$\mathrm{Ni}$

C

$\mathrm{C}$

C

$\mathrm{C}$

C

$\mathrm{C}$

$\mathrm{H}$

$\mathrm{H}$

$\mathrm{H}$

$\mathrm{H}$

$\mathrm{Br}$

C

$\mathrm{H}$

$\mathrm{H}$

$\mathrm{H}$

C

C

$\mathrm{H}$

$\mathrm{H}$

C

$\mathrm{C}$

$\mathrm{H}$

$\mathrm{H}$

$\mathrm{H}$

$\mathrm{H}$

C

$\mathrm{O}$

$\mathrm{O}$

C

$\mathrm{H}$

$\mathrm{H}$

$\mathrm{H}$

C
$-0.70885300$

$-1.24483000$

$-1.89610100$

$-2.16732100$

$-0.66454600$

$-0.98791700$

$-2.48431900$

$-2.64997600$

0.05941900

$-0.52434400$

$-3.19959700$

$-2.82026900$

$-3.75426900$

$-2.94278800$

$-3.95454800$

$-4.65387600$

$-3.39782500$

$-2.17804400$

$-1.99446100$

$-2.45966600$

$-0.91900900$

$-4.58451500$

$-4.36058600$

$-5.48321800$

$-4.82275800$

$-0.88358500$

0.30943500

0.43211200

1.36426000

2.61634700

2.94917300

3.32800000

2.53264100

$-3.07585300$
0.14705700

1.78858300

4.29971600

2.64953700

2.20939000

3.44813800

3.89422800

2.34801100

1.56706900

3.74950600

4.54885800

$-0.80394300$

1.23963800

1.98338900

0.99398000

1.71782400

$-0.02491200$

$-0.74684800$

$-1.69522200$

$-0.99484800$

0.02684000

$-0.99900500$

$-1.92088900$

$-0.54124300$

$-1.28249200$

1.02282400

$-0.51564500$

$-1.61723100$

0.32562700

$-0.11832900$

$-1.03315100$

0.69439600

$-0.31888800$

0.34142300
$-0.57870400$

$-1.32173600$

$-2.46436300$

$-0.70363400$

$-2.53086100$

$-3.10117200$

$-1.26102700$

0.22788500

$-3.04317600$

$-4.04526600$

$-0.75420200$

$-0.96925700$

2.61779100

2.64672800

1.56344300

3.03745500

3.41373900

2.77092400

3.29785200

1.72926500

2.72736400

3.37142500

3.93231600

3.81530600

2.33371700

2.28385000

3.26174800

3.78269100

3.10523400

3.63300800

3.12270700

3.44974100

4.71113000

4.87009200 


$\begin{array}{rrrr}\mathrm{H} & -2.80788200 & -0.55417600 & 5.45404700 \\ \mathrm{H} & -2.22925100 & 1.04502000 & 4.92543900 \\ \mathrm{H} & -3.94314700 & 0.81719500 & 5.35588200 \\ \mathrm{H} & -2.14577500 & 5.26933500 & -2.90300400 \\ \mathrm{C} & 1.91805400 & -0.05487300 & 0.22480200 \\ \mathrm{C} & 3.23670700 & 1.71961500 & 0.05094300 \\ \mathrm{C} & 1.76402000 & 2.12619400 & -0.21846900 \\ \mathrm{~N} & 1.07964600 & 0.81491500 & -0.22173900 \\ \mathrm{H} & 3.86903600 & 1.74652500 & -0.84684300 \\ \mathrm{H} & 1.65283600 & 2.58424300 & -1.21014400 \\ \mathrm{C} & -0.17012500 & -2.98623800 & 0.48790000 \\ \mathrm{C} & 1.10969000 & -3.46479000 & 1.23019700 \\ \mathrm{C} & 1.39119700 & -1.40003200 & 0.46494500 \\ \mathrm{~N} & 0.15424500 & -1.58177900 & 0.17420700 \\ \mathrm{H} & -1.03875700 & -2.99360000 & 1.16171000 \\ \mathrm{H} & 1.54008500 & -4.38632800 & 0.81930200 \\ \mathrm{H} & 3.70988500 & 2.28831900 & 0.86064600 \\ \mathrm{H} & 0.97232800 & -3.54497000 & 2.31511900 \\ \mathrm{O} & 3.15693500 & 0.32031400 & 0.48095700 \\ \mathrm{O} & 2.08356900 & -2.38589600 & 1.00914200 \\ \mathrm{C} & 1.16967300 & 3.04017600 & 0.84834400 \\ \mathrm{H} & 1.70793900 & 4.00030900 & 0.85657900 \\ \mathrm{H} & 0.11077000 & 3.23374000 & 0.63235500 \\ \mathrm{H} & 1.25358600 & 2.57358300 & 1.84101800 \\ \mathrm{C} & -0.49536400 & -3.77215400 & -0.77759400 \\ \mathrm{H} & -0.72883600 & -4.81669000 & -0.52017200 \\ \mathrm{H} & -1.36432200 & -3.32438100 & -1.27830300 \\ \mathrm{H} & 0.36048200 & -3.76478200 & -1.47090700 \\ \mathrm{H} & & \end{array}$

UB3LYP-D3(BJ)/def2-SVP-CPCM(THF)

Zero-point correction=

0.689324 (Hartree/Particle)

Thermal correction to Energy=

0.734093

Thermal correction to Enthalpy=

0.735038

Thermal correction to Gibbs Free Energy=

Sum of electronic and zero-point Energies=

Sum of electronic and thermal Energies=

Sum of electronic and thermal Enthalpies=

Sum of electronic and thermal Free Energies=

$\mathrm{HF}=-5921.8794522$

0.606265

$-5921.190128$

$-5921.145359$

$-5921.144415$

$-5921.273188$

\section{${ }^{2} \mathrm{~F}^{\prime}$-TS-H ${ }^{R}-B^{C F 3}$-Conf2}

$\begin{array}{lrrr}\mathrm{Ni} & -1.40461300 & 1.04075000 & -0.23681400 \\ \mathrm{C} & -1.83144800 & 0.77974100 & 1.62486400 \\ \mathrm{C} & -1.44466200 & 1.68637600 & 4.25465600 \\ \mathrm{C} & -0.77235400 & 0.27962500 & 2.40411600\end{array}$




\begin{tabular}{|c|c|c|c|}
\hline $\mathrm{C}$ & -2.74785200 & 1.66885100 & 2.21623100 \\
\hline $\mathrm{C}$ & -2.55352400 & 2.12216100 & 3.51653900 \\
\hline $\mathrm{C}$ & -0.56228100 & 0.75249400 & 3.69854300 \\
\hline $\mathrm{H}$ & -0.10387600 & -0.47890800 & 1.99820000 \\
\hline $\mathrm{H}$ & -3.59812200 & 2.03168700 & 1.63715700 \\
\hline $\mathrm{H}$ & -3.26081600 & 2.82715500 & 3.95554200 \\
\hline $\mathrm{H}$ & 0.28855400 & 0.39102500 & 4.27804800 \\
\hline $\mathrm{C}$ & -2.87592700 & -0.99060700 & -1.04301500 \\
\hline $\mathrm{H}$ & -2.12216500 & -1.77910000 & -1.13324000 \\
\hline $\mathrm{C}$ & -2.88921000 & -0.50536800 & 0.41422700 \\
\hline $\mathrm{H}$ & -3.82809900 & -0.00866400 & 0.66135300 \\
\hline $\mathrm{H}$ & -2.60503800 & -0.19098400 & -1.75017100 \\
\hline $\mathrm{C}$ & -4.23450000 & -1.56604400 & -1.54044400 \\
\hline $\mathrm{C}$ & -5.31352600 & -0.47353900 & -1.57748900 \\
\hline $\mathrm{H}$ & -6.24631600 & -0.87242300 & -2.00748100 \\
\hline $\mathrm{H}$ & -5.55045300 & -0.09694200 & -0.57028300 \\
\hline $\mathrm{H}$ & -4.98635100 & 0.38626400 & -2.18088700 \\
\hline $\mathrm{C}$ & -4.70208100 & -2.73144000 & -0.65400200 \\
\hline $\mathrm{H}$ & -3.92865800 & -3.51189400 & -0.57985100 \\
\hline $\mathrm{H}$ & -4.94746600 & -2.39797100 & 0.36562300 \\
\hline $\mathrm{H}$ & -5.61055700 & -3.19075000 & -1.07547600 \\
\hline $\mathrm{C}$ & -3.99206000 & -2.08538200 & -2.96789900 \\
\hline $\mathrm{H}$ & -4.92054200 & -2.49132100 & -3.40021100 \\
\hline $\mathrm{H}$ & -3.63519300 & -1.27846000 & -3.62810700 \\
\hline $\mathrm{H}$ & -3.23675600 & -2.88778900 & -2.97473700 \\
\hline $\mathrm{C}$ & -2.62188700 & -1.63285600 & 1.35735100 \\
\hline $\mathrm{O}$ & -1.76983300 & -2.48681200 & 1.19878700 \\
\hline $\mathrm{O}$ & -3.42160900 & -1.59950300 & 2.43355700 \\
\hline $\mathrm{C}$ & -3.16326000 & -2.56716700 & 3.45226800 \\
\hline $\mathrm{H}$ & -2.14928400 & -2.43250600 & 3.85777200 \\
\hline $\mathrm{H}$ & -3.90993200 & -2.39235000 & 4.23539500 \\
\hline $\mathrm{H}$ & -3.25748900 & -3.58822000 & 3.05511200 \\
\hline $\mathrm{Br}$ & -2.92666400 & 2.63515600 & -1.35025300 \\
\hline $\mathrm{C}$ & 1.51023400 & 1.74589200 & 0.02299500 \\
\hline $\mathrm{C}$ & 2.47003400 & 3.63227700 & 0.68036500 \\
\hline $\mathrm{C}$ & 0.94865200 & 3.84443200 & 0.44797900 \\
\hline $\mathrm{N}$ & 0.46997700 & 2.48026700 & 0.12577000 \\
\hline $\mathrm{H}$ & 3.11465700 & 4.30484200 & 0.10248500 \\
\hline $\mathrm{H}$ & 0.77418200 & 4.46230200 & -0.45085500 \\
\hline $\mathrm{C}$ & 0.51559500 & -1.57219500 & -1.08437000 \\
\hline $\mathrm{C}$ & 1.99297200 & -1.78391300 & -0.68042400 \\
\hline $\mathrm{C}$ & 1.40056000 & 0.32940800 & -0.35419400 \\
\hline $\mathrm{N}$ & 0.25869500 & -0.19375800 & -0.61300400 \\
\hline $\mathrm{H}$ & -0.14335200 & -2.25263200 & -0.52937400 \\
\hline
\end{tabular}




$\begin{array}{lrrr}\mathrm{H} & 2.62046200 & -2.23561900 & -1.45694800 \\ \mathrm{H} & 2.74422400 & 3.68035500 & 1.74473300 \\ \mathrm{H} & 2.10481500 & -2.34474800 & 0.25820200 \\ \mathrm{O} & 2.71663100 & 2.27015700 & 0.23393900 \\ \mathrm{O} & 2.48498400 & -0.42946800 & -0.43696400 \\ \mathrm{C} & 0.23136100 & 4.51709200 & 1.62807700 \\ \mathrm{H} & 0.32613800 & 3.84215000 & 2.49925100 \\ \mathrm{C} & 0.23142300 & -1.69569700 & -2.59808400 \\ \mathrm{H} & -0.79806900 & -1.33327300 & -2.73959900 \\ \mathrm{C} & 0.91995700 & 5.84695200 & 1.96014400 \\ \mathrm{H} & 0.45088000 & 6.32291000 & 2.83242600 \\ \mathrm{H} & 1.98732900 & 5.72251300 & 2.19817400 \\ \mathrm{H} & 0.84131700 & 6.54755500 & 1.11152300 \\ \mathrm{C} & -1.25964200 & 4.70440900 & 1.32439800 \\ \mathrm{H} & -1.68486200 & 3.72797400 & 1.06518500 \\ \mathrm{H} & -1.36320400 & 5.32018200 & 0.41306700 \\ \mathrm{C} & -2.05969200 & 5.33263100 & 2.46426400 \\ \mathrm{H} & -1.83053400 & 6.40181400 & 2.59520700 \\ \mathrm{H} & -3.14105100 & 5.24879600 & 2.27290700 \\ \mathrm{H} & -1.84900000 & 4.83019400 & 3.42084500 \\ \mathrm{C} & 1.14602900 & -0.81081500 & -3.44881900 \\ \mathrm{H} & 1.09839100 & 0.24028200 & -3.12577900 \\ \mathrm{H} & 2.19754900 & -1.13824300 & -3.40656800 \\ \mathrm{H} & 0.83824400 & -0.83511400 & -4.50366700 \\ \mathrm{C} & 0.25694500 & -3.17715900 & -3.00835600 \\ \mathrm{H} & -0.38919600 & -3.73892500 & -2.31155100 \\ \mathrm{H} & 1.27579100 & -3.58245000 & -2.87068600 \\ \mathrm{C} & -0.20718200 & -3.43916300 & -4.44040600 \\ \mathrm{H} & 0.47005100 & -2.98948600 & -5.18255100 \\ \mathrm{H} & -0.25186200 & -4.51973400 & -4.64683900 \\ \mathrm{~F} & -1.21365300 & -3.02277300 & -4.61071400 \\ \mathrm{~F} & -1.18250400 & 2.27877400 & 5.60584700 \\ \mathrm{~F} & -0.61670300 & 3.50936400 & 5.50664500 \\ \mathrm{~F} & -2.31196200 & 2.44370900 & 6.32125800 \\ \mathrm{H} & -0.34366900 & 1.53276600 & 6.34616500\end{array}$

UB3LYP-D3(BJ)/def2-SVP-CPCM(THF)

Zero-point correction=

0.689454 (Hartree/Particle)

Thermal correction to Energy=

0.733978

0.734922

Thermal correction to Enthalpy=

0.607791

$-5921.186304$

$-5921.141781$

$-5921.140837$

$-5921.267968$ 


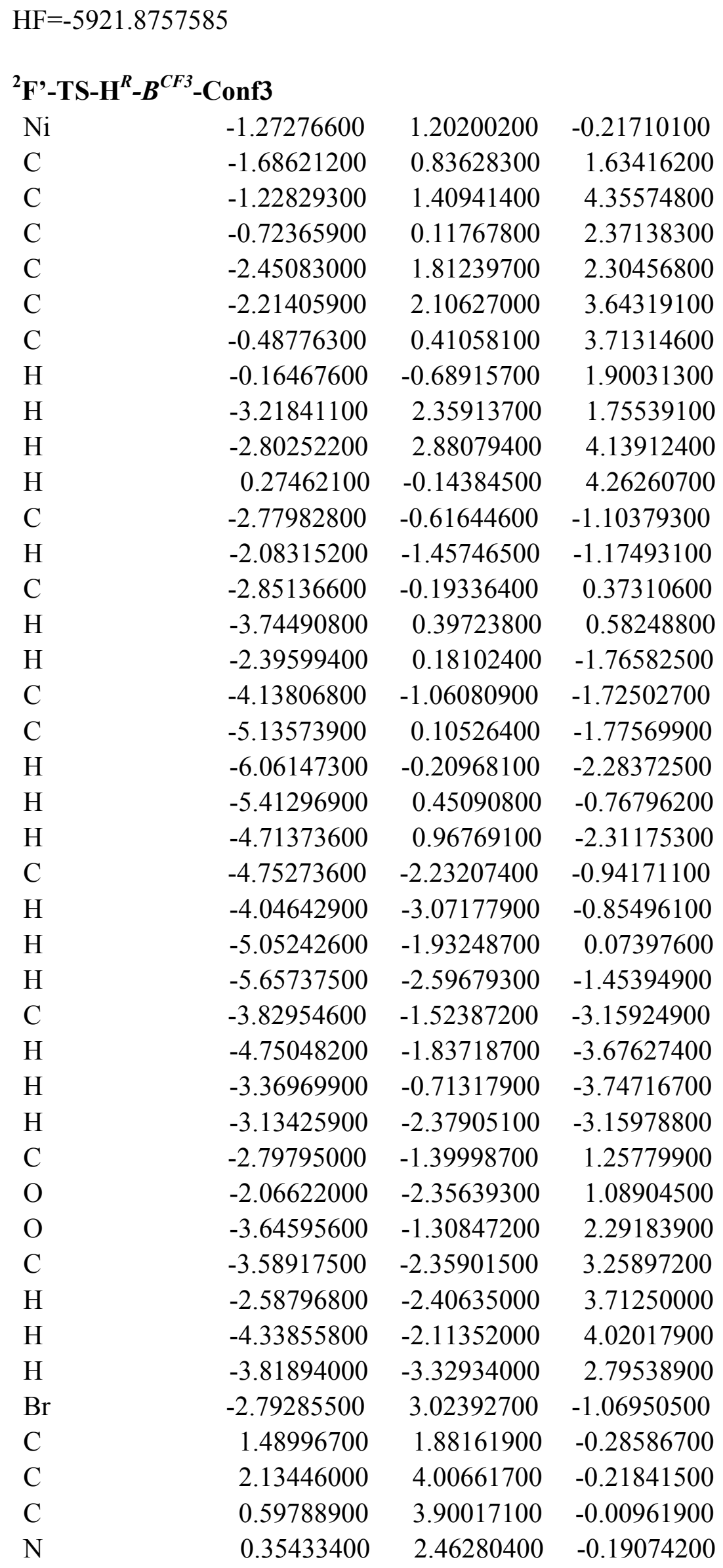




\begin{tabular}{|c|c|c|c|}
\hline $\mathrm{H}$ & 2.41012000 & 4.46511600 & -1.17753200 \\
\hline $\mathrm{H}$ & 0.04997400 & 4.42661900 & -0.80165700 \\
\hline $\mathrm{C}$ & 0.67466800 & -1.60070800 & -0.84951700 \\
\hline $\mathrm{C}$ & 2.16581200 & -1.68972600 & -0.44049800 \\
\hline $\mathrm{C}$ & 1.49157300 & 0.42165400 & -0.44551700 \\
\hline $\mathrm{N}$ & 0.36682500 & -0.17640000 & -0.60337800 \\
\hline $\mathrm{H}$ & 0.03795200 & -2.21108000 & -0.19380400 \\
\hline $\mathrm{H}$ & 2.80280800 & -2.23412200 & -1.14665400 \\
\hline $\mathrm{H}$ & 2.66734200 & 4.51210000 & 0.59593100 \\
\hline $\mathrm{H}$ & 2.31177100 & -2.08994400 & 0.57260700 \\
\hline $\mathrm{O}$ & 2.59507800 & 2.61507800 & -0.25351900 \\
\hline $\mathrm{O}$ & 2.60753900 & -0.29423800 & -0.42347600 \\
\hline $\mathrm{C}$ & 0.07012500 & 4.37973200 & 1.35679100 \\
\hline $\mathrm{H}$ & -0.98925400 & 4.07643800 & 1.36895300 \\
\hline $\mathrm{C}$ & 0.38633300 & -1.98560700 & -2.31880400 \\
\hline $\mathrm{H}$ & -0.66306300 & -1.70821800 & -2.49998500 \\
\hline $\mathrm{C}$ & 0.77582100 & 3.69324800 & 2.52612700 \\
\hline $\mathrm{H}$ & 0.29868000 & 3.96339300 & 3.47834300 \\
\hline $\mathrm{H}$ & 0.72584400 & 2.59908600 & 2.44096600 \\
\hline $\mathrm{H}$ & 1.83604400 & 3.98780900 & 2.59630300 \\
\hline $\mathrm{C}$ & 0.12572800 & 5.91036800 & 1.48432700 \\
\hline $\mathrm{H}$ & 1.17898000 & 6.24635900 & 1.46079400 \\
\hline $\mathrm{H}$ & -0.24160200 & 6.16717100 & 2.49224500 \\
\hline $\mathrm{C}$ & -0.68854200 & 6.67794600 & 0.44213300 \\
\hline $\mathrm{H}$ & -1.72550300 & 6.30748000 & 0.39759300 \\
\hline $\mathrm{H}$ & -0.72149000 & 7.75201000 & 0.68247400 \\
\hline $\mathrm{H}$ & -0.26404300 & 6.58589700 & -0.57025600 \\
\hline $\mathrm{C}$ & 1.24326000 & -1.20370800 & -3.31738900 \\
\hline $\mathrm{H}$ & 1.13923500 & -0.11813700 & -3.16916000 \\
\hline $\mathrm{H}$ & 2.31177100 & -1.46220900 & -3.23834200 \\
\hline $\mathrm{H}$ & 0.93079200 & -1.41784500 & -4.34921400 \\
\hline $\mathrm{C}$ & 0.49138800 & -3.51052100 & -2.48475500 \\
\hline $\mathrm{H}$ & -0.10405700 & -3.98602600 & -1.68632400 \\
\hline $\mathrm{H}$ & 1.53582300 & -3.83083300 & -2.31816500 \\
\hline $\mathrm{C}$ & 0.01022800 & -4.02731000 & -3.83998500 \\
\hline $\mathrm{H}$ & 0.63686700 & -3.66174000 & -4.66787600 \\
\hline $\mathrm{H}$ & 0.03221900 & -5.12764800 & -3.87085000 \\
\hline $\mathrm{H}$ & -1.02594900 & -3.70697700 & -4.03890300 \\
\hline $\mathrm{C}$ & -0.93544000 & 1.78434900 & 5.77646900 \\
\hline $\mathrm{F}$ & -2.05386300 & 2.08801600 & 6.46471400 \\
\hline $\mathrm{F}$ & -0.13679500 & 2.87791300 & 5.85756200 \\
\hline $\mathrm{F}$ & -0.30525900 & 0.80387900 & 6.44949700 \\
\hline
\end{tabular}

UB3LYP-D3(BJ)/def2-SVP-CPCM(THF)

Zero-point correction=

0.688670 (Hartree/Particle) 
Thermal correction to Energy=

Thermal correction to Enthalpy=

Thermal correction to Gibbs Free Energy=

Sum of electronic and zero-point Energies=

Sum of electronic and thermal Energies=

Sum of electronic and thermal Enthalpies=

Sum of electronic and thermal Free Energies=

$\mathrm{HF}=-5921.8791022$

\section{${ }^{2} F^{\prime}$-TS-H ${ }^{R}-B^{C F 3}$-Conf4}

$\mathrm{Ni}$

$\mathrm{C}$

C

$\mathrm{C}$

$\mathrm{C}$

C

$\mathrm{C}$

$\mathrm{H}$

$\mathrm{H}$

$\mathrm{H}$

$\mathrm{H}$

$\mathrm{C}$

$\mathrm{H}$

$\mathrm{C}$

$\mathrm{H}$

$\mathrm{H}$

C

C

$\mathrm{H}$

$\mathrm{H}$

$\mathrm{H}$

C

$\mathrm{H}$

$\mathrm{H}$

$\mathrm{H}$

C

$\mathrm{H}$

$\mathrm{H}$

$\mathrm{H}$

C

$\mathrm{O}$

$\mathrm{O}$

C

$\mathrm{H}$
$-1.33949400$

$-1.66275700$

1.40341200

1.04445100

1.74532000

0.43259300

1.96824100

2.32807800

0.79068600

$-0.33812700$

2.42683400

3.06444200

0.32363800

$-0.66077900$

$-1.41673700$

$-0.19281600$

0.33889300

0.15469700

$-1.26890900$

$-0.20837200$

$-0.62324600$

0.13276300

0.67753800

$-2.46568600$

$-3.22674300$

$-2.15982400$

$-2.93784900$

$-1.74923700$

$-2.17957500$

$-0.91598700$

$-2.52287700$

$-1.32764700$

$-2.21273500$

$-1.26140600$

$-2.23413700$

$-2.13748400$
0.733336

0.734280

0.607040

$-5921.190432$

$-5921.145766$

$-5921.144822$

$-5921.272062$
$-0.39650900$

1.48161800

4.16268100

2.20372400

2.14872000

3.46782700

3.52644000

1.73557700

1.61238800

3.96219400

4.06488800

$-1.16840300$

$-1.24560200$

0.29057500

0.52597500

$-1.86543300$

$-1.71220200$

$-1.75291900$

$-2.22167400$

$-0.74362300$

$-2.32326900$

$-0.86197100$

$-0.78432800$

0.15795500

$-1.31477200$

$-3.14367500$

$-3.60472000$

$-3.77887800$

$-3.15067600$

1.23243600

1.07123600

2.31179500

3.33271200

3.72716500 
H

$\mathrm{H}$

$\mathrm{Br}$

C

C

C

$\mathrm{N}$

$\mathrm{H}$

$\mathrm{H}$

C

C

C

$\mathrm{N}$

$\mathrm{H}$

$\mathrm{H}$

$\mathrm{H}$

$\mathrm{H}$

O

O

C

$\mathrm{H}$

C

$\mathrm{H}$

C

$\mathrm{H}$

$\mathrm{H}$

$\mathrm{H}$

C

$\mathrm{H}$

$\mathrm{H}$

C

$\mathrm{H}$

$\mathrm{H}$

$\mathrm{H}$

C

H

$\mathrm{H}$

$\mathrm{H}$

C

$\mathrm{H}$

$\mathrm{H}$

C

$\mathrm{H}$

$\mathrm{H}$ $\begin{array}{ll}-3.88354100 & -2.02661700\end{array}$

$-3.29072900-3.25236000$

$-3.02260000 \quad 3.04959700$

$1.44630300 \quad 2.16501900$

1.98143800

0.45651200

0.28527400

2.21760800

$-0.12940500$

0.89175200

2.39565500

1.54771400

0.47832600

0.31908800

3.06081400

2.50221000

2.59444600

2.50991900

2.72159100

$-0.07833600$

$-1.12135800$

0.59167300

$-0.46619500$

1.41848600

1.08340100

1.30830200

2.49089100

0.73941400

1.73334200

0.73772500

$-0.35723800$

$-1.34431700$

$-0.18331000$

$-0.42360800$

0.67326600

0.19309900

0.67663800

1.71825400

$-0.09884600$

0.93538700

$-0.46262800$

$-0.96858300$

$-1.98637800$

$-1.05095600$
4.32444800

4.14862500

2.69456900

4.76525500

4.61282200

$-1.40904800$

$-1.34743000$

0.69787300

0.00096400

$-1.99094500$

$-1.88939100$

4.88117900

$-1.66082800$

2.95733000

0.07662500

4.65539400

4.30025200

$-1.95447500$

$-1.71596700$

$-1.26631500$

$-1.59723900$

$-0.17158400$

$-1.50704900$

$-3.48521300$

$-3.76840400$

$-3.80390500$

$-4.24100400$

$-4.04666600$

$-5.32766000$

$-3.94602800$

4.05021300

4.33292500

2.95254900

4.39900600

6.19054500

6.57795000

6.46783100

6.87377100

6.45117100

7.95384900
4.12233400

2.94126800

$-1.27819500$

$-0.62586600$

$-0.65352000$

$-0.41283200$

$-0.52716700$

$-1.63112200$

$-1.21691300$

$-0.92261900$

$-0.54959800$

$-0.70178000$

$-0.80400300$

$-0.18886000$

$-1.23159600$

0.13498200

0.48487100

$-0.65328200$

$-0.64079100$

0.94145800

0.97905500

$-2.33810400$

$-2.53079000$

$-3.42663400$

$-4.42132200$

$-3.38978100$

$-3.34820600$

$-2.37616600$

$-1.98388800$

$-3.43190000$

$-1.62408700$

$-2.07439800$

$-1.65946500$

$-0.56598500$

2.12711400

3.07439700

2.08409400

2.17290000

1.00936300

0.95386900

2.01309000

$-0.04660000$

$-0.05528600$

0.15105100 


$\begin{array}{lrrr}\mathrm{H} & -0.55799900 & 6.76138700 & -1.06269100 \\ \mathrm{C} & -0.85238200 & 2.19902300 & 5.55609100 \\ \mathrm{~F} & -1.96528100 & 2.43929200 & 6.27710500 \\ \mathrm{~F} & -0.11931600 & 1.30026200 & 6.23876400 \\ \mathrm{~F} & -0.14346000 & 3.35536500 & 5.56262800\end{array}$

UB3LYP-D3(BJ)/def2-SVP-CPCM(THF)

Zero-point correction=

0.689511 (Hartree/Particle)

Thermal correction to Energy=

0.734150

Thermal correction to Enthalpy=

0.735094

Thermal correction to Gibbs Free Energy=

0.607734

$-5921.190816$

$-5921.146177$

$-5921.145233$

$-5921.272594$

Sum of electronic and thermal Free Energies= $\mathrm{HF}=-5921.8803271$

$\begin{array}{lrrr}{ }^{2} \mathbf{F}^{\prime}-\mathbf{T S}-\mathbf{H}^{\boldsymbol{R}} \boldsymbol{- B}^{\boldsymbol{C} \boldsymbol{F} \mathbf{}} \text {-Conf5 } & & \\ \mathrm{Ni} & -1.40120300 & 1.24041200 & -0.22186400 \\ \mathrm{C} & -1.82410400 & 0.83531400 & 1.62402700 \\ \mathrm{C} & -1.48091500 & 1.41755300 & 4.35766300 \\ \mathrm{C} & -0.82231700 & 0.18996000 & 2.37702100 \\ \mathrm{C} & -2.68755400 & 1.73071700 & 2.28365000 \\ \mathrm{C} & -2.50861900 & 2.03148900 & 3.63133000 \\ \mathrm{C} & -0.64336700 & 0.48894800 & 3.72541200 \\ \mathrm{H} & -0.18222200 & -0.55760600 & 1.91087300 \\ \mathrm{H} & -3.48971300 & 2.21315400 & 1.72308800 \\ \mathrm{H} & -3.16533100 & 2.75493700 & 4.11724300 \\ \mathrm{H} & 0.15877900 & 0.00513400 & 4.28557300 \\ \mathrm{C} & -2.87185000 & -0.79679100 & -1.11224300 \\ \mathrm{H} & -2.12941200 & -1.60006900 & -1.16088400 \\ \mathrm{C} & -2.96383500 & -0.33129400 & 0.34404400 \\ \mathrm{H} & -3.87413400 & 0.23519300 & 0.54664100 \\ \mathrm{H} & -2.53566700 & 0.00366700 & -1.79107800 \\ \mathrm{C} & -4.20752100 & -1.33403000 & -1.70739300 \\ \mathrm{C} & -5.25338200 & -0.21360800 & -1.80656300 \\ \mathrm{H} & -6.16595700 & -0.58614000 & -2.29925200 \\ \mathrm{H} & -5.54431200 & 0.16326600 & -0.81391900 \\ \mathrm{H} & -4.86655500 & 0.64152900 & -2.38034000 \\ \mathrm{C} & -4.76763500 & -2.49360900 & -0.86807000 \\ \mathrm{H} & -4.02462400 & -3.29713600 & -0.74883200 \\ \mathrm{H} & -5.07357000 & -2.16173000 & 0.13578900 \\ \mathrm{H} & -5.65830400 & -2.92071900 & -1.35616600 \\ \mathrm{C} & -3.88170800 & -1.84810200 & -3.11991400 \\ \mathrm{H} & -4.78902400 & -2.22352500 & -3.61954000\end{array}$




\begin{tabular}{|c|c|c|c|}
\hline $\mathrm{H}$ & -3.45724900 & -1.04635700 & -3.74554800 \\
\hline $\mathrm{H}$ & -3.15063900 & -2.67191800 & -3.08285600 \\
\hline $\mathrm{C}$ & -2.81952200 & -1.48118600 & 1.28571200 \\
\hline $\mathrm{O}$ & -2.03068900 & -2.39767600 & 1.14986600 \\
\hline $\mathrm{O}$ & -3.65282800 & -1.39179600 & 2.33306300 \\
\hline $\mathrm{C}$ & -3.49459500 & -2.37132400 & 3.36073100 \\
\hline $\mathrm{H}$ & -2.48598700 & -2.30617200 & 3.79557800 \\
\hline $\mathrm{H}$ & -4.24912200 & -2.14010200 & 4.12140600 \\
\hline $\mathrm{H}$ & -3.64988700 & -3.38487700 & 2.96359300 \\
\hline $\mathrm{Br}$ & -2.96399400 & 2.87802700 & -1.25049700 \\
\hline $\mathrm{C}$ & 1.44259900 & 1.96256100 & -0.26680200 \\
\hline $\mathrm{C}$ & 2.22703800 & 4.03810600 & -0.22572800 \\
\hline $\mathrm{C}$ & 0.69550100 & 4.03168500 & 0.03796900 \\
\hline $\mathrm{N}$ & 0.34554300 & 2.60515700 & -0.12662600 \\
\hline $\mathrm{H}$ & 2.50031500 & 4.49001200 & -1.18888300 \\
\hline $\mathrm{H}$ & 0.16136900 & 4.60102700 & -0.73659200 \\
\hline $\mathrm{C}$ & 0.60710900 & -1.51424400 & -0.89153400 \\
\hline $\mathrm{C}$ & 2.09145600 & -1.60936800 & -0.46406500 \\
\hline $\mathrm{C}$ & 1.41474600 & 0.50207900 & -0.44068500 \\
\hline $\mathrm{N}$ & 0.29508500 & -0.09632600 & -0.61599300 \\
\hline $\mathrm{H}$ & -0.03606900 & -2.14078300 & -0.25794300 \\
\hline $\mathrm{H}$ & 2.73801000 & -2.14575600 & -1.16752100 \\
\hline $\mathrm{H}$ & 2.81129600 & 4.50349800 & 0.57833700 \\
\hline $\mathrm{H}$ & 2.22307000 & -2.02097100 & 0.54654600 \\
\hline $\mathrm{O}$ & 2.59341000 & 2.62616600 & -0.28570300 \\
\hline $\mathrm{O}$ & 2.53159600 & -0.21419600 & -0.42498200 \\
\hline $\mathrm{C}$ & 0.30127300 & 4.59147000 & 1.42217300 \\
\hline $\mathrm{H}$ & 0.83687500 & 5.55631600 & 1.50196500 \\
\hline $\mathrm{C}$ & 0.34014300 & -1.85833600 & -2.37518300 \\
\hline $\mathrm{H}$ & -0.69837000 & -1.55176600 & -2.57036900 \\
\hline $\mathrm{C}$ & -1.19845100 & 4.87712100 & 1.48948800 \\
\hline $\mathrm{H}$ & -1.48104700 & 5.29987000 & 2.46453300 \\
\hline $\mathrm{H}$ & -1.49667600 & 5.59426100 & 0.70949400 \\
\hline $\mathrm{H}$ & -1.77632900 & 3.95930900 & 1.32762800 \\
\hline $\mathrm{C}$ & 0.79463500 & 3.67757100 & 2.55469700 \\
\hline $\mathrm{H}$ & 1.85534500 & 3.42349700 & 2.38206500 \\
\hline $\mathrm{H}$ & 0.24531100 & 2.72680200 & 2.50338300 \\
\hline $\mathrm{C}$ & 0.65903000 & 4.27017100 & 3.95551600 \\
\hline $\mathrm{H}$ & -0.39293100 & 4.44384000 & 4.22566900 \\
\hline $\mathrm{H}$ & 1.07390800 & 3.58509900 & 4.70984200 \\
\hline $\mathrm{H}$ & 1.19289700 & 5.23166300 & 4.03925200 \\
\hline $\mathrm{C}$ & 1.23422400 & -1.07433400 & -3.33913500 \\
\hline $\mathrm{H}$ & 1.15897800 & 0.00951500 & -3.16329100 \\
\hline $\mathrm{H}$ & 2.29363000 & -1.36571300 & -3.25348600 \\
\hline
\end{tabular}




$\begin{array}{lrrr}\mathrm{H} & 0.93011400 & -1.25073900 & -4.38051300 \\ \mathrm{C} & 0.41496900 & -3.38131800 & -2.57212500 \\ \mathrm{H} & -0.22642000 & -3.85834300 & -1.81101200 \\ \mathrm{H} & 1.44284800 & -3.73128800 & -2.36641100 \\ \mathrm{C} & -0.01698500 & -3.85750000 & -3.95836300 \\ \mathrm{H} & 0.65926000 & -3.49679700 & -4.74844700 \\ \mathrm{H} & -0.02691000 & -4.95726400 & -4.00965900 \\ \mathrm{H} & -1.03212800 & -3.50128900 & -4.19951300 \\ \mathrm{C} & -1.30648800 & 1.71038600 & 5.81743000 \\ \mathrm{~F} & -1.74917600 & 2.93971100 & 6.14821900 \\ \mathrm{~F} & -0.01346000 & 1.63771800 & 6.19903600 \\ \mathrm{~F} & -1.98090500 & 0.83789200 & 6.59976300\end{array}$

UB3LYP-D3(BJ)/def2-SVP-CPCM(THF)

Zero-point correction $=$

Thermal correction to Energy=

0.689968 (Hartree/Particle)

Thermal correction to Enthalpy=

0.734447

0.735392

Thermal correction to Gibbs Free Energy=

Sum of electronic and zero-point Energies=

0.609158

$-5921.189751$

Sum of electronic and thermal Energies=

$-5921.145271$

$-5921.144327$

$-5921.270561$

Sum of electronic and thermal Free Energies=

$\mathrm{HF}=-5921.8797183$

\section{${ }^{2} F^{\prime}-T S-H^{S}-B^{C F 3}$-Conf1}

$\begin{array}{lrrr}\mathrm{Ni} & -0.82619500 & 0.87376900 & -0.28106300 \\ \mathrm{C} & -1.90446500 & 1.49558700 & 1.17765500 \\ \mathrm{C} & -2.29358900 & 3.32873300 & 3.26108400 \\ \mathrm{C} & -1.43687100 & 1.20734700 & 2.46819900 \\ \mathrm{C} & -2.63589400 & 2.67589000 & 0.95386000 \\ \mathrm{C} & -2.81610500 & 3.59308100 & 1.98703900 \\ \mathrm{C} & -1.62035800 & 2.12466500 & 3.50170300 \\ \mathrm{H} & -0.88494600 & 0.28620100 & 2.66465400 \\ \mathrm{H} & -3.03853800 & 2.87863900 & -0.03943000 \\ \mathrm{H} & -3.35113700 & 4.52504600 & 1.79925500 \\ \mathrm{H} & -1.20972800 & 1.91374300 & 4.49104400 \\ \mathrm{Br} & -1.03932700 & 2.21171100 & -2.37383600 \\ \mathrm{C} & -2.34849900 & -1.49361400 & 0.84621400 \\ \mathrm{H} & -1.58924600 & -1.33997500 & 1.62519700 \\ \mathrm{C} & -2.75125600 & -0.17554200 & 0.21527500 \\ \mathrm{H} & -3.60919300 & 0.27278400 & 0.71484600 \\ \mathrm{H} & -1.88162800 & -2.11436000 & 0.07266000 \\ \mathrm{C} & -3.51527700 & -2.29585600 & 1.47964300 \\ \mathrm{C} & -2.94557200 & -3.65017700 & 1.93091400 \\ \mathrm{H} & -3.72226600 & -4.26604300 & 2.41217200\end{array}$




\begin{tabular}{|c|c|c|c|}
\hline $\mathrm{H}$ & -2.54689200 & -4.21583300 & 1.07290000 \\
\hline $\mathrm{H}$ & -2.12565900 & -3.51292700 & 2.65488900 \\
\hline $\mathrm{C}$ & -4.63392400 & -2.53324300 & 0.45223900 \\
\hline $\mathrm{H}$ & -5.10340700 & -1.58916300 & 0.13363300 \\
\hline $\mathrm{H}$ & -4.24531400 & -3.03543700 & -0.44725100 \\
\hline $\mathrm{H}$ & -5.42693100 & -3.16531700 & 0.88312400 \\
\hline $\mathrm{C}$ & -4.07614300 & -1.55076900 & 2.70130800 \\
\hline $\mathrm{H}$ & -4.88506700 & -2.13118300 & 3.17323300 \\
\hline $\mathrm{H}$ & -3.29171700 & -1.38360600 & 3.45677500 \\
\hline $\mathrm{H}$ & -4.49256200 & -0.56817900 & 2.42873400 \\
\hline $\mathrm{C}$ & -3.09164100 & -0.25268600 & -1.23872800 \\
\hline $\mathrm{O}$ & -2.66934500 & -1.07697800 & -2.02912900 \\
\hline $\mathrm{O}$ & -4.00321100 & 0.67714500 & -1.57048400 \\
\hline $\mathrm{C}$ & -4.37033100 & 0.74905300 & -2.94985500 \\
\hline $\mathrm{H}$ & -4.77293400 & -0.21449900 & -3.29461200 \\
\hline $\mathrm{H}$ & -5.13800400 & 1.52883000 & -3.01940100 \\
\hline $\mathrm{H}$ & -3.49409800 & 1.02241300 & -3.55435100 \\
\hline $\mathrm{C}$ & 2.04717100 & 1.12723700 & 0.25068300 \\
\hline $\mathrm{C}$ & 3.02526700 & 3.12036700 & 0.16849300 \\
\hline $\mathrm{C}$ & 1.48031100 & 3.27598700 & 0.22082800 \\
\hline $\mathrm{N}$ & 1.02244600 & 1.88224600 & 0.14787000 \\
\hline $\mathrm{H}$ & 3.45472300 & 3.40003800 & -0.80290600 \\
\hline $\mathrm{H}$ & 1.10289800 & 3.79367200 & -0.67306200 \\
\hline $\mathrm{C}$ & 0.83131400 & -2.26413600 & -0.14451300 \\
\hline $\mathrm{C}$ & 2.34076000 & -2.49892600 & 0.12753700 \\
\hline $\mathrm{C}$ & 1.85927600 & -0.33212900 & 0.20465200 \\
\hline $\mathrm{N}$ & 0.67736500 & -0.81834200 & 0.10358400 \\
\hline $\mathrm{H}$ & 0.22111300 & -2.81978300 & 0.58082100 \\
\hline $\mathrm{H}$ & 2.86590300 & -3.04538000 & -0.66503900 \\
\hline $\mathrm{H}$ & 3.56042500 & 3.64138200 & 0.97096600 \\
\hline $\mathrm{H}$ & 2.53526800 & -2.97859600 & 1.09640800 \\
\hline $\mathrm{O}$ & 3.24976400 & 1.68306600 & 0.34381800 \\
\hline $\mathrm{O}$ & 2.90262400 & -1.15134000 & 0.18517400 \\
\hline $\mathrm{C}$ & 0.91503500 & 3.97985900 & 1.47081800 \\
\hline $\mathrm{H}$ & -0.17090700 & 3.80704600 & 1.42899800 \\
\hline $\mathrm{C}$ & 0.34234800 & -2.61731900 & -1.56502200 \\
\hline $\mathrm{H}$ & -0.70192000 & -2.26997800 & -1.61488100 \\
\hline $\mathrm{C}$ & 1.11541800 & -1.86600900 & -2.65218300 \\
\hline $\mathrm{H}$ & 0.70141700 & -2.08841200 & -3.64587400 \\
\hline $\mathrm{H}$ & 1.03852900 & -0.77707300 & -2.51241400 \\
\hline $\mathrm{H}$ & 2.18323500 & -2.14089000 & -2.67236200 \\
\hline $\mathrm{C}$ & 0.33684400 & -4.14244500 & -1.75543000 \\
\hline $\mathrm{H}$ & -0.15702100 & -4.59919600 & -0.87955500 \\
\hline $\mathrm{H}$ & 1.37350000 & -4.52616100 & -1.75507500 \\
\hline
\end{tabular}




$\begin{array}{lrrr}\text { C } & -0.37893500 & -4.60576700 & -3.02399400 \\ \mathrm{H} & 0.13308000 & -4.26046700 & -3.93533700 \\ \mathrm{H} & -0.42815800 & -5.70486800 & -3.07028400 \\ \mathrm{H} & -1.41096000 & -4.21947500 & -3.05535700 \\ \mathrm{C} & 1.44078300 & 3.37637500 & 2.77565000 \\ \mathrm{H} & 0.87024800 & 3.75497400 & 3.63389500 \\ \mathrm{H} & 1.33591700 & 2.28182100 & 2.78131300 \\ \mathrm{H} & 2.50150300 & 3.62553000 & 2.94407300 \\ \mathrm{C} & 1.14786200 & 5.49599100 & 1.37708600 \\ \mathrm{H} & 0.80069100 & 5.84118400 & 0.38784600 \\ \mathrm{H} & 2.23322700 & 5.70471600 & 1.40906500 \\ \mathrm{C} & 0.43980900 & 6.30384400 & 2.46441600 \\ \mathrm{H} & 0.80781400 & 6.05347300 & 3.47097000 \\ \mathrm{H} & 0.59472900 & 7.38371400 & 2.31449200 \\ \mathrm{H} & -0.64533000 & 6.11592100 & 2.45614900 \\ \mathrm{C} & -2.45404900 & 4.31318900 & 4.38052600 \\ \mathrm{~F} & -1.29993200 & 4.48368200 & 5.06696900 \\ \mathrm{~F} & -2.83427200 & 5.52944200 & 3.94504300 \\ \mathrm{~F} & -3.37296500 & 3.91930200 & 5.28804200\end{array}$

UB3LYP-D3(BJ)/def2-SVP-CPCM(THF)

Zero-point correction=

0.689599 (Hartree/Particle)

Thermal correction to Energy=

0.734283

0.735227

Thermal correction to Gibbs Free Energy=

Sum of electronic and zero-point Energies=

0.607733

$-5921.192377$

$-5921.147694$

$-5921.146750$

Sum of electronic and thermal Enthalpies=

$-5921.274243$

$\mathrm{HF}=-5921.8819765$

$\begin{array}{lrrr}{ }^{2} \mathbf{F}^{\prime}-\mathbf{T S}-\mathbf{H}^{\boldsymbol{S}}-\boldsymbol{B}^{\boldsymbol{C F} \boldsymbol{3}} \text {-Conf2 } & & \\ \mathrm{Ni} & -0.79270500 & 0.87234400 & -0.28770200 \\ \mathrm{C} & -1.89849200 & 1.52525000 & 1.13560400 \\ \mathrm{C} & -2.31182300 & 3.39932600 & 3.17681400 \\ \mathrm{C} & -1.47025400 & 1.25127500 & 2.44242000 \\ \mathrm{C} & -2.60985700 & 2.71069100 & 0.87359300 \\ \mathrm{C} & -2.79757400 & 3.64996900 & 1.88469800 \\ \mathrm{C} & -1.66526400 & 2.18964700 & 3.45580000 \\ \mathrm{H} & -0.94306600 & 0.32293200 & 2.67040500 \\ \mathrm{H} & -2.98835600 & 2.89939900 & -0.13167600 \\ \mathrm{H} & -3.31724100 & 4.58492700 & 1.66899400 \\ \mathrm{H} & -1.29287100 & 1.98719700 & 4.46151800 \\ \mathrm{Br} & -0.95374600 & 2.21175000 & -2.38945500 \\ \mathrm{C} & -2.33284600 & -1.47485200 & 0.81909600\end{array}$


H

C

$\mathrm{H}$

$\mathrm{H}$

C

C

$\mathrm{H}$

$\mathrm{H}$

$\mathrm{H}$

C

$\mathrm{H}$

$\mathrm{H}$

$\mathrm{H}$

C

$\mathrm{H}$

$\mathrm{H}$

$\mathrm{H}$

C

$\mathrm{O}$

$\mathrm{O}$

C

$\mathrm{H}$

$\mathrm{H}$

$\mathrm{H}$

C

C

C

$\mathrm{N}$

$\mathrm{H}$

$\mathrm{H}$

C

C

C

$\mathrm{N}$

$\mathrm{H}$

$\mathrm{H}$

$\mathrm{H}$

$\mathrm{H}$

$\mathrm{O}$

$\mathrm{O}$

C

$\mathrm{H}$

C

$\mathrm{H}$

\begin{tabular}{rrr}
-1.58506800 & -1.31688800 & 1.60855500 \\
-2.72910400 & -0.15964400 & 0.17735500 \\
-3.59293600 & 0.29143500 & 0.66447000 \\
-1.85218900 & -2.09658400 & 0.05480300 \\
-3.50676500 & -2.27804200 & 1.43785000 \\
-2.94059000 & -3.62987800 & 1.90087300 \\
-3.72264700 & -4.24602100 & 2.37300300 \\
-2.52877900 & -4.19712400 & 1.05013700 \\
-2.13115600 & -3.48882400 & 2.63582000 \\
-4.60994600 & -2.52050300 & 0.39506100 \\
-5.07700500 & -1.57824000 & 0.06758200 \\
-4.20726200 & -3.02374100 & -0.49760100 \\
-5.40758600 & -3.15342700 & 0.81602700 \\
-4.08672100 & -1.53089000 & 2.64924700 \\
-4.90149100 & -2.11145800 & 3.11080000 \\
-3.31372000 & -1.36057700 & 3.41571900 \\
-4.50069200 & -0.54970000 & 2.36803500 \\
-3.04819300 & -0.24003800 & -1.28111200 \\
-2.61799700 & -1.06879300 & -2.06246100 \\
-3.94792300 & 0.69516400 & -1.62987700 \\
-4.29087600 & 0.76765200 & -3.01548600 \\
-4.68923000 & -0.19507400 & -3.36731500 \\
-5.05563100 & 1.54898400 & -3.09841600 \\
-3.40365900 & 1.03939200 & -3.60456600 \\
2.07574800 & 1.09845000 & 0.26417400 \\
3.04292800 & 3.09797600 & 0.19414000 \\
1.49857300 & 3.24506800 & 0.25483600 \\
1.04863600 & 1.85031200 & 0.16323000 \\
3.46441100 & 3.37846900 & -0.78065200 \\
1.11558000 & 3.76588600 & -0.63258300 \\
0.86393900 & -2.29216000 & -0.15175300 \\
2.37317300 & -2.52767200 & 0.12387100 \\
1.89041800 & -0.36112700 & 0.20712300 \\
0.70947600 & -0.84706700 & 0.09644500 \\
0.25132700 & -2.84727400 & 0.57241800 \\
2.90106100 & -3.06913800 & -0.67035300 \\
3.58118000 & 3.62237000 & 0.99233000 \\
2.56499500 & -3.01319500 & 1.09033900 \\
3.27477000 & 1.66082600 & 0.36814800 \\
\hline .93440700 & -1.18018600 & 0.19127500 \\
\hline
\end{tabular}




$\begin{array}{lrrr}\mathrm{C} & 1.15131900 & -1.89337900 & -2.65859100 \\ \mathrm{H} & 0.73882600 & -2.11514100 & -3.65306100 \\ \mathrm{H} & 1.07347900 & -0.80469100 & -2.51743500 \\ \mathrm{H} & 2.21939300 & -2.16742200 & -2.67759000 \\ \mathrm{C} & 0.37554300 & -4.17145900 & -1.76330800 \\ \mathrm{H} & -0.11998900 & -4.62901100 & -0.88878800 \\ \mathrm{H} & 1.41297300 & -4.55306800 & -1.75976000 \\ \mathrm{C} & -0.33517800 & -4.63674600 & -3.03397100 \\ \mathrm{H} & 0.17884800 & -4.29038600 & -3.94377700 \\ \mathrm{H} & -0.38156300 & -5.73598100 & -3.08016600 \\ \mathrm{H} & -1.36810300 & -4.25311500 & -3.06875400 \\ \mathrm{C} & 1.49109500 & 3.31359200 & 2.81228300 \\ \mathrm{H} & 0.94209800 & 3.71328400 & 3.67664800 \\ \mathrm{H} & 1.38182200 & 2.21925100 & 2.83153000 \\ \mathrm{H} & 2.55624100 & 3.55883200 & 2.95456100 \\ \mathrm{C} & 1.10233900 & 5.44303200 & 1.49348700 \\ \mathrm{H} & 2.17692300 & 5.69852800 & 1.44253800 \\ \mathrm{H} & 0.74618200 & 5.82837700 & 2.46298100 \\ \mathrm{C} & 0.33990000 & 6.14711700 & 0.37093500 \\ \mathrm{H} & -0.72999300 & 5.88275100 & 0.39865800 \\ \mathrm{H} & 0.41944700 & 7.24118900 & 0.46624400 \\ \mathrm{H} & 0.71931300 & 5.87958800 & -0.62793500 \\ \mathrm{C} & -2.39841500 & 4.45346300 & 4.23816600 \\ \mathrm{~F} & -1.25269100 & 5.18107700 & 4.31414200 \\ \mathrm{~F} & -3.39063100 & 5.33305500 & 4.01501600 \\ \mathrm{~F} & -2.59012600 & 3.93245800 & 5.46525700 \\ \mathrm{H} & -\mathrm{s}\end{array}$

UB3LYP-D3(BJ)/def2-SVP-CPCM(THF)

Zero-point correction=

0.689275 (Hartree/Particle)

Thermal correction to Energy=

0.734101

Thermal correction to Enthalpy=

0.735045

0.606568

Thermal correction to Gibbs Free Energy=

Sum of electronic and zero-point Energies=

$-5921.192282$

$-5921.147455$

$-5921.146511$

Sum of electronic and thermal Enthalpies=

$-5921.274988$

$\mathrm{HF}=-5921.8815562$

$\begin{array}{lrrr}{ }^{\mathbf{2}} \mathbf{F} \text { - } \mathbf{T S}-\mathbf{H}^{\boldsymbol{S}} \mathbf{- B}^{\boldsymbol{C} \boldsymbol{F} \mathbf{3}} \text {-Conf3 } & & \\ \mathrm{Ni} & -0.46516300 & 0.33638200 & -0.04343600 \\ \mathrm{C} & -1.30856200 & 0.71486800 & 1.63206500 \\ \mathrm{C} & -1.38726100 & 2.24632000 & 3.97952700 \\ \mathrm{C} & -0.64745700 & 0.27284600 & 2.78699700 \\ \mathrm{C} & -2.07088400 & 1.89747600 & 1.68182400 \\ \mathrm{C} & -2.09556700 & 2.66542100 & 2.84284400\end{array}$




\begin{tabular}{|c|c|c|c|}
\hline $\mathrm{C}$ & -0.67684800 & 1.04063000 & 3.95099800 \\
\hline $\mathrm{H}$ & -0.07219900 & -0.65450300 & 2.77517000 \\
\hline $\mathrm{H}$ & -2.62568900 & 2.21529800 & 0.79832400 \\
\hline $\mathrm{H}$ & -2.66181600 & 3.59793700 & 2.86622000 \\
\hline $\mathrm{H}$ & -0.12741800 & 0.70837600 & 4.83353200 \\
\hline $\mathrm{Br}$ & -1.02320800 & 1.94752900 & -1.86580900 \\
\hline $\mathrm{C}$ & -1.74879300 & -2.18533200 & 0.94778800 \\
\hline $\mathrm{H}$ & -0.86135200 & -2.13679900 & 1.59357400 \\
\hline $\mathrm{C}$ & -2.23506100 & -0.79225300 & 0.58859600 \\
\hline $\mathrm{H}$ & -3.02107600 & -0.46662900 & 1.26971300 \\
\hline $\mathrm{H}$ & -1.43690700 & -2.68720700 & 0.02439200 \\
\hline $\mathrm{C}$ & -2.80148300 & -3.07585200 & 1.65839800 \\
\hline $\mathrm{C}$ & -2.18054900 & -4.47057400 & 1.83576400 \\
\hline $\mathrm{H}$ & -2.87403800 & -5.14779200 & 2.35970500 \\
\hline $\mathrm{H}$ & -1.93730200 & -4.92157200 & 0.86098200 \\
\hline $\mathrm{H}$ & -1.25005500 & -4.41738700 & 2.42447200 \\
\hline $\mathrm{C}$ & -4.07568200 & -3.19399100 & 0.80611000 \\
\hline $\mathrm{H}$ & -4.58329300 & -2.22322600 & 0.69148700 \\
\hline $\mathrm{H}$ & -3.84371800 & -3.57297400 & -0.20139500 \\
\hline $\mathrm{H}$ & -4.79285700 & -3.88562500 & 1.27652300 \\
\hline $\mathrm{C}$ & -3.14806100 & -2.50042100 & 3.04093000 \\
\hline $\mathrm{H}$ & -3.87434000 & -3.14696800 & 3.55898000 \\
\hline $\mathrm{H}$ & -2.24956200 & -2.42298000 & 3.67373400 \\
\hline $\mathrm{H}$ & -3.59471400 & -1.49604800 & 2.97070100 \\
\hline $\mathrm{C}$ & -2.82216400 & -0.67018600 & -0.78342100 \\
\hline $\mathrm{O}$ & -2.55198000 & -1.36762500 & -1.74340200 \\
\hline $\mathrm{O}$ & -3.78037300 & 0.27166800 & -0.81273900 \\
\hline $\mathrm{C}$ & -4.39142700 & 0.52448000 & -2.07965200 \\
\hline $\mathrm{H}$ & -4.85229400 & -0.39153500 & -2.47698200 \\
\hline $\mathrm{H}$ & -5.15712300 & 1.28846500 & -1.90013500 \\
\hline $\mathrm{H}$ & -3.63900900 & 0.89863500 & -2.78772700 \\
\hline $\mathrm{C}$ & 2.38610400 & 0.68940100 & 0.03855900 \\
\hline $\mathrm{C}$ & 3.26112600 & 2.72796500 & 0.08783700 \\
\hline $\mathrm{C}$ & 1.75841600 & 2.77414000 & 0.47389300 \\
\hline $\mathrm{N}$ & 1.33554600 & 1.38539100 & 0.24431700 \\
\hline $\mathrm{H}$ & 3.46482800 & 3.16028200 & -0.90131100 \\
\hline $\mathrm{H}$ & 1.18976100 & 3.40450200 & -0.22201800 \\
\hline $\mathrm{C}$ & 1.16292200 & -2.63029000 & -0.72110600 \\
\hline $\mathrm{C}$ & 2.69587900 & -2.85868500 & -0.70425100 \\
\hline $\mathrm{C}$ & 2.21805100 & -0.74774300 & -0.21476400 \\
\hline $\mathrm{N}$ & 1.03479800 & -1.24753400 & -0.21451700 \\
\hline $\mathrm{H}$ & 0.66961000 & -3.31099600 & -0.01741700 \\
\hline $\mathrm{H}$ & 3.10494200 & -3.26434200 & -1.63732500 \\
\hline $\mathrm{H}$ & 3.93497500 & 3.17166100 & 0.82981400 \\
\hline
\end{tabular}




$\begin{array}{lrrr}\mathrm{H} & 3.03246700 & -3.47218200 & 0.14259500 \\ \mathrm{O} & 3.56696800 & 1.29634200 & 0.00518300 \\ \mathrm{O} & 3.24972200 & -1.51917600 & -0.52175000 \\ \mathrm{C} & 1.45237800 & 3.20602400 & 1.92231400 \\ \mathrm{H} & 0.39523400 & 2.95287500 & 2.07926700 \\ \mathrm{C} & 0.49174400 & -2.75663700 & -2.10629900 \\ \mathrm{H} & -0.56121500 & -2.46998600 & -1.95742200 \\ \mathrm{C} & 1.08439100 & -1.78360700 & -3.12945900 \\ \mathrm{H} & 0.56604100 & -1.89137700 & -4.09435600 \\ \mathrm{H} & 0.96023500 & -0.73818500 & -2.80942400 \\ \mathrm{H} & 2.15616200 & -1.96761800 & -3.31251400 \\ \mathrm{C} & 0.51775100 & -4.20735100 & -2.61535300 \\ \mathrm{H} & 1.56294400 & -4.54535900 & -2.73902200 \\ \mathrm{H} & 0.08824200 & -4.20737100 & -3.63102100 \\ \mathrm{C} & -0.25298700 & -5.20608600 & -1.75132600 \\ \mathrm{H} & -1.29868300 & -4.88506000 & -1.61576800 \\ \mathrm{H} & -0.26721300 & -6.20247700 & -2.21959900 \\ \mathrm{H} & 0.18961400 & -5.32583000 & -0.74975900 \\ \mathrm{C} & 2.27600800 & 2.43769800 & 2.95769700 \\ \mathrm{H} & 1.90760400 & 2.66747400 & 3.96758900 \\ \mathrm{H} & 2.19369300 & 1.34973100 & 2.81876300 \\ \mathrm{H} & 3.34285500 & 2.71277200 & 2.92407600 \\ \mathrm{C} & 1.58857200 & 4.72509100 & 2.11084800 \\ \mathrm{H} & 2.62417100 & 5.03905000 & 1.88375100 \\ \mathrm{H} & 1.43812900 & 4.93473700 & 3.18250200 \\ \mathrm{C} & 0.59596500 & 5.55838400 & 1.30007200 \\ \mathrm{H} & -0.44007400 & 5.24421500 & 1.50781100 \\ \mathrm{H} & 0.68077400 & 6.62703700 & 1.55148300 \\ \mathrm{H} & 0.75684900 & 5.46625000 & 0.21427700 \\ \mathrm{C} & -1.30765800 & 3.12462100 & 5.19037400 \\ \mathrm{~F} & -2.34894200 & 3.97029000 & 5.28624300 \\ \mathrm{~F} & -1.25585100 & 2.41783800 & 6.33572100 \\ \mathrm{~F} & -18908900 & 3.89744200 & 5.17570600\end{array}$

UB3LYP-D3(BJ)/def2-SVP-CPCM(THF)

Zero-point correction=

Thermal correction to Energy=

0.688714 (Hartree/Particle)

0.733456

Thermal correction to Enthalpy=

0.734400

Thermal correction to Gibbs Free Energy=

Sum of electronic and zero-point Energies=

Sum of electronic and thermal Energies=

0.607045

$-5921.192079$

$-5921.147337$

$-5921.146393$

Sum of electronic and thermal Enthalpies=

$-5921.273748$

Sum of electronic and thermal Free Energies= $\mathrm{HF}=-5921.880793$ 


\begin{tabular}{|c|c|c|c|}
\hline \multicolumn{4}{|c|}{${ }^{2} F^{\prime}-\mathrm{TS}-\mathrm{H}^{S}-B^{C F 3}-\mathrm{Conf} 4$} \\
\hline $\mathrm{Ni}$ & -1.16203000 & 1.12868200 & -0.45476700 \\
\hline $\mathrm{C}$ & -2.38953100 & 1.98314200 & 0.75763500 \\
\hline $\mathrm{C}$ & -3.34645500 & 4.25889400 & 2.09609200 \\
\hline $\mathrm{C}$ & -1.91449300 & 2.30600800 & 2.03770100 \\
\hline $\mathrm{C}$ & -3.37896500 & 2.79371400 & 0.17022900 \\
\hline $\mathrm{C}$ & -3.84397300 & 3.92909600 & 0.82754700 \\
\hline $\mathrm{C}$ & -2.38308700 & 3.44198500 & 2.69929600 \\
\hline $\mathrm{H}$ & -1.15125500 & 1.68999000 & 2.51539000 \\
\hline $\mathrm{H}$ & -3.78438500 & 2.52131000 & -0.80599800 \\
\hline $\mathrm{H}$ & -4.59235900 & 4.56445900 & 0.35111900 \\
\hline $\mathrm{H}$ & -1.98193500 & 3.70248000 & 3.68025600 \\
\hline $\mathrm{Br}$ & -1.44628300 & 1.25920400 & -2.91152500 \\
\hline $\mathrm{C}$ & 1.52113300 & 2.34599400 & -0.13132400 \\
\hline $\mathrm{C}$ & 2.24423800 & 4.20188400 & -1.11415400 \\
\hline $\mathrm{C}$ & 0.74127600 & 3.96558200 & -1.42910200 \\
\hline $\mathrm{N}$ & 0.42746800 & 2.74711700 & -0.65336700 \\
\hline $\mathrm{H}$ & 2.88233900 & 4.22496200 & -2.00620500 \\
\hline $\mathrm{H}$ & 0.60344000 & 3.69705100 & -2.48822300 \\
\hline $\mathrm{C}$ & 0.96902200 & -1.00413500 & 1.14007000 \\
\hline $\mathrm{C}$ & 2.42639100 & -0.72687600 & 1.58431800 \\
\hline $\mathrm{C}$ & 1.57196500 & 1.05022700 & 0.56231900 \\
\hline $\mathrm{N}$ & 0.52179700 & 0.31814000 & 0.65296500 \\
\hline $\mathrm{H}$ & 0.36077600 & -1.29901400 & 2.00353900 \\
\hline $\mathrm{H}$ & 3.16303500 & -1.44426600 & 1.20339700 \\
\hline $\mathrm{H}$ & 2.42366900 & 5.10320800 & -0.51117900 \\
\hline $\mathrm{H}$ & 2.53341500 & -0.63193900 & 2.67352400 \\
\hline $\mathrm{O}$ & 2.63733400 & 3.04777600 & -0.31440400 \\
\hline $\mathrm{O}$ & 2.72964100 & 0.57556300 & 0.99956800 \\
\hline $\mathrm{C}$ & -0.16565000 & 5.16834900 & -1.10937100 \\
\hline $\mathrm{H}$ & 0.35758400 & 6.04960500 & -1.52681900 \\
\hline $\mathrm{C}$ & 0.79360700 & -2.06189900 & 0.03079600 \\
\hline $\mathrm{H}$ & -0.26918800 & -2.01926400 & -0.25535600 \\
\hline $\mathrm{C}$ & -2.29924100 & -0.86564100 & 1.63822900 \\
\hline $\mathrm{H}$ & -1.64983200 & -0.26824000 & 2.29036200 \\
\hline $\mathrm{C}$ & -2.84968100 & -0.02209200 & 0.50632800 \\
\hline $\mathrm{H}$ & -3.82462000 & 0.39314700 & 0.75615500 \\
\hline $\mathrm{H}$ & -1.67722200 & -1.65847800 & 1.20684600 \\
\hline $\mathrm{C}$ & -3.37753700 & -1.52758200 & 2.53594000 \\
\hline $\mathrm{C}$ & -4.34493200 & -2.37466700 & 1.69316900 \\
\hline $\mathrm{H}$ & -5.06284600 & -2.90136800 & 2.34211800 \\
\hline $\mathrm{H}$ & -4.92818200 & -1.75559400 & 0.99345400 \\
\hline $\mathrm{H}$ & -3.80022800 & -3.12733900 & 1.10226800 \\
\hline C & -4.16008000 & -0.45639000 & 3.31245400 \\
\hline
\end{tabular}




\begin{tabular}{|c|c|c|c|}
\hline $\mathrm{H}$ & -3.48550700 & 0.15289100 & 3.93489100 \\
\hline $\mathrm{H}$ & -4.70177400 & 0.22716100 & 2.63980500 \\
\hline $\mathrm{H}$ & -4.90563900 & -0.92441600 & 3.97500200 \\
\hline $\mathrm{C}$ & -2.64393300 & -2.43857800 & 3.53311600 \\
\hline $\mathrm{H}$ & -3.35445100 & -2.92758900 & 4.21878700 \\
\hline $\mathrm{H}$ & -2.08191600 & -3.22753400 & 3.00712800 \\
\hline $\mathrm{H}$ & -1.92783000 & -1.86282700 & 4.14214700 \\
\hline $\mathrm{C}$ & -3.04143000 & -0.77081600 & -0.77664900 \\
\hline $\mathrm{O}$ & -2.41126800 & -1.74859300 & -1.13451100 \\
\hline $\mathrm{O}$ & -4.08990700 & -0.28569000 & -1.46309200 \\
\hline $\mathrm{C}$ & -4.37076300 & -0.90072200 & -2.72231600 \\
\hline $\mathrm{H}$ & -4.56588900 & -1.97524100 & -2.59240500 \\
\hline $\mathrm{H}$ & -5.26248400 & -0.39690100 & -3.11369600 \\
\hline $\mathrm{H}$ & -3.52149100 & -0.75729300 & -3.40450000 \\
\hline $\mathrm{C}$ & 1.60968000 & -1.73752300 & -1.22333600 \\
\hline $\mathrm{H}$ & 1.40813100 & -2.47145000 & -2.01637900 \\
\hline $\mathrm{H}$ & 1.33928900 & -0.75076900 & -1.62878300 \\
\hline $\mathrm{H}$ & 2.69546200 & -1.75116300 & -1.03164600 \\
\hline $\mathrm{C}$ & 1.06579400 & -3.46514100 & 0.59593800 \\
\hline $\mathrm{H}$ & 0.51145100 & -3.56994500 & 1.54536200 \\
\hline $\mathrm{H}$ & 2.13550400 & -3.56575600 & 0.85546400 \\
\hline $\mathrm{C}$ & 0.65666000 & -4.60003700 & -0.34252500 \\
\hline $\mathrm{H}$ & 1.25177300 & -4.60386800 & -1.26867700 \\
\hline $\mathrm{H}$ & 0.79305100 & -5.58006000 & 0.14067400 \\
\hline $\mathrm{H}$ & -0.40399200 & -4.50827100 & -0.62834500 \\
\hline $\mathrm{C}$ & -1.50696600 & 5.03381900 & -1.83172600 \\
\hline $\mathrm{H}$ & -2.17171500 & 5.88072300 & -1.60802600 \\
\hline $\mathrm{H}$ & -1.36060300 & 4.99581200 & -2.92203000 \\
\hline $\mathrm{H}$ & -2.01489600 & 4.10786800 & -1.53512800 \\
\hline $\mathrm{C}$ & -0.30666800 & 5.37095800 & 0.40605700 \\
\hline $\mathrm{H}$ & 0.69525800 & 5.34846400 & 0.86983800 \\
\hline $\mathrm{H}$ & -0.84787900 & 4.51045100 & 0.82365400 \\
\hline $\mathrm{C}$ & -1.00400800 & 6.66654700 & 0.81375700 \\
\hline $\mathrm{H}$ & -2.04575900 & 6.69840800 & 0.46254000 \\
\hline $\mathrm{H}$ & -1.03303600 & 6.76641000 & 1.90917200 \\
\hline $\mathrm{H}$ & -0.48317100 & 7.54920700 & 0.40599300 \\
\hline $\mathrm{C}$ & -3.87762900 & 5.45768700 & 2.82402500 \\
\hline $\mathrm{F}$ & -4.94507000 & 5.15674700 & 3.59666500 \\
\hline $\mathrm{F}$ & -4.28186000 & 6.42831200 & 1.97959000 \\
\hline 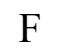 & -2.95711400 & 6.00352800 & 3.6456740 \\
\hline
\end{tabular}

UB3LYP-D3(BJ)/def2-SVP-CPCM(THF)

Zero-point correction=

0.689915 (Hartree/Particle)

Thermal correction to Energy=

0.734361

Thermal correction to Enthalpy=

0.735306 
Thermal correction to Gibbs Free Energy=

Sum of electronic and zero-point Energies=

Sum of electronic and thermal Energies=

Sum of electronic and thermal Enthalpies=

Sum of electronic and thermal Free Energies=

$\mathrm{HF}=-5921.8801328$

$\begin{array}{lrrr}\text { Scheme S10 } & & & \\ \text { F } \boldsymbol{R - l a c} & & & \\ \mathrm{Ni} & -1.68577800 & 1.30158000 & -0.41516100 \\ \mathrm{C} & -1.54801800 & 1.10075000 & 1.48047500 \\ \mathrm{C} & -1.16061700 & 1.16287900 & 4.24968000 \\ \mathrm{C} & -0.64275000 & 0.21909400 & 2.07338100 \\ \mathrm{C} & -2.28338700 & 1.99208600 & 2.26579300 \\ \mathrm{C} & -2.08016800 & 2.03007400 & 3.64851600 \\ \mathrm{C} & -0.44841400 & 0.25512600 & 3.46176500 \\ \mathrm{H} & -0.08236800 & -0.49758500 & 1.47178300 \\ \mathrm{H} & -3.02180000 & 2.65121400 & 1.80448500 \\ \mathrm{H} & -2.65039000 & 2.73535600 & 4.25900100 \\ \mathrm{H} & 0.26350000 & -0.43417500 & 3.92368900 \\ \mathrm{H} & -1.00883600 & 1.18787500 & 5.33146600 \\ \mathrm{Br} & -3.07739600 & 3.07509700 & -1.51462600 \\ \mathrm{C} & 1.12478900 & 2.08431600 & -0.76260100 \\ \mathrm{C} & 1.60862100 & 4.24603900 & -0.54579500 \\ \mathrm{C} & 0.07886600 & 4.02355700 & -0.41345000 \\ \mathrm{~N} & -0.04192700 & 2.55270900 & -0.52509300 \\ \mathrm{H} & 1.89311100 & 4.91408600 & -1.36787800 \\ \mathrm{H} & -0.46561000 & 4.44856600 & -1.27004600 \\ \mathrm{C} & 0.99242000 & -1.51836700 & -1.26245500 \\ \mathrm{C} & 2.51996300 & -1.22826600 & -1.23463000 \\ \mathrm{C} & 1.34942200 & 0.64461900 & -0.97167300 \\ \mathrm{~N} & 0.38130600 & -0.18583300 & -1.04209700 \\ \mathrm{H} & 0.71283100 & -2.16611900 & -0.41671500 \\ \mathrm{H} & 3.02750800 & -1.49207200 & -2.17283000 \\ \mathrm{H} & 2.07238700 & 4.58950400 & 0.39015800 \\ \mathrm{H} & 3.04229100 & -1.69455700 & -0.38891900 \\ \mathrm{O} & 2.14798300 & 2.92241000 & -0.83967700 \\ \mathrm{O} & 2.60535000 & 0.21383500 & -1.07128800 \\ \mathrm{C} & -4.00229900 & -1.54704800 & -2.24737900 \\ \mathrm{C} & -5.25442800 & -2.00117300 & -1.48021100 \\ \mathrm{H} & -6.04291400 & -2.30366900 & -2.18736500 \\ \mathrm{H} & -5.04614200 & -2.86158200 & -0.82600800 \\ \mathrm{H} & -5.66392800 & -1.19271300 & -0.85554900 \\ \mathrm{C} & & -2.69550700 & -3.14018500\end{array}$

0.609731

$-5921.190218$

$-5921.145771$

$-5921.144827$

$-5921.270402$ 


\begin{tabular}{|c|c|c|c|}
\hline $\mathrm{H}$ & -2.62160400 & -2.38544600 & -3.72721700 \\
\hline $\mathrm{H}$ & -3.22559900 & -3.58895300 & -2.55924300 \\
\hline $\mathrm{H}$ & -4.28639100 & -2.99673000 & -3.85111500 \\
\hline $\mathrm{C}$ & -4.35535100 & -0.35703800 & -3.15448200 \\
\hline $\mathrm{H}$ & -5.11267800 & -0.65511400 & -3.89682700 \\
\hline $\mathrm{H}$ & -4.75494600 & 0.49536500 & -2.58727600 \\
\hline $\mathrm{H}$ & -3.46649600 & -0.00097700 & -3.69986200 \\
\hline $\mathrm{C}$ & -2.41723700 & -2.19641500 & -0.26493900 \\
\hline $\mathrm{C}$ & -2.85411400 & -1.11828200 & -1.27594700 \\
\hline $\mathrm{C}$ & -3.22140300 & 0.01845100 & -0.32239200 \\
\hline $\mathrm{C}$ & -3.62935800 & -0.66215300 & 0.93976900 \\
\hline $\mathrm{O}$ & -3.13284600 & -1.92976900 & 0.94899800 \\
\hline $\mathrm{H}$ & -2.64783300 & -3.22417700 & -0.57219400 \\
\hline $\mathrm{H}$ & -1.99940100 & -0.85342700 & -1.91519000 \\
\hline $\mathrm{H}$ & -4.00461800 & 0.70584300 & -0.64891900 \\
\hline $\mathrm{O}$ & -4.33357000 & -0.26502800 & 1.83618100 \\
\hline $\mathrm{H}$ & -1.34234800 & -2.12879300 & -0.05027300 \\
\hline $\mathrm{C}$ & -0.51630300 & 4.57291000 & 0.87309000 \\
\hline $\mathrm{H}$ & -0.32881400 & 5.65634300 & 0.92973300 \\
\hline $\mathrm{H}$ & -1.60016600 & 4.40656400 & 0.88799000 \\
\hline $\mathrm{H}$ & -0.06968700 & 4.08817600 & 1.75371300 \\
\hline $\mathrm{C}$ & 0.52316900 & -2.15975200 & -2.56344400 \\
\hline $\mathrm{H}$ & 1.04712200 & -3.11543700 & -2.71686400 \\
\hline $\mathrm{H}$ & -0.55438200 & -2.36405800 & -2.54431200 \\
\hline $\mathrm{H}$ & 0.73787000 & -1.50162000 & -3.41945500 \\
\hline \multicolumn{4}{|c|}{ UB3LYP-D3(BJ)/def2-SVP-CPCM(THF) } \\
\hline \multicolumn{3}{|c|}{ Zero-point correction $=$} & 0.493978 (Hartree/Particle) \\
\hline \multicolumn{3}{|c|}{ Thermal correction to Energy= } & 0.525909 \\
\hline \multicolumn{3}{|c|}{ Thermal correction to Enthalpy= } & 0.526853 \\
\hline \multicolumn{3}{|c|}{ Thermal correction to Gibbs Free Energy= } & 0.427734 \\
\hline \multicolumn{3}{|c|}{ Sum of electronic and zero-point Energies $=$} & -5347.637996 \\
\hline \multicolumn{3}{|c|}{ Sum of electronic and thermal Energies $=$} & -5347.606066 \\
\hline \multicolumn{3}{|c|}{ Sum of electronic and thermal Enthalpies $=$} & -5347.605122 \\
\hline \multicolumn{3}{|c|}{ Sum of electronic and thermal Free Energies= } & -5347.704241 \\
\hline \multicolumn{4}{|c|}{$\mathrm{HF}=-5348.1319749$} \\
\hline \multicolumn{4}{|c|}{ F'-TS-H $^{R \text {-lac }}$} \\
\hline $\mathrm{Ni}$ & -1.71375600 & 1.57770000 & -0.22051200 \\
\hline $\mathrm{C}$ & -1.61539400 & 0.94554500 & 1.59196700 \\
\hline $\mathrm{C}$ & -0.43038200 & 1.02030200 & 4.14014300 \\
\hline $\mathrm{C}$ & -0.63159500 & 0.00865800 & 1.94269100 \\
\hline $\mathrm{C}$ & -2.04194800 & 1.88532100 & 2.54533700 \\
\hline $\mathrm{C}$ & -1.43900200 & 1.93148200 & 3.80364800 \\
\hline $\mathrm{C}$ & -0.03511800 & 0.05617700 & 3.20763300 \\
\hline
\end{tabular}




\begin{tabular}{|c|c|c|c|}
\hline $\mathrm{H}$ & -0.30803100 & -0.74903300 & 1.22947000 \\
\hline $\mathrm{H}$ & -2.84937400 & 2.57485300 & 2.29668200 \\
\hline $\mathrm{H}$ & -1.76425100 & 2.68311900 & 4.52743100 \\
\hline $\mathrm{H}$ & 0.74221900 & -0.66903400 & 3.46141000 \\
\hline $\mathrm{H}$ & 0.03361100 & 1.05247200 & 5.12852500 \\
\hline $\mathrm{Br}$ & -3.32978600 & 3.36933500 & -0.93654000 \\
\hline $\mathrm{C}$ & 1.04880400 & 2.33582500 & -0.70103200 \\
\hline $\mathrm{C}$ & 1.61581000 & 4.45978900 & -0.35329500 \\
\hline $\mathrm{C}$ & 0.08649500 & 4.26914700 & -0.15173900 \\
\hline $\mathrm{N}$ & -0.09155500 & 2.82454300 & -0.39366000 \\
\hline $\mathrm{H}$ & 1.87533000 & 5.16000500 & -1.15707400 \\
\hline $\mathrm{H}$ & -0.49106000 & 4.79677000 & -0.92637300 \\
\hline $\mathrm{C}$ & 0.65206100 & -1.17897200 & -1.53096100 \\
\hline $\mathrm{C}$ & 2.17696500 & -1.06019800 & -1.27439000 \\
\hline $\mathrm{C}$ & 1.17718700 & 0.90469500 & -1.01708000 \\
\hline $\mathrm{N}$ & 0.14355300 & 0.16337000 & -1.15789300 \\
\hline $\mathrm{H}$ & 0.20261100 & -1.93035800 & -0.86433800 \\
\hline $\mathrm{H}$ & 2.79485400 & -1.41867600 & -2.10722700 \\
\hline $\mathrm{H}$ & 2.13724300 & 4.74595500 & 0.57116600 \\
\hline $\mathrm{H}$ & 2.50074200 & -1.53654400 & -0.33800700 \\
\hline $\mathrm{O}$ & 2.10455700 & 3.13805700 & -0.74044800 \\
\hline $\mathrm{O}$ & 2.39250000 & 0.37327700 & -1.13495100 \\
\hline $\mathrm{C}$ & -3.70935600 & -1.80338000 & -1.20363700 \\
\hline $\mathrm{C}$ & -5.21090300 & -1.86701700 & -0.87599200 \\
\hline $\mathrm{H}$ & -5.77187400 & -2.21625000 & -1.75709000 \\
\hline $\mathrm{H}$ & -5.42945600 & -2.55990200 & -0.04970700 \\
\hline $\mathrm{H}$ & -5.60957400 & -0.87686600 & -0.60575600 \\
\hline $\mathrm{C}$ & -3.20261600 & -3.20700700 & -1.57568600 \\
\hline $\mathrm{H}$ & -2.14212600 & -3.18122200 & -1.87291500 \\
\hline $\mathrm{H}$ & -3.29872100 & -3.91310500 & -0.73621900 \\
\hline $\mathrm{H}$ & -3.77925200 & -3.61620200 & -2.41987900 \\
\hline $\mathrm{C}$ & -3.50830000 & -0.86339100 & -2.40723000 \\
\hline $\mathrm{H}$ & -3.88152900 & -1.33668200 & -3.32854300 \\
\hline $\mathrm{H}$ & -4.04812200 & 0.08723300 & -2.28608500 \\
\hline $\mathrm{H}$ & -2.44704400 & -0.61644700 & -2.55278400 \\
\hline $\mathrm{C}$ & -3.16033000 & -2.00632600 & 1.34044400 \\
\hline $\mathrm{C}$ & -2.86851000 & -1.28567800 & 0.00991900 \\
\hline $\mathrm{C}$ & -3.16878600 & 0.15975200 & 0.37329000 \\
\hline $\mathrm{C}$ & -4.11007000 & 0.08619400 & 1.53301700 \\
\hline $\mathrm{O}$ & -4.07874800 & -1.16456800 & 2.05599900 \\
\hline $\mathrm{H}$ & -3.63391900 & -2.98868500 & 1.21825600 \\
\hline $\mathrm{H}$ & -1.81326600 & -1.42017900 & -0.25672600 \\
\hline $\mathrm{H}$ & -3.65739500 & 0.75062200 & -0.41241300 \\
\hline $\mathrm{O}$ & -4.84607800 & 0.93448600 & 1.97425600 \\
\hline
\end{tabular}




\begin{tabular}{|c|c|c|c|}
\hline $\mathrm{H}$ & -2.25426100 & -2.12782700 & 1.95053000 \\
\hline $\mathrm{C}$ & -0.42310300 & 4.68833300 & 1.21914500 \\
\hline $\mathrm{H}$ & -0.22992300 & 5.76059500 & 1.37689400 \\
\hline $\mathrm{H}$ & -1.50466400 & 4.51297500 & 1.28220800 \\
\hline $\mathrm{H}$ & 0.07610700 & 4.11568600 & 2.01510000 \\
\hline $\mathrm{C}$ & 0.31434200 & -1.51406700 & -2.98122700 \\
\hline $\mathrm{H}$ & 0.69915400 & -2.51414300 & -3.23232100 \\
\hline $\mathrm{H}$ & -0.76998000 & -1.51074200 & -3.14715000 \\
\hline $\mathrm{H}$ & 0.77177600 & -0.78112200 & -3.66378500 \\
\hline \multicolumn{4}{|c|}{ UB3LYP-D3(BJ)/def2-SVP-CPCM(THF) } \\
\hline \multicolumn{3}{|c|}{ Zero-point correction $=$} & 0.493840 (Hartree/Particle) \\
\hline \multicolumn{3}{|c|}{ Thermal correction to Energy= } & 0.525002 \\
\hline \multicolumn{3}{|c|}{ Thermal correction to Enthalpy= } & 0.525946 \\
\hline \multicolumn{3}{|c|}{ Thermal correction to Gibbs Free Energy= } & 0.429437 \\
\hline \multicolumn{3}{|c|}{ Sum of electronic and zero-point Energies $=$} & -5347.630303 \\
\hline \multicolumn{3}{|c|}{ Sum of electronic and thermal Energies $=$} & -5347.599141 \\
\hline \multicolumn{3}{|c|}{ Sum of electronic and thermal Enthalpies $=$} & -5347.598196 \\
\hline \multicolumn{3}{|c|}{ Sum of electronic and thermal Free Energies= } & -5347.694705 \\
\hline \multicolumn{4}{|c|}{$\mathrm{HF}=-5348.1241424$} \\
\hline \multicolumn{4}{|c|}{$\mathbf{F}^{, \text {s-lac }}$} \\
\hline $\mathrm{Ni}$ & -0.93175100 & 0.98925000 & 0.27779800 \\
\hline $\mathrm{C}$ & -1.12577200 & 1.20947400 & 2.16683300 \\
\hline $\mathrm{C}$ & -1.28008200 & 1.72078500 & 4.91282400 \\
\hline $\mathrm{C}$ & -0.33012800 & 0.48053800 & 3.05335800 \\
\hline $\mathrm{C}$ & -1.99993800 & 2.19109400 & 2.64220100 \\
\hline $\mathrm{C}$ & -2.07317100 & 2.44593500 & 4.01656300 \\
\hline $\mathrm{C}$ & -0.40883200 & 0.74043600 & 4.42888100 \\
\hline $\mathrm{H}$ & 0.34967500 & -0.29283000 & 2.69054300 \\
\hline $\mathrm{H}$ & -2.62548300 & 2.75226600 & 1.94513300 \\
\hline $\mathrm{H}$ & -2.75738100 & 3.21434300 & 4.38635600 \\
\hline $\mathrm{H}$ & 0.21627300 & 0.17019900 & 5.12132300 \\
\hline $\mathrm{H}$ & -1.34281500 & 1.91807800 & 5.98559000 \\
\hline $\mathrm{Br}$ & -1.94350400 & 2.85447600 & -1.09751200 \\
\hline $\mathrm{C}$ & 1.99970400 & 1.21294400 & 0.26769500 \\
\hline $\mathrm{C}$ & 2.91326200 & 3.24141200 & 0.36280700 \\
\hline $\mathrm{C}$ & 1.36337000 & 3.35285900 & 0.31249700 \\
\hline $\mathrm{N}$ & 0.94640500 & 1.93955500 & 0.27175600 \\
\hline $\mathrm{H}$ & 3.42091900 & 3.71276900 & -0.48782800 \\
\hline $\mathrm{H}$ & 1.02317400 & 3.81423000 & -0.62724500 \\
\hline $\mathrm{C}$ & 1.02447500 & -2.27396000 & -0.06324800 \\
\hline $\mathrm{C}$ & 2.51180000 & -2.38108900 & 0.36080600 \\
\hline $\mathrm{C}$ & 1.89217900 & -0.25641900 & 0.21151300 \\
\hline $\mathrm{N}$ & 0.75388500 & -0.82407100 & 0.07703000 \\
\hline
\end{tabular}




$\begin{array}{lrrr}\mathrm{H} & & & \\ \mathrm{H} & 3.37107000 & -2.82705300 & 0.62476800 \\ \mathrm{H} & 3.34050600 & 3.61380800 & 1.30444200 \\ \mathrm{H} & 2.64771100 & -2.73008000 & 1.39467600 \\ \mathrm{O} & 3.18341700 & 1.80532100 & 0.29866000 \\ \mathrm{O} & 2.99016700 & -1.00226100 & 0.30016400 \\ \mathrm{C} & -4.73517300 & -0.96837100 & 1.66833100 \\ \mathrm{C} & -5.32410700 & 0.44829200 & 1.78534100 \\ \mathrm{H} & -6.41711600 & 0.39927600 & 1.91290100 \\ \mathrm{H} & -4.90213100 & 0.97904500 & 2.65354600 \\ \mathrm{H} & -5.12269200 & 1.05669800 & 0.89100400 \\ \mathrm{C} & -5.12519700 & -1.75310000 & 2.93293100 \\ \mathrm{H} & -4.76813400 & -2.79404100 & 2.90389300 \\ \mathrm{H} & -4.70989000 & -1.27685500 & 3.83592300 \\ \mathrm{H} & -6.22081800 & -1.78611500 & 3.04164000 \\ \mathrm{C} & -5.31688100 & -1.66880300 & 0.42980300 \\ \mathrm{H} & -6.41274400 & -1.74377900 & 0.51410000 \\ \mathrm{H} & -5.09557500 & -1.11321100 & -0.49463000 \\ \mathrm{H} & -4.92183400 & -2.68981600 & 0.31330100 \\ \mathrm{C} & -2.43473800 & -2.19285400 & 1.45475900 \\ \mathrm{C} & -3.17695300 & -0.85699300 & 1.60697200 \\ \mathrm{C} & -2.66066800 & -0.10449200 & 0.38464600 \\ \mathrm{C} & -2.32541700 & -1.17026900 & -0.60076300 \\ \mathrm{O} & -2.18754900 & -2.35680400 & 0.04498400 \\ \mathrm{H} & -1.46348300 & -2.16620100 & 1.97342300 \\ \mathrm{H} & -2.87258200 & -0.38494200 & 2.54699700 \\ \mathrm{H} & -3.31746800 & 0.66138500 & -0.03346100 \\ \mathrm{O} & -2.14825600 & -1.08743100 & -1.79757800 \\ \mathrm{H} & -2.99296400 & -3.07187900 & 1.79917600 \\ \mathrm{C} & 0.74829800 & 4.10220900 & 1.48536400 \\ \mathrm{H} & 1.12385300 & 5.13686900 & 1.50240700 \\ \mathrm{H} & -0.34365000 & 4.12521700 & 1.38166400 \\ \mathrm{H} & 1.00116800 & 3.61520100 & 2.43912800 \\ \mathrm{H} & -96686700 & -2.73162500 & -1.49721700 \\ \mathrm{HB} & -3.81450700 & -1.57785100 \\ \mathrm{H} & -2.51907300 & -1.79269400 \\ \mathrm{H} & -2.21280600 & -2.19271800\end{array}$

UB3LYP-D3(BJ)/def2-SVP-CPCM(THF)

Zero-point correction=

Thermal correction to Energy=

0.494551 (Hartree/Particle)

0.526241

Thermal correction to Enthalpy=

0.527185

Thermal correction to Gibbs Free Energy=

Sum of electronic and zero-point Energies $=$

0.429690

$-5347.642565$

$-5347.610876$ 
Sum of electronic and thermal Enthalpies=

Sum of electronic and thermal Free Energies= $\mathrm{HF}=-5348.1371164$

\section{F'-TS-H $^{S \text {-lac }}$}

\begin{tabular}{|c|c|c|c|}
\hline $\mathrm{Ni}$ & -0.77053900 & 0.99055600 & 0.10588900 \\
\hline $\mathrm{C}$ & -1.33473500 & 0.98147000 & 1.95441000 \\
\hline $\mathrm{C}$ & -1.10383100 & 1.78655900 & 4.64248000 \\
\hline $\mathrm{C}$ & -0.52205000 & 0.25960400 & 2.84598900 \\
\hline $\mathrm{C}$ & -2.05778500 & 2.08789900 & 2.43016800 \\
\hline $\mathrm{C}$ & -1.93531400 & 2.48938200 & 3.76244700 \\
\hline $\mathrm{C}$ & -0.39789000 & 0.67181000 & 4.17644700 \\
\hline $\mathrm{H}$ & 0.03024500 & -0.61645100 & 2.50432700 \\
\hline $\mathrm{H}$ & -2.70444800 & 2.64202700 & 1.74805900 \\
\hline $\mathrm{H}$ & -2.49399100 & 3.36084600 & 4.11389200 \\
\hline $\mathrm{H}$ & 0.25499500 & 0.11308600 & 4.85227900 \\
\hline $\mathrm{H}$ & -1.01287800 & 2.10012700 & 5.68492300 \\
\hline $\mathrm{Br}$ & -1.95727100 & 2.77461600 & -1.18076500 \\
\hline $\mathrm{C}$ & 2.10480900 & 1.26666900 & 0.27953200 \\
\hline $\mathrm{C}$ & 2.99077700 & 3.29602400 & 0.50319300 \\
\hline $\mathrm{C}$ & 1.45000000 & 3.39662500 & 0.31135100 \\
\hline $\mathrm{N}$ & 1.05102000 & 1.98530800 & 0.18520400 \\
\hline $\mathrm{H}$ & 3.57033600 & 3.78321100 & -0.29097800 \\
\hline $\mathrm{H}$ & 1.19679000 & 3.89420100 & -0.63773200 \\
\hline $\mathrm{C}$ & 1.06561200 & -2.19394100 & -0.10182600 \\
\hline $\mathrm{C}$ & 2.55337300 & -2.34259400 & 0.31483500 \\
\hline $\mathrm{C}$ & 1.98488400 & -0.19926800 & 0.20309600 \\
\hline $\mathrm{N}$ & 0.83548800 & -0.73802300 & 0.03650500 \\
\hline $\mathrm{H}$ & 0.40009700 & -2.72345000 & 0.59389200 \\
\hline $\mathrm{H}$ & 3.15407300 & -2.92769300 & -0.39280600 \\
\hline $\mathrm{H}$ & 3.32653200 & 3.65936400 & 1.48459000 \\
\hline $\mathrm{H}$ & 2.68569400 & -2.73682500 & 1.33238500 \\
\hline $\mathrm{O}$ & 3.27780100 & 1.86243500 & 0.44545800 \\
\hline $\mathrm{O}$ & 3.06056200 & -0.97252800 & 0.30817900 \\
\hline $\mathrm{C}$ & -4.83448500 & -1.01943600 & 1.74994200 \\
\hline $\mathrm{C}$ & -5.41350200 & 0.39859200 & 1.89606500 \\
\hline $\mathrm{H}$ & -6.51294900 & 0.35765400 & 1.94502500 \\
\hline $\mathrm{H}$ & -5.04838800 & 0.88179200 & 2.81623600 \\
\hline $\mathrm{H}$ & -5.14629900 & 1.04607800 & 1.04776900 \\
\hline $\mathrm{C}$ & -5.34541800 & -1.86849400 & 2.92706800 \\
\hline $\mathrm{H}$ & -4.99317400 & -2.90994600 & 2.87330200 \\
\hline $\mathrm{H}$ & -5.01526500 & -1.44736600 & 3.89041300 \\
\hline $\mathrm{H}$ & -6.44638200 & -1.89497600 & 2.92923800 \\
\hline $\mathrm{C}$ & -5.31266100 & -1.63625500 & 0.42539300 \\
\hline
\end{tabular}

$-5347.609932$

$-5347.707427$ 


$\begin{array}{lrrr}\mathrm{H} & -6.41252100 & -1.69330800 & 0.40924800 \\ \mathrm{H} & -5.00260900 & -1.03335100 & -0.44195600 \\ \mathrm{H} & -4.92560100 & -2.65710700 & 0.28396500 \\ \mathrm{C} & -2.54483000 & -2.27859900 & 1.65498200 \\ \mathrm{C} & -3.27567600 & -0.93776200 & 1.83695400 \\ \mathrm{C} & -2.65003700 & -0.10835800 & 0.71432500 \\ \mathrm{C} & -2.27233500 & -1.12067500 & -0.31489700 \\ \mathrm{O} & -2.15842700 & -2.33989700 & 0.26831500 \\ \mathrm{H} & -1.63476100 & -2.33022800 & 2.27021100 \\ \mathrm{H} & -3.04633300 & -0.53315300 & 2.82995500 \\ \mathrm{H} & -3.25278600 & 0.70000400 & 0.29866000 \\ \mathrm{O} & -2.07589400 & -0.97828500 & -1.50491000 \\ \mathrm{H} & -3.15992700 & -3.16147000 & 1.86766900 \\ \mathrm{C} & 0.71159900 & 4.08750000 & 1.44943200 \\ \mathrm{H} & 1.06160100 & 5.12646400 & 1.54900400 \\ \mathrm{H} & -0.36581900 & 4.09373700 & 1.24026700 \\ \mathrm{H} & 0.88026500 & 3.56133000 & 2.40136200 \\ \mathrm{C} & 0.77641000 & -2.65401700 & -1.52817800 \\ \mathrm{H} & 0.93676400 & -3.74038900 & -1.60811000 \\ \mathrm{H} & -0.26128600 & -2.42183500 & -1.79975200 \\ \mathrm{H} & 1.45014700 & -2.14914700 & -2.23886400 \\ \mathrm{H} & \end{array}$

UB3LYP-D3(BJ)/def2-SVP-CPCM(THF)

Zero-point correction=

0.494413 (Hartree/Particle)

Thermal correction to Energy=

0.525435

Thermal correction to Enthalpy=

0.526379

Thermal correction to Gibbs Free Energy=

0.430536

Sum of electronic and zero-point Energies $=$

$-5347.633252$

$-5347.602230$

$-5347.601285$

Sum of electronic and thermal Enthalpies=

$-5347.697129$

$\mathrm{HF}=-5348.1276648$

$\begin{array}{lrrr}\mathbf{F}^{, R \text {-amide }} & & & \\ \mathrm{Ni} & -1.35427400 & 1.06602900 & -0.24325800 \\ \mathrm{C} & -1.44692500 & 1.19709000 & 1.66613800 \\ \mathrm{C} & -1.43939900 & 1.70235600 & 4.43020200 \\ \mathrm{C} & -0.39797500 & 0.73341400 & 2.46281000 \\ \mathrm{C} & -2.50024100 & 1.90411700 & 2.25678100 \\ \mathrm{C} & -2.49857000 & 2.15258600 & 3.63430000 \\ \mathrm{C} & -0.38839800 & 0.99446300 & 3.83942800 \\ \mathrm{H} & 0.41997300 & 0.15370500 & 2.02918600 \\ \mathrm{H} & -3.31911500 & 2.27722200 & 1.63687100 \\ \mathrm{H} & -3.32643900 & 2.70648200 & 4.08571500 \\ \mathrm{H} & 0.44151000 & 0.63216300 & 4.45235100\end{array}$


$\mathrm{H}$

C

$\mathrm{H}$

C

$\mathrm{H}$

$\mathrm{H}$

C

C

$\mathrm{H}$

$\mathrm{H}$

$\mathrm{H}$

C

$\mathrm{H}$

$\mathrm{H}$

$\mathrm{H}$

C

$\mathrm{H}$

$\mathrm{H}$

$\mathrm{H}$

C

$\mathrm{O}$

$\mathrm{Br}$

C

C

C

$\mathrm{N}$

$\mathrm{H}$

$\mathrm{H}$

C

C

C

$\mathrm{N}$

$\mathrm{H}$

$\mathrm{H}$

$\mathrm{H}$

$\mathrm{H}$

$\mathrm{O}$

$\mathrm{O}$

$\mathrm{N}$

$\mathrm{H}$

C

C

C

C
$-1.43558200$

$-2.74455800$

$-2.00720000$

$-2.73600500$

$-3.63208800$

$-2.42697800$

$-4.10594600$

$-5.16239100$

$-6.10164600$

$-5.39182000$

$-4.81715300$

$-4.61140300$

$-3.85930800$

$-4.84306300$

$-5.53186900$

$-3.86154300$

$-4.79141800$

$-3.49089900$

$-3.11441400$

$-2.49248200$

$-1.72104600$

$-2.90052400$

1.35078200

1.86225200

0.31799600

0.17816100

2.21122400

$-0.16829500$

0.82794900

2.30555700

1.46540300

0.39533200

0.20535800

2.99944900

2.27067200

2.45407500

2.40321500

2.63567600

$-3.22277800$

$-3.75984600$

$-3.22119600$

$-3.75570000$

$-1.81001400$

$-4.15792500$
1.89818900

$-0.85881700$

$-1.66851500$

$-0.33914400$

0.24117100

$-0.07141700$

$-1.40650400$

$-0.29380900$

$-0.68113400$

0.10715800

0.54849200

$-2.54641300$

$-3.34486900$

$-2.18926300$

$-2.98604400$

$-1.95020700$

$-2.35258000$

$-1.15716400$

$-2.76066800$

$-1.42760200$

$-2.37160200$

2.76261300

1.95133100

4.10417700

3.91079300

2.45181000

4.70919300

4.37275700

$-1.59503400$

$-1.55439300$

0.49395900

$-0.19311700$

$-2.23782700$

$-2.06842300$

4.50521900

$-1.90717300$

2.75398900

$-0.12656800$

$-1.32728000$

$-0.47646200$

$-2.26705900$

$-3.63218300$

$-2.41541700$

$-1.66515200$
5.50518500

$-1.50355000$

$-1.56618500$

$-0.07567200$

0.16800900

$-2.20925100$

$-2.02549400$

$-2.08896000$

$-2.51681400$

$-1.08997000$

$-2.70710100$

$-1.12701400$

$-1.03514600$

$-0.11251100$

$-1.54472400$

$-3.44261100$

$-3.87662100$

$-4.11238800$

$-3.43222000$

0.93794100

0.72588000

$-1.31317000$

$-0.40485900$

$-0.19837900$

$-0.15716200$

$-0.30563600$

$-1.04462500$

$-1.02980300$

$-0.94100800$

$-0.48428100$

$-0.55864300$

$-0.72636500$

$-0.30562600$

$-1.16023200$

0.73958000

0.54666600

$-0.36861800$

$-0.50199800$

2.07655800

2.19108300

3.21306200

2.75047900

3.80305300

4.26773200 


\begin{tabular}{|c|c|c|c|}
\hline $\mathrm{H}$ & -4.77747500 & -3.53179700 & 2.35244400 \\
\hline $\mathrm{H}$ & -3.11252800 & -4.04463700 & 1.96188300 \\
\hline $\mathrm{H}$ & -3.77934600 & -4.33915800 & 3.59436700 \\
\hline $\mathrm{H}$ & -1.43837400 & -1.44319000 & 4.15449900 \\
\hline $\mathrm{H}$ & -1.83111000 & -3.11376800 & 4.65428100 \\
\hline $\mathrm{H}$ & -1.11695800 & -2.79955800 & 3.04400100 \\
\hline $\mathrm{H}$ & -4.20973300 & -2.32100900 & 5.14895700 \\
\hline $\mathrm{H}$ & -3.79298300 & -0.67791500 & 4.59336400 \\
\hline $\mathrm{H}$ & -5.17810400 & -1.54737600 & 3.86804700 \\
\hline $\mathrm{C}$ & -0.34436400 & 4.42716800 & 1.11227000 \\
\hline $\mathrm{H}$ & -0.17614200 & 5.51144900 & 1.20269800 \\
\hline $\mathrm{H}$ & -1.42395600 & 4.23717500 & 1.07118700 \\
\hline $\mathrm{H}$ & 0.06615000 & 3.92582600 & 2.00147800 \\
\hline $\mathrm{C}$ & 0.67412300 & -1.98128500 & -2.41143700 \\
\hline $\mathrm{H}$ & 0.97804400 & -3.02953800 & -2.55471100 \\
\hline $\mathrm{H}$ & -0.36761000 & -1.87501600 & -2.73918500 \\
\hline $\mathrm{H}$ & 1.30547600 & -1.34488600 & -3.05171200 \\
\hline \multicolumn{4}{|c|}{ UB3LYP-D3(BJ)/def2-SVP-CPCM(THF) } \\
\hline \multicolumn{3}{|c|}{ Zero-point correction $=$} & 0.610541 (Hartree/Particle) \\
\hline \multicolumn{3}{|c|}{ Thermal correction to Energy= } & 0.648224 \\
\hline \multicolumn{3}{|c|}{ Thermal correction to Enthalpy= } & 0.649169 \\
\hline \multicolumn{3}{|c|}{ Thermal correction to Gibbs Free Energy= } & 0.539207 \\
\hline \multicolumn{3}{|c|}{ Sum of electronic and zero-point Energies $=$} & -5446.775954 \\
\hline \multicolumn{3}{|c|}{ Sum of electronic and thermal Energies $=$} & -5446.738271 \\
\hline \multicolumn{3}{|c|}{ Sum of electronic and thermal Enthalpies $=$} & -5446.737327 \\
\hline \multicolumn{3}{|c|}{ Sum of electronic and thermal Free Energies $=$} & -5446.847288 \\
\hline \multicolumn{4}{|c|}{$\mathrm{HF}=-5447.3864951$} \\
\hline \multicolumn{4}{|c|}{ F'$^{\prime}$-TS-H ${ }^{R \text {-amide }}$} \\
\hline $\mathrm{Ni}$ & -1.30266500 & 1.18714200 & -0.48527900 \\
\hline $\mathrm{C}$ & -1.68921800 & 0.95386600 & 1.39586600 \\
\hline $\mathrm{C}$ & -1.25676400 & 1.82735900 & 4.04563400 \\
\hline $\mathrm{C}$ & -0.62499400 & 0.43736100 & 2.15798300 \\
\hline $\mathrm{C}$ & -2.56609600 & 1.87260400 & 2.00220200 \\
\hline $\mathrm{C}$ & -2.34694100 & 2.30787900 & 3.31105500 \\
\hline $\mathrm{C}$ & -0.39894900 & 0.88904000 & 3.45941400 \\
\hline $\mathrm{H}$ & 0.01885200 & -0.33485500 & 1.73875800 \\
\hline $\mathrm{H}$ & -3.40324600 & 2.27153200 & 1.42629200 \\
\hline $\mathrm{H}$ & -3.03031100 & 3.03580500 & 3.75654500 \\
\hline $\mathrm{H}$ & 0.44683400 & 0.49021200 & 4.02570900 \\
\hline $\mathrm{H}$ & -1.08509800 & 2.16942900 & 5.06881700 \\
\hline $\mathrm{C}$ & -2.66104200 & -0.78092000 & -1.24519100 \\
\hline $\mathrm{H}$ & -1.90699900 & -1.57310000 & -1.29051200 \\
\hline $\mathrm{C}$ & -2.73579100 & -0.29013900 & 0.21098700 \\
\hline
\end{tabular}




\begin{tabular}{|c|c|c|c|}
\hline $\mathrm{H}$ & -3.68037900 & 0.22998800 & 0.38636200 \\
\hline $\mathrm{H}$ & -2.34639900 & 0.00859800 & -1.95296100 \\
\hline $\mathrm{C}$ & -3.99330000 & -1.34719600 & -1.82284800 \\
\hline $\mathrm{C}$ & -5.09232400 & -0.27489600 & -1.85771400 \\
\hline $\mathrm{H}$ & -5.99139000 & -0.66906600 & -2.35866800 \\
\hline $\mathrm{H}$ & -5.39170300 & 0.03717000 & -0.84506300 \\
\hline $\mathrm{H}$ & -4.75460100 & 0.62581200 & -2.39086900 \\
\hline $\mathrm{C}$ & -4.48083100 & -2.55824600 & -1.01146800 \\
\hline $\mathrm{H}$ & -3.69990000 & -3.33019900 & -0.93644700 \\
\hline $\mathrm{H}$ & -4.76960200 & -2.27423300 & 0.01078200 \\
\hline $\mathrm{H}$ & -5.36452600 & -3.00759400 & -1.49290100 \\
\hline $\mathrm{C}$ & -3.68782900 & -1.79823000 & -3.26152000 \\
\hline $\mathrm{H}$ & -4.58910100 & -2.20945600 & -3.74393400 \\
\hline $\mathrm{H}$ & -3.33084300 & -0.95425700 & -3.87376400 \\
\hline $\mathrm{H}$ & -2.91082700 & -2.57988100 & -3.27552800 \\
\hline $\mathrm{C}$ & -2.55648300 & -1.45471700 & 1.16355300 \\
\hline $\mathrm{O}$ & -1.71425000 & -2.33381200 & 0.96350900 \\
\hline $\mathrm{Br}$ & -2.91823500 & 2.85487400 & -1.48097100 \\
\hline $\mathrm{C}$ & 1.42515600 & 2.01274900 & -0.44121300 \\
\hline $\mathrm{C}$ & 1.98452000 & 4.13327500 & -0.07590700 \\
\hline $\mathrm{C}$ & 0.43842600 & 3.99134800 & -0.19572600 \\
\hline $\mathrm{N}$ & 0.26481500 & 2.55126700 & -0.44320300 \\
\hline $\mathrm{H}$ & 2.43317800 & 4.76331500 & -0.85466000 \\
\hline $\mathrm{H}$ & 0.05683300 & 4.52766500 & -1.07846900 \\
\hline $\mathrm{C}$ & 0.80368600 & -1.50480400 & -1.05088100 \\
\hline $\mathrm{C}$ & 2.28958100 & -1.51558300 & -0.61054900 \\
\hline $\mathrm{C}$ & 1.50448200 & 0.55910200 & -0.62973800 \\
\hline $\mathrm{N}$ & 0.41792100 & -0.09317000 & -0.82765400 \\
\hline $\mathrm{H}$ & 0.17835200 & -2.13229700 & -0.40015400 \\
\hline $\mathrm{H}$ & 2.96111500 & -2.01622200 & -1.31899800 \\
\hline $\mathrm{H}$ & 2.30905300 & 4.48098800 & 0.91489700 \\
\hline $\mathrm{H}$ & 2.44174000 & -1.92232600 & 0.39955000 \\
\hline $\mathrm{O}$ & 2.49707200 & 2.77492000 & -0.25439200 \\
\hline $\mathrm{O}$ & 2.65572800 & -0.09978100 & -0.56771700 \\
\hline $\mathrm{N}$ & -3.39160600 & -1.45295100 & 2.22977800 \\
\hline $\mathrm{H}$ & -3.97838300 & -0.63401800 & 2.33924500 \\
\hline $\mathrm{C}$ & -3.38299200 & -2.41514700 & 3.34922200 \\
\hline $\mathrm{C}$ & -3.69216400 & -3.82585700 & 2.82407500 \\
\hline $\mathrm{C}$ & -2.02325400 & -2.38955600 & 4.06501300 \\
\hline $\mathrm{C}$ & -4.48623400 & -1.96378600 & 4.31344500 \\
\hline $\mathrm{H}$ & -4.67358400 & -3.84500700 & 2.32531900 \\
\hline $\mathrm{H}$ & -2.92744400 & -4.14168600 & 2.10291900 \\
\hline $\mathrm{H}$ & -3.71264000 & -4.54400200 & 3.65832300 \\
\hline $\mathrm{H}$ & -1.81203700 & -1.38273600 & 4.45383800 \\
\hline
\end{tabular}




\begin{tabular}{|c|c|c|c|}
\hline $\mathrm{H}$ & -2.02596600 & -3.09956900 & 4.90661200 \\
\hline $\mathrm{H}$ & -1.22010000 & -2.66657300 & 3.36981200 \\
\hline $\mathrm{H}$ & -4.53756200 & -2.64405100 & 5.17582800 \\
\hline $\mathrm{H}$ & -4.28564100 & -0.94805500 & 4.69109700 \\
\hline $\mathrm{H}$ & -5.46918200 & -1.96462400 & 3.81589200 \\
\hline $\mathrm{C}$ & -0.33664500 & 4.44173500 & 1.03526900 \\
\hline $\mathrm{H}$ & -0.15849000 & 5.51214100 & 1.22176900 \\
\hline $\mathrm{H}$ & -1.41010900 & 4.28059800 & 0.87306900 \\
\hline $\mathrm{H}$ & -0.02928500 & 3.86809200 & 1.92286400 \\
\hline $\mathrm{C}$ & 0.61041700 & -1.88985300 & -2.51663900 \\
\hline $\mathrm{H}$ & 0.88020900 & -2.94658100 & -2.66562000 \\
\hline $\mathrm{H}$ & -0.43405100 & -1.75264300 & -2.82545800 \\
\hline $\mathrm{H}$ & 1.24740000 & -1.27146600 & -3.16854500 \\
\hline \multicolumn{4}{|c|}{ UB3LYP-D3(BJ)/def2-SVP-CPCM(THF) } \\
\hline \multicolumn{3}{|c|}{ Zero-point correction $=$} & 0.610028 (Hartree/Particle) \\
\hline \multicolumn{3}{|c|}{ Thermal correction to Energy= } & 0.647107 \\
\hline \multicolumn{3}{|c|}{ Thermal correction to Enthalpy= } & 0.648051 \\
\hline \multicolumn{3}{|c|}{ Thermal correction to Gibbs Free Energy= } & 0.539816 \\
\hline \multicolumn{3}{|c|}{ Sum of electronic and zero-point Energies $=$} & -5446.761502 \\
\hline \multicolumn{3}{|c|}{ Sum of electronic and thermal Energies $=$} & -5446.724423 \\
\hline \multicolumn{3}{|c|}{ Sum of electronic and thermal Enthalpies $=$} & -5446.723479 \\
\hline \multicolumn{3}{|c|}{ Sum of electronic and thermal Free Energies $=$} & -5446.831714 \\
\hline \multicolumn{4}{|c|}{$\mathrm{HF}=-5447.3715297$} \\
\hline \multicolumn{4}{|c|}{$\mathbf{F}^{, S \text {-amide }}$} \\
\hline $\mathrm{Ni}$ & -1.18521400 & 0.93543900 & -0.04971200 \\
\hline $\mathrm{C}$ & -1.39992600 & 1.31799100 & 1.82461100 \\
\hline $\mathrm{C}$ & -1.48687400 & 2.08963000 & 4.52123700 \\
\hline $\mathrm{C}$ & -0.47342800 & 0.79866900 & 2.73326400 \\
\hline $\mathrm{C}$ & -2.37644500 & 2.21467900 & 2.26527700 \\
\hline $\mathrm{C}$ & -2.41800700 & 2.60002300 & 3.61118500 \\
\hline $\mathrm{C}$ & -0.51403300 & 1.18763800 & 4.07877900 \\
\hline $\mathrm{H}$ & 0.28688900 & 0.08429100 & 2.40892800 \\
\hline $\mathrm{H}$ & -3.10021800 & 2.62468700 & 1.55803500 \\
\hline $\mathrm{H}$ & -3.18603200 & 3.30194200 & 3.94768900 \\
\hline $\mathrm{H}$ & 0.21976400 & 0.78178400 & 4.78059000 \\
\hline $\mathrm{H}$ & -1.52125000 & 2.39006500 & 5.57129900 \\
\hline $\mathrm{Br}$ & -2.35895400 & 2.88799100 & -1.32081300 \\
\hline $\mathrm{C}$ & -2.48056100 & -1.48572500 & 1.12801000 \\
\hline $\mathrm{H}$ & -1.74010600 & -1.23746500 & 1.89841100 \\
\hline $\mathrm{C}$ & -2.76252500 & -0.28727500 & 0.23236000 \\
\hline $\mathrm{H}$ & -3.54017000 & 0.36796000 & 0.63806900 \\
\hline $\mathrm{H}$ & -2.03946500 & -2.27443200 & 0.50246000 \\
\hline $\mathrm{C}$ & -3.71691600 & -2.07280900 & 1.86178600 \\
\hline
\end{tabular}




\begin{tabular}{|c|c|c|c|}
\hline $\mathrm{C}$ & -3.25512400 & -3.33787200 & 2.60272700 \\
\hline $\mathrm{H}$ & -4.08542900 & -3.79290000 & 3.16733600 \\
\hline $\mathrm{H}$ & -2.87227300 & -4.09199600 & 1.89574200 \\
\hline $\mathrm{H}$ & -2.44836900 & -3.10477700 & 3.31709100 \\
\hline $\mathrm{C}$ & -4.82874200 & -2.44661200 & 0.86837000 \\
\hline $\mathrm{H}$ & -5.21758800 & -1.56165800 & 0.34187000 \\
\hline $\mathrm{H}$ & -4.45810400 & -3.14981700 & 0.10686900 \\
\hline $\mathrm{H}$ & -5.67428500 & -2.92058000 & 1.39339800 \\
\hline $\mathrm{C}$ & -4.25506000 & -1.06177200 & 2.88748900 \\
\hline $\mathrm{H}$ & -5.09858300 & -1.49152300 & 3.45226500 \\
\hline $\mathrm{H}$ & -3.47169600 & -0.77325600 & 3.60651500 \\
\hline $\mathrm{H}$ & -4.61409100 & -0.14059900 & 2.40375000 \\
\hline $\mathrm{C}$ & -3.12763600 & -0.71295700 & -1.17998100 \\
\hline $\mathrm{O}$ & -2.66632900 & -1.73664900 & -1.69633200 \\
\hline $\mathrm{C}$ & 1.72369100 & 1.35204300 & -0.19633300 \\
\hline $\mathrm{C}$ & 2.53894500 & 3.42069400 & -0.28942900 \\
\hline $\mathrm{C}$ & 0.98643900 & 3.45613800 & -0.19972100 \\
\hline $\mathrm{N}$ & 0.63589900 & 2.02463300 & -0.18910200 \\
\hline $\mathrm{H}$ & 2.93675600 & 3.82269000 & -1.23021500 \\
\hline $\mathrm{H}$ & 0.54060400 & 3.89756200 & -1.10322000 \\
\hline $\mathrm{C}$ & 0.83565300 & -2.17371300 & -0.11160200 \\
\hline $\mathrm{C}$ & 2.37339700 & -2.21430800 & 0.11937100 \\
\hline $\mathrm{C}$ & 1.67416700 & -0.11996200 & -0.13887700 \\
\hline $\mathrm{N}$ & 0.54820900 & -0.72538800 & -0.16604800 \\
\hline $\mathrm{H}$ & 0.29848000 & -2.59397300 & 0.75146300 \\
\hline $\mathrm{H}$ & 2.91225300 & -2.80868000 & -0.63010800 \\
\hline $\mathrm{H}$ & 3.03437400 & 3.90742500 & 0.56153100 \\
\hline $\mathrm{H}$ & 2.65990500 & -2.54031600 & 1.12857500 \\
\hline $\mathrm{O}$ & 2.87918900 & 1.99847000 & -0.24072700 \\
\hline $\mathrm{O}$ & 2.79564600 & -0.82339100 & -0.02721400 \\
\hline $\mathrm{N}$ & -4.03357800 & 0.08269700 & -1.80189100 \\
\hline $\mathrm{H}$ & -4.12868800 & 1.01037100 & -1.39516700 \\
\hline $\mathrm{C}$ & -4.42989300 & -0.01943200 & -3.22035400 \\
\hline $\mathrm{C}$ & -3.19991800 & 0.13361000 & -4.13244200 \\
\hline $\mathrm{C}$ & -5.13006800 & -1.36319900 & -3.47136100 \\
\hline $\mathrm{C}$ & -5.41107500 & 1.13201200 & -3.47407900 \\
\hline $\mathrm{H}$ & -2.70403800 & 1.09629800 & -3.93762800 \\
\hline $\mathrm{H}$ & -2.48149900 & -0.67560000 & -3.94466700 \\
\hline $\mathrm{H}$ & -3.50212500 & 0.09784100 & -5.19093000 \\
\hline $\mathrm{H}$ & -6.01097800 & -1.46470300 & -2.81816400 \\
\hline $\mathrm{H}$ & -5.46630300 & -1.42279500 & -4.51826300 \\
\hline $\mathrm{H}$ & -4.44683400 & -2.19724800 & -3.26814500 \\
\hline $\mathrm{H}$ & -5.75014500 & 1.11885500 & -4.52037900 \\
\hline $\mathrm{H}$ & -6.29499200 & 1.04292500 & -2.82274400 \\
\hline
\end{tabular}




$\begin{array}{lrrr}\mathrm{H} & -4.92991700 & 2.10306900 & -3.27806600 \\ \mathrm{C} & 0.44281300 & 4.17571100 & 1.02783400 \\ \mathrm{H} & 0.75409300 & 5.23156400 & 1.00792400 \\ \mathrm{H} & -0.65330400 & 4.12826100 & 1.03140800 \\ \mathrm{H} & 0.81661900 & 3.71060400 & 1.95268700 \\ \mathrm{C} & 0.37713600 & -2.87123000 & -1.38718500 \\ \mathrm{H} & 0.56717900 & -3.95328600 & -1.31130400 \\ \mathrm{H} & -0.69601100 & -2.69653400 & -1.54447200 \\ \mathrm{H} & 0.93123400 & -2.48136300 & -2.25636700\end{array}$

UB3LYP-D3(BJ)/def2-SVP-CPCM(THF)

Zero-point correction=

Thermal correction to Energy=

Thermal correction to Enthalpy=

Thermal correction to Gibbs Free Energy=

Sum of electronic and zero-point Energies=

Sum of electronic and thermal Energies=

Sum of electronic and thermal Enthalpies=

Sum of electronic and thermal Free Energies=

$\mathrm{HF}=-5447.3824072$

F'-TS-H ${ }^{S \text {-amide }}$

$\mathrm{Ni}$

$\mathrm{C}$

C

$\mathrm{C}$

$\mathrm{C}$

$\mathrm{C}$

$\mathrm{C}$

$\mathrm{H}$

$\mathrm{H}$

$\mathrm{H}$

$\mathrm{H}$

$\mathrm{H}$

$\mathrm{Br}$

$\mathrm{C}$

$\mathrm{H}$

C

$\mathrm{H}$

$\mathrm{H}$

C

C

$\mathrm{H}$

$\mathrm{H}$

$\mathrm{H}$

$\begin{array}{rrr}-0.89698600 & 0.90882500 & -0.28613000 \\ -1.81341300 & 1.27561500 & 1.37786100 \\ -1.92849500 & 2.84976800 & 3.71766100 \\ -1.06321100 & 0.92810900 & 2.51229600 \\ -2.65158300 & 2.40585300 & 1.44325600 \\ -2.70128300 & 3.18632200 & 2.59766700 \\ -1.10834400 & 1.71971000 & 3.66618900 \\ -0.41007400 & 0.05380400 & 2.49389000 \\ -3.24583900 & 2.68446200 & 0.57110900 \\ -3.34995800 & 4.06593000 & 2.62357700 \\ -0.49403100 & 1.44590900 & 4.52810000 \\ -1.97142700 & 3.46082400 & 4.62216000 \\ -1.58111200 & 2.64805800 & -1.97606300 \\ -2.29592700 & -1.56720400 & 1.03777200 \\ -1.52302300 & -1.41072900 & 1.80005900 \\ -2.69484100 & -0.25531200 & 0.37933100 \\ -3.59329500 & 0.15191100 & 0.84203500 \\ -1.85303400 & -2.20642700 & 0.26452000 \\ -3.45762400 & -2.34054800 & 1.71253700 \\ -2.88553600 & -3.67257200 & 2.22390000 \\ -3.66136800 & -4.26867700 & 2.73119900 \\ -2.48497800 & -4.27471700 & 1.39212000 \\ -2.06659600 & -3.50195600 & 2.94189800\end{array}$

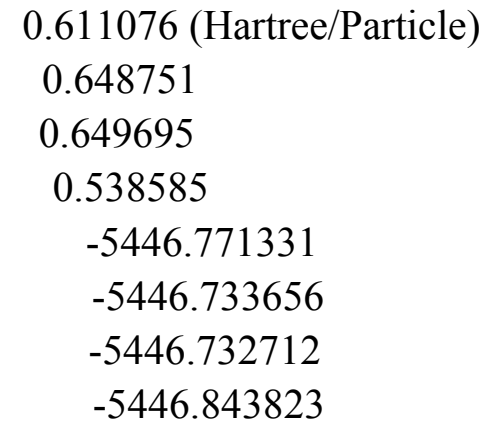




\begin{tabular}{|c|c|c|c|}
\hline $\mathrm{C}$ & -4.58232100 & -2.62948700 & 0.70521700 \\
\hline $\mathrm{H}$ & -5.04816200 & -1.70436300 & 0.33380700 \\
\hline $\mathrm{H}$ & -4.19837800 & -3.18065100 & -0.16735600 \\
\hline $\mathrm{H}$ & -5.37501400 & -3.23627700 & 1.17215900 \\
\hline $\mathrm{C}$ & -4.01372300 & -1.54390100 & 2.90404200 \\
\hline $\mathrm{H}$ & -4.81145200 & -2.10936600 & 3.41225000 \\
\hline $\mathrm{H}$ & -3.22249900 & -1.33170400 & 3.64086900 \\
\hline $\mathrm{H}$ & -4.44341900 & -0.57942000 & 2.59048500 \\
\hline $\mathrm{C}$ & -3.00966500 & -0.44970300 & -1.10178600 \\
\hline $\mathrm{O}$ & -2.40382800 & -1.26675700 & -1.80358700 \\
\hline $\mathrm{C}$ & 1.97390400 & 1.16826400 & 0.18848400 \\
\hline $\mathrm{C}$ & 2.88676700 & 3.18827500 & 0.37421700 \\
\hline $\mathrm{C}$ & 1.37585500 & 3.30429400 & 0.02146800 \\
\hline $\mathrm{N}$ & 0.95086100 & 1.89679700 & -0.04190200 \\
\hline $\mathrm{H}$ & 3.55464600 & 3.62425400 & -0.37949900 \\
\hline $\mathrm{H}$ & 1.22880300 & 3.73007100 & -0.98316800 \\
\hline $\mathrm{C}$ & 0.86869000 & -2.27931600 & -0.07421600 \\
\hline $\mathrm{C}$ & 2.36446000 & -2.44654400 & 0.31098800 \\
\hline $\mathrm{C}$ & 1.82264000 & -0.29491500 & 0.17878500 \\
\hline $\mathrm{N}$ & 0.66643900 & -0.81555200 & -0.00023700 \\
\hline $\mathrm{H}$ & 0.22317300 & -2.75939000 & 0.67391700 \\
\hline $\mathrm{H}$ & 2.94647000 & -3.01603900 & -0.42553500 \\
\hline $\mathrm{H}$ & 3.12850700 & 3.59847600 & 1.36520600 \\
\hline $\mathrm{H}$ & 2.51469900 & -2.87291500 & 1.31247200 \\
\hline $\mathrm{O}$ & 3.14651500 & 1.74981000 & 0.41957400 \\
\hline $\mathrm{O}$ & 2.88246000 & -1.08266300 & 0.33177600 \\
\hline $\mathrm{N}$ & -4.07372500 & 0.25794100 & -1.54701700 \\
\hline $\mathrm{H}$ & -4.48759800 & 0.91547500 & -0.89846000 \\
\hline $\mathrm{C}$ & -4.71654300 & 0.15016100 & -2.87252500 \\
\hline $\mathrm{C}$ & -3.70797300 & 0.35061500 & -4.01347800 \\
\hline $\mathrm{C}$ & -5.38786300 & -1.22862500 & -2.99146400 \\
\hline $\mathrm{C}$ & -5.77297500 & 1.26063100 & -2.92262900 \\
\hline $\mathrm{H}$ & -3.19819000 & 1.31766400 & -3.90777800 \\
\hline $\mathrm{H}$ & -2.94806400 & -0.44010000 & -3.99913000 \\
\hline $\mathrm{H}$ & -4.23772300 & 0.32087600 & -4.97855900 \\
\hline $\mathrm{H}$ & -6.14365200 & -1.36345800 & -2.20199200 \\
\hline $\mathrm{H}$ & -5.88400400 & -1.33106900 & -3.96919900 \\
\hline $\mathrm{H}$ & -4.63655300 & -2.02476600 & -2.89541100 \\
\hline $\mathrm{H}$ & -6.31535000 & 1.22651600 & -3.87853800 \\
\hline $\mathrm{H}$ & -6.50830900 & 1.14523400 & -2.10945300 \\
\hline $\mathrm{H}$ & -5.29766800 & 2.24962800 & -2.82885000 \\
\hline $\mathrm{C}$ & 0.54800500 & 4.09694700 & 1.02297800 \\
\hline $\mathrm{H}$ & 0.90882500 & 5.13594400 & 1.07237300 \\
\hline $\mathrm{H}$ & -0.50418500 & 4.10000300 & 0.71204500 \\
\hline
\end{tabular}




$\begin{array}{rrrr}\mathrm{H} & 0.60979800 & 3.64895500 & 2.02618500 \\ \mathrm{C} & 0.51311900 & -2.79687500 & -1.46393300 \\ \mathrm{H} & 0.61610700 & -3.89270300 & -1.49748400 \\ \mathrm{H} & -0.51948700 & -2.51486700 & -1.71102500 \\ \mathrm{H} & 1.18669100 & -2.35989200 & -2.21867200\end{array}$

UB3LYP-D3(BJ)/def2-SVP-CPCM(THF)

Zero-point correction=

0.610694 (Hartree/Particle)

Thermal correction to Energy=

0.647891

Thermal correction to Enthalpy=

0.648835

Thermal correction to Gibbs Free Energy=

0.539282

Sum of electronic and zero-point Energies $=$

$-5446.757133$

$-5446.719936$

$-5446.718991$

Sum of electronic and thermal Enthalpies=

Sum of electronic and thermal Free Energies=

$-5446.828545$

$\mathrm{HF}=-5447.3678264$

\section{Scheme S11}

\section{Styrene}

$\begin{array}{lrrr}\mathrm{C} & -4.38102900 & -3.76073900 & -0.90984900 \\ \mathrm{H} & -3.49745700 & -3.63618700 & -1.54203700 \\ \mathrm{C} & -4.33613000 & -4.37463900 & 0.28219400 \\ \mathrm{H} & -5.26602100 & -4.45840500 & 0.85649000 \\ \mathrm{H} & -5.32021200 & -3.35704700 & -1.29571800 \\ \mathrm{C} & -3.15779700 & -4.96773900 & 0.93771400 \\ \mathrm{C} & -3.30956400 & -5.56196800 & 2.20475500 \\ \mathrm{C} & -1.87455400 & -4.96947000 & 0.35433300 \\ \mathrm{C} & -2.22334300 & -6.13698300 & 2.86869600 \\ \mathrm{H} & -4.29764200 & -5.57012500 & 2.67266700 \\ \mathrm{C} & -0.78980500 & -5.54300200 & 1.01552000 \\ \mathrm{H} & -1.72253100 & -4.51773600 & -0.62798500 \\ \mathrm{C} & -0.95777500 & -6.12993100 & 2.27648100 \\ \mathrm{H} & -2.36659200 & -6.59189000 & 3.85181900 \\ \mathrm{H} & 0.19647400 & -5.53363600 & 0.54505800 \\ \mathrm{H} & -0.10533200 & -6.57856300 & 2.79192300\end{array}$

UB3LYP-D3(BJ)/def2-SVP-CPCM(THF)

Zero-point correction=

0.133407 (Hartree/Particle)

Thermal correction to Energy=

0.140095

Thermal correction to Enthalpy=

0.141039

Thermal correction to Gibbs Free Energy=

0.102310

Sum of electronic and zero-point Energies=

$-309.325631$

Sum of electronic and thermal Energies=

$-309.318943$

Sum of electronic and thermal Enthalpies=

$-309.317999$

Sum of electronic and thermal Free Energies=

$-309.356729$

$\mathrm{HF}=-309.4590384$ 
TS-Giese Addition ${ }^{\text {sty }}$

$\begin{array}{lrrr}\mathrm{C} & -2.21402700 & -1.39806100 & 0.87653700 \\ \mathrm{H} & -1.79611000 & -1.22371300 & 1.86890500 \\ \mathrm{C} & -2.66357400 & -0.35345200 & 0.13036100 \\ \mathrm{H} & -2.73148100 & 0.62998900 & 0.60813100 \\ \mathrm{H} & -2.03052100 & -2.38253900 & 0.44230100 \\ \mathrm{C} & -4.12243600 & -2.42245300 & 2.02664600 \\ \mathrm{C} & -3.39227400 & -3.50097800 & 2.76463500 \\ \mathrm{H} & -4.09347300 & -4.10719400 & 3.37723600 \\ \mathrm{H} & -2.88739200 & -4.19923100 & 2.07711700 \\ \mathrm{H} & -2.64082600 & -3.08872700 & 3.45770100 \\ \mathrm{C} & -4.95639000 & -2.82770600 & 0.85186000 \\ \mathrm{H} & -5.19893500 & -1.96472300 & 0.21179400 \\ \mathrm{H} & -4.45571000 & -3.58827400 & 0.23201300 \\ \mathrm{H} & -5.92132000 & -3.26530800 & 1.18606000 \\ \mathrm{C} & -4.59976600 & -1.24297500 & 2.81489000 \\ \mathrm{H} & -5.51166600 & -1.49772900 & 3.39534800 \\ \mathrm{H} & -3.84377100 & -0.89702400 & 3.53823500 \\ \mathrm{H} & -4.86281800 & -0.39974000 & 2.15581800 \\ \mathrm{C} & -3.14047500 & -0.40484700 & -1.24942700 \\ \mathrm{C} & -3.64879900 & 0.76749800 & -1.85071000 \\ \mathrm{C} & -3.12002200 & -1.58394400 & -2.02937900 \\ \mathrm{C} & -4.11830500 & 0.76502300 & -3.16473600 \\ \mathrm{H} & -3.67418700 & 1.69194700 & -1.26722100 \\ \mathrm{C} & -3.58880900 & -1.58570300 & -3.34113300 \\ \mathrm{H} & -2.73402800 & -2.51039800 & -1.60043900 \\ \mathrm{C} & -4.09257500 & -0.41250200 & -3.91929600 \\ \mathrm{H} & -4.50751900 & 1.68764100 & -3.60283000 \\ \mathrm{H} & -3.56269400 & -2.51145400 & -3.92172400 \\ \mathrm{H} & -4.46014000 & -0.41828400 & -4.94805500\end{array}$

UB3LYP-D3(BJ)/def2-SVP-CPCM(THF)

Zero-point correction=

Thermal correction to Energy=

Thermal correction to Enthalpy=

Thermal correction to Gibbs Free Energy=

Sum of electronic and zero-point Energies=

Sum of electronic and thermal Energies=

Sum of electronic and thermal Enthalpies=

Sum of electronic and thermal Free Energies= $\mathrm{HF}=-467.162415$

\section{Int-Giese Addition ${ }^{\text {sty }}$}

C

$-2.47691900-1.50670300$
0.250915 (Hartree/Particle)

0.264257

0.265201

0.209273

$-466.911500$

$-466.898158$

$-466.897214$

$-466.953142$ 


$\begin{array}{lrrr}\mathrm{H} & -1.67085900 & -1.18085700 & 1.52989800 \\ \mathrm{C} & -2.90454600 & -0.33920500 & 0.02361800 \\ \mathrm{H} & -3.01019700 & 0.62172800 & 0.53686500 \\ \mathrm{H} & -2.04654600 & -2.29817400 & 0.21398300 \\ \mathrm{C} & -3.59049300 & -2.15414000 & 1.73373100 \\ \mathrm{C} & -2.94876300 & -3.28540900 & 2.55062500 \\ \mathrm{H} & -3.69814100 & -3.78414800 & 3.18649400 \\ \mathrm{H} & -2.50402100 & -4.04821300 & 1.89085400 \\ \mathrm{H} & -2.15150300 & -2.89935600 & 3.20651500 \\ \mathrm{C} & -4.70385400 & -2.73108700 & 0.84695900 \\ \mathrm{H} & -5.17074800 & -1.94831600 & 0.22965500 \\ \mathrm{H} & -4.31197000 & -3.50643100 & 0.16899100 \\ \mathrm{H} & -5.49139400 & -3.19182800 & 1.46516900 \\ \mathrm{C} & -4.18315900 & -1.10697000 & 2.68915300 \\ \mathrm{H} & -4.92283600 & -1.57086100 & 3.36151700 \\ \mathrm{H} & -3.39857900 & -0.64966900 & 3.31432900 \\ \mathrm{H} & -4.69127300 & -0.30050200 & 2.13800100 \\ \mathrm{C} & -3.21979400 & -0.35243800 & -1.35766300 \\ \mathrm{C} & -3.63360500 & 0.85411500 & -2.00335100 \\ \mathrm{C} & -3.15108900 & -1.52745300 & -2.16724800 \\ \mathrm{C} & -3.95478600 & 0.88076200 & -3.35336300 \\ \mathrm{H} & -3.69589800 & 1.76964800 & -1.40899700 \\ \mathrm{C} & -3.47480000 & -1.48895900 & -3.51819200 \\ \mathrm{H} & -2.84250800 & -2.47257600 & -1.71902800 \\ \mathrm{C} & -3.87928400 & -0.29010600 & -4.12608700 \\ \mathrm{H} & -4.26873900 & 1.81951500 & -3.81700600 \\ \mathrm{H} & -3.41385700 & -2.40468300 & -4.11202400 \\ \mathrm{H} & -4.13283000 & -0.26805400 & -5.18826500 \\ \mathrm{H} & -\mathrm{B} 3 \mathrm{~s}\end{array}$

UB3LYP-D3(BJ)/def2-SVP-CPCM(THF)

Zero-point correction=

Thermal correction to Energy=

0.255459 (Hartree/Particle)

0.267758

0.268702

Thermal correction to Enthalpy=

0.216395

$-466.953351$

$-466.941052$

$-466.940108$

$-466.992415$

Sum of electronic and thermal Free Energies=

$\mathrm{HF}=-467.2088102$

$\begin{array}{cccc}\mathbf{F}^{, R \text {-sty }} & & & \\ \mathrm{Ni} & -1.88038400 & 0.87120700 & 0.05444400 \\ \mathrm{C} & -1.92580400 & 0.96817000 & 1.96984800 \\ \mathrm{C} & -1.86389600 & 1.27249600 & 4.76246500 \\ \mathrm{C} & -1.09893700 & 0.15323700 & 2.74944200\end{array}$




\begin{tabular}{|c|c|c|c|}
\hline $\mathrm{C}$ & -2.72687900 & 1.93189200 & 2.59162900 \\
\hline $\mathrm{C}$ & -2.69592400 & 2.08344000 & 3.98352200 \\
\hline $\mathrm{C}$ & -1.06392700 & 0.30768800 & 4.14080000 \\
\hline $\mathrm{H}$ & -0.49114900 & -0.62568000 & 2.28376800 \\
\hline $\mathrm{H}$ & -3.38103000 & 2.56650600 & 1.98832600 \\
\hline $\mathrm{H}$ & -3.32769300 & 2.83837700 & 4.46043500 \\
\hline $\mathrm{H}$ & -0.41343900 & -0.33478300 & 4.74113800 \\
\hline $\mathrm{C}$ & -3.48741700 & -0.79641500 & -1.26633200 \\
\hline $\mathrm{H}$ & -2.80054200 & -1.64662400 & -1.37154200 \\
\hline $\mathrm{C}$ & -3.51427800 & -0.31405000 & 0.17663500 \\
\hline $\mathrm{H}$ & -4.27391800 & 0.45837500 & 0.33461000 \\
\hline $\mathrm{H}$ & -3.08603500 & 0.00377800 & -1.91311700 \\
\hline $\mathrm{C}$ & -4.85696200 & -1.19861000 & -1.89711500 \\
\hline $\mathrm{C}$ & -5.84063000 & -0.01931800 & -1.86014200 \\
\hline $\mathrm{H}$ & -6.76091700 & -0.27149900 & -2.41165500 \\
\hline $\mathrm{H}$ & -6.13069200 & 0.23342100 & -0.82884300 \\
\hline $\mathrm{H}$ & -5.39787200 & 0.88268000 & -2.30933900 \\
\hline $\mathrm{C}$ & -5.47156600 & -2.40486100 & -1.17157700 \\
\hline $\mathrm{H}$ & -4.78175500 & -3.26375500 & -1.16681700 \\
\hline $\mathrm{H}$ & -5.71664300 & -2.16890000 & -0.12611700 \\
\hline $\mathrm{H}$ & -6.40083400 & -2.71782900 & -1.67557300 \\
\hline $\mathrm{C}$ & -4.57924200 & -1.57941200 & -3.36084000 \\
\hline $\mathrm{H}$ & -5.50685000 & -1.88906500 & -3.86896000 \\
\hline $\mathrm{H}$ & -4.15498200 & -0.72850300 & -3.91809600 \\
\hline $\mathrm{H}$ & -3.86363000 & -2.41569800 & -3.42394900 \\
\hline $\mathrm{Br}$ & -3.15427000 & 2.81652500 & -1.05272100 \\
\hline $\mathrm{C}$ & 1.01753200 & 1.33580400 & -0.23125000 \\
\hline $\mathrm{C}$ & 1.78063600 & 3.42678800 & -0.25319100 \\
\hline $\mathrm{C}$ & 0.23321400 & 3.41676600 & -0.09979400 \\
\hline $\mathrm{N}$ & -0.08340900 & 1.97691500 & -0.13467600 \\
\hline $\mathrm{H}$ & 2.13061200 & 3.89452500 & -1.18256600 \\
\hline $\mathrm{H}$ & -0.26306500 & 3.88076000 & -0.96501200 \\
\hline $\mathrm{C}$ & 0.31026200 & -2.23074900 & -0.33593200 \\
\hline $\mathrm{C}$ & 1.85435600 & -2.19628500 & -0.15495400 \\
\hline $\mathrm{C}$ & 1.02263900 & -0.13645900 & -0.26073200 \\
\hline $\mathrm{N}$ & -0.06876300 & -0.80087200 & -0.31412600 \\
\hline $\mathrm{H}$ & -0.16893800 & -2.72177600 & 0.52371600 \\
\hline $\mathrm{H}$ & 2.40033700 & -2.69751000 & -0.96551000 \\
\hline $\mathrm{H}$ & 2.29760300 & 3.87644000 & 0.60567400 \\
\hline $\mathrm{H}$ & 2.19215500 & -2.58644800 & 0.81460100 \\
\hline $\mathrm{O}$ & 2.15516700 & 2.01501200 & -0.30191700 \\
\hline $\mathrm{O}$ & 2.18631800 & -0.77941100 & -0.20162400 \\
\hline $\mathrm{C}$ & -0.27327300 & 4.07553100 & 1.17602400 \\
\hline $\mathrm{H}$ & 0.01492900 & 5.13812900 & 1.18508400 \\
\hline
\end{tabular}




\begin{tabular}{|c|c|c|c|}
\hline $\mathrm{H}$ & -1.36683800 & 4.00408000 & 1.22218200 \\
\hline $\mathrm{H}$ & 0.14966800 & 3.58452400 & 2.06565700 \\
\hline $\mathrm{C}$ & -0.13152500 & -2.91063200 & -1.62772900 \\
\hline $\mathrm{H}$ & 0.17887800 & -3.96650500 & -1.62185700 \\
\hline $\mathrm{H}$ & -1.22328500 & -2.87516400 & -1.74340300 \\
\hline $\mathrm{H}$ & 0.32243600 & -2.41597300 & -2.50031600 \\
\hline $\mathrm{H}$ & -1.84249500 & 1.38804100 & 5.84909000 \\
\hline $\mathrm{C}$ & -3.56970400 & -1.33394600 & 1.25113900 \\
\hline $\mathrm{C}$ & -4.29776300 & -1.06933100 & 2.42855000 \\
\hline $\mathrm{C}$ & -2.90603900 & -2.57087100 & 1.16423200 \\
\hline $\mathrm{C}$ & -4.34088400 & -1.98470300 & 3.47827800 \\
\hline $\mathrm{H}$ & -4.82375200 & -0.11696700 & 2.51864000 \\
\hline $\mathrm{C}$ & -2.94281200 & -3.49095000 & 2.21410800 \\
\hline $\mathrm{H}$ & -2.35899600 & -2.82577400 & 0.25855500 \\
\hline $\mathrm{C}$ & -3.65560600 & -3.20191700 & 3.38161000 \\
\hline $\mathrm{H}$ & -4.91053500 & -1.74731800 & 4.38035800 \\
\hline $\mathrm{H}$ & -2.41347800 & -4.44240600 & 2.11653100 \\
\hline $\mathrm{H}$ & -3.68424900 & -3.92002100 & 4.20457800 \\
\hline \multicolumn{4}{|c|}{ UB3LYP-D3(BJ)/def2-SVP-CPCM(THF) } \\
\hline \multicolumn{3}{|c|}{ Zero-point correction $=$} & 0.552457 (Hartree/Particle) \\
\hline \multicolumn{3}{|c|}{ Thermal correction to Energy= } & 0.586504 \\
\hline \multicolumn{3}{|c|}{ Thermal correction to Enthalpy= } & 0.587449 \\
\hline \multicolumn{3}{|c|}{ Thermal correction to Gibbs Free Energy= } & 0.483440 \\
\hline \multicolumn{3}{|c|}{ Sum of electronic and zero-point Energies $=$} & -5351.980912 \\
\hline \multicolumn{3}{|c|}{ Sum of electronic and thermal Energies $=$} & -5351.946865 \\
\hline \multicolumn{3}{|c|}{ Sum of electronic and thermal Enthalpies $=$} & -5351.945921 \\
\hline \multicolumn{3}{|c|}{ Sum of electronic and thermal Free Energies $=$} & -5352.049930 \\
\hline \multicolumn{4}{|c|}{$\mathrm{HF}=-5352.5333692$} \\
\hline \multicolumn{4}{|c|}{$\mathrm{F}^{\prime}$-TS-H ${ }^{R-\mathrm{sty}}$} \\
\hline $\mathrm{Ni}$ & -1.25306700 & 1.26683100 & -0.46626300 \\
\hline $\mathrm{C}$ & -1.67101600 & 1.07101200 & 1.40824500 \\
\hline $\mathrm{C}$ & -1.27838200 & 1.91985300 & 4.07031800 \\
\hline $\mathrm{C}$ & -0.65877500 & 0.50008300 & 2.20153800 \\
\hline $\mathrm{C}$ & -2.51072100 & 2.04066400 & 1.98275200 \\
\hline $\mathrm{C}$ & -2.31110400 & 2.46476100 & 3.29943900 \\
\hline $\mathrm{C}$ & -0.45255600 & 0.93509200 & 3.51210100 \\
\hline $\mathrm{H}$ & -0.03216300 & -0.29624800 & 1.79677200 \\
\hline $\mathrm{H}$ & -3.31018000 & 2.48048300 & 1.38274500 \\
\hline $\mathrm{H}$ & -2.96677200 & 3.23001400 & 3.72407000 \\
\hline $\mathrm{H}$ & 0.35242900 & 0.49347400 & 4.10613400 \\
\hline $\mathrm{H}$ & -1.12484500 & 2.24906100 & 5.10080500 \\
\hline $\mathrm{C}$ & -2.62063500 & -0.83894700 & -1.15185200 \\
\hline $\mathrm{H}$ & -1.84026100 & -1.60877100 & -1.10535100 \\
\hline
\end{tabular}




\begin{tabular}{|c|c|c|c|}
\hline $\mathrm{C}$ & -2.83647800 & -0.22426600 & 0.23608600 \\
\hline $\mathrm{H}$ & -3.69271100 & 0.45350800 & 0.22620800 \\
\hline $\mathrm{H}$ & -2.26064800 & -0.08270400 & -1.87379000 \\
\hline $\mathrm{C}$ & -3.88078800 & -1.46579300 & -1.82921600 \\
\hline $\mathrm{C}$ & -5.03112500 & -0.44961500 & -1.89898700 \\
\hline $\mathrm{H}$ & -5.85574100 & -0.85003400 & -2.51058700 \\
\hline $\mathrm{H}$ & -5.43916300 & -0.23111500 & -0.90004200 \\
\hline $\mathrm{H}$ & -4.69640300 & 0.50293100 & -2.33711000 \\
\hline $\mathrm{C}$ & -4.35735300 & -2.73155700 & -1.09943900 \\
\hline $\mathrm{H}$ & -3.54655700 & -3.47193200 & -1.00988000 \\
\hline $\mathrm{H}$ & -4.71938400 & -2.50917400 & -0.08636300 \\
\hline $\mathrm{H}$ & -5.18427900 & -3.19892600 & -1.65915300 \\
\hline $\mathrm{C}$ & -3.46015400 & -1.84829600 & -3.25917200 \\
\hline $\mathrm{H}$ & -4.29949800 & -2.30769500 & -3.80541400 \\
\hline $\mathrm{H}$ & -3.12961100 & -0.96283600 & -3.82583900 \\
\hline $\mathrm{H}$ & -2.62979100 & -2.57339900 & -3.24822500 \\
\hline $\mathrm{Br}$ & -2.85740000 & 2.84402000 & -1.62005300 \\
\hline $\mathrm{C}$ & 1.52538300 & 2.05640400 & -0.51830100 \\
\hline $\mathrm{C}$ & 2.05571800 & 4.19796600 & -0.22847500 \\
\hline $\mathrm{C}$ & 0.51232000 & 4.03037400 & -0.33418400 \\
\hline $\mathrm{N}$ & 0.35719200 & 2.57745500 & -0.51516100 \\
\hline $\mathrm{H}$ & 2.49032300 & 4.81973000 & -1.02146000 \\
\hline $\mathrm{H}$ & 0.11957600 & 4.51674400 & -1.24085000 \\
\hline $\mathrm{C}$ & 1.07938100 & -1.50439800 & -1.04825800 \\
\hline $\mathrm{C}$ & 2.59905100 & -1.40928600 & -0.74660900 \\
\hline $\mathrm{C}$ & 1.65707300 & 0.60027000 & -0.67427100 \\
\hline $\mathrm{N}$ & 0.60861200 & -0.12647200 & -0.79387300 \\
\hline $\mathrm{H}$ & 0.58519000 & -2.18713500 & -0.34174400 \\
\hline $\mathrm{H}$ & 3.23414800 & -1.84115100 & -1.53036800 \\
\hline $\mathrm{H}$ & 2.38050300 & 4.56920500 & 0.75387000 \\
\hline $\mathrm{H}$ & 2.87563500 & -1.82877100 & 0.23102200 \\
\hline $\mathrm{O}$ & 2.58646300 & 2.84467900 & -0.38366400 \\
\hline $\mathrm{O}$ & 2.85684700 & 0.02564900 & -0.69749000 \\
\hline $\mathrm{C}$ & -0.26463800 & 4.53115200 & 0.87531700 \\
\hline $\mathrm{H}$ & -0.09644500 & 5.61092500 & 1.00941800 \\
\hline $\mathrm{H}$ & -1.33708000 & 4.35314000 & 0.72484300 \\
\hline $\mathrm{H}$ & 0.05021600 & 4.00412400 & 1.78867400 \\
\hline $\mathrm{C}$ & 0.76655700 & -1.92562100 & -2.48218300 \\
\hline $\mathrm{H}$ & 1.10652700 & -2.95799300 & -2.65543200 \\
\hline $\mathrm{H}$ & -0.31267200 & -1.87681800 & -2.67783800 \\
\hline $\mathrm{H}$ & 1.27776100 & -1.26321900 & -3.19797700 \\
\hline $\mathrm{C}$ & -2.95202100 & -1.21196100 & 1.34611500 \\
\hline $\mathrm{C}$ & -3.93081200 & -1.03366500 & 2.34014200 \\
\hline $\mathrm{C}$ & -2.09828500 & -2.32264900 & 1.45748700 \\
\hline
\end{tabular}




$\begin{array}{llll}\mathrm{C} & -4.05444300 & -1.92935500 & 3.40278900 \\ \mathrm{H} & -4.59897400 & -0.17182500 & 2.27485900 \\ \mathrm{C} & -2.21573100 & -3.21973500 & 2.52024600 \\ \mathrm{H} & -1.32538100 & -2.48973700 & 0.70672900 \\ \mathrm{C} & -3.19522000 & -3.02946300 & 3.50019400 \\ \mathrm{H} & -4.82634700 & -1.76867600 & 4.15968000 \\ \mathrm{H} & -1.53712500 & -4.07417300 & 2.58324900 \\ \mathrm{H} & -3.28878100 & -3.73191900 & 4.33171900\end{array}$

UB3LYP-D3(BJ)/def2-SVP-CPCM(THF)

Zero-point correction=

Thermal correction to Energy=

0.552764 (Hartree/Particle)

0.585950

0.586894

Thermal correction to Enthalpy=

0.485231

Thermal correction to Gibbs Free Energy=

Sum of electronic and zero-point Energies=

$-5351.966081$

$-5351.932895$

$-5351.931951$

Sum of electronic and thermal Enthalpies=

Sum of electronic and thermal Free Energies=

$-5352.033614$

$\mathrm{HF}=-5352.5188452$

\section{G-TS-F $^{\text {S-sty }}$}

$\begin{array}{lrrr}\mathrm{Ni} & -1.39228000 & 1.64295300 & -0.98849200 \\ \mathrm{C} & -1.93741600 & 2.18239200 & 0.83819000 \\ \mathrm{C} & -2.76687600 & 3.03241900 & 3.42572700 \\ \mathrm{C} & -1.10457300 & 2.04449900 & 1.96241300 \\ \mathrm{C} & -3.19537400 & 2.77654100 & 1.05088200 \\ \mathrm{C} & -3.61010400 & 3.19565900 & 2.32140400 \\ \mathrm{C} & -1.50590000 & 2.45588400 & 3.24040600 \\ \mathrm{H} & -0.11002700 & 1.59590400 & 1.86279300 \\ \mathrm{H} & -3.87573900 & 2.91094400 & 0.20405900 \\ \mathrm{H} & -4.59691700 & 3.65036300 & 2.45125800 \\ \mathrm{H} & -0.83369500 & 2.32463100 & 4.09390400 \\ \mathrm{Br} & -3.01401800 & 2.27009600 & -2.68168700 \\ \mathrm{C} & 1.32246200 & 2.29853700 & -0.68139100 \\ \mathrm{C} & 2.03431500 & 4.40013200 & -0.83752800 \\ \mathrm{C} & 0.54760500 & 4.29871500 & -1.29117400 \\ \mathrm{~N} & 0.26769500 & 2.86183400 & -1.14385400 \\ \mathrm{H} & 2.71769000 & 4.72089400 & -1.63381700 \\ \mathrm{H} & 0.44651900 & 4.55477900 & -2.35770000 \\ \mathrm{C} & 0.32284100 & -1.15752600 & -0.24058600 \\ \mathrm{C} & 1.71805000 & -1.14822000 & 0.44024900 \\ \mathrm{C} & 1.24385000 & 0.86418300 & -0.36895100 \\ \mathrm{~N} & 0.12322800 & 0.26793800 & -0.56371600 \\ \mathrm{H} & -0.46387700 & -1.47343600 & 0.45910100 \\ \mathrm{H} & 2.41157200 & -1.89719800 & 0.03841900\end{array}$




\begin{tabular}{|c|c|c|c|}
\hline $\mathrm{H}$ & 2.17389300 & 5.03183200 & 0.05041500 \\
\hline $\mathrm{H}$ & 1.67034000 & -1.22963200 & 1.53498700 \\
\hline $\mathrm{O}$ & 2.40303400 & 3.03241400 & -0.46404000 \\
\hline $\mathrm{O}$ & 2.26079300 & 0.17652400 & 0.12821100 \\
\hline $\mathrm{C}$ & -3.41186100 & -0.87840100 & 1.66024200 \\
\hline $\mathrm{H}$ & -2.96440500 & 0.05745500 & 2.02793400 \\
\hline $\mathrm{C}$ & -3.51267000 & -0.78807000 & 0.17627500 \\
\hline $\mathrm{H}$ & -3.84271400 & 0.16586300 & -0.24220800 \\
\hline $\mathrm{H}$ & -2.73160800 & -1.69196100 & 1.96229500 \\
\hline $\mathrm{C}$ & -4.76268700 & -1.07796100 & 2.42026900 \\
\hline $\mathrm{C}$ & -5.35878800 & -2.45558800 & 2.09777600 \\
\hline $\mathrm{H}$ & -6.31384400 & -2.59958900 & 2.62917500 \\
\hline $\mathrm{H}$ & -5.55123700 & -2.56545100 & 1.01935800 \\
\hline $\mathrm{H}$ & -4.67831700 & -3.26694700 & 2.40372900 \\
\hline $\mathrm{C}$ & -5.76240800 & 0.02008300 & 2.02635500 \\
\hline $\mathrm{H}$ & -5.33895700 & 1.01979300 & 2.20490700 \\
\hline $\mathrm{H}$ & -6.03353500 & -0.04702600 & 0.96117200 \\
\hline $\mathrm{H}$ & -6.68931700 & -0.07284100 & 2.61556500 \\
\hline $\mathrm{C}$ & -4.47012100 & -0.98147400 & 3.92511400 \\
\hline $\mathrm{H}$ & -5.38824500 & -1.13440400 & 4.51568800 \\
\hline $\mathrm{H}$ & -3.73724000 & -1.74407800 & 4.23650400 \\
\hline $\mathrm{H}$ & -4.05928400 & 0.00806300 & 4.18276300 \\
\hline $\mathrm{C}$ & -0.42943400 & 5.13663400 & -0.47596600 \\
\hline $\mathrm{H}$ & -0.20920000 & 6.20705200 & -0.60637800 \\
\hline $\mathrm{H}$ & -1.45800500 & 4.94159000 & -0.81029900 \\
\hline $\mathrm{H}$ & -0.36302100 & 4.88140100 & 0.59189700 \\
\hline $\mathrm{C}$ & 0.25689000 & -2.00146800 & -1.50858800 \\
\hline $\mathrm{H}$ & 0.36764700 & -3.06677800 & -1.25939400 \\
\hline $\mathrm{H}$ & -0.71065500 & -1.86599400 & -2.00815900 \\
\hline $\mathrm{H}$ & 1.06067700 & -1.71419700 & -2.20467000 \\
\hline $\mathrm{H}$ & -3.08763900 & 3.35406900 & 4.42011900 \\
\hline $\mathrm{C}$ & -3.26899200 & -1.83382200 & -0.74940000 \\
\hline $\mathrm{C}$ & -3.42007200 & -1.58894000 & -2.14965600 \\
\hline $\mathrm{C}$ & -2.87430800 & -3.14924600 & -0.35908000 \\
\hline $\mathrm{C}$ & -3.19934400 & -2.59111800 & -3.08455100 \\
\hline $\mathrm{H}$ & -3.69411000 & -0.58240300 & -2.47435300 \\
\hline $\mathrm{C}$ & -2.65732400 & -4.14411500 & -1.30531200 \\
\hline $\mathrm{H}$ & -2.74503700 & -3.38033200 & 0.69871100 \\
\hline $\mathrm{C}$ & -2.81798200 & -3.87976600 & -2.67402100 \\
\hline $\mathrm{H}$ & -3.31953500 & -2.37256600 & -4.14885100 \\
\hline $\mathrm{H}$ & -2.35726200 & -5.14268800 & -0.97699100 \\
\hline $\mathrm{H}$ & -2.64476200 & -4.66625600 & -3.4118980 \\
\hline
\end{tabular}

UB3LYP-D3(BJ)/def2-SVP-CPCM(THF)

Zero-point correction=

0.549596 (Hartree/Particle) 
Thermal correction to Energy=

Thermal correction to Enthalpy=

Thermal correction to Gibbs Free Energy=

Sum of electronic and zero-point Energies=

Sum of electronic and thermal Energies=

Sum of electronic and thermal Enthalpies=

Sum of electronic and thermal Free Energies=

$\mathrm{HF}=-5352.5212715$

$\mathrm{F}^{, S \text {-sty }}$

$\mathrm{Ni}$

$\mathrm{C}$

C

$\mathrm{C}$

$\mathrm{C}$

$\mathrm{C}$

$\mathrm{C}$

$\mathrm{H}$

$\mathrm{H}$

$\mathrm{H}$

$\mathrm{H}$

$\mathrm{H}$

$\mathrm{Br}$

$\mathrm{C}$

$\mathrm{H}$

$\mathrm{C}$

$\mathrm{H}$

$\mathrm{H}$

C

C

$\mathrm{H}$

$\mathrm{H}$

$\mathrm{H}$

C

$\mathrm{H}$

$\mathrm{H}$

$\mathrm{H}$

C

$\mathrm{H}$

$\mathrm{H}$

$\mathrm{H}$

C

C

C
$-1.15616300$

$-1.31931900$

$-1.37959900$

$-0.44611900$

$-2.22821500$

$-2.25852900$

$-0.47154400$

0.26519900

$-2.91113700$

$-2.97597700$

0.22269200

$-1.40410000$

$-2.20401600$

$-2.51746700$

$-1.77010400$

$-2.79291600$

$-3.54458700$

$-2.08287100$

$-3.75025500$

$-3.28337900$

$-4.10719300$

$-2.91677600$

$-4.87233000$

$-5.27654000$

$-4.50789700$

$-5.70457100$

$-4.27099000$

$-5.11586500$

$-3.48038600$

$-4.62261700$

1.78994400

2.71932800

1.18794700
$-2.46358600$
1.11368300

1.38005600

1.94970700

0.73001000

2.31344100

2.59673500

1.01550400

$-0.01271800$

2.83106900

3.32695600

0.50277000

2.17006100

3.19925500

$-1.33901900$

$-1.19409600$

$-0.02718800$

0.58017900

$-2.05947200$

$-2.00626200$

$-3.35546200$

$-3.87314600$

$-4.01859500$

$-3.21551600$

$-1.30701300$

$-2.84011700$

$-2.80413900$

$-1.13023700$

$-1.62082200$

$-0.94764300$

$-0.15062500$

1.23293800

3.23461300

3.37549300
$-2.25064000$
0.583574

0.584518

0.480175

$-5351.971676$

$-5351.937698$

$-5351.936754$

$-5352.041097$

$-0.02849100$

1.87442600

4.63430600

2.75510100

2.38810500

3.75910100

4.12716100

2.38726800

1.71093400

4.14434700

4.79900000

5.70447300

$-1.12233000$

0.89004200

1.68097000

0.16207200

0.67461100

0.18361400

1.55684400

2.12803200

2.64589700

1.32728400

2.85170100

0.53689400

0.14234000

$-0.32003200$

1.00200900

2.70788800

3.21807800

3.45340600

2.35005600

$-0.21845700$

$-0.50411700$

$-0.29996800$ 


$\begin{array}{lrrr}\mathrm{N} & 0.74347600 & 1.96798500 & -0.25394500 \\ \mathrm{H} & 3.05109900 & 3.48562600 & -1.52105100 \\ \mathrm{H} & 0.70259800 & 3.85492600 & -1.16106900 \\ \mathrm{C} & 0.84324700 & -2.25517700 & 0.21893800 \\ \mathrm{C} & 2.38430100 & -2.28571100 & 0.43421000 \\ \mathrm{C} & 1.69005300 & -0.22654600 & -0.04311100 \\ \mathrm{~N} & 0.56531500 & -0.83135300 & -0.07590500 \\ \mathrm{H} & 0.31316000 & -2.50826400 & 1.15077200 \\ \mathrm{H} & 2.90899100 & -2.91215100 & -0.30090500 \\ \mathrm{H} & 3.31491200 & 3.79266900 & 0.22976300 \\ \mathrm{H} & 2.68473800 & -2.57860000 & 1.44872700 \\ \mathrm{O} & 2.98158900 & 1.81137600 & -0.29875300 \\ \mathrm{O} & 2.80318600 & -0.90882300 & 0.22486400 \\ \mathrm{C} & 0.78787700 & 4.10809500 & 0.97564100 \\ \mathrm{H} & 1.14673300 & 5.14819600 & 0.93506700 \\ \mathrm{H} & -0.30517900 & 4.11045600 & 1.07145700 \\ \mathrm{H} & 1.21981300 & 3.61699600 & 1.86132100 \\ \mathrm{C} & 0.37864400 & -3.19393700 & -0.88909000 \\ \mathrm{H} & 0.68614000 & -4.22437600 & -0.65364300 \\ \mathrm{H} & -0.71435900 & -3.18581400 & -0.99068900 \\ \mathrm{H} & 0.82507000 & -2.90920300 & -1.85417800 \\ \mathrm{C} & -3.09463100 & -0.12207000 & -1.28998200 \\ \mathrm{C} & -4.22872100 & 0.52208600 & -1.83024200 \\ \mathrm{C} & -2.24463000 & -0.81134400 & -2.17983800 \\ \mathrm{C} & -4.51646700 & 0.45427200 & -3.18926400 \\ \mathrm{H} & -4.88476500 & 1.08107300 & -1.16031200 \\ \mathrm{C} & -2.52054000 & -0.86134100 & -3.54974700 \\ \mathrm{H} & -1.35037300 & -1.29885100 & -1.79625800 \\ \mathrm{C} & -3.65982100 & -0.23564600 & -4.05974400 \\ \mathrm{H} & -5.40929200 & 0.94833700 & -3.58042800 \\ \mathrm{H} & -1.84159300 & -1.39630100 & -4.21836600 \\ \mathrm{H} & -3.88245100 & -0.28101900 & -5.12846300 \\ \mathrm{HB} & -\mathrm{d} & \end{array}$

UB3LYP-D3(BJ)/def2-SVP-CPCM(THF)

Zero-point correction=

Thermal correction to Energy=

0.552686 (Hartree/Particle)

0.586666

Thermal correction to Enthalpy=

0.587610

Thermal correction to Gibbs Free Energy=

0.483678

Sum of electronic and zero-point Energies $=$

$-5351.978924$

$-5351.944944$

$-5351.944000$

Sum of electronic and thermal Enthalpies=

$-5352.047933$

$\mathrm{HF}=-5352.5316103$

F'-TS-H $^{S \text {-sty }}$ 


\begin{tabular}{|c|c|c|c|}
\hline $\mathrm{Ni}$ & -1.02293600 & 1.12110600 & -0.27746900 \\
\hline $\mathrm{C}$ & -1.80637500 & 1.32593100 & 1.49513500 \\
\hline $\mathrm{C}$ & -1.75565400 & 2.66635600 & 3.98020800 \\
\hline $\mathrm{C}$ & -0.98316200 & 0.87352500 & 2.53791100 \\
\hline $\mathrm{C}$ & -2.63476500 & 2.43982600 & 1.72861000 \\
\hline $\mathrm{C}$ & -2.60336400 & 3.10602500 & 2.95384300 \\
\hline $\mathrm{C}$ & -0.94630500 & 1.54799900 & 3.76472300 \\
\hline $\mathrm{H}$ & -0.33696800 & 0.00540500 & 2.39319700 \\
\hline $\mathrm{H}$ & -3.28945200 & 2.79592200 & 0.93070000 \\
\hline $\mathrm{H}$ & -3.24705300 & 3.97591100 & 3.11029900 \\
\hline $\mathrm{H}$ & -0.27739300 & 1.19255200 & 4.55323900 \\
\hline $\mathrm{H}$ & -1.73399600 & 3.18767600 & 4.94011500 \\
\hline $\mathrm{Br}$ & -1.75609900 & 3.08264700 & -1.70273200 \\
\hline $\mathrm{C}$ & -2.35737900 & -1.47072300 & 0.91599700 \\
\hline $\mathrm{H}$ & -1.55916300 & -1.37558700 & 1.66172200 \\
\hline $\mathrm{C}$ & -2.76949000 & -0.10644800 & 0.37626800 \\
\hline $\mathrm{H}$ & -3.63920400 & 0.27037400 & 0.91217900 \\
\hline $\mathrm{H}$ & -1.94254400 & -2.07408600 & 0.09950400 \\
\hline $\mathrm{C}$ & -3.50250200 & -2.28001600 & 1.57709200 \\
\hline $\mathrm{C}$ & -2.92785800 & -3.65430200 & 1.95828100 \\
\hline $\mathrm{H}$ & -3.68914500 & -4.27824100 & 2.45376400 \\
\hline $\mathrm{H}$ & -2.57399800 & -4.19626400 & 1.06603200 \\
\hline $\mathrm{H}$ & -2.07544500 & -3.54896400 & 2.64942600 \\
\hline $\mathrm{C}$ & -4.67350300 & -2.47569700 & 0.60161300 \\
\hline $\mathrm{H}$ & -5.14531100 & -1.51925800 & 0.33131200 \\
\hline $\mathrm{H}$ & -4.33506500 & -2.95405800 & -0.33157600 \\
\hline $\mathrm{H}$ & -5.44924400 & -3.11618700 & 1.05189700 \\
\hline $\mathrm{C}$ & -3.99300800 & -1.57242800 & 2.85091100 \\
\hline $\mathrm{H}$ & -4.77728500 & -2.16640800 & 3.34714300 \\
\hline $\mathrm{H}$ & -3.16731300 & -1.43030600 & 3.56641200 \\
\hline $\mathrm{H}$ & -4.41952700 & -0.58070900 & 2.63369300 \\
\hline $\mathrm{C}$ & 1.88154300 & 1.14767700 & 0.05868900 \\
\hline $\mathrm{C}$ & 2.84858700 & 3.15279700 & 0.08462700 \\
\hline $\mathrm{C}$ & 1.30940400 & 3.29664600 & -0.05636300 \\
\hline $\mathrm{N}$ & 0.86185300 & 1.89216500 & -0.14550000 \\
\hline $\mathrm{H}$ & 3.40325900 & 3.47622700 & -0.80686400 \\
\hline $\mathrm{H}$ & 1.02928200 & 3.79306000 & -0.99593100 \\
\hline $\mathrm{C}$ & 0.85588700 & -2.34050900 & 0.08221600 \\
\hline $\mathrm{C}$ & 2.37079300 & -2.42652500 & 0.42384500 \\
\hline $\mathrm{C}$ & 1.74306900 & -0.31406500 & 0.11828800 \\
\hline $\mathrm{N}$ & 0.61416900 & -0.88594400 & -0.06436800 \\
\hline $\mathrm{H}$ & 0.24984300 & -2.69956800 & 0.92762400 \\
\hline $\mathrm{H}$ & 2.95162200 & -2.98389600 & -0.32475300 \\
\hline $\mathrm{H}$ & 3.25469000 & 3.65203000 & 0.97445800 \\
\hline
\end{tabular}




$\begin{array}{lrrr}\mathrm{H} & 2.57542600 & -2.83223200 & 1.42351300 \\ \mathrm{O} & 3.06874100 & 1.71792800 & 0.24201500 \\ \mathrm{O} & 2.82208000 & -1.04571300 & 0.39995600 \\ \mathrm{C} & 0.63111700 & 4.00940100 & 1.10706700 \\ \mathrm{H} & 0.99754300 & 5.04545000 & 1.17502400 \\ \mathrm{H} & -0.45413400 & 4.02487500 & 0.94797000 \\ \mathrm{H} & 0.83683700 & 3.49498400 & 2.05815200 \\ \mathrm{C} & 0.47782600 & -3.12448500 & -1.16998100 \\ \mathrm{H} & 0.75603100 & -4.18223000 & -1.04459400 \\ \mathrm{H} & -0.60325500 & -3.08355700 & -1.35871000 \\ \mathrm{H} & 1.00349100 & -2.72683500 & -2.05175300 \\ \mathrm{C} & -3.08565600 & -0.04554800 & -1.08516600 \\ \mathrm{C} & -4.24709700 & 0.62797700 & -1.51850300 \\ \mathrm{C} & -2.27219800 & -0.65075700 & -2.06793400 \\ \mathrm{C} & -4.58649900 & 0.68669800 & -2.86656500 \\ \mathrm{H} & -4.88901700 & 1.10599800 & -0.77466900 \\ \mathrm{C} & -2.60815600 & -0.58123500 & -3.42249200 \\ \mathrm{H} & -1.36820500 & -1.17902300 & -1.77140400 \\ \mathrm{C} & -3.76645900 & 0.08410000 & -3.82948000 \\ \mathrm{H} & -5.49714400 & 1.20738000 & -3.17302700 \\ \mathrm{H} & -1.95854000 & -1.05578600 & -4.16213600 \\ \mathrm{H} & -4.03277600 & 0.13209800 & -4.88793300 \\ \mathrm{UB} & -\mathrm{P}-\mathrm{P} & \end{array}$

UB3LYP-D3(BJ)/def2-SVP-CPCM(THF)

Zero-point correction=

0.552549 (Hartree/Particle)
0.585845
0.586790
0.485042
-5351.965523
-5351.932227
-5351.931283
-5352.033030

Thermal correction to Energy=

Thermal correction to Enthalpy=

Thermal correction to Gibbs Free Energy=

Sum of electronic and zero-point Energies=

Sum of electronic and thermal Energies=

Sum of electronic and thermal Enthalpies=

Sum of electronic and thermal Free Energies= $\mathrm{HF}=-5352.5180721$

\section{Scheme S12}

$\begin{array}{lrrr}\text { G-TS-F }{ }^{\boldsymbol{R}} \text {-Conf2 } & & & \\ \mathrm{Ni} & -0.58740300 & 1.03748700 & -1.05976800 \\ \mathrm{C} & -0.35275200 & 1.03331200 & -3.01235300 \\ \mathrm{C} & -0.03316300 & 1.07393300 & -5.83778200 \\ \mathrm{C} & -1.16690900 & 1.83607600 & -3.83411600 \\ \mathrm{C} & 0.64644200 & 0.27594900 & -3.65033000 \\ \mathrm{C} & 0.80559400 & 0.28531800 & -5.04168900 \\ \mathrm{C} & -1.01762600 & 1.85817900 & -5.22687100 \\ \mathrm{H} & -1.95918300 & 2.44799600 & -3.38759500 \\ \mathrm{H} & 1.31228400 & -0.35421500 & -3.05252400\end{array}$


$\mathrm{H}$

$\mathrm{H}$

$\mathrm{H}$

C

$\mathrm{H}$

C

$\mathrm{H}$

$\mathrm{H}$

C

C

$\mathrm{H}$

$\mathrm{H}$

$\mathrm{H}$

C

$\mathrm{H}$

$\mathrm{H}$

$\mathrm{H}$

C

$\mathrm{H}$

$\mathrm{H}$

$\mathrm{H}$

C

$\mathrm{O}$

$\mathrm{O}$

C

$\mathrm{H}$

$\mathrm{H}$

$\mathrm{H}$

$\mathrm{Br}$

C

C

C

$\mathrm{N}$

$\mathrm{H}$

$\mathrm{H}$

C

C

C

$\mathrm{N}$

$\mathrm{H}$

$\mathrm{H}$

$\mathrm{H}$

$\mathrm{H}$

O
1.58469400

$-0.32527100$

2.48308300

1.07947400

$-1.42328900$

$-2.36408000$

$-0.58626000$

0.42706700

$-0.90725000$

$-1.77538600$

$-0.49883200$

$-0.75050100$

0.16649900

0.06633300

$-2.51851700$

$-3.42245700$

$-1.87987000$

$-2.82734400$

$-2.68168300$

$-2.96185400$

$-2.17079500$

$-3.60888200$

$-1.17429000$

$-2.22578700$

$-0.43928100$

$-0.88172600$

$-0.80006900$

$-0.21402500$

$-1.92321300$

1.63867200

1.11453400

3.00065000

3.25667500

1.92906500

3.22319000

3.52126800

$-2.07514300$

$-2.38745500$

$-0.28553400$

$-0.62615400$

$-2.24701900$

$-3.24910000$

3.52378700

$-2.50002000$

1.56379500
$-5.50825200$

$-5.83806900$

$-6.92494100$

$-3.49279000$

$-3.07897100$

$-2.37733900$

$-2.56101600$

$-4.00230600$

$-4.57249900$

$-5.26331000$

$-6.06935400$

$-4.55839200$

$-5.70354200$

$-3.92200800$

$-3.39391000$

$-3.19101400$

$-4.68243800$

$-5.61088500$

$-6.40794700$

$-6.07975700$

$-5.14549300$

$-1.08440100$

$-0.65943800$

$-0.39382000$

0.92626200

1.56745800

1.29852100

0.91963900

0.93517500

0.04549600

0.20901000

$-0.54369200$

$-0.52750800$

$-0.38623300$

$-1.59711800$

$-0.12783000$

0.87860800

0.18175300

$-0.34728800$

0.30477600

0.59674000

1.17424200

1.91362200

0.48958200 


\begin{tabular}{|c|c|c|c|}
\hline $\mathrm{O}$ & 2.29429000 & -1.19492300 & 0.83881200 \\
\hline $\mathrm{C}$ & 0.95039000 & 4.31238800 & 0.09218100 \\
\hline $\mathrm{H}$ & 1.45524100 & 5.29037100 & 0.08591100 \\
\hline $\mathrm{H}$ & 0.00826600 & 4.40012800 & -0.46735600 \\
\hline $\mathrm{H}$ & 0.70659700 & 4.03877100 & 1.12941000 \\
\hline $\mathrm{C}$ & 0.41764200 & -2.82961600 & -1.44792900 \\
\hline $\mathrm{H}$ & 0.21858600 & -3.89999800 & -1.28802000 \\
\hline $\mathrm{H}$ & -0.31215100 & -2.44213500 & -2.16813500 \\
\hline $\mathrm{H}$ & 1.42583100 & -2.71867000 & -1.87678800 \\
\hline \multicolumn{4}{|c|}{ UB3LYP-D3(BJ)/def2-SVP-CPCM(THF) } \\
\hline \multicolumn{3}{|c|}{ Zero-point correction $=$} & 0.511160 (Hartree/Particle) \\
\hline \multicolumn{3}{|c|}{ Thermal correction to Energy= } & 0.545252 \\
\hline \multicolumn{3}{|c|}{ Thermal correction to Enthalpy= } & 0.546196 \\
\hline \multicolumn{3}{|c|}{ Thermal correction to Gibbs Free Energy= } & 0.440485 \\
\hline \multicolumn{3}{|c|}{ Sum of electronic and zero-point Energies $=$} & -5348.803524 \\
\hline \multicolumn{3}{|c|}{ Sum of electronic and thermal Energies $=$} & -5348.769432 \\
\hline \multicolumn{3}{|c|}{ Sum of electronic and thermal Enthalpies $=$} & -5348.768488 \\
\hline \multicolumn{3}{|c|}{ Sum of electronic and thermal Free Energies $=$} & -5348.874199 \\
\hline \multicolumn{4}{|c|}{$H F=-5349.3146838$} \\
\hline \multicolumn{4}{|c|}{ G-TS-F ${ }^{, S}$-Conf2 } \\
\hline $\mathrm{Ni}$ & -0.27584700 & -0.36460900 & 0.61566700 \\
\hline $\mathrm{C}$ & -1.12223700 & -1.76981200 & -0.48789100 \\
\hline $\mathrm{C}$ & -2.39857600 & -3.81892800 & -1.99393600 \\
\hline $\mathrm{C}$ & -0.42164300 & -2.52615900 & -1.44368100 \\
\hline $\mathrm{C}$ & -2.48776100 & -2.06652500 & -0.31811300 \\
\hline $\mathrm{C}$ & -3.12154600 & -3.07775300 & -1.05289400 \\
\hline $\mathrm{C}$ & -1.04243800 & -3.53615800 & -2.19105700 \\
\hline $\mathrm{H}$ & 0.64385200 & -2.33814700 & -1.62196700 \\
\hline $\mathrm{H}$ & -3.07475800 & -1.49367100 & 0.40679800 \\
\hline $\mathrm{H}$ & -4.18389700 & -3.28631200 & -0.89202400 \\
\hline $\mathrm{H}$ & -0.46665100 & -4.10601500 & -2.92695300 \\
\hline $\mathrm{Br}$ & -1.88816800 & 1.24590500 & 1.45358100 \\
\hline $\mathrm{H}$ & -2.88801500 & -4.60810700 & -2.57112800 \\
\hline $\mathrm{C}$ & 2.46112200 & -0.89506100 & 0.12140200 \\
\hline $\mathrm{C}$ & 3.63809900 & -2.42595100 & 1.22290900 \\
\hline $\mathrm{C}$ & 2.19754800 & -2.33088900 & 1.80411800 \\
\hline $\mathrm{N}$ & 1.59797500 & -1.24353800 & 1.00515100 \\
\hline $\mathrm{H}$ & 4.41945000 & -2.11329900 & 1.92778300 \\
\hline $\mathrm{H}$ & 2.22700900 & -2.00591200 & 2.85539800 \\
\hline $\mathrm{C}$ & 0.68858200 & 1.50399800 & -1.93866900 \\
\hline $\mathrm{C}$ & 2.08159500 & 1.47202300 & -2.63331300 \\
\hline $\mathrm{C}$ & 2.06583100 & 0.11051900 & -0.87568000 \\
\hline $\mathrm{N}$ & 0.87857500 & 0.58767200 & -0.80294400 \\
\hline
\end{tabular}




$\begin{array}{lrrr}\mathrm{H} & -0.08311300 & 1.06021300 & -2.58857300 \\ \mathrm{H} & 2.61148000 & 2.43311700 & -2.57357700 \\ \mathrm{H} & 3.87779800 & -3.41786300 & 0.81724300 \\ \mathrm{H} & 2.05310600 & 1.12691600 & -3.67406200 \\ \mathrm{O} & 3.64849800 & -1.47960300 & 0.10728400 \\ \mathrm{O} & 2.85710900 & 0.49609400 & -1.86480200 \\ \mathrm{C} & 1.38474500 & -3.61460700 & 1.69388800 \\ \mathrm{H} & 1.87194900 & -4.41072700 & 2.27840900 \\ \mathrm{H} & 0.37330800 & -3.45459800 & 2.08900800 \\ \mathrm{H} & 1.31334700 & -3.93790800 & 0.64436900 \\ \mathrm{C} & 0.23725000 & 2.88541100 & -1.48506400 \\ \mathrm{H} & 0.08942200 & 3.54122800 & -2.35655700 \\ \mathrm{H} & -0.70847500 & 2.80863000 & -0.93005400 \\ \mathrm{H} & 0.99211200 & 3.34112500 & -0.82542200 \\ \mathrm{C} & -0.19905200 & -0.32541300 & 4.31756300 \\ \mathrm{H} & -0.00465000 & 0.71868400 & 4.02036600 \\ \mathrm{C} & -1.65058200 & -0.59188400 & 4.17364600 \\ \mathrm{H} & -2.39217800 & 0.12763200 & 4.52183000 \\ \mathrm{H} & 0.34578400 & -0.97482000 & 3.61803800 \\ \mathrm{C} & 0.39445200 & -0.53967600 & 5.74473300 \\ \mathrm{C} & 1.89454300 & -0.21748000 & 5.67927900 \\ \mathrm{H} & 2.36751000 & -0.34842000 & 6.66591500 \\ \mathrm{H} & 2.41221400 & -0.88144200 & 4.96774500 \\ \mathrm{H} & 2.06459200 & 0.82212900 & 5.35569700 \\ \mathrm{C} & 0.20158500 & -1.99917900 & 6.18260100 \\ \mathrm{H} & -0.86649500 & -2.25646300 & 6.25828800 \\ \mathrm{H} & 0.66153600 & -2.69402800 & 5.46261900 \\ \mathrm{H} & 0.66139100 & -2.17164800 & 7.16922800 \\ \mathrm{C} & -0.28868300 & 0.40098200 & 6.74858500 \\ \mathrm{H} & 0.15908300 & 0.29198900 & 7.74962600 \\ \mathrm{H} & -0.18053900 & 1.45431300 & 6.44227400 \\ \mathrm{H} & -1.36433500 & 0.18246200 & 6.83779500 \\ \mathrm{C} & -2.13744800 & -1.82139800 & 3.58869600 \\ \mathrm{O} & -1.43325400 & -2.74973000 & 3.21017800 \\ \mathrm{O} & -3.48683600 & -1.86136000 & 3.50765700 \\ \mathrm{C} & -4.05219000 & -3.06227100 & 2.98571700 \\ \mathrm{H} & -3.79084700 & -3.92475600 & 3.61831600 \\ \mathrm{H} & -5.13880700 & -2.91364500 & 2.98278700 \\ \mathrm{H} & -3.69272900 & -3.25403700 & 1.96444100 \\ \mathrm{H} & & \end{array}$

UB3LYP-D3(BJ)/def2-SVP-CPCM(THF)

Zero-point correction=

Thermal correction to Energy=

0.511052 (Hartree/Particle)

Thermal correction to Enthalpy=

0.545183

0.546127

Thermal correction to Gibbs Free Energy=

0.440113 
Sum of electronic and zero-point Energies=

Sum of electronic and thermal Energies=

Sum of electronic and thermal Enthalpies=

Sum of electronic and thermal Free Energies= $\mathrm{HF}=-5349.3158248$
$-5348.804773$

$-5348.770642$

$-5348.769698$

$-5348.875711$ 


\section{References}

1. Liu, L.; Lee, W.; Yuan, M.; Acha, C.; Geherty, M. B.; Williams, B.; Gutierrez, O., Intra- and intermolecular Fe-catalyzed dicarbofunctionalization of vinyl cyclopropanes. Chem. Sci. 2020, 11 (12), 3146-3151.

2. Primer, D. N.; Molander, G. A., Enabling the Cross-Coupling of Tertiary Organoboron Nucleophiles through Radical-Mediated Alkyl Transfer. J. Am. Chem. Soc. 2017, 139 (29), 9847-9850.

3. Zhao, J.; Nanjo, T.; de Lucca, E. C.; White, M. C., Chemoselective methylene oxidation in aromatic molecules. Nat. Chem. 2019, 11 (3), 213-221.

4. Jin, M.; Adak, L.; Nakamura, M., Iron-Catalyzed Enantioselective Cross-Coupling Reactions of $\alpha$-Chloroesters with Aryl Grignard Reagents. J. Am. Chem. Soc. 2015, 137 (22), 7128-7134.

5. (a) Lee, C.; Yang, W.; Parr, R. G., Development of the Colle-Salvetti CorrelationEnergy Formula into a Functional of the Electron Density. Phys. Rev. B 1988, 37 (2), 785-789. (b) Becke, A. D., Density-Functional Thermochemistry. III. The Role of Exact Exchange. J. Chem. Phys.1993, 98 (7), 5648-5652.

6. Grimme, S.; Ehrlich, S.; Goerigk, L., Effect of the damping function in dispersion corrected density functional theory. J. Comp. Chem. 2011, 32 (7), 1456-1465.

7. (a) Weigend, F.; Ahlrichs, R. Balanced basis sets of split valence, triple zeta valence and quadruple zeta valence quality for $\mathrm{H}$ to $\mathrm{Rn}$ : Design and assessment of accuracy. Phys. Chem. Chem. Phys. 2005, 7 (18), 3297-3305. (b) Weigend, F. Accurate Coulombfitting basis sets for H to Rn. Phys. Chem. Chem. Phys. 2006, 8 (9), 1057-1065.

8. Cossi, M.; Rega, N.; Scalmani, G.; Barone, V. Energies, structures, and electronic properties of molecules in solution with the C-PCM solvation model. J. Comp. Chem. 2003, 24 (6), 669-681.

9. Gaussian 16, Revision C.01, M. J. Frisch, G. W. Trucks, H. B. Schlegel, G. E. Scuseria, M. A. Robb, J. R. Cheeseman, G. Scalmani, V. Barone, G. A. Petersson, H. Nakatsuji, X. Li, M. Caricato, A. V. Marenich, J. Bloino, B. G. Janesko, R. Gomperts, B. Mennucci, H. P. Hratchian, J. V. Ortiz, A. F. Izmaylov, J. L. Sonnenberg, D. 
Williams-Young, F. Ding, F. Lipparini, F. Egidi, J. Goings, B. Peng, A. Petrone, T. Henderson, D. Ranasinghe, V. G. Zakrzewski, J. Gao, N. Rega, G. Zheng, W. Liang, M. Hada, M. Ehara, K. Toyota, R. Fukuda, J. Hasegawa, M. Ishida, T. Nakajima, Y. Honda, O. Kitao, H. Nakai, T. Vreven, K. Throssell, J. A. Montgomery, Jr., J. E. Peralta, F. Ogliaro, M. J. Bearpark, J. J. Heyd, E. N. Brothers, K. N. Kudin, V. N. Staroverov, T. A. Keith, R. Kobayashi, J. Normand, K. Raghavachari, A. P. Rendell, J. C. Burant, S. S. Iyengar, J. Tomasi, M. Cossi, J. M. Millam, M. Klene, C. Adamo, R. Cammi, J. W. Ochterski, R. L. Martin, K. Morokuma, O. Farkas, J. B. Foresman, and D. J. Fox, Gaussian, Inc., Wallingford CT, 2019.

10. CYLview, 1.0b; Legault, C. Y., Université de Sherbrooke, 2009 (http://www.cylview.org).

11. Lu, T.; Chen, F., Multiwfn: A multifunctional wavefunction analyzer. J. Comp. Chem. 2012, 33 (5), 580-592.

12. Humphrey, W.; Dalke, A.; Schulten, K., VMD - Visual Molecular Dynamics. J. Mol. Graphics 1996, 14 (1), 33-38. 


\section{NMR Spectra}

\section{4; ${ }^{1} \mathrm{H}-\mathrm{NMR}\left(600 \mathrm{MHz}, \mathrm{CDCl}_{3}\right)$}
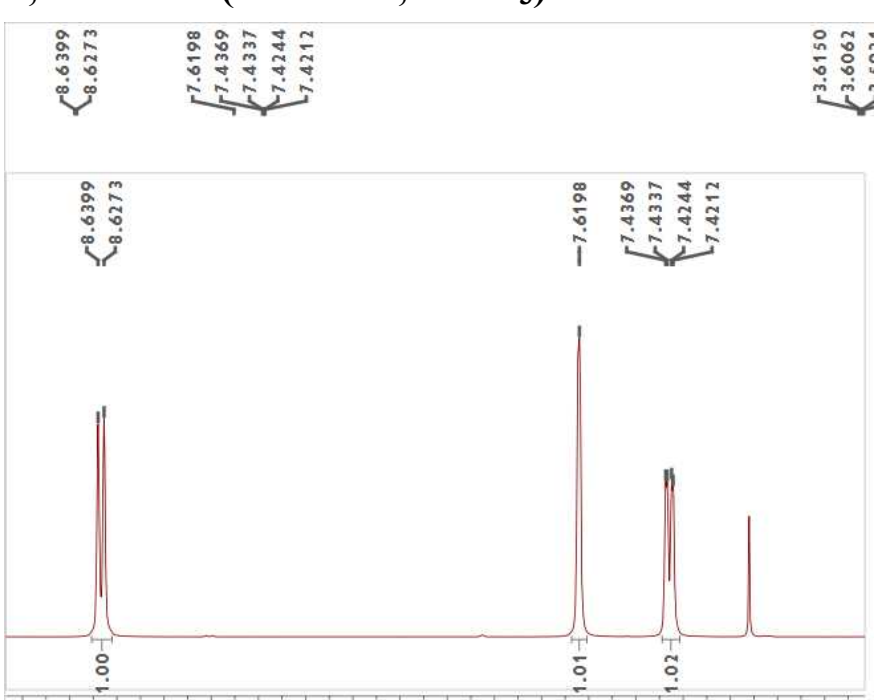

高产

象

$\begin{array}{llllllllllllllllllllll}8.8 & 8.7 & 8.6 & 8.5 & 8.4 & 8.3 & 8.2 & 8.1 & 8.0 & 7.9 & 7.8 & 7.7 & 7.6 & 7.5 & 7.4 & 7.3 & 7.2 & 7.1\end{array}$<smiles>CC(C)(C)OC(=O)C(CBr)c1ccnc(C(F)(F)F)c1</smiles>
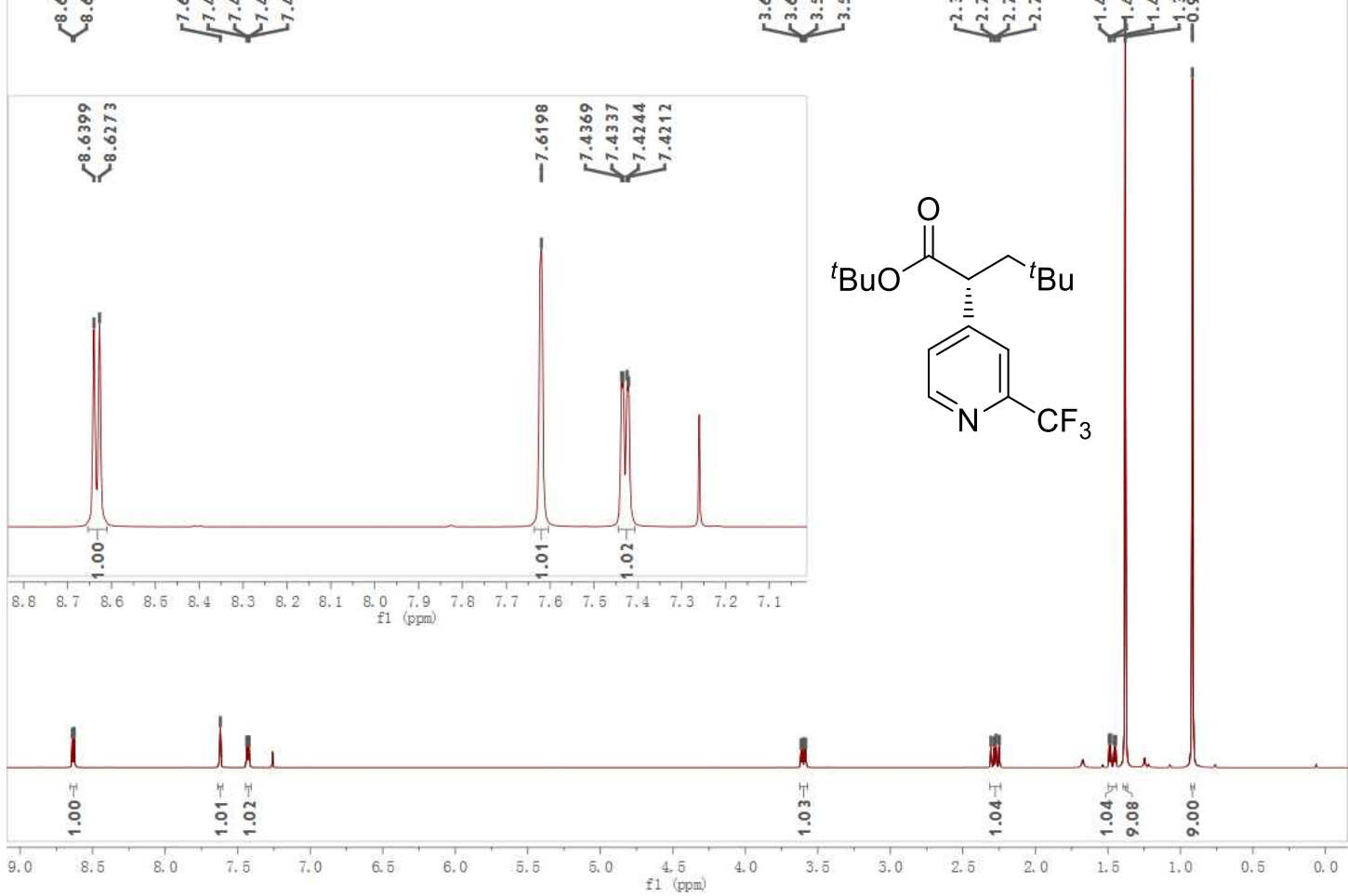

$4 ;{ }^{19}$ F-NMR (375 MHz, CDCl ${ }_{3}$ )

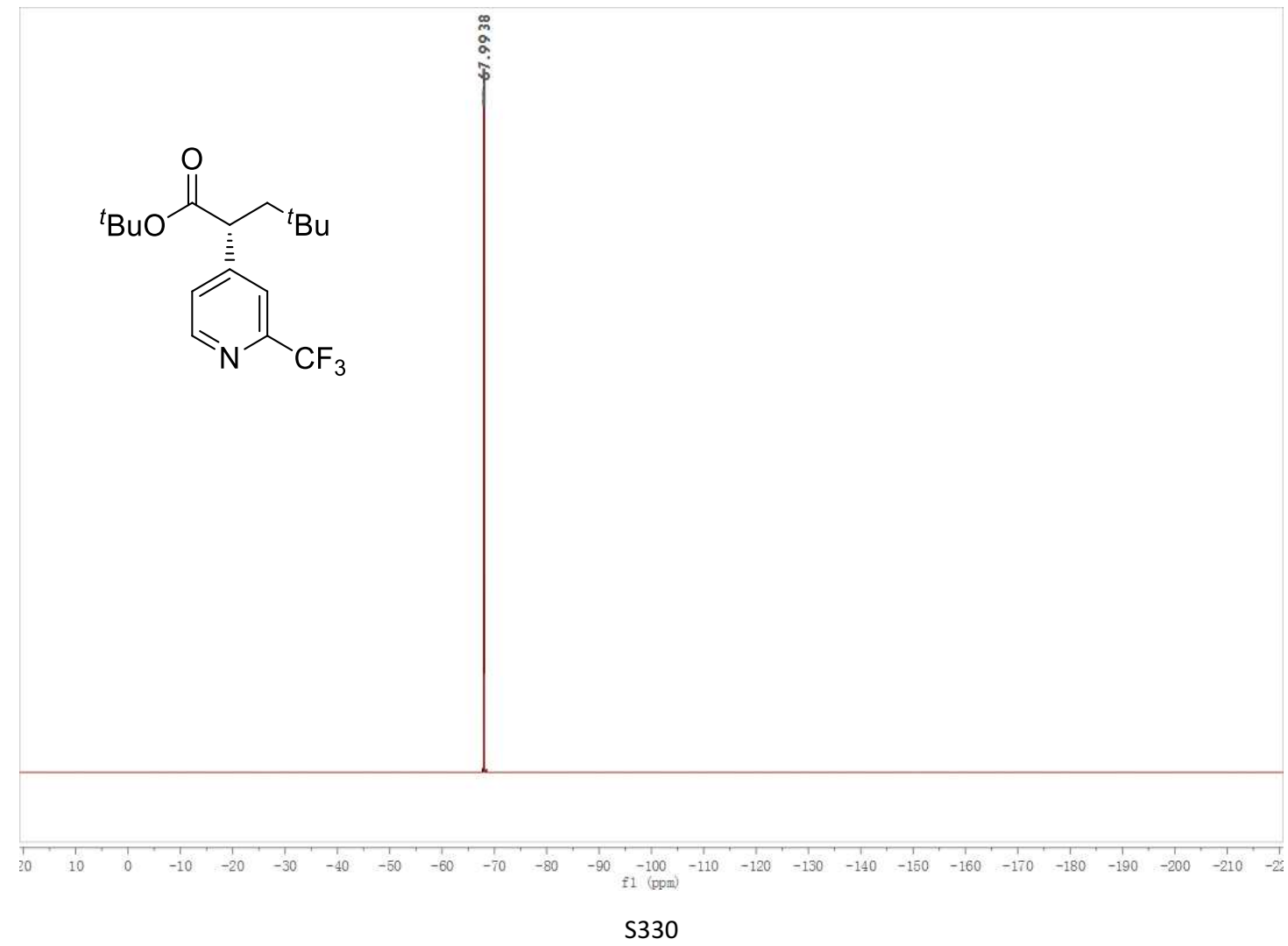


4; ${ }^{13} \mathrm{C}-\mathrm{NMR}\left(100 \mathrm{MHz}, \mathrm{CDCl}_{3}\right)$

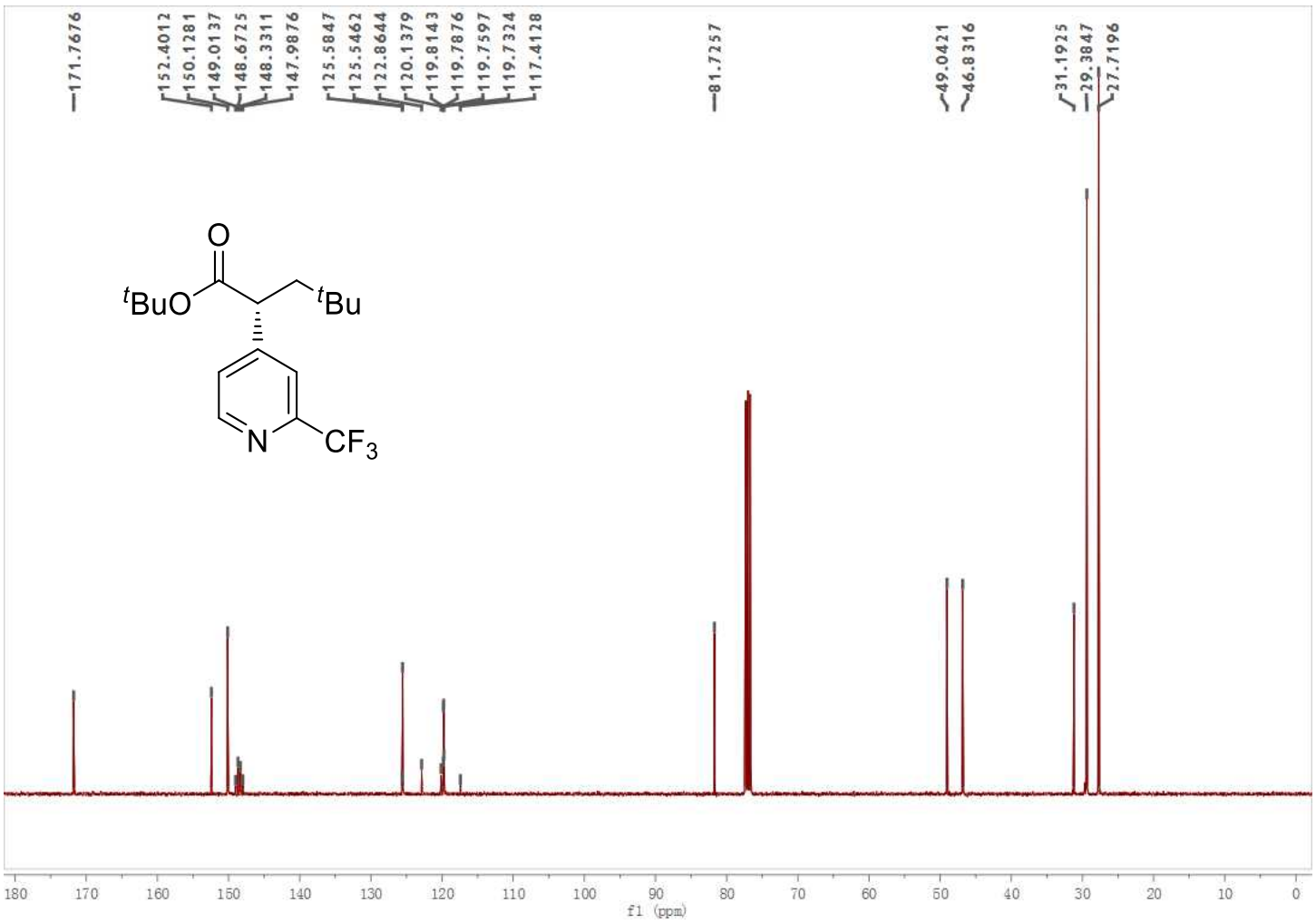

5; ${ }^{1} \mathrm{H}-\mathrm{NMR}\left(600 \mathrm{MHz}, \mathrm{CDCl}_{3}\right)$

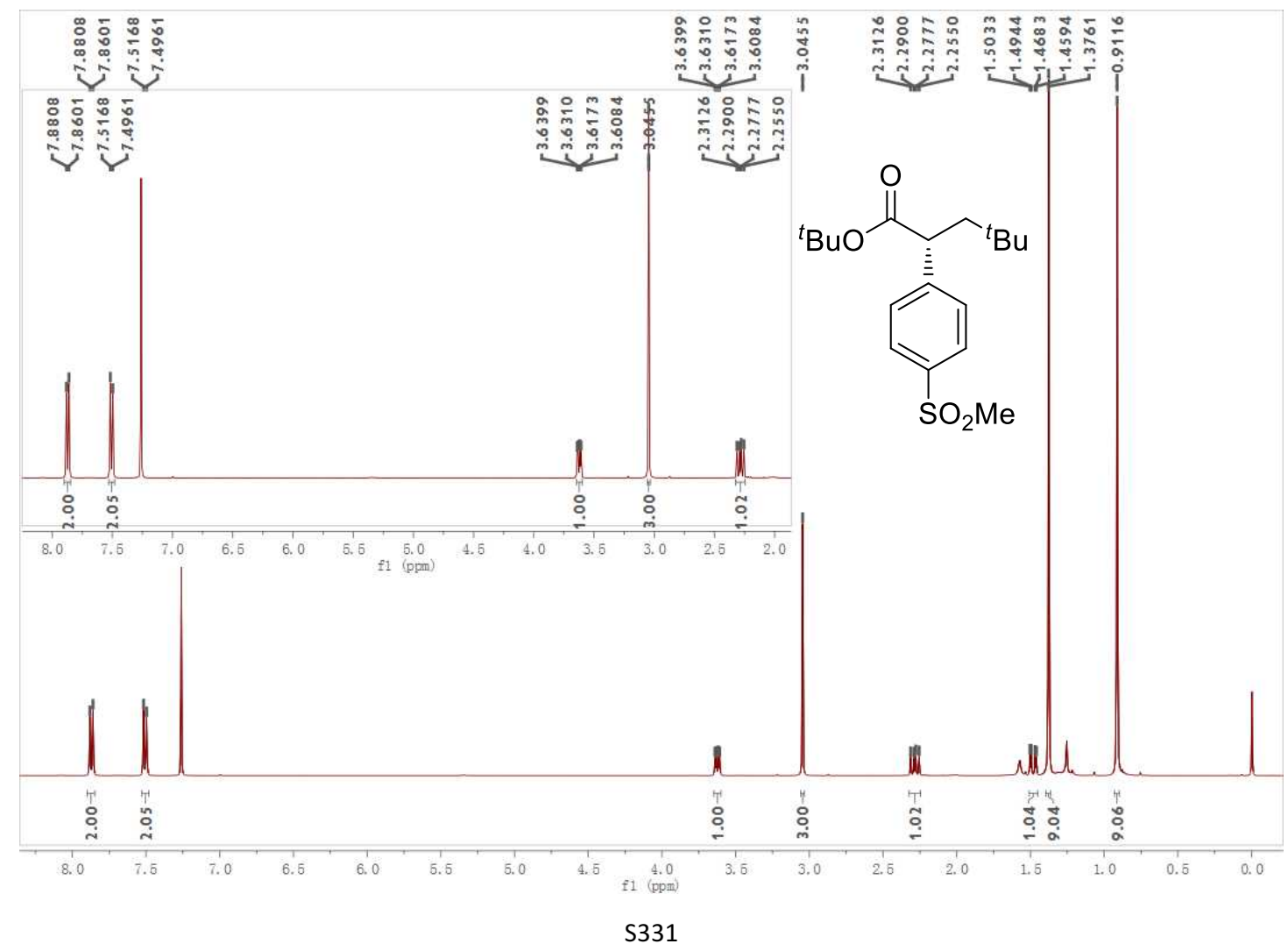


5; ${ }^{13} \mathrm{C}-\mathrm{NMR}\left(100 \mathrm{MHz}, \mathrm{CDCl}_{3}\right)$

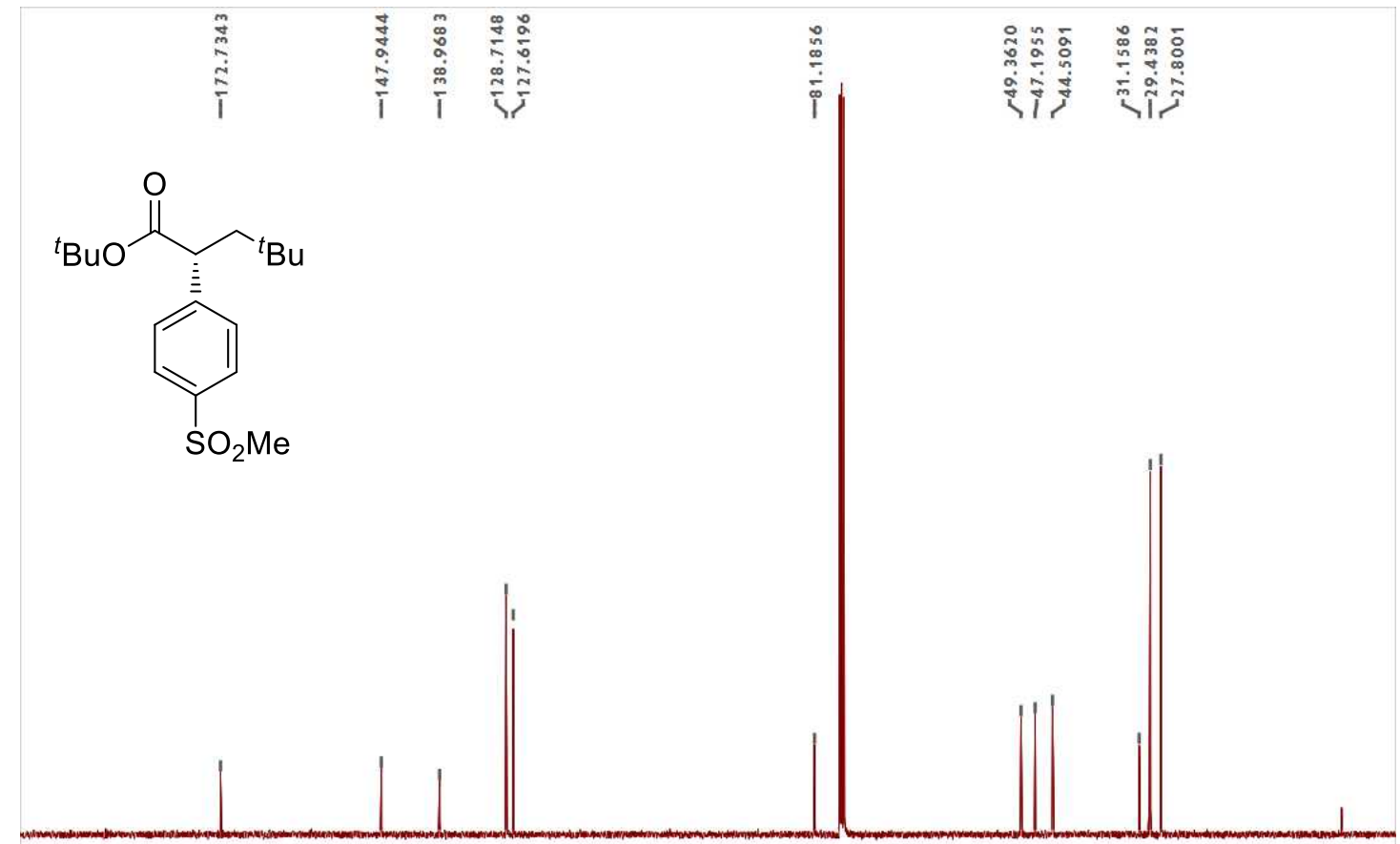

6; ${ }^{1} \mathrm{H}-\mathrm{NMR}$ (400 MHz, $\mathrm{CDCl}_{3}$ )

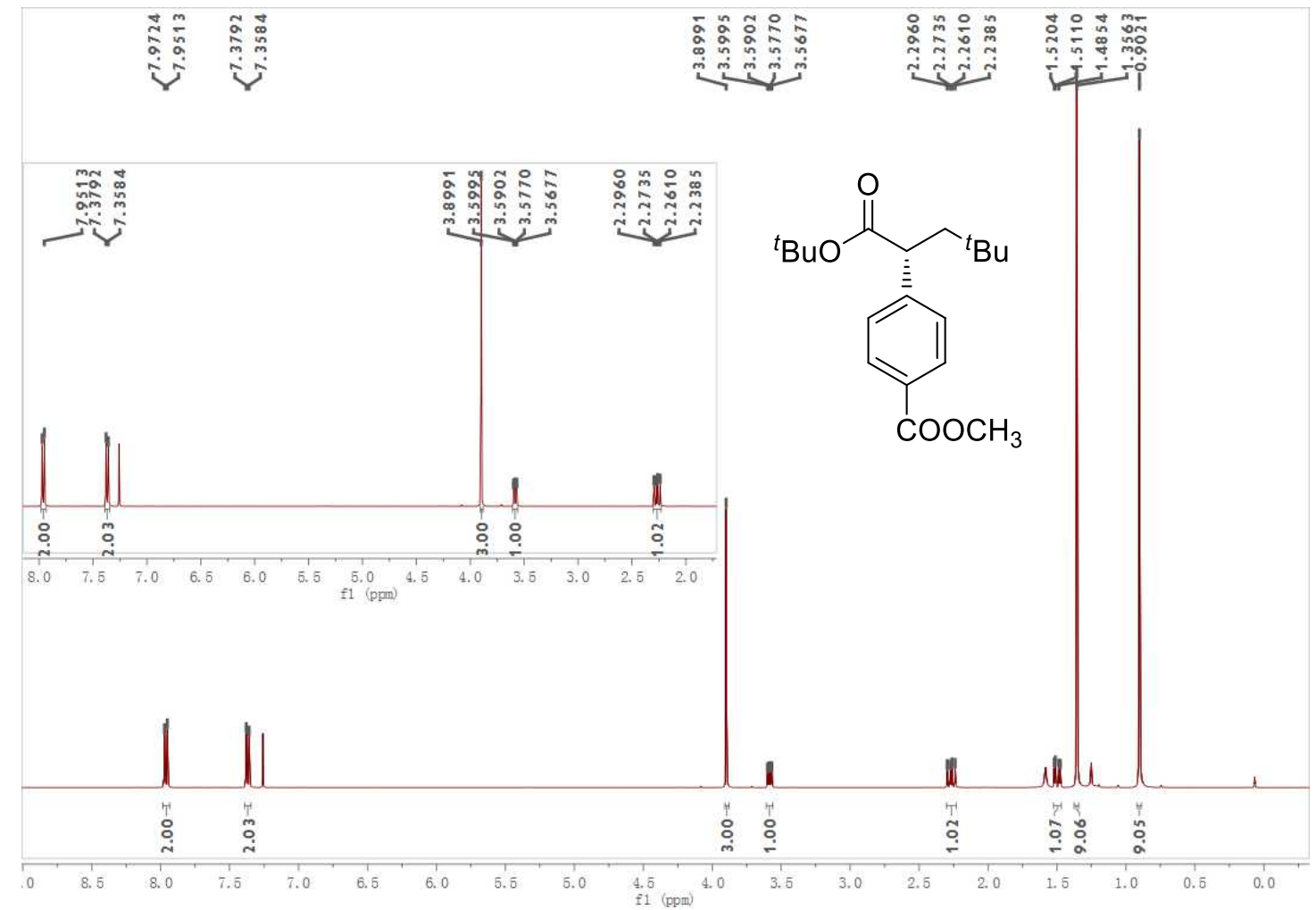


6; ${ }^{13} \mathrm{C}-\mathrm{NMR}\left(100 \mathrm{MHz}, \mathrm{CDCl}_{3}\right)$

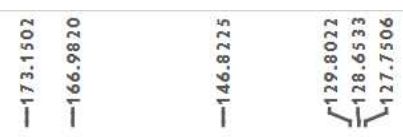<smiles>CC(=O)c1ccc([C@H](C[18O])C(=O)O[Na])cc1</smiles>

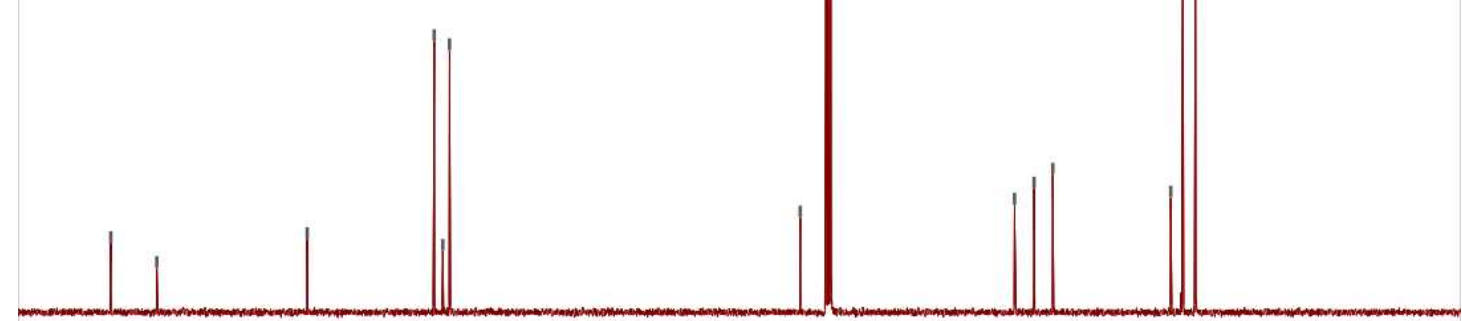

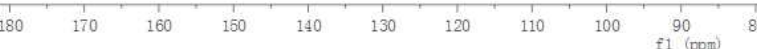

\section{7; ${ }^{1} \mathrm{H}-\mathrm{NMR}$ (400 $\mathrm{MHz}, \mathrm{CDCl}_{3}$ )}

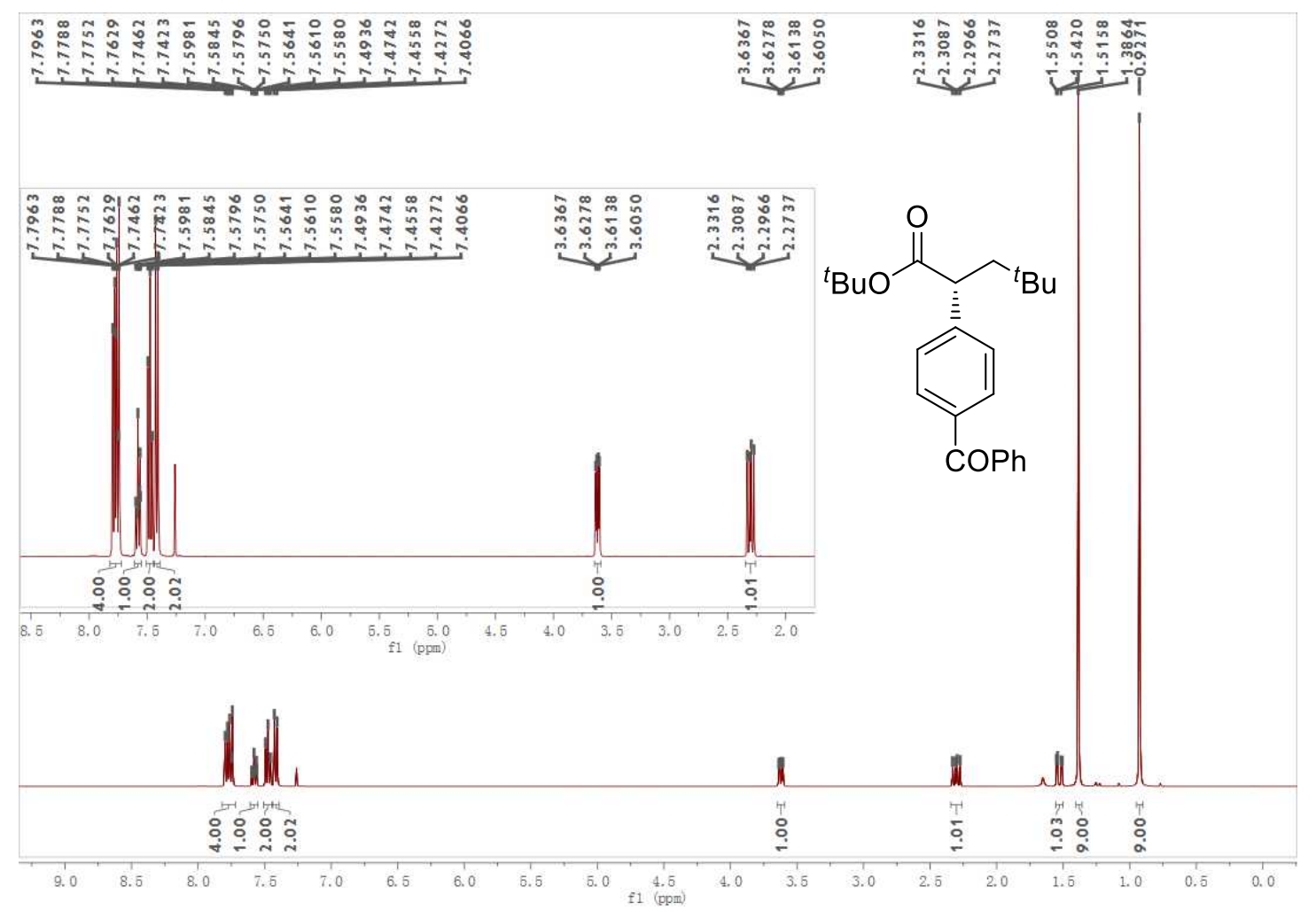


7; ${ }^{13} \mathrm{C}$-NMR (100 MHz, $\mathrm{CDCl}_{3}$ )

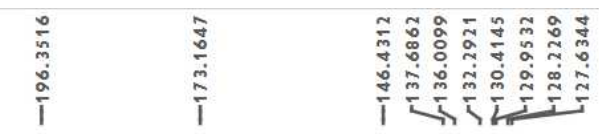<smiles>CC(C)(C)OC(=O)C(C[18O])c1ccc(C(=O)c2ccccc2)cc1</smiles>
Bu
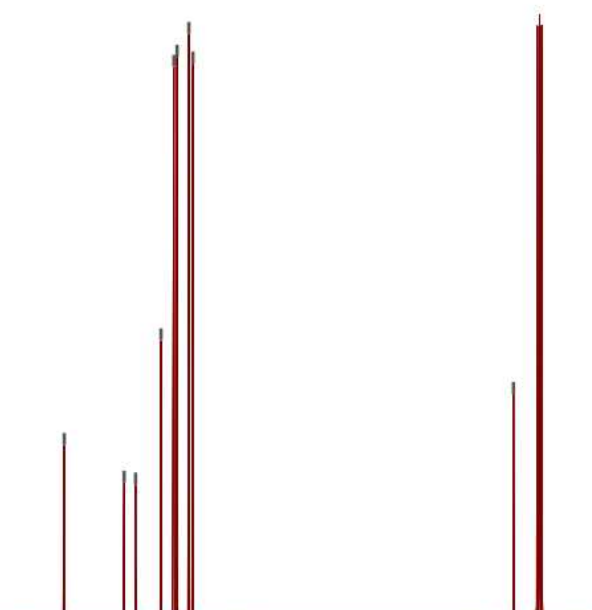

$\begin{array}{lllllllllll}200 & 190 & 180 & 170 & 160 & 160 & 140 & 130 & 120 & 110 & 100 \\ \mathrm{f} 1 & 1(\mathrm{ppm})\end{array}$

8; ${ }^{1} \mathrm{H}-\mathrm{NMR}\left(400 \mathrm{MHz}, \mathrm{CDCl}_{3}\right)$

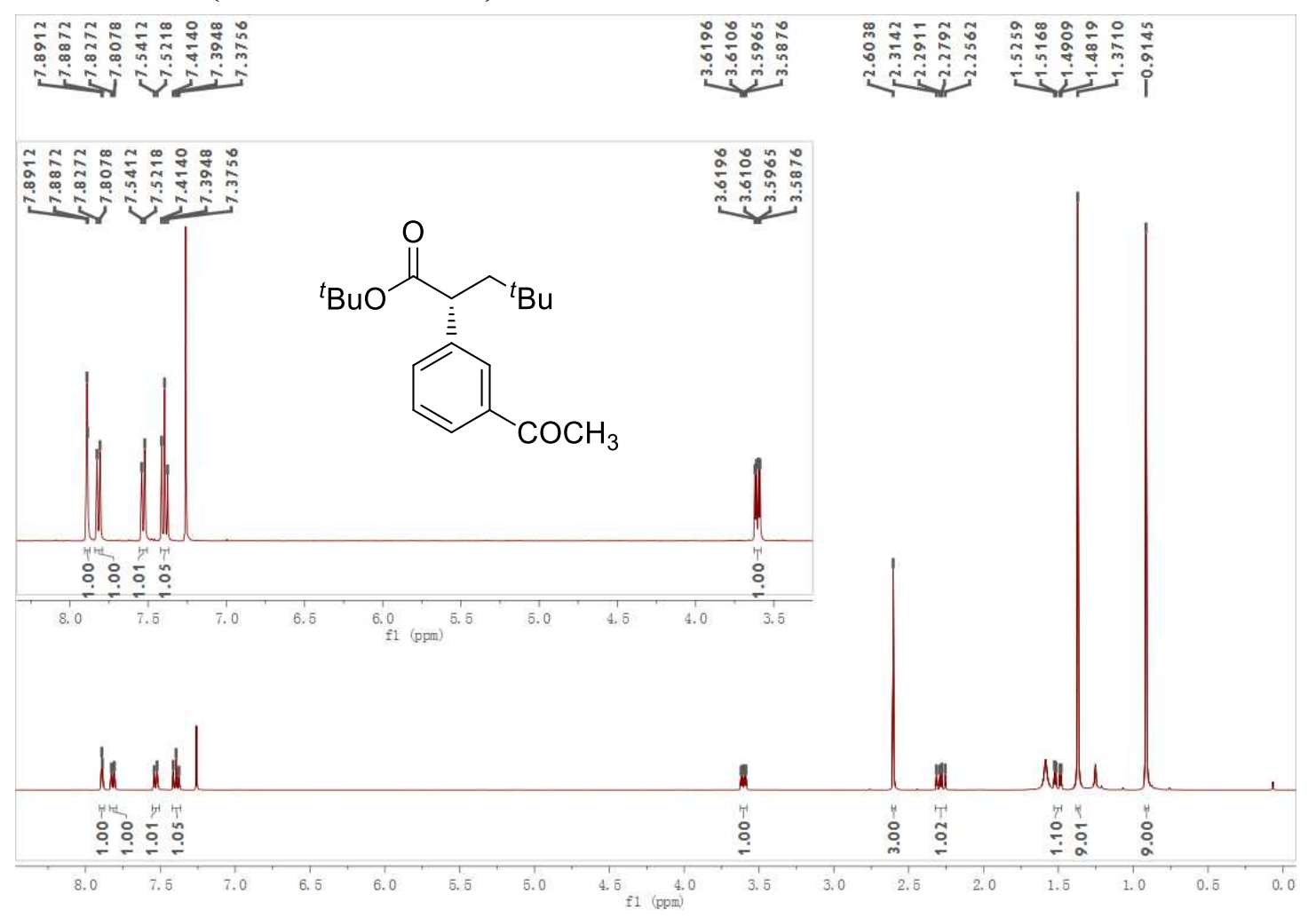

8; ${ }^{13} \mathrm{C}-\mathrm{NMR}\left(100 \mathrm{MHz}, \mathrm{CDCl}_{3}\right)$ 


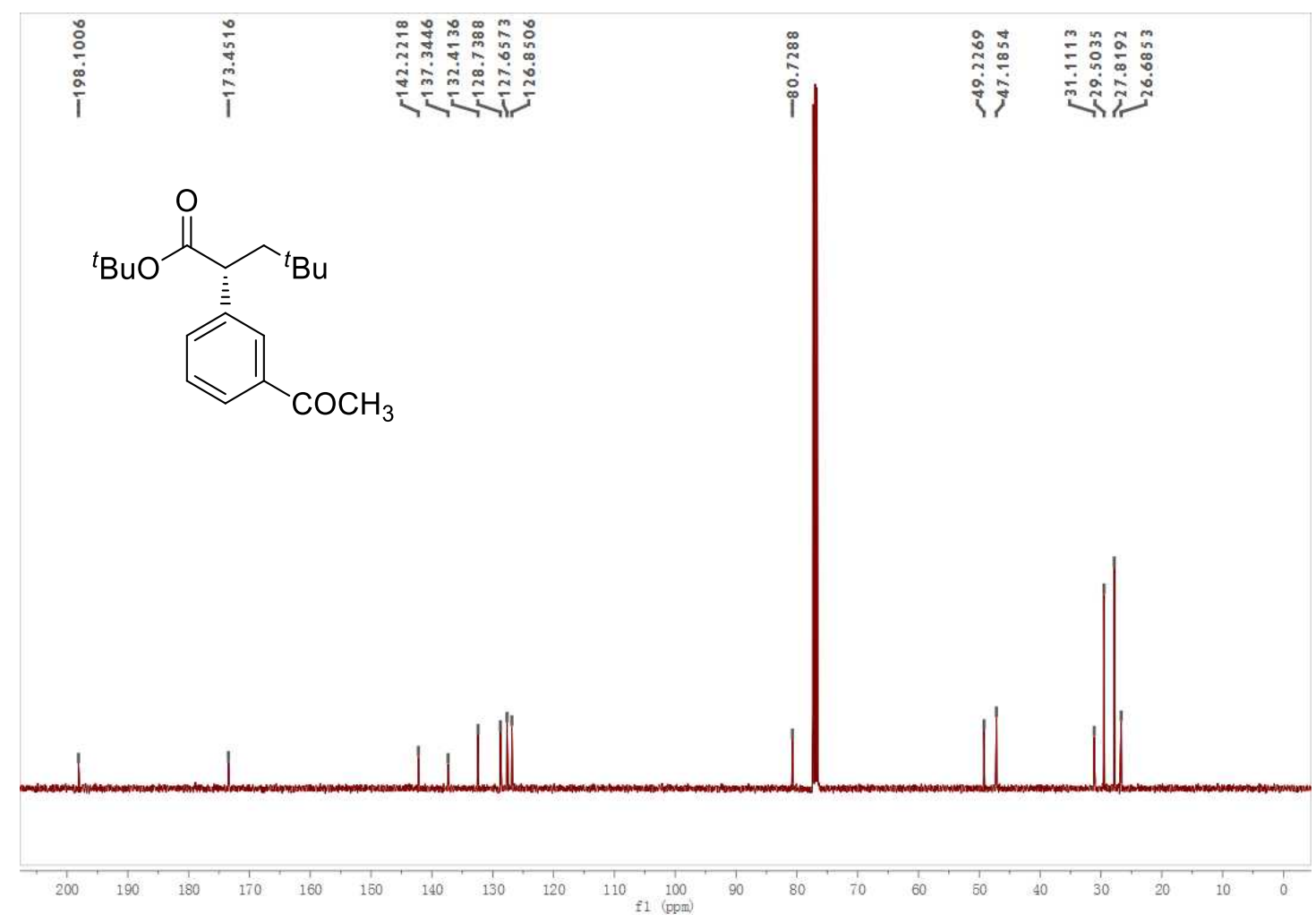

9; ${ }^{1} \mathrm{H}-\mathrm{NMR}$ (400 MHz, $\mathrm{CDCl}_{3}$ )

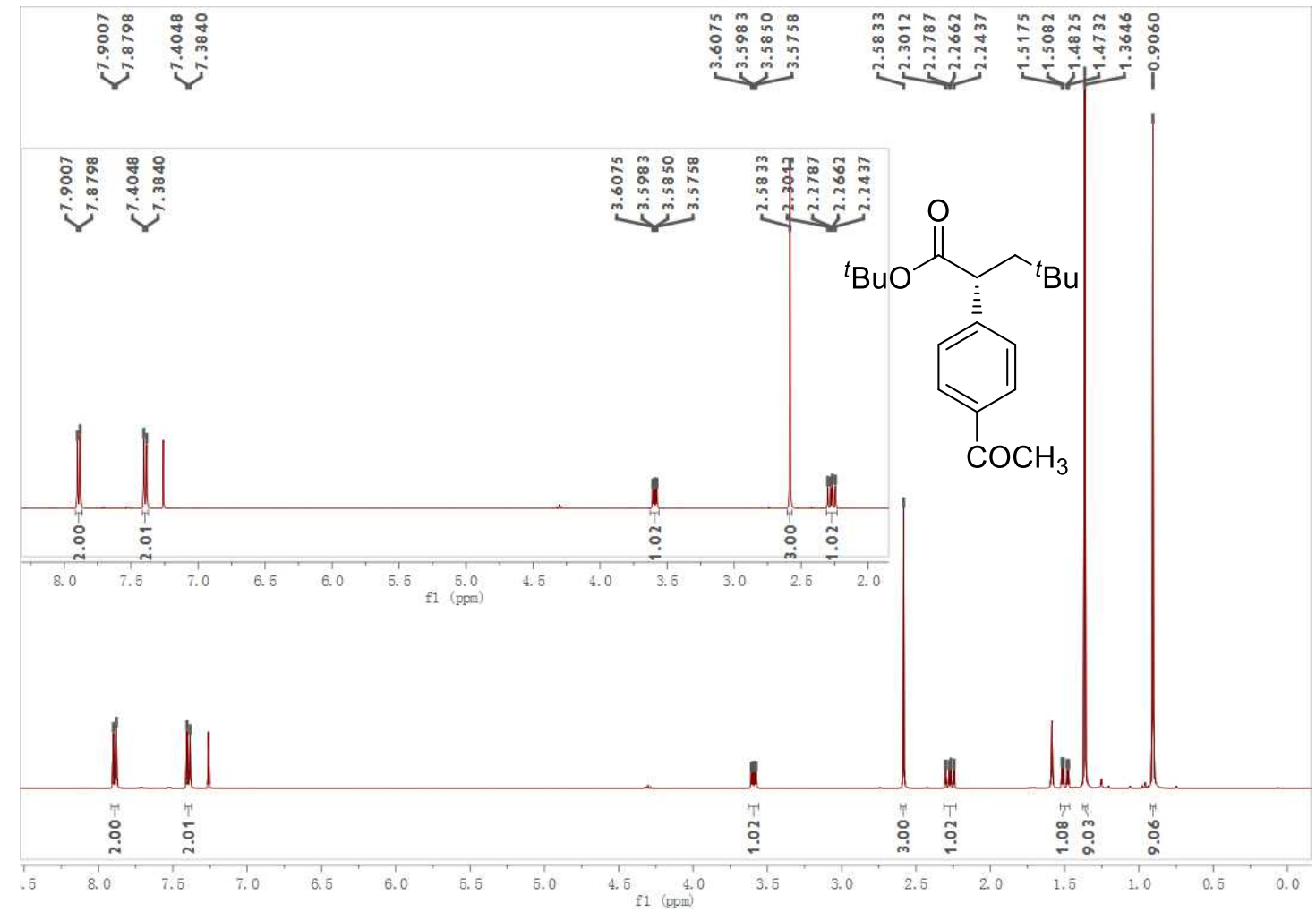


9; ${ }^{13} \mathrm{C}-\mathrm{NMR}\left(100 \mathrm{MHz}, \mathrm{CDCl}_{3}\right)$

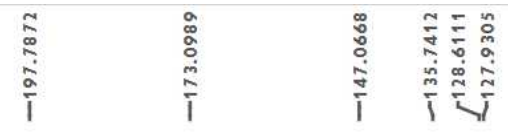<smiles>CC(=O)OC(=O)C(CBr)c1ccc(C(C)(C)C)cc1</smiles>

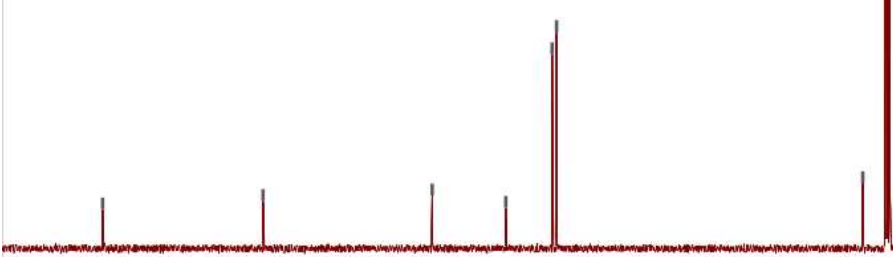

$210 \quad 200$

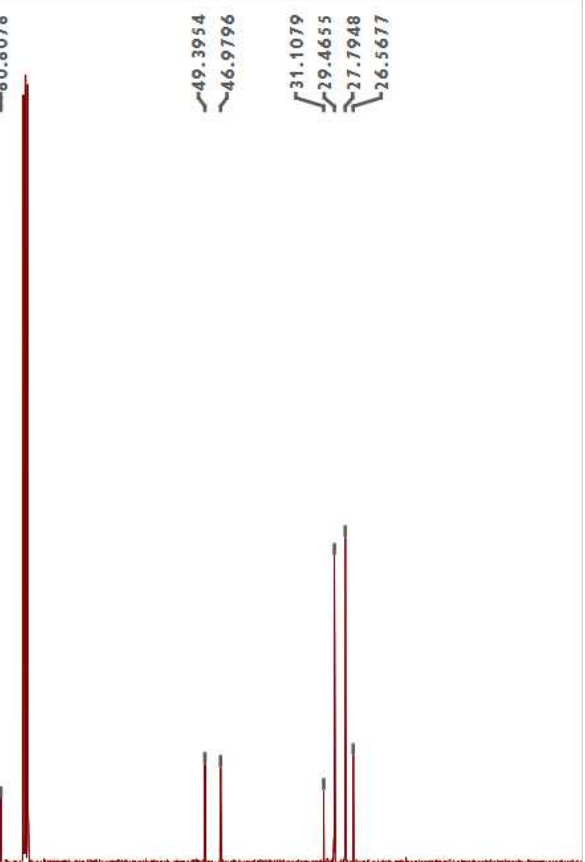

10; ${ }^{1} \mathrm{H}-\mathrm{NMR}$ (400 MHz, $\mathrm{CDCl}_{3}$ )

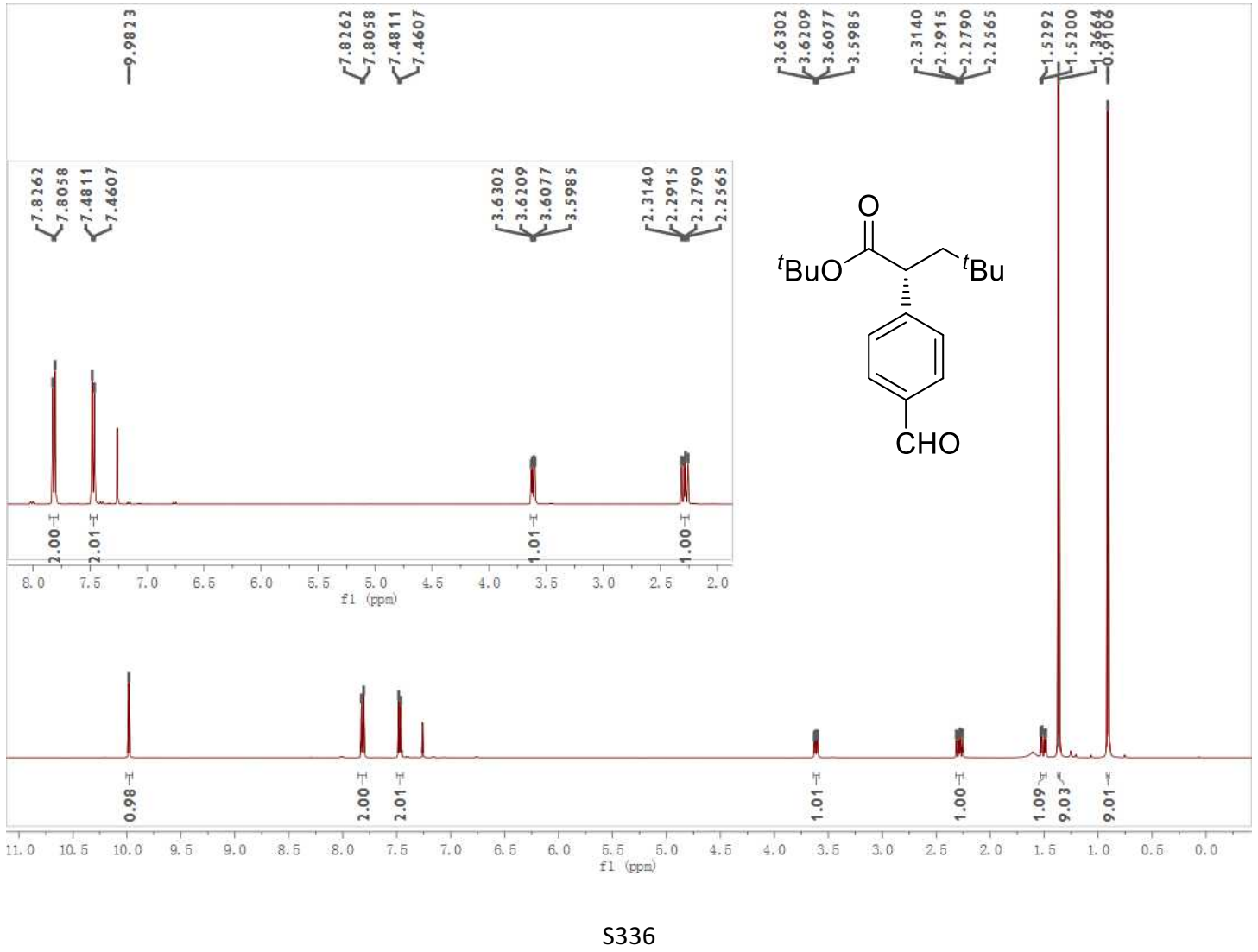


10; ${ }^{13} \mathrm{C}-\mathrm{NMR}\left(100 \mathrm{MHz}, \mathrm{CDCl}_{3}\right)$

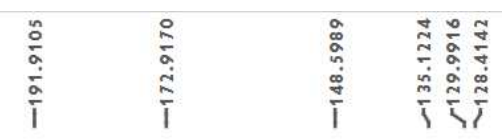

(CHO

11; ${ }^{1} \mathrm{H}-\mathrm{NMR}\left(400 \mathrm{MHz}, \mathrm{CDCl}_{3}\right)$

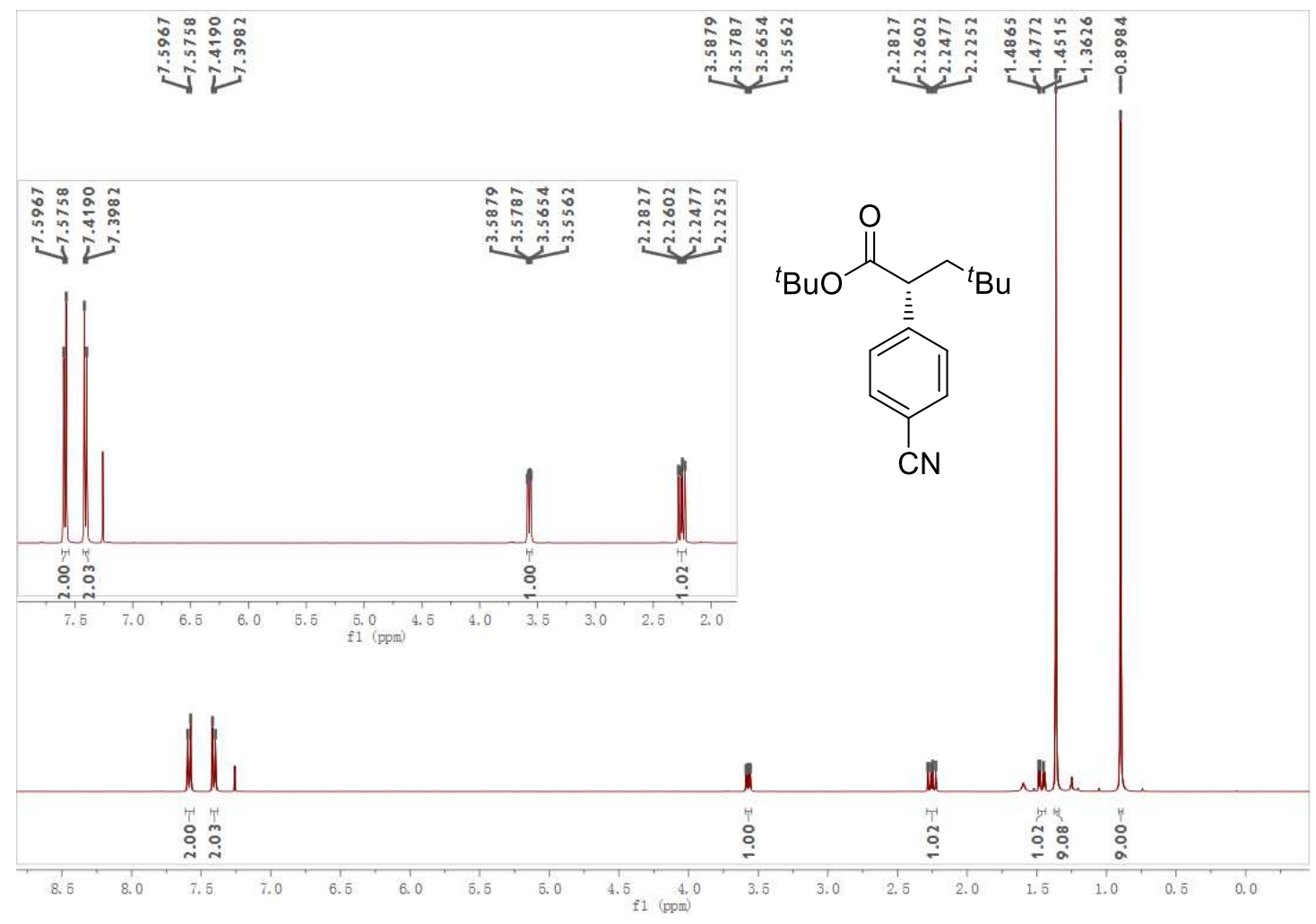


11; ${ }^{13} \mathrm{C}$-NMR (100 MHz, $\mathrm{CDCl}_{3}$ )

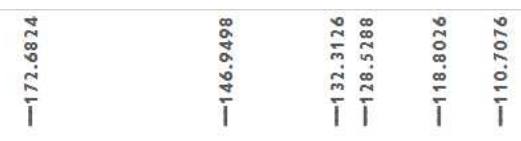<smiles>CCCOC(=O)C(CC(C)CC)c1ccc(C#N)cc1</smiles>

12; ${ }^{1} \mathrm{H}-\mathrm{NMR}$ (400 MHz, $\mathrm{CDCl}_{3}$ )

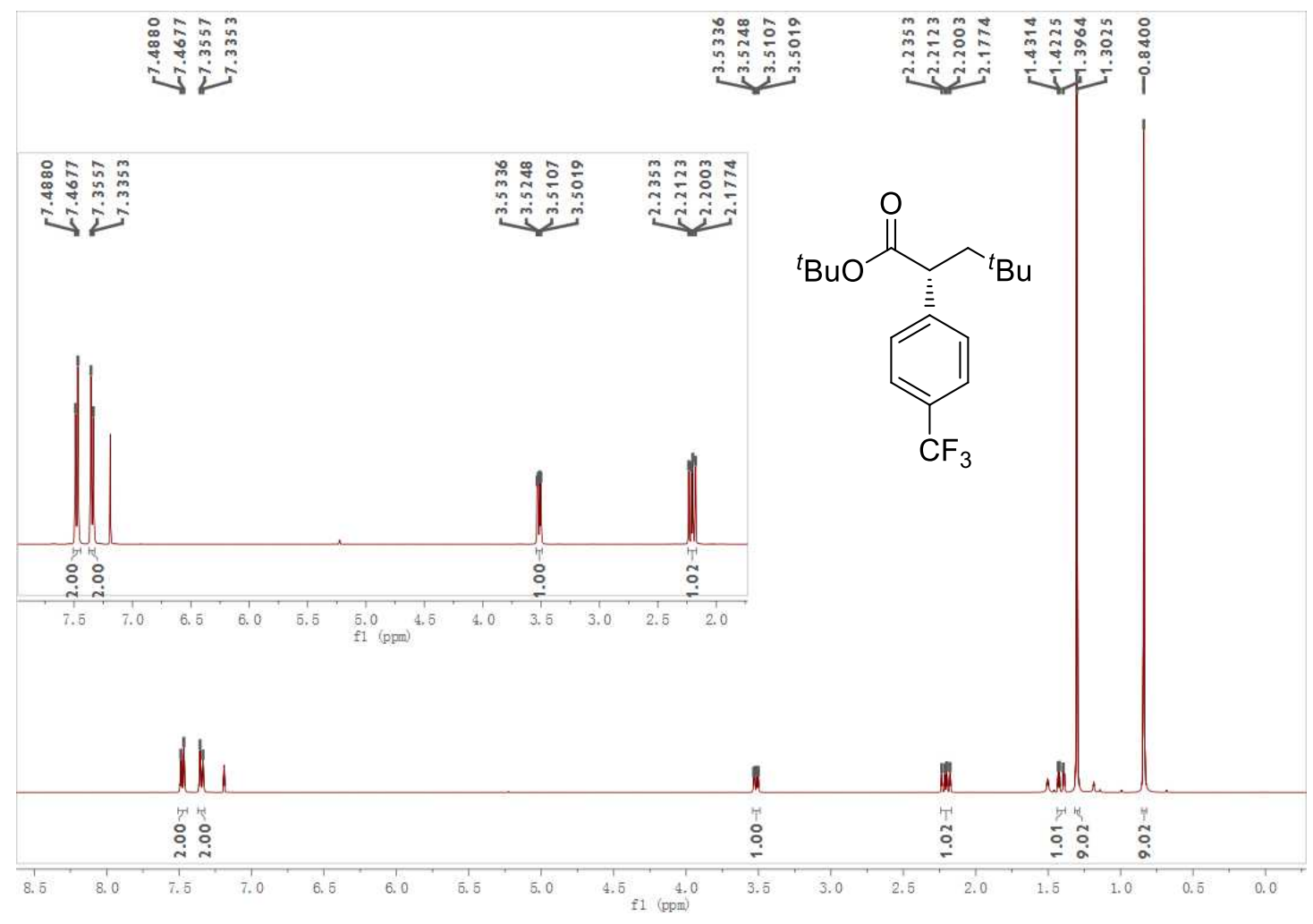


12; ${ }^{19}$ F-NMR (375 MHz, $\left.\mathrm{CDCl}_{3}\right)$

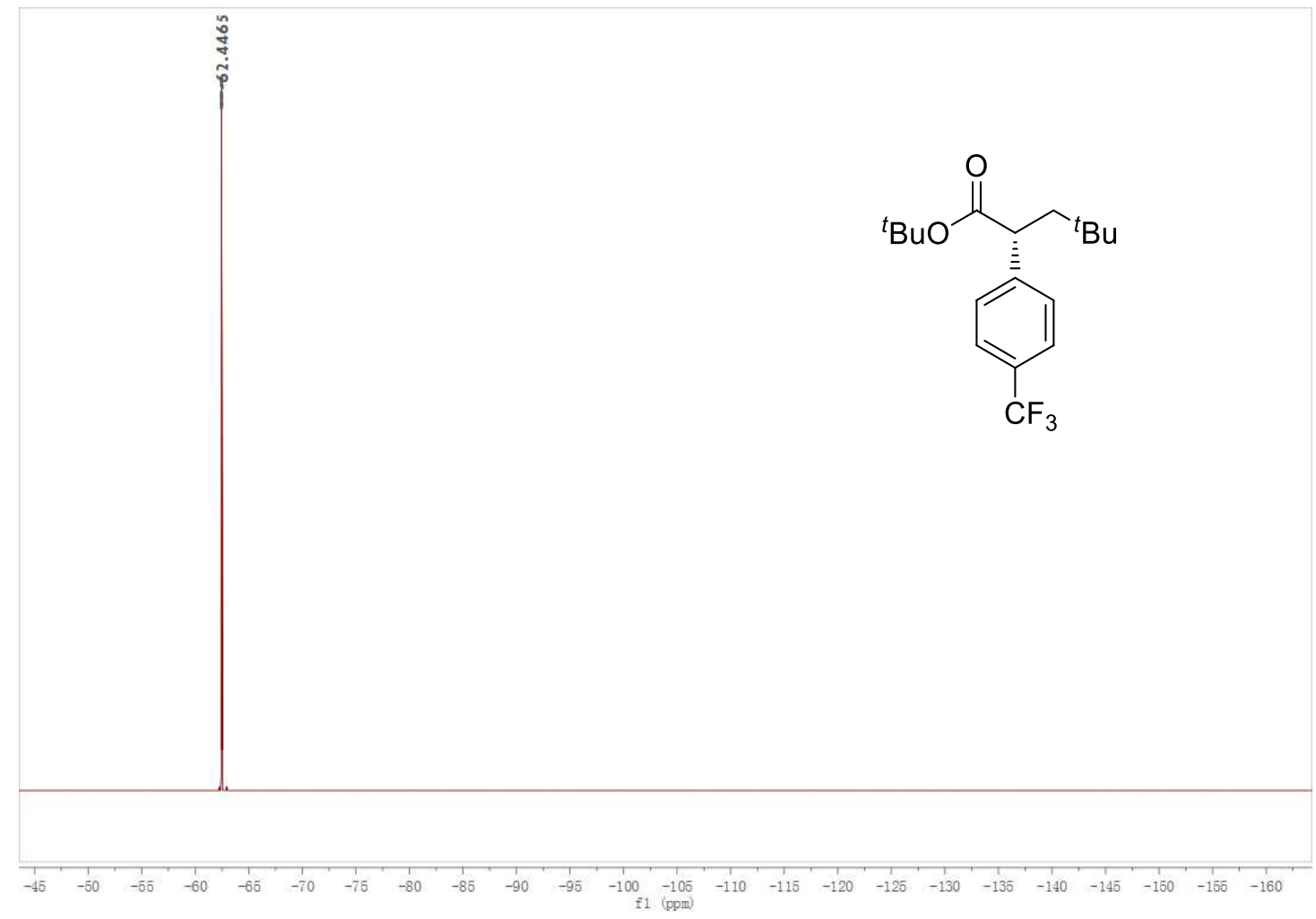

12; ${ }^{13} \mathrm{C}-\mathrm{NMR}\left(100 \mathrm{MHz}, \mathrm{CDCl}_{3}\right)$

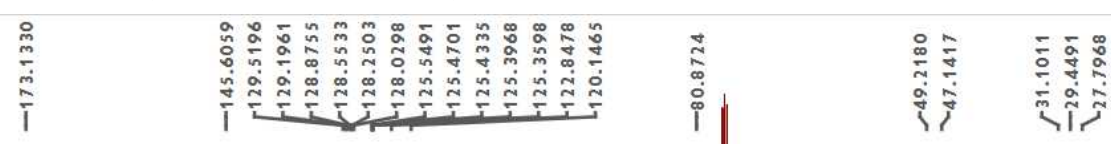<smiles>CC(C)(C)CC(CCBr)c1ccc(C(F)(F)F)cc1</smiles>

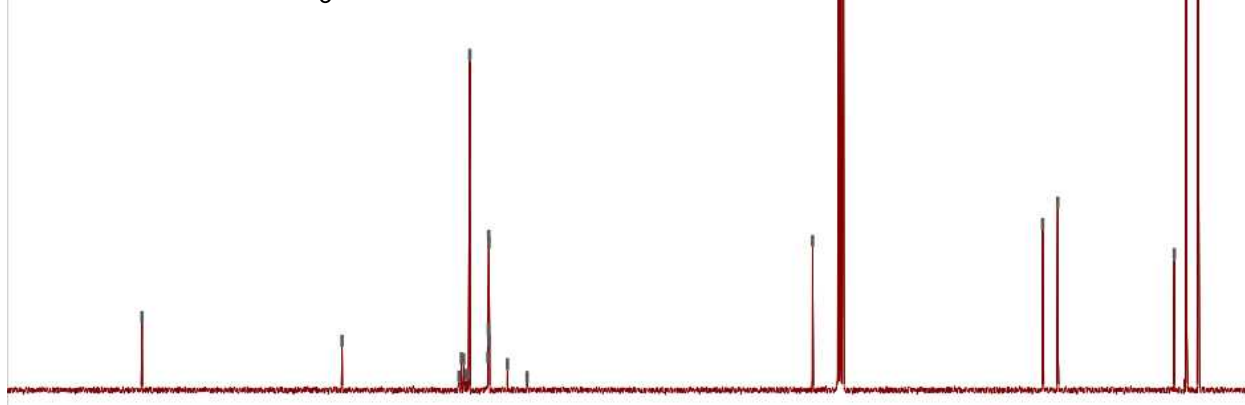

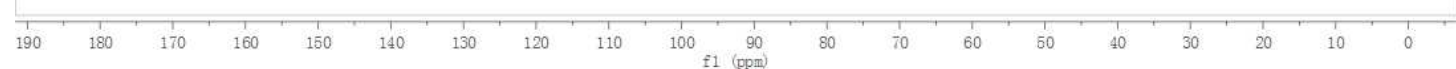


13; ${ }^{1} \mathrm{H}-\mathrm{NMR}$ (400 MHz, $\mathrm{CDCl}_{3}$ )

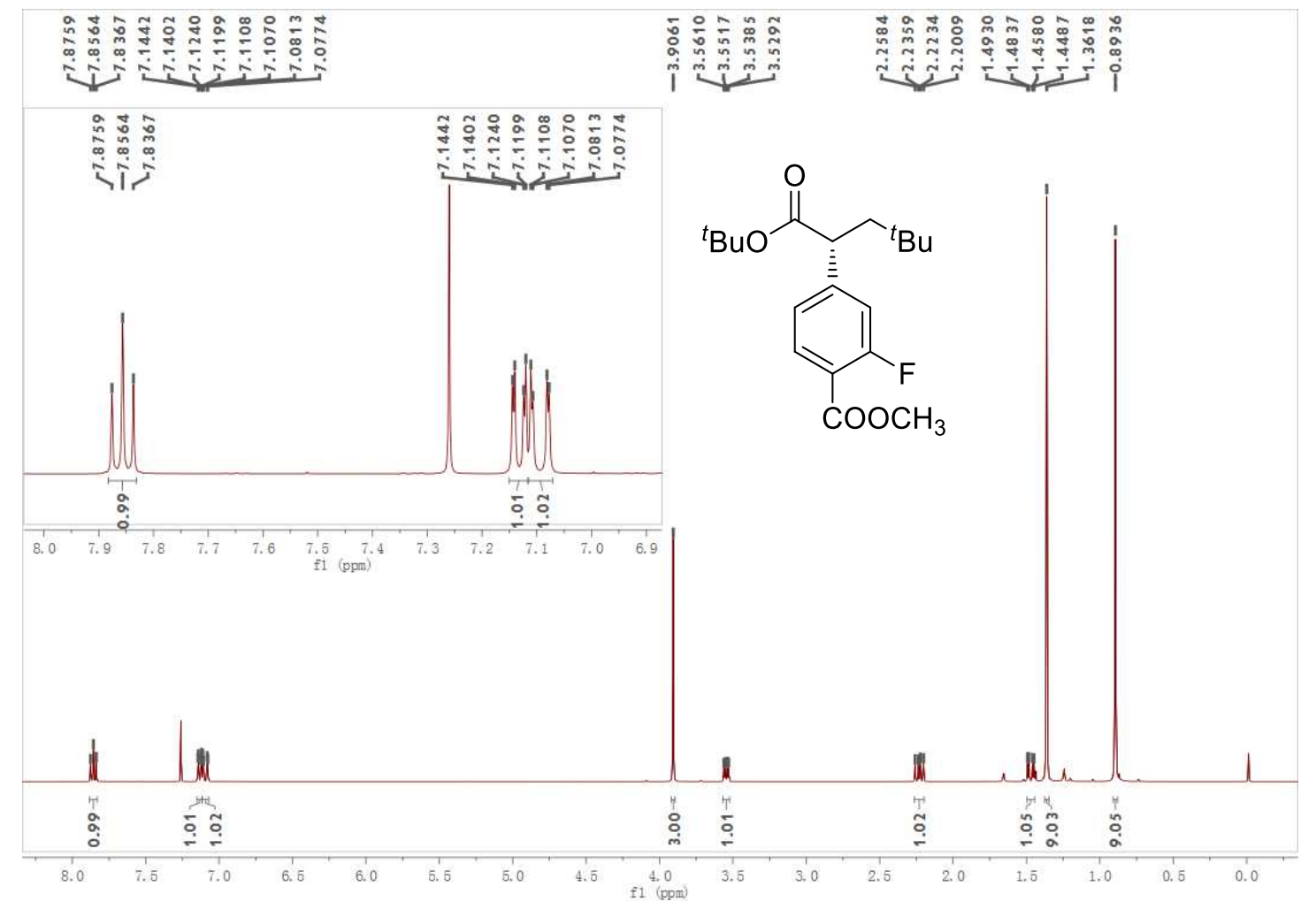

13; ${ }^{19}$ F-NMR (375 MHz, $\left.\mathrm{CDCl}_{3}\right)$

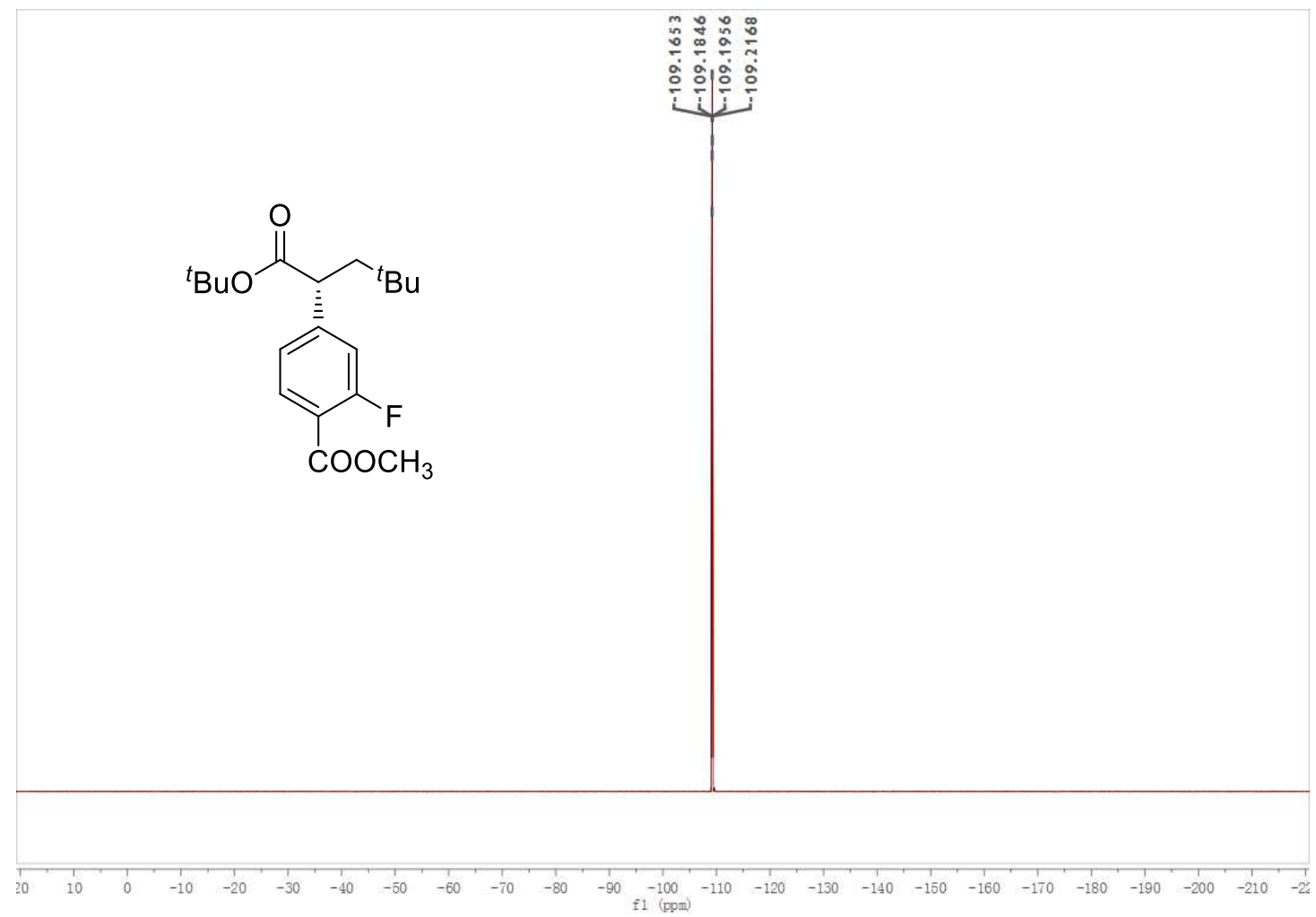


13; ${ }^{13} \mathrm{C}$-NMR (100 MHz, $\left.\mathrm{CDCl}_{3}\right)$

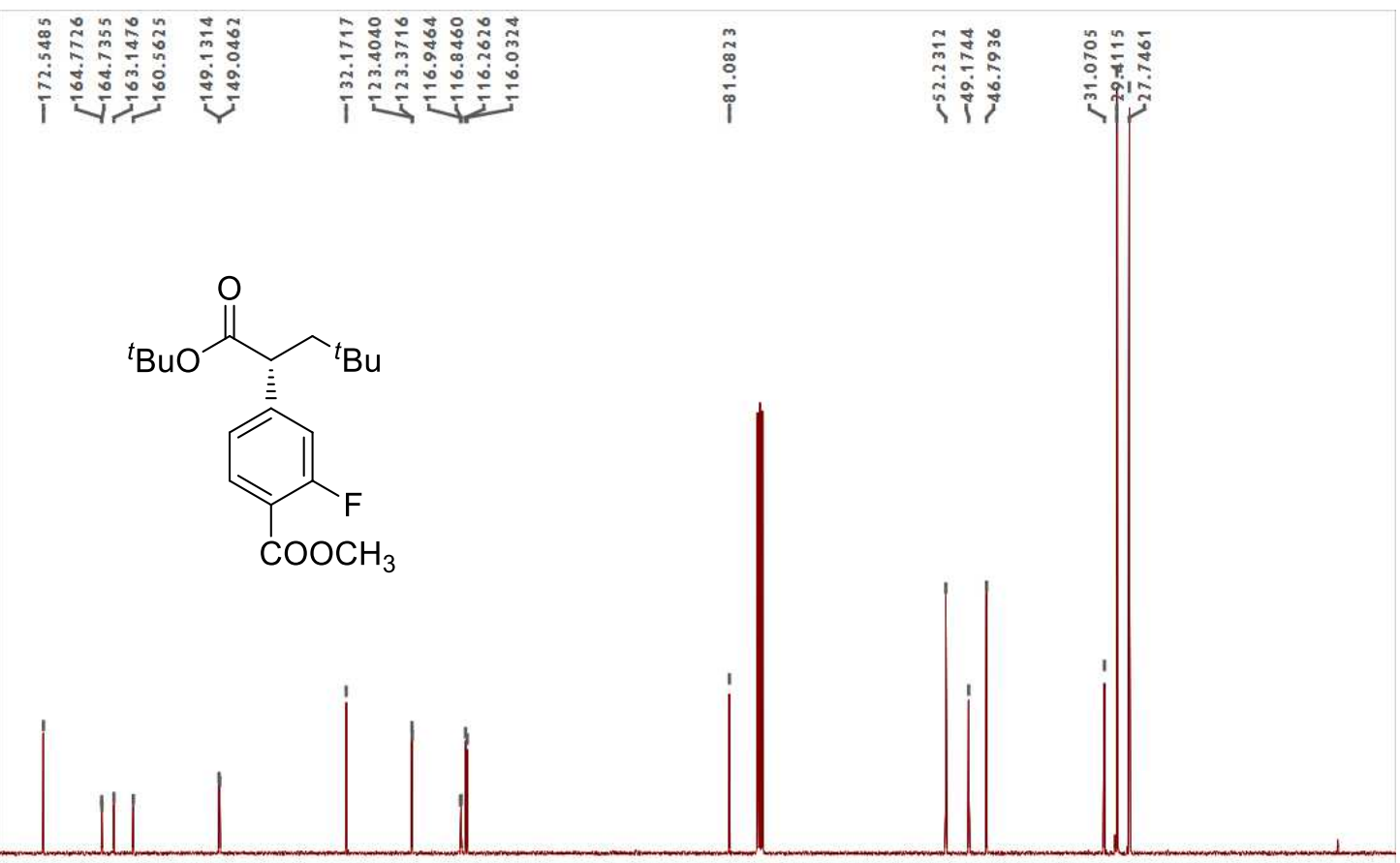

14; ${ }^{1} \mathrm{H}-\mathrm{NMR}$ (400 MHz, $\mathrm{CDCl}_{3}$ )

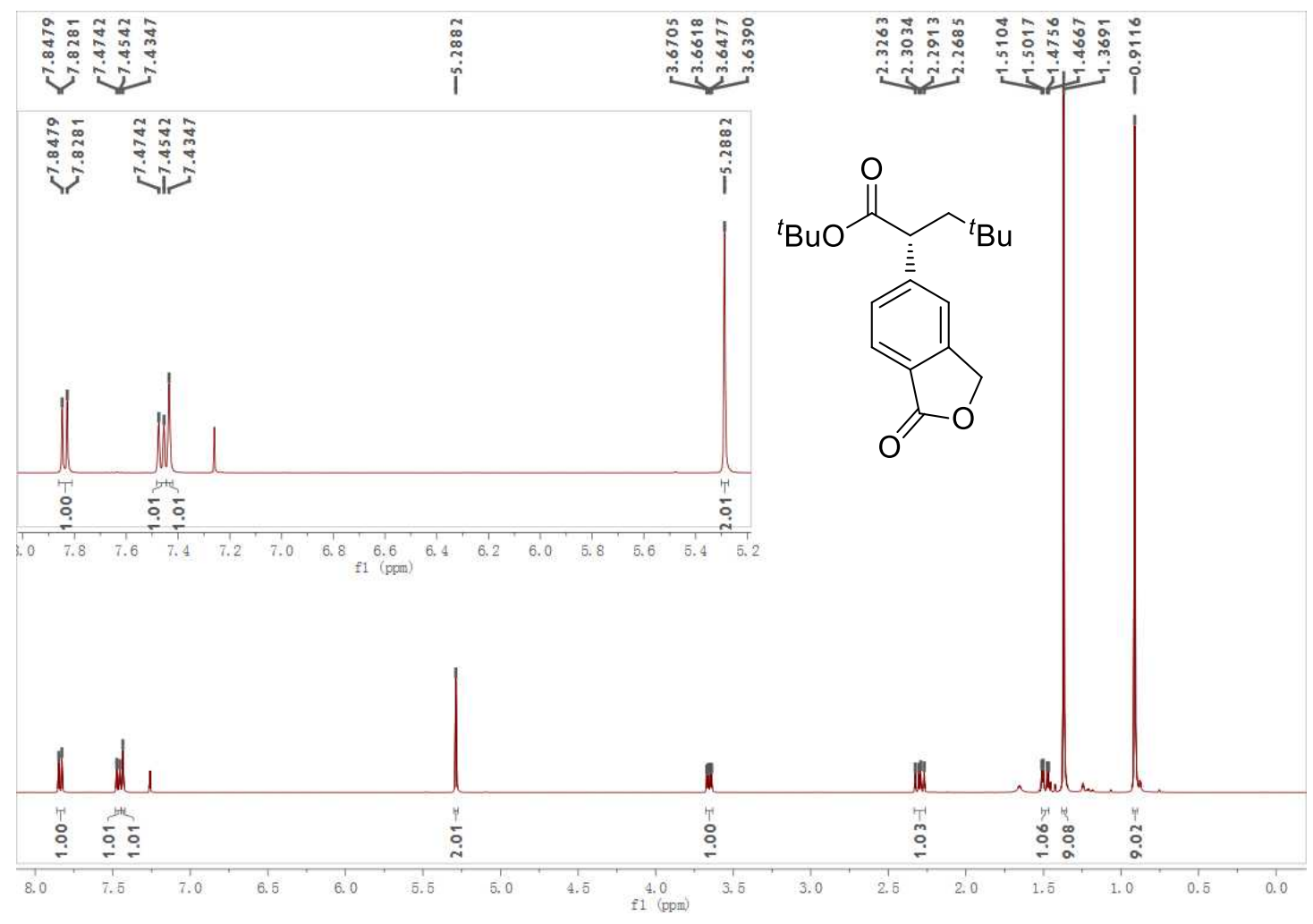


14; ${ }^{13} \mathrm{C}$-NMR (100 MHz, $\left.\mathrm{CDCl}_{3}\right)$

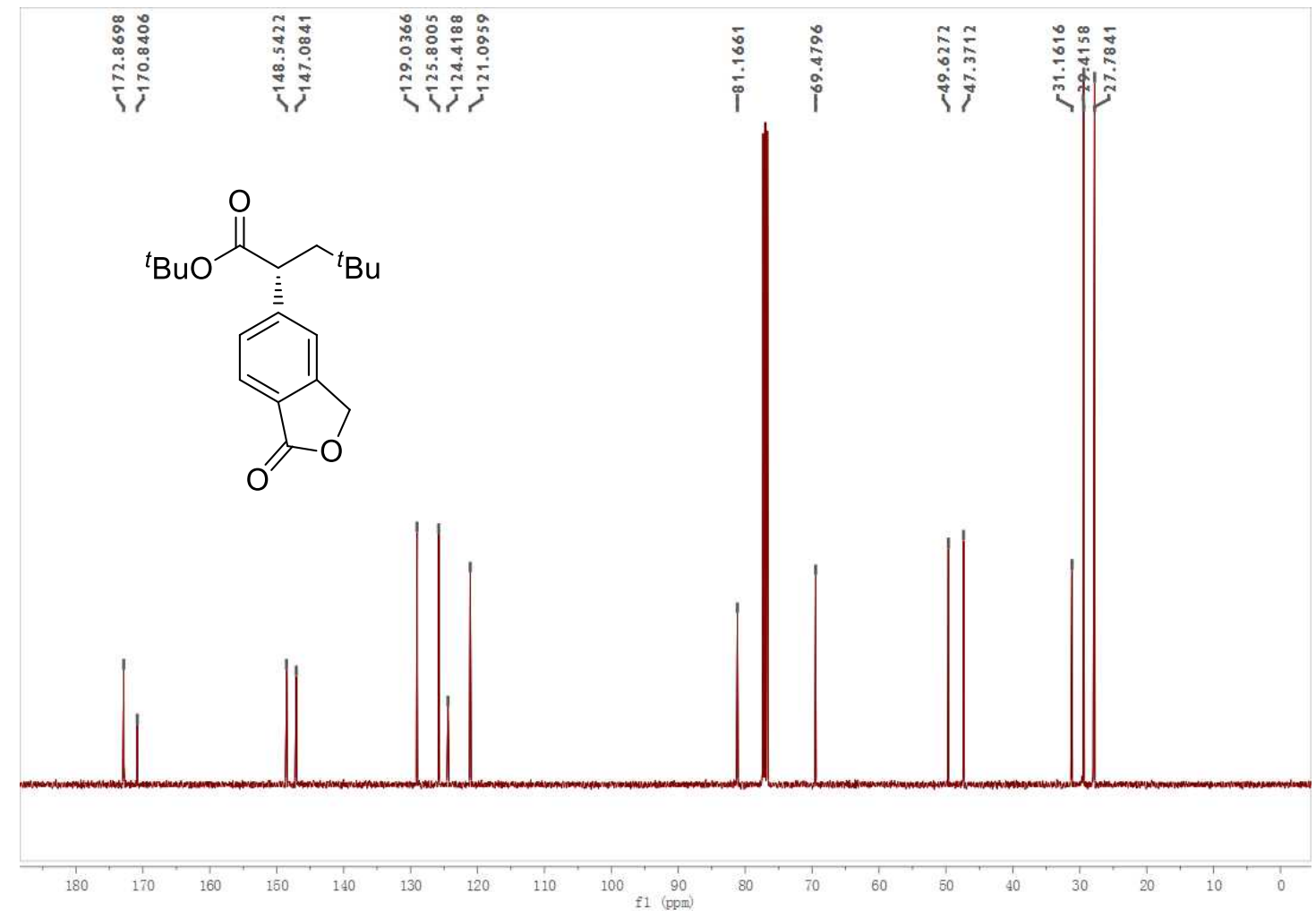

15; ${ }^{1} \mathrm{H}-\mathrm{NMR}$ (400 MHz, $\mathrm{CDCl}_{3}$ )

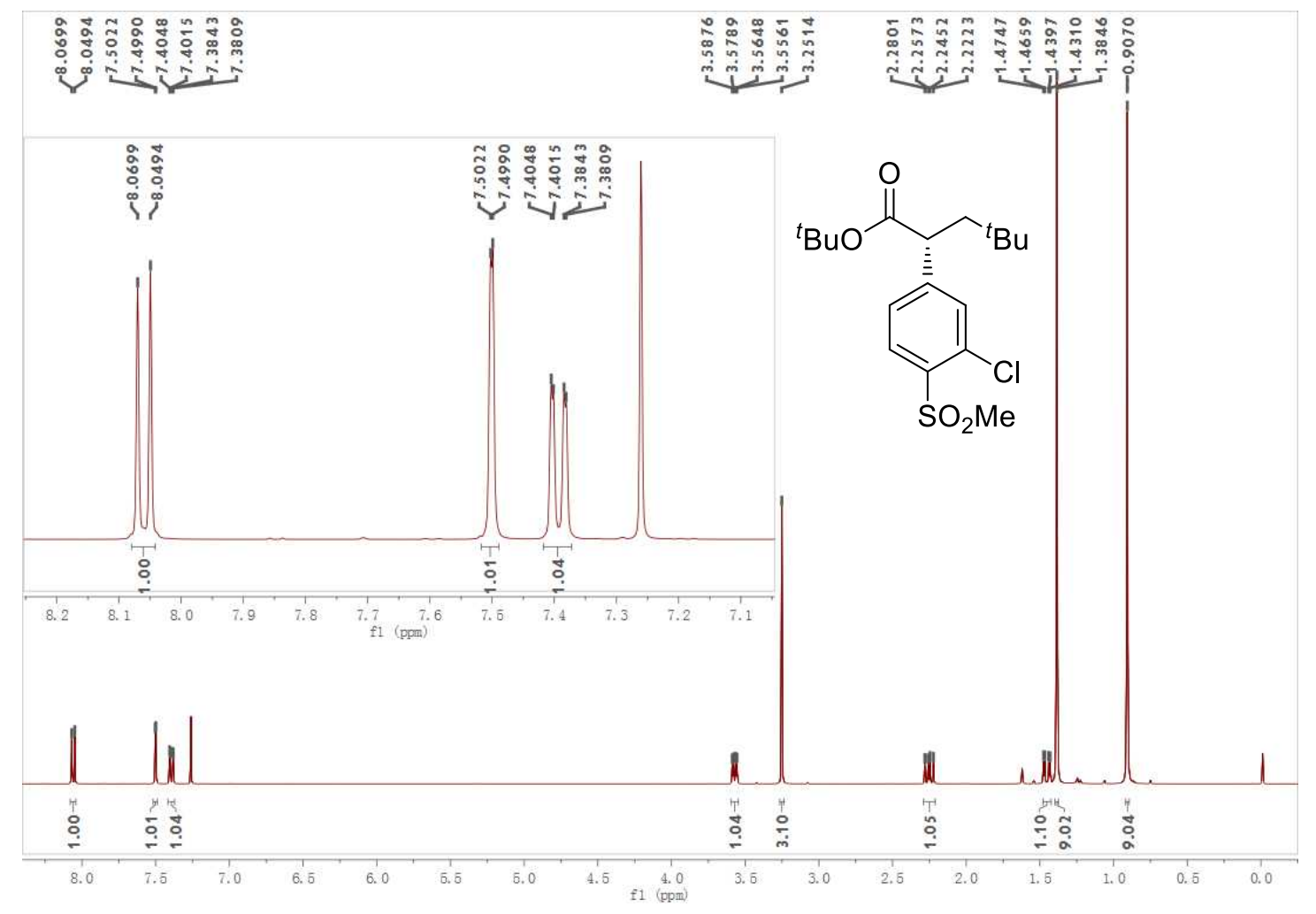


$15 ;{ }^{13} \mathrm{C}-\mathrm{NMR}\left(100 \mathrm{MHz}, \mathrm{CDCl}_{3}\right)$

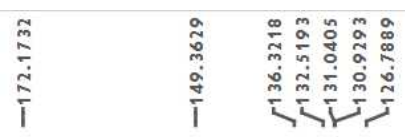

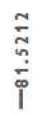

0
0
0<smiles>CC(C)(C)OC(=O)C(CBr)c1ccc(OS(=O)(=O)OC(C)(C)C)c(Cl)c1</smiles>

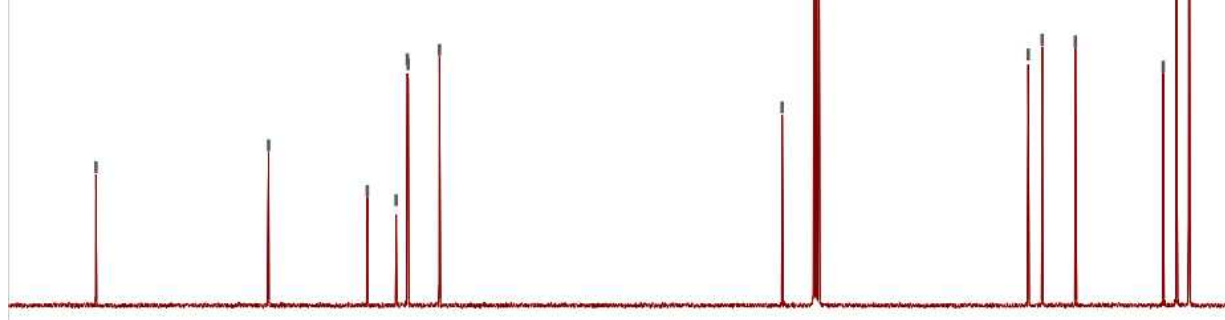

$\begin{array}{llllllllll}180 & 170 & 160 & 150 & 140 & 130 & 120 & 110 & 100 & 90 \\ \mathrm{f1}(\mathrm{ppm})\end{array}$

16; ${ }^{1} \mathrm{H}-\mathrm{NMR}\left(400 \mathrm{MHz}, \mathrm{CDCl}_{3}\right)$

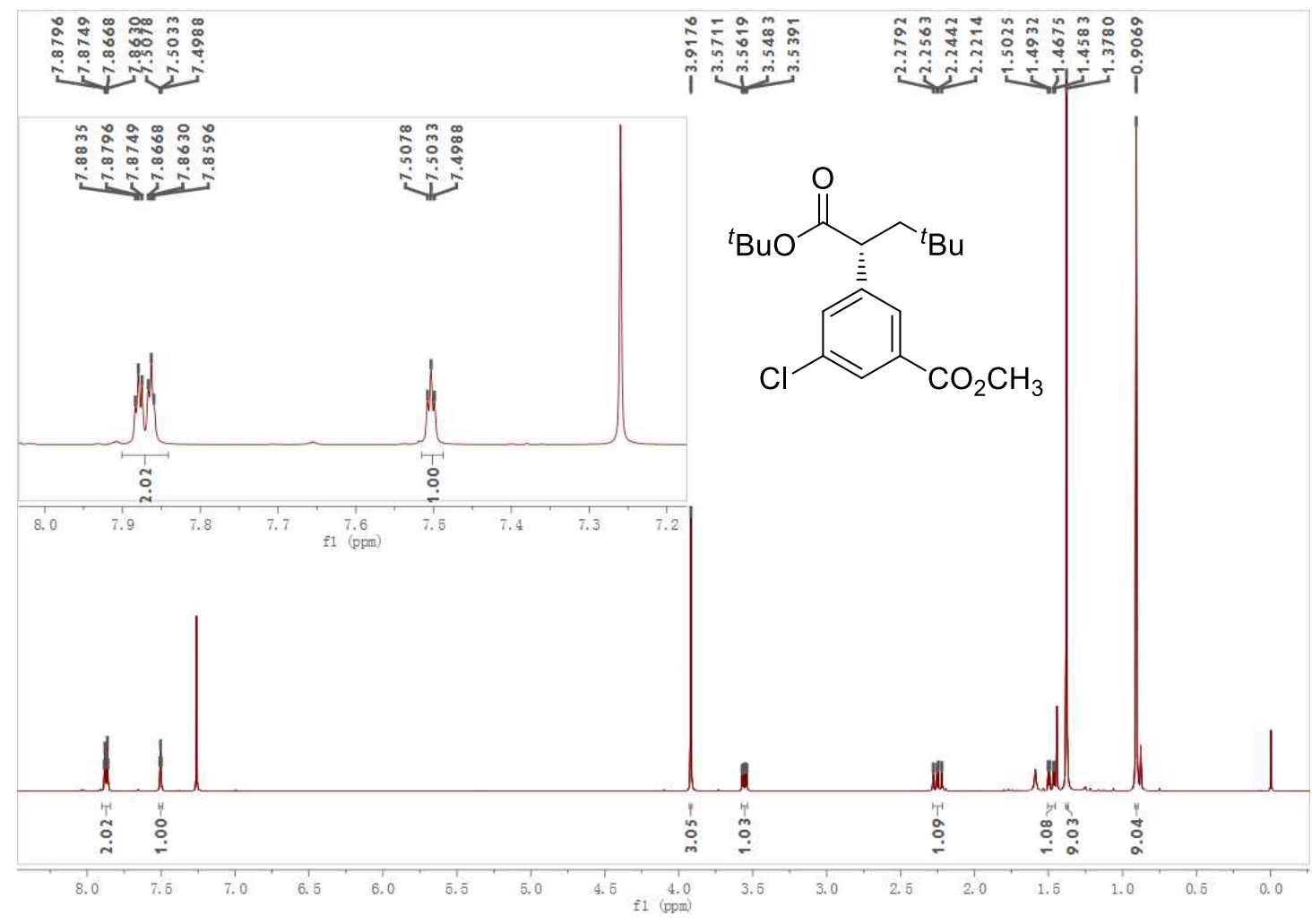


16; ${ }^{13} \mathrm{C}-\mathrm{NMR}$ (100 MHz, $\left.\mathrm{CDCl}_{3}\right)$

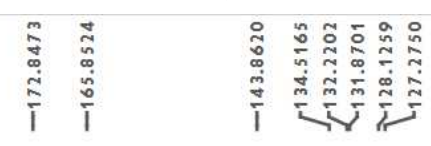<smiles>CCCCCC(=O)OC(C)(C)C</smiles><smiles>COC(=O)c1cccc(Cl)c1</smiles>

17; ${ }^{1} \mathrm{H}-\mathrm{NMR}$ (400 MHz, $\mathrm{CDCl}_{3}$ )

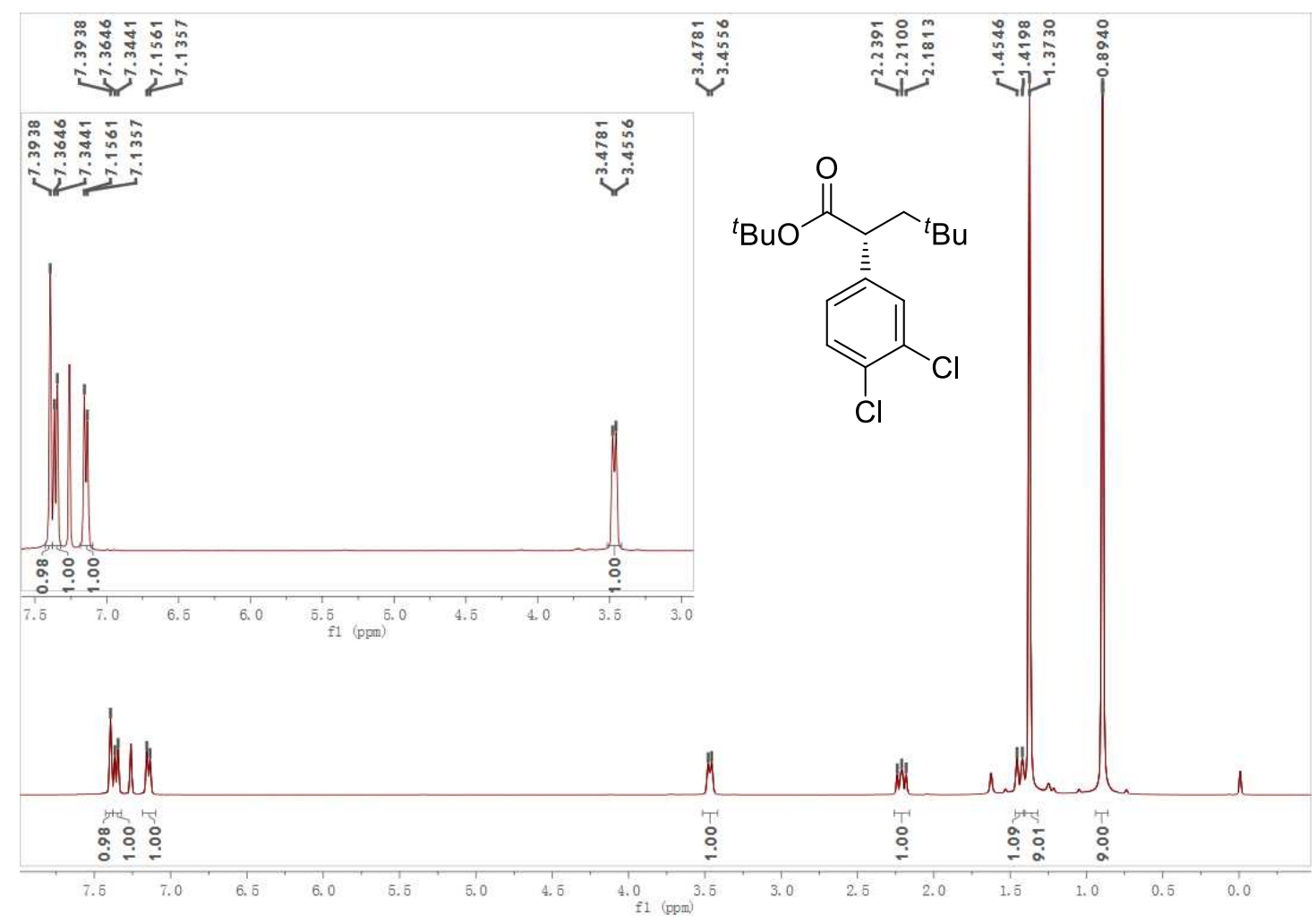


17; ${ }^{13} \mathrm{C}-\mathrm{NMR}\left(100 \mathrm{MHz}, \mathrm{CDCl}_{3}\right)$

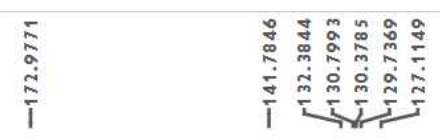

$\approx$
$\vdots$
$\vdots$
$\vdots$
$i$<smiles>CCCCC(=O)OC(=O)CCCCCC(C)(C)C</smiles>

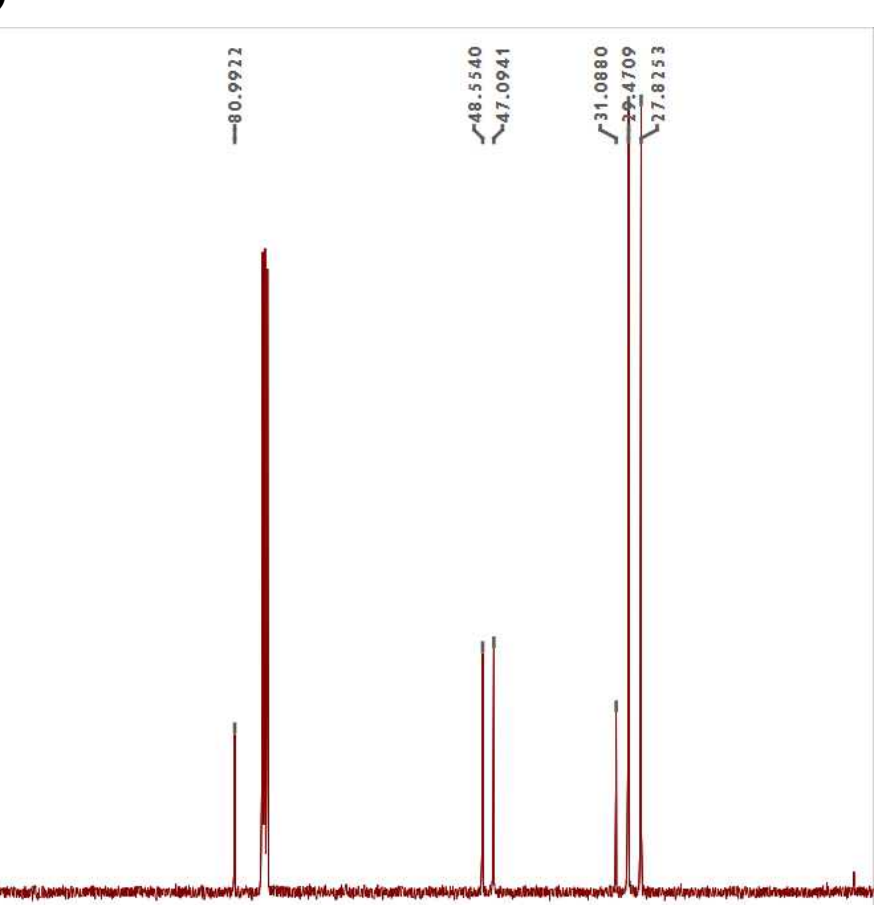

$\begin{array}{llllllllll}180 & 170 & 160 & 150 & 140 & 130 & 120 & 110 & 100 & 90 \\ \mathrm{f} 1(\mathrm{ppm}) & \end{array}$

18; ${ }^{1} \mathrm{H}-\mathrm{NMR}\left(400 \mathrm{MHz}, \mathrm{CDCl}_{3}\right)$

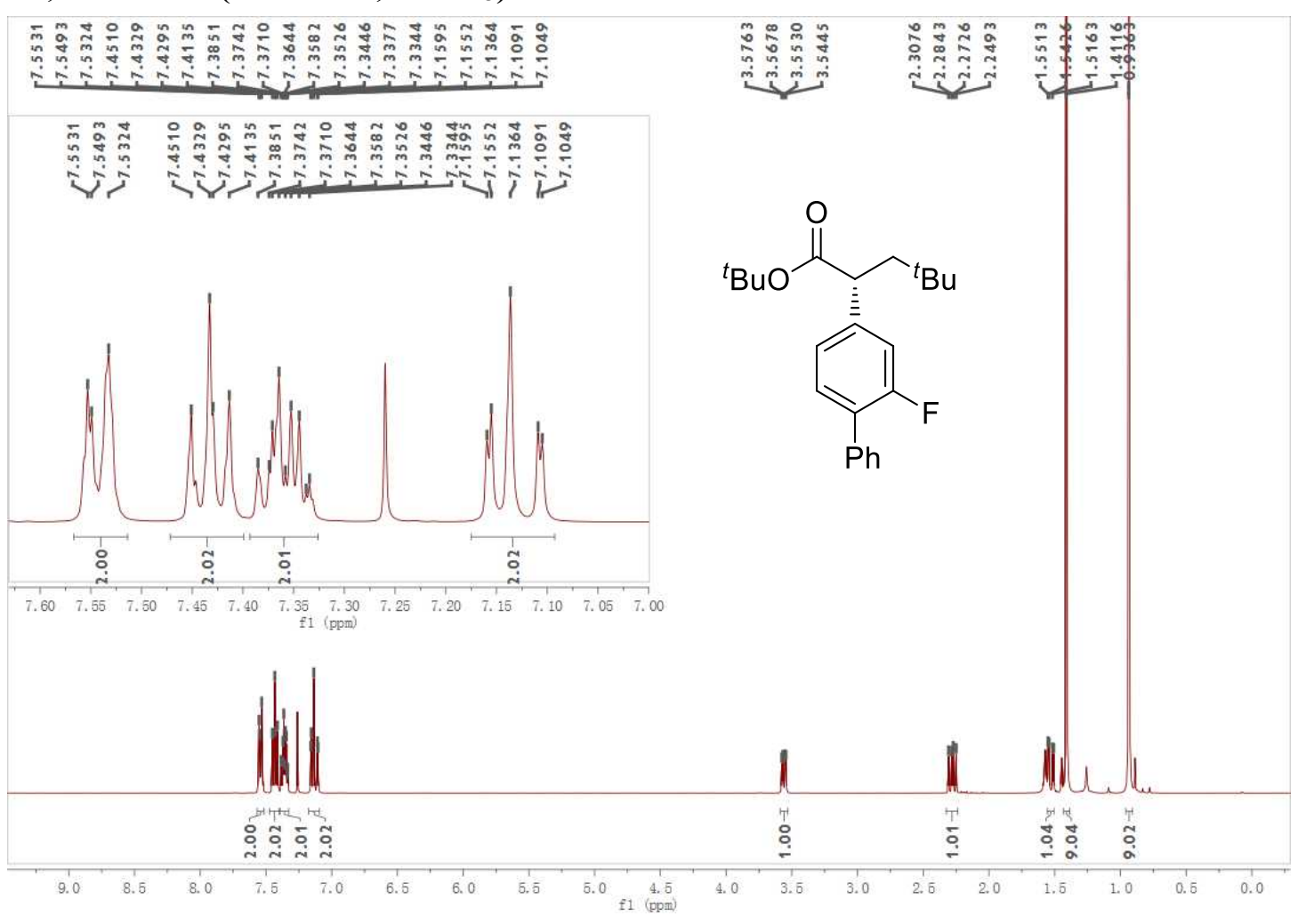


18; ${ }^{19}$ F-NMR (375 MHz, $\left.\mathrm{CDCl}_{3}\right)$

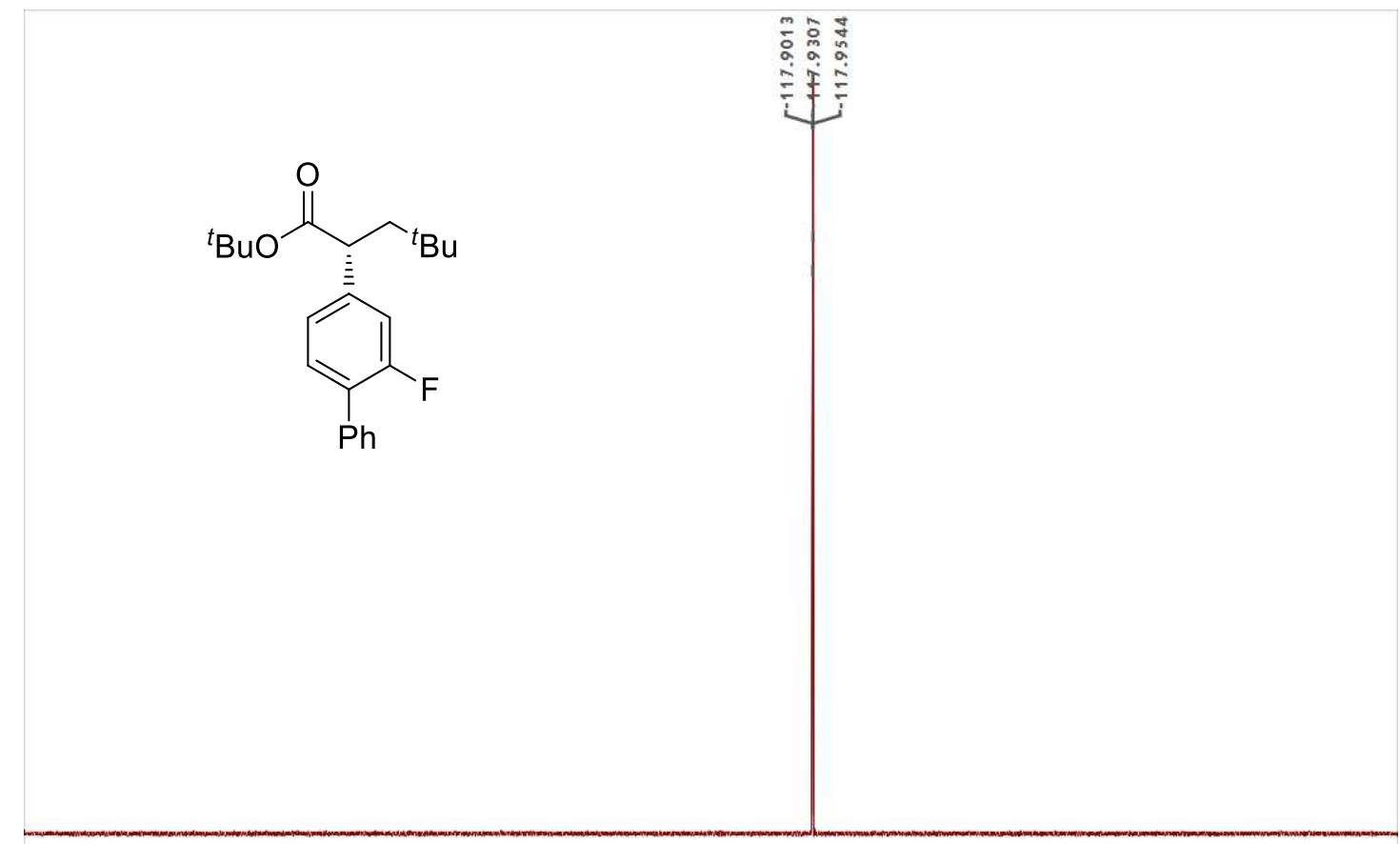

18; ${ }^{13} \mathrm{C}-\mathrm{NMR}\left(100 \mathrm{MHz}, \mathrm{CDCl}_{3}\right)$

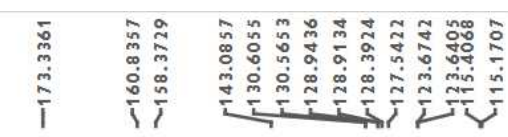

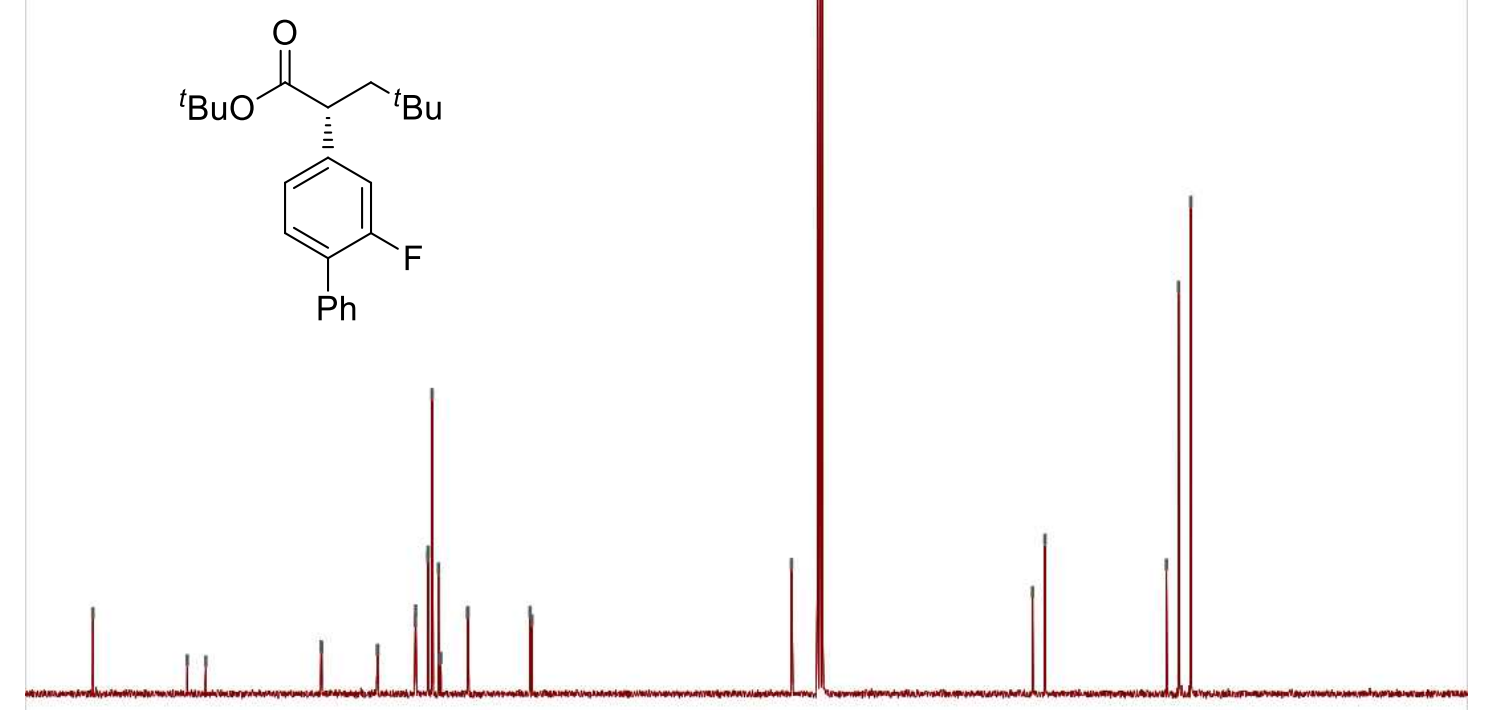

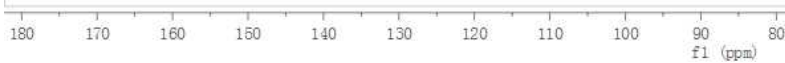


19; ${ }^{1} \mathrm{H}-\mathrm{NMR}$ (400 MHz, $\mathrm{CDCl}_{3}$ )

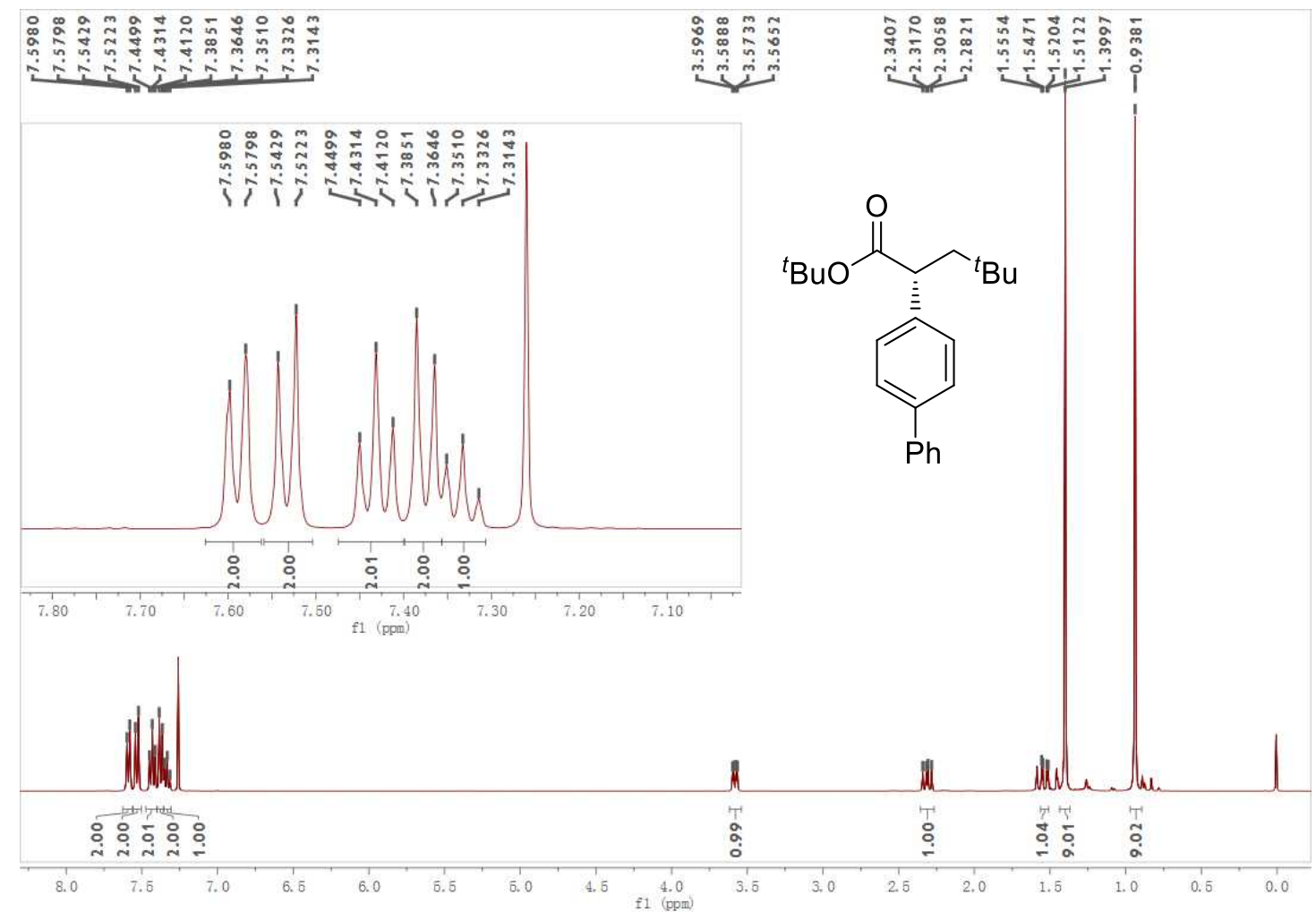

19; ${ }^{13} \mathrm{C}$-NMR (100 MHz, $\left.\mathrm{CDCl}_{3}\right)$

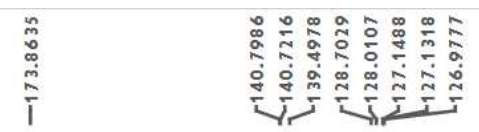<smiles>CC(C)(C)CC(C(=O)OC(C)(C)C)c1ccc(-c2ccccc2)cc1</smiles> 
20; ${ }^{1} \mathrm{H}-\mathrm{NMR}$ (400 MHz, $\mathrm{CDCl}_{3}$ )

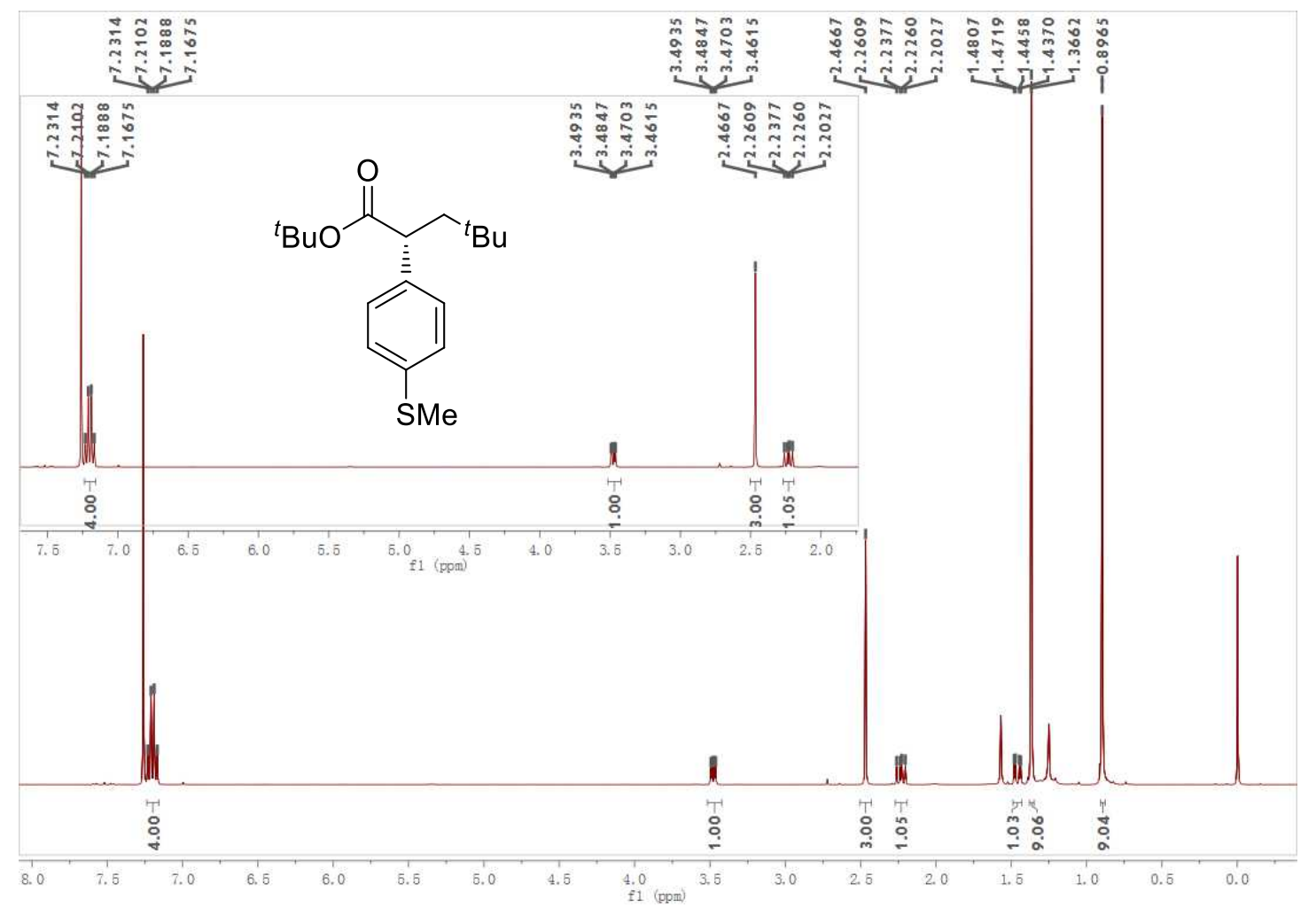

20; ${ }^{13} \mathrm{C}$-NMR (100 MHz, $\mathrm{CDCl}_{3}$

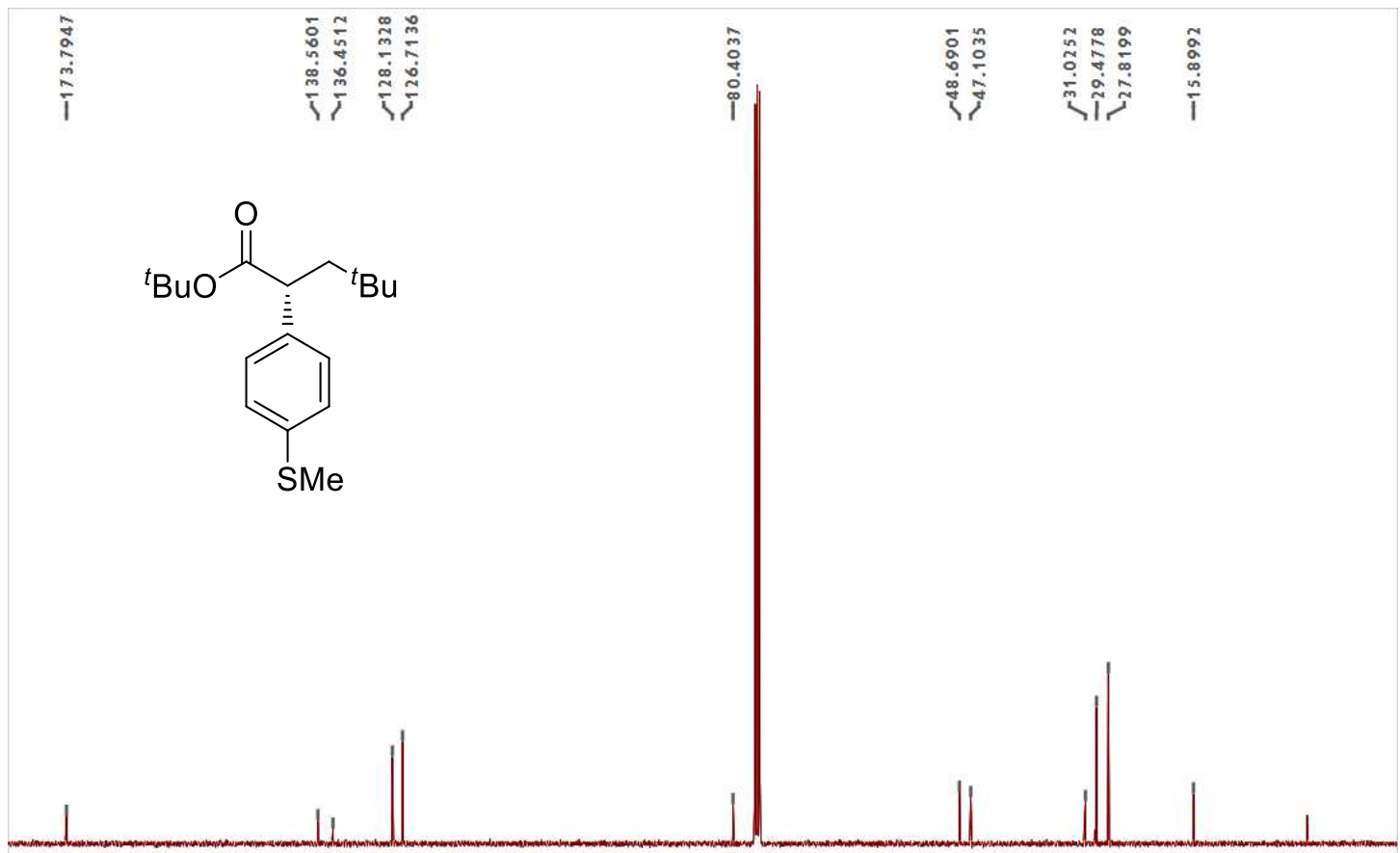


21; ${ }^{1} \mathrm{H}-\mathrm{NMR}$ (400 MHz, $\mathrm{CDCl}_{3}$ )

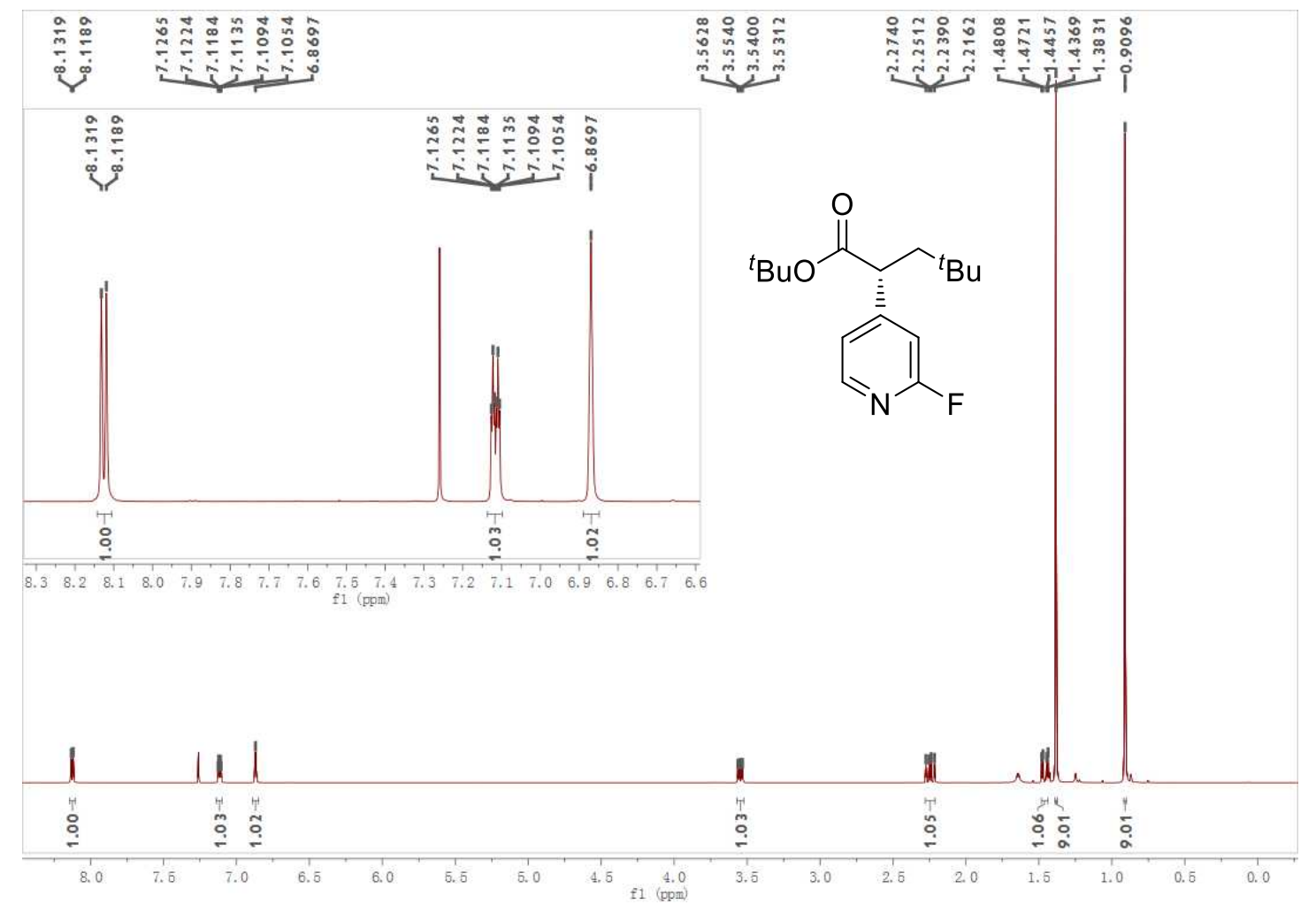

21; ${ }^{19}$ F-NMR (375 MHz, $\mathrm{CDCl}_{3}$ )

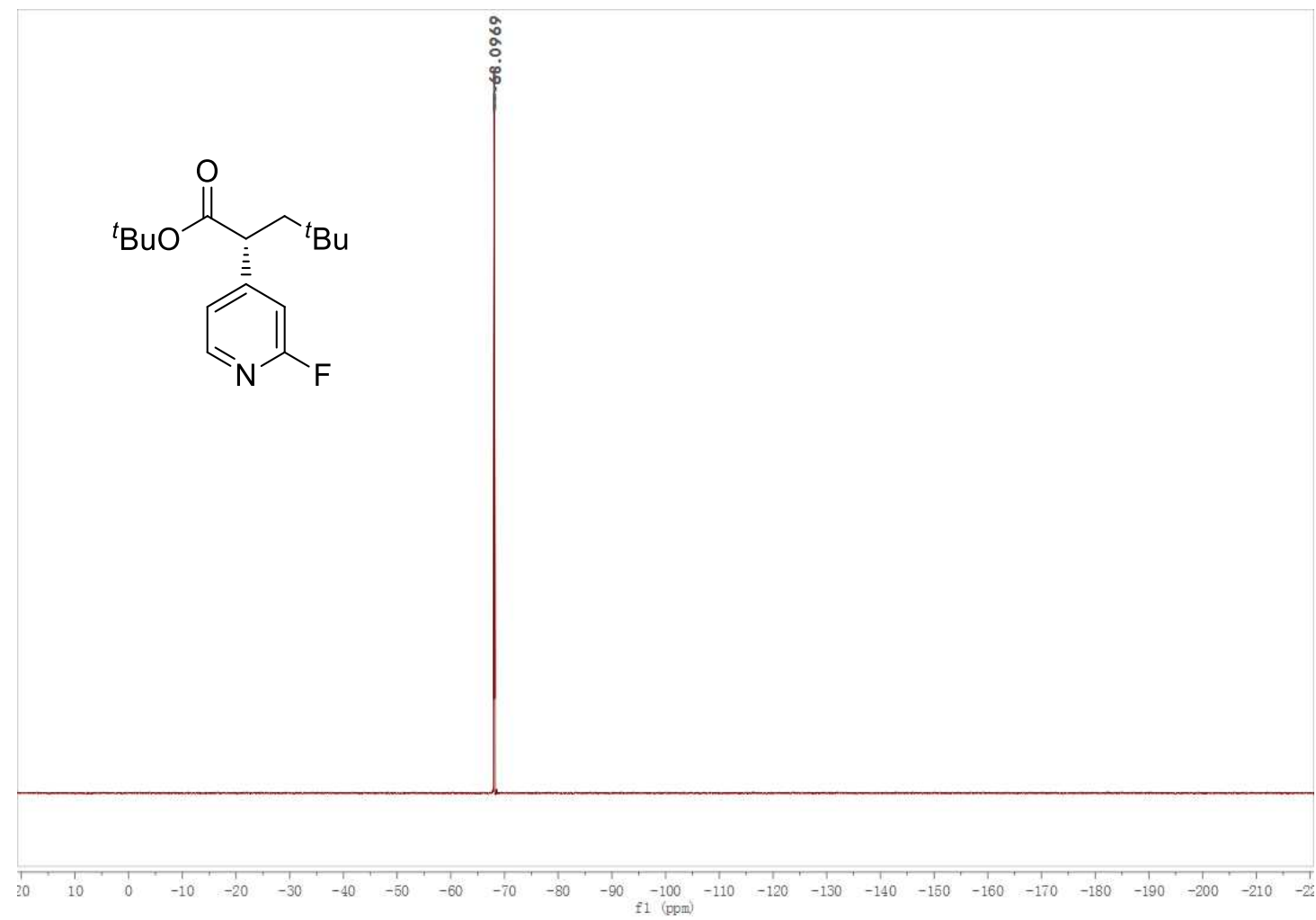


21; ${ }^{13} \mathrm{C}-\mathrm{NMR}\left(100 \mathrm{MHz}, \mathrm{CDCl}_{3}\right)$

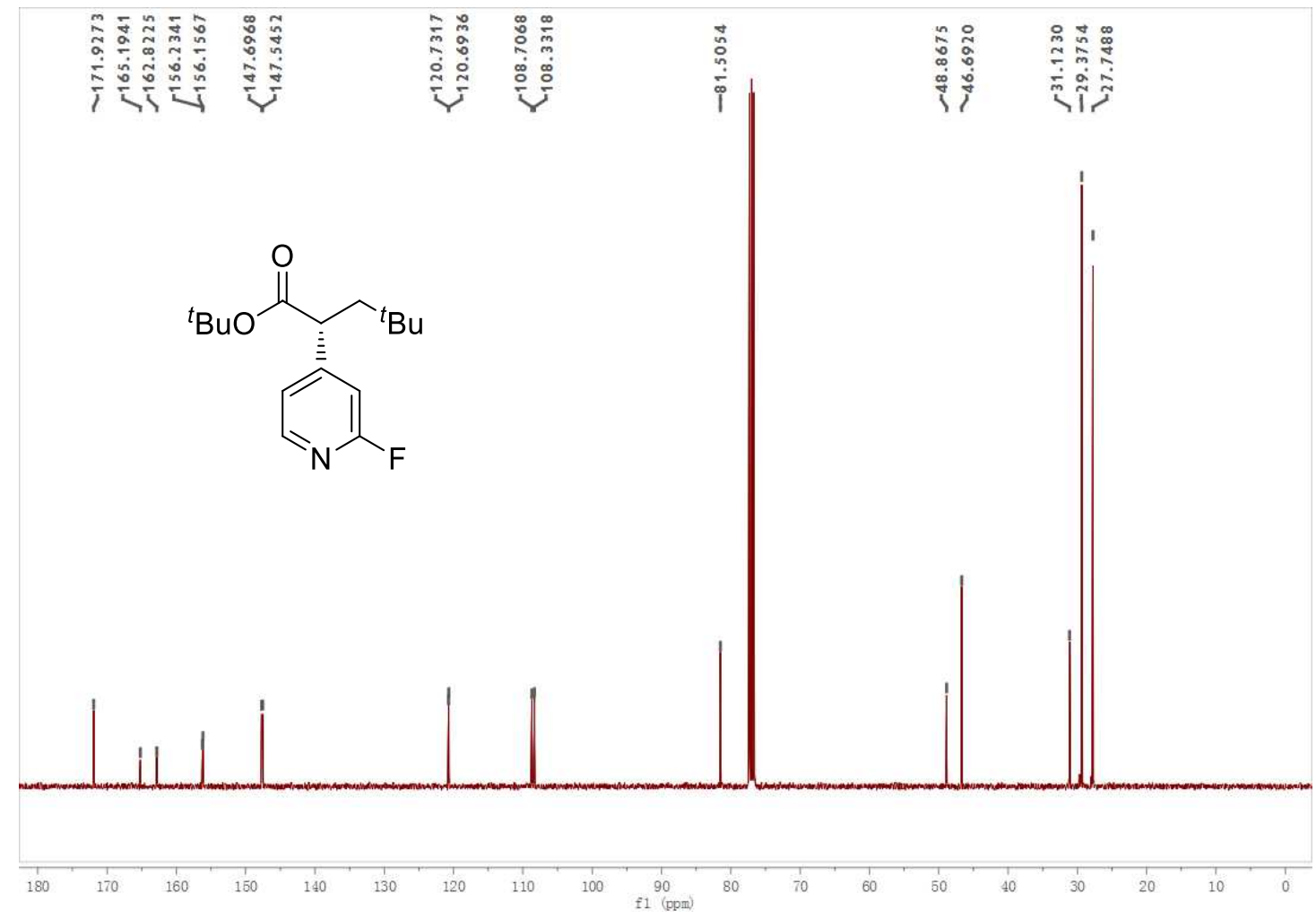

22; ${ }^{1} \mathrm{H}-\mathrm{NMR}\left(400 \mathrm{MHz}, \mathrm{CDCl}_{3}\right)$

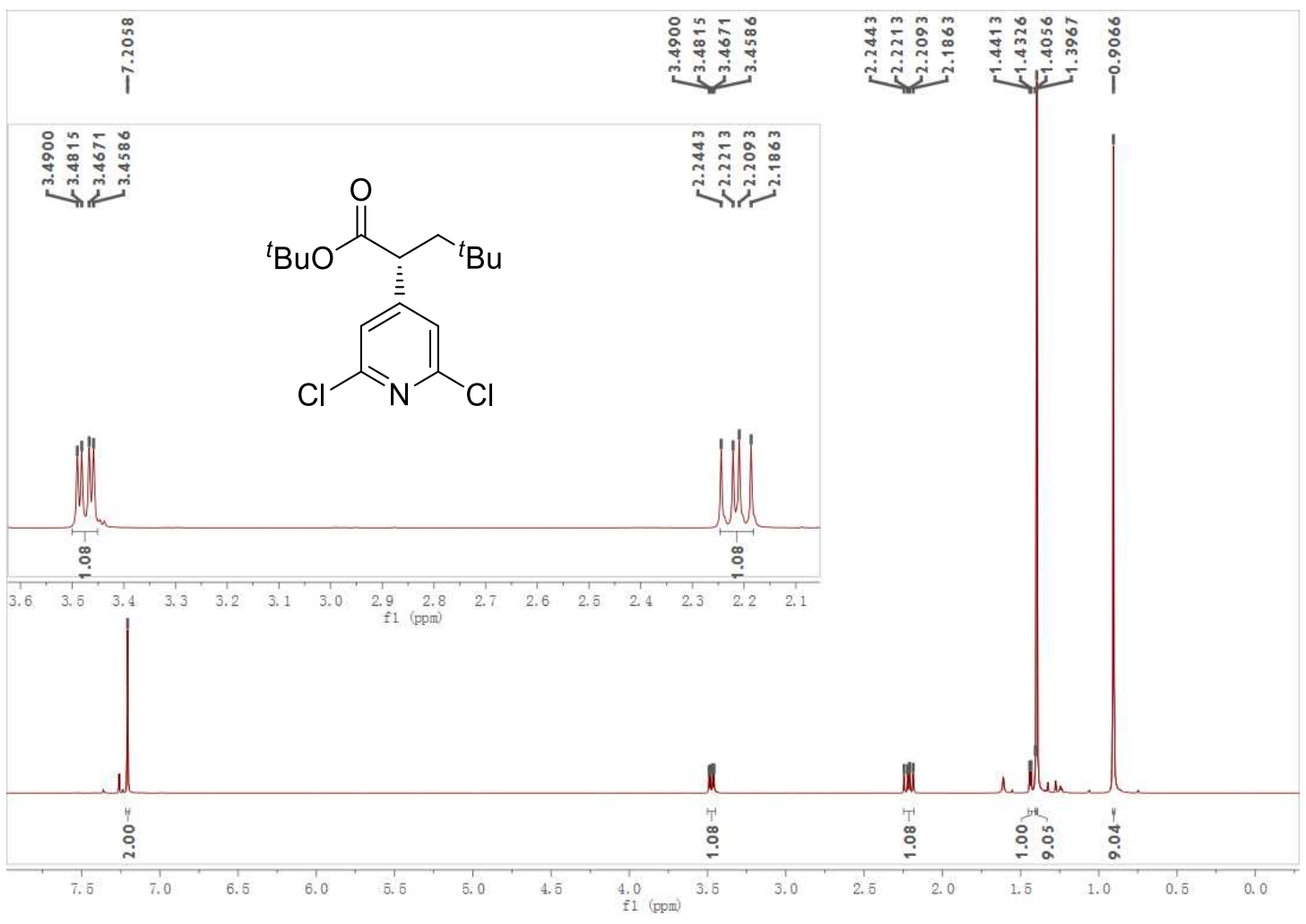


22; ${ }^{13} \mathrm{C}$-NMR (100 MHz, $\left.\mathrm{CDCl}_{3}\right)$

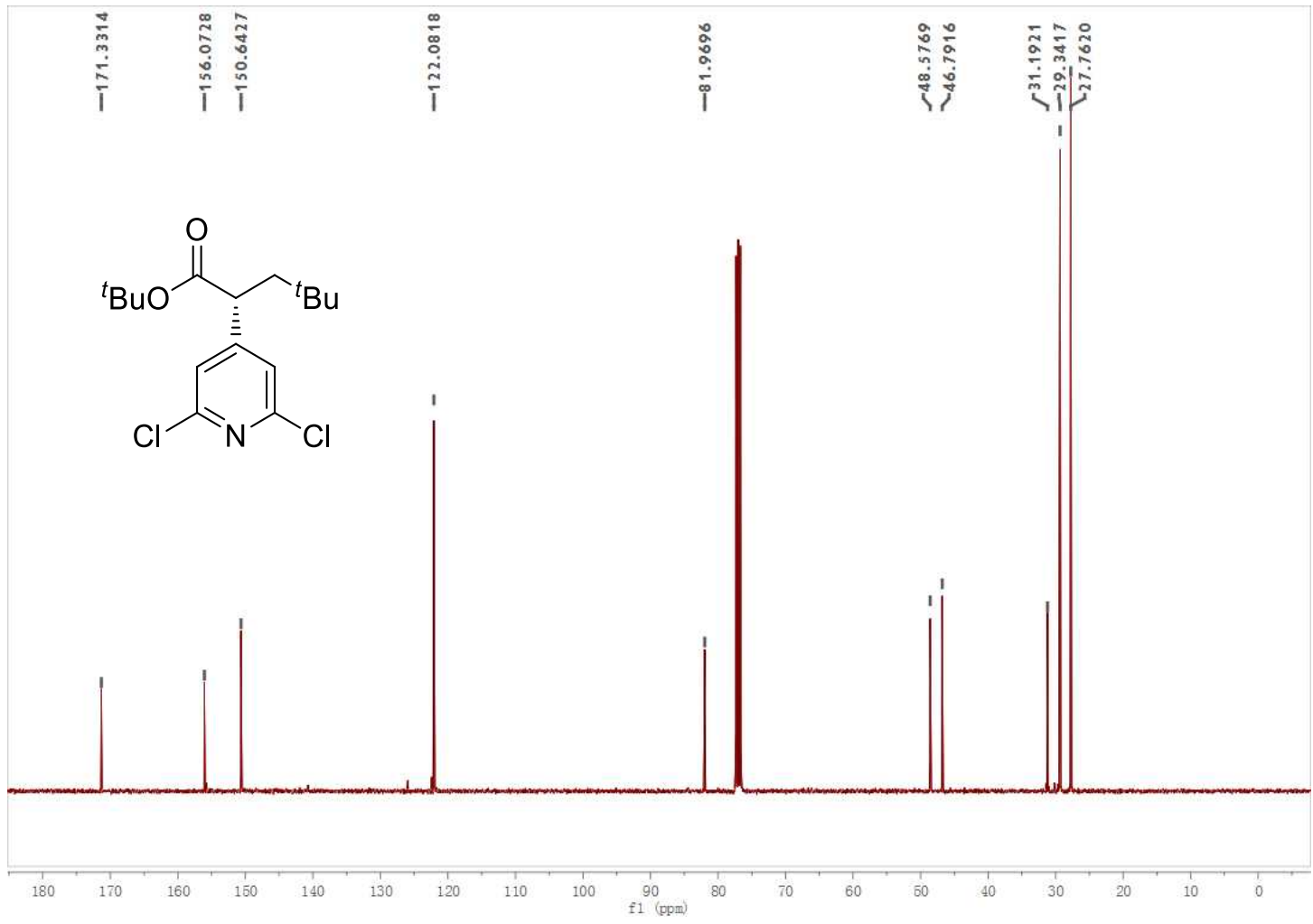

23; ${ }^{1} \mathrm{H}-\mathrm{NMR}\left(400 \mathrm{MHz}, \mathrm{CDCl}_{3}\right)$

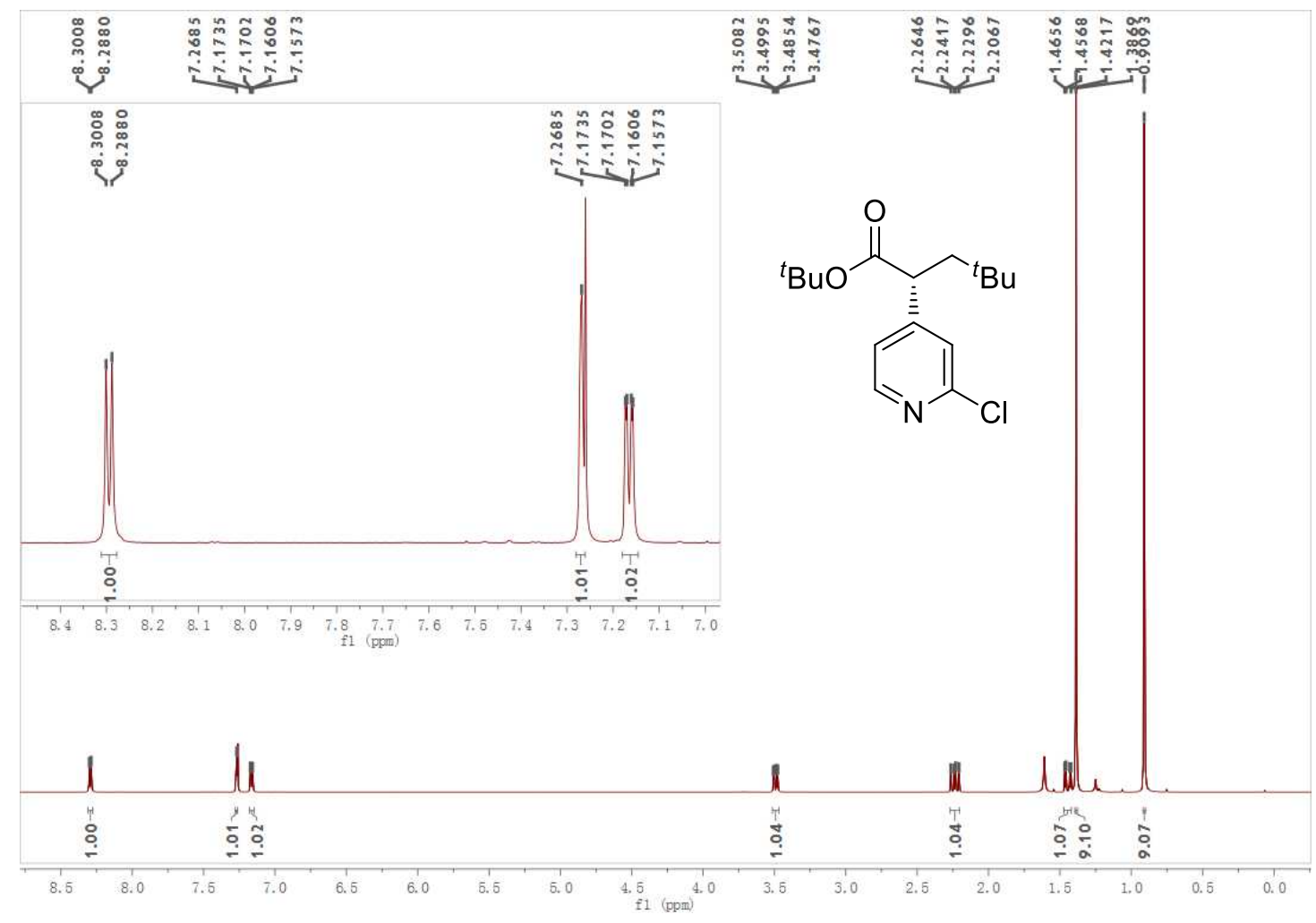


23; ${ }^{13} \mathrm{C}-\mathrm{NMR}\left(100 \mathrm{MHz}, \mathrm{CDCl}_{3}\right)$

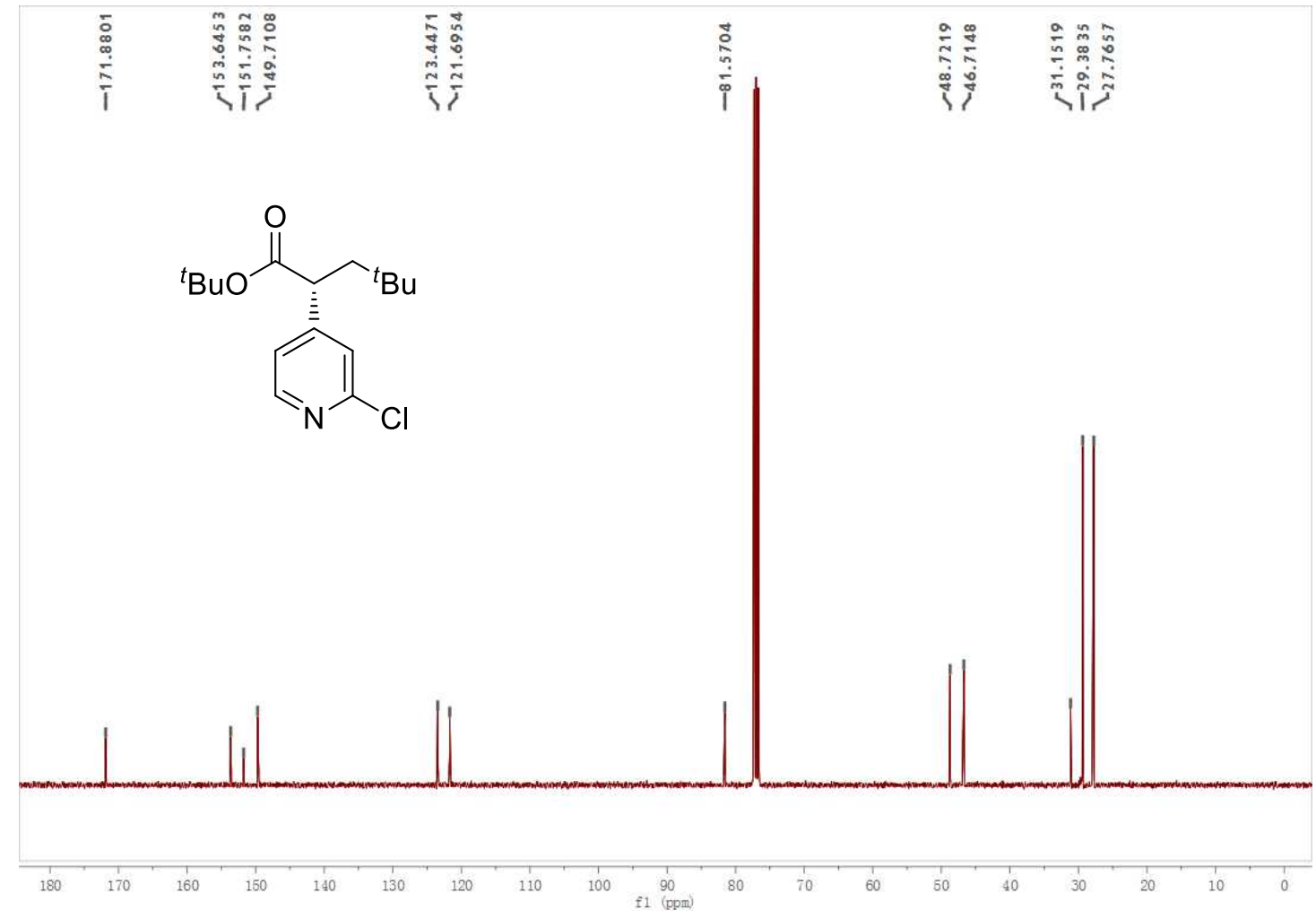

24; ${ }^{1} \mathrm{H}-\mathrm{NMR}\left(400 \mathrm{MHz}, \mathrm{CDCl}_{3}\right)$

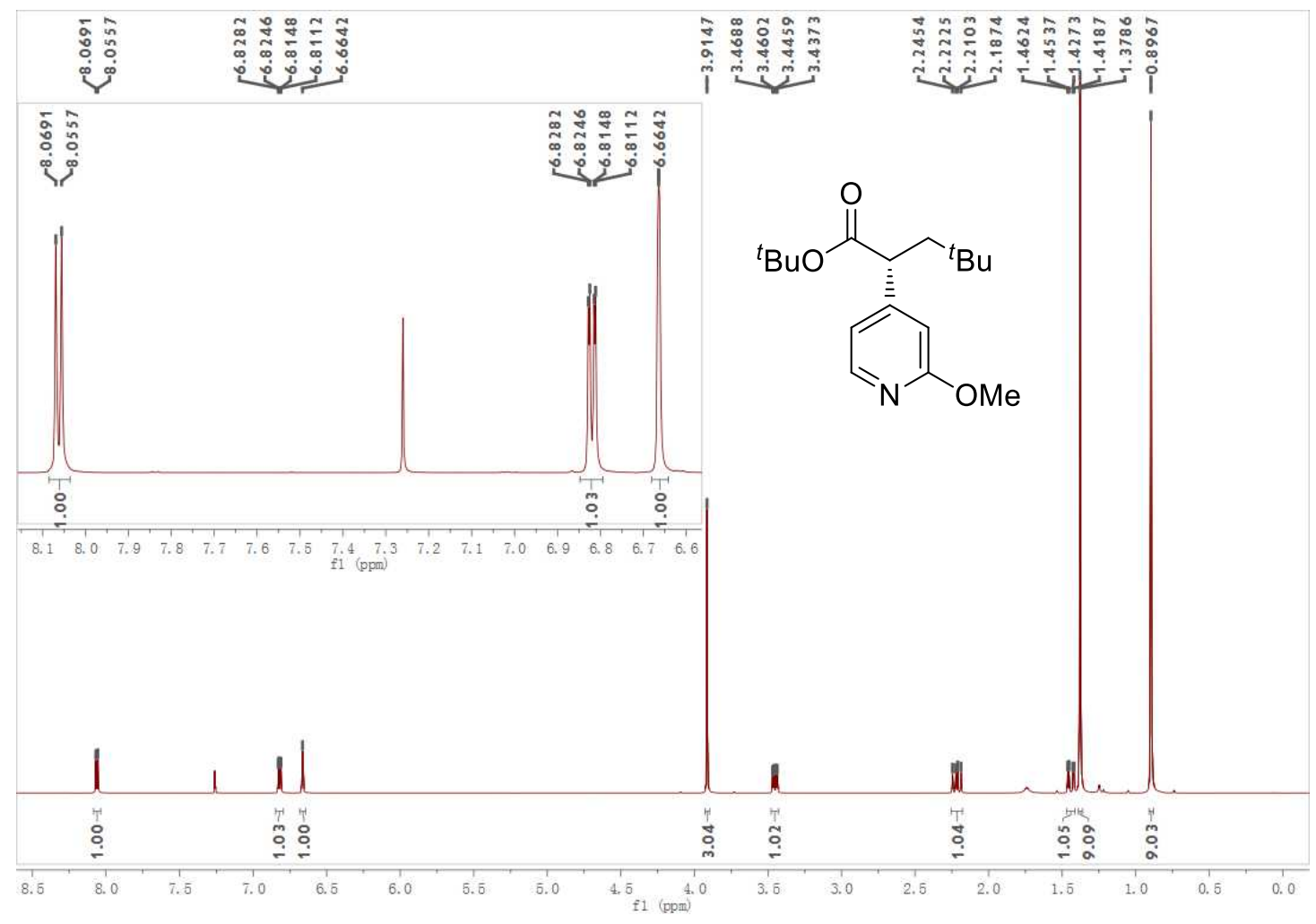


24; ${ }^{13} \mathrm{C}-\mathrm{NMR}\left(100 \mathrm{MHz}, \mathrm{CDCl}_{3}\right)$

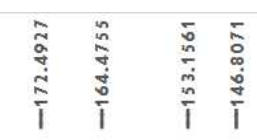

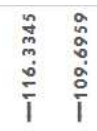

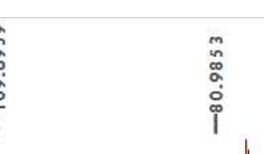

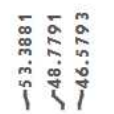<smiles>COc1cc([C@@H](CBr)C(=O)[18OH])ccn1</smiles>

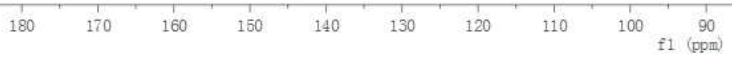

25; ${ }^{1} \mathrm{H}-\mathrm{NMR}\left(400 \mathrm{MHz}, \mathrm{CDCl}_{3}\right)$

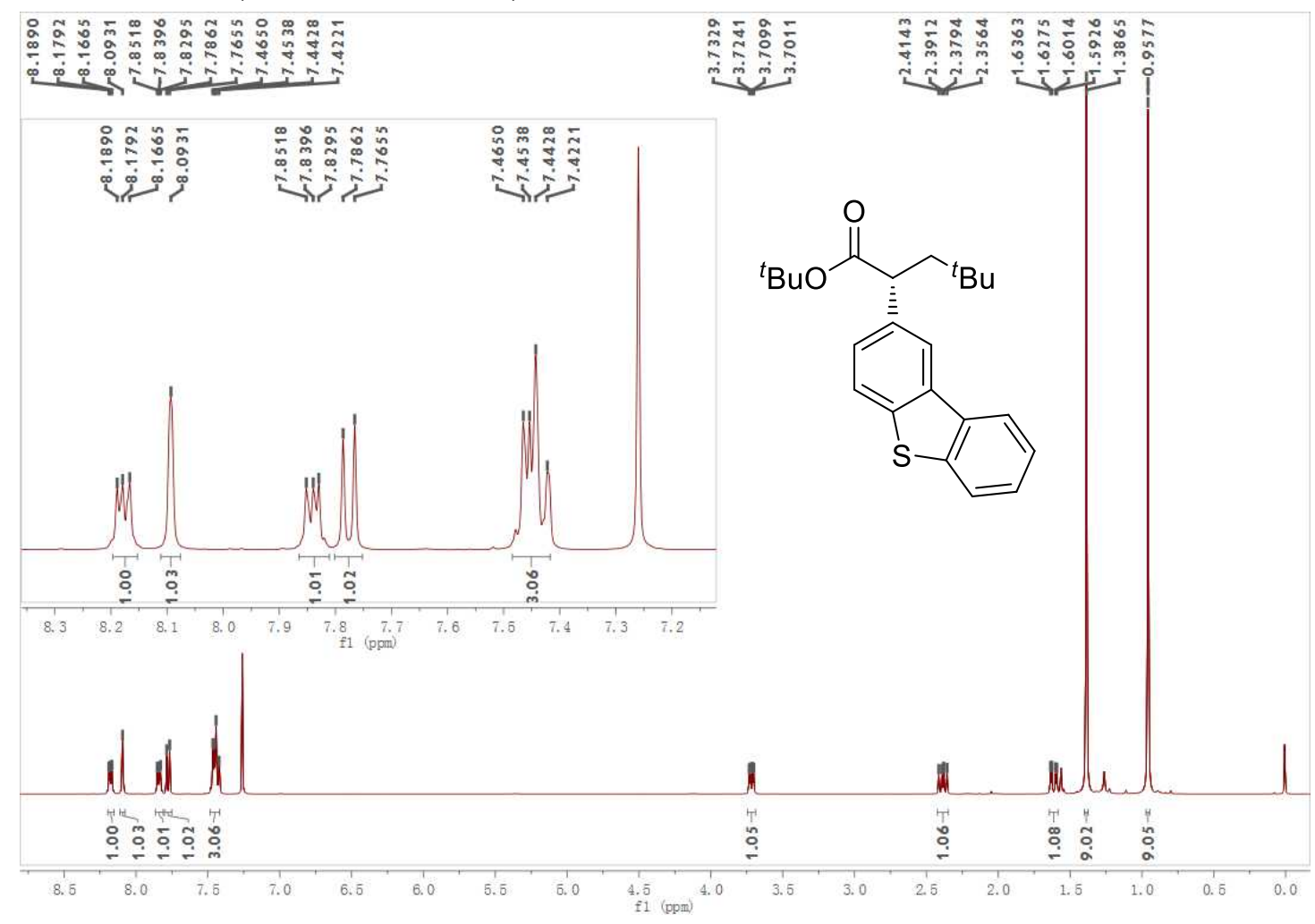


25; ${ }^{13} \mathrm{C}-\mathrm{NMR}$ (100 MHz, $\left.\mathrm{CDCl}_{3}\right)$

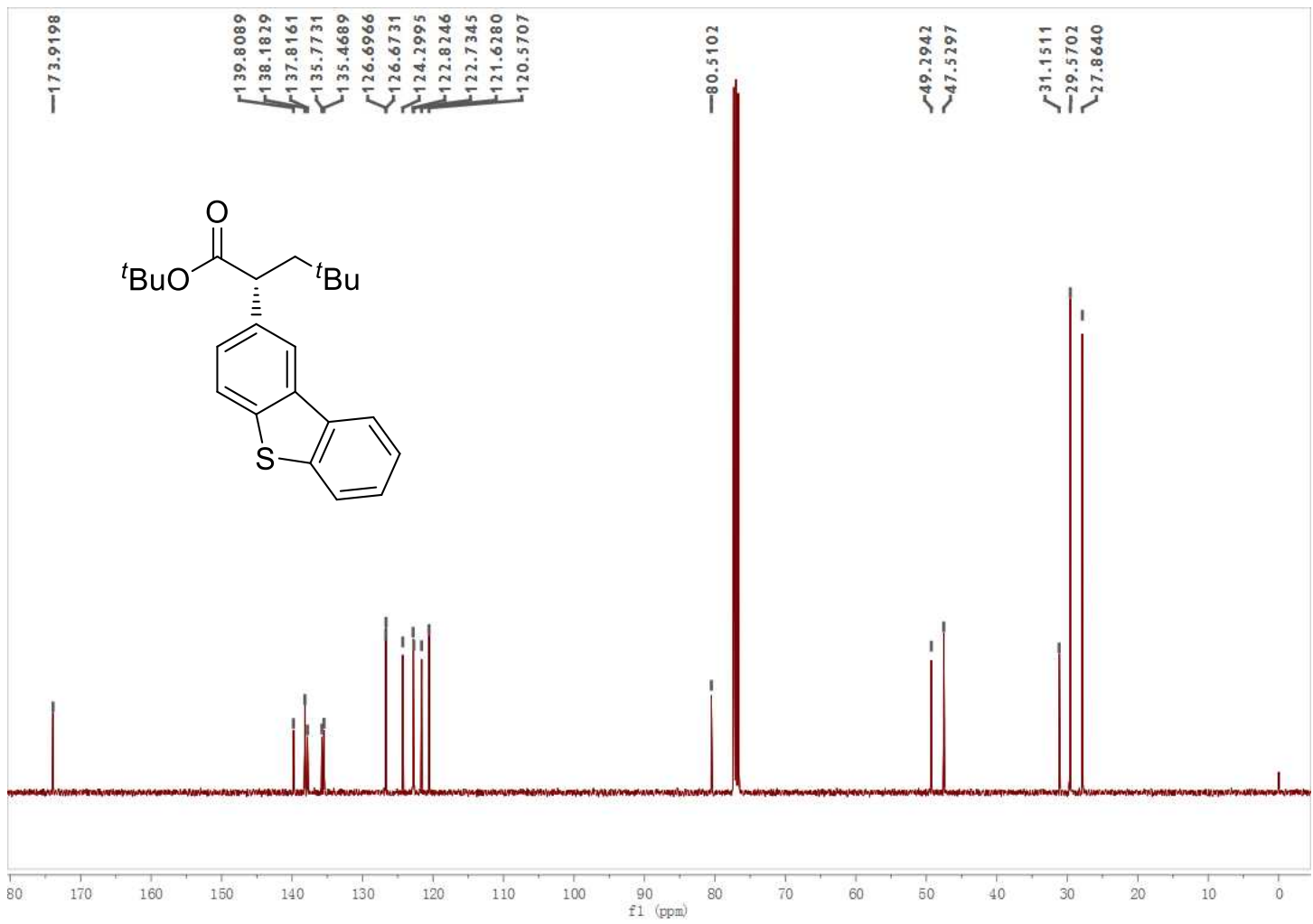

26; ${ }^{1} \mathrm{H}-\mathrm{NMR}$ (400 MHz, $\mathrm{CDCl}_{3}$ )
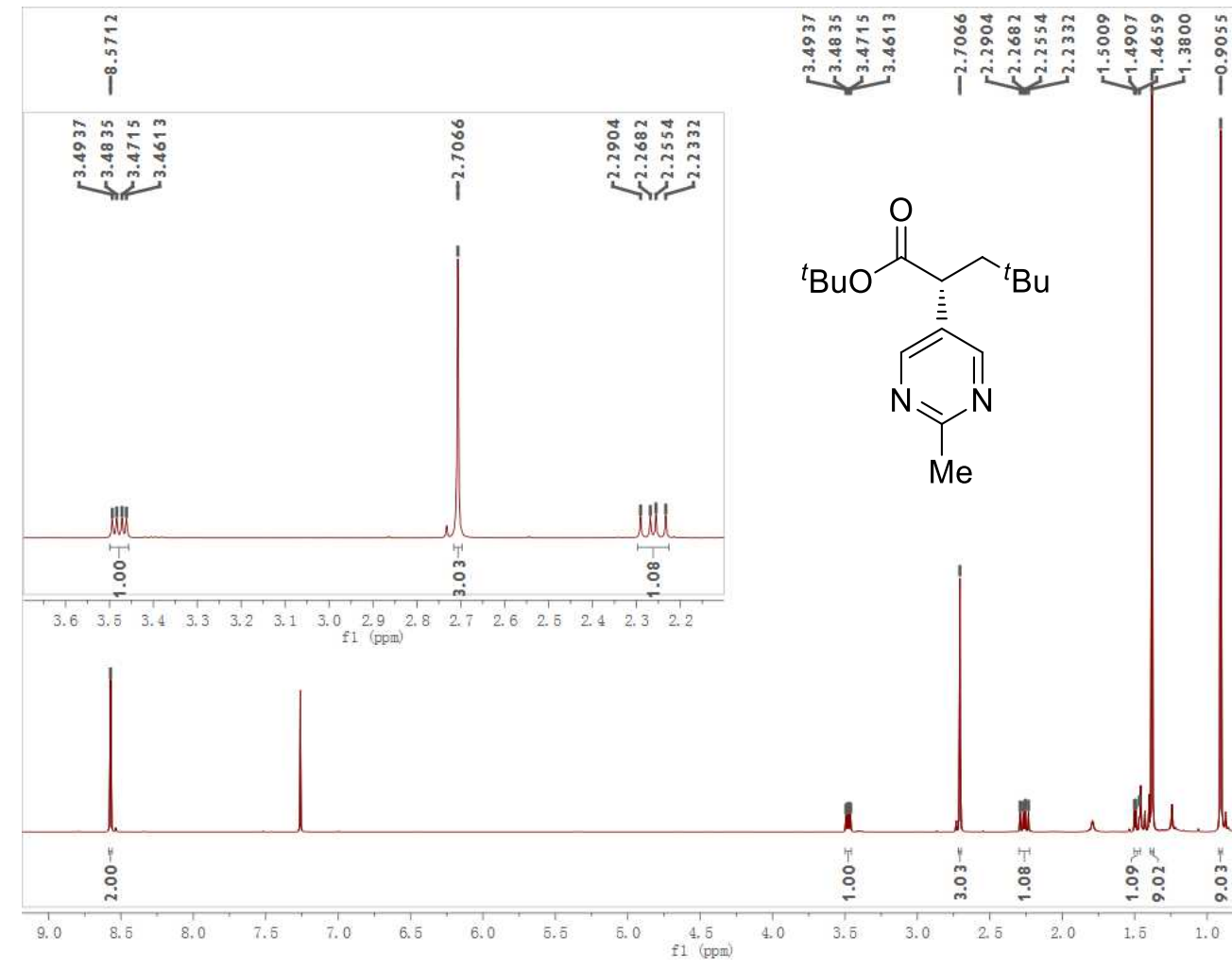
26; ${ }^{13} \mathrm{C}-\mathrm{NMR}\left(100 \mathrm{MHz}, \mathrm{CDCl}_{3}\right)$

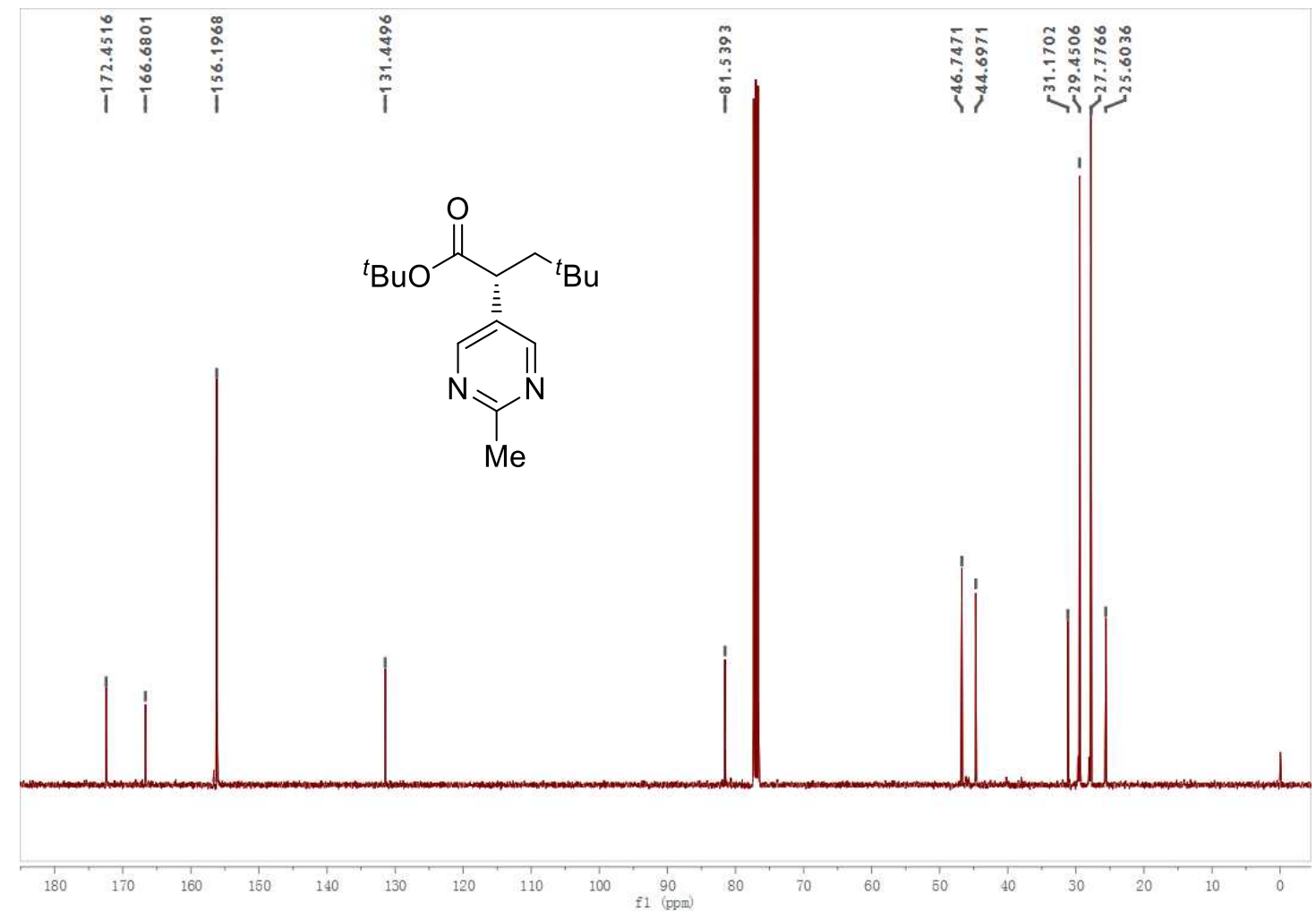

27; ${ }^{1} \mathrm{H}-\mathrm{NMR}\left(400 \mathrm{MHz}, \mathrm{CDCl}_{3}\right.$ )

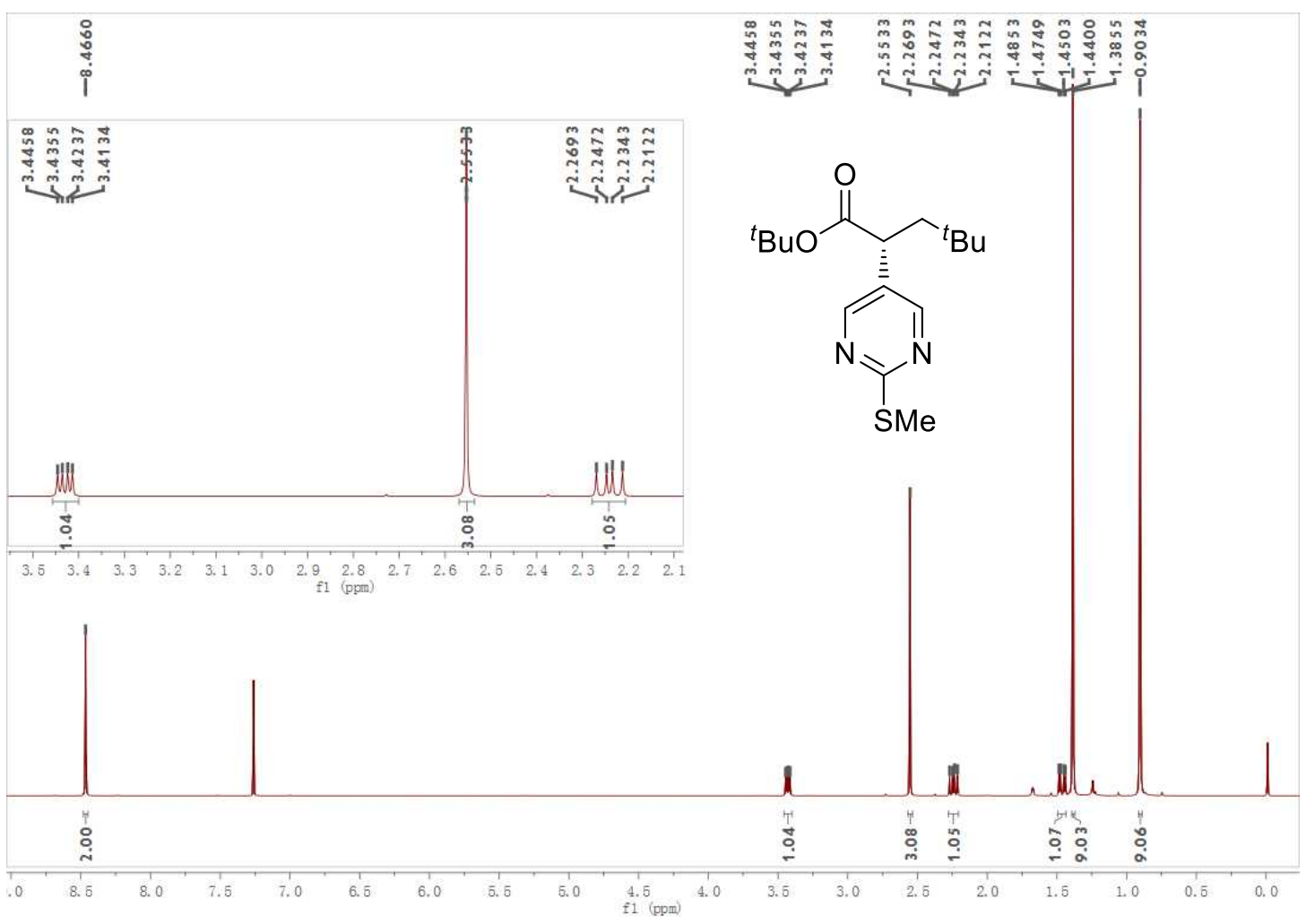


27; ${ }^{13} \mathrm{C}-\mathrm{NMR}\left(100 \mathrm{MHz}, \mathrm{CDCl}_{3}\right)$

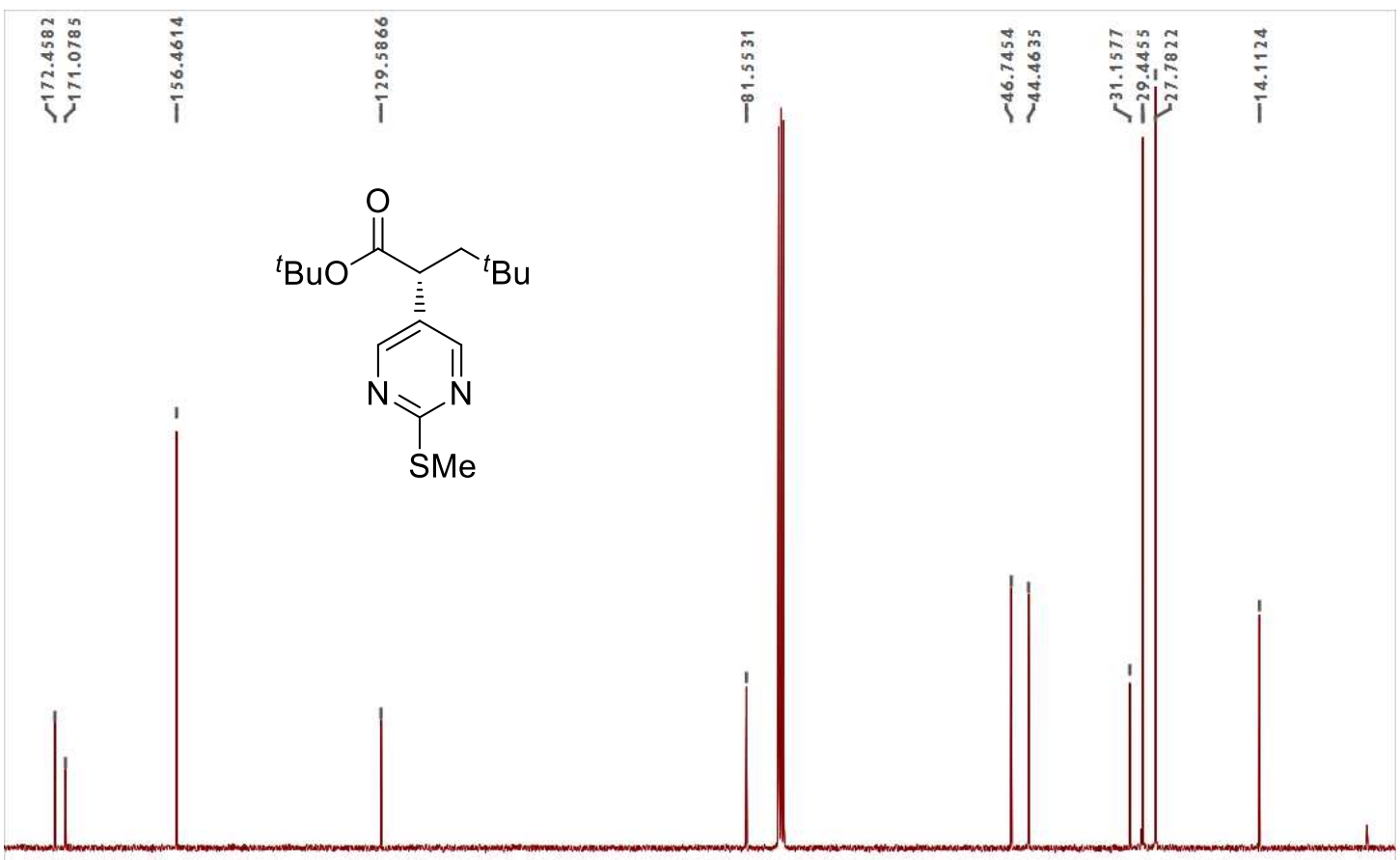

28; ${ }^{1} \mathrm{H}-\mathrm{NMR}\left(400 \mathrm{MHz}, \mathrm{CDCl}_{3}\right)$

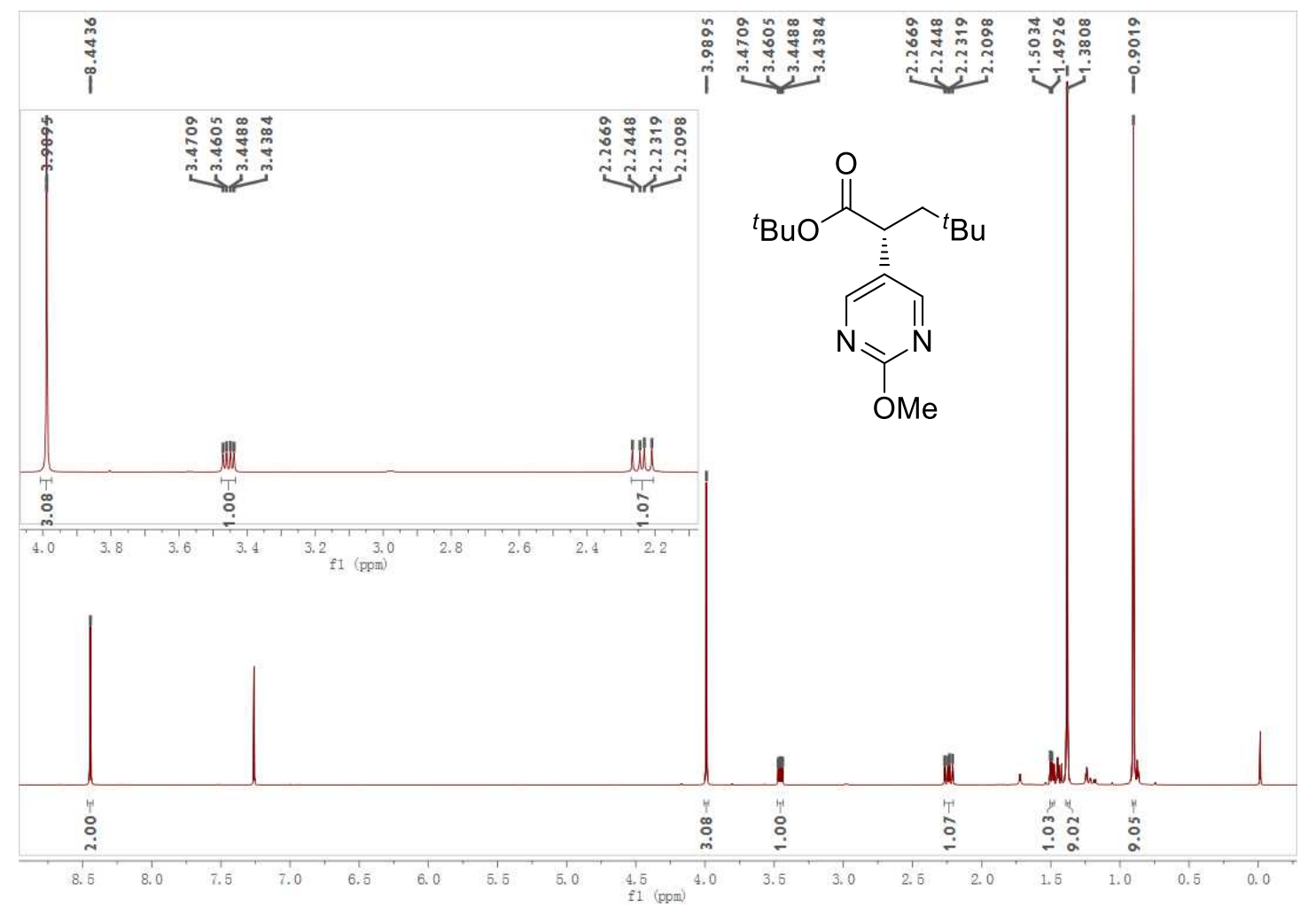


28; ${ }^{13} \mathrm{C}-\mathrm{NMR}\left(100 \mathrm{MHz}, \mathrm{CDCl}_{3}\right)$

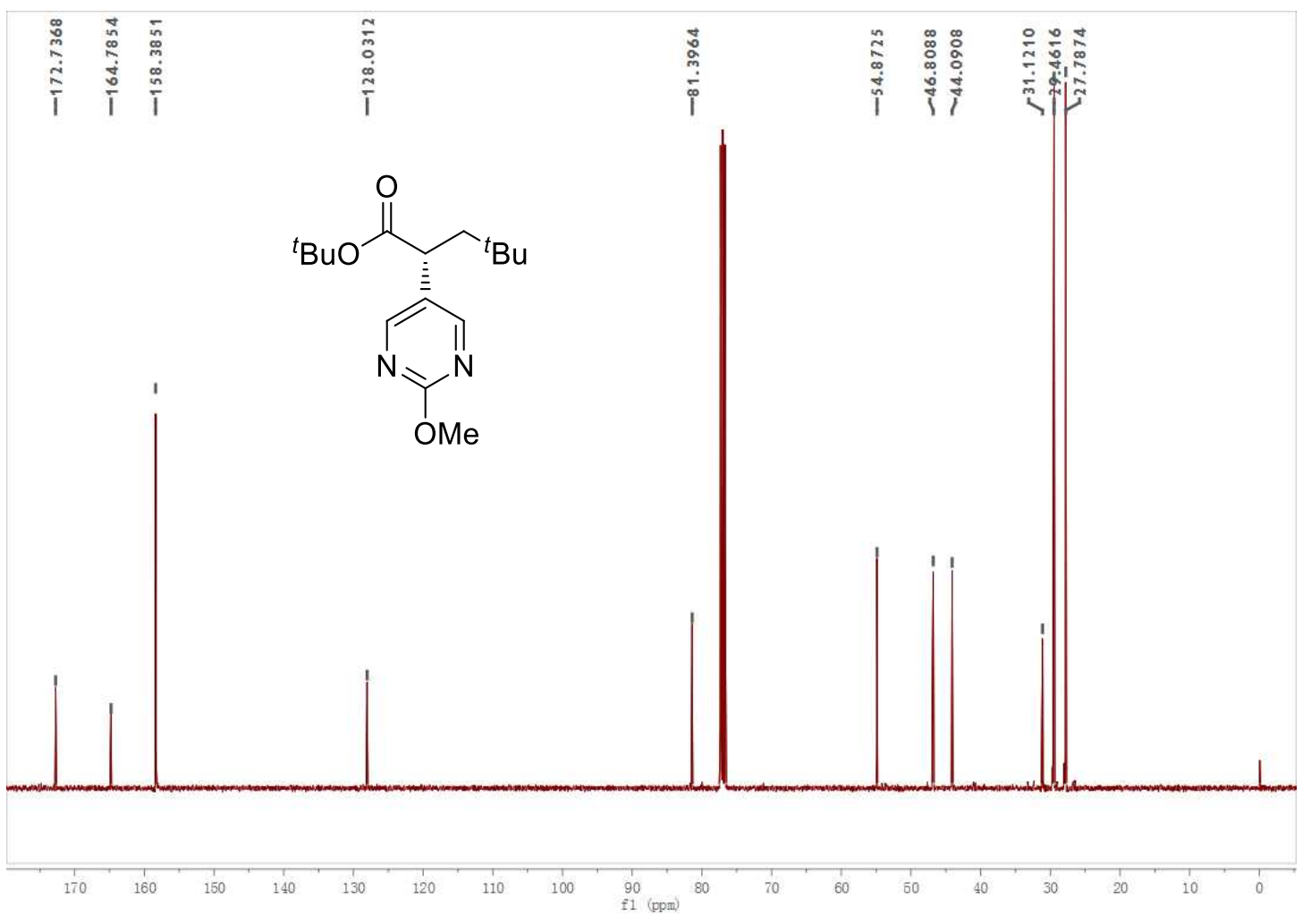

29; ${ }^{1} \mathrm{H}-\mathrm{NMR}$ (400 MHz, $\mathrm{CDCl}_{3}$ )

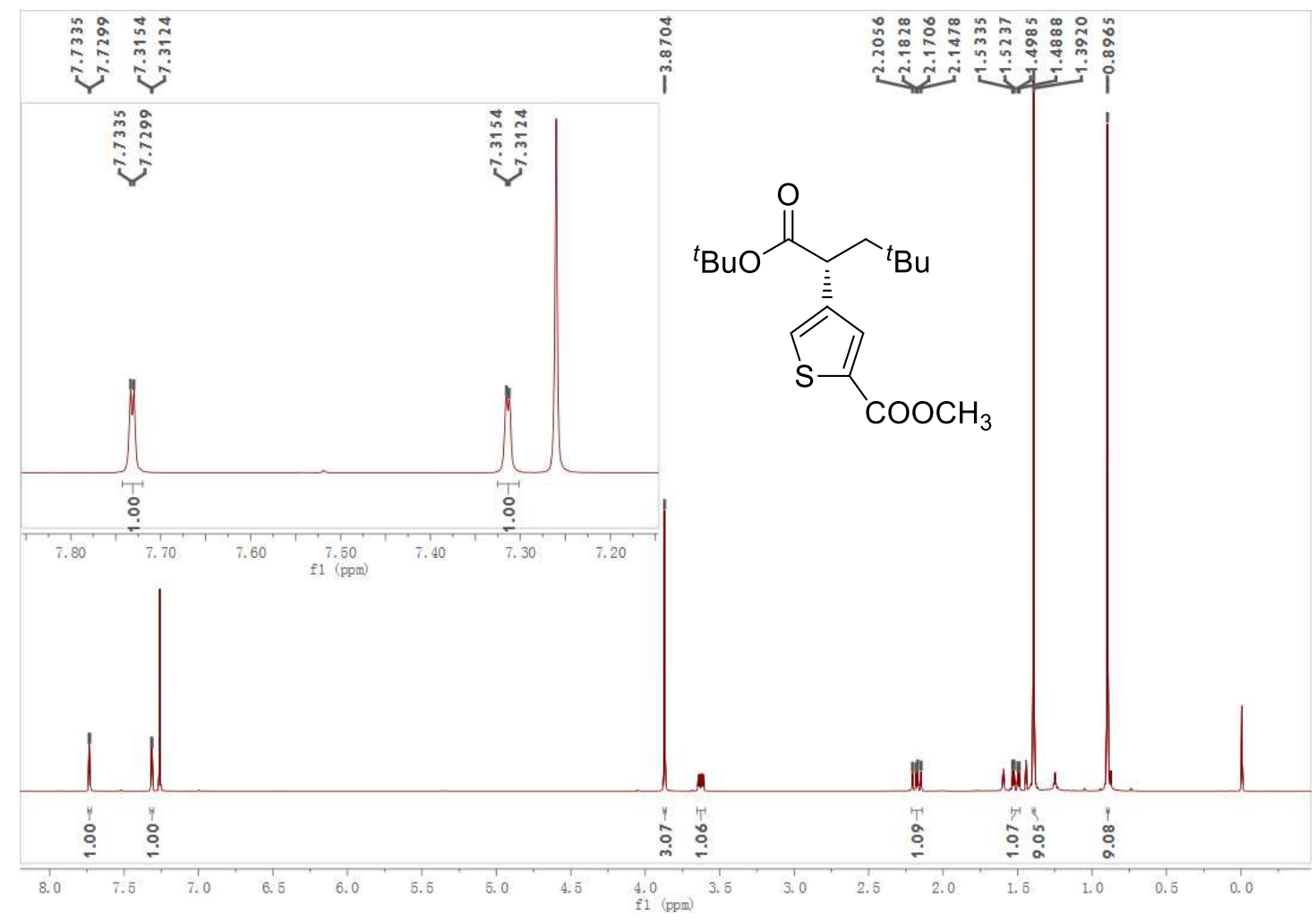


29; ${ }^{13} \mathrm{C}-\mathrm{NMR}\left(100 \mathrm{MHz}, \mathrm{CDCl}_{3}\right)$

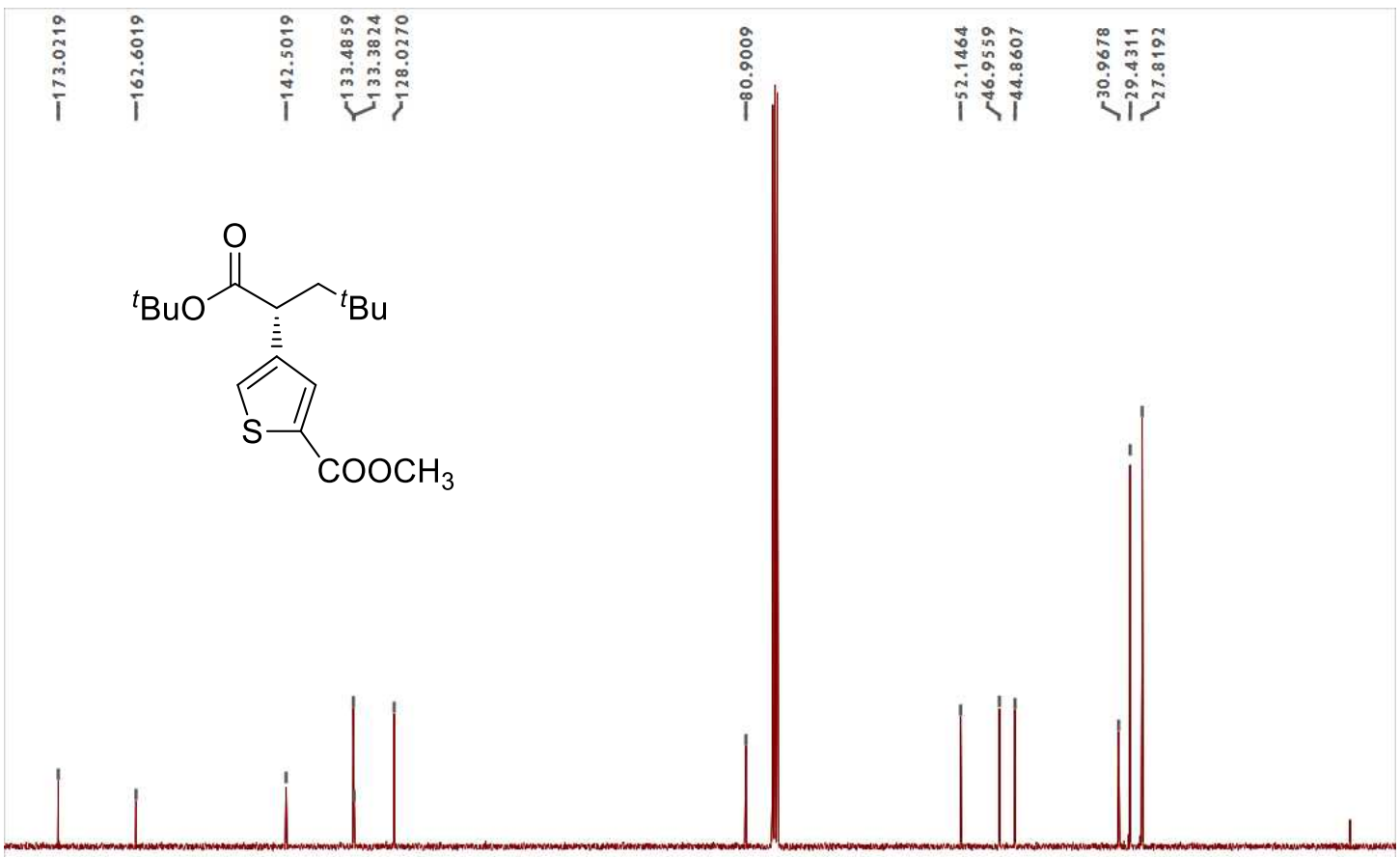

30; ${ }^{1} \mathrm{H}-\mathrm{NMR}$ (400 MHz, $\mathrm{CDCl}_{3}$ )

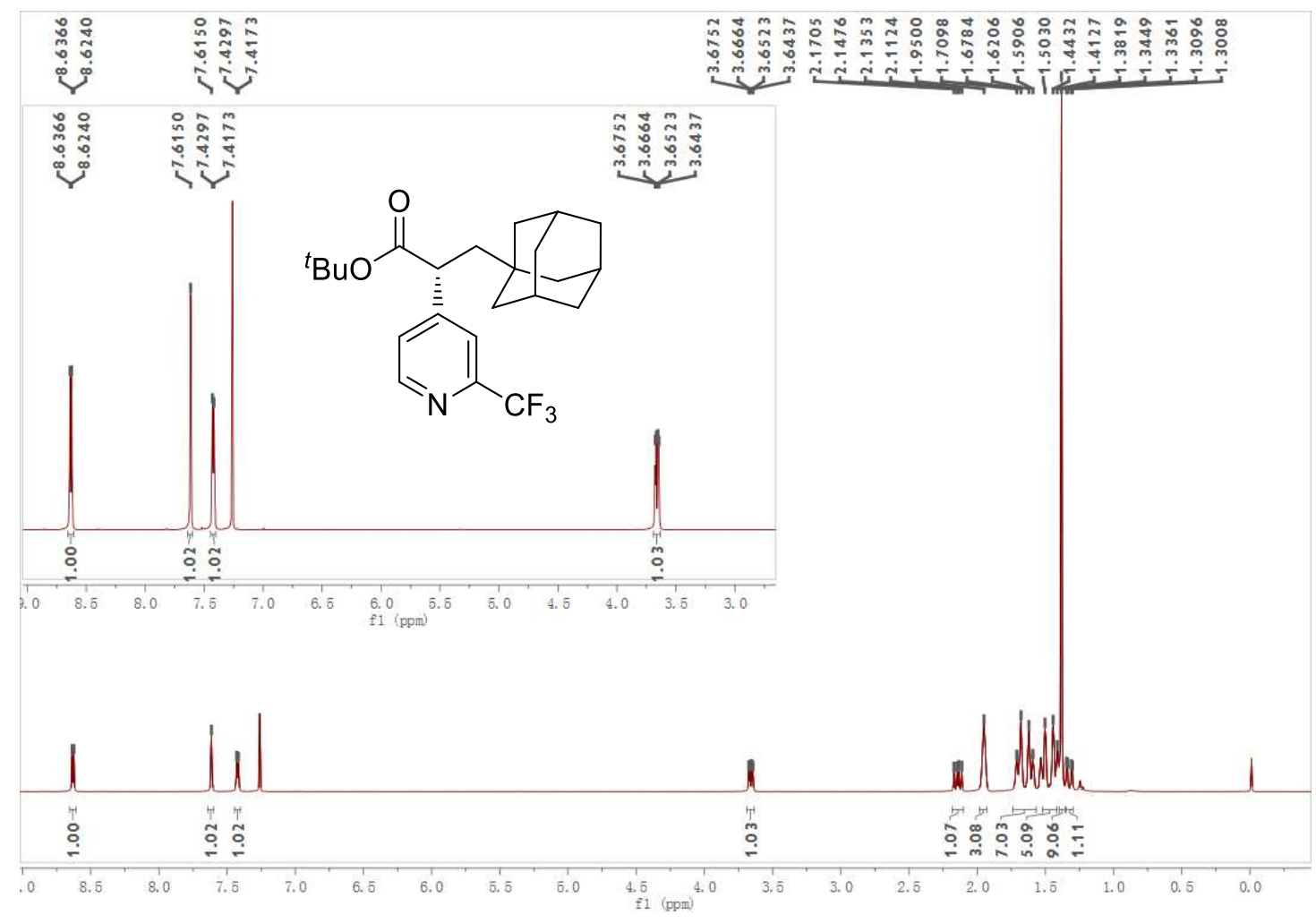


30; ${ }^{19}$ F-NMR (375 MHz, $\mathrm{CDCl}_{3}$ )

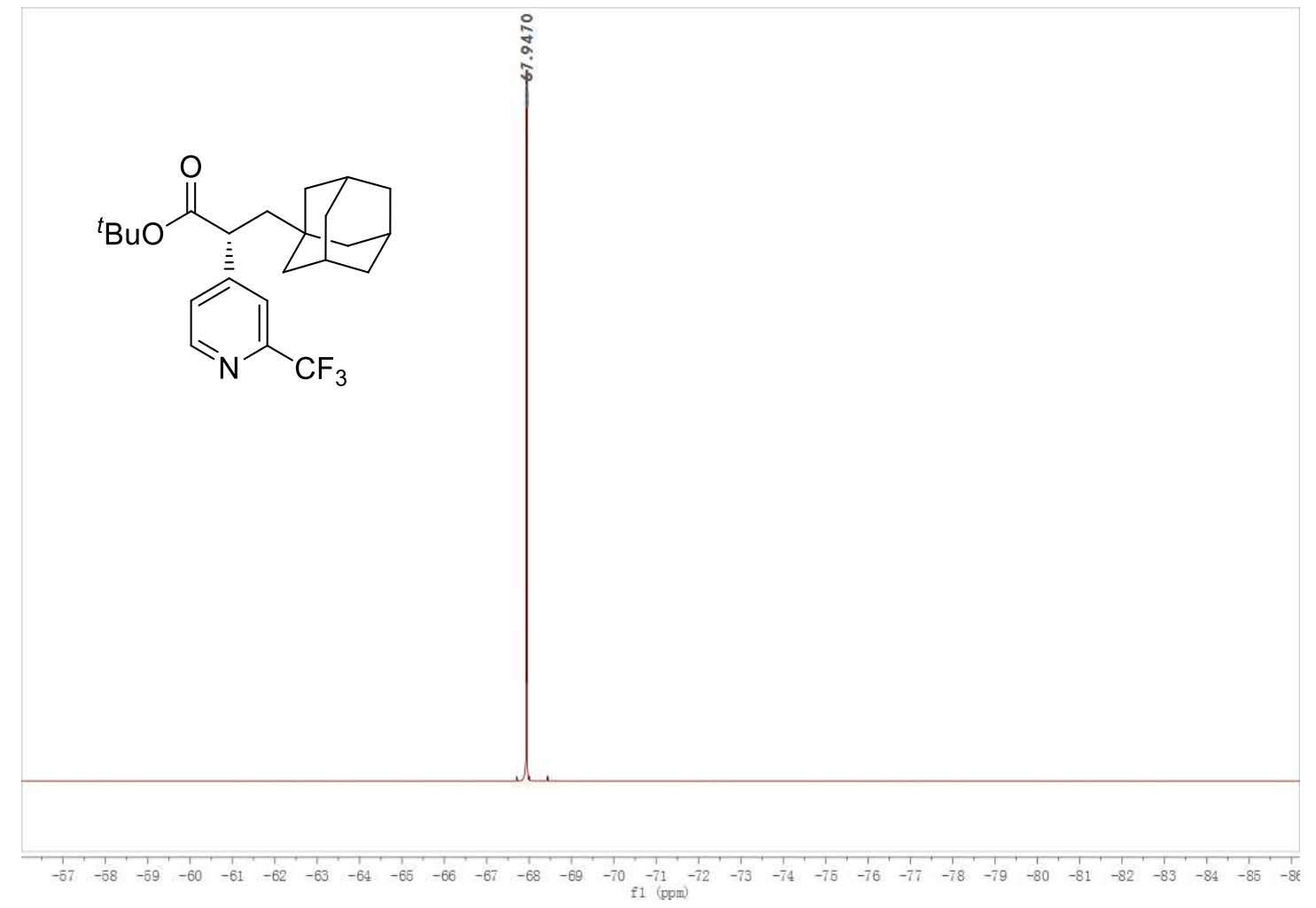

30; ${ }^{13} \mathrm{C}$-NMR (100 MHz, $\left.\mathrm{CDCl}_{3}\right)$

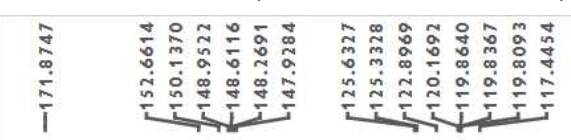<smiles>CCC(C)OC(=O)[C@H](CC12CC3CC(CC(C3)C1)C2)c1ccnc(C(F)(F)F)c1</smiles>
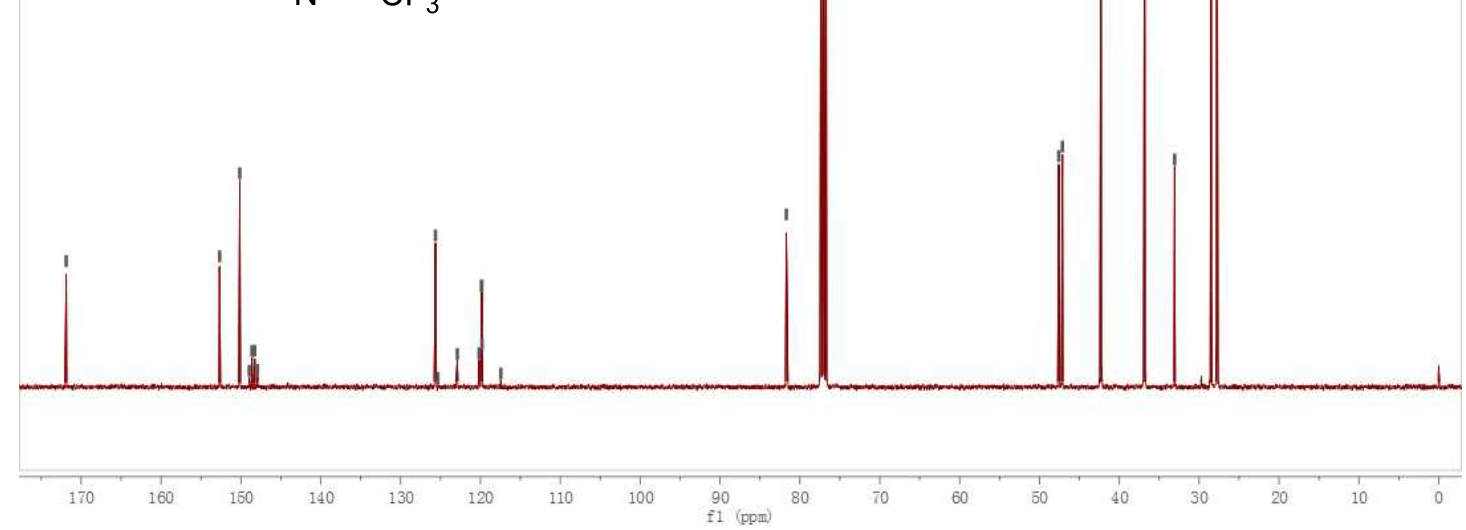
31; ${ }^{1} \mathrm{H}-\mathrm{NMR}$ (400 MHz, $\mathrm{CDCl}_{3}$ )

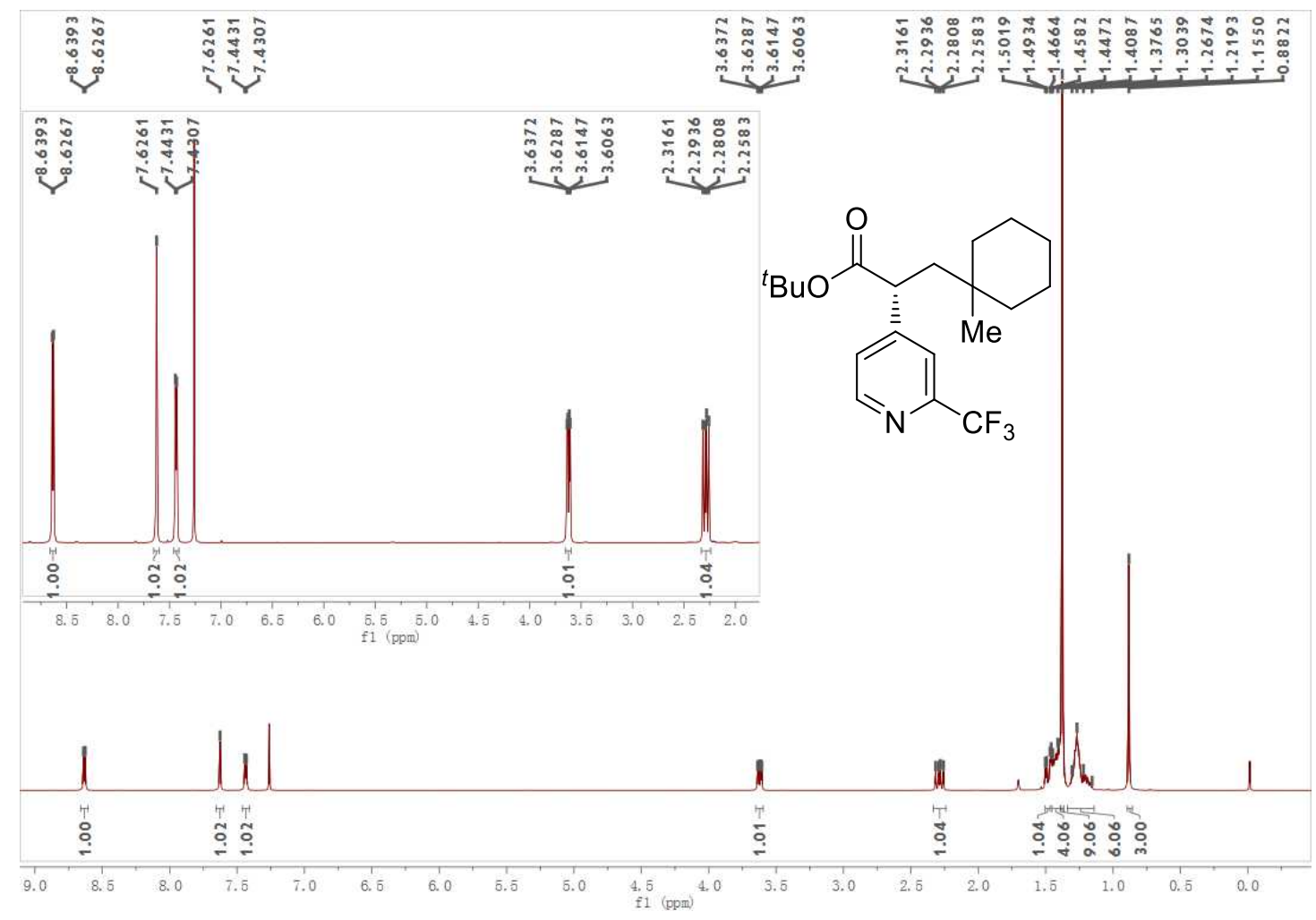

31; ${ }^{19}$ F-NMR (375 MHz, $\left.\mathrm{CDCl}_{3}\right)$

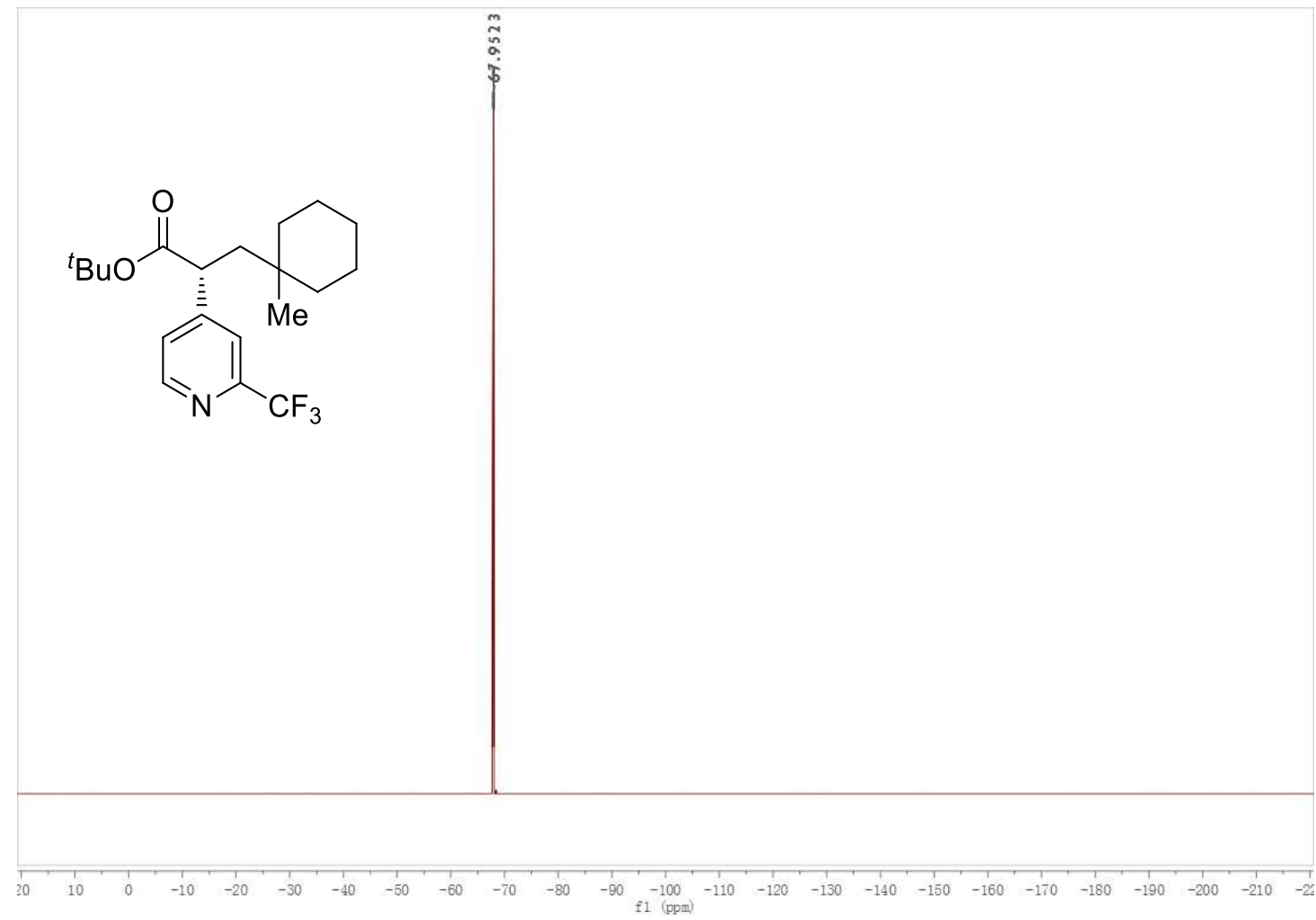


31; ${ }^{13} \mathrm{C}-\mathrm{NMR}\left(100 \mathrm{MHz}, \mathrm{CDCl}_{3}\right)$

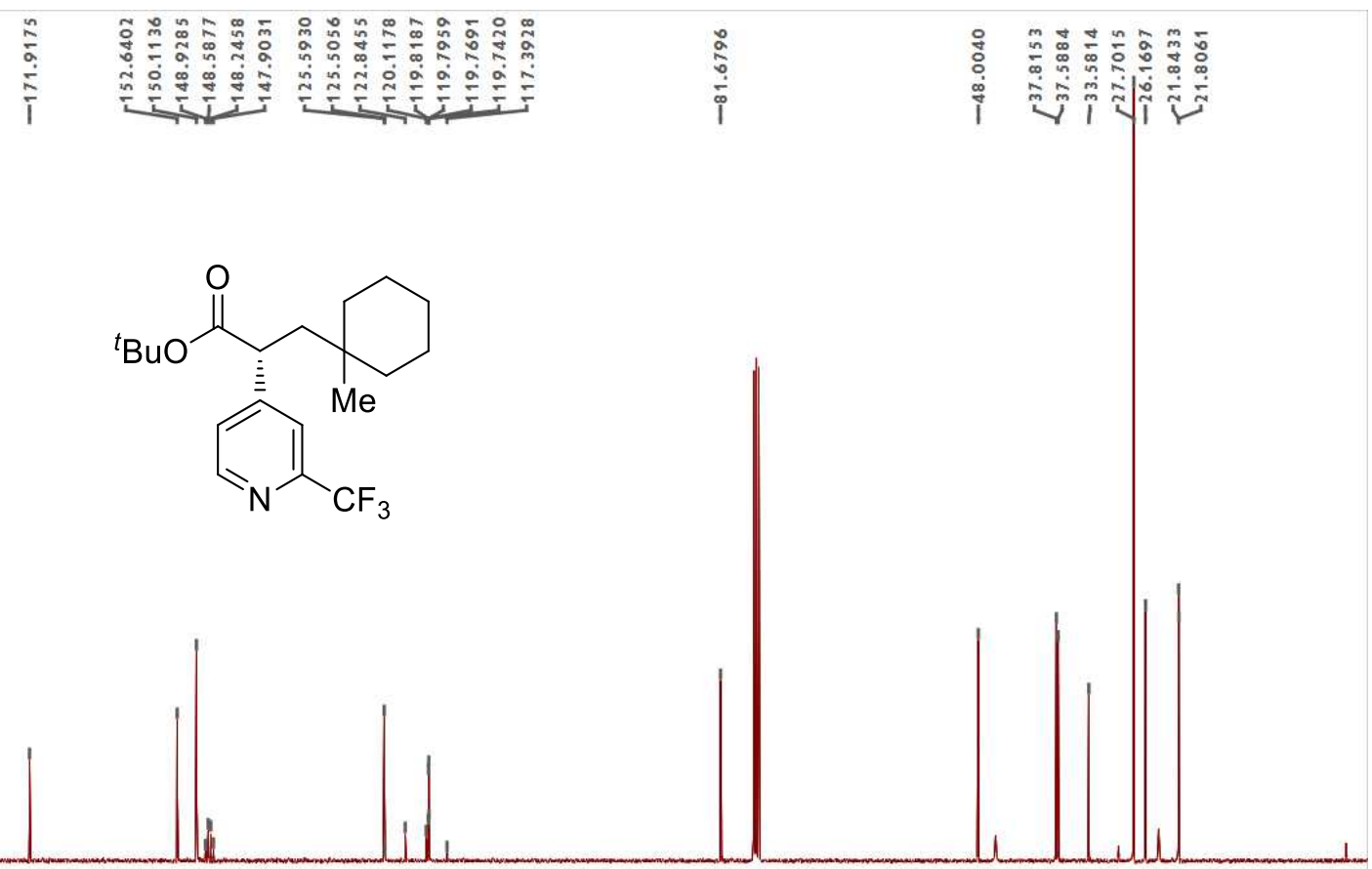

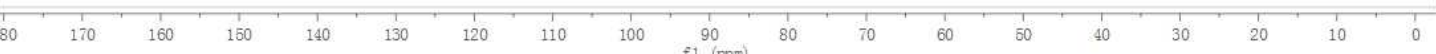

32; ${ }^{1} \mathrm{H}-\mathrm{NMR}\left(400 \mathrm{MHz}, \mathrm{CDCl}_{3}\right)$

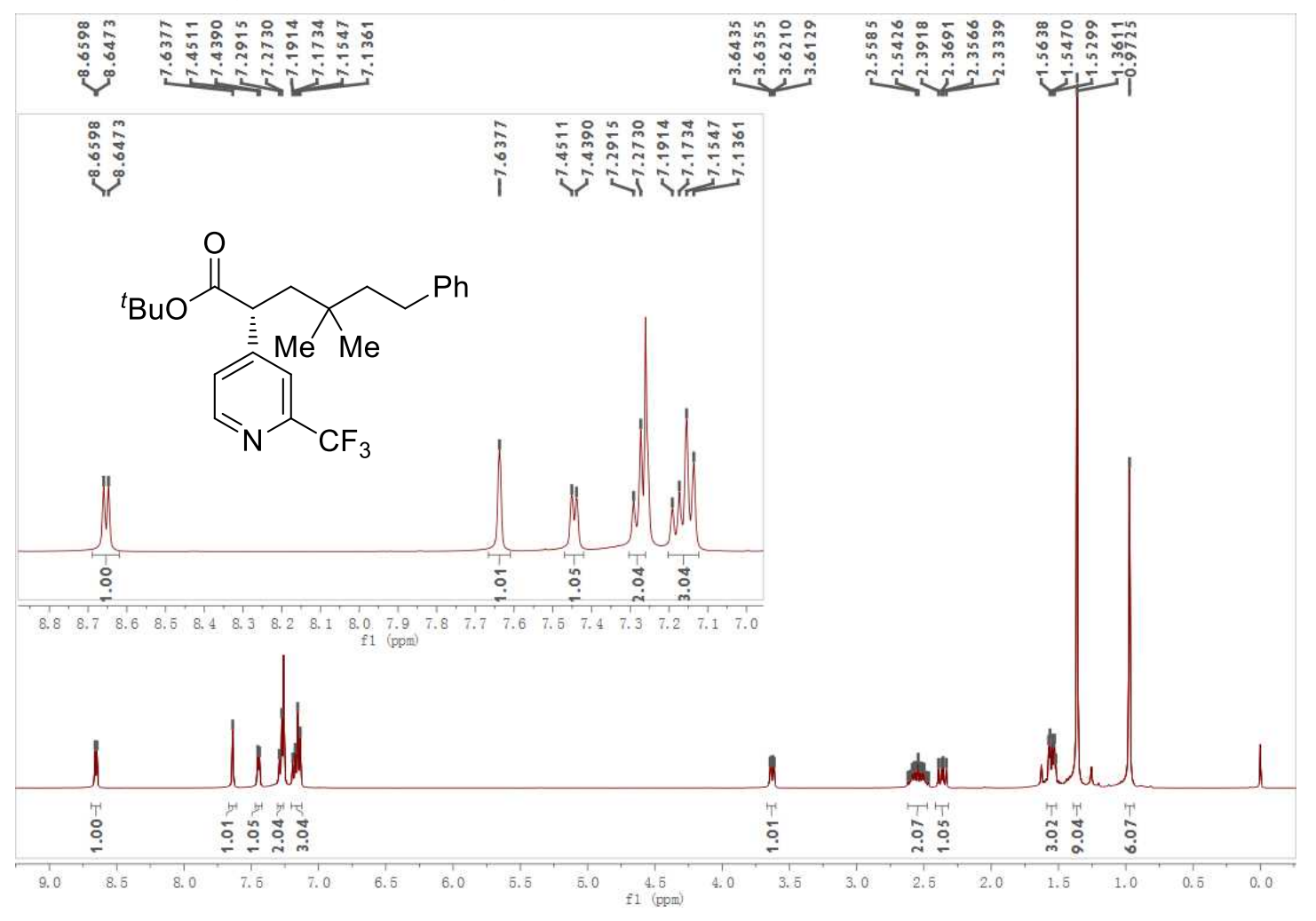


32; ${ }^{19}$ F-NMR (375 $\left.\mathrm{MHz}, \mathrm{CDCl}_{3}\right)$

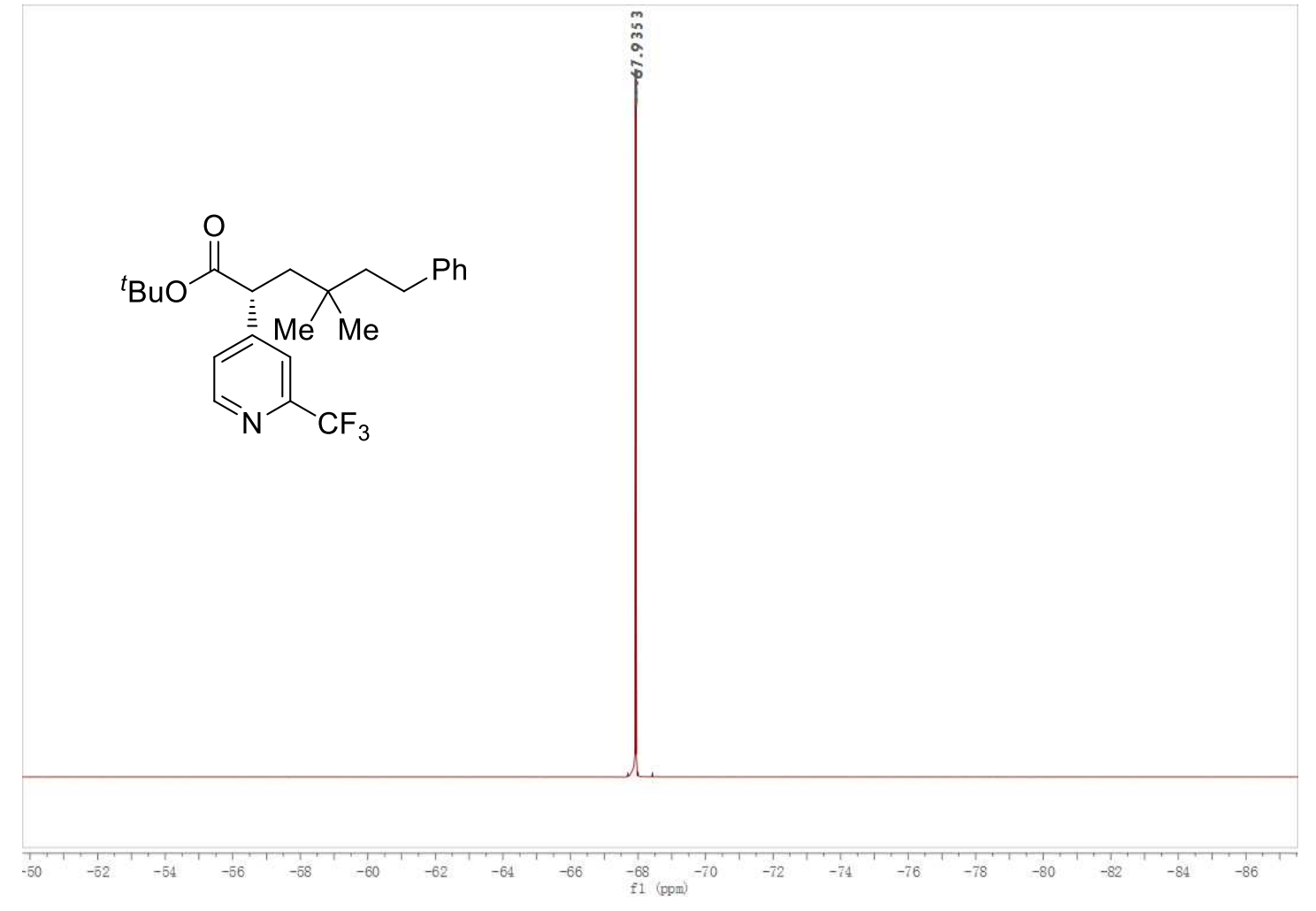

32; ${ }^{13} \mathrm{C}-\mathrm{NMR}\left(100 \mathrm{MHz}, \mathrm{CDCl}_{3}\right)$

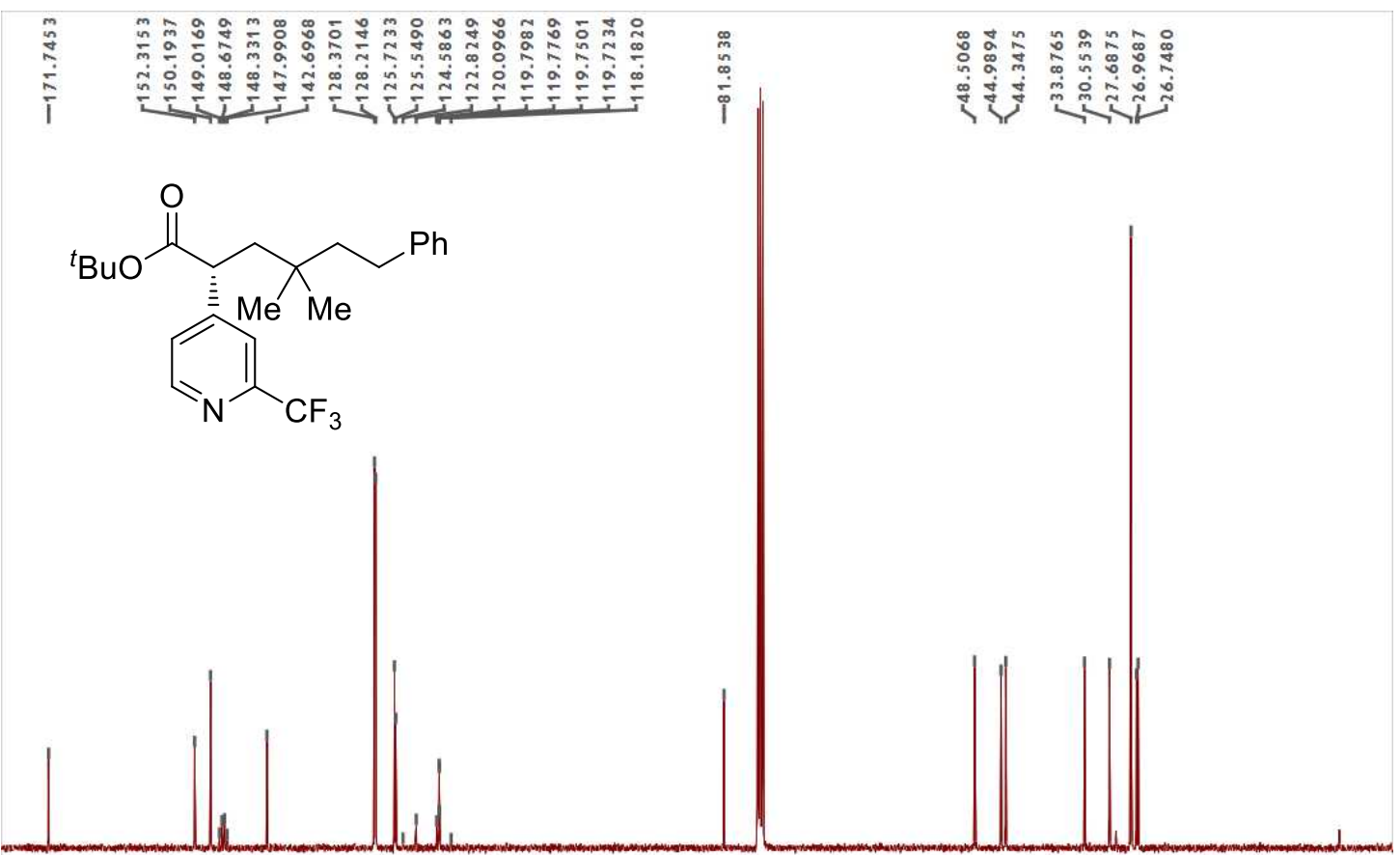

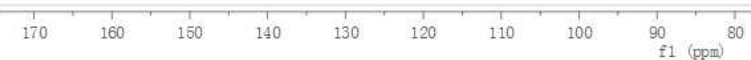


33; ${ }^{1} \mathrm{H}-\mathrm{NMR}$ (400 $\mathrm{MHz}, \mathrm{CDCl}_{3}$ )

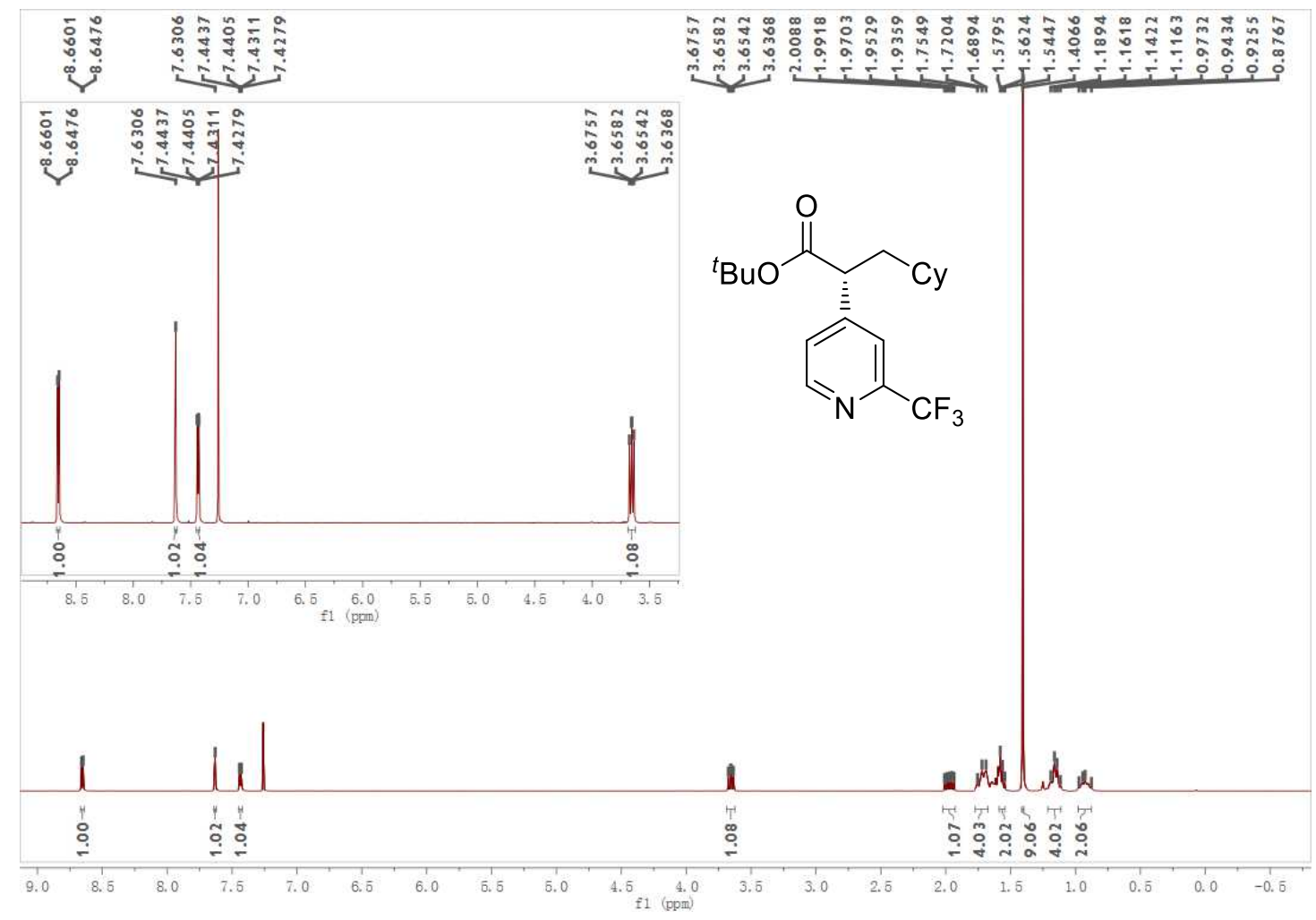

33; ${ }^{19}$ F-NMR (375 MHz, $\left.\mathrm{CDCl}_{3}\right)$

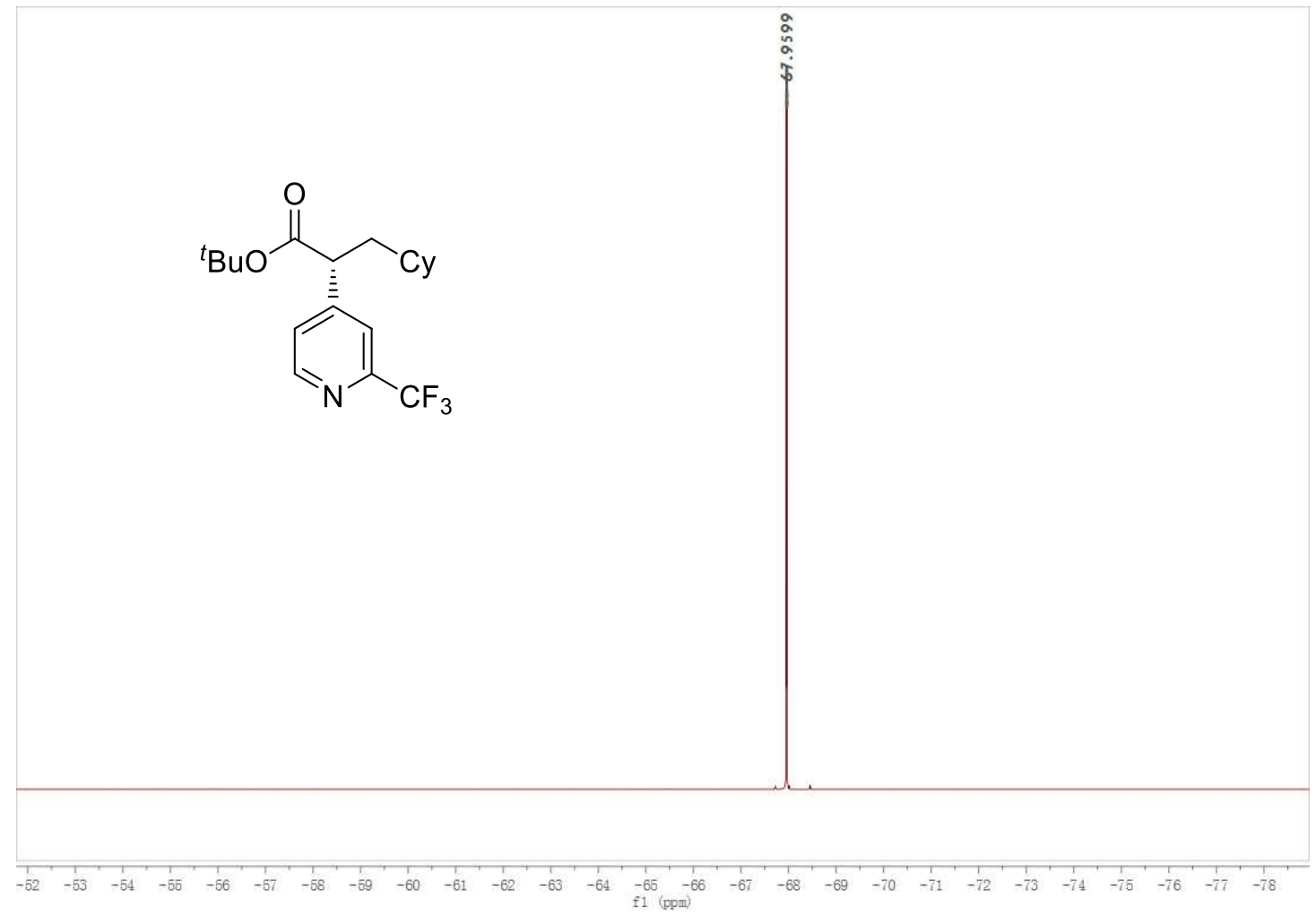


33; ${ }^{13} \mathrm{C}-\mathrm{NMR}$ (100 MHz, $\left.\mathrm{CDCl}_{3}\right)$

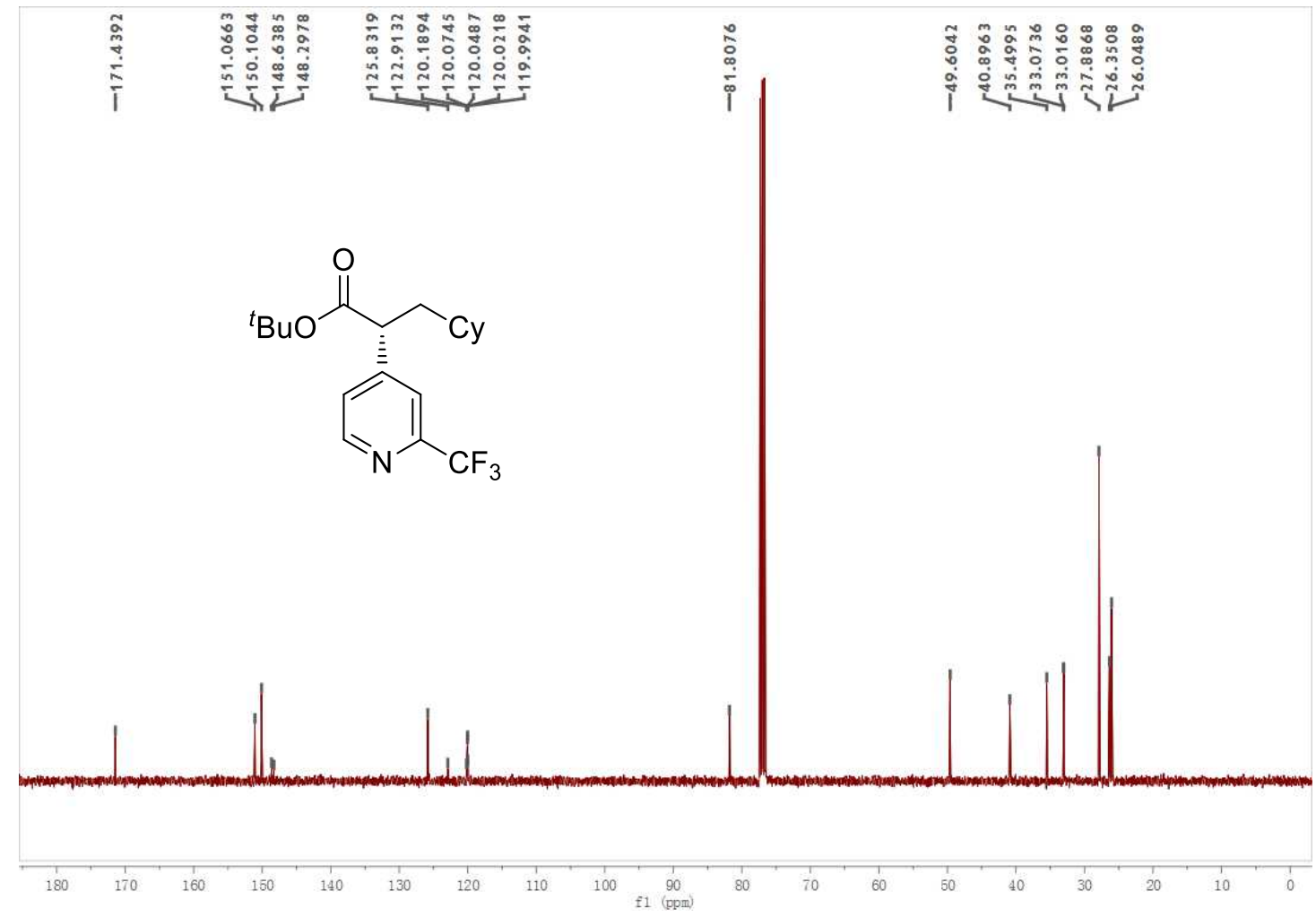

34; ${ }^{1} \mathrm{H}-\mathrm{NMR}$ (400 MHz, $\mathrm{CDCl}_{3}$ )

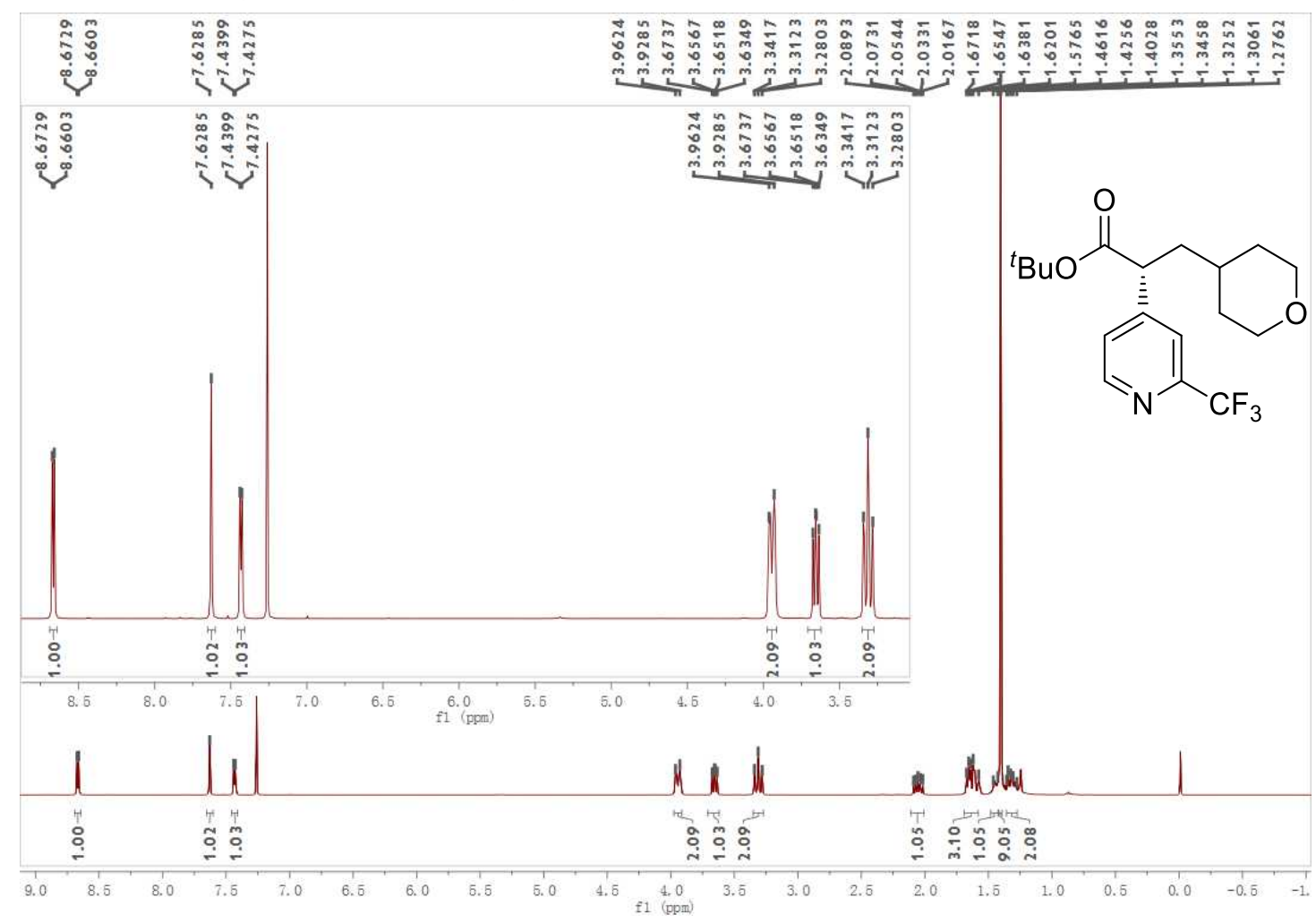


34; ${ }^{19}$ F-NMR (375 MHz, $\mathrm{CDCl}_{3}$ )

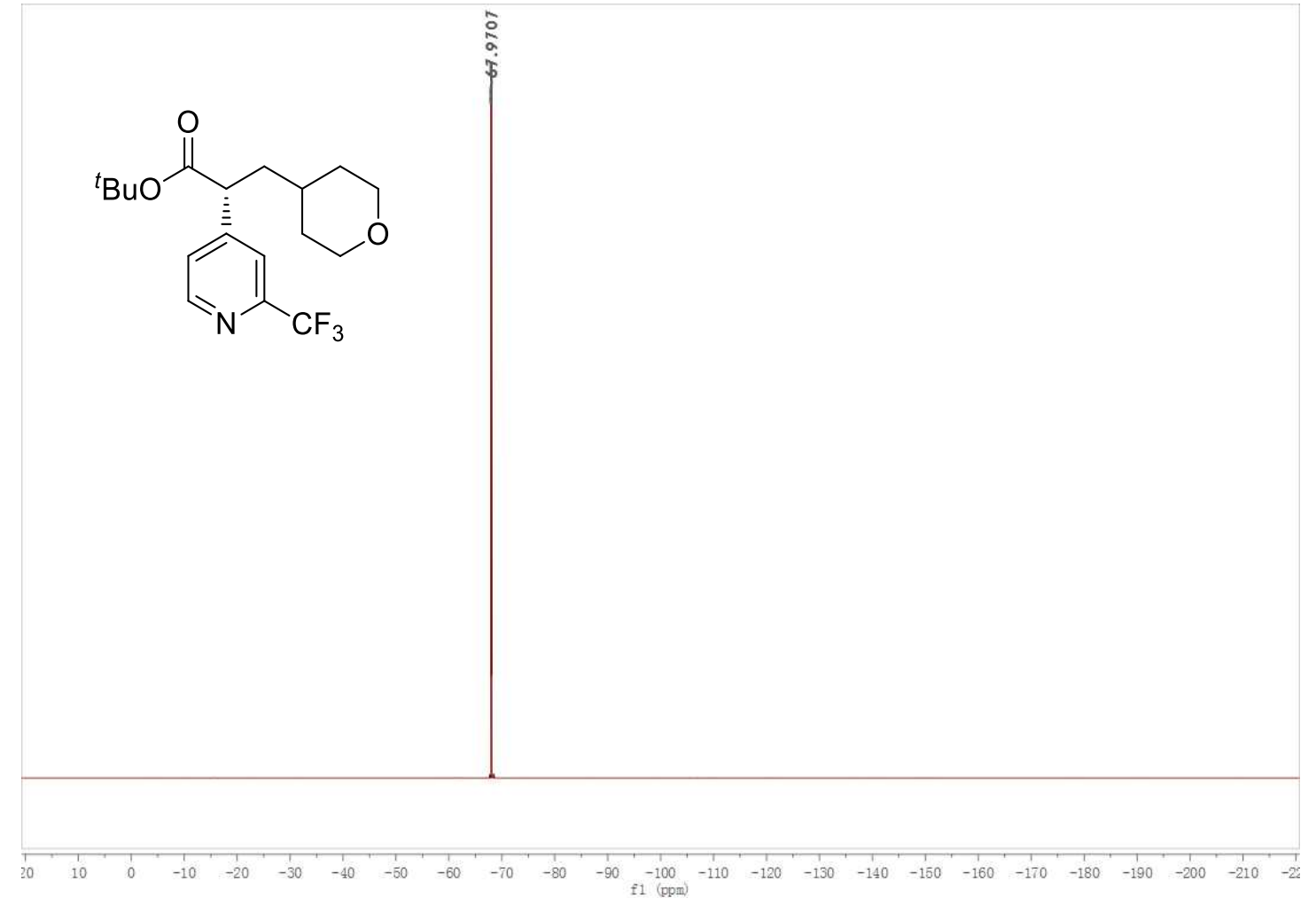

34; ${ }^{13} \mathrm{C}-\mathrm{NMR}$ (100 MHz, $\mathrm{CDCl}_{3}$ )

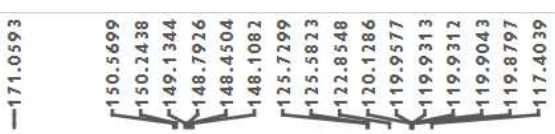<smiles>CC(C)(C)OC(=O)[C@H](CC1CCOCC1)c1ccnc(C(F)(F)F)c1</smiles>

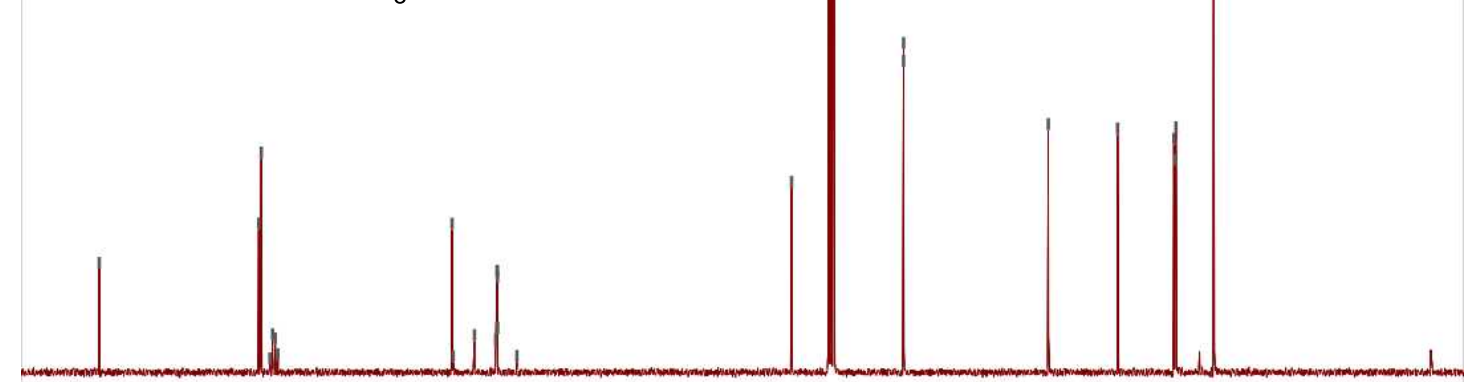

180
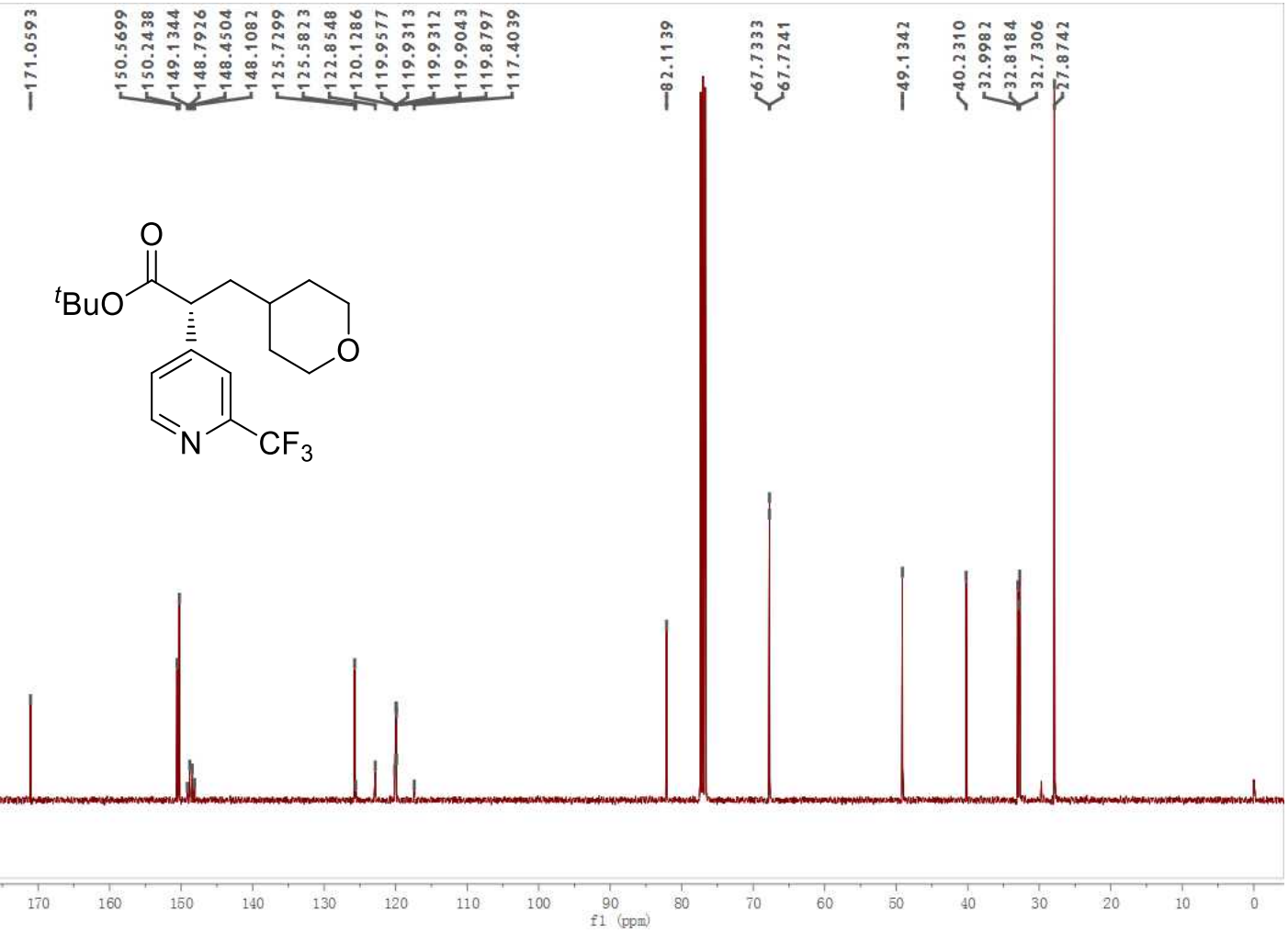
35; ${ }^{1} \mathrm{H}-\mathrm{NMR}$ (400 $\mathrm{MHz}, \mathrm{CDCl}_{3}$ )

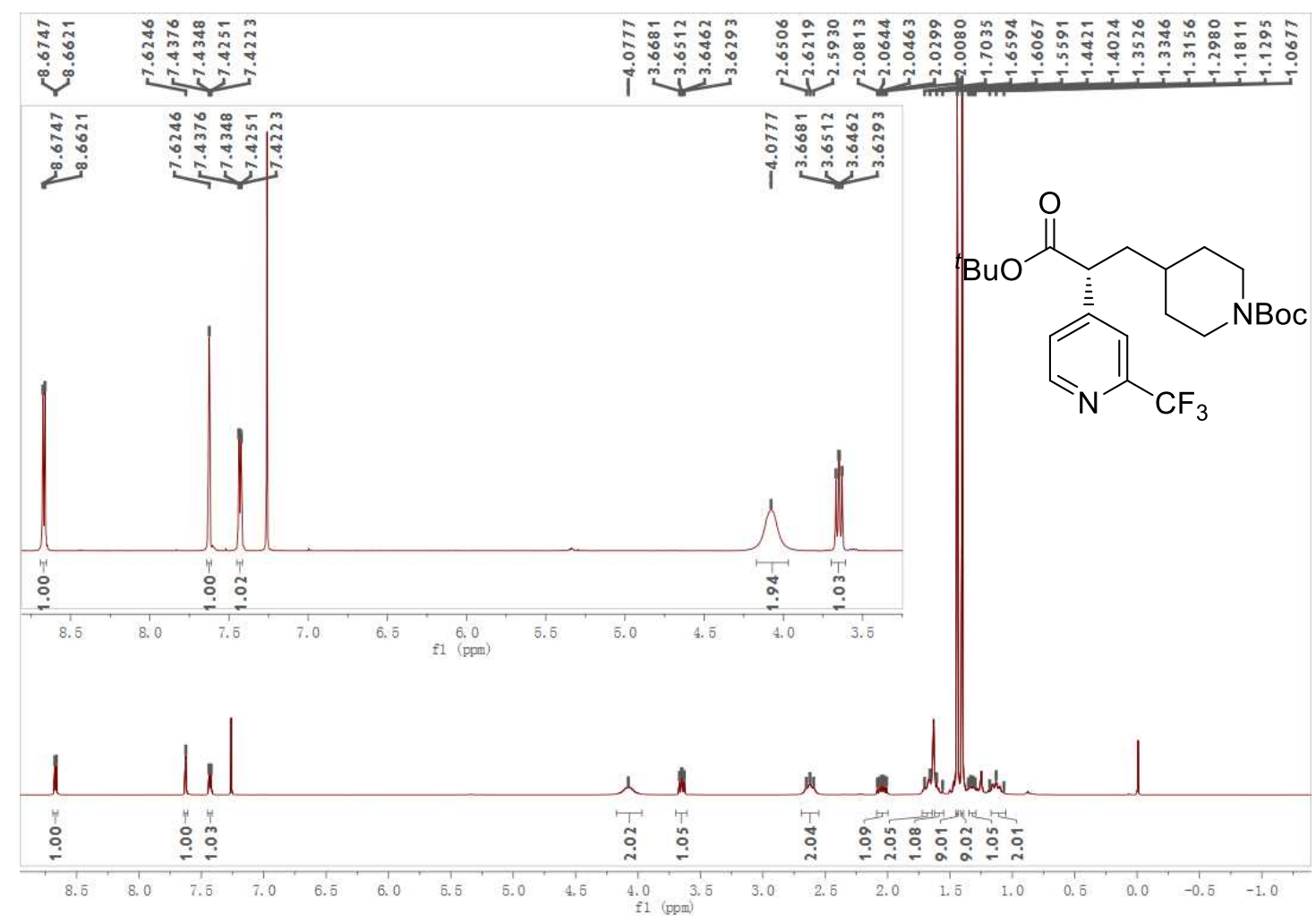

35; ${ }^{19}$ F-NMR (375 MHz, $\left.\mathrm{CDCl}_{3}\right)$

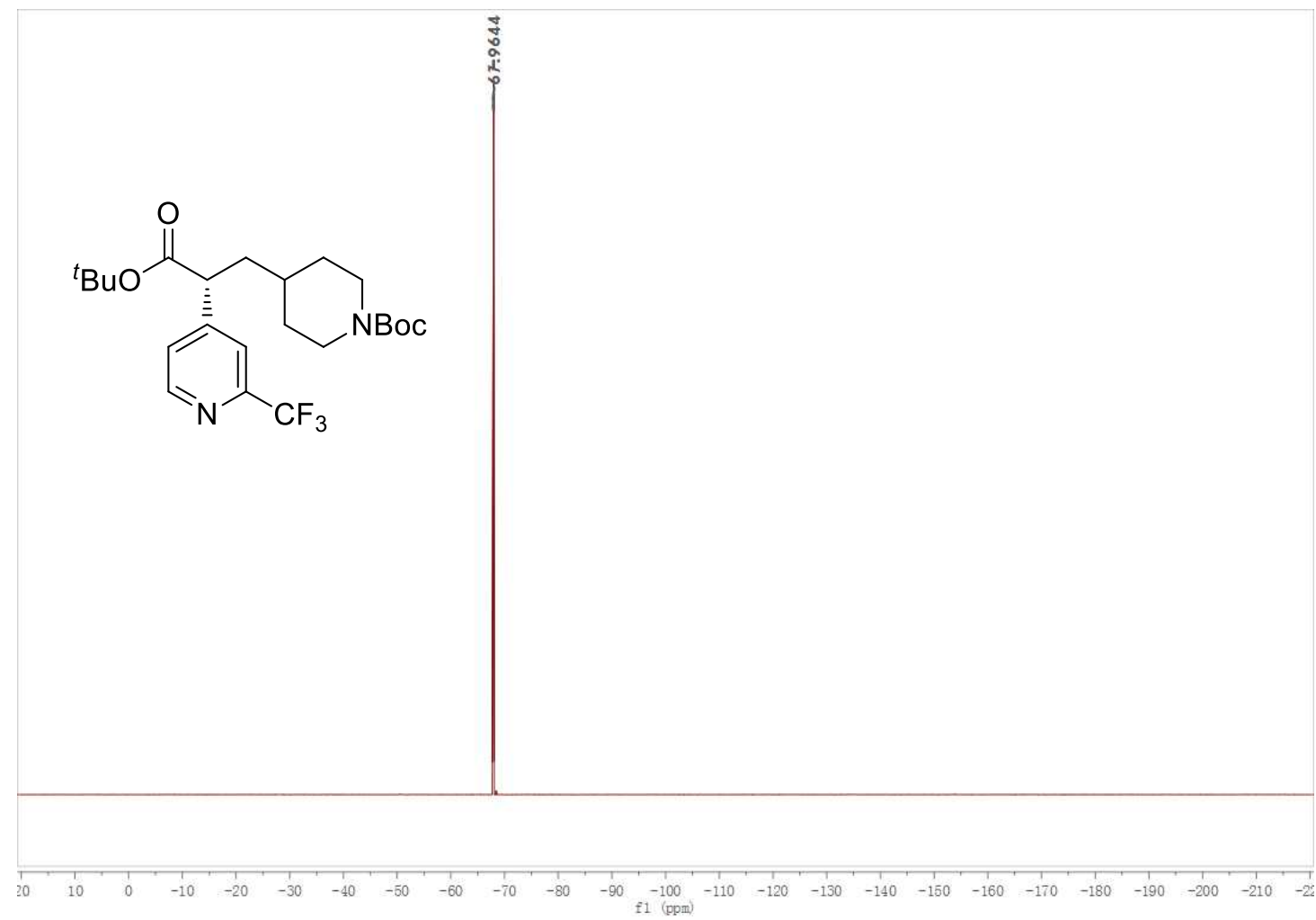


35; ${ }^{13} \mathrm{C}-\mathrm{NMR}\left(100 \mathrm{MHz}, \mathrm{CDCl}_{3}\right)$

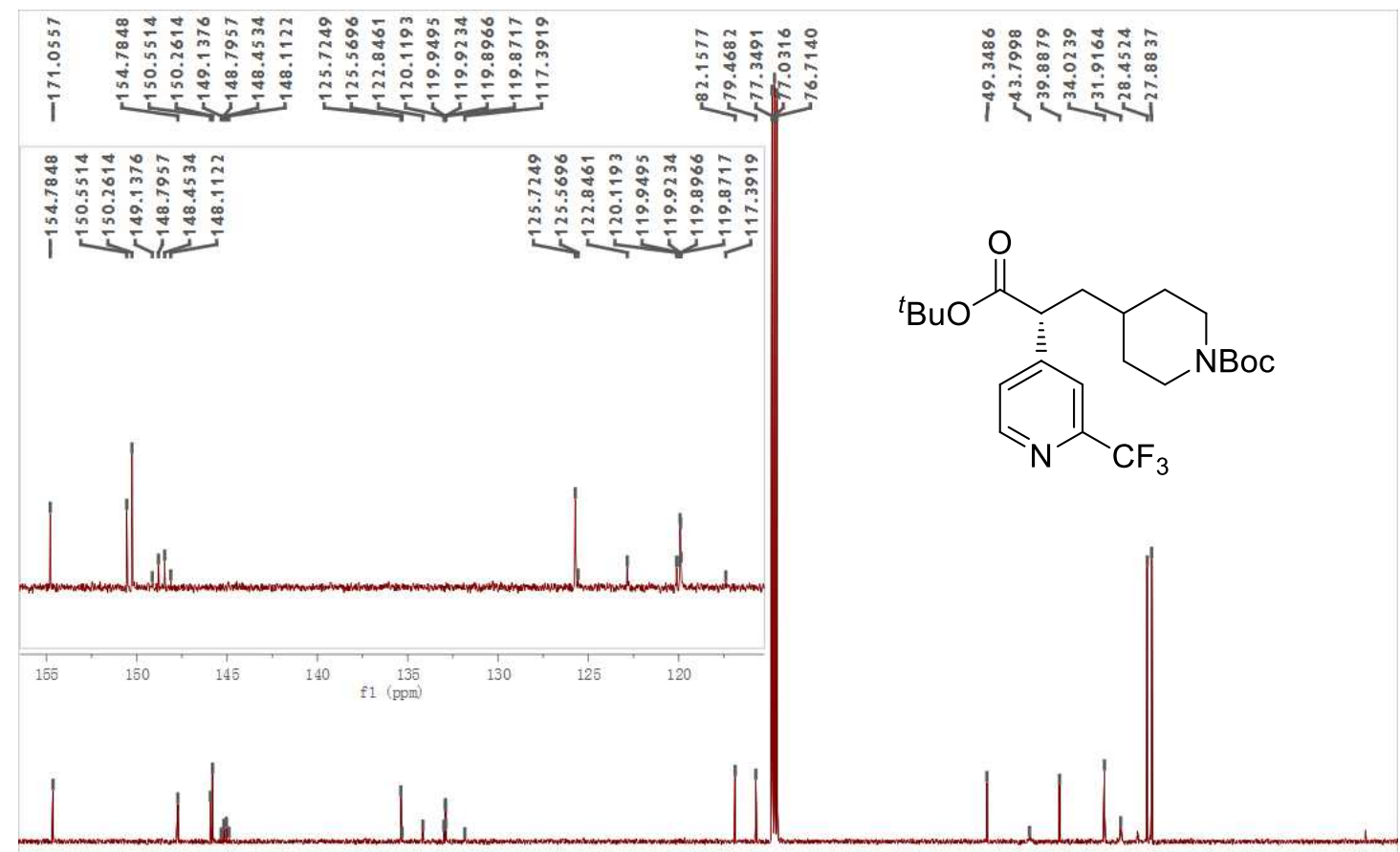

36; ${ }^{1} \mathrm{H}-\mathrm{NMR}\left(400 \mathrm{MHz}, \mathrm{CDCl}_{3}\right.$ )

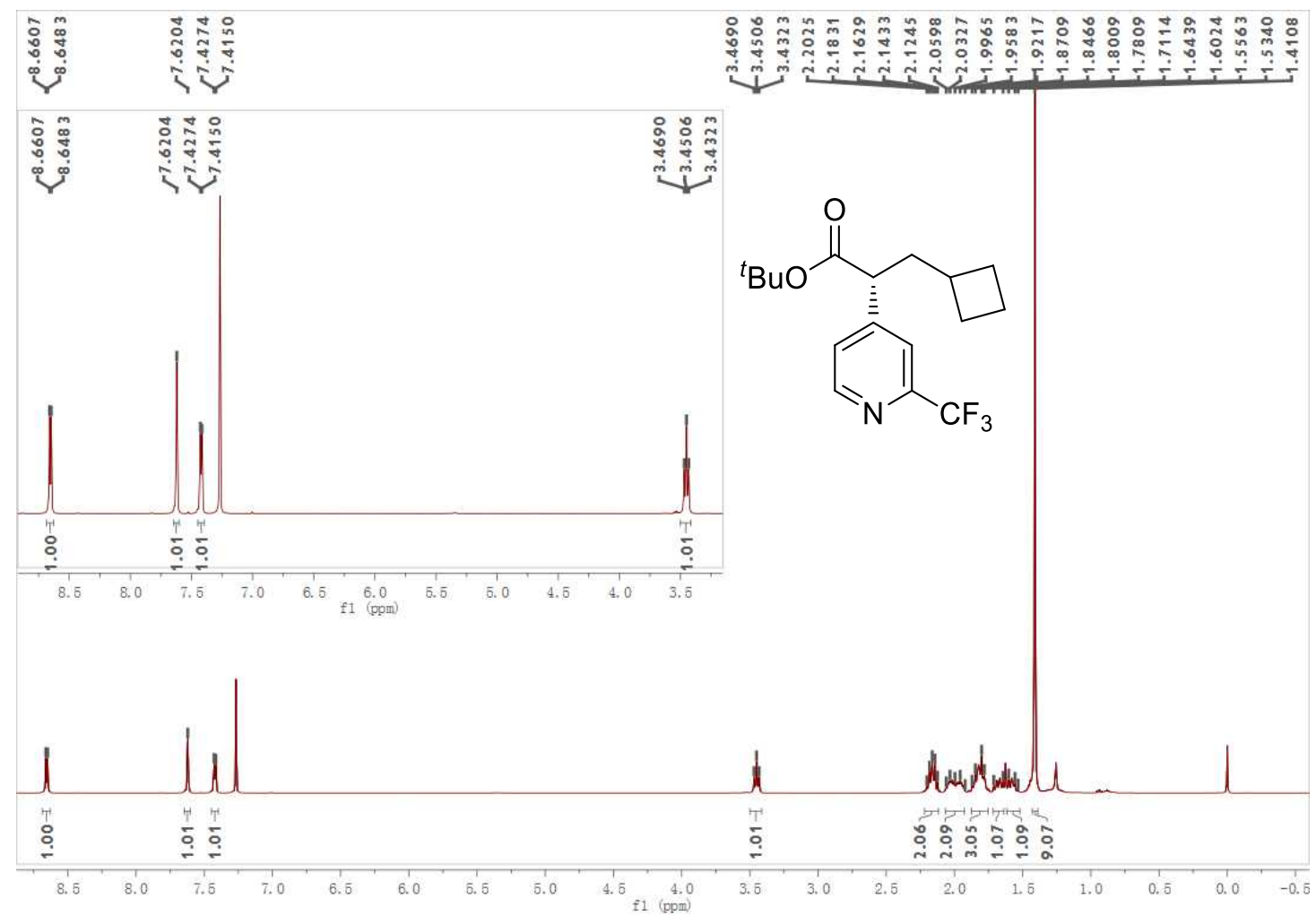


36; ${ }^{19}$ F-NMR (375 MHz, $\mathrm{CDCl}_{3}$ )

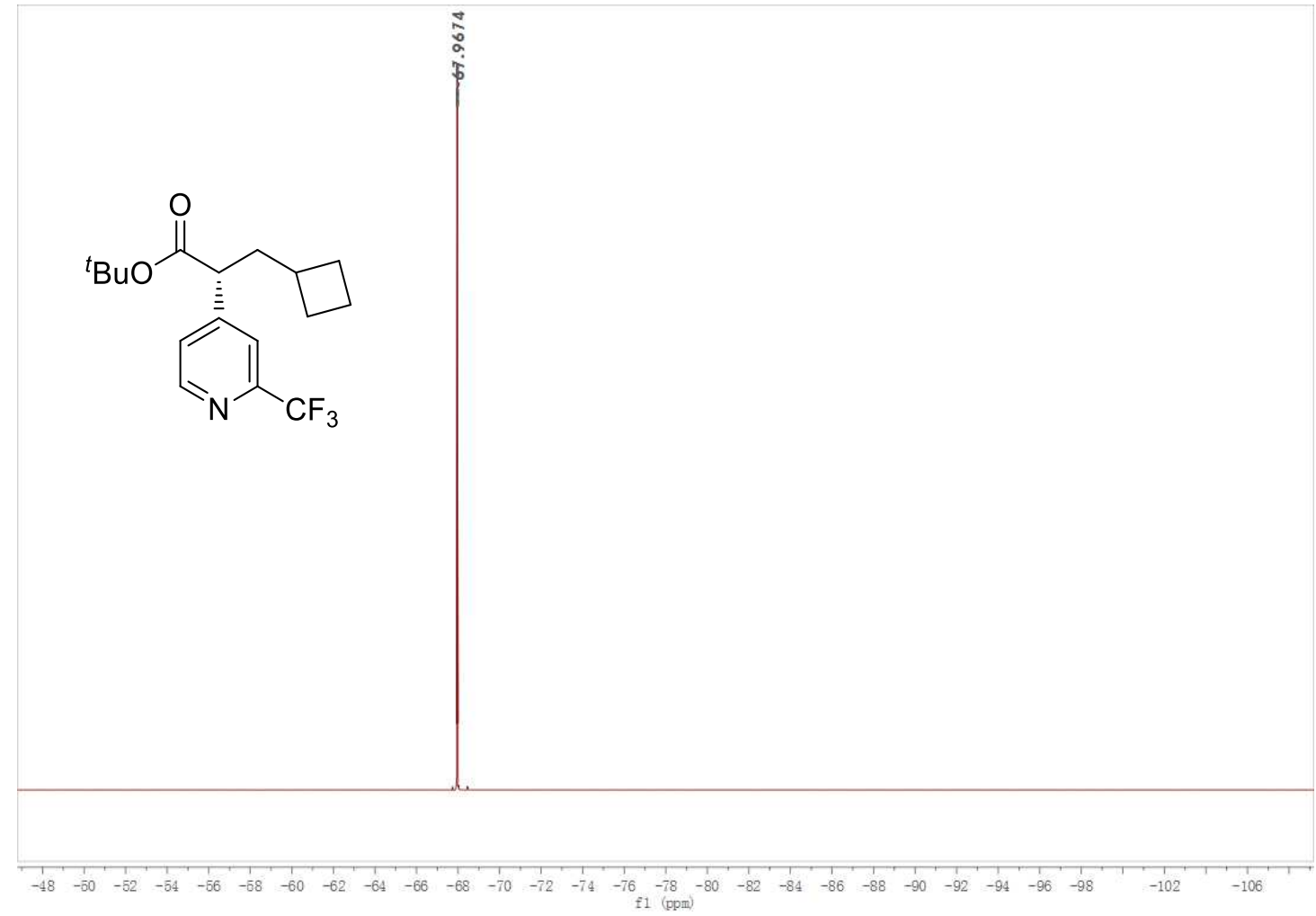

36; ${ }^{13} \mathrm{C}-\mathrm{NMR}\left(100 \mathrm{MHz}, \mathrm{CDCl}_{3}\right)$

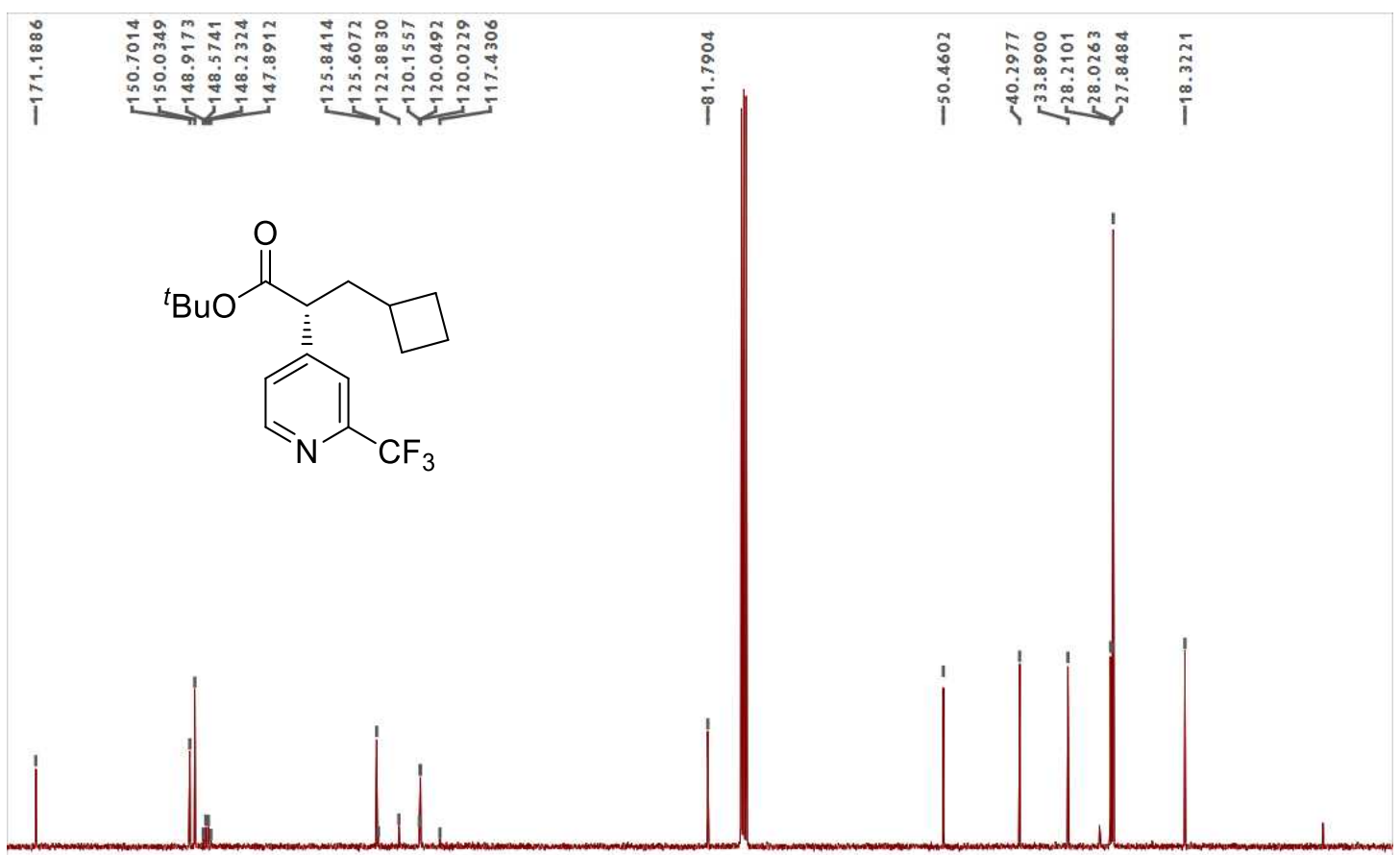

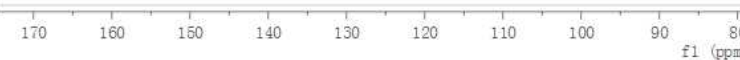


37; ${ }^{1} \mathrm{H}-\mathrm{NMR}$ (400 $\mathrm{MHz}, \mathrm{CDCl}_{3}$ )

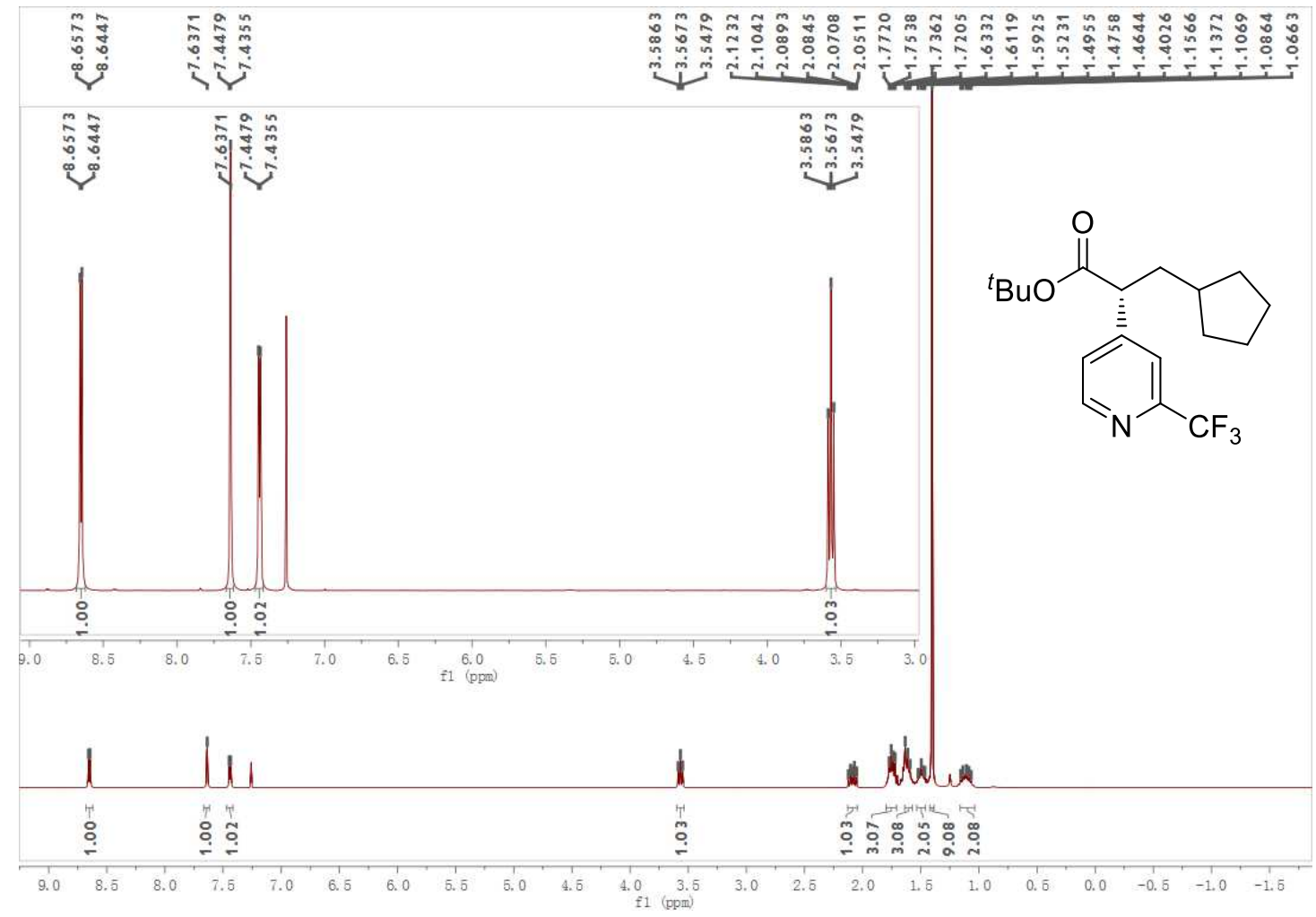

37; ${ }^{19}$ F-NMR (375 MHz, $\mathrm{CDCl}_{3}$ )

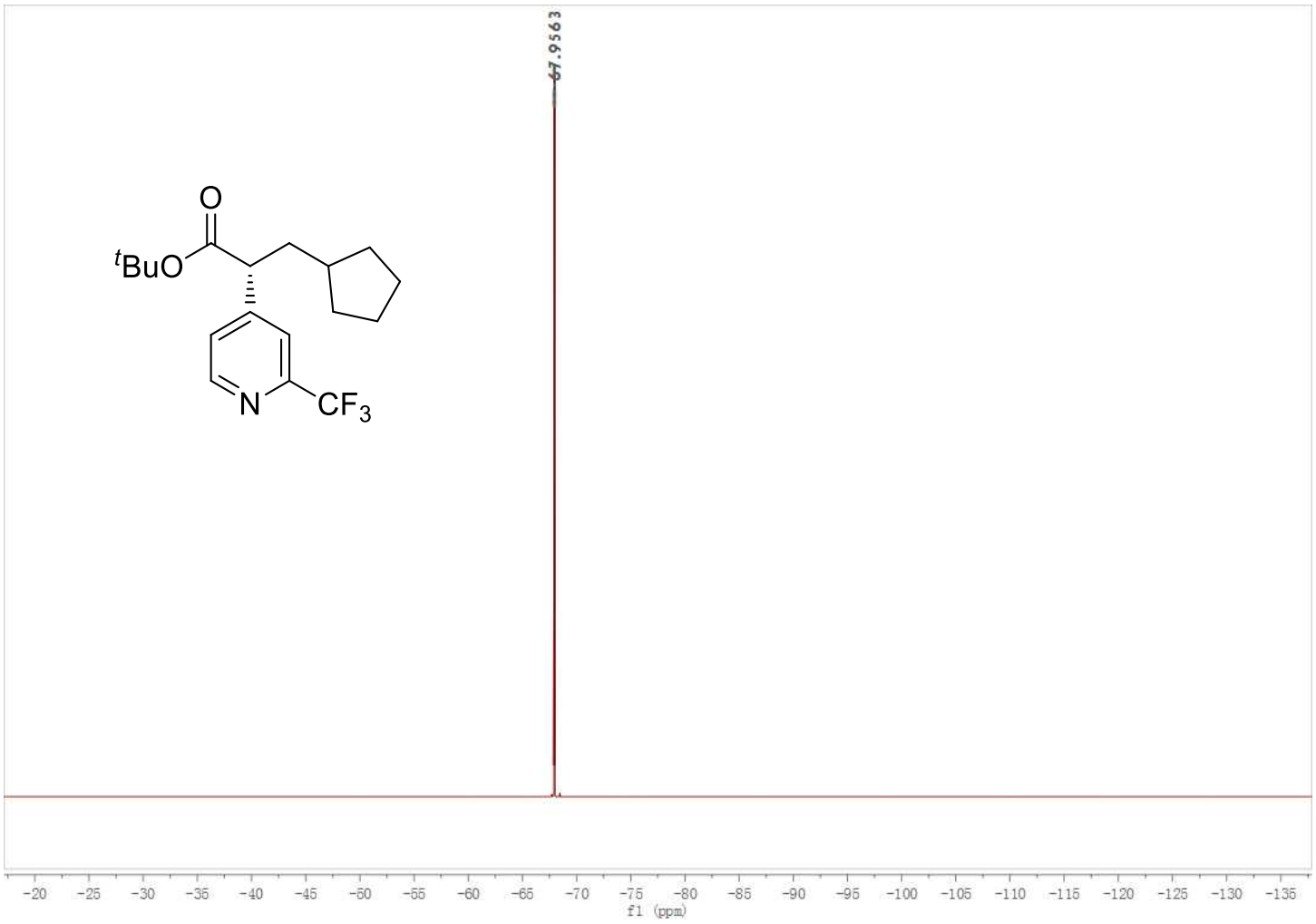


37; ${ }^{13} \mathrm{C}-\mathrm{NMR}\left(100 \mathrm{MHz}, \mathrm{CDCl}_{3}\right)$

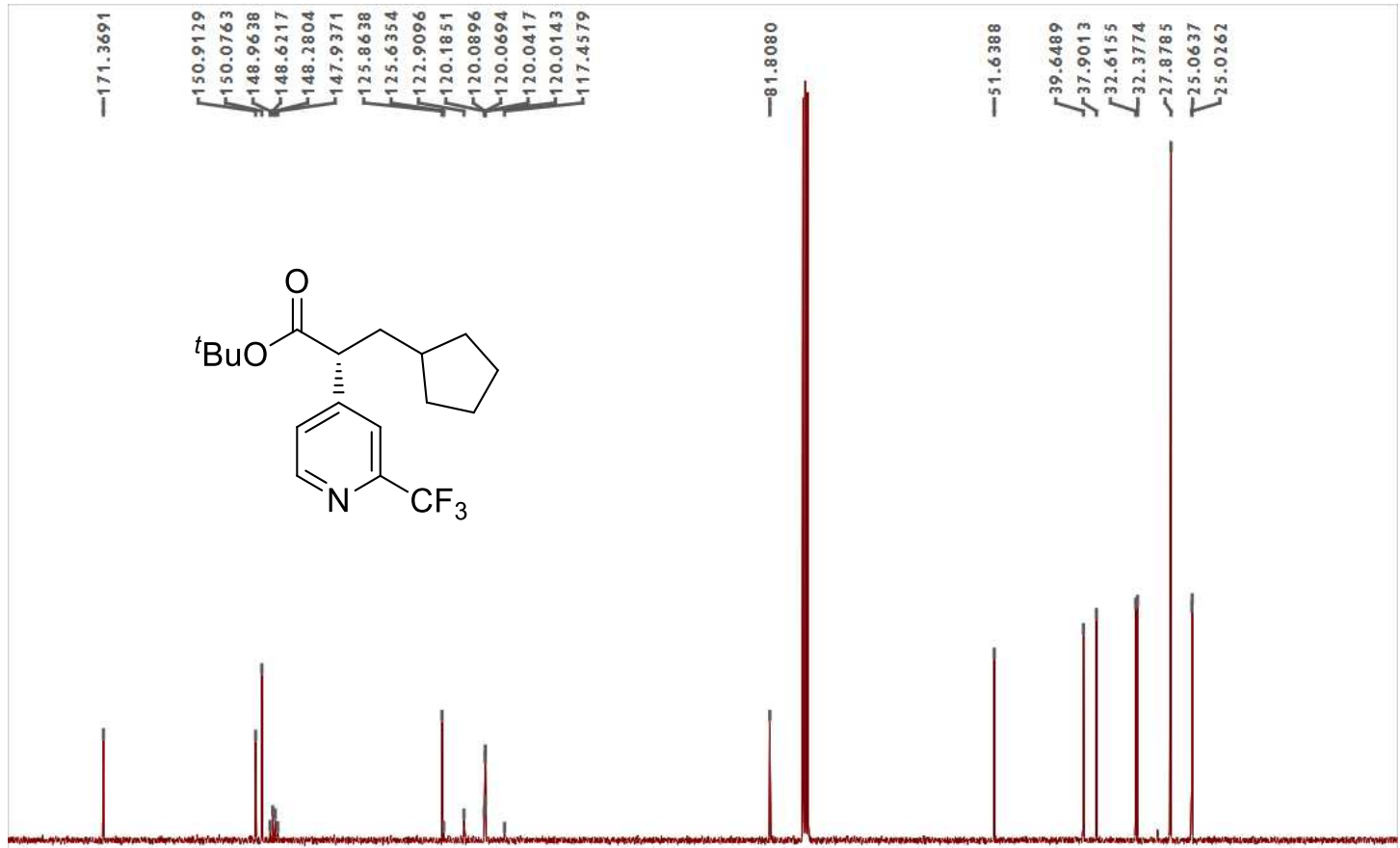

$\begin{array}{llllllllll}1 & 1 & 1 & 1 & 1 & 1 & 1 & 1 & 1 & 1 \\ 80 & 170 & 160 & 150 & 140 & 130 & 120 & 110 & 100 & 90 \\ \mathrm{f} 1(\mathrm{ppm})\end{array}$

38; ${ }^{1} \mathrm{H}-\mathrm{NMR}\left(400 \mathrm{MHz}, \mathrm{CDCl}_{3}\right.$ )

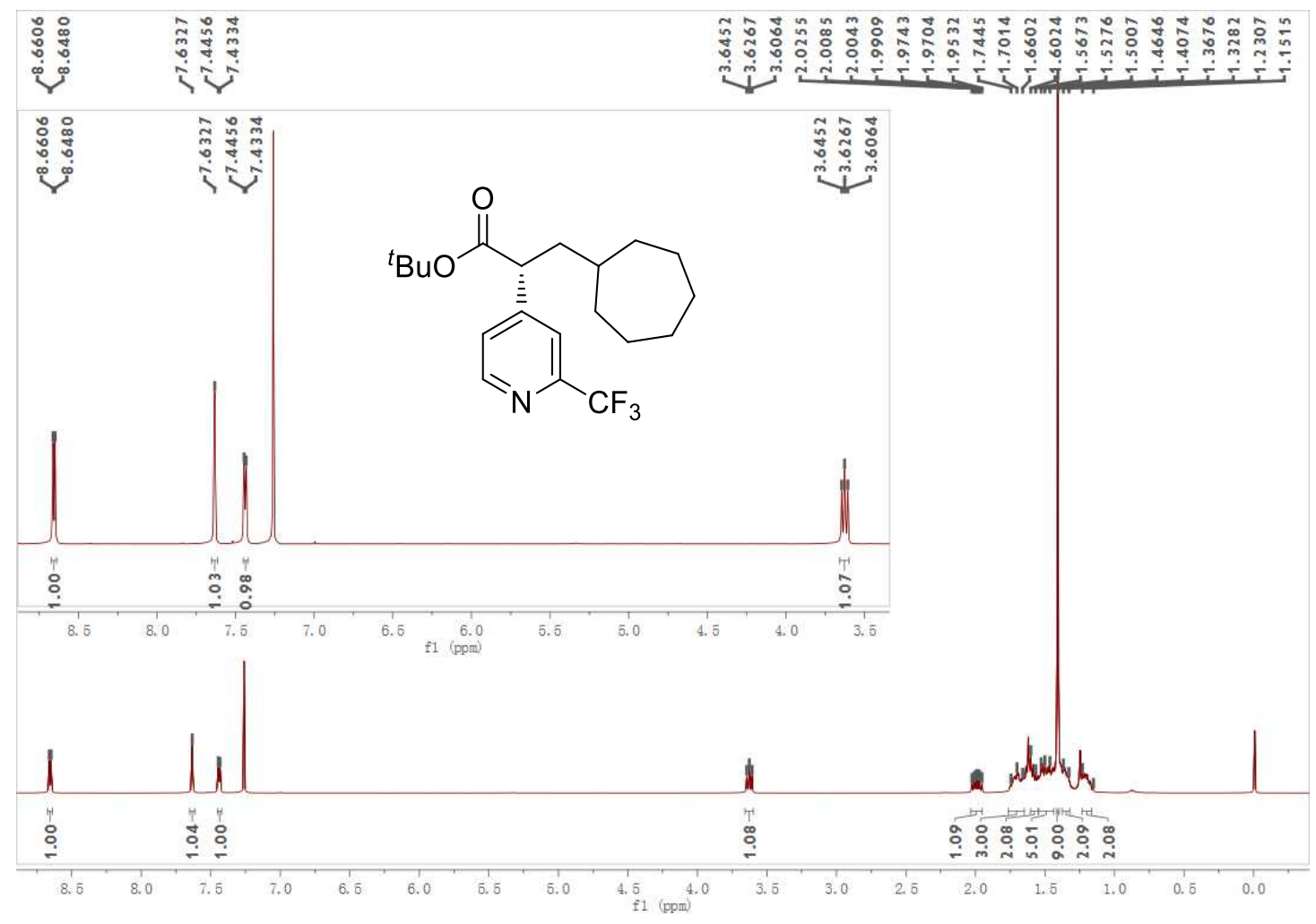


38; ${ }^{19}$ F-NMR (375 MHz, $\mathrm{CDCl}_{3}$ )

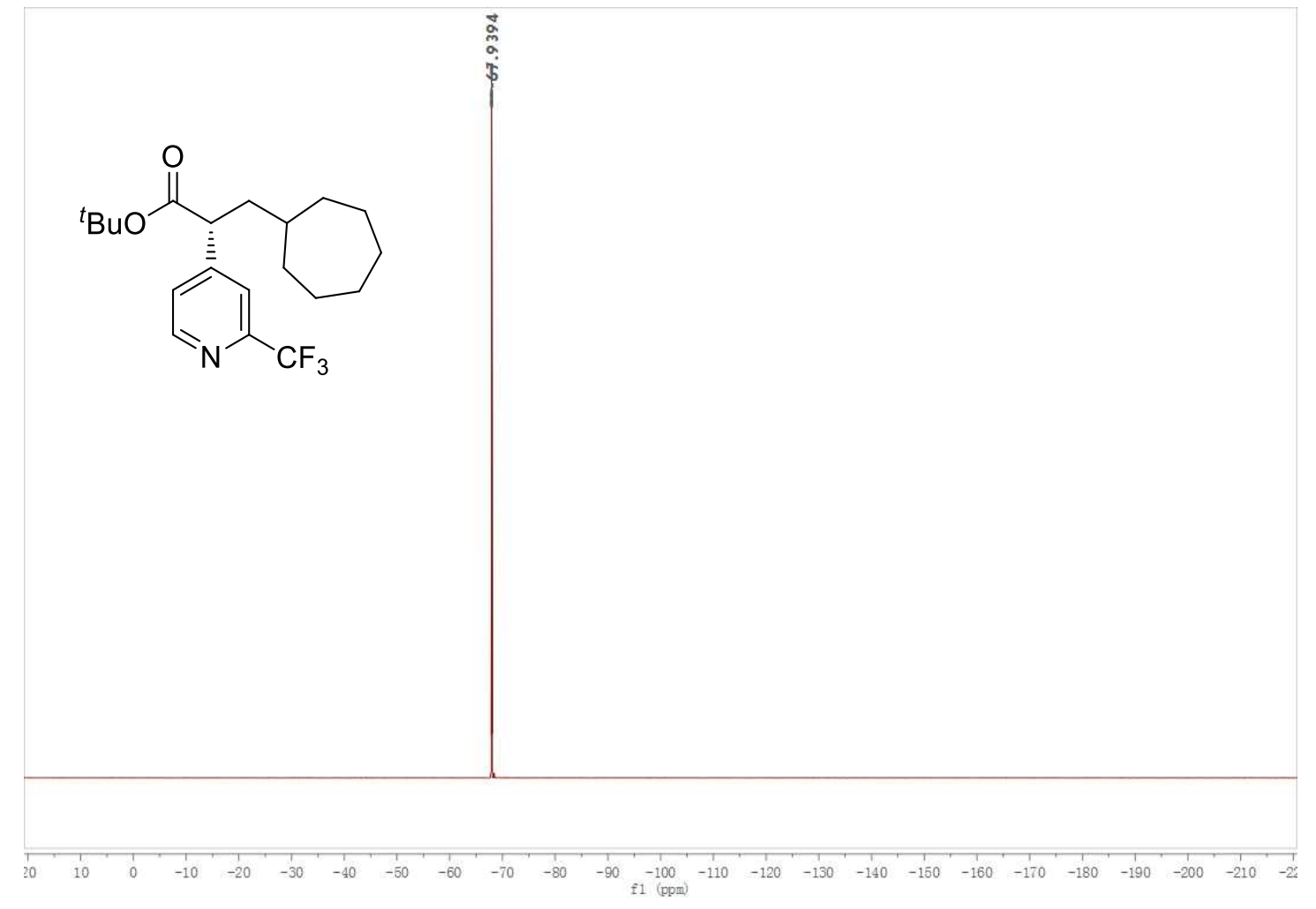

38; ${ }^{13} \mathrm{C}-\mathrm{NMR}$ (100 MHz, $\left.\mathrm{CDCl}_{3}\right)$

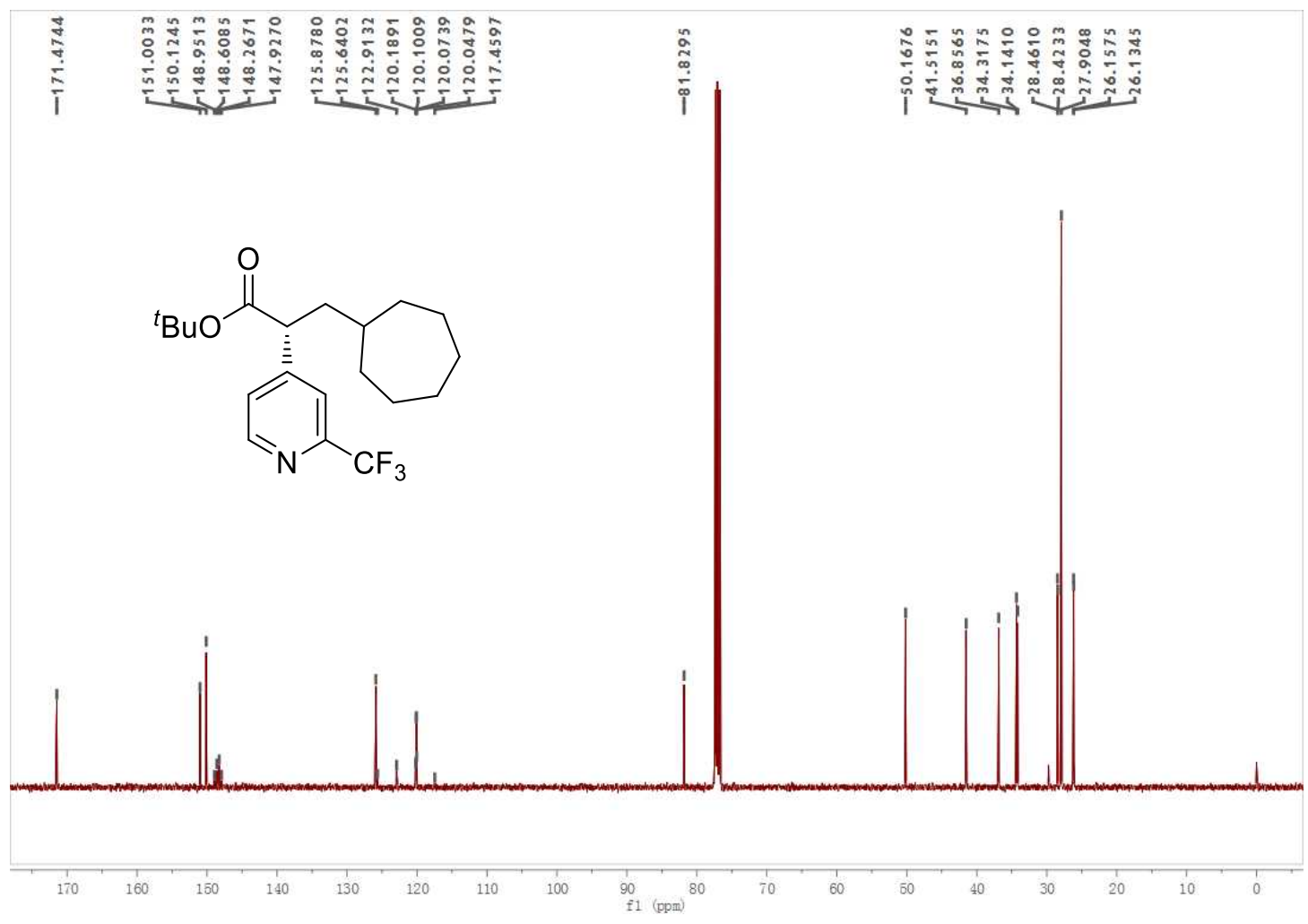


39; ${ }^{1} \mathrm{H}-\mathrm{NMR}$ (400 $\mathrm{MHz}, \mathrm{CDCl}_{3}$ )

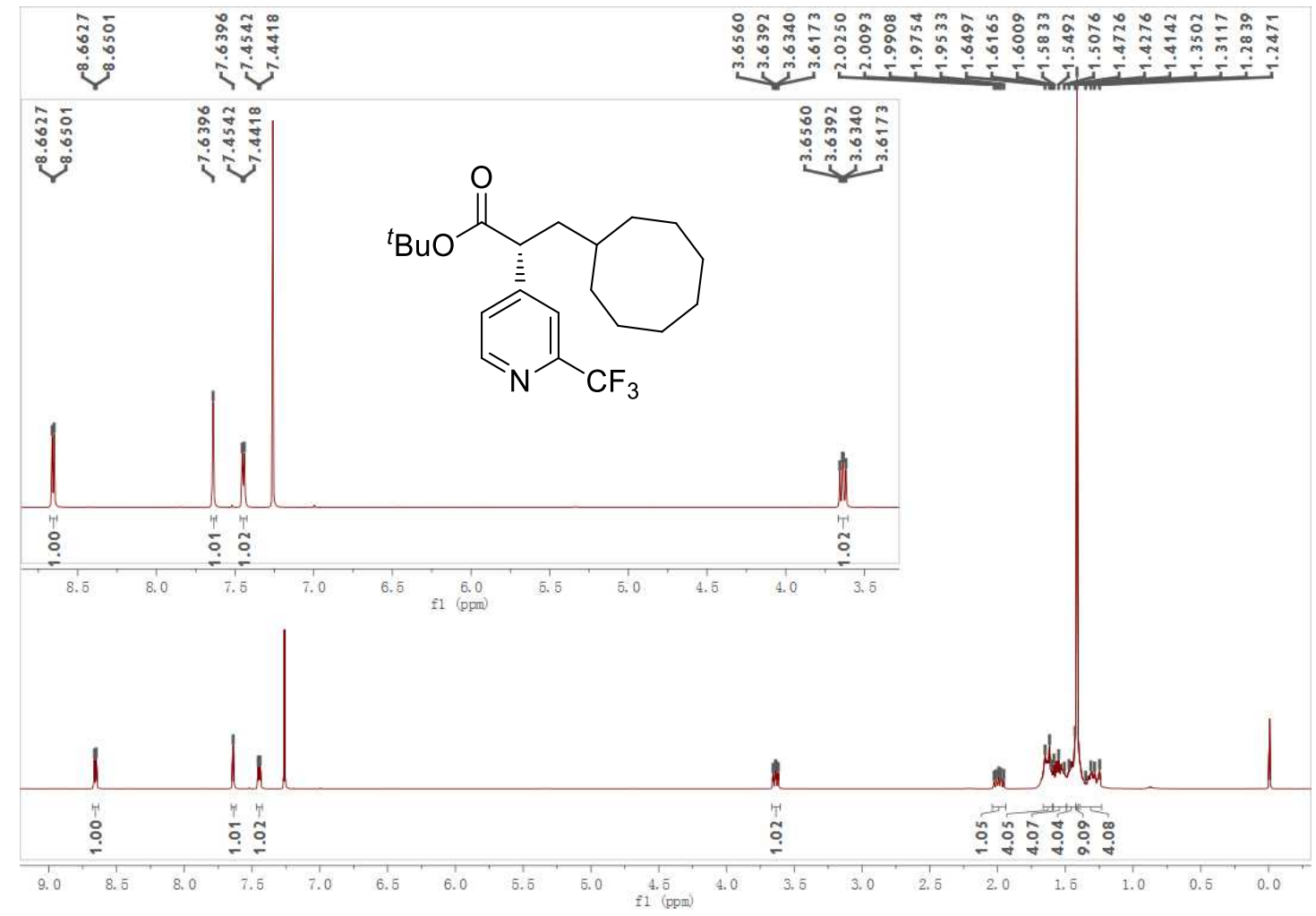

39; ${ }^{19}$ F-NMR (375 MHz, $\left.\mathrm{CDCl}_{3}\right)$

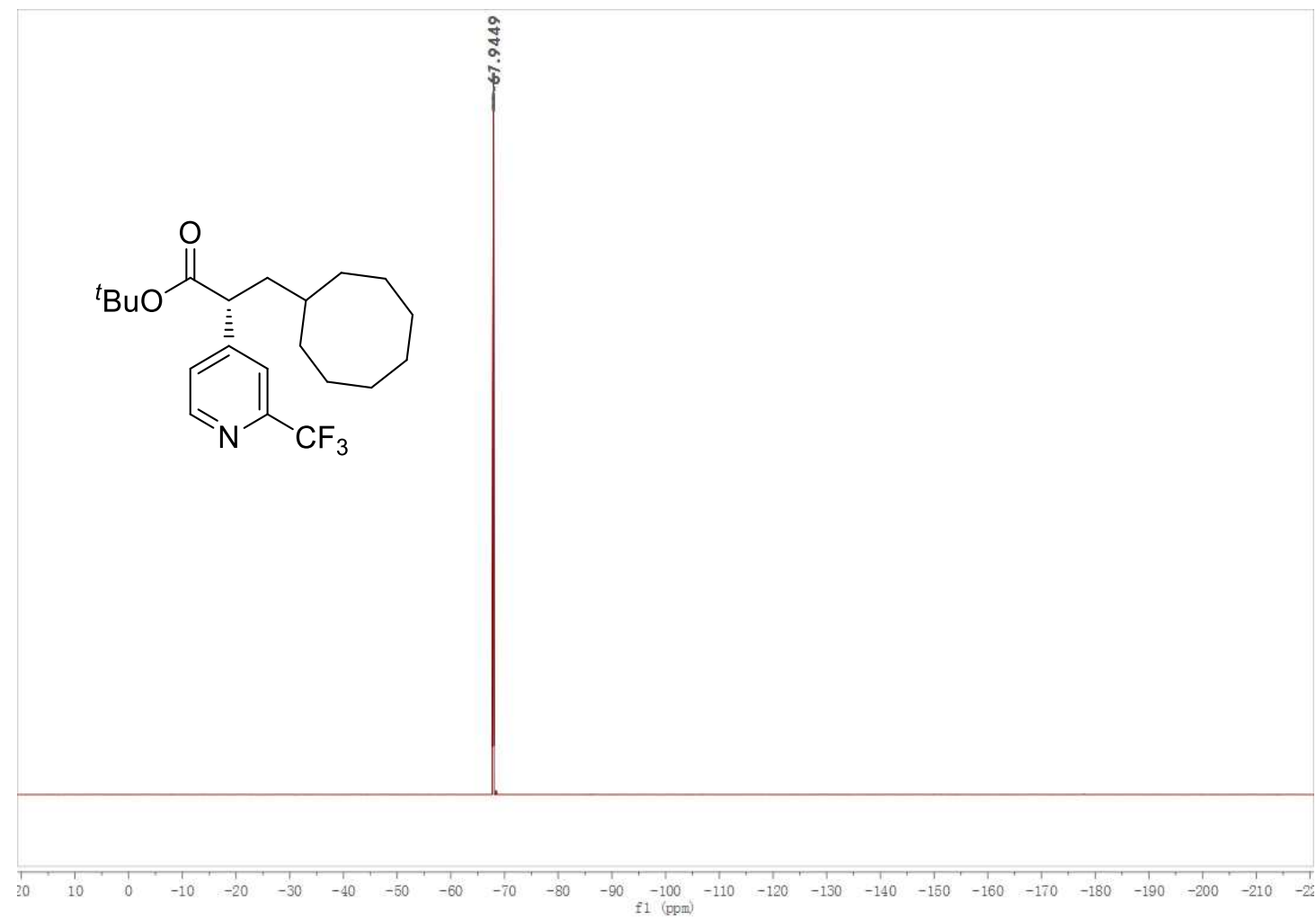


39; ${ }^{13} \mathrm{C}-\mathrm{NMR}$ (100 MHz, $\mathrm{CDCl}_{3}$ )

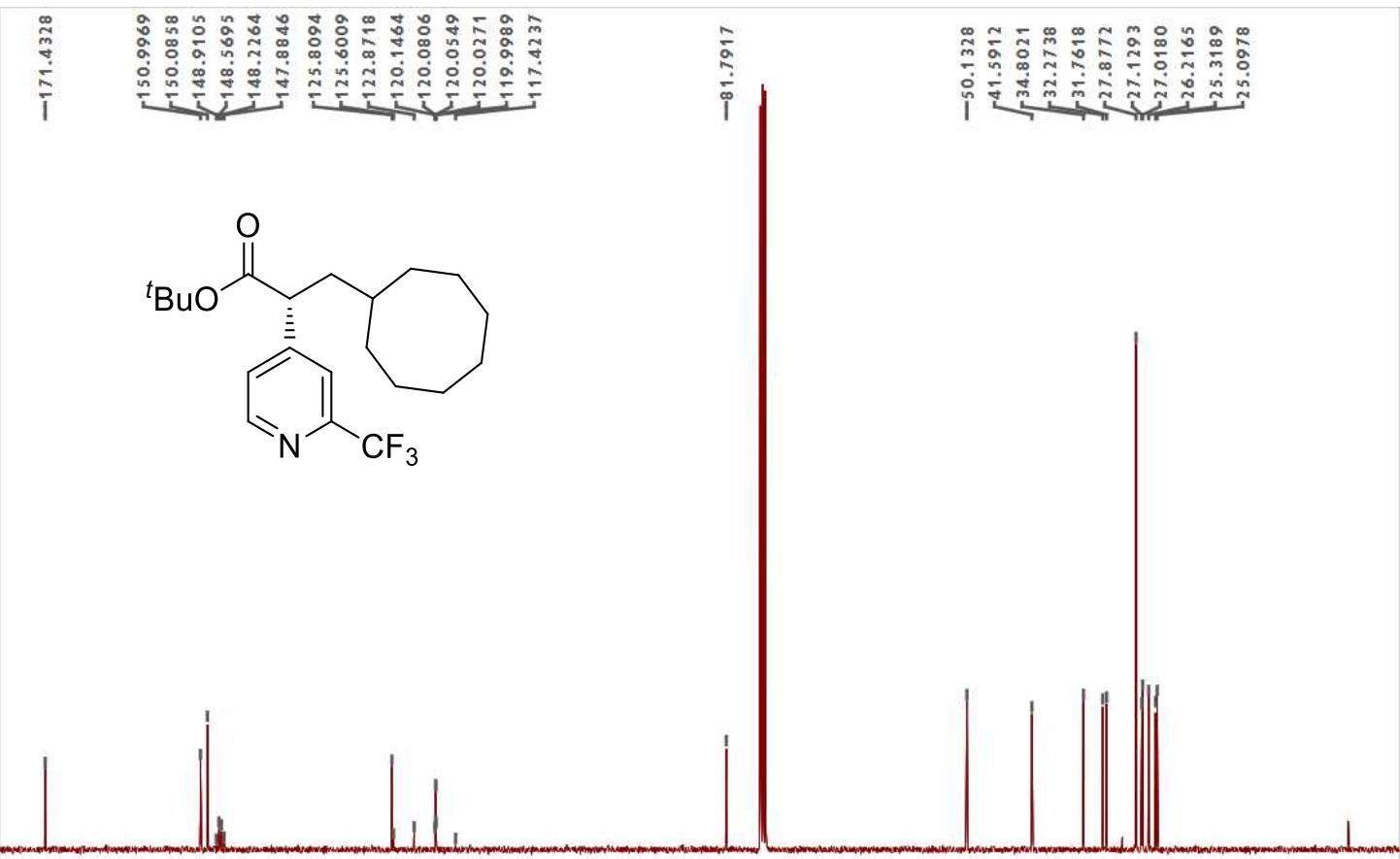

40; ${ }^{1} \mathrm{H}-\mathrm{NMR}\left(400 \mathrm{MHz}, \mathrm{CDCl}_{3}\right)$

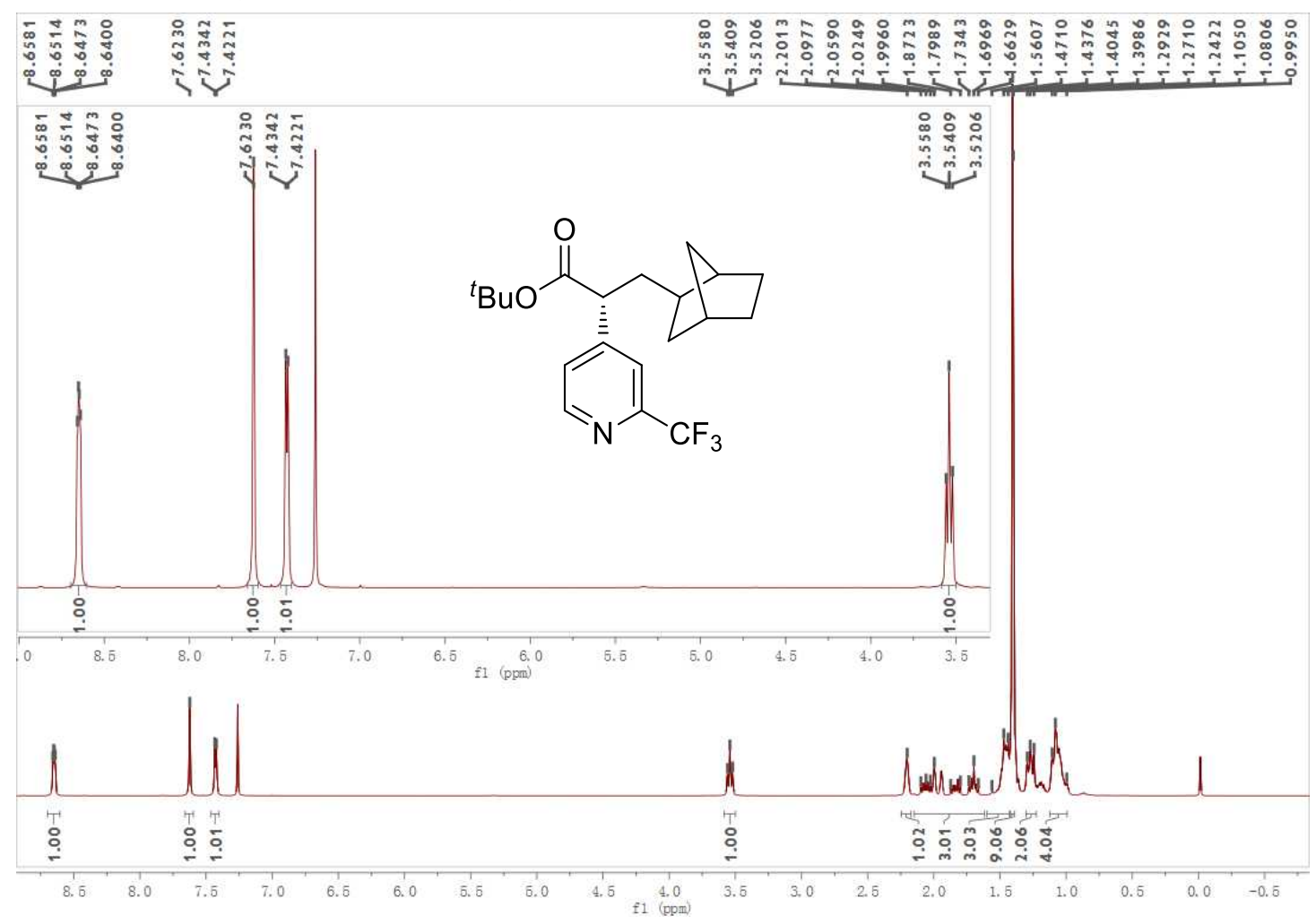


40; ${ }^{19}$ F-NMR (375 MHz, $\mathrm{CDCl}_{3}$ )

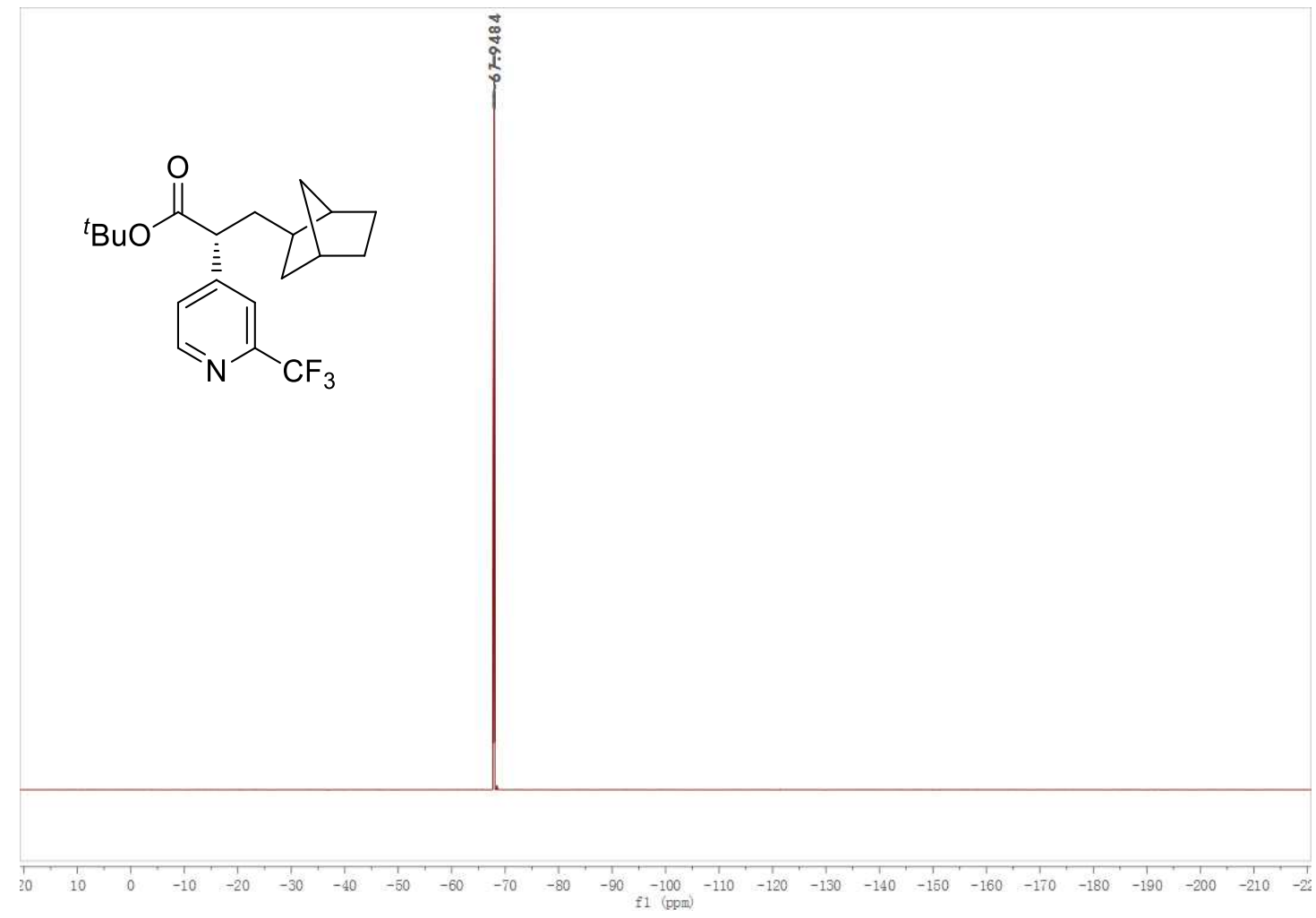

40; ${ }^{13} \mathrm{C}-\mathrm{NMR}\left(100 \mathrm{MHz}, \mathrm{CDCl}_{3}\right)$

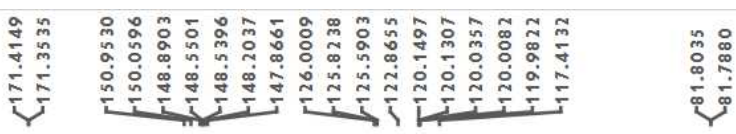<smiles>CC(C)(C)OC(=O)[C@H](CC12CCC(C1)C2)c1ccnc(C(F)(F)F)c1</smiles>

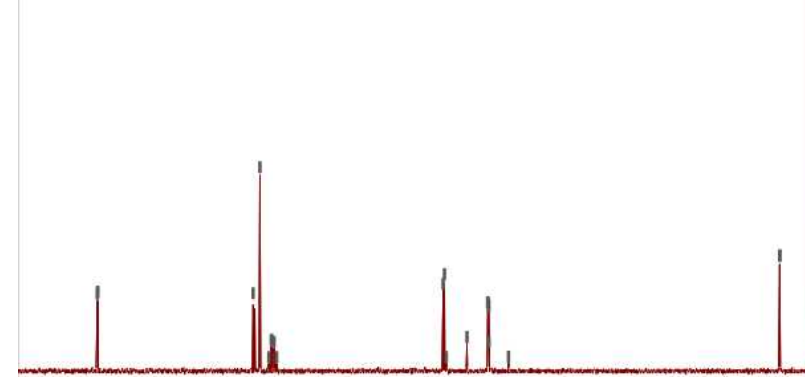

$\frac{1}{180}$

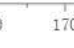

160
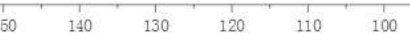

f1 (ppm) 
41; ${ }^{1} \mathrm{H}-\mathrm{NMR}\left(400 \mathrm{MHz}, \mathrm{CDCl}_{3}\right)$

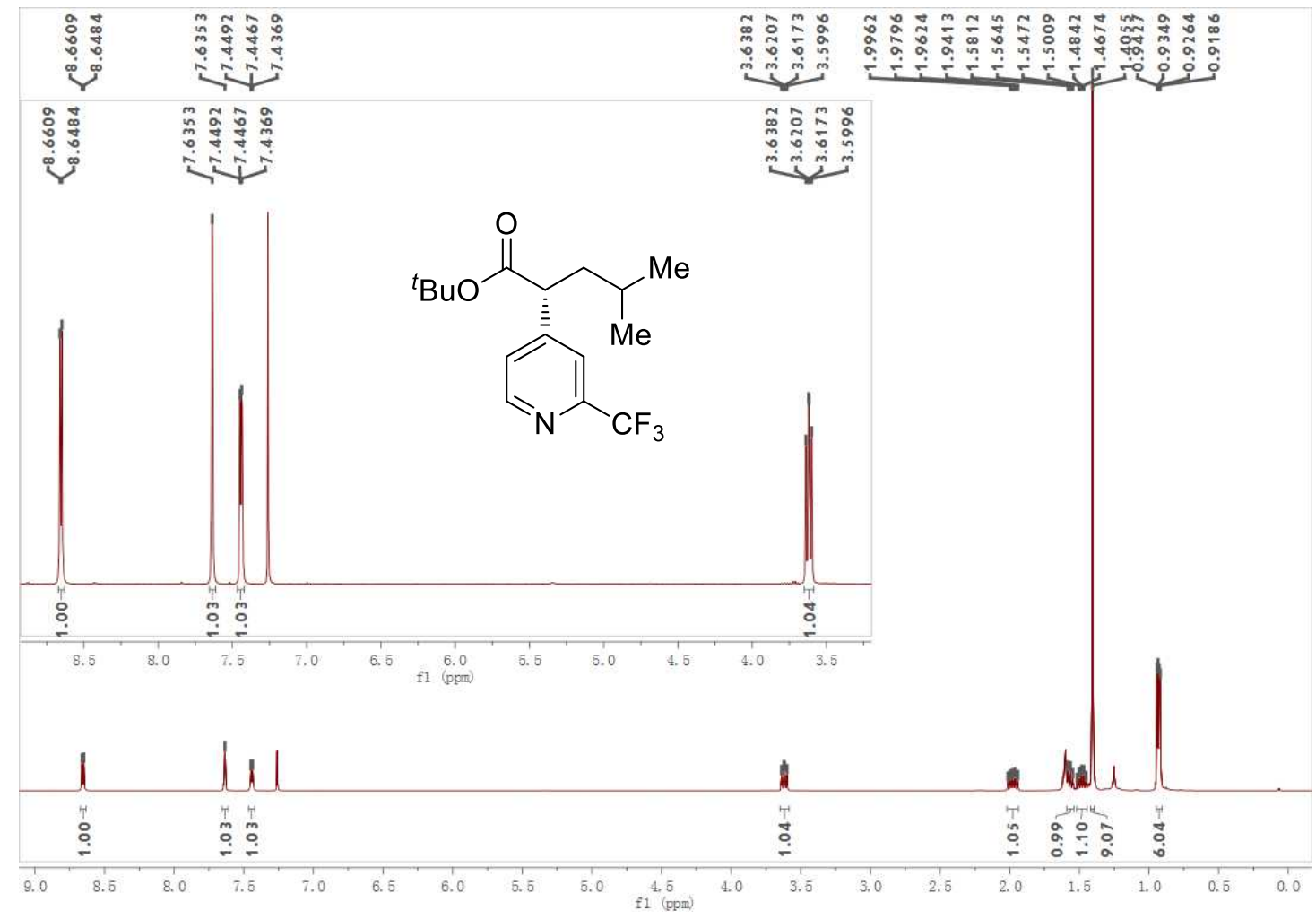

41; ${ }^{19}$ F-NMR (375 MHz, $\mathrm{CDCl}_{3}$ )

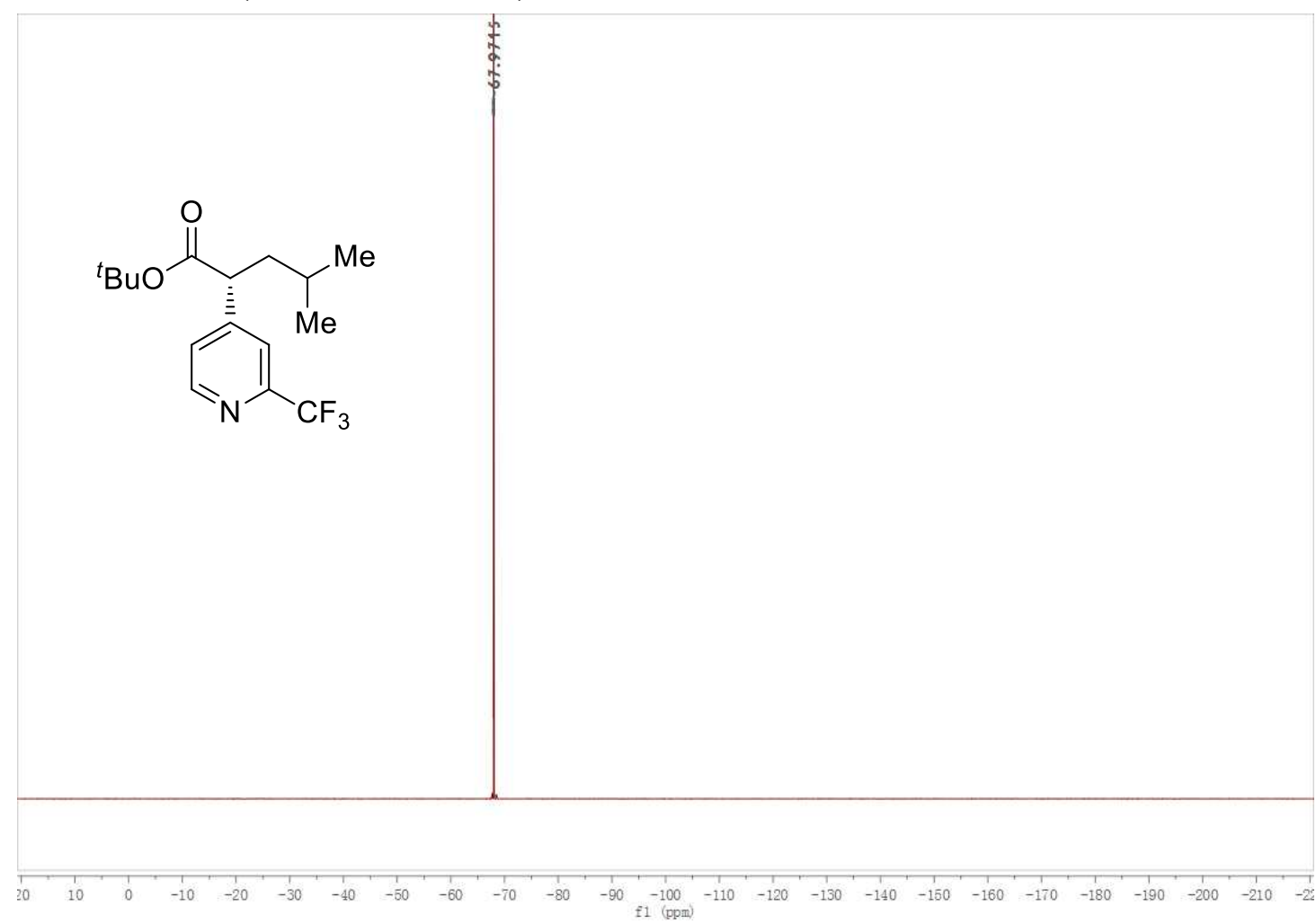


41; ${ }^{13} \mathrm{C}-\mathrm{NMR}$ (100 MHz, $\left.\mathrm{CDCl}_{3}\right)$

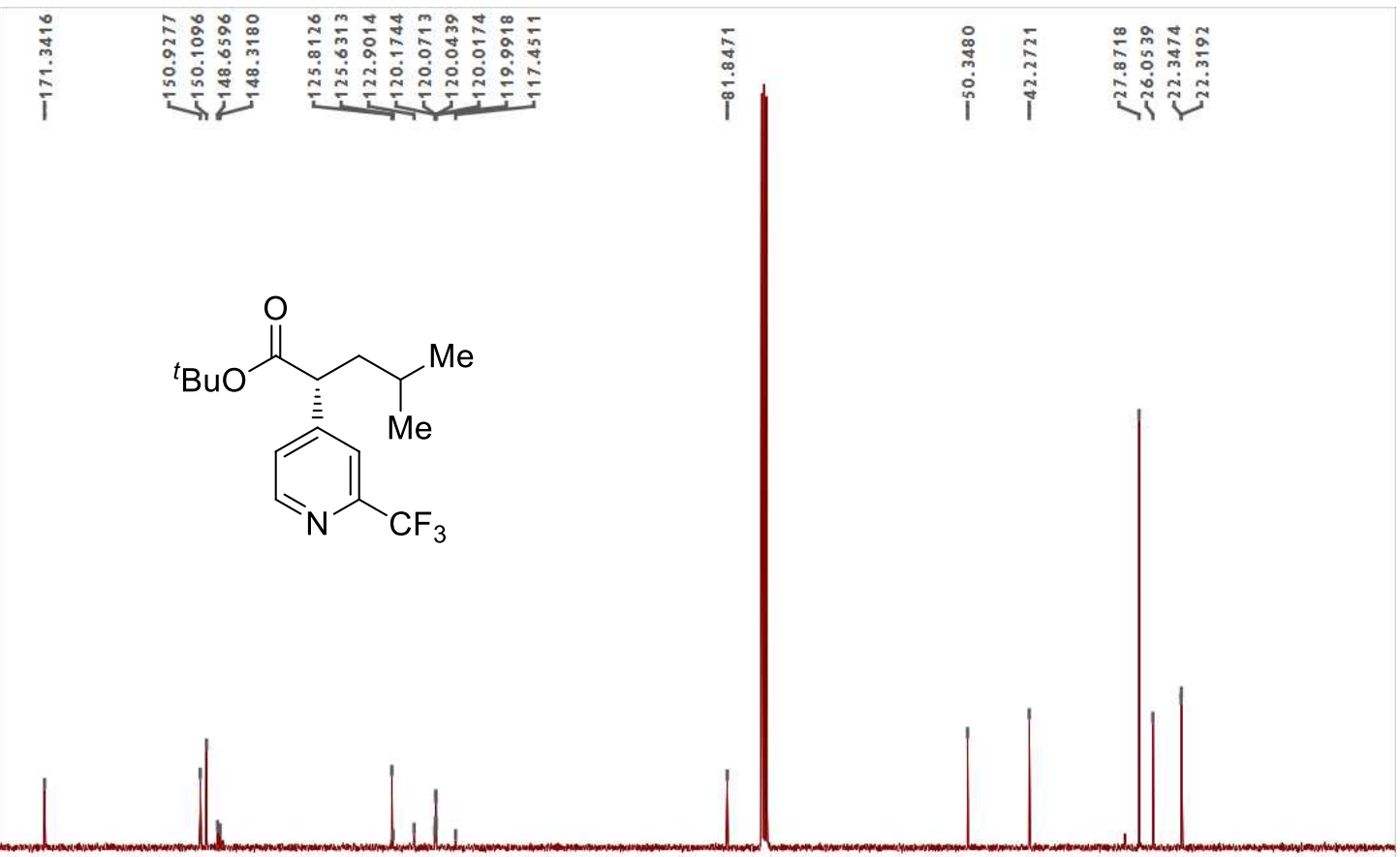

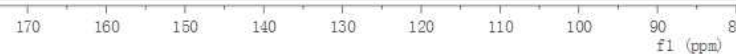

42; ${ }^{1} \mathrm{H}-\mathrm{NMR}\left(400 \mathrm{MHz}, \mathrm{CDCl}_{3}\right)$

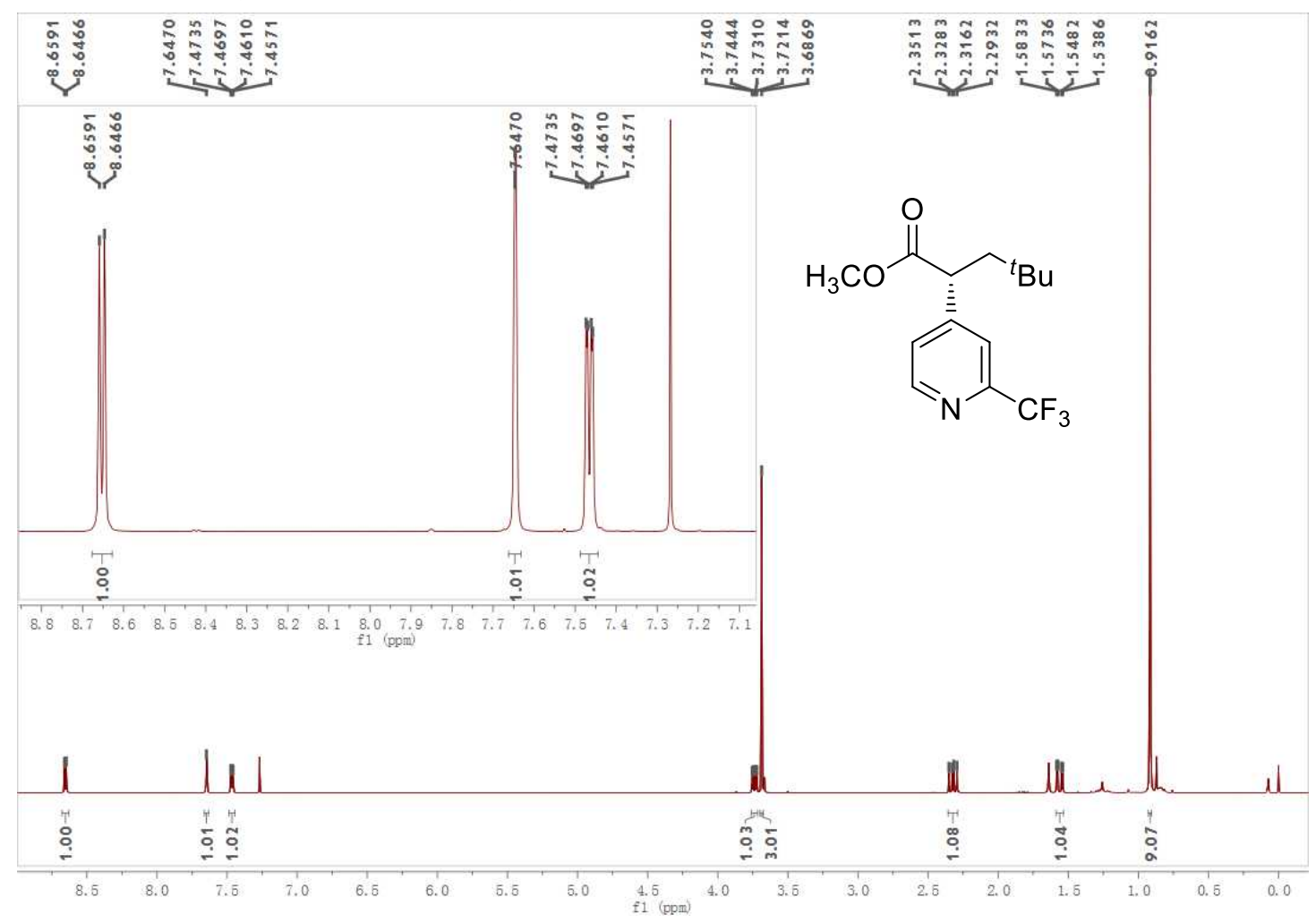


42; ${ }^{19}$ F-NMR (375 MHz, $\mathrm{CDCl}_{3}$ )

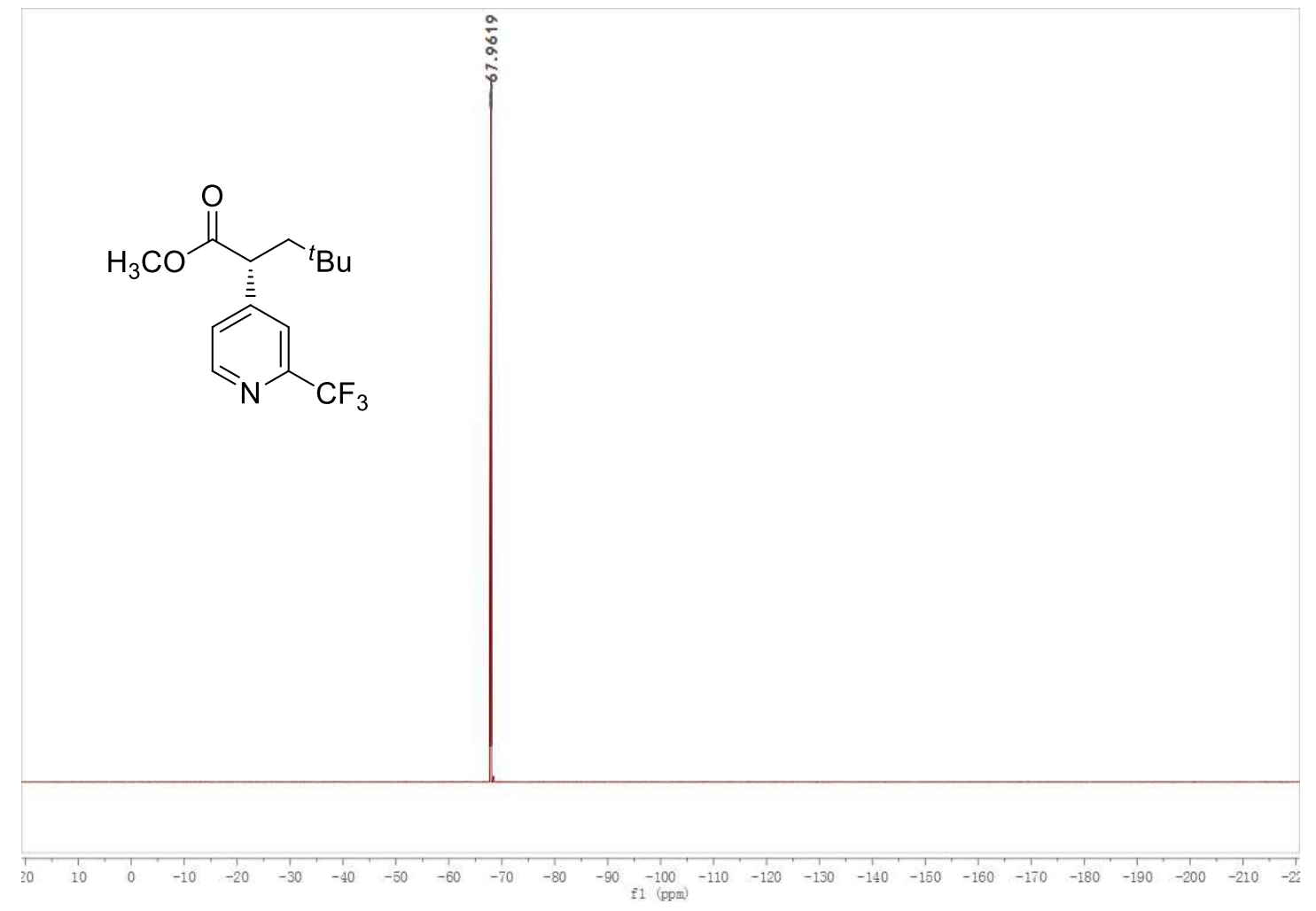

42; ${ }^{13} \mathrm{C}-\mathrm{NMR}\left(100 \mathrm{MHz}, \mathrm{CDCl}_{3}\right)$

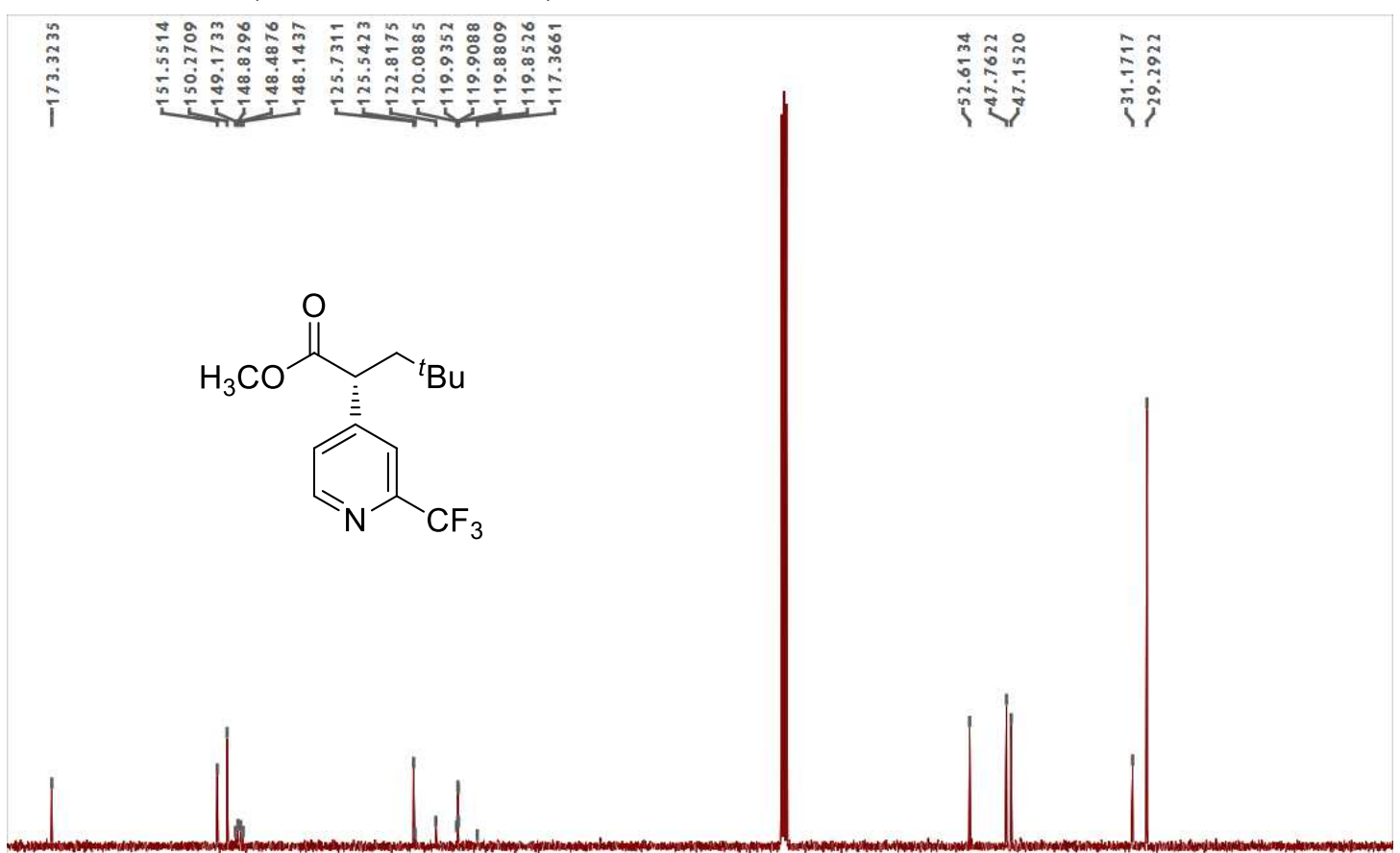


43; ${ }^{1} \mathrm{H}-\mathrm{NMR}$ (400 MHz, $\left.\mathrm{CDCl}_{3}\right)$

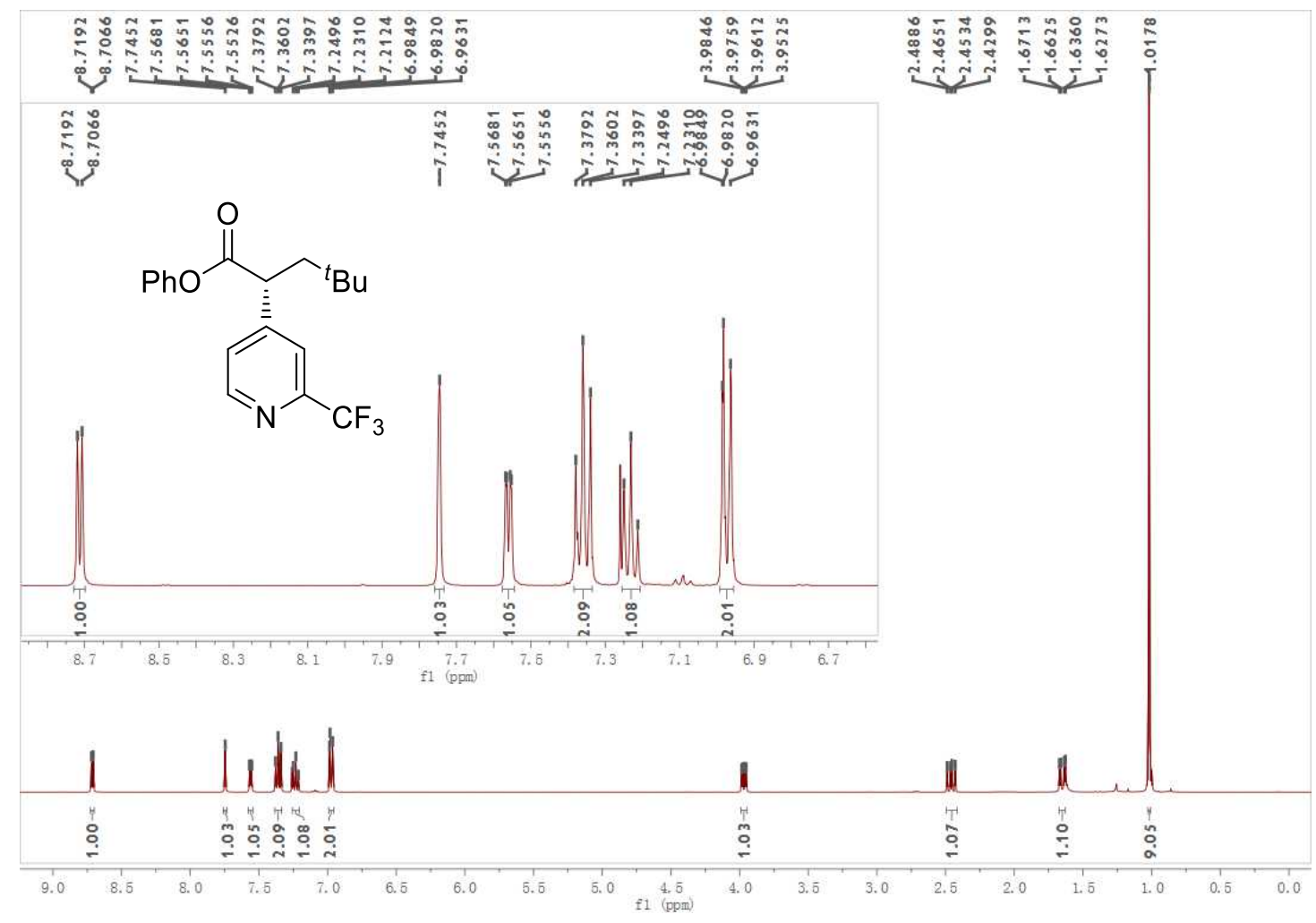

43; ${ }^{19}$ F-NMR (375 MHz, $\mathrm{CDCl}_{3}$ )

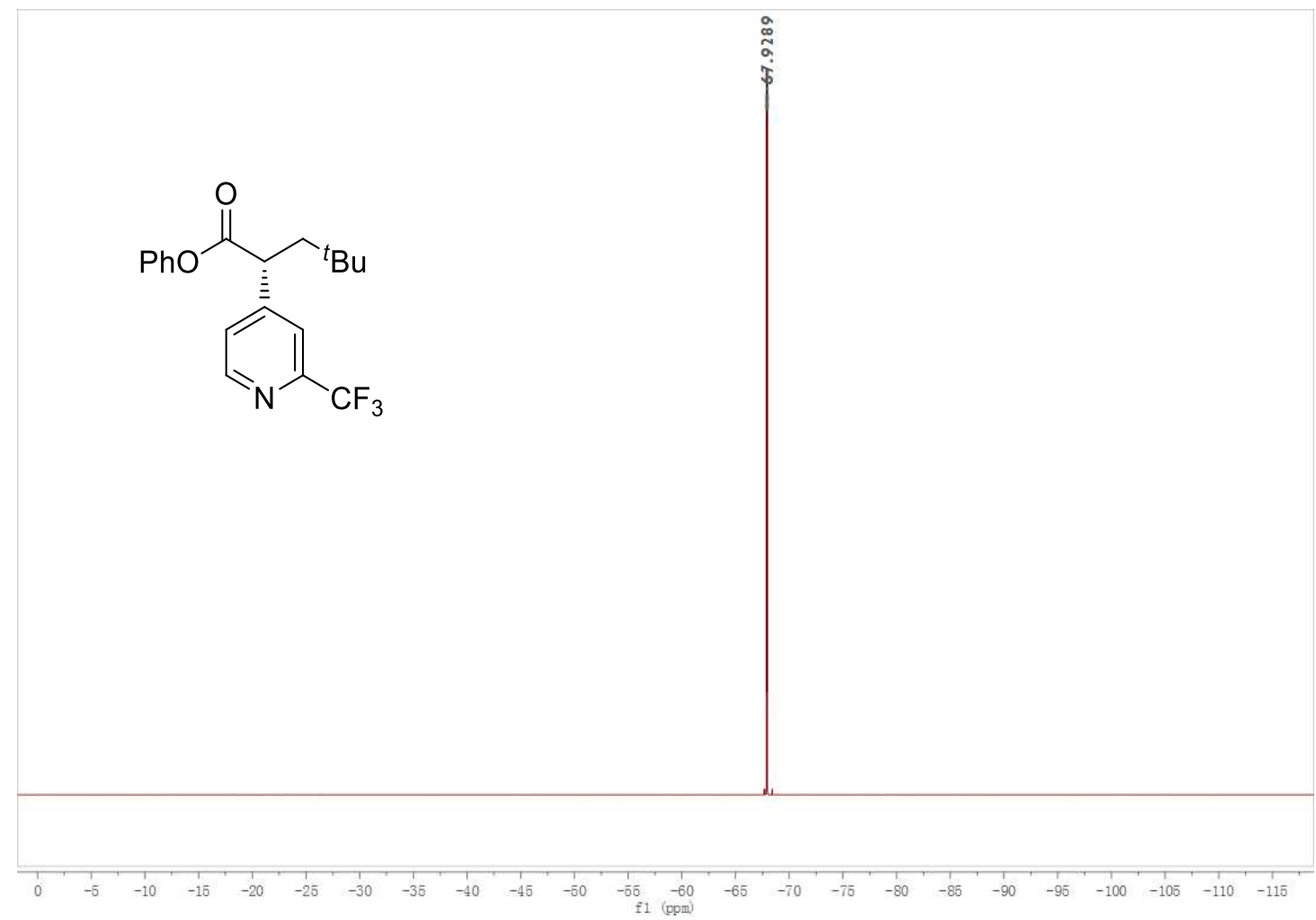


43; ${ }^{13} \mathrm{C}-\mathrm{NMR}\left(100 \mathrm{MHz}, \mathrm{CDCl}_{3}\right)$

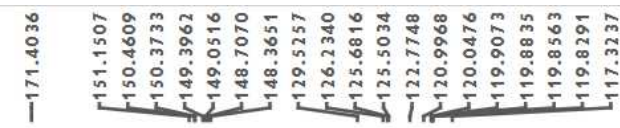



(

望

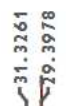

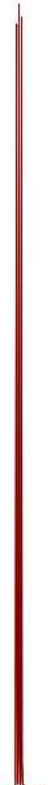

44; ${ }^{1} \mathrm{H}-\mathrm{NMR}\left(400 \mathrm{MHz}, \mathrm{CDCl}_{3}\right.$ )

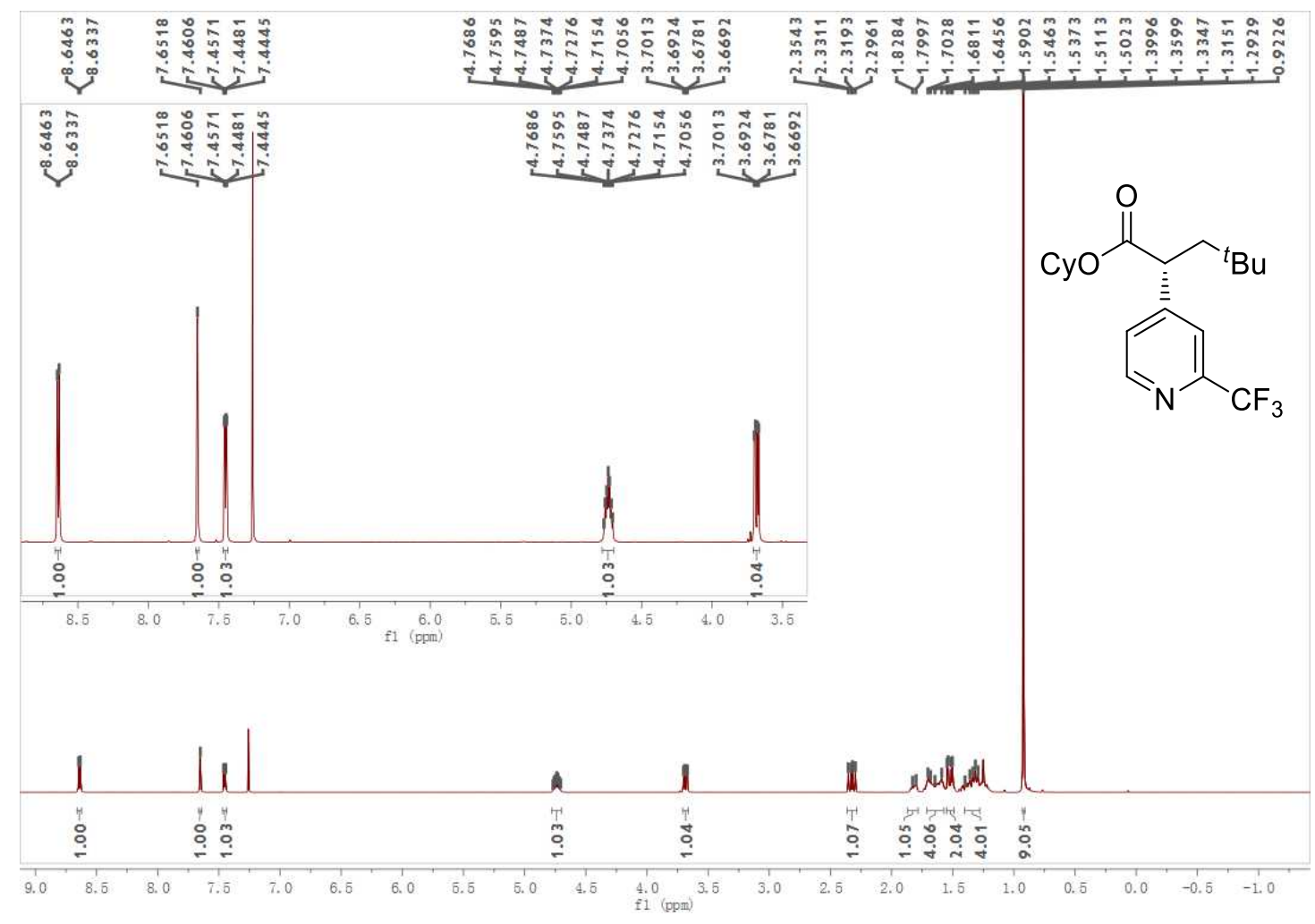


44; ${ }^{19}$ F-NMR (375 MHz, $\mathrm{CDCl}_{3}$ )

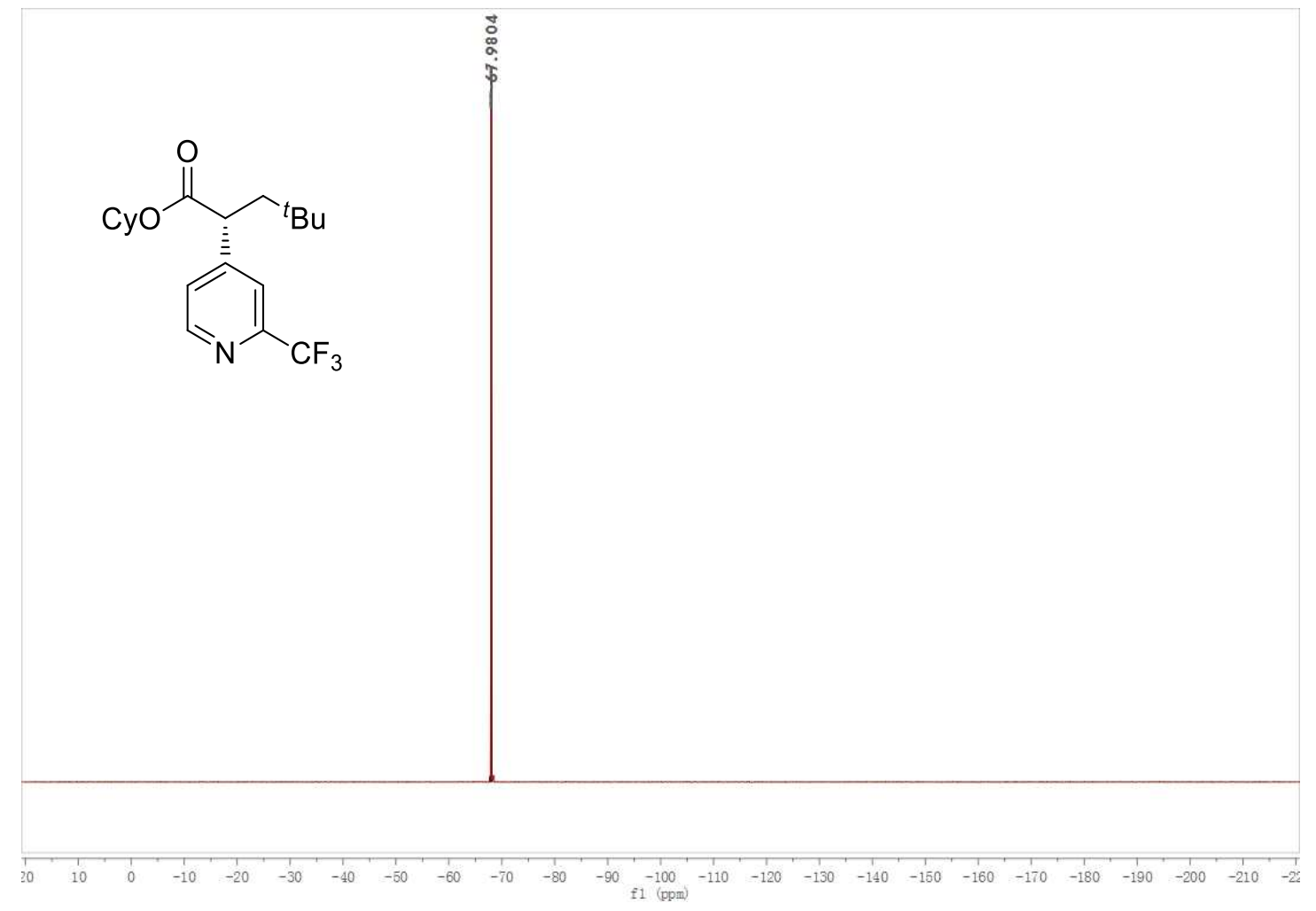

44; ${ }^{13} \mathrm{C}-\mathrm{NMR}$ (100 MHz, $\left.\mathrm{CDCl}_{3}\right)$

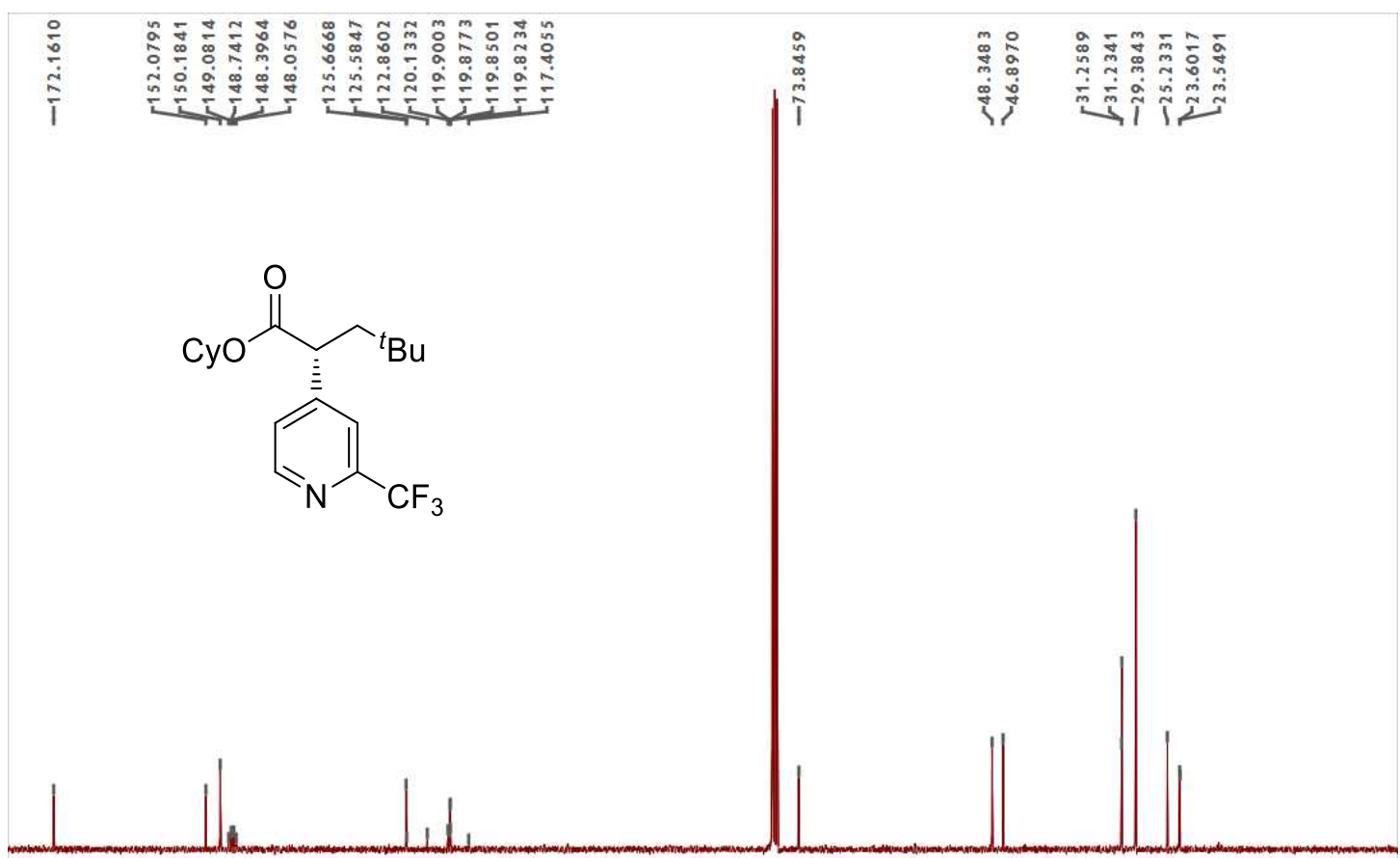

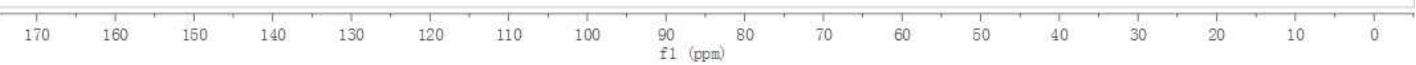


45; ${ }^{1} \mathrm{H}-\mathrm{NMR}$ (400 $\mathrm{MHz}, \mathrm{CDCl}_{3}$ )

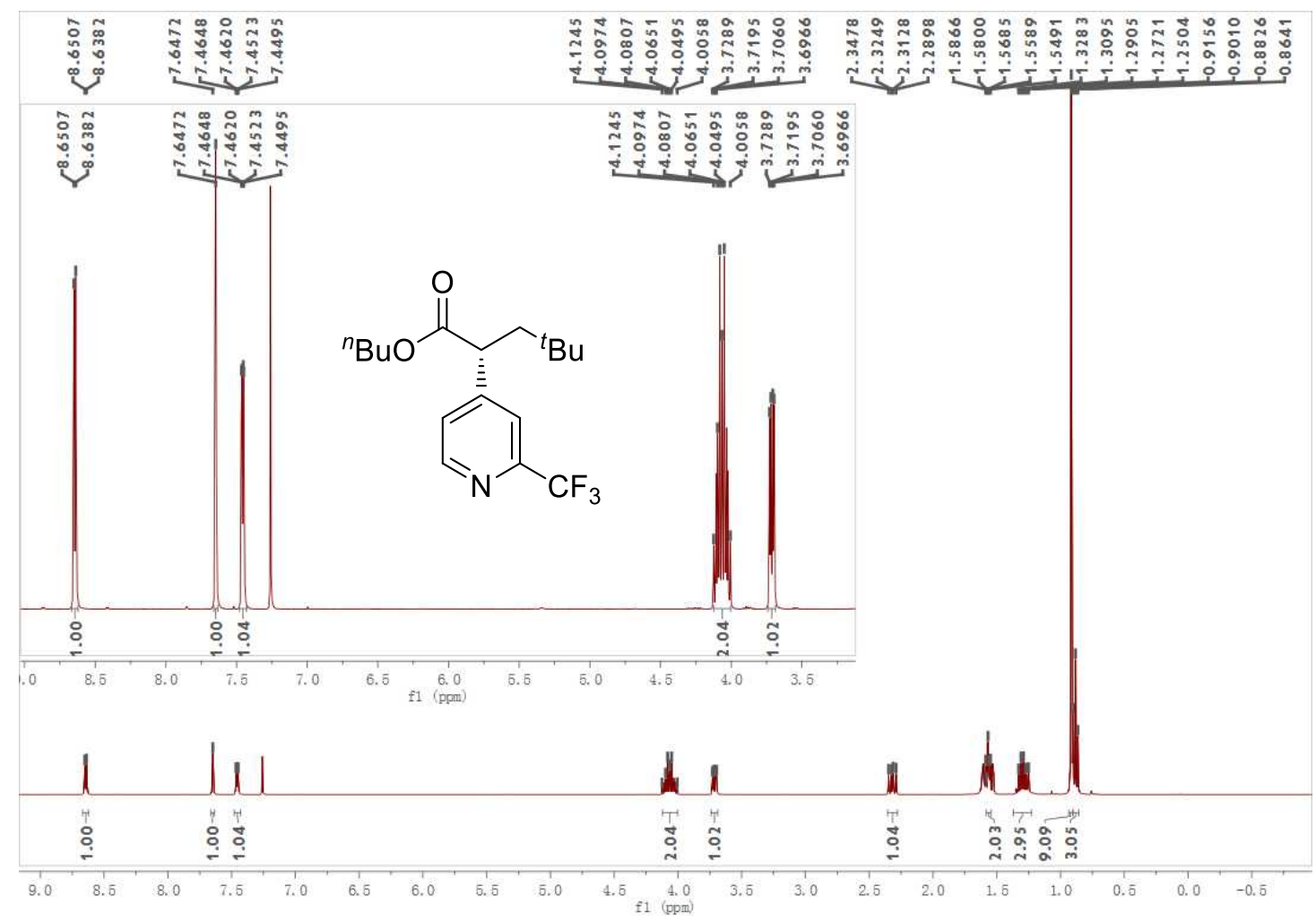

45; ${ }^{19}$ F-NMR (375 MHz, $\mathrm{CDCl}_{3}$ )

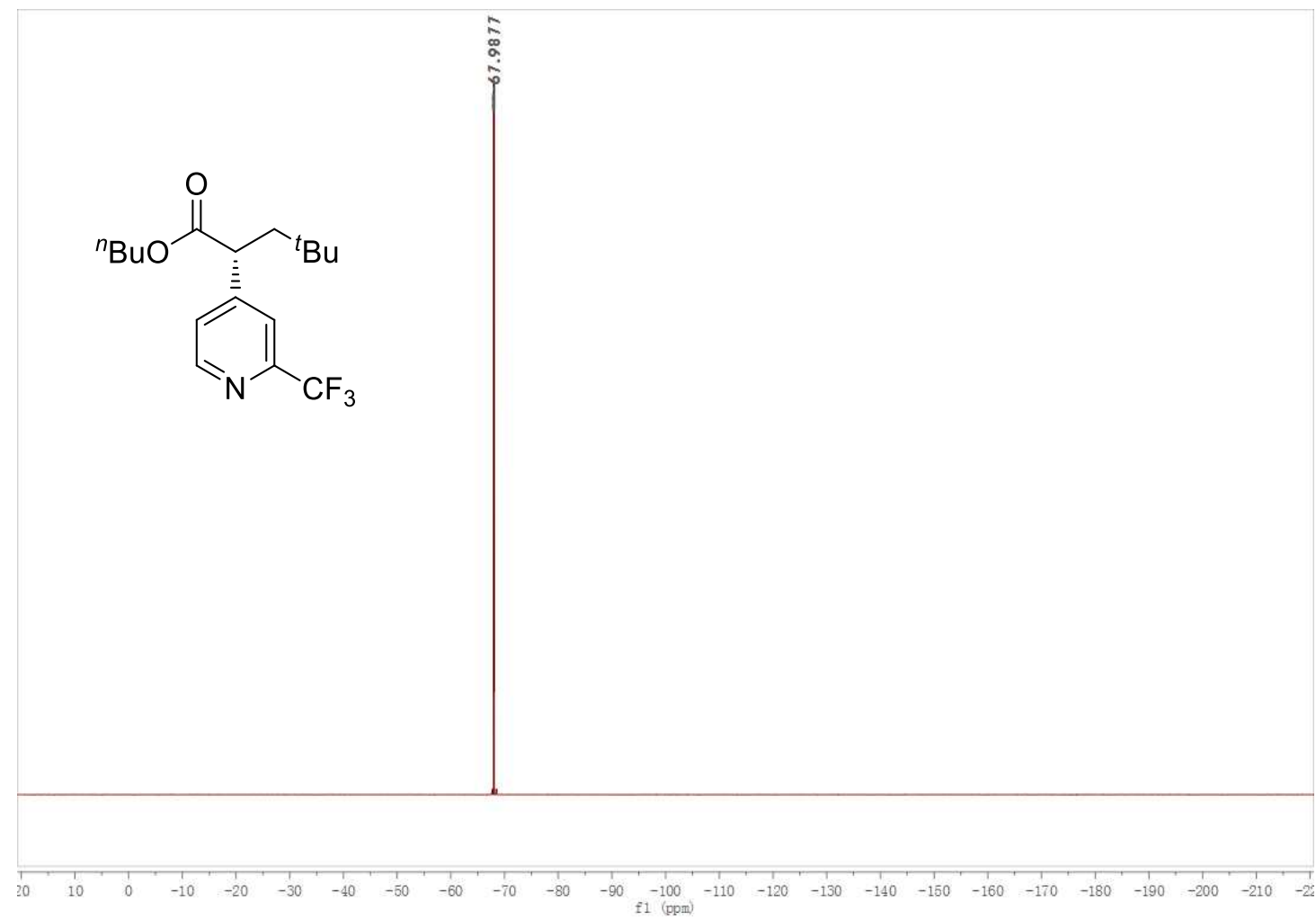


45; ${ }^{13} \mathrm{C}-\mathrm{NMR}\left(100 \mathrm{MHz}, \mathrm{CDCl}_{3}\right)$

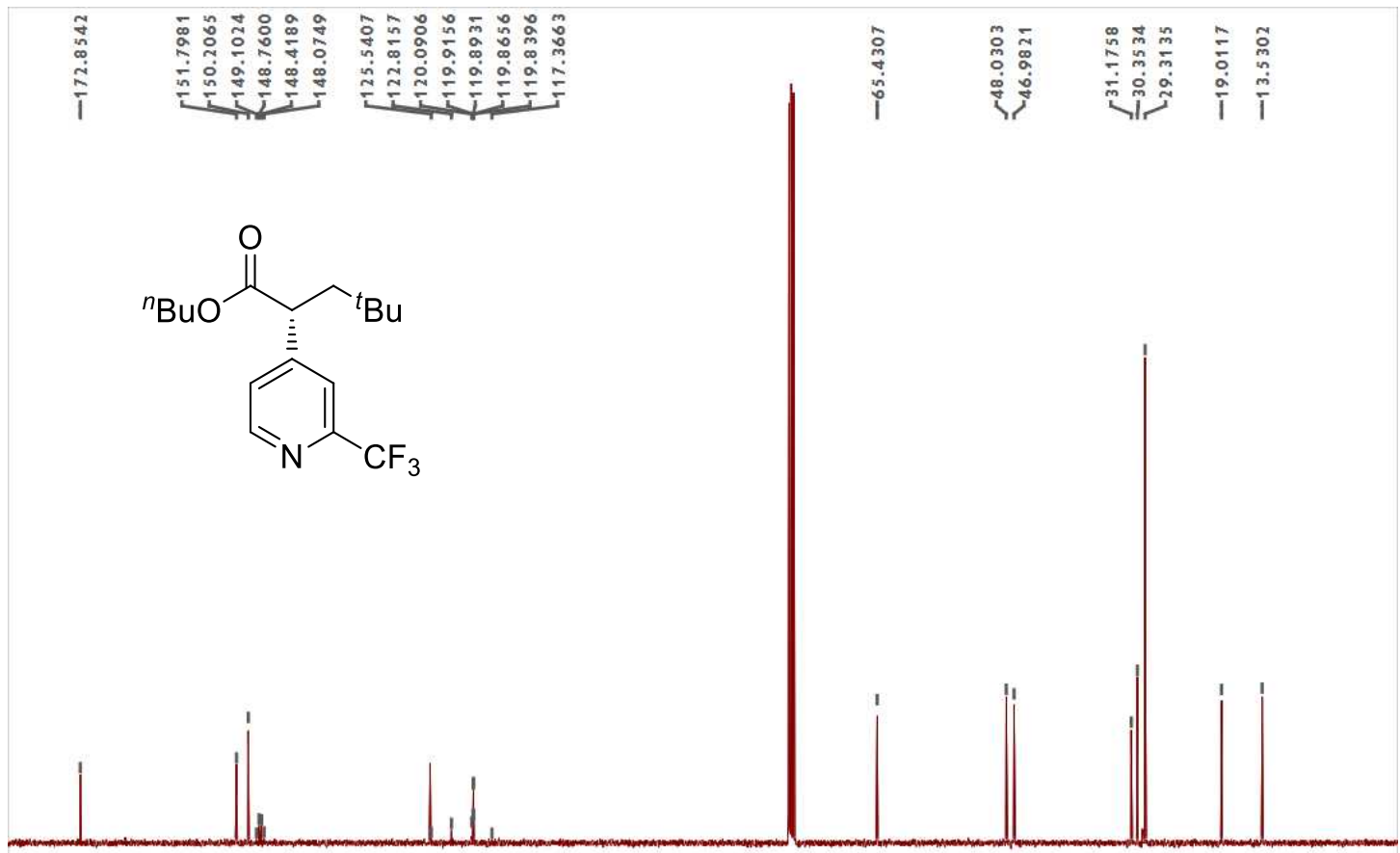

46; ${ }^{1} \mathrm{H}-\mathrm{NMR}\left(400 \mathrm{MHz}, \mathrm{CDCl}_{3}\right)$

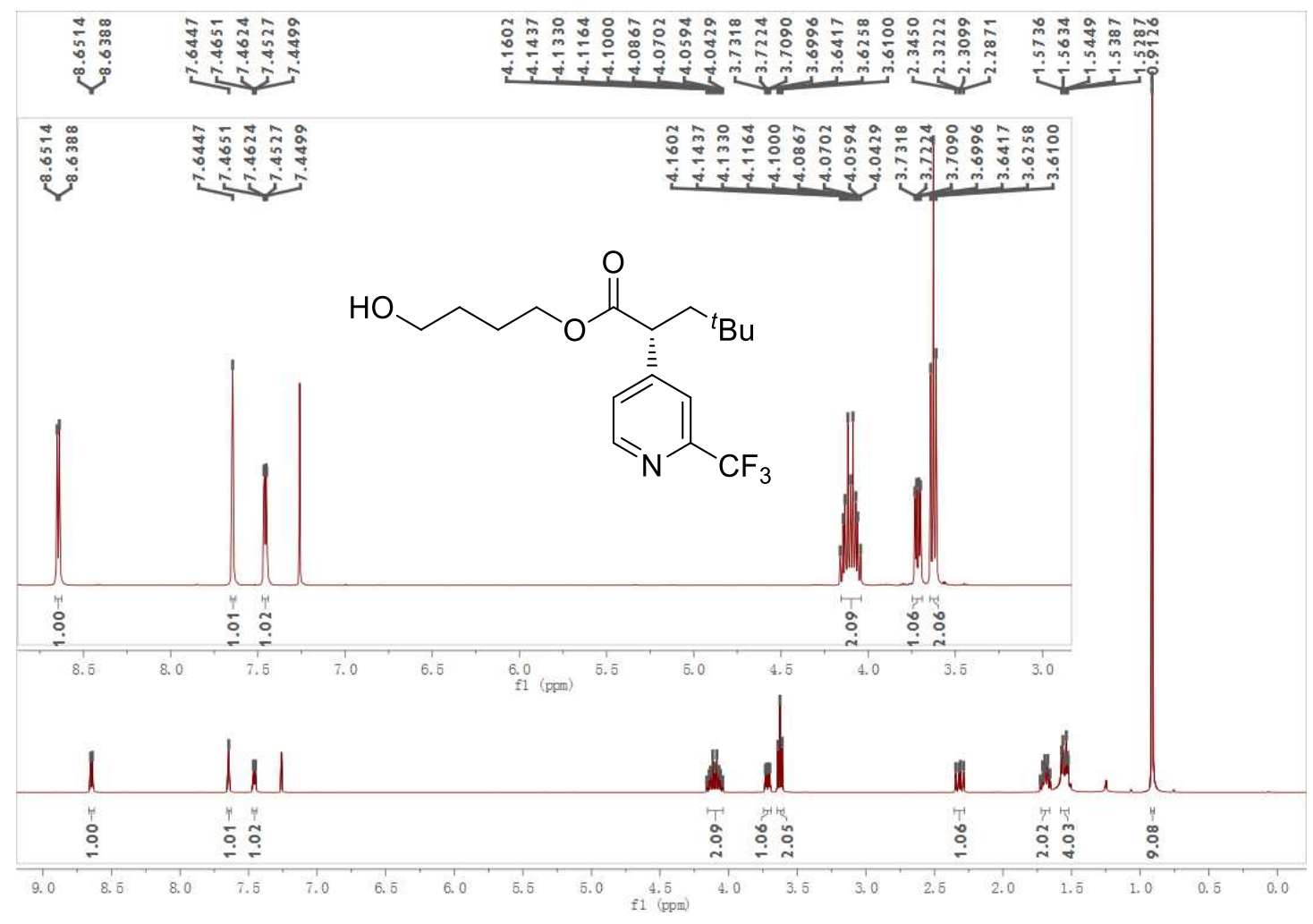


46; ${ }^{19}$ F-NMR (375 MHz, $\mathrm{CDCl}_{3}$ )

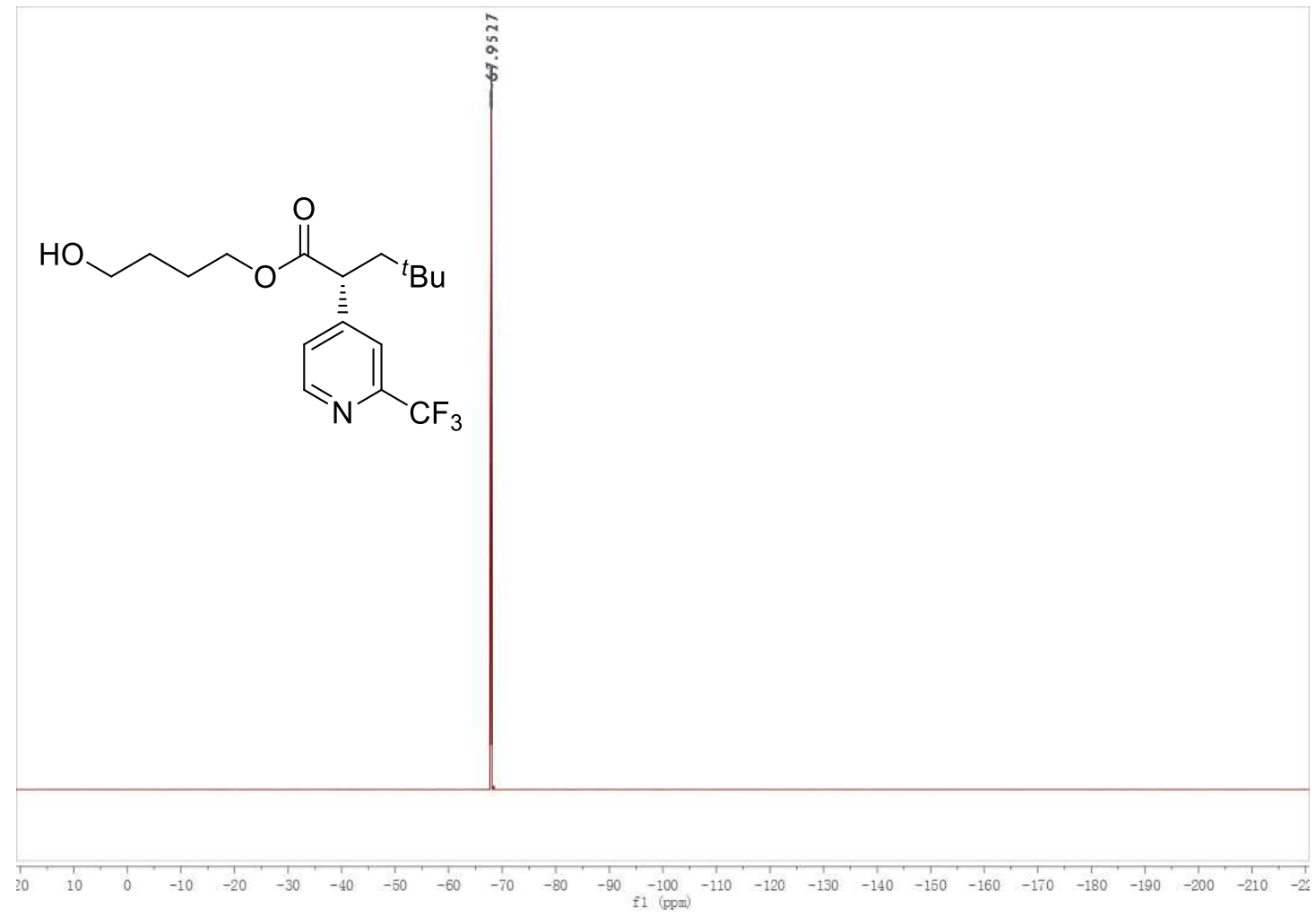

46; ${ }^{13} \mathrm{C}-\mathrm{NMR}\left(100 \mathrm{MHz}, \mathrm{CDCl}_{3}\right)$
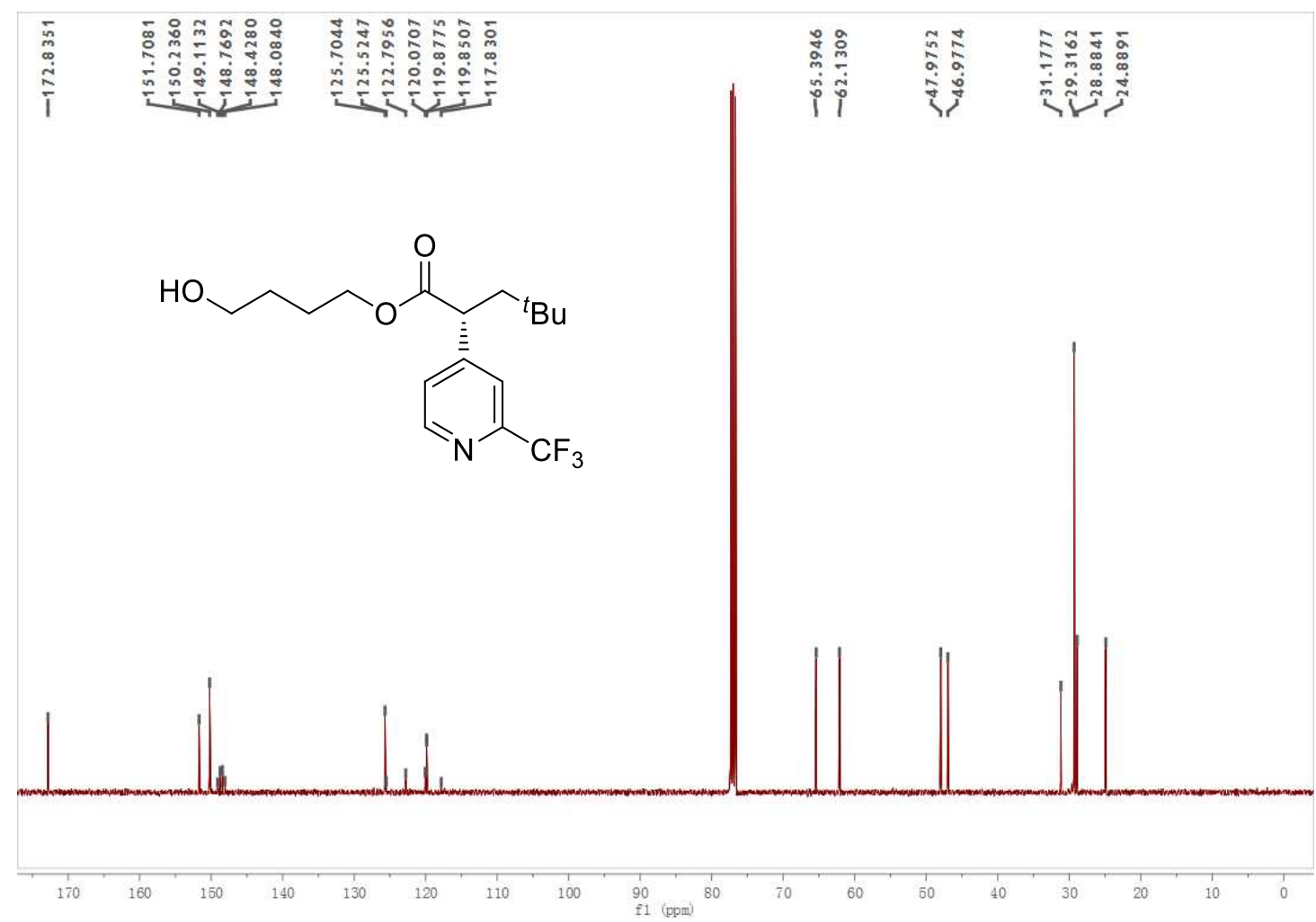
47; ${ }^{1} \mathrm{H}-\mathrm{NMR}$ (400 $\mathrm{MHz}, \mathrm{CDCl}_{3}$ )

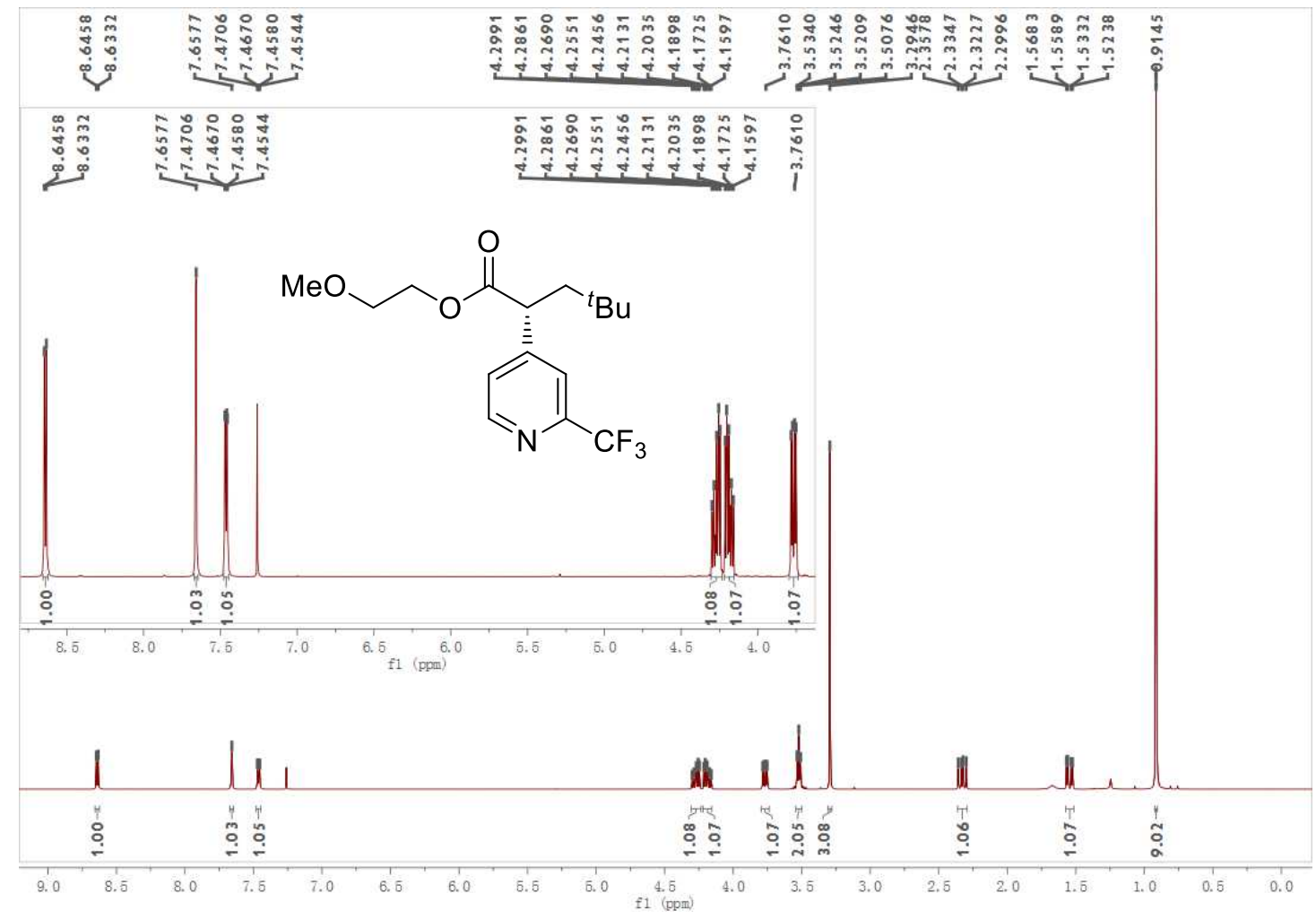

47; ${ }^{19}$ F-NMR (375 MHz, $\mathrm{CDCl}_{3}$ )

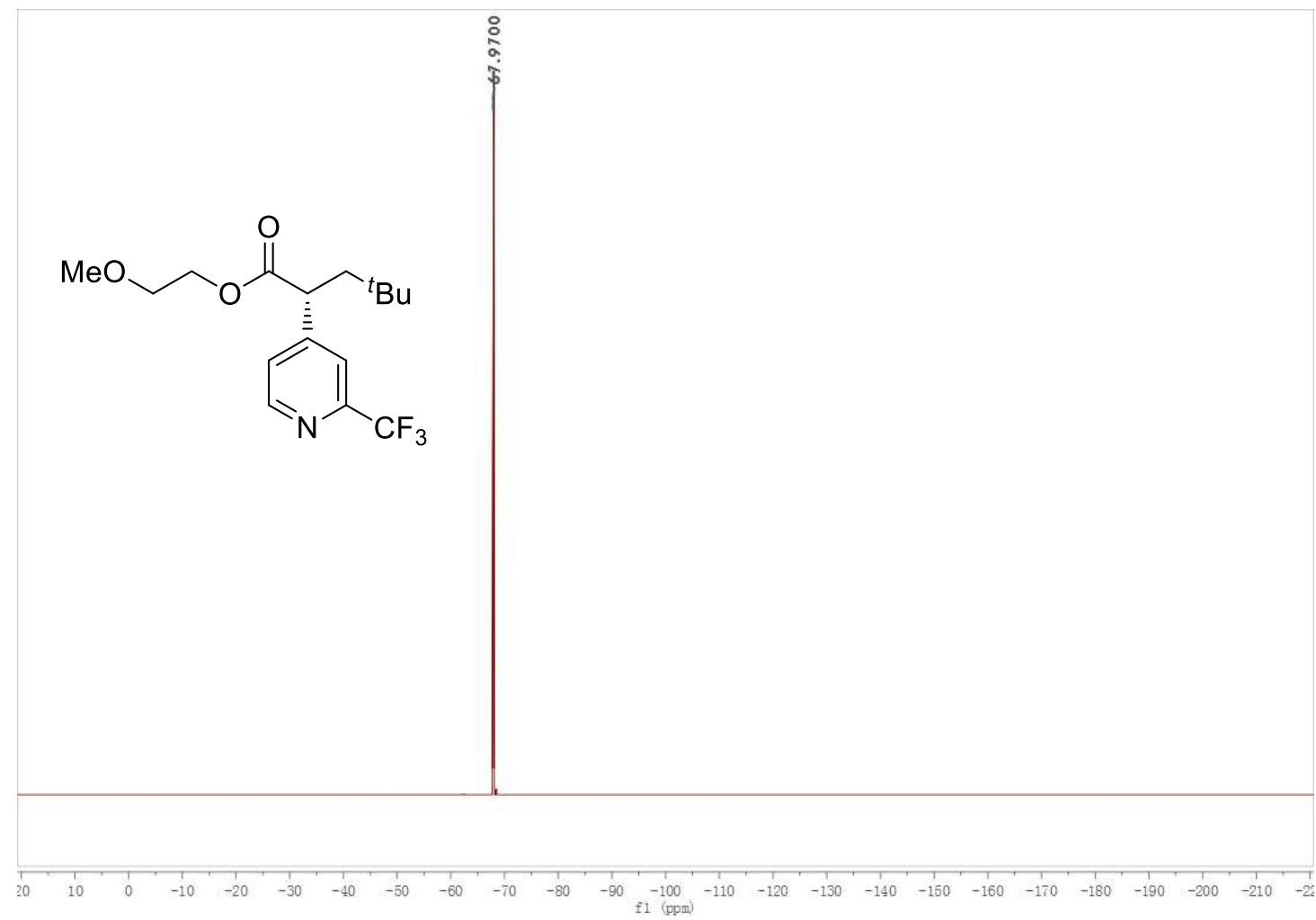


47; ${ }^{13} \mathrm{C}-\mathrm{NMR}\left(100 \mathrm{MHz}, \mathrm{CDCl}_{3}\right)$

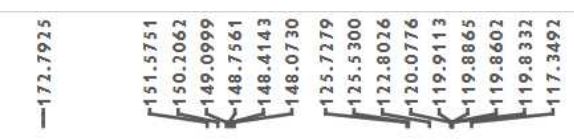

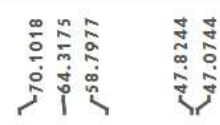<smiles>COCCOC(=O)[C@H](CCBr)c1ccnc(C(F)(F)F)c1</smiles>

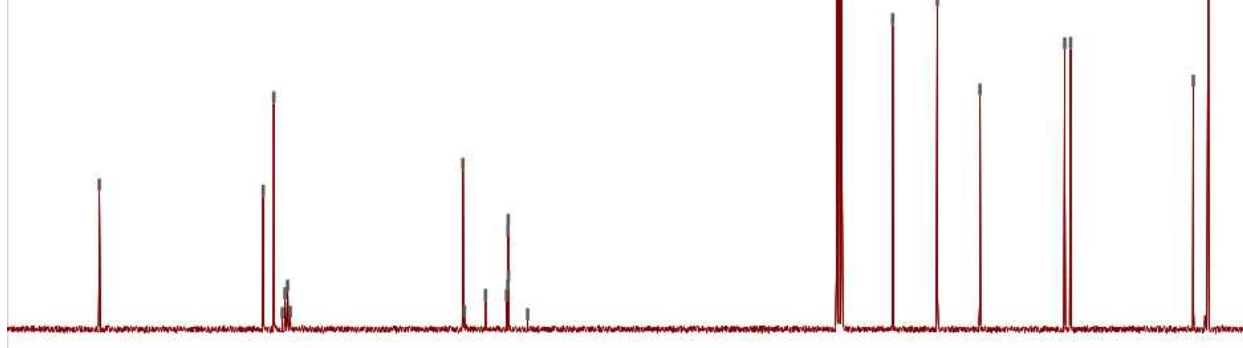

48; ${ }^{1} \mathrm{H}-\mathrm{NMR}$ (400 MHz, $\mathrm{CDCl}_{3}$ )

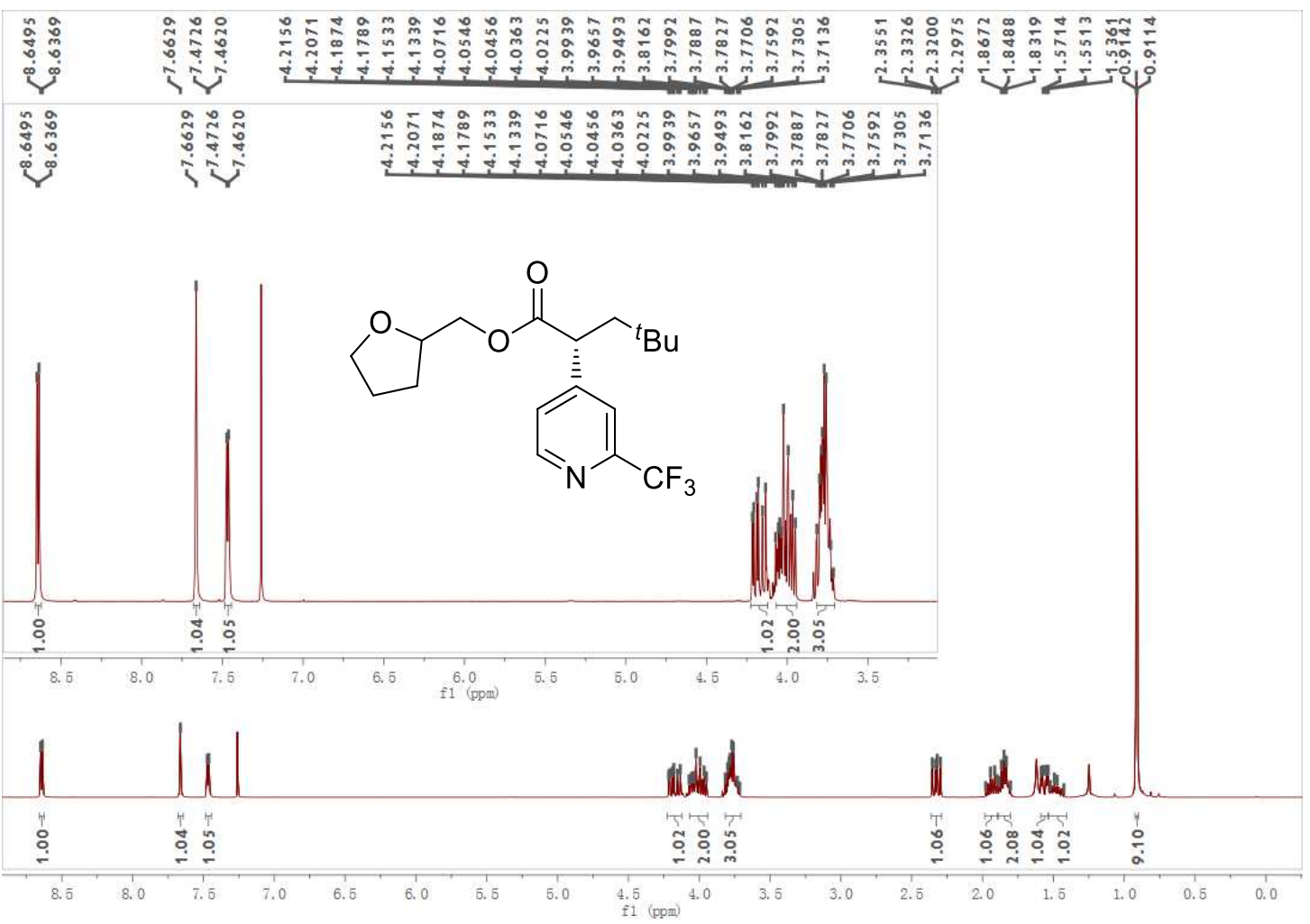


48; ${ }^{19}$ F-NMR (375 MHz, $\left.\mathrm{CDCl}_{3}\right)$

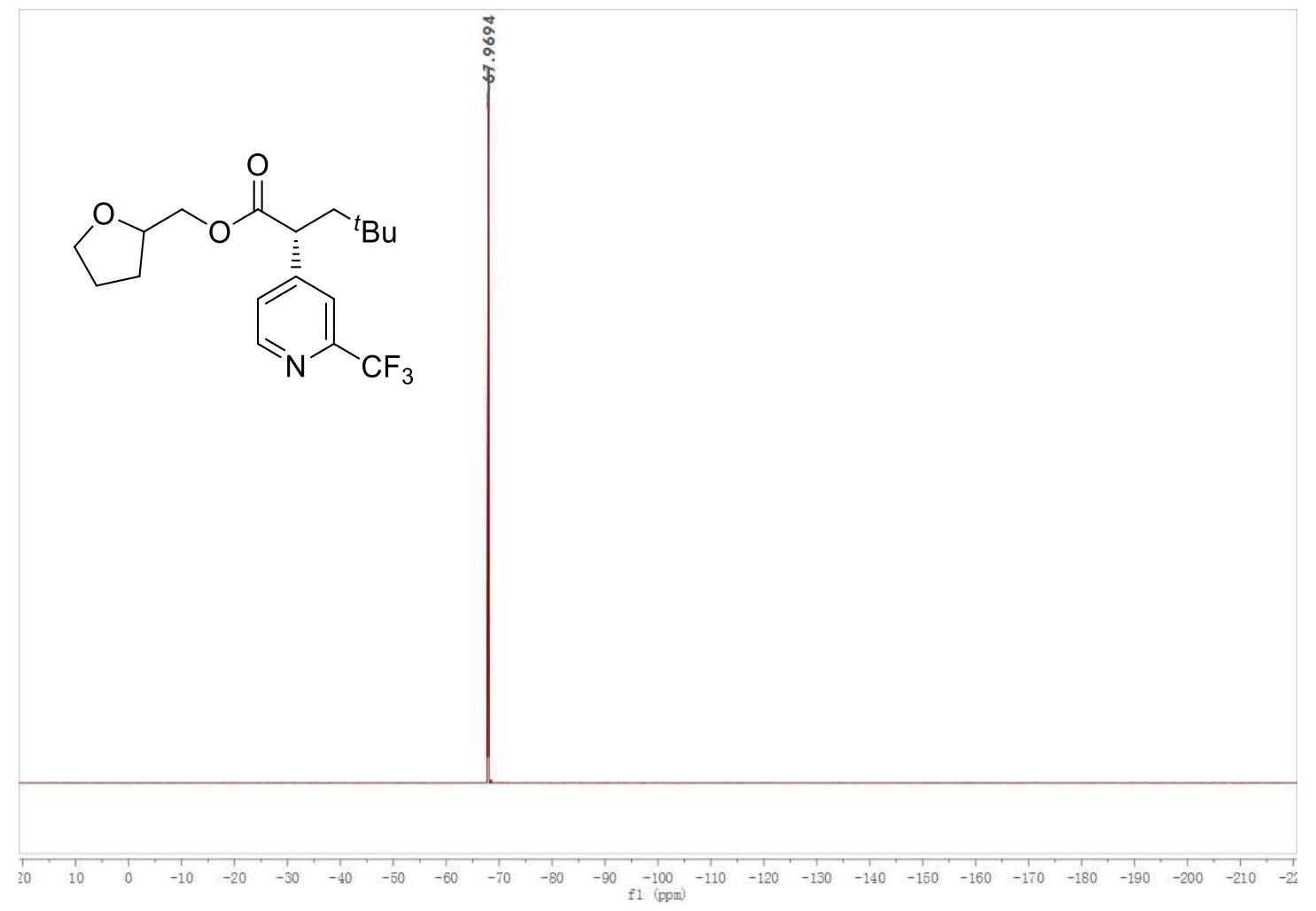

48; ${ }^{13} \mathrm{C}-\mathrm{NMR}$ (100 MHz, $\mathrm{CDCl}_{3}$ )
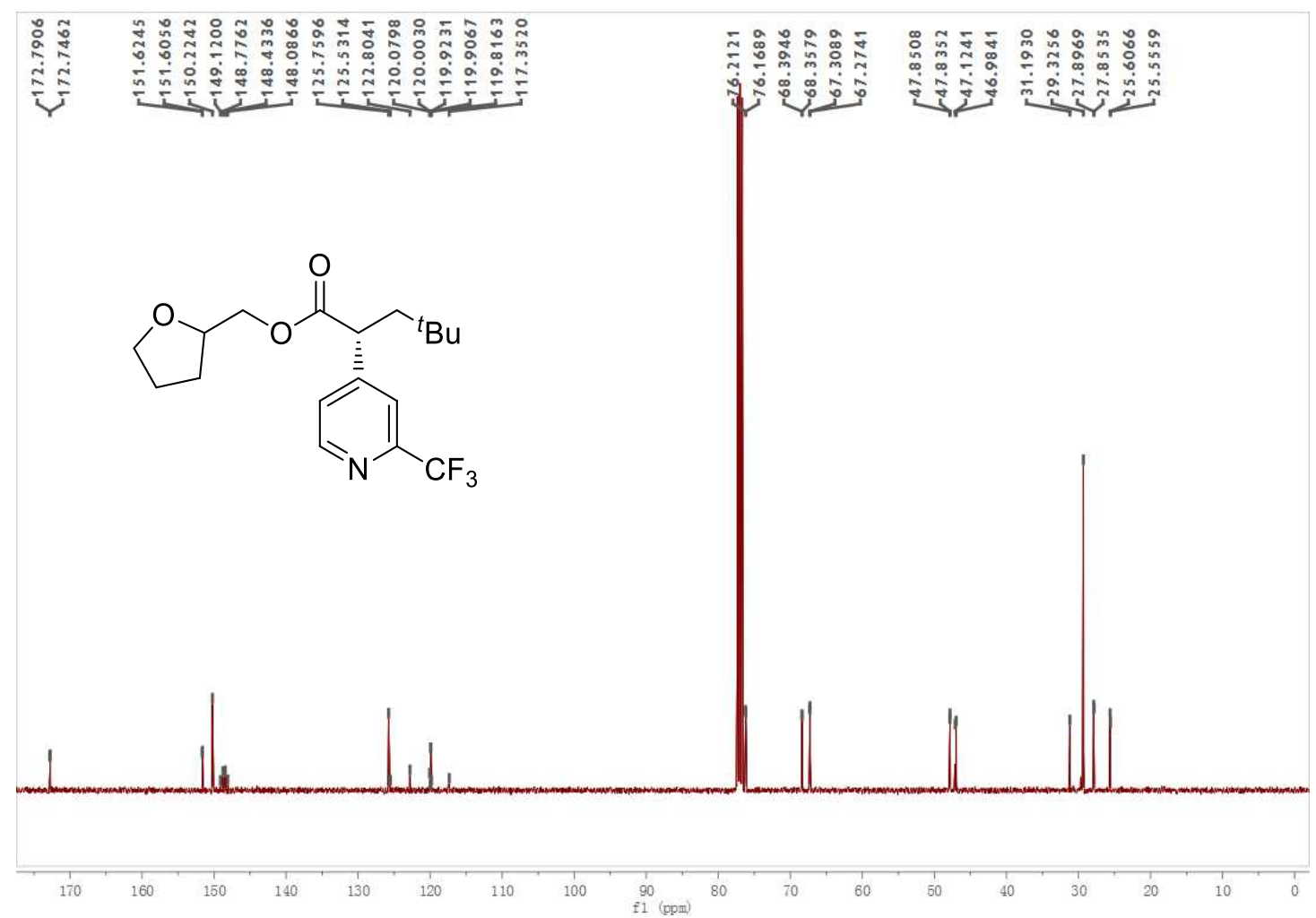
49; ${ }^{1} \mathrm{H}-\mathrm{NMR}$ (400 $\left.\mathrm{MHz}, \mathrm{CDCl}_{3}\right)$

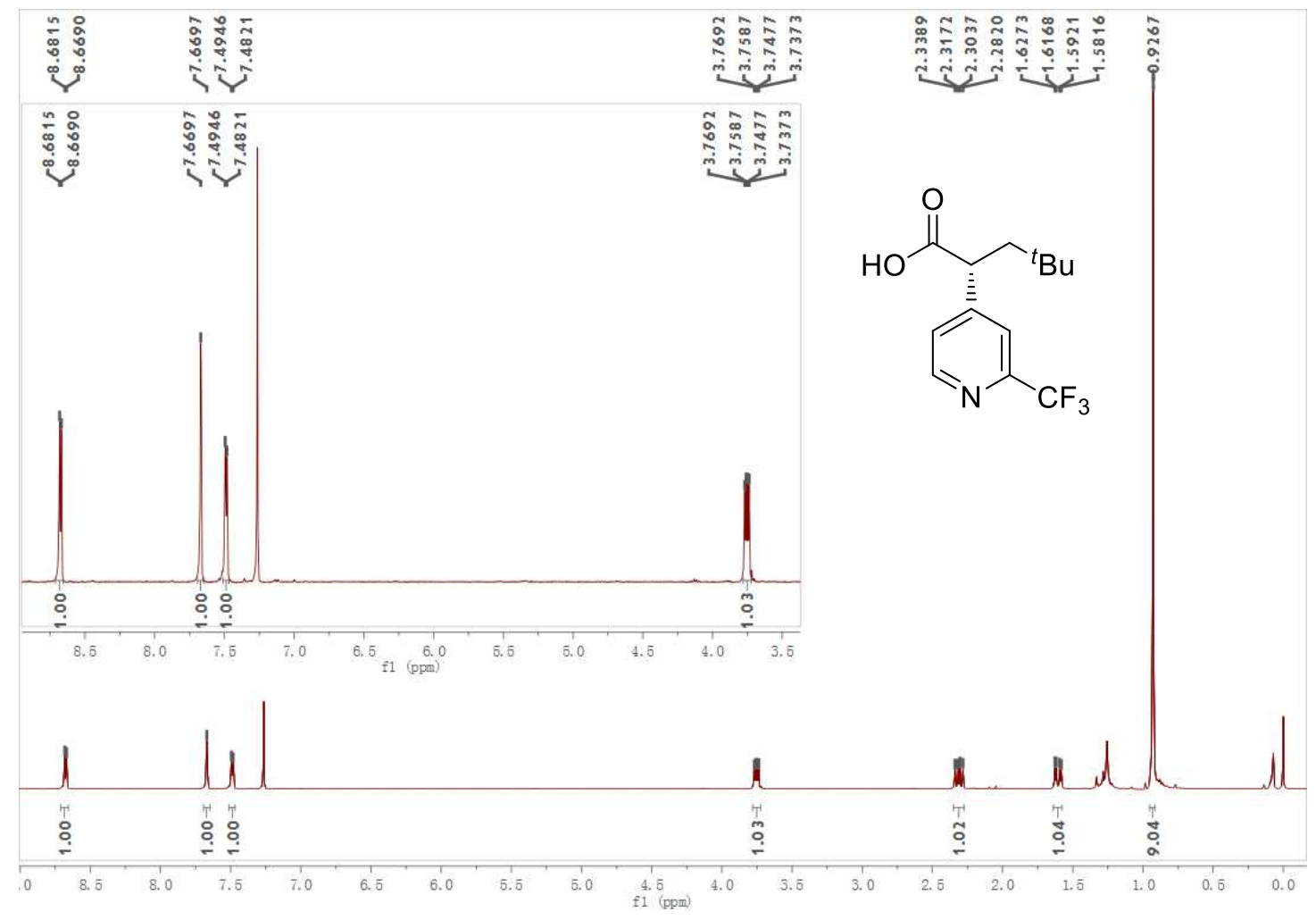

49; ${ }^{19}$ F-NMR (375 MHz, $\mathrm{CDCl}_{3}$ )

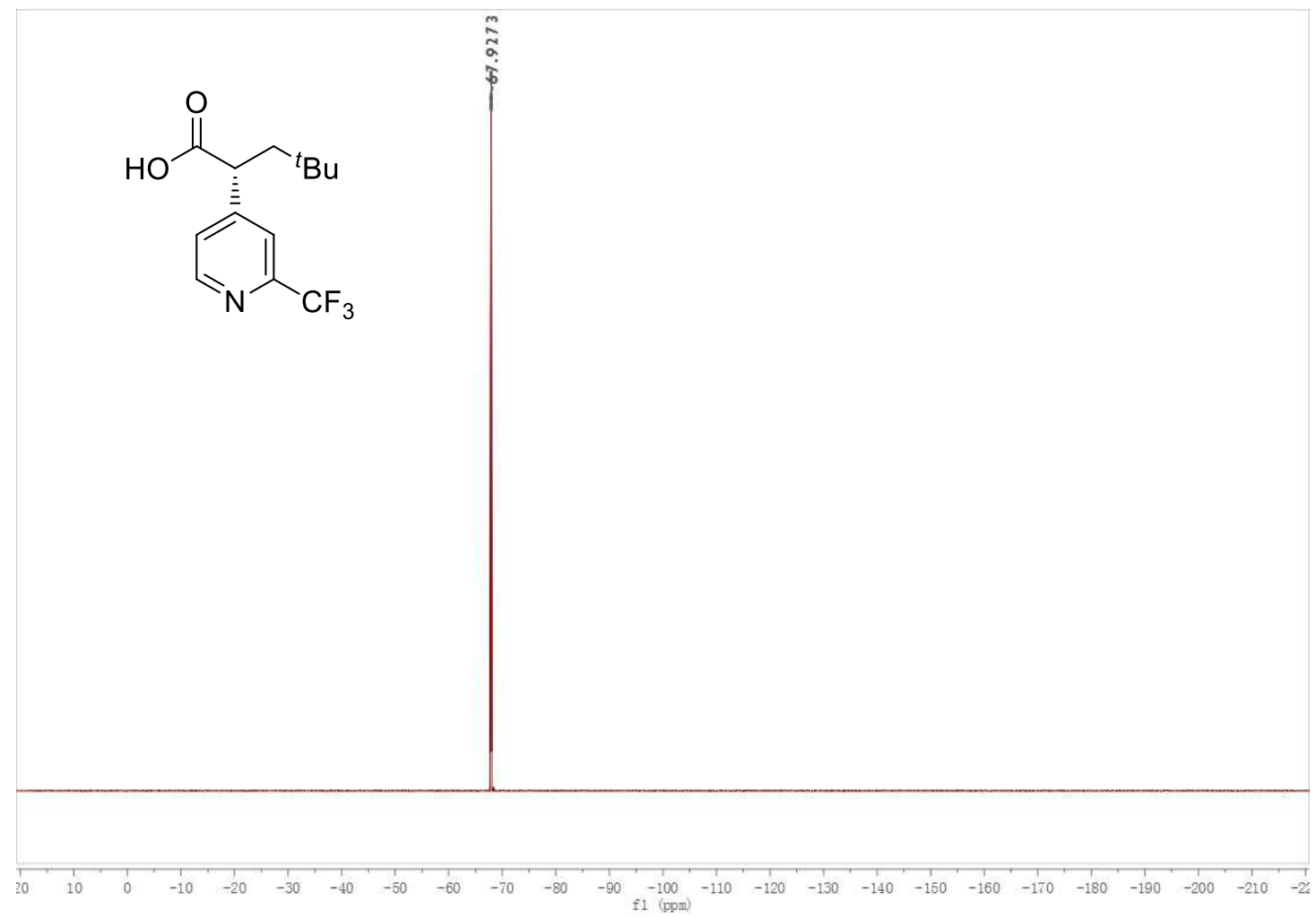


49; ${ }^{13} \mathrm{C}-\mathrm{NMR}$ (100 MHz, $\left.\mathrm{CDCl}_{3}\right)$

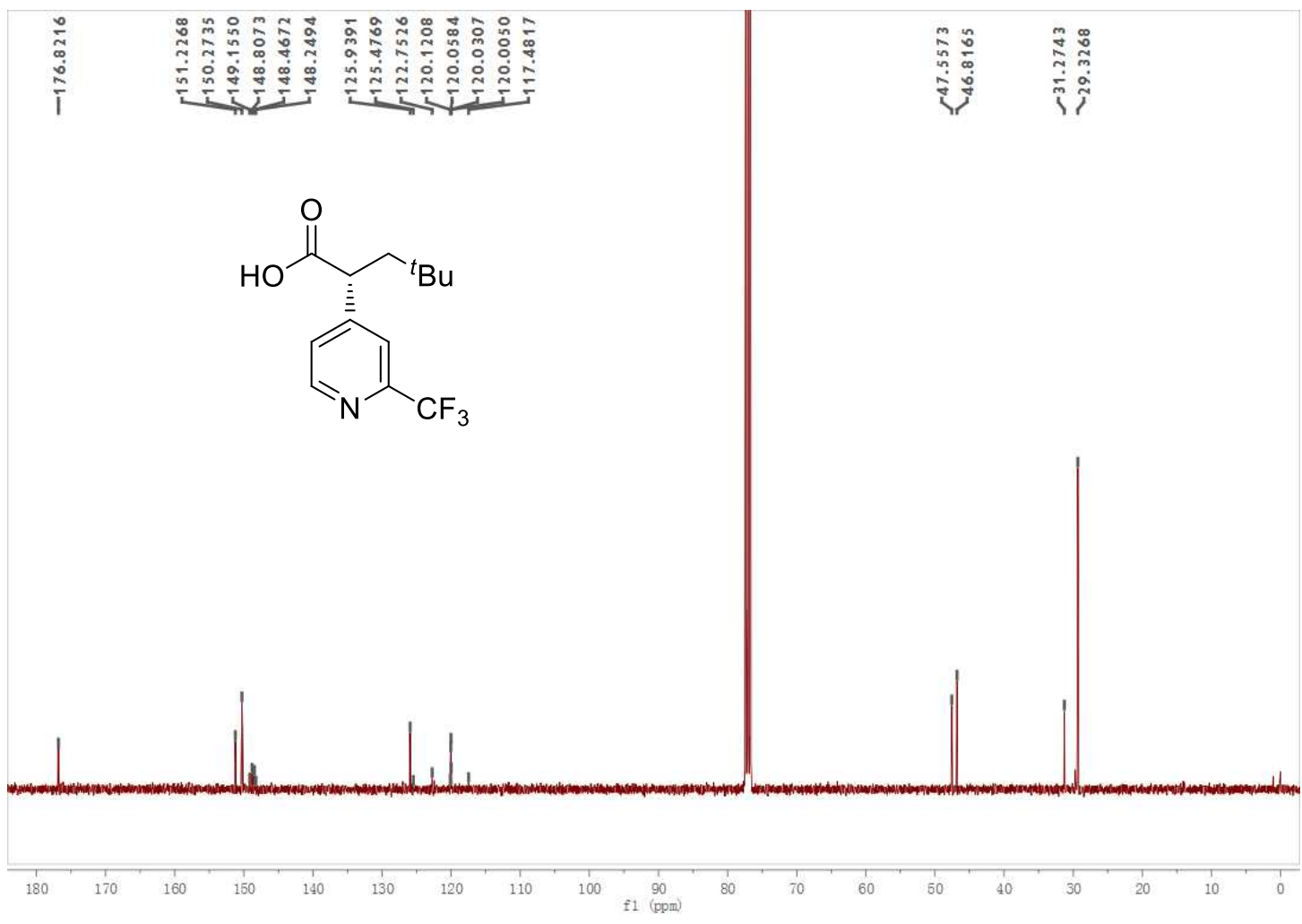

50; ${ }^{1} \mathrm{H}-\mathrm{NMR}\left(400 \mathrm{MHz}, \mathrm{CDCl}_{3}\right)$

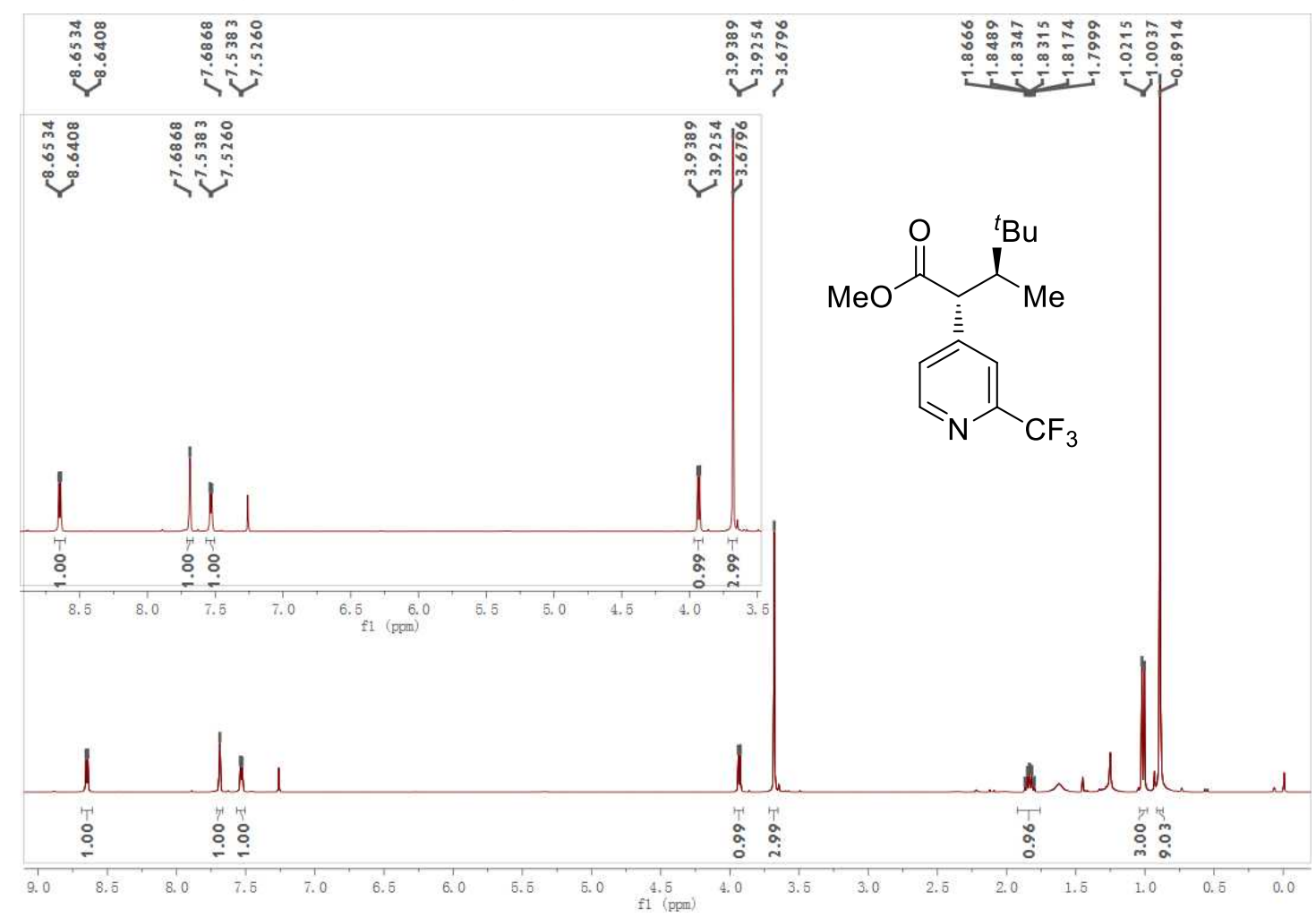


50; ${ }^{19}$ F-NMR (375 MHz, $\mathrm{CDCl}_{3}$ )

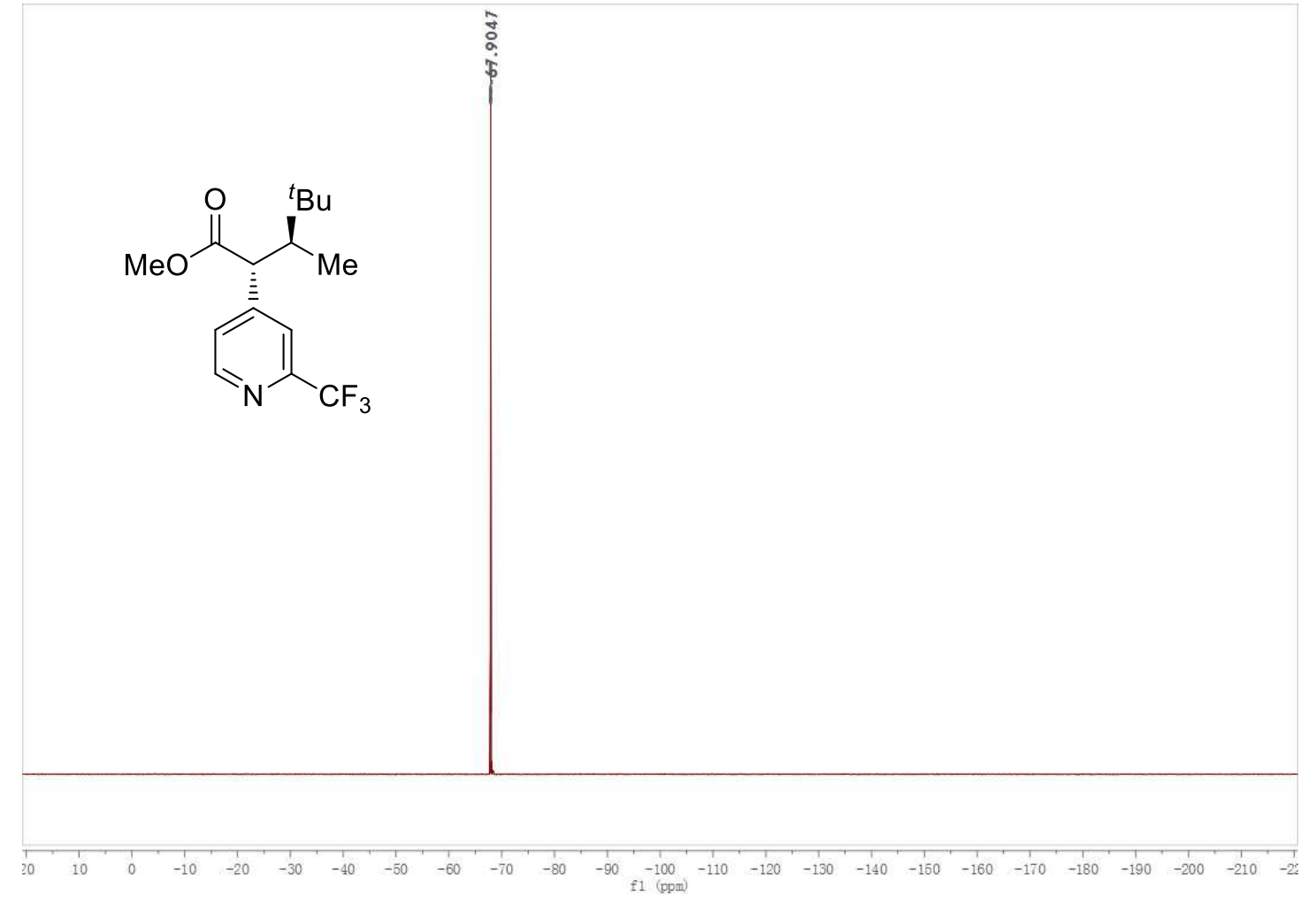

50; ${ }^{13} \mathrm{C}-\mathrm{NMR}\left(100 \mathrm{MHz}, \mathrm{CDCl}_{3}\right)$

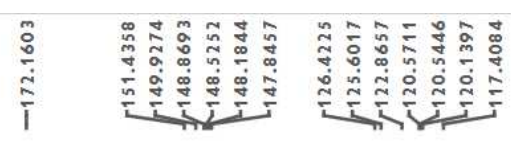

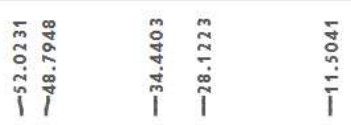<smiles>CCC(C)[C@H](C(=O)OC)c1ccnc(C(F)(F)F)c1</smiles>

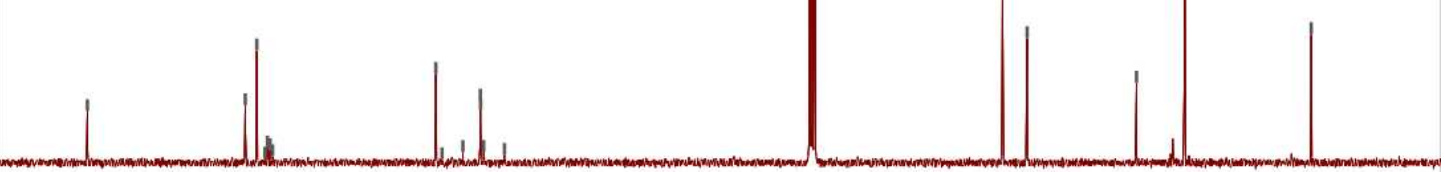

$\begin{array}{llllllllll}180 & 170 & 160 & 150 & 140 & 130 & 120 & 110 & 100 & 90 \\ \mathrm{f} 1(\mathrm{ppm})\end{array}$ 
50; COSY

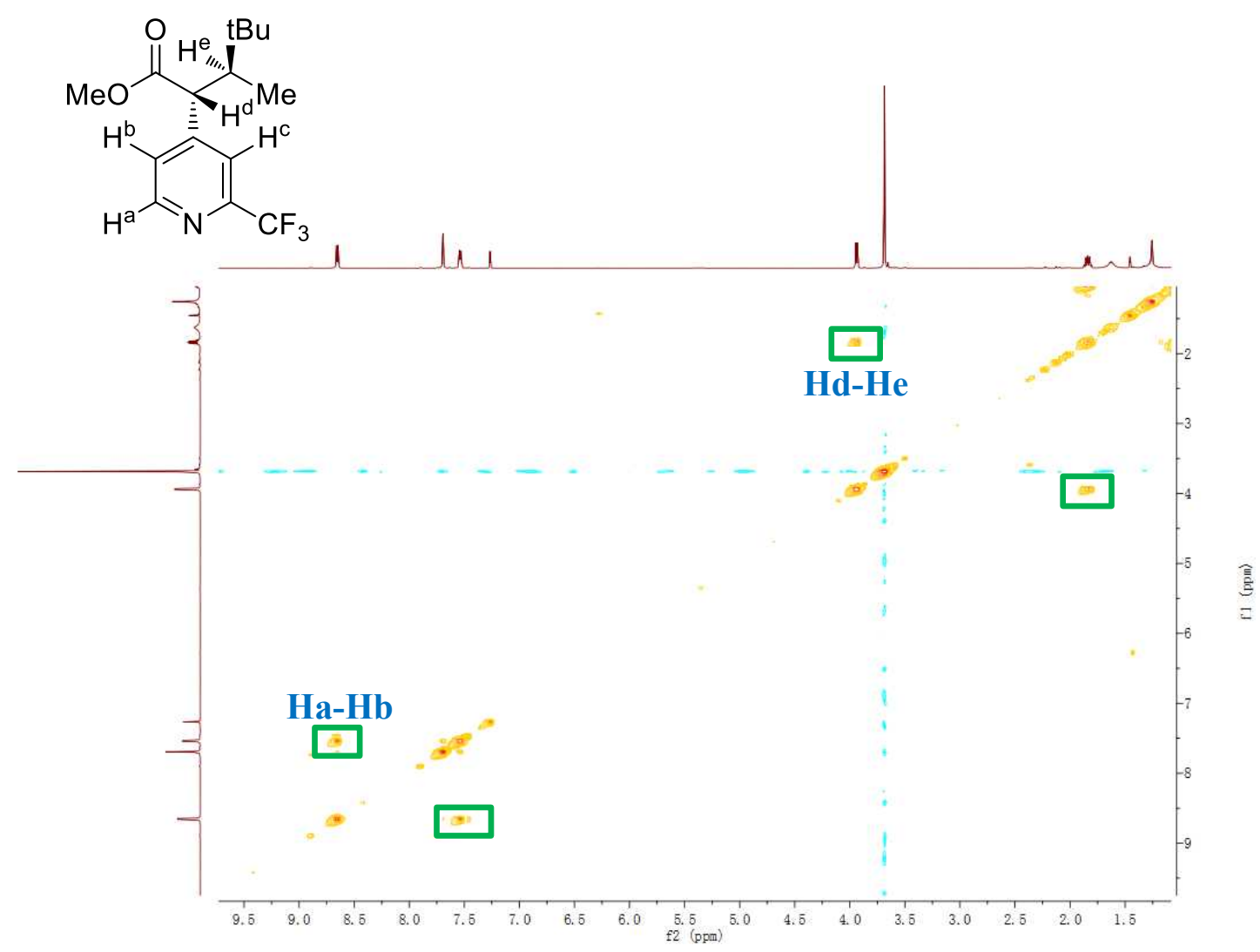

50; NOESY

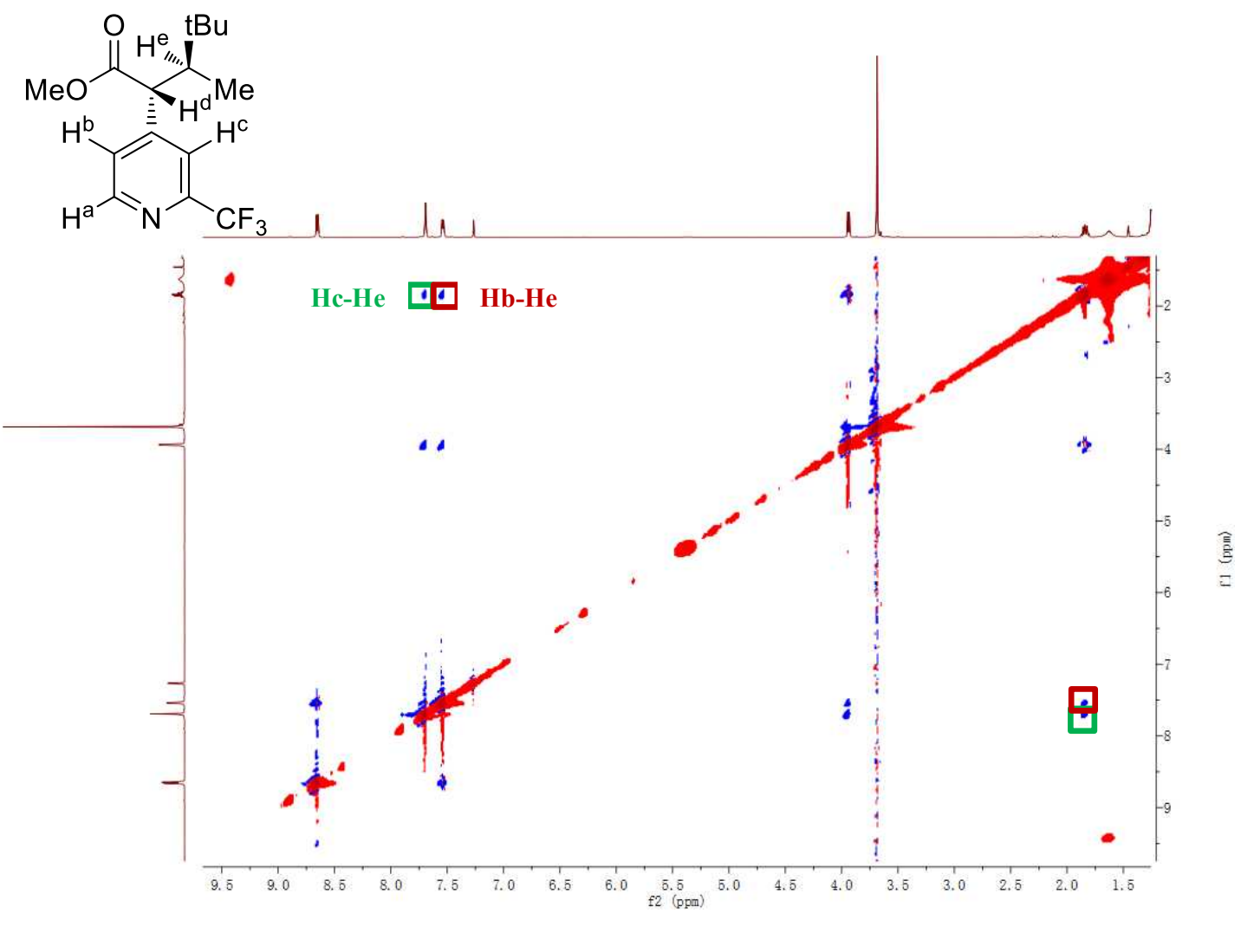


51; ${ }^{1} \mathrm{H}-\mathrm{NMR}$ (400 MHz, $\mathrm{CDCl}_{3}$ )

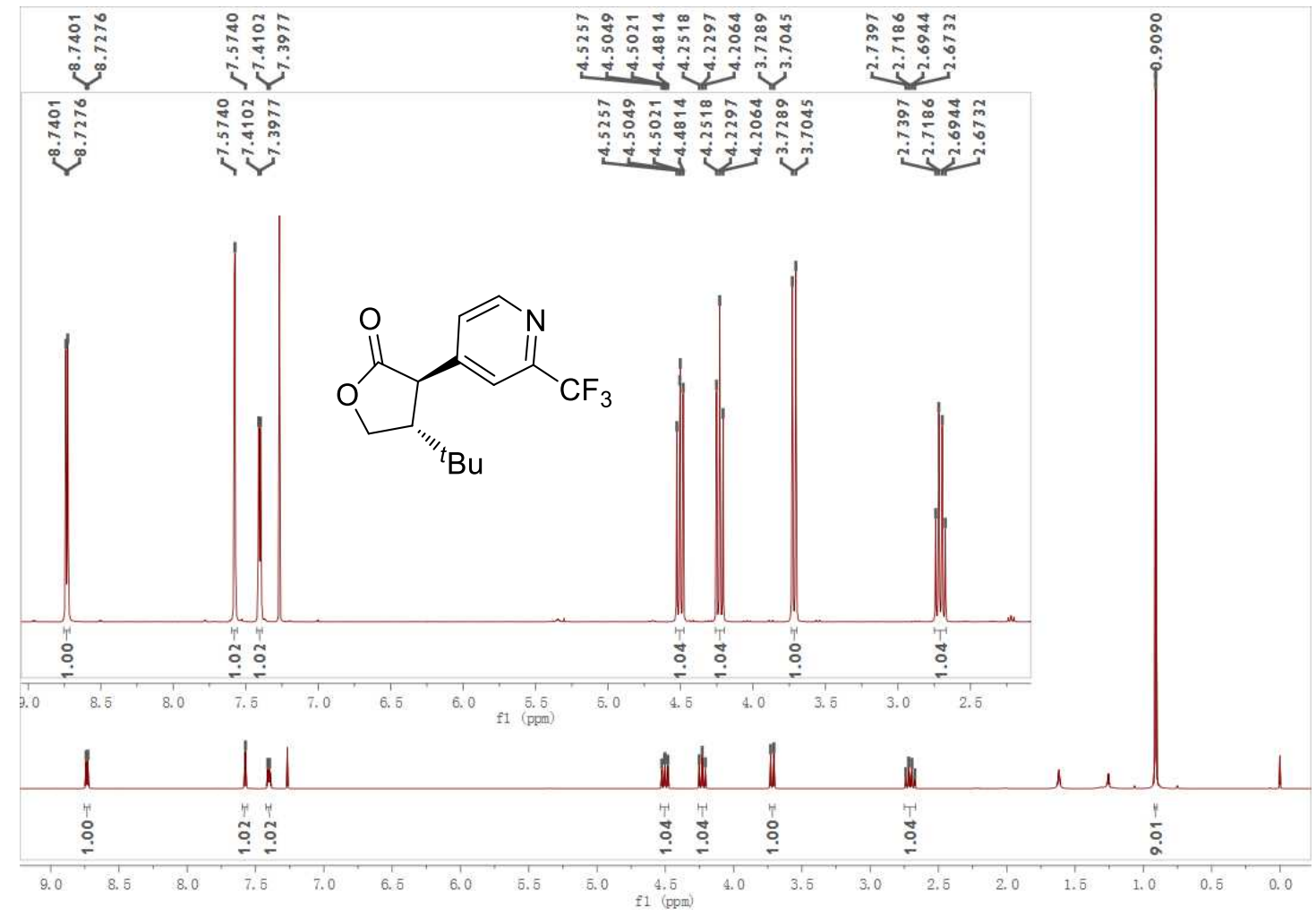

51; ${ }^{19}$ F-NMR (375 MHz, $\left.\mathrm{CDCl}_{3}\right)$

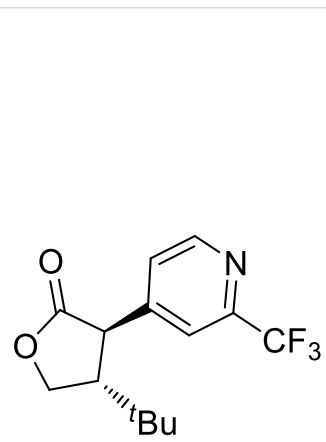

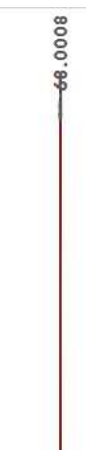


51; ${ }^{13} \mathrm{C}-\mathrm{NMR}$ (100 MHz, $\left.\mathrm{CDCl}_{3}\right)$

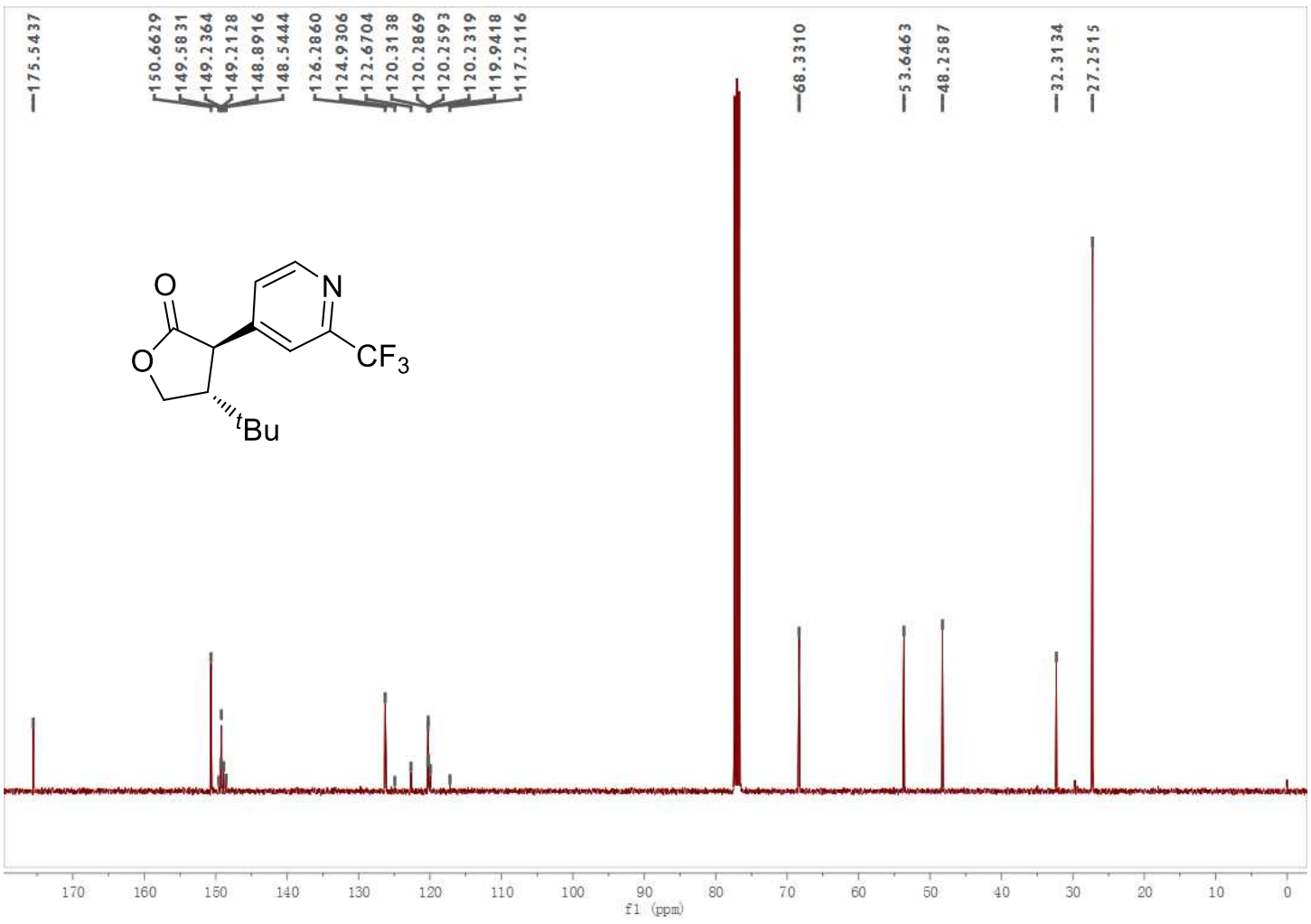

51; COSY

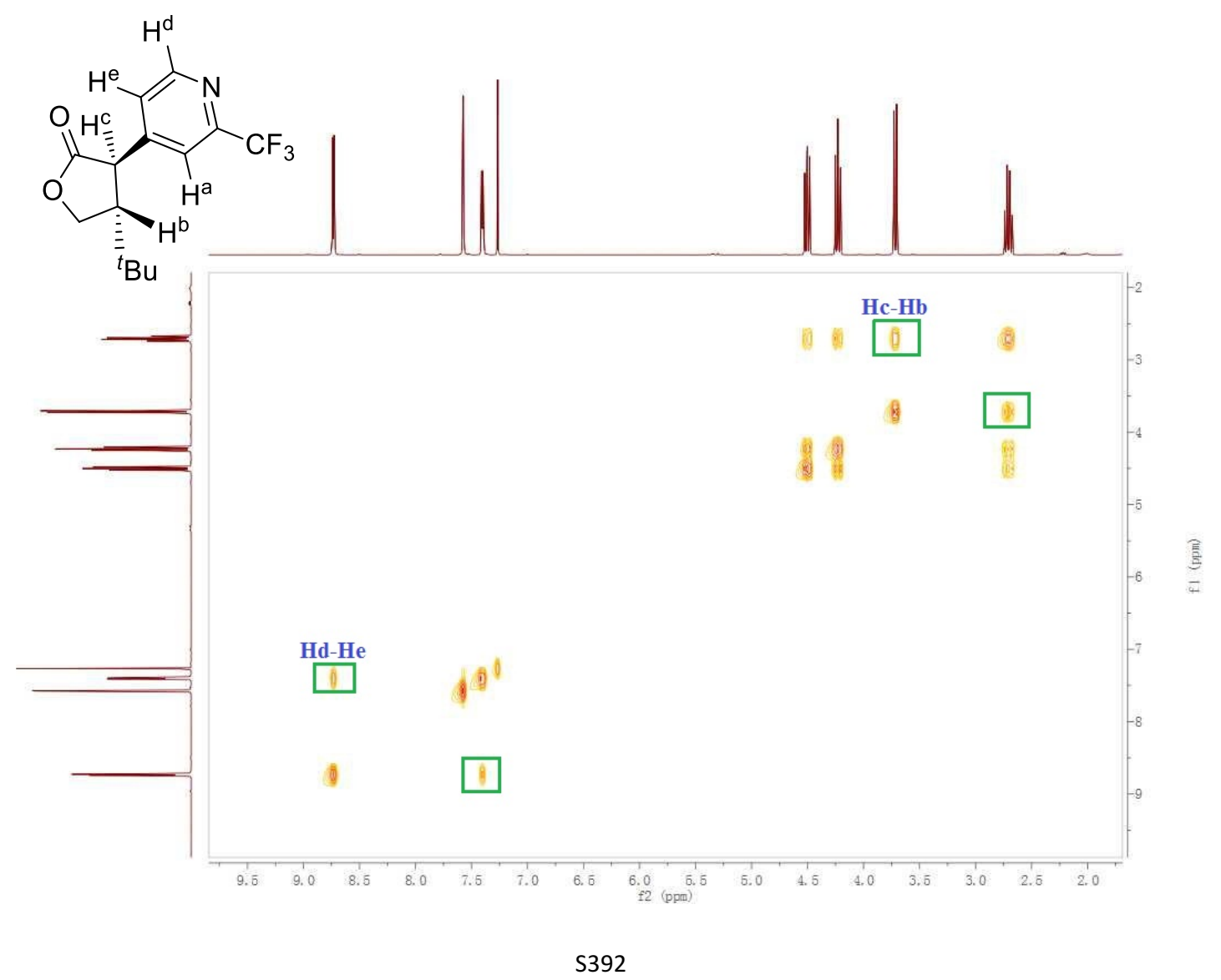


51; NOESY

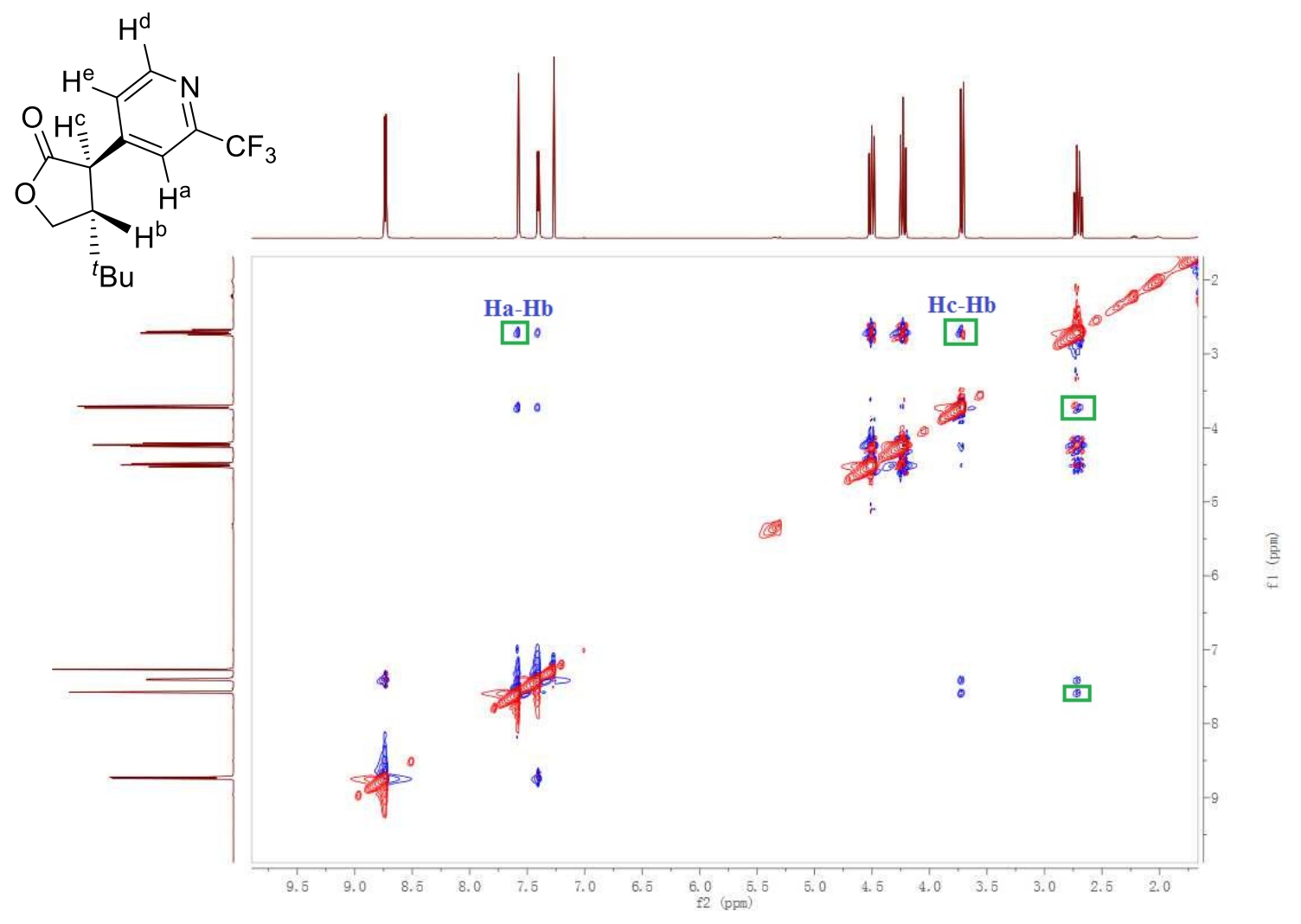

52; ${ }^{1} \mathrm{H}-\mathrm{NMR}$ (400 MHz, $\mathrm{CDCl}_{3}$ )

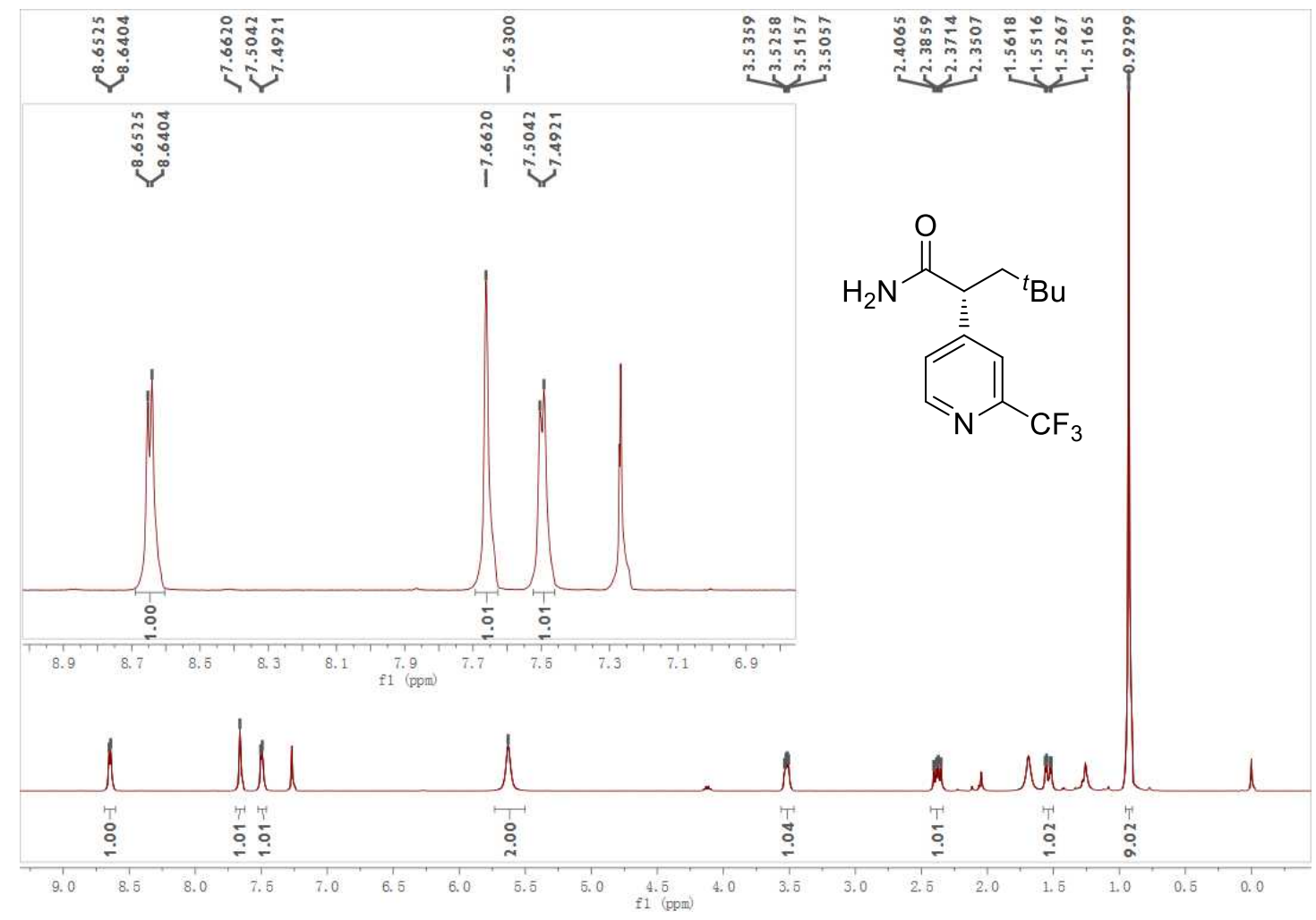


52; ${ }^{19}$ F-NMR (375 $\left.\mathrm{MHz}, \mathrm{CDCl}_{3}\right)$

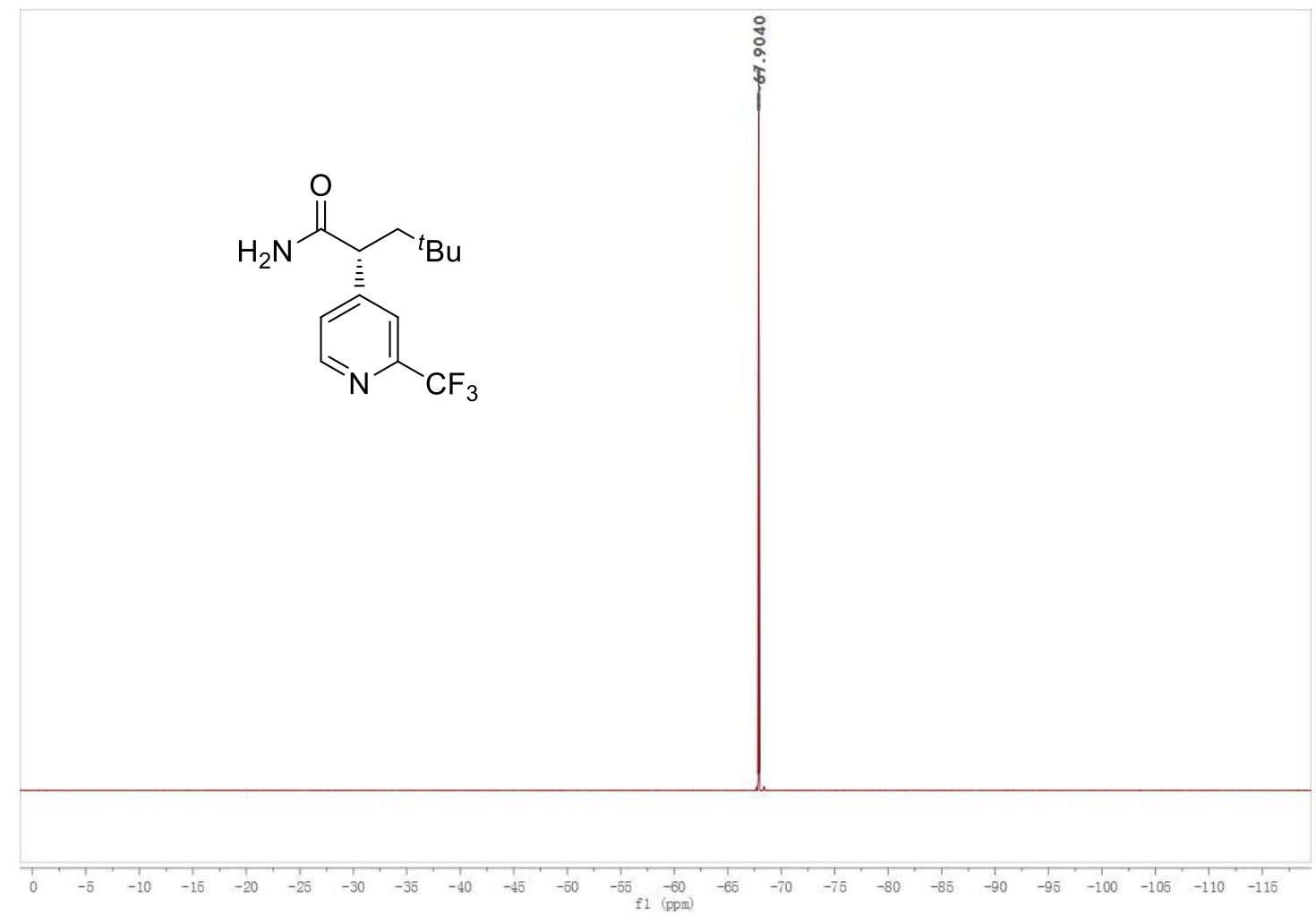

52; ${ }^{13} \mathrm{C}$-NMR (100 MHz, $\left.\mathrm{CDCl}_{3}\right)$

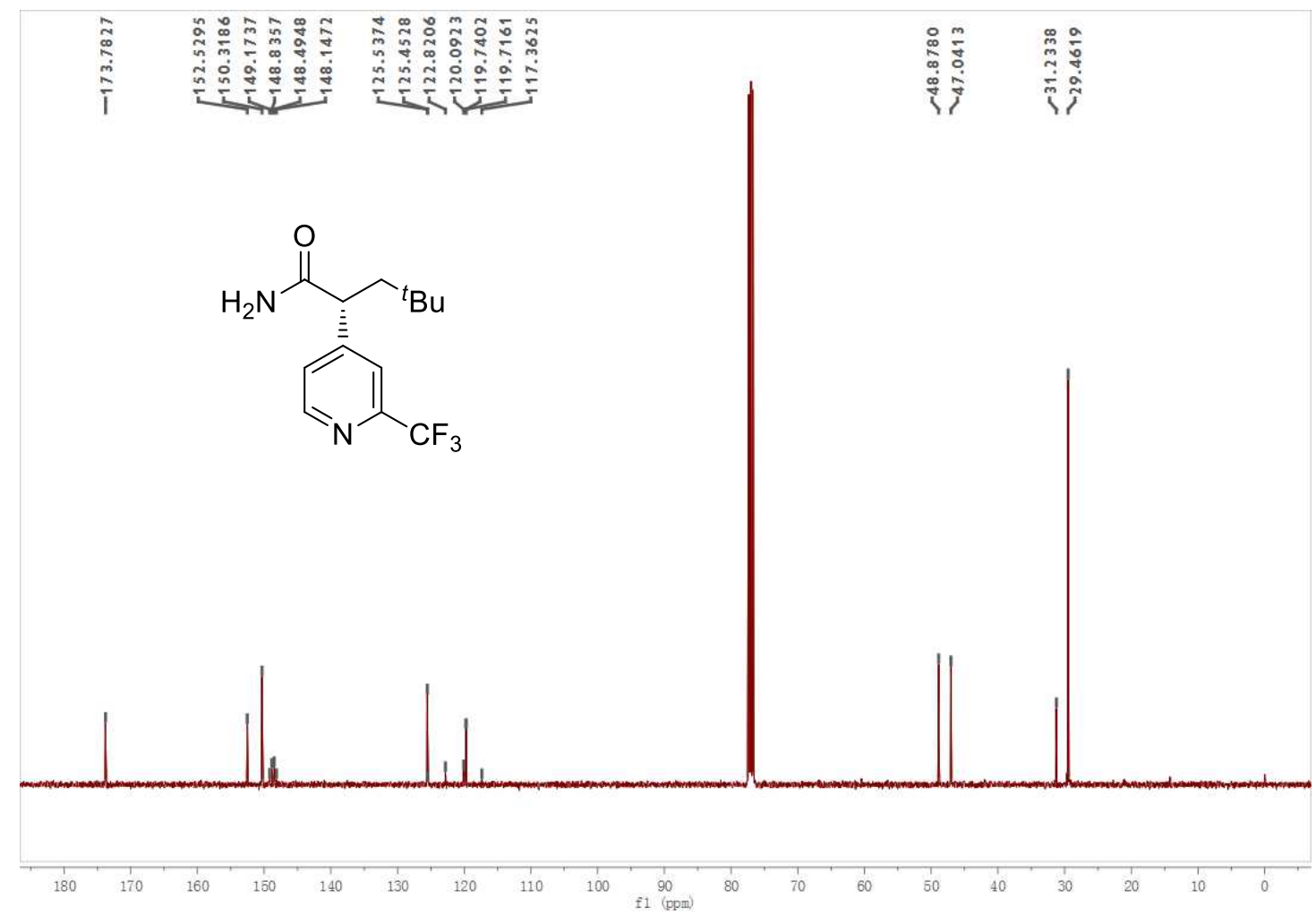


53; ${ }^{1} \mathrm{H}-\mathrm{NMR}$ (400 $\left.\mathrm{MHz}, \mathrm{CDCl}_{3}\right)$

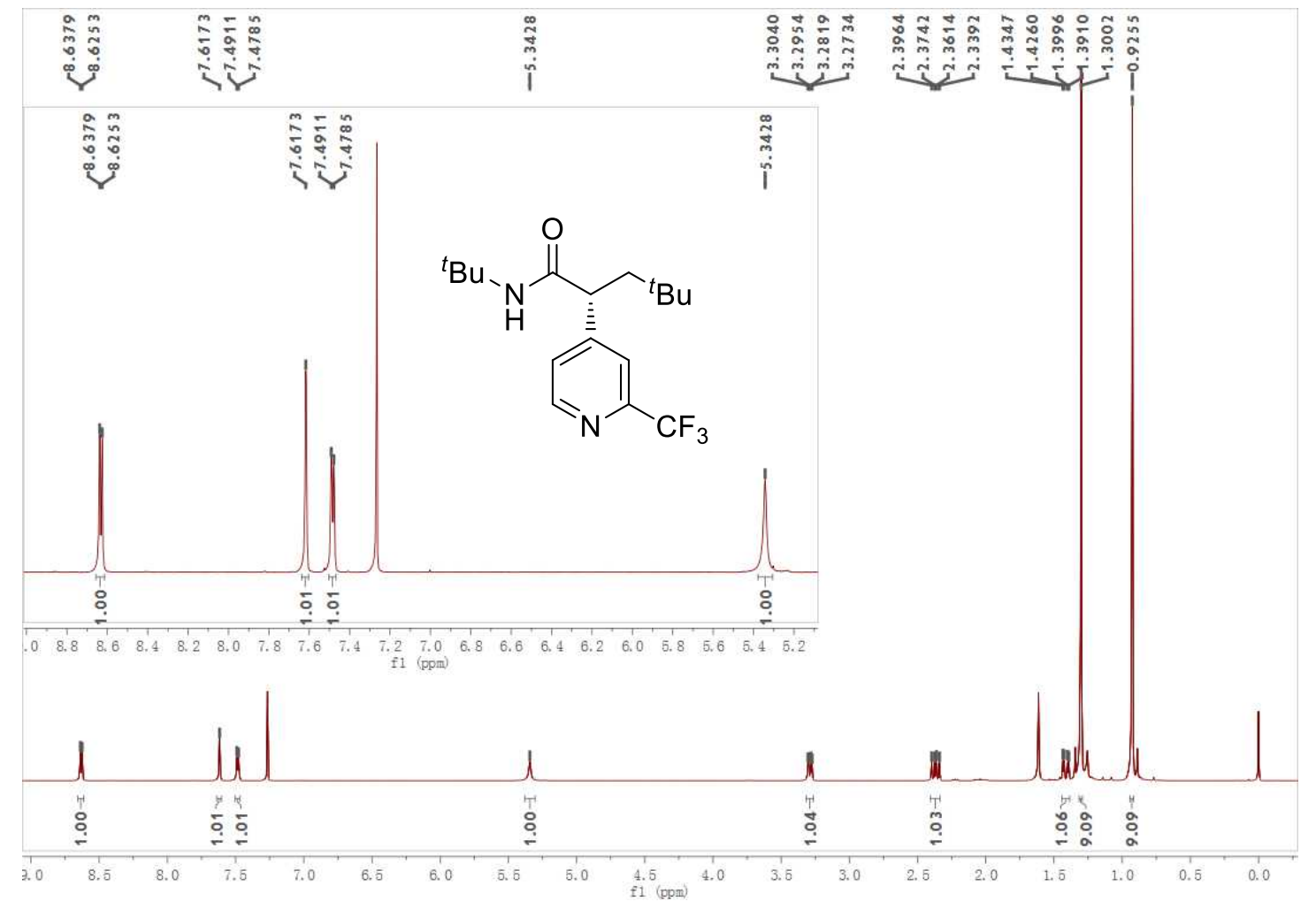

53; ${ }^{19}$ F-NMR (375 MHz, $\left.\mathrm{CDCl}_{3}\right)$

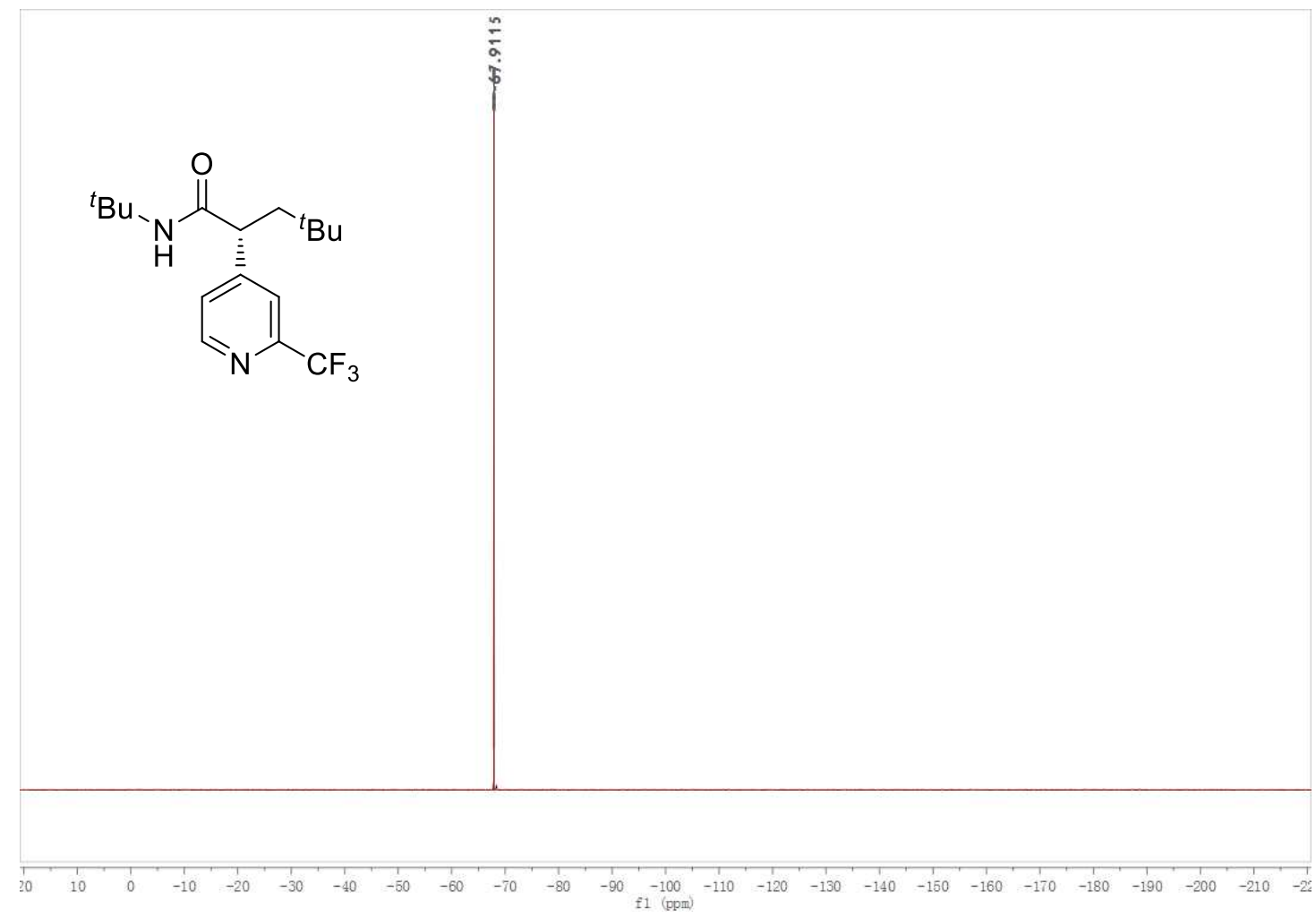


53; ${ }^{13} \mathrm{C}-\mathrm{NMR}\left(100 \mathrm{MHz}, \mathrm{CDCl}_{3}\right)$

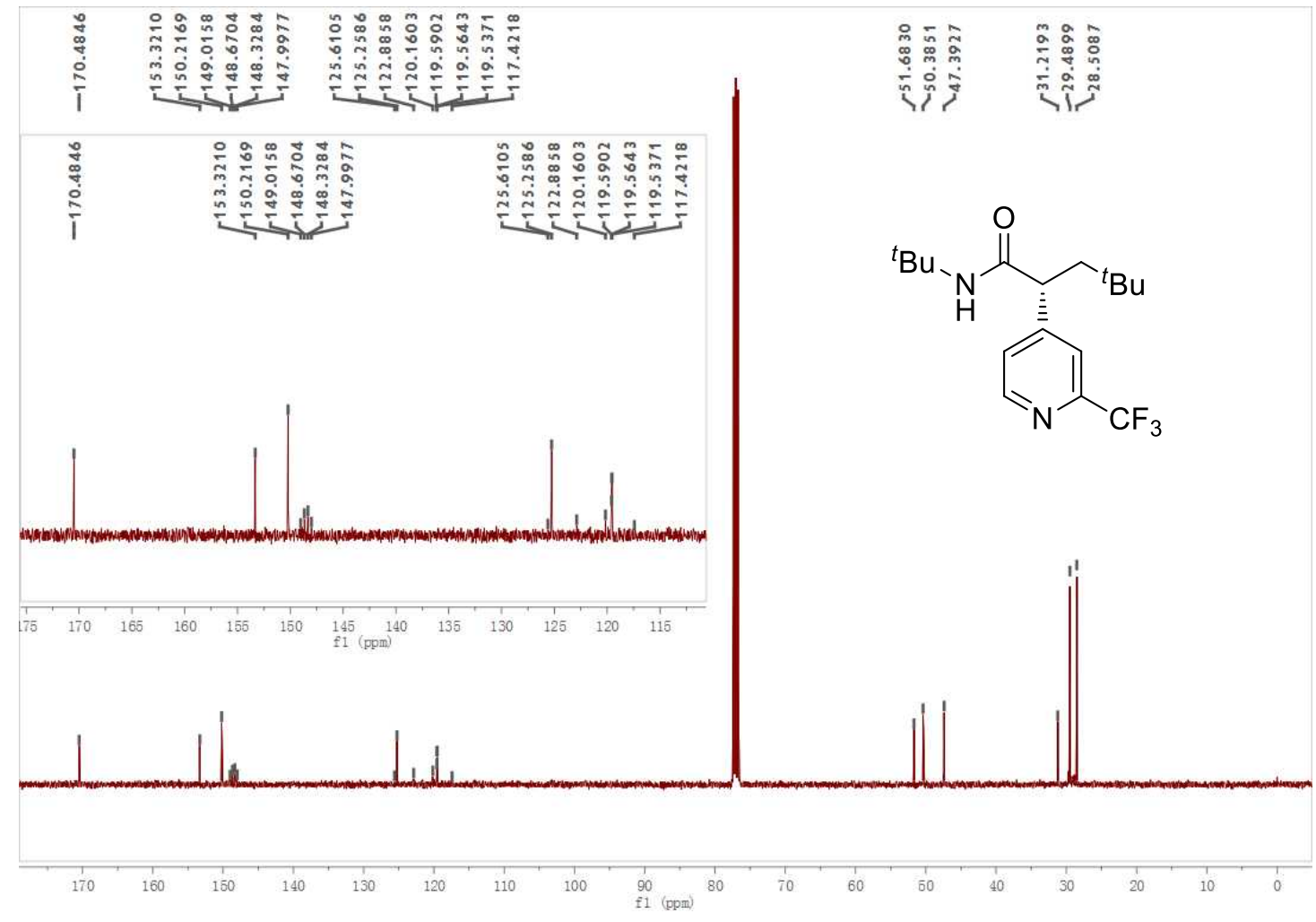

54; ${ }^{1} \mathrm{H}-\mathrm{NMR}$ (400 MHz, $\mathrm{CDCl}_{3}$ )

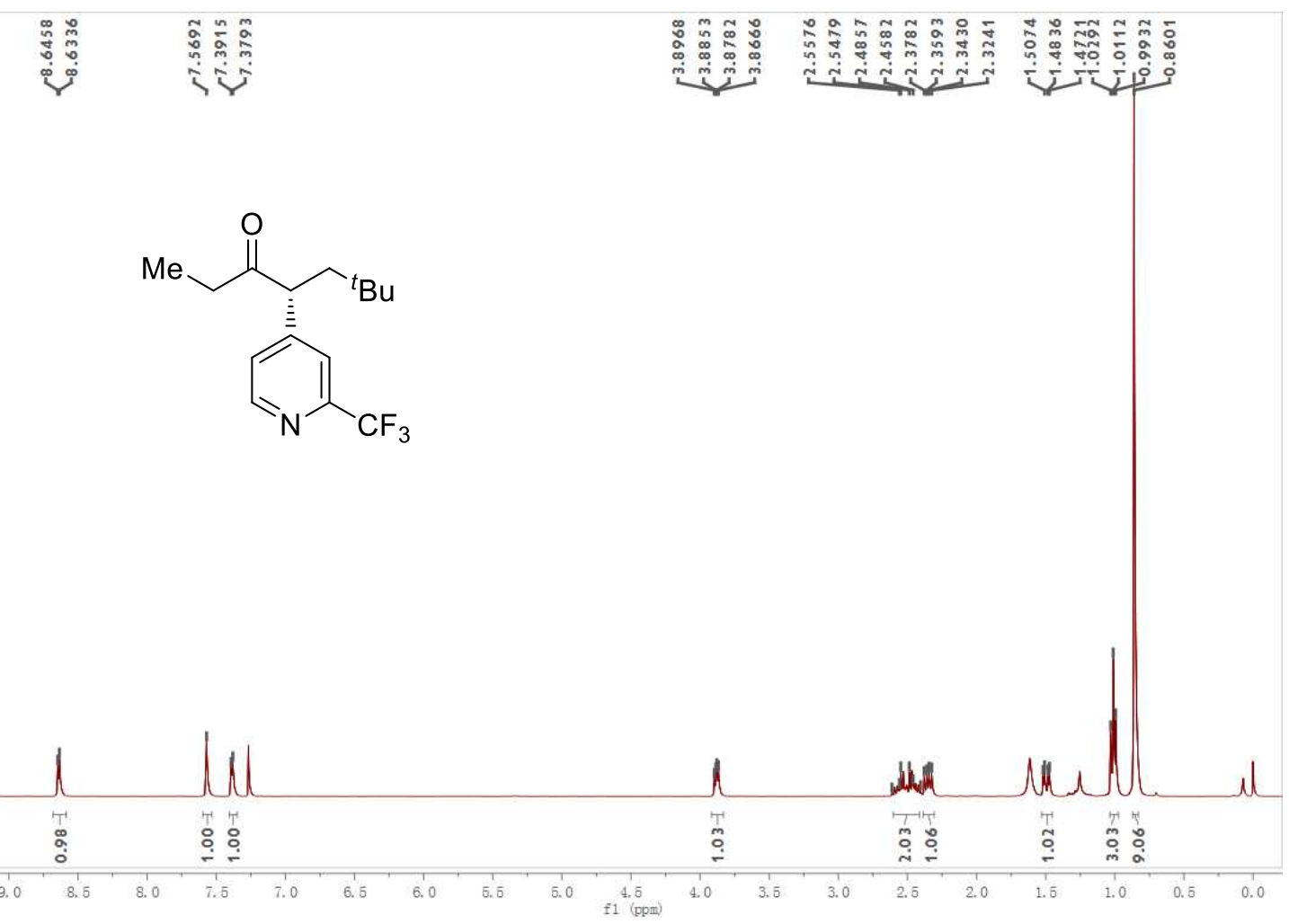


54; ${ }^{19}$ F-NMR (375 MHz, $\mathrm{CDCl}_{3}$ )

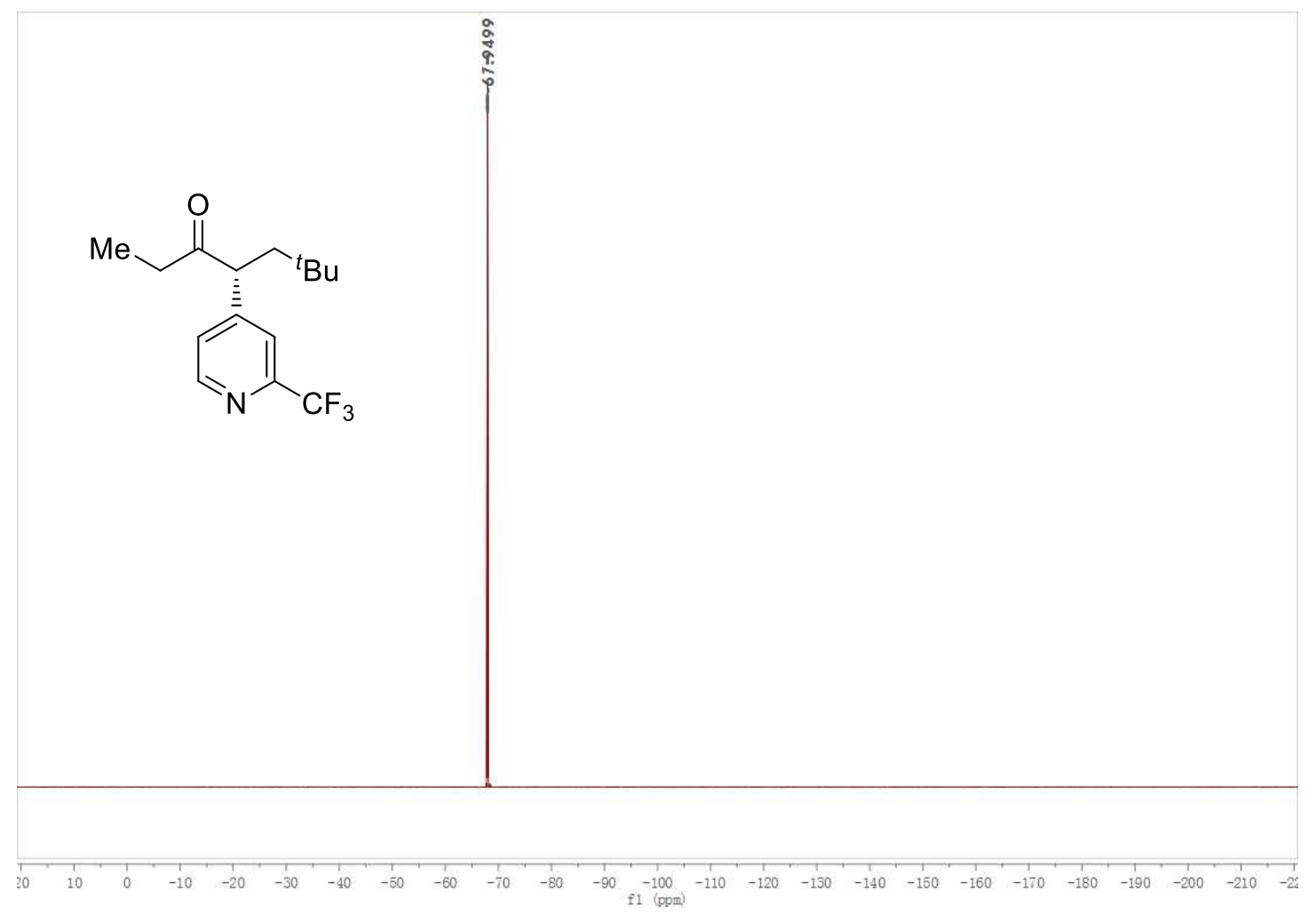

54; ${ }^{13} \mathrm{C}$-NMR (100 MHz, $\left.\mathrm{CDCl}_{3}\right)$

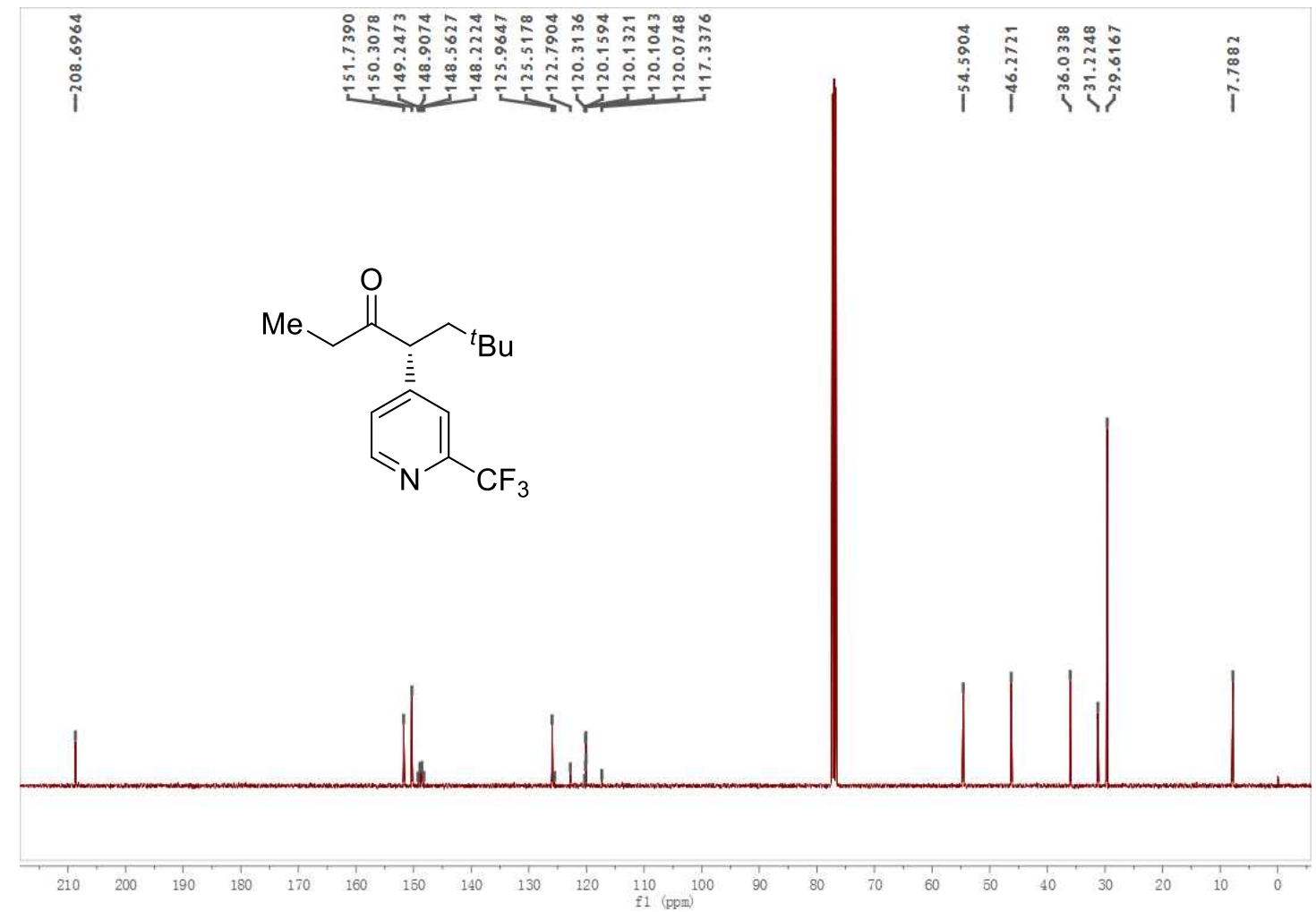


55; ${ }^{1} \mathrm{H}-\mathrm{NMR}$ (400 $\mathrm{MHz}, \mathrm{CDCl}_{3}$ )

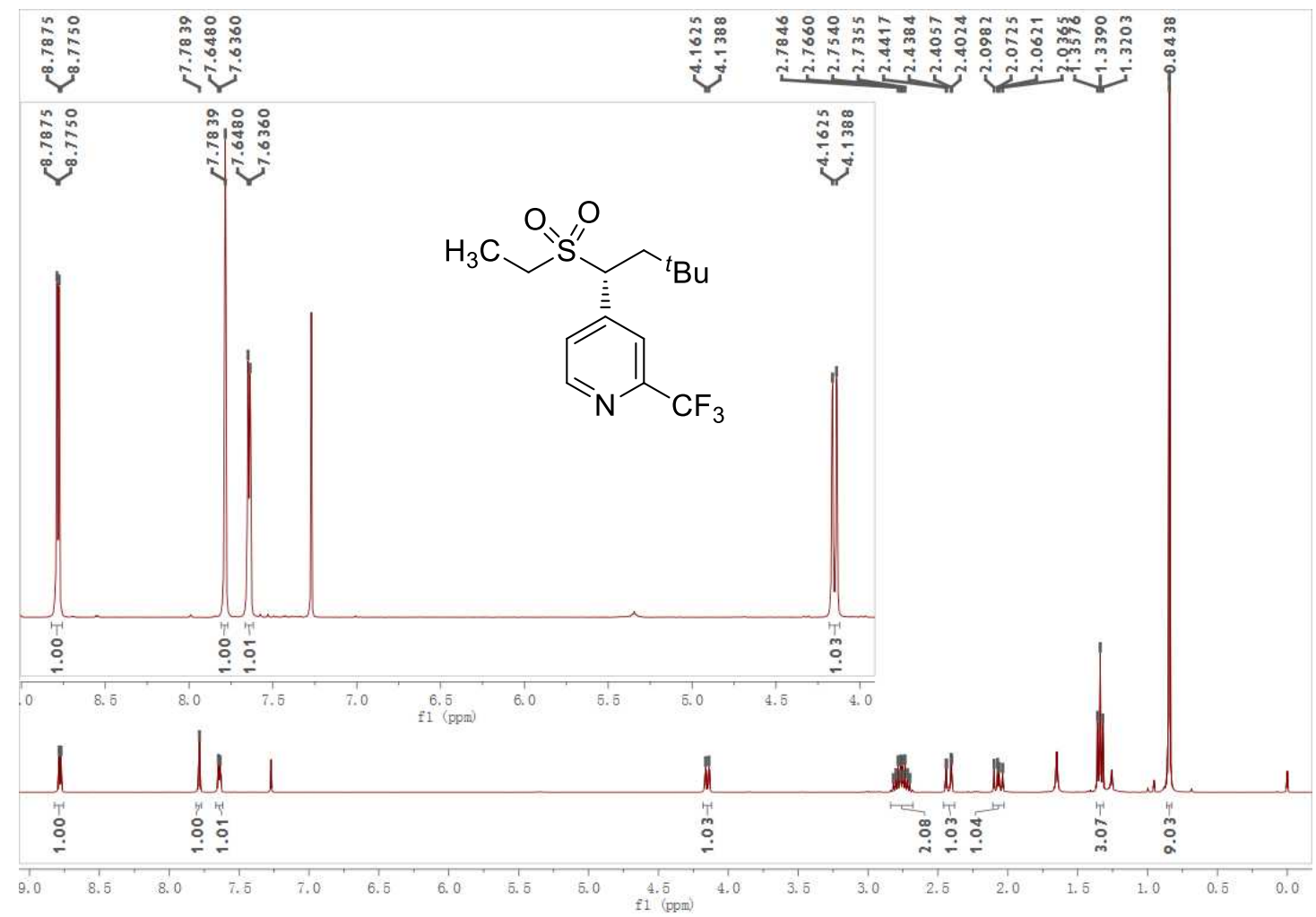

55; ${ }^{19}$ F-NMR (375 MHz, $\mathrm{CDCl}_{3}$ )

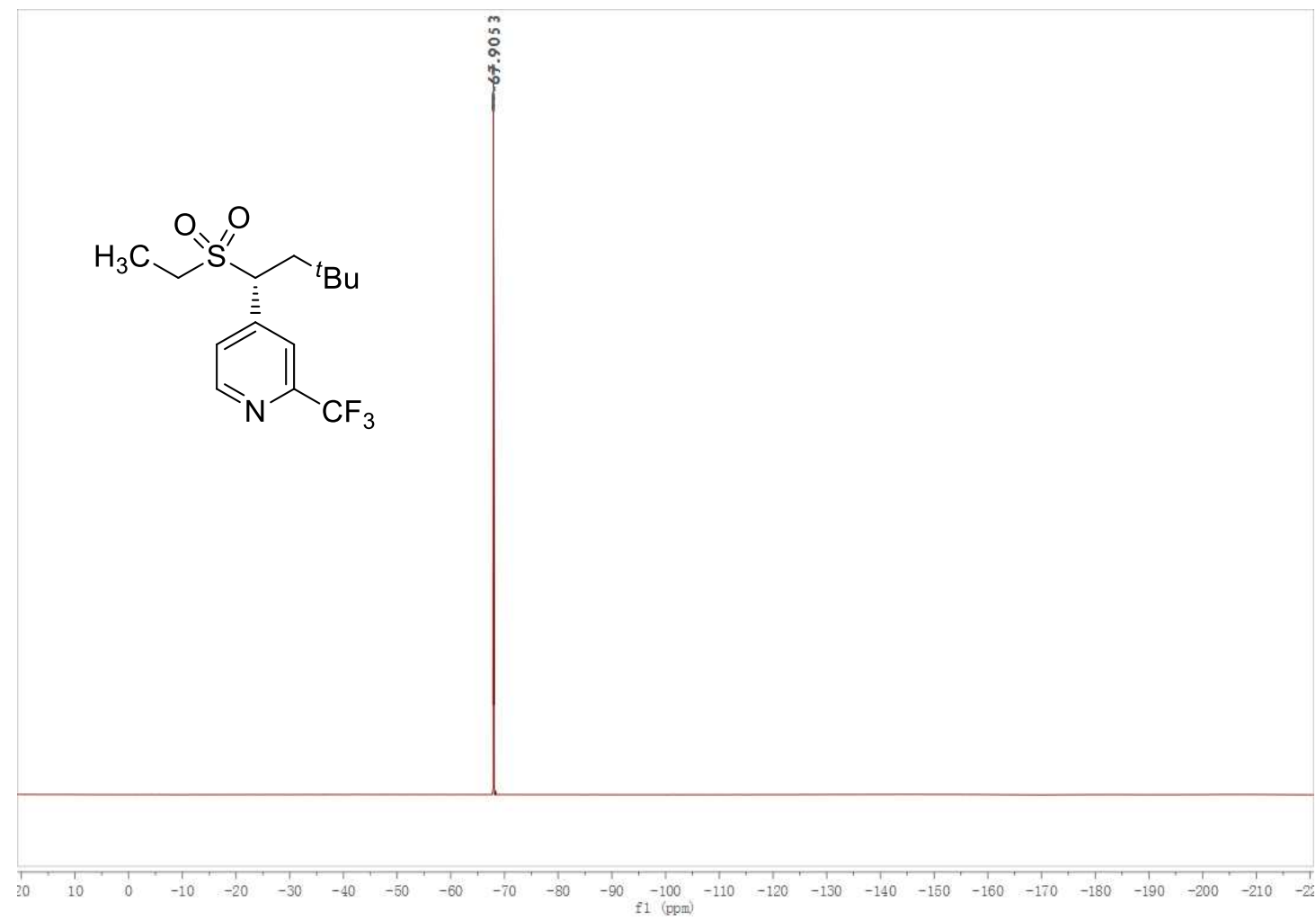


55; ${ }^{13} \mathrm{C}-\mathrm{NMR}\left(100 \mathrm{MHz}, \mathrm{CDCl}_{3}\right)$

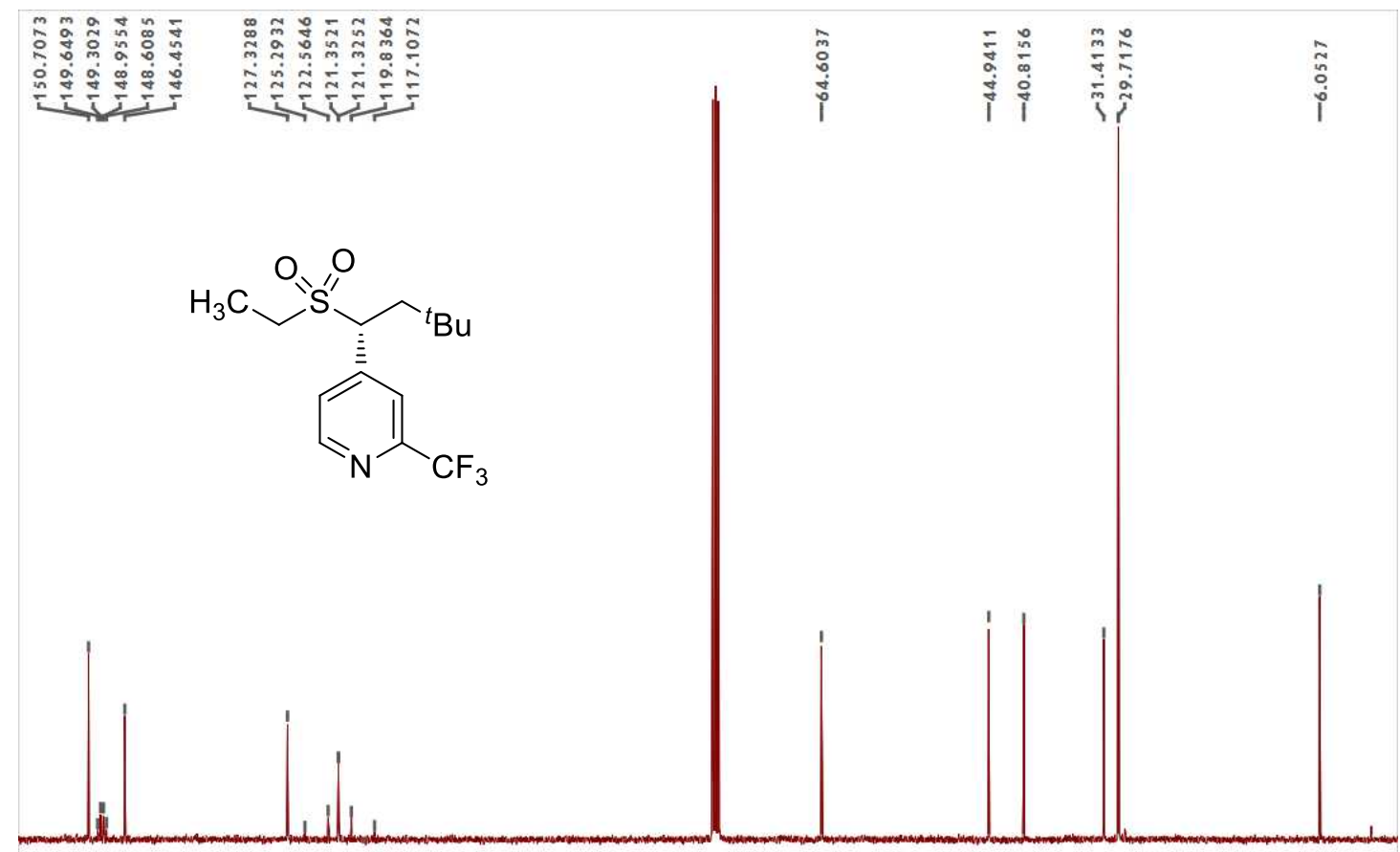

56; ${ }^{1} \mathrm{H}-\mathrm{NMR}$ (400 MHz, $\mathrm{CDCl}_{3}$ )

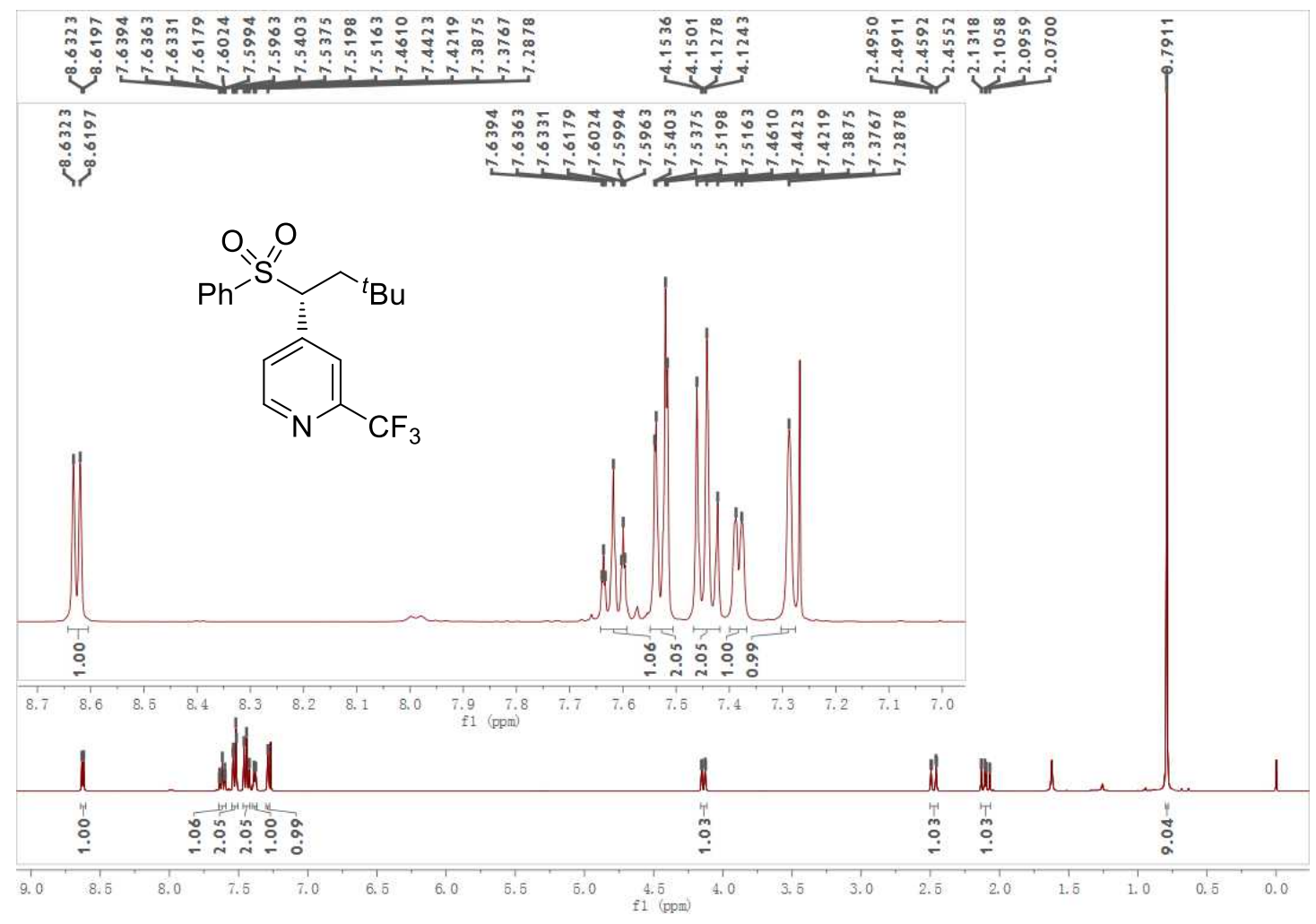


56; ${ }^{19}$ F-NMR (375 MHz, $\mathrm{CDCl}_{3}$ )

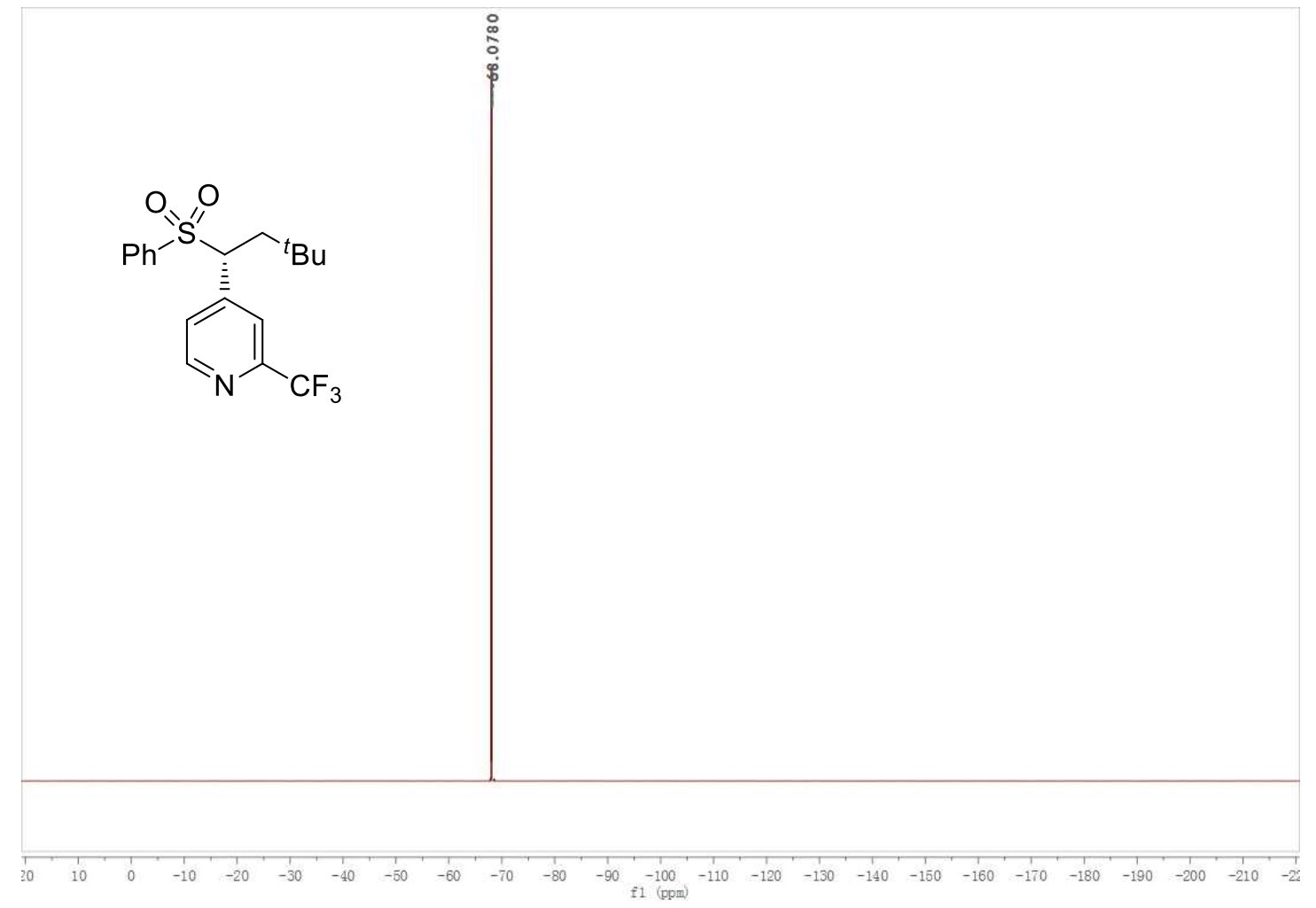

56; ${ }^{13} \mathrm{C}-\mathrm{NMR}\left(100 \mathrm{MHz}, \mathrm{CDCl}_{3}\right)$

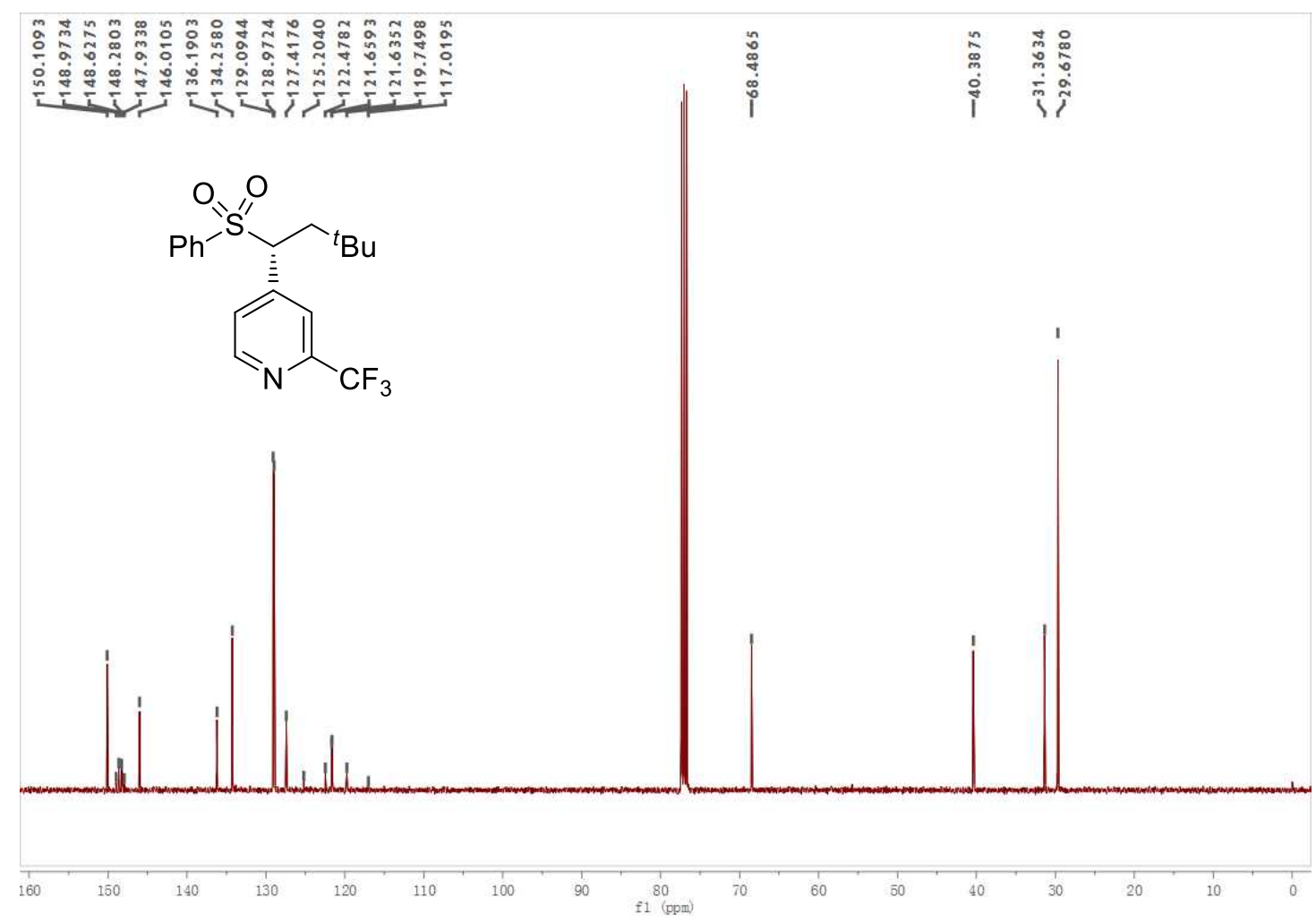


57; ${ }^{1} \mathrm{H}-\mathrm{NMR}$ (400 MHz, $\mathrm{CDCl}_{3}$ )

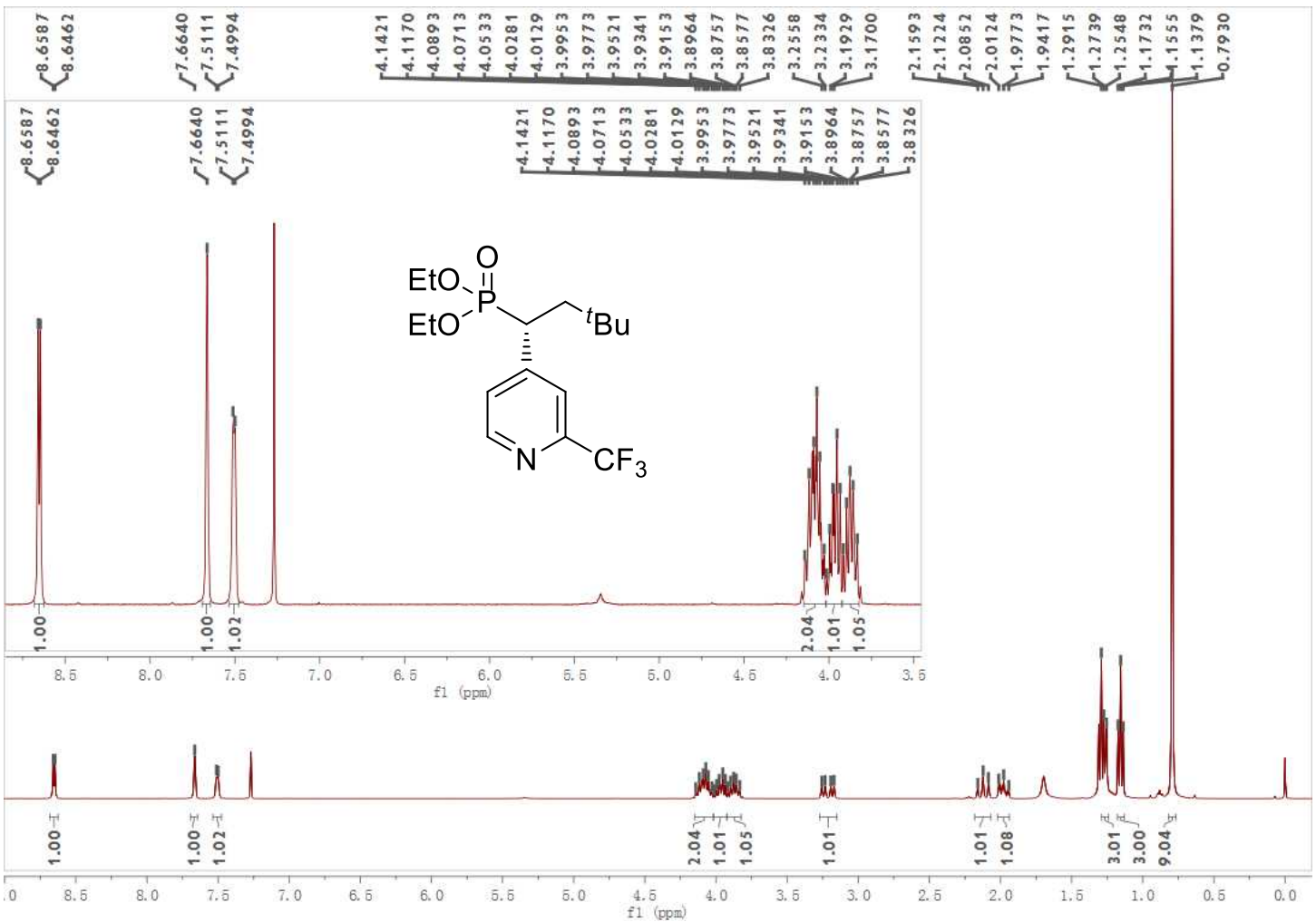

57; ${ }^{19}$ F-NMR (375 MHz, $\left.\mathrm{CDCl}_{3}\right)$

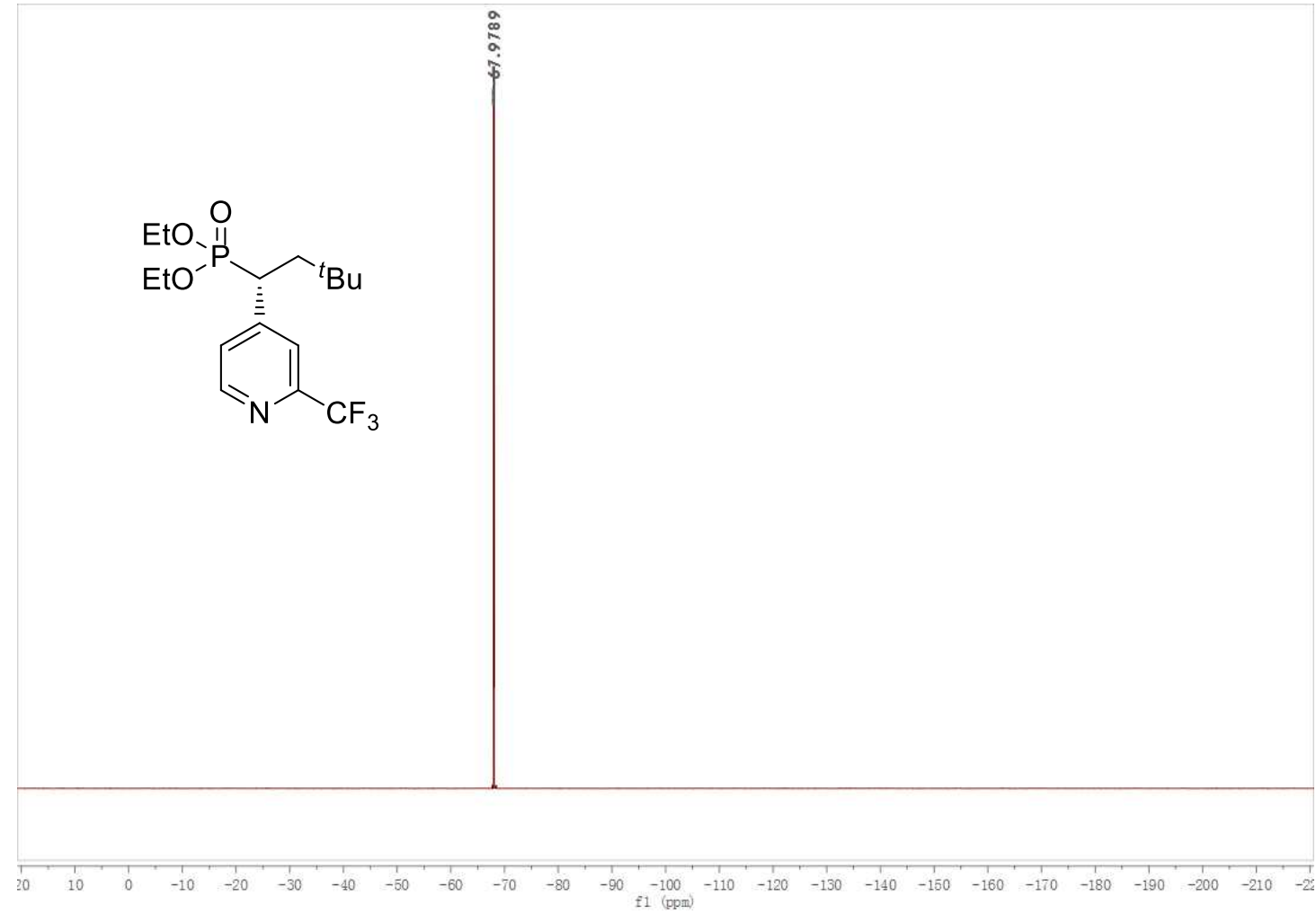


57; ${ }^{13} \mathrm{C}$-NMR (100 MHz, $\mathrm{CDCl}_{3}$ )

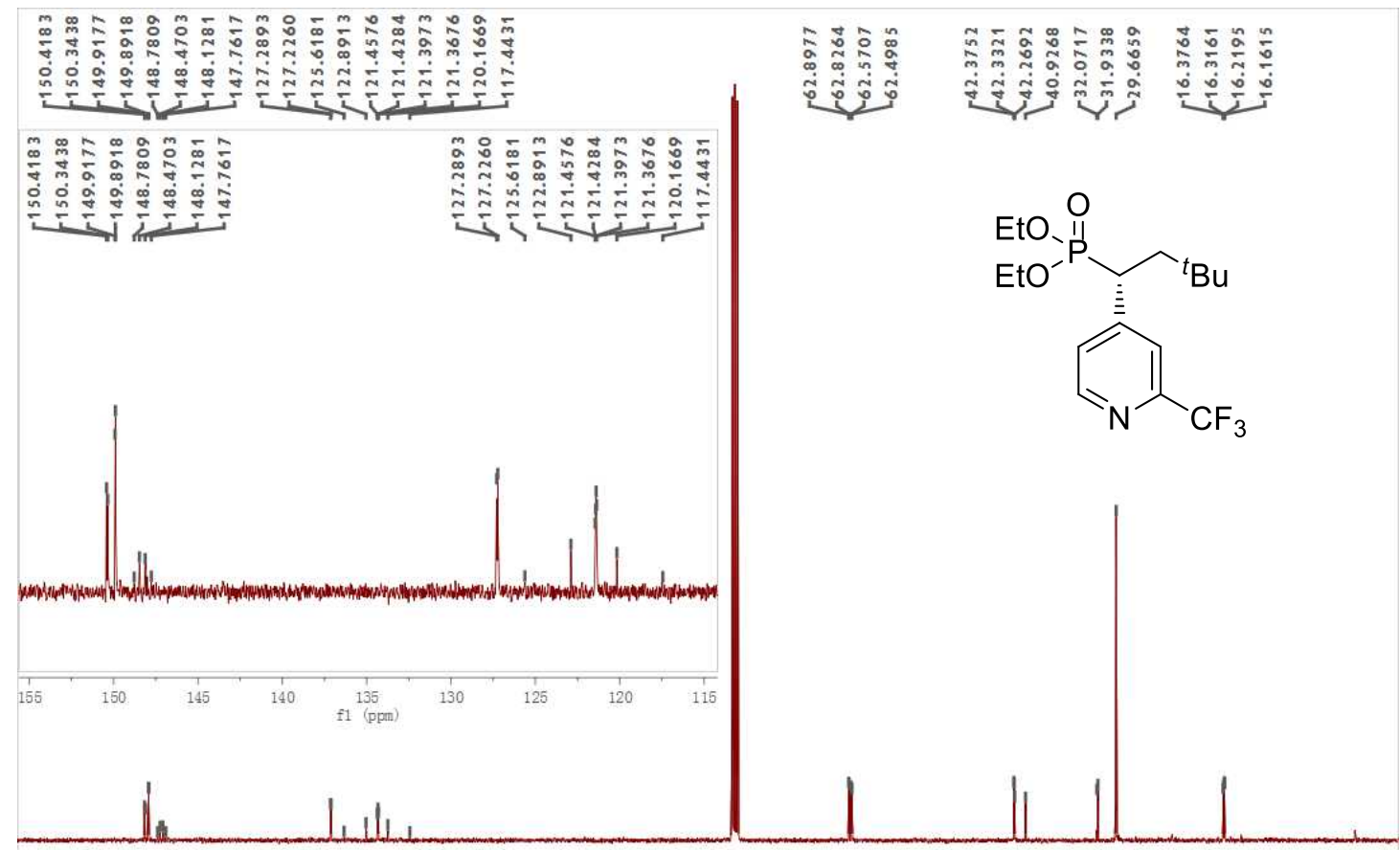

58; ${ }^{1} \mathrm{H}-\mathrm{NMR}\left(400 \mathrm{MHz}, \mathrm{CDCl}_{3}\right)$

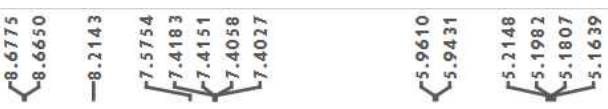

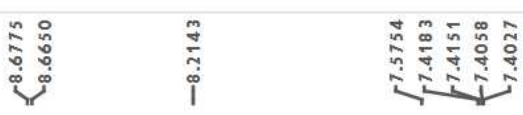
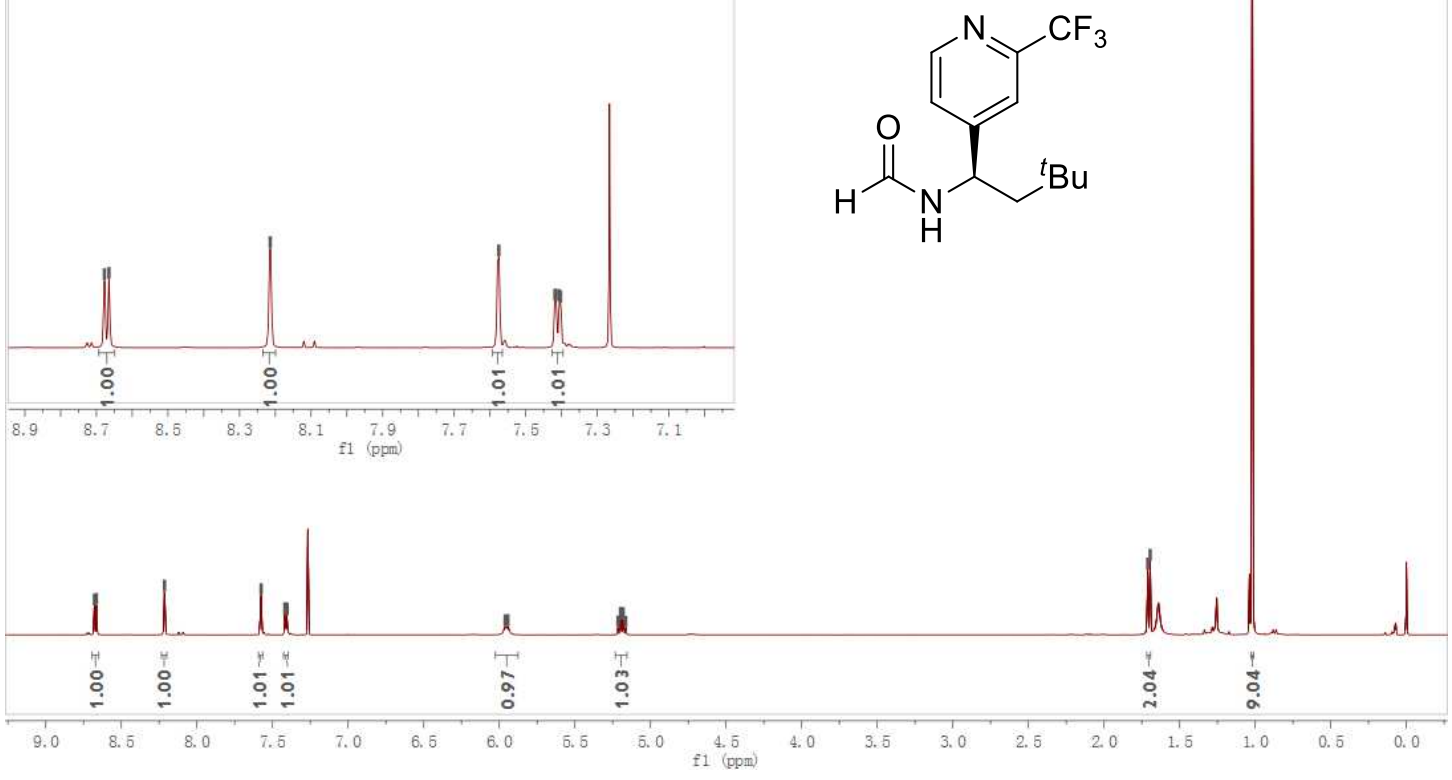
58; ${ }^{19}$ F-NMR (375 MHz, $\mathrm{CDCl}_{3}$ )

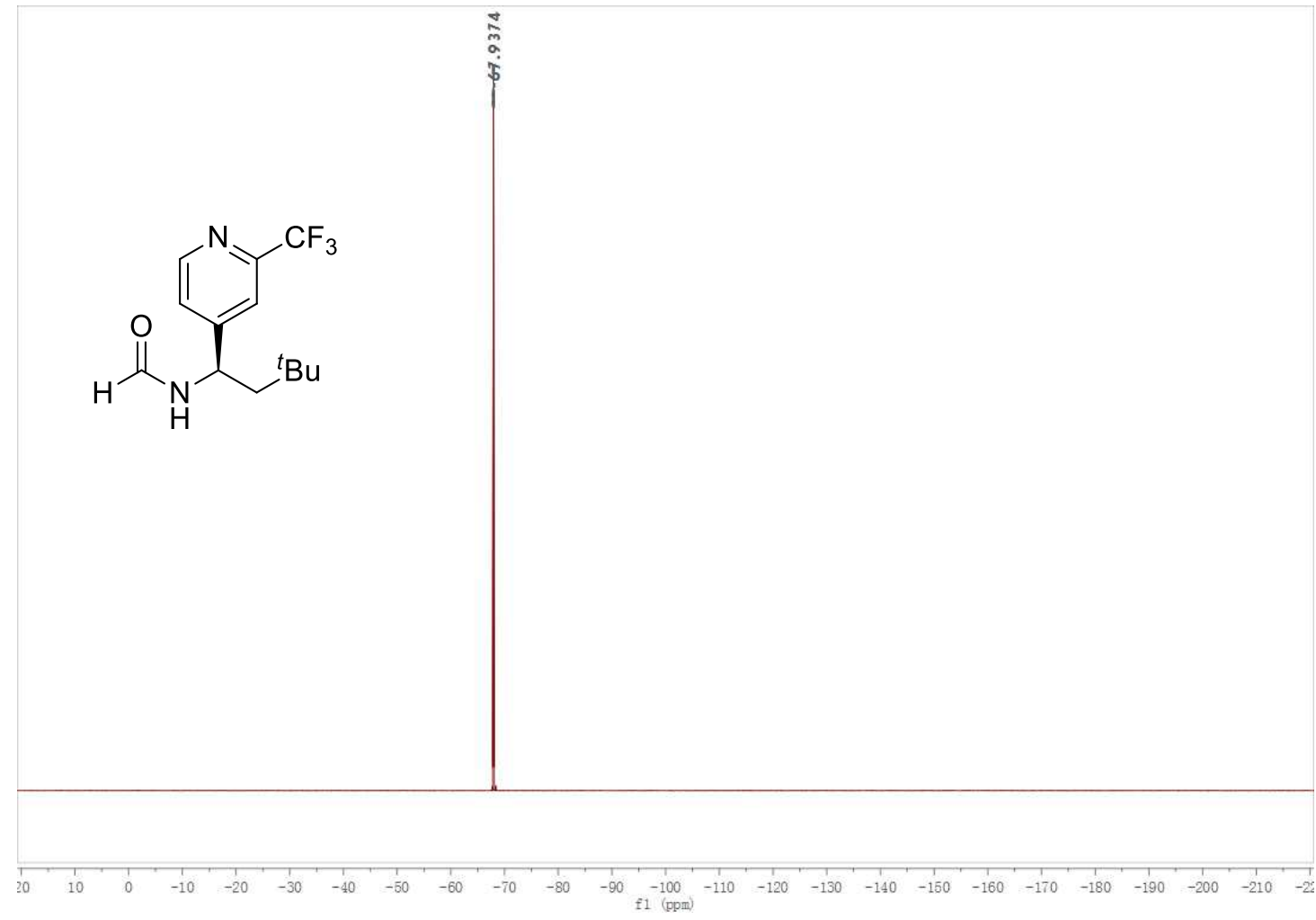

58; ${ }^{13} \mathrm{C}-\mathrm{NMR}\left(100 \mathrm{MHz}, \mathrm{CDCl}_{3}\right)$

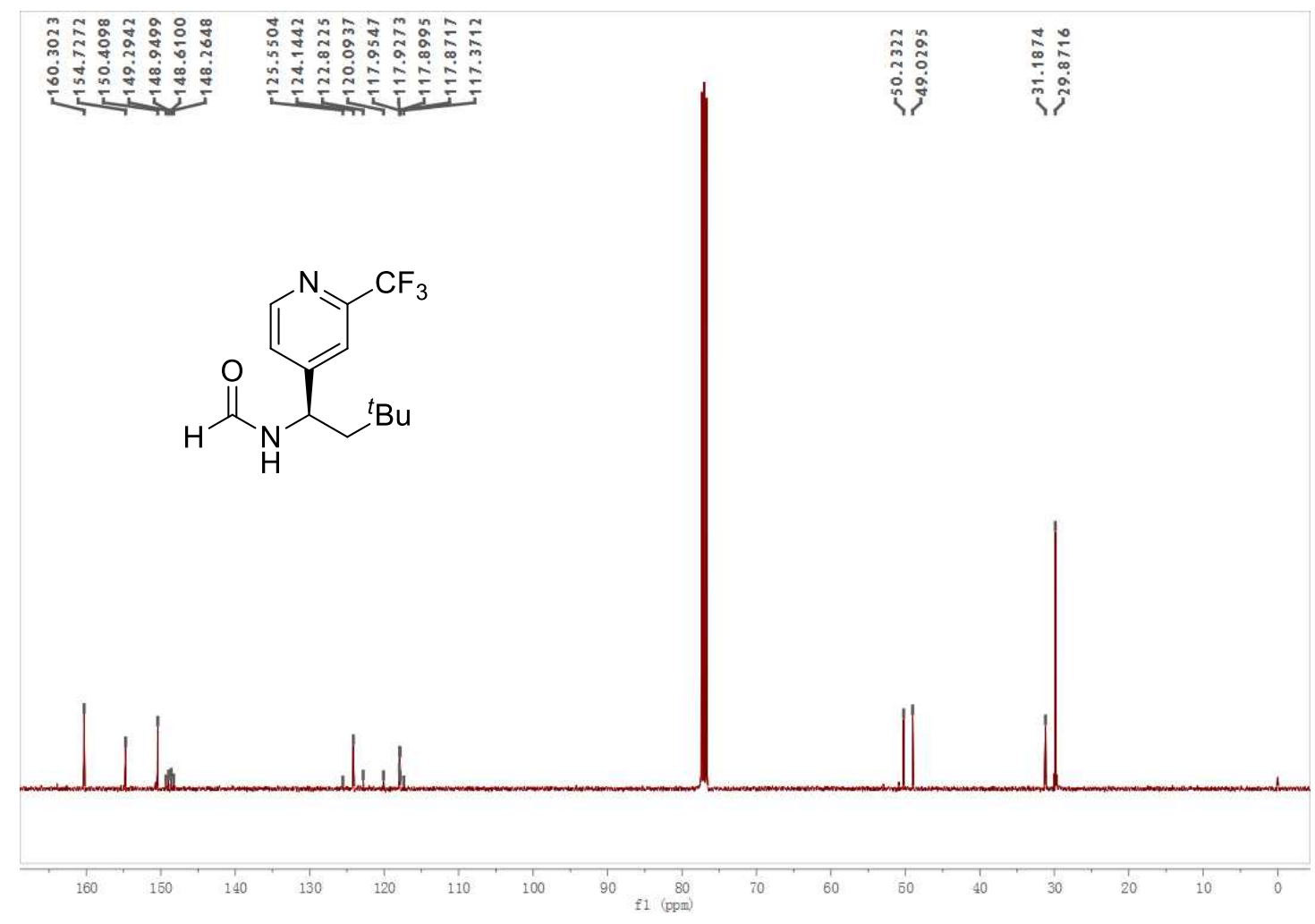


59; ${ }^{1} \mathrm{H}-\mathrm{NMR}$ (400 MHz, $\left.\mathrm{CDCl}_{3}\right)$

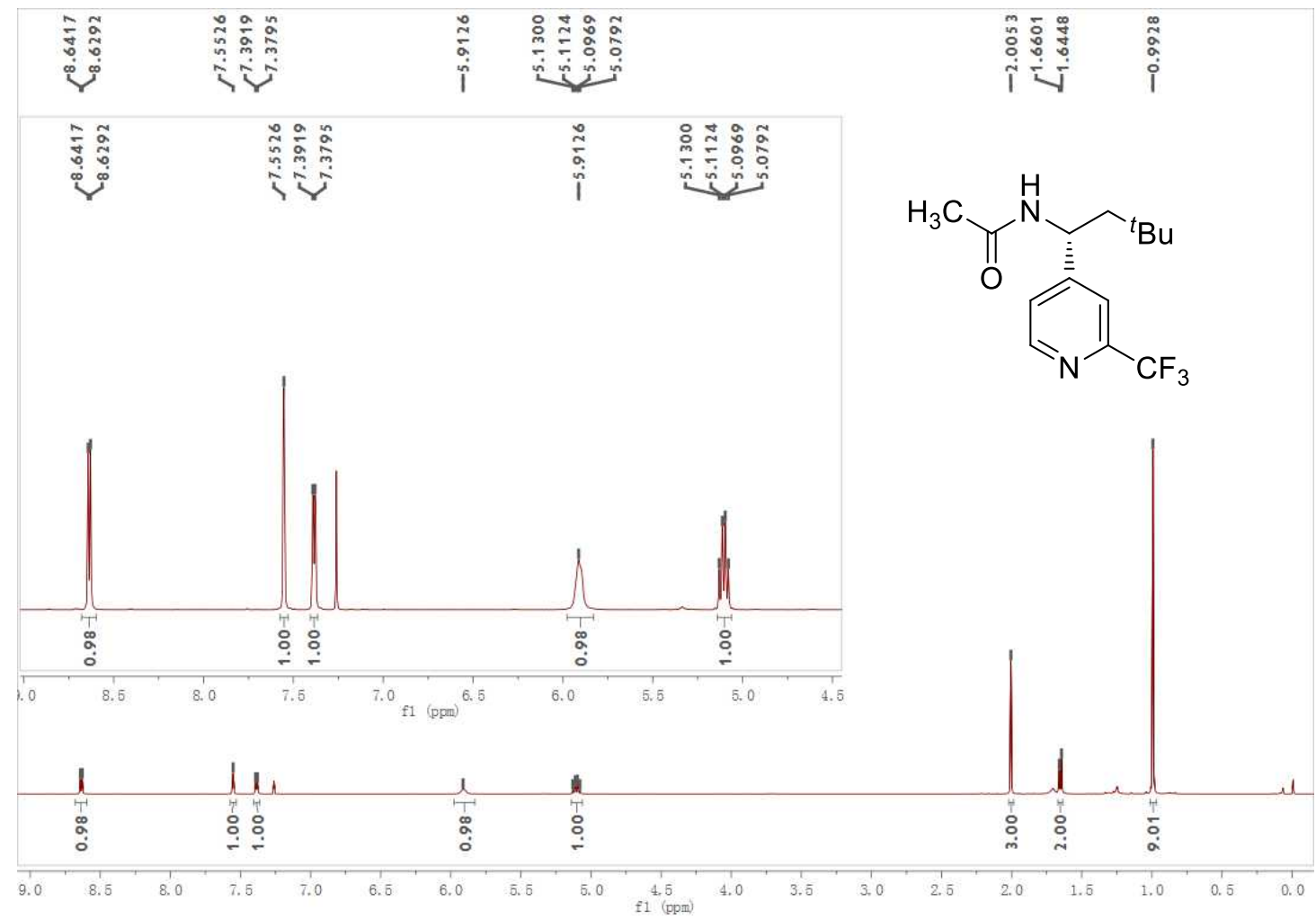

59; ${ }^{19}$ F-NMR (375 MHz, $\mathrm{CDCl}_{3}$ )

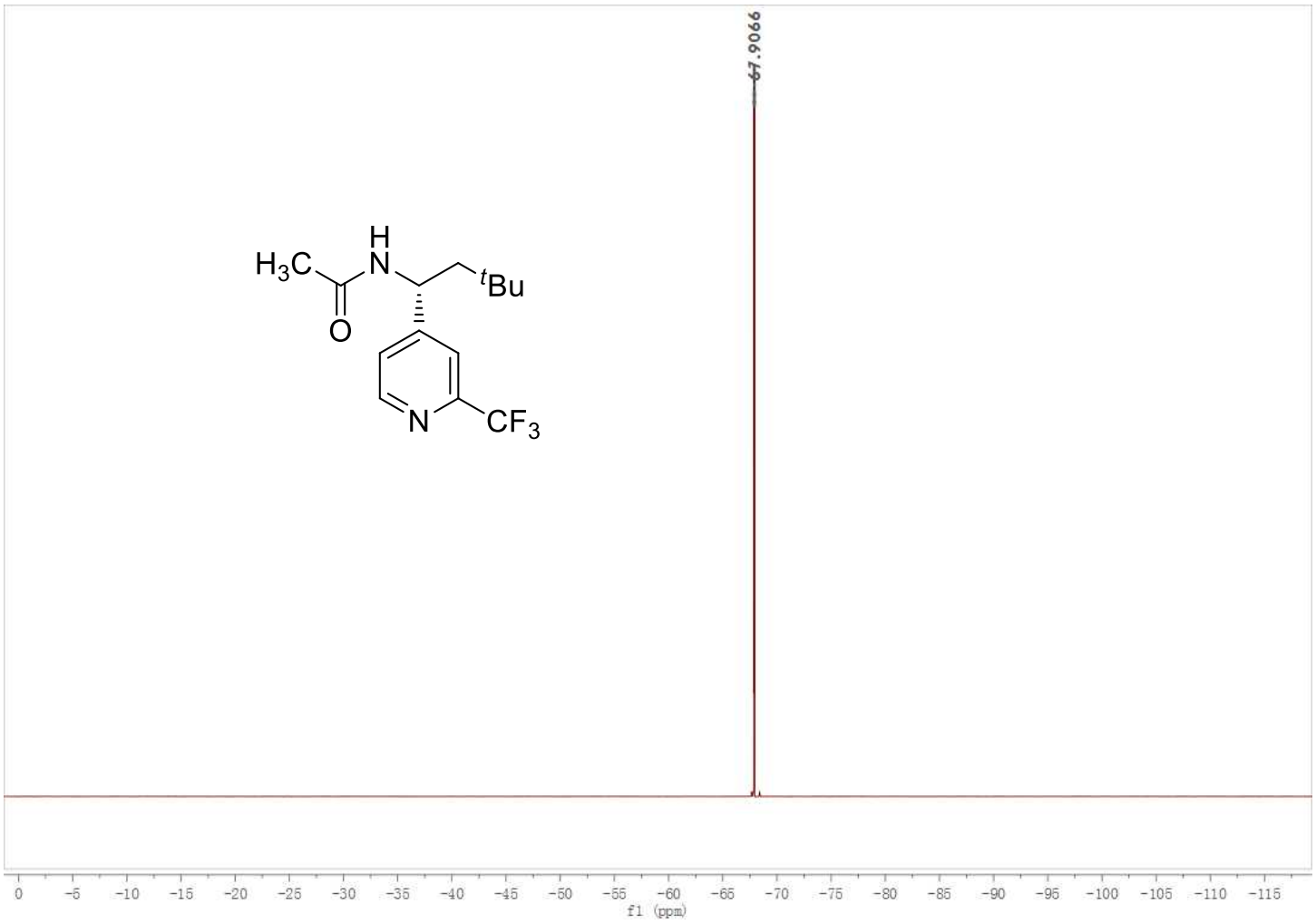


59; ${ }^{13} \mathrm{C}-\mathrm{NMR}\left(100 \mathrm{MHz}, \mathrm{CDCl}_{3}\right)$

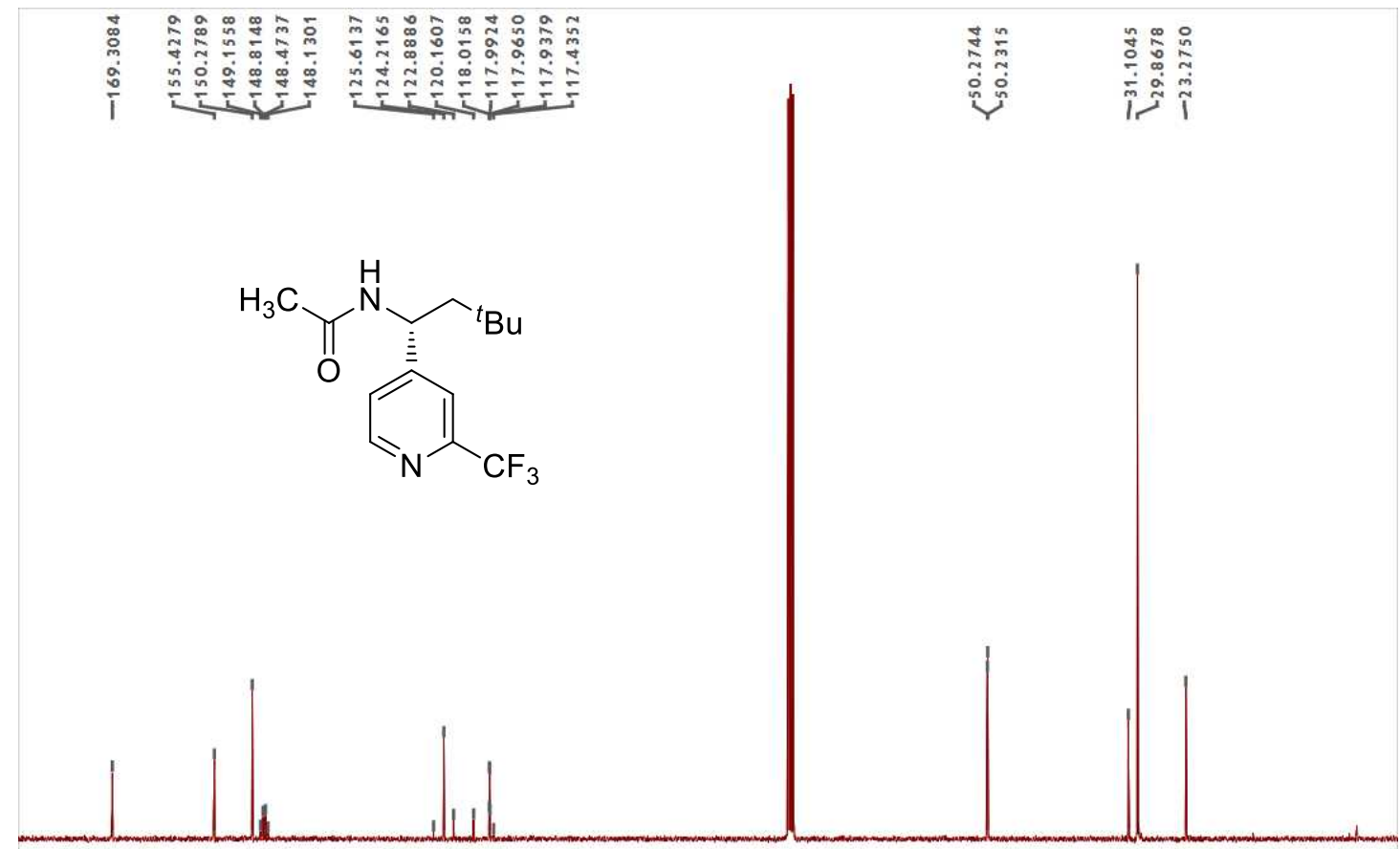

60; ${ }^{1} \mathrm{H}-\mathrm{NMR}\left(400 \mathrm{MHz}, \mathrm{CDCl}_{3}\right)$

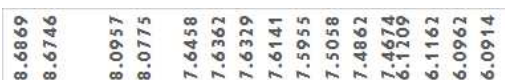

نู

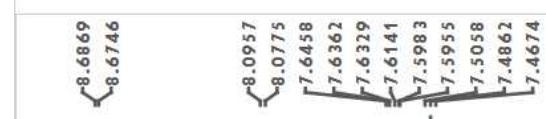

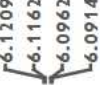

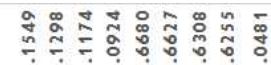

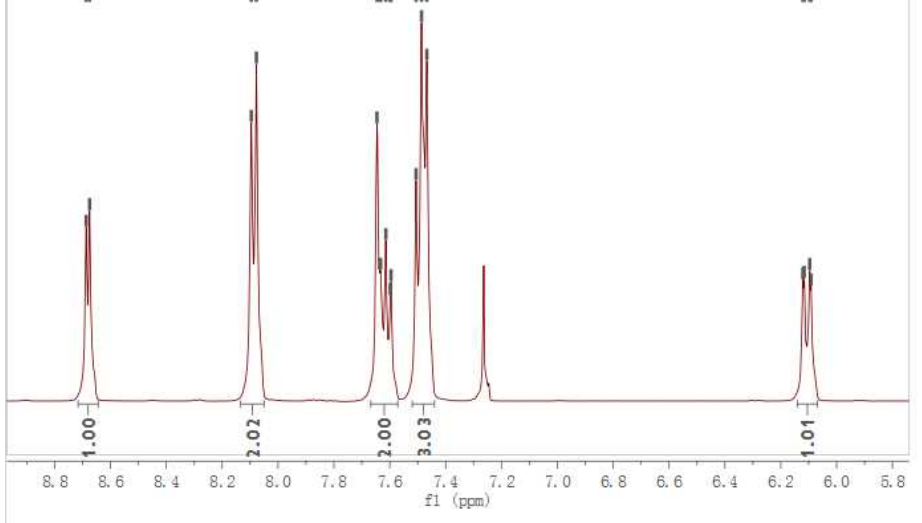

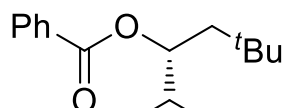

0<smiles>FC(F)(F)c1ccccn1</smiles>

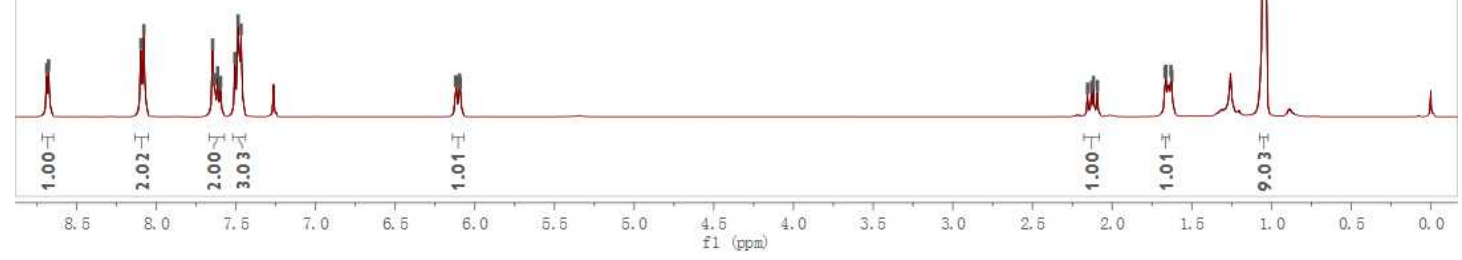


60; ${ }^{19}$ F-NMR (375 MHz, $\left.\mathrm{CDCl}_{3}\right)$

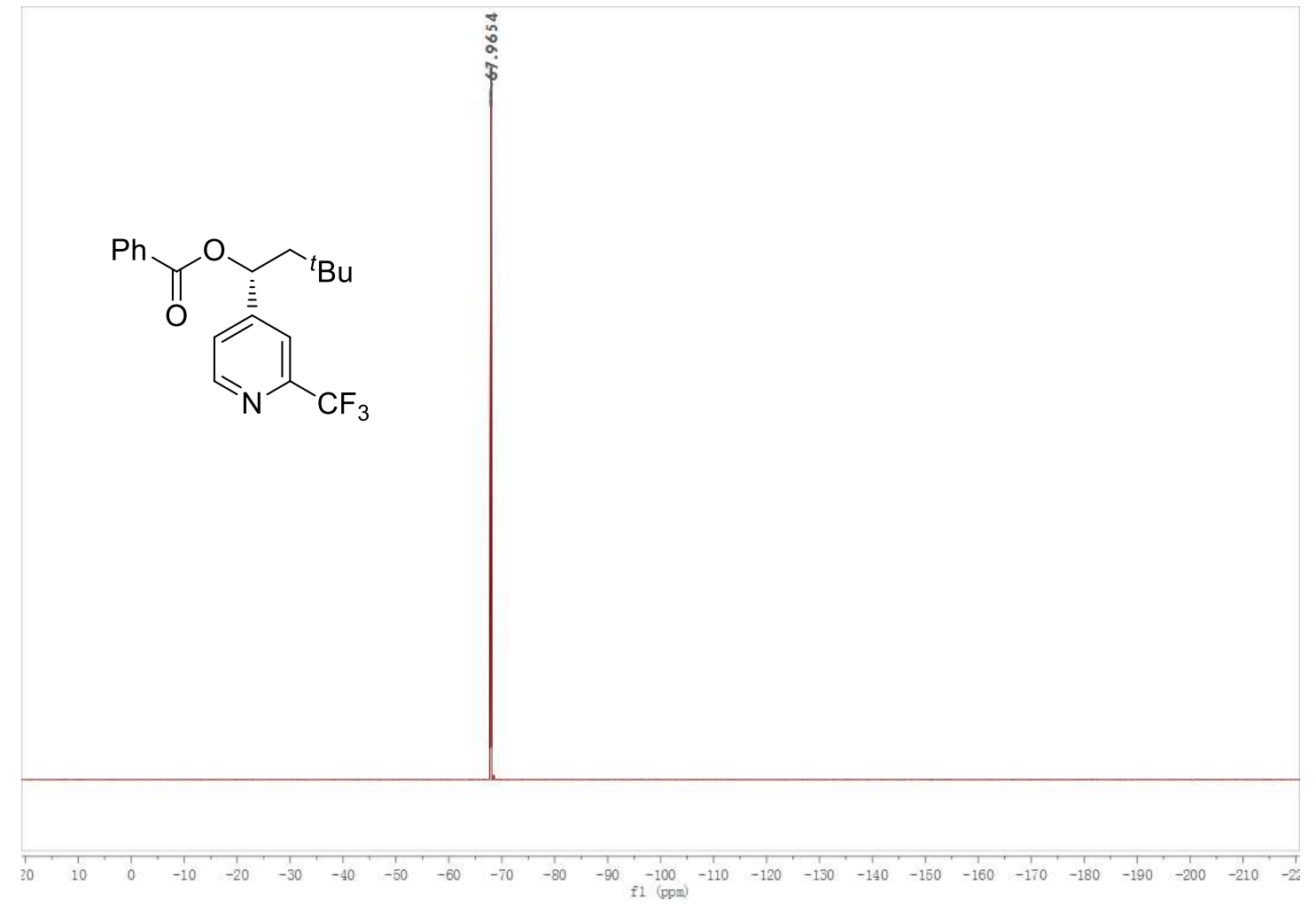

60; ${ }^{13} \mathrm{C}-\mathrm{NMR}$ (100 MHz, $\left.\mathrm{CDCl}_{3}\right)$
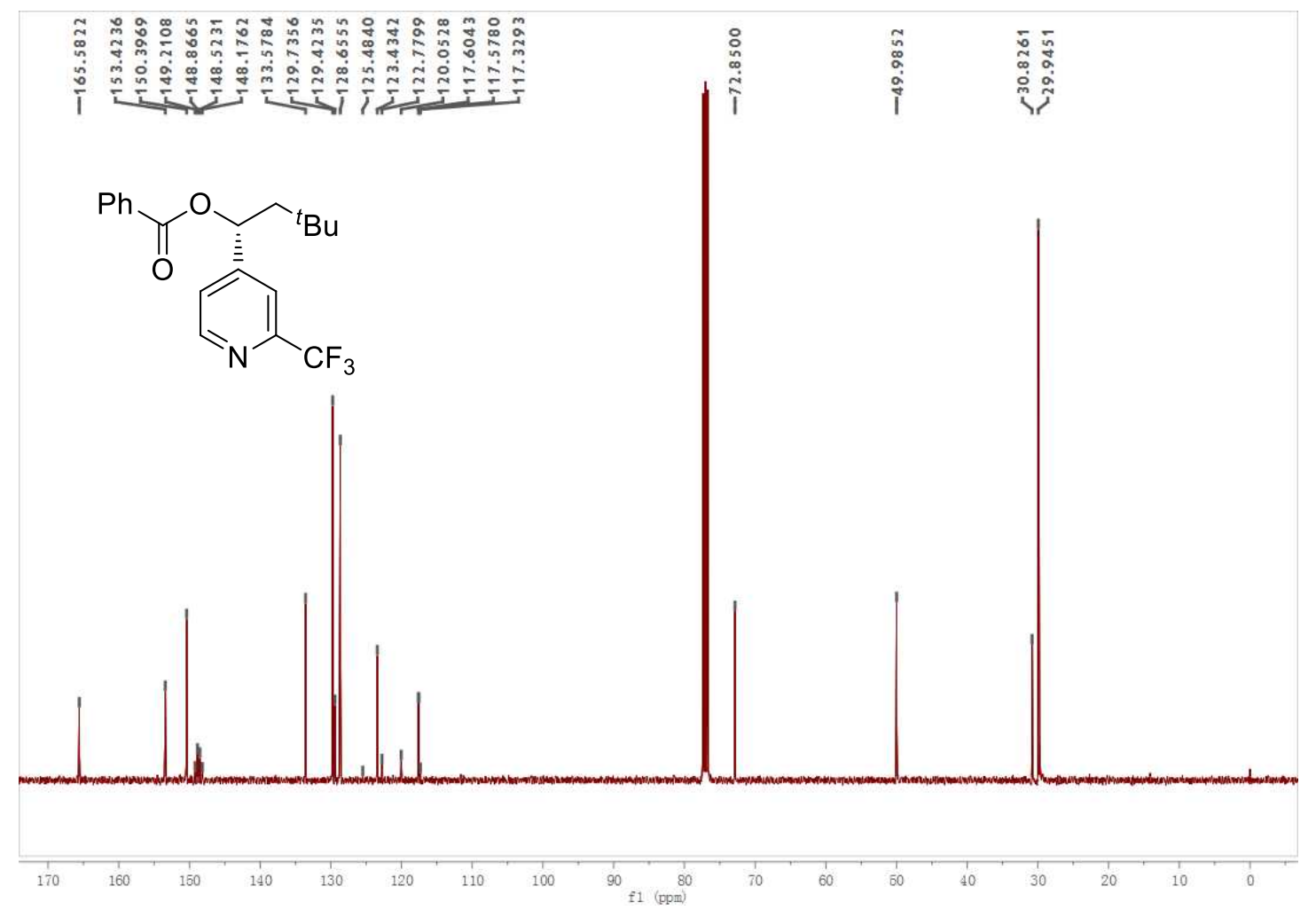
61; ${ }^{1} \mathrm{H}-\mathrm{NMR}\left(400 \mathrm{MHz}, \mathrm{CDCl}_{3}\right)$

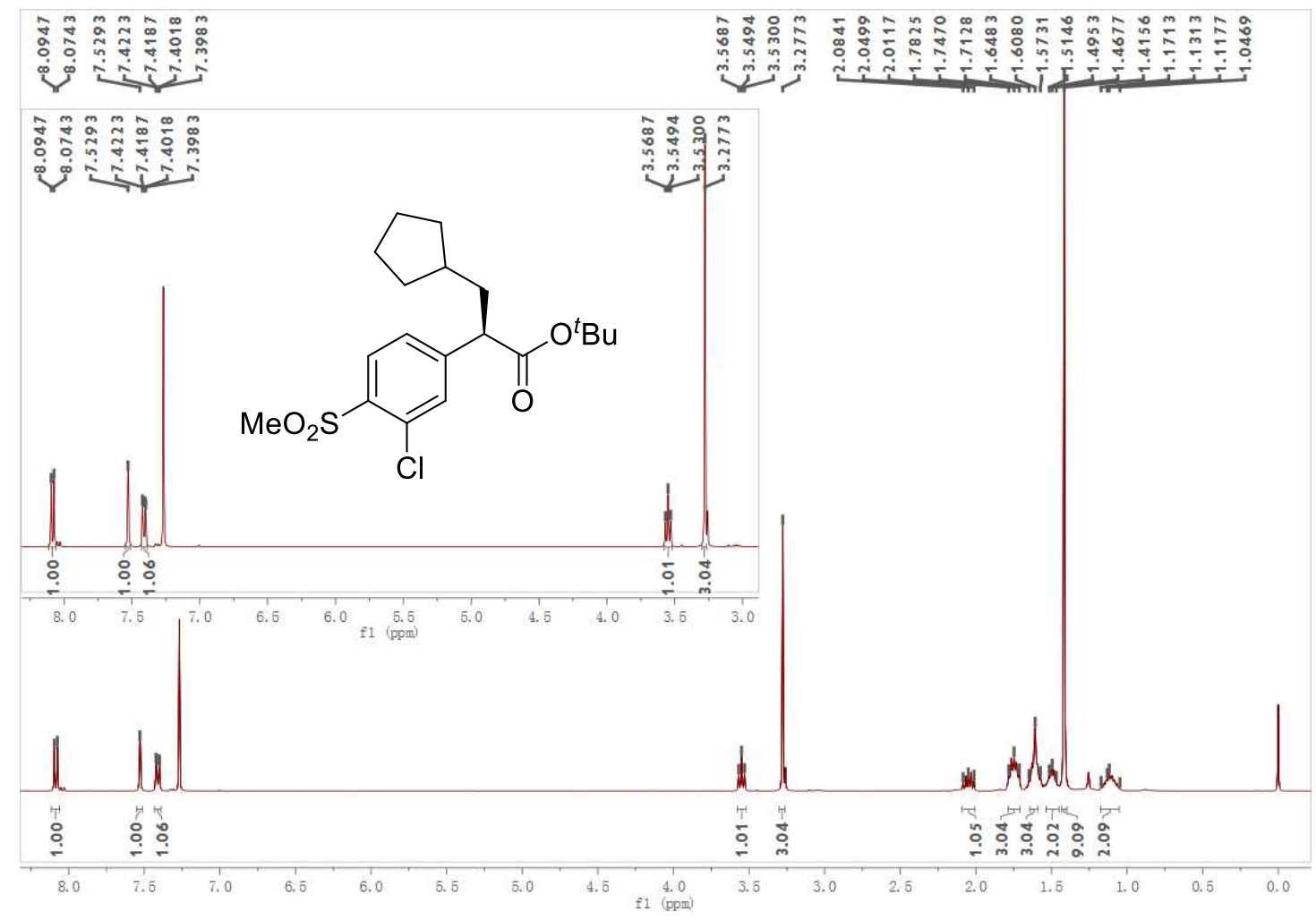

61; ${ }^{13} \mathrm{C}-\mathrm{NMR}\left(100 \mathrm{MHz}, \mathrm{CDCl}_{3}\right)$

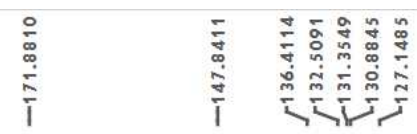

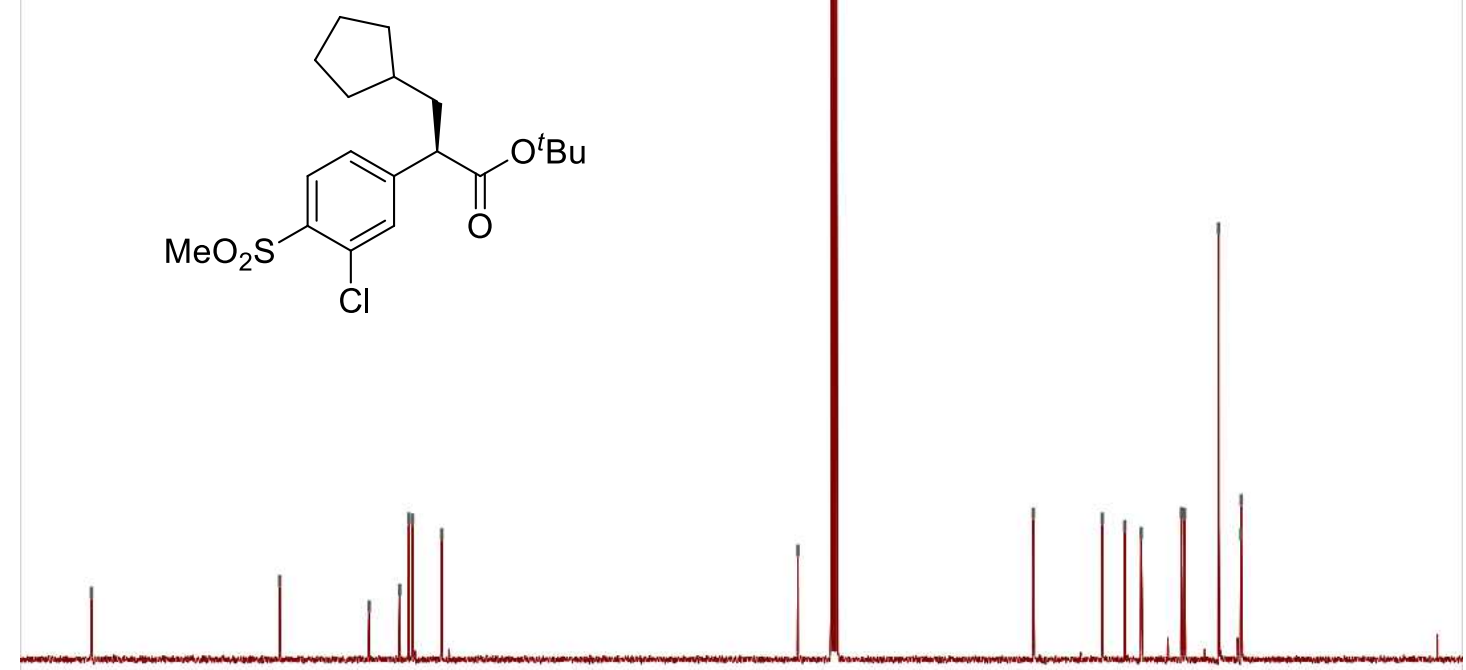

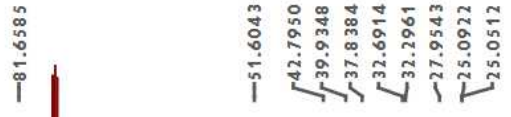

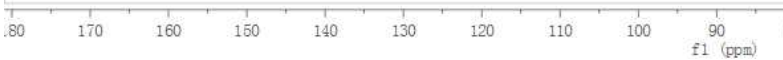


62; ${ }^{1} \mathrm{H}-\mathrm{NMR}$ (400 MHz, $\mathrm{CDCl}_{3}$ )

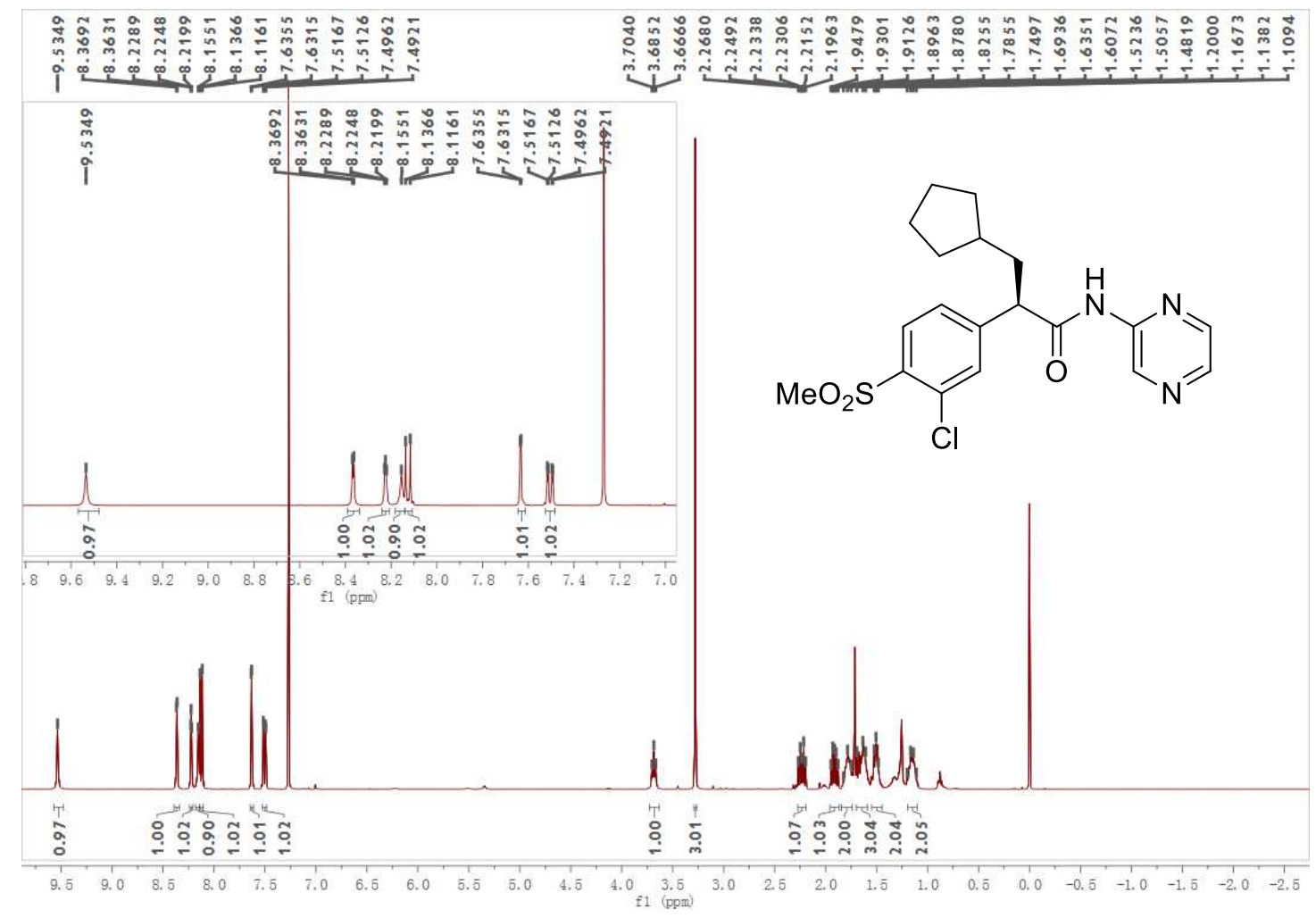

63; ${ }^{1} \mathrm{H}-\mathrm{NMR}$ (400 MHz, $\mathrm{CDCl}_{3}$ )

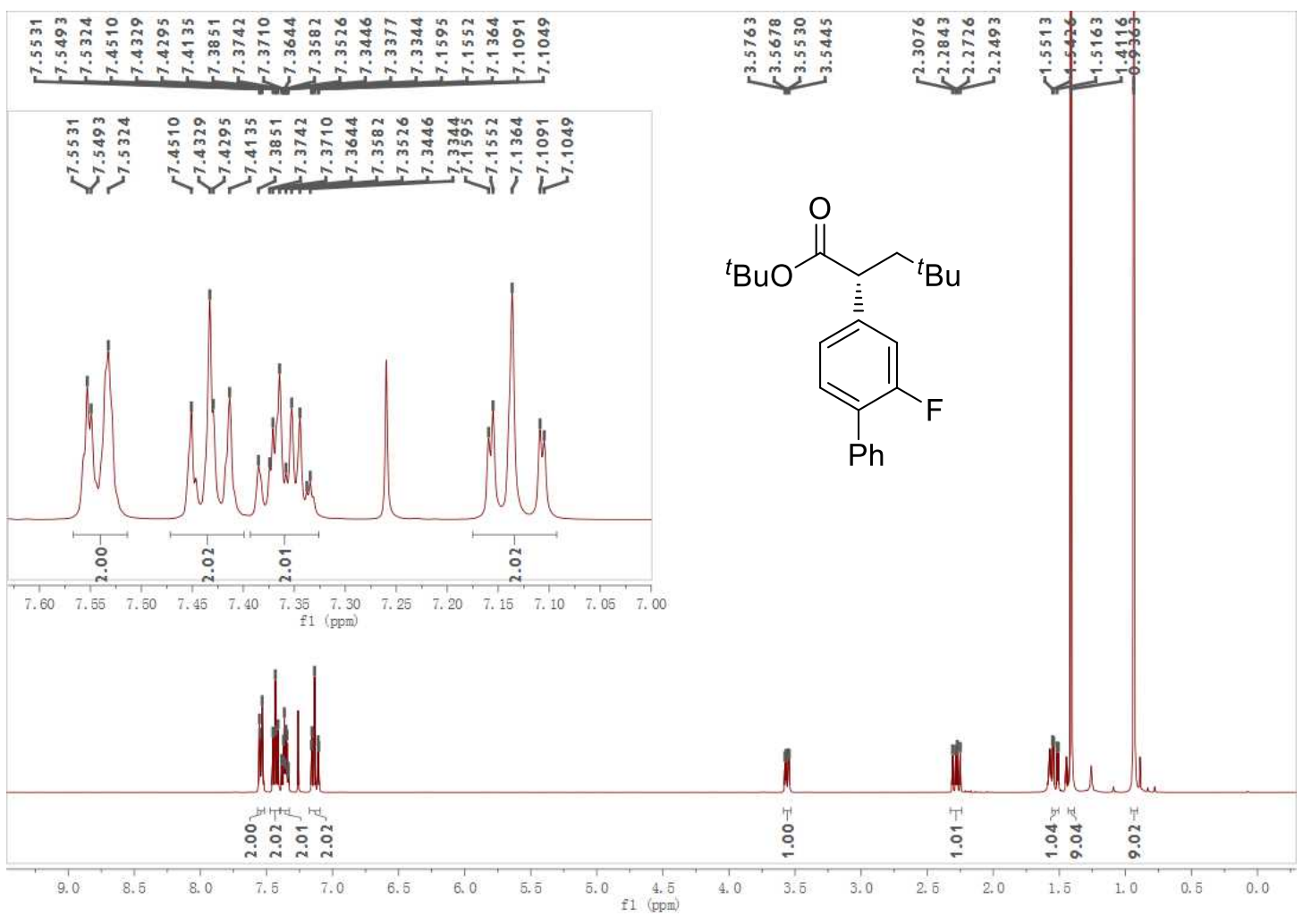




\section{3; ${ }^{19}$ F-NMR (375 MHz, $\left.\mathrm{CDCl}_{3}\right)$}

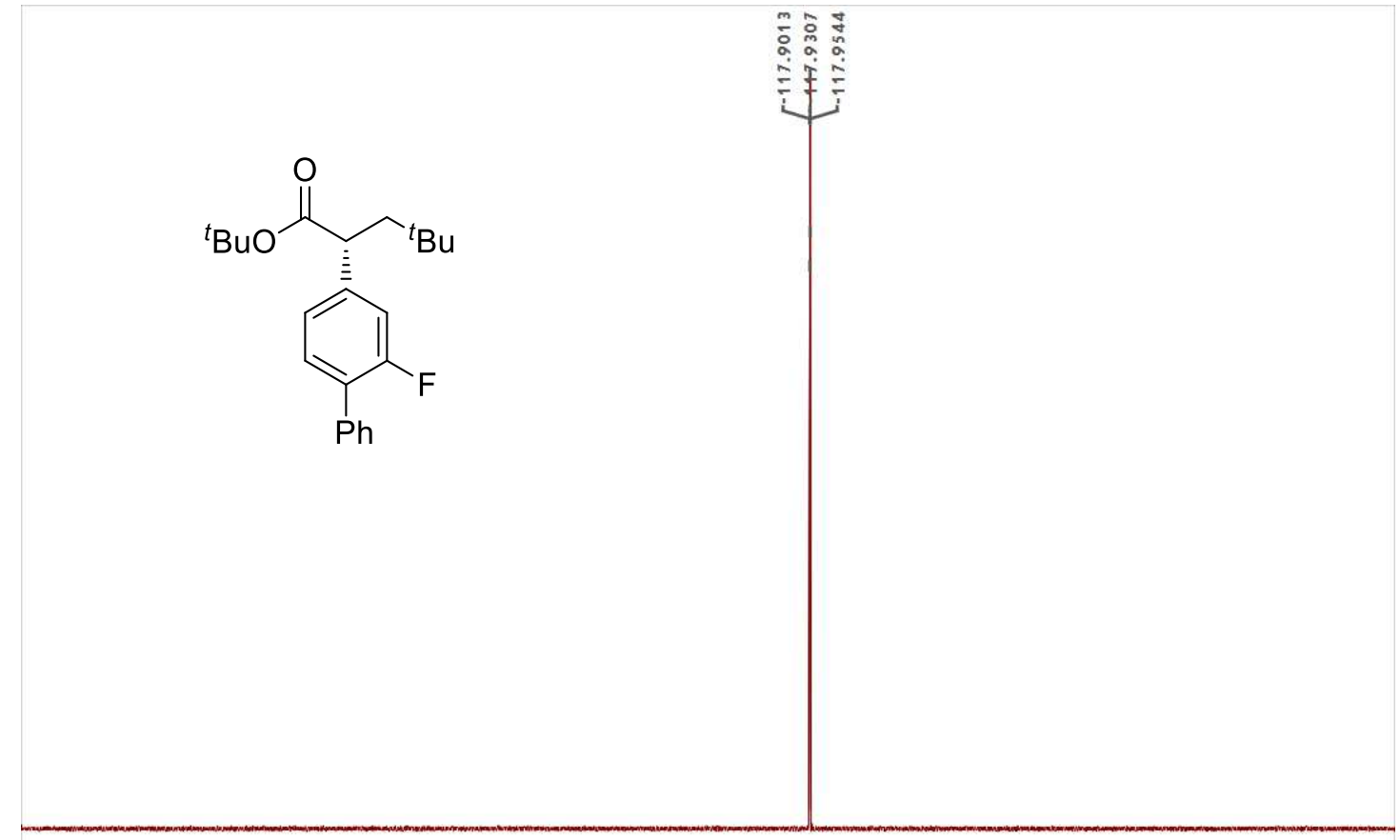

63; ${ }^{13} \mathrm{C}-\mathrm{NMR}\left(100 \mathrm{MHz}, \mathrm{CDCl}_{3}\right)$

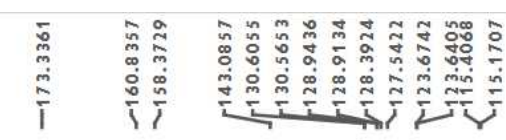

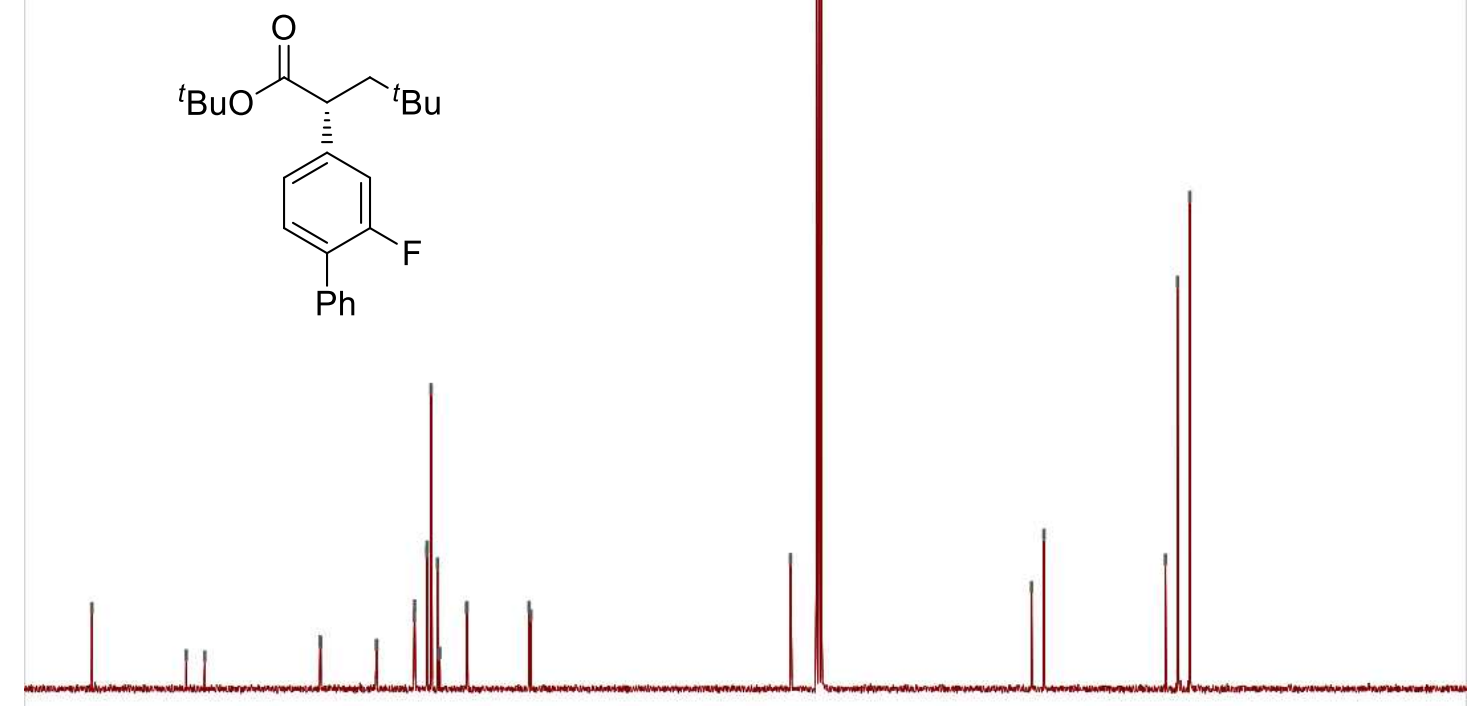

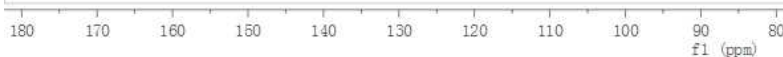


64; ${ }^{1} \mathrm{H}-\mathrm{NMR}$ (400 MHz, $\mathrm{CDCl}_{3}$ )

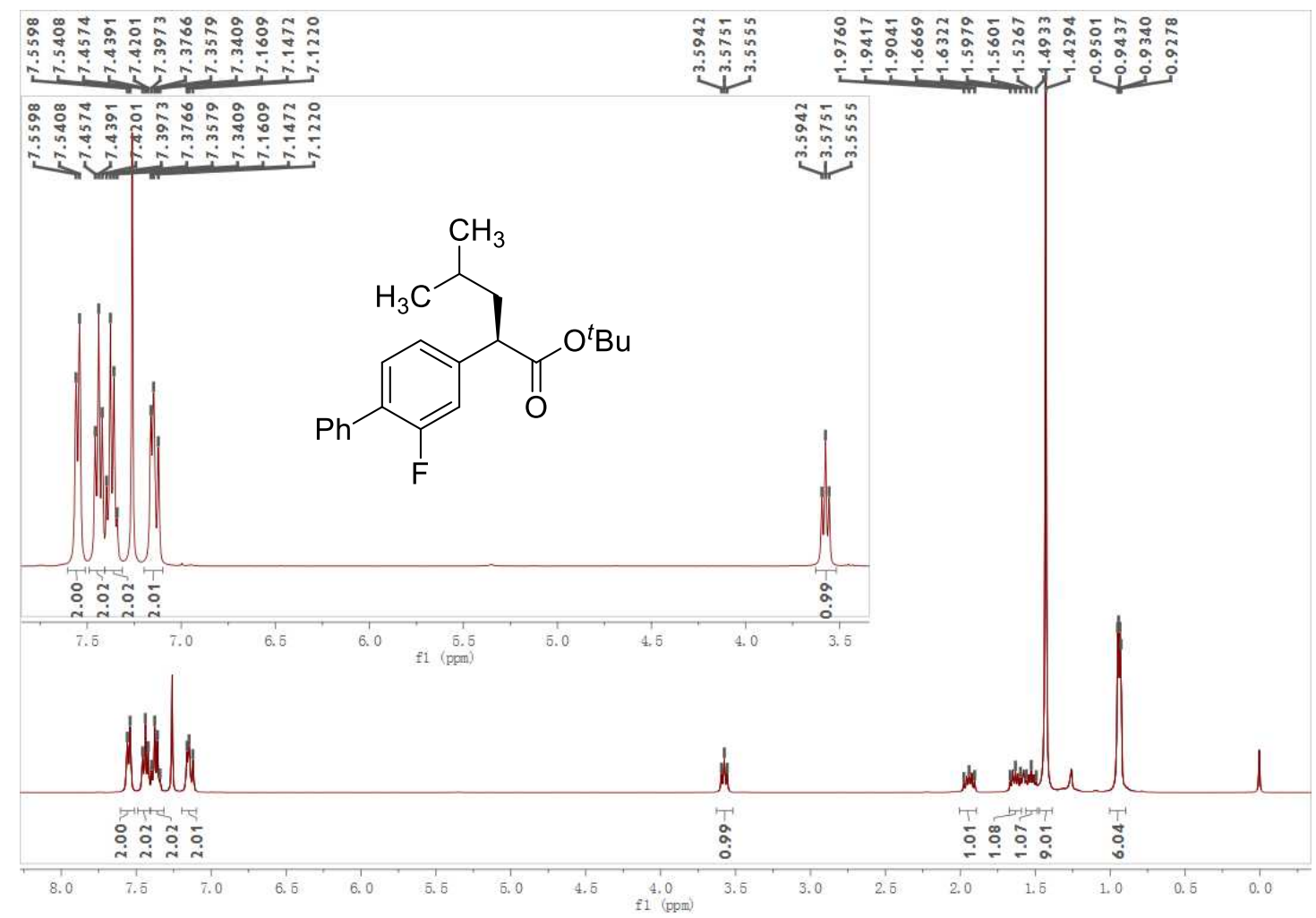

64; ${ }^{19}$ F-NMR (375 MHz, $\left.\mathrm{CDCl}_{3}\right)$

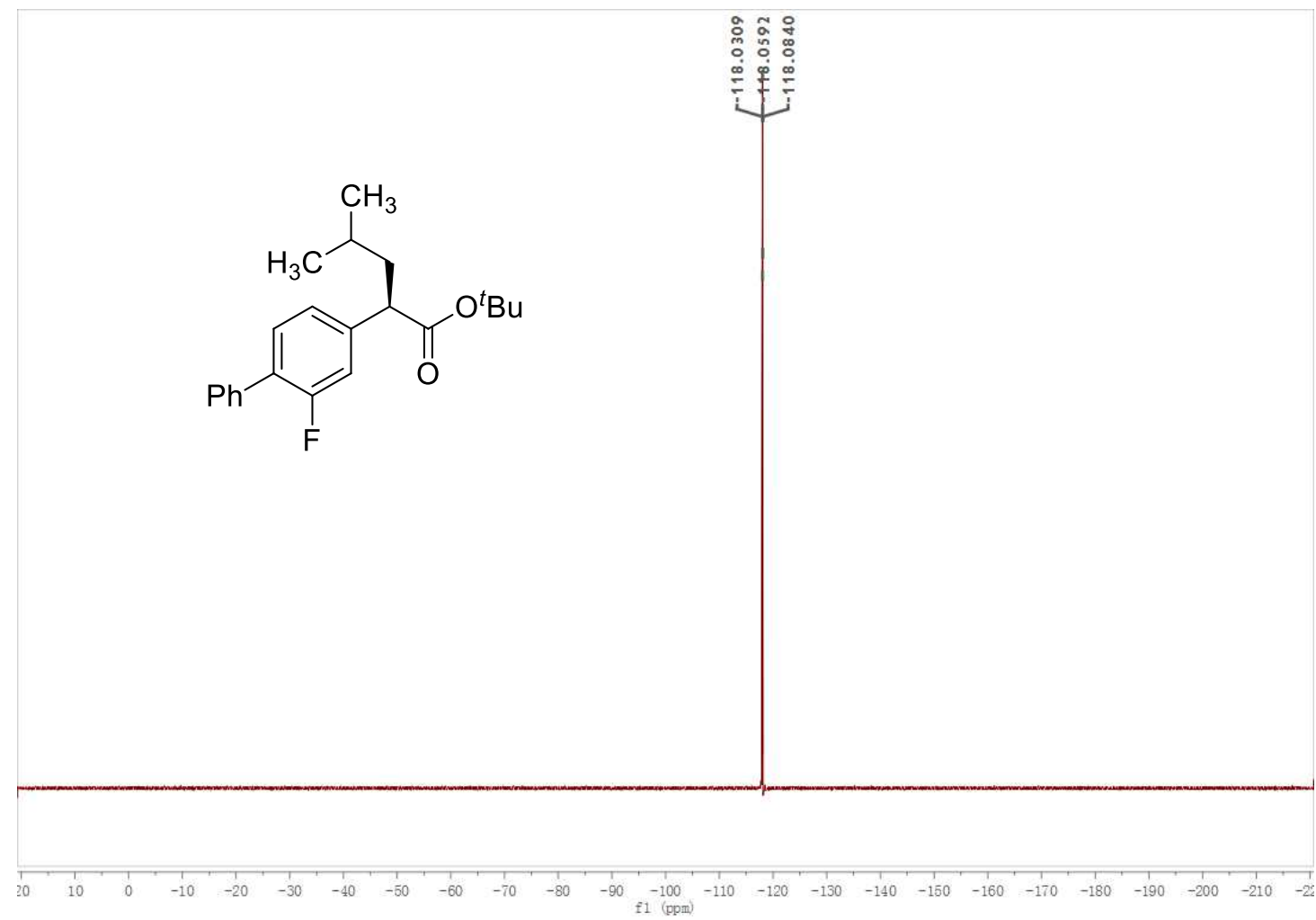


64; ${ }^{13} \mathrm{C}-\mathrm{NMR}\left(100 \mathrm{MHz}, \mathrm{CDCl}_{3}\right)$<smiles>CCCCOC(=O)C(CC(C)C)c1ccc(-c2ccccc2)c(F)c1</smiles>

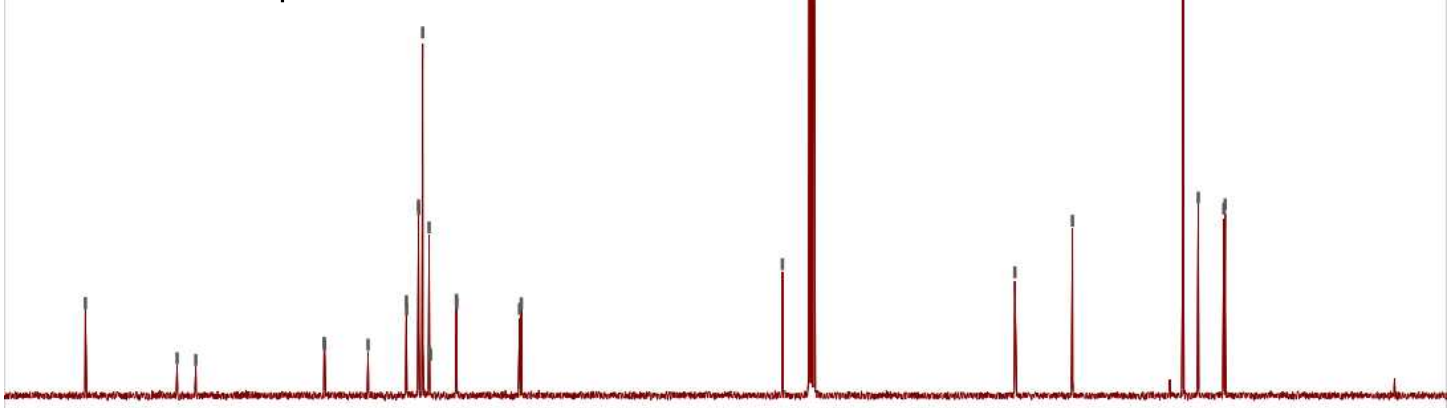

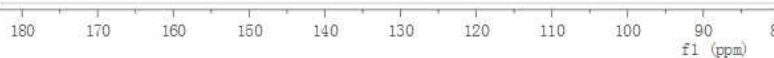

65; ${ }^{1} \mathrm{H}-\mathrm{NMR}\left(400 \mathrm{MHz}, \mathrm{CDCl}_{3}\right)$

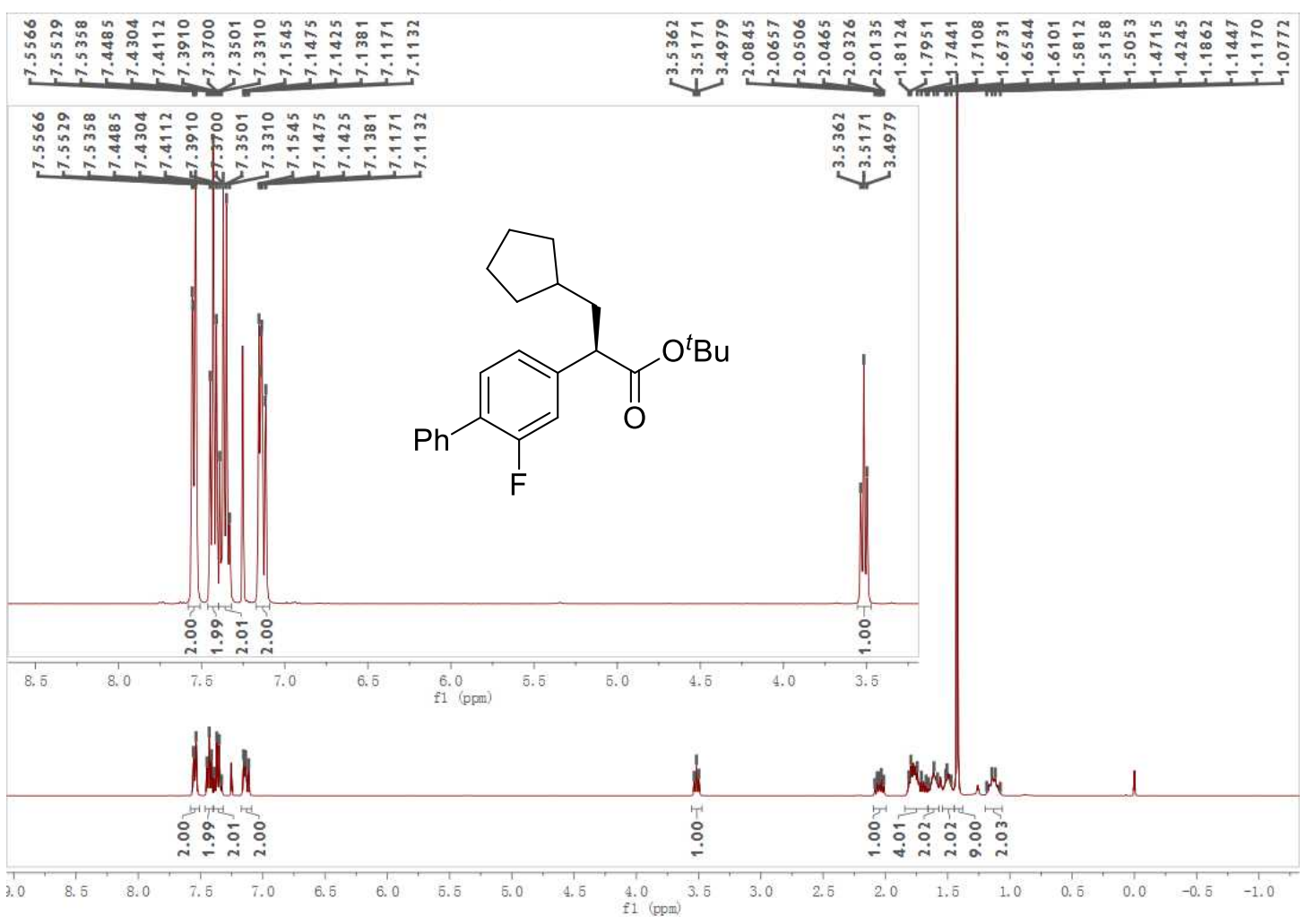


65; ${ }^{19}$ F-NMR (375 $\left.\mathrm{MHz}, \mathrm{CDCl}_{3}\right)$
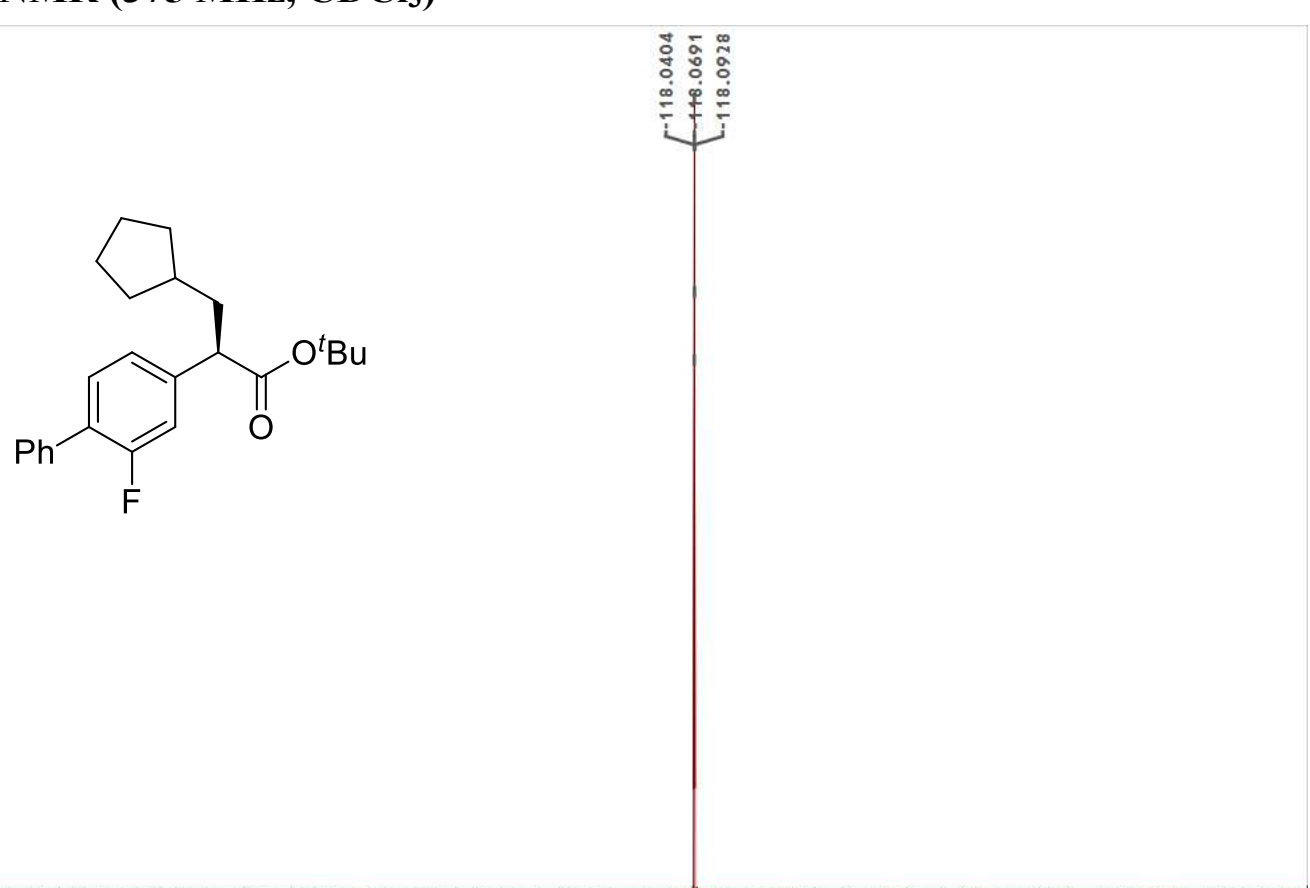

65; ${ }^{13} \mathrm{C}-\mathrm{NMR}\left(100 \mathrm{MHz}, \mathrm{CDCl}_{3}\right)$

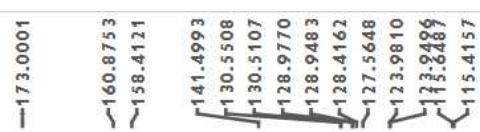

(c)

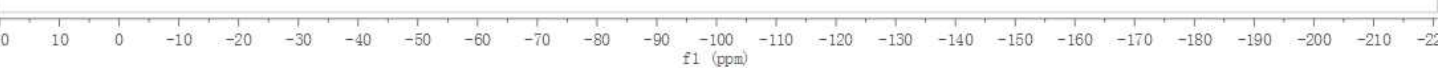

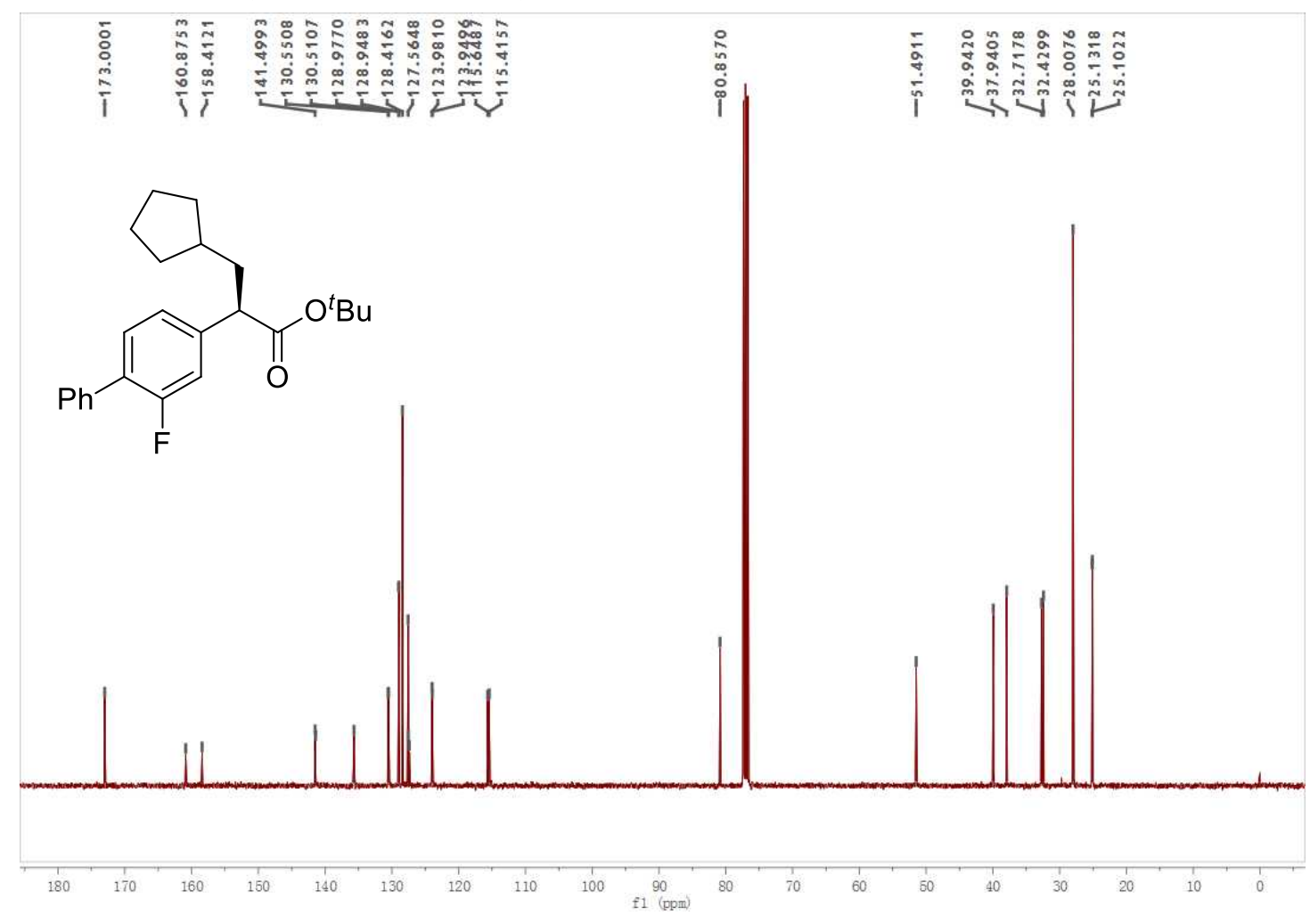


66; ${ }^{1} \mathrm{H}-\mathrm{NMR}$ (400 MHz, $\mathrm{CDCl}_{3}$ )

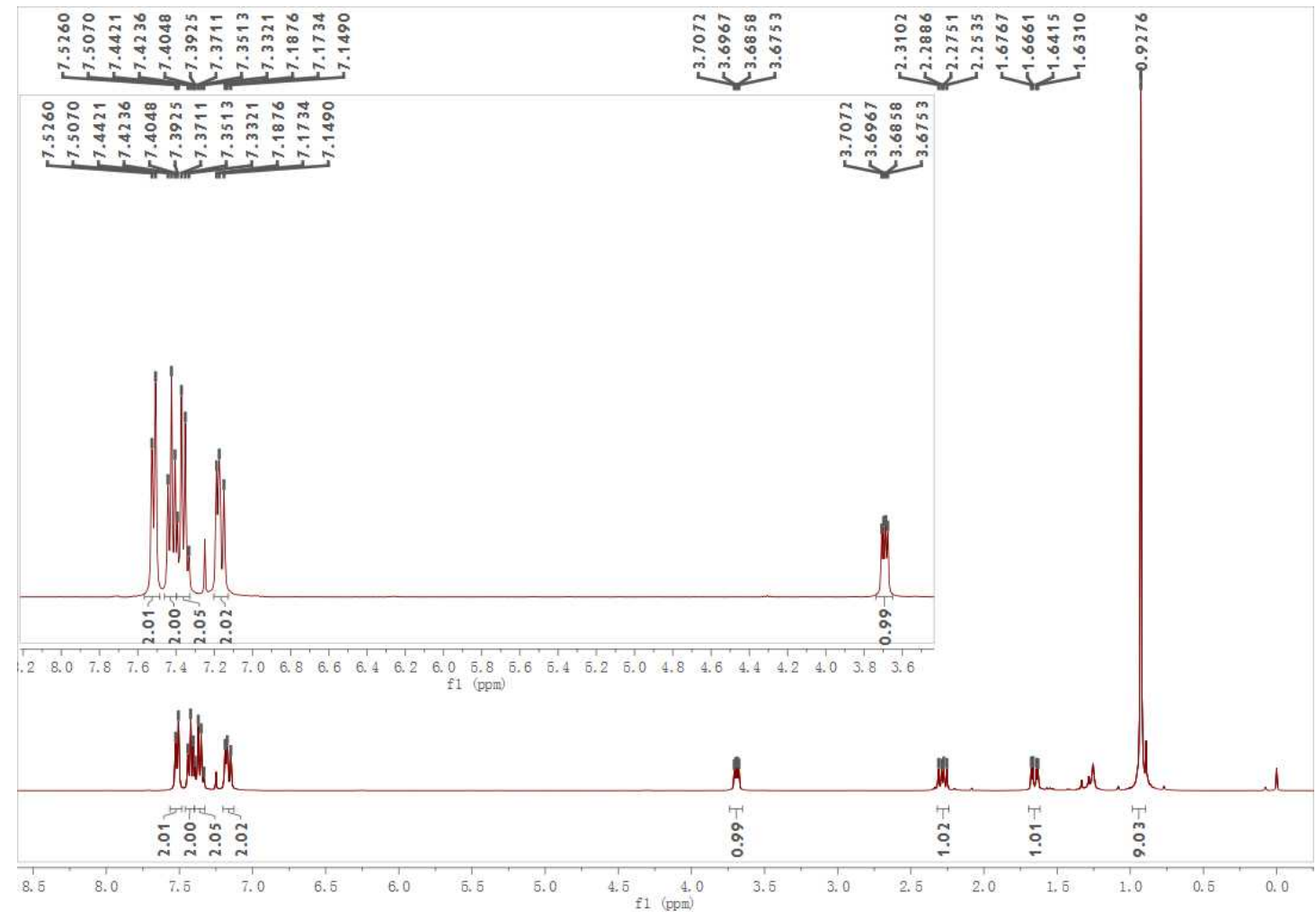

66; ${ }^{19}$ F-NMR (375 MHz, $\left.\mathrm{CDCl}_{3}\right)$

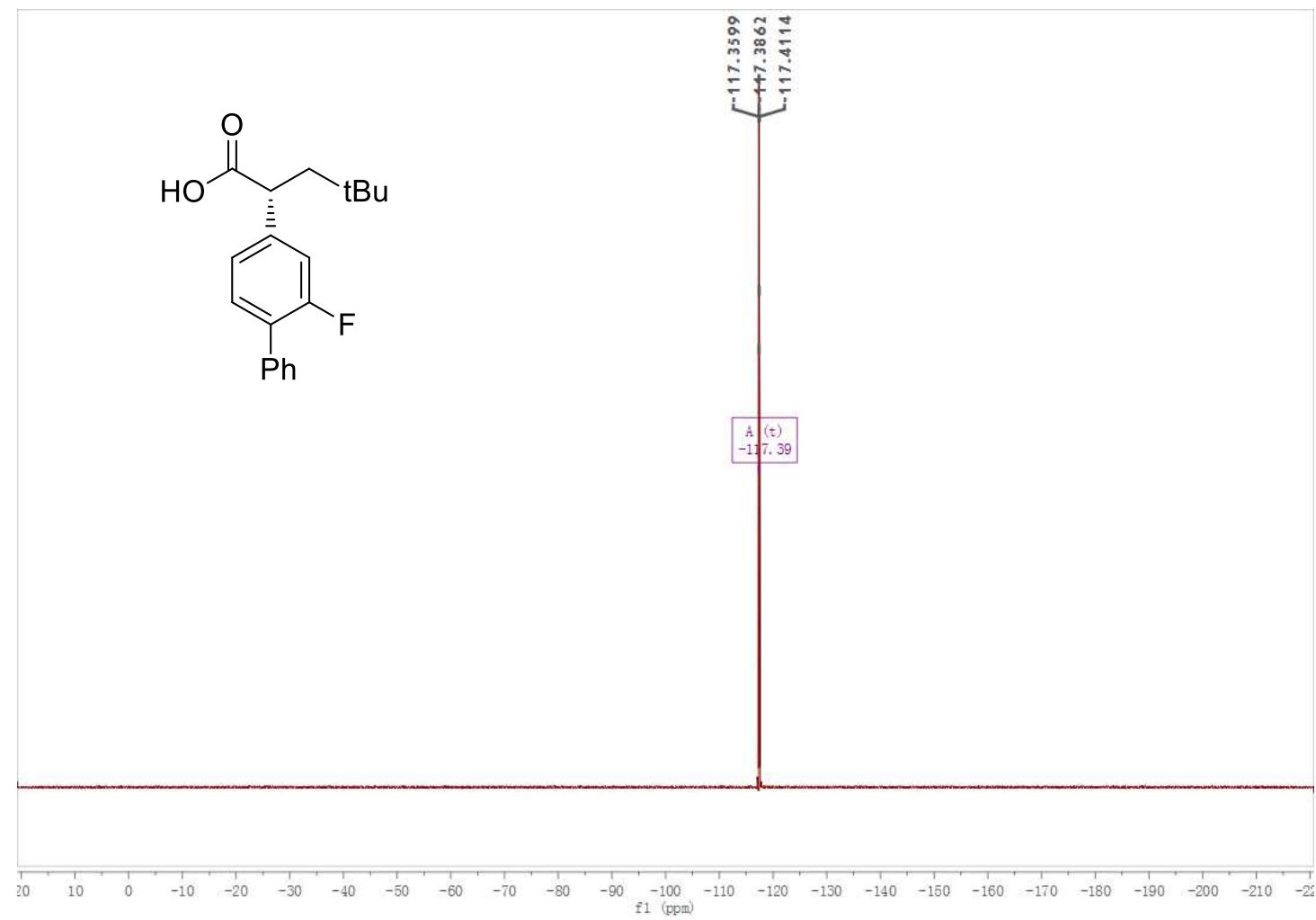


66; ${ }^{13} \mathrm{C}-\mathrm{NMR}$ (100 MHz, $\left.\mathrm{CDCl}_{3}\right)$

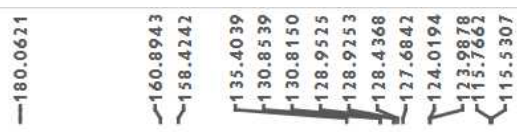

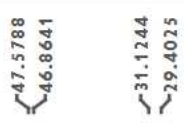<smiles>CC(C)CC(C(=O)O)c1ccc(-c2ccccc2)c(F)c1</smiles>

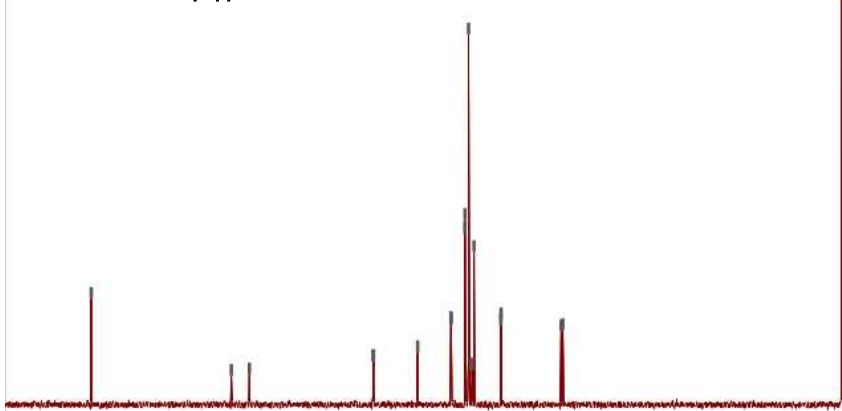

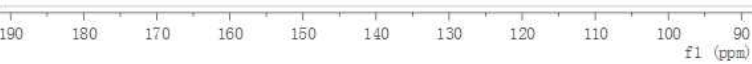

\section{7; ${ }^{1} \mathrm{H}-\mathrm{NMR}$ (400 MHz, $\mathrm{CDCl}_{3}$ )}

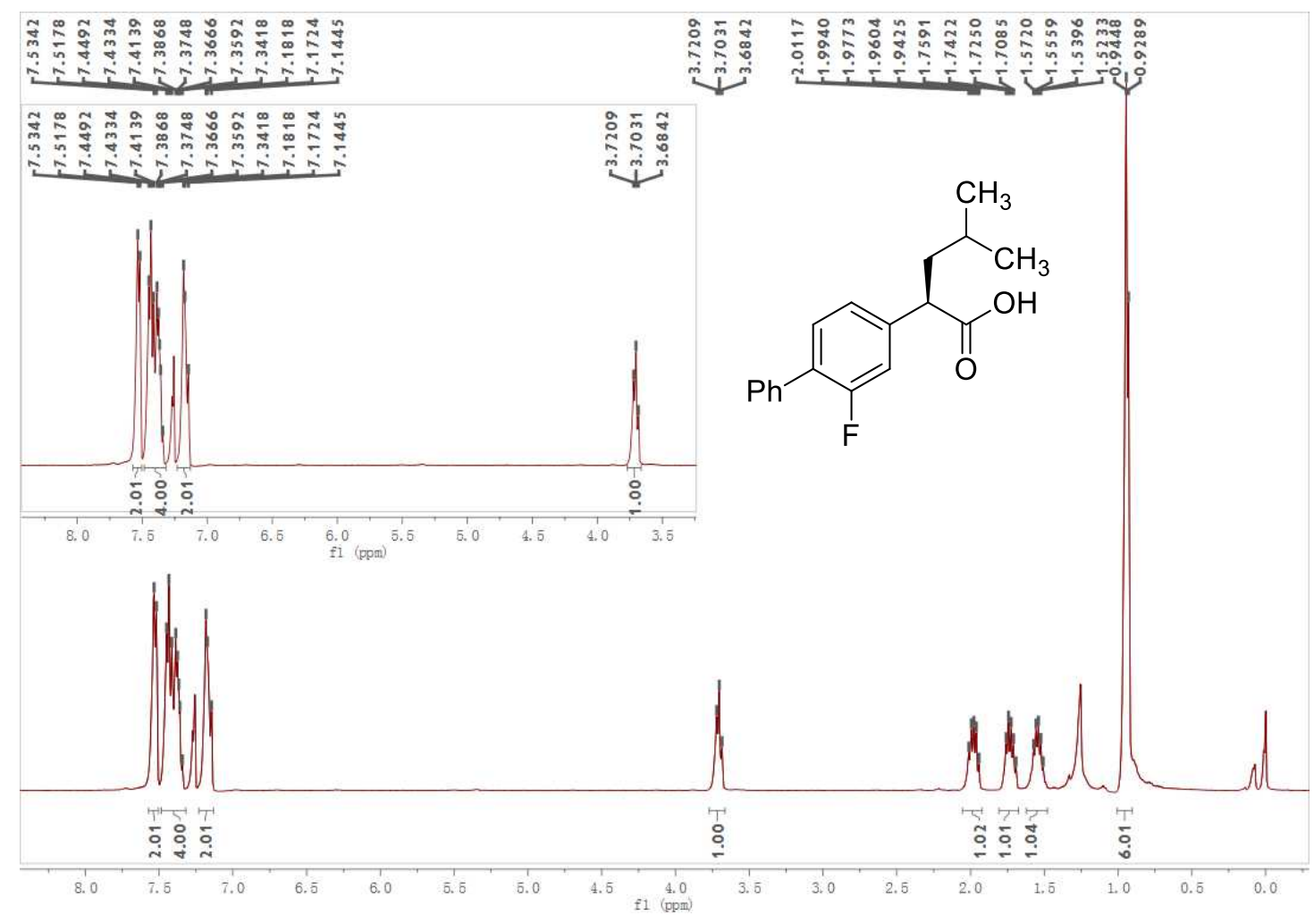


<smiles>CC(C)CC(C(=O)O)c1ccc(-c2ccccc2)c(F)c1</smiles> 
68; ${ }^{1} \mathrm{H}-\mathrm{NMR}\left(400 \mathrm{MHz}, \mathrm{CDCl}_{3}\right)$

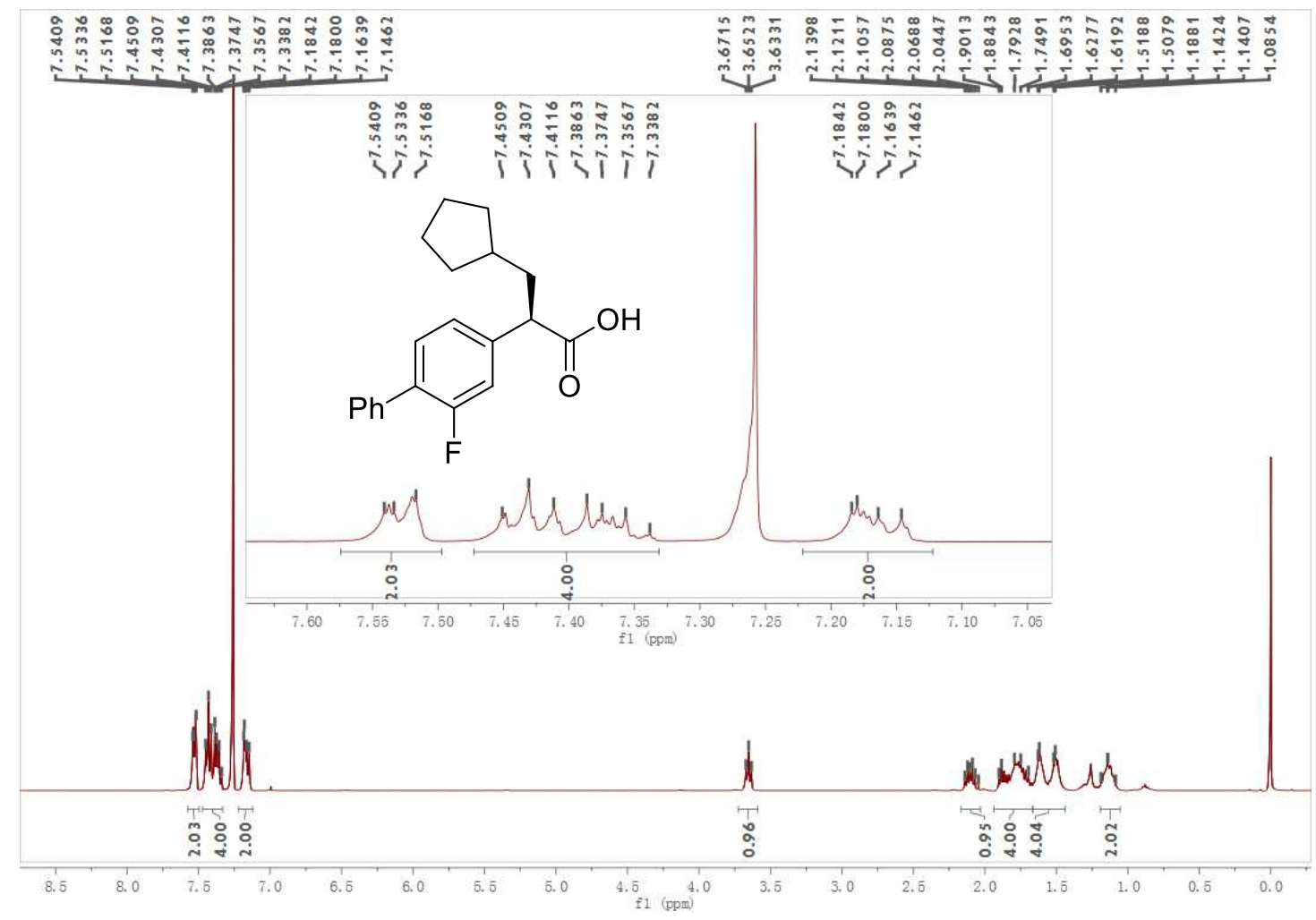

$68 ;{ }^{19}$ F-NMR (375 MHz, $\left.\mathrm{CDCl}_{3}\right)$<smiles>O=C(O)C(CC1CCCC1)c1ccc(-c2ccccc2)c(F)c1</smiles>

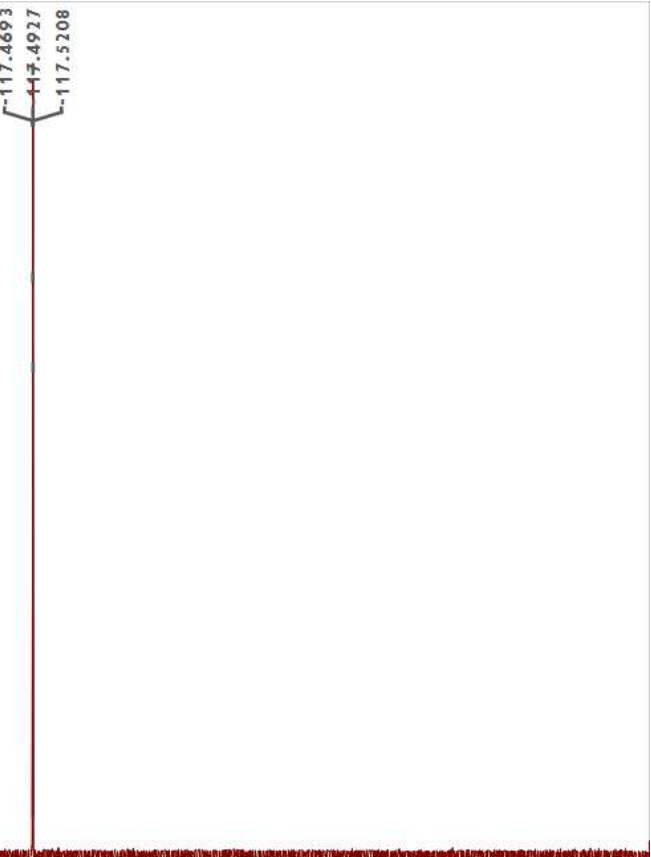

\begin{tabular}{llllllllllllllllllllllllll}
\hline 0 & 10 & 0 & -10 & -20 & -30 & -40 & -50 & -60 & -70 & -80 & -90 & -100 & -110 & -120 & -130 & -140 & -150 & -160 & -170 & -180 & -190 & -200 & -210 & -22 \\
\hline
\end{tabular} 
68; ${ }^{13} \mathrm{C}-\mathrm{NMR}\left(100 \mathrm{MHz}, \mathrm{CDCl}_{3}\right)$

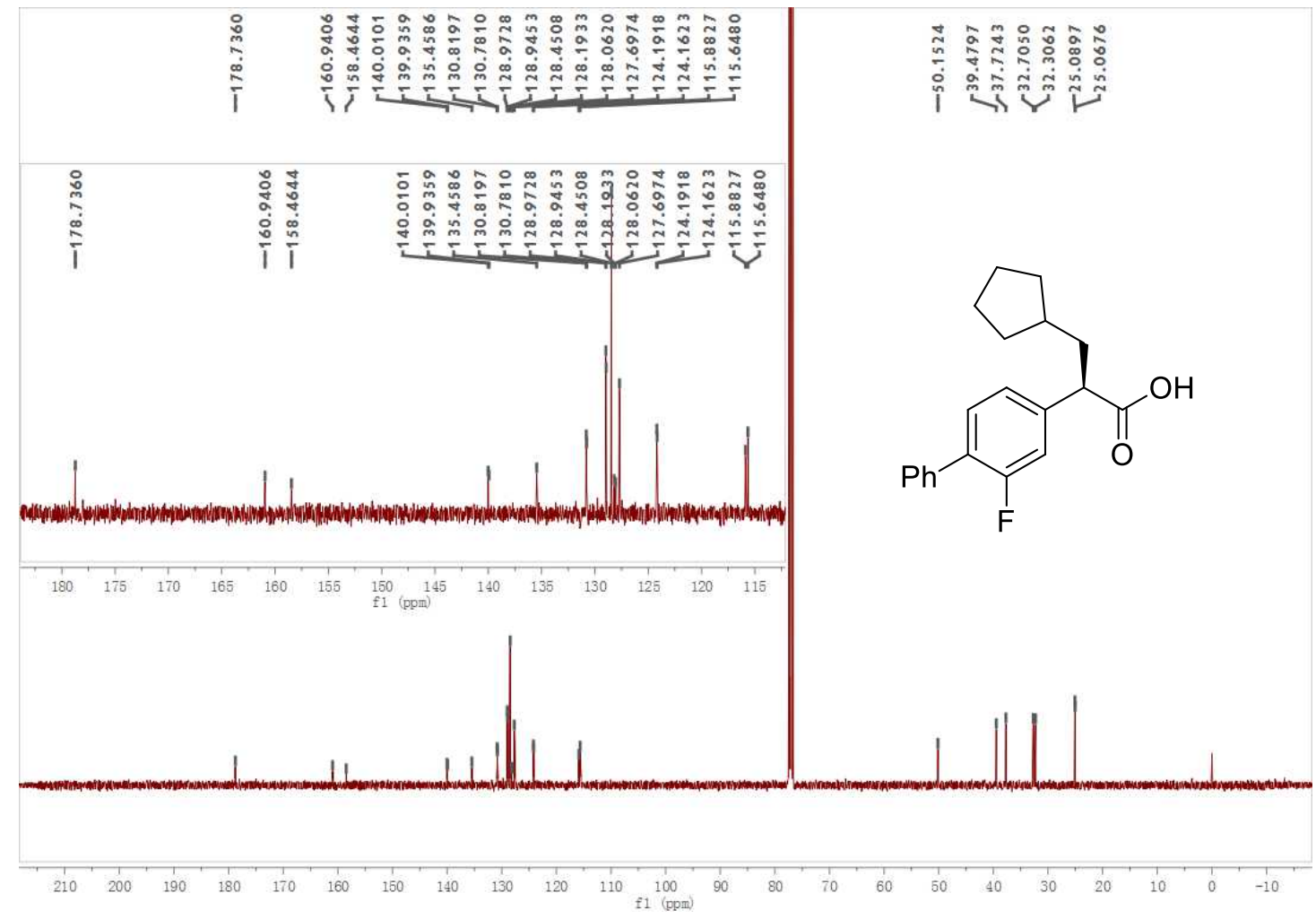

69; ${ }^{1} \mathrm{H}-\mathrm{NMR}$ (400 MHz, $\mathrm{CDCl}_{3}$ )

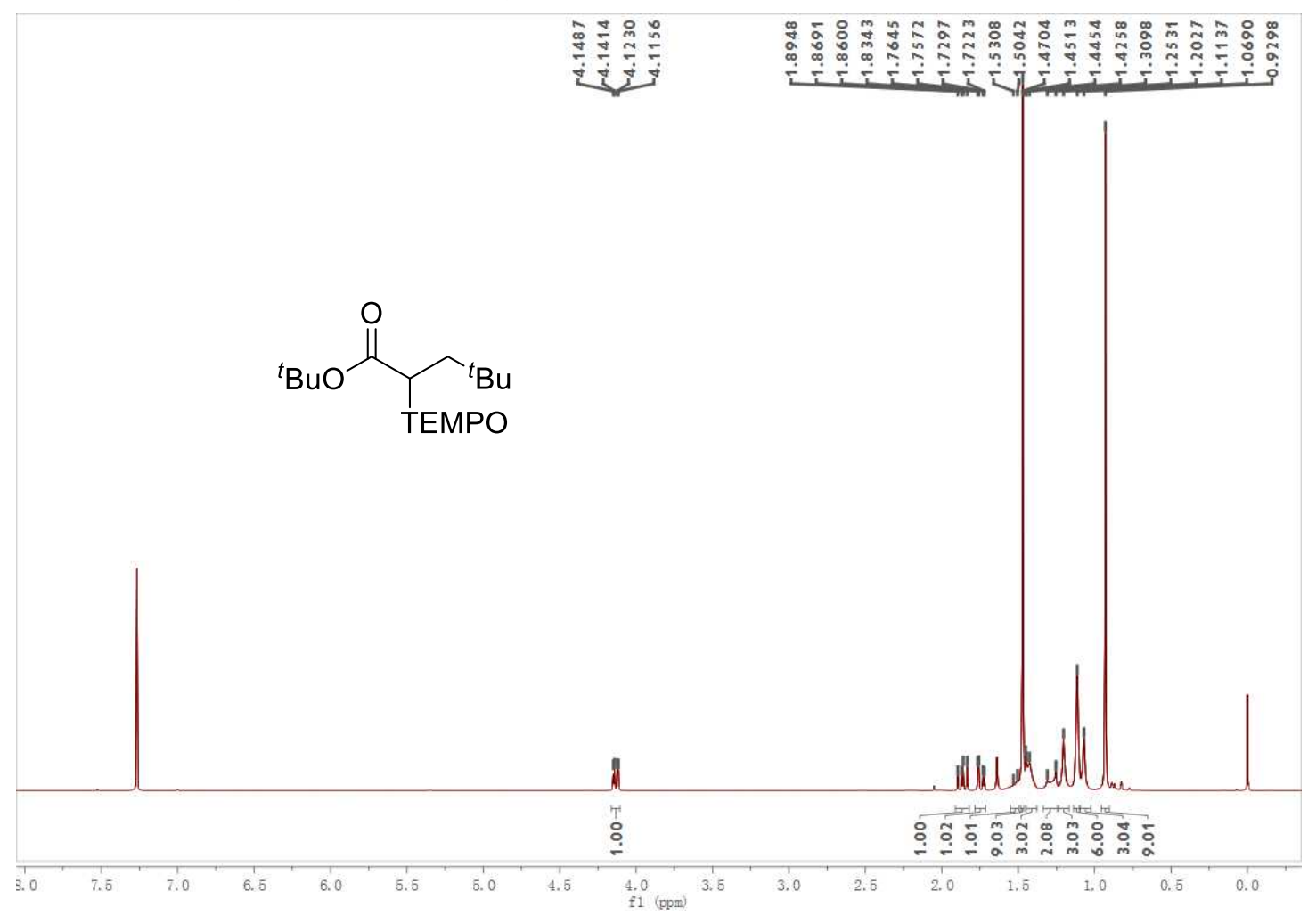


69; ${ }^{13} \mathrm{C}-\mathrm{NMR}\left(100 \mathrm{MHz}, \mathrm{CDCl}_{3}\right)$

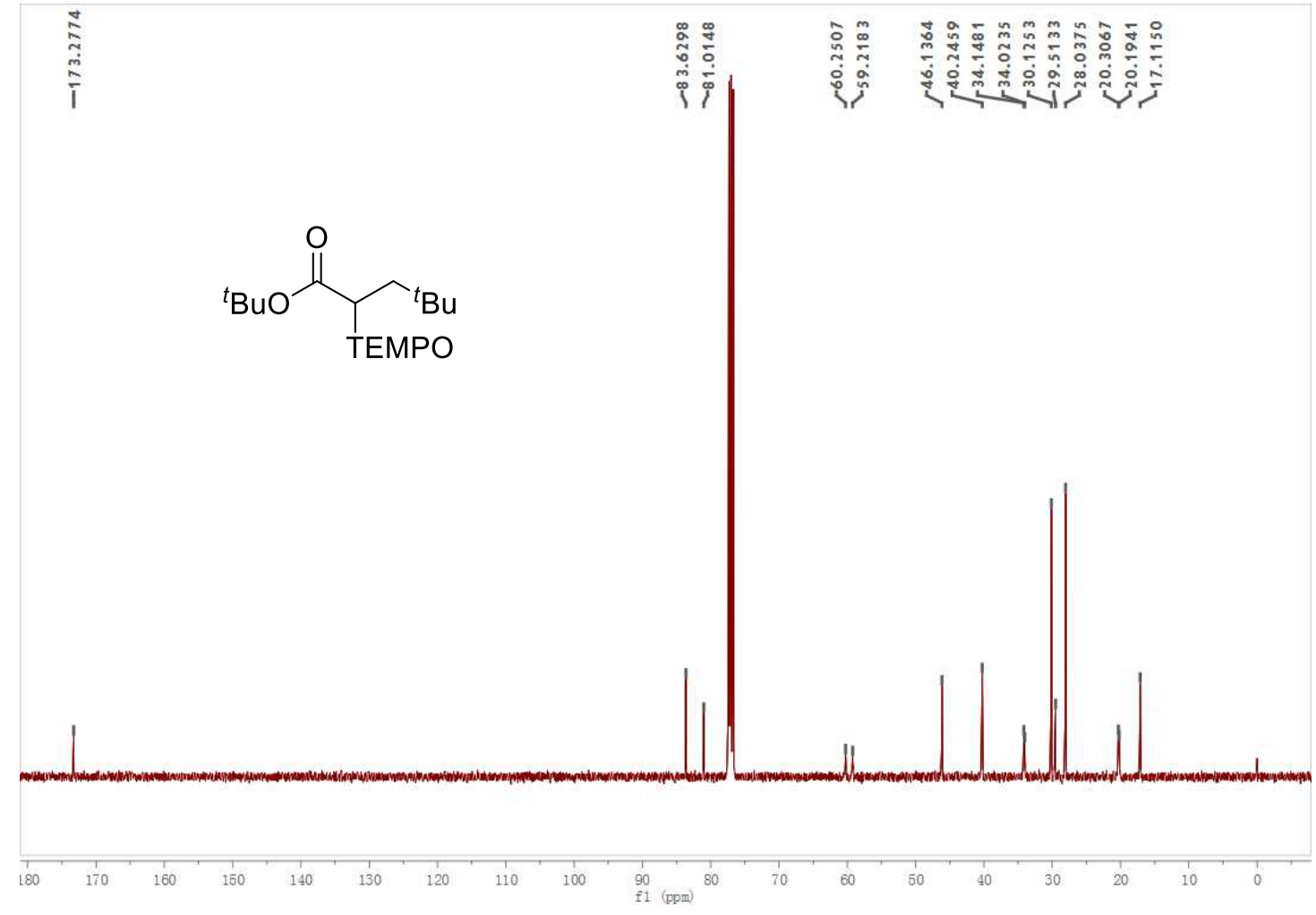

71; ${ }^{1} \mathrm{H}-\mathrm{NMR}\left(400 \mathrm{MHz}, \mathrm{CDCl}_{3}\right)$

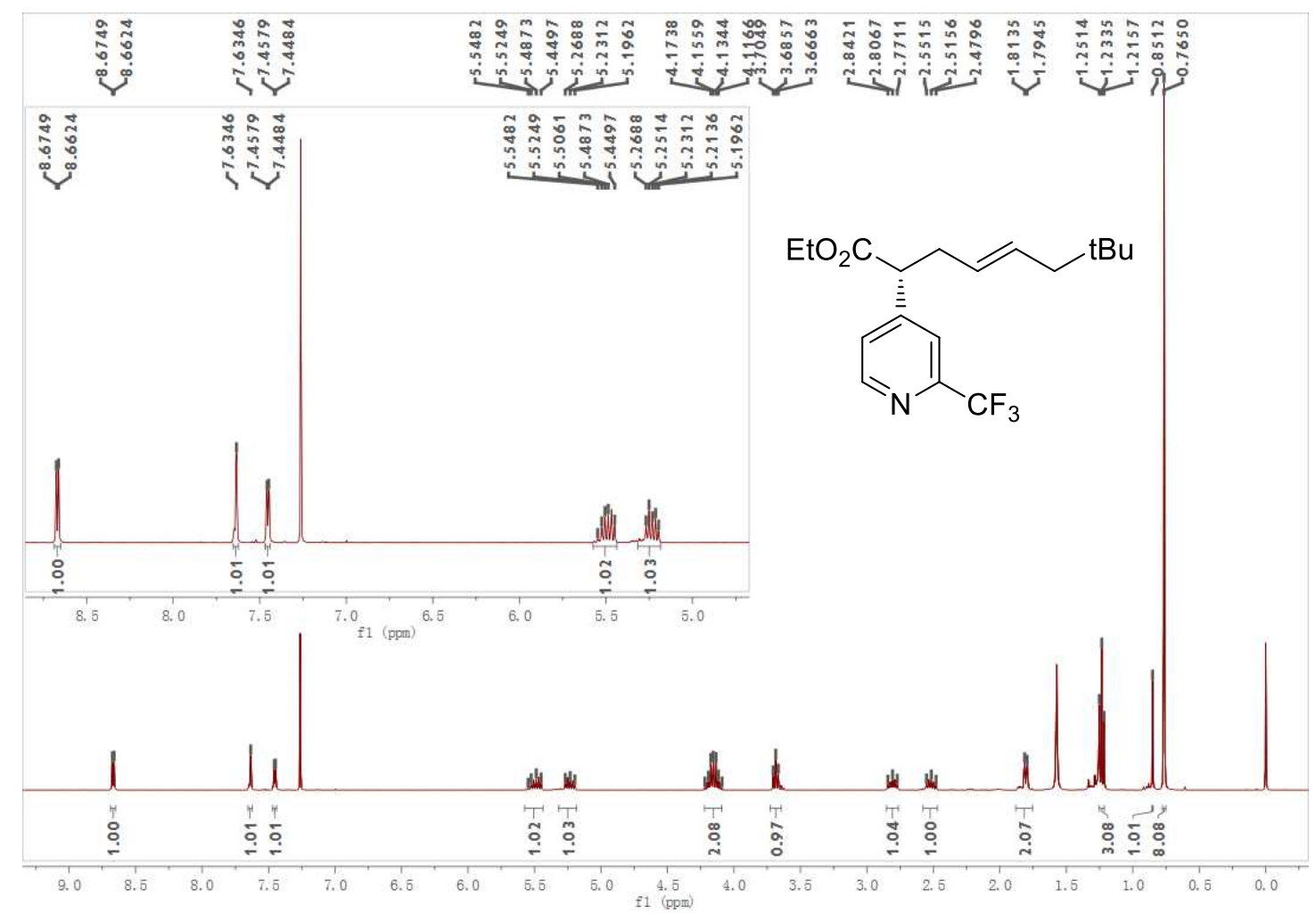




\section{1; ${ }^{19}$ F-NMR (375 MHz, $\left.\mathrm{CDCl}_{3}\right)$}

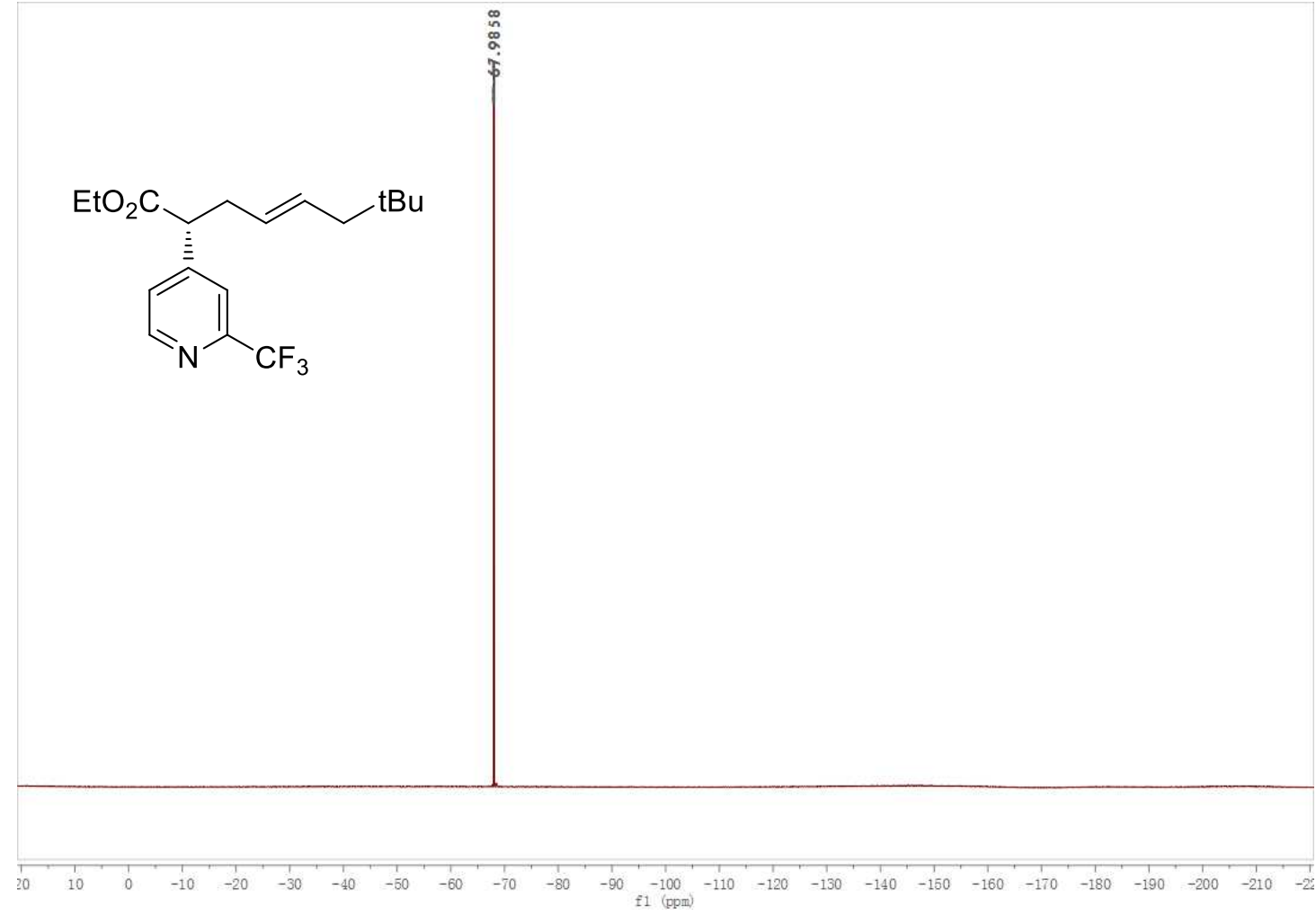

71; ${ }^{13} \mathrm{C}-\mathrm{NMR}$ (100 MHz, $\left.\mathrm{CDCl}_{3}\right)$

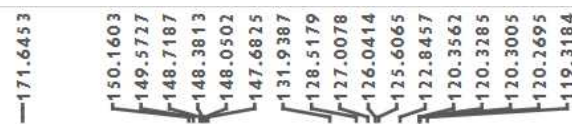

$\mathrm{EtO}_{2} \mathrm{C} \sim \mathrm{tBu}^{\mathrm{tBu}}$<smiles>Cc1ccnc(C(F)(F)F)c1</smiles>

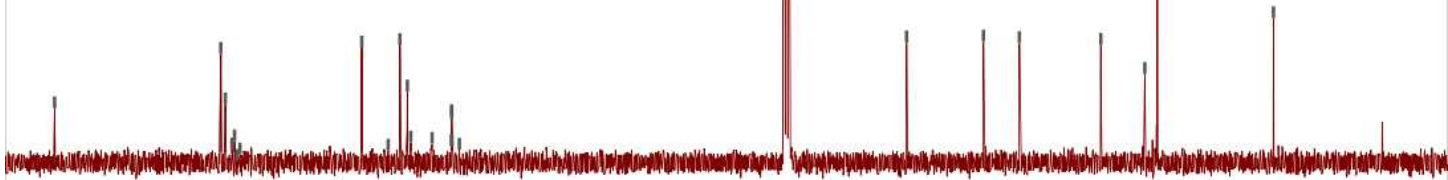

$\begin{array}{lllllllll}170 & 160 & 160 & 140 & 130 & 120 & 110 & 100 & 90 \\ & & & & & & & 80\end{array}$ 
S72; ${ }^{1} \mathrm{H}-\mathrm{NMR}\left(400 \mathrm{MHz}, \mathrm{CDCl}_{3}\right)$

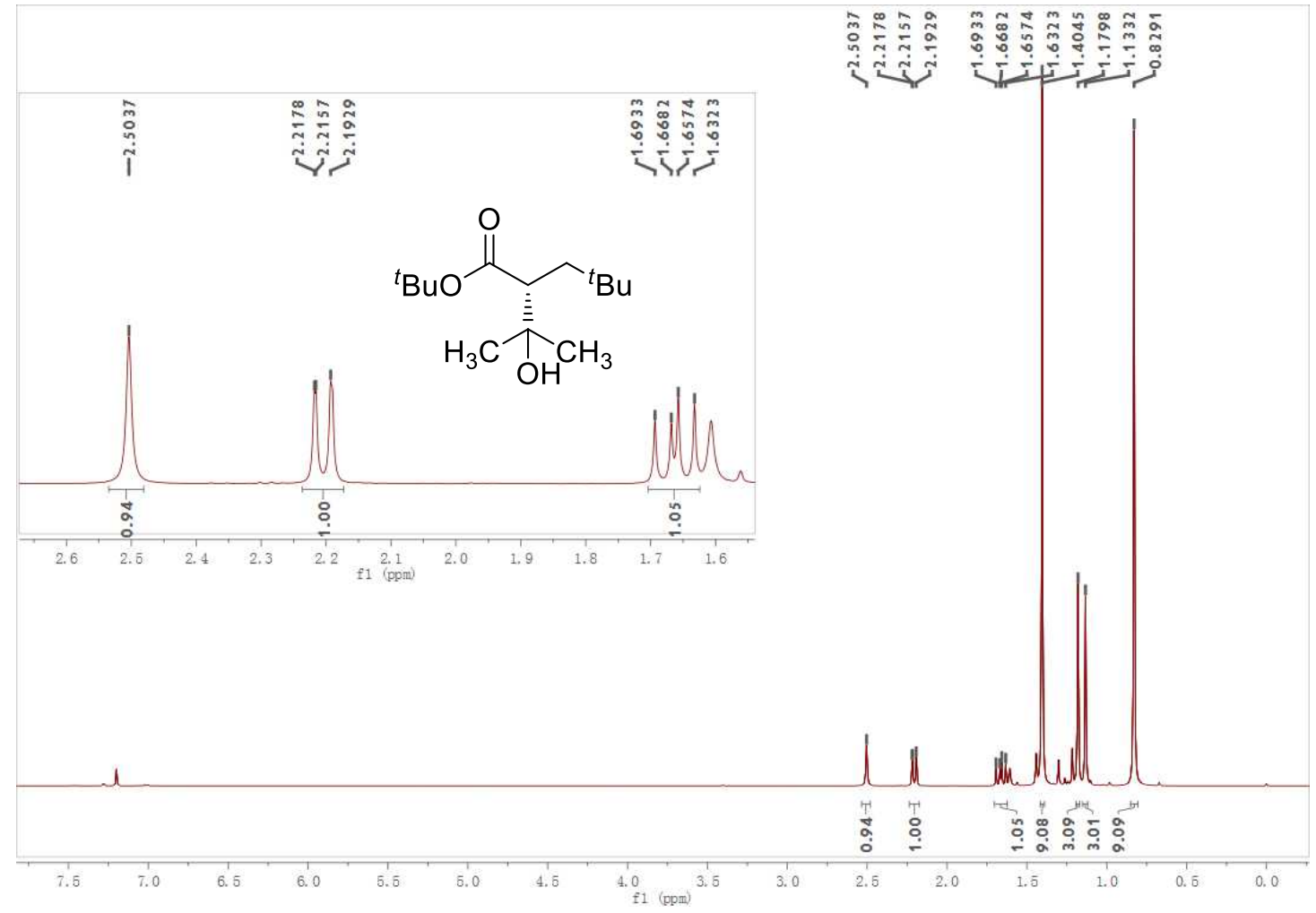

S72; ${ }^{13}$ C-NMR (100 MHz, $\left.\mathrm{CDCl}_{3}\right)$

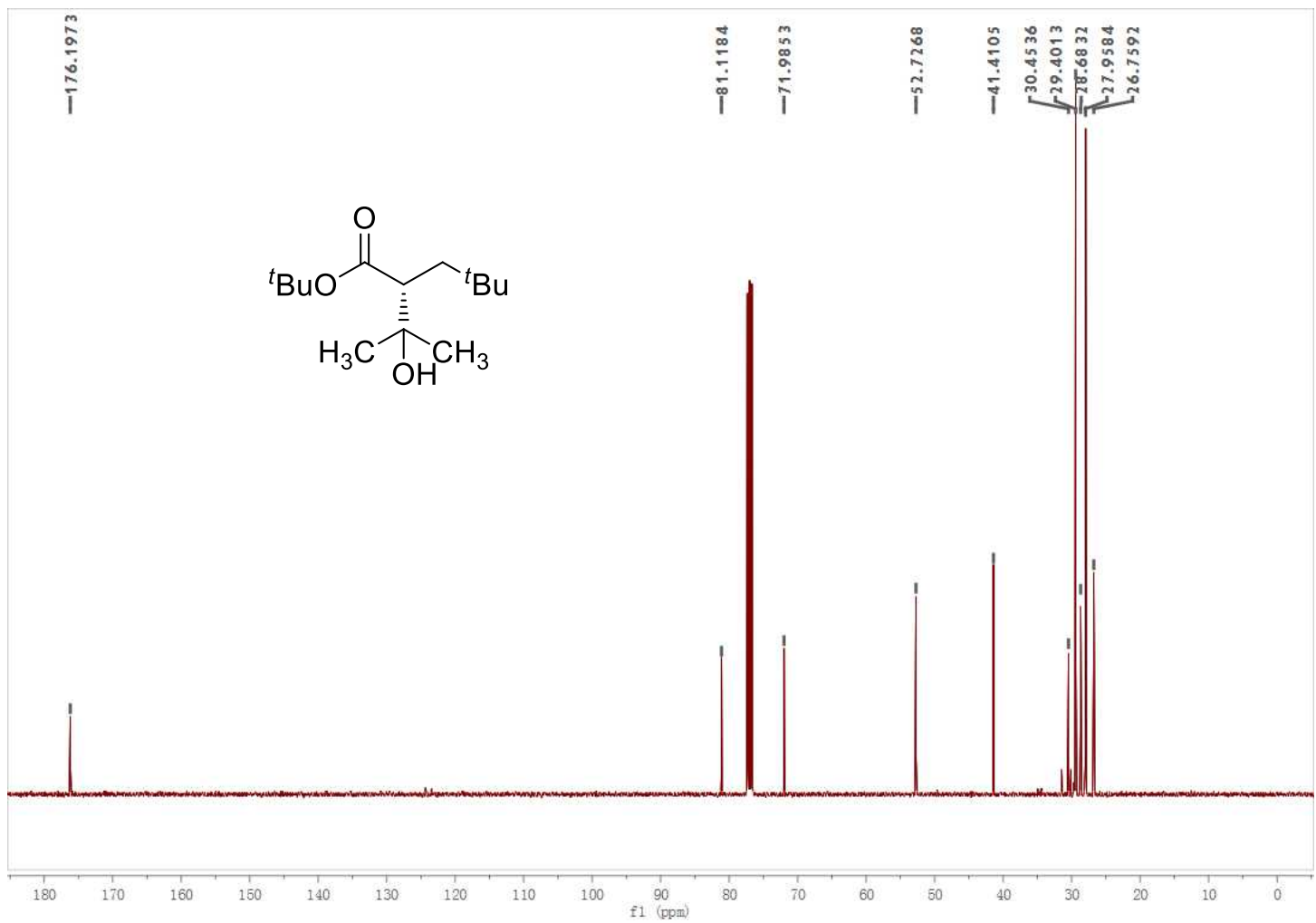




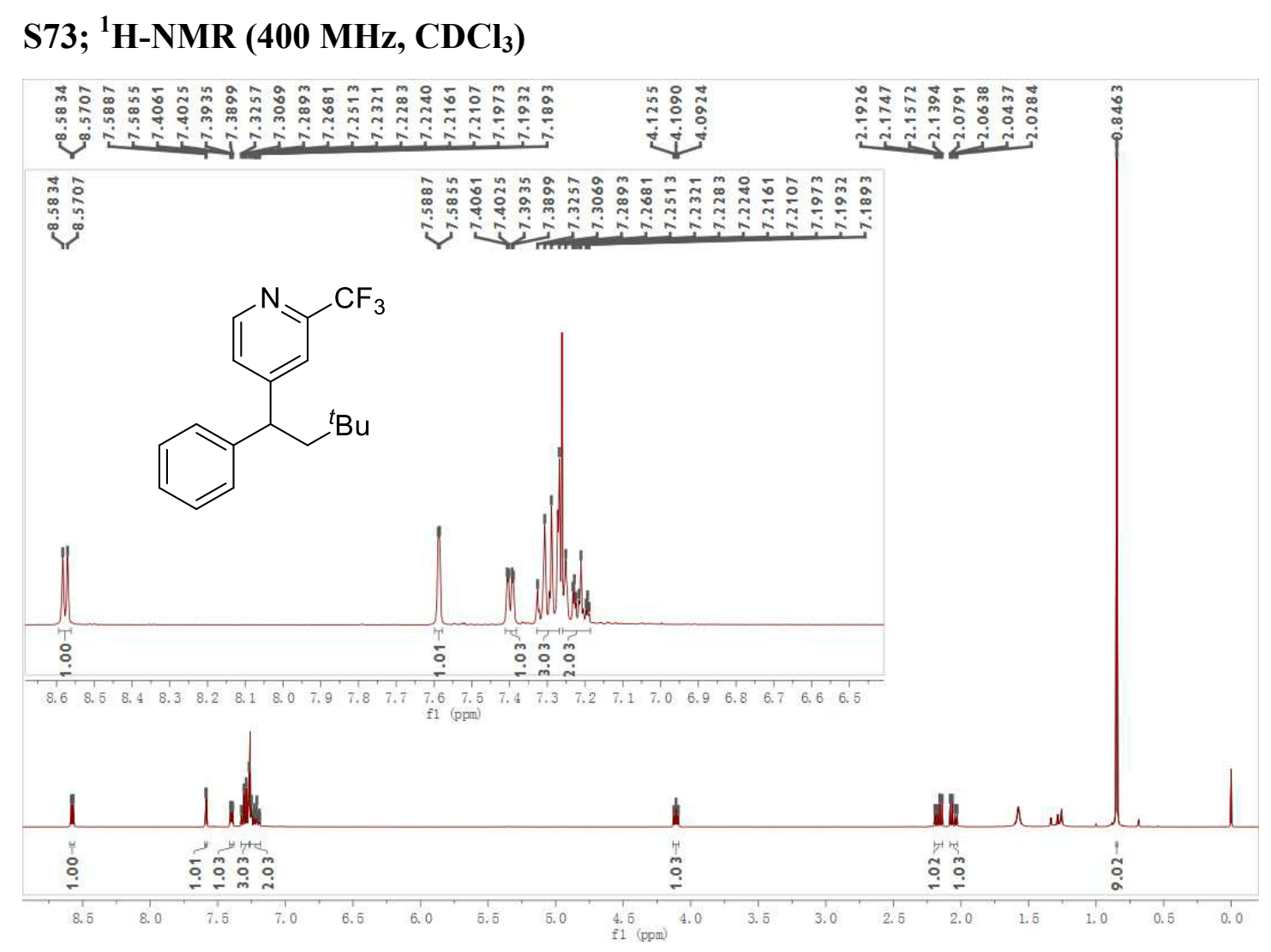

S73; ${ }^{19}$ F-NMR (375 MHz, $\left.\mathrm{CDCl}_{3}\right)$<smiles>CC(C)(C)CC(c1ccccc1)c1ccnc(C(F)(F)F)c1</smiles>

$\$ 421$ 
S73; ${ }^{13}$ C-NMR (100 MHz, $\left.\mathrm{CDCl}_{3}\right)$

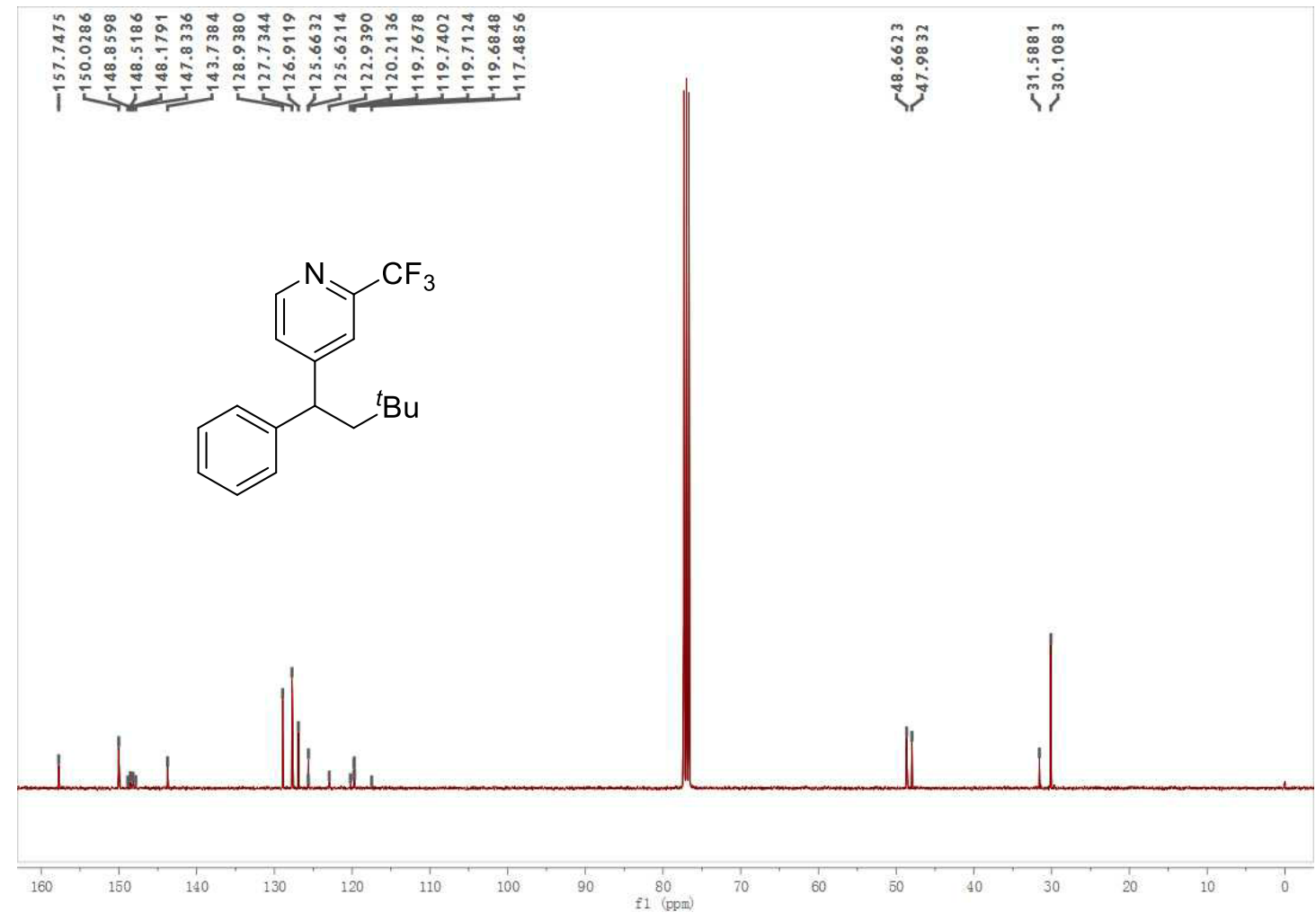

S74; ${ }^{1}$ H-NMR (400 MHz, $\mathrm{CDCl}_{3}$ )

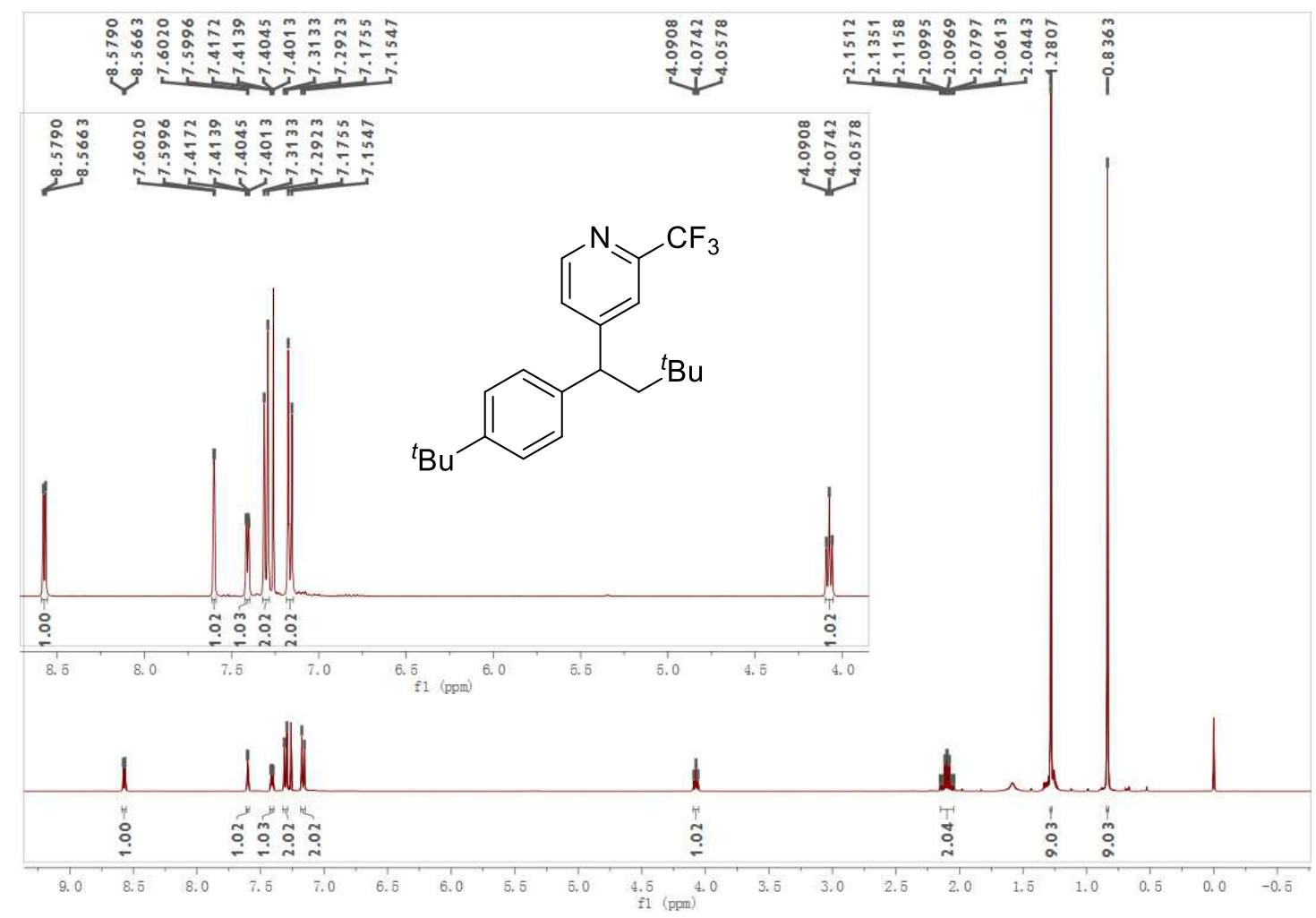


S74; ${ }^{19}$ F-NMR (375 MHz, $\mathrm{CDCl}_{3}$ )

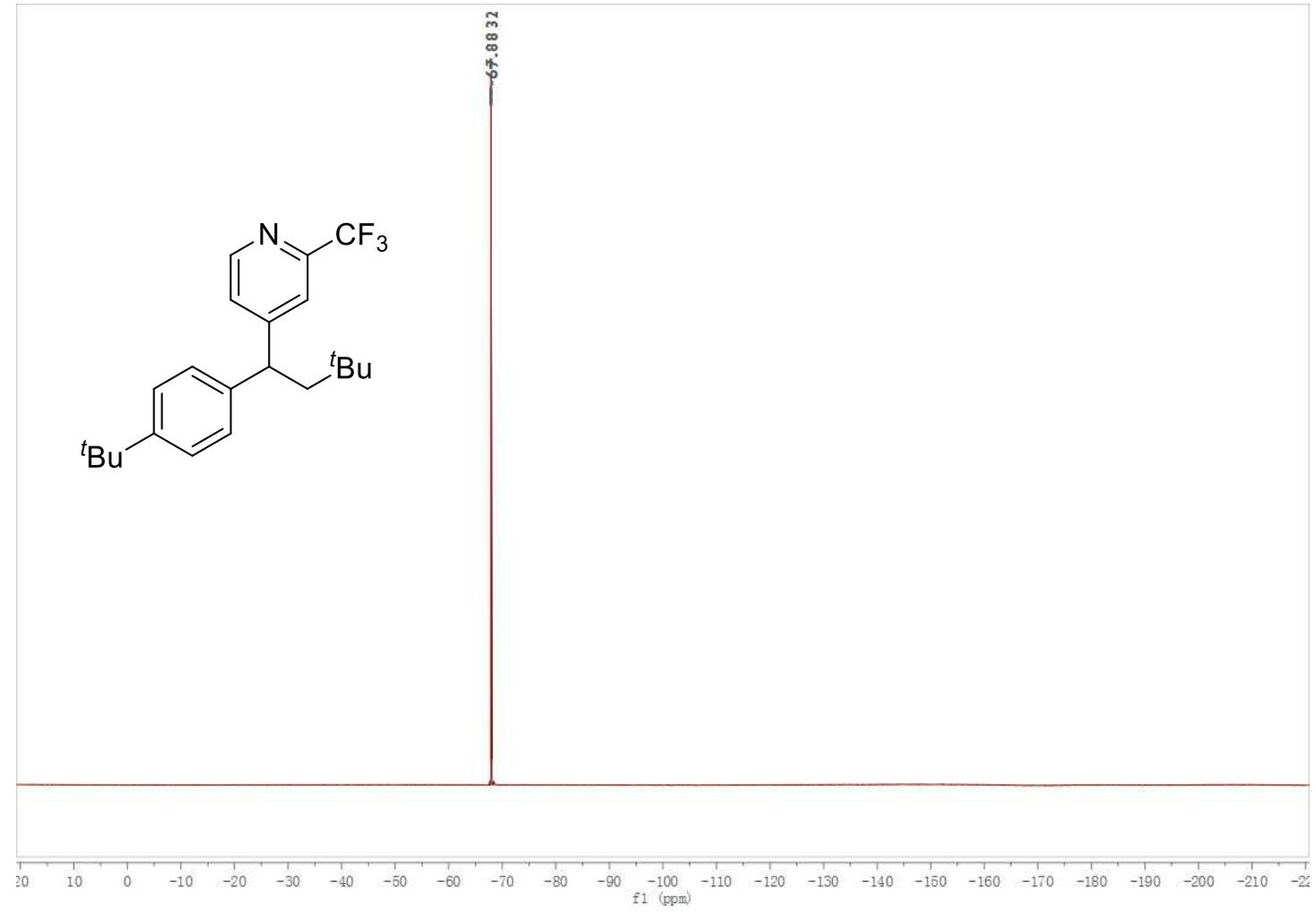

S74; ${ }^{13}$ C-NMR (100 MHz, $\left.\mathrm{CDCl}_{3}\right)$
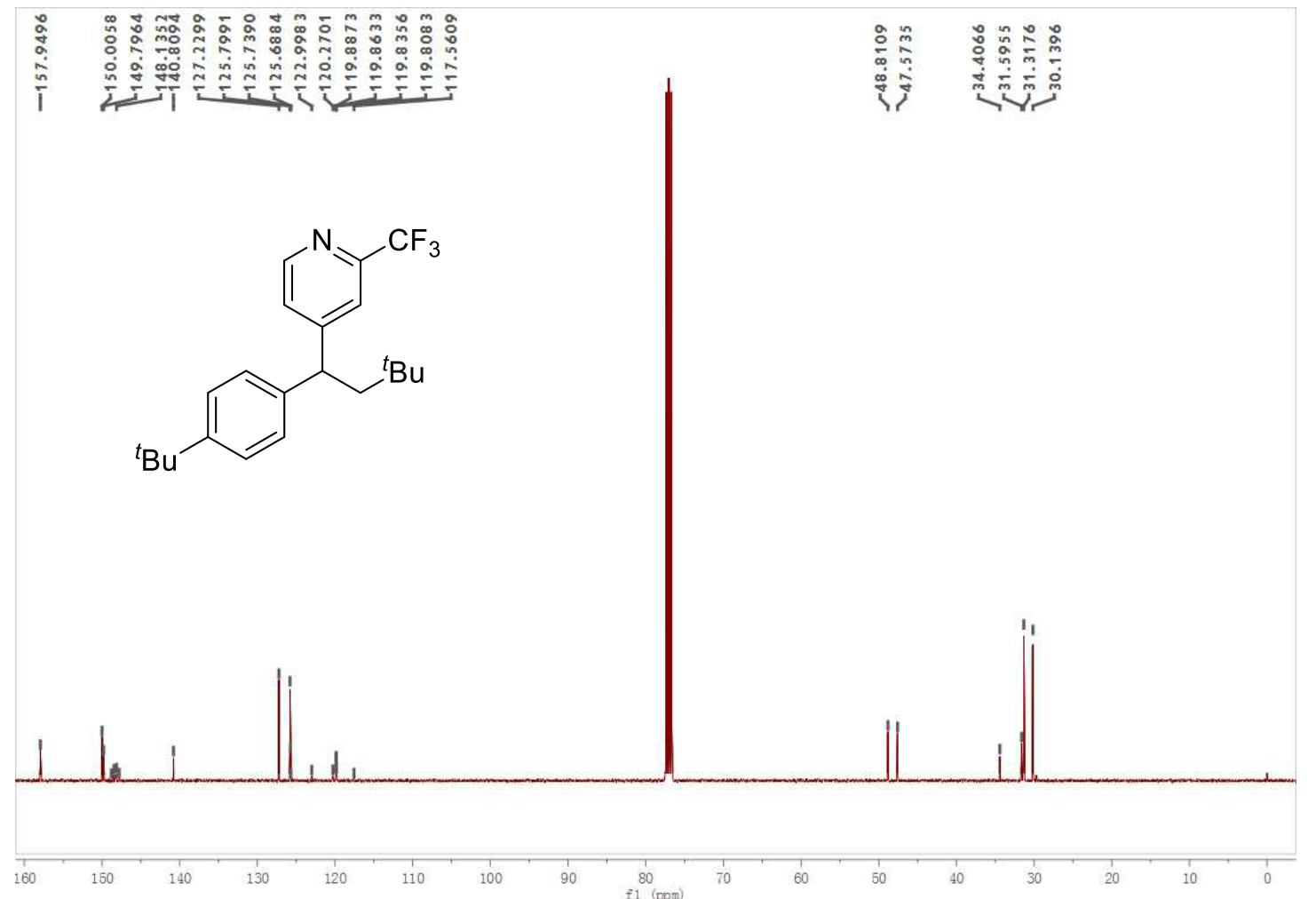
S75; ${ }^{1}$ H-NMR (400 MHz, $\mathrm{CDCl}_{3}$ )

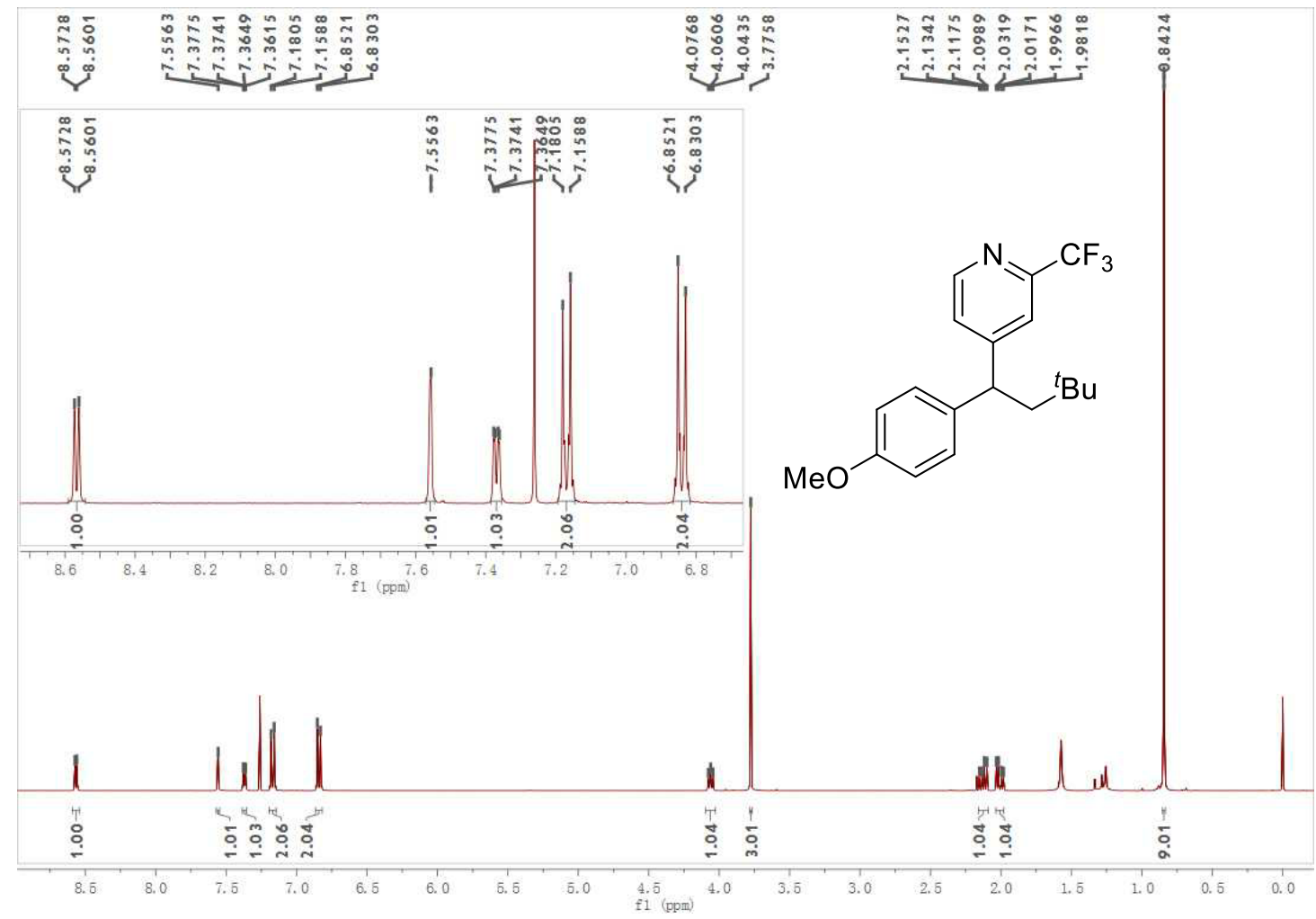

S75; ${ }^{19}$ F-NMR (375 MHz, $\left.\mathrm{CDCl}_{3}\right)$

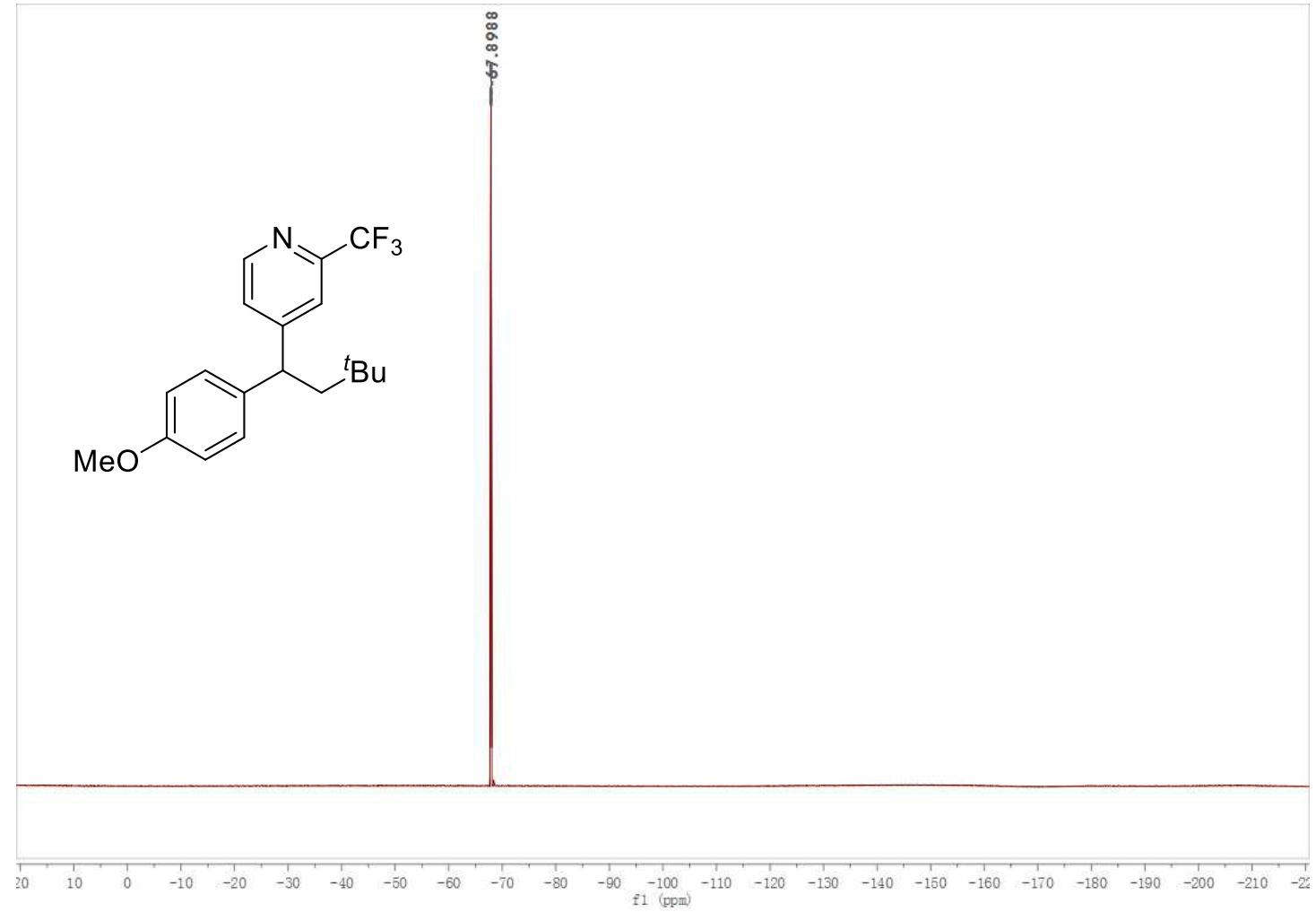


S75; ${ }^{13}$ C-NMR (100 MHz, $\left.\mathrm{CDCl}_{3}\right)$

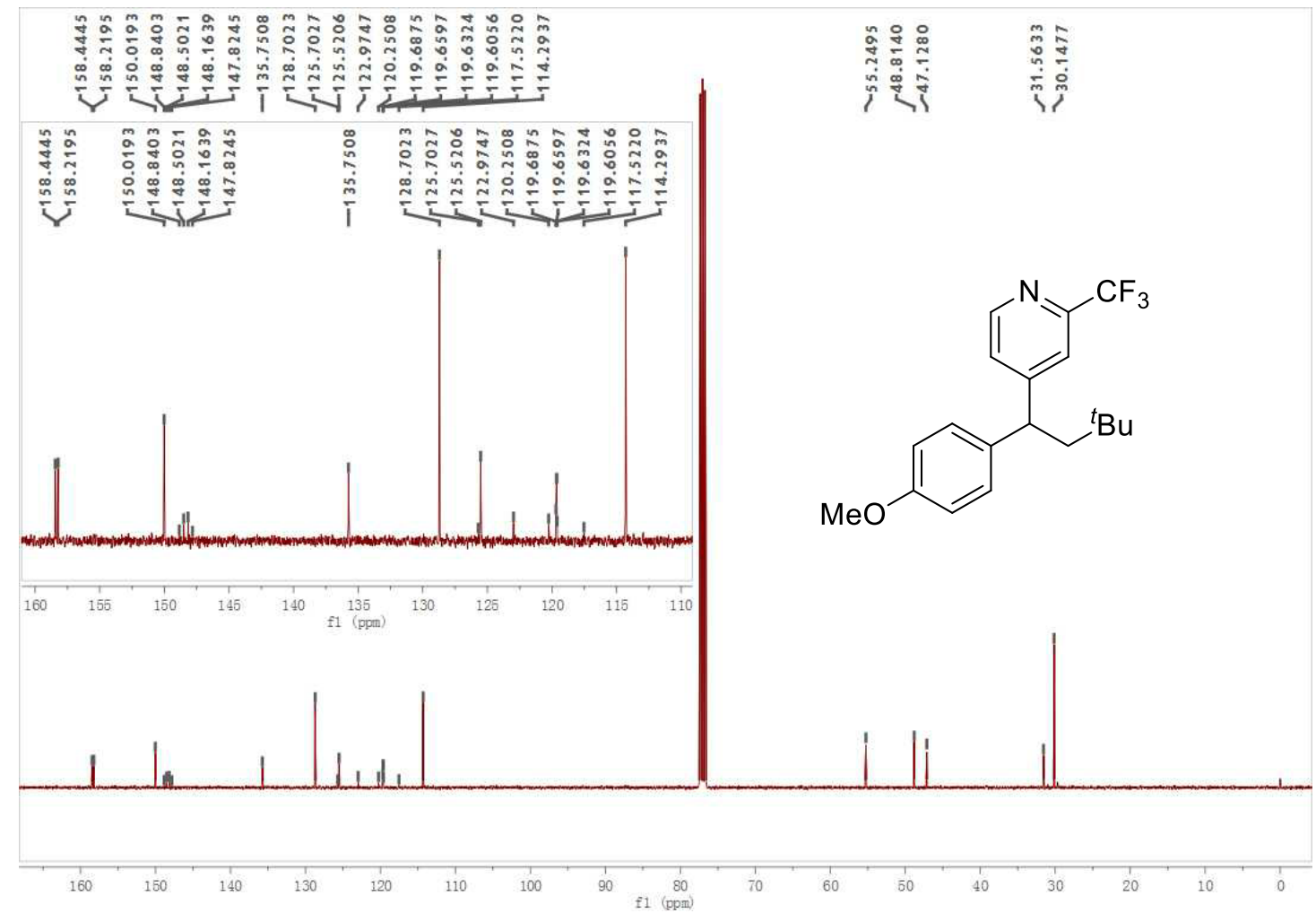

S76; ${ }^{1}$ H-NMR (400 MHz, $\mathrm{CDCl}_{3}$ )

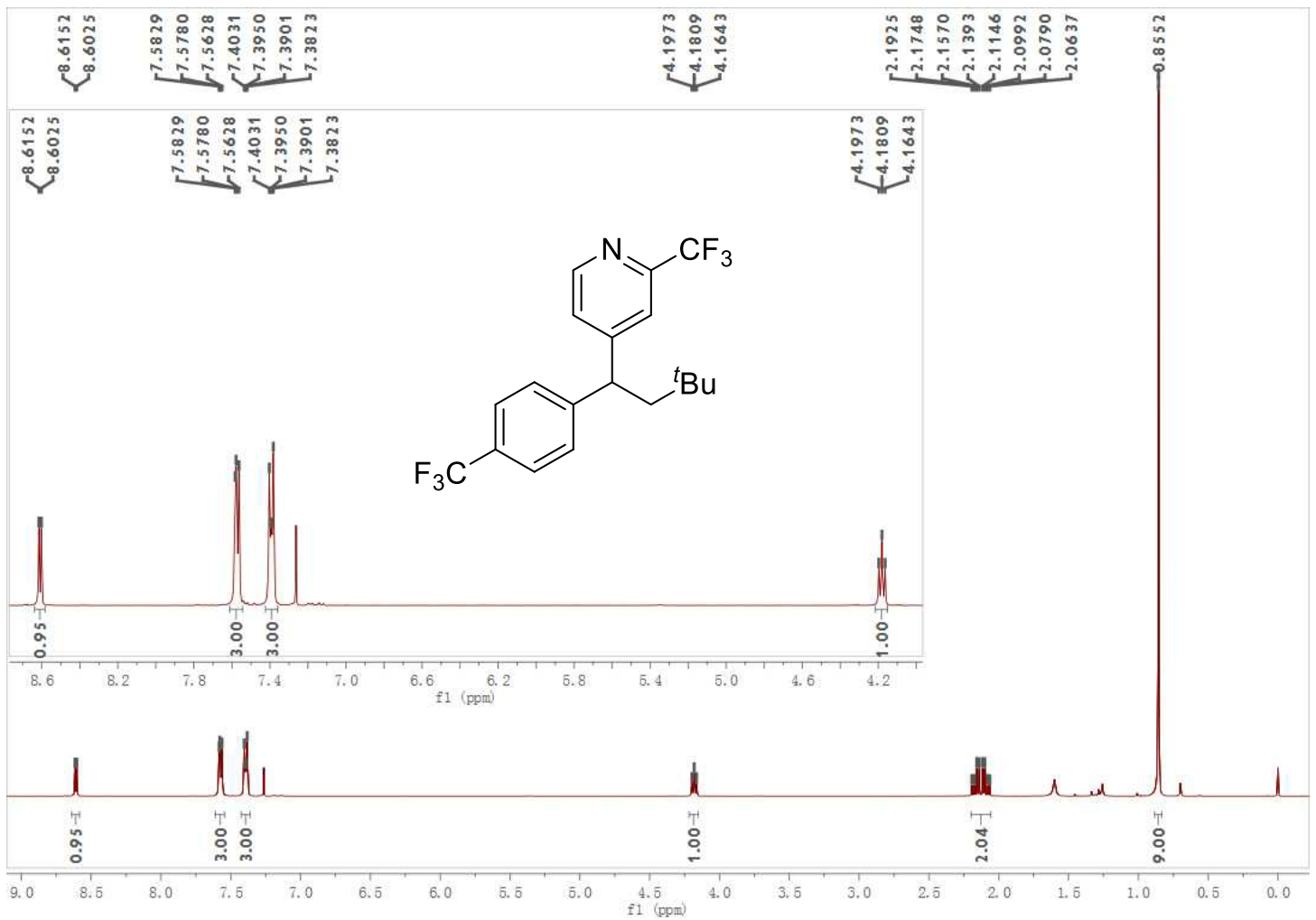


S76; ${ }^{19}$ F-NMR (375 MHz, $\mathrm{CDCl}_{3}$ )

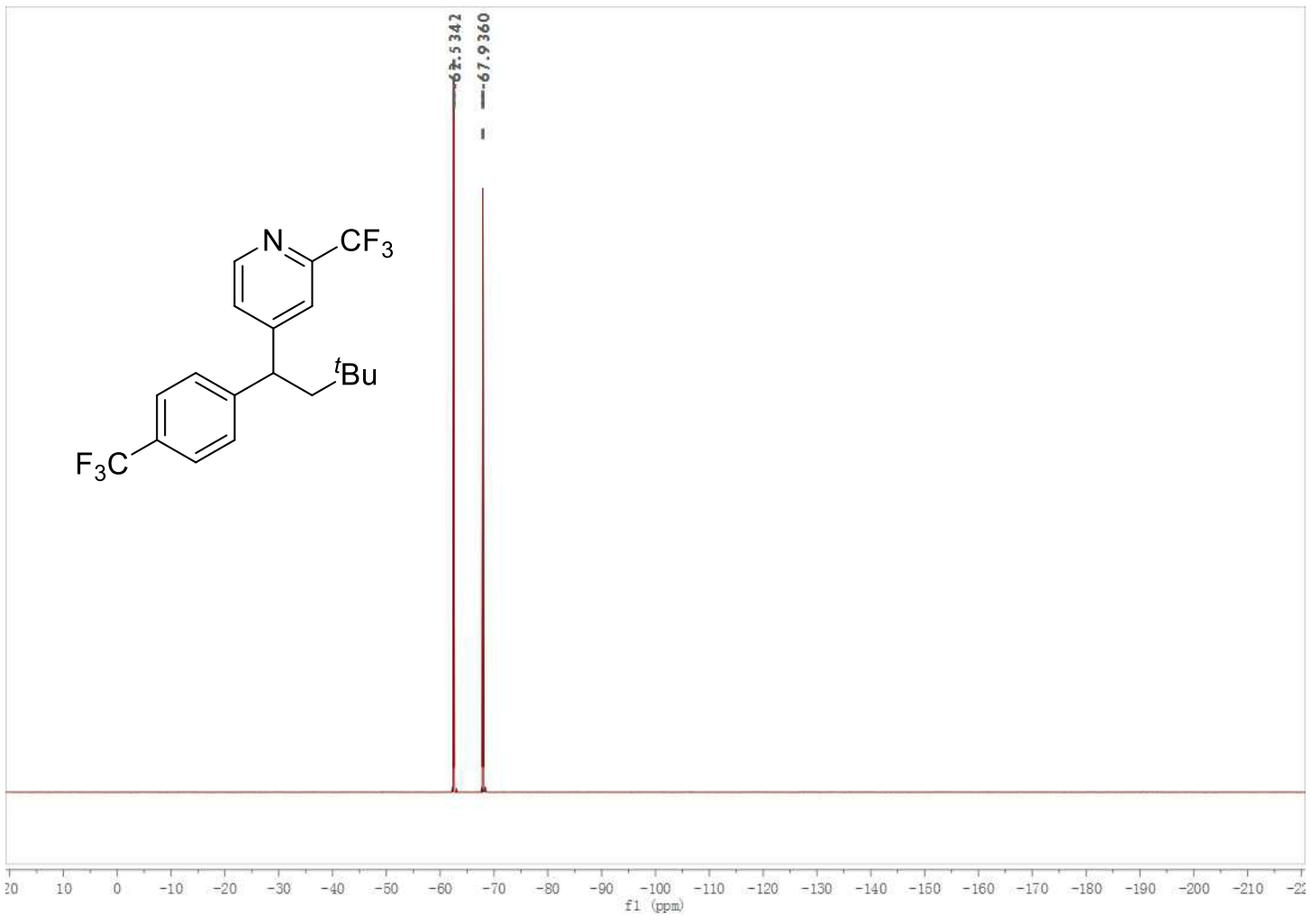

S76; ${ }^{13} \mathrm{C}-\mathrm{NMR}\left(100 \mathrm{MHz}, \mathrm{CDCl}_{3}\right)$

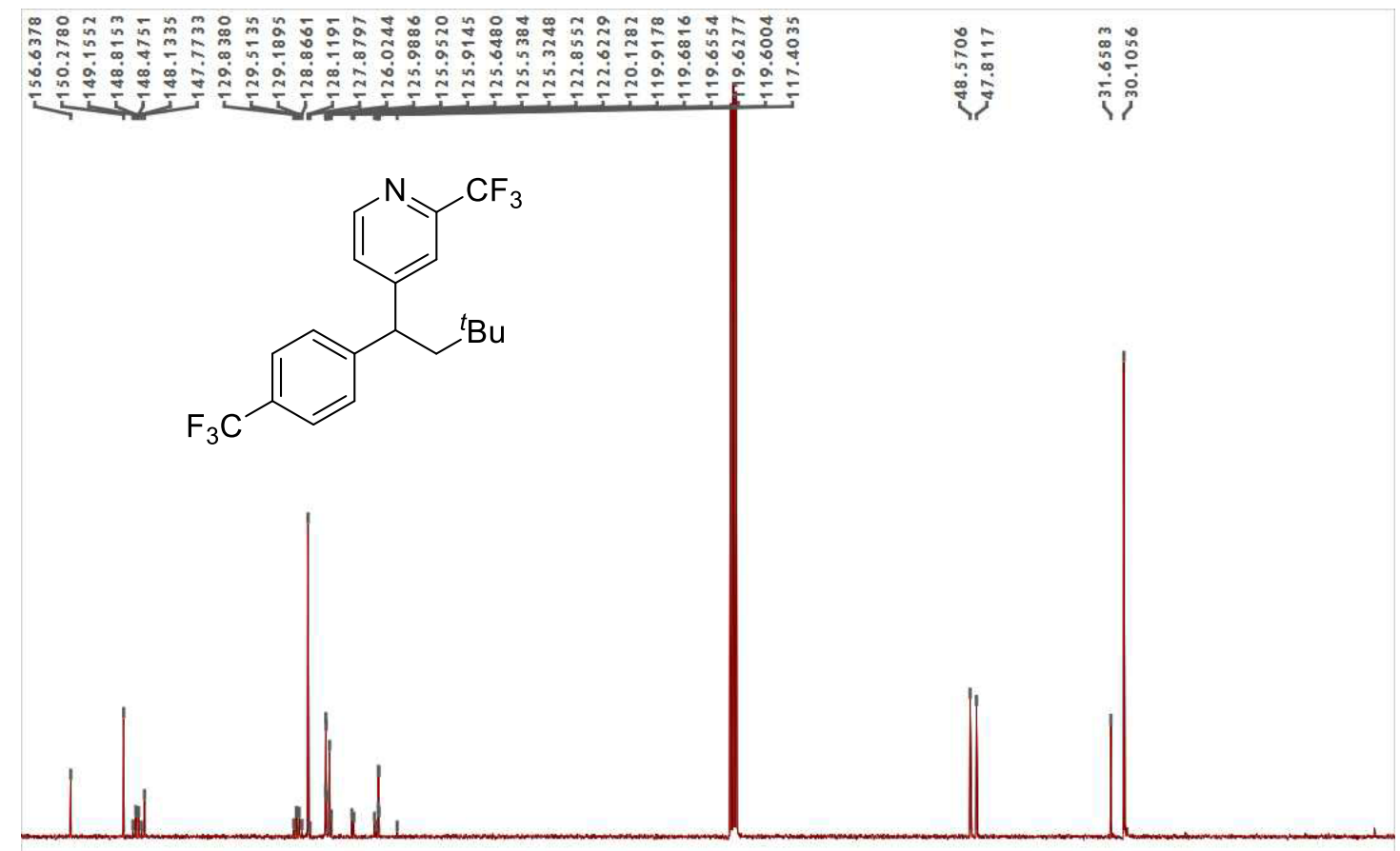

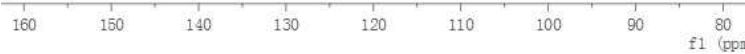

